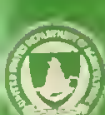

United Stales

Departinent of

Agriculture

Soil

Conservation

Service

SCS-TP-159

\section{National List of Scientific Plant Names}

Volume 2 Synonymy 


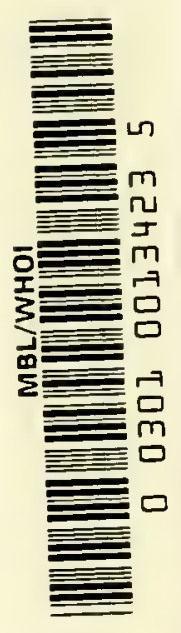




\section{Contents}

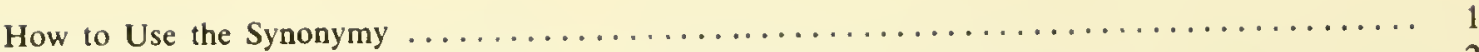

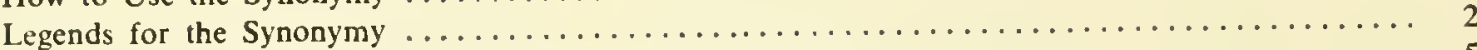

Synonymy for the National List of Scientific Plant Names $\ldots \ldots \ldots \ldots \ldots \ldots \ldots \ldots \ldots \ldots$ 

The synonymy lists all genera that were included in source manuals or were accepted for the U.S. regions by the Smithsonian Institution. The synonomy does not include all accepted names, so do not use it unless you have first looked in the list for the region you are concerned with.

If you have checked the list covering the appropriate region and have not found the name you were seeking, look it up alphabetically by genus in the synonymy. Within the genus, species are also listed alphabetically. When you look up the species, you will find one of the following cases:

"Accepted" will not appear to the far right, and one or more synonyms will be listed after the species. Symbols between the name you looked up and its synonym will explain the relationship between the two names. If the synonym is part of the same genus, you may use the family name and number to look up the synonym (using the instructions to volume I).

If the synonym is in a different genus, you will need to look up that genus in the Guide to Family Numbers (volume 1, p. 7). For example, had you looked up Rhus radicans in volume I to see if it were the accepted name for poison ivy, you would not have found it. Then you would look up Rhus radicans in the synonymy and find Toxicodendron radicans listed as a synonym. Then you could look up Toxicodendron in the Guide to Family Numbers and proceed as described on page I of volume I.
If the name you are looking up is a subspecies or variety, a synonym may not be listed. If one is not listed, check to see if the species, listed above the subspecies or variety, has "Accepted" written in the right-hand column. If it does, the species is the accepted name. For example, Abronia angustifolia var. arizonica (p. 5) is not an accepted name and has no synonym listed. However, the species $A$. angustifolia is accepted, so that is the name you should use.

Also, if a synonym is listed for a species, varieties and subspecies under that species will take the synonym of the species unless a separate synonym is shown for them. For example, Acer grandentatum var. brachypterum (p. 7) has no synonym listed. Acer grandentatum is not accepted, but a synonym is listed. That synonym would also be used for A. grandentatum var. brachypterum. Acer grandentatum var. sinuosum, however, has a separate synonym listed, which is the correct synonym.

In rare instances, you may find one of two other possibilities. If the plant is not listed, the name was not included in our sources and the consultants did not consider it a valid name. If the species is listed but no synonym is given, then it was included in our sources, our consultants considered it invalid for a United States plant, but they could not determine the valid synonym. If you encounter either of these possibilities and the plant is important on your site, you probably should submit a herbarium specimen for identification by a local or national authority. 
Symbol (Garrison-Skovlin-Poulton System)

Basic five-letter symbols, consisting of the first five letters, are used for the generic name. If the name has fewer than five letters, "+" signs are added to make a five-letter symbol. For example, for fir trees, Abies, the symbol is ABIES; for wheatgrasses, Agropyron, the symbol is AGROP; for bluegrasses, Poa, the symbol is POA + +; and for maples, Acer, the symbol is ACER + .

Tiebreakers are added to the basic five-letter symbol if needed. For example, the symbol CHRYS is the first five letters of several genera-Chrysopsis, Chrysopogon. Chrysothamnus, and Chrysanthemum. The genus symbol for the first one is CHRYS and for the others CHRYS2, CHRYS3, and CHRYS4.

The symbol for a species is the first two letters of the genus and the first two of the species. For example, the symbol for Kentucky bluegrass, Poa pratensis, is POPR. All other plants having the same four-letter symbol have tiebreakers in numeric sequence, starting with 2. Examples: POPR2, POPR 3, and so forth.

For varieties or subspecies, the first letter of the variety in a trinomial or quadrinomial or of the subspecies in a trinonial is added to the basic four-letter plant name symbol.

Example: Pinus ponderosa variety arizonica has the fiveletter symbol PIPOA.

\section{Genus/Species}

The species is indented below the genus, and the infraspecies name below the species. If the infraspecies is a subspecies, the letter $S$ precedes it. If a variety is assigned to a subspecies, its position in the list reffects that assignment. The letter $\mathrm{X}$ precedes the name of a hybrid taxon. Thus, $X$ AGROELYMUS is a hybrid genus, and "X STELLOIDES" is a hybrid species under the genus QUERCUS. Under Eriophorum is the species angustifolium; under the species is the subspecies subarticum; and under subarticum is the variety coloratum. So the full name is the quadrinomial, Eriophorum angustifolium ssp. subarticum var. coloratum.

\section{Synonym}

The synonyms listed are almost always accepted names. Several symbols are sed to clarify further the relationship of plant names and their synonyms.

\#-Our consultants determined that the names had the same type (the specimen on which the original description and name of a taxon have been based).

(-The name above this symbol has a type that is included in the description of the following name. A name preceding this sign cannot be an accepted name.

)-The description for the name above the symbol includes the type of the name after the sign.

$=-$ The two names linked by this symbol have been used for the same plant by at least one source. If the name is used correctly and we know it, the \# sign will be used.

$>$-One or more sources have used the name above the sign to include plants covered by other sources' descriptions for the name following the sign.

$<-$ Plants covered by the description of the plant name above the sign have been included in the description of the name after the sign by one or more sources.

$\mathrm{N}$-Some sources or consultants believe the name above the sign has been misapplied to the name after it.

* - The two names have been used for the same plant, but our consultants could not determine if another sign could be substituted.

Occasionally, more than one synonym appears with one or more of the above symbols. The synonyms are accepted, but the plant specimen could be either of the accepted names.

\section{Author}

An asterisk following an author means that the Smithsonian Institution studied the original description fully enough to confirm that it is accurately cited and that it complies with the International Code of Botanical Nomenclature (1978, International Association for Plant Taxonomy, Utrecht, Netherlands) . 


\section{Source Manuals}

Letters, numbers, or symbols in this column correspond to the letters in parentheses in the following list of publications, which includes all major sources that our consultants used. Sources that are listed but do not have symbols in parentheses are mainly checklists. Numbers to the left of the sources give the region to which the source applies.

1-0 Little, E. L., Jr., 1979. Checklist of United States trees. U.S. Dept. Agr., Agr. Handb. 541.

1,3 (F) Fernald, M. L. 1970. Gray's manual of botany, 8th ed. Rev. R. C. Rollins. Van Nostrand Reinhold.

1,3 (B) Gleason, H. A. 1963. The new Britton and Brown illustrated flora of the northeastern United States and adjacent Canada. Hafner Publishing.

1.3 (G) Gleason, H. A., and Arthur Cronquist. 1963. Manual of vascular plants of northeastern United States and adjacent Canada. D. Van Nostrand. southeastern flora and Ferns of the southeastern states. Science Press Printing. Thorne, R. F. 1954: The vascular plants of southwestern Georgia. Am. Midl. Naturalist $52: 257$.

Sharp, A. J., et al. 1956-1960. A preliminary checklist of monocots in Tennessee and $A$ preliminary checklist of dicots in Tennessee. A. J. Sharp.

Radford, A. E., et al. 1968. Manual of the vascular flora of the Carolinas. Univ. North Carolina.

(L) Long, R. R., and Olga Lakela. 1971. A flora of tropical Florida. Univ. Miami. Allen, C. M., et al. 1975. A vascular flora of St. Helena and West Feliciana Parishes. Univ. Southwestern Louisiana. Balogh, Pamela. 1976. A vegetational survey of Barksdale Air Force Base. U.S. Dept. Air Force 2d Civil Engr. Sqdn. Lakela, Olga, et al. 1976. Plants of the Tampa Bay area, 3 d ed. Banyan Books. Smith, E. B. 1978. An atlas and annotated list of the vascular plants of Arkansas. Edwin B. Smith.

(M) Steyermark, J. A. 1963. Flora of Missouri. lowa State Univ.
McGregor, R. L., et al. 1977. Atlas of the flora of the Great Plains. Iowa State Univ. Van Bruggen, Theodore. 1976. The vascular plants of South Dakota. Iowa State Univ. Dorn, Robert D. 1977. Flora of the Black Hills. Robert D. Dorn and Jane L. Dorn.

(Y) Dorn, R. D. 1977. Manual of the vascular plants of Wyoming. Garland Publishing. Barkley, T. M. 1968. A manual of the flowering plants of Kansas. Kansas State Univ. Endow. Assoc.

(R) Weber, W. A. 1976. Rocky Mountain flora, 5th ed. Colorado Assoc. Univ. Press.

Weber, W. A., and B. C. Johnston. 1976. Natural history inventory of Colorado, 1 . Univ. Colorado Mus.

(T) Correll, D. S., and M. C. Johnston. 1970, 1972. Manual of the vascular plants of Texas. Texas Res. Fdn. Supp.: Am. Midl. Naturalist 88:490. (6) Waterfall, U. T. 1972. Keys to the flora of Oklahoma, 5th ed. U. T. Waterfall.

(Z) Kearney, T. H., and R. H. Peebles. 1960. Arizona flora, 2d ed. Rev. J. T. Howell and Elizabeth McClintock. Univ. California. Martin, W. C., and E. F. Castetter. 1970. A checklist of the gymnosperms and angiosperms of New Mexico.

Dittmer, H. J., et al., 1954. The ferns and fern allies of New Mexico. Univ. New Mexico.

McDougall, W. B. 1973. Seed plants of northern Arizona. Mus. Northern Arizona.

Holmgren, A. H., and J. L. Reveal. 1966. Checklist of the vascular plants of the Intermountain Region. U.S. Dept. Agr. For. Serv. Res. Paper INT-32. DICOTS ONLY

\section{(t) Cronquist, Arthur, et al. 1972- . Vascular} plants of the Intermountain West, 1, 6. Hafner Publishing and Columbia Univ.

(U) Welsh, S. L., and Glen Moore. 1973. Utah plants, 3d ed. Brigham Young Univ.

Beatley, J. C. 1976. Vascular plants of the Nevada Test Site and central-southern Nevada. U.S. Energy Res. Dev. Adm.

\section{Hitchcock, C. L., and Arthur Cronquist. 1973.} Flora of the Pacific Northwest. Univ. Washington.

Peck, Morton Eaton. 1961. A manual of the higher plants of Oregon, 2d ed. Binfords and Mort. Munz, P. A., and D. D. Keck. 1959, 1968. A California flora. Univ. California. Supp.: P. A. Munz. California. Univ. California. 
(A) Hulten, Eric. 1968, 1973. Flora of Alaska and neighboring territories. Stanford Univ. Supp.: Botaniska Notiser 126, 459.

A

A

$\mathbf{H}$

$\mathbf{H}$

$\mathrm{H}$

C

C

C

(@) Liogier, Br. Alain. 1965-1967. Nomenclatural changes and additions to Britton and Wilson's "Flora of Porto Rico and the Virgin Islands" and Further changes and additions to the flora of Porto Rico and the Virgin Islands. Rhodora $67: 315 ; 69: 372$.

C

C

C

(V) Little, E. L., Jr., et al. 1974. Trees of Puerto

Rico and the Virgin Islands. U.S. Dept. Agr.

Hbk. 449.

C

C

C

C

Alaska trees and shrubs. U.S. Dept. Agr. For. Serv. Hbk. 410.

Alaska and adjacent parts of Canada. Brigham Young Univ.

Hawaiian pteridophyta. Bishop Mus. Bull. 25. Wagner, W. H., Jr. 1950. Ferns naturalized in Hawaii. Occas. Papers Bishop Mus. 20:95. the flowering plants in the Hawaiian Islands. Pacific Tropical Bot. Garden Mem. 1. Botany of Porto Rico and the Virgin Islands and Supp. New York Acad. Sci.

Hitchcock, A. S. 1936. Manual of the grasses of the West Indies. U.S. Dept. Agr. Misc. Little, E. L., Jr., and F. H. Wadsworth. 1964. Common trees of Puerto Rico and the Virgin Islands. U.S. Dept. Agr. Hbk. 249.

Woodbury, R. C., et al. 1971. The flora of Desecheo Island. J. Agr. Univ. Puerto Rico 55:478.

Fosberg, F. R. 1976. Revisions in the flora of St. Croix. Rhodora 78:79.

Woodbury, R. O., and E. L. Little, Jr. 1976. Flora of Buck Island National Monument. U.S. Dept. Agr. For. Serv. Res. Paper ITF-19. Woodbury, R. C. et al. 1977. The flora of Mona and Monito Islands. Univ. Puerto Rico Agr. Exp. Sta. Little, E. L., Jr., et al. 1976. Flora of Virgin Gorda. U.S. Dept. Agr. For. Serv. Res. Paper ITF-21.

\section{Habit}

The status-native (N) or introduced (I) - refers to the region or regions nominally covered by the particular part of the checklist: the United States and Canada, Hawaii, or the Caribbean region. If we do not know the status, an asterisk appears instead of $\mathbf{N}$ or $\mathbf{I}$. A blank space appears in the first position of the Habit column, in place of any of those three symbols, for a species of which only varieties other than the type variety have grown spontaneously in the United States. Combinations of codes such as AP, ST, or $\mathrm{ZE}$ indicate a variable growth cycle, habit, or special babitat.

The codes are as follows:

\begin{tabular}{|c|c|c|}
\hline $\mathrm{N}$ Native & - & Saprophytic \\
\hline Introduced & $\$$ & Succulent \\
\hline Annual & W & Woody \\
\hline Biennial & $\mathrm{H}$ & Partly Woody \\
\hline Perennial & G & Grasslike (Families \\
\hline Submersed & & $036,037,053)$ \\
\hline Emergent & V & Vine \\
\hline Floating & F or ; & Herbaceous (other \\
\hline $\begin{array}{l}\text { Epiphytic (Indicated } \\
\text { only if on trees) }\end{array}$ & & $\begin{array}{l}\text { than G or V) } \\
\text { Shrub }\end{array}$ \\
\hline Parasitic & $\mathrm{T}$ & Tree \\
\hline
\end{tabular}

\section{Family Name/No.}

Family names follow the system used by the United States National Herbarium, Smithsonian Institution, Washington, D.C. 


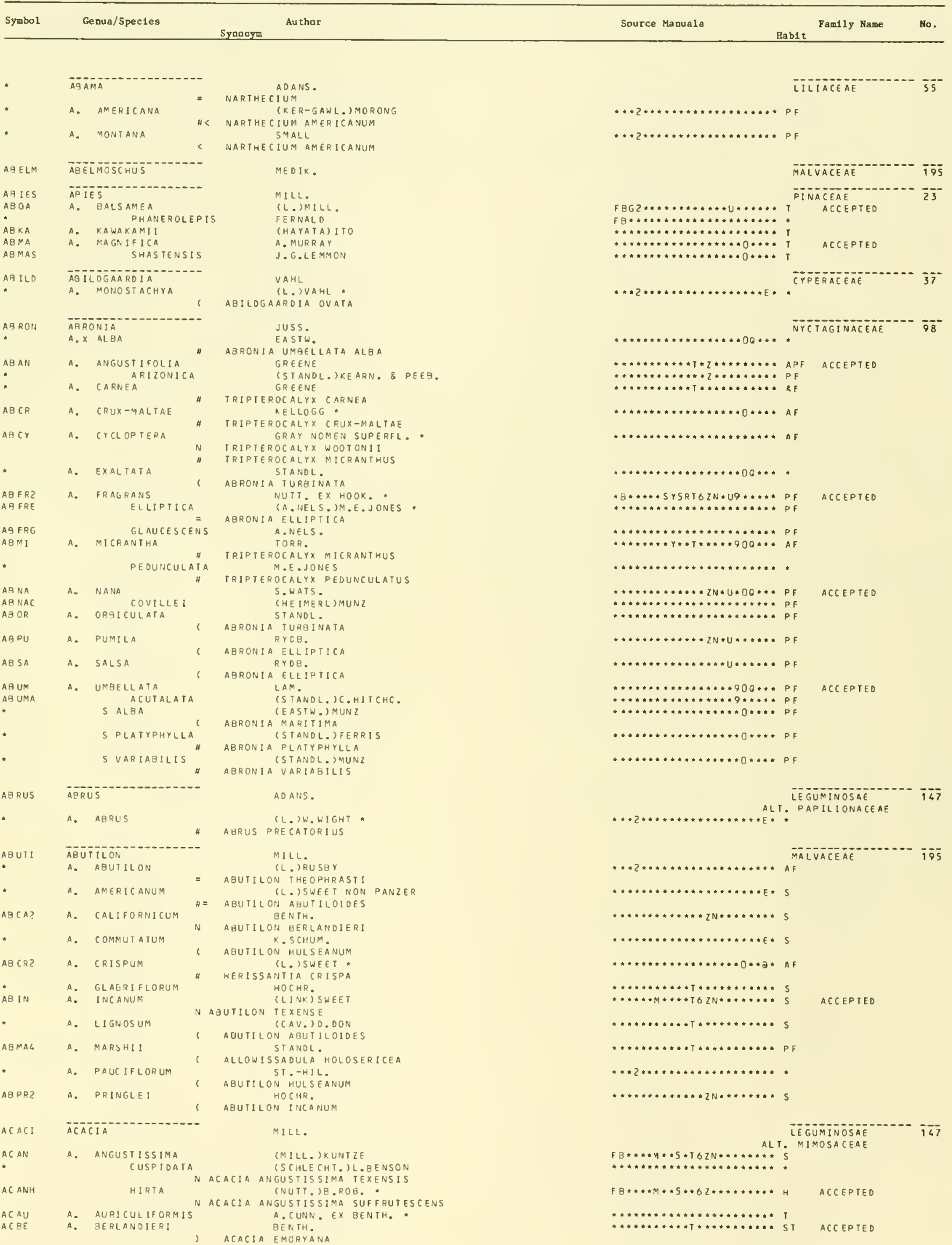




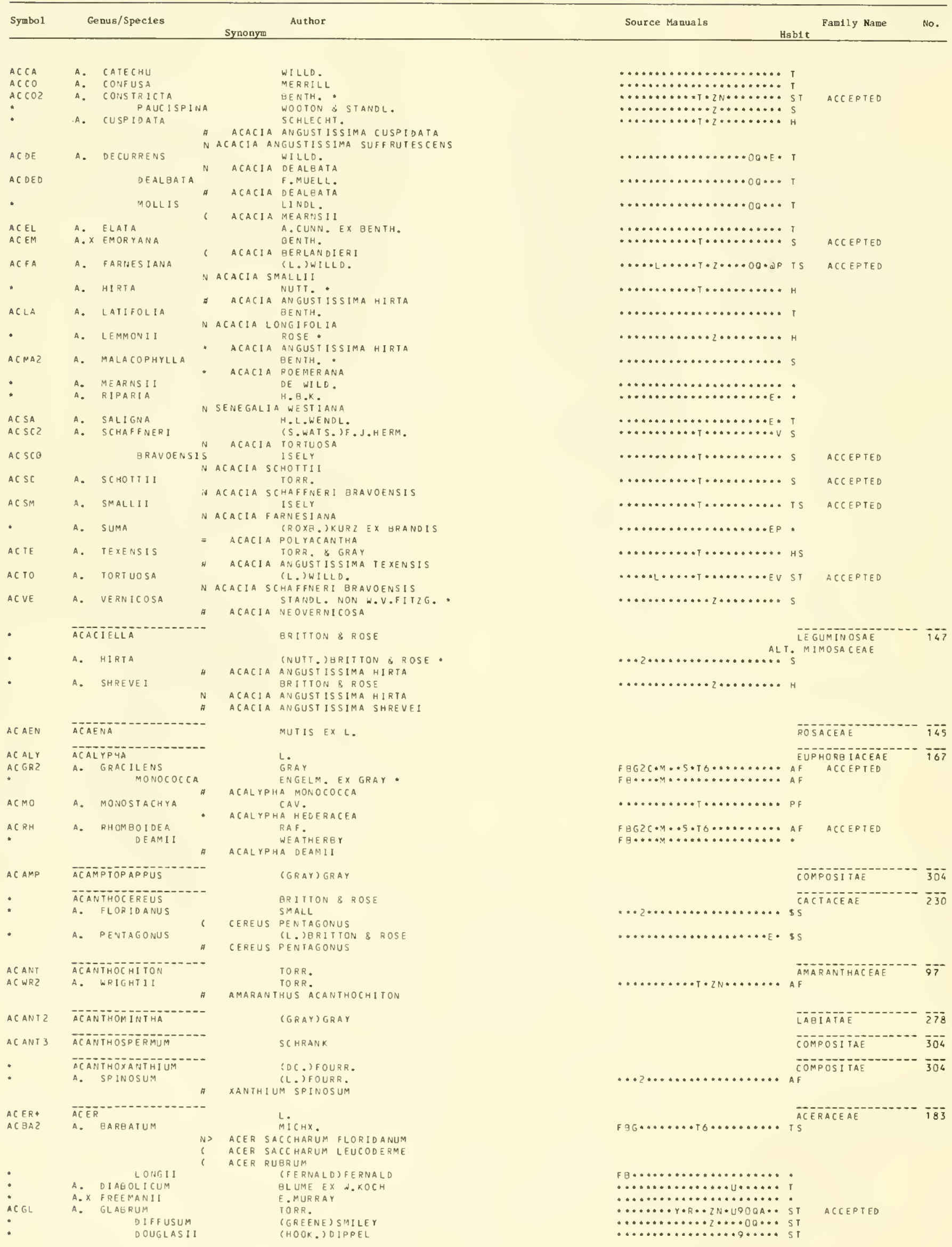




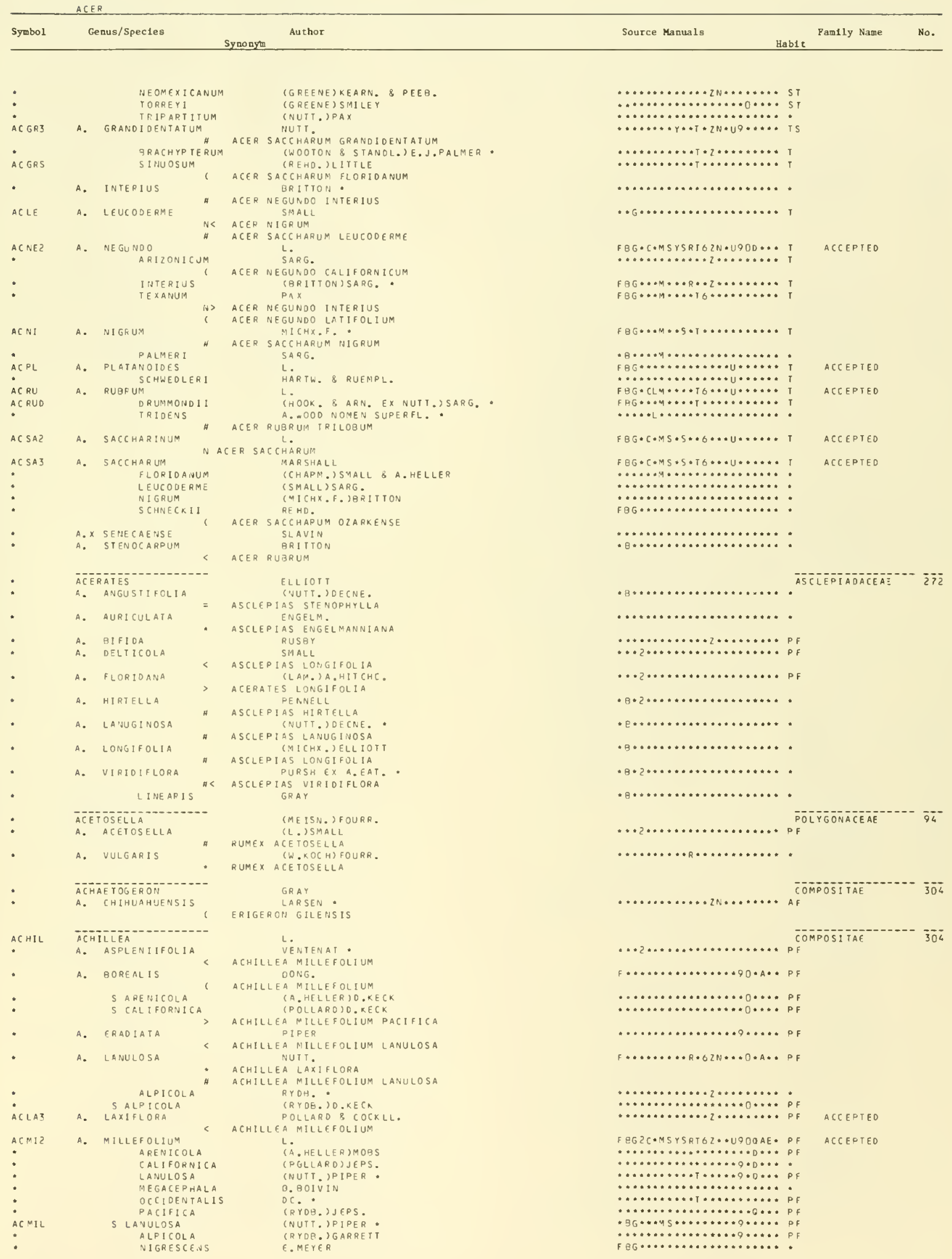


ACHILLEA

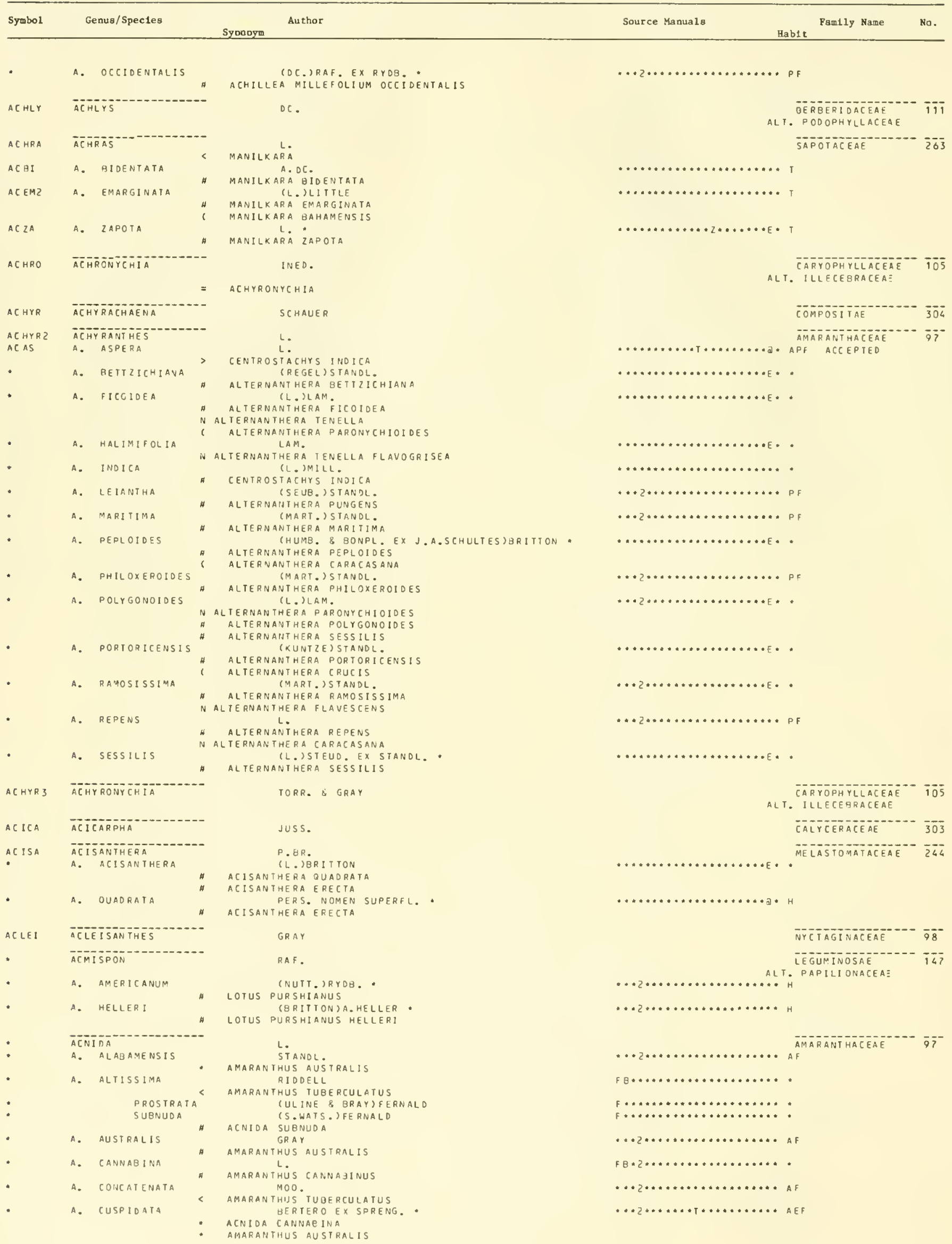




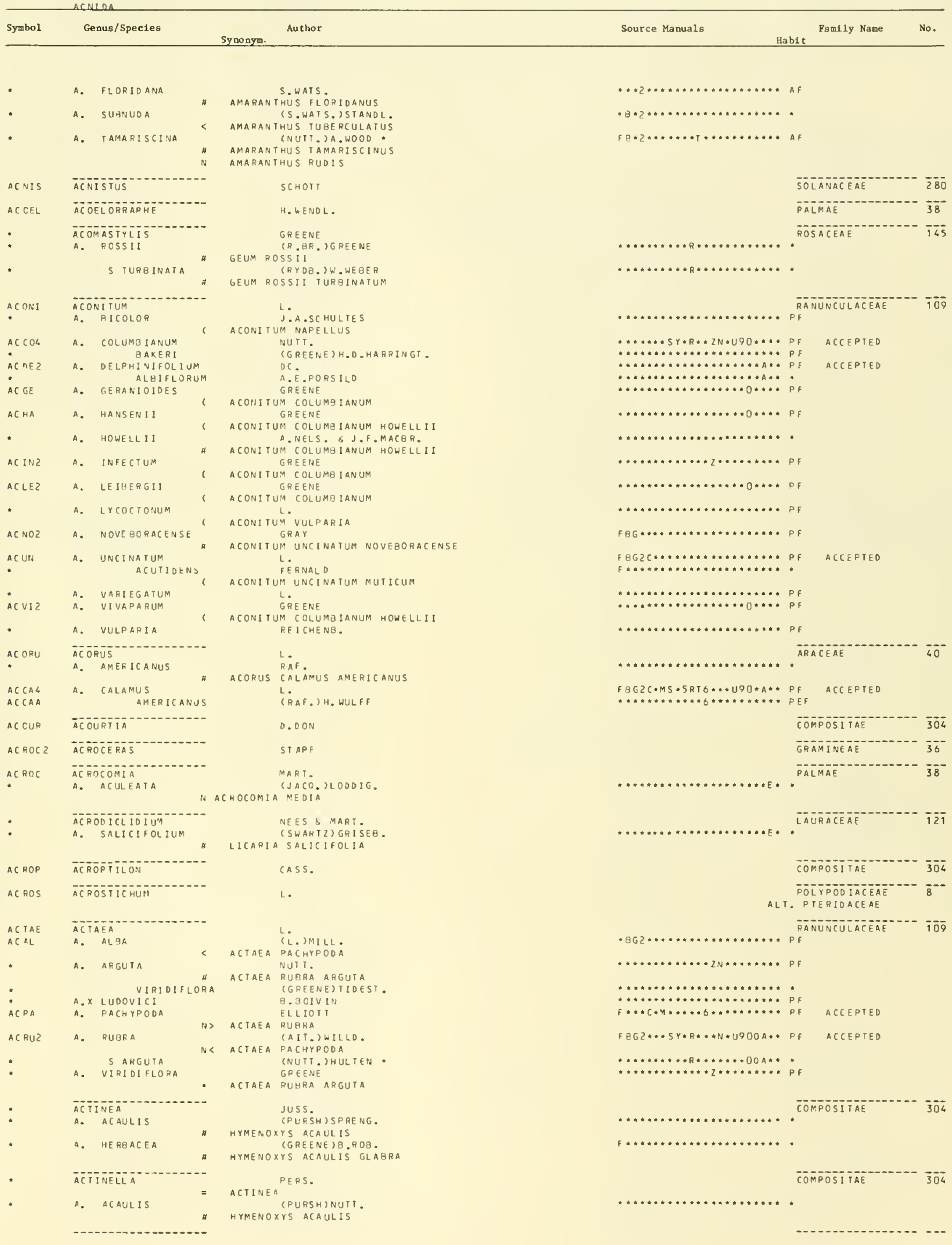


NUTT.

ACTINOMERIS A. ALTERNIFOLIA

CL. . OOC.

VERBESINA ALTERNIF OLIA

VERBES IN A WALTER I

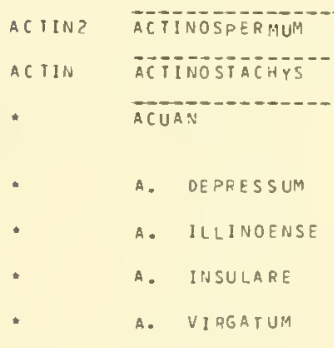

$\triangle D$ ANS

ADEL !

ADANSONIA

AOELIA

A. BERNARDIA

ADENANTHERA

A. PEREGR INA

AOENOCALYMNA

A. ALLIACEUM

ADENOCAULOOH

AOENOPHORUS

AOENOPLEA

A. LINDLEYANA

AOENOPOFCES

A. QUXIFOLIA

ADENOFIMA

A. PUNICEA

ADENOROPIUM

A. GOSSYPIfOLIUM

A. HASTATUM

A. MULTIFIDUM

a.

AOENOSTEMA

ADENOSTEMMA

A. LAVENI

ADENOSTOMA

ADHATODA

A. LITHOSPERMIFOLIA

AOTANTOPSIS

\section{ADIANTUM}

A. CAPILLUS-VEIVERIS

a. CUNEATUM

A. PEDATUM

SUBPUMILUM

ALEUTICUIM

AD I CEA

AOIY.F

A. PUMILA

ADIPERA

A. BICAPSULARIS

a. CORYagosa

MACQ. NEES

(L. TRAF.

\section{ELLIOTT \\ WALLICH \\ MEDIK.}

DE SMANTHUS

(HUMB. \& BONPL. EX WILLD.) KUNTZE.

DESMANTHUS VIRGATUS DEPRESSUS

(MICHX.) KUNTZE

DESMANTHUS ILLINOENSIS

TRITION \& ROSE

DESMANTHUS VIRGATUS

(L.) MEOIK.

DESMANTHUS VIRGATUS

L.

L. .

BERNAROIA DICHOTOMA

L.

(L.) SPEG.

PIPIADENI A PERE GRINA

MART. EX MEISN.

(LAM.)MIERS

PACHYPTERA ALLIACEA

HOOK.
GAUD.

RADLK.

(FORTUNE)SMALL

BUDDLEJA LINDLEYANA

SMALL

(CAV.) SMALL

TETRAPTERIS BUXIFOLIA

RAF.

(SWARTZ) RAF.

POHL

(1.) $\mathrm{POHL}$

JATROPHA GOSSYPIIIOLIA

(JACQ.) BRITION O P.WILSON

JATROPHA INIEGERRIMA HASTATA

(L.) POHL

IATROPHA MULTIFIDA

OESPORTES
ADENOSTEMMA

J. \& G.FORST.

(i. \& KUNIZE

ADENOSTEMMA VISCOSUM

HOOK. \& AFN.

MILL.

FEE

L.

L.

(UNDERW.) FERNALD

LANGSD. FISCH.

SOIANTUM FAOOLIANUM

L.

W.H.WAGNER

(RUPF.) CALOER B R.L.TAYLOR

A OIKE

RAF. EX ERITION \& A.BR. NOMEN SUPERFL. *

RAF. -

PILEA PUMILA

RAF.

(L.)BRITTON \& ROSE

CASSIA UICAPSULARIS

(LAM.)BRITTON \& ROSE.

CASSIA COAYMBOSA
COMPOSITAE

304

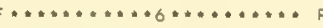

COMPOSITAE

SCHIZAEACEAE

LEGUMINOSAE

ALT. MIMOSA CEAE

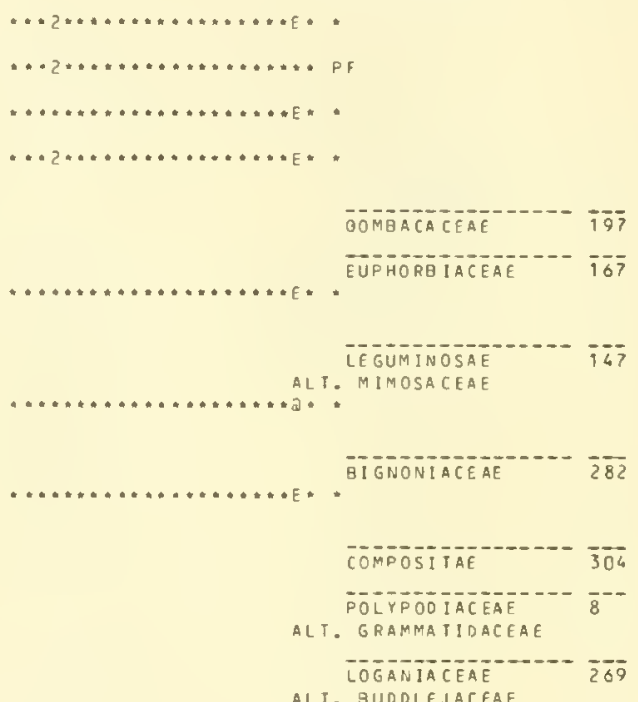

ALP. BUDDLEJACEAE

$5 \overline{9}$

SH

AALPIGHIACEAE

EUPHORB IACEAE

EUPWORATACEAE

COMPOSITAE

COMPOSITAE

ROSACEAE

ACANTHACEAE

POLYPOOIACEAE

ALI. SINOPTERIDACEAE

POLYPODTACEAE ALI ADIANTACEAE

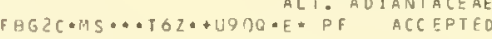

$\ldots \ldots \ldots \ldots \ldots \ldots+\ldots \ldots \ldots$

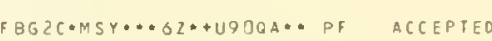

$\ldots \ldots \ldots \ldots \ldots \ldots \ldots \ldots \ldots \ldots$

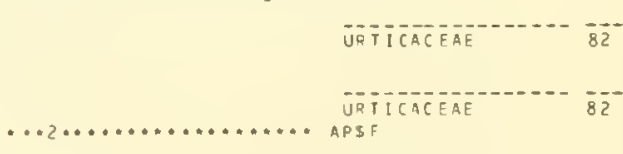

LEGUMINOSAE ALT. CAESALPIN]ACEAE 


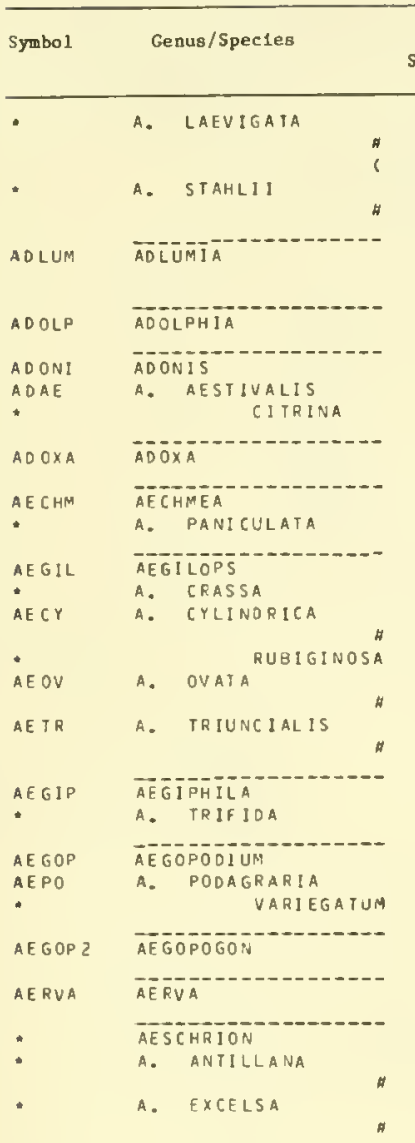

AESCH

AE AM

AEEV

AE IN

AEVI?

AEVI3

AESCU

AE AR

*

AF G

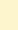

AE O

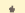

AETHU AETHUSA

* AFZELIA

a. CASSIOIDES

A. PECTINATA

$$
\text { PENINSULARIS }
$$

AGALINIS

A. CADDOENSIS

- a. erecta agalinis SiRICIIfolia
(walter)penNel

Syoooya.

(WILLD.)BRITION \& ROSE

CASSIA LAEVIGATA

CASSIA FLORIBUNDA

TRBANIBRIYTON \& ROSE

STAHLII

RAF. EX DC.

ME ISN.

L.

HOF FM.

L.

RU1 2 \& PAVON

RU12 \& PAVON

L.

Boiss.

HOST

TRITI CUM CYLINDRICUM

POPOVA

TRITICUM OVATUM

TRITIGUM IRUNCIALE

JACQ.
SWARTL

L.

L.H.BAILEY

HUMB. \& BONPL. EX WILLO.

FORSSK.

VELL.

(E GGERS) SMALL

PICRASMA ANTI LLANA

(SWARTZ) KUNTZE

PICRASIMA EXCELSA

L.

L.

AESCHYNOMENE VILLOSA

[. WRIGHT

AESCHYNOMENE INDICA

L.

AESCHYNOMENE EVENIA

AESCHYNOMENE VIRGINICA

UROAN

AESCHYNOMENE GRACILIS

POIR.

AESCHYNOMENE AMERI CANA

(L.) B.S.P.

AESCHYNOMENE INOICA

L.

BUCKLEY

SARG.

AESCULUS PAVIA

AESCULUS SYLVATICA

WI LLD.

SARG.

RE HD.

AESCULUS GLABRA ARGUTA gOOIH EX DIPPEL (SPACH) SCHELLE LINDL.

AESCULUS SYLVATICA MARSHALL

AESCULUS SYLVATICA

AESCULUS FLAVA

KOEHNE

L.

J.F.GMEL.

SEYMERIA

(WALTER) J.F.GMEL.

SEYMERIA CASSIOIDES

(PURSH) XUVTZE

SEYMERIA PECTINATA

ENNEL

RAF.

PENNELL

AGALINIS STRICIIFOLIA

- a. erecta agalinis STRICIIfOLIa
(WALTER)PENNELL

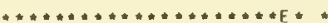

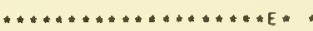

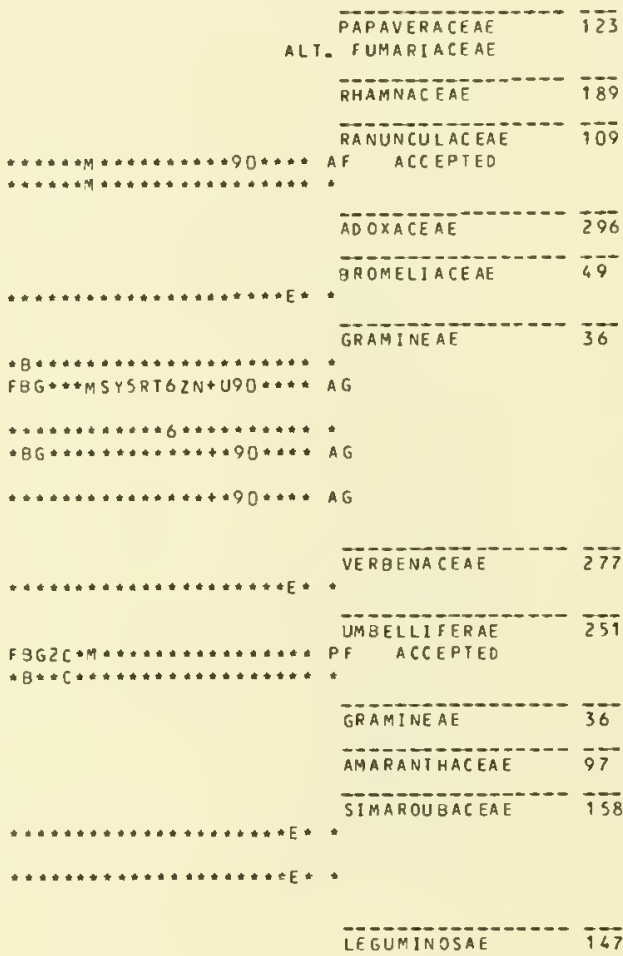

ALI. PAPILIONACEA

i PF ALCEPTED

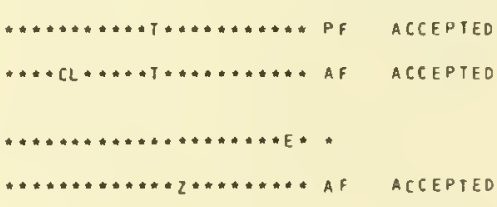

FBG $2 C \ldots \ldots \ldots \ldots \ldots \ldots \ldots * * *$ ACCEPTEO

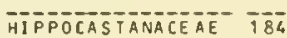

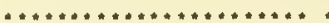

$F B G \ldots+\cdots \ldots \ldots \ldots \ldots \ldots \ldots$

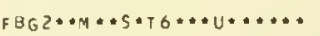

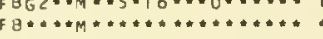
F $8 * \cdots * * * * 5 * \cdots * * * * * * * *$

ACCEPTED

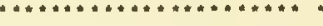

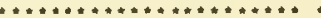

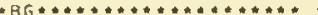

f $8 G 2 C \cdot M * \ldots \ldots \ldots * U * \ldots \ldots$

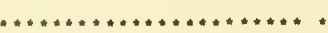

UMBELLIFERAE SCROPHULARIACEAE

$\ldots 2 \ldots \ldots \ldots \ldots \ldots+\ldots \ldots$ A

...2,................ A

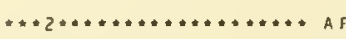

SETOPHULARIACEAE

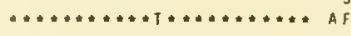

< agalints ogtusifolia 


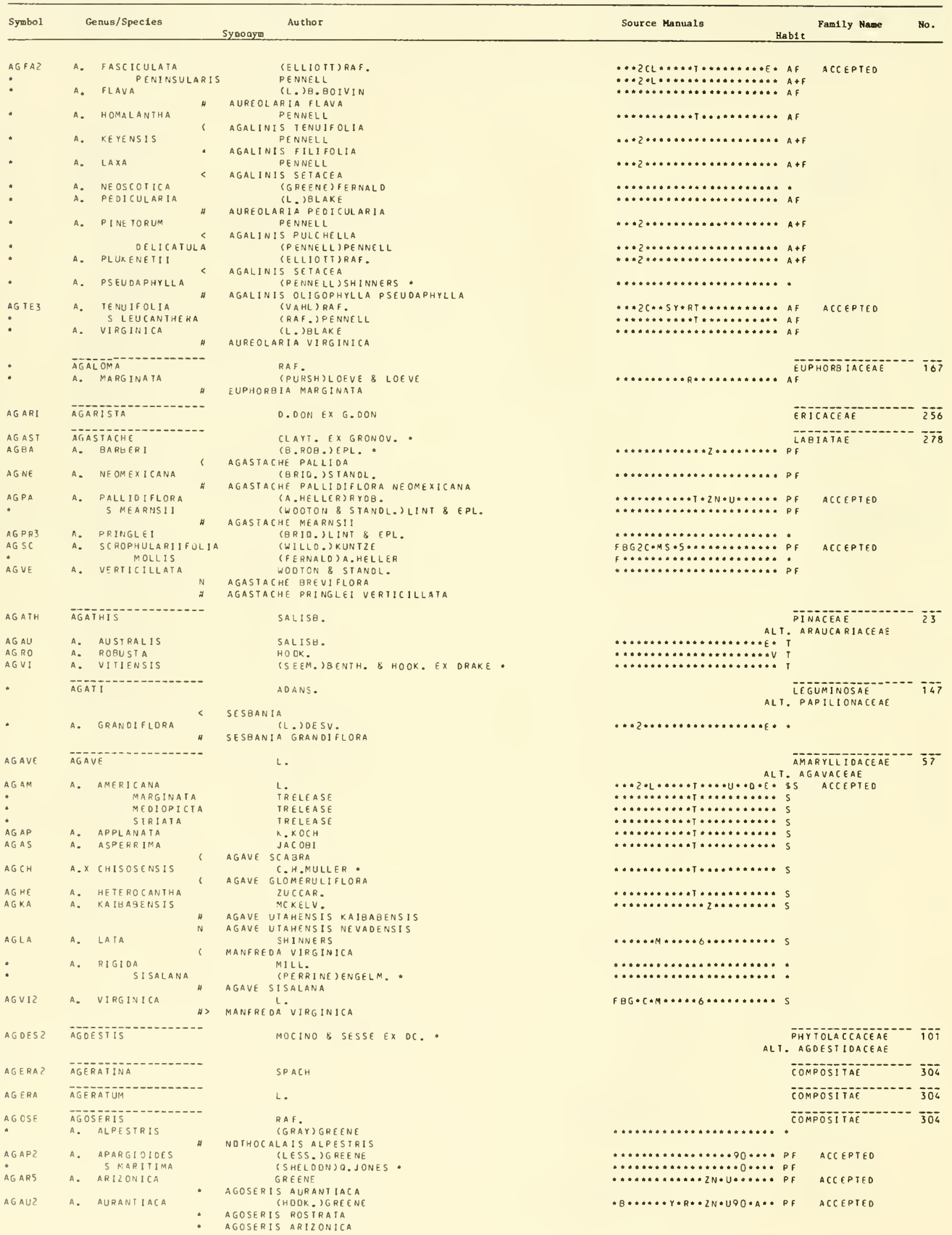




\begin{tabular}{|c|c|c|}
\hline Symbo 1 & \multicolumn{2}{|c|}{ Geous/Species } \\
\hline • & A. & BAPBELLULATA \\
\hline • & A. & CUSP IDATA \\
\hline • & A. & GASPENSIS \\
\hline $\begin{array}{l}A G G L \\
A G G L L\end{array}$ & A. & $\begin{array}{l}\text { GLAUCA } \\
\text { LACIIIIATA }\end{array}$ \\
\hline$A G G L P$ & & PARVIFLOR \\
\hline • & A. & GRAM IN I FOLIA \\
\hline AG HEZ & A. & $\begin{array}{l}\text { HETEROPHYLLA } \\
\text { CRENULATA }\end{array}$ \\
\hline : & & $\begin{array}{l}\text { RYMAPLEUR } \\
\text { TURG IOA }\end{array}$ \\
\hline • & A. & HIRSUTA \\
\hline * & A. & LACINIATA \\
\hline * & A. & PLEGEIA \\
\hline$A G R O 2$ & A. & ROSIRATA \\
\hline
\end{tabular}

$\begin{array}{ll}\text { AGRIM } & \text { AGFIMONIA } \\ \text { A. PLATYCARPA } & \text { A. PUBESCENS } \\ \text { AGPUR } & \text { A. PUMILACARPA }\end{array}$

AGROE X AGROELYMUS

AGROH $\times$ AGROHORDEUM

$A G R O P 3 \times A G R O P O G O N$

AGROPZ AGROFYRON

AGAL A. ALBICANS

A.X ANDINUN

$A G \theta A Z$

A. BAKERI

$A G B O$ A. BOREALE

$A G B O A$

AGBOH

$A G C A 2$

A. X BREVIF OLIUM

A. CANINUM

MAJUS

S MAJUS

ANOINUM

LATI GLUHE

UNILATERALE

AGCR A. CRISTATUM

$A G O R$

A. DASYSTACHYUH

- seder a. ofeseriorum

AGEL? A. ELMERI

AGGR3 A. GRIFIITHSII

AGIN A. INERNE

aglaz a. latiglume
Synonym

Authn $\mathrm{r}$

Source Manuals

Famlly Name

Ho.

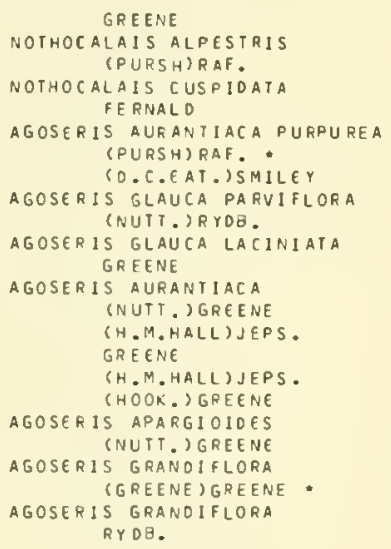

GREENE

NOTHOCALAIS ALPESTRIS

(PURSH)RAF.

NOTHOCALAIS CUSPIDATA

FERNAL D

AGOSERIS AURANTIACA PURPUREA (PURSH)RAF. .

(O.C.EAT.) SMILEY

AGOSERIS GLAUCA PARVIFLORA

CNUTT. JRYDA.

AGOSERIS GLAUCA LACINIATA GREENE

AGOSERIS AURANTIACA

(NUTT, ) GREENE

(H. M. HALL) JEPS.

GREENE

(H.M. HALL)JEPS

GHOOR.) GREENE

AGOSERIS APARGIOIDES

(NUTT, GREENE

AGOSERIS GRANOIFLORA

(GREENE ) GREENE

AGOSERIS GRANOIFLORA

RYDB.

AGOSERIS AURANTIACA

WALLR.

AGRIMONIA MICROCARPA

WALLR.

AGRIMONIA MICROCARPA

MUHL. EX BRITTON \& a, Gr. *

AGRIMONIA MICROCARPA

$$
\text { E. G.CAMUS EX A.CAMUS }
$$

E.G. CAMUS EX A.CAMUS

FOURN.

GAERTN.

SCRIBN. \& J.G.SMITH

AGROPYRON DASYSIACHYUM

AGROPYRON TRACHYCAULUM

(SCRIBN. \& J.G.SMITH)RYOB.

AGROPYRON TRACHYCAULUM

E. NELS.

AGROPYRON SCRIENERI

AGROPYRON CANINUM

AGROPYRON TRACHYCAULUM

STURC?.SDROBOY

ROEGNERIA BOREALIS

(SLRI3N. \& HERRILL)MELOERIS

(POLUNIN) MELOERIS

AGROPYRON VIOLACEUM

SCRIBN.

AGROPYRON TRACHYCAULUM

IL. JOEAUV.

AGROPYRON $\triangle A K E R I$

AGROPYRON VIOLACEUM

(VASEY) C.HITCHC. NON BAUMG. *

AGROPYRON TRACHYCAULUM MAJUS

(VASEY)C.HITCHC.

AGROPYRON TRACHYCAULUM MAJUS

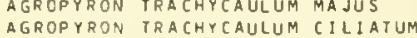

AGROPYRON TRACHYCAULUM (ILIATUM
ISCRIEN. \& J.G.SMITH)C. HITCHC.

AGROPYRON TRACHYCAULUM GLAUCUM

AGROPYRON TRACHYCAULUM LATIGLUME

(SCRIBN. \& J.G.SMITH) C.HITCHC.

TORON PSEUDOREPENS

AGROPYRON VIOLACEUM

$$
\begin{aligned}
& \text { RON VIOLACEUM } \\
& \text { (VASEY)C. HITCHC. }
\end{aligned}
$$

AGROPYRON TRA GHYCAULUM UN ILATERALE

(L.) GAERTN. *

AGROPYRON DESERTORUM

AGROPYRON PECIINIF ORM

AGROPYRON SIBIRICUM

$$
\text { CHOOK.) SCRION. }
$$

AGROPYRON ELMER I

AGROPYRON ALBICANS

AGROPYRON PSEUDOREPENS

(J.M.GILLETT \& H.A.SENN)E.G.VOSS *

(A.HITCHC.) B.BOIVIN

(FISCH. EX LINK)J.A.SCHULTES

AGROPYRON CRISTATUM

AGROPYRON PECTINIFORM

AGROPYRON SIEIRICUN

$$
\text { SCRIEN. }
$$

AGROPYRON DASYSTACHYUM

SCRIBN. \& J.G.SMITH

AGROPYRON ALOICANS GRIFFITHSII

AGROPYRON OASYSTACHYUM

(SCRIBN. \& J.G.SMITH)RYDB.

OSCRIBN. SPICATUM INERME

AGROPYRON SPICATUM INERME

(SCRIEN. J J.G.SMIYH)RYDB
AGROPYRCN TRACHYCAULUM LATIGLUME

AGROPYRON CANINUM LATIGLUME

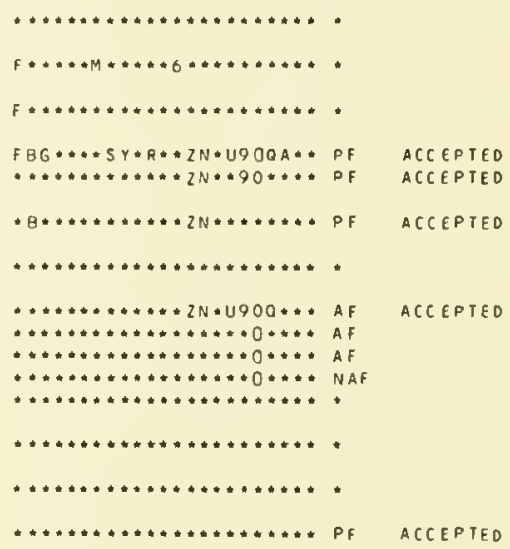

ROSACEAE 145

F $3 G 2 C \cdot 45.5 \ldots 6 \ldots \ldots \ldots \ldots$ PF ACCEPTEO

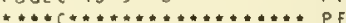

. $P F$

GRAMINEAE

$* \ldots \ldots \ldots \ldots \ldots \ldots+\ldots \ldots \ldots * * P G$

$* B G \ldots \ldots \ldots \ldots+* * * * * * * P G$

ACCEPTED

ACCEPTEO

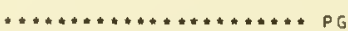

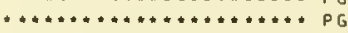

$\ldots+* * * * * * * * * \cdots+* * * * * * * P G$

$\# * * * * \Delta \gamma+\cdots * * * * 9 * * * * P G$

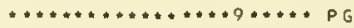

.................... be

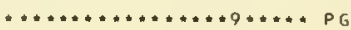

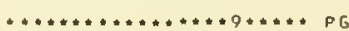

$* * \ldots+* * * * *+\cdots * 9+* * * P G$

F $3 G * * 4 S Y * R T 6 \cdots+* 9 * * * * P G$

$F B G \cdots * B Y R * 2 N+U 9 O \cdots * P G$

ACCEPTED

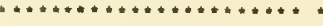

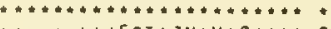

$\ldots \ldots \ldots \ldots 5 R T+2 N * U * 0 \ldots \ldots P G$

ACCEPTEO

ACCEPTED 


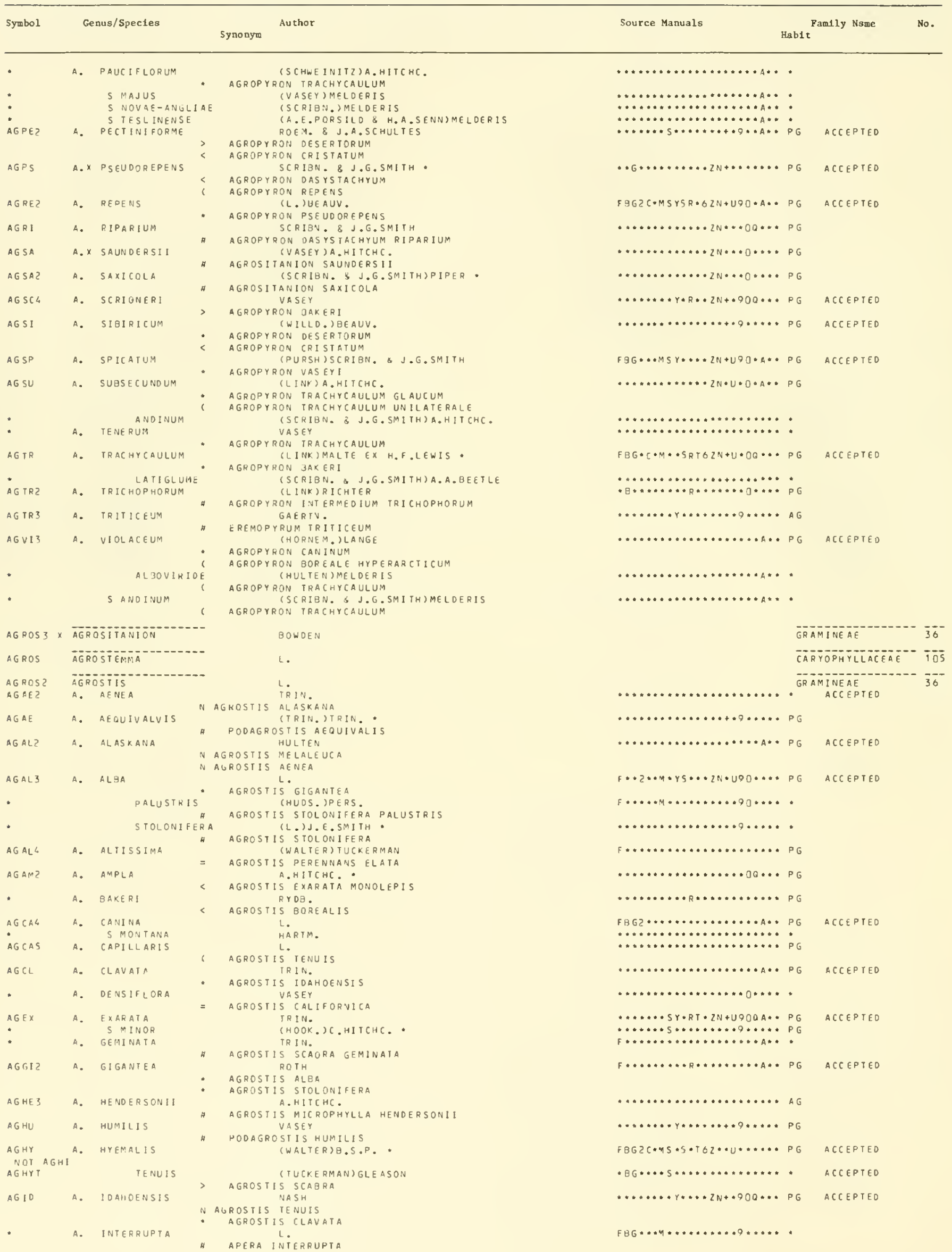




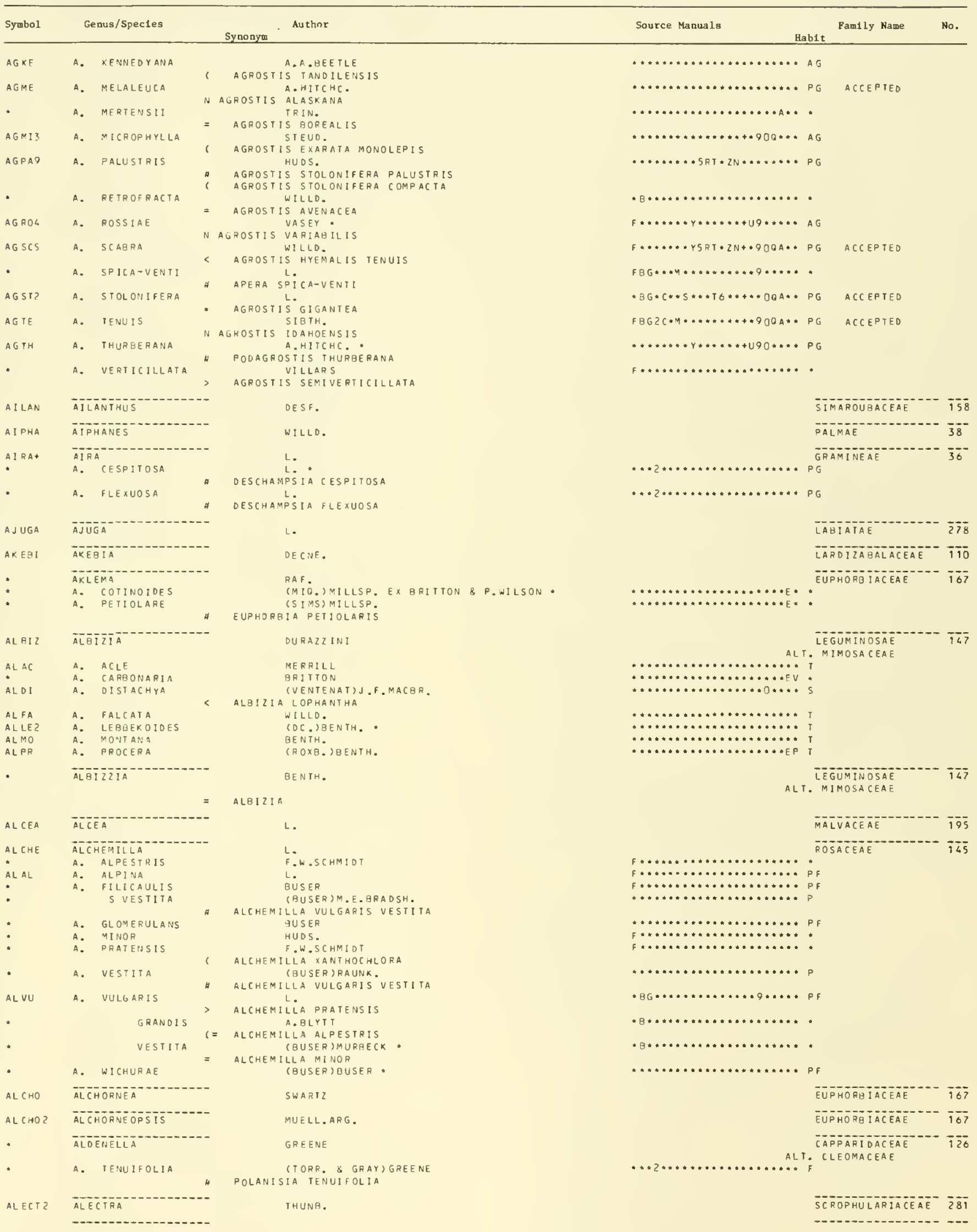




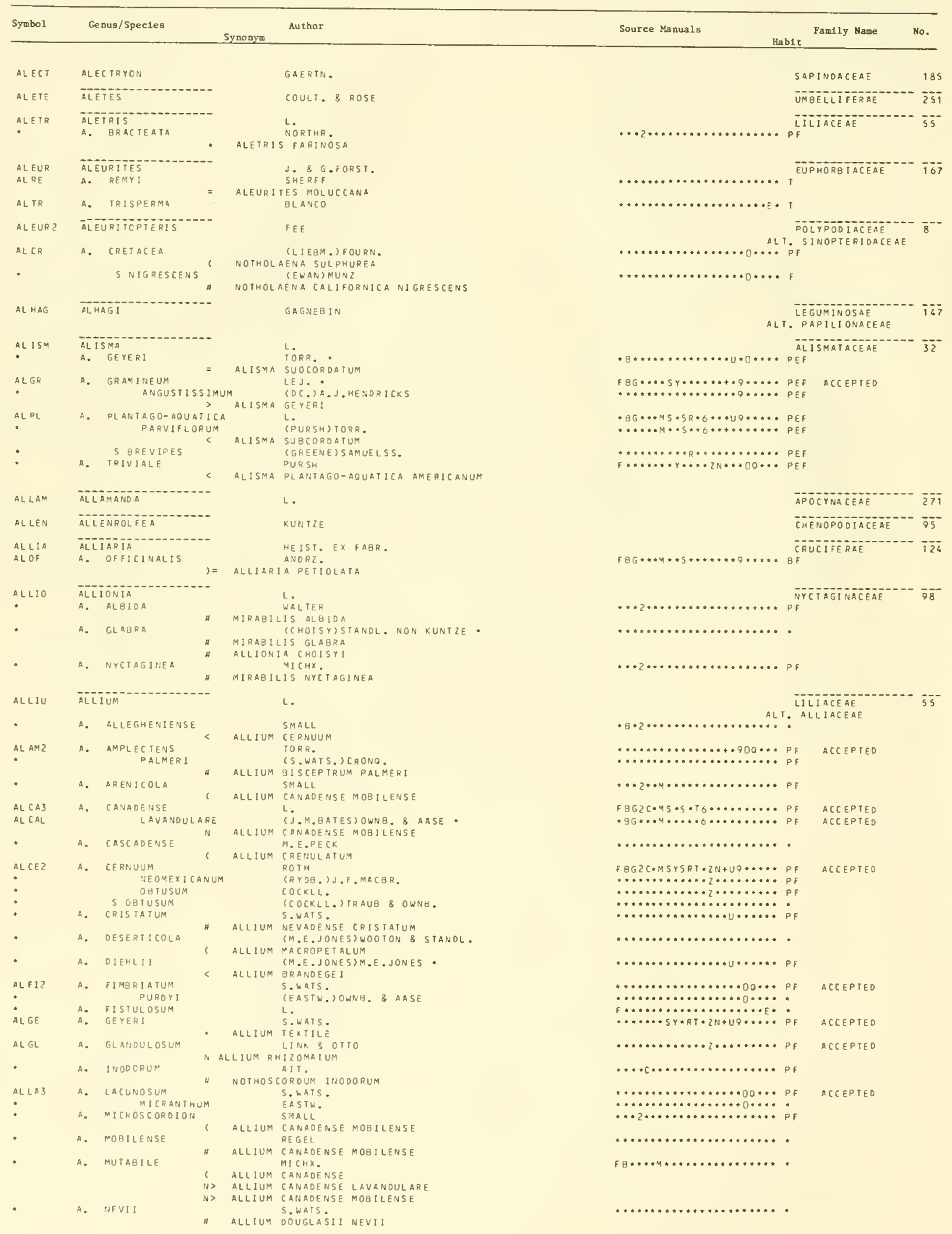




\begin{tabular}{|c|c|c|c|c|c|c|}
\hline Syrbol 1 & Genus/Specles & Synonym Author & Source Manuals & Hablt & Family Name & No. \\
\hline
\end{tabular}

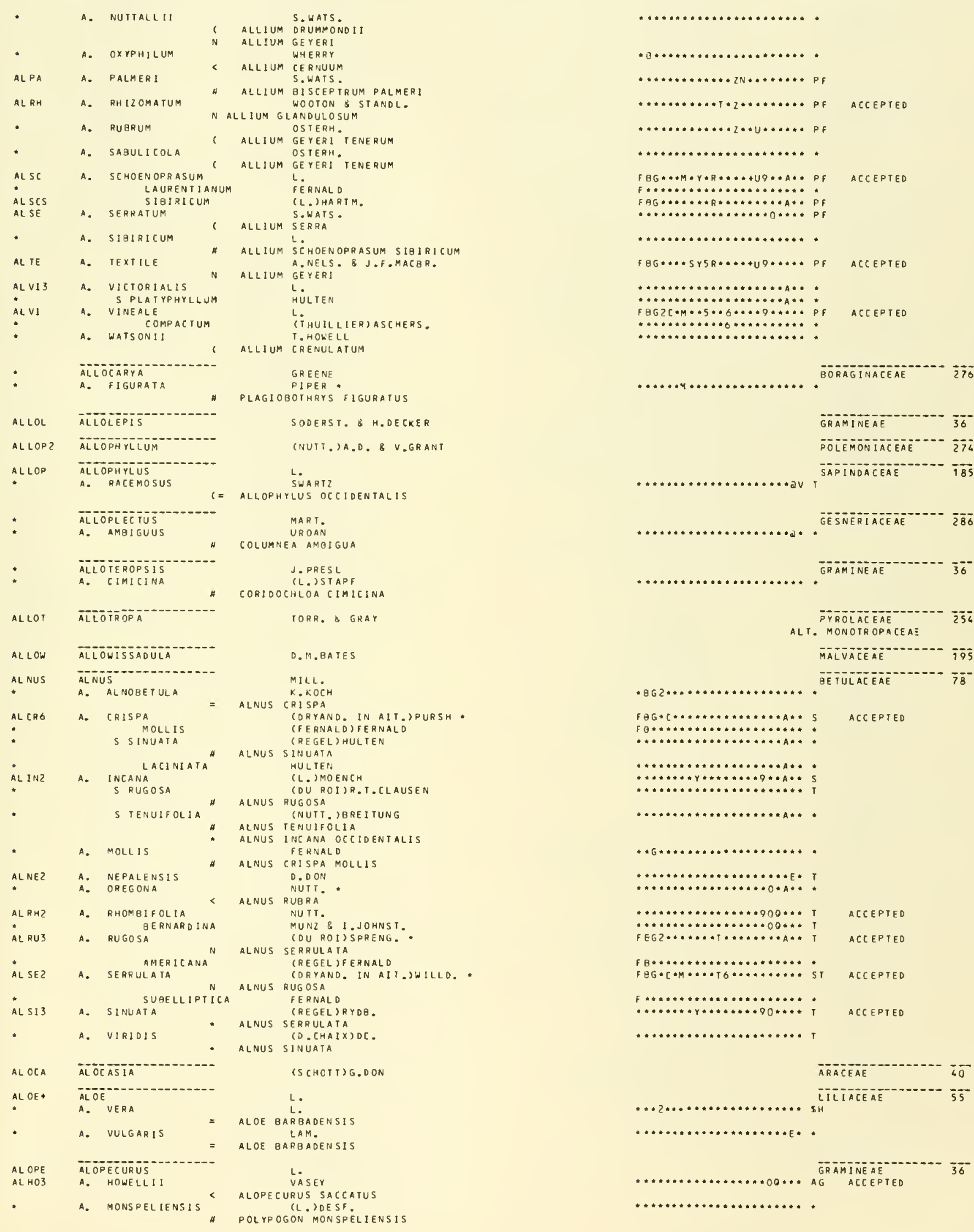




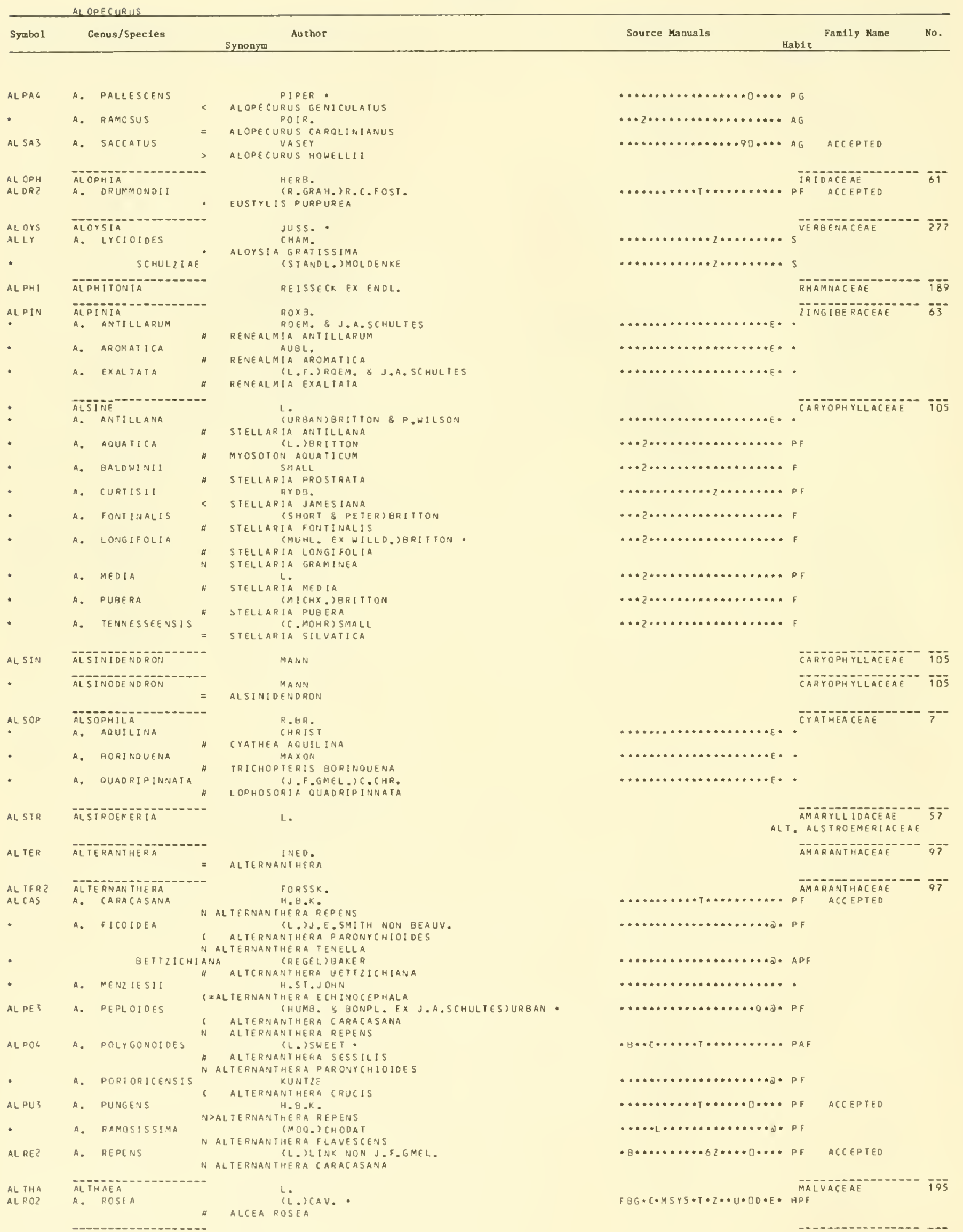




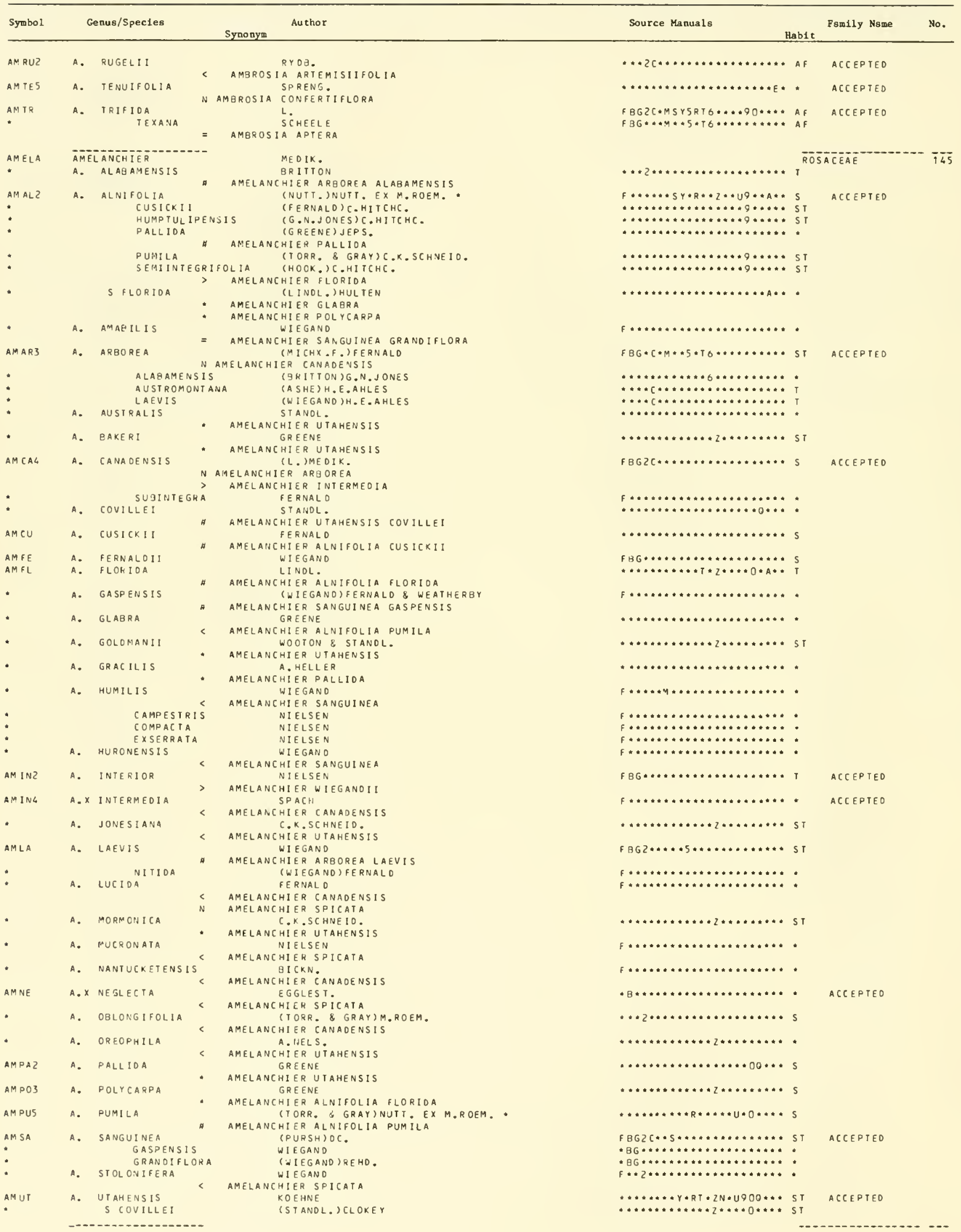




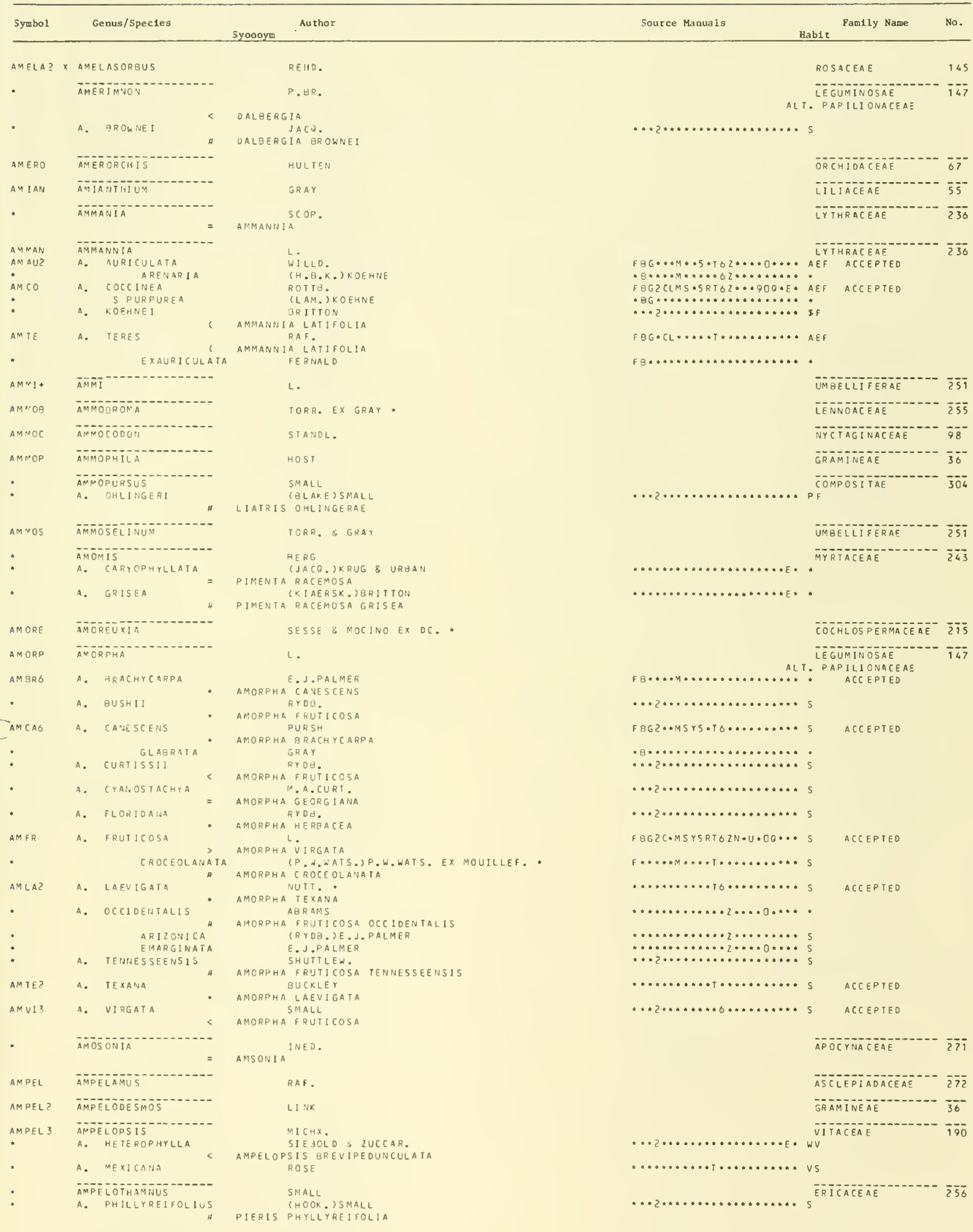




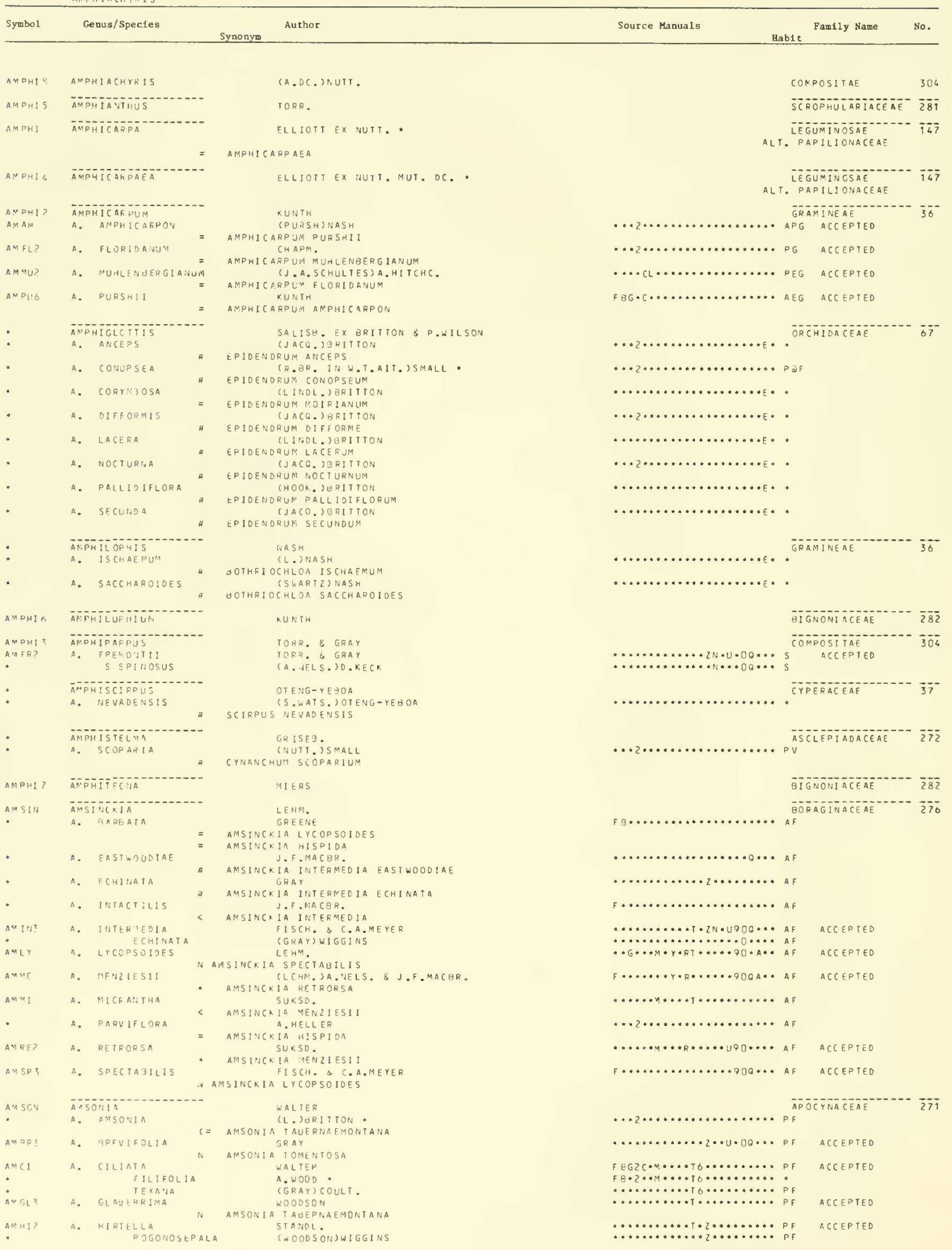




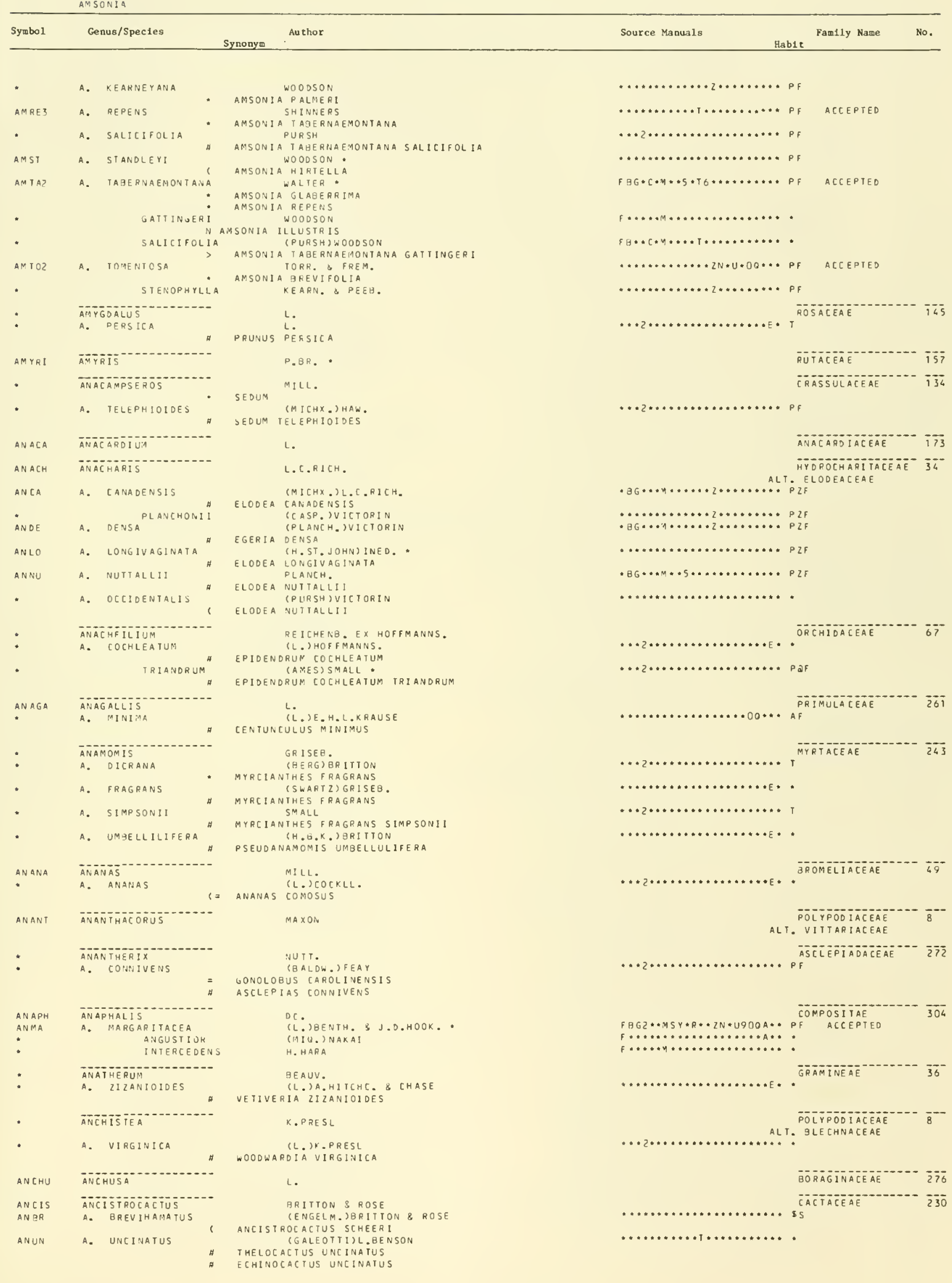




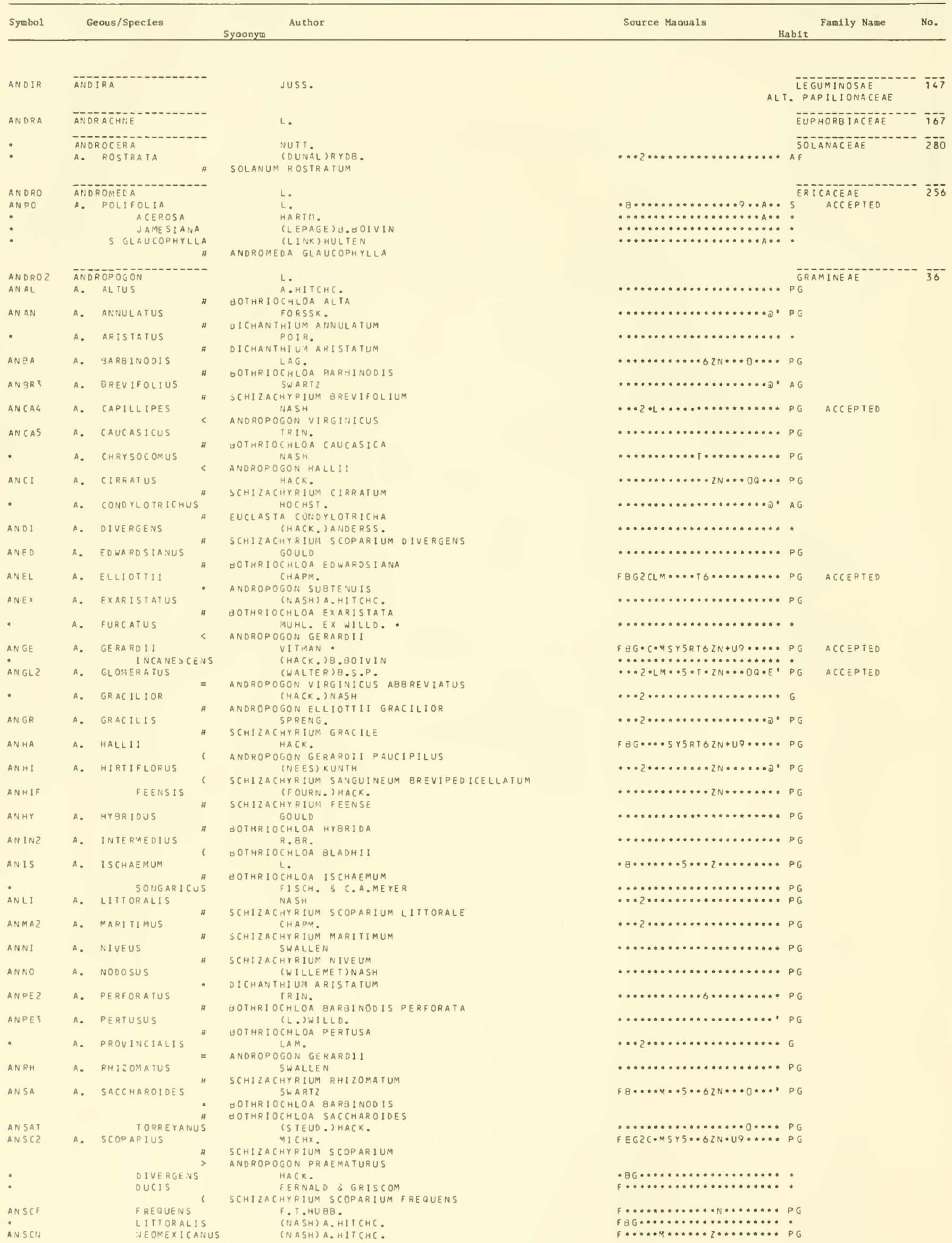


ANOROPOGON

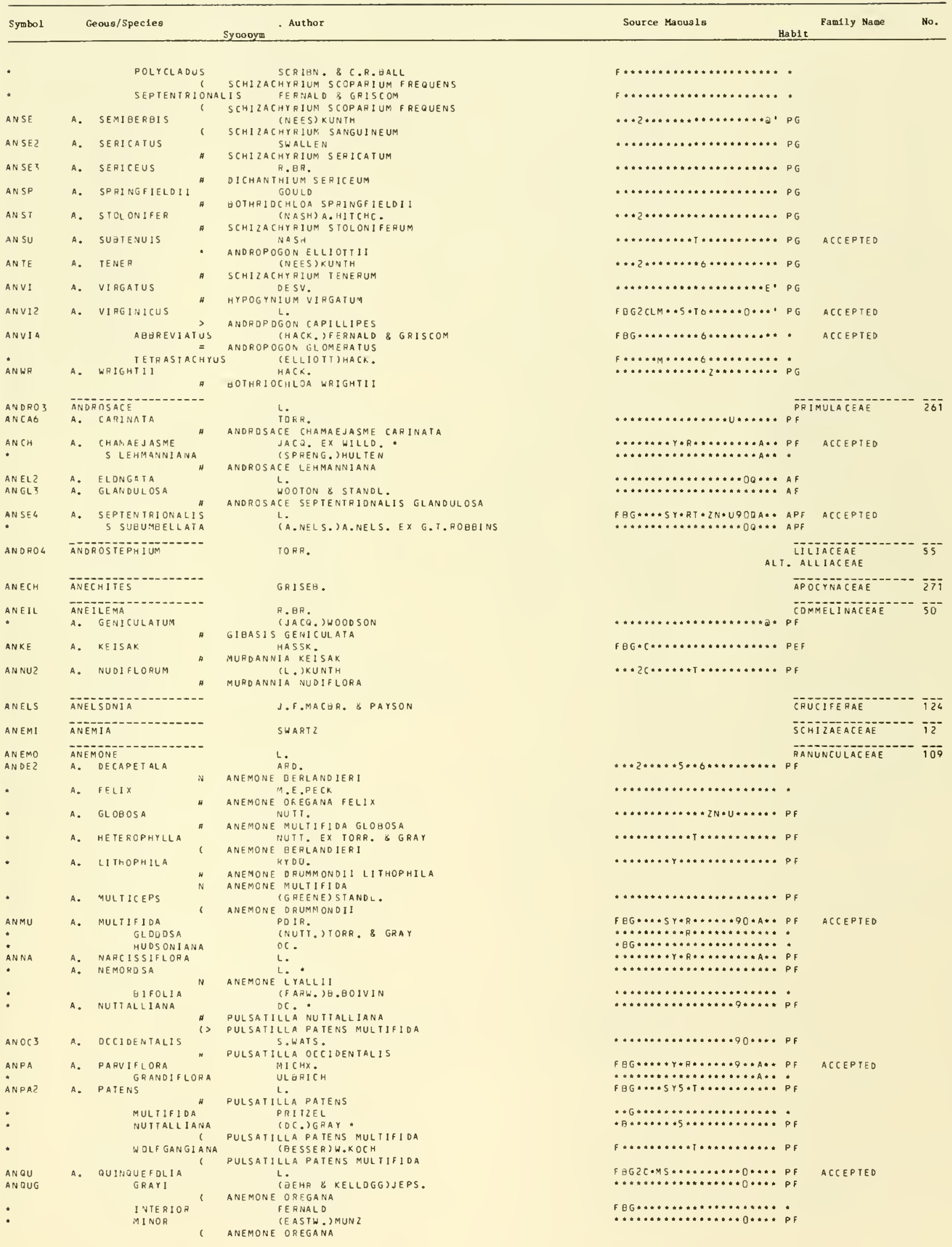




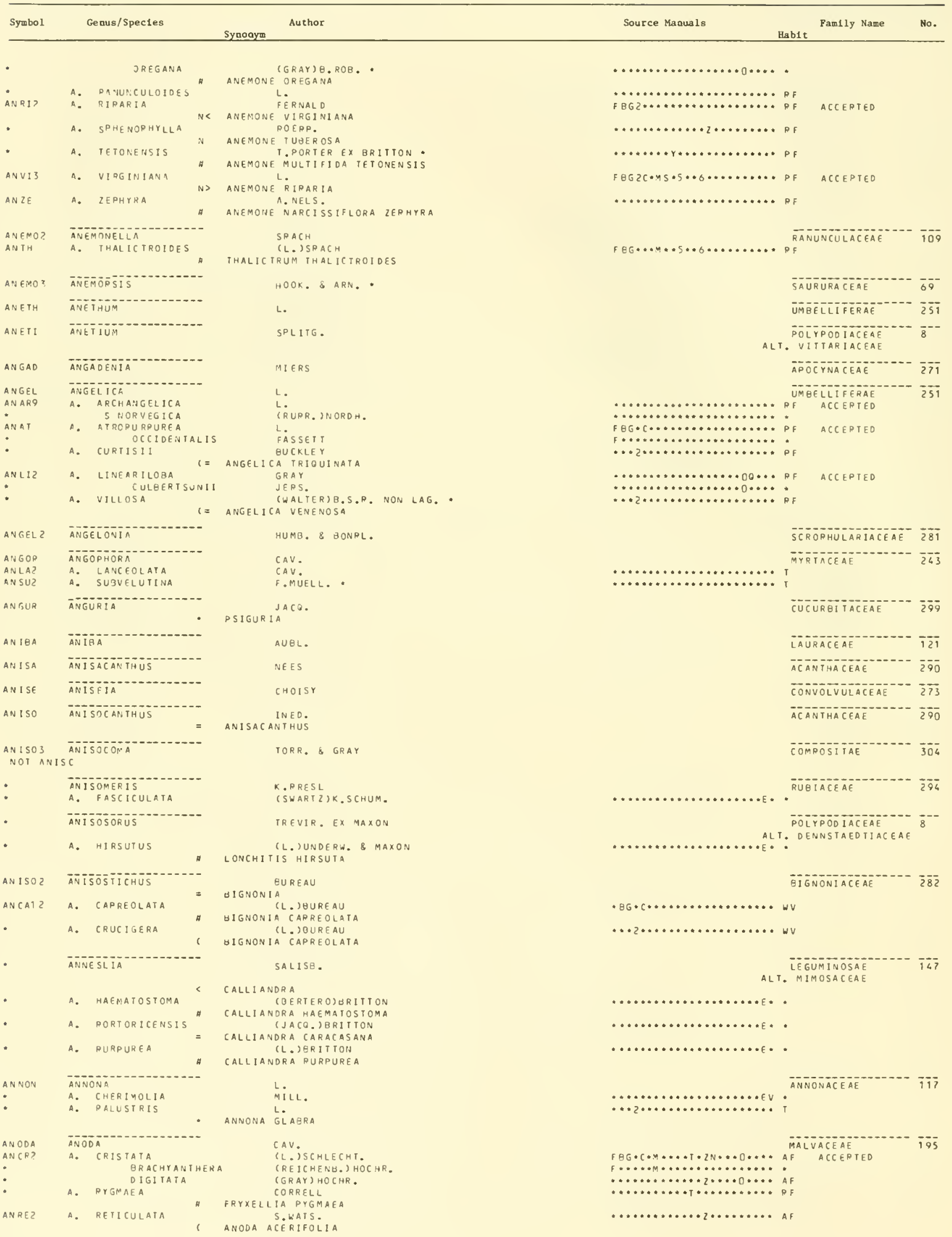




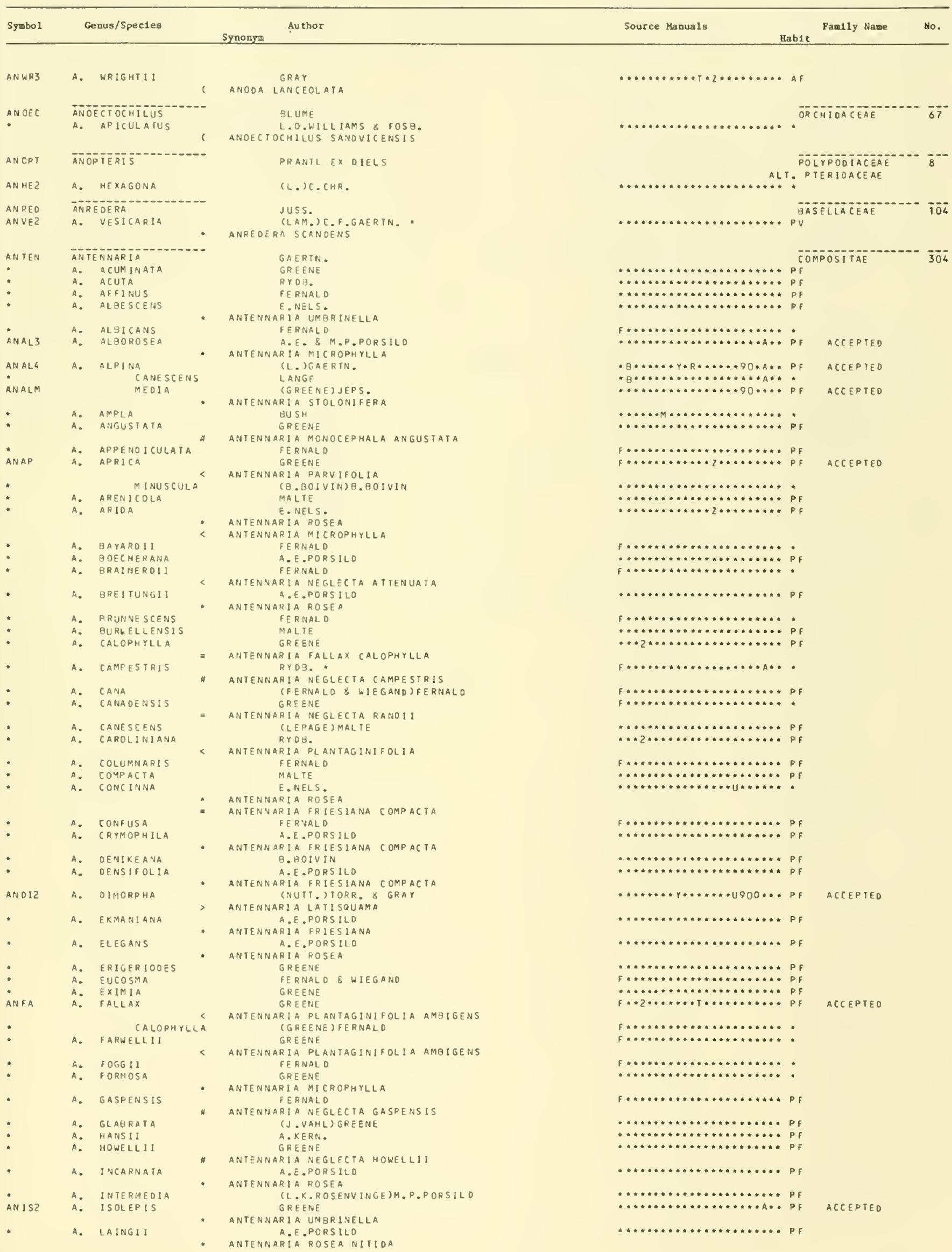




\begin{tabular}{|c|c|c|c|c|c|}
\hline Symbol & & nus/Species & Syaonym & Source Maquala & Bamily Name \\
\hline ANLAB & A. & LANATA & $\begin{array}{l}\text { (HOOK. ) GREENE } \\
\text { ANTENNARIA CARPATICA LANATA }\end{array}$ & $* * \ldots+\cdots+\gamma+\ldots \ldots+\cdots, \ldots \ldots+p F$ & \\
\hline ANLAG & A. & LAIISQUAMA & $\begin{array}{l}\text { PIPER } \\
\text { ANTENNARIA DIMORPHA }\end{array}$ & 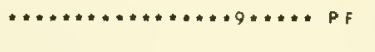 & ACCEPTEO \\
\hline ANLE3 & A. & $\begin{array}{l}\text { LEONTOPODIODES } \\
\text { LEUCHIPPI! }\end{array}$ & $\begin{array}{l}\text { CODY } \\
\text { M.P.PORSILD }\end{array}$ & $* * \cdots * * * * *+* * * * \cdots \cdots * P F$ & \\
\hline ANLUZ & A. & LUZULOIDES & $\begin{array}{l}\text { TORR. } 8 \text { GRAY } \\
\text { ANTENNARIA III CROCEPHALA } \\
\text { ANTENNARIA MONOCEPHALA }\end{array}$ & $* \ldots * * * * * Y * * * * * *$ U9O****PF & ACCEPIED \\
\hline ANMAS & A. & MARC INATA & $\begin{array}{l}\text { GREENE } \\
\text { ANTENNARIA NEGLECTA } \\
\text { ANTENNARIA RECURVA }\end{array}$ & $* * * * \cdots \cdots+T \cdot Z N \cdots O Q \cdots P F$ & ACCEPTED \\
\hline - & A. & MEOIA & $\begin{array}{l}\text { GREENE } \\
\text { ANTENNARIA ALPINA MEDIA }\end{array}$ & 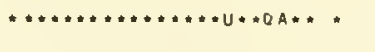 & \\
\hline * & A. & MEGACEPHALA & $\begin{array}{l}\text { FERNAL D } \\
\text { ANTENNARIA FRIESIANA MEGACEPHALA }\end{array}$ & 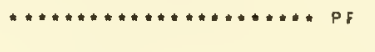 & \\
\hline ANM12 & A. & MICROCEPHALA & $\begin{array}{l}\text { GRAY } \\
\text { ANTENNARIA LUZULOIDES }\end{array}$ & 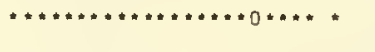 & $A C C E P T E D$ \\
\hline ANMI 3 & A. & MICROPHYLLA & $\begin{array}{l}\text { RYDB. } \\
\text { ANTENNARIA ROSEA } \\
\text { ANTENNARIA ALBOROSEA }\end{array}$ & $F * G \cdots \cdots * Y * \cdots \cdots \cdots * \cup Q \cdots \cdots * P F$ & ACCEPTED \\
\hline AN MOZ & A. & MONOCEPHALA & $\begin{array}{l}\text { OC } \\
\text { ANTENNARIA LUZULOIDES }\end{array}$ & 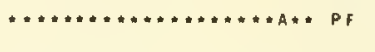 & ACCEPIED \\
\hline - & A. & MUNDA & $\begin{array}{l}\text { FERNAL } \\
\text { ANTENNARIA PL ANTAGINI FOLIA AMBIGENS }\end{array}$ & $F * * \cdots \cdots * * * \cdots+\cdots \cdots * * * *$ & \\
\hline ANNE & A. & NEGLECTA & $\begin{array}{l}\text { GREENE } \\
\text { ANTENNARIA OBOVATA } \\
\text { ANTENNAR!A MARGINATA }\end{array}$ & $F B G * \# M S Y S F * 6 * * \cup 90 * A * F F$ & ACCEPTED \\
\hline * & & S HOWELL II & (GREENE) HULTEN & $* \ldots \ldots \cdots \cdots \cdots \cdots * \cdots \cdots \cdots * * A * *$ & \\
\hline * & A. & NEOALASKANA & A.E.PORSILD & 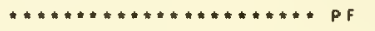 & \\
\hline * & A. & NEOD $101 C A$ & $\begin{array}{l}\text { GREEPE } \\
\text { ANTENNARIA NEGLECTA ATTENUATA }\end{array}$ & 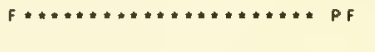 & \\
\hline - & & ATTENUATA & $\begin{array}{l}\text { FERNALD } \\
\text { ANTENNARIA NEGLECTA ATTENUATA }\end{array}$ & $F+\cdots+\cdots+\cdots+\cdots+\cdots+\cdots+\cdots *$ & \\
\hline * & & CHLOROPHYLL. & FERNAL D & 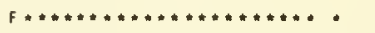 & \\
\hline$\dot{*}$ & & GRANDIS & $\begin{array}{l}\text { FERNAL D } \\
\text { FEFNALD }\end{array}$ & 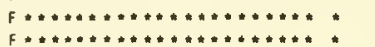 & \\
\hline AN $0 B$ & A. & OBOVATA & $\begin{array}{l}\text { E.NELS } \\
\text { ANTENNARIA NEGLECTA }\end{array}$ & $\ldots \ldots \ldots \ldots \ldots \ldots \ldots \ldots \ldots+\cdots, p F$ & ACCEPTED \\
\hline ANPA3 & A. & PALLIDA & $\begin{array}{l}\text { E. NELS - } \\
\text { ANTENNAR IA UMBRINELLA }\end{array}$ & 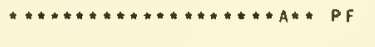 & ACCEPTED \\
\hline$\cdot$ & A. & PAFL IN I I & $\begin{array}{l}\text { FEFNALD } \\
\text { ANTENNARIA PL ANTAGINIFOLIA AMBIGENS }\end{array}$ & $F \cdots \ldots \ldots+\cdots+\cdots \cdots+\cdots \cdots+\cdots F$ & \\
\hline * & & ARNOGLOSSA & $\begin{array}{l}\text { (GREENE) FERNALI } \\
\text { ANTENNARIA PLANTAGINIFOLIA AGNOGLOSSA }\end{array}$ & $F+\cdots+\cdots+\cdots \ldots+\cdots+\cdots \cdots+*$ & \\
\hline ANPAL & A. & PAFVIFOLIA $>$ & $\begin{array}{l}\text { NUTT } \\
\text { ANTENNARI A APRICA }\end{array}$ & $\bullet B G * \cdots \cdot S Y * R * B 2 N \cdot U 9 * \ldots+P F$ & ACCEPTED \\
\hline * & & BRACTEOSA & (RYDB.)A.NELS. * & 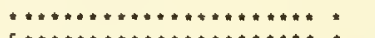 & \\
\hline * & A. & PEASEI & $F E R N A L D$ & $F \cdots+\ldots+\ldots+\ldots+\cdots+\cdots+\cdots$ & \\
\hline ANPEG & A. & PEDICELLATA & $\begin{array}{l}\text { GEEENE } \\
\text { ANTENNARI A RA CEMOSA }\end{array}$ & $\cdots+\cdots+\cdots+\infty+\cdots+\ldots+\infty+\infty$ & ACC EPTED \\
\hline$\cdot$ & A. & PEDUNCULATA & $\begin{array}{l}\text { A. E.PORSILD } \\
\text { FEFNALD }\end{array}$ & 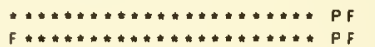 & \\
\hline * & A. & PETALOIDEA & ANTENNARIA NEGLECTA & & \\
\hline * & & SCARIOSA & FERNAL D & $F+\ldots \ldots+\ldots \ldots \ldots \ldots+\cdots \cdots *$ & \\
\hline - & & SUSCORYMBDS & FER VAL D & 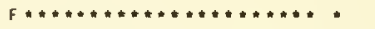 & \\
\hline * & A. & PH ILONIPHA & $\begin{array}{l}\text { A.E.POESILD } \\
\text { ANTENNARIA MONOCEPHALA PHILONIPHA }\end{array}$ & 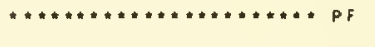 & \\
\hline $\begin{array}{l}\text { ANPL } \\
\text { AN PLA }\end{array}$ & A. & $\begin{array}{l}\text { PLANTAGINIFOLIA } \\
\text { AMBIGENS }\end{array}$ & $\begin{array}{l}\text { (L.)RICHARDS. } \\
\text { (GREENE) CRONQ. }\end{array}$ & 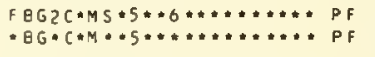 & $\begin{array}{l}\text { ACCEPTED } \\
\text { ACCEPTED }\end{array}$ \\
\hline & & $>$ & ANTENNARIA FALLAX & & \\
\hline : & & PETIOLATA & (FERWALD)A. HELLER & 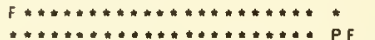 & \\
\hline ANPU & A. & $\begin{array}{l}\text { PORSILDII } \\
\text { PULCHERRIMA }\end{array}$ & $\begin{array}{l}\text { E. EKMAN } \\
\text { (HOOK.) GREENE }\end{array}$ & 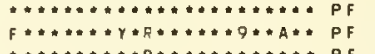 & ACCEFTED \\
\hline - & & S ANAPHALOIDES & $\begin{array}{l}\text { (FYDB.) W.WEBER } \\
\text { RNTENNAR IA AN WPHALOIOES }\end{array}$ & 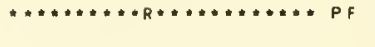 & \\
\hline - & A. & PYGMAEA & FERNALD & 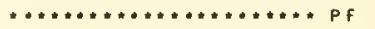 & \\
\hline ANRE 4 & A. & RECURVA & $\begin{array}{l}\text { GREENE } \\
\text { ANTENNARIA MARGINATA }\end{array}$ & $\cdots \cdots \cdots \cdots+\cdots+2 \cdots \cdots \cdots \cdots P F$ & $A C C E P T E D$ \\
\hline * & A. & REFLEXA & $\begin{array}{l}\text { E. NELS. } \\
\text { ANTENNARI A ALPINA } \\
\text { ANTENNARIA UMBRINELLA }\end{array}$ & 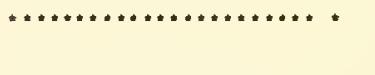 & \\
\hline AN RO2 & A. & ROSEA $><$ & $\begin{array}{l}\text { GREENE. } \\
\text { ANTENNAR A MICRDPHYLLA }\end{array}$ & 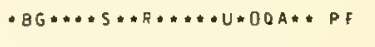 & ACCEPTED \\
\hline * & & IMBRICATA & 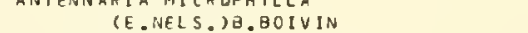 & 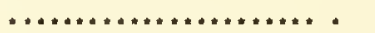 & \\
\hline * & A. & ROUSSEAUI & A.E.PORSILO & 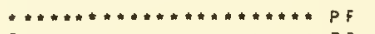 & \\
\hline - & A. & RUPICOLA & $\begin{array}{l}\text { FERNALD } \\
\text { ANTENNARIA NEGLECTA ATTENUATA }\end{array}$ & 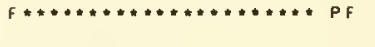 & \\
\hline • & A. & RUSSELLII & $\begin{array}{l}\text { 9.BOIVIN } \\
\text { SOIV }\end{array}$ & 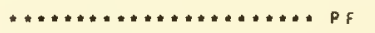 & \\
\hline . & A. & SANS ONII & GREENE & 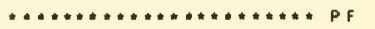 & \\
\hline - & A. & SHUMAG INENS I S & $\begin{array}{c}\text { A.E.PORSILD } \\
\text { ANTENNARIA MONOCEPHALA }\end{array}$ & 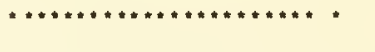 & \\
\hline - & A. & SORNBOFGCRI & FERNALD & $\| \cdots \cdots \cdots \cdots \cdots+\cdots+\cdots \cdots, P F$ & \\
\hline - & A. & SPATHULATA & FERNAL O & $f * \hbar * * * \ldots \ldots \ldots \ldots \ldots \ldots \ldots+p q$ & \\
\hline ANST3 & A. & STOL ON I FERA & $\begin{array}{l}\text { A.E.PORSILD } \\
\text { ANTENNARIA ALPINA MEDIA }\end{array}$ & $* * * * \ldots \ldots \ldots \ldots \ldots * * * * A * * P F$ & ACCEP TED \\
\hline • & A. & ST RAMINEA & FERNAL D & $F \ldots \ldots \ldots \ldots \ldots \ldots \ldots+\cdots * \ldots F$ & \\
\hline - & A. & SUBV IS $\cos A$ & $\begin{array}{l}\text { FEFNALD } \\
\text { ANIENNARIA RDSEA NITIDA }\end{array}$ & $F * * \ldots * * \ldots \ldots \ldots \ldots \ldots * * A * *$ PF & \\
\hline - & A. & TWEEOSMUIRII & 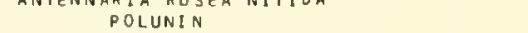 & 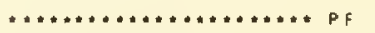 & \\
\hline AN UM & A. & UMBRINELLA & $\begin{array}{l}\text { RYOB. } \\
\text { ANTENNARIA ISOLEPIS }\end{array}$ & $\bullet B * * * * Y * \cdots 2 N * \cup 90 \cdots *$ PF & $A C C E P T E D$ \\
\hline & & ( & ANTENNARIA PALLIDA & & \\
\hline * & A. & UNGAVENSIS & MALTE & 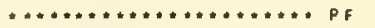 & \\
\hline • & A. & VEXILLIFERA & FERNALD & 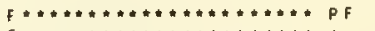 & \\
\hline * & A. & VIFGINICA & $\begin{array}{l}\text { ST } E \text { BS. } \\
\text { S }\end{array}$ & $F \cdots+\cdots+\cdots+\cdots+\cdots+\cdots+\cdots+\cdots *$ & \\
\hline - & & ARGILLICOLA & $\begin{array}{l}\text { ANTENNAR I A NEGLECTA ARGILLICOLA } \\
\text { STEBB. } \\
\text { ANTENNARIA NEGLECTA ARGILLICOLA }\end{array}$ & $f * \ldots+* *+\ldots+\cdots+\ldots+\ldots+\cdots *$ & \\
\hline
\end{tabular}




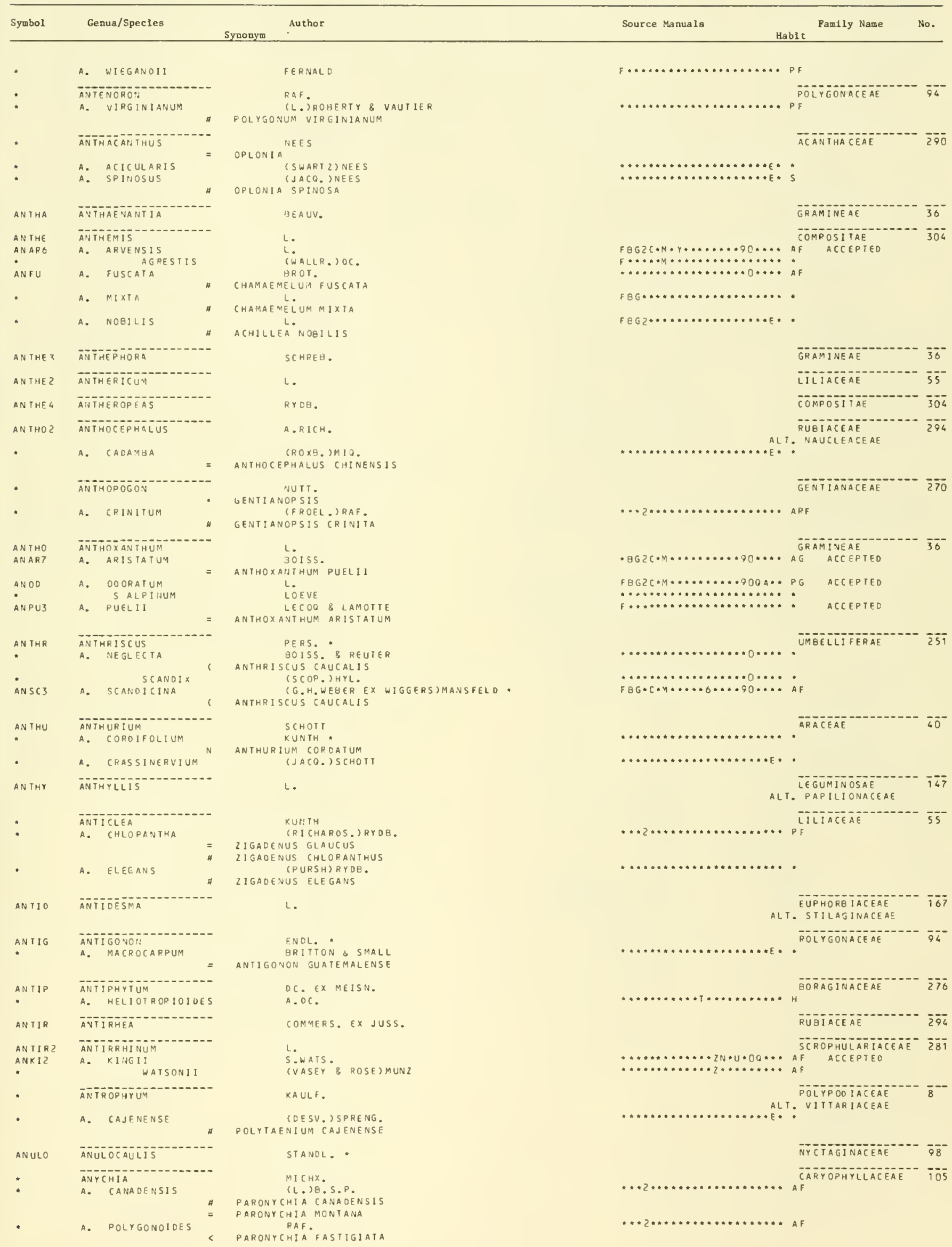




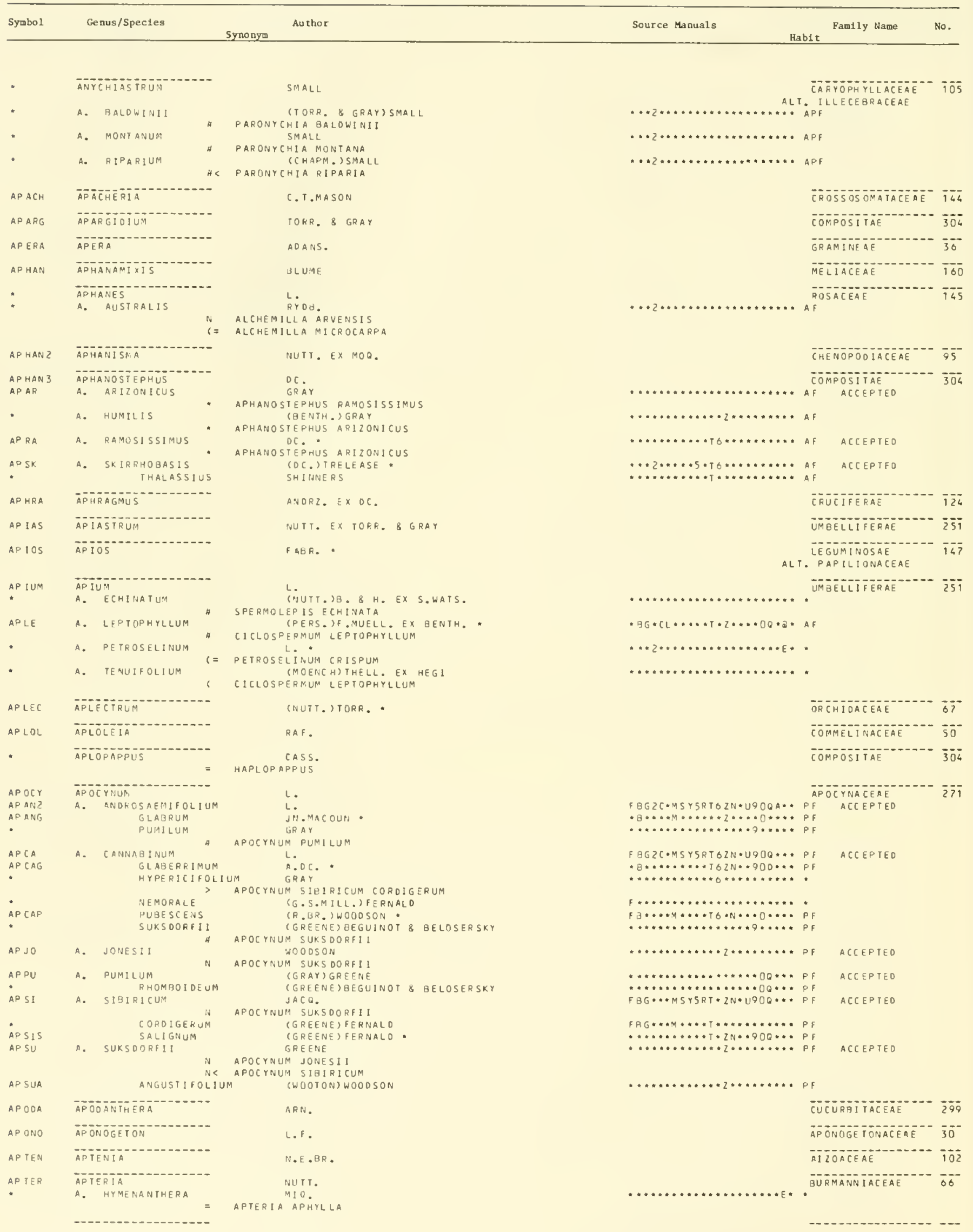




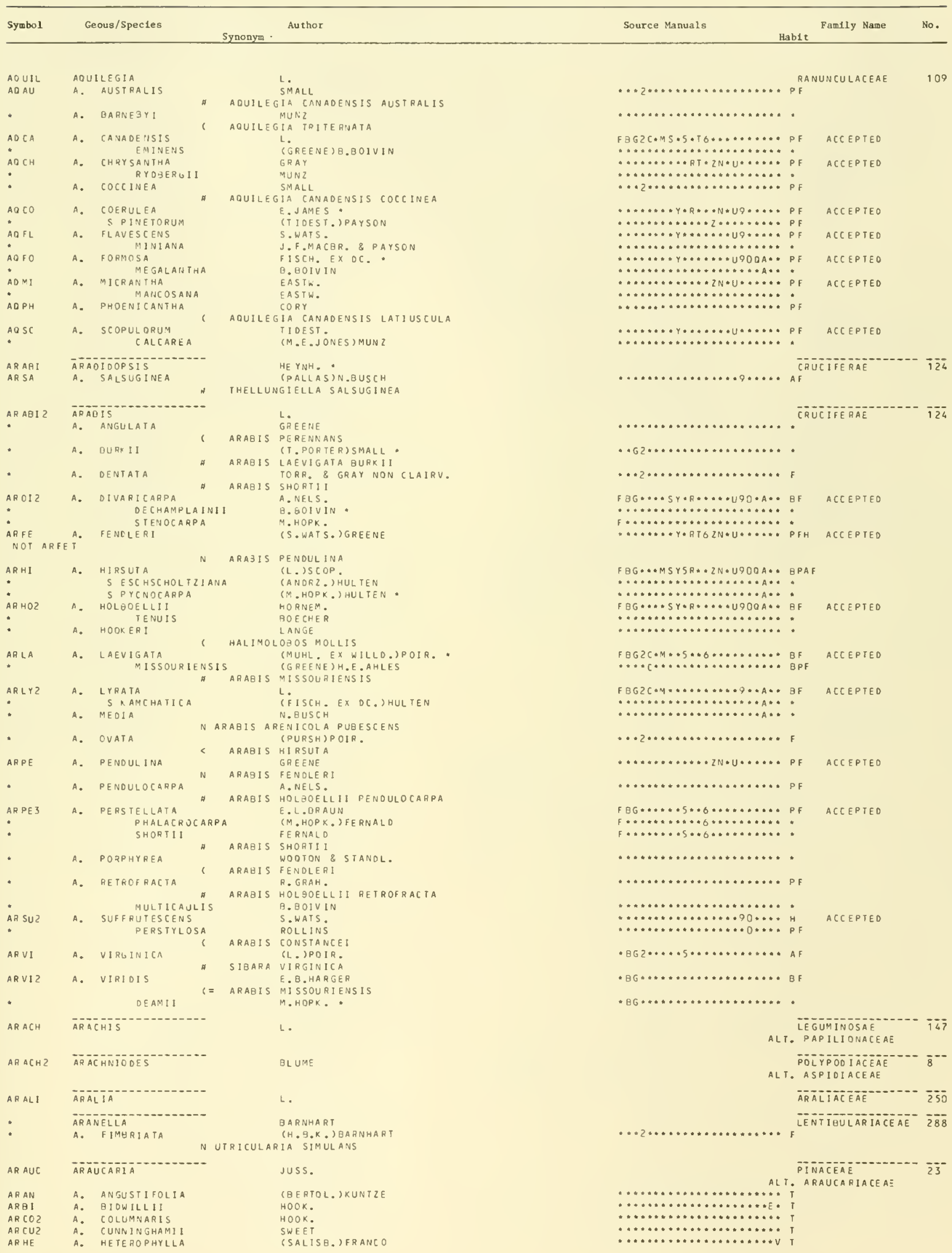




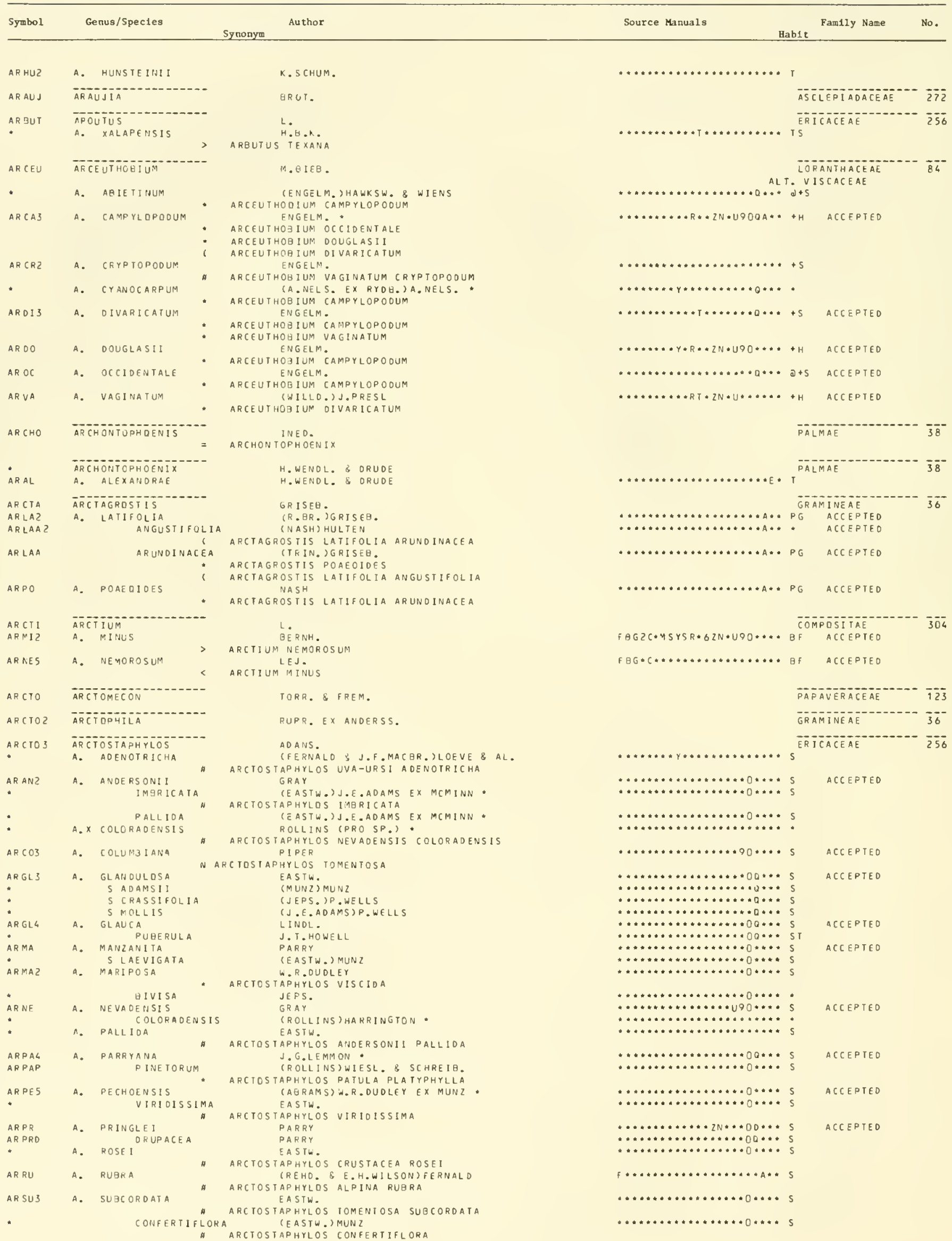


ARCTOSTAPHYLOS

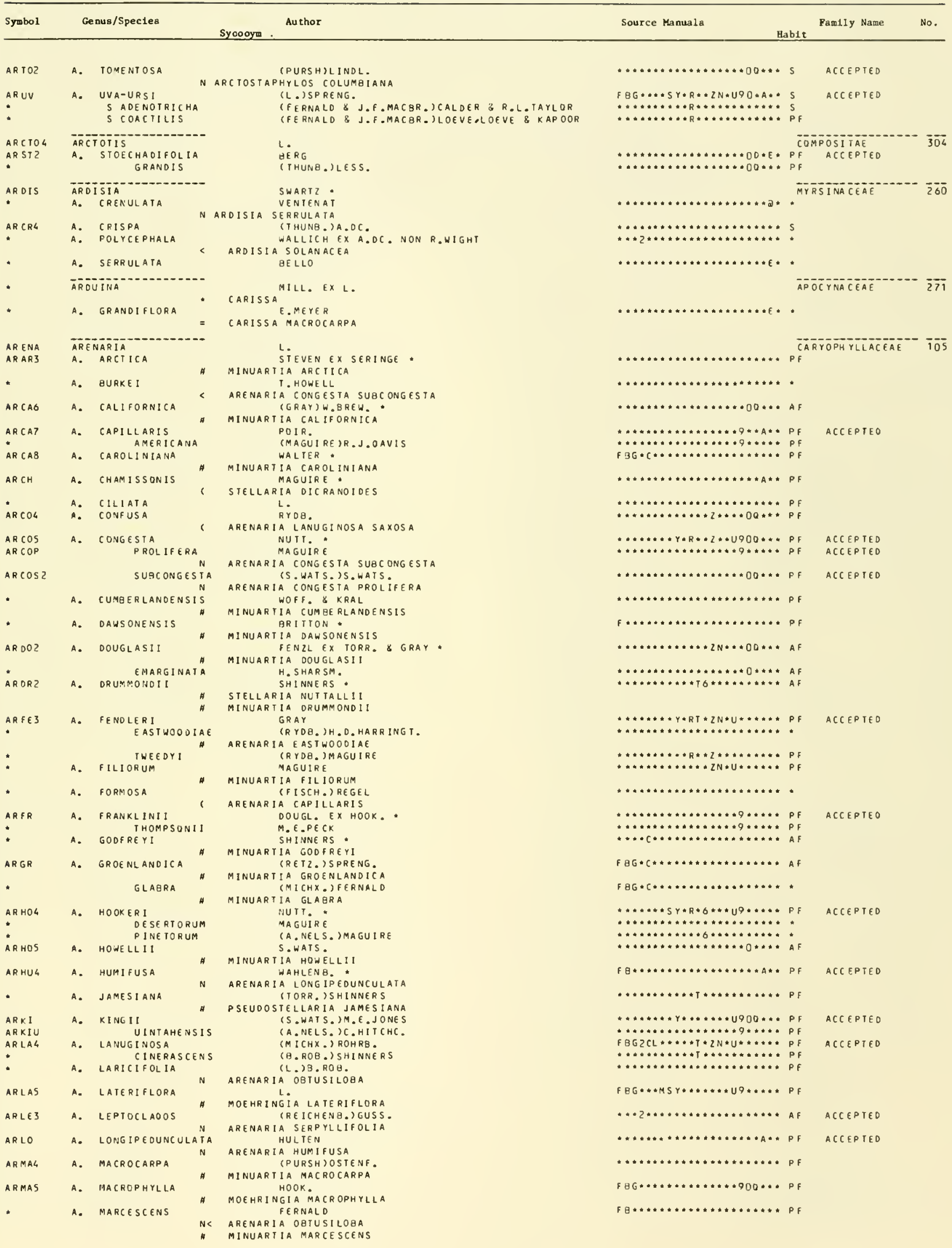




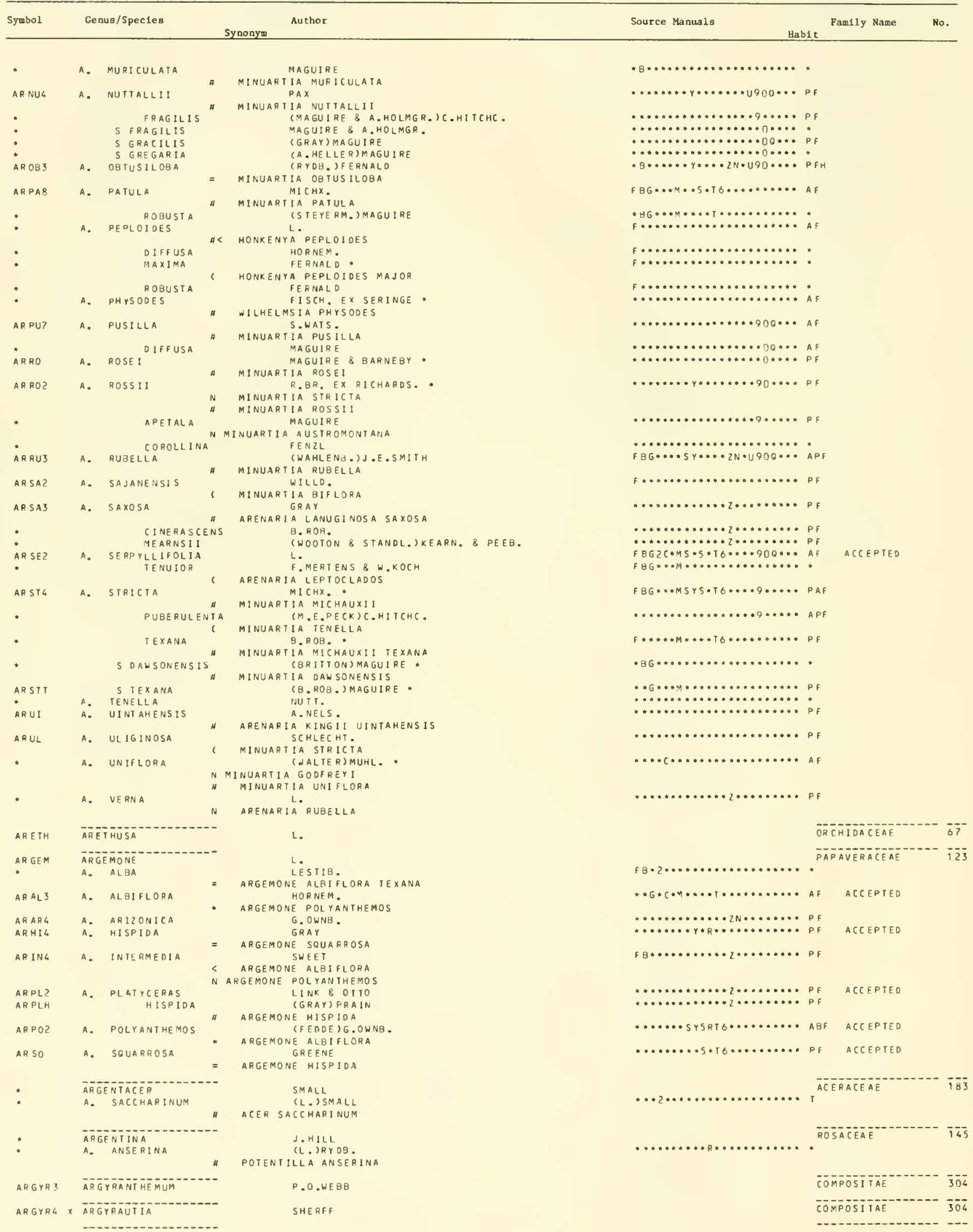




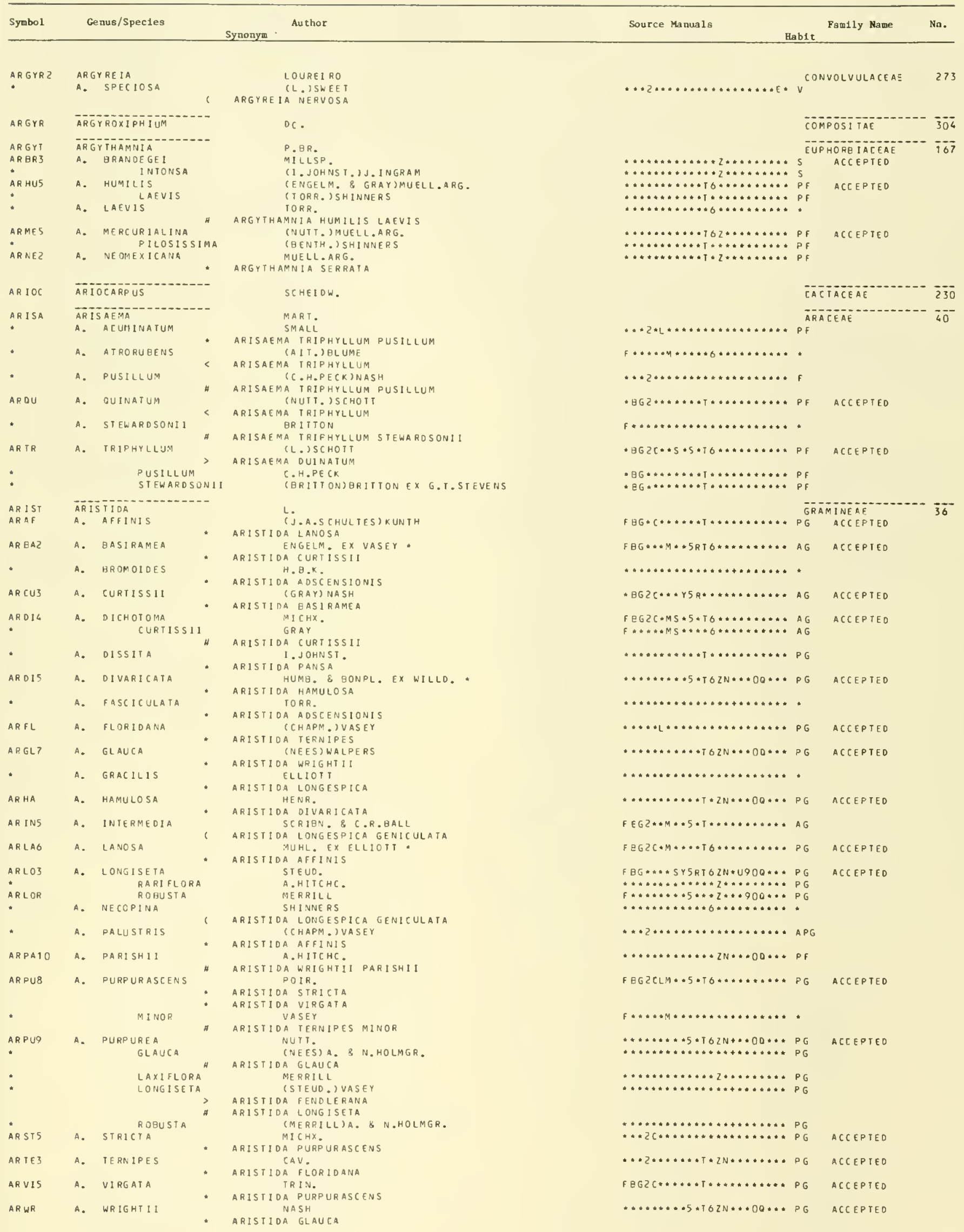




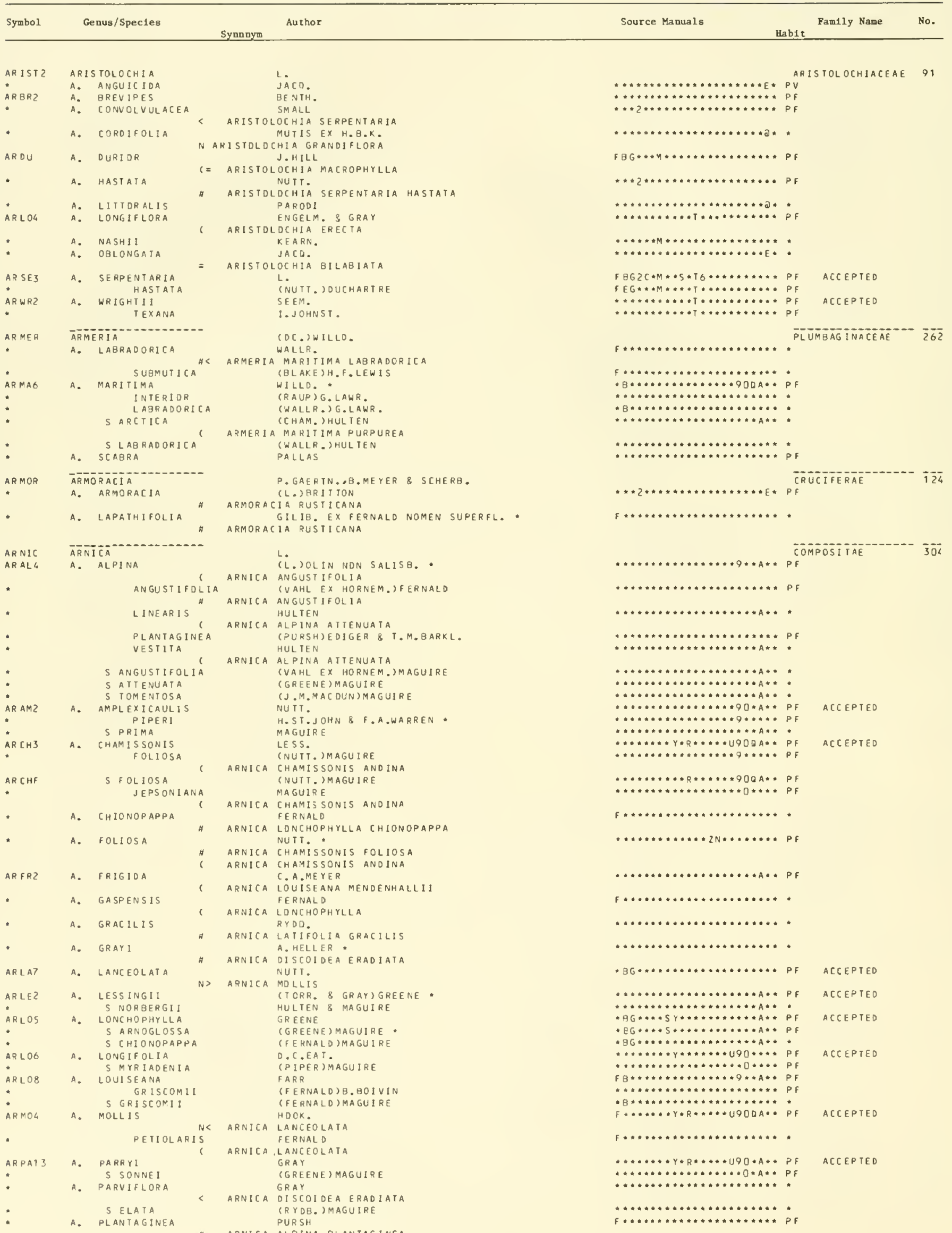


ARNICA

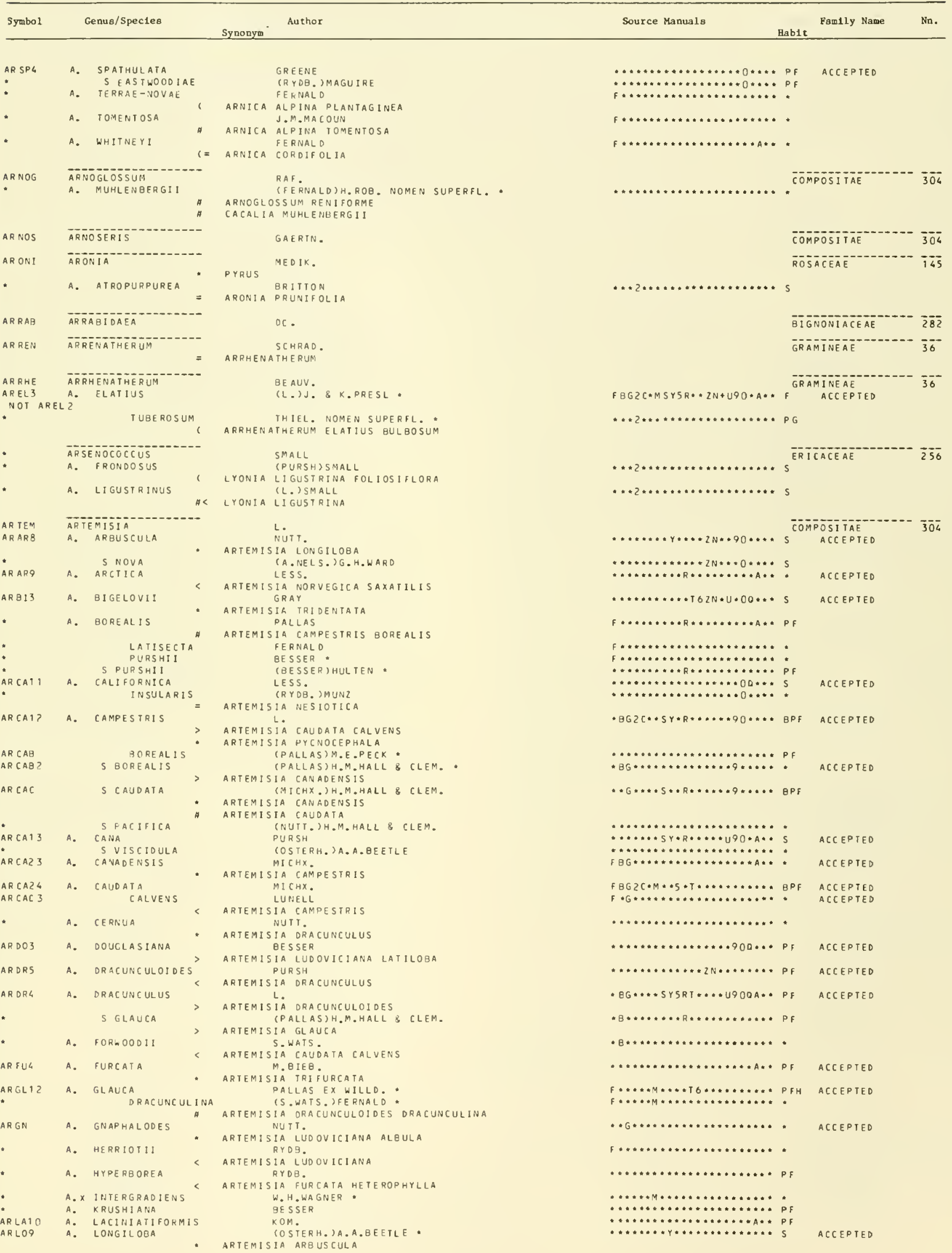


ARTEMISIA

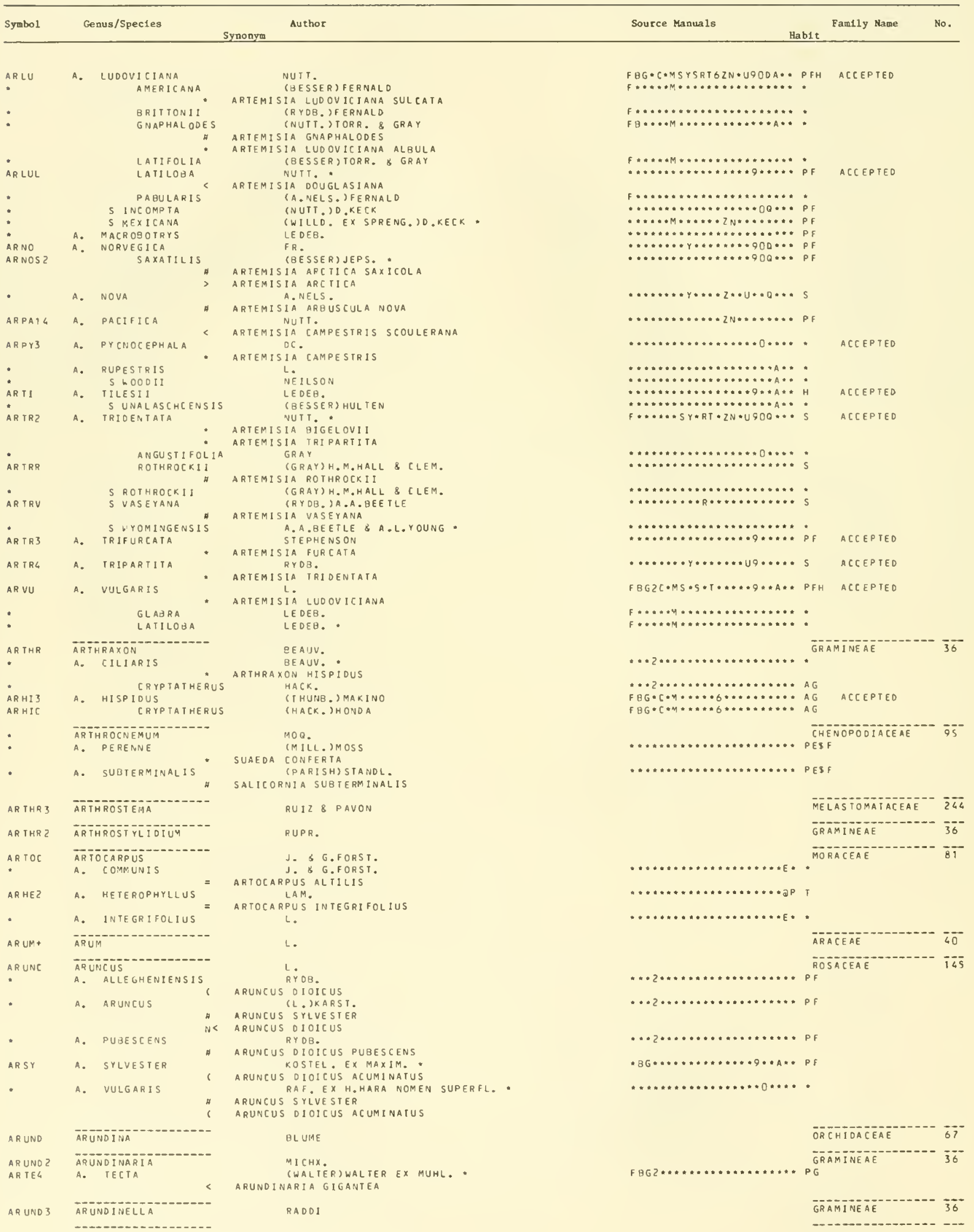




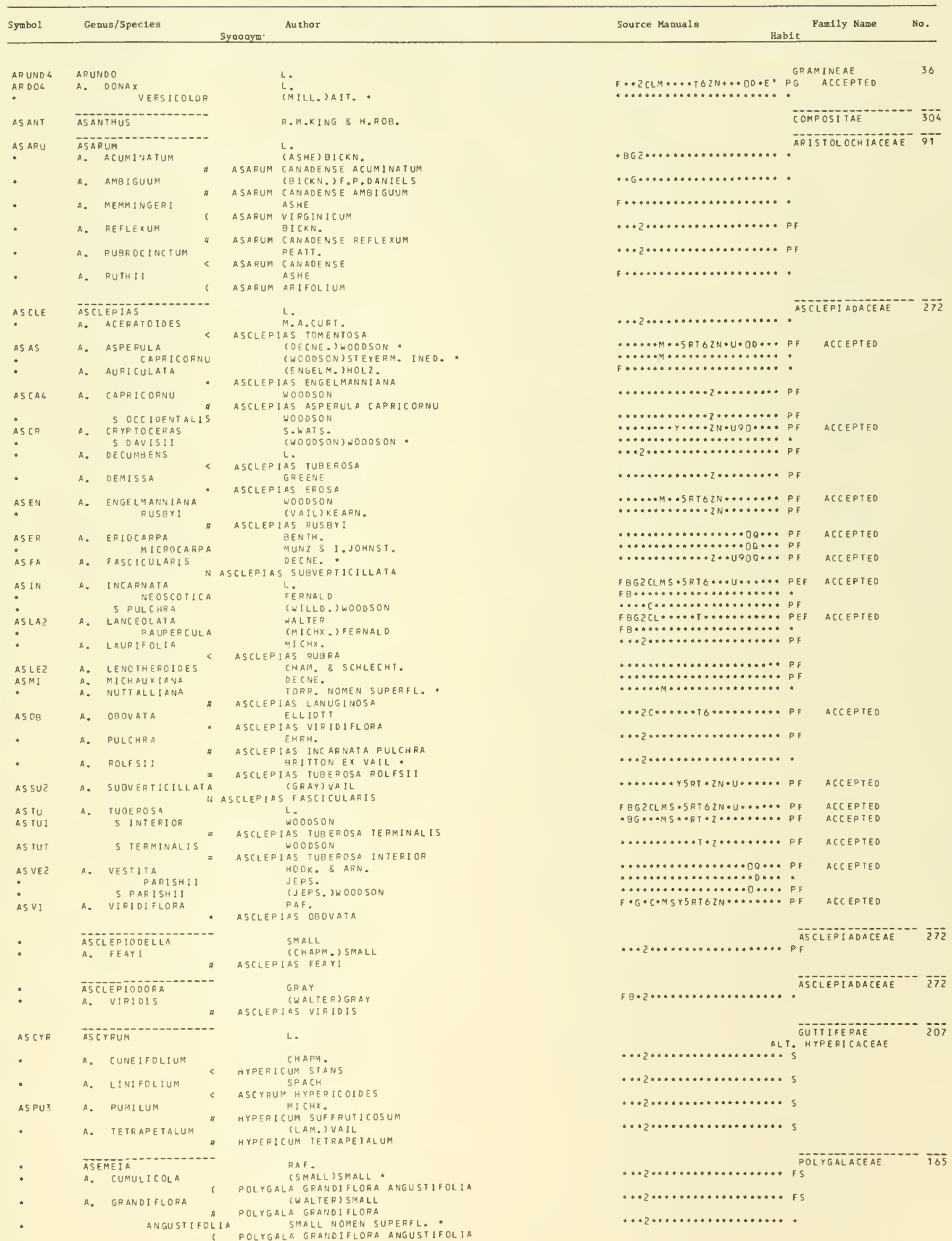




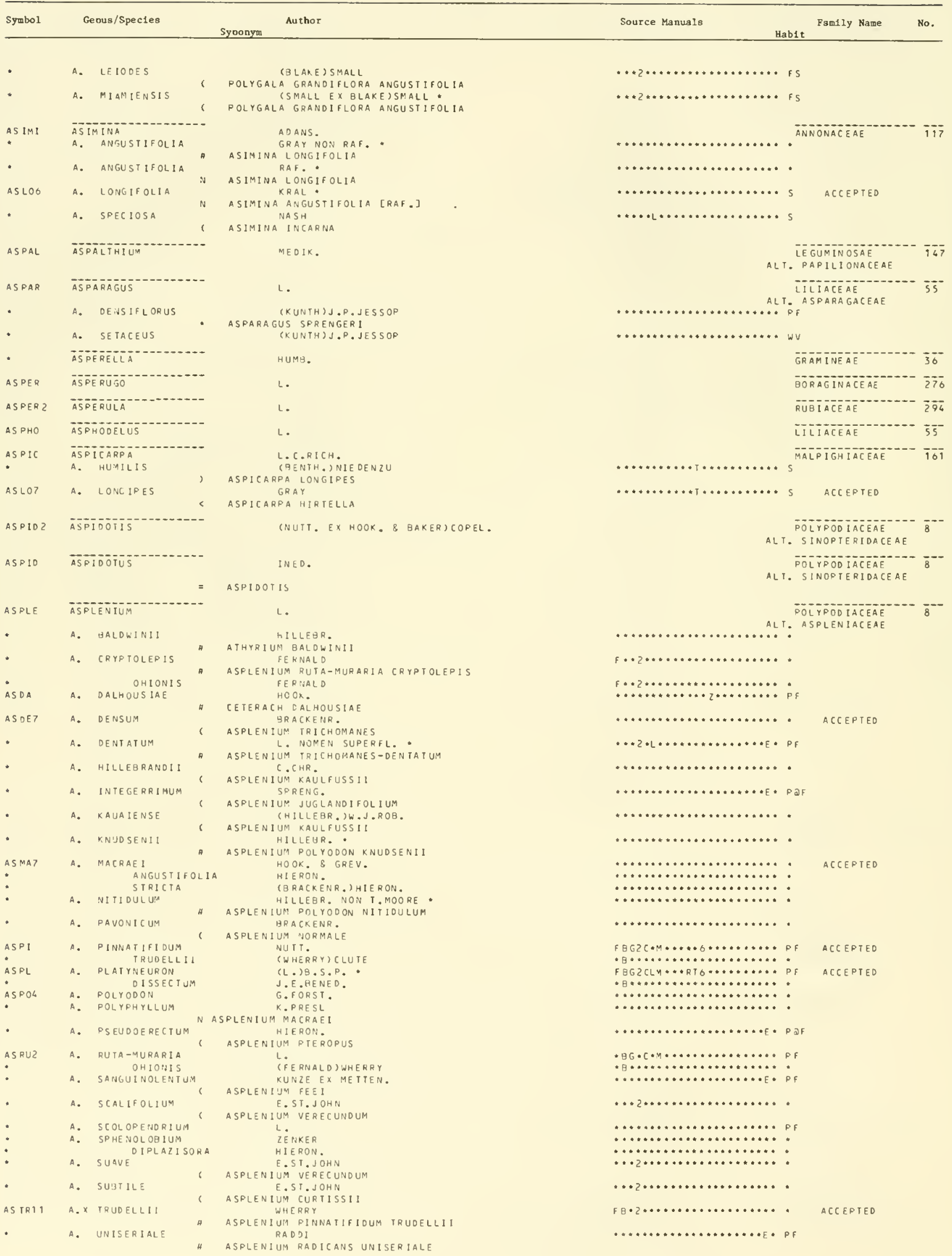




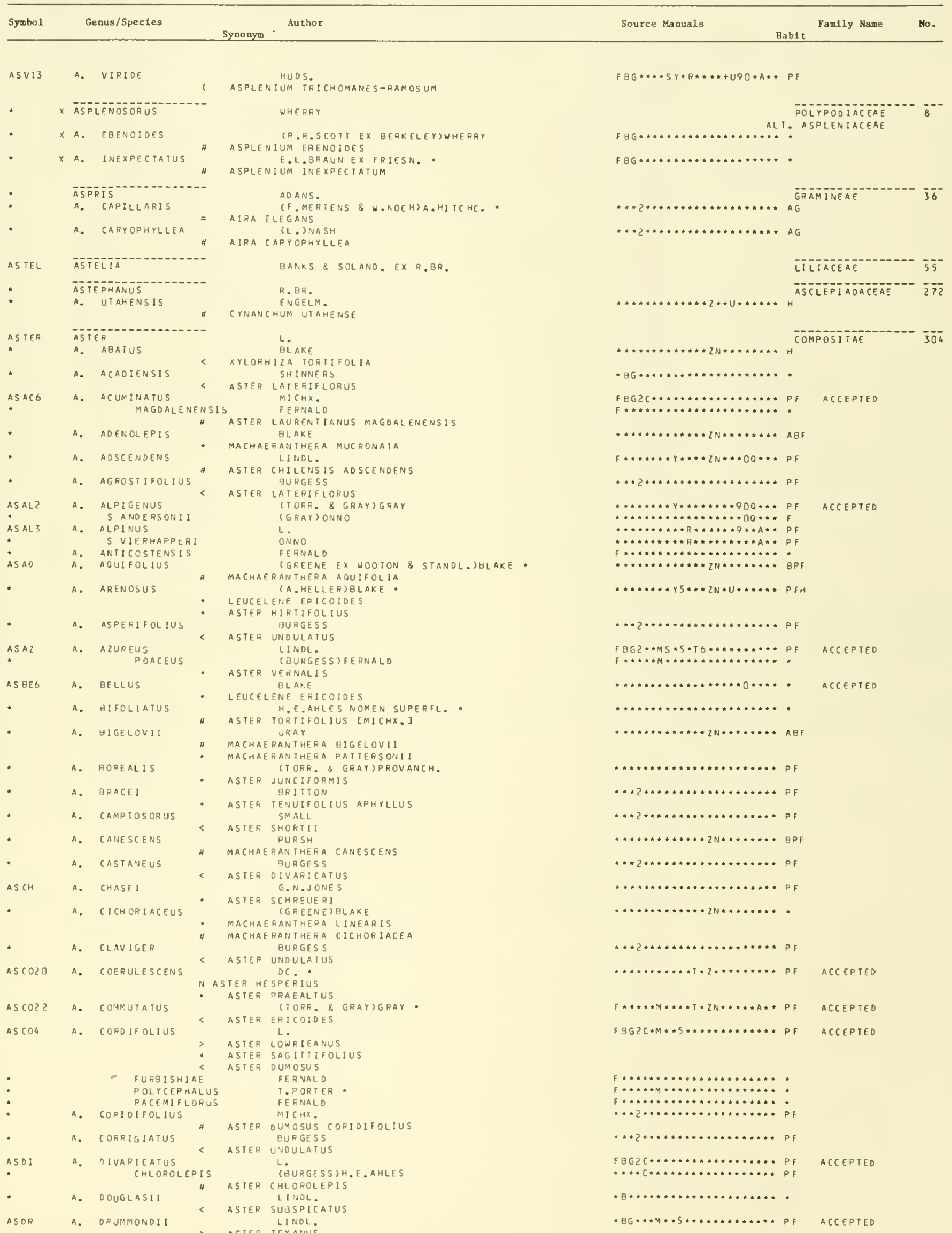




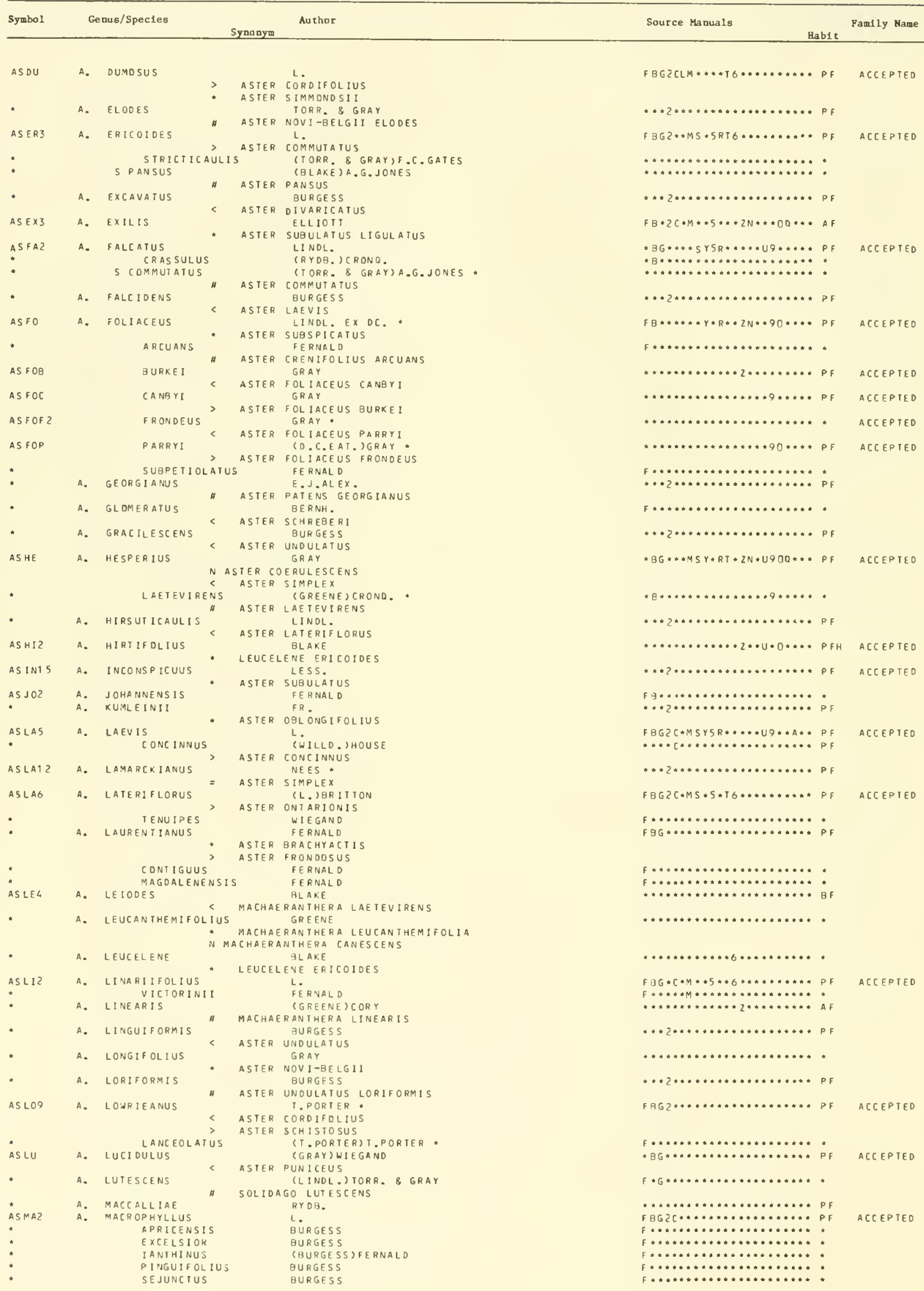




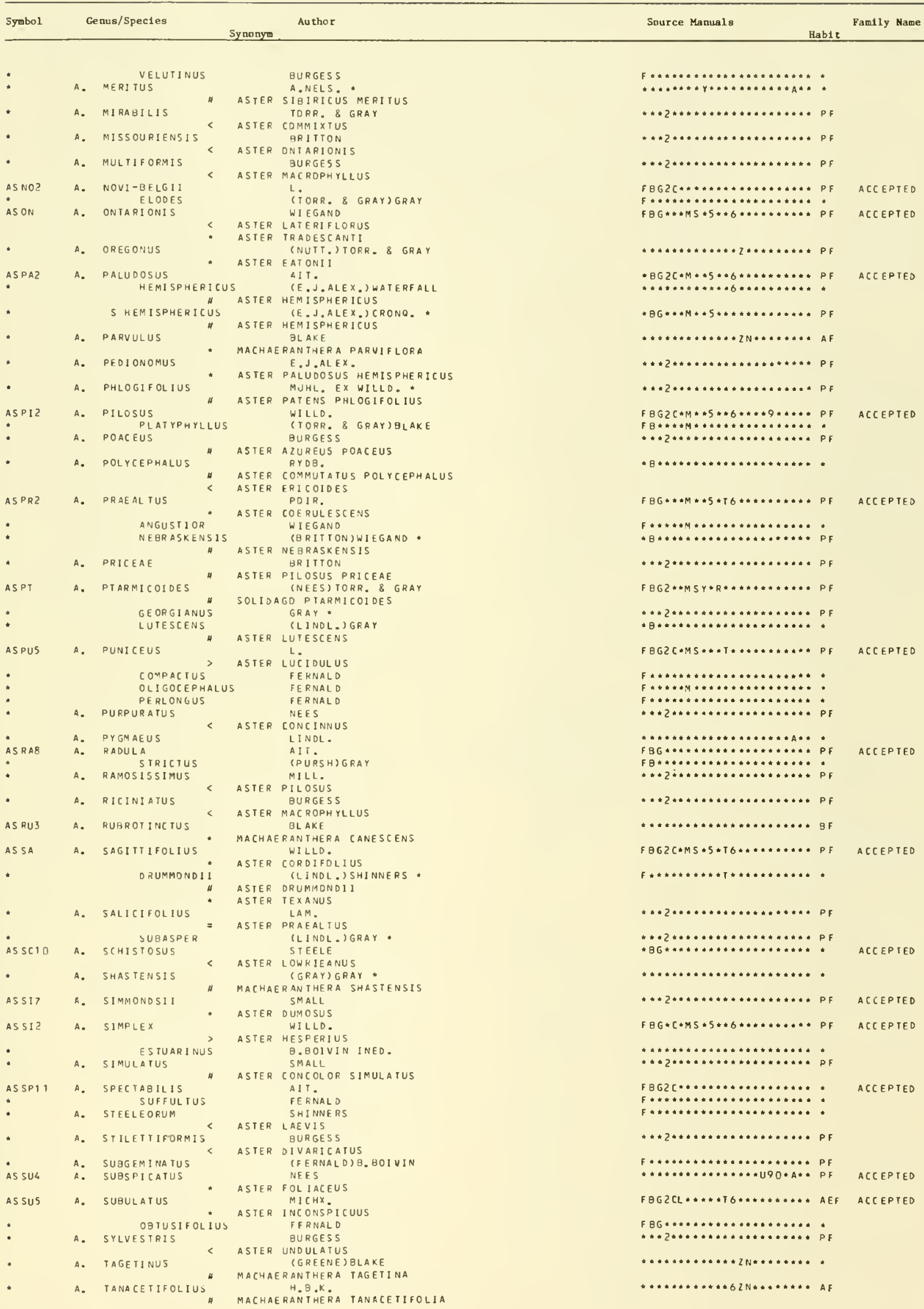




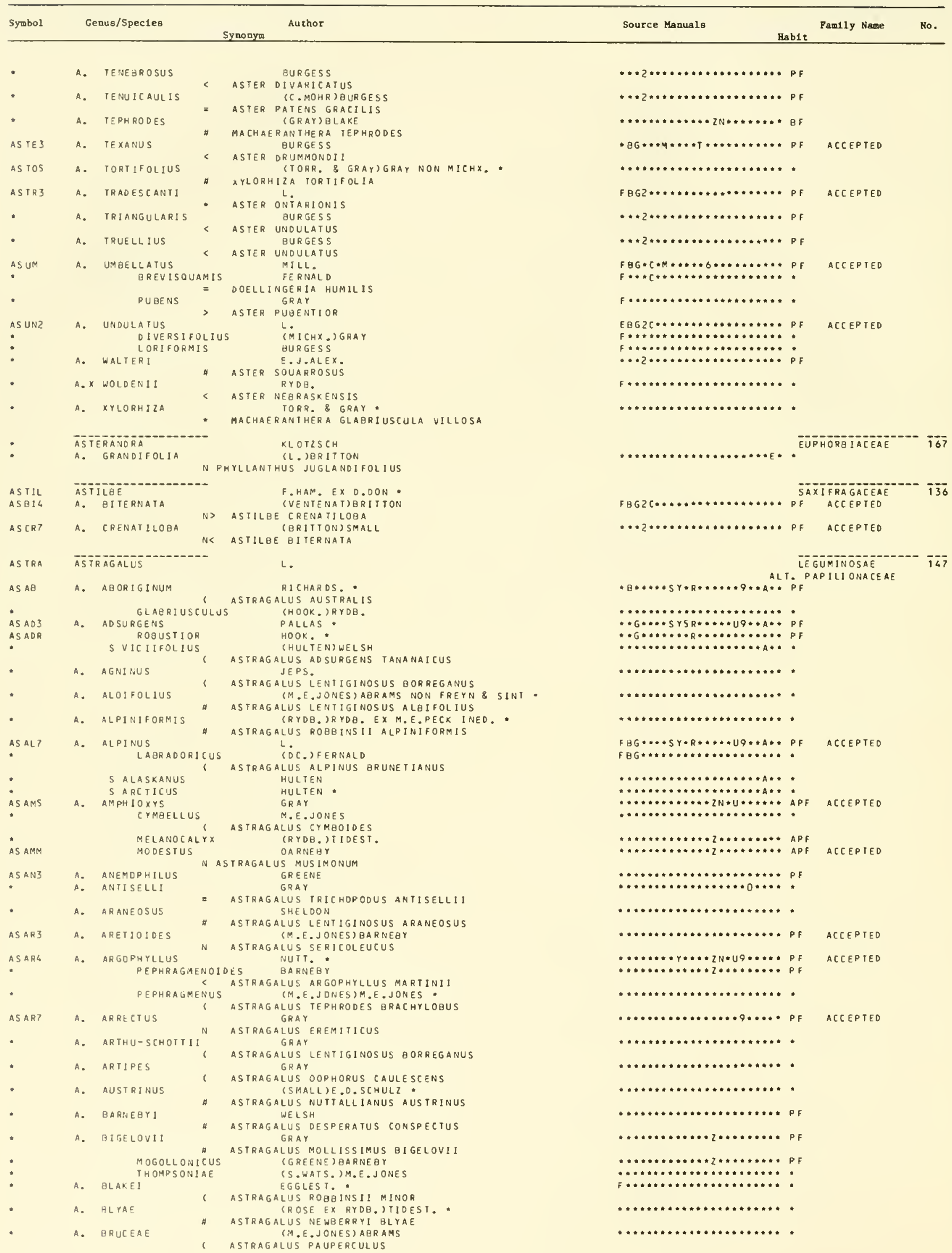




\begin{tabular}{|c|c|c|c|c|c|c|}
\hline Symba1 & & us/Spec1es & Author & Source Manuals & Family Name & Nn. \\
\hline A5 CA11 & A. & CANADENSIS & L. & $F G G * 6 * M S Y S R T G \cdots \cdots \cup O O A \cdots P F$ & ACCEPTEO & \\
\hline * & & CAROLINIANUS & (L.)M.E.JONES & $F_{\theta} \ldots \ldots \ldots \ldots \ldots \ldots \ldots \ldots \ldots$ & & \\
\hline - & & LONGILOBUS & FASSETT & 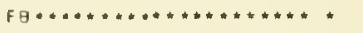 & & \\
\hline - & A. & CANOELARIUS & $\begin{array}{l}\text { SHELDON } \\
\text { ASTRAGALUS PURSHII TINCTUS }\end{array}$ & $\ldots \ldots \ldots \ldots \ldots+\cdots+\ldots \ldots+\cdots+\ldots+*$ & & \\
\hline AS CA 9 & A. & CANOVIRENS & $\begin{array}{l}\text { (RYOB. BARNEBY } \\
\text { ASTRAGALUS COLTONII MOABENS IS }\end{array}$ & $\cdots \ldots \ldots \ldots \ldots+2 \cdots \cdots \cdots F$ & ACCEPTEO & \\
\hline ASCA13 & A. & CARM IN IS & BARNEBY & $\ldots \ldots * \ldots \ldots \ldots \ldots \ldots \ldots \ldots \ldots$ PF & & \\
\hline * & A. & CAROLINIANUS & $\begin{array}{l}\text { L. } \\
\text { ASTRAGALUS CANAOENSIS CAROLINIANUS }\end{array}$ & $\cdots 2 \cdots \cdots \cdots \cdots \cdots \cdots, F$ & & \\
\hline - & A. & CARYOCARPUS & $\begin{array}{l}\text { KER-GAWL. } \\
\text { ASTRAGALUS CRASSICARPUS }\end{array}$ & $F \cdots+\cdots * \cdots \cdots+\cdots+\cdots+\cdots+* * *$ & & \\
\hline ASCAIC & A. & CASE I & $\begin{array}{l}\text { GRAY } \\
\text { TRAGALUS COLUMBIANUS }\end{array}$ & $\cdots * \ldots+\ldots \ldots \ldots \ldots \ldots 00 \ldots+$ PF & ACCEPTEO & \\
\hline $\begin{array}{l}\text { ASCA1S } \\
\text { ASCAC }\end{array}$ & A. & $\begin{array}{l}\text { CASTANE IFORMIS } \\
\text { CONSOBRINUS }\end{array}$ & $\begin{array}{l}\text { S. WAIS. } \\
\text { BARNEBY }\end{array}$ & 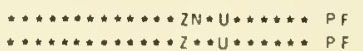 & ACCEPTED & \\
\hline & & 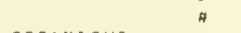 & ASTRAGALUS CONSOGRINUS & & & \\
\hline ASCE & A. & CERAMICUS & SHELDON & $\ldots \ldots+S Y 5 R=62 N * U 9 \ldots \ldots P F$ & ACCEPTEO & \\
\hline * & & IMPERFECTUS & $\begin{array}{l}\text { SHELOON NOMEN SUPERFL. } \\
\text { ASTRAGALUS CERAMICUS FILIFOLIUS }\end{array}$ & $\ldots+*+\ldots+\cdots+\cdots+\cdots+\cdots+\infty$ & & \\
\hline - & A. & CHANOONNETII & $\begin{array}{l}\text { LUNELL. } \\
\text { ASTRAGALUS ADSURGENS ROBUSTIOR }\end{array}$ & $\bullet, \ldots \ldots \ldots \ldots \ldots \ldots \ldots \ldots$ & & \\
\hline * & A. & CHLORIDAE & $\begin{array}{l}\text { (M E. J ONES) TIOEST. } \\
\text { ASTRAGALUS TEPHRODES CHLORIDAE }\end{array}$ & $\ldots \ldots+\ldots \ldots+\cdots+\cdots \ldots+\cdots+\cdots+$ & & \\
\hline - & A. & CHUCKWALLAE & $\begin{array}{l}\text { ABRAMS } \\
\text { ASTRAGALUS NUTANS }\end{array}$ & $\ldots \ldots \ldots+\cdots+\ldots+\ldots+\cdots \cdots \cdots$ & & \\
\hline - & A. & CINERASCENS & $\begin{array}{l}\text { (RYDB. TIDEST. } \\
\text { ASTRAGALUS IETRAPTERUS CINERASCENS }\end{array}$ & $\ldots \ldots+\ldots \ldots \ldots \ldots+\ldots \ldots \ldots$ & & \\
\hline As $c 09$ & A. & COLUMBIANUS N AS & $\begin{array}{l}\text { BARNEBY } \\
\text { IRAGALUS CASEI }\end{array}$ & $\ldots \ldots \ldots \ldots \ldots \ldots+\cdots \ldots+P F$ & ACCEPTED & \\
\hline * & A. & COPIFRTIFLORUS & $\begin{array}{l}\text { GRAY } \\
\text { ASTRAGALUS FLAVUS CANDICANS }\end{array}$ & $\cdots \ldots \ldots+\cdots * \ldots+2 \ldots+\ldots \ldots+$ & & \\
\hline - & & FLAVIFL URUS & $\begin{array}{l}\text { (KUNTZE) M. E. JONES } \\
\text { ASTRAGALUS FLAVUS }\end{array}$ & $\cdots+\cdots \cdots+\cdots \cdots+\cdots+\cdots+\infty$ & & \\
\hline A S $\mathrm{CO}_{2} 2$ & A. & CONVALLARIUS N & $\begin{array}{l}\text { GREENE } \\
\text { ASTRAGALUS DIVERSIFOLIUS }\end{array}$ & $\cdots \cdots \cdots \cdots \cdots+2 * \bullet \cup 9 \cdots \ldots$ PF & ACCEPTED & \\
\hline - & & FOLIOLATUS & $\begin{array}{l}\text { BARNEBY } \\
\text { ASTRAGALUS TI TANOPHILUS }\end{array}$ & $\cdots+\ldots \ldots \ldots \ldots+2 * * * * * * * * P F$ & & \\
\hline - & & XIPHOIDES & $\begin{array}{c}\text { BARNEBY } \\
\text { ASTRAGALUS XIPHOIDES }\end{array}$ & $\ldots \ldots \ldots+\ldots \pi *+2 \ldots \ldots+\ldots+P F$ & & \\
\hline * & A. & COOPERI & $\begin{array}{l}\text { GRAY NOMEN SUPERFL. } \\
\text { ASTRAGALUS NEGLECTUS }\end{array}$ & 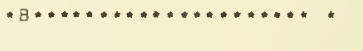 & & \\
\hline AS 5018 & A. & COTTAMII & $\begin{array}{l}\text { WELSH } \\
\text { ASTRAGALUS MONUMENTALIS }\end{array}$ & 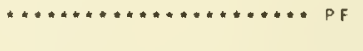 & ACCEPTED & \\
\hline - & A. & COULTERI & $\begin{array}{l}\text { BENTH. } \\
\text { ASTRAGALUS LENTIGINOSUS COULTERI } \\
\text { ASTRAGALUS LENTIGINOSUS BORREGANUS }\end{array}$ & $\cdots \cdots+\ldots \ldots \ldots+\ldots \ldots+\ldots \ldots \ldots \ldots$ & & \\
\hline - & A. & CURTILCBUS & $\begin{array}{l}\text { ASTRAGALUS LENTIGINOSUS BORREGANUS } \\
\text { TIDEST. } \\
\text { ASTRAGALUS TEPHRODES GRACHYLOEUS } \\
\text { ASTRAGALUS AMPHIOXYS }\end{array}$ & $\ldots \ldots \ldots \ldots \ldots \ldots \ldots \ldots \ldots$ & & \\
\hline * & A. & DANICUS & $\begin{array}{l}\text { RETZ: } \\
\text { ASTRAGALUS AGRESTIS }\end{array}$ & 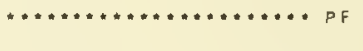 & & \\
\hline * & A. & OASYGLOTTIS & $\begin{array}{l}\text { FISCH. EX OC. NON PALLAS } \\
\text { ASTRAGALUS AGRESTIS }\end{array}$ & $\cdots \cdots \cdots \cdots R+\cdots \cdots * \cdots \cdot \cdots P F$ & & \\
\hline - & A. & DATILENSIS & $\begin{array}{l}\text { (AYDQ.) TIDEST. } \\
\text { ASTRAGALUS HUMISTRATUS }\end{array}$ & $\cdots \ldots \ldots \ldots \ldots \ldots \ldots \ldots \ldots \ldots \ldots \ldots$ & & \\
\hline * & A. & DECLMBENS & $\begin{array}{l}\text { (NUTT. EX TORR. \& GRAY)GRAY - } \\
\text { ASTRAGALUS MISER DECUMBENS }\end{array}$ & $\| n+\ldots \ldots \ldots \ldots \ldots \ldots \ldots \ldots$ & & \\
\hline * & & CRISPATUS & (M.E. JONES) CRONQ. 8 BARNEBY - & 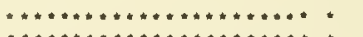 & & \\
\hline - & & DECURRENS & (RYDB.) CRONQ. & $\cdots \cdots \cdots \cdots \cdots \cdots$ & & \\
\hline * & & OBLONGIFULIUS & (RYDO.) CRONQ. & $\ldots \ldots \ldots \ldots \ldots \ldots \ldots \ldots \ldots$ & & \\
\hline * & & SEROIINUS & (GRAY)M.E.JONES - & $\cdots+\ldots \ldots \ldots \ldots \ldots \ldots \ldots+\cdots *$ & & \\
\hline AS OEZ & A. & $\begin{array}{l}\text { DESPERATUS } \\
\text { PETROPHILUS }\end{array}$ & $\begin{array}{l}\text { M.E.JONES } \\
\text { M.E.JONES }\end{array}$ & $\because \cdots \cdots \cdots \cdots \cdots+2 N \cdot U \cdots \cdots \cdots * F$ & ACCEPTED & \\
\hline ASDI3 & A. & $\begin{array}{l}\text { DIOYMOCARPUS } \\
\text { OALEOIDES }\end{array}$ & HOOK. \& ARN. & $\cdots \cdots \cdots \cdots \cdots+\cdots \cdots 00 \cdots$ AF & ACCEPTED & \\
\hline - & 4. & DIHOLCOS & $\begin{array}{l}\text { TI OEST. } \\
\text { ASTRAGALUS BISULCATUS }\end{array}$ & $\ldots \ldots \ldots \ldots \ldots \ldots+\ldots \ldots+\ldots+\cdots$ & & \\
\hline * & A. & OIPHYSUS & $\begin{array}{l}\text { GRAY } \\
\text { ASTRAGALUS LENTIGINOSUS ALgIFLORUS }\end{array}$ & $\ldots+\ldots \ldots \ldots \ldots \ldots+\ldots \ldots *$ & & \\
\hline . & A. & DISPERMUS & $\begin{array}{l}\text { GRAY } \\
\text { ASTRAGALUS DIOYMOCARPUS OISPERMUS }\end{array}$ & $\ldots \ldots \ldots \ldots \ldots \ldots \ldots \ldots \ldots \ldots$ & & \\
\hline • & A. & DIURNUS & $\begin{array}{l}\text { S. WATS } \\
\text { ASTRAGALUS DIAPHANUS }\end{array}$ & $\ldots \ldots \ldots \ldots \ldots \ldots \ldots \ldots \ldots$ & & \\
\hline AS DIS & A. & DIVERSIFOLIUS N & $\begin{array}{l}\text { GRAY } \\
\text { ASTRAGALUS CONVALLARIUS }\end{array}$ & $\cdots \cdots \cdots \cdots r \cdots \cdots \cdots * U S * \cdots * P F$ & ACCEPTED & \\
\hline • & A. & DREFANOLOBUS & $\begin{array}{l}\text { GRAY } \\
\text { ASTRAGALUS DIAPHANUS }\end{array}$ & 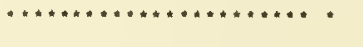 & & \\
\hline ASEG & A. & EGGLESTONII & $\begin{array}{l}\text { (RYDE. KEARN. \& PEEB. } \\
\text { ASTRAGALUS RUSBY! }\end{array}$ & $\ldots \ldots \ldots \ldots \ldots+2 N \cdots * * \ldots * P F$ & ACCEPTED & \\
\hline AS EP & A. & EPISCOPUS & $\begin{array}{l}\text { S. WATS. } \\
\text { ASTRAGALUS LANCEARIUS }\end{array}$ & $\cdots * \cdots * \cdots \cdots * n \cdot U \cdot \ldots \ldots * P F$ & ACCEPTEO & \\
\hline ASERG & A. & EREM ITICUS & $\begin{array}{l}\text { SHELDON } \\
\text { ASTRAGALUS }\end{array}$ & $\ldots \ldots \ldots \ldots \ldots+2 N \cdot U q \ldots \ldots \cdot P F$ & ACCEPIED & \\
\hline - & & MALHESRENSIS & $\begin{array}{l}\text { (A. HELLER) BARNEBY } \\
\text { ASTRAGALUS CUSICKII }\end{array}$ & $\ldots \ldots \ldots \ldots \ldots \ldots \ldots \ldots \ldots \ldots$ & & \\
\hline - & & SPENCIANUS & 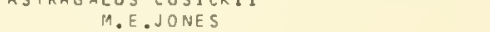 & $\ldots \ldots \ldots \ldots \ldots \ldots \ldots \ldots \ldots+\ldots *$ & & \\
\hline AS EU2 & A. & Eucosmus & B. คOB. & $F B G \cdots * \cdots Y \cdot R \cdots \cdots \cdots q \cdots A \cdots P F$ & ACCEPTED & \\
\hline * & & FAC I NOR UIA & FERNALD & $F+\ldots \ldots \ldots \ldots \ldots \ldots \ldots \ldots \ldots$ & & \\
\hline - & A. & FAMELICUS & $\begin{array}{l}\text { SHELOON } \\
\text { ASTRAGALUS HALLII FALLAX }\end{array}$ & $\cdots \cdots \cdots+\cdots+\cdots, 2+\ldots \cdots \cdots+P F$ & & \\
\hline - & A. & FERN AL D I 1 & $\begin{array}{c}\text { (R YOB. ) H. F. LEWIS } \\
\text { ASTRAGALUS ROBGINSII FERNALOII }\end{array}$ & $f \cdots \ldots \ldots+\ldots \ldots \ldots+\ldots \ldots$ & & \\
\hline AS FL2 & A. & FLEXUOSUS & (HOOK.)DOUGL. EX G.DON & 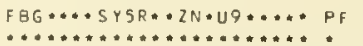 & ACGEPTED & \\
\hline : & A. & $\begin{array}{l}\text { SIERRAE-BLAN } \\
\text { FORWOODII }\end{array}$ & $\begin{array}{ll}\text { AE } & \text { SRYDB. BBARNEBY " } \\
& \text { S.WATS. }\end{array}$ & $\ldots+\ldots+\ldots+\cdots$ & & \\
\hline . & & WALLOWENSIS & (RYDB. )M.E. PECK & \#... & & \\
\hline
\end{tabular}




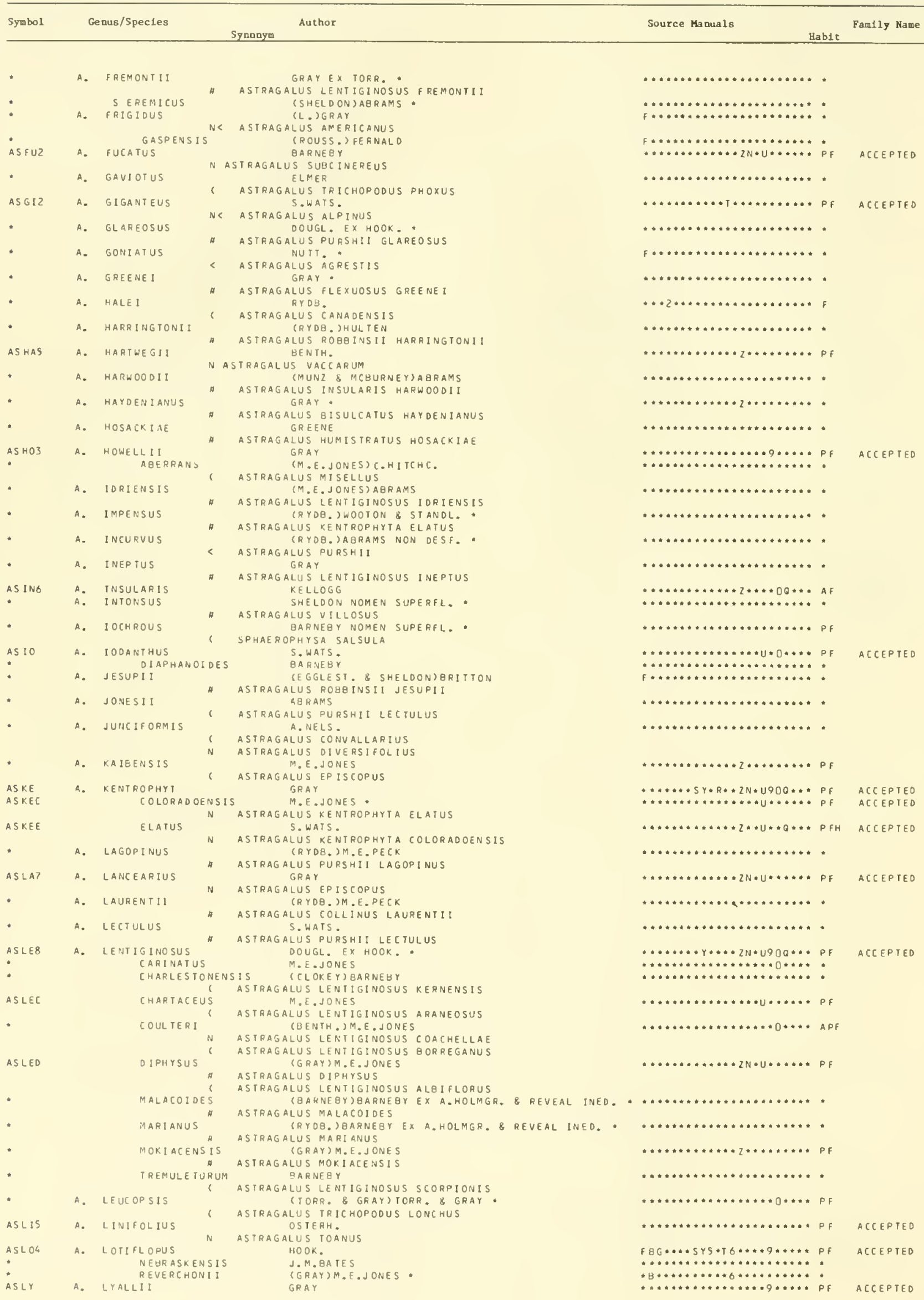


ASTRAGALUS

\begin{tabular}{|c|c|c|c|c|c|c|}
\hline Symbol & & nua/Speciea & Author & Source Manuals & Family Name & No. \\
\hline$\cdot$ & & CARICINUS & M.E.JONES & $\ldots \ldots \ldots \ldots \ldots \ldots \ldots \ldots \ldots \ldots$ & & \\
\hline & & यAcosuc & ASTRAGALUS CARICINUS & & & \\
\hline - & A. & if ACDOU GALII & $\begin{array}{l}\text { SHELOON } \\
\text { ASTRAGALUS LENTIGINOSUS OIPHYSUS }\end{array}$ & 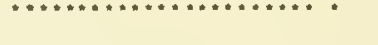 & & \\
\hline$\cdot$ & A. & MACGREGORII & $\begin{array}{l}\text { (RYOB. TIOEST. } \\
\text { ASTRAGALUS FILIPES }\end{array}$ & $\ldots \ldots \ldots \ldots \ldots \ldots \ldots \ldots \ldots \ldots$ & & \\
\hline - & A. & MACOUN II & $\begin{array}{l}\text { RYOB. } \\
\text { ASTRAGALUS ROBEINS I: MINOR }\end{array}$ & $\ldots+\ldots+\ldots+\cdots \cdots \cdots \ldots \ldots+$ & & \\
\hline AS MAL & A. & $\begin{array}{l}\text { MAGDALENAE } \\
\text { NIVEUS }\end{array}$ & $\begin{array}{l}\text { GREENE } \\
\text { (RYDB. BARNEBY }\end{array}$ & $\because \cdots \cdots \cdots \cdots \cdots \cdots \cdots \cdots 0 Q \cdots P F$ & & \\
\hline ASMAN & & $\begin{array}{l}\text { NIVEUS } \\
\text { MATTHE WS I }\end{array}$ & $\begin{array}{l}\text { (RYDB. ) BARNEBY } \\
\text { ASTRAGALUS NIVEUS } \\
\text { S. WATS. }\end{array}$ & $\ldots \ldots \ldots \ldots \ldots \ldots+\ldots \ldots+\ldots b^{2}$ & & \\
\hline * & A. & MATTHE WSII & $\begin{array}{l}\text { S. 'AATS. } \\
\text { ASTRAGALUS MOLLISSIMUS MATTHEWSII }\end{array}$ & 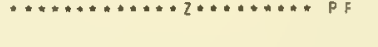 & & \\
\hline - & A. & MENSANUS & $\begin{array}{l}\text { (M. E. JONES) ABRAMS } \\
\text { ASTRAGALUS ATRATUS MENSANUS }\end{array}$ & $\ldots \ldots \ldots * \ldots \ldots+\cdots * \cdots \ldots \ldots * * *$ & & \\
\hline - & A. & MENZIESII & $\begin{array}{l}\text { GRAY NOMEN SUPERFL. } \\
\text { ASTRAGALUS NUTTALLII }\end{array}$ & 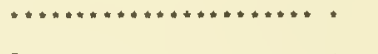 & & \\
\hline * & A. & MEXICANUS & $\begin{array}{l}\text { A.OC. } \\
\text { ASTRAGALUS CRASSICARPUS BERLANDIERI }\end{array}$ & $F \cdots \cdots+\cdots \cdots \cdots \cdots \cdots \cdots$ & & \\
\hline • & & TRICHOCALYX & (NUTT.)FERNALD & $F \cdots \cdots \cdots n+\cdots \cdots+\cdots \cdots \cdots \cdots \cdots$ & & \\
\hline $\begin{array}{l}\text { ASM17 } \\
\text { ASN1G }\end{array}$ & A. & $\begin{array}{l}\text { MINTHOR INIAE } \\
\text { GRACILIOH }\end{array}$ & $\begin{array}{l}\text { (RYOB. ) JEPS. } \\
\text { (BARNEBY) OARNEBY }\end{array}$ & 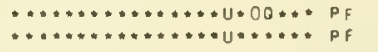 & $\begin{array}{l}\text { ACCEPTED } \\
\text { ACCEPTED }\end{array}$ & \\
\hline$\cdot$ & A. & MORTONII & $\begin{array}{l}\text { ASTRAGALUS ENSIFORMIS } \\
\text { NUTY. } \\
\text { ASIRAGALUS CANADENSIS MORTONI! }\end{array}$ & $\ldots \ldots \ldots \ldots \ldots \ldots \ldots \ldots \ldots \ldots+$ & & \\
\hline As sur & A. & MUSIMONUM & $\begin{array}{l}\text { BARNEBY } \\
\text { ASTRAGALUS AMPHIOXYS MODESTUS }\end{array}$ & $\cdots \cdots \cdots \cdots \cdots+\cdots \cdots \cdots \cdots+P F$ & ACCEPTED & \\
\hline ASNE3 & A. & NELS ON! ANUS & $\begin{array}{l}\text { BARNEBY } \\
\text { ASTRAGALUS PECTINATUS }\end{array}$ & $\cdots \ldots \ldots \ldots \ldots \ldots \cdots \cup \ldots \ldots$ PF & ACCEPTED & \\
\hline ASNEO & A. & $\begin{array}{l}\text { NEWEERRYI } \\
\text { WAROIANUS }\end{array}$ & $\begin{array}{l}\text { GRAY } \\
\text { BARNEBY }\end{array}$ & $\cdots \ldots \cdots \cdots \cdots \cdots+2 N * 4900 * \cdots P F$ & ACCEPTED & \\
\hline & & a & ASTRAGALUS LOANUS & & & \\
\hline$\cdot$ & A. & NIGRICALYCIS & $\begin{array}{l}\text { (M. E. JONES) ABRAMS } \\
\text { ASTRAGALUS LENTIGINOSUS NIGRICALYGIS }\end{array}$ & $* * \cdots+\cdots+\ldots+\cdots+\cdots+\ldots \ldots+$ & & \\
\hline - & A. & NIVEUS & $\begin{array}{l}\text { (RYOQ.) BARNEBY } \\
\text { ASTRAGALUS MAGDALENAE PEIRSONII }\end{array}$ & 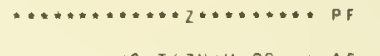 & & \\
\hline $\begin{array}{l}\text { ASNUL } \\
\text { AS NUT }\end{array}$ & A. & $\begin{array}{l}\text { NUTTALLIANUS } \\
\text { TRICHOCARP }\end{array}$ & US OC." \& GRAY & 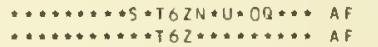 & $\begin{array}{l}\text { ACCEPTED } \\
\text { ACCEPTED }\end{array}$ & \\
\hline & & (1) & ASTRAGALUS NUTTALLIANUS AUSTRINUS & & & \\
\hline * & A. & OCCIDENTALIS & $\begin{array}{l}\text { (S. WATS.)M.E. JONES } \\
\text { ASTRAGALUS ROBBINSII OCCIDENTALIS }\end{array}$ & $\cdots \cdots \cdots \cdots+\cdots \cdots \cdots+\cdots \cdots+\cdots$ & & \\
\hline * & A. & OPHIOGENES & $\begin{array}{l}\text { BARNEOY } \\
\text { ASIRAGALUS PURSHII OPHIOGENES }\end{array}$ & $\cdots+\cdots+\cdots+\cdots+\cdots+\cdots+\cdots+\cdots *$ & & \\
\hline - & A. & OWYHEENSIS & $\begin{array}{l}\text { A.NELS. \& J. F. MACBR. } \\
\text { ASTRAGALUS ATRATUS OWYHEENS IS }\end{array}$ & $\cdots \cdots+\ldots \ldots+\ldots \ldots \ldots \ldots \ldots+\cdots$ & & \\
\hline - & A. & PALANS & $\begin{array}{l}\text { M. E.JONES } \\
\text { ASTRAGALUS LENTIGINOSUS PALANS }\end{array}$ & $\cdots+\cdots \cdots \cdots \cdots \cdots \cdots+\cdots$ & & \\
\hline - & A. & PANGUICENSIS & $\begin{array}{l}\text { (M. E.JONES)M. E. JONES } \\
\text { ASTRAGALUS ARGOPHYLLUS PANGUICENSIS }\end{array}$ & $\cdots \cdots \cdots+\cdots+\cdots+\cdots \cdots \cdots \cdots+$ & & \\
\hline - & A. & PARISH II & $\begin{array}{l}\text { GRAY } \\
\text { ASTRAGALUS DOUGLASII PARISHII }\end{array}$ & $\cdots+\cdots+\cdots+\ldots+\cdots+\cdots+\cdots+$ & & \\
\hline - & A. & PARVIFLORUS & $\begin{array}{l}\text { (PURSH)MACMIL. NON LAM.* } \\
\text { ASTRAGALUS GRACILIS } \\
\text { ASTRAGALUS EUCOSMUS }\end{array}$ & $\ldots \ldots \ldots \ldots+\ldots \ldots+\ldots+\ldots+\cdots$ & & \\
\hline ASPA14 & A. & $\begin{array}{l}\text { PATTERSONII } \\
\text { PRAELONGUS }\end{array}$ & $\begin{array}{l}\text { C ASTRAGALUS EUCOSMUS } \\
\text { GRAY. } \\
\text { (SHELOON)M.E.JONES NOMEN SUPERFL. * }\end{array}$ & $\because \cdots \cdots \cdots \cdots+\cdots 2 N \cdot U \cdots \cdots+P F H$ & ACCEPTEO & \\
\hline ASPES & A. & PECTINATUS & $\begin{array}{l}\text { ASTRAGALUS PRAELONGUS } \\
\text { (HOOK. OOOUGL. EX G.OON * } \\
\text { ASTRAGALUS NELSONIANUS }\end{array}$ & $\ldots \ldots \ldots 5 \times 5 \ldots \ldots * 0,0 * *$ PF & ACCEPTED & \\
\hline - & A. & PE IRSONII & $\begin{array}{l}\text { ASTRAGALUS NELSONIANUS } \\
\text { MUNZ \& MCBURNEY } \\
\text { ASTRAGALUS MAGOALENAE PEIRSONII }\end{array}$ & $\ldots \ldots \ldots+\ldots \ldots \ldots \ldots+\ldots+*$ & & \\
\hline * & A. & PEPHRAGMENUS & $\begin{array}{l}\text { M. E. JONES } \\
\text { ASTRAGALUS TEPHRODES ERACHYLOSUS }\end{array}$ & $\ldots \ldots \ldots \ldots \ldots \ldots \ldots \ldots \ldots$ & & \\
\hline - & A. & PICTUS & $\begin{array}{l}\text { (GRAY)GRAY NON BOISS. B GALLLARDOT } \\
\text { ASTRAGALUS CERAMICUS }\end{array}$ & $\ldots+\cdots+\ldots \ldots+2 \ldots \ldots+\cdots+$ & & \\
\hline ASPL? & & ANGUSTUS & M.E.JONES & $\ldots \ldots \ldots \ldots \ldots \ldots+2 \ldots \ldots \ldots+P_{P F}$ & & \\
\hline ASPL? & A. & PLATTENSIS & $\begin{array}{l}\text { NUTT. } \\
\text { ASTRAGALUS TENIESSEENSIS }\end{array}$ & 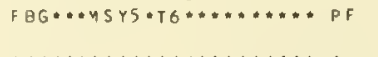 & ACCEPTEO & \\
\hline$*$ & A. & PLUMBEUS & $\begin{array}{l}\text { BaRnEB NON GONiSCH. } \\
\text { astragalus MOLYBOENUS }\end{array}$ & 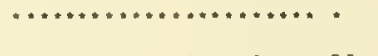 & & \\
\hline ASPRG & A. & $\begin{array}{l}\text { PREUSSI ! } \\
\text { LATUS }\end{array}$ & $\begin{array}{l}\text { GRAY } \\
\text { M.E.JONES }\end{array}$ & $\because \cdots * \cdots \cdots \cdots * * 2 N * U * 0 Q * \cdots P F F$ & ACCEPIEO & \\
\hline & & N & $\begin{array}{l}\text { ASTRAGALUS PRAELONGUS LONCHOPUS } \\
\text { ASTRAGALUS LENTIGINOSUS LATUS }\end{array}$ & & & \\
\hline AS PUO & A. & PURSHII & DOUGL. EX HOOK. & 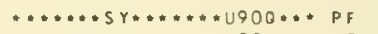 & ACCEPTED & \\
\hline ASPUL & & LAGOPINUS & $\begin{array}{l}\text { (RYDB.) BARNEBY } \\
\text { ASTRAGALUS PURSHI! LECTULUS }\end{array}$ & $\ldots \ldots \ldots \ldots \ldots \cdots \cdots 90 \cdots \cdots P F$ & ACC EPTEO & \\
\hline AS PUL Z & & LECTULUS NS & $\begin{array}{l}\text { ASTRAGALUS PURSHT LECTULUS } \\
\text { (S. WATS.)M.E.JONES } \\
\text { S ASTRAGALUS PURSHII LAGOP!NUS }\end{array}$ & $\ldots \ldots \ldots \ldots \ldots \cdots 00 \cdots P F$ & ACCEPTED & \\
\hline * & & $\begin{array}{r}\text { LONGILOAUS } \\
6=\end{array}$ & $\begin{array}{l}\quad \text { ASTRAGALUS PURSHII LAGOPINUS } \\
\text { M. E.JONES } \\
=\text { ASTRAGALUS PURSHII TINETUS }\end{array}$ & $\ldots \ldots \ldots \ldots \ldots \ldots \ldots \ldots \ldots$ & & \\
\hline - & A. & RECEDENS & $\begin{array}{l}\text { (RYOB, C.L.PORTER } \\
\text { ASTRAGALUS PRAELONGUS }\end{array}$ & $\ldots \ldots \ldots+\ldots+\ldots \ldots \ldots+\ldots \ldots$ & & \\
\hline - & A. & REMULCUS & $\begin{array}{l}\text { ASTRAGALUS TEPHRODES BRACHYLOBUS } \\
\text { M. JONES }\end{array}$ & $+\ldots \ldots+\cdots+\cdots+\ldots \ldots \ldots$ & & \\
\hline - & & CHLORIOAE & $\begin{array}{l}\text { M. E. JONES } \\
\text { ASTRAGALUS TEPHRODES CHLORIDAE }\end{array}$ & $\ldots \ldots \ldots \ldots+\cdots \ldots \ldots \ldots+p^{2}$ & & \\
\hline AS RE 5 & A. & REVENTUS & GRAY Th Th & $\ldots \ldots \ldots \ldots \ldots \ldots, q \ldots \ldots P F$ & ACEEPTED & \\
\hline- & & CONJUNGTUS & $\begin{array}{l}\text { (S.WATS.) M. E, JONES } \\
\text { ASTRAGALUS CONJUNCTUS }\end{array}$ & $\ldots \ldots \ldots \ldots \ldots \ldots+\ldots+\ldots+\cdots$ & & \\
\hline$\cdot$ & & SHELDONI:" & $\begin{array}{l}\text { (RYOB.) C.HITCHC. } \\
\text { ASTRAGALUS SHELOONII }\end{array}$ & $\cdots+\cdots+\cdots+\ldots+\ldots \ldots+\ldots+\cdots$ & & \\
\hline AS RO & A. & ROAB 1 NS 11 & $\begin{array}{l}\text { ASTRAGALOS SHE LORAY } \\
\text { (OAKES GRAY }\end{array}$ & $F$ F $\cdots \cdots Y \cdots \cdots \cdots * Q * * A * P F$ & ACLEPTED & \\
\hline - & & QLAKEI & $\begin{array}{l}\text { (EGGLEST.) BARNEBY } \\
\text { ASTRAGALUS ROBBINS II MINOR }\end{array}$ & $\theta G \ldots \ldots \ldots \ldots \ldots \ldots \ldots \ldots \ldots$ & & \\
\hline & & $\sharp$ & ASTRAGALUS BLAKE: & & & \\
\hline * & & FERNALOII & ( $R$ YOB.) BARNEBY & $\cdots * * \cdots \cdots \cdots * * \cdots * P F$ & & \\
\hline ASROM & & MINOR N & $\begin{array}{l}\text { (HOOK.)BARNEBY } \\
\text { ASIRAGALUS ROBBINSII OCEIOENTALIS }\end{array}$ & $\cdots \cdots+\ldots \ldots \ldots+\cdots, q * A * P F$ & ACCEPTED & \\
\hline
\end{tabular}




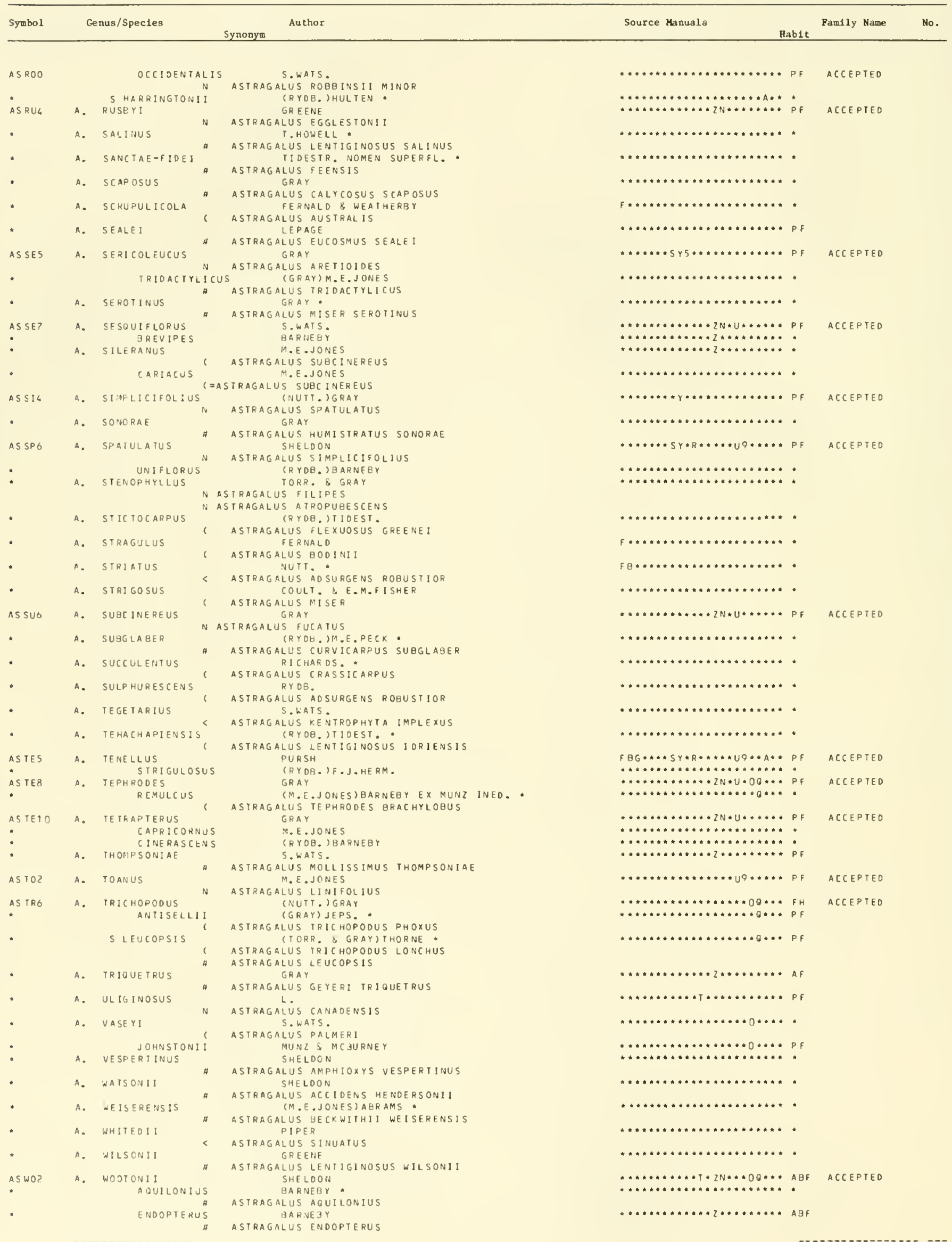


ASTRANTHIUM

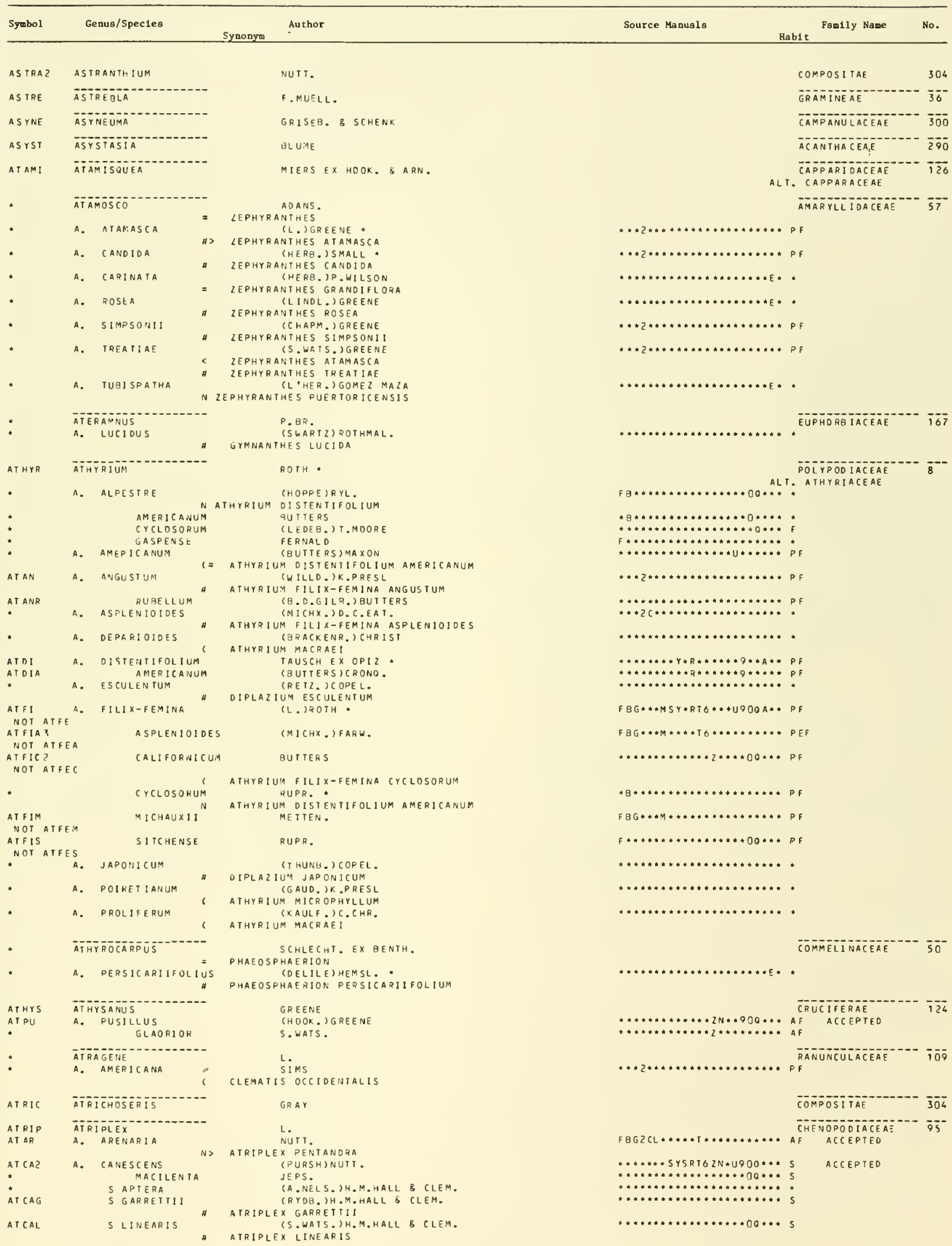




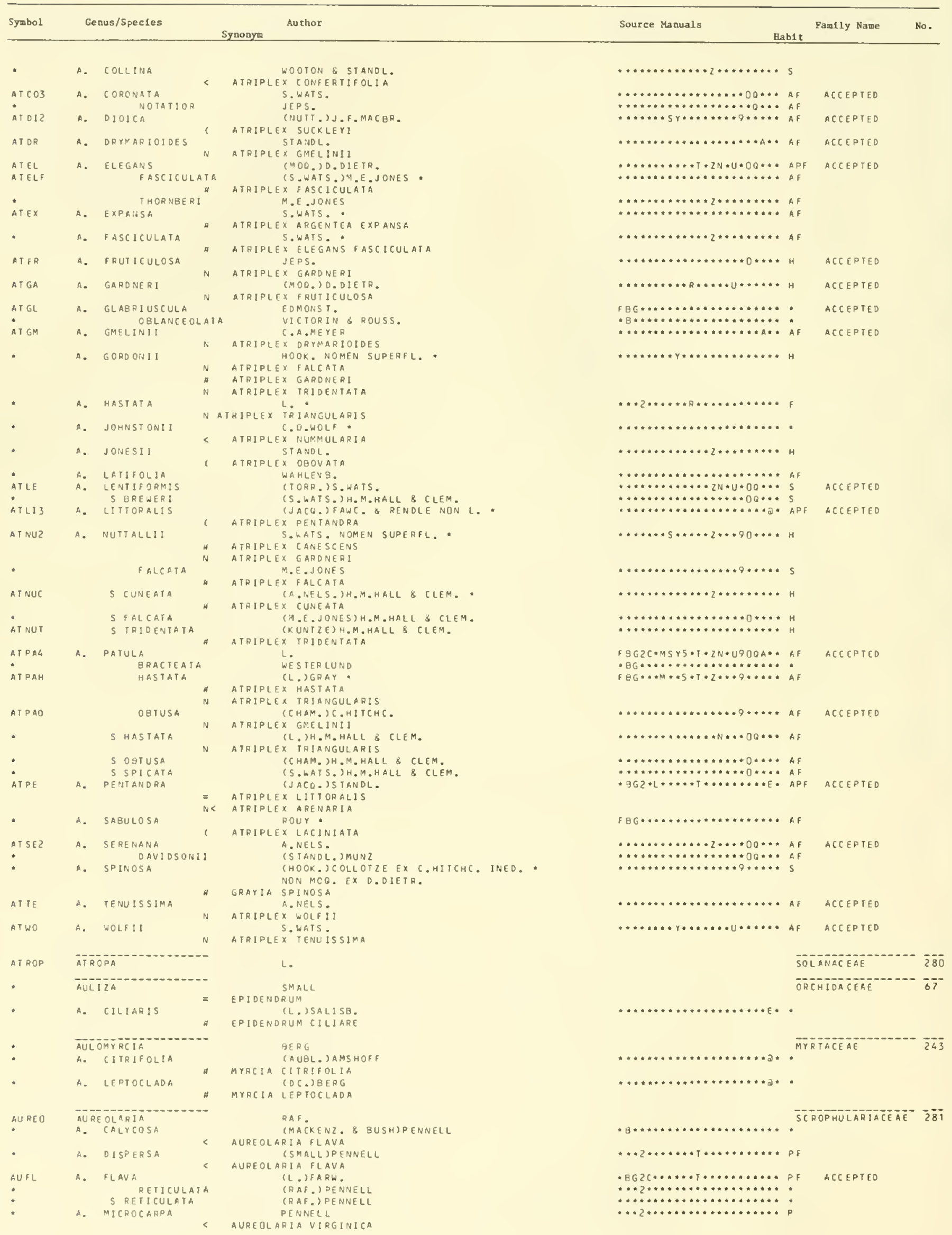




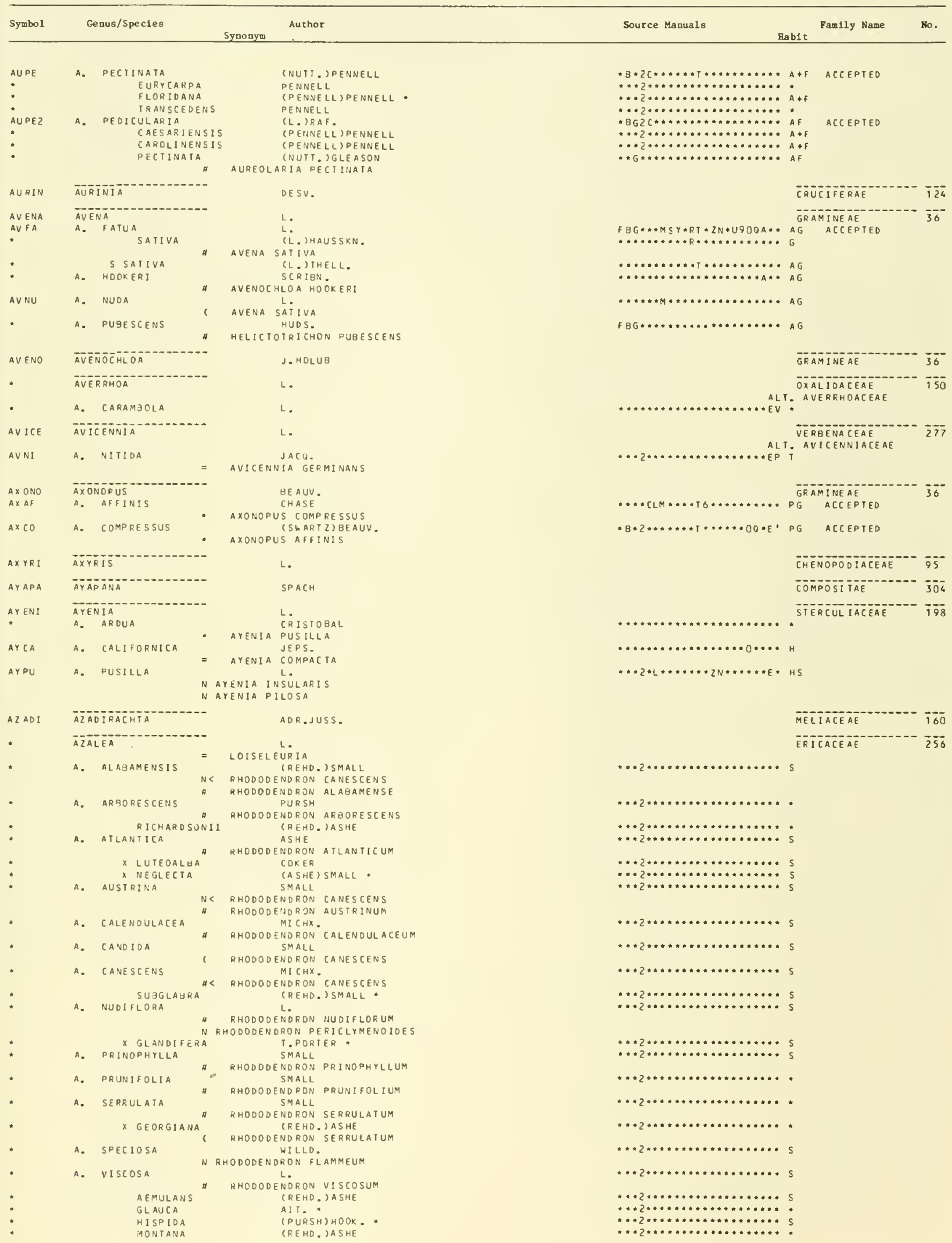




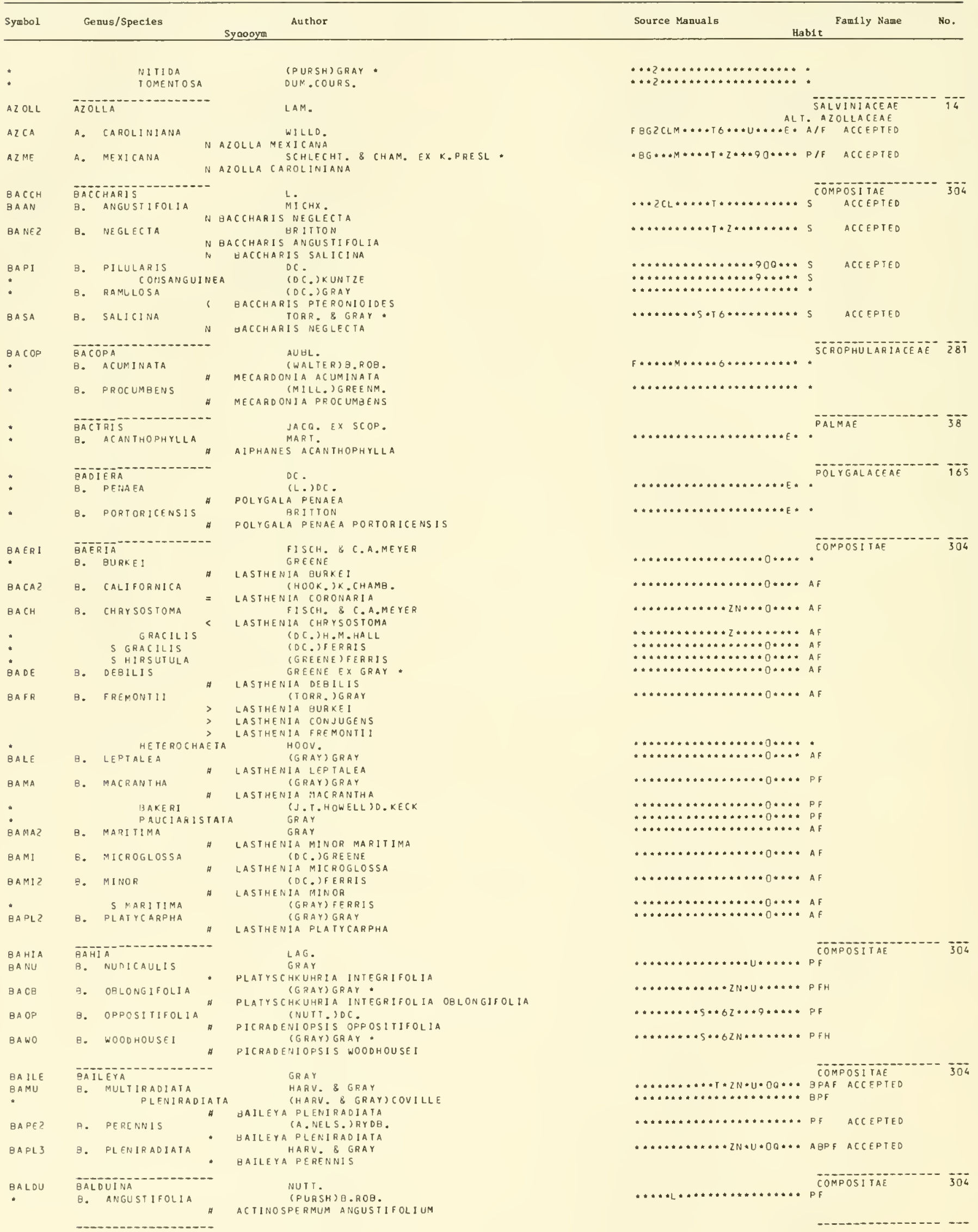




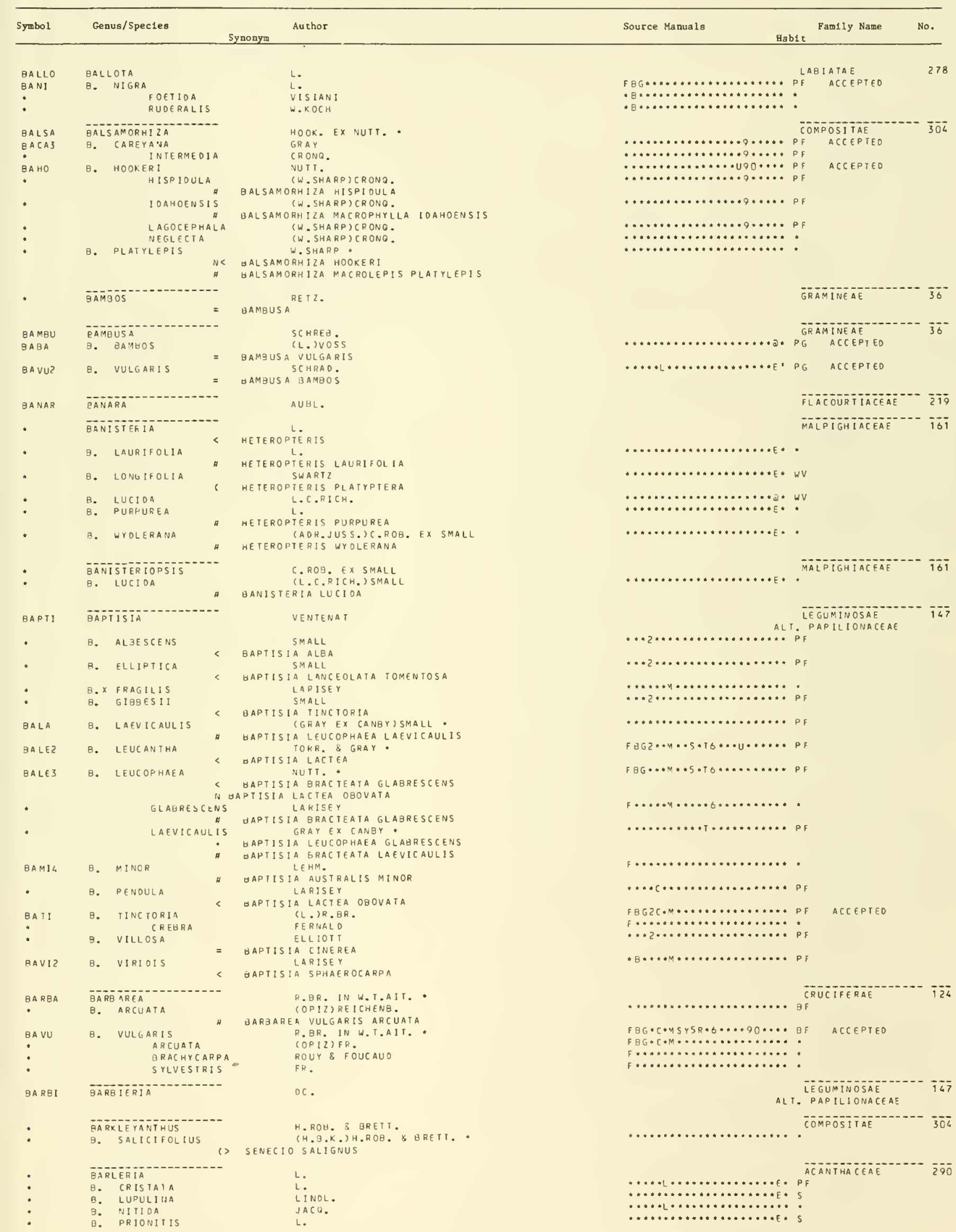




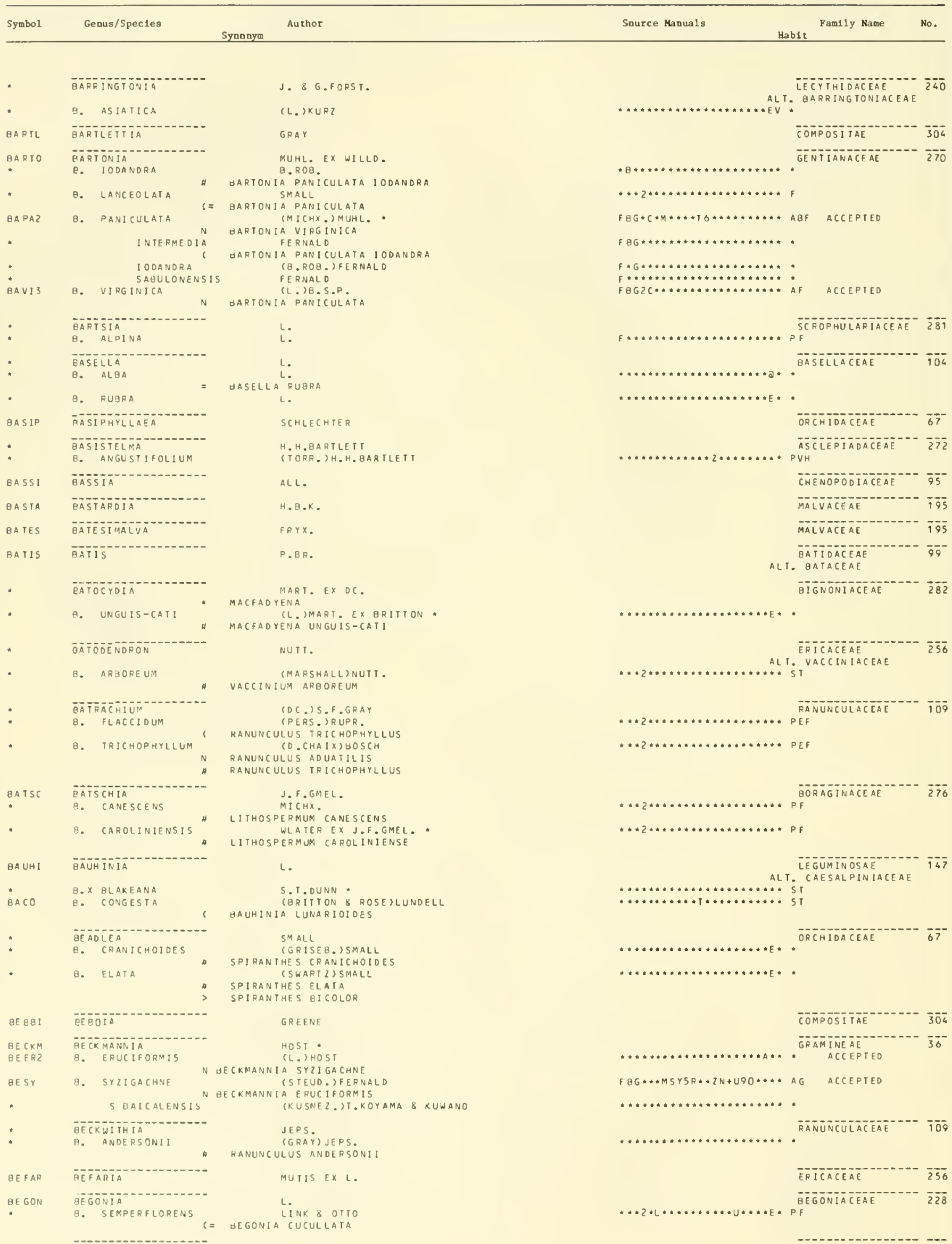




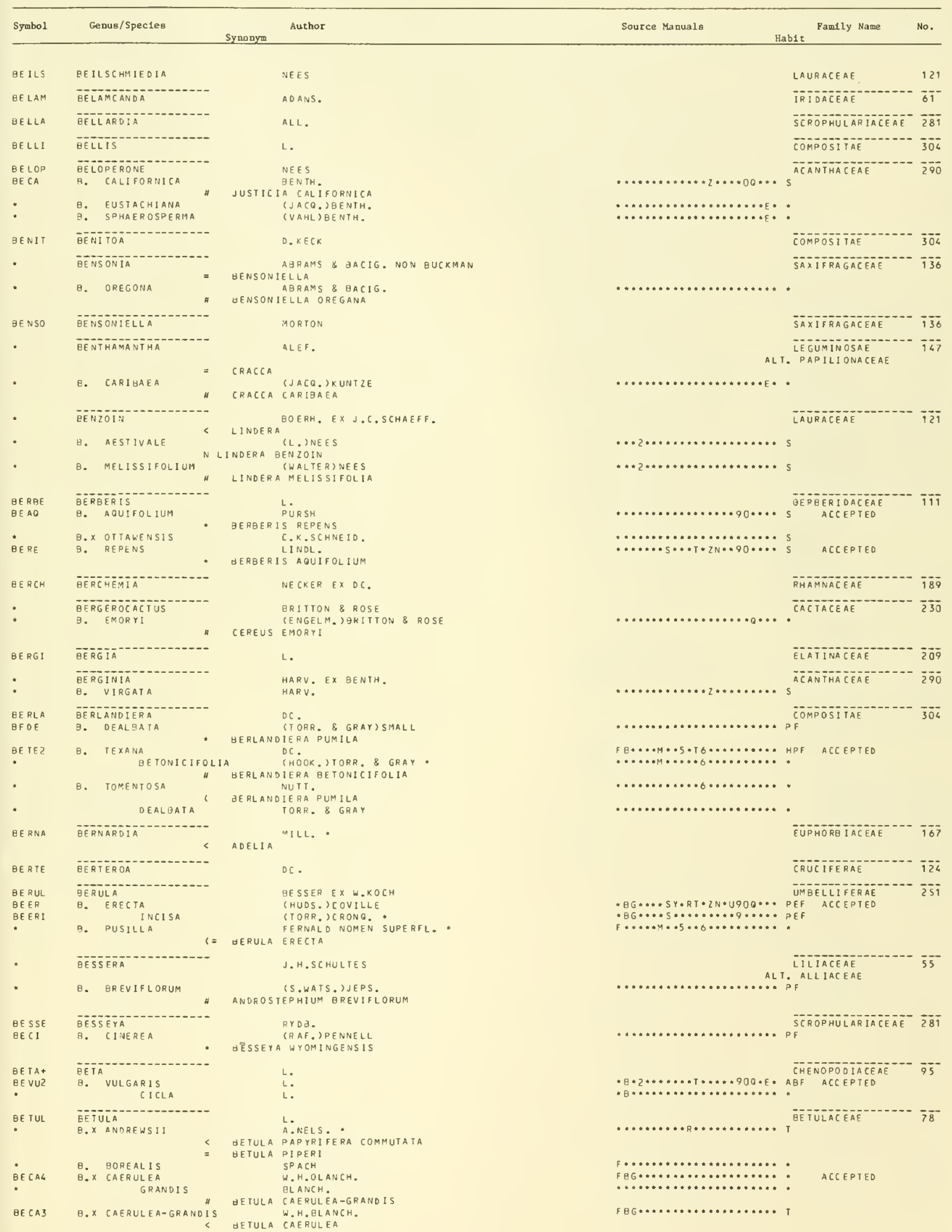


BE TULA

\begin{tabular}{|c|c|c|c|c|c|c|c|c|}
\hline Symbol & & aus/Species & Synonym & Author & Source Manusls & Habit & Family Name & No. \\
\hline • & 8. $x$ & CORDIFOLIA & BETULA & $\begin{array}{l}\text { RESEL } \\
\text { PAPYRIFER CORDIFOLIA }\end{array}$ & 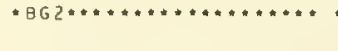 & $\cdot$ & & \\
\hline 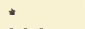 & B. $x$ & DJIILLYI & & LEPAGE & $\ldots \ldots \ldots \ldots \ldots \ldots+\ldots+\ldots \ldots+\hbar \hbar$ & $\mathrm{T}$ & & \\
\hline$B \in E A$ & 9. $x$ & FASTWOODIAE & $N \quad \forall E T U L A O C$ & $\begin{array}{l}\text { SARG. } \\
\text { CCI DENTAL IS }\end{array}$ & $\ldots \ldots \ldots \ldots \ldots \ldots \ldots \ldots \ldots$ & $\mathrm{T}$ & $A C C E P T E O$ & \\
\hline$\bullet$ & B. & FONTINALIS & $=\quad \forall E T U L A$ & $\begin{array}{l}\text { SARG. } \\
\text { OCCIDENTALIS }\end{array}$ & $\cdots \cdots \cdots \cdots \cdots+R * 2 * \cdots \cdots 00 * \cdots$ & 1 & & \\
\hline$*$ & & INOPINA & & JEPS. & $\ldots \ldots \ldots \ldots \ldots \ldots \ldots+0 \cdots \cdots$ & $\cdot$ & & \\
\hline * & $\forall \cdot$ & GL ANDUL IF ERA & a $\quad \forall E T U L A$ & $\begin{array}{l}\text { (REGEL) B.T. BUTLER } \\
\text { GLANOULOSA GLANDULIFERA }\end{array}$ & 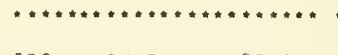 & * & & \\
\hline BE GL & B. & $\begin{array}{l}\text { GLANDULOSA } \\
\text { GLANDULIF }\end{array}$ & $F \in R A$ & $\begin{array}{l}\text { MICHX. } \\
\text { ( REGEL) GLEASON }\end{array}$ & $F B G * \cdots S Y \star R * \ldots * \cup 90 * A * *$ & $\begin{array}{l}5 \\
1\end{array}$ & ACCEPTED & \\
\hline * & & HALLII & $\nexists \quad$ BETULA & $\begin{array}{l}\text { PUMILA GLANDUL IFERA } \\
\text { (T. HOWELL) C. HITCHC. }\end{array}$ & $\star * \star * * * \ldots \ldots * * \ldots+* * 9+\ldots \ldots$ & s & & \\
\hline$B E K E$ & 9. & KENAICA & a $\quad \forall$ ETULA & $\begin{array}{l}\text { W. HEVANS } \\
\text { PAPYRIFERA KENAICA }\end{array}$ & 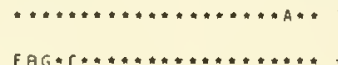 & 1 & & \\
\hline • & B. & LUIEA & $=\quad B E T U L A$ & $\begin{array}{l}\text { MICHX.F. } \\
\text { ALLEGHANIENSIS }\end{array}$ & $F B G \cdot[\ldots \ldots * \ldots \ldots \ldots \ldots \ldots$ & $*$ & & \\
\hline$:$ & ө. & $\begin{array}{l}\text { MACROLEPI } \\
\text { MIChAUXII }\end{array}$ & & $\begin{array}{l}\text { FE RNAL D } \\
\text { SPACH }\end{array}$ & $\begin{array}{l}F B G \ldots \ldots \ldots \ldots \ldots \ldots \ldots \ldots \\
F, G+\cdots, \ldots\end{array}$ & it & & \\
\hline$*$ & 9. $x$ & MINCR & A $\quad \forall E T U L A$ & $\begin{array}{l}\text { (TUCKERMAN) FERNALO } \\
\text { PAPYE! FERA MINOR }\end{array}$ & 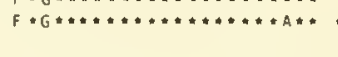 & ? & & \\
\hline BE NA & B. & $\begin{array}{l}\text { NANA } \\
\text { SEXILIS }\end{array}$ & " & (SUKATSCH.) HULTEN & $\because \ldots * \cdots \cdots * \cdots \cdots \cdots+* A * *$ & 5 & ACCEPTED & \\
\hline$\cdot$ & в. & NEOALASKAHA & \# BETULA & $\begin{array}{l}\text { SAFG. } \\
\text { PAPYRIFERA NEOALASKANA }\end{array}$ & 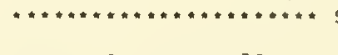 & 5 & & \\
\hline$B \in O C 2$ & 日. & OCCIOENTALIS & $\begin{array}{l}N \text { BETULA EA } \\
N \text { GETULA PA }\end{array}$ & $\begin{array}{l}\text { HOOK. } \\
\text { ASTWOODIAE } \\
\text { APYRIFERA COMMUTATA }\end{array}$ & $\cdots \cdots \cdots 5 Y \cdots \cdots+2 N * U 90 * A * *$ & $5 \mathrm{~T}$ & ACCEPTED & \\
\hline$B E O C F$ & & $\begin{array}{l}\text { FECUNDA } \\
\text { INOPINA }\end{array}$ & $=\quad \forall E T U L A$ & $\begin{array}{l}\text { FERNALD } \\
\text { FONTINALIS INOPINA }\end{array}$ & 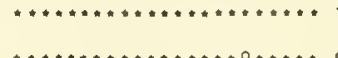 & $2 \mathrm{~T}$ & & \\
\hline QE PA & B. & $\begin{array}{l}\text { INOP INA } \\
\text { PAPYRI FERA }\end{array}$ & & $\begin{array}{l}\text { SJEPS. ) C. HITCHC. } \\
\text { MARSHALL }\end{array}$ & 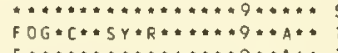 & $\begin{array}{l}1 \\
5\end{array}$ & $A C C E P T E D$ & \\
\hline$B E P A C$ & & COMMUTATA & ${ }^{4} N$ BEIULA OC & $\begin{array}{l}\text { (REGEL) FERNALD } \\
\text { CCIDENTALIS }\end{array}$ & $F \cdots \cdots \cdots \cdots+\cdots+q \cdots A \cdots$ & $T$ & ACCEPTED & \\
\hline * & & ELOSATA & 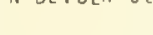 & (FERNALD) SARG. & $f_{\ldots} \ldots \ldots \ldots \ldots \ldots \ldots \ldots \ldots$ & - & & \\
\hline BEPAH & & HUMILIS & - $\quad$ eetula & $\begin{array}{l}\text { (REGEL)FERNALD \& RAUP } \\
\text { NEOALASKANA }\end{array}$ & $\cdots \ldots+\ldots \star \ldots+\cdots+\ldots+\cdots+\cdots$ & I & & \\
\hline * & & MACROSTAC & CHYA & FERNALD & 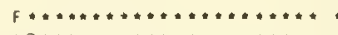 & $\cdot$ & & \\
\hline - & & MINOR & & TUCKERMAN & 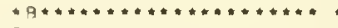 & * & & \\
\hline : & & PENSILIS & & FERNALD & $F \cdots \cdots \cdots \cdots+\cdots \cdots * \cdots$ & : & & \\
\hline : & B. & $\begin{array}{l}\text { S HUMILIS } \\
\text { PUBESCENS }\end{array}$ & & $\begin{array}{l}\text { (REGEL) HULTEN } \\
\text { EHFH. }\end{array}$ & $\because \cdots \cdots+\cdots, \cdots \cdots \cdots \cdots A$, & $\dot{s}$ & & \\
\hline BE PUL & 8. & PUMILA & & L. & FBG $\ldots \ldots \ldots \ldots \ldots \ldots \ldots$ & 5 & ALCEPTED & \\
\hline * & & GLABRA & & $R \in G E L$ & 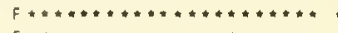 & $\cdot$ & & \\
\hline$\cdot$ & & RENIFOLIA & & FERNALO & $F \ldots \ldots \ldots \ldots \ldots \ldots \ldots \ldots \ldots$ & - & & \\
\hline • & BIAN & VICAEA & & TODARO & $A L T$ & $\begin{array}{r}\text { LEG } \\
\text { T. CA }\end{array}$ & $\begin{array}{l}\text { SUMINOSAE } \\
\text { AESALPINIACEAE }\end{array}$ & $7 \overrightarrow{47}$ \\
\hline • & B. & SEPIARIA & $\begin{array}{l}c=\quad \text { CAESALP } \\
\Rightarrow \quad C A E S A L P\end{array}$ & $\begin{array}{l}\text { (ROXB.) TOOARO } \\
\text { PINIA OECAPETALA } \\
\text { PINIA SEPIARIA }\end{array}$ & $\cdots \cdots \cdots \cdots * \cdots * \cdots * \cdots * * E_{*}$ & 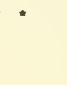 & & \\
\hline * & $\bar{B} \mathrm{I} C \overline{\mathrm{Cu}}$ & JCULLA & ADLUM IA & BORKH. & ALT & $\begin{array}{l}\overline{P A} \bar{P} \\
T .\end{array}$ & JMVERACEAE & $\overline{123}$ \\
\hline • & e. & CA VA DENSIS & a DICENTR & $\begin{array}{l}\text { (GOLDIE)MILLSP. } \\
\text { RA CANAOENSIS }\end{array}$ & $* \cdots 2 \ldots \ldots \ldots \ldots+\ldots+\ldots \ldots$ & PF & & \\
\hline - & B. & CUCULLARIA & * DICENTR & RL O MILLSP. & $\cdots * 2 * * \cdots+\cdots * \ldots+\ldots+\cdots *$ & PF & & \\
\hline - & 8. & EXINI I A & H OICENTR & $\begin{array}{l}\text { (KER-GAWL.) MILLSP. } \\
R A E X I M I A\end{array}$ & $\cdots+2 * \ldots+\cdots+\cdots \cdots+\cdots \cdots \cdots+\ldots$ & PF & & \\
\hline BI DEN & $\overline{O I D E}$ & 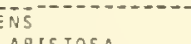 & -- & L. & & COM & APOSI I AE & $\overline{304}$ \\
\hline BI AR & в. & ARISTOSA & - $\quad$ IOENS & $\begin{array}{l}\text { (MICHX.)BRITTON } \\
\text { POLYLEPIS RETRORSA }\end{array}$ & 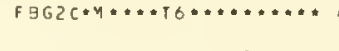 & $A B F$ & ACCEPTED & \\
\hline BIBE & P. & BECK $I]$ & a MEgaloo & $\begin{array}{l}\text { TORR. } \\
\text { OONTA BECXII }\end{array}$ & 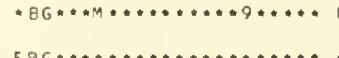 & P ZF & & \\
\hline 81813 & 9. & BIDE WTOIDES & $>\quad$ SIOENS & $\begin{array}{l}\text { (NUIT.)BRITTON } \\
\text { MARIANA }\end{array}$ & 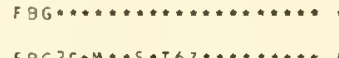 & 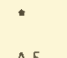 & $A C C E P T E D$ & \\
\hline $8 I P I 2$ & 8. & PIPIUHA IA & - cosmos & EIPINNATUS & 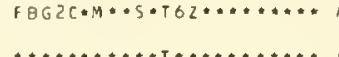 & AF & & \\
\hline - & & 31 TERNATO & $O I D E S$ & SHERFF & 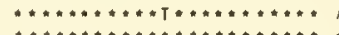 & Af & & \\
\hline$\cdot$ & B. & CAUDATA & COSMOS & $\begin{array}{l}\text { SH.B.K.) SCH.BIP. } \\
\text { CAUDATUS }\end{array}$ & $\cdots+\ldots+\ldots \ldots \ldots+\ldots+\ldots$ & 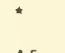 & & \\
\hline$B$ I CE & B. & $\begin{array}{l}\text { CERIAUA } \\
\text { ELLIPTICA }\end{array}$ & * $\quad$ IDENS & $\begin{array}{l}\text { L. } \\
\text { LAEVIS } \\
\text { WIEGAND }\end{array}$ & 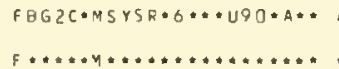 & AF & $A C C E P T E D$ & \\
\hline$\cdot$ & & & HIOENS & FRONDOSA & & & & \\
\hline$\theta 1 \cos$ & B. & COMOSA & $<\quad \forall I D E N S$ & $\begin{array}{l}\text { (GRAY) WIEGAND } \\
\text { TRIPARTIIA }\end{array}$ & $F B G Z \cdots M S Y * \ldots \ldots \ldots \ldots \ldots$ & Af & ACCEPIED & \\
\hline$\theta$ I $\cos$ & B. & CONN AT A & A IDENS & $\begin{array}{l}\text { MUHL. EX WILLD. } \\
\text { TRIPARTITA }\end{array}$ & FBG $\ldots$ MS $\ldots \ldots \ldots \ldots 0 \cdots$ & * & $A C C E P I E D$ & \\
\hline B I CO & 8. & CORONATA & - $\quad$ IDENS & $\begin{array}{l}\text { (L B)BRITTON } \\
\text { MITIS }\end{array}$ & 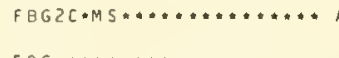 & AF & $A C C E P T E D$ & \\
\hline$B ! \in A$ & B. & EATONII & > BIDENS & $\begin{array}{l}\text { FERVALD } \\
\text { HETERODOXA }\end{array}$ & $F O G \ldots \ldots \ldots \ldots \ldots \ldots \ldots \ldots$ & $\cdot$ & $A C C E P T E D$ & \\
\hline - & & INTERSTE: & & FASSETT & $f \cdots \ldots+\ldots \ldots \ldots \ldots \ldots \ldots$ & * & & \\
\hline BI HE? & 8. & HE TERODOXA & BIOENS & $\begin{array}{l}\text { (FERNA LO) FERNALD \& H.ST. JOHN } \\
\text { EATUHI I }\end{array}$ & $F+\ldots+\ldots+\ldots \ldots+\ldots+\ldots+\cdots$ & $*$ & ACCEPIED & \\
\hline$\cdot$ & 8. & INVOLUCRAIA & - $\quad$ IDENS & $\begin{array}{l}\text { SNUTT. SORITTON } \\
\text { POLYLEPIS }\end{array}$ & $\cdots+2 \cdots \ldots+\ldots \ldots+\cdots \cdots$ & F & & \\
\hline BI LA & B. & LAEVIS & - BIDENS & SL. SO.S.P. & 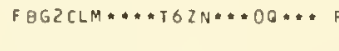 & PAF & ACCEPTEO & \\
\hline $81 M A 3$ & B. & MARIANA & (5) & OLAKE & $F \ldots \ldots \ldots+\ldots \ldots \ldots \ldots \ldots \ldots$ & * & ACCEPTEO & \\
\hline BI MI & 9. & MITIS & $\forall$ IOENS & $\begin{array}{l}\text { BI DENIOIDES } \\
\text { SMI GHX.) SHERFF }\end{array}$ & $F B G Z C L \ldots \ldots+T \ldots \ldots \ldots \cdots \cdots$ & AF & $A C C E P T E D$ & \\
\hline
\end{tabular}


AI DENS

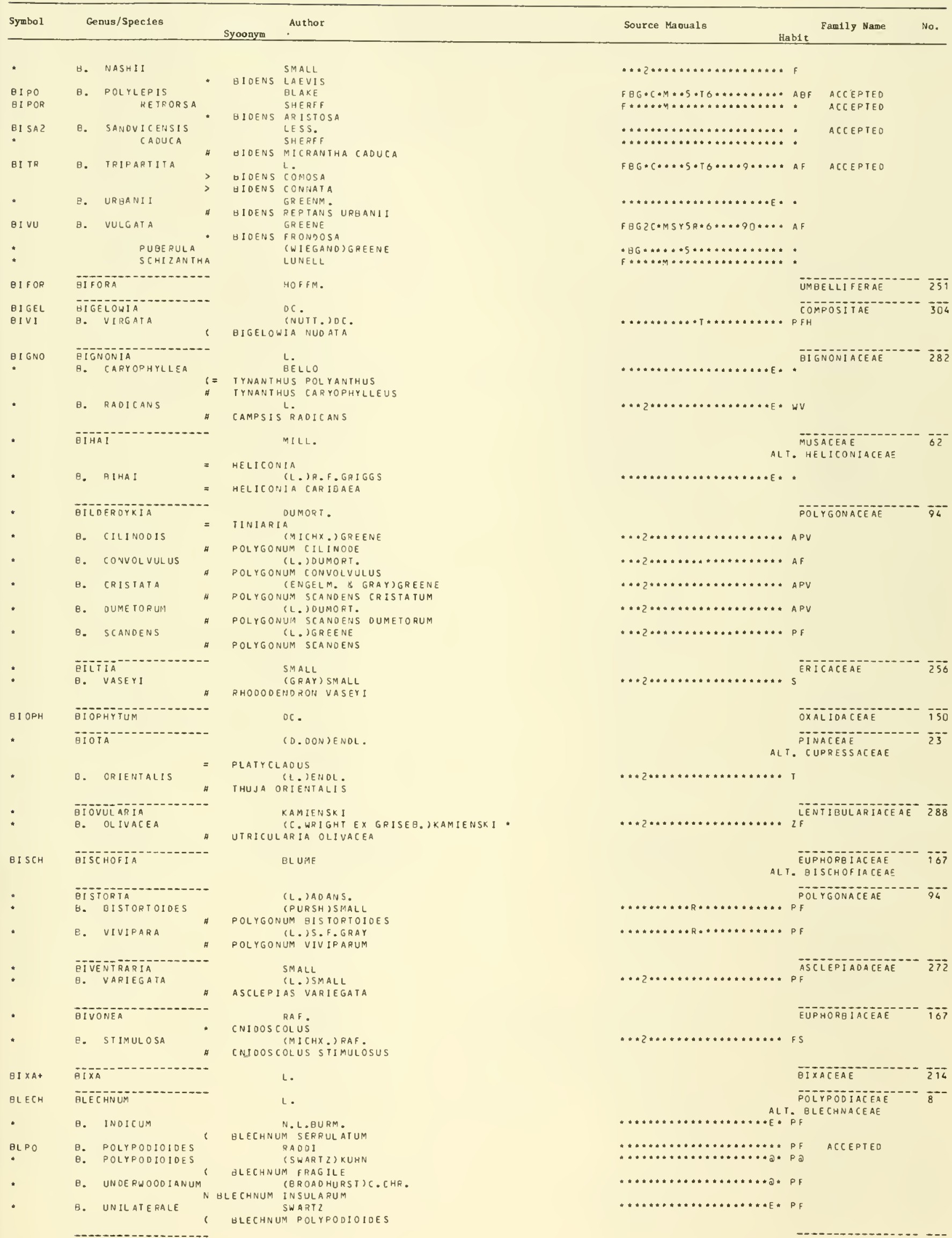




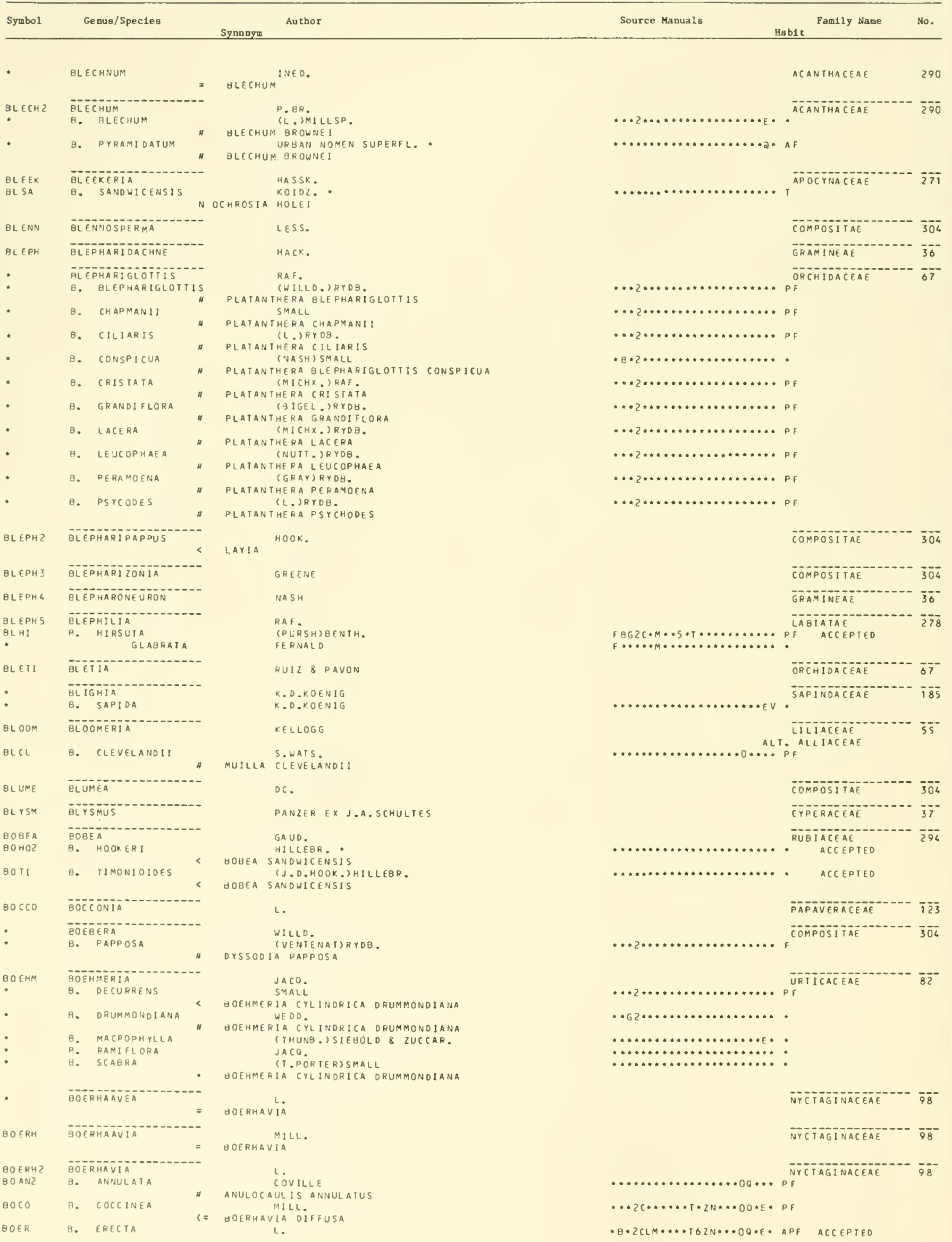




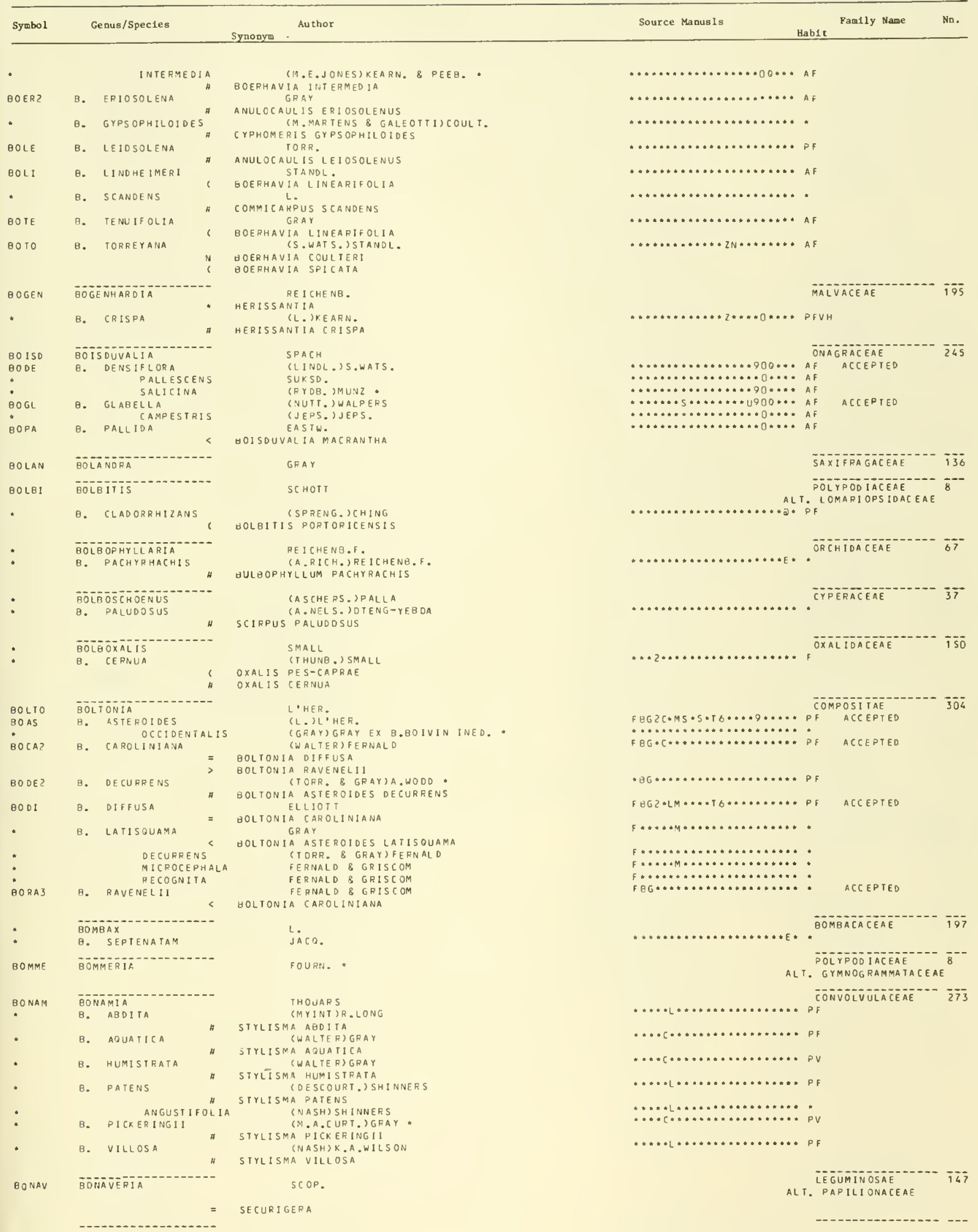




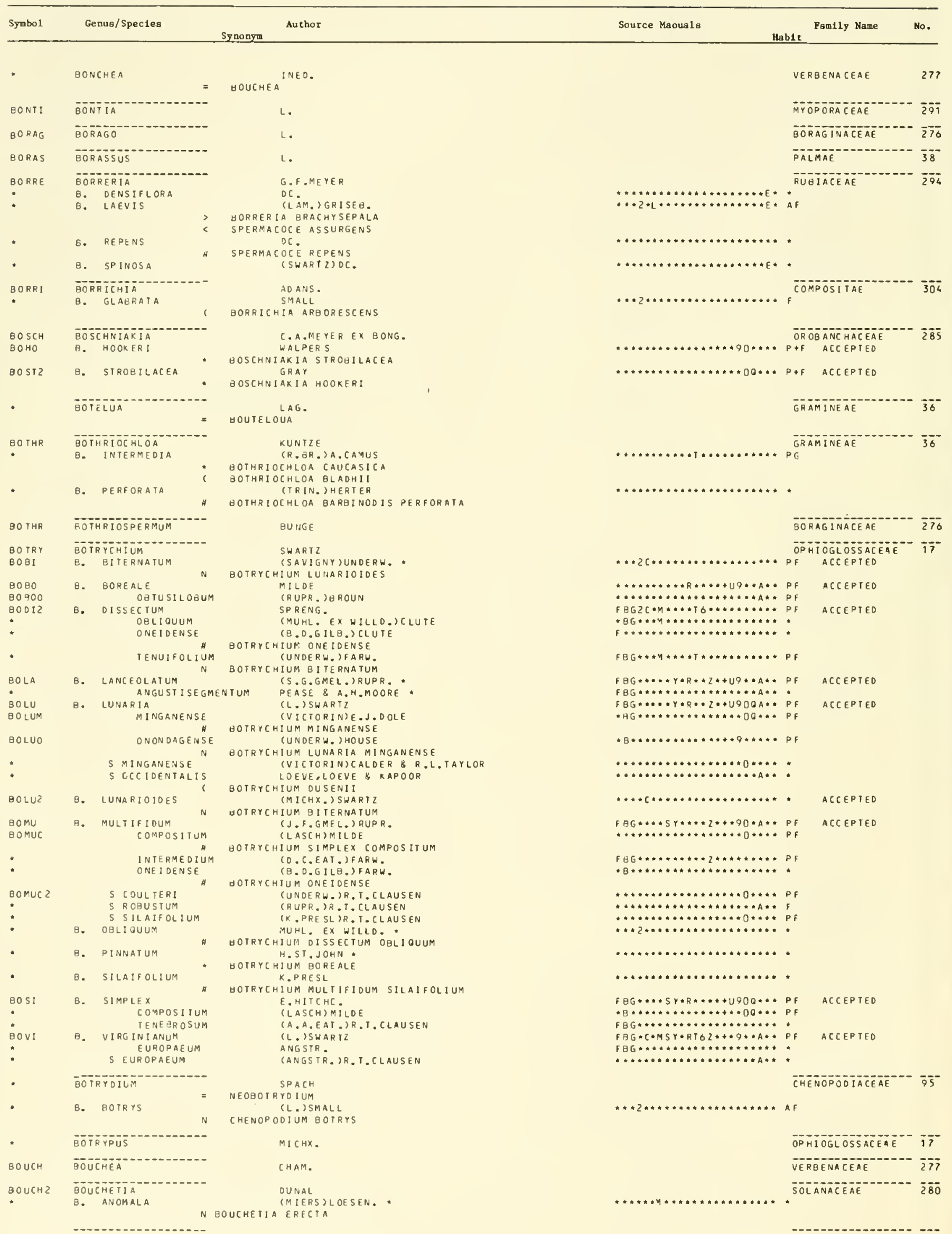


BOUGAINVILLEA

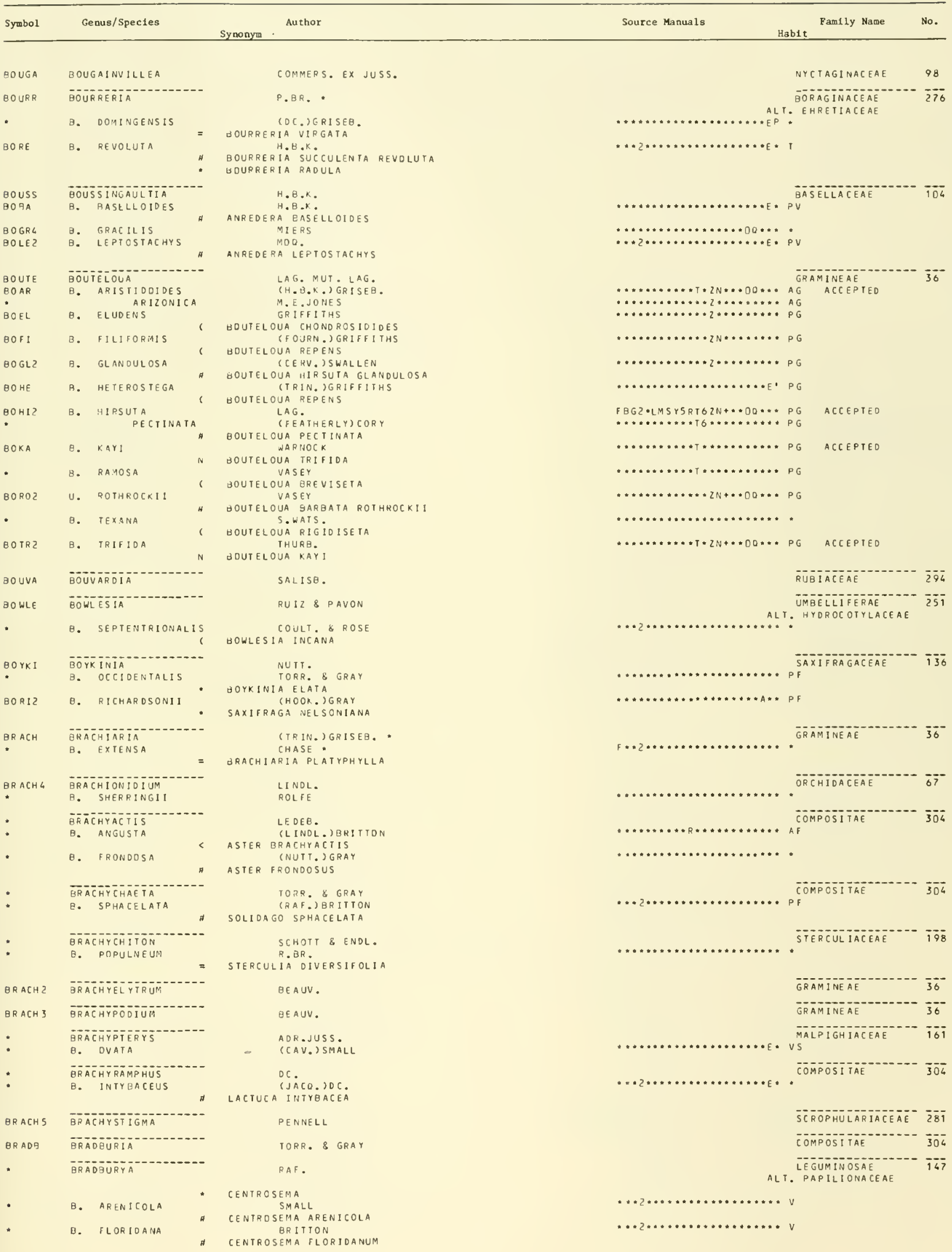




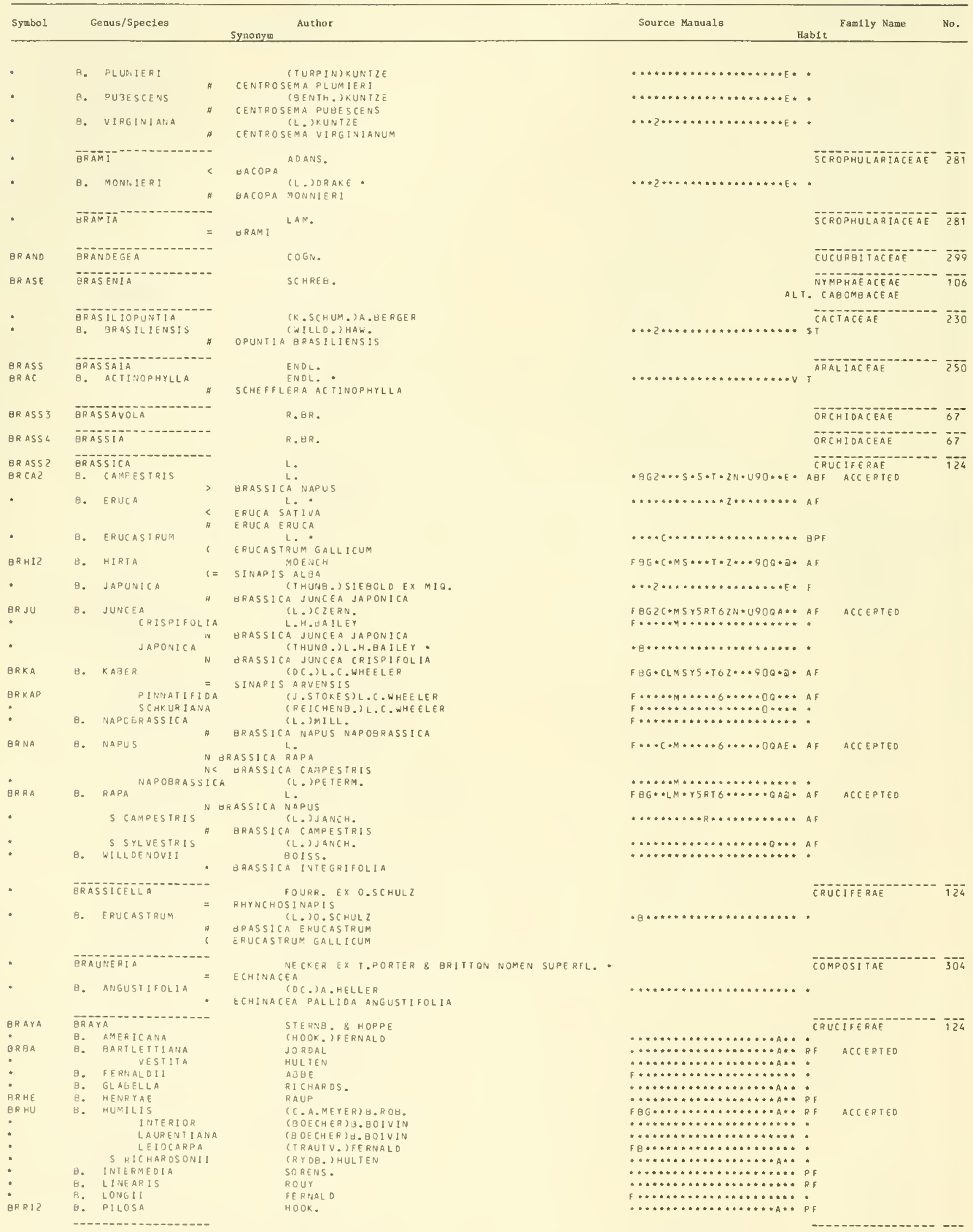


BRAYULINEA

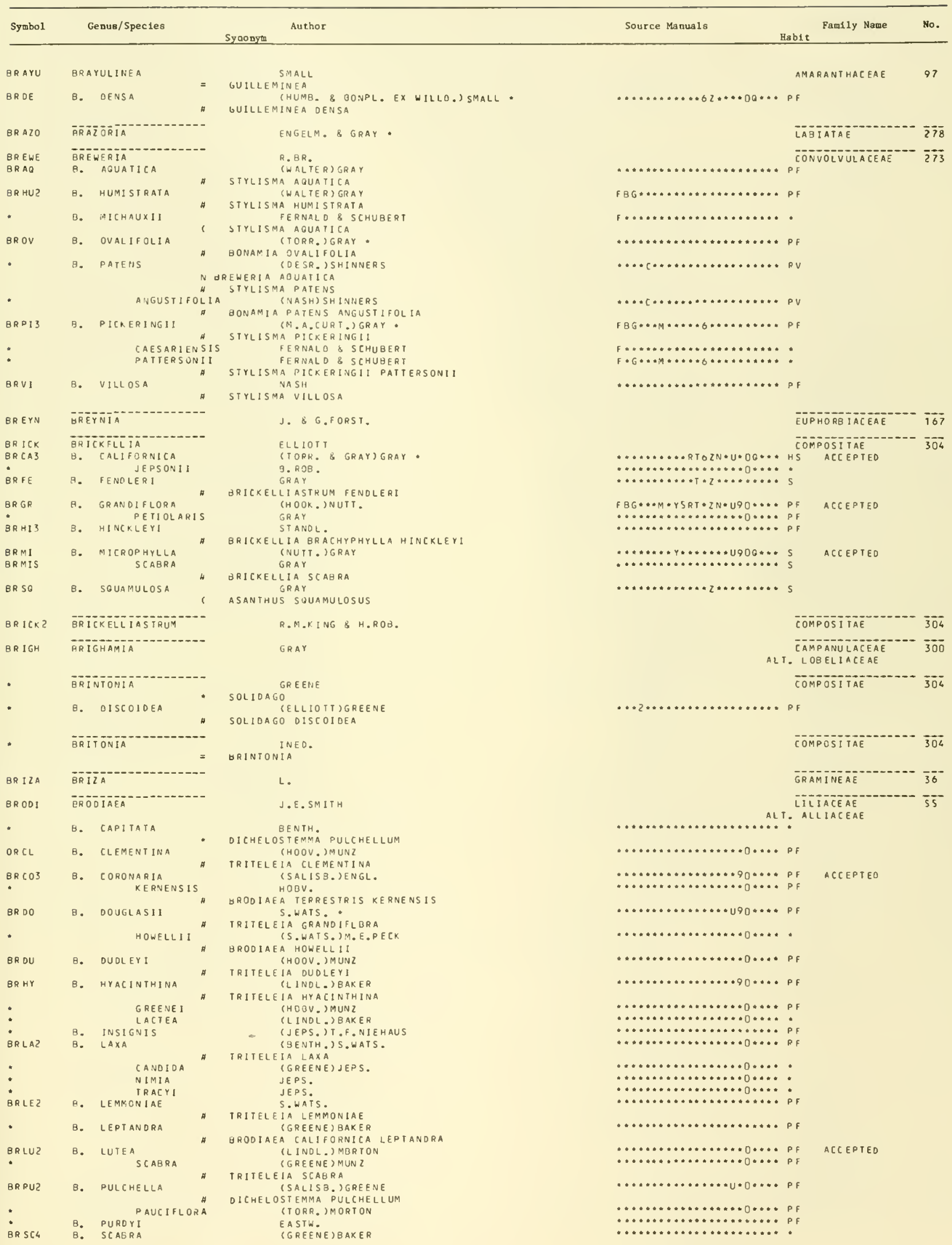




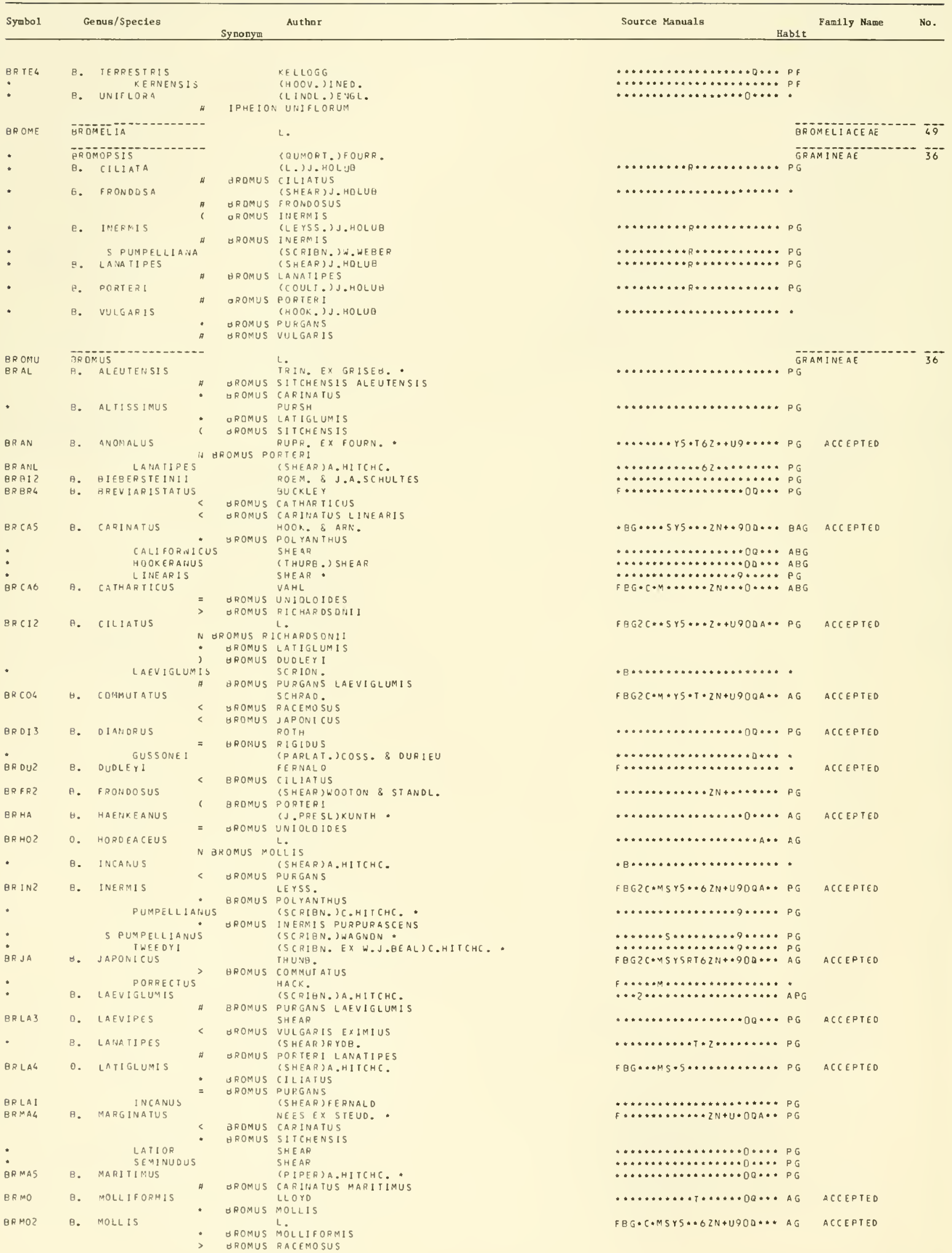


BROMUS

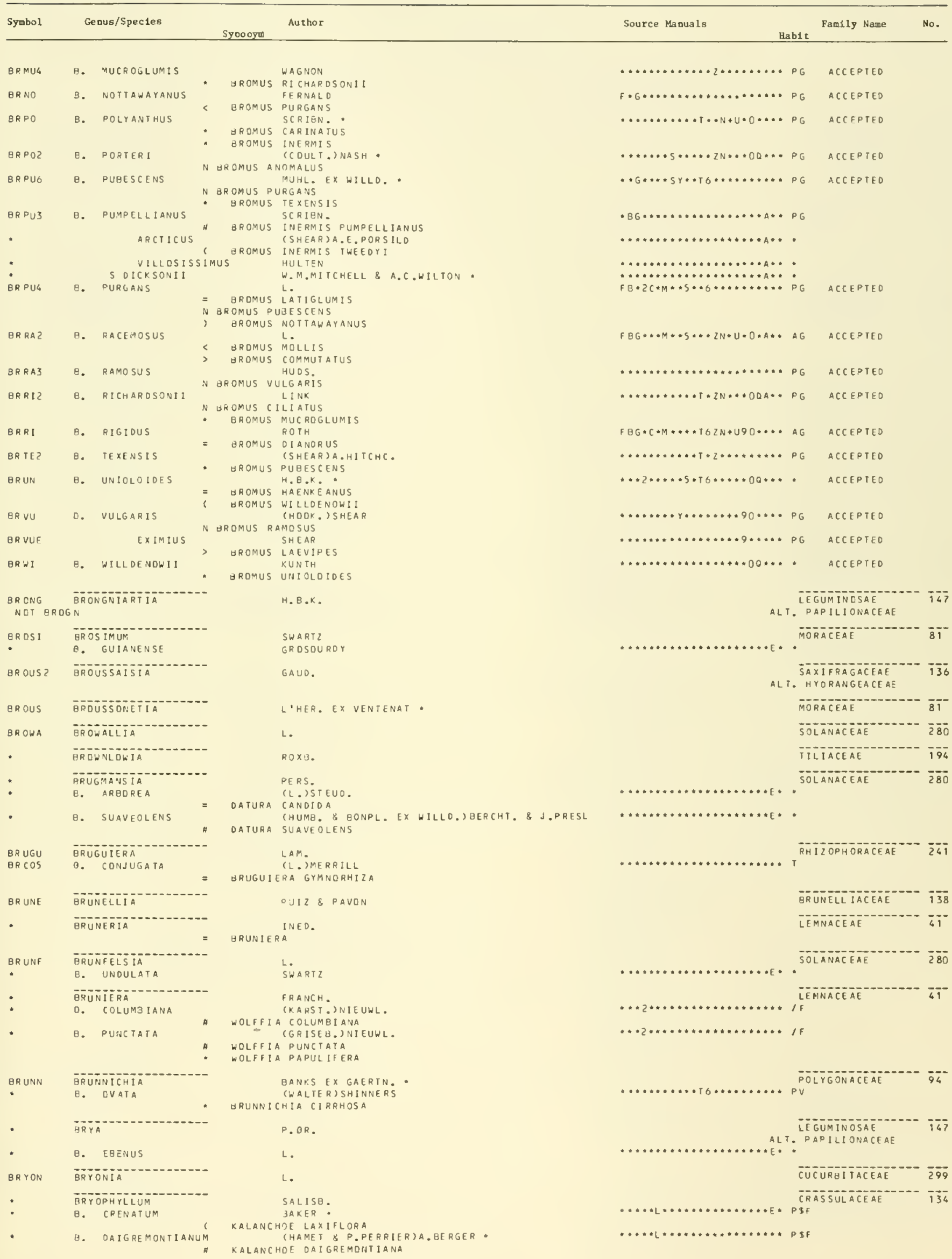




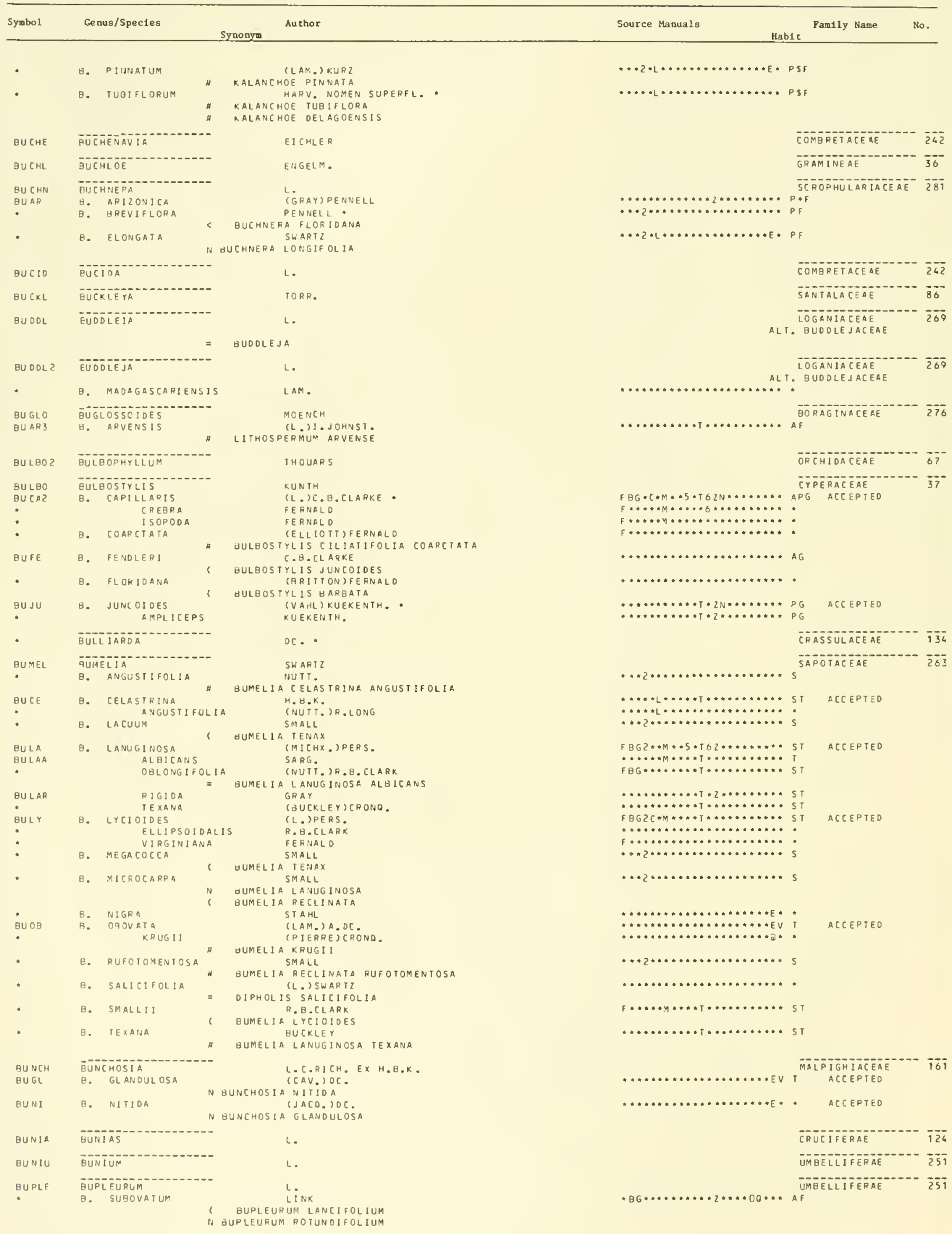




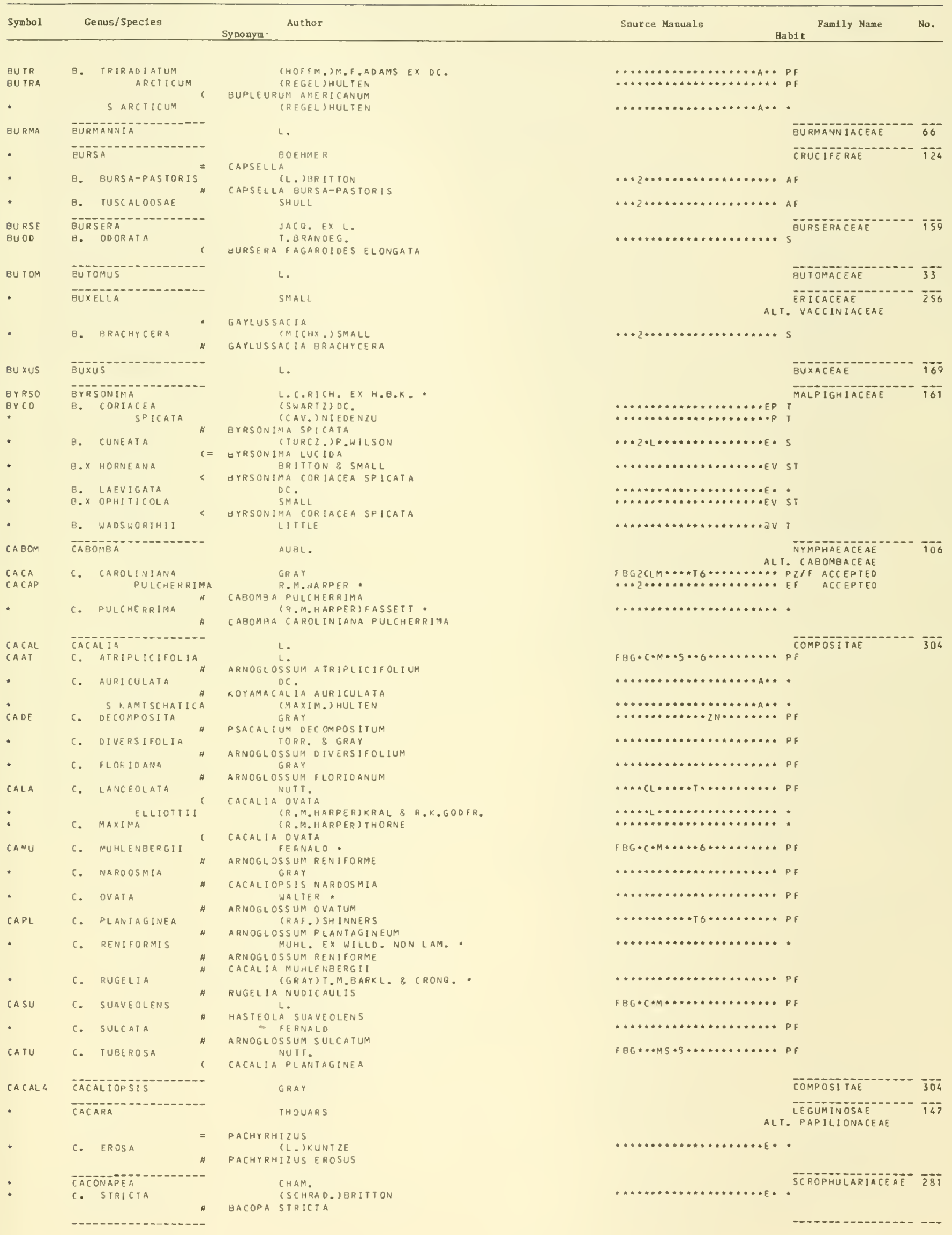




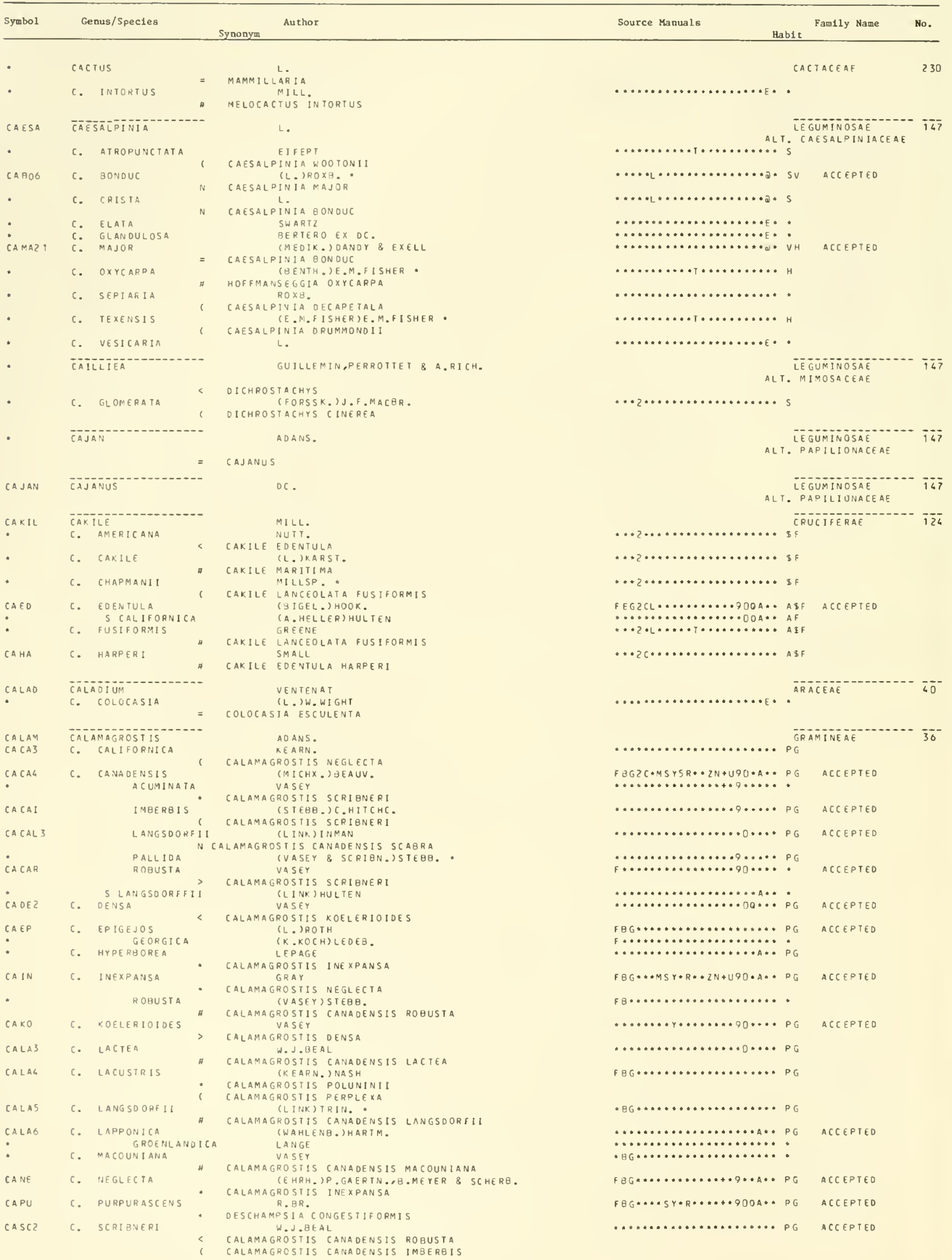




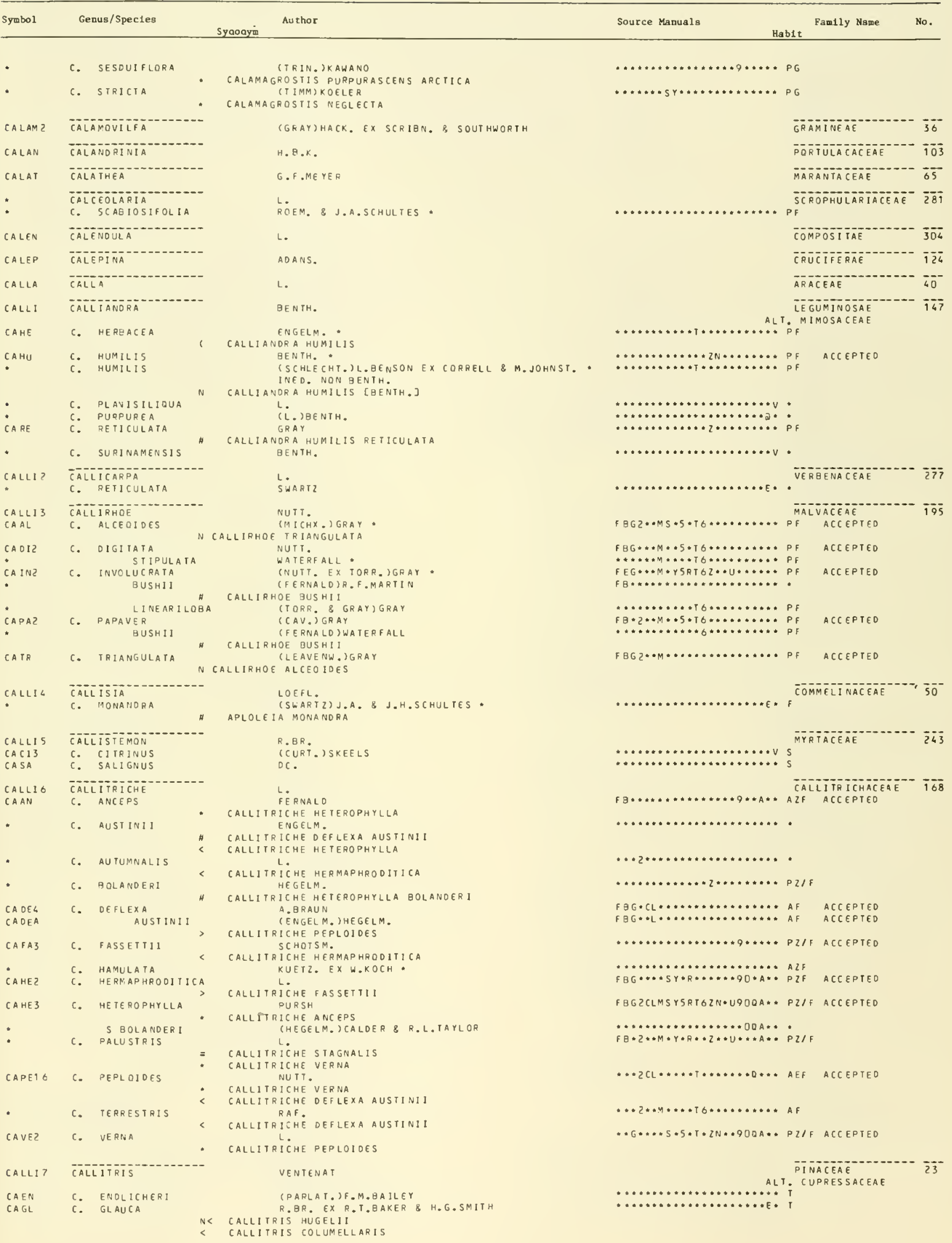




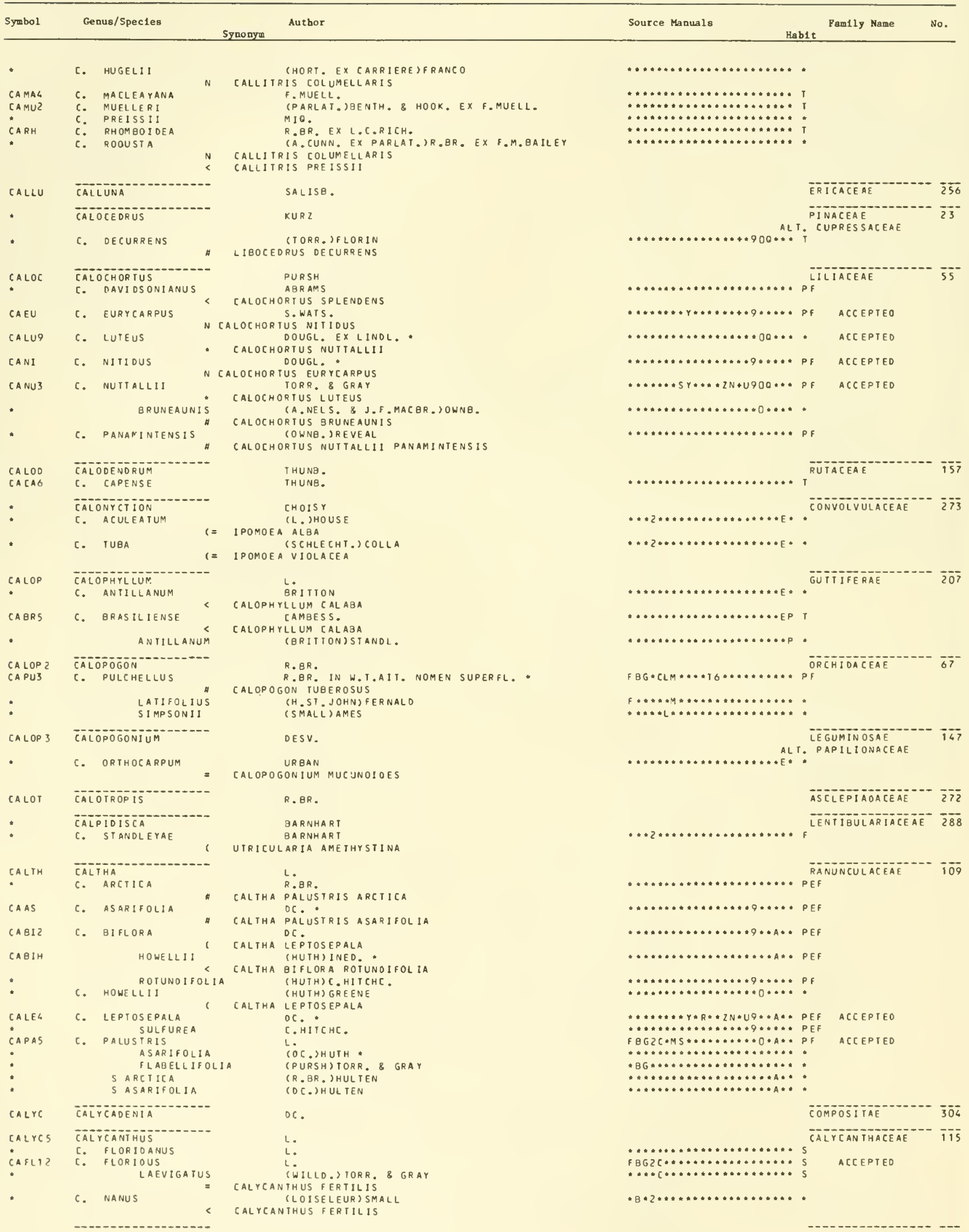




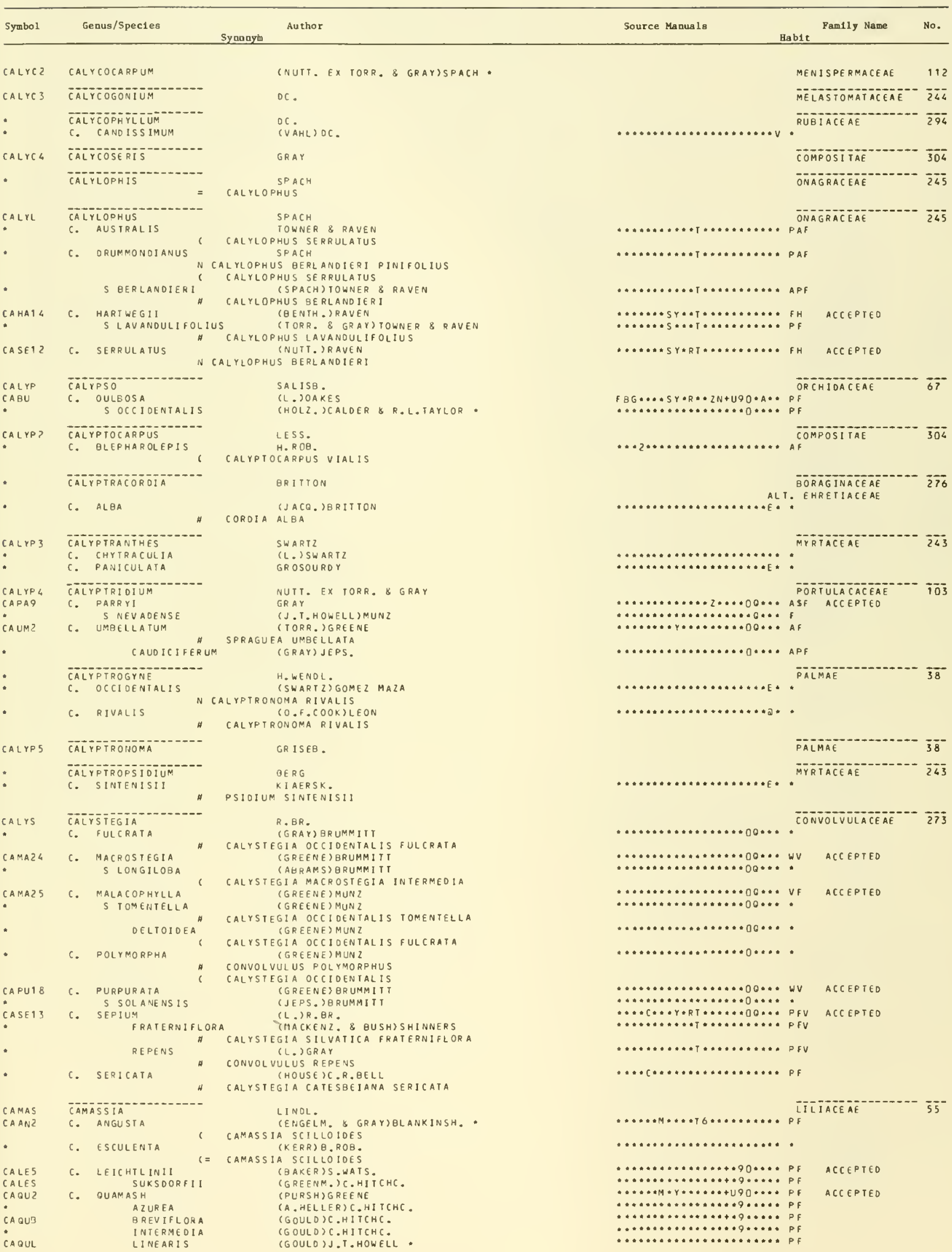




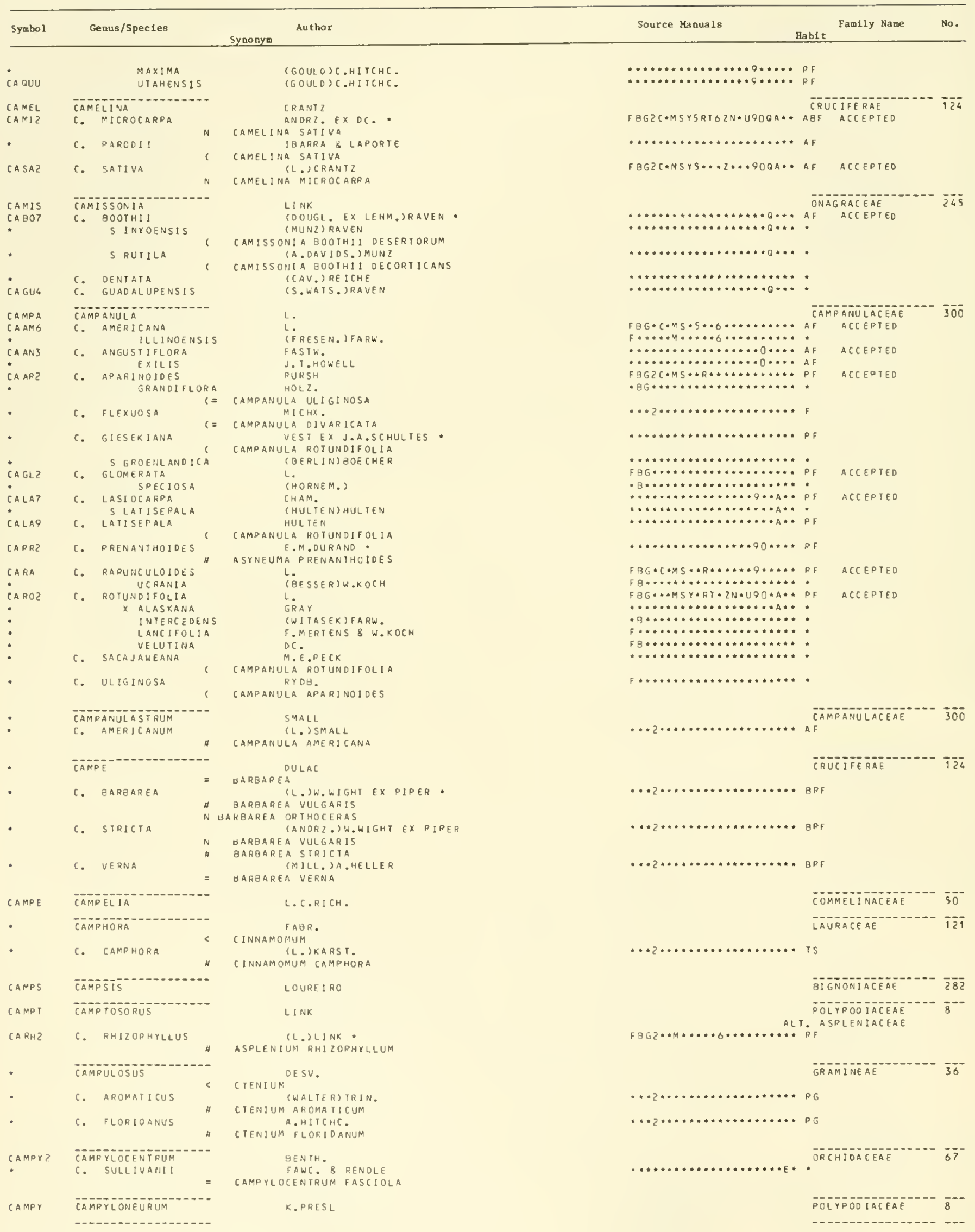




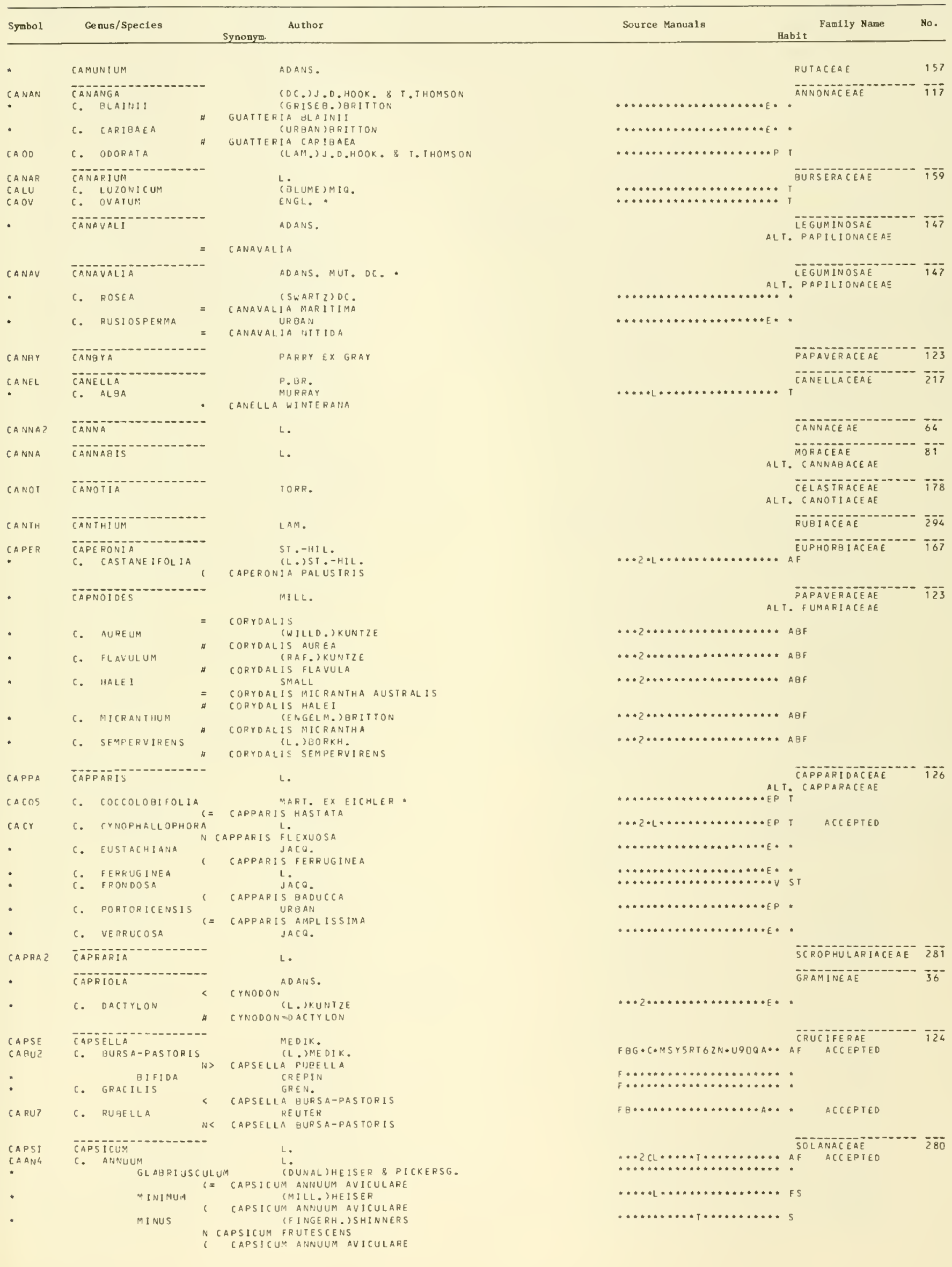




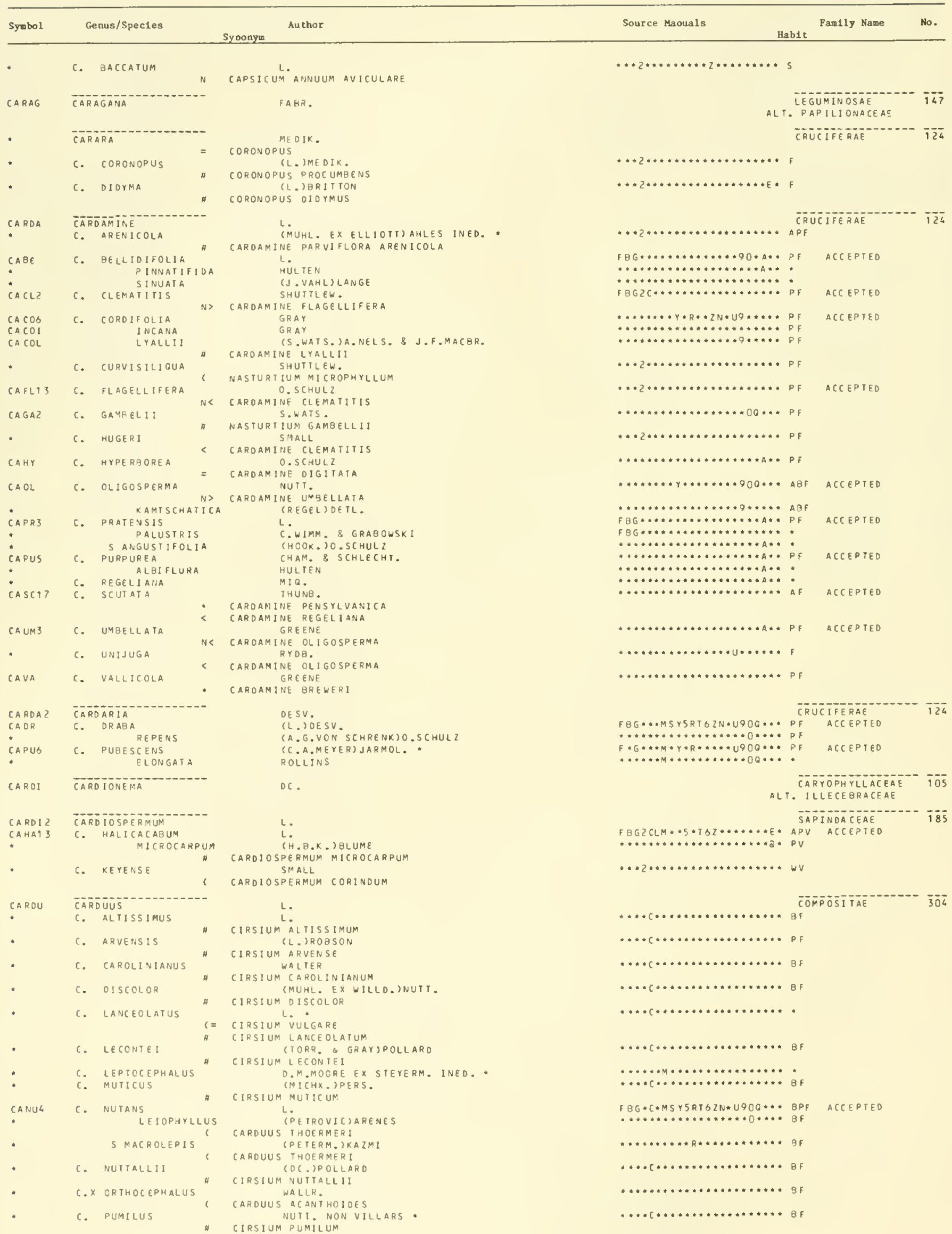




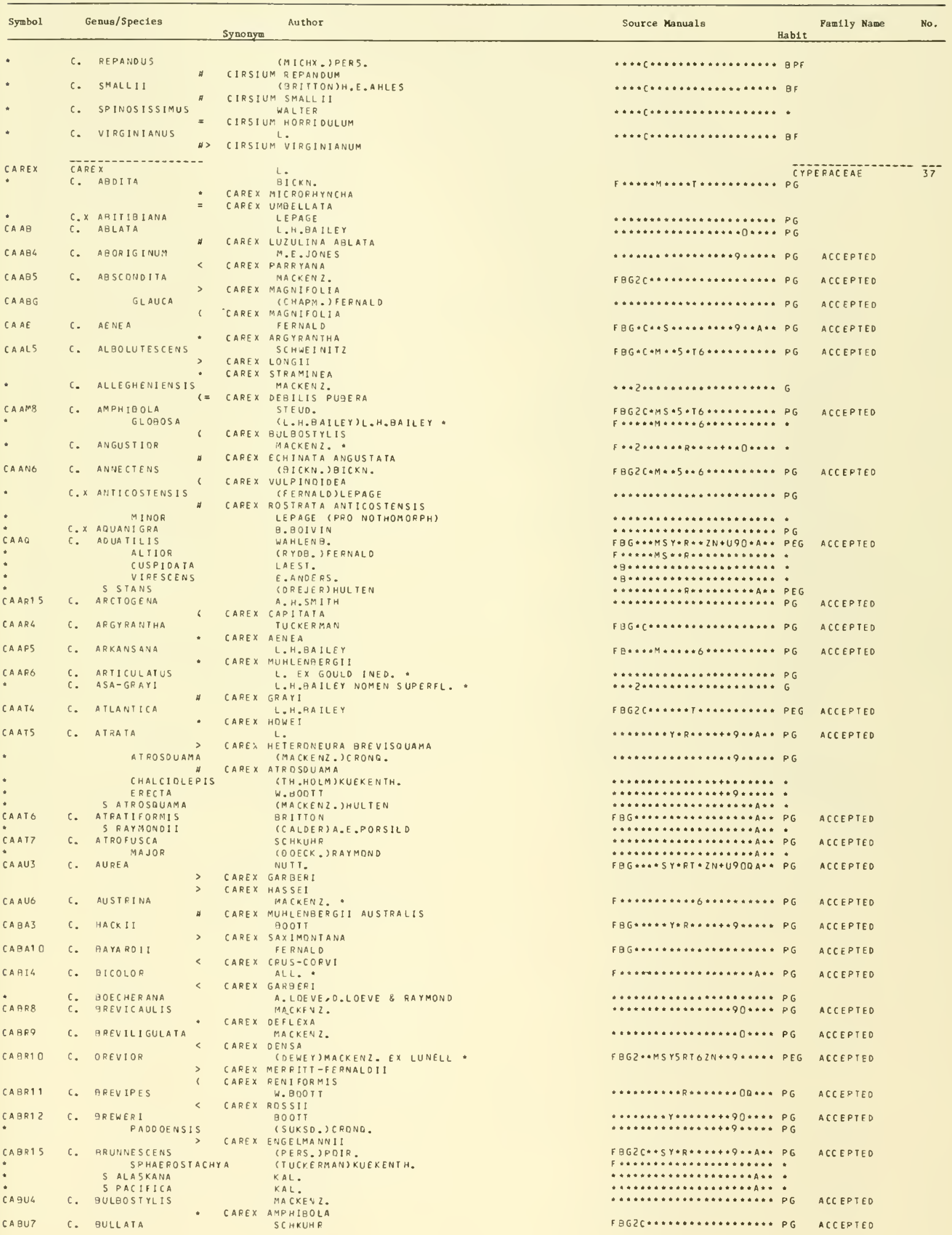




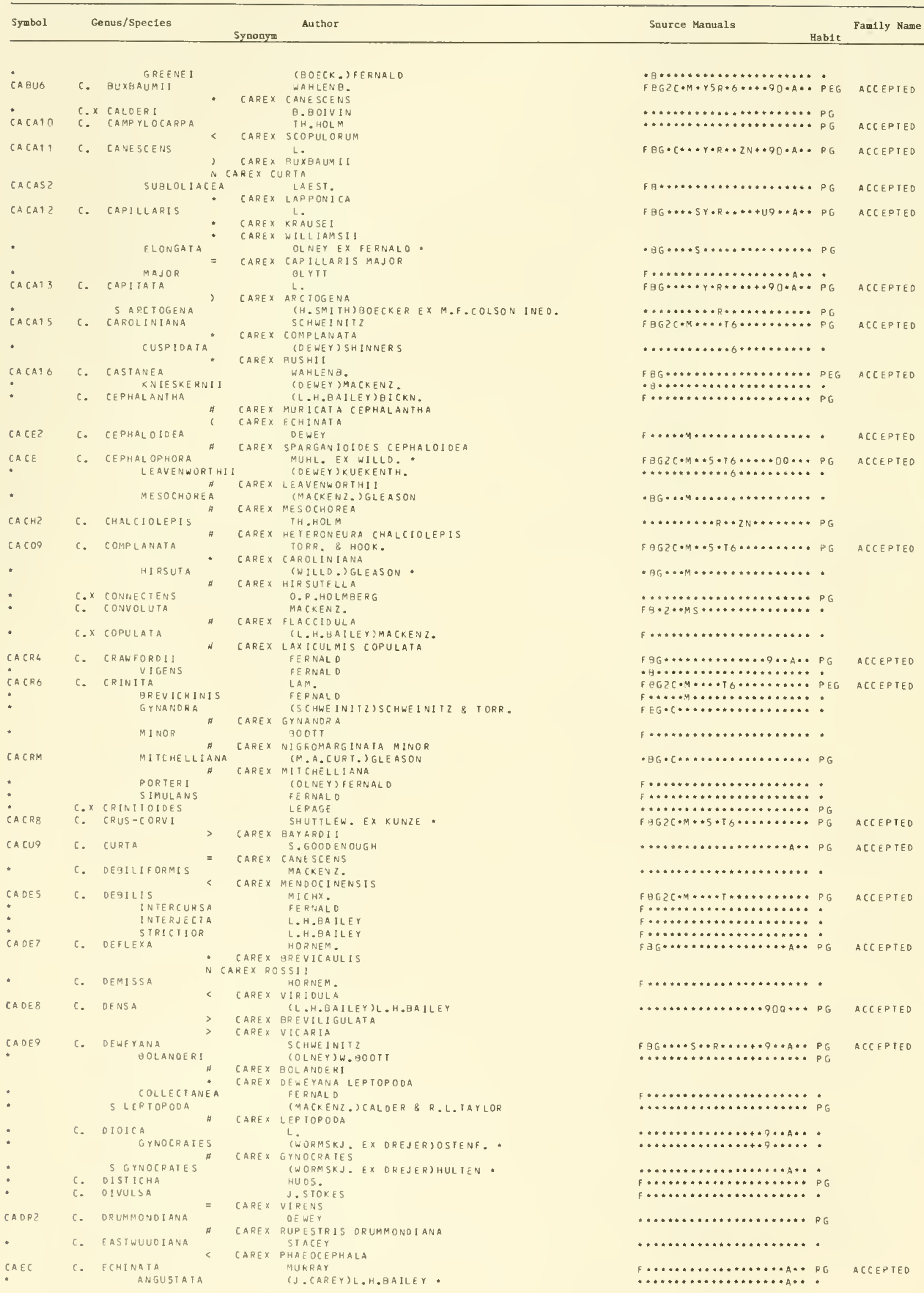




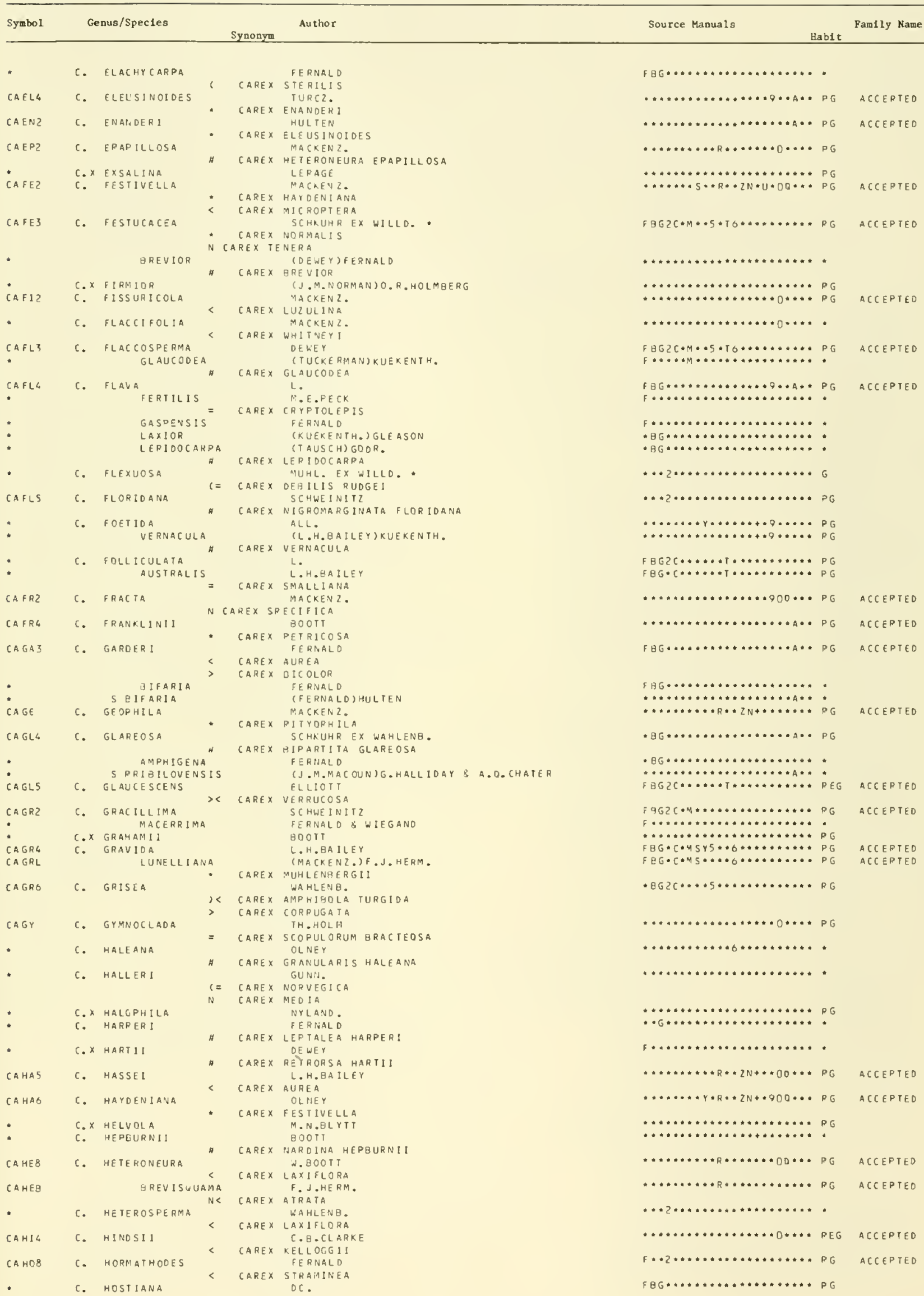




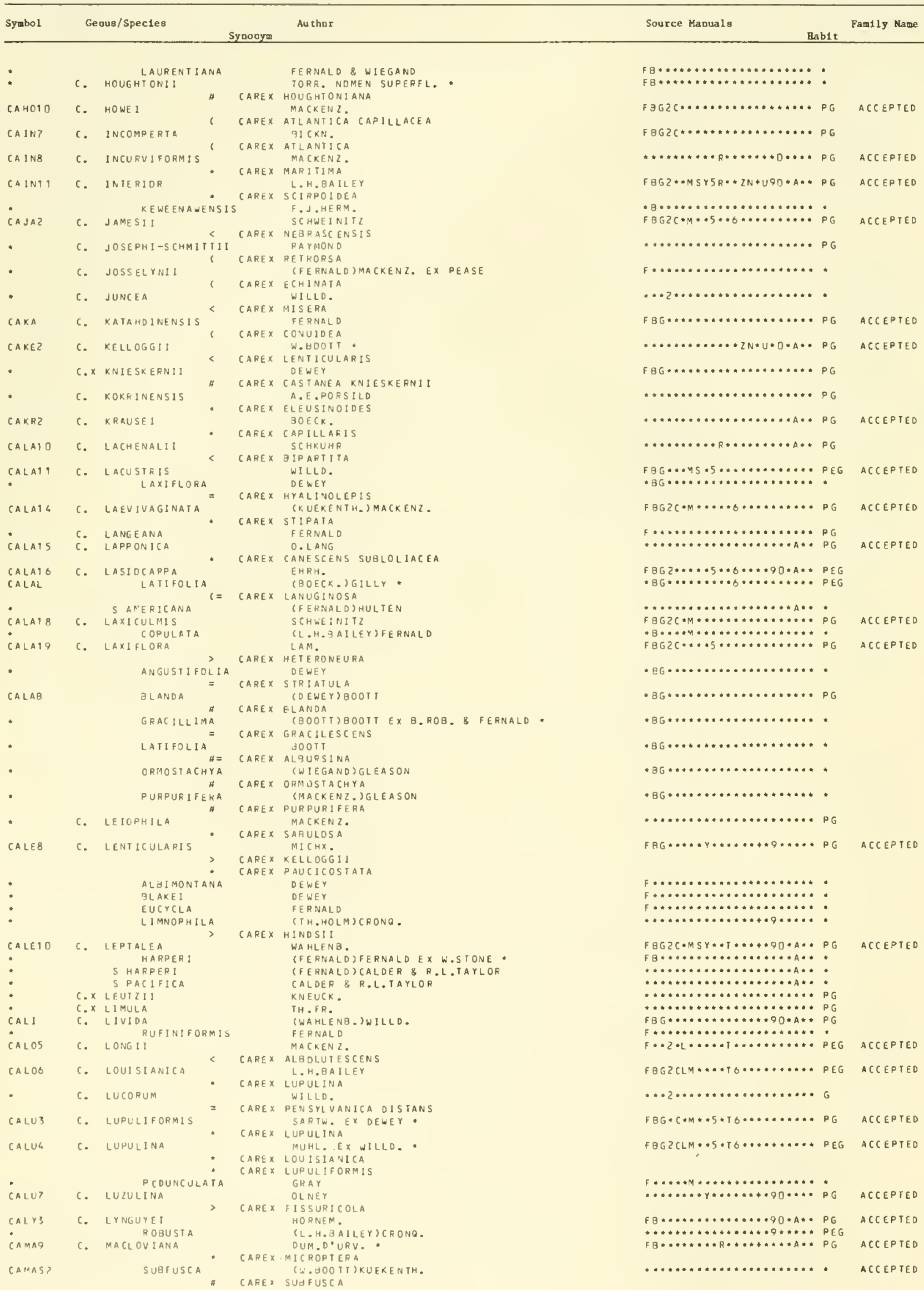




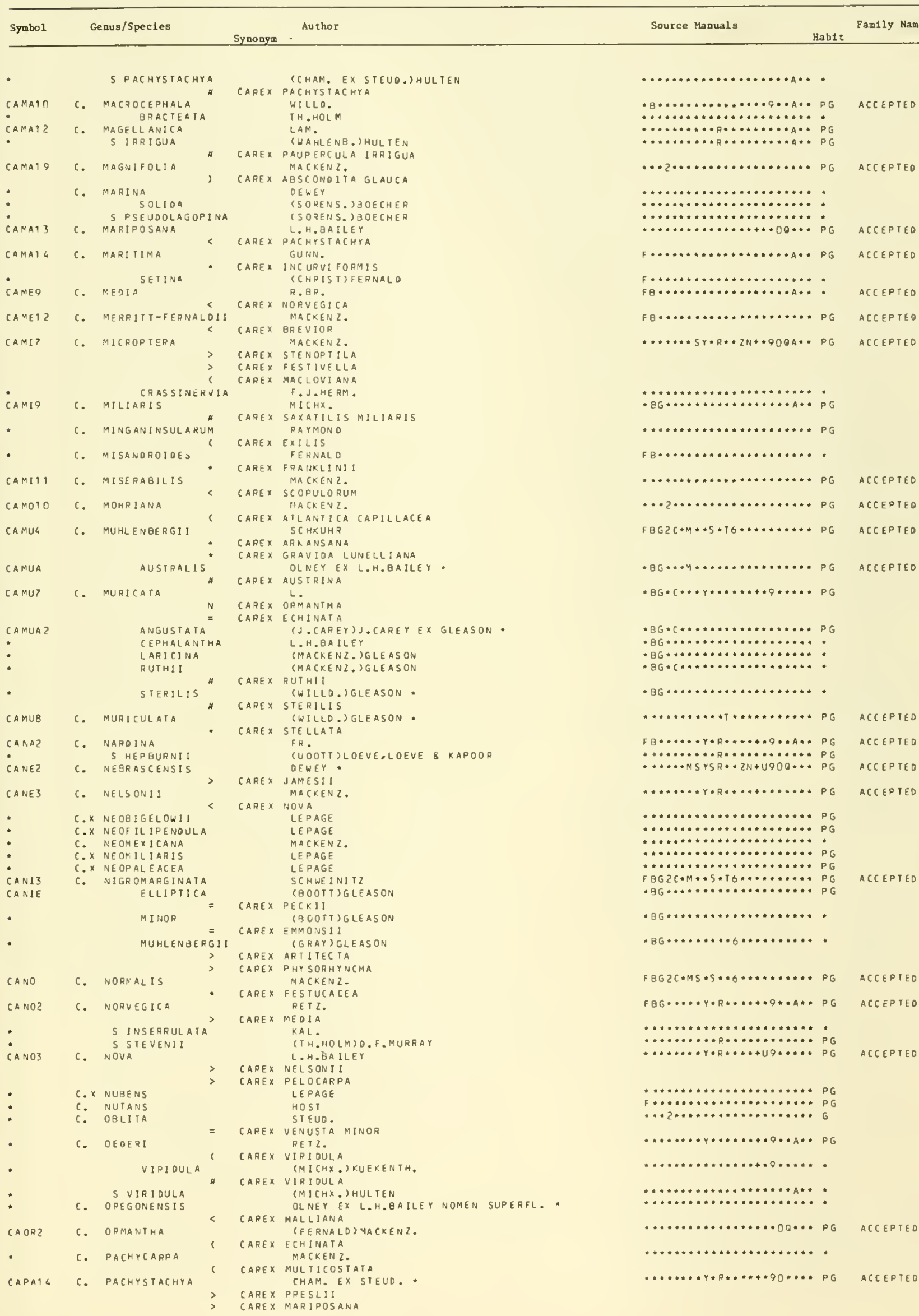




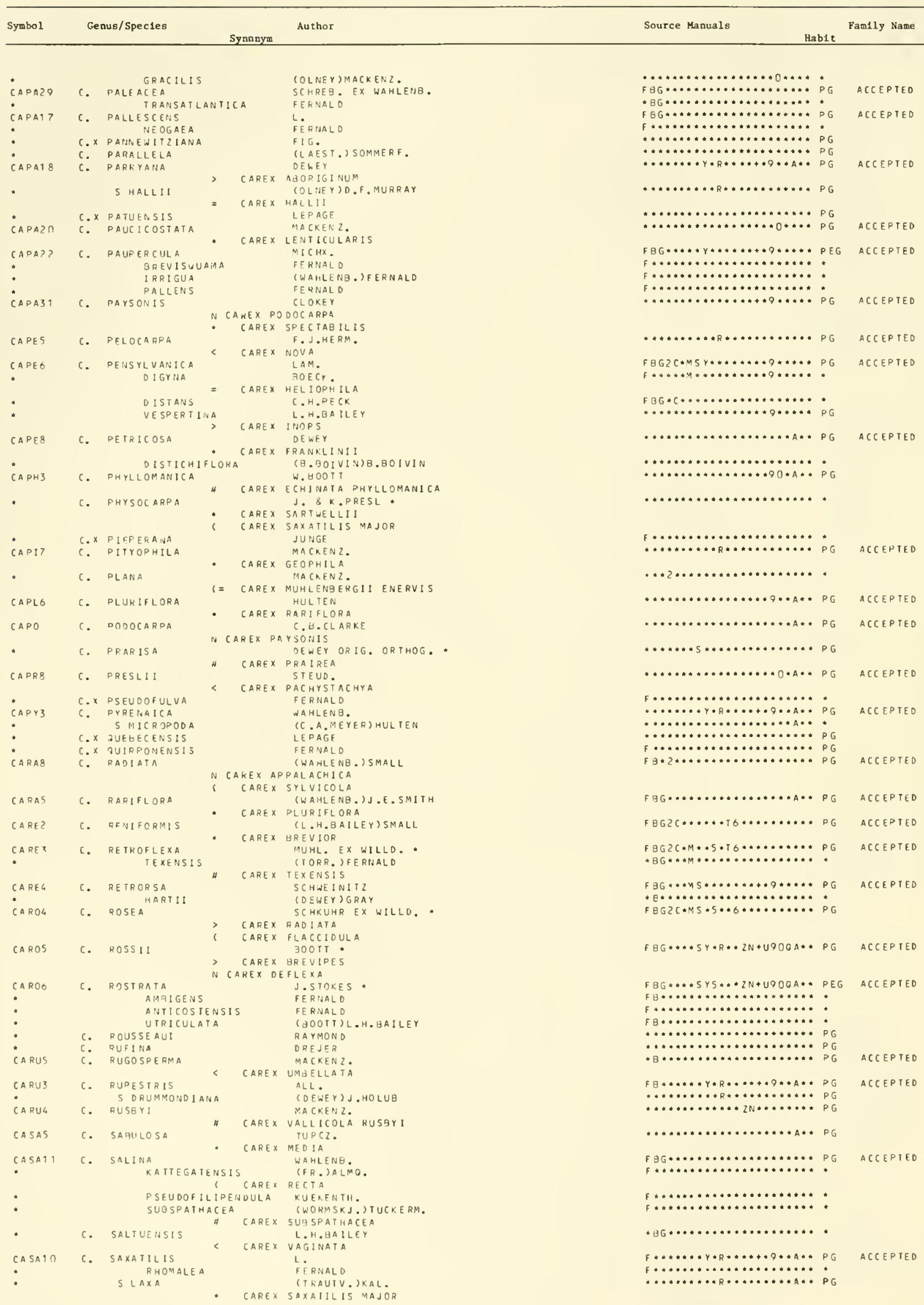




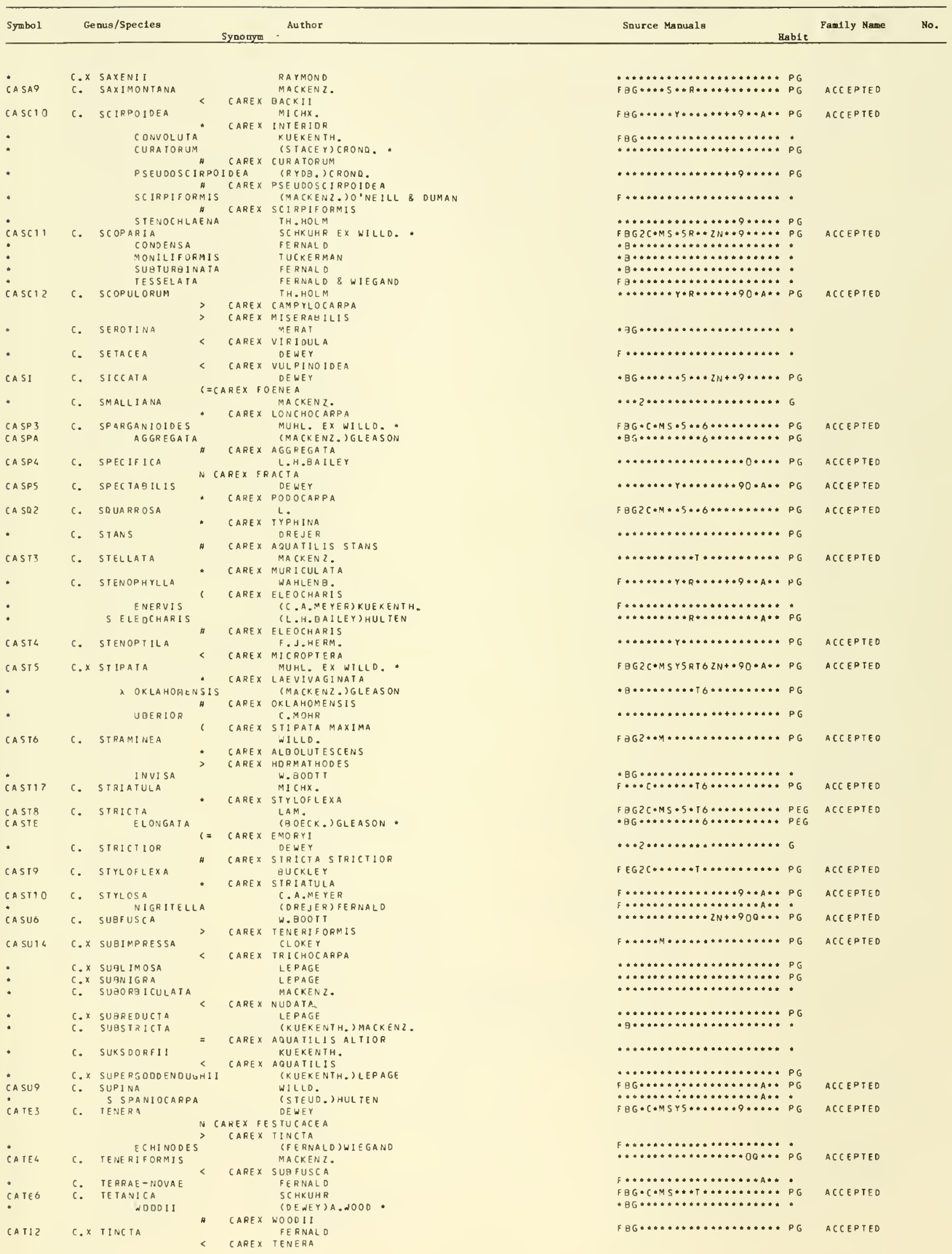




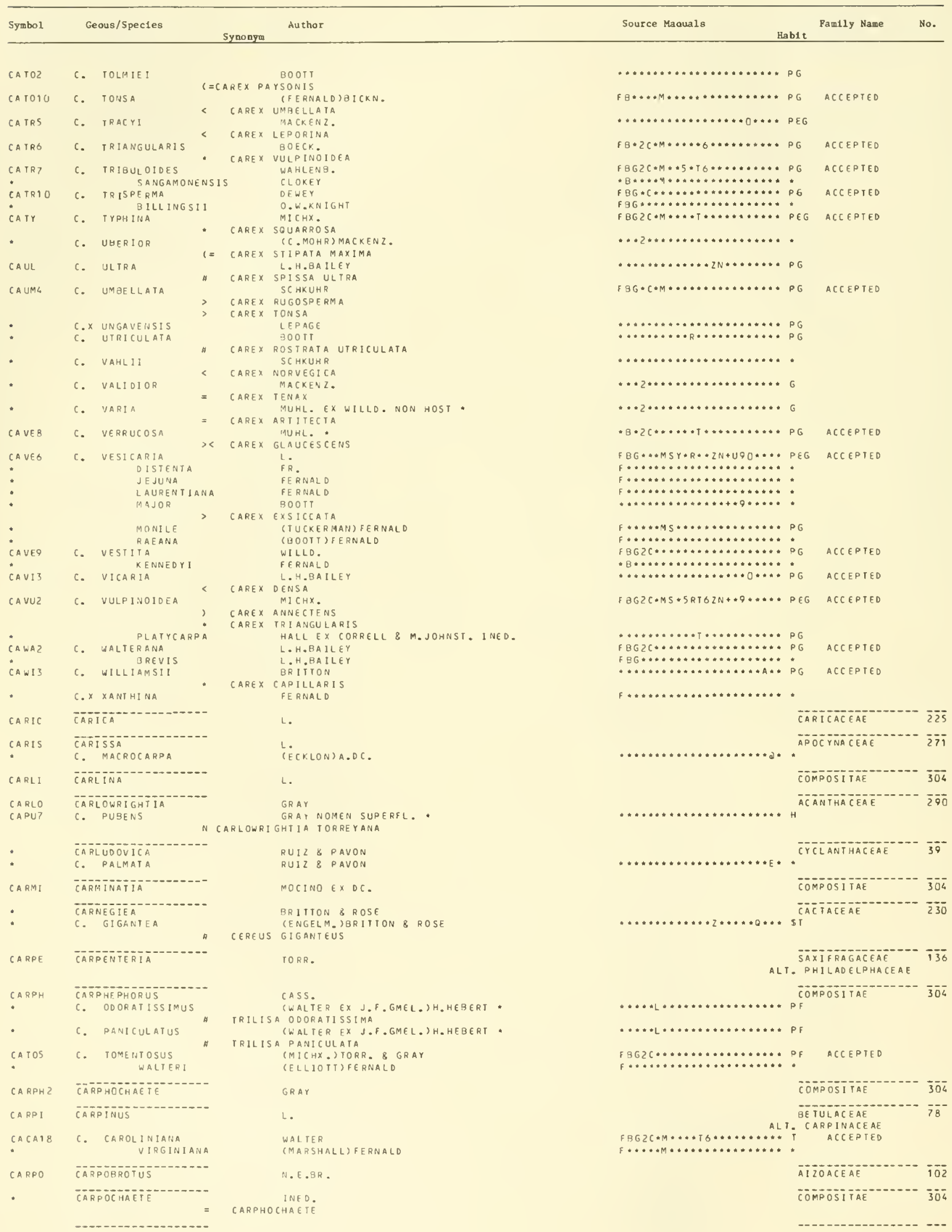




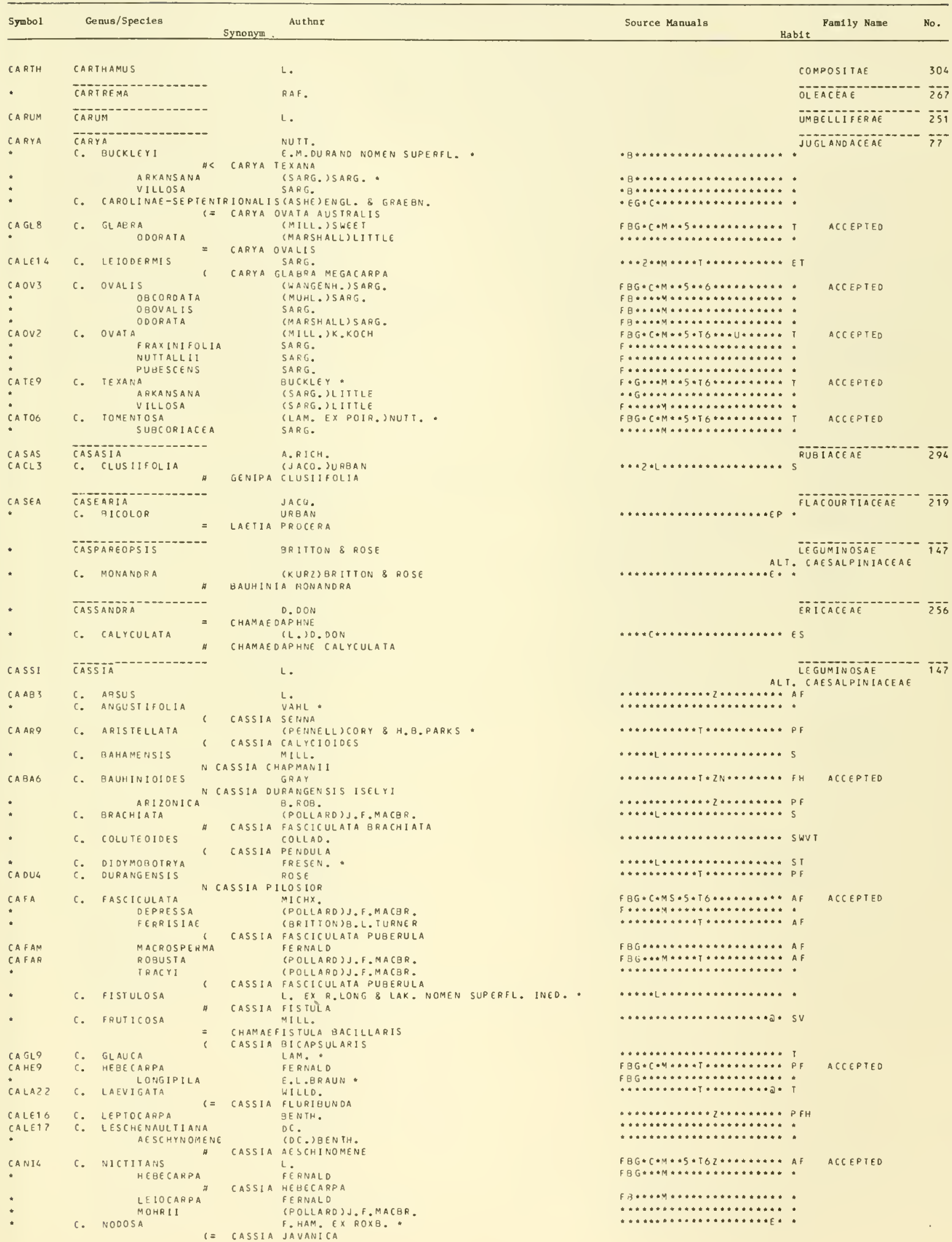




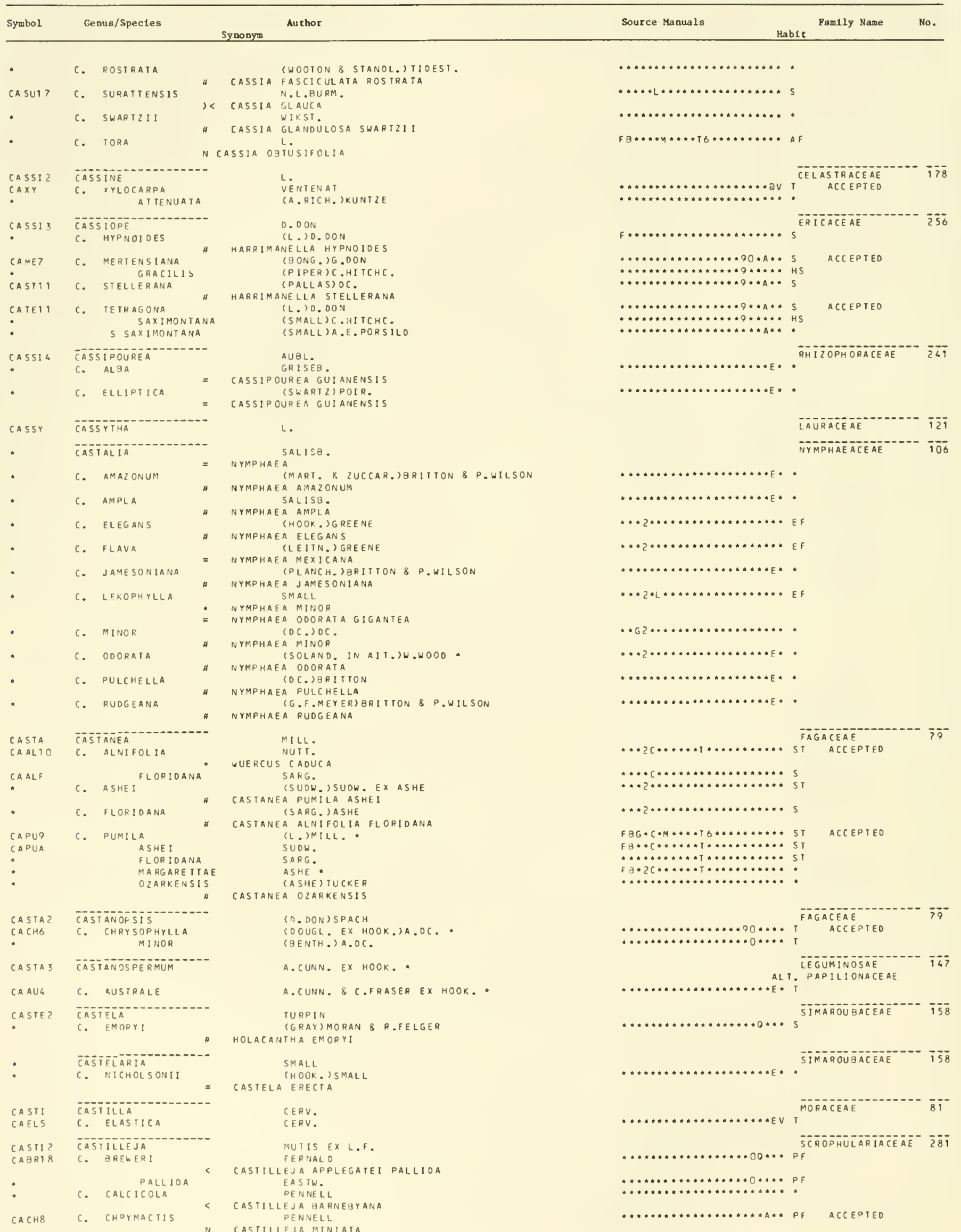




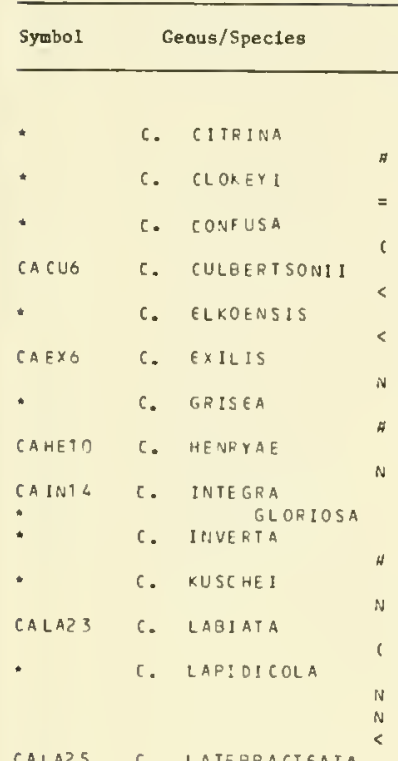

CALAZ 5 C. LATEERAGTEATA

- c. lauta

CALAZT C. LAXA

CALEI9 C. LEINARDII

CALIG C. LINARIIFOLIA

- t. Magnistylis

CAMAIG

CANE8

CAME 17

[AN11 ?

CAMI13

*

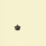

CADALO

CAPAZ 6 C. PARVIFLORA

CAPAZ 7 C. PAYPEAE

CAFI C. PILOSA

EAPR11 S JUSSELII

CAPR11 C. PRUINOSA

CAPRG S GLEASONIL

IAPS3 C. PSITTACINA

C. PYRAMIDALIS

CA ROQ

CA SE 4

-

CA V

CA

CA W I

CA SUA

CA CUS

CALI8

CAMO

CAPAC 8

CA ST: 3

CA SU13

c. SUBEROSA

Synonym

Author

Source Manuals

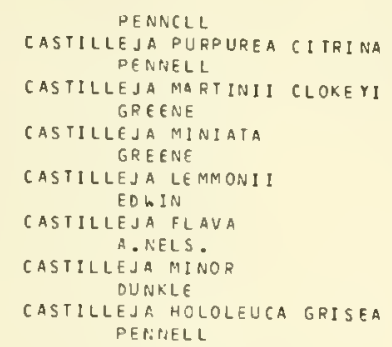

PENPELL
CASTILLEJA PURPUREA LINDHEIMERI

$$
\text { L.: }
$$$$
\text { STICYM.M. }
$$

CASUARINA EQUISETIFOL I S.MOORE IN AIT. OTTO A.DIETR: LESCHEN. EX MIQ.
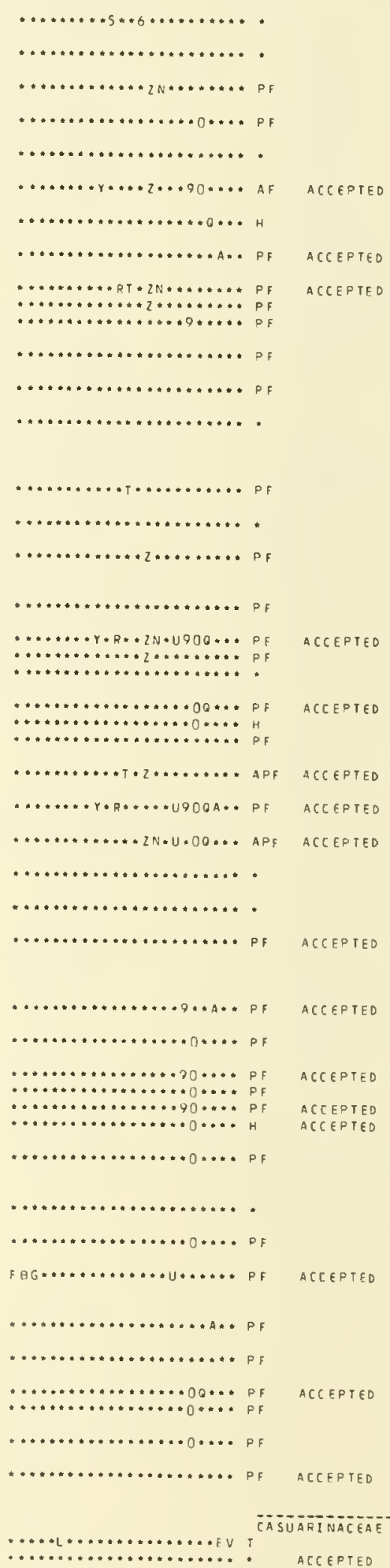

CASUARINACEAE G8

ACCEPTED 


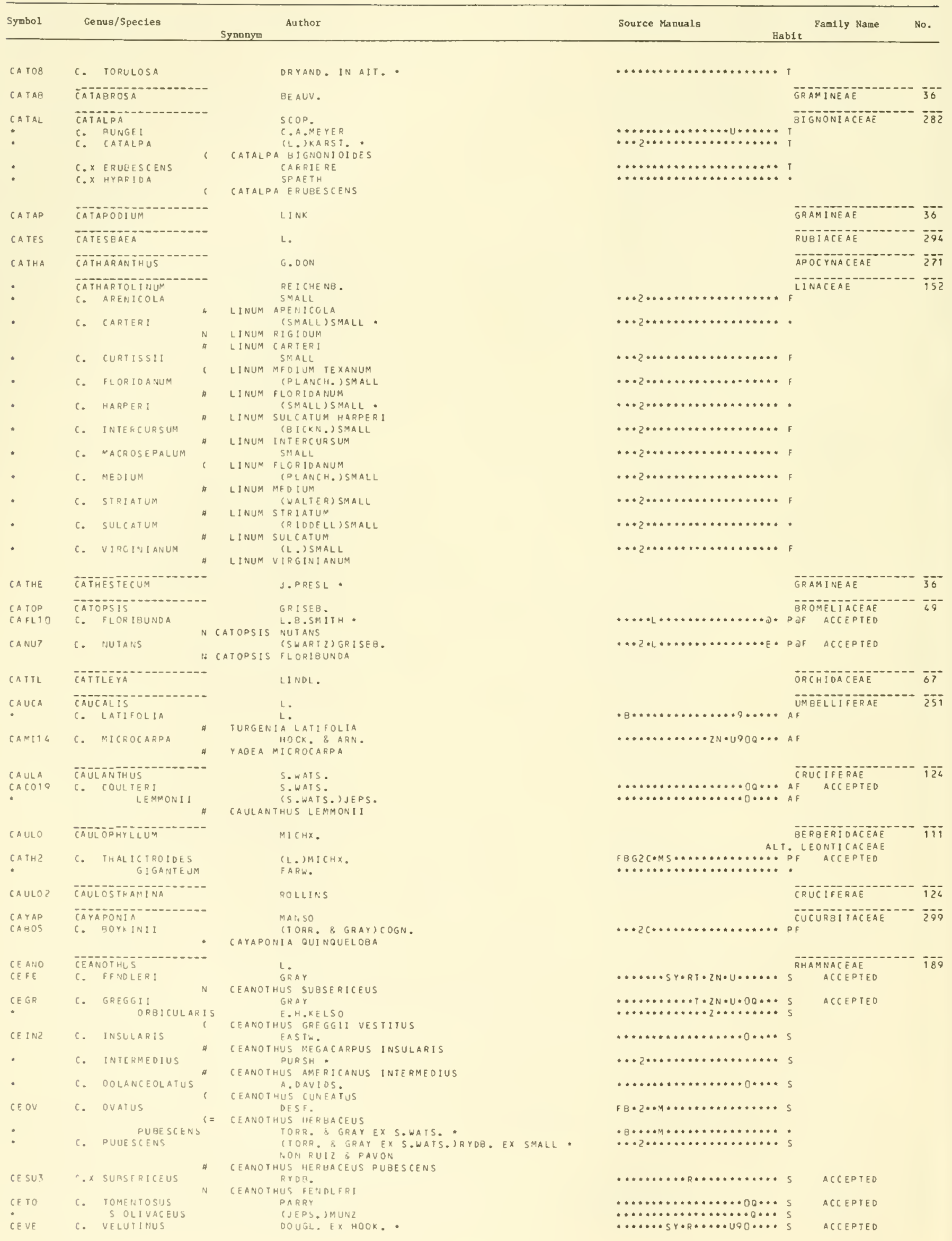




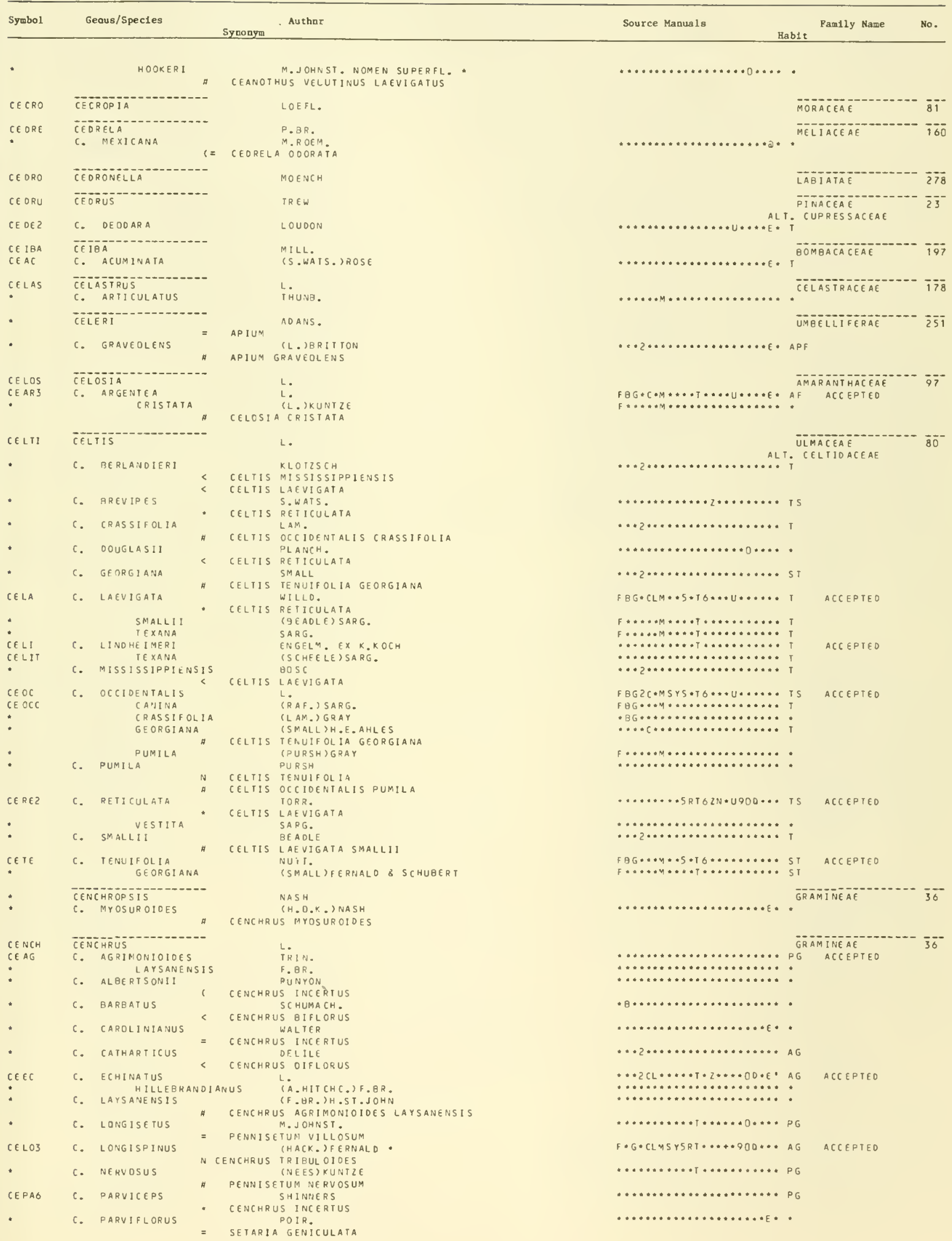




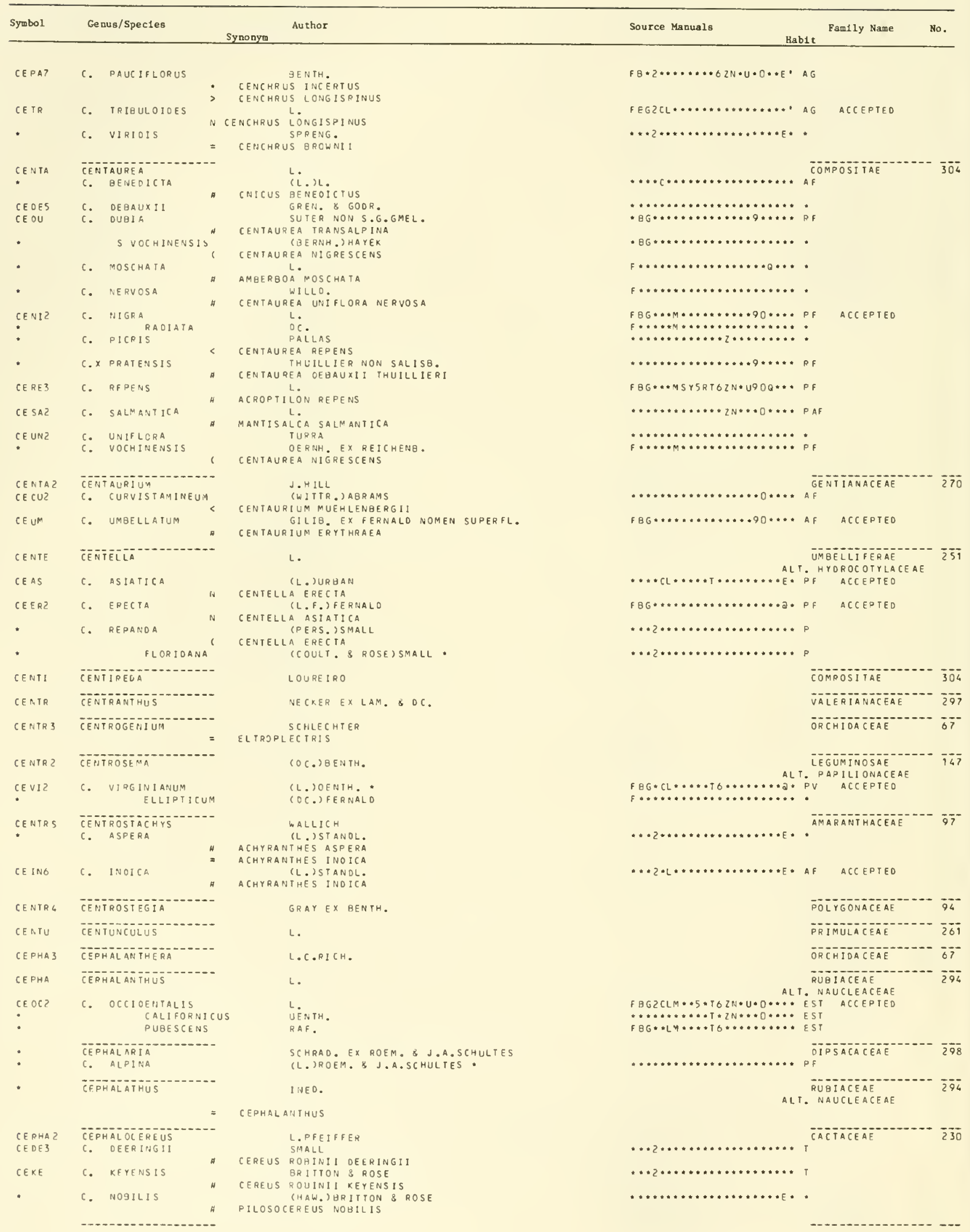


CERASTIUM

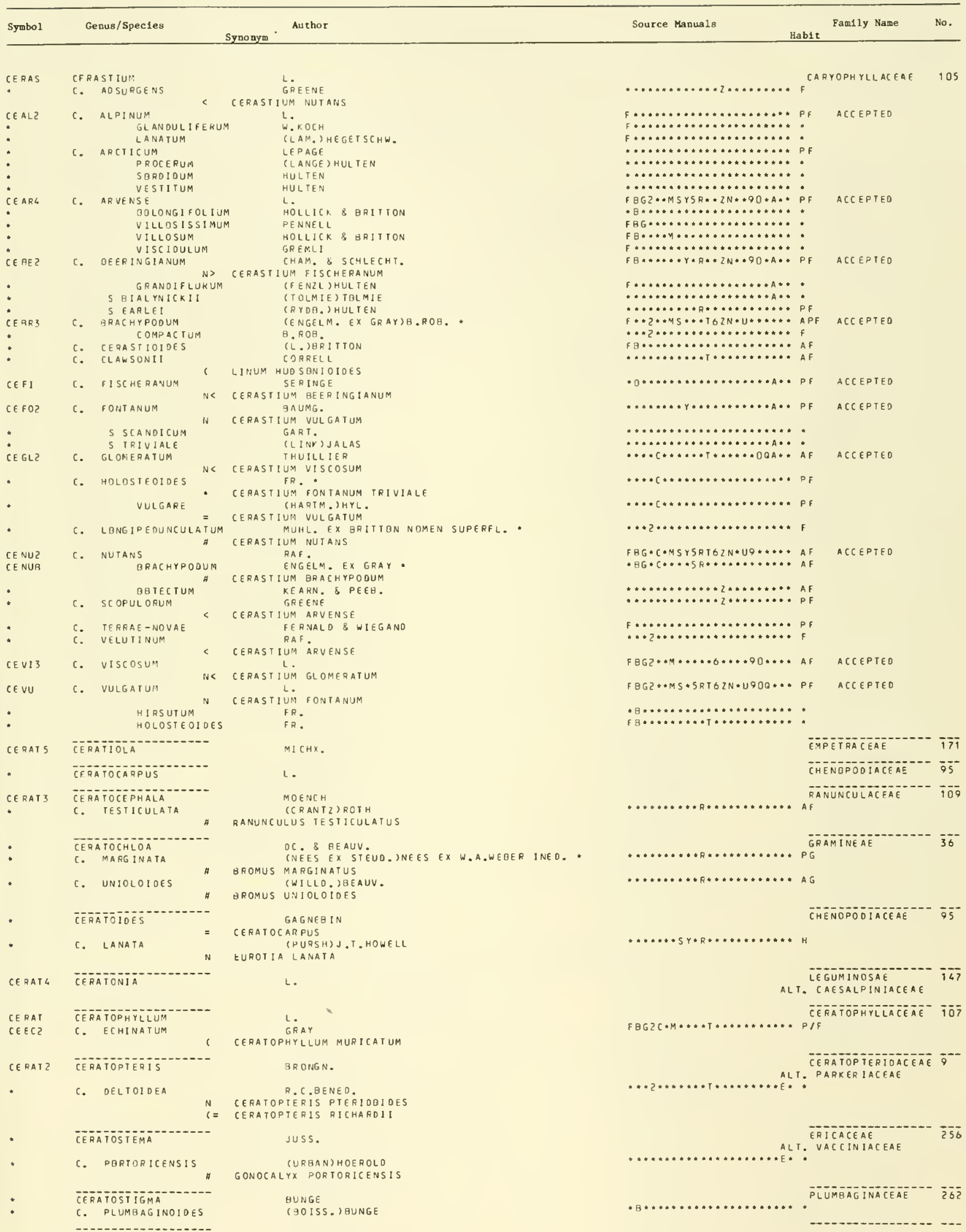




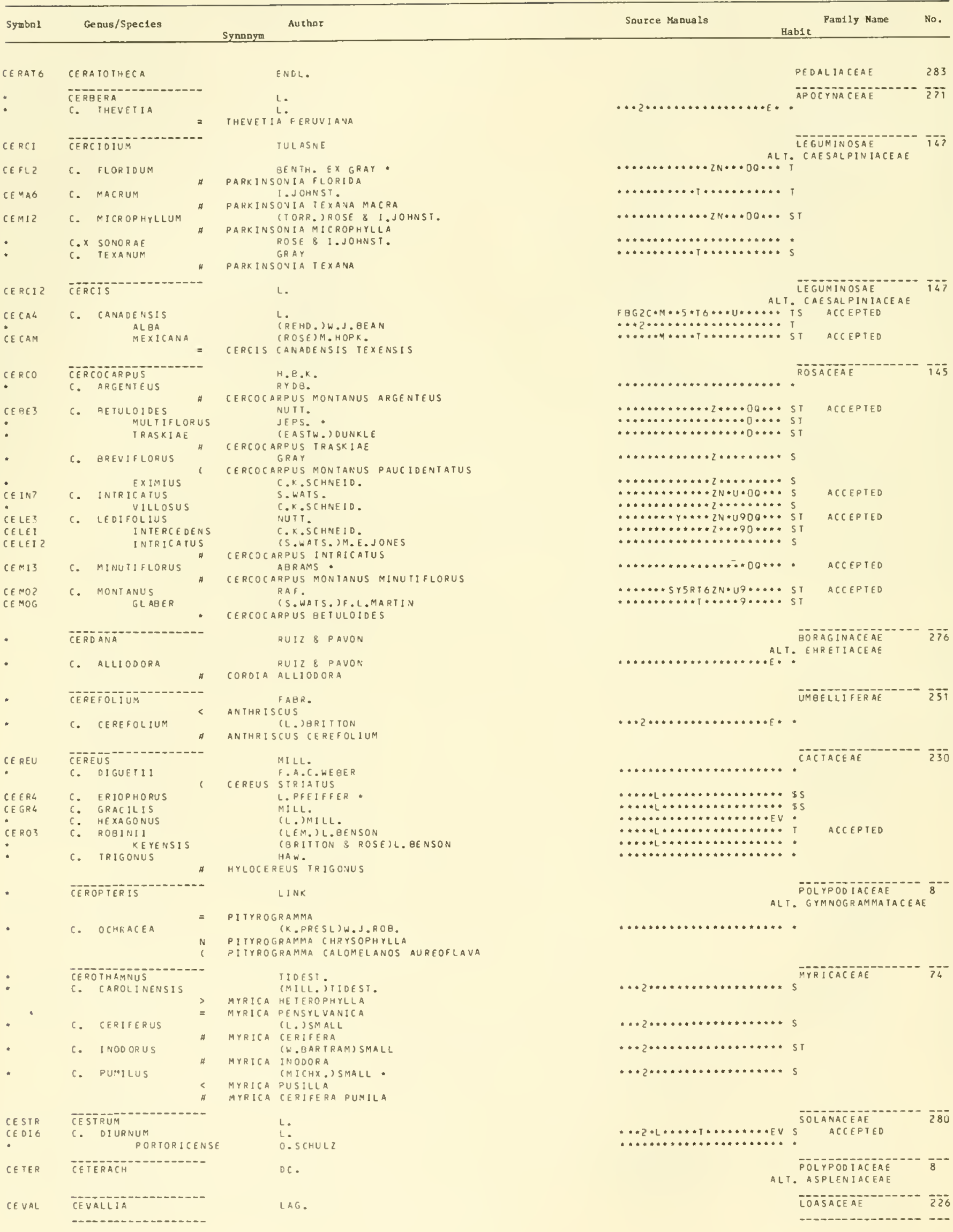




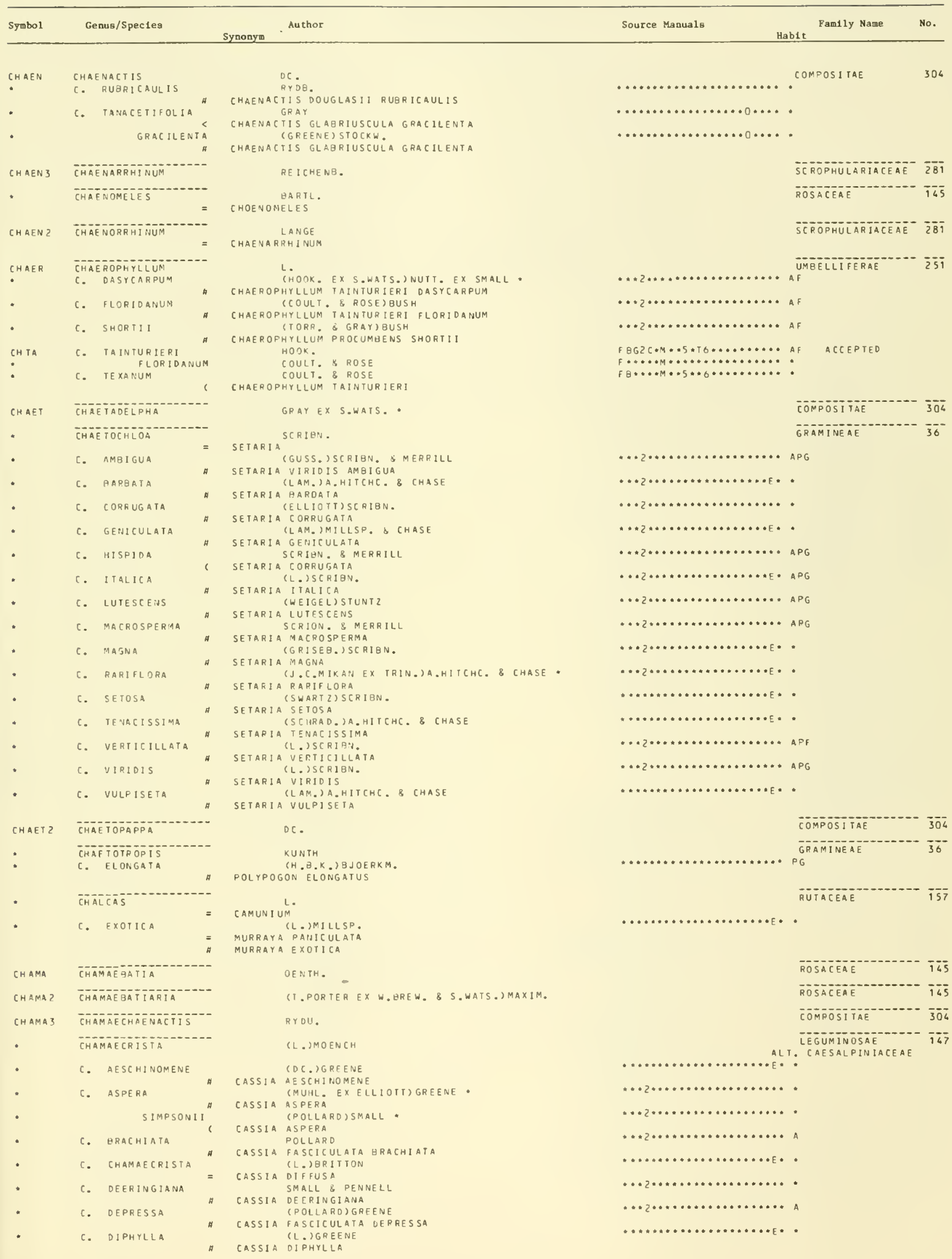




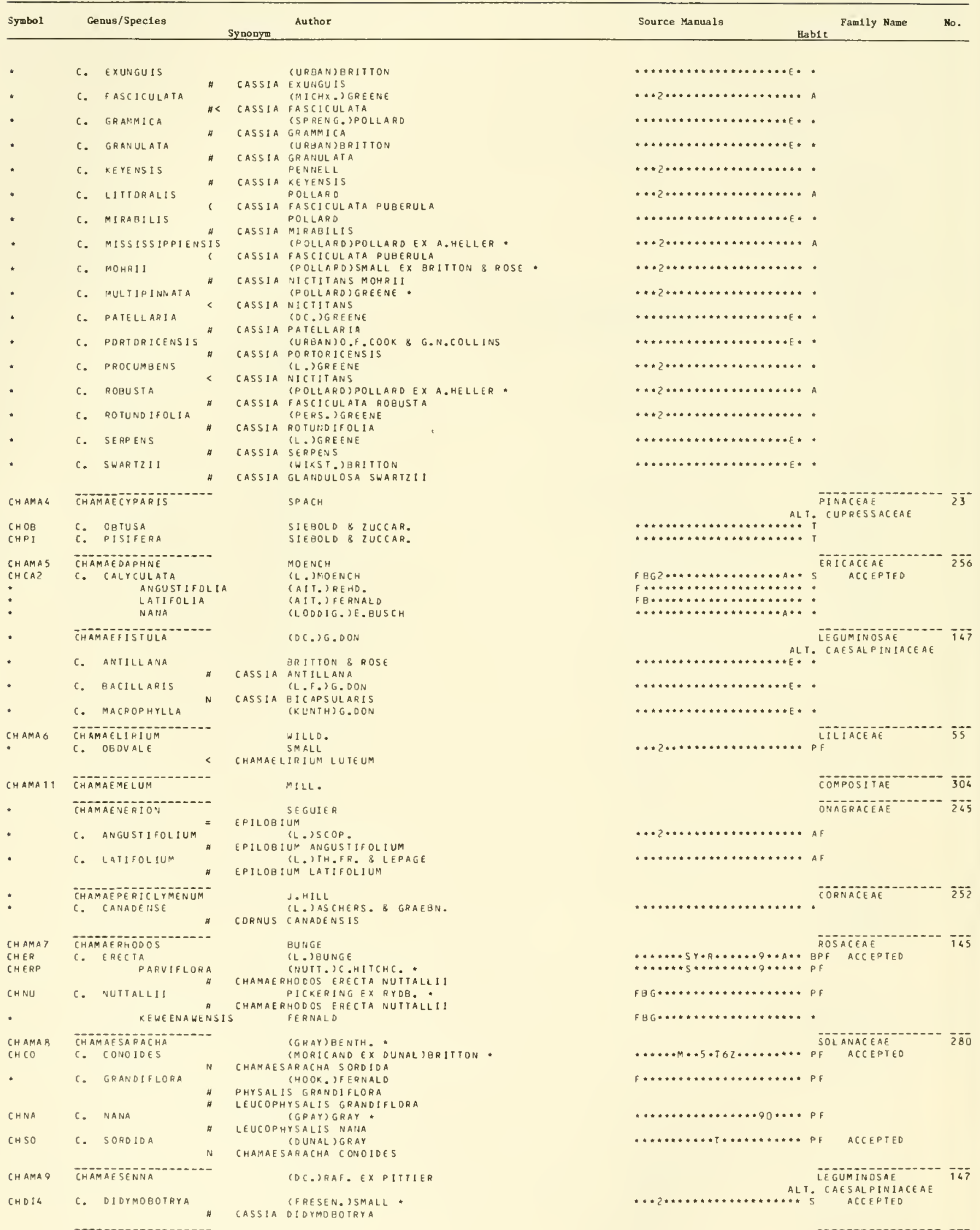




Symbol Genus/Spectes

CHAMA 10 CHAMAESYCE

C. ADENOPTERA

$S$ PERGAMENA

C. ADHAERENS

C. AOICIOIDES

c. AL gOMARGINATA

c. AMMANNIOIDES

C. ANEGADENSIS

c. ARTICULATA

c. BERTERIANA

c. BLOOGETTII

c. BOMBENSIS

C. BRACHYPODA

c. BUXIFOLIA

C. CHIOGENES

c. CONFERTA

c. COROIFOLIA

6. DELTOIDEA

5 SERPYLLUM

c. FENOLERI

c. GARBER:

c. GEMELLA

C. GEYERI

c. GLYPTOSPERMA

c. HIRTA

c. HUMISTRATA

c. HYPERICIFOLIA

C. HYSSOPIFOLIA

c. INGALLSII

c. KEYENSIS

c. LASIOCARPA

c. LAIA

c. MACULATA

c. MATHEWSII

c. MENDEZ I I

c. MESEMBRIANTHEM

c. MICROMERA

c. MISSURICA

C. MONENSIS

c. MOSIERI

c. NASHII

c. NUTANS

c. OPHTHALMICA

C. PARRYI

c. POLYGONIFOLIA

c. PORTORICENSIS

c. PROSTRATA
Syoonym

Author

RAF.

(GERTOL.) SMALL

I A PERGAMENA

EUPHORPIA PERGAMENA

SMALL

EUPHOREIA DELTOIOEA

SMALL

EUPHORBIA GARBERI

- (TORR. \& GRAY) SMALL

EUPHORBIA ALBOMARGINATA

EUPHORBIA AMMANNIOIDES

MILLSP.

EUPHOREIA TURPINII

(AUBL.)BRITTON
EUPHOREI A ARTICULATA

(BALB. EX SPRENG.) MILLSP.

EUPHORBIA QERTERIANA

(ENGELM. EX A. HITCHC.)SMALL .
EUPHORBIA BLOQGETTII

CJACQ.) DUGAND

N EUPHORBIA AMMANNLIDES

SMALL

( euphorbia garberi

(LAM.) SMALL

$=$ EUPHQRAIA MESEMGRIANTHEMIFOLIA SMALL

EUPHORBIA BLOOGETIII

SMALL

EUPHORBIA COMFERTA

(ALLLOTT)SMALL

EUPHORBIA COR OI FOLIA

(ENGELM. EX CHAPM. ISMALL.

EUPHORBIA OELTOIDEA

(SMALL) BURCH

EUPHOREIA OELTOIOEA

(TORR. \& GRAY ISMALL

EUPHOREIA FENDLERI

SENGELM. EX CHAPM. 'SMALL *

EUPHORBIA GARBERI

¿LAG.) SMALL

EUPHOREIA OPHTHALMICA

(ENGELM.) SMALL

EUPHORBIA GEYER L

$$
\text { CENGELM. ISMALL }
$$

EUPHORBIA GLYPTOSPERMA

(L.) MILLSP.

EUPHORBIA HIRTA

EUPHORBIA OPHTHALMICA

(ENGELM.)SMALL

EUPHBRBIA HUMISTRATA

EUPHOREIA HYPERICIFOLIA

EUPHORBIA MACULATA

$$
\text { CL. ISIMALL }
$$

EUPHORQ IA HYSSOPIFOLIA

EUPHORBIA NUTANS

SMALL

= EUPHORBIA AMMANNIOLOES

SMALL
CHAMAESYCE PORTERANA KEYENSIS

SYC PORTERANA KEYI
(KLOTZSCH)ARTH.

EUPHORBIA LASIOCARPA

(ENGELM.) SMALL

EUPHORBIA LATA

(L.)SMALL

E UPHORBIA MACULATA

SHALL

EUPHORBIA MACULATA

(BOISS.)MILLSP.

EUPHORBIA MENDE ZII

lia (JaCQ.) OUGand.

EUPHORBIA MESEMBR I ANTHEMI FOL JA
(BOISS.) WOOTON \& STANOL.

EUPHORBIA MICROMERA

(RAF.) SHINNERS

EUPHORBIA MISSURICA

MILLSP.

EUPHORRIA PERGAMENA

EUPHORBIA GA

EUPHORBIA GARBER!
SMALL

EUPHORBIA BLODGETTII

(LAG.) SMAL

EUPHORBIA NUTANS

EUPHORBIA HYPERICIFOL IA

EUPHORBIA OPHTHALMICA

N CHAMAESYCE HIRTA

SENGELM.) RYOB.

EUPHORBIA PARRYI

EUPHORBIA POLYGONIFOLIA

(URSAN)MILLSP
EUPHORBIA IURPINII

EUPHORBIA IURPINI I
(AIT, ) SMALL

* euphorbia prostrata
Source Manuals

Pantly Name

No.

Hab1t

EUPHORB IACEAE

167

$\cdots \cdot 2 * L * \ldots * * * \cdots * \cdots * * E * b$

$\ldots \ldots$............. b

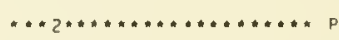

$* \star \cdot 2 \ldots \ldots \ldots \ldots \ldots \ldots \ldots+\ldots s$

$* \ldots+\ldots * \ldots * \ldots \ldots \ldots \ldots \ldots \ldots *$

$* n * * 4 * \ldots+* * \ldots * \ldots \ldots \ldots * *$ P

$\ldots \ldots \ldots \ldots \ldots \ldots \ldots \ldots \ldots$

$\ldots \ldots \ldots \ldots \ldots \ldots \ldots \ldots \ldots+$ EV $5 T$

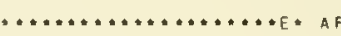

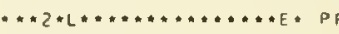

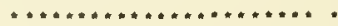

$\ldots * 2 \ldots \ldots \ldots \ldots \ldots \ldots \ldots \ldots * * p$

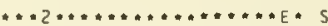

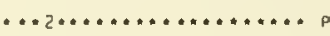

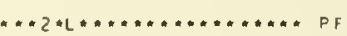

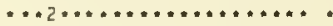

$\ldots * 2 * L \ldots \ldots \ldots \ldots \ldots \ldots \ldots \ldots P F$

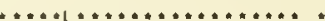

$* n * \ldots \ldots \ldots+R \ldots \ldots \ldots \ldots \ldots+P F$

**2*L**************PF

**2*............. p

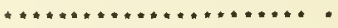

$\ldots \ldots \ldots+* * R \cdot \ldots \ldots \ldots \ldots \ldots$ A
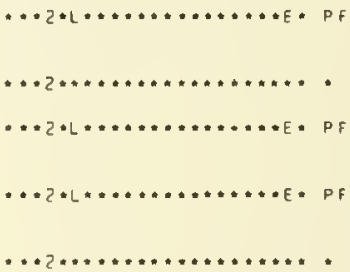

$\ldots * 2 * \ldots \ldots \ldots \ldots \ldots \ldots \ldots * p$
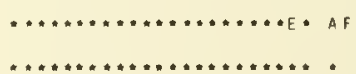

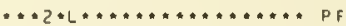

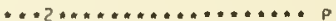

$\ldots \ldots \ldots \ldots \ldots \ldots \ldots \ldots \ldots \ldots \ldots \ldots$ PF

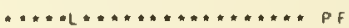
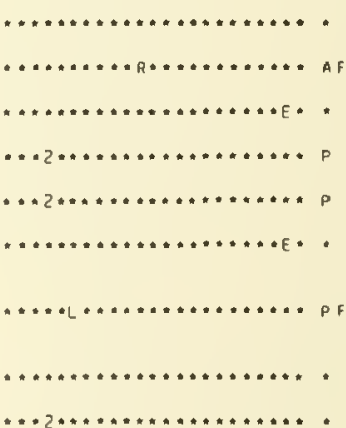

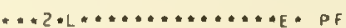




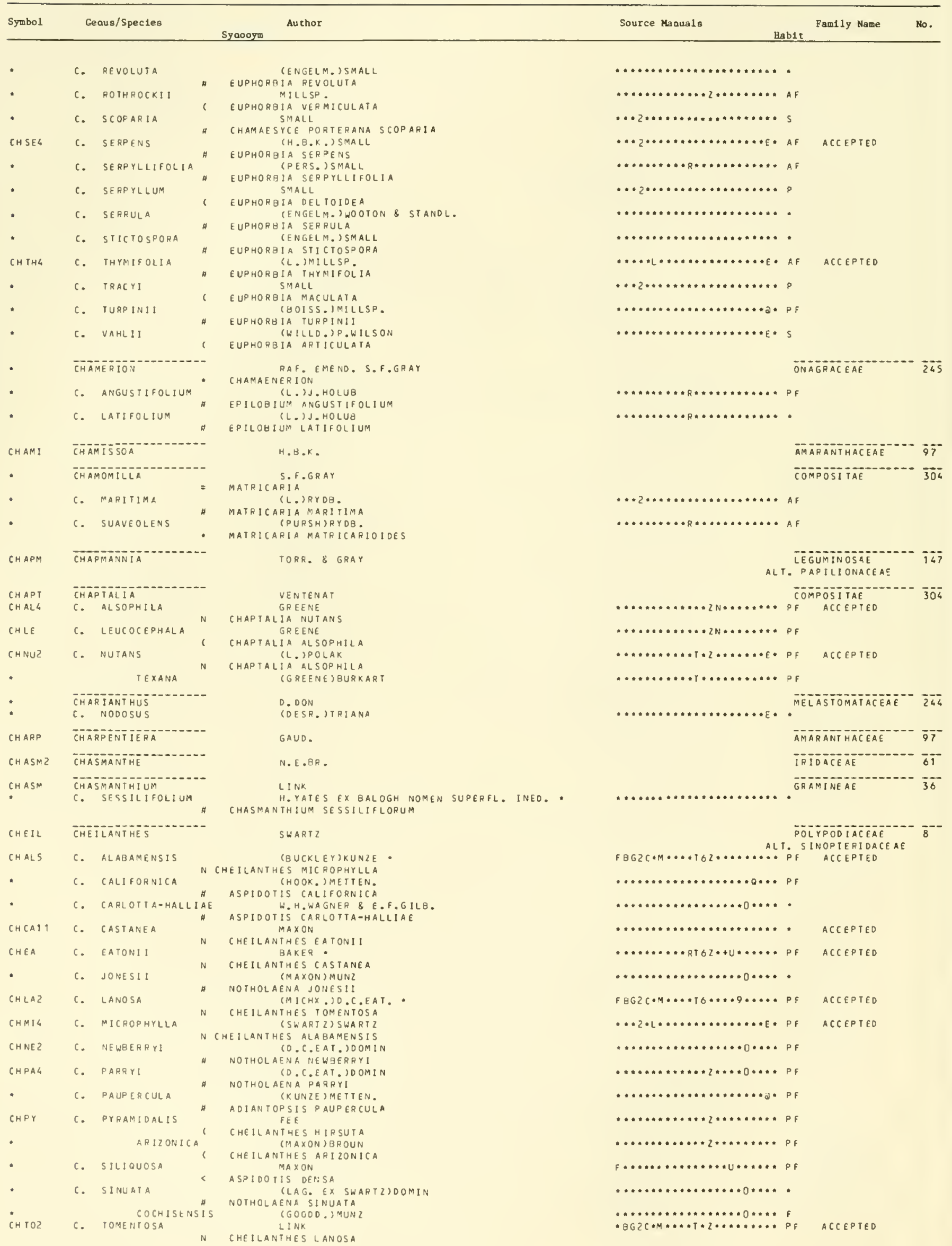




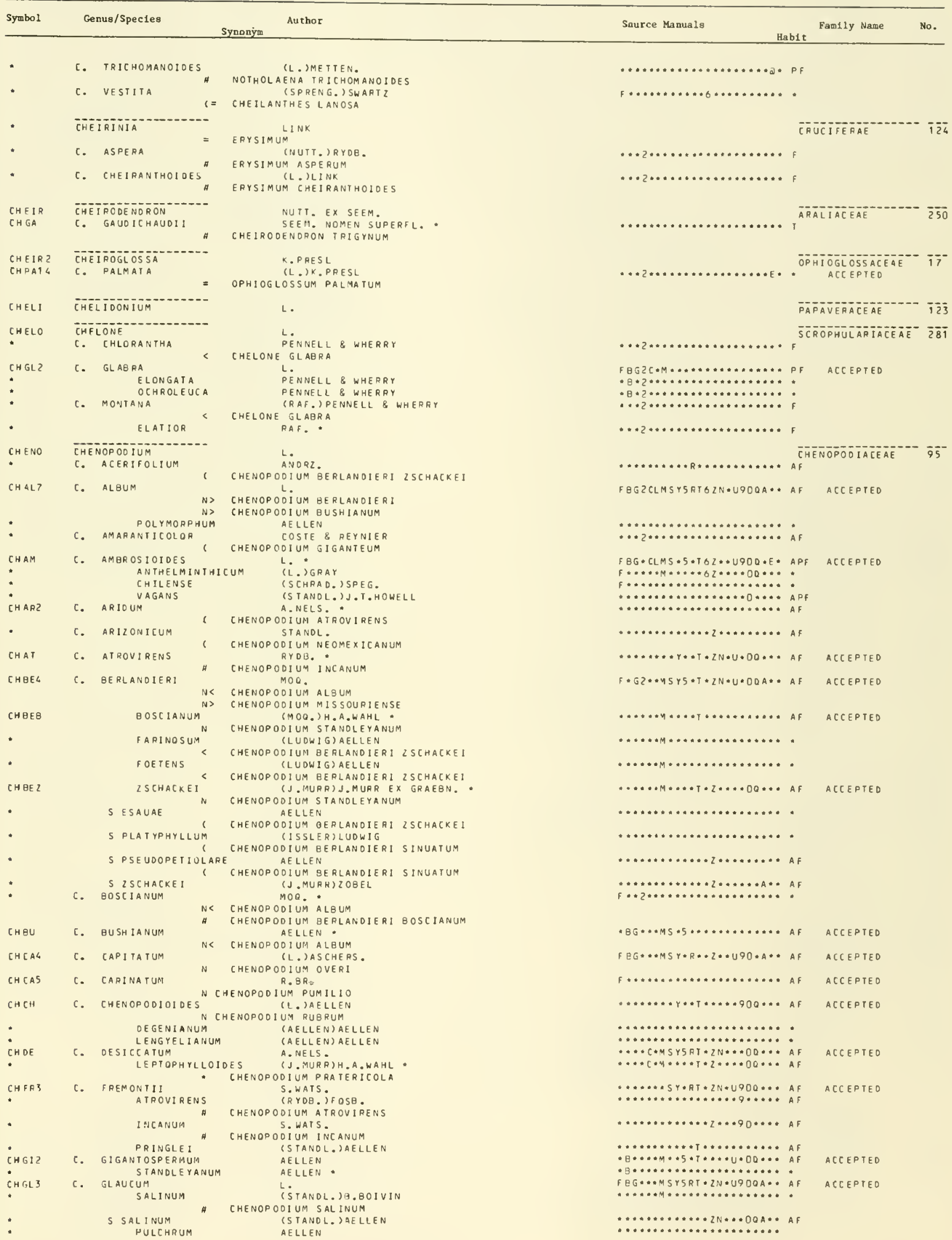




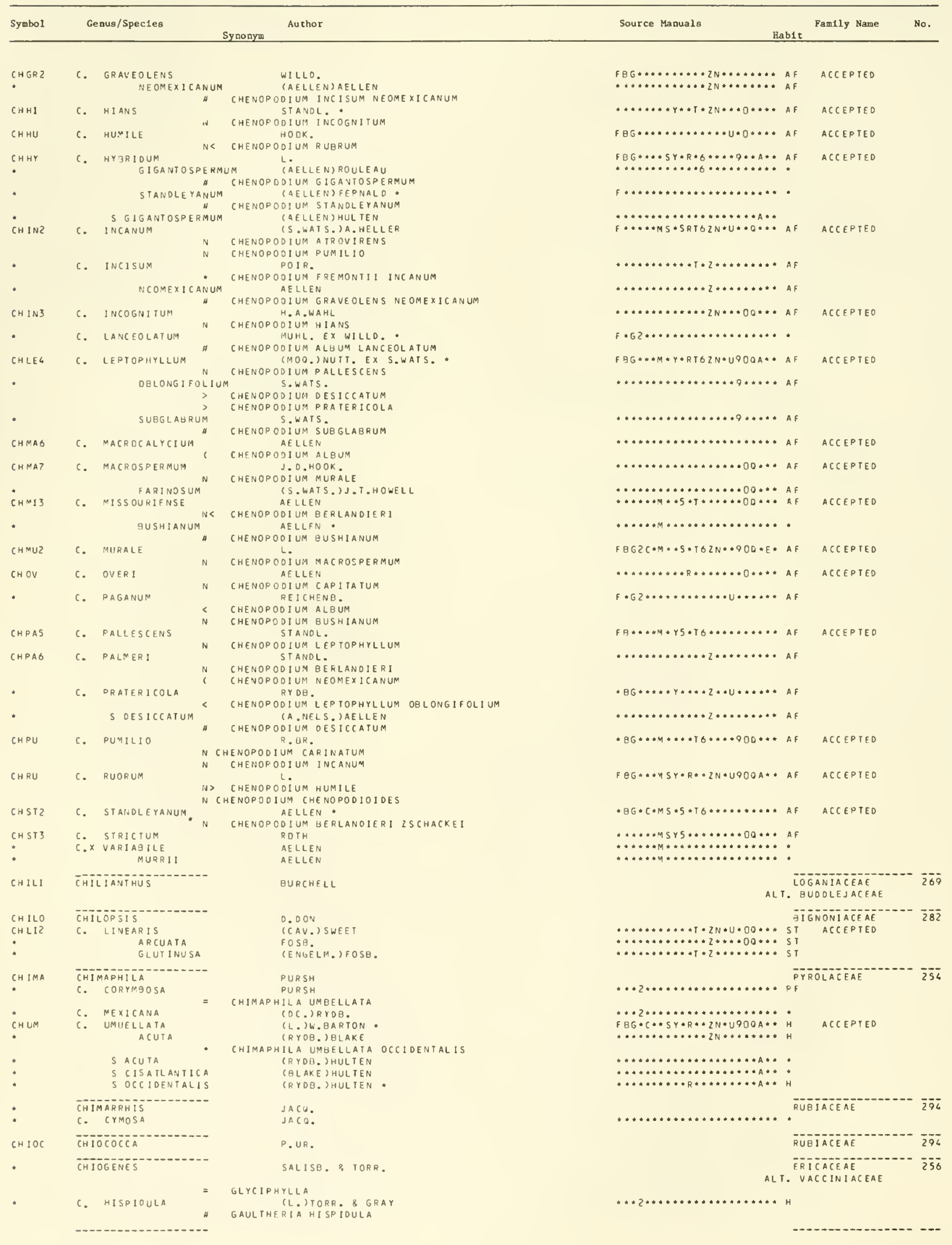




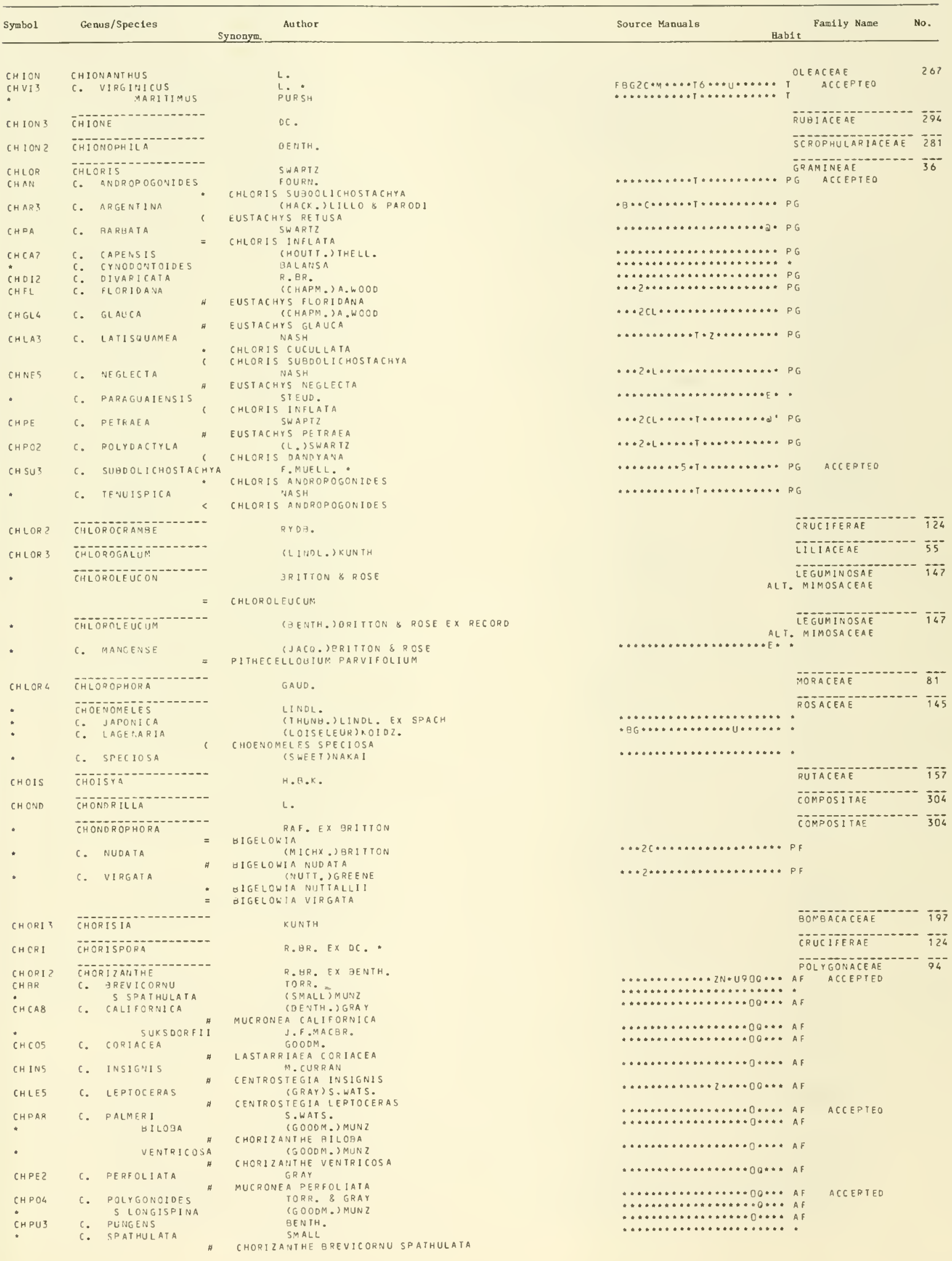




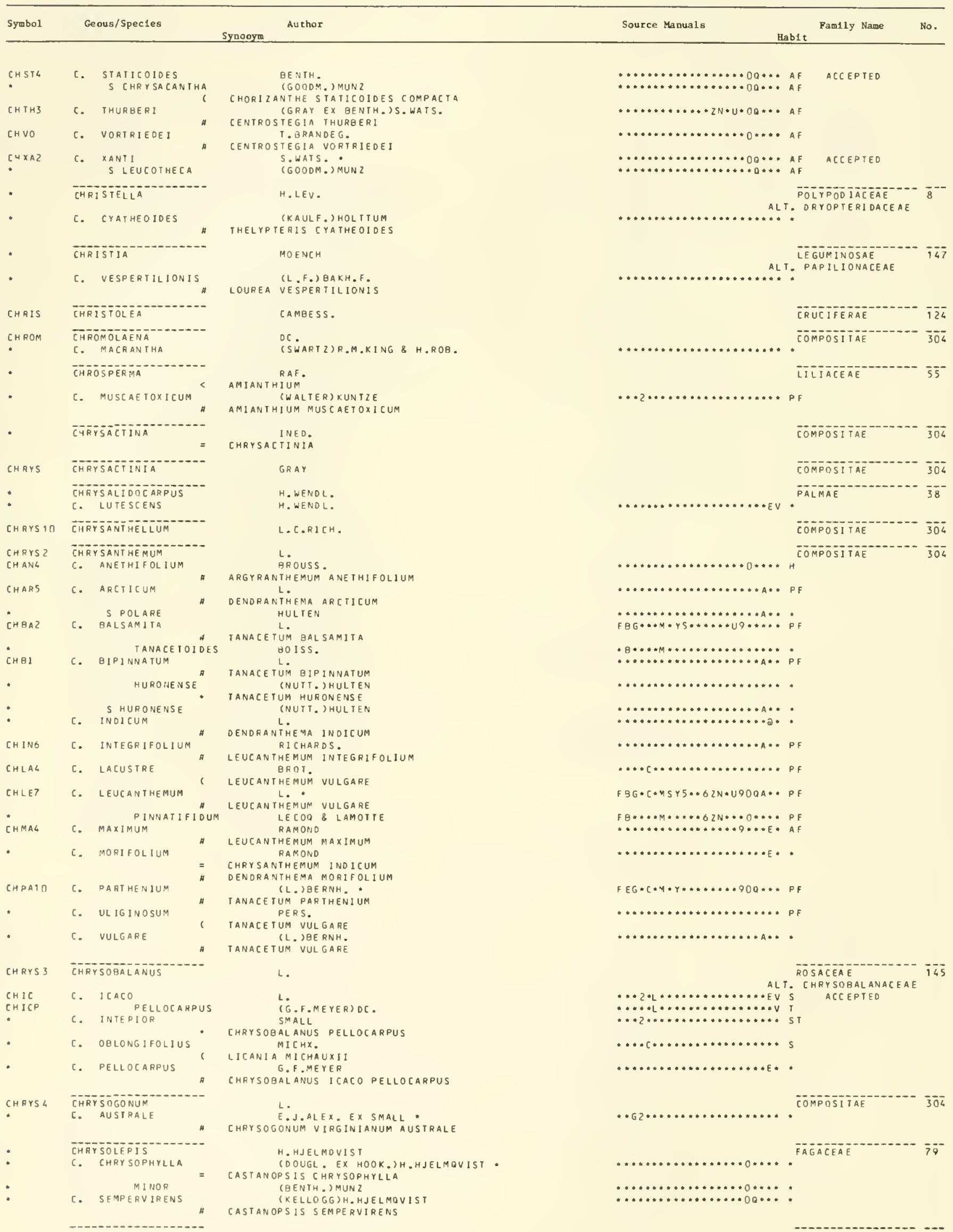


Source Manualo

Fsmily Nam

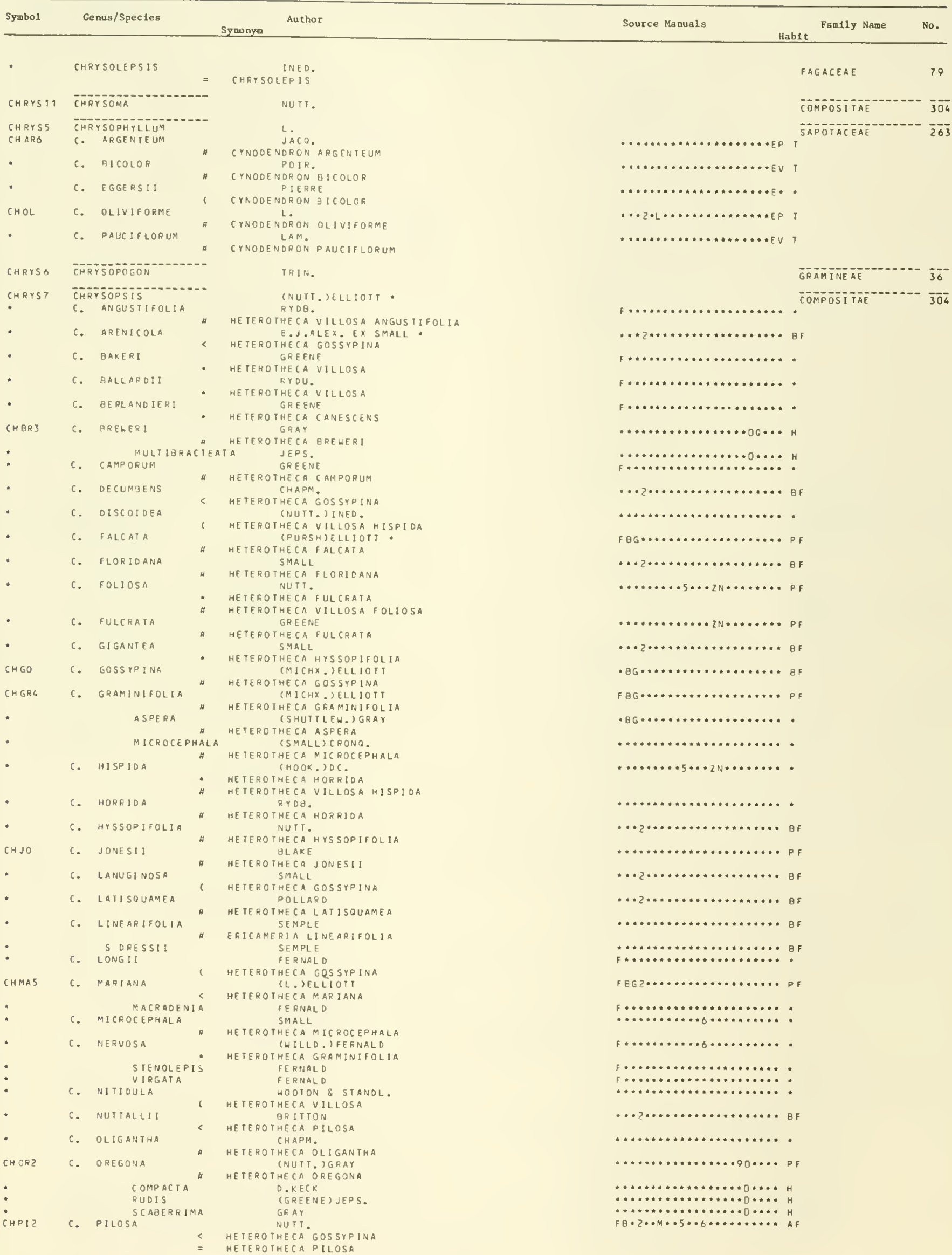




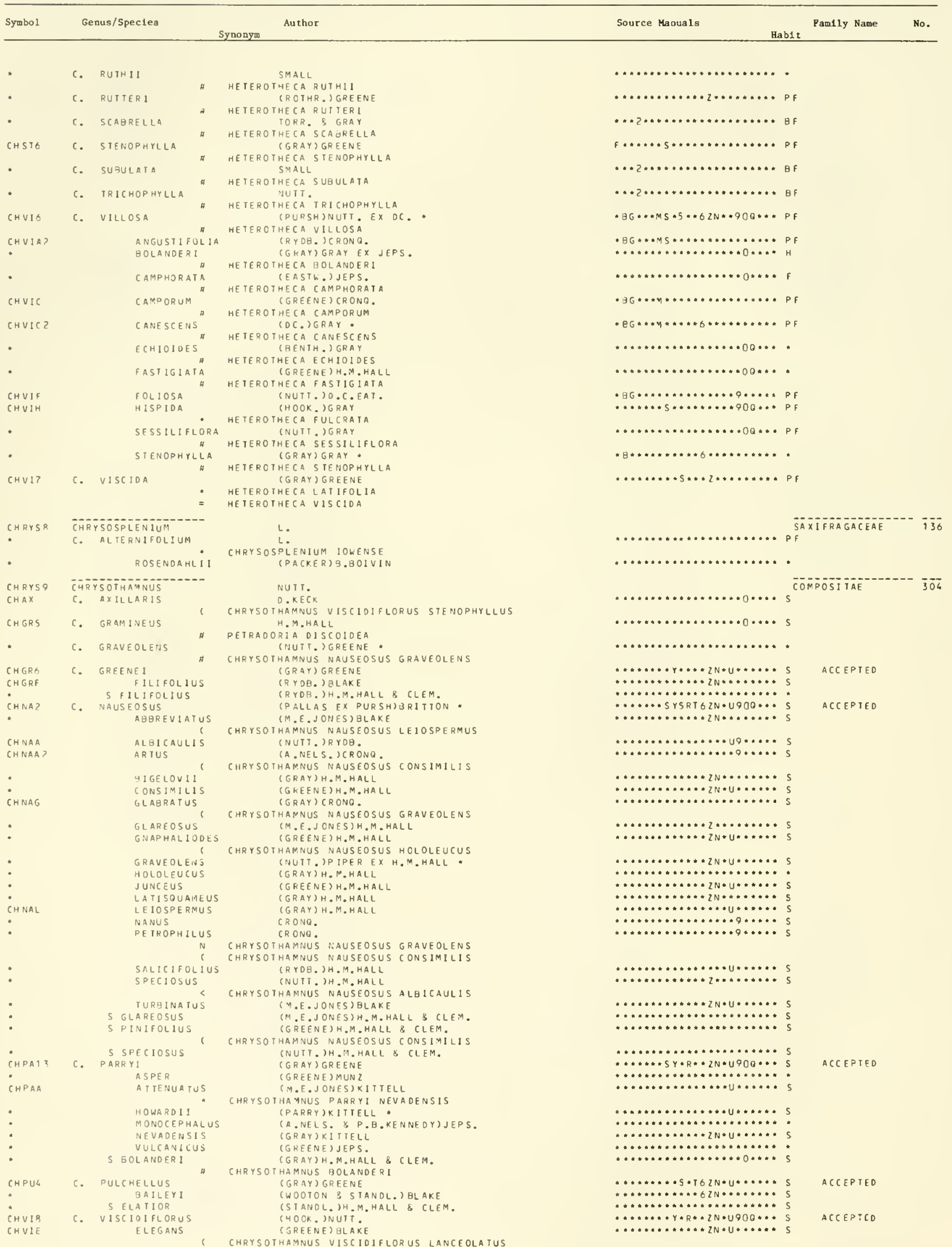




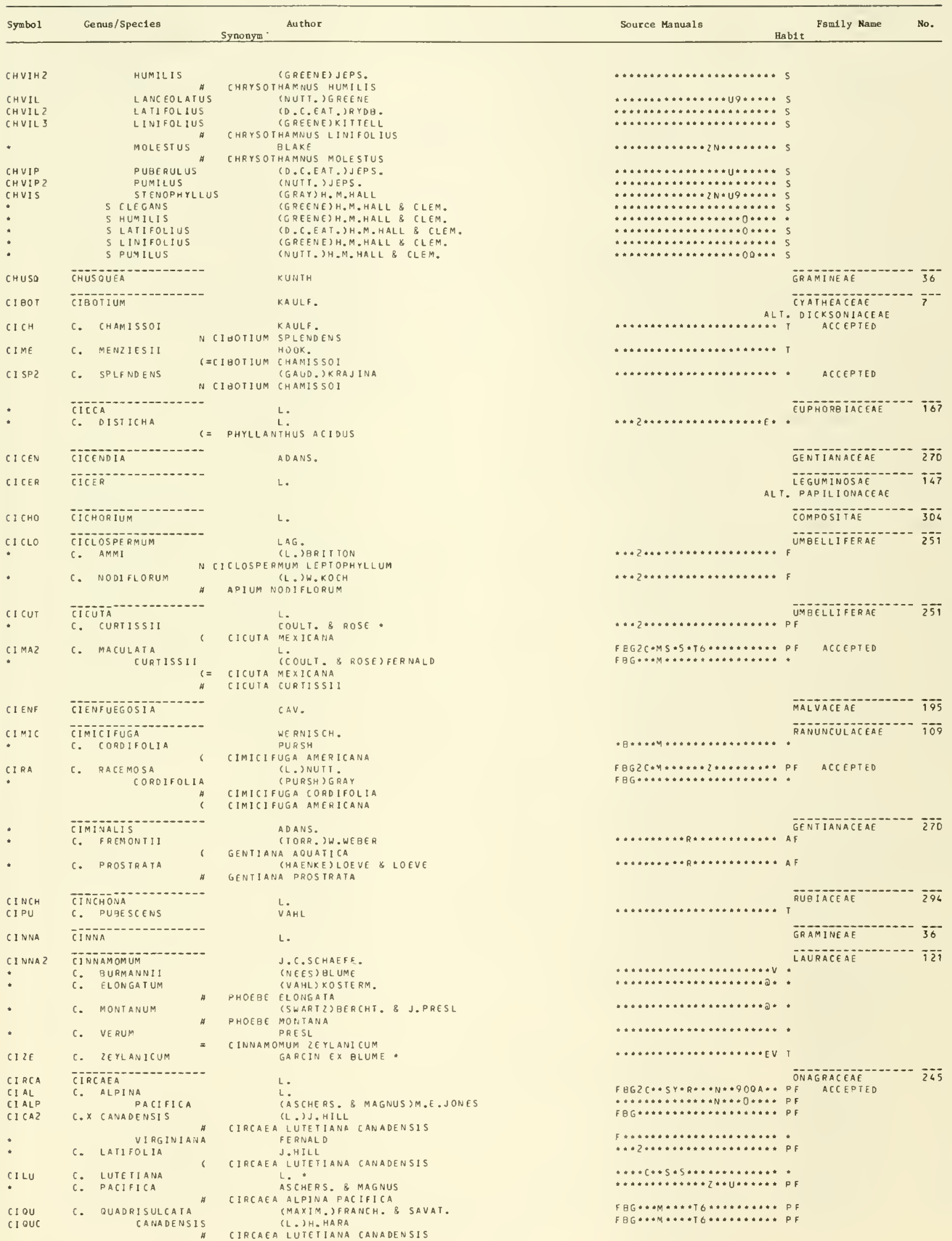




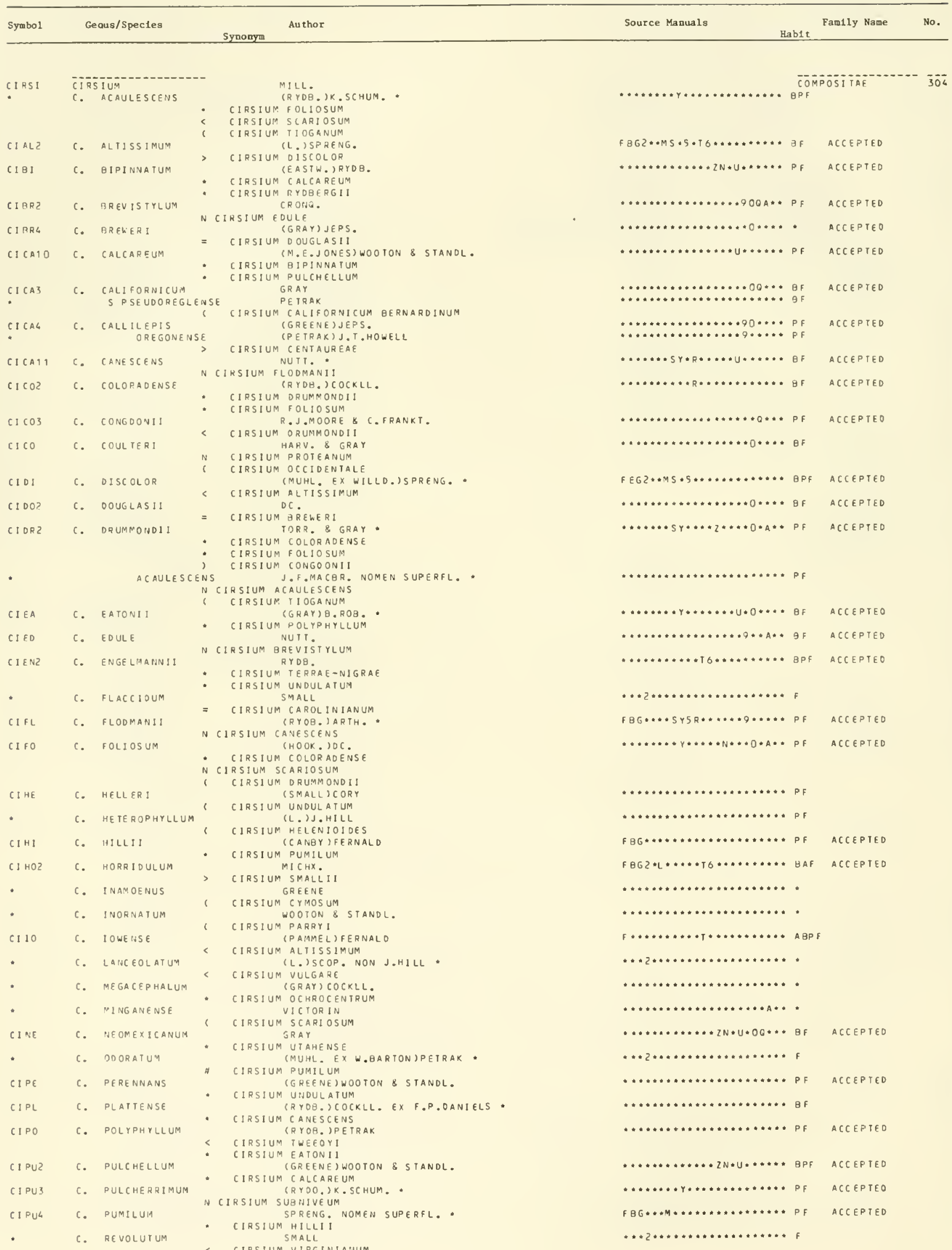




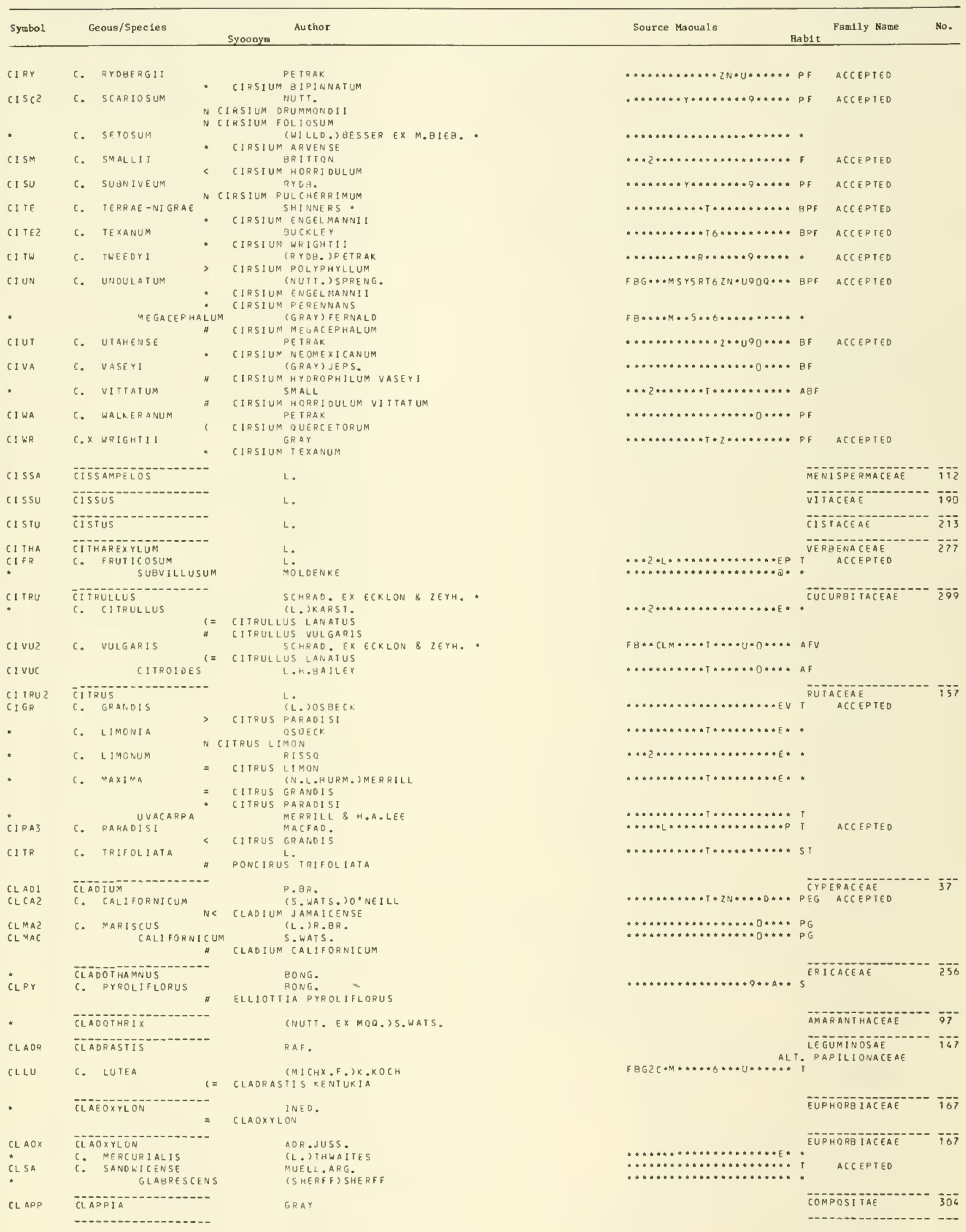




\begin{tabular}{|c|c|}
\hline Symbol & Genus/Specles \\
\hline CLARK & CLARKIA \\
\hline CLAM & C. AM OENA \\
\hline - & CAURINA \\
\hline - & LINOLEYI \\
\hline CLOE & C. DEFLEXA \\
\hline • & C. ELEGANS \\
\hline CLN! & C. NITENS \\
\hline * & C. BUADRI VULNERA \\
\hline CLRU & C. RUBICUNOA \\
\hline CLVI & $\begin{array}{l}\text { S ELASOALEI } \\
\text { C. VIMINEA }\end{array}$ \\
\hline
\end{tabular}

.

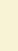

CLAY

CLAC

CLACC

CLAOZ

CLCA

.

*

$+$

.

-

$C L L A 2$

\section{CLAVENNA}

c. TEIRANDRA

\section{GLATYTONATA}

c. ACUTIFOLIA

GRAMINIFOLIA

C. UELLIOIFOLIA

c. DOSTOCXII

c. CAROLINIANA

c. CHAMISSOI

c. DICHOIOMA

c. DIFFUSA

c. FL AGELLARIS

c. FONTANA

c. HOWELLII

c. LanceOLATA

CHRYSANIHA

FLAVA

MULTISCAPA

PEIRSONII

SESSILIFOLIA

C. LINEARIS

C. MEDIA

c. PARUIFOLIA

5 FLAGELLARIS"

CLPE

c. PERFOLIATA OEPRESSA

PARVIFLORA

c. ROBUSTA

c. ROSEA

c. SIMSII

c. SPATHULATA

E IIGUA

TENU: $O L$ IA VIRIDIS

CLTU

C. TUBEROSA

CZUKCZORUM

... CLEISTES

BIEARIA
BIFATA

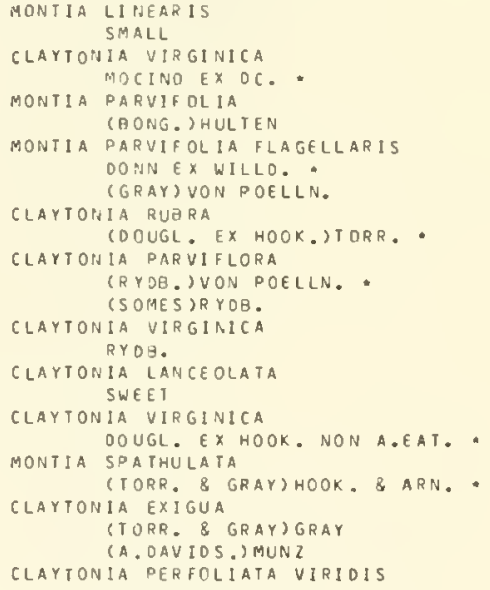

CLEMATIS VIORNA

Source Manuals

Famtly Name

No.

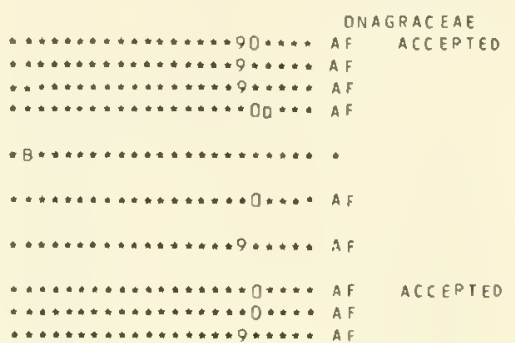

. ACC $\overline{10} \overline{3}$
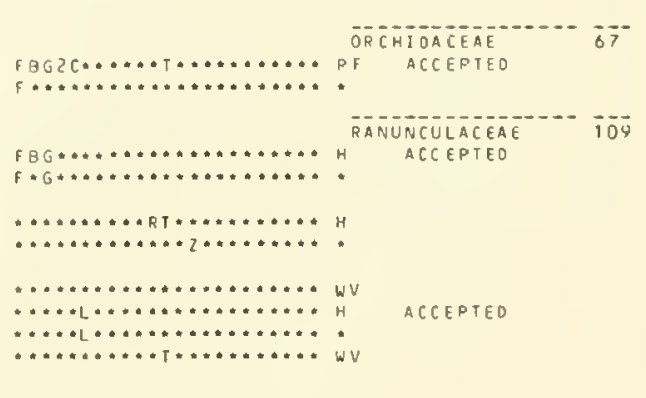

ACCEPIEO

ACCEPIEO

PF ACCEPTED ACCEPIED

\section{A CEPTEO}

P

, 


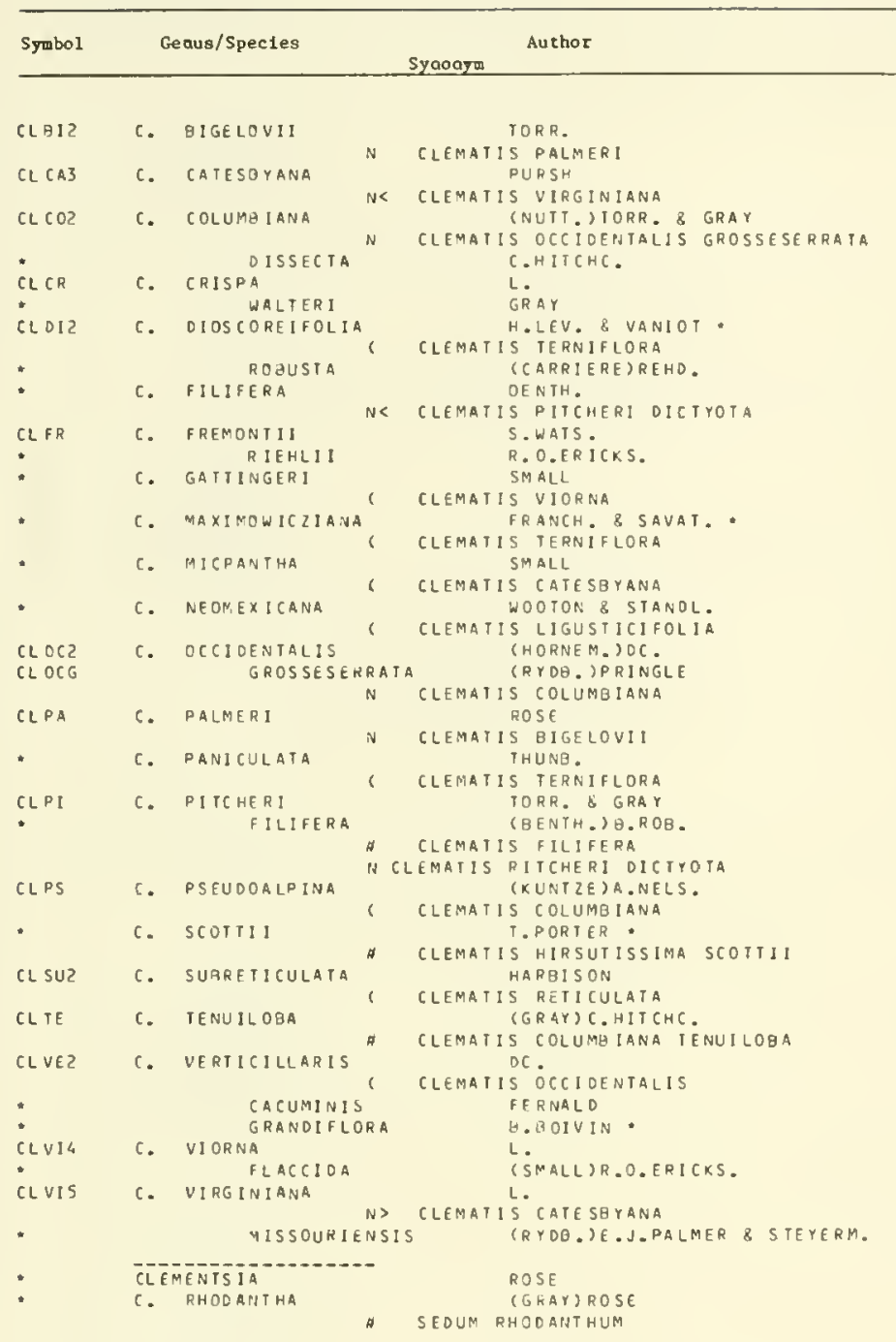

CL EOM

\begin{tabular}{|c|c|c|}
\hline • & $c$. & CILIATA \\
\hline CL HA & C. & HASSLERANA \\
\hline 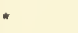 & c. & HOUT IEANA \\
\hline * & c. & I COSANDRA \\
\hline $\begin{array}{l}\text { CLSE } \\
\text { CLSEA }\end{array}$ & c. & $\begin{array}{l}\text { SERRULA A A } \\
\text { ANGUSTA }\end{array}$ \\
\hline L so & c. & SONORAE \\
\hline CL SPL & c. & SPECIOSA \\
\hline CLSP3 & c. & SPINOSA \\
\hline
\end{tabular}

CLEOM 2

CLHI2

CL MA 3

*

CLNA

CLOA

CLPL?

:

CLERM

- c. carinifera

CLHAL C. HAWAIIENSIS GRANDIS

L.

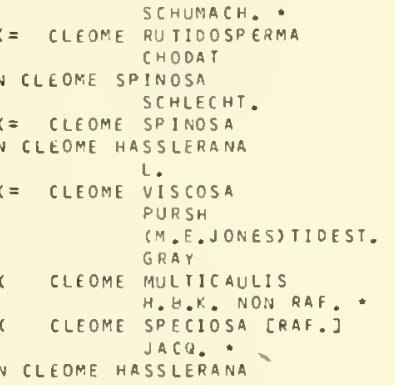
iv CLEOME HASSLERANA or.

CLEOMELL A MAL BRIDEANA LA MAC BRI

CLEOMELLA HILLMANI

PAYSON

EASTW.

A STW.

TORR. \& FREM.

A. NELS.

S. WATS.

(PAYSON)E. CRUM EX JEPS. *

E. CRUM EX JEPS. INED.

GAUD.

$H . L E V$.

(HILLEBR.) J.F.ROCK

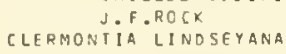

CLERMONIIA PARVIFLORA GRANDIS
Source Mausls

Habit

Family Name

No.
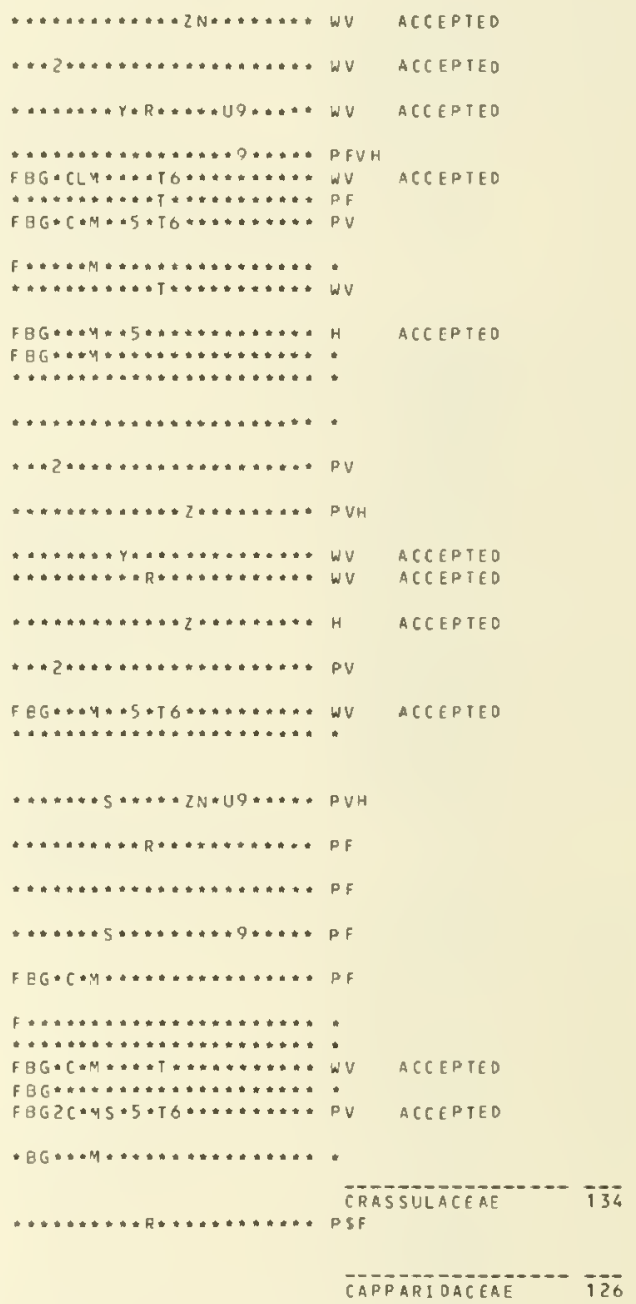
ALT. CLEOMACEAE
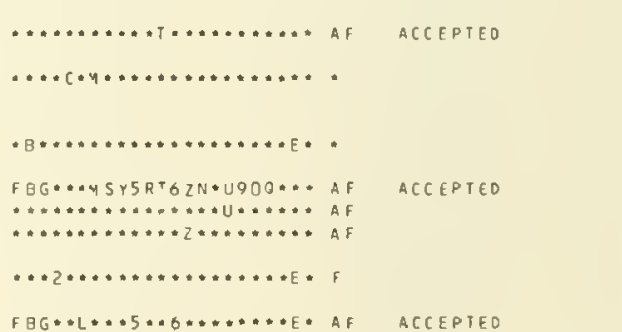

FBG* * * * $5 * 0 * \cdots * \cdots E$ AF ACCEPTED

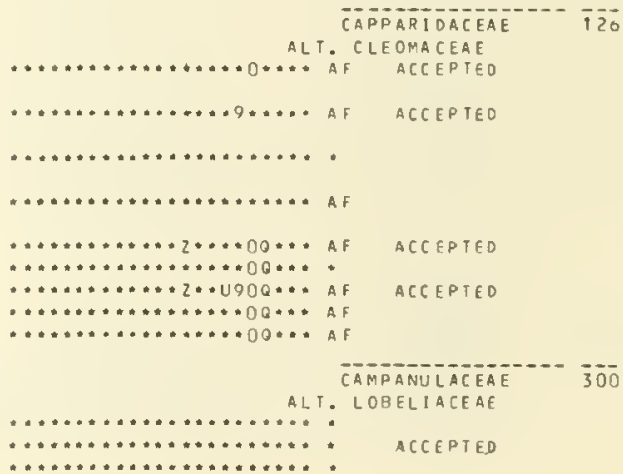




\begin{tabular}{|c|c|}
\hline Symbol & Genus/Species \\
\hline CLERO & CLEROOENDRON \\
\hline CLEROZ & CLERODENORUM \\
\hline - & C. FOEIIDUM \\
\hline • & C. FRAGRANS \\
\hline CLETH & CLETHRA \\
\hline • & C. TOMENTOSA \\
\hline CLEYE & CLE EYYERA \\
\hline CLIBA & CLIBADIUM \\
\hline CLIDE & CLIDEMIA \\
\hline$C L I F T$ & CLIFTONIA \\
\hline : & CLINOPOOIUM \\
\hline * & C. ASHE ! \\
\hline • & C. COCCINEUM \\
\hline • & C. DENTATUM \\
\hline • & C. GE ORGI AIJUM \\
\hline • & C. GLABELLUM \\
\hline - & C. MACROCALYX \\
\hline - & C. NEPETA \\
\hline • & C. VULGARE \\
\hline
\end{tabular}

CLINT TLINTONIA

CL I TO

CLODO

CLUSI
CLKR

CNEMI

CNEOR

CNICU

CNIDI

[NAJ

CNIDO

cocet

$\operatorname{coc} \mathrm{C}$

$\operatorname{coccos}$

cocco

COD18

-

-

-

$\cos w$

.

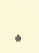

$\operatorname{coccos}$
CLEROQENDRUM

$$
\text { BUNGE }
$$

CLERODENORUM BUNGE

VENTENAT

CLEROCENDRUM PHILIPPINUM

$$
\text { LAM. }
$$

CLETHRA ALNIFOLIA TOMENTOSA

-
H
N
H
H
H

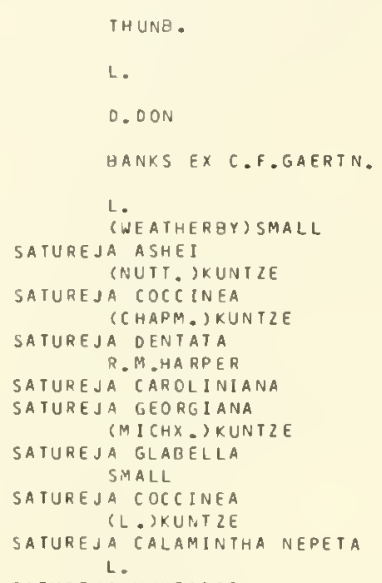

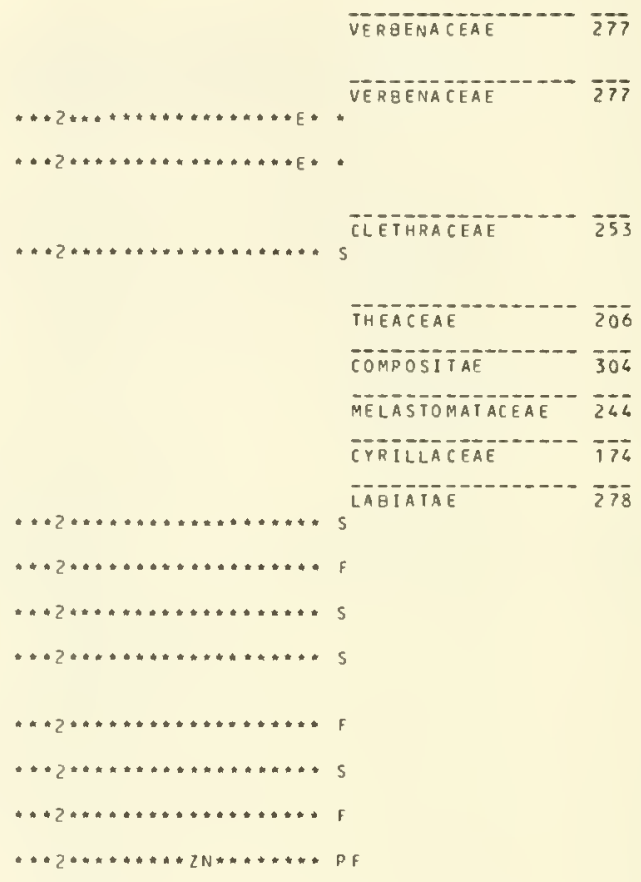

LILIACEAE 55

LEGUMINOSAE-O-- T-T ALT. PAPILIONACEAE

EUTTIEATEAE

CYATTEATCEAEE

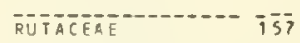

COMPOSITAE $3 \overline{04}$

PM

EUPHOREIACEAE

GUCURETtÁCEAE

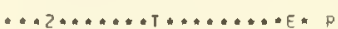

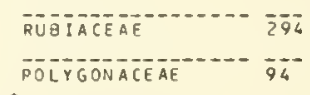

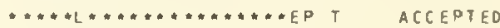
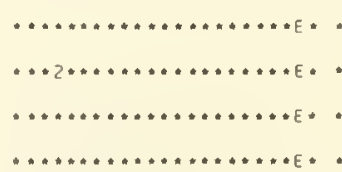

$\ldots \ldots \ldots \ldots \ldots \ldots \ldots$. $\ldots \ldots \ldots$ T ACCEPTED

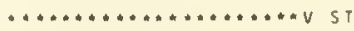
POLYGONACEAE PALMAE 


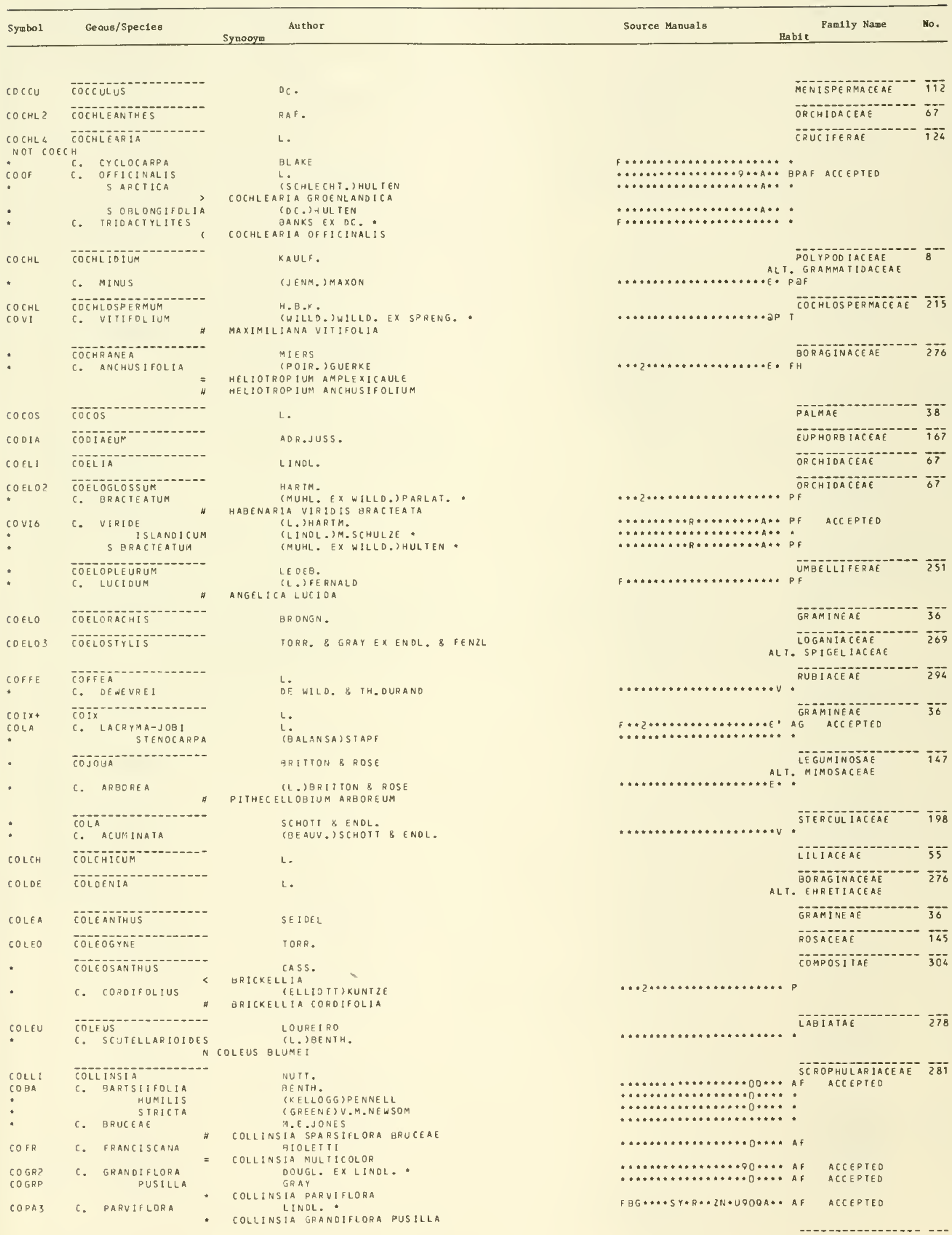




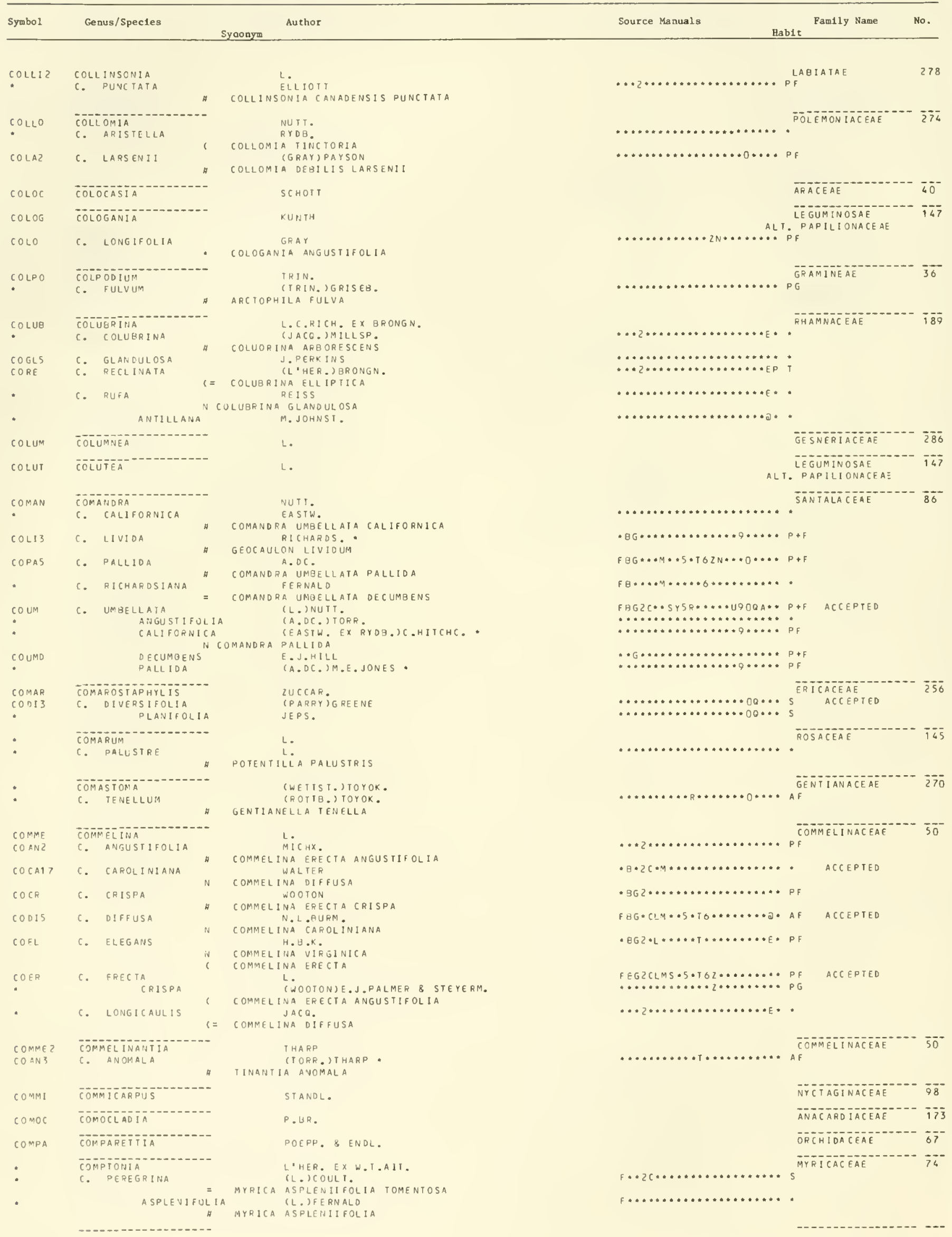




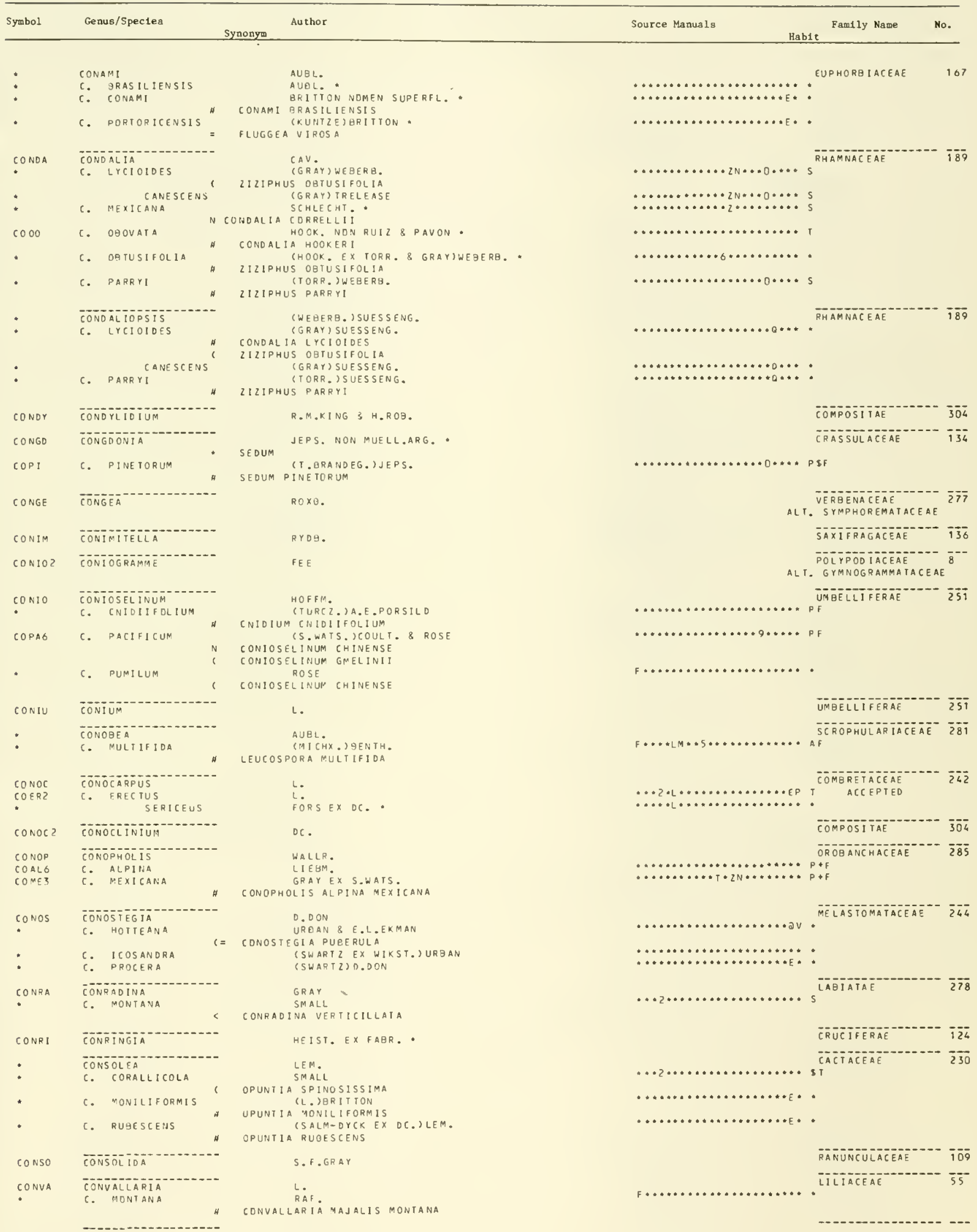




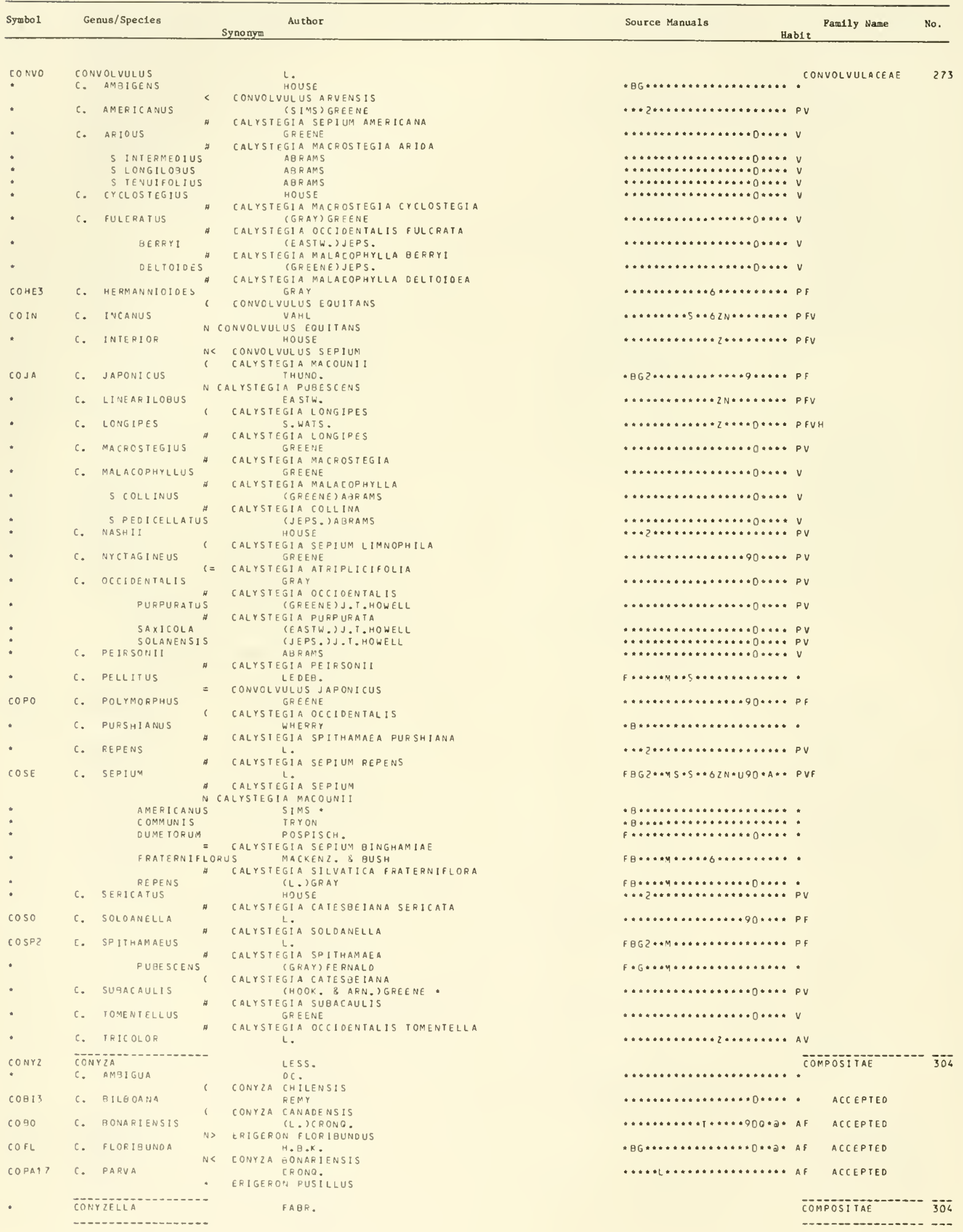


COOPER IA

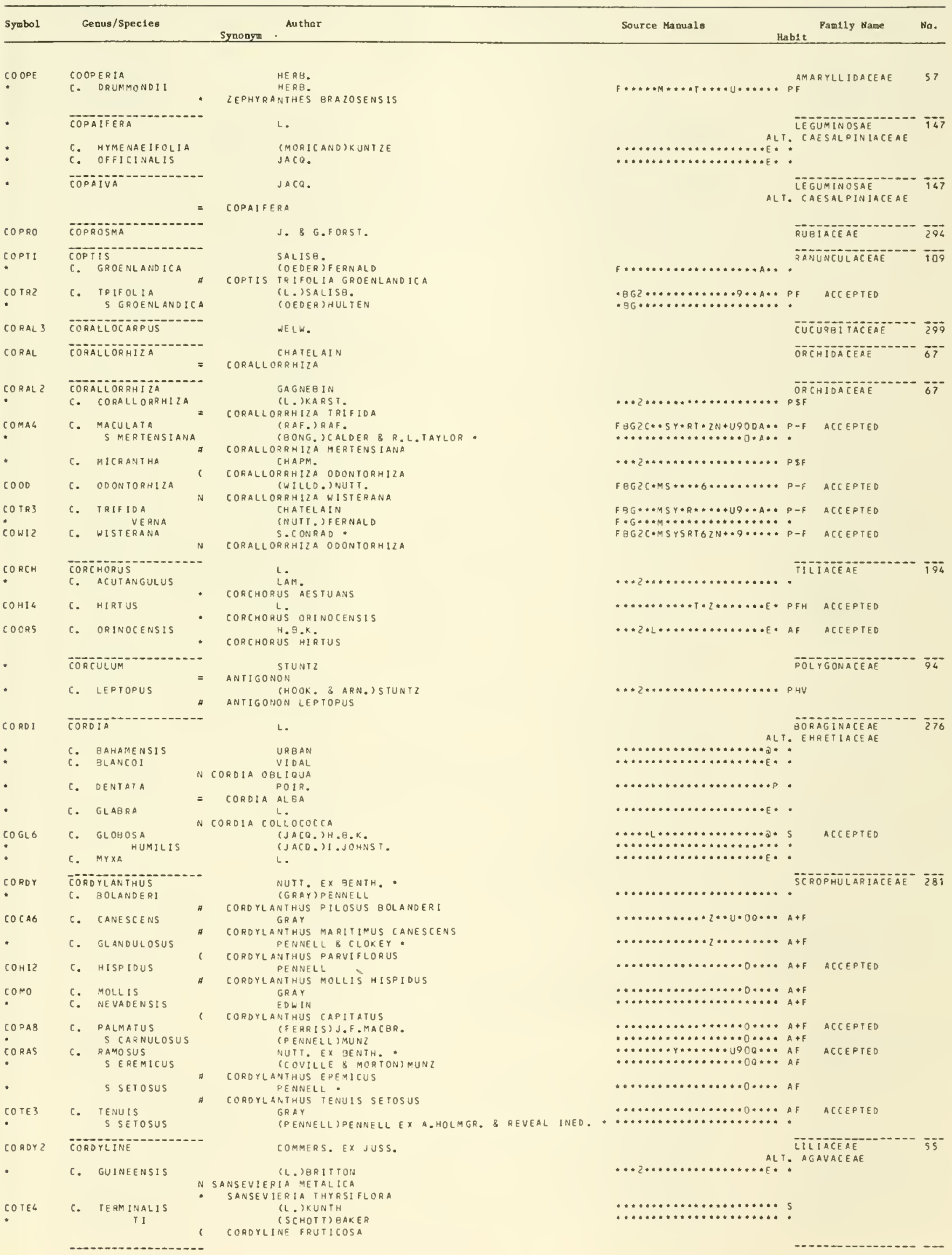




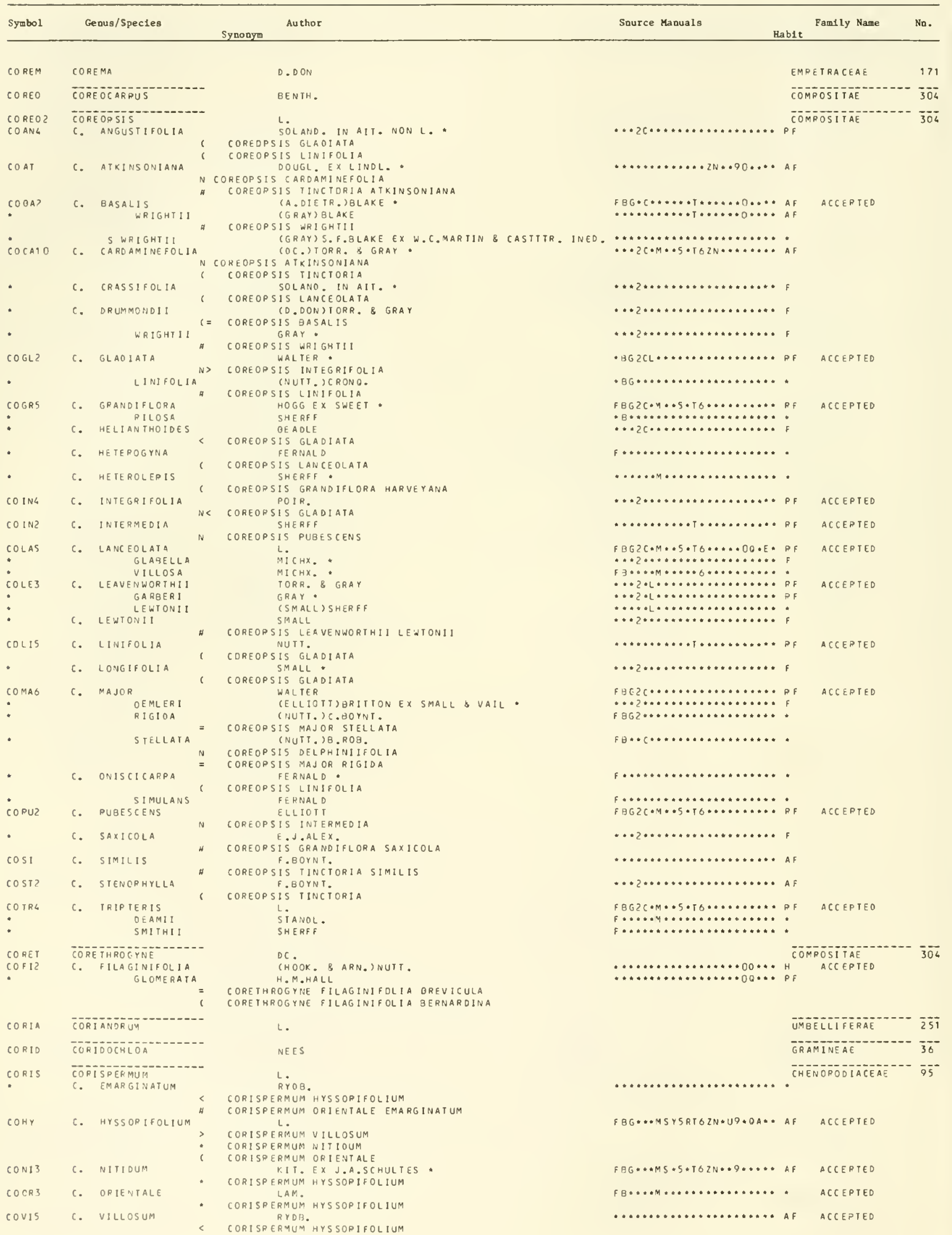




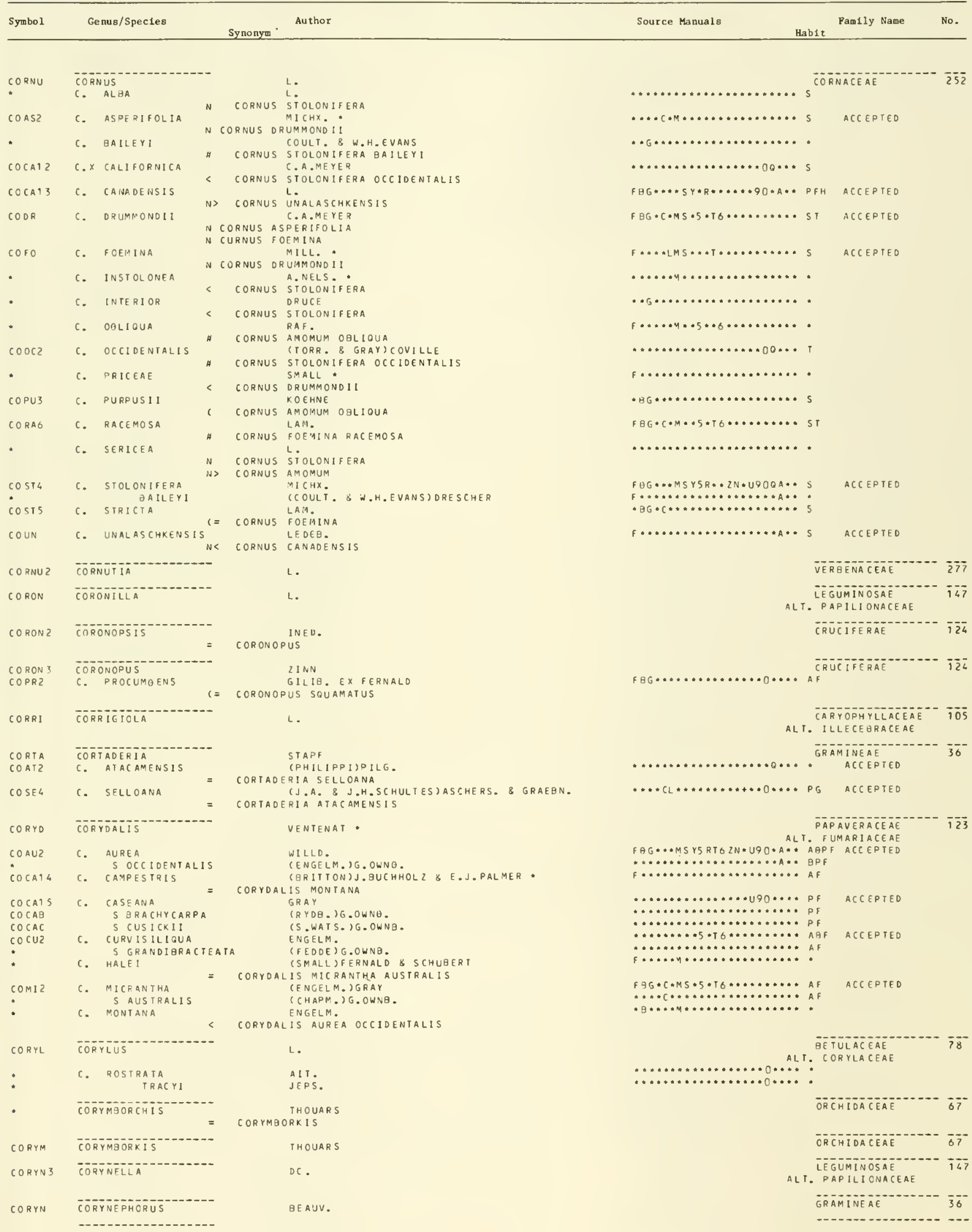




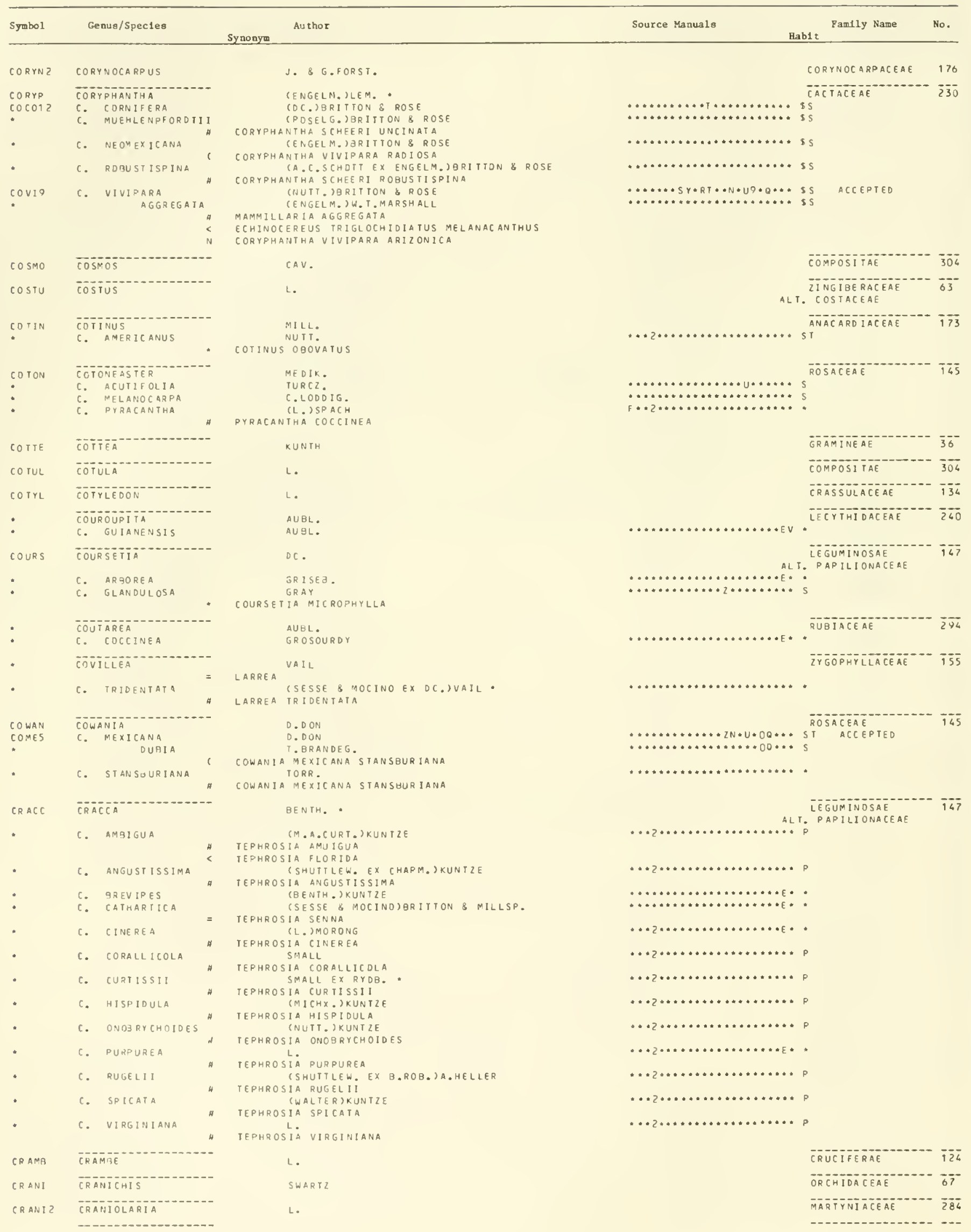




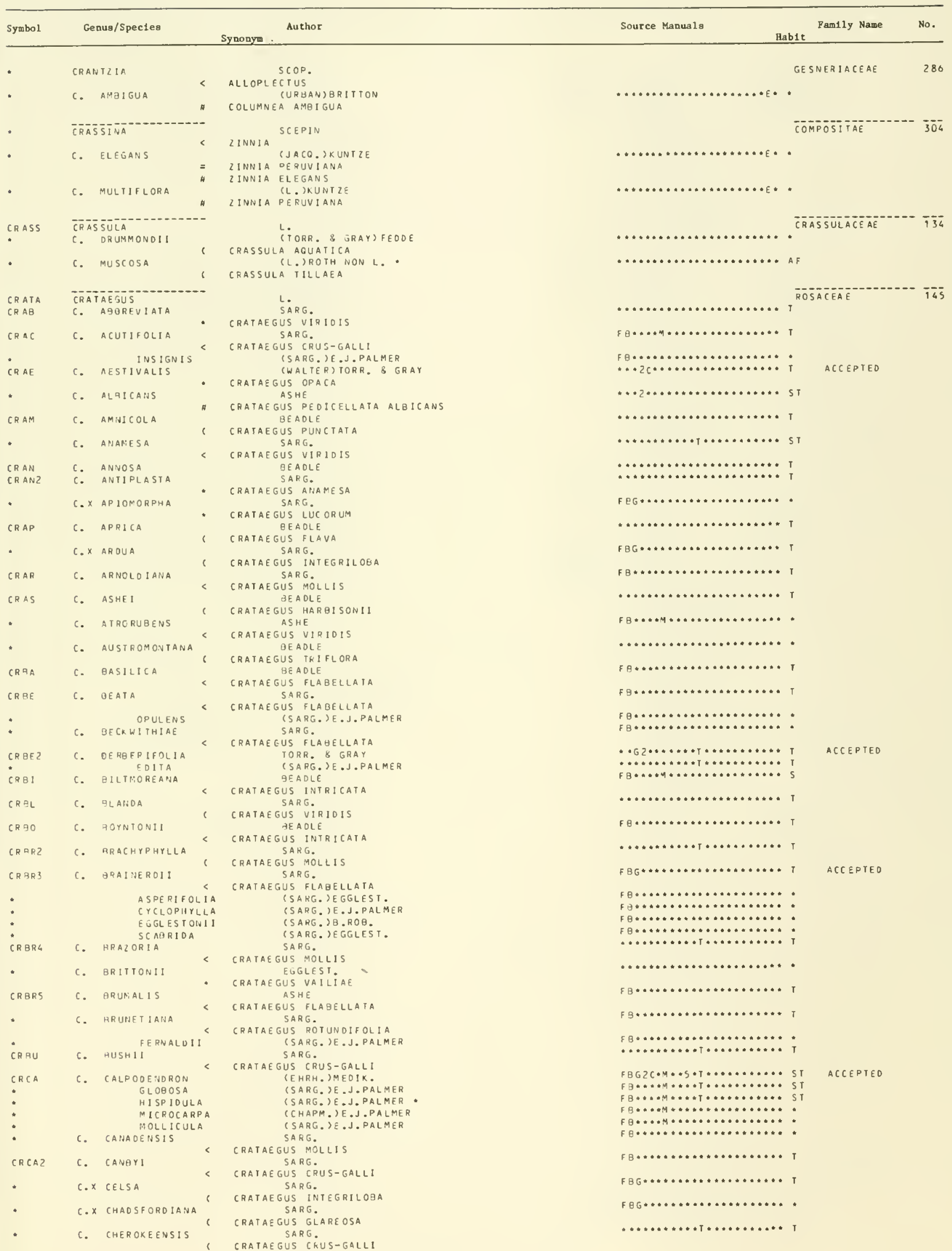




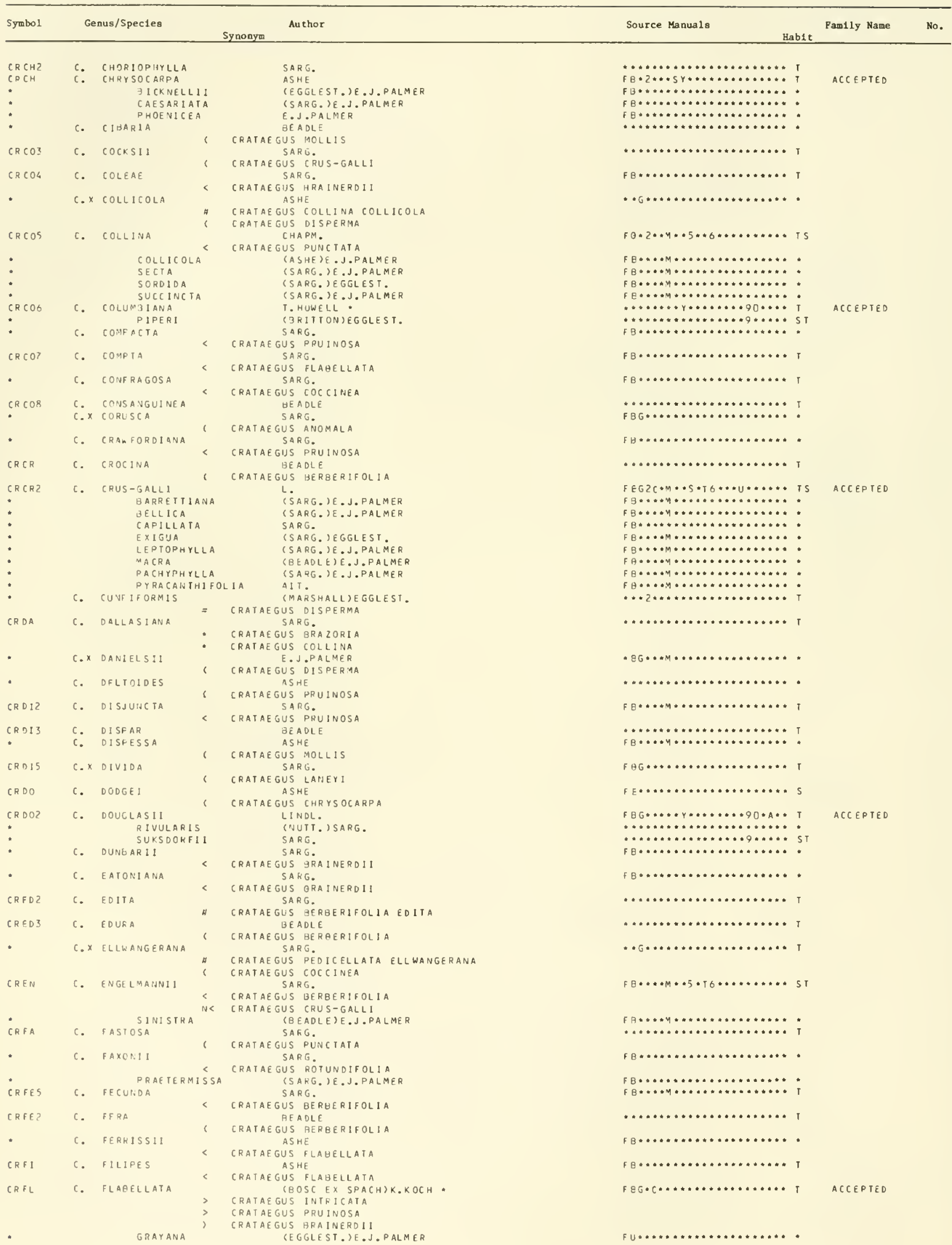




\begin{tabular}{|c|c|c|c|c|c|c|}
\hline Symbo 1 & & nus/Specles & Synonym & Source Manuala & Pam1ly Name & No. \\
\hline CRFL3 & c. & FLORIDANA & $\begin{array}{c}\text { SARg. } \\
\text { - cratagus finva }\end{array}$ & 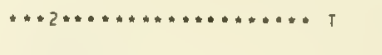 & & \\
\hline • & c. & FLOR IFERA & $\begin{array}{l}\text { SARG. } \\
\text { ( CRATAEGUS PUNCTATA }\end{array}$ & $\ldots \ldots \ldots \ldots \ldots \ldots \ldots \ldots$ & & \\
\hline * & c. & FOETIOA & $\begin{array}{l}\text { - crshe } \\
\text { c tatagus intricata }\end{array}$ & $f \theta \cdots \ldots \ldots \ldots \ldots \ldots \ldots \ldots \cdots$ & & \\
\hline CRFO & c. & FOMT ANES IANA & $\begin{array}{l}\text { (SFACH) STEUD. } \\
\text { CRATAEGUS CRUS-GALLi }\end{array}$ & 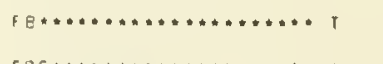 & & \\
\hline - & c. $x$ & FORHOSA & $\begin{array}{l}\text { SARG. } \\
\text { C CRATAEgus HAEMocarpa }\end{array}$ & $F B G \ldots \ldots \ldots+\ldots \ldots+\ldots+\ldots \ldots \ldots$ & & \\
\hline • & c. & FORTUNATA & $\begin{array}{l}\text { SARg. } \\
<\text { CRATAEgUS Intricata }\end{array}$ & $F s \cdots \ldots \ldots \ldots \ldots \ldots \ldots \ldots$ & & \\
\hline * & c. & FRAKXLI NENS:S & $\begin{array}{l}\text { SARG. } \\
\text { < CRATAEGUS PRUINOSA }\end{array}$ & $F, \ldots \ldots \ldots \ldots \ldots \ldots \ldots \ldots \ldots$ & & \\
\hline * & $c . x$ & FRETALIS & $\begin{array}{l}\text { SARG. } \\
\text { c CRATAEGUS LUC OR UM }\end{array}$ & $F B G \ldots \ldots \ldots \ldots \ldots \ldots \ldots \ldots \ldots$ & & \\
\hline CR GA & c. & $\begin{array}{l}\text { GATTINGERI } \\
\text { RIGIDA }\end{array}$ & $\begin{array}{c}\text { ASHE } \\
<\quad \text { CRATAEGUS PRUINOSA } \\
\text { E.J.PALMER }\end{array}$ & $\varepsilon_{B} \ldots \ldots+\ldots \ldots \ldots \ldots \ldots \ldots$ & & \\
\hline : & c. & $\begin{array}{l}\text { RIGIDA } \\
\text { GAUDENS }\end{array}$ & $\begin{array}{r}\text { E.J.PALMER } \\
\text { SARG. } \\
<\quad \text { CRATAEGUS PRUINOSA }\end{array}$ & $F B \ldots \ldots \ldots \ldots \ldots \ldots \ldots$ & & \\
\hline CRGE & c. & GE ORGI ANA & $\begin{array}{l}\text { SARG. } \\
\text { CRATAEGUS PRLINOSA }\end{array}$ & $\cdots \cdots \cdots \cdots \ldots \ldots+\ldots \ldots \ldots \ldots+$ T & & \\
\hline CR GL & c. & GLABRI USCULA & $\begin{array}{l}\text { SARG. } \\
\text { < CRATAEGUS VIRIDIS }\end{array}$ & $\ldots \ldots \ldots \ldots \ldots+\ldots \ldots \ldots \ldots *$ T & & \\
\hline$*$ & c. & GRAV I DA & $\begin{array}{l}\text { BEADLE } \\
\text { CRATAEGUS MOLLIS }\end{array}$ & $\ldots \ldots \ldots \ldots \ldots \ldots \ldots \ldots \ldots \ldots \ldots \cdots$ & & \\
\hline CRGR & c. & GRAVIS & $\begin{array}{c}\text { ASHE } \\
<\quad \text { CRATAEGUS FLABELLATA } \\
\text { GEADLE }\end{array}$ & $\mathrm{FB} \cdots \cdots \cdots \cdots+\ldots+\cdots \cdots \cdots \cdots \cdots+\mathrm{T}$ & & \\
\hline * & c. & GREGALIS & & $\ldots \ldots \ldots \ldots \ldots \ldots \ldots \ldots$ & & \\
\hline CRGR? & c. & $\begin{array}{l}\text { GREGGIANA } \\
\text { HAGERER I }\end{array}$ & $\begin{array}{l}\text { EGGLES T } \\
\text { CRATAEGUS MOLLiS } \\
\text { SARG. }\end{array}$ & 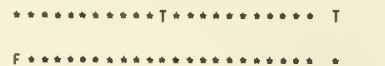 & ACCEPTED & \\
\hline CRHA & c. & HAGE RE RI & $\begin{array}{l}\text { SARG. } \\
\text { CRATAEGUS FLA GELLATA } \\
\text { E. J.PALMER }\end{array}$ & $28 \cdots$ & & \\
\hline CRHA & c. & HANN IBALENS IS & $\begin{array}{c}\text { E. J PALMER } \\
\text { CRATAEGUS CPUS-GALLI }\end{array}$ & 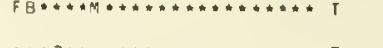 & & \\
\hline CRHA? & c. & HARE ISONII & $\begin{array}{l}\text { Beadle } \\
\text { - crataegus CRUS-galli } \\
\text { sarg. }\end{array}$ & $\cdots 2 \cdots \cdots \cdots \cdots \cdots \cdots \cdots \cdots$ & ACCEPIED & \\
\hline$\cdot$ & c.x & $\begin{array}{l}\text { HARRYI } \\
\text { HARVEYANA }\end{array}$ & $\begin{array}{l}\text { SARG. } \\
\text { ( CRATAEGUS DESUETa } \\
\text { SARG. } \\
\text { CRATAEGUS INTRICATa }\end{array}$ & $\ldots \ldots \ldots \ldots \ldots \ldots \ldots \ldots \ldots \ldots \ldots$ & & \\
\hline - & c. ' & HELVINA & $\begin{array}{l}\text { - CRATAEGUS INTRICATA } \\
\text { ASHE } \\
\text { C CRATAEGUS CRUS-GALLI }\end{array}$ & $\ldots \ldots \ldots \ldots \ldots \ldots \ldots \ldots \ldots \ldots \ldots$ & & \\
\hline * & c. & H[LLI] & $\begin{array}{l}\text { SARG } \\
<\text { CRATAEGUS COCCINEA }\end{array}$ & $8, \ldots \ldots \ldots \ldots \ldots \ldots \ldots$ & & \\
\hline$\cdot$ & C. $x$ & HIRTIFLORA & $\begin{array}{l}\text { SARG. } \\
\text { C CRATAEGUS LETIERMANI }\end{array}$ & 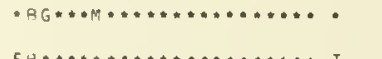 & & \\
\hline$\cdot$ & & AMICTA & $\begin{array}{r}\text { (ASHE) E. J.PALMER } \\
\text { CRATAEGUS DUROBRIVENS IS }\end{array}$ & (a) & & \\
\hline : & & $\begin{array}{l}\text { CHIPPE } \sim A E \\
\text { MAGNIFLOR }\end{array}$ & $\begin{array}{ll}\text { NSIS } & \text { (SARG.)E.J.PALMER } \\
\text { A } & \text { (SARG.)E.J.PALMER }\end{array}$ & $f \beta \cdots \ldots \ldots \ldots \ldots \ldots \ldots$ & & \\
\hline - & & VILLIPES & ASHE & $f 8 \ldots \ldots \ldots \ldots \ldots \ldots \ldots \ldots$ & & \\
\hline$C P: G$ & c. & IGNAVA & $\forall \subseteq A D L E$ & $\ldots \ldots+\ldots \ldots \ldots+\ldots+\ldots \ldots \ldots+, T$ & & \\
\hline$\cdot$ & c. & LNAN IS & $\begin{array}{l}\text { BEADLE } \\
\text { \& CRATAEGUS IIITRICATA }\end{array}$ & $\cdots+\ldots \ldots \ldots+\ldots \ldots+\ldots \ldots \ldots *$ & & \\
\hline C R IN? & c. & INGE NS & $\begin{array}{l}\text { beadle } \\
\text { - crataegus aminicola }\end{array}$ & 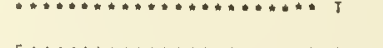 & & \\
\hline - & c. & INSOLENS & $\begin{array}{l}\text { SARG. } \\
\text { ( CRATAEGUS CHRYSOCARPA }\end{array}$ & $F \cdots \cdots \cdots \cdots \cdots \cdots \cdots \cdots \cdots \cdots$ & & \\
\hline - & c. & LNTERIOR & $\begin{array}{l}\text { BEADLE } \\
\text { C CRATAEGUS VIRIDIS }\end{array}$ & $\ldots \ldots \ldots \ldots \ldots \ldots \ldots+\ldots \ldots \ldots *$ & & \\
\hline CRIN3 & c. & INTR I CATA & $\begin{array}{l}\text { LANGE } \\
\text { C CRATAEGUS TRI IFLORA } \\
\text { GRATAGGUS FLABELLATA }\end{array}$ & $F B G 2 \cdots \ldots \ldots \ldots \ldots \cdots \cdots+\ldots$ & ACCEPTED & \\
\hline - & & STRAMIIVEA & (BEADLE)E.J.PALMER & $F \theta * \ldots \ldots+\cdots+\ldots+\cdots \ldots \ldots$ & & \\
\hline - & c. & INVISA & $\begin{array}{l}\text { SARG. } \\
\text { < CRATAEgUS Mullis }\end{array}$ & $\cdots \cdots \cdots \cdots+T \cdots \cdots \cdots \cdots+$ T & & \\
\hline$C R \backslash R$ & c. & IRACUNDA & $\begin{array}{l}\text { 3EROLE } \\
<\text { CRATAEgus FLABELLATA }\end{array}$ & $F, \ldots \ldots \ldots \ldots \ldots \ldots \ldots \ldots+1$ & & \\
\hline CRIRZ & & $\begin{array}{l}\text { SILVICOLA } \\
\text { IRRASA }\end{array}$ & $\begin{array}{l}\text { E.J.PALMER } \\
\text { SARG. }\end{array}$ & $f_{\theta}+\ldots \ldots \ldots \ldots \ldots \ldots \ldots \ldots$ & & \\
\hline$C R I R Z$ & c. & IRRASA & ( CRATAEGUS CHRYSOCARPA & & & \\
\hline : & c. & $\begin{array}{l}\text { 3LANGHARD } \\
\text { ITEFATA }\end{array}$ & $\begin{array}{l}\text { ISARG. IEGGLEST. } \\
\text { SARG. } \\
\text { C CRATAEGUS FLABELLATA }\end{array}$ & $F_{B} \beta \ldots \ldots \ldots \ldots \ldots \ldots \ldots \ldots \ldots$ & & \\
\hline - & c. & $J A C K: 1$ & $\begin{array}{c}\text { SARG. } \\
<\text { Crataegus Rotundfolia }\end{array}$ & FR $\ldots \ldots \ldots \ldots \ldots \ldots \ldots \ldots$ & & \\
\hline$C R J E$ & c. & JESUPII & $\begin{array}{l}\text { SARg. } \\
\text { < cRataegus PRUjnosa }\end{array}$ & $F, \ldots \ldots \ldots \ldots \ldots \ldots \ldots$ & & \\
\hline * & c. & JONES I AE & $\begin{array}{l}\text { SARG. } \\
\text { CRAIAEGUS CHRYSOCARPA }\end{array}$ & $F g \ldots \ldots * \ldots \ldots \ldots \ldots \ldots \ldots \ldots$ r & & \\
\hline - & c. $x$ & KELLERMANII & $\begin{array}{l}\text { SARG. } \\
\text { CRATAEGUS DUROBRIVENS IS }\end{array}$ & r G $_{\ldots} \ldots \ldots \ldots \ldots \ldots \ldots \ldots \ldots$ & & \\
\hline - & $c . x$ & KNIESRERNIANA & $\begin{array}{l}\text { SARG. } \\
\text { C CRATAEGUS LUC ORUM }\end{array}$ & $F_{B}, \ldots \ldots \ldots \ldots \ldots \ldots \ldots \ldots$ & & \\
\hline CRLA & c. 1 & LACERA & $\begin{array}{l}\text { Sarg. } \\
\text { - crataegus mollis }\end{array}$ & $\ldots \ldots+\cdots+\cdots+\ldots+\cdots+\cdots+\cdots T$ & & \\
\hline * & c. $\times$ : & LAFTIFICA & $\begin{array}{l}\text { SARG. } \\
\text { ( CRATAEGUS PERSIMILIS }\end{array}$ & $F_{B}, \ldots \ldots \ldots \ldots \ldots \ldots \ldots$ & & \\
\hline - & C. L & LANATA & $\begin{array}{l}\text { JEADLE } \\
\text { ( CRATAEGUS FLAVA }\end{array}$ & $\ldots \ldots \ldots \ldots \ldots \ldots \ldots \ldots \ldots \ldots$ & & \\
\hline
\end{tabular}




\begin{tabular}{|c|c|c|c|c|c|c|}
\hline Symbol & & nus/Species & Synonyw & Source Manuals & Family Name & No. \\
\hline CRLA3 & 6. & LANUGINOSA & $\begin{array}{l}\text { SARG. } \\
\text { C CRATAEGUS MOLLIS }\end{array}$ & $F g \cdots \cdots 4 \cdots 5 \cdots 6 \ldots \ldots \ldots \ldots$ is & & \\
\hline - & c. & LA TEBR OSA & $\begin{array}{l}\text { SARG. } \\
\text { CRATAEGUS PUNCTATA }\end{array}$ & $\mathrm{F} \mathrm{E} \cdots \cdots+\cdots \cdots \cdots \cdots \cdots \cdots \cdots \cdots$ & & \\
\hline - & C. & LAXIFLORA & $\begin{array}{l}\text { SARG. } \\
<\quad \text { CRATAEGUS SUCCULENTA }\end{array}$ & $F B \cdots \ldots \ldots \ldots \ldots \ldots+\ldots \ldots *$ & & \\
\hline • & C. $x$ & LECTA & $\begin{array}{l}\text { SARG. } \\
\text { C CRATAEGUS LOCUPLES }\end{array}$ & F $\mathrm{Bg} \cdots \cdots \cdots \ldots \ldots \ldots \ldots \ldots \ldots \ldots$ & & \\
\hline CRLE & c. & LEIOPHYLLA & $\begin{array}{c}\text { SARG. } \\
<\quad \text { CRATAEGUS PRUINOSA } \\
5 \text { SAPG. }\end{array}$ & 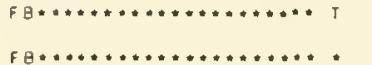 & & \\
\hline 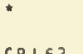 & $c$ & LEMI NG TONENS IS & $\begin{array}{l}\text { 5APG. } \\
\text { C CRATAEGUS FLABELLATA } \\
\text { E.J.PALMER }\end{array}$ & 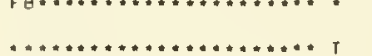 & & \\
\hline CRLEZ & c. & $\begin{array}{l}\text { LEONENSIS } \\
\text { LETCHWORTHIANA }\end{array}$ & $\begin{array}{c}\text { E. J.PALMER } \\
\text { SARG. } \\
\text { CRATAEGUS COC CINEA }\end{array}$ & $f+\ldots \ldots+\ldots$ & & \\
\hline - & c. & LEVIS & $\begin{array}{l}\text { SARG. } \\
<\quad \text { CRATAEGUS FLABELLATA }\end{array}$ & $f \theta \cdots \ldots \ldots \ldots \ldots \ldots \ldots \ldots$ & & \\
\hline CRLI & $c$ & LIMARIA & $\begin{array}{l}\text { SARG. } \\
\text { C CRATAEGUS MOLLIS } \\
\text { SARG. }\end{array}$ & 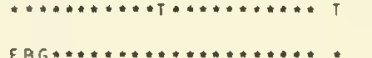 & & \\
\hline * & $c \cdot x$ & LITICRALIS & $\begin{array}{l}\text { SARG. } \\
\text { ( CRATAEGUS PILOSA } \\
\text { SARG. }\end{array}$ & $\begin{array}{l}F R G \cdots \ldots \ldots \ldots \ldots \ldots \ldots \ldots \\
\ldots \ldots 2 \ldots \ldots \ldots \ldots \ldots \ldots \ldots \ldots \ldots\end{array}$ & & \\
\hline * & c. & $\begin{array}{l}\text { LUCULENTA } \\
\text { MACAULEYAE }\end{array}$ & $\begin{array}{c}<\text { GRATAEGUS AESIIVALIS } \\
\text { SARG. }\end{array}$ & 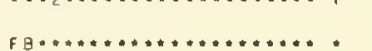 & & \\
\hline CRMA ? & c. & $\begin{array}{l}\text { MACAULEYAE } \\
\text { MACKENZII }\end{array}$ & $\begin{array}{c}<\quad \text { CRATAEGUS BRAINERDII } \\
\text { SARG. }\end{array}$ & F & & \\
\hline - & & ASPERA & $\begin{array}{l}\text { CRATAEGUS PRUINOSA } \\
\text { (SARG.)E.J.PALMER }\end{array}$ & $F B \cdots \ldots M \ldots \ldots \ldots \ldots \ldots \ldots \ldots$ & & \\
\hline - & & BRACTEATA & (SARG.)E. J.PALMER & $f \beta \cdots \cdots M \cdots 5 \cdots 6 \cdots \cdots \cdots \cdots$ & & \\
\hline CRMA & $c$ & MACROSPERMA & $\begin{array}{c}\text { ASHE } \\
\text { CRATAEGUS FLABELLAIA }\end{array}$ & $F B \cdot 2 \ldots \ldots \ldots \ldots \ldots \ldots \ldots+* * T$ & & \\
\hline : & & ACUIILOBA & (SARG.) EGGLEST. & 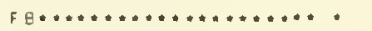 & & \\
\hline • & & DEMISSA & ( SARG.)EGGLEST. & F $\beta \ldots \ldots \ldots \ldots \ldots \ldots \ldots$ & & \\
\hline * & & MATURA & ( SARG. ) EGGLEST. & $F 9 \cdots \ldots \ldots \ldots \ldots \ldots \ldots \ldots$ & & \\
\hline • & & PENTANDNA & (SARG.) EGGLEST. & $f \mathrm{~g} \cdots \ldots \ldots+\cdots \cdots \cdots \cdots+\cdots \cdots$ & & \\
\hline : & & ROANENS IS & (ASHE) E. J.PALMER & $f \theta \ldots \ldots \ldots \ldots \ldots \ldots \ldots \ldots$ & & \\
\hline - & c. & MALCIDES & $\begin{array}{l}\text { SARG. } \\
\text { CRATAEGUS AESIIVAL IS }\end{array}$ & $\cdots * 2 \cdots \cdots \cdots \cdots \cdots+\cdots \cdots \cdots+$ & & \\
\hline CRMAL & c. & MARGARETTAE & $\begin{array}{c}\text { ASHE } \\
\text { ( CRATAEGUS CHRYSOCARPA }\end{array}$ & $F B * 2 \cdots y+\cdots \cdots \cdots \cdots \cdots \cdots \cdot T$ & & \\
\hline - & & ANGUSTI I FO & LIIA E.J.PALMER & $f_{B} \ldots \ldots \ldots \ldots \ldots \ldots \ldots \ldots$ & & \\
\hline : & & $\begin{array}{l}\text { 3RON NEI } \\
\text { MEIOPHYLL }\end{array}$ & $\begin{array}{l}\text { (BRITTON)SARG. } \\
\text { (SARG, E SPALMER }\end{array}$ & $f \beta \cdots \cdots M \cdots \cdots \cdots \cdots$ & & \\
\hline * & c. $x$ & NEDIA & $\begin{array}{l}\text { SARG. } \\
\text { CRATAEGUS HAEMOCARPA }\end{array}$ & $f B G \ldots \ldots+\cdots \cdots+\cdots$ & & \\
\hline • & c. $x$ & MEMERANACEA & $\begin{array}{l}\text { SARG. } \\
\text { CRATAEGUS GLAREOSA }\end{array}$ & $F_{\theta G} \ldots \ldots \ldots \ldots \ldots \ldots \ldots \ldots$ & & \\
\hline 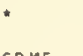 & c. $\times$ & MENANDI ANA & $\begin{array}{l}\text { SARG. } \\
\text { CRATAEGUS INTEGRILOEA }\end{array}$ & FBG $\ldots \ldots \ldots \ldots \ldots \ldots \ldots \ldots \cdots$ & & \\
\hline CRME & c. & RENDOSA & $\begin{array}{l}\text { GEADLE } \\
\text { [RATAEGUS INTRICATA }\end{array}$ & $\cdots \cdot \cdots \cdots \cdots \cdots \cdots \cdots \cdots+\cdots$ & & \\
\hline • & c. & MERCERENSIS & $\begin{array}{l}\text { SARG. } \\
\text { CRATAEGUS ROTUNDIFOLIA }\end{array}$ & f $8, \ldots \ldots \ldots \ldots \ldots \ldots \ldots \ldots$ & & \\
\hline CRME? & c. & MERIDIANA & $\begin{array}{l}\text { BEADLE } \\
\text { CRATAEGUS FLAVA }\end{array}$ & $\ldots \ldots \ldots \ldots \ldots \ldots \ldots \ldots \ldots \ldots+\varphi_{T}$ & & \\
\hline CRME3 & c. & MFRIDIONALIS & SARG. & $\ldots \ldots \ldots \ldots \ldots \ldots \ldots \ldots \ldots+\cdots$ & & \\
\hline • & $c \cdot x$ & YERITA & $\begin{array}{l}\text { SARG. } \\
\text { CRATAEGUS LUC ORUM }\end{array}$ & FBg $\ldots \ldots \ldots \ldots \ldots \ldots \ldots \ldots$ & & \\
\hline - & c. 1 & MICHAUXII & $\begin{array}{c}\text { PERS. } \\
\text { CRATAEGUS FLAVA }\end{array}$ & 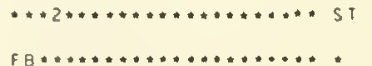 & & \\
\hline - & c. 1 & MILLER I & $\begin{array}{l}\text { SARG. } \\
\text { CRATAEGUS PRUINOSA } \\
\text { BEADLE }\end{array}$ & 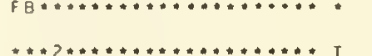 & & \\
\hline C RMO & c. & MOHRII & $\begin{array}{c}\text { BEADLE } \\
\text { CRATAEGUS CRUS-GALLI } \\
\text { SCHEELE. }\end{array}$ & 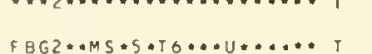 & ACCEPTED & \\
\hline CRMOZ & c. & MOLL IS & $\begin{array}{l}\text { CRATAEGUS TEXANA } \\
\text { CRATAEGUS GREGG IANA }\end{array}$ & 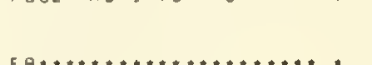 & & \\
\hline CRMOS & c. & $\begin{array}{l}\text { SERA } \\
\text { MONOGYNA }\end{array}$ & $\begin{array}{l}\text { (SARg.) JegGlest. } \\
\text { Jaco. } \\
\text { - crataegus oxyacantha }\end{array}$ & 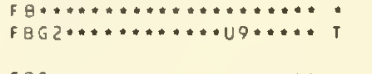 & ACCEPTED & \\
\hline - & C. $x$ & NEOEAXTERI & C SARG. & $\mathrm{fBg} \ldots \ldots \ldots \ldots \ldots+\cdots+\ldots \ldots *$ & & \\
\hline - & c. & WEOBUSHIL & $\begin{array}{l}\text { SARG. } \\
<\text { CRATAEGUS INTRICATA }\end{array}$ & $F B \cdots \cdots+\cdots \ldots \ldots \ldots \ldots$ & & \\
\hline CRNI & $6 . x$ & NIIIOA & $\begin{array}{l}\text { IENGELM. 2SARG. } \\
\text { - crataegus VIRIDIS }\end{array}$ & $F B G \cdots \cdots 4 * \ldots \cdots \cdots \cdots \cdots+* * * T$ & ACCEPTED & \\
\hline CRNO & c. & NOELENSIS & $\begin{array}{l}\text { SARG. } \\
\text { ( CRATAEGUS MOLLIS }\end{array}$ & $\varepsilon_{\theta} \ldots \ldots, \ldots \ldots \ldots \ldots \ldots \ldots+1$ & & \\
\hline - & c.x & NUDA & $\begin{array}{l}\text { SARG. } \\
\text { CRATAEGUS PERSIMILIS }\end{array}$ & f $\theta G \cdots \cdots \cdots \ldots \ldots \cdots \cdots \cdots \cdots$ & & \\
\hline - & c. & OAKESI ANA & $\begin{array}{l}\text { EGGLEST } \\
\text { CRATAEGIJS CHRYSOCARPA }\end{array}$ & $F B \cdots \ldots \ldots \ldots \ldots \ldots \ldots \ldots$ & & \\
\hline " & c. & OHIOENSIS & $\begin{array}{l}\text { SARG. } \\
\text { CRATAEGUS CRUS - GALLI }\end{array}$ & $f_{B} \ldots \ldots \ldots \ldots \ldots \ldots \ldots \ldots$ & & \\
\hline CA OP & c. & $O P A C A$ & $\begin{array}{l}\text { hoOK. \& arn. } \\
\text { - cratagus aestivalis }\end{array}$ & $\cdots \ldots \cdots+\cdots \cdots+\ldots \ldots \cdots \cdots \in$ ET & ACCEPTED & \\
\hline - & c. & OPERIA & $\begin{array}{l}\text { ASHE } \\
\text { ( CRATAEGUS CRUS-GALLI }\end{array}$ & $\ldots \ldots \ldots \ldots \ldots \ldots \ldots \ldots \ldots \ldots$ & & \\
\hline CR OP Z & c. & OPIYA & $\begin{array}{c}\text { GEADLE } \\
\text { \& CRATAEGUS PULCHERRIMA }\end{array}$ & $\ldots \ldots \ldots \ldots \ldots \ldots \ldots \ldots \ldots \ldots+\tau$ & & \\
\hline CROU & c. & OUACHITENSIS & $\begin{array}{l}\text { E.J PPALMER } \\
\text { ( CRATAEGUS INTRICATA }\end{array}$ & $\ldots \ldots \ldots \ldots \ldots \ldots \ldots \ldots \ldots \ldots, \tau$ & & \\
\hline CRO $\times 2$ & C. & OXYACA I THA & - crataegus monogrna & $F \in G \ldots \ldots \ldots \ldots \ldots$ U $\ldots \ldots \ldots$ is & ACCEPTED & \\
\hline$C P P A$ & c. & PADIFOLIA & $\begin{array}{l}\text { SARG, } \\
\text { CRATAEGUS INTRICATA }\end{array}$ & $F \theta \ldots \ldots m \ldots \ldots \ldots \ldots \ldots \ldots \ldots r$ & & \\
\hline ; & c. & $\begin{array}{l}\text { I NCARNATA } \\
\text { PAGENS IS }\end{array}$ & $\begin{array}{l}\text { SARG. } \\
\text { SARG. }\end{array}$ & 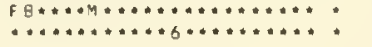 & & \\
\hline
\end{tabular}




Source Manusla Hablt Fomily Name No.

\begin{tabular}{|c|c|c|c|c|c|}
\hline Symbol & Geor & us/Spec1es & & oonym & Source Manusla \\
\hline CRPA? & c. & PALMERI & ( & $\begin{array}{l}\text { SARG. } \\
\text { CRATAEGUS CRUS-GALLI }\end{array}$ & $F B \ldots \ldots M+5 \ldots \ldots \ldots \ldots \ldots+1$ \\
\hline CRPA3 & c. & PANDA & & BEADLE & $\because \ldots \ldots \ldots \cdots \cdots$ \\
\hline * & c. $x$ & PAUS I ACA & & $\begin{array}{l}\text { ASHE } \\
\text { CRATAEGUS PUNCTATA PAUSIACA }\end{array}$ & 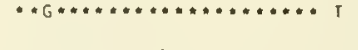 \\
\hline • & c. & PEARSONII & & $\begin{array}{l}\text { ASHE } \\
\text { CRATAEGUS FLAVA }\end{array}$ & 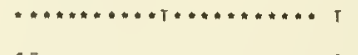 \\
\hline CRPE & c. & PEDICELLATA & & $\begin{array}{l}\text { SARG. } \\
\text { CRATAEGUS COCCINEA }\end{array}$ & fE $\ldots \ldots \ldots \ldots \ldots \ldots \ldots \ldots \ldots$ I \\
\hline : & & $\begin{array}{l}\text { ALBICANS } \\
\text { ELLWANGER }\end{array}$ & ANA & $\begin{array}{l}\text { (ASHE) E.J.PALMER } \\
\text { (SARG.) EGGLEST. }\end{array}$ & $f \mathrm{f} \ldots \ldots \ldots \ldots \ldots \ldots \ldots \ldots \ldots \ldots$ \\
\hline * & & ROBESONIA & NA & (SARG.)E.J.PALMER & f \\
\hline CRPE? & c. & PENITA & & $\begin{array}{l}\text { BEAOLE } \\
\text { CRATAEGUS AMNICOLA }\end{array}$ & 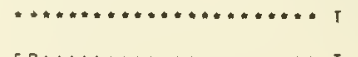 \\
\hline CRPE 3 & c. & PENNSYLVANICA & & $\begin{array}{l}\text { ASHE } \\
\text { CRATAEGUS COC CINEA }\end{array}$ & $\mathrm{F} B \cdots \cdots \cdots \cdots \cdots+\ldots$ \\
\hline CRPE 4 & c. & PEORIENSIS & & $\begin{array}{l}\text { SARG. } \\
\text { CRATAEGUS PLINCTATA }\end{array}$ & 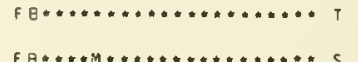 \\
\hline CRPES & c. & PERMIXTA & & $\begin{array}{c}\text { E.J.PALMER } \\
\text { CRATAE GUS CRUS-GALLI }\end{array}$ & 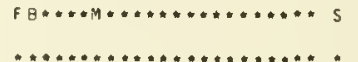 \\
\hline * & c. & PINETORUM & & $\begin{array}{l}\text { BEADLE } \\
\text { CRATAEGUS PULCHERRIMA }\end{array}$ & $* * \ldots \ldots \ldots \ldots \ldots \ldots \ldots \ldots \ldots * *$ \\
\hline • & c. & PINGU IS & & $\begin{array}{l}\text { SARG. } \\
\text { CRATAEGUS BRAINERDII }\end{array}$ & $\mathrm{f}$ \\
\hline * & c. & PIPER I & " & $\begin{array}{l}\text { BRITTON } \\
\text { CRATAEGUS COLUMBIANA PIPERI } \\
\text { CRATAEGUS CHRYSOCARPA }\end{array}$ & $\ldots \ldots \ldots \ldots \ldots \ldots \ldots \ldots \ldots \ldots \ldots$ \\
\hline * & c. & PLATYCARPA & $<$ & $\begin{array}{l}\text { SAFG. } \\
\text { CRATAEGUS PRUINOSA } \\
\text { SAFG. }\end{array}$ & 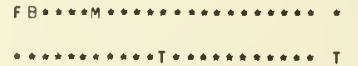 \\
\hline CRPO & c. & $\begin{array}{l}\text { POLIOPHYLLA } \\
\text { POPULIFOLIA }\end{array}$ & i & $\begin{array}{l}\text { SAFG. } \\
\text { CRATAEGUS VIRIDIS } \\
\text { WALTER }\end{array}$ & 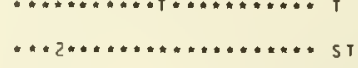 \\
\hline CRPOZ & c. & POPULNEA & $<$ & $\begin{array}{l}\text { CRATAEGUS FLAEELLAIA } \\
\text { ASHE } \\
\text { CRATAEGUS FLABELLATA }\end{array}$ & F \\
\hline CRPO3 & c. & PORTER I & & $\begin{array}{l}\text { BRITTON } \\
\text { CRATAEGUS PRUINOSA }\end{array}$ & $k_{B} \ldots \ldots \ldots \ldots \ldots \ldots \ldots \ldots+c$ \\
\hline ERPR & c. & $\begin{array}{l}\text { CAERULESC } \\
\text { PRINGLE } 1\end{array}$ & ENS & $\begin{array}{l}\text { (SARG.)E.J.PALMER } \\
\text { SARG. } \\
\text { CRATAEGUS COCCINEA }\end{array}$ & 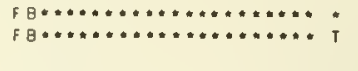 \\
\hline - & & $E \times C L U S A$ & & $\begin{array}{l}\text { CRATAEGUS COC CINEA } \\
\text { (SARG. )EGGLEST. }\end{array}$ & $F B \cdots * \cdots \cdots \cdots \cdots \cdots$ \\
\hline CRPRZ & c. & $\begin{array}{l}\text { LOBULATA } \\
\text { PRUINOSA }\end{array}$ & & $\begin{array}{l}\text { (SARG.)EGGLEST. } \\
\text { (H.L.WENOL.)K, KOCH }\end{array}$ & 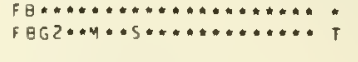 \\
\hline * & & 3RACHYPOO & ' & $\begin{array}{l}\text { CRATAEGUS FLABELLATA } \\
\text { (SARG.) E. J.PALMER }\end{array}$ & $F B \cdots \ldots M \cdots \cdots \cdots \cdots \cdots \cdots \cdots *$ \\
\hline$*$ & & DELA WAREN & vSIS & (SARG.) E.J.PALMER & $F B \cdots \ldots \ldots \ldots \ldots \ldots \ldots \ldots$ \\
\hline$*$ & & OISSONA & & (SARG * EGGLEST. & F $\theta \ldots \ldots \ldots \ldots \ldots \ldots \ldots \ldots \ldots$ \\
\hline 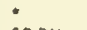 & & LATISEPAL & & (ASHE) EGGLEST. & 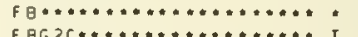 \\
\hline CRPU & c. & PUNC TA TA & & JACQ. & 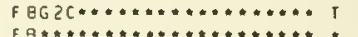 \\
\hline & & AUREA & & AITITON & 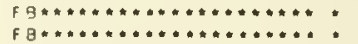 \\
\hline * & & $\begin{array}{l}\text { CAVE SCENS } \\
\text { MICROPHYL }\end{array}$ & LA & $\begin{array}{l}\text { BRITTON } \\
\text { SARG. }\end{array}$ & 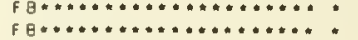 \\
\hline * & & PAUS IACA & & (A SHE)E. J.PALMER & $f_{\theta} \ldots \ldots \ldots \ldots \ldots \ldots \ldots \ldots \ldots$ \\
\hline - & $c \cdot x$ & PUTATA & $i$ & $\begin{array}{l}\text { SARG. } \\
\text { [RATAEGUS GLAREOSA }\end{array}$ & $f \theta G+\cdots \cdots \cdots \cdots \ldots \ldots \ldots \ldots \ldots$ \\
\hline CRPU? & c. & PUT TAM I ANA & $<$ & $\begin{array}{l}\text { SARG. } \\
\text { CRATAEGUS COCCINEA }\end{array}$ & $F \theta * \ldots \ldots \ldots \ldots \ldots \ldots \ldots+1$ \\
\hline CRPY & ᄃ. & PYRACANTHOIDES & $<$ & $\begin{array}{l}\text { BEADLE } \\
\text { CRATAEGUS CRUS - GALLI } \\
\text { CRATAEGUS BERBER:FOLIA }\end{array}$ & $f 8 * \cdots * \eta \ldots \ldots+\ldots \ldots \ldots \ldots$ T \\
\hline * & & ARBOREA & & (SEADLE)E. J.PALMER & $f B \cdots \cdots M \cdots \ldots \ldots \ldots \ldots$ \\
\hline CRRA & c. & RA VENE LII & & SARG. & $\cdots \ldots \ldots \ldots \ldots \ldots \ldots \ldots \ldots *$ I \\
\hline * & c. & RAVIDA & & AS HE & 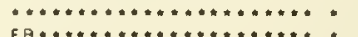 \\
\hline • & c. & RECEDENS & $<$ & $\begin{array}{l}\text { SARG. } \\
\text { CRATAEGUS PUNCTATA }\end{array}$ & 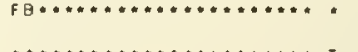 \\
\hline CRRE & c. & RECURVA & 1 & $\begin{array}{l}\text { BEAOLE } \\
\text { CRATAEGUS FLAVA }\end{array}$ & $\ldots \ldots \ldots \ldots \ldots \ldots \ldots+\ldots \ldots \ldots+\cdots, T$ \\
\hline CRRE? & c. & REGALIS & & $\begin{array}{l}\text { BEAOLE } \\
\text { CRATAEGUS CRUS-GALLI }\end{array}$ & $F \theta \cdots \cdots M+5 \cdots \cdots \cdots \cdots \cdots * T$ \\
\hline - & & PAFADOXA & & (SARG.) E.J.PALMER & $F B * \ldots M \cdots 5 * \ldots \ldots \cdots \cdots \cdots+T$ \\
\hline CRRE 3 & c. & RE VE RC HON II & & SARG. & $F B * \cdots M * 5 * T 6 \cdots \ldots \ldots \ldots$ S T \\
\hline • & & DISCOLOH & & (SARG.)E.J.PALMER & $F B \cdots \cdots 4 \cdots 5 \cdots 6 \cdots \ldots \ldots \ldots$ \\
\hline • & & STEVENSIA & ANA & (SARG.)E.J.PALMER & $\because \ldots \ldots \ldots s \cdots 6 \cdot \ldots \cdots \cdots \cdots+\cdots$ \\
\hline - & c. & RHODELLA & 6 & $\begin{array}{l}\text { ASHE } \\
\text { CRATAEGUS UNIFL ORA }\end{array}$ & $\cdots \cdots \cdots+\cdots+\cdots+\cdots+\cdots+\cdots \cdots \cdots$ \\
\hline CRRI & c. & RIVULARIS & & $\begin{array}{l}\text { NUTT. } \\
\text { CRATAEGUS DOUGLASII RIVULARIS }\end{array}$ & $\cdots \cdots \cdots \cdots *+1 \cdot 2 n \cdot U \cdots \cdots * * 1$ \\
\hline$\cdot$ & $c . x$ & ROBI NS ONII & ( & $\begin{array}{l}\text { SARG. } \\
\text { CRATAEGUS SUCCULENTA }\end{array}$ & $F \cdots \cdots \cdots \cdots \cdots \cdots \cdots \cdots \cdots$ \\
\hline CRRO & & ROBUR & 6 & $\begin{array}{l}\text { BEAOLE } \\
\text { CRATAEGUS PULCHERRIMA }\end{array}$ & $\ldots \ldots \ldots \ldots \ldots \ldots \ldots \ldots \ldots \ldots \ldots+T$ \\
\hline - & $c . x$ & ROTUNOATA & & $\begin{array}{l}\text { SARG. } \\
\text { CRATAEGUS IMMANIS }\end{array}$ & fBg $\ldots \ldots \ldots \ldots \ldots \ldots \ldots \ldots$ \\
\hline CRRO2 & c. & ROTUNDIF OLIA & $>$ & $\begin{array}{l}\text { MOENCH NON LAM. } \\
\text { CRATAEGUS DOOGEI } \\
\text { CRATAEGUS CHRYSOCARPA }\end{array}$ & $\cdots G \cdots \cdots \cdots \cdots \cdots \cdots \cdots \cdots * c$ \\
\hline CRRU & c. & RUBELLA & $\langle>$ & $\begin{array}{c}\text { CRATAEGUS CHRYSOCARPA } \\
\text { BEADLE } \\
\text { CRATAEGUS INTRICATA }\end{array}$ & $F B \cdots \cdots \cdots \cdots \cdots \cdots \cdots \cdots \cdots+s$ \\
\hline * & $6 . x$ & RUBR OC ARNEA & c & $\begin{array}{l}\text { SARG. } \\
\text { CRAIAEGUS RANDIANA }\end{array}$ & $F B G \ldots \ldots \ldots \ldots \ldots \ldots \ldots \ldots \ldots$ \\
\hline CRRUZ & c. & RUGOSA & $<$ & $\begin{array}{l}\text { ASHE } \\
\text { CRATAEGUS PRUINOSA }\end{array}$ & $F B \cdots \cdots+\ldots \ldots \ldots \ldots \ldots \ldots+$ T \\
\hline CRSA & c. & SABINEANA & $<$ & $\begin{array}{l}\text { ASHE } \\
\text { CRATAEGUS CRUS-GALLI }\end{array}$ & $\cdots \ldots \ldots \cdots \cdots+T \ldots \ldots \ldots \ldots \ldots+T$ \\
\hline CRSA3 & c. & SARGENTII & & $\begin{array}{l}\text { BEADLE } \\
\text { CRATAEGUS VENUSTA }\end{array}$ & $\ldots \ldots \ldots \ldots \ldots \ldots \ldots \ldots \ldots \ldots \ldots+r$ \\
\hline$*$ & c. & SCHIZOPHYLLA & & $\begin{array}{l}\text { EGGLEST. } \\
\text { CRATAEGUS CRUS-GALLI }\end{array}$ & $F B \cdots \ldots \ldots \ldots \ldots \ldots \ldots \ldots \ldots$ \\
\hline
\end{tabular}




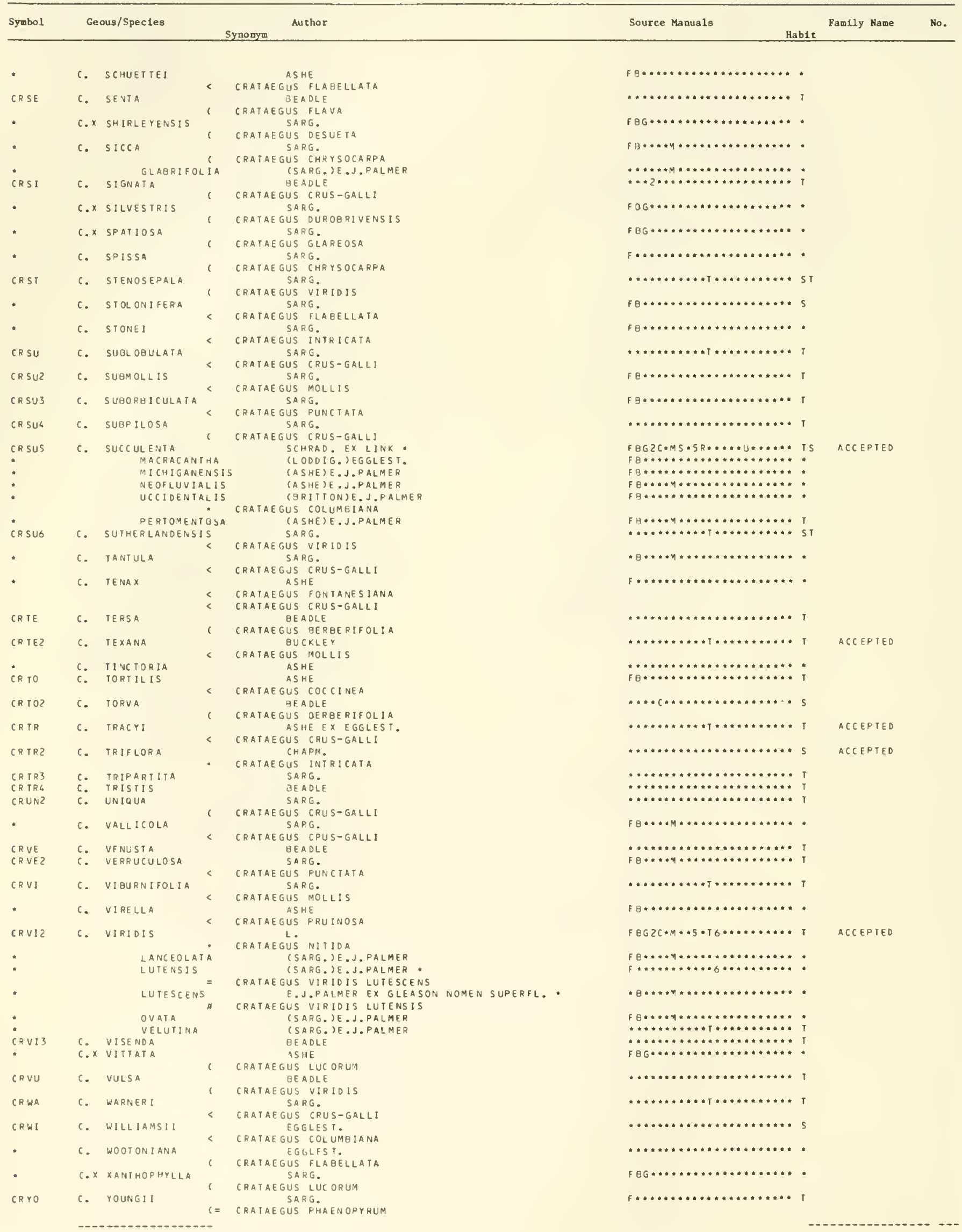




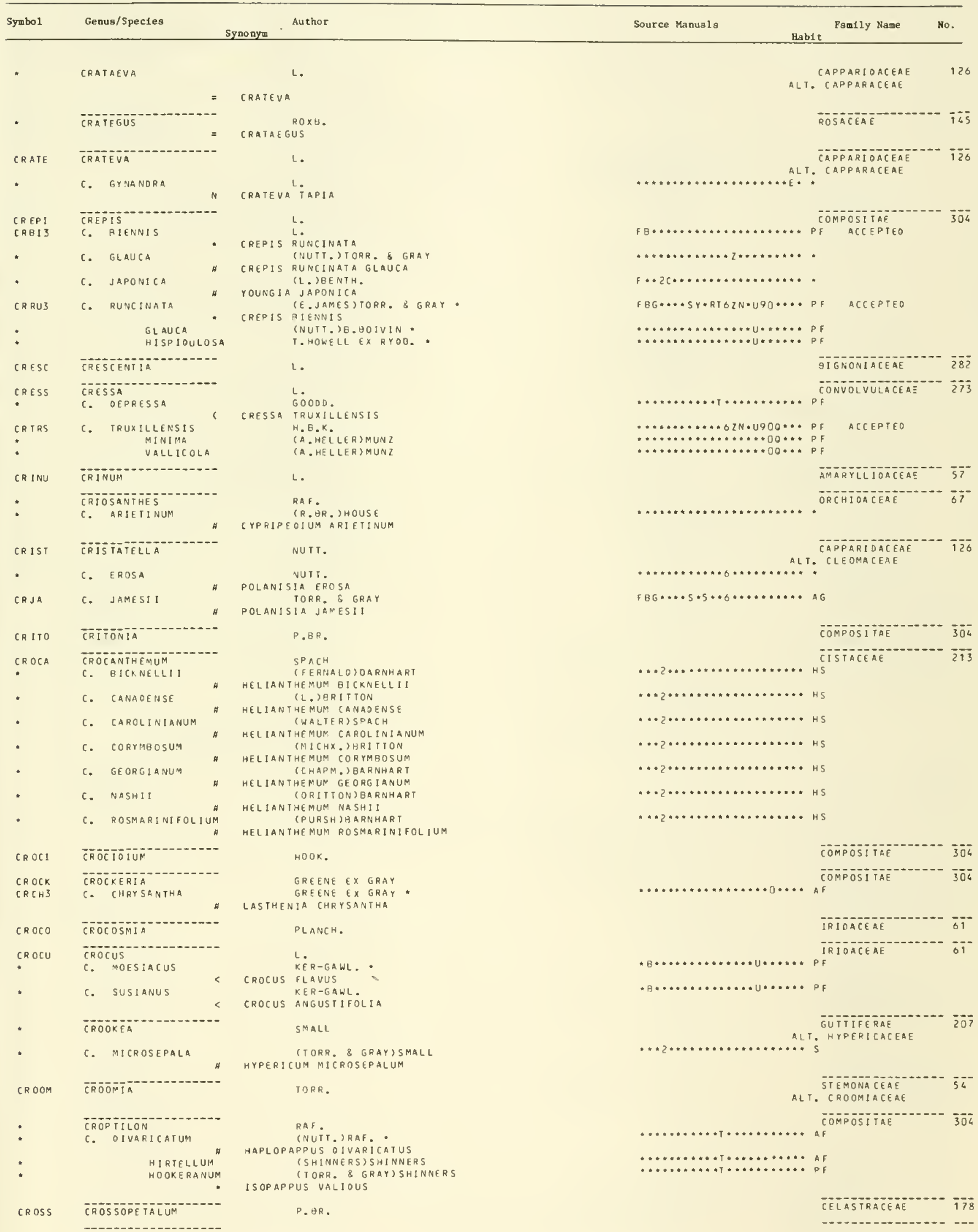




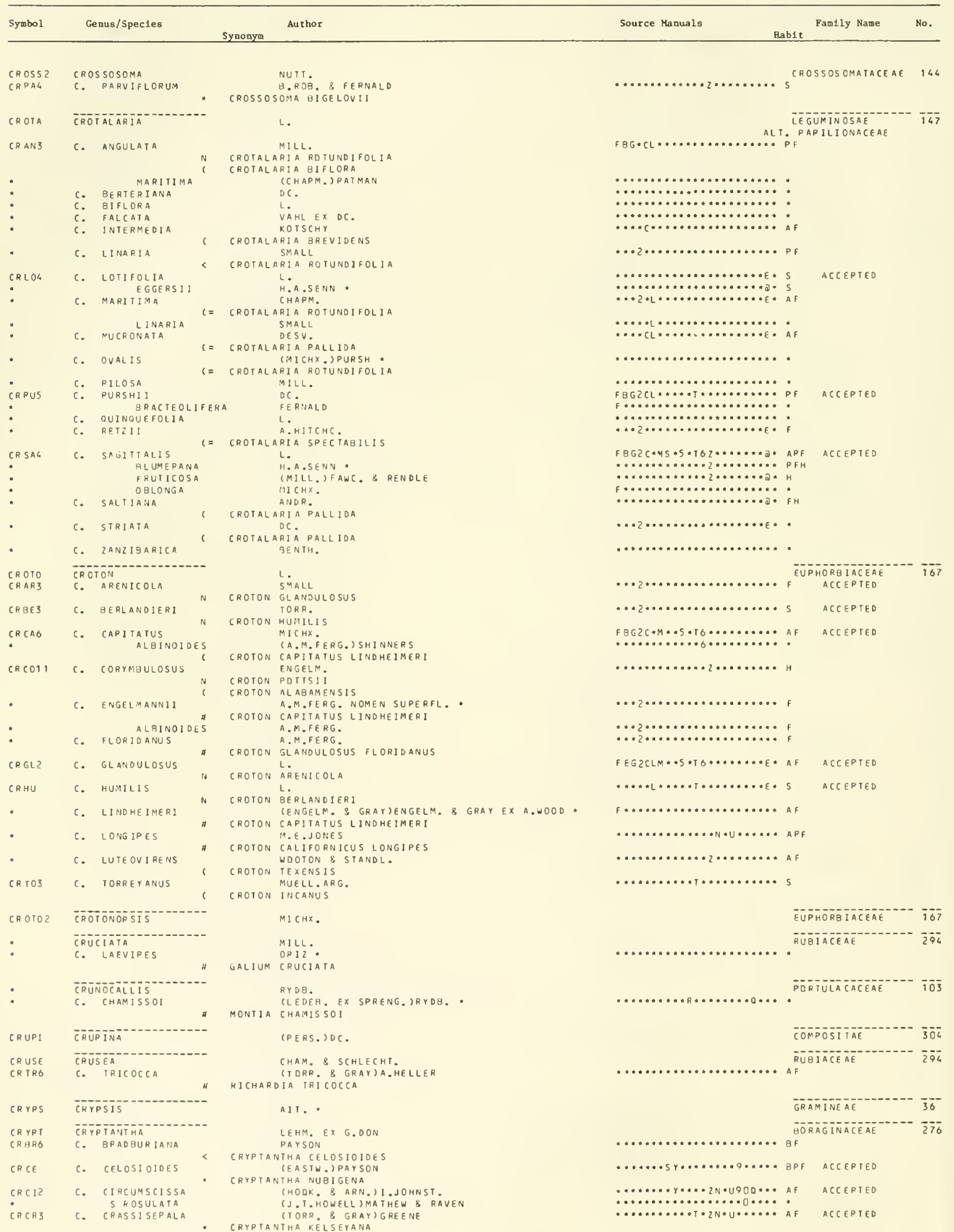




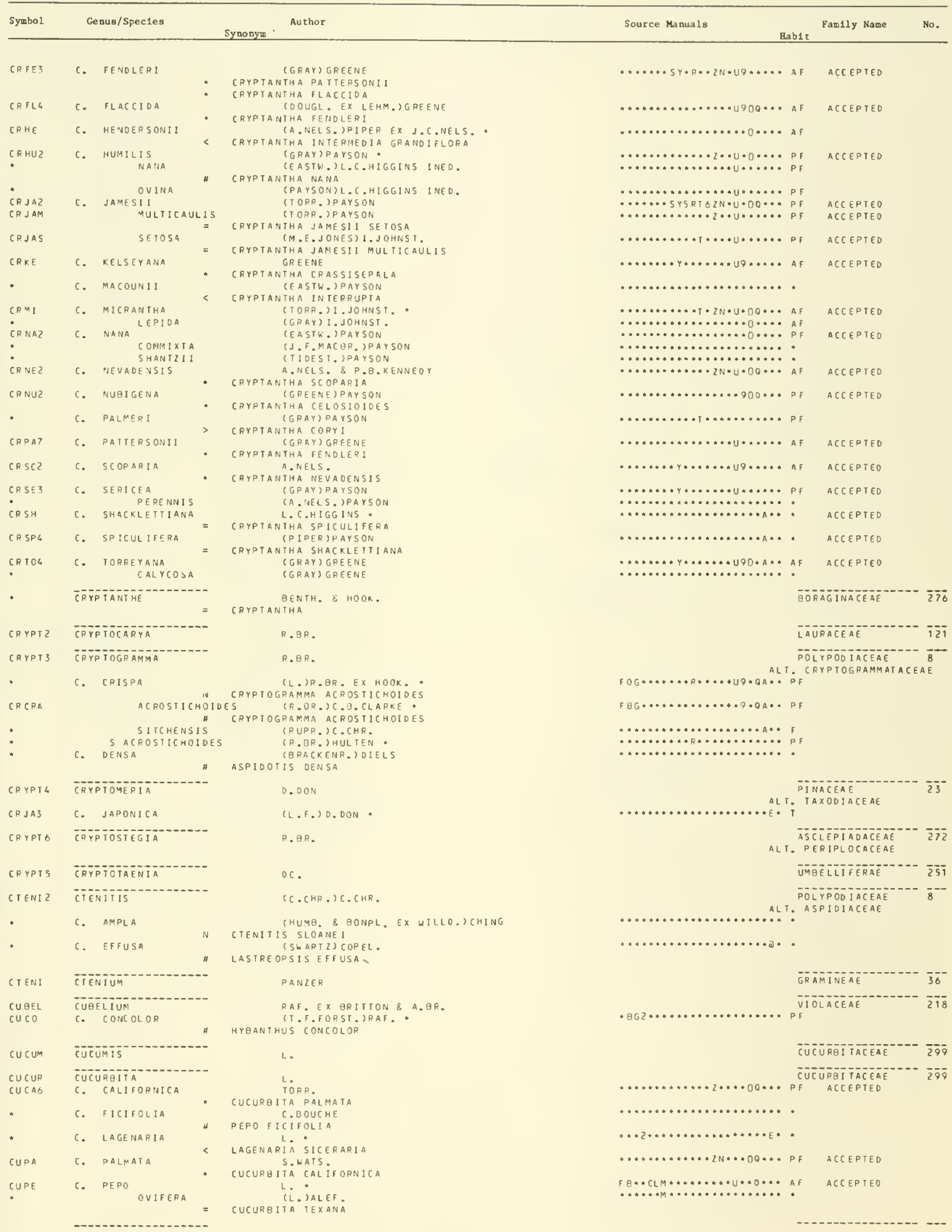




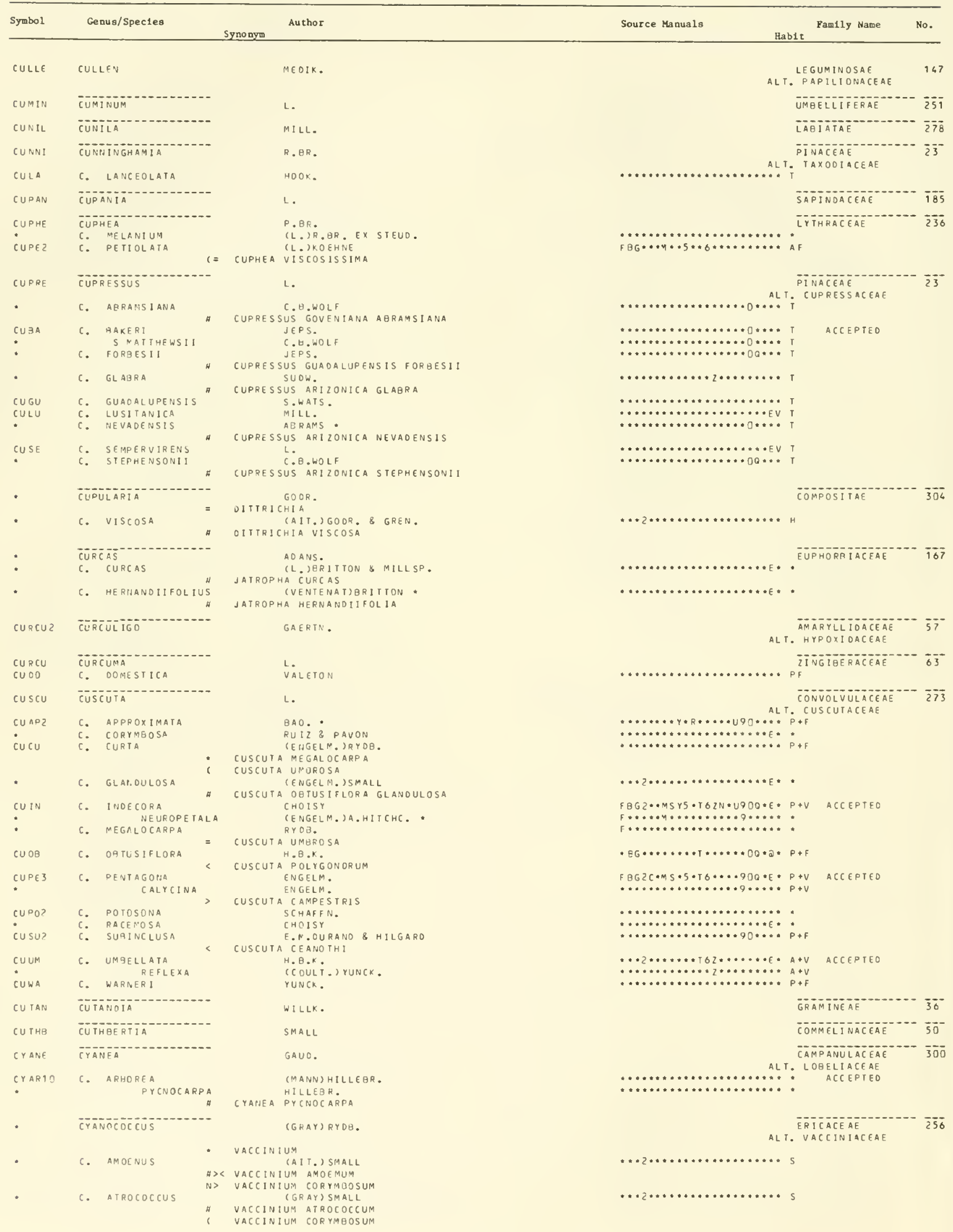




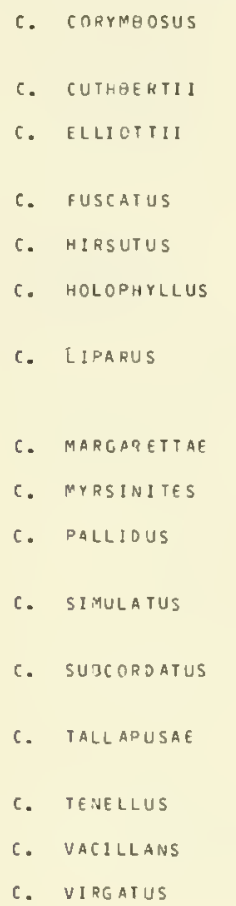

CL. IRYOQ.
VACCINIUM CORYMAOS VACEINIUM CORYM DOSUM SMALL
SACEM CIN VACCINIUM CORYMBOSUM VACCINIUM ELLIOITII VACCINIUM CORYMUOSUM (AIT.) SMALL VACCINIUY FUSCATUM VACCINIUY FUS CATUM
(BUCKLEY)SMALL VACCINIUM HIRSUTUM SYALL VACCINIUM FUSCATUM VACCINIUM CORYMBOSUM SMALL

VACCINIUM CAESARIENSE VACCINIUM CORYMOOSUM VACCINIUM PALLIDUM$$
\text { VACCIN! UM PALLIDUM }
$$

VACCINIUME) SMAL UACCINIUM PALLI DUM VACCINIUM MYRSINITES (AIIT.) SMALL VACCINIUM VAGILLANS VACCINIUIA PALLIDUM SMRLL VACCINIUM SIMULATUM VACCINIUM CONSTABLAE VACCINIUM ALT O-MONTANUM VACCINIUM PALLI DUM
COVILLE EX SMALL. VACCINIUM ALTO-MONTANUM VACCINIUM PALLI OUM (AI T.) SMALL
VACCINIUM TENELLUM (KALM) RYDB. VACCINIUM VACILLANS (AIT,) SMALL VACCINIUM AUS IRALE VACCINIUM CORYMOOSUM

- C. TENERA
TRICHOPTERIS PUBESCENS
(J.E.SMITH)T.MOORE
J.E.SMITH (CHRIST)OOMIN CYATHEA PARVULA TRICHOPTERIS BORINQUENA MAXON CYATHEA FURFURACEA $M A X O N$ ALSOPHILA BROOKS: MAXON ALSOPHILA DRYOPIEROIDES (KARST.) OOMIN CYATHEA ANDINA (L.) J.E. SMITH CNEMIDARIA HORRIDA SPRENG. EX KUHN NEPHELEA PORTORICENSIS METTEN. EX KUHN

C. TENERA (J.E.SMITH)T.MOORE CYATHULA c. PROSTRATA CYCLADENTA
C. HUMILIS TOMENTOSA VENUSTA

CYCLAZ CYCLANTHERA CrCLODONA c. ALAUAMENSE C VATHEA BRIITONIANA BL UME (L.) EL UME BENTH. BENTH. (GRAY) GRAY (EASTW.) WOOOSON EX MUNZ. SCHRAD.

SMALL (VAIL)SMALL MATELEA ALABAMENSIS

CYCLOLOMA MOQ.

CYCLO CYCLOPELTIS

J. SM!TH

K.PRESL -

CY CL OROG OAN
CRANICHOIDES

C. ELATUM (SWARTZ) SCHLECHTER

(GRISEB.) SCHLECHTER

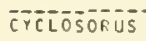

c. DENTATUS

c. PARASITICUS

c. IRUNCATUS
\#

(SWARTZ) SCHLECHTER

LI NK

(FORSSK.) CHING

THELYPTERIS DENTAIA

* Thel ILPTER IS PARASItica

CPOir. JFarw.
* thelypteris truncata
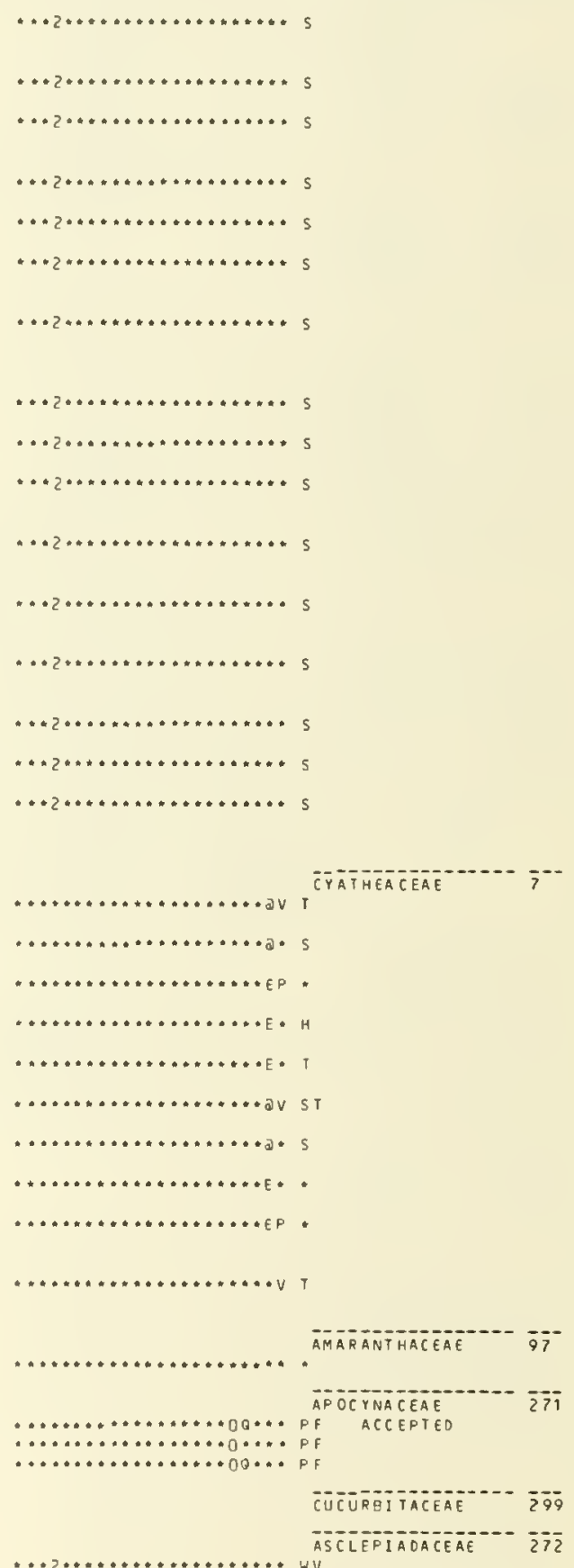

CHENOPODIACEAE 95

POLYPODIACEAE ALT. ASPIDIACEAE

ORCHIDACEAE $P F$

$P F$

POLYPODIACEAE ALT. THELYPIERIOACEAE 


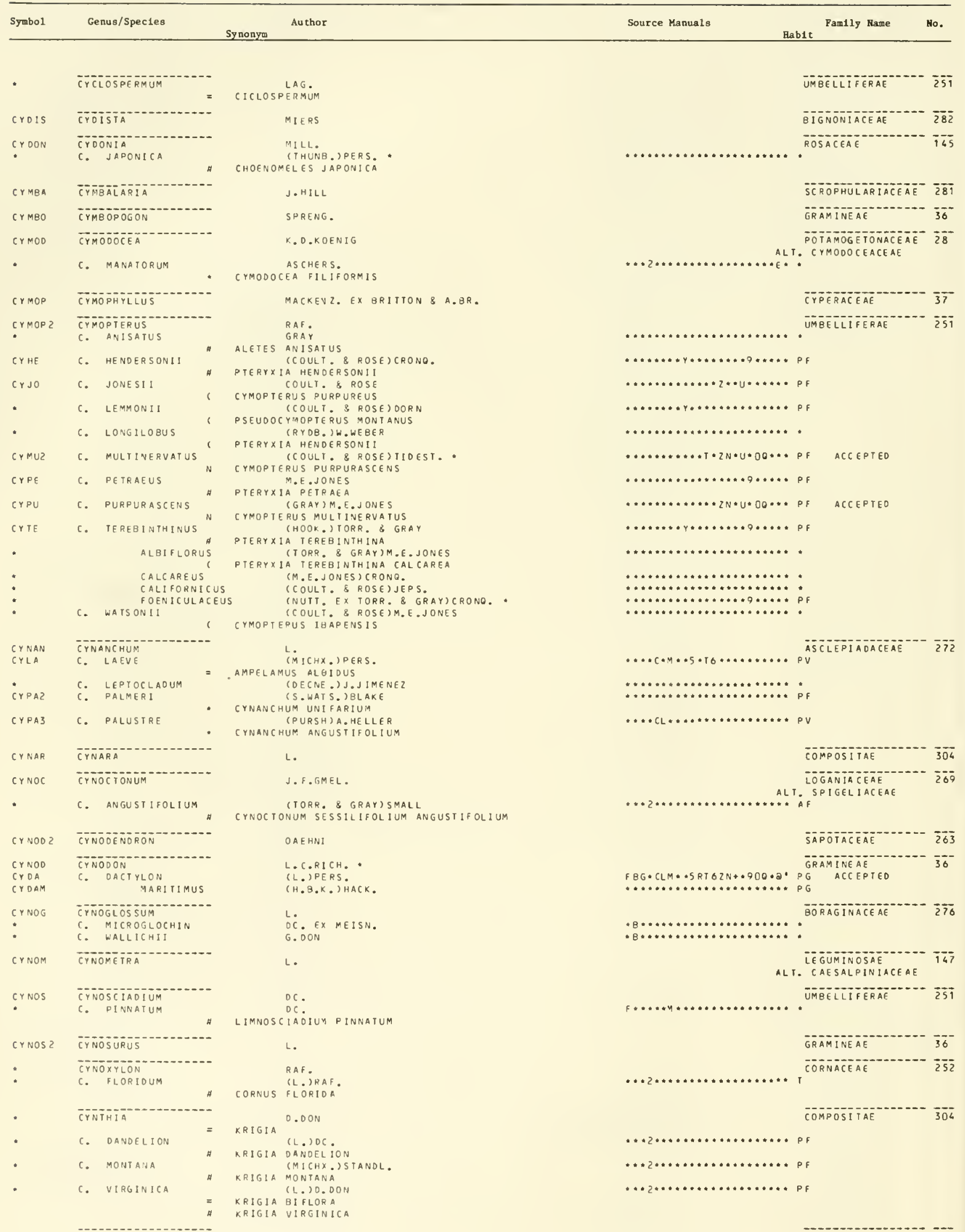




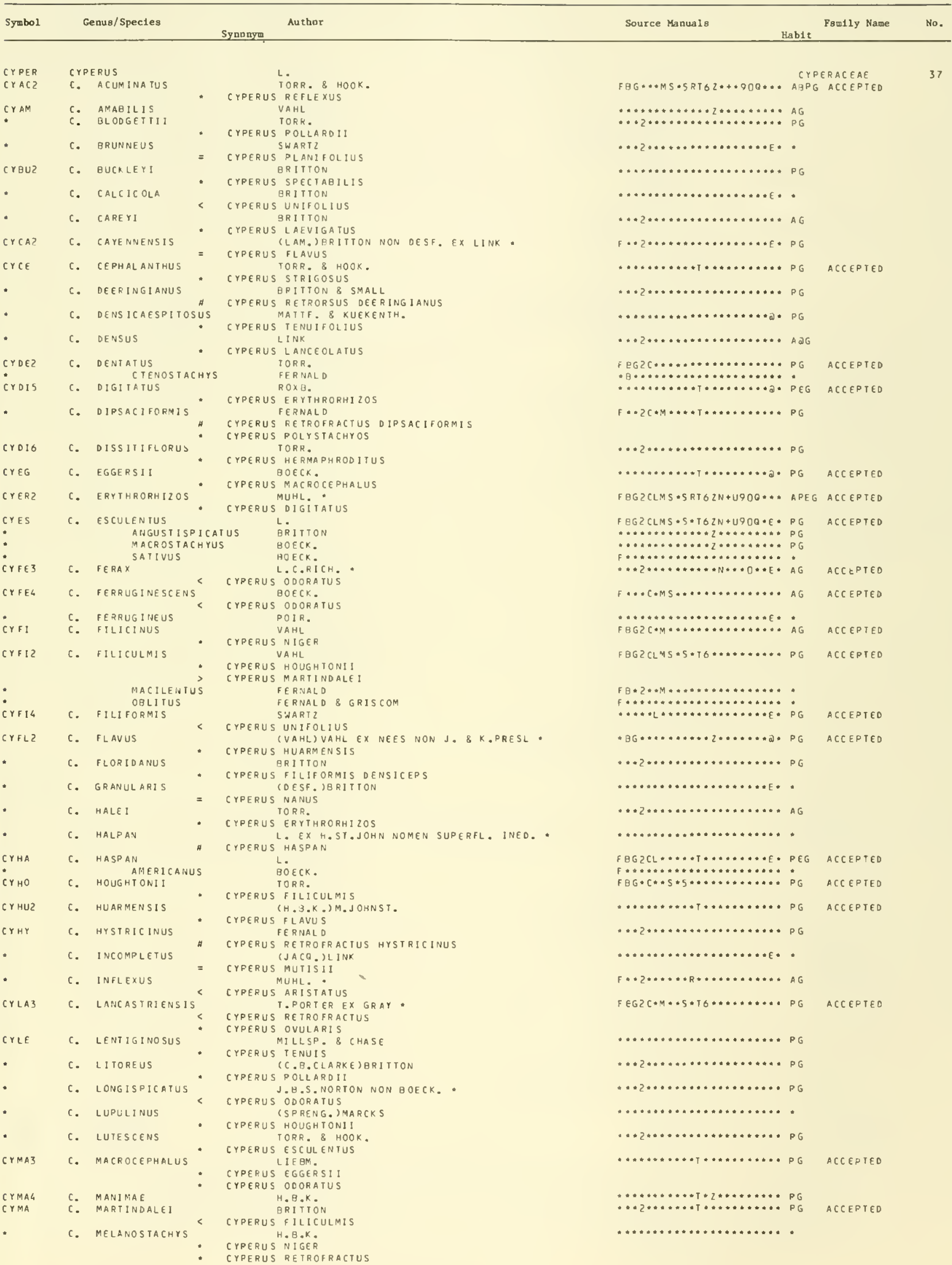




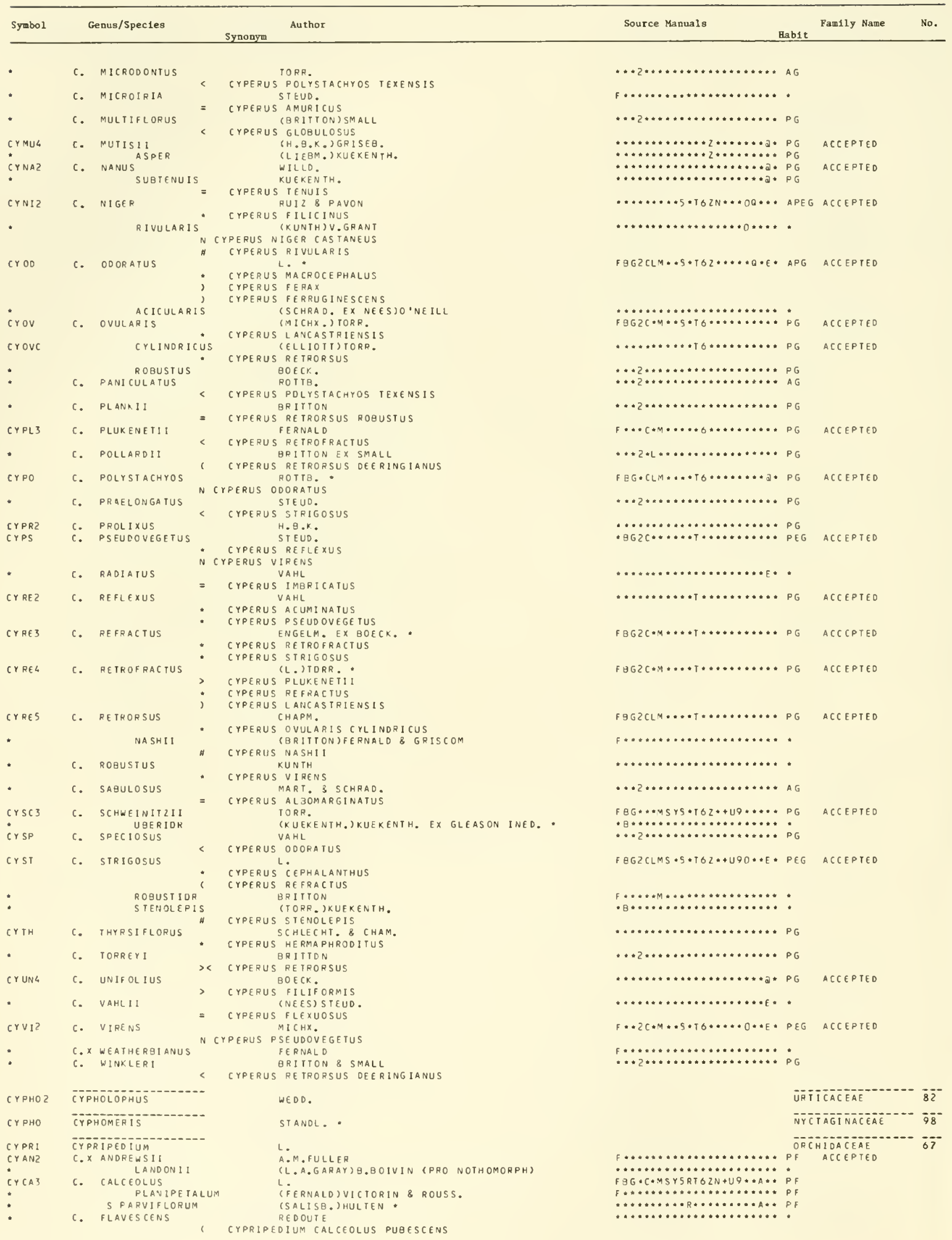




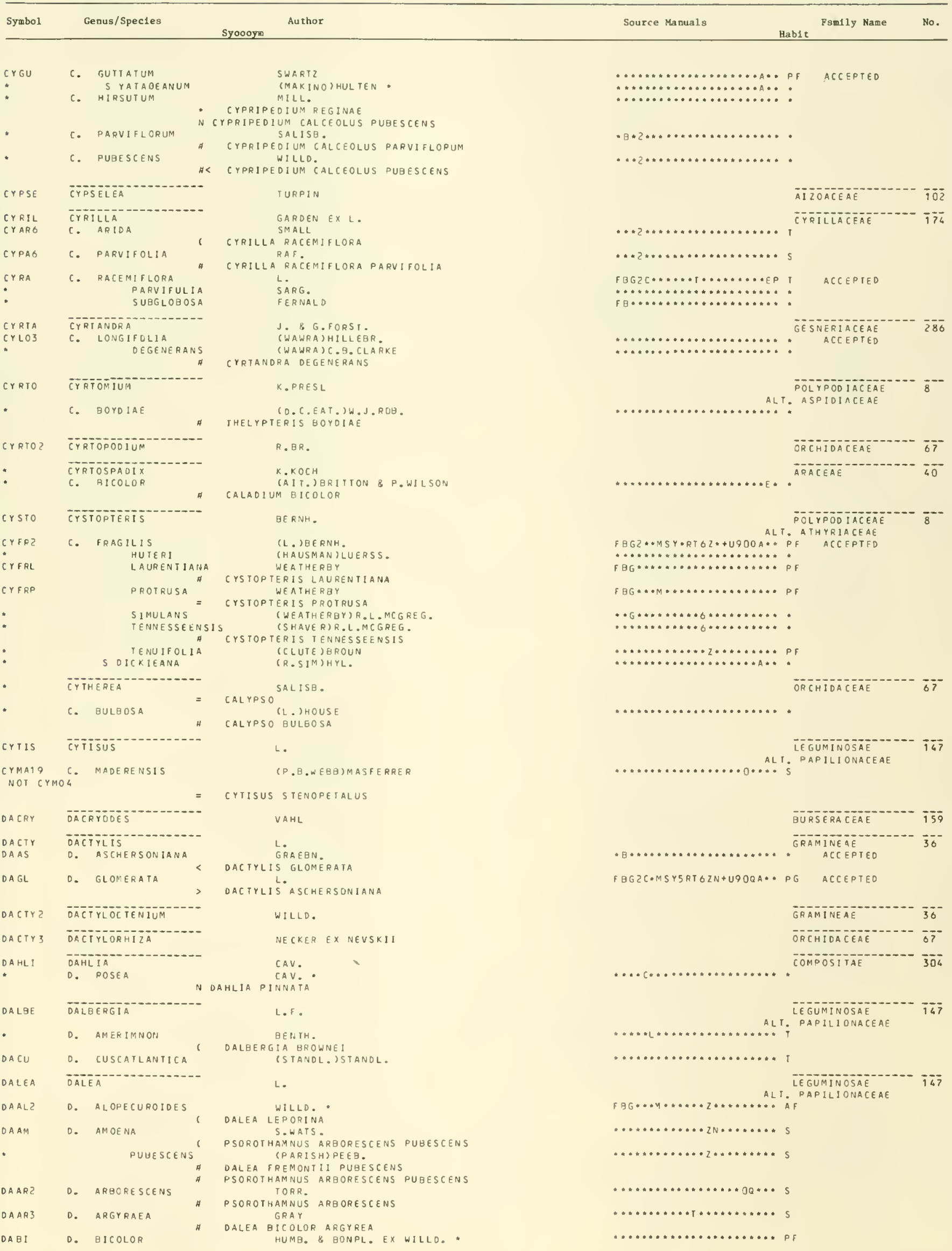




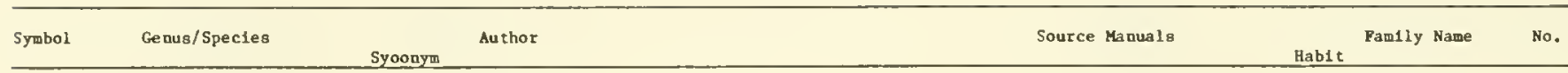

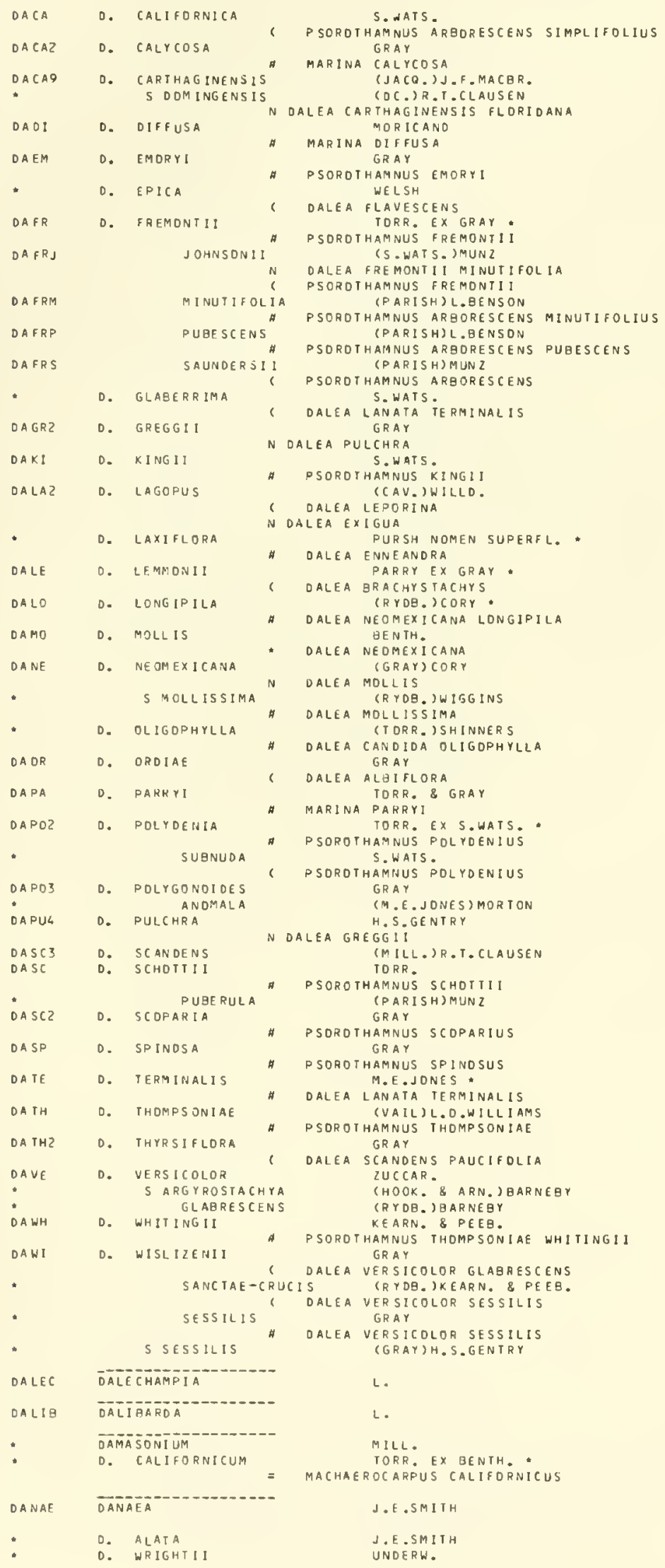

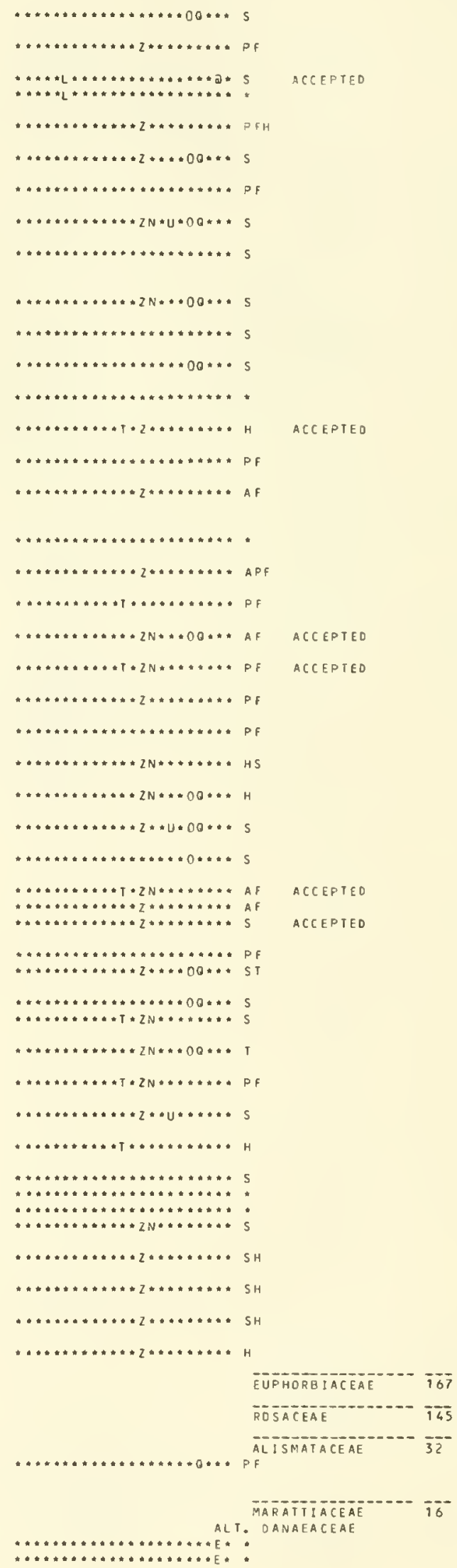




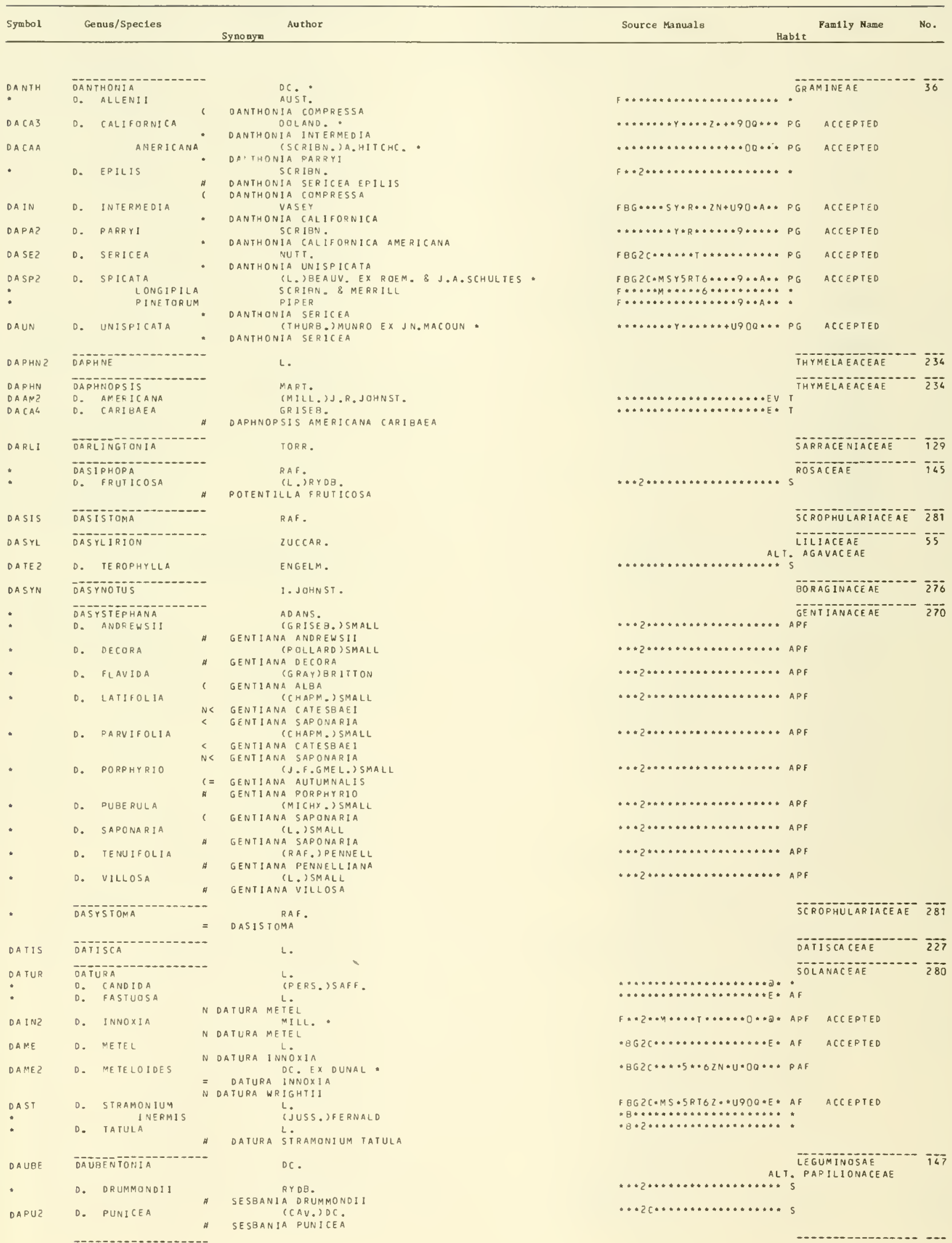




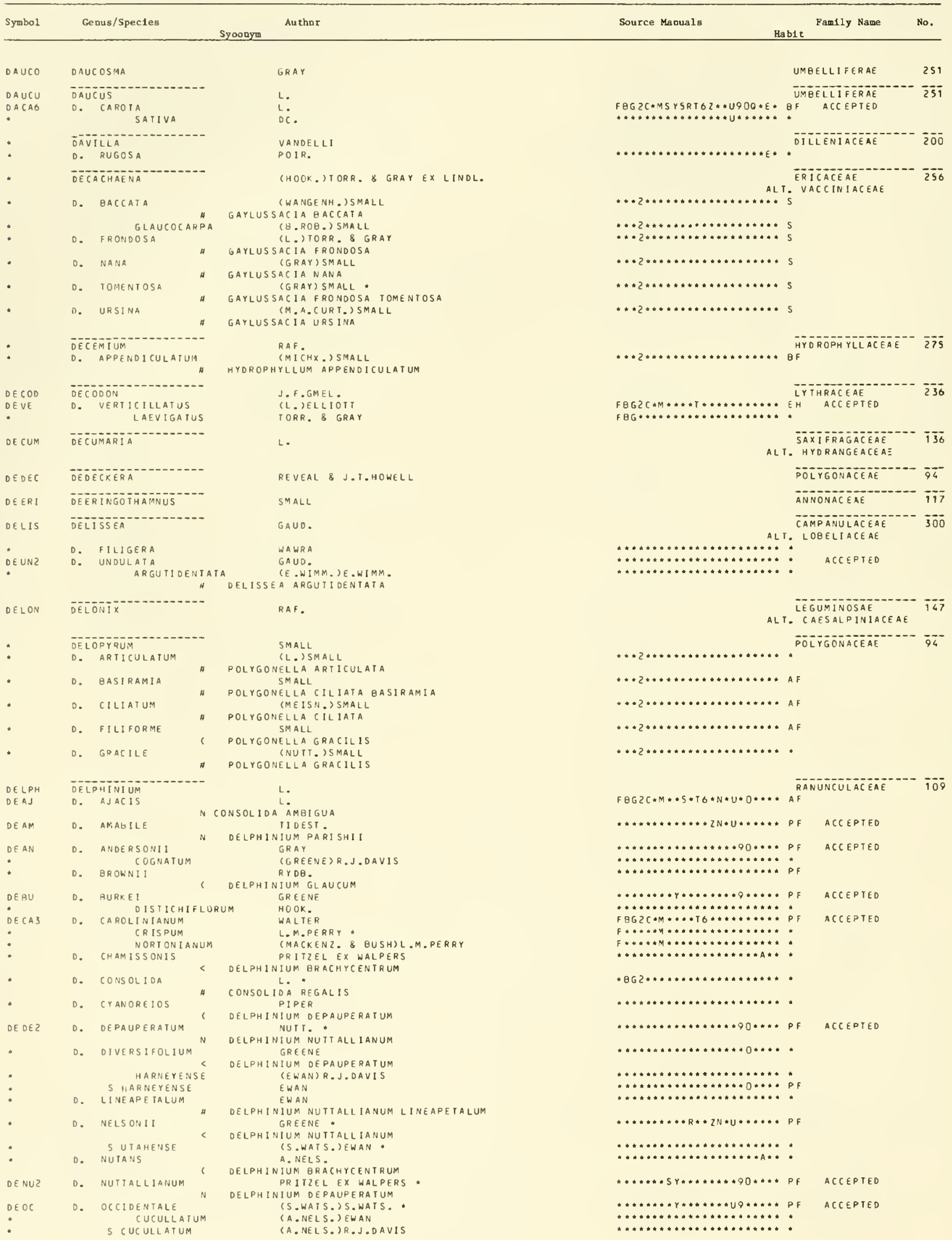




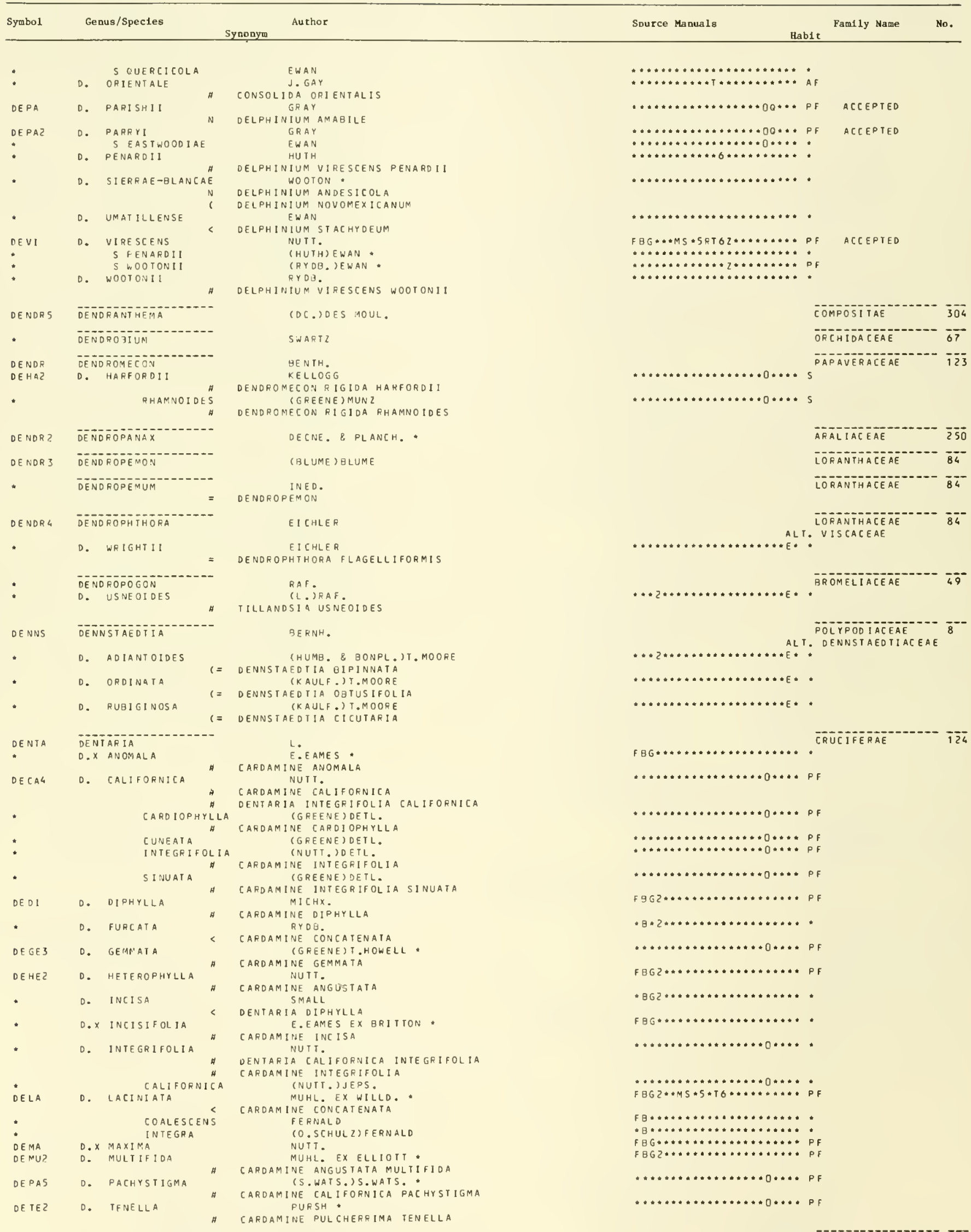




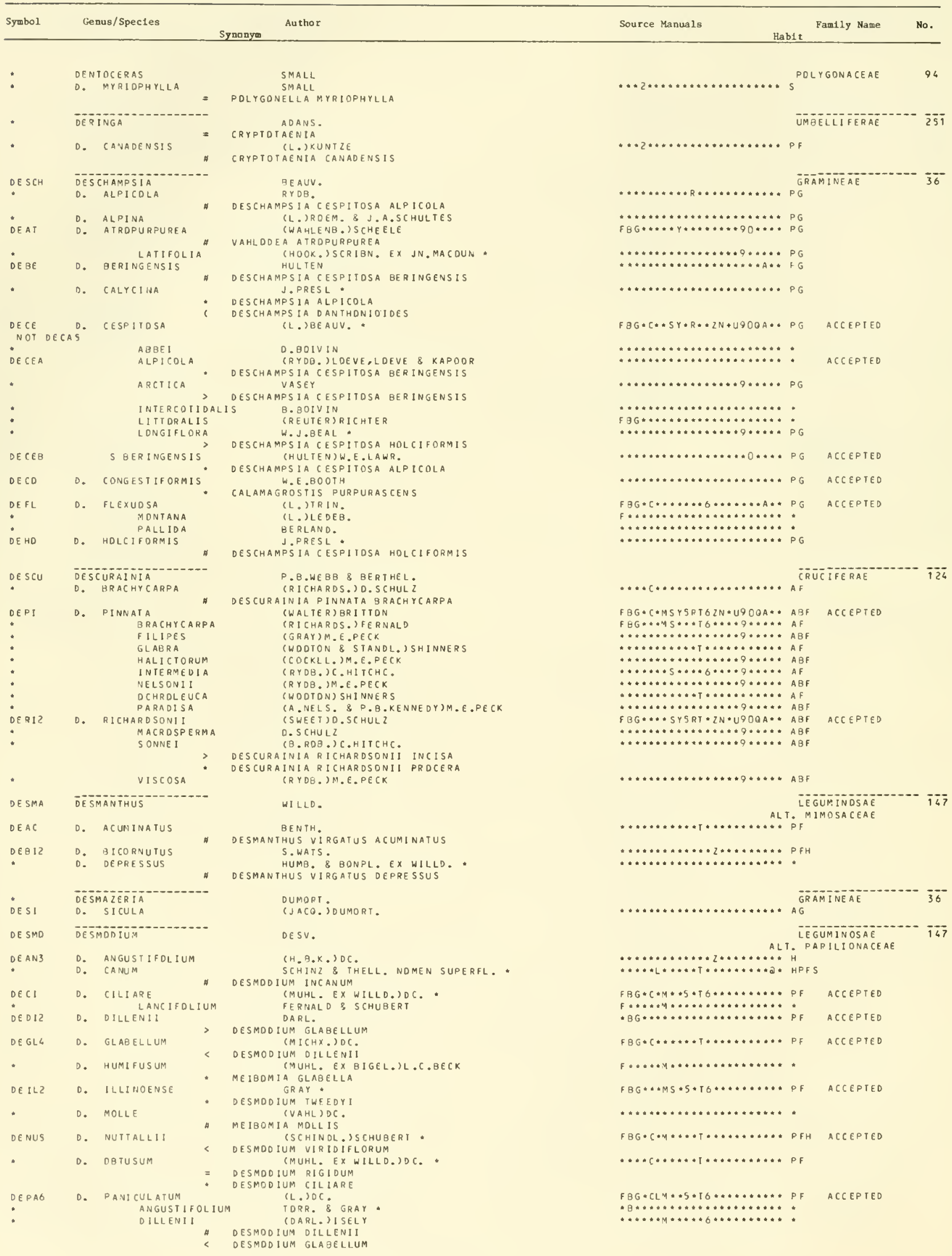




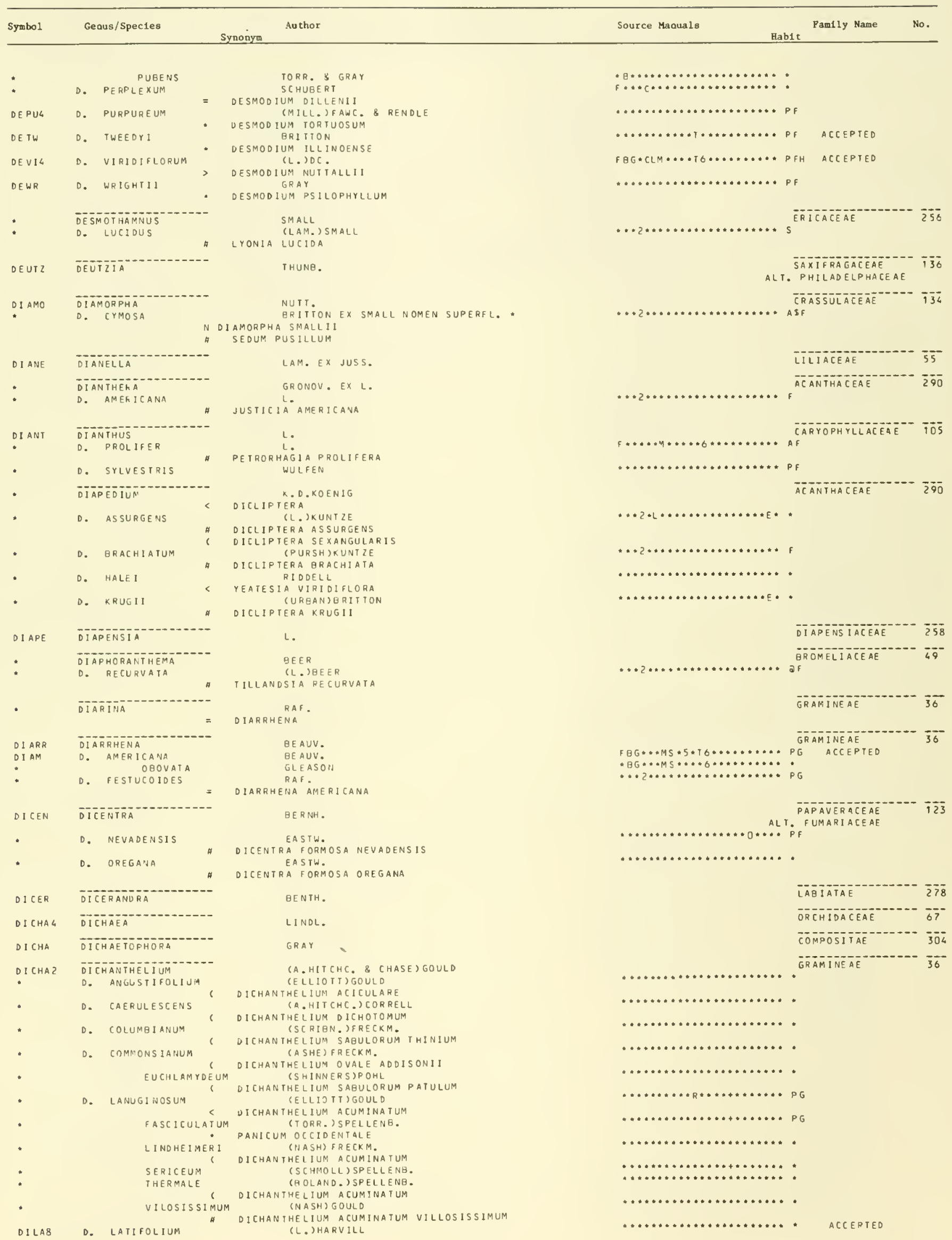




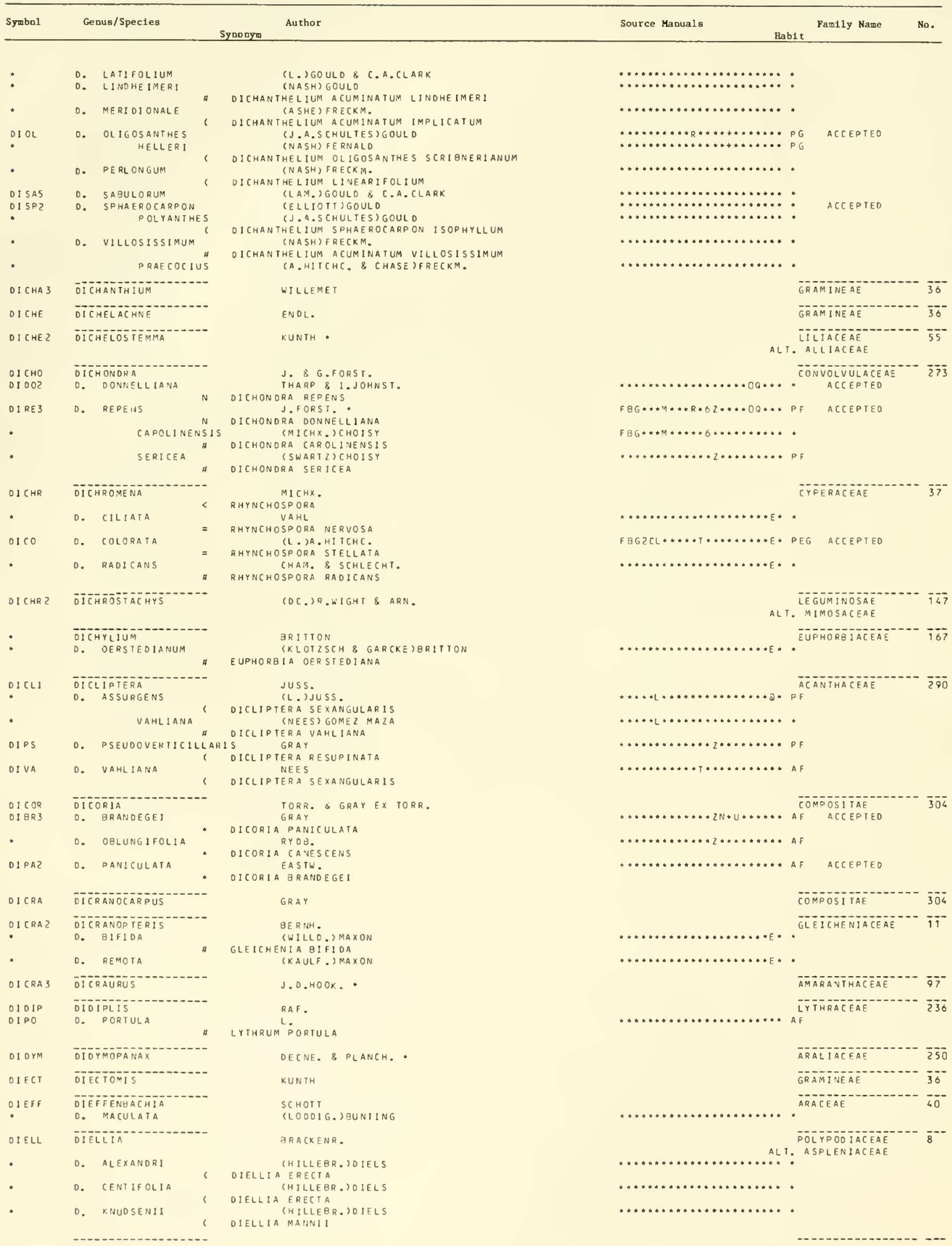




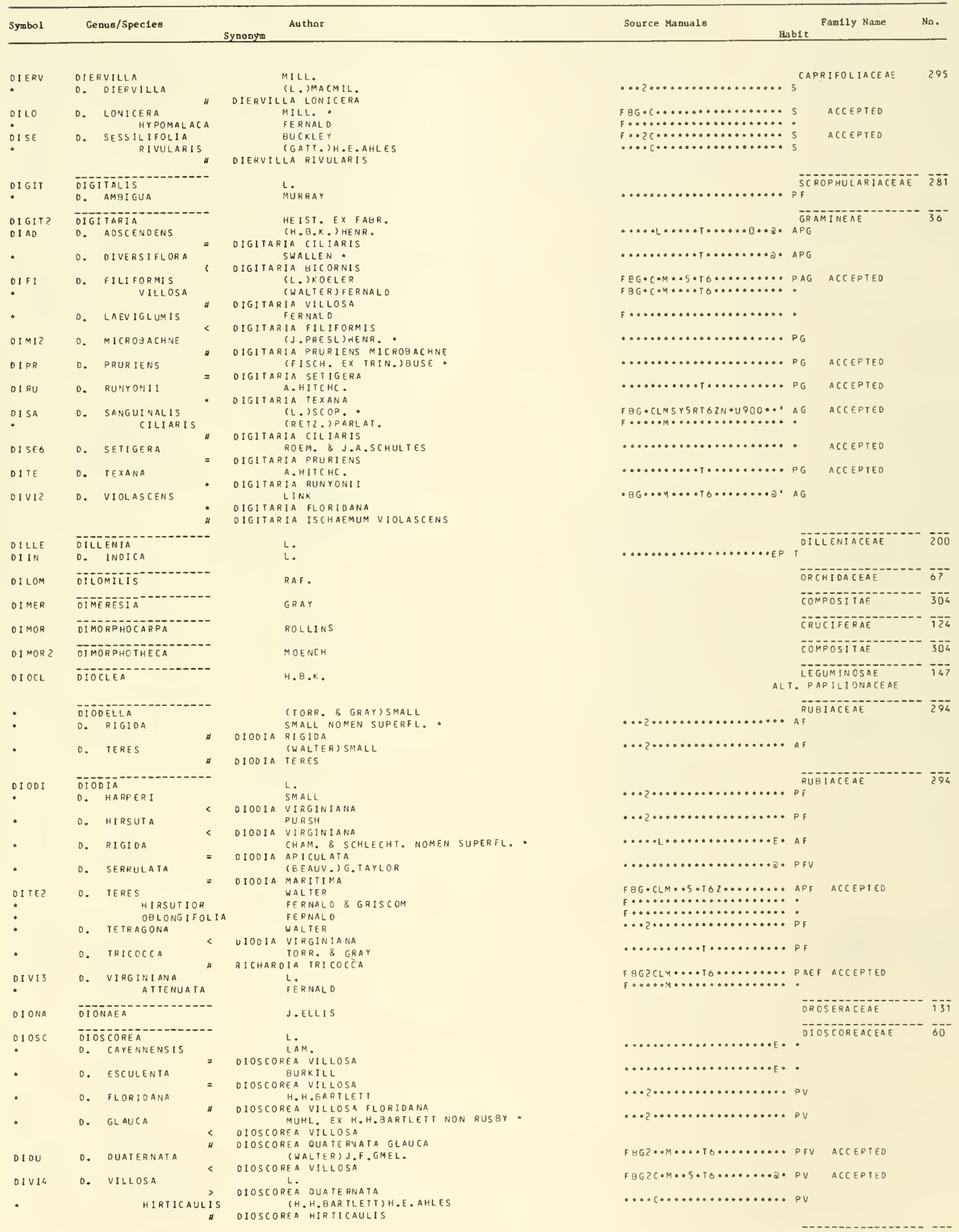




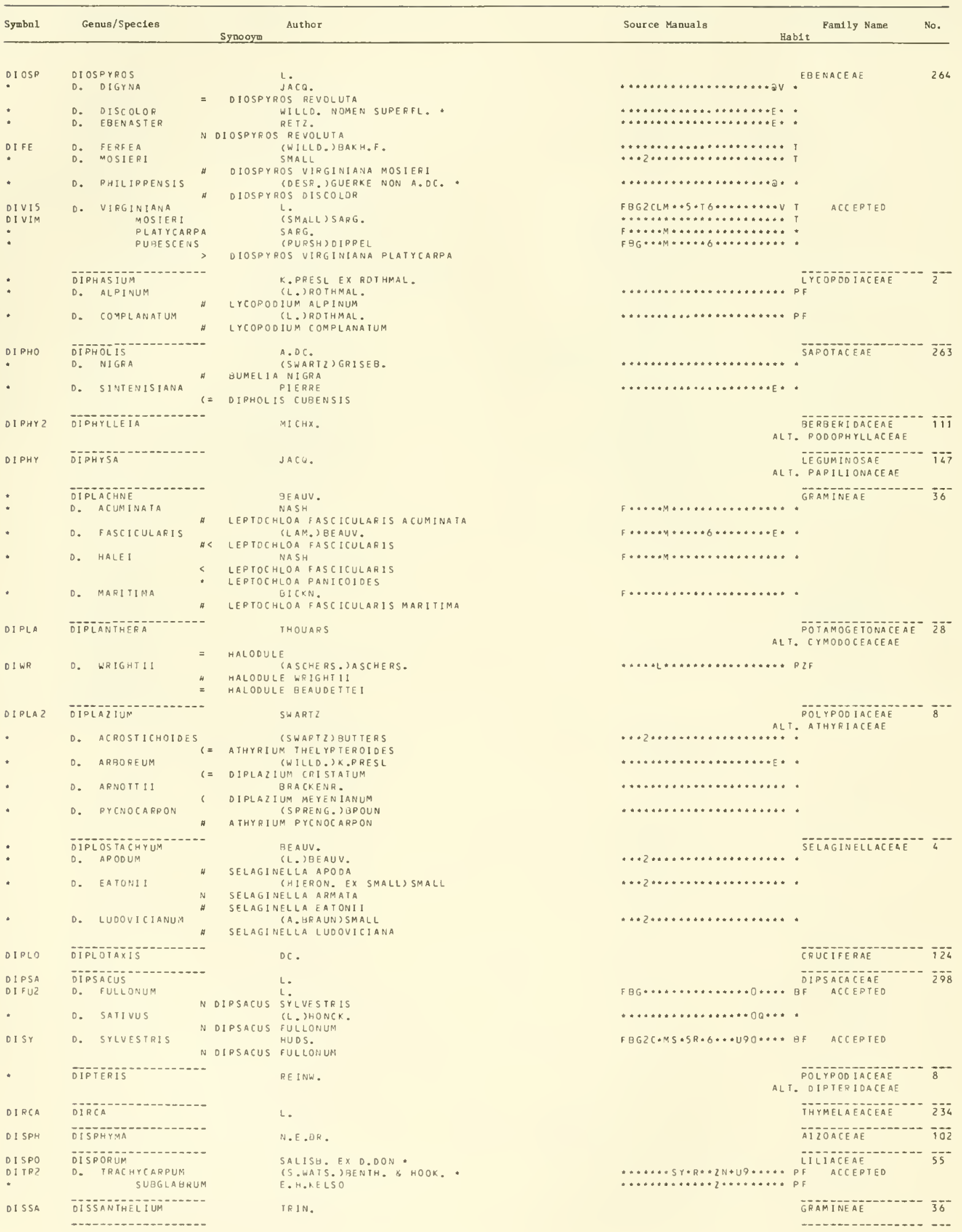




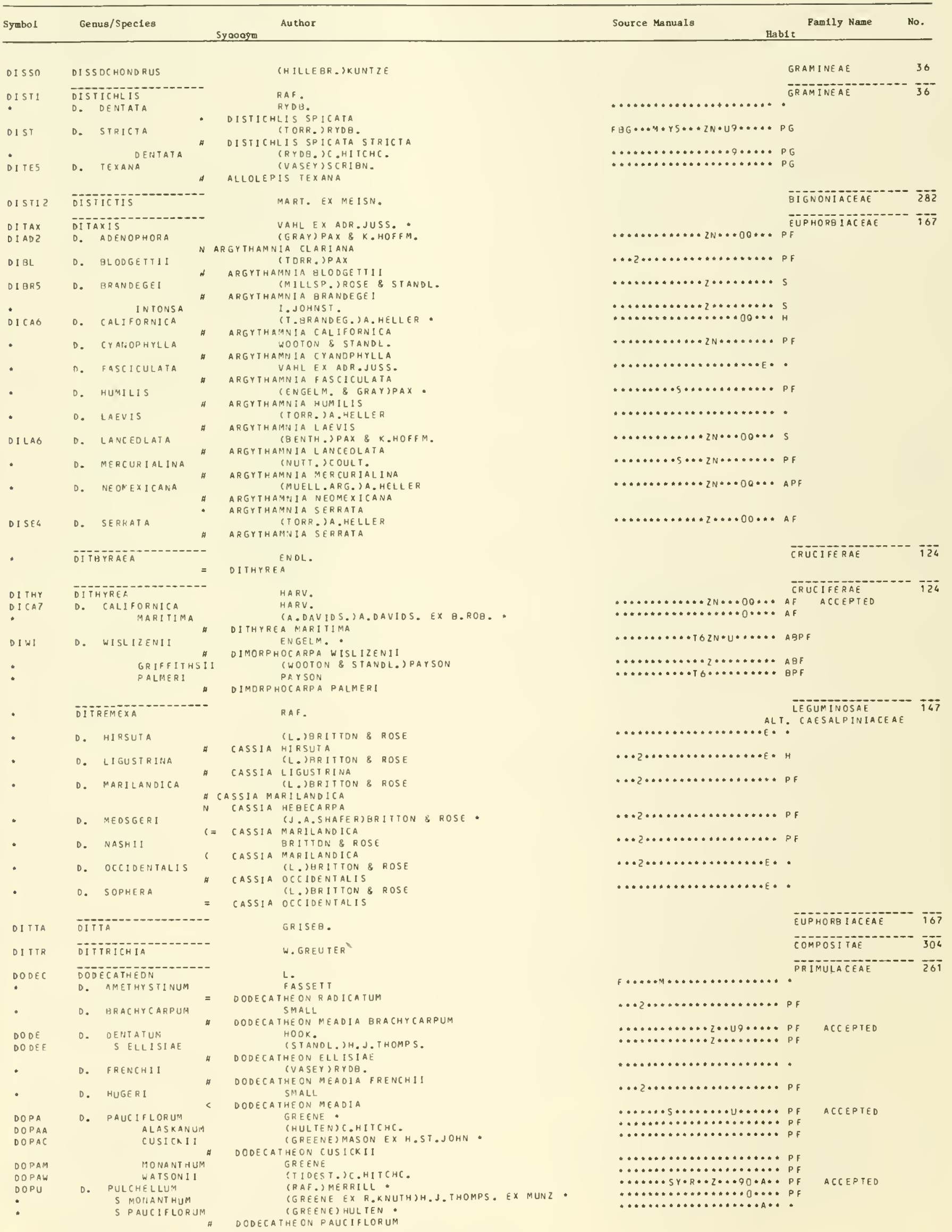




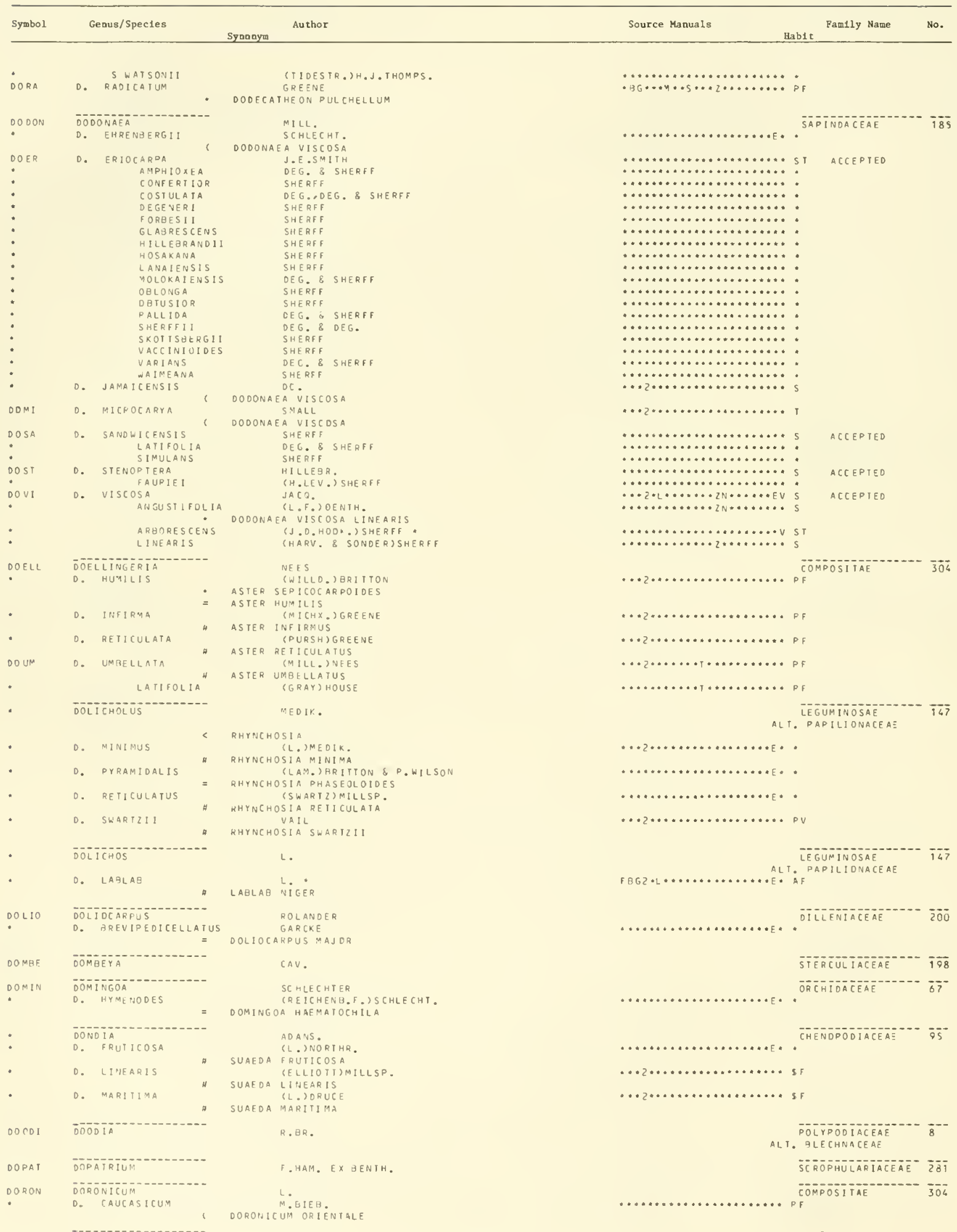




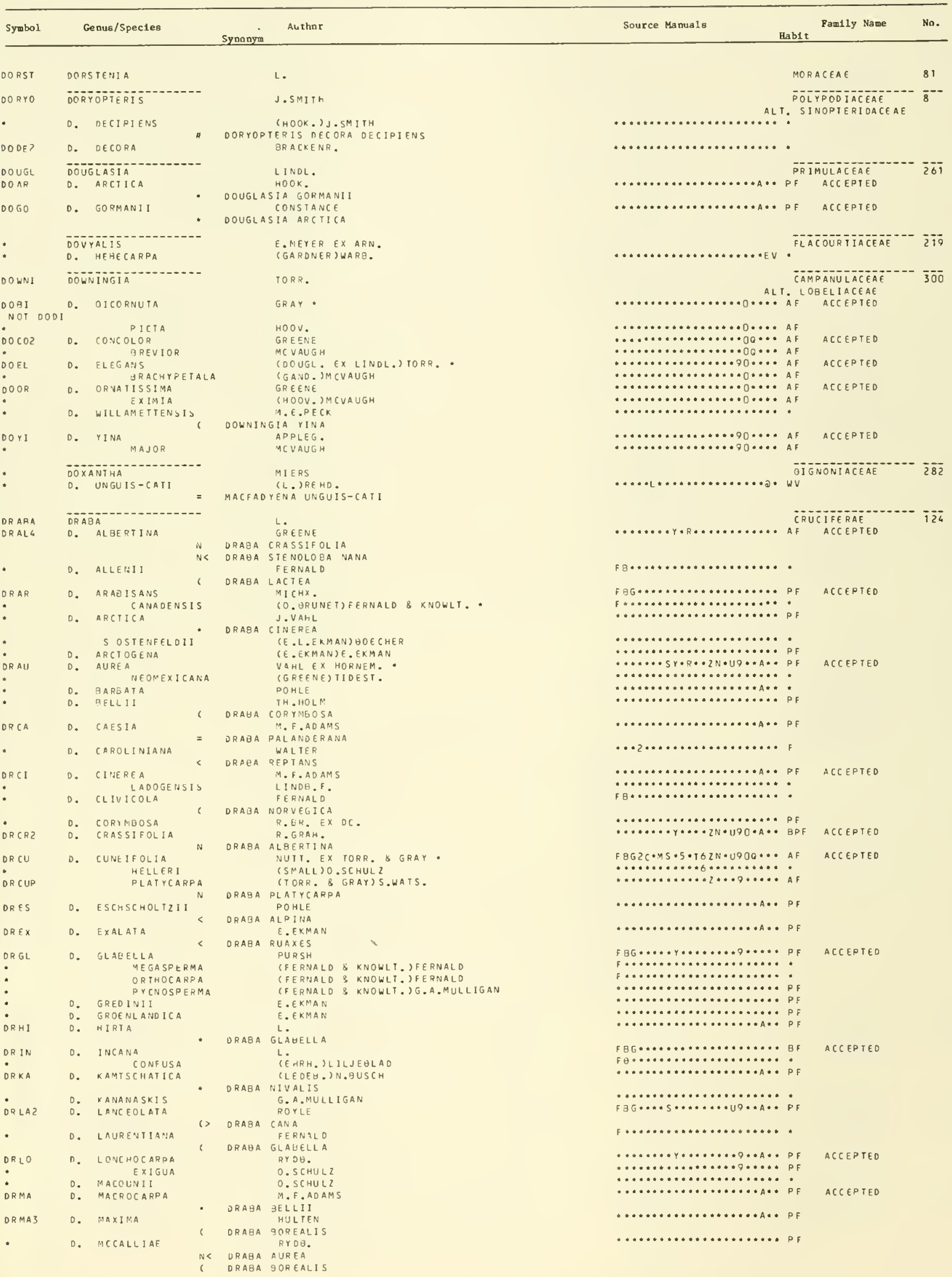




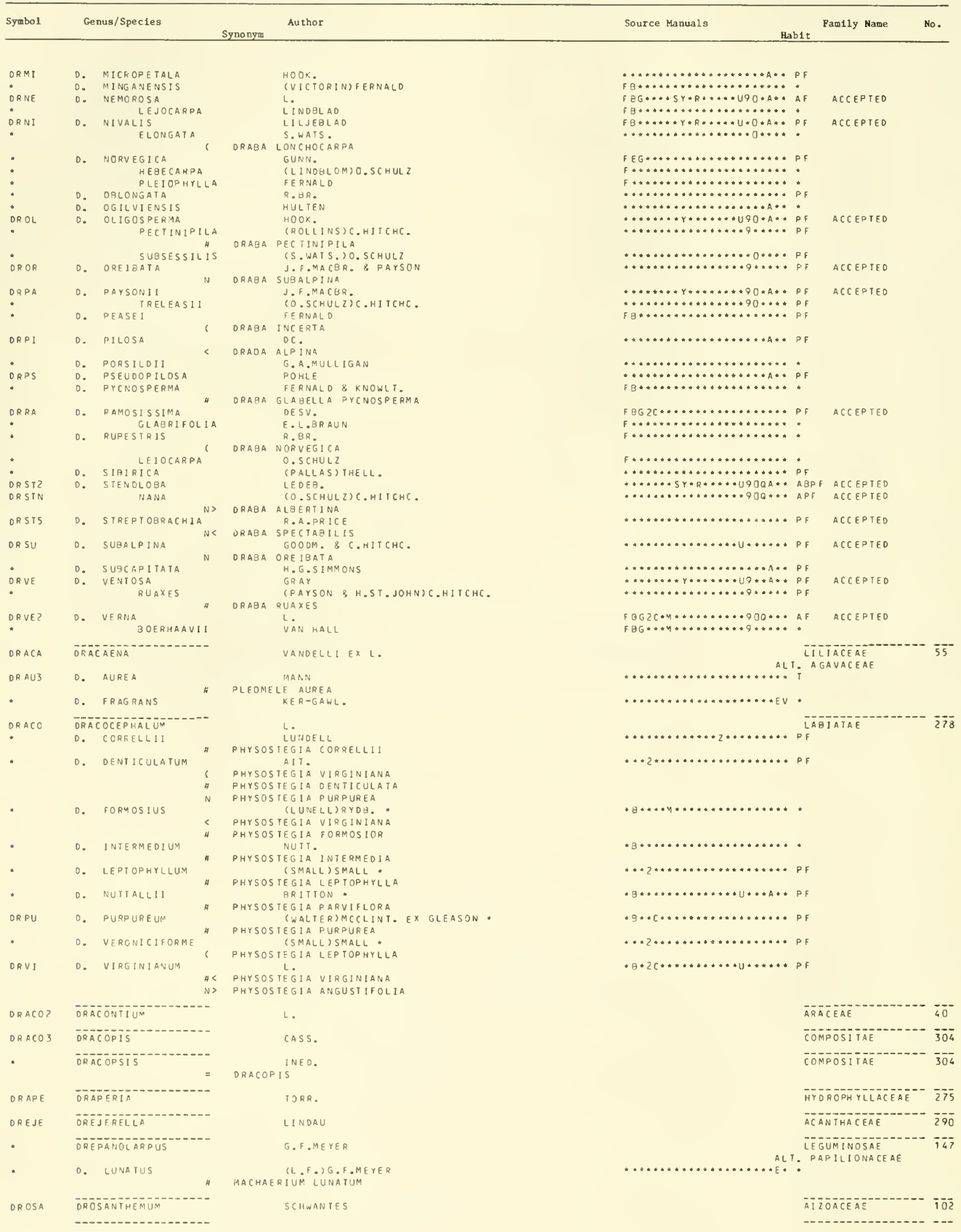




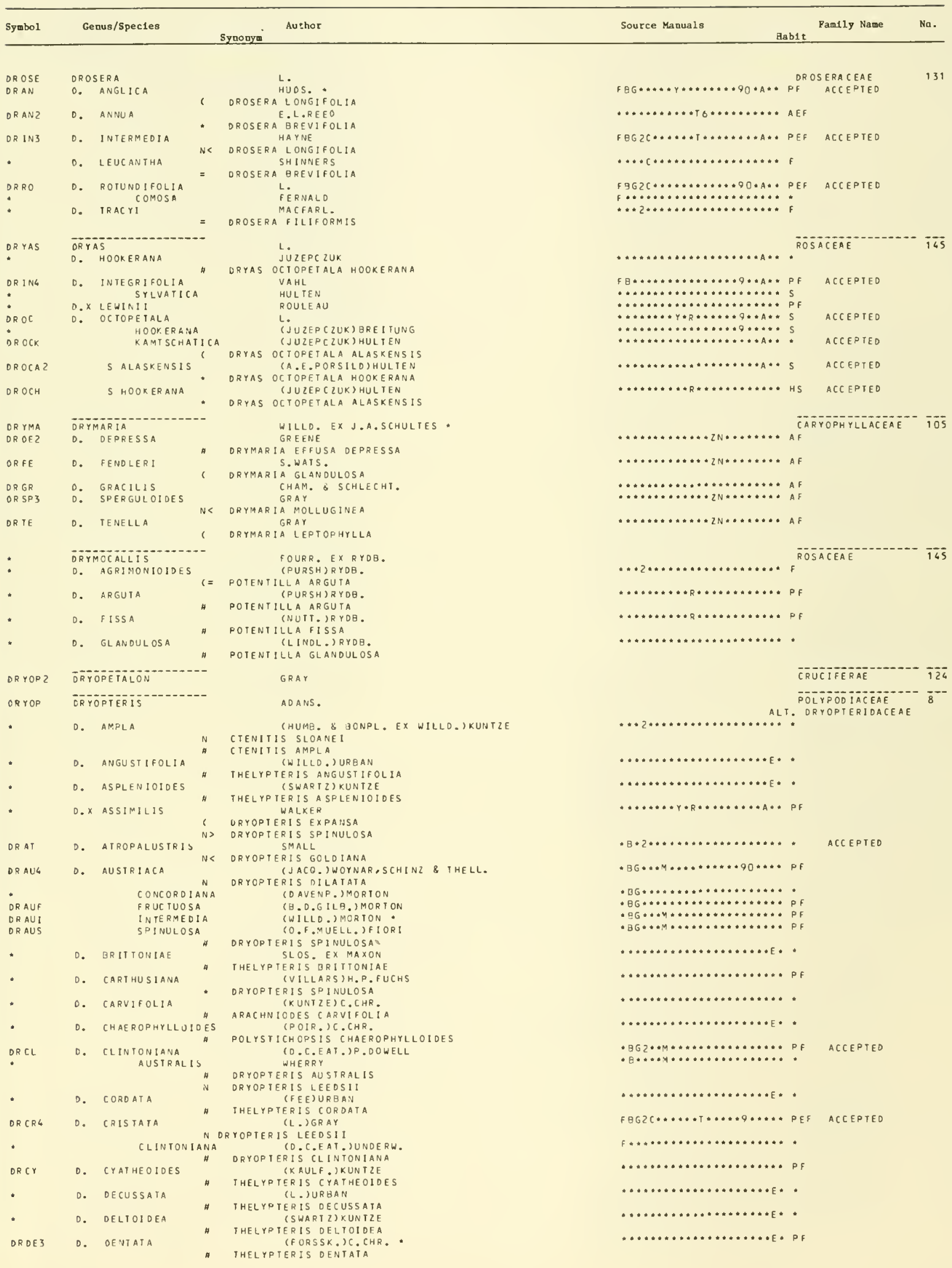




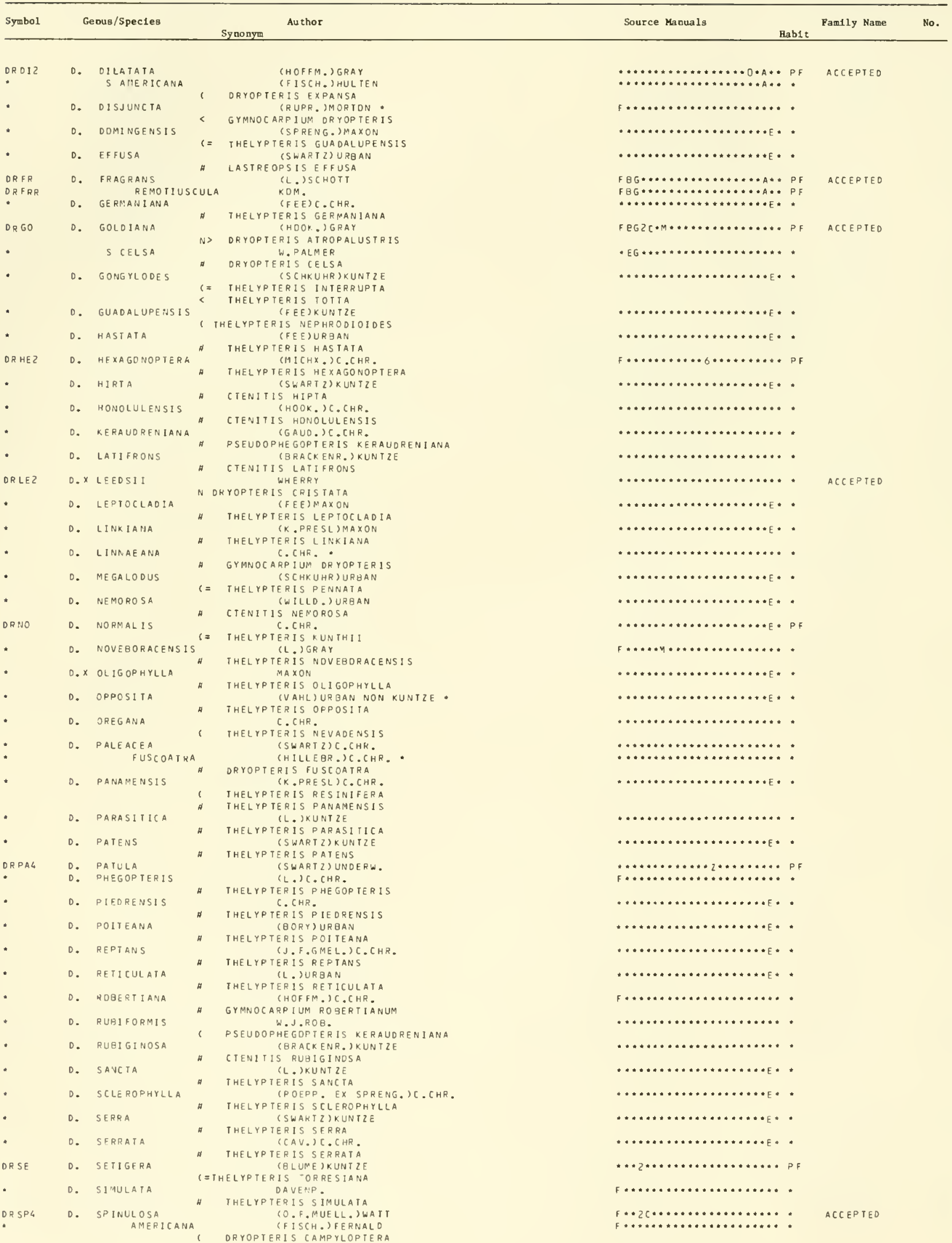




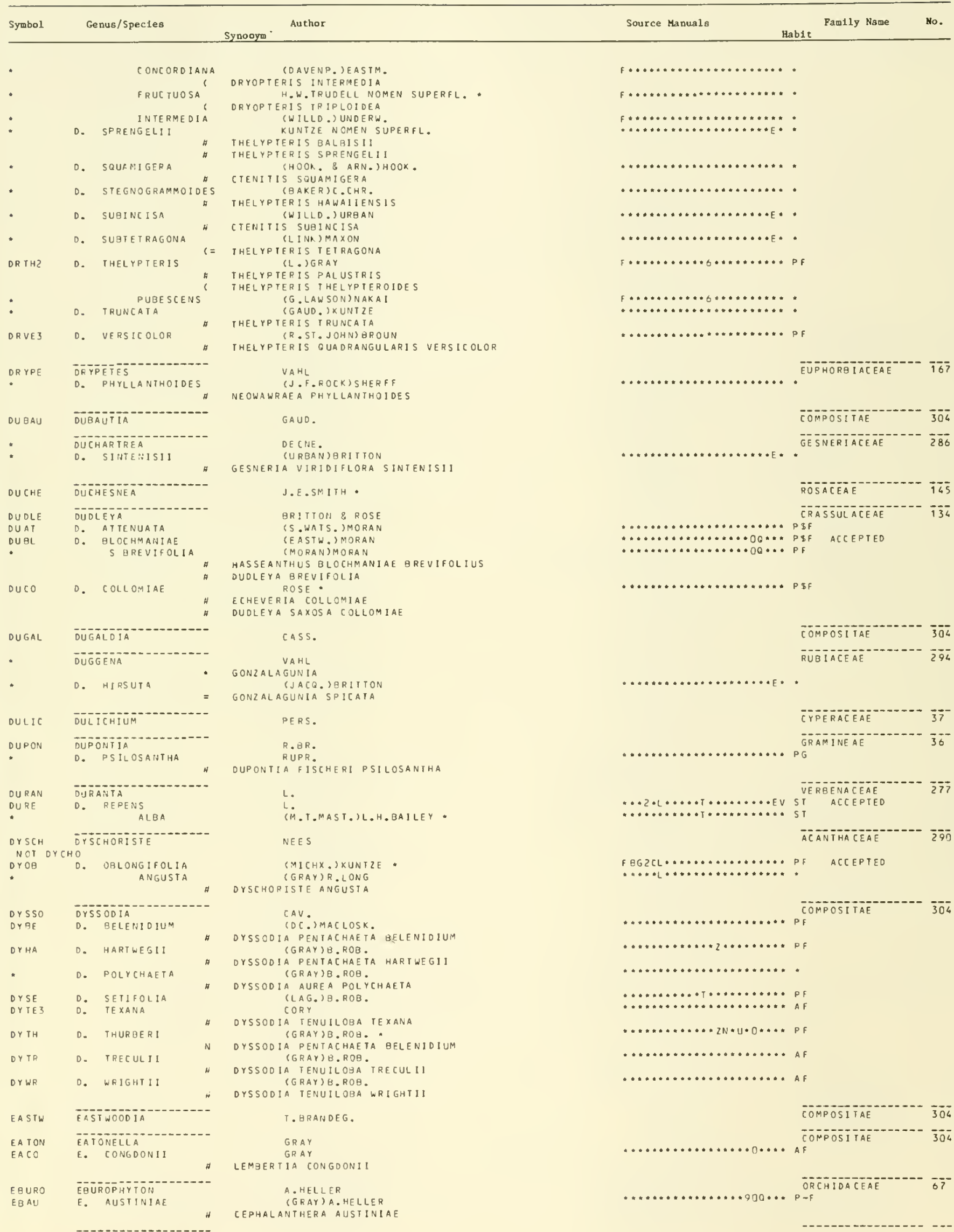




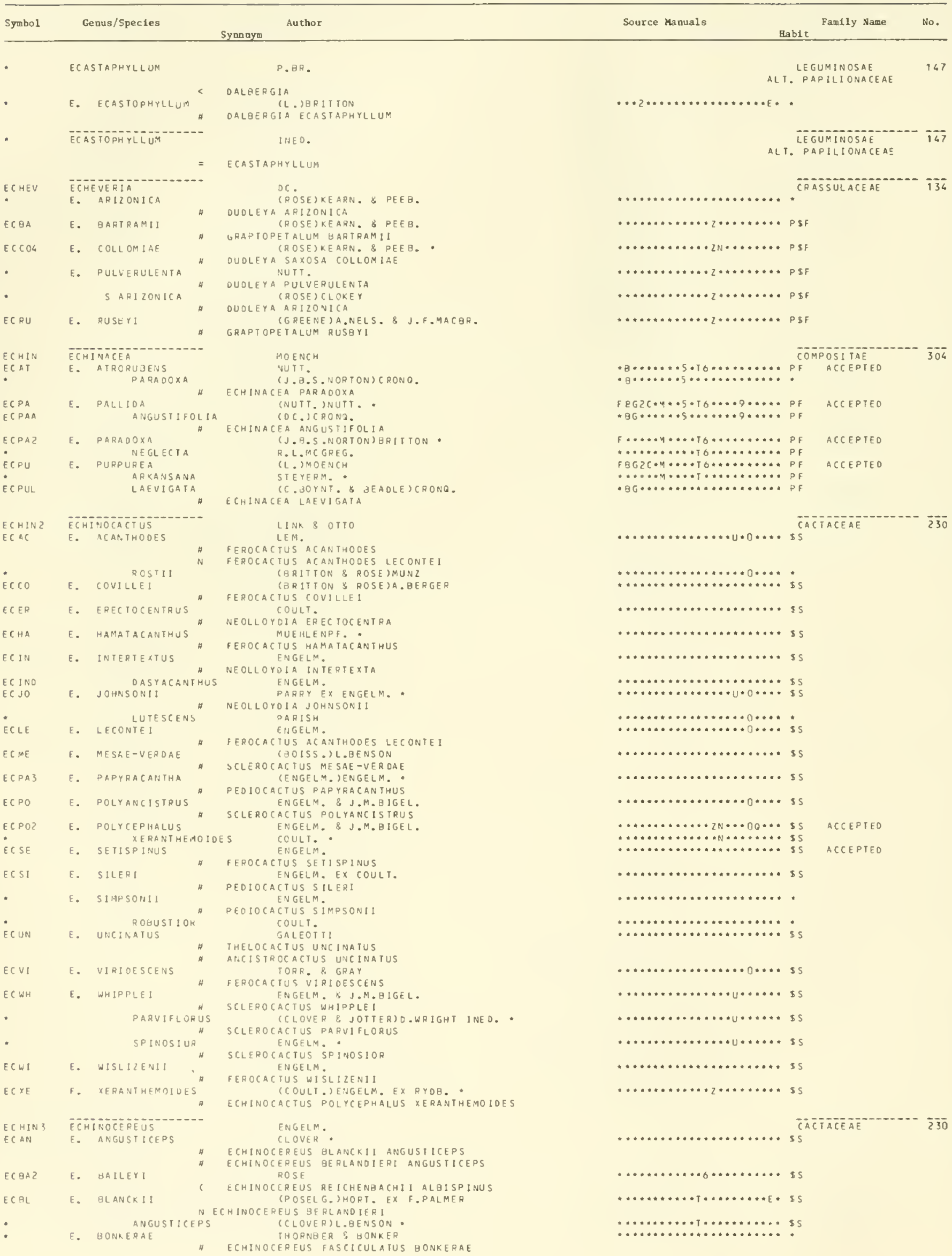




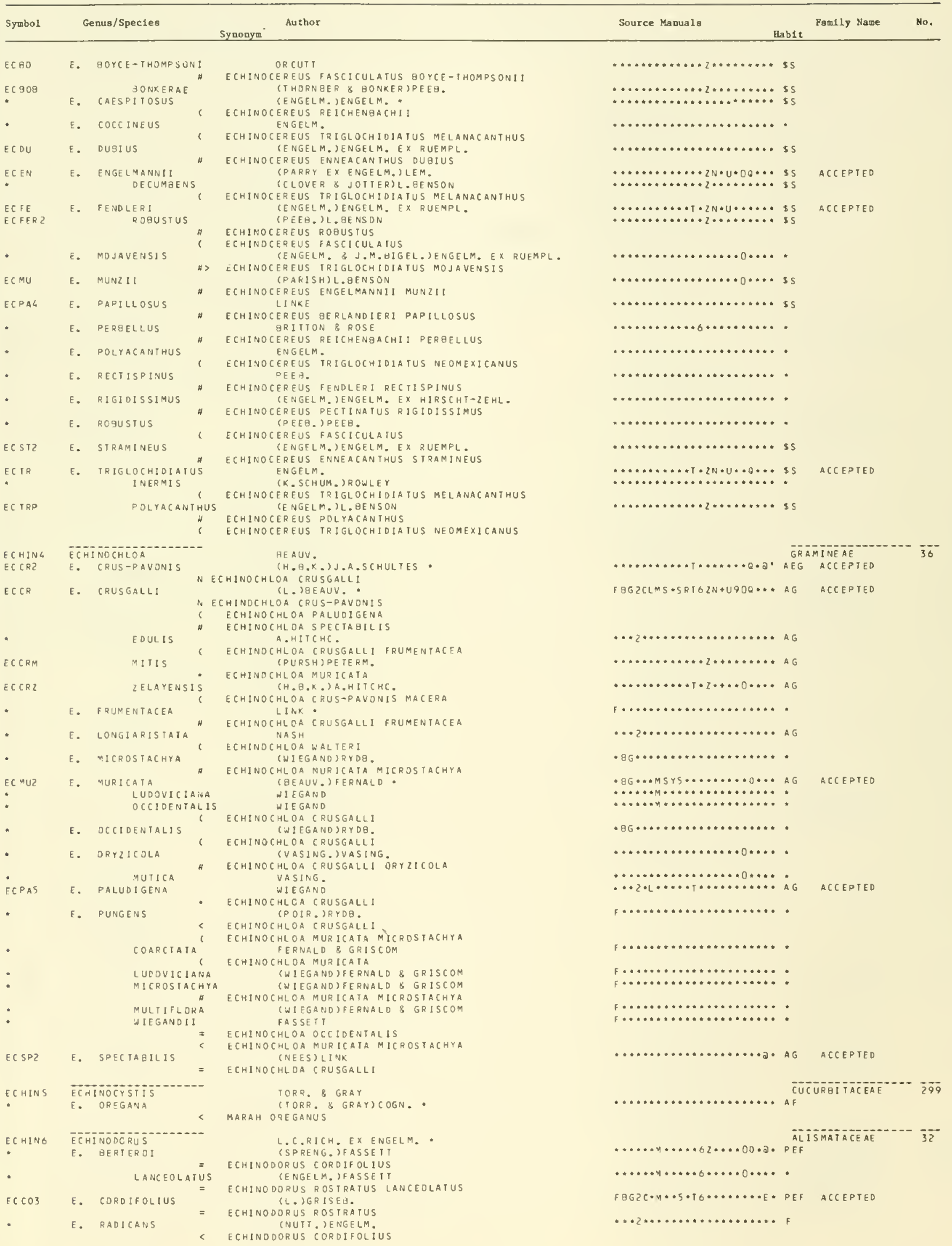


ECHINODORUS

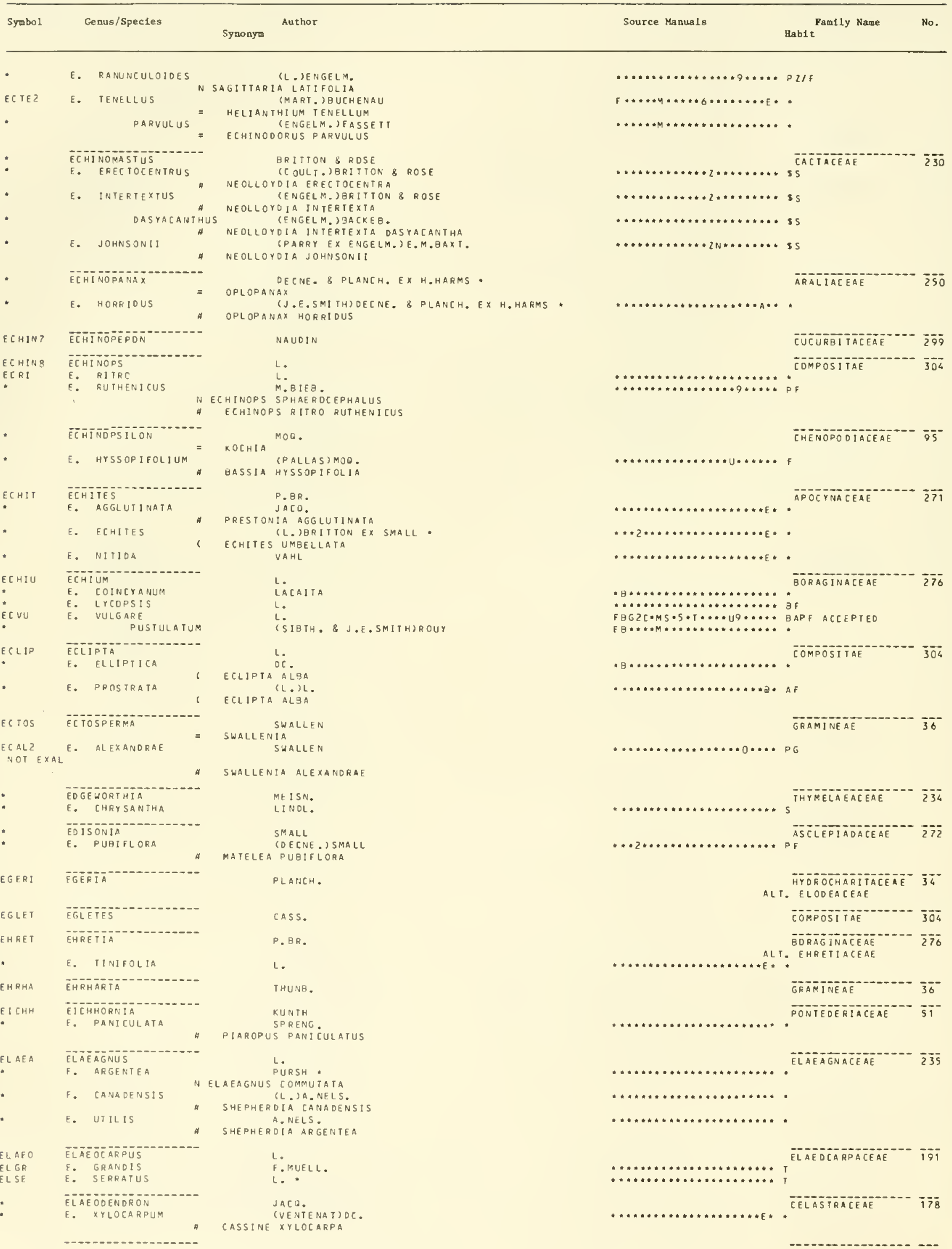




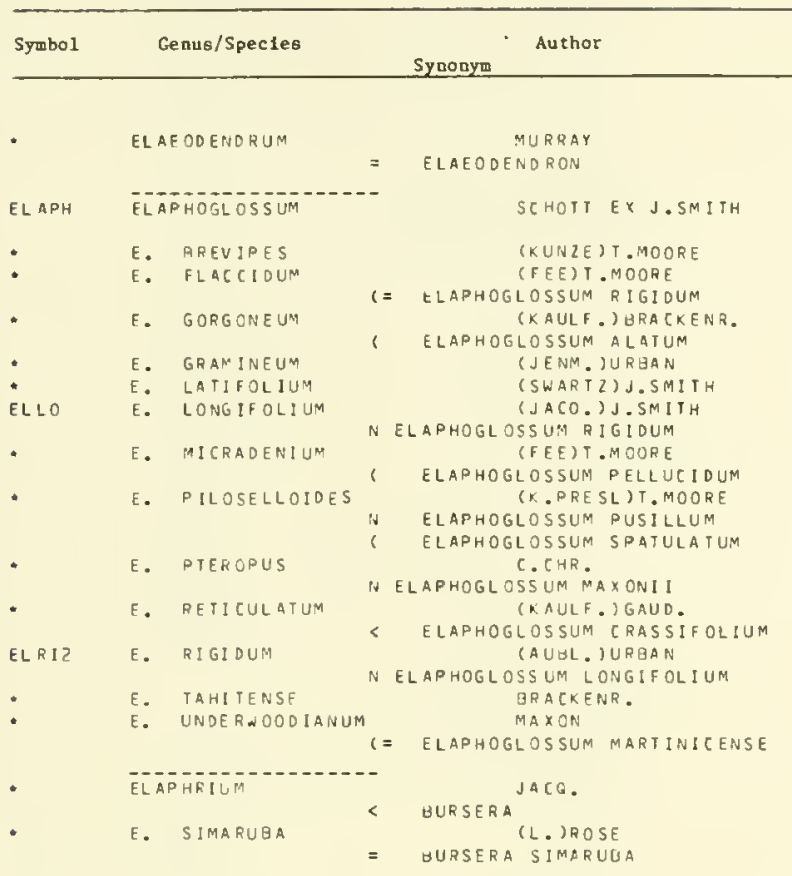

\section{ELATI}

\section{ELATINE}

E. RUIGELLA

EL IR

ELTRE$$
\begin{aligned}
& \text { E. TRIANDRA Nelatine } \\
& \text { TRIAMNORA } \\
& \text { SCHKuHR } \\
& \text { (PURSH) }
\end{aligned}
$$$$
\begin{aligned}
& \text { HYOH. } \\
& \text { N ELATINE AMERICANA } \\
& \text { N ELATINE TRIANDRA }
\end{aligned}
$$$$
\text { ameajcana a elatine apericanset }
$$$$
\begin{aligned}
& \text { 3RACHYSPERMA ELATINE APERICANA } \\
& \text { (GRAY)FASSETI }
\end{aligned}
$$$$
\text { * ELATINE GRACHYSPERMA }
$$

\section{ELEOC
ELAC}

$$
\text { . }
$$

ELEOCOHARIS

E. ACILULARIS

\section{BELLA}

GRACILESCENS PADICANS SUBMERSA

E. AC UMINATA

E. AMgigens

E. ArETHICola

ELAT

e. atropurpurea

E. SICULOR

elar e. tarittonit

EL CAB

E. calva

e. capillacea

E. capitata

elcaz e. cartiaea

- e. carolina

ELCOS

ELCOZ

:

.$$
\text { • }
$$

* eleocharis obtusa oetonsa
Source Manuals

Fan1ly Name

No. Habit

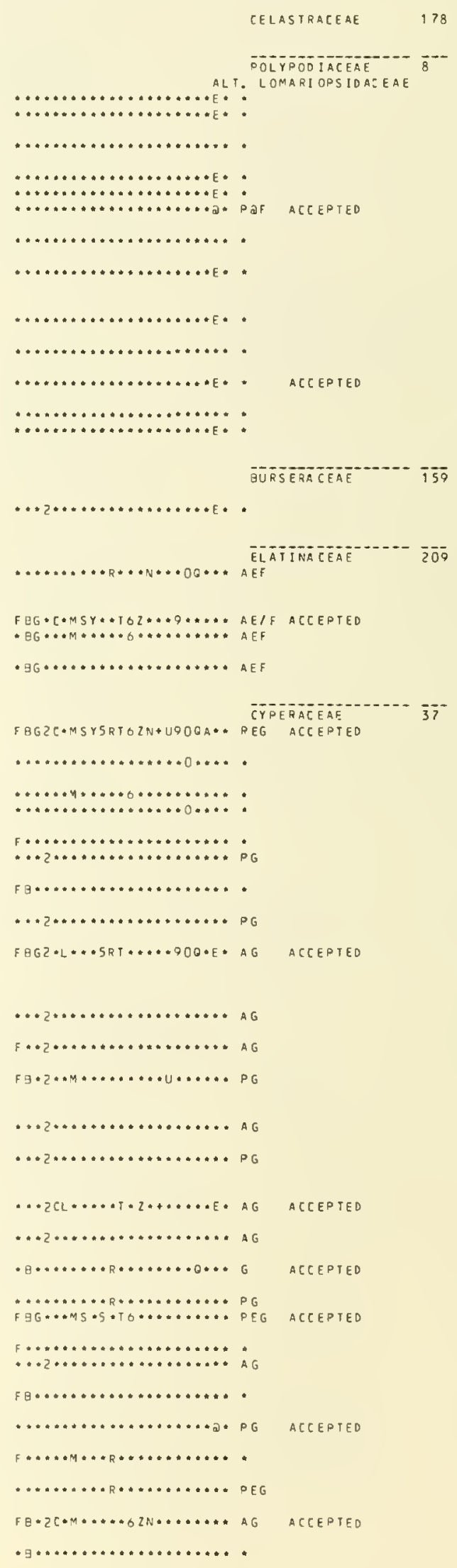




\begin{tabular}{|c|c|c|c|c|c|c|c|}
\hline Symbol & & Genus/Species & Synonym & Source Manuals & HabIt & Pamily Name & No. \\
\hline EL ER & $E$. & ERYTHROPODA & $\begin{array}{l}\text { SiEUOD. } \\
\text { - elleocharis palustris }\end{array}$ & 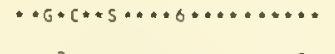 & PG & ACCEPTED & \\
\hline - & E. & FLACCIOA & $\begin{array}{l}\text { (REICHENB.) URBAN } \\
=\quad \text { ELEOCHARIS FLAVESCENS }\end{array}$ & $\cdots * 2 \cdots \cdots \cdots \cdots \cdots \cdots \cdots+\omega_{*}$ & - & & \\
\hline EL FL & $\varepsilon$. & $\begin{array}{l}\text { FLAVESCENS } \\
\text { OLIVACEA }\end{array}$ & $\begin{array}{l}\text { (PUIR, UURBAN } \\
\text { (TORR. )GLEASON }\end{array}$ & $F 8 G+C+\ldots \times 5 * T * \cdots * 90 \cdots E *$ & $P G$ & ACCEPTEO & \\
\hline EL GE & $E$. & GENICULATA & $\begin{array}{l}\text { ELEOCHARIS OLIVAGEA } \\
\text { (L. )ROEM. \& J.A.SCHULTES } \\
\text { N ELEOCHARIS ELEGANS } \\
=\text { ELHARIS ATROPURPUREA } \\
=\text { ELEOCHARIS CARIBAEA }\end{array}$ & $F B G * \ldots \ldots 5 * 06 * \cdots+00 * E$ & $A E G$ & ACCEPTED & \\
\hline * & E. & MACOUNII & $\begin{array}{c}\text { FERNALD } \\
<\quad \text { ELEOCHARIS INTERMEDIA }\end{array}$ & $F \ldots \ldots \ldots \ldots \ldots \ldots \ldots+\ldots+$ & $\cdot$ & & \\
\hline ELMA5 & E. & MACROSIACHYA & $\begin{array}{l}\text { BRITTON. } \\
* \quad \text { ELEOCHARIS PALUSTRIS } \\
\text { ELEOCHARIS XYRIOIFORMIS }\end{array}$ & $F B+2 \cdots M S \cdots R T O Z N * U * 00 \cdots$ & PEG & ACCEPIED & \\
\hline ELNIL & $E$. & $\begin{array}{l}\text { FILICULMIS } \\
\text { MINUTIFLORA }\end{array}$ & $\begin{array}{l}\text { SORR. } \\
\text { N ELEOCHARIS MICROCARPA }\end{array}$ & $\ldots \ldots \ldots \ldots \ldots \ldots \ldots \ldots+\varepsilon$ & $A G$ & ACCEPTED & \\
\hline $\begin{array}{l}\text { ELMO2 } \\
\text { EL } P O B\end{array}$ & $E$. & $\begin{array}{l}\text { MONIEVIDENSIS } \\
\text { BOLANOERI }\end{array}$ & $\begin{array}{l}\text { KUNTH } \\
\text { (GRAY) V.GRANT }\end{array}$ & $* * \cdots * * \cdots+62 \ldots \cdots 00 \cdots *$ & $P G$ & $\begin{array}{l}\text { ACCEPTED } \\
\text { ACCEPTED }\end{array}$ & \\
\hline & & & ELEOCHARIS EOLANOERI & & & & \\
\hline * & & OISCIFORIA & $\begin{array}{l}\text { IS (PARISH)V.GRANT } \\
\text { a ELEOCHARIS OISCIFORMIS }\end{array}$ & $\cdots \cdots \cdots \cdots \cdots \cdots \cdots \cdots+\cdots \cdots$ & - & & \\
\hline * & & PARISHII & $\begin{array}{l}\text { (BRITTON)V.GRANT } \\
\text { * ELEOCHARIS PARISHII }\end{array}$ & $\cdots \cdots \cdots \cdots \cdots \cdots+\cdots+\cdots \cdots+$ & $\cdot$ & & \\
\hline ELMUZ & E. & MUTA TA & $\begin{array}{l}\text { IL. MROEM. \& J.A.SCHULTES } \\
\text { * ELEOCHARIS GUAORANGULATA }\end{array}$ & $\cdots \ldots \ldots \ldots \ldots \ldots \ldots \ldots \cdots$, & PEG & ACCEPIED & \\
\hline ELN12 & E. & NIGRESCENS & $\begin{array}{l}\text { (NEES) STEUD. } \\
\text { - ELEOCHARIS ATROPURPUREA } \\
\text { FERNAL D }\end{array}$ & 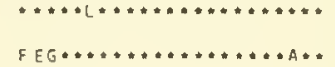 & AG & $\begin{array}{l}\text { ACCEPTEO } \\
\text { ACCEPTED }\end{array}$ & \\
\hline ELNI & E. & NITIDA & $\begin{array}{l}\text { ELEOGHARIS TENUIS BOREALIS } \\
\text { (ROTH) J.A.SCHULTES }\end{array}$ & $* * 2 \cdots * \cdots \cdots \cdots \cdots \cdots+\cdots$ & & ALCETIEO & \\
\hline - & E. & NODULOSA & $\begin{array}{l}\text { (ROTH) J.A.SCHULTES } \\
\text { " ELEOCHARIS MONTANA NODULOSA } \\
\text { (WILLD.) J.A.SCHULTES. }\end{array}$ & & $A P E G$ & & \\
\hline EL Ca2 & E. & OE TUSA & ALIS FERHALD J.A.SCHULTES - & 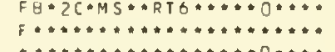 & APEG & ACCEPTED & \\
\hline EL O8E & & ENGELMANN & $\begin{array}{l}\text { (S TEUO.) GILLY } \\
\text { \# ELEOCHAR!S ENGELMANNII }\end{array}$ & $* \cdots \cdots+\cdots \cdots+\cdots+\cdots+* 0 *+\cdots$ & $A G$ & $A C C E P T E D$ & \\
\hline : & & $\begin{array}{l}\text { JEJUNA } \\
\text { LANCEOLAT }\end{array}$ & $\begin{array}{l}\text { FERNALO } \\
\text { IFERNALO)GILLY } \\
\text { ELEOCHARIS LANCEOLATA }\end{array}$ & 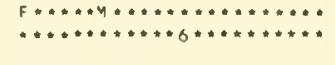 & : & & \\
\hline • & & OVATA & $\begin{array}{l}\text { " ELEOCHARIS LANCEOLATA } \\
\text { (ROTH) DRAPAL. \& MOHLENB. EX VAN QRUG. INED. * } \\
\text { "ELEOCHARIS OVATA }\end{array}$ & $\ldots \ldots \ldots+\ldots \ldots \ldots \ldots \ldots \ldots$ & A G & & \\
\hline 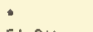 & & PEASEI & SVENS. & $F \ldots \ldots \ldots \ldots \ldots \ldots \ldots \ldots$ & $\cdot$ & & \\
\hline ELOV & E. & OVAIA & $\begin{array}{l}\text { (ROTH)ROEM. \& J.A.SCHULTES * } \\
>\quad \text { LLEOCHARIS ENGELMANNI] }\end{array}$ & $F B G \ldots+M * Y S \ldots \ldots+\cdots, \ldots \ldots$ & AEG & ACCEPTED & \\
\hline ELPAZ & E. & $\begin{array}{l}\text { HEUSER ! } \\
\text { PALMER I }\end{array}$ & $\begin{array}{c}\text { UeChTR. } \\
\text { SVENS. } \\
\text { * Eleochar!S MONTEVIDENSIS }\end{array}$ & $\ldots \ldots \ldots \ldots \ldots+\cdots+\cdots+\ldots$ & $\dot{P G}$ & & \\
\hline ELPA3 & E. & PALUSTRIS & $\begin{array}{ll}\text { ( } & \text { ELEOCHARIS ROEM. \& J.A.SCHULTES } \\
\text { ) ELEOCHARIS UNIGLUMIS } \\
\text { 3 ELEOCHARIS SMALLII }\end{array}$ & $F B G * C * M+Y 5 \cdots+\cdots+90 * A * *$ & PEG & ACCEPTED & \\
\hline - & & $M A J O R$ & SONDER & $F * \ldots+\ldots+\ldots \ldots \ldots \ldots \ldots \ldots$ & $\cdot$ & & \\
\hline ELPAS & E. & $\begin{array}{l}\text { PARVULA } \\
\text { COLORADOE: }\end{array}$ & $\begin{array}{l}\text { (ROEM. \& J.A.SCHULTES)LINK EX BLUFF \&FINGERH. } \\
\text { (JRITTON)A.A.BEETLE }\end{array}$ & $\because F B G+C * M S * S+T 6 Z * * 90 \cdots \cdots$ & PAEG & ACCEPTED & \\
\hline ELPAO & E. & $\begin{array}{l}\text { PAUCIFLORA } \\
\text { OERNAR DIN }\end{array}$ & $\begin{array}{l}\text { (LIGHTF.)LINK } \\
\text { IAUNZ \& I.JOHNST.JSVENS. } \\
\text { * ELEOCHARIS OERNAROINA }\end{array}$ & $F B G \cdots \cdots+\cdots \cdots+2 n+490 \cdots \cdots$ & $P G$ & ACCEPTEO & \\
\hline - & & FERN AL DII & SVENS. & $F \theta \ldots \ldots \ldots \ldots \ldots \ldots$ & $\cdot$ & & \\
\hline - & E. & PRATICOLA & $\begin{array}{l}\text { BritTon } \\
\text { - eleocharis FLAVESCENS }\end{array}$ & $\cdots+2+\ldots \ldots \ldots \ldots \ldots \ldots \ldots$ & $A G$ & & \\
\hline - & E. & PROL IFERA & $\begin{array}{l}\text { TORR. } \\
\text { - ELEOCHARIS VIVIPARA } \\
<\quad \text { ELEOCHARIS GALDWINII }\end{array}$ & $\cdots+2 \cdots \cdots \cdots \cdots \cdots+\cdots+\cdots+*$ & $A G$ & & \\
\hline ELQU & E. & DUAD RANGULA IA & $\begin{array}{l}\text { (MICHX.) ROEM. \& J.A.SCHULTES } \\
\text { - ELEOCHARIS MUTATA }\end{array}$ & $F \operatorname{eg} 2 C * M \cdots \cdots+16 \cdots \cdots 0 \cdots \cdots$ & PEG & ACCEPTED & \\
\hline * & E. & DUINGUEFLORA & $\begin{array}{l}\text { (F. HARTMAHN)O.SCHWARZ } \\
=\quad \text { ELEOCHARIS PAUCIFLORA }\end{array}$ & $\cdots \cdots \cdots \cdots R \cdots \cdots O O A A$ & $P G$ & & \\
\hline • & & 5 FERNALDII & (SVENS.) HULTEN & $\cdots \ldots \ldots \ldots \ldots \ldots \ldots \ldots \ldots A+$ & : & & \\
\hline - & F. & $S I$ I $A$ LEX & $\begin{array}{l}\text { (ELLIOTI)A.OIETR. } \\
=\quad \text { ELEOCHARIS TORTILIS }\end{array}$ & $* * 2 * \cdots \cdots \cdots \cdots+\cdots \cdots \cdots \cdots$ & $A G$ & & \\
\hline ELSI & E. & SINTENISI I & $\begin{array}{l}\text { AOECK } \\
\text { NELEOCHARIS ATROPURPUREA }\end{array}$ & $\cdots \cdots \cdots \cdots+\cdots+\cdots \cdots \cdots \cdots+\cdots$ & $A G$ & ACCEPTED & \\
\hline EL SM & E. & SMALLII & $\begin{array}{c}\text { BRITION } \\
<\quad \text { ELEOCHARIS PALUSTRIS }\end{array}$ & $F g \ldots \ldots$ ns $\ldots \ldots \ldots \ldots \ldots \ldots$ & $P G$ & ACCEPTED & \\
\hline ELTE & E. & TENU IS & $\begin{array}{l}\text { (WILLO.) J.A.SEHULTES } \\
\text { - ELEOCHARIS COMPRESSA }\end{array}$ & $F B G * C * M \cdots 5 * 16 \cdots \cdots+9 \cdots \cdots$ & $P G$ & ACCEPTED & \\
\hline EL TEB & & GOREAL IS & $\begin{array}{l}\text { (SVENS.) GLEASON } \\
\text { ELEOCHARIS NITIOA }\end{array}$ & $\Delta g G * * * * * * * * \cdots * * 9 * \ldots \ldots$ & $\cdot$ & ACCEPTED & \\
\hline • & E. & TORREYANA & $\begin{array}{c}\text { OOECK } \\
<\quad \text { ELEOCHARIS MICROCARPA }\end{array}$ & $* * * 2 * \cdots+\ldots+\ldots \ldots \ldots \ldots \ldots \ldots$ & $A G$ & & \\
\hline ELTU & E. & $\begin{array}{l}\text { TUBERCULOSA } \\
\text { PUANICOEN }\end{array}$ & $\begin{array}{l}\text { IMICHX . JROEM. \& J.A.SCHULTES } \\
\text { FERNALO }\end{array}$ & 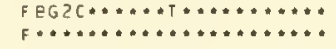 & $P G$ & ACCEPTED & \\
\hline - & E. & UNCI AL IS & $\begin{array}{l}\text { CHAPM. } \\
\text { - EleOCHARIS MINIMA }\end{array}$ & $\cdots+2 * \ldots \ldots+\cdots \cdots+\cdots+\cdots$ & $A G$ & & \\
\hline ELUN & E. & UNIGLUMIS & $\begin{array}{l}\text { (LINK) JAA.SCHULIES } \\
<\quad \text { ELEOCHARIS PALUSTRIS }\end{array}$ & $F \cdots \cdots+\cdots \cdots+\cdots \cdots \cdots+\cdots \cdots A \cdots$ & $P G$ & ACCEPTED & \\
\hline ELXY & E. & XYRIOIFORMIS & $\begin{array}{l}\text { FERNALD \& A BRACKETT * } \\
\text { - ELEOCHARIS MACROSTACHYA }\end{array}$ & 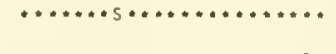 & $P G$ & ACCEPTED & \\
\hline 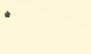 & E. & YUNG UENSIS & $\begin{array}{c}\text { QR!TTON } \\
=\quad \text { ELEOCHARIS SINIEHISII }\end{array}$ & $* \ldots+\ldots \ldots \ldots \ldots+\cdots \cdots \cdots E$ & - & & \\
\hline
\end{tabular}




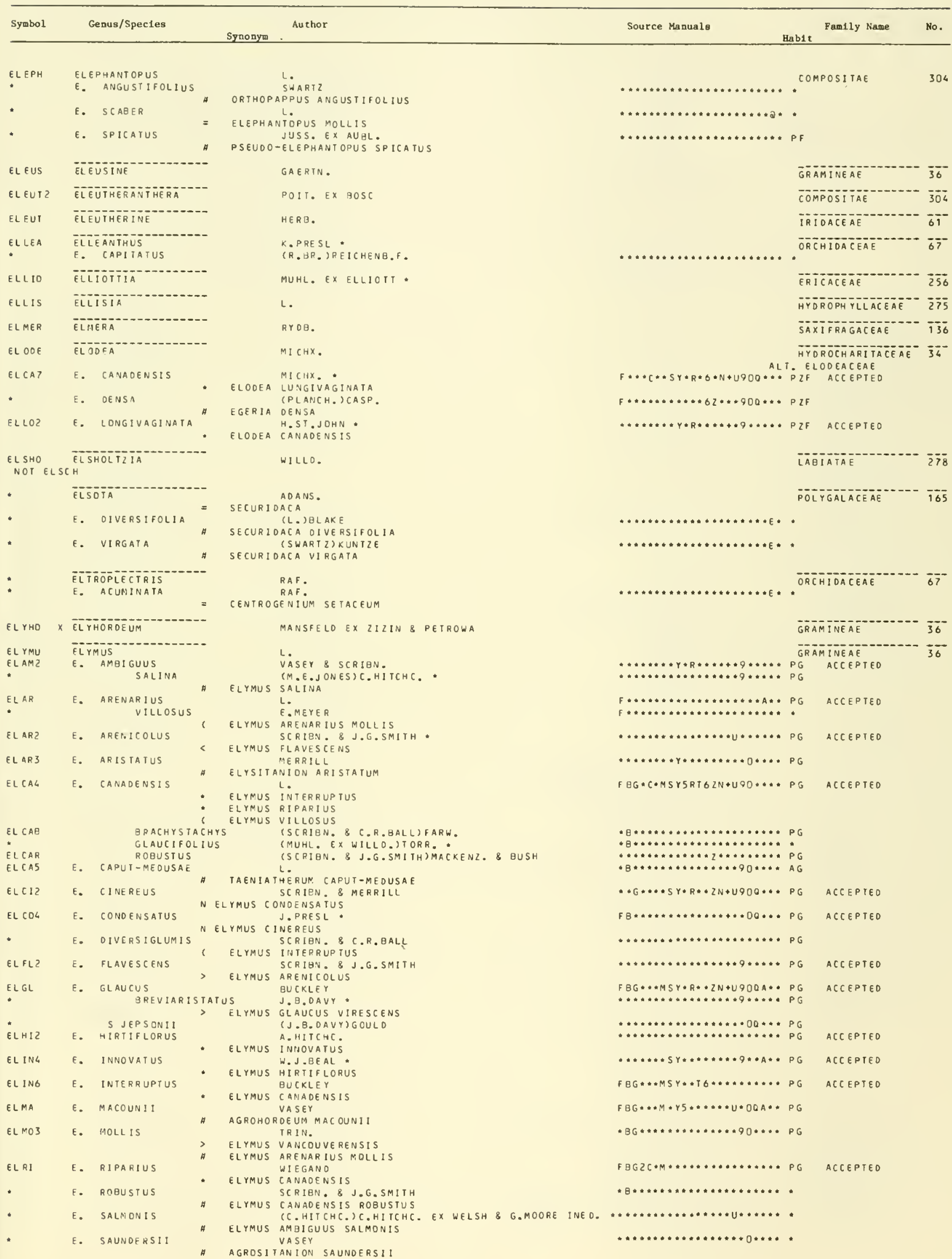




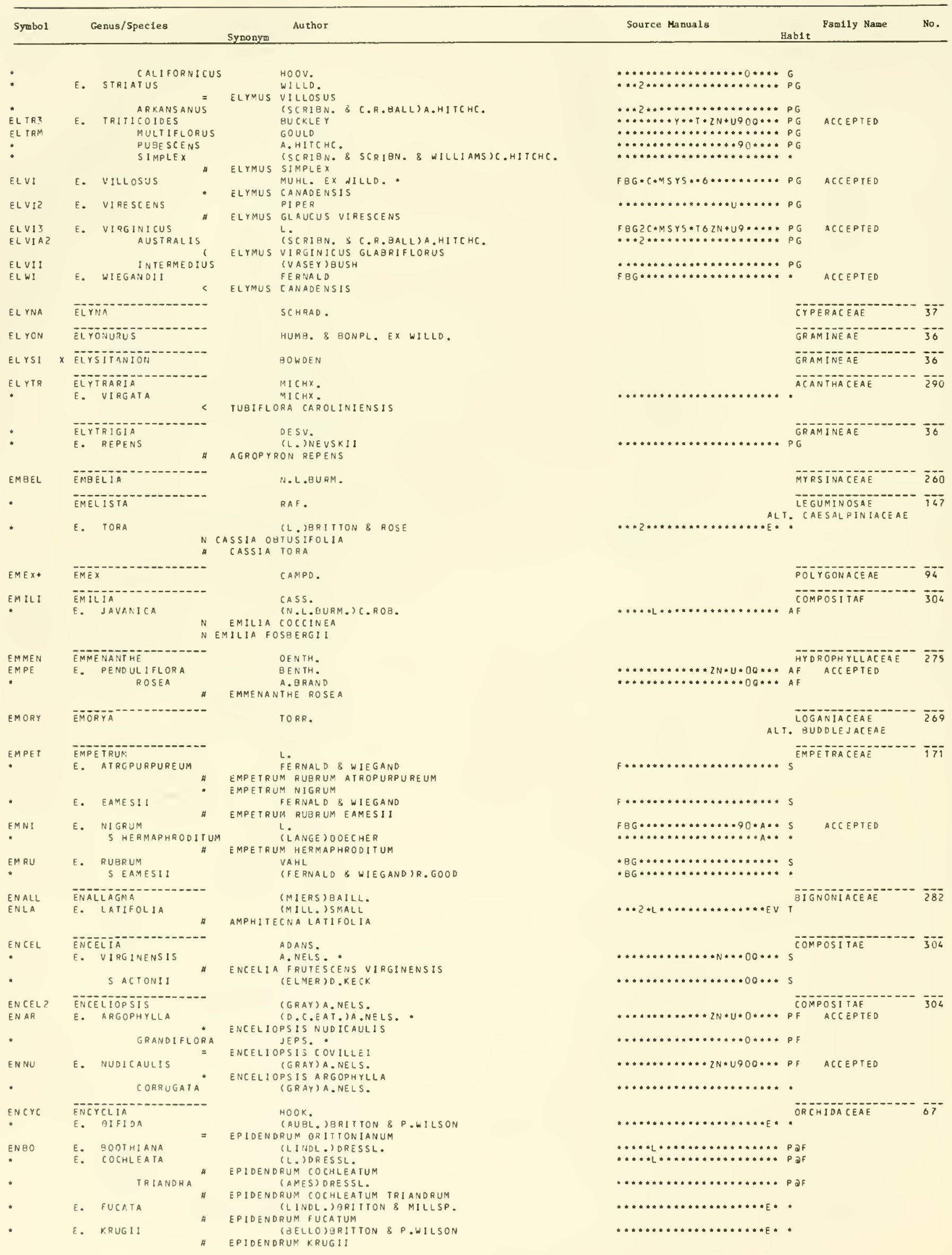


ENCYCLIA

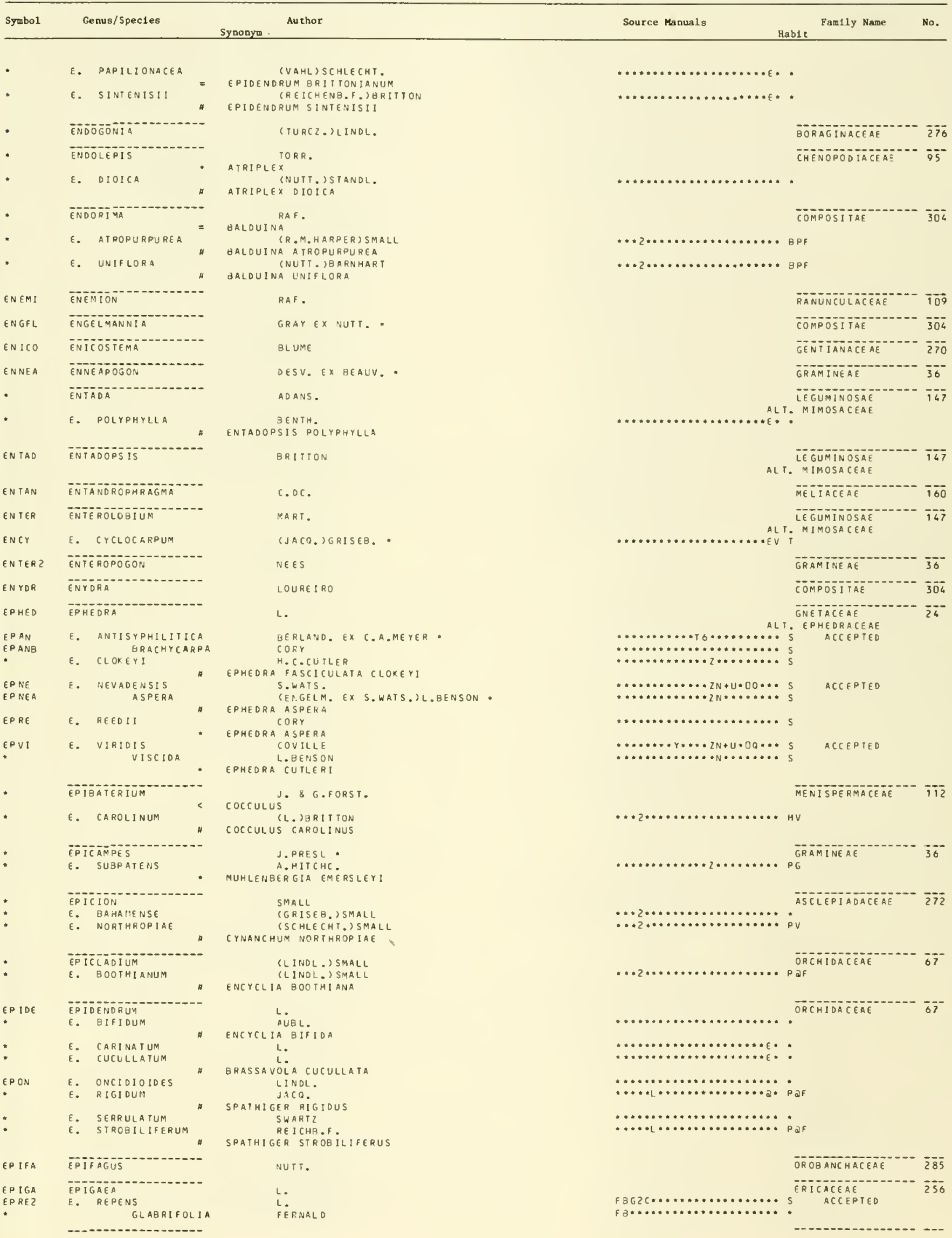




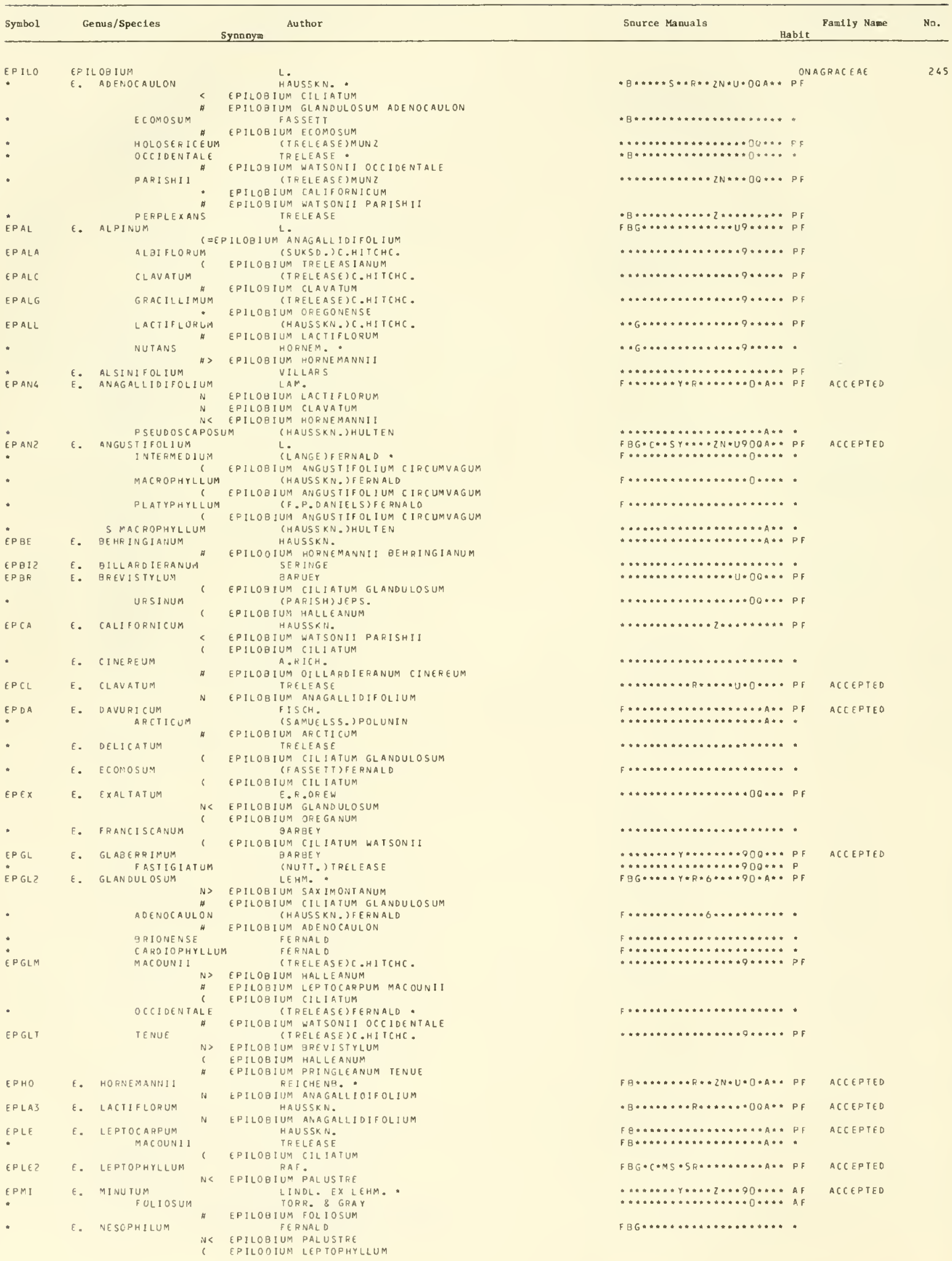




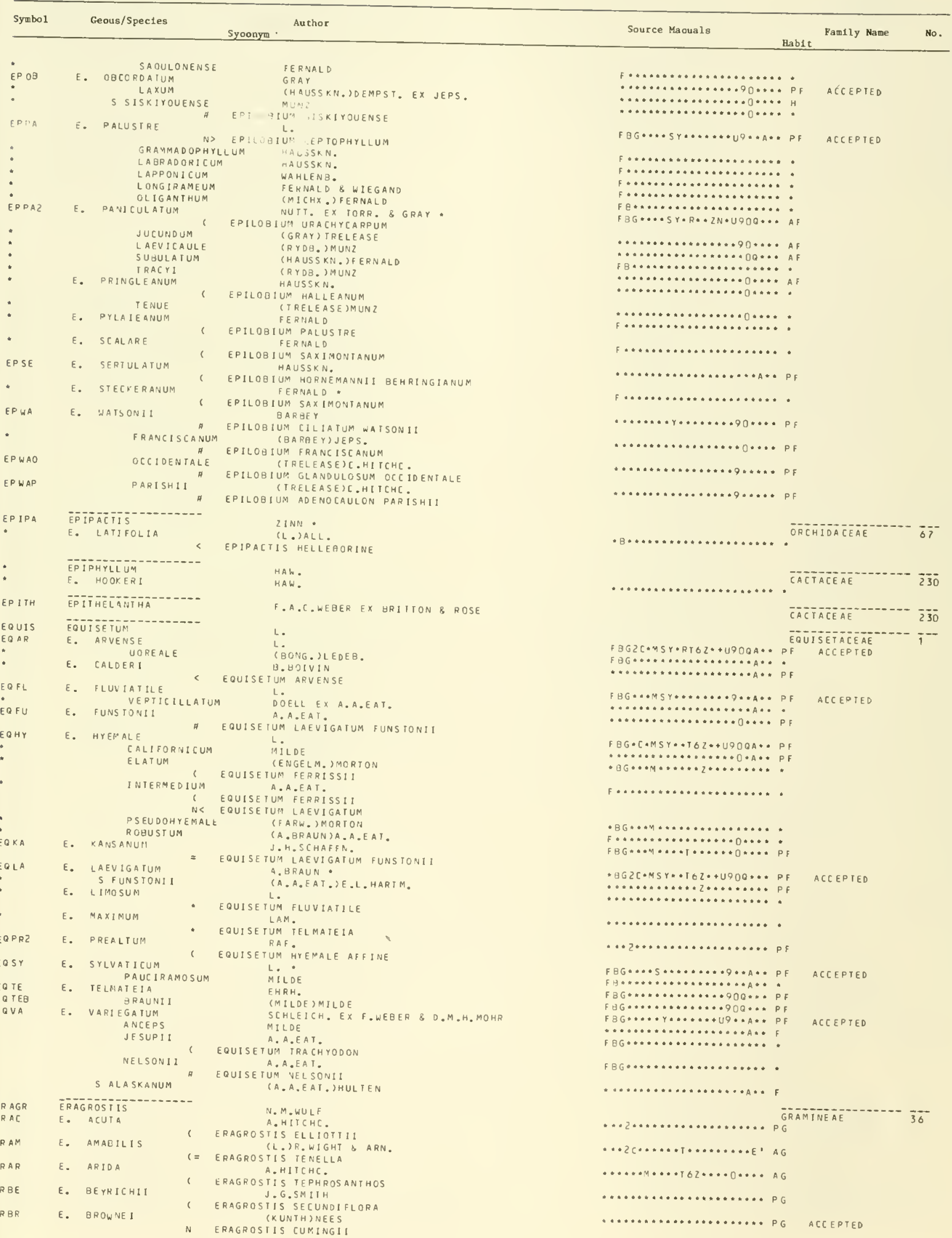




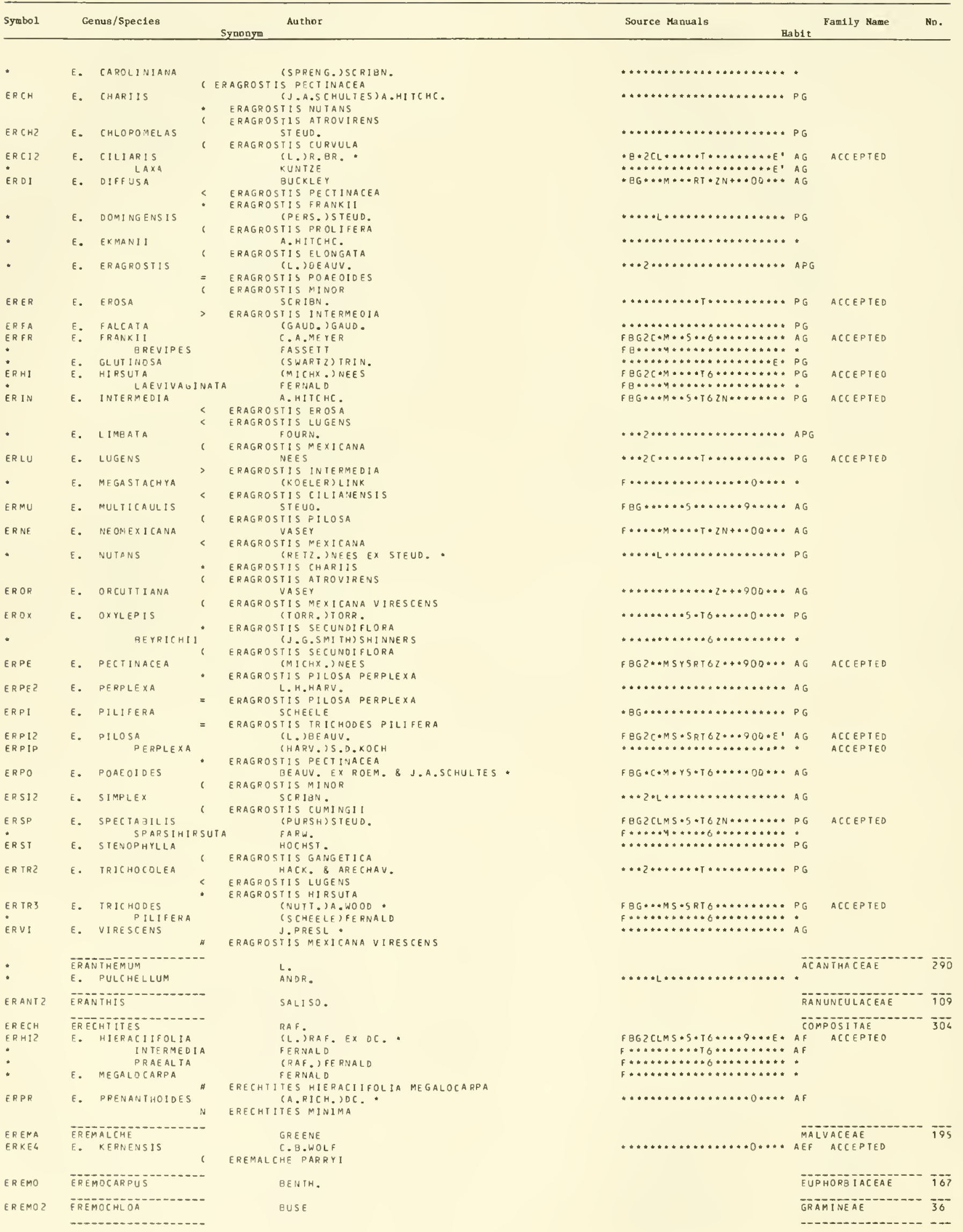




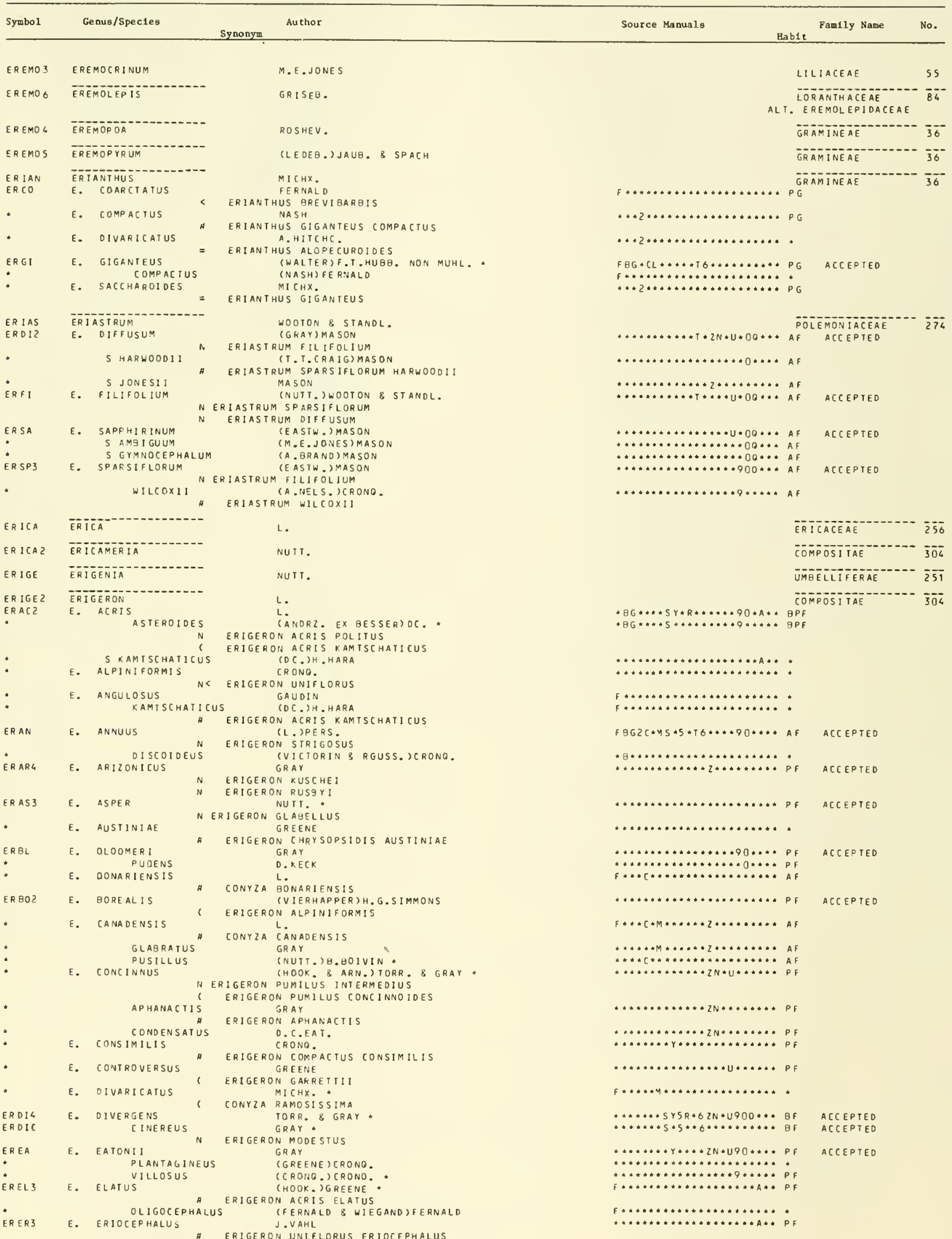




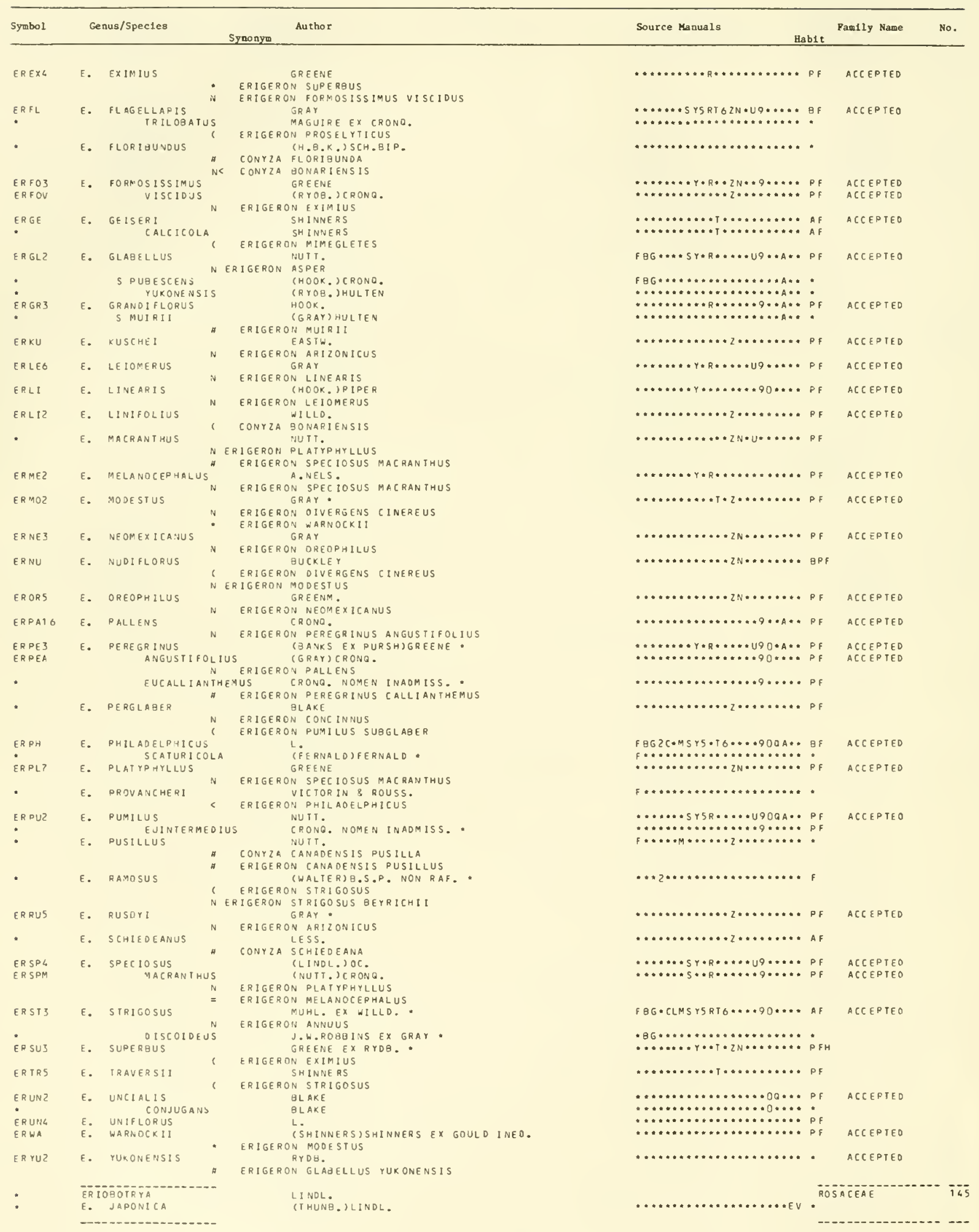




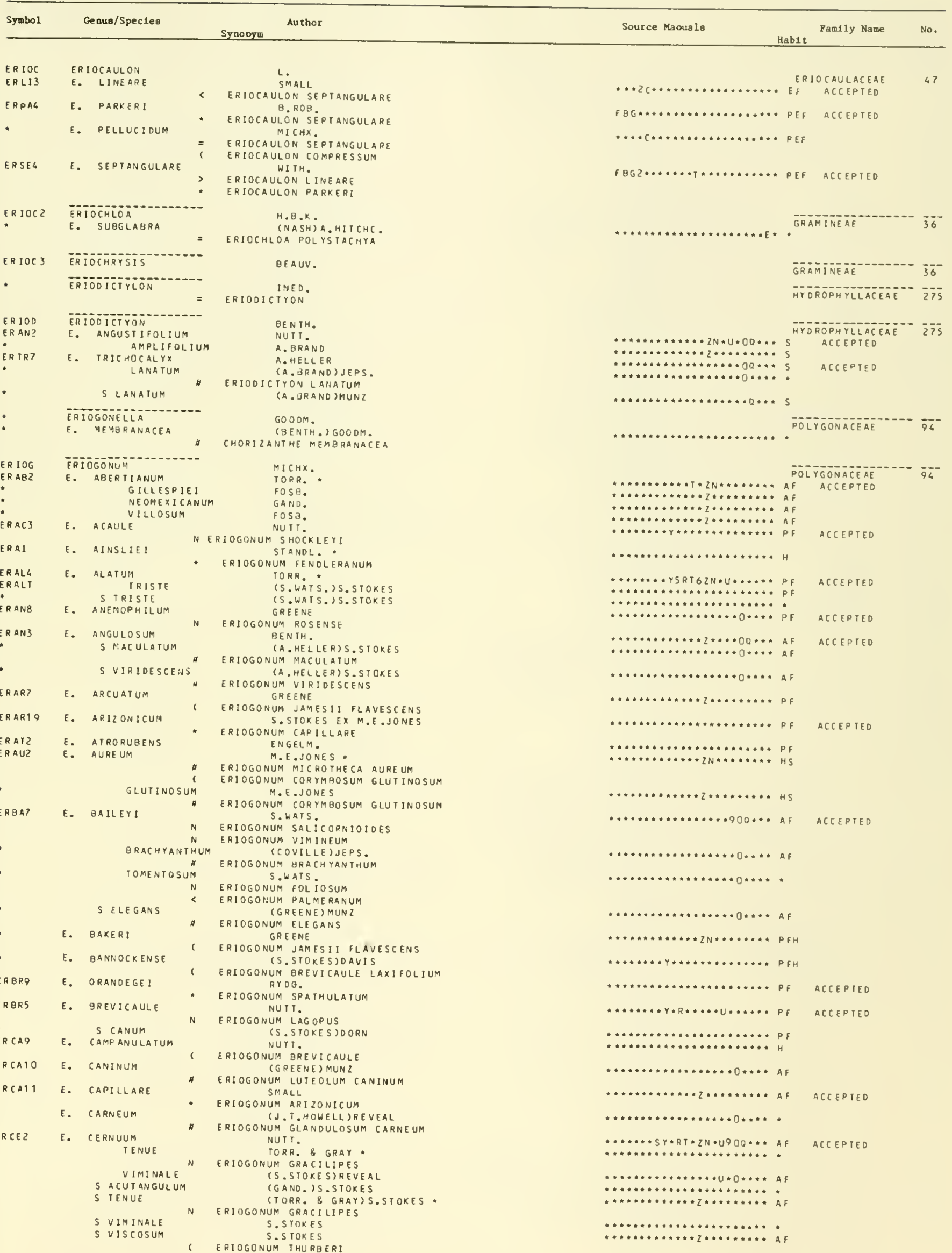




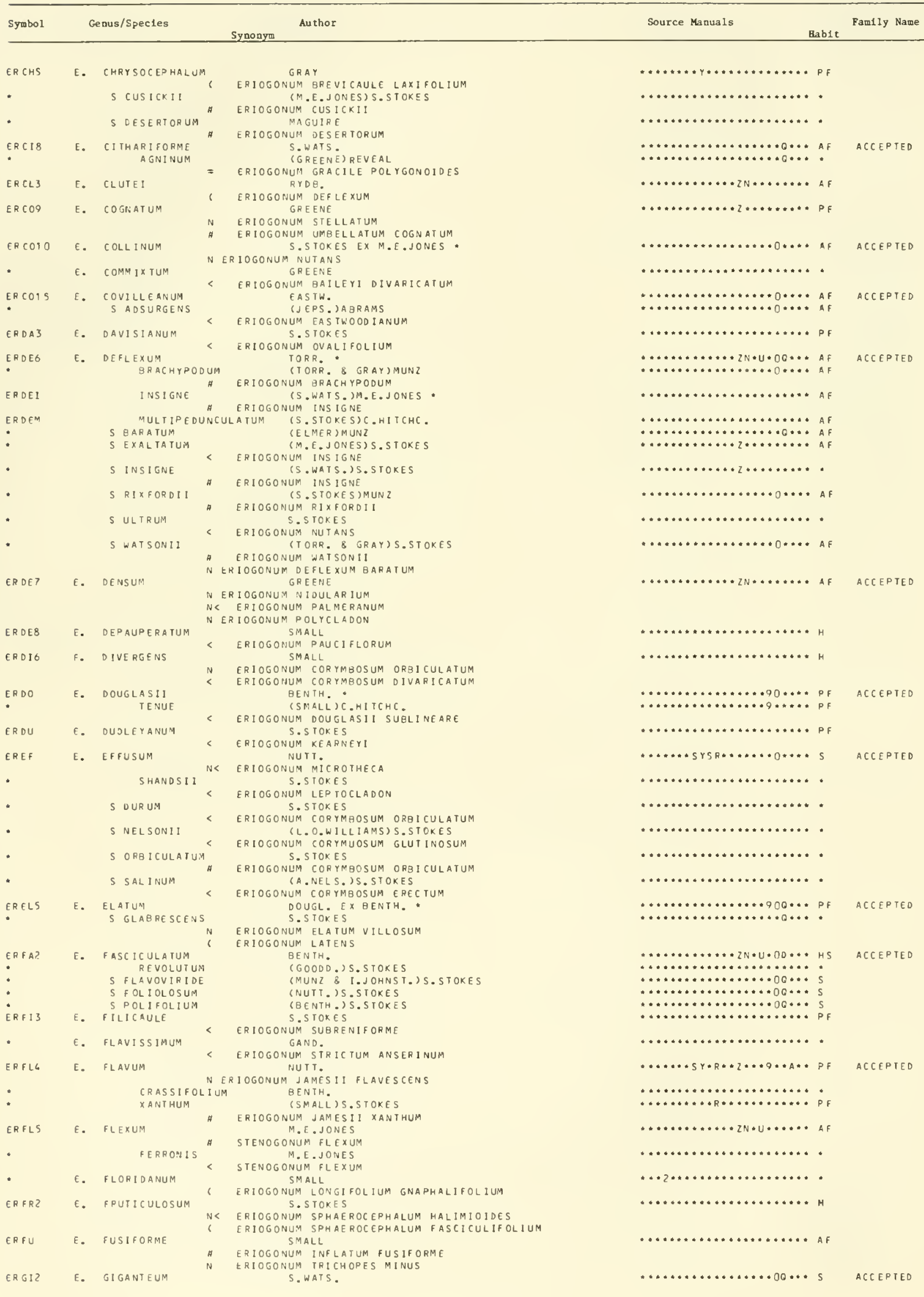




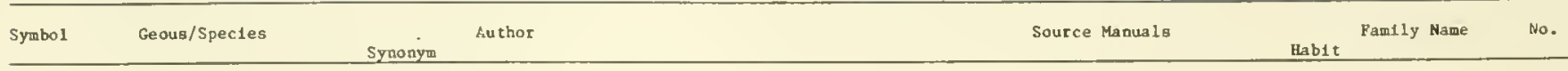

$\begin{array}{lll}\text { - S cOMPACTUM } & \text { (DUNKLE)MUNz } \\ \text { S FORIOSULA } & \text { (M.CURRAN)RAVEN }\end{array}$

ERGLG E. GLANQULOSUM

ERGLE

N STENOGONUM FLEXUM

(NUTT.) NUTT, EX BENTH.

ERGL6 E. GRACILEREUM J.HOWELL

E. GRACTLE BENTH.

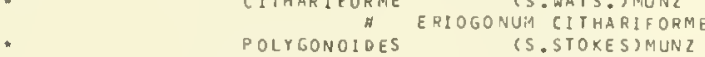

ergRtz E. GRANDE GREENE

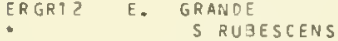

5 TIMORUM

ERHE E. HEERMANNII

$S$ ARGENSE
5 FLOCCOSUM

5 HUMILIUS

S SULCATUM
ERHE? E. HERACLEOIOES

* minus

ERHO6 E. HOFFMANMII

5 ROBUSTIUS

ERHD?

E. HOWELL! !

$$
\text { SUBRACEM SUM }
$$

ERIN3 E. INERME

ERING E. INFLATUM

- contiguuia

ERKE3

ERKE?

ERKE

:

*

ERLAQ

ERLAS

*

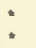$$
\text { . }
$$

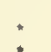$$
:
$$$$
\text { : }
$$

E. LEUCOPHYLLUM

ERlos los longIFOLIUM
*
LINOHEJMERI

LINOHEIMERI
PLANTAGINEUm
* macoougaliI

ERMAL

ERME 3

ERMEL

PULCHRUM
E. MENSICOLA

ERMI 4$$
\text { - }
$$$$
*
$$

ERMIL ?

E. MARI FOLIUM I N CANUM

E. MEARNSII

E. MICROTHECA CONFERT IFL N CRISPUS

FRI SCANUM

IDAHOENSE LAXI FLORUM MACOOUGAL :

$S$ AUREUM

EXPANSUM

S CONFERTIFLORUM

5 INTERMEOIUM

ERMO3 E. MOHAVENiSF

S AMPULLACEUM
(GREENE) MUN 2

( REVEAL) MUNZ

E. M. DURAND \& HILGARD

(M. E. JONES) MUN $Z$

(MUNZ) MUNZ

S. STOKES

(S. WATS.) IAUNZ

NUTT.

ERIOGONUM HERACLEOIDES ANGUSTIFOLIUM

S. 5 TOKES

(5. STOKES)MUN

S.STOKES

ERIOGONUM HEERMANNII ARGENSE S. STOKES

ERIOGONUM HEERMANNII SUBRACEMOSUM

(S. WATS.) JEPS.

(GOODM.) MUNZ

TORR. \& FREM.

ERIOGONUM CONTIGUUM

TIOEST.

(S.STOKES)S, STOKES

PORTER EX S.WATS. J.T. HOWELL

ERIOGONUM WRIGHTII OLANCHENSE

(MUNZ \& 1.JOHNST.) MUNZ

(IAUNZ \& 1.JOHNST.) S.STOKES

(S. WATS.)S.STOKES

ERIOGONUM GRACILIPES

(T. BRANDEG., MUUN?

RYOB

J.E.SMITH

(BENTH.) S.STOKES

ERIOGONUM LATIFOLIUM OECURRENS

(JEPS.) S. STOKES

S. STORES

(GREENE)S.STOKES

ERIOGONUM GRANDE
(GREENE) MUNZ

(GREENE) MUNZ

ERIOGONUM NUDUM

(GREENE) S. STOKES EX MUNZ INED. *

(S. WATS, S. STOKES

(A. HELLER)S.STOKES

(GREENE) S. STOKES

ERIOGONUM NUDUM OBLONGIFOLIUM

ER:OGONUM HAVARDI?

TUTT.

GAND.

ENGELM. \& GRAY

GANO.

ERIOGONUIM MICROTHECA FOLIOSUM

ERIOGONUM MICROTHECA MACDOUGALII

TORR. \& GRAY

(TORR. \& GRAY)M.E. JONES

ERIOGONUM INCANUM

ERIOGONUM ERICIFOLIUM

$$
\text { (EASTW.) KEARN. QP PEEB. }
$$

ERIOGONUM PANAMINTENSE MENSICOLA

NUTT.

ERIOGONUM EFFUSUM

UN (BENTH.) TORR. \& GRAY

ERIOGONUM MICROTHECA LAXIFLORUM

(L.O.WILLIAMS) S.STOKES

ERIOGONUM CORYMBOSUM GLUTINOSUM

(M.E. SONES) S. STOKES

ERIOGONUM MICROTHECA FOLIOSUM

$$
\text { ( RYDB. ) S.STOKES }
$$

$$
\text { HOOK. }
$$

ERIOGONUM MICROTHECA PANAMINTENSE

(GAND.)S.STOKES

ERIOGONUM MICROTHECA FOLIOSUM

ERLOGONUM CORYMBOSUM GLUTINOSUM S. STOKES

ERIOGONUM MICROTHECA AMBI GUUM (BENTH.) S. STOKE S

ERIOGONUM MICROTHECA LAXIFLORUM

S. STOKES

ERIOGONUM MICROTHECA FOLIOSUM

S. WATS .

ERIOGONUM AMPULLALEUM STOKES
(EASTW.) S. STOKES $\ldots \ldots \ldots \ldots \ldots \ldots \ldots \ldots \ldots+\ldots * \ldots, s$

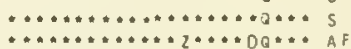
$\ldots \ldots \ldots \ldots \ldots \ldots \ldots \ldots 0 \ldots \ldots$ A

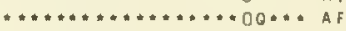
$\ldots \ldots \ldots \ldots \ldots \ldots \ldots D_{*} \ldots$ AF

$\ldots \ldots \ldots \ldots \ldots \ldots \ldots \ldots \ldots \ldots$.

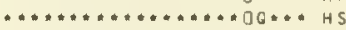

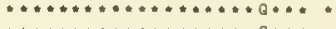

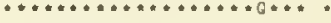

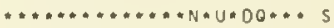

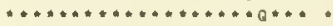
$\ldots \ldots \ldots \ldots \ldots \ldots+\ldots, \ldots, \ldots$ $\ldots \ldots \ldots \ldots+\ldots \ldots+\ldots+\ldots+\ldots$

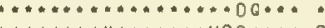
$* * * * * *+4 * * * * * U 90 * * * P F$

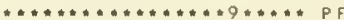

$\ldots \ldots \ldots \ldots \ldots \ldots \ldots 00 \ldots A_{F}$

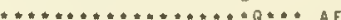
$\cdots \ldots \ldots * \ldots * \cdots+\ldots \ldots \ldots \ldots{ }_{H}$

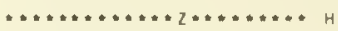

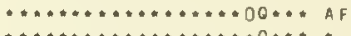

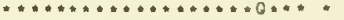
$\cdots \cdots \cdots+Y \cdots+2 N \cdot U \cdot 0 Q \cdots \cdot P A F$ ................... apf

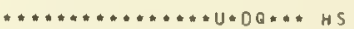
$\ldots \ldots \ldots \ldots \ldots \ldots \ldots \ldots \ldots * \ldots$ $\ldots \ldots+\ldots, \ldots$

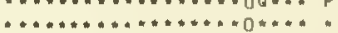

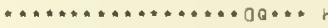
$\ldots \ldots \ldots \ldots \ldots \ldots \ldots O \ldots H_{H}$

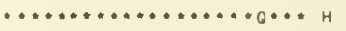
$\ldots \ldots \ldots \ldots r \ldots \ldots \ldots \ldots \ldots$ PF

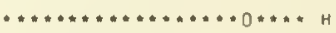

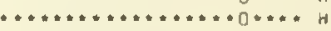

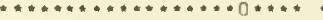
$\ldots \ldots \ldots \ldots \ldots \ldots \ldots \ldots 0 \ldots \ldots \% H$

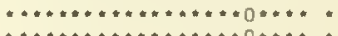

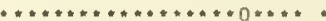

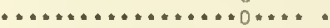
$\because \cdots \cdots+\ldots \ldots+\cdots *+0 * \cdots *$

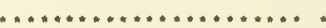

$F 日 \cdots * * M *+5 * T 6 \ldots \ldots \ldots \ldots \ldots$ F

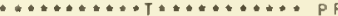

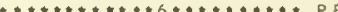

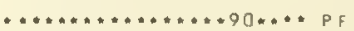
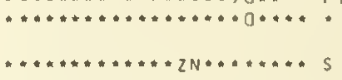

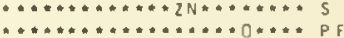

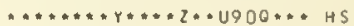

ACCEPTEO
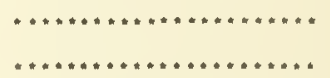

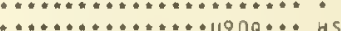

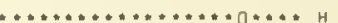

ACLEPIEO
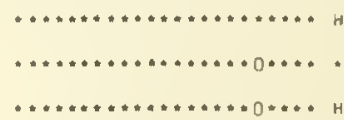

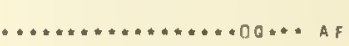

ACCEPTED

ACEEPTED

ACCEPTEO

ACC EPTEO

ACGEPTEO

ACC EPTED

ACCEPIEO

ACEEPTEO

ACCEPTEO

ACCEPTED

ACCEPTEO

ACC EPIEO

ACEEPTEO

................ AF 


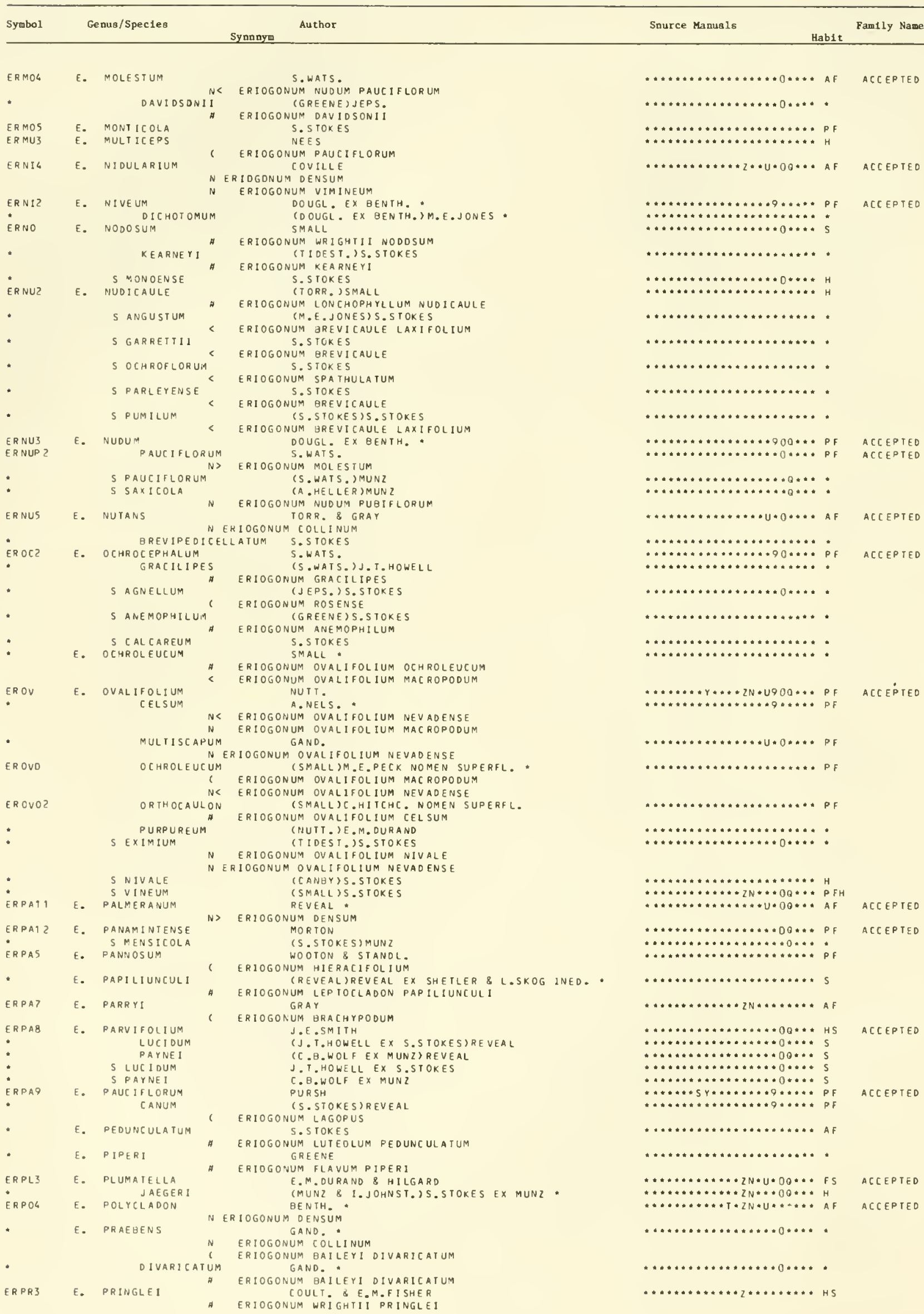




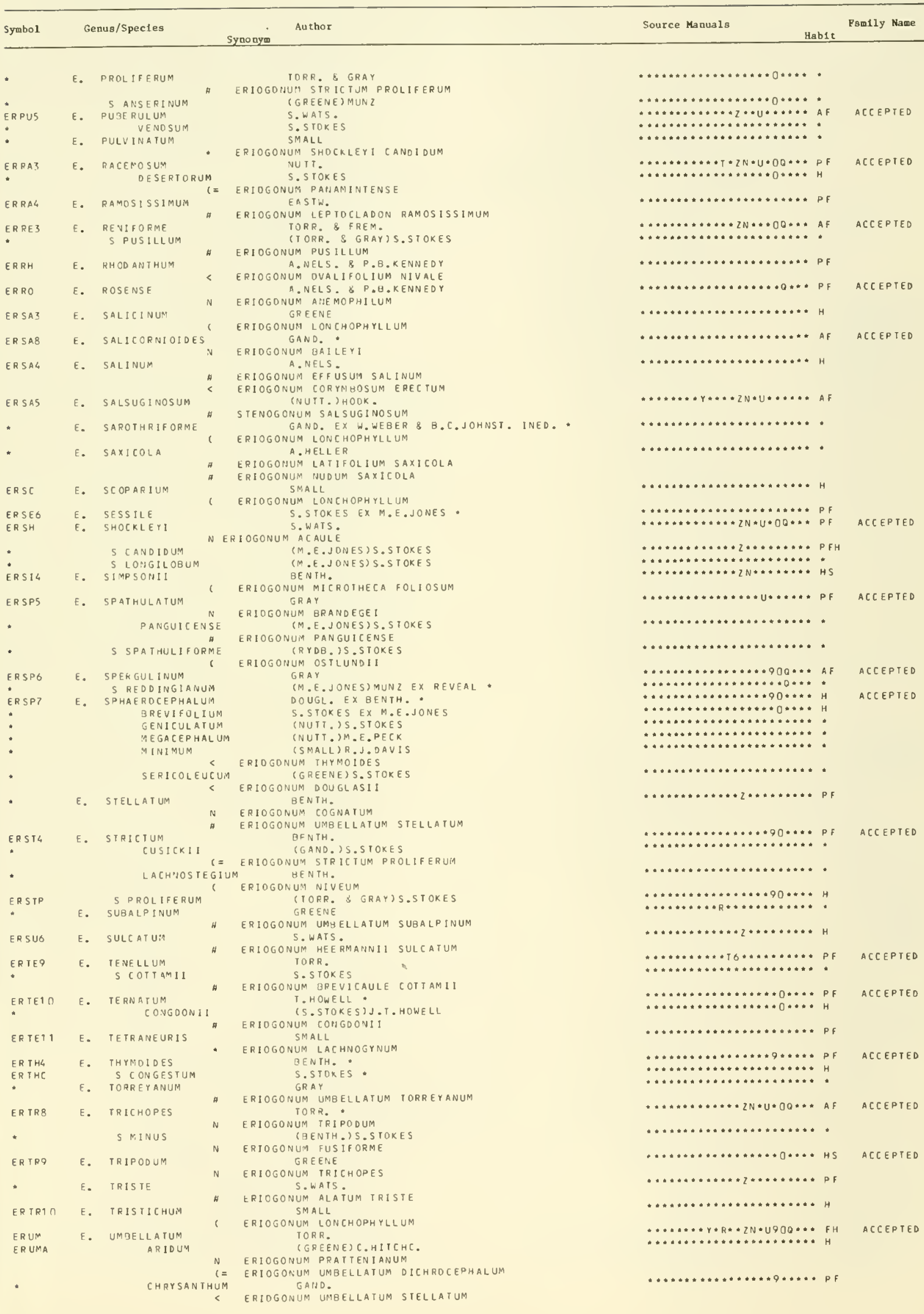




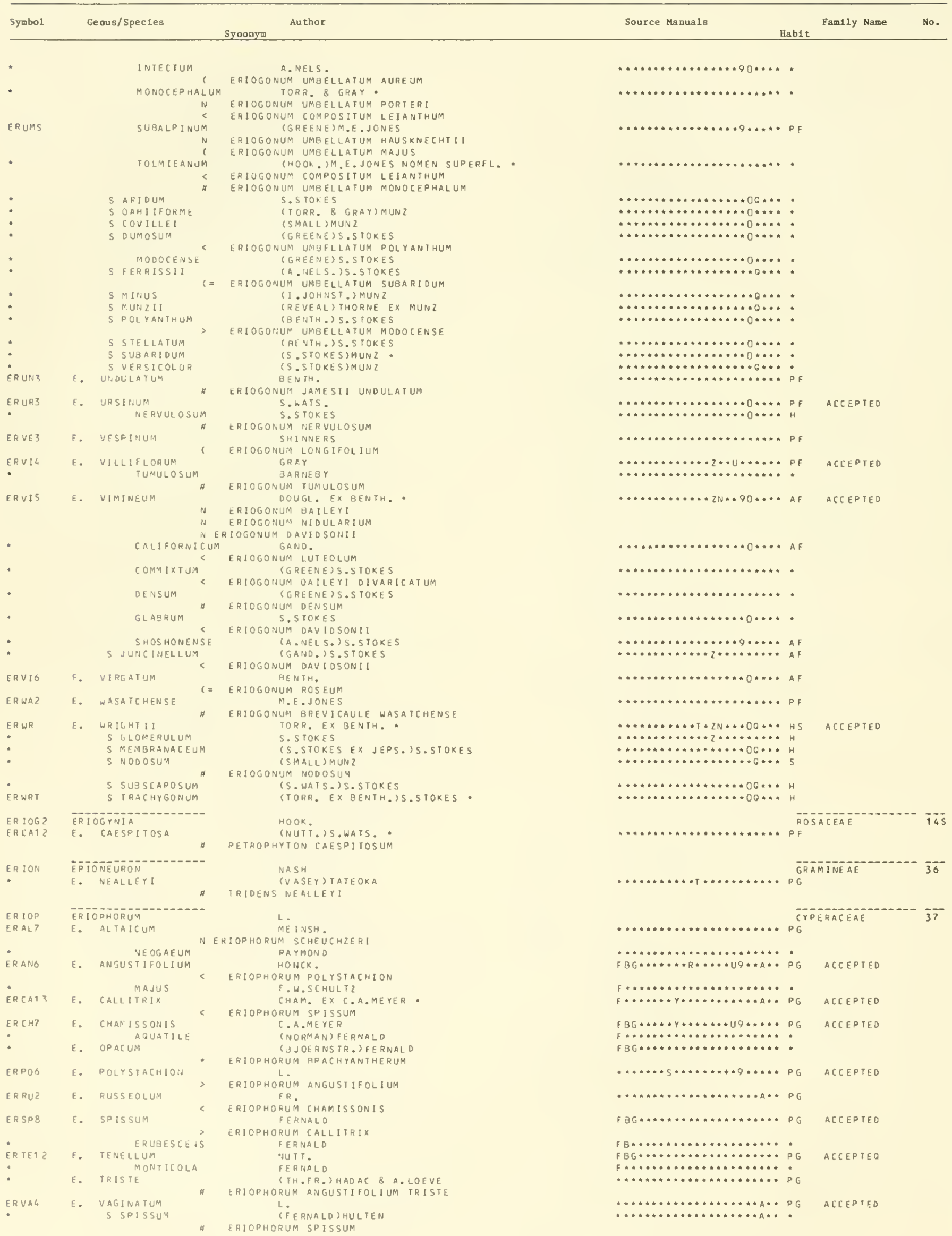




\begin{tabular}{|c|c|c|c|c|c|}
\hline Symbol & Genus/Species & Author & Source Manuala & Fam1ly Name & No. \\
\hline ERVIQ & $\begin{array}{r}\text { E. VIRIDICARINATUM } \\
\text { FELL OWS } I\end{array}$ & $\begin{array}{l}\text { (ENGELM. ) FERNALD } \\
\text { FERNALD }\end{array}$ & 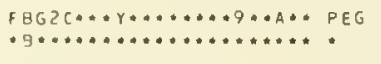 & ACCEPTED & \\
\hline ER $10 P_{2}$ & ERIOPHYLLUM & LAG. & COM & POSI TAE & $\overrightarrow{304}$ \\
\hline ERCO1B & E. CONFERTIFLORUA & (OC, GRAY & $\cdots \cdots \cdots+\cdots \cdots+2 * *+00 * \cdots+H$ & ACCEP TED & \\
\hline - & DISCOIDEUM & (RYDB. MUUZ & $\cdots \cdots \cdots \cdots \cdots \cdots \cdots \cdots$ & & \\
\hline : & LAXIFLORUM & $\begin{array}{l}\text { GRAY } \\
\text { (RYDU, ) MUNZ }\end{array}$ & $* * \cdots+\cdots \cdots \cdots+\cdots+00 * * s$ & & \\
\hline * & IRIFIDUM & (NUTT.) GRAY & $\cdots \ldots \ldots \ldots \ldots \ldots \ldots \ldots+\cdots \cdots$ & & \\
\hline ERLAG & E. LANATUM TUNE ATUM & $\begin{array}{l}\text { (PURSH) J FOREES } \\
\text { (KELLOGG) JEPS. }\end{array}$ & 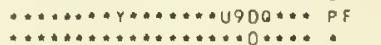 & ACCEPTED & \\
\hline$E R L A ?$ & E. LANOSUM & $\begin{array}{l}\text { ERIOPHYLLUM LANAIUM INTEGRIFOLIUM } \\
\text { (GRAY) GRAY }\end{array}$ & $\cdots \cdots \cdots \cdots \cdots \cdots+Z N+U * O D * \cdots A F$ & & \\
\hline - & E. TENUIF OLI UN & $\begin{array}{l}\text { ANTHEROPEAS LANOSUM } \\
\text { (DC.)RYOH. }\end{array}$ & $\cdots \cdots \cdots \cdots \cdots \cdots \cdots+Z * \cdots \cdots * \cdots * H$ & & \\
\hline ERWAL & E. WALLACE I & $\begin{array}{c}\text { ERIOPHYLLUM CONFERTIFLORUM } \\
\text { (GRAY) GRAY }\end{array}$ & $\cdots \ldots \ldots \cdots \cdots \cdots \cdots Z N \cdot U \cdot 00 \cdots A F$ & & \\
\hline (n) & RUBELLUM & ANTHEROPEAS WALLACEI & & & \\
\hline • & E. YUKONENSIS & $\begin{array}{l}\text { (GRAY) GRAY } \\
\text { RYDB. }\end{array}$ & 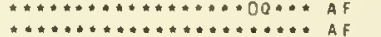 & & \\
\hline ER 105 & ERIOSORUS & FEE & $A L T \cdot \overrightarrow{P O L}$ & $\begin{array}{l}\text { YPOD IACEAE } \\
\text { MNOG RAMMATA CEAE }\end{array}$ & 8 \\
\hline ER ITH & ERITHALIS & P.UR. & $\overline{R U B}$ & $\overline{I A C E} \overline{A E}$ & $\overline{29 \overline{4}}$ \\
\hline ERITR & ERTTRICHIUM & SCHRAD. EX GAUDIN - & $\overline{B O} \bar{R}$ & AGINACEAE & $\overline{2} \overline{76}$ \\
\hline ERAR 10 & E. ARETIOIDES & $\begin{array}{l}\text { (CHAM.) A . DC : } \\
\text { ERITRICHIUM NANUM ELONGATUM }\end{array}$ & $\cdots \ldots \ldots \ldots \cdots R \cdots \cdots \cdots \cdots \cdots A, \cdots P F$ & & \\
\hline ERCH8 & E. CHAMISSONIS & $\begin{array}{l}\text { ERITRICHIUM NANUM ARETIOIOES } \\
\text { A. DC. } \\
\text { EKITRICHIUA ARETIDIDES }\end{array}$ & $\cdots \cdots \cdots \cdots \cdots \cdots \cdots \cdots \cdots A * P F$ & & \\
\hline EREL? & E. ELDNGATUM & $\begin{array}{l}\text { (R YDB. ) W. WIGHT } \\
\text { ERITRICHIUM NANUM ELONGATUM }\end{array}$ & $\ldots \ldots \ldots \ldots \ldots \ldots \ldots \ldots \ldots, P F$ & & \\
\hline ERELA & ARGENTEUM & (W.WIGHT) I. JOHNST. • & $* \cdots * * * * * \ldots * * \ldots * \ldots \ldots \ldots \ldots \rho p q$ & & \\
\hline ERNA & $\begin{array}{ll}E . & \text { NANUM } \\
\text { E. RUPESTRE }\end{array}$ & (VILLARS) SCHRAD. & $\cdots \cdots \star \cdots * \gamma \ldots \cdots \cdots * U q * \star * \cdots P F$ & & \\
\hline • & $\begin{array}{l}\text { E. RUPESTRE } \\
-\end{array}$ & (PALLAS)BUNGE & $\cdots \ldots \ldots \ldots \ldots \ldots+\ldots \ldots \ldots, p_{F}$ & & \\
\hline ERNOO & ERNOOEA & SWARTZ & 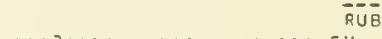 & IACEAE & $\overline{2} \overline{94}$ \\
\hline • & E. ANGUSTA & $\begin{array}{l}\text { SMALL } \\
\text { ERNODEA LITTORALIS ANGUSTA }\end{array}$ & $\cdots 2 \ldots \ldots \cdots \cdots \cdots+\cdots \cdots \cdots+5 v$ & & \\
\hline ERLIS & $\begin{array}{l}\text { E. LITTORALIS } \\
\text { ANGUSTA }\end{array}$ & $\begin{array}{l}\text { SWARTL } \\
\text { (SMALL)R.LONG }\end{array}$ & $* * 2 * L * \cdots * \cdots * * * * * * * E * 5$ & ACCEPIED & \\
\hline ERODI & ERODIUM & L'HER: EX SOLAND. IN AIT. * & $\overline{G E} \bar{R}$ & ANIACEAE & $\overline{949}$ \\
\hline : & $\begin{array}{l}\text { E. CICONIUM } \\
\text { E. LACINIATUM }\end{array}$ & $\begin{array}{l}\text { (L, I) 'HER, } \\
\text { (CAV.)WILLD. }\end{array}$ & 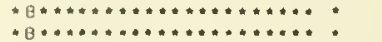 & & \\
\hline ERMO? & E. MOSCHATUM & (L.)L'HER. EX SOLAND. IN AIT. & $F B G 2 * \ldots \cdots \cdots+\cdots 90 \cdots A F$ & ACCEPTED & \\
\hline - & PRAECOX & LANGE & 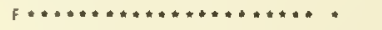 & & \\
\hline - & E. STEPHENI ANUM & WI LLO. & $* 8 \ldots \ldots+\ldots \ldots \ldots \ldots+\cdots \ldots \ldots$ & & \\
\hline - & EROTEUM & $\begin{array}{l}\text { SWARTZ } \\
\text { FREZIERA }\end{array}$ & ( 50 & ACEAE & 200 \\
\hline * & E. ALBOPUNCTATUM & $\begin{array}{l}\text { CGRISEB. BBRIITON } \\
\text { CLEYERA ALBOPUNCTATA }\end{array}$ & $\cdots+\cdots \ldots \cdots \cdots \cdots \cdots \cdots \cdots * \cdots_{*} \cdot$ & & \\
\hline ERRAZ & ERRAZURIZIA & PHILIPPI & ALT. PAA & $\begin{array}{l}\text { UMINOSAE } \\
\text { PILI ONACEAE }\end{array}$ & 977 \\
\hline ERUCA & $\begin{array}{l}\text { ERUCA } \\
\text { E. ERUCA }\end{array}$ & MILL. E TTION & CRU & CIFERAE & $\sqrt[124]{24}$ \\
\hline & Co & $\begin{array}{l}\text { (L.DBRITTON } \\
\text { ERUCA SATIVA }\end{array}$ & $\cdots+2 \cdots+\ldots \ldots+\cdots \cdots \cdots+\cdots f$ & & \\
\hline ER VES & E. VESICARIA , & $\begin{array}{l}\text { EL.) CAV. } \\
\text { ERUCA SATIVA }\end{array}$ & $* B G * \ldots \ldots \ldots \ldots \ldots \ldots \cdots * \cdots A F$ & & \\
\hline ERUCA Z & ERUCASTRUM & K.PRESL . & $\bar{C} \overline{R U}$ & CIFE RAE & $\overline{1} \overline{2} \overline{4}$ \\
\hline ER YNG & ERYNGIUM & L. & $\ldots \ldots \ldots \ldots \ldots \ldots \ldots$ & ELLIFERAE & $\overline{2} \overline{s i}$ \\
\hline ERAO & E. AQUATICUM & ERYNGIUM YUCCCIFOLIUM & 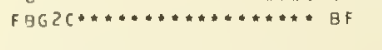 & ACCEPIED & \\
\hline ERAR14 & E. ARTICULATUM N & $\begin{array}{l}\text { HOOK. } \\
\text { ERYNGI UM PLANUM }\end{array}$ & $\ldots \ldots \cdots \cdots \cdots \cdots \cdots \cdot+\cdots 0 * \cdots p F$ & ACCEPIED & \\
\hline ERFL6 & E. FLORIDANUM < & $\begin{array}{l}\text { COULT. \& ROSE } \\
\text { ERYNGIUM AQUAII CUM RAVENELII }\end{array}$ & 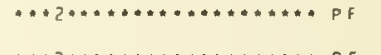 & & \\
\hline ERLUG & E. LUDOVICIANUM & $\begin{array}{l}\text { MORONG } \\
\text { ERYNGIUM INTEGRIFOLIUM }\end{array}$ & $\cdots \cdot \cdots \cdots \cdots \cdots \cdots \cdots \cdots \cdot P F$ & & \\
\hline ERPLS & E. PLANUM & $\begin{array}{l}\text { L. } \\
\text { ERYNGIUM ARTI CULATUM }\end{array}$ & $F \ldots \ldots * s \cdots \cdots \cdots \cdots+q \ldots \ldots P F$ & ACCEPTED & \\
\hline ERPRS & $\begin{array}{l}\text { E. PROSTRATUM } \\
\text { DISJUNCTUM }\end{array}$ & $\begin{array}{l}\text { NUTT. EX OC. } \\
\text { FERNALD }\end{array}$ & 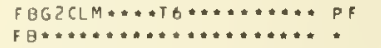 & ACCEPTED & \\
\hline ERRAG & E. RAVENELII & $\begin{array}{l}\text { GRAY } \\
\text { ERYNGIUM AQUATI CUM RAVENELI I }\end{array}$ & $\cdots+\cdots \ldots \ldots \ldots \ldots * \ldots \ldots+p q$ & & \\
\hline ERSY & E. SYNCHAETUM & $\begin{array}{l}\text { (GRAYEX COULT. \& ROSE) COULT, \& ROSE * } \\
\text { ERYNGIUM YUCCIFOLIUM SYNCHAETUM }\end{array}$ & $\cdots \cdot \ldots \ldots \ldots \cdots \cdots \cdots \cdots \cdot P F$ & & \\
\hline ERVAS & $\begin{array}{l}\text { E. VASEYI } \\
\text { VALLICOLA, }\end{array}$ & $\begin{array}{c}\text { COULT. \& ROSE } \\
\text { (JEPS.) MUNZ } \\
\text { ERYNGIUM VASEYI CASTRENSE }\end{array}$ & 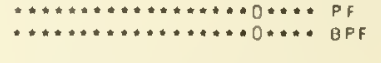 & ACCEPTED & \\
\hline ERVIIO & E. VIRGINIANUM & $\begin{array}{l}\text { ERYNGIUM VASEYI CASTRENSE } \\
\text { LAM. } \\
\text { ERYNGIUM AGUATI GUM }\end{array}$ & $\cdots+2 \ldots \ldots \ldots \ldots \ldots \ldots \ldots * P F$ & & \\
\hline ER YU & E. YUCCIFOLIUM & ERYNGIUM AQUATI CUM & $F B G * C L M *+5 * T 6 * \ldots \ldots \ldots \ldots P F$ & ACCEPTED & \\
\hline ERYSI & ERYSIMUM & L. & $\overline{C R U}$ & $\bar{C} \overline{Y F E} \overline{R A E}$ & $\overline{124}$ \\
\hline - & E. AMOENUM & $\begin{array}{l}\text { (GREENE) RYDB. } \\
\text { ERYSIMUM NIVALE }\end{array}$ & $\cdots \cdots \cdots \cdots \cdots R \cdots \cdots \cdots \cdots \cdots * P_{F}$ & & \\
\hline ERAN7 & E. ANGUSTATUM & RYOB. & 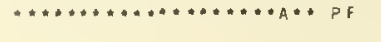 & & \\
\hline
\end{tabular}




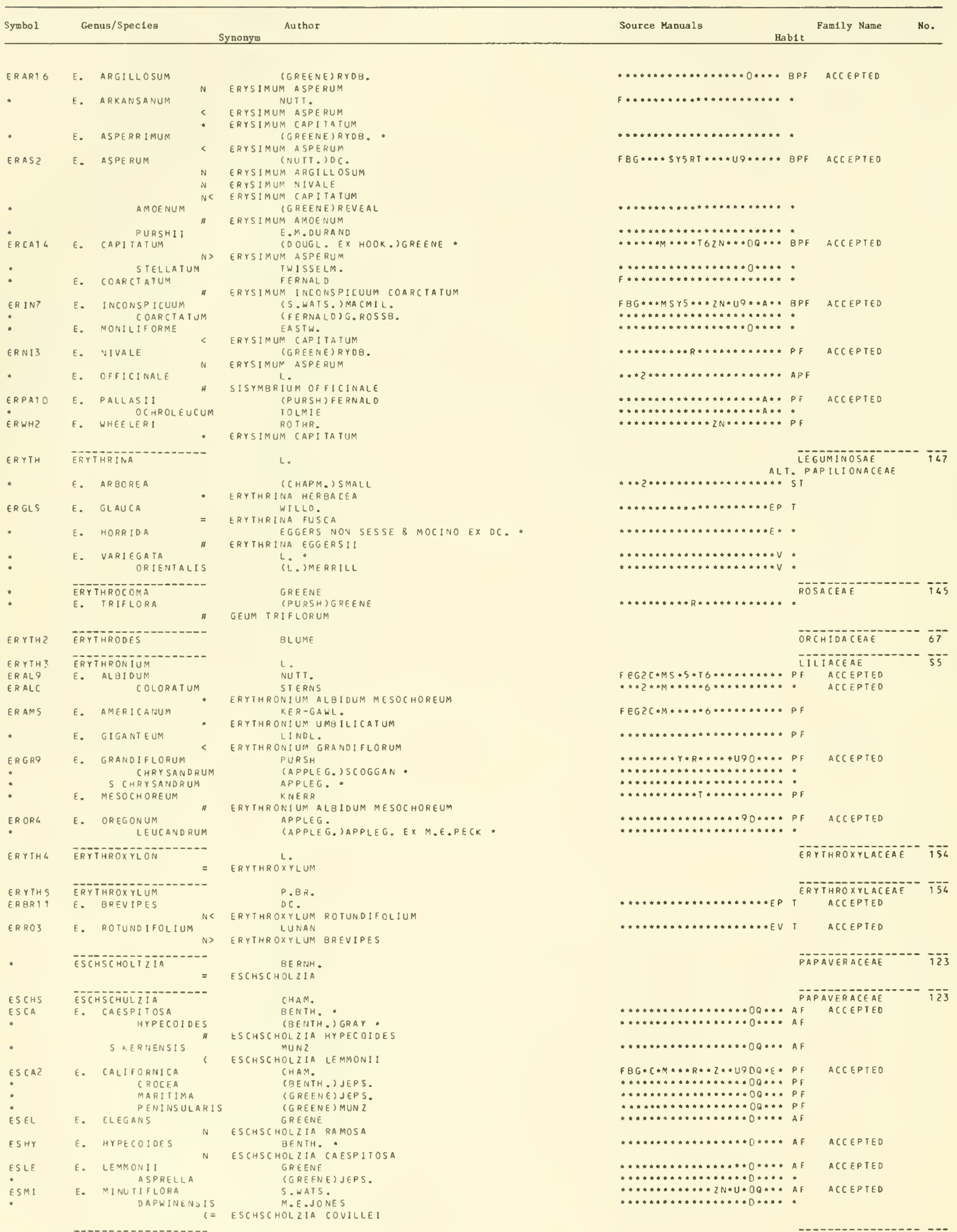




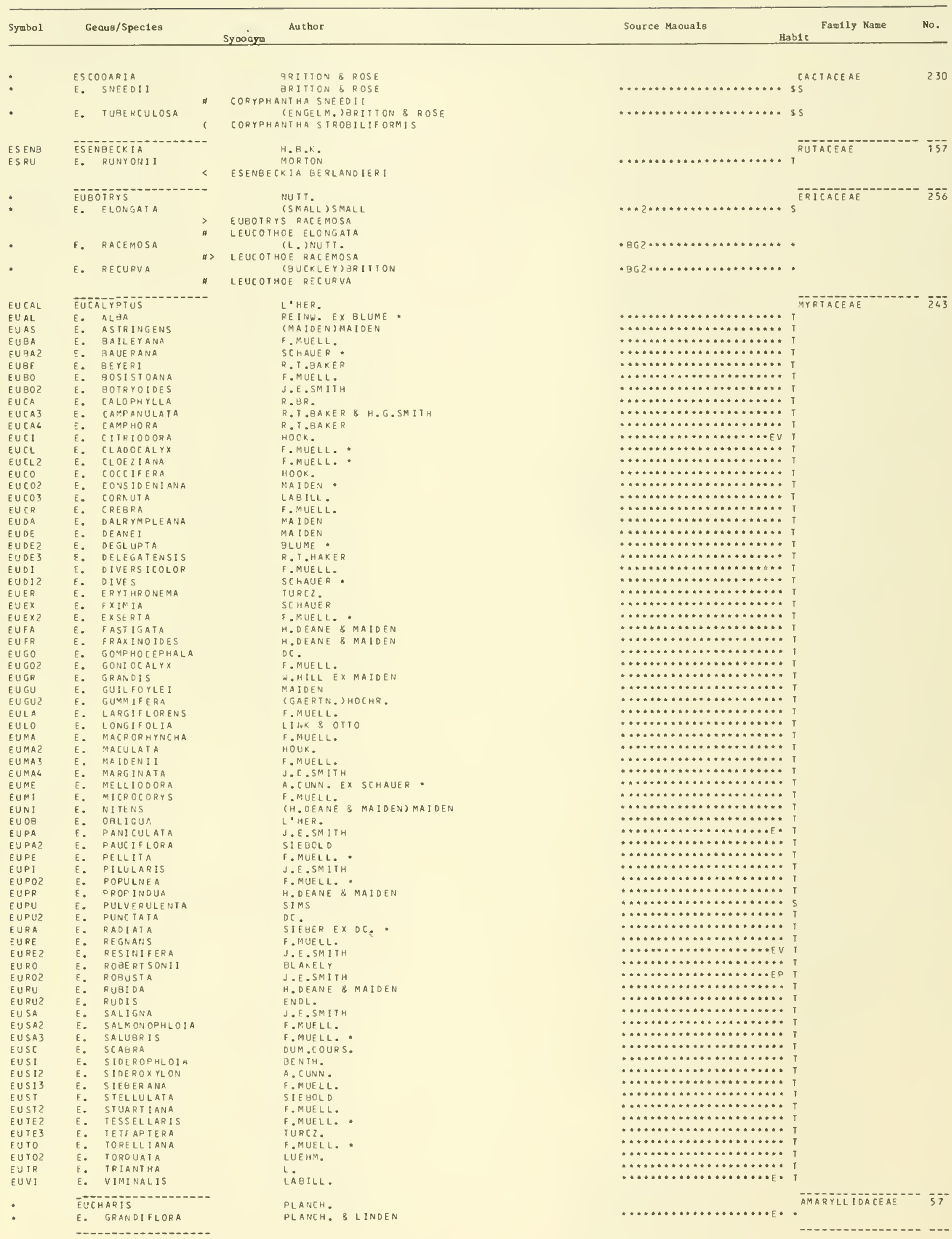




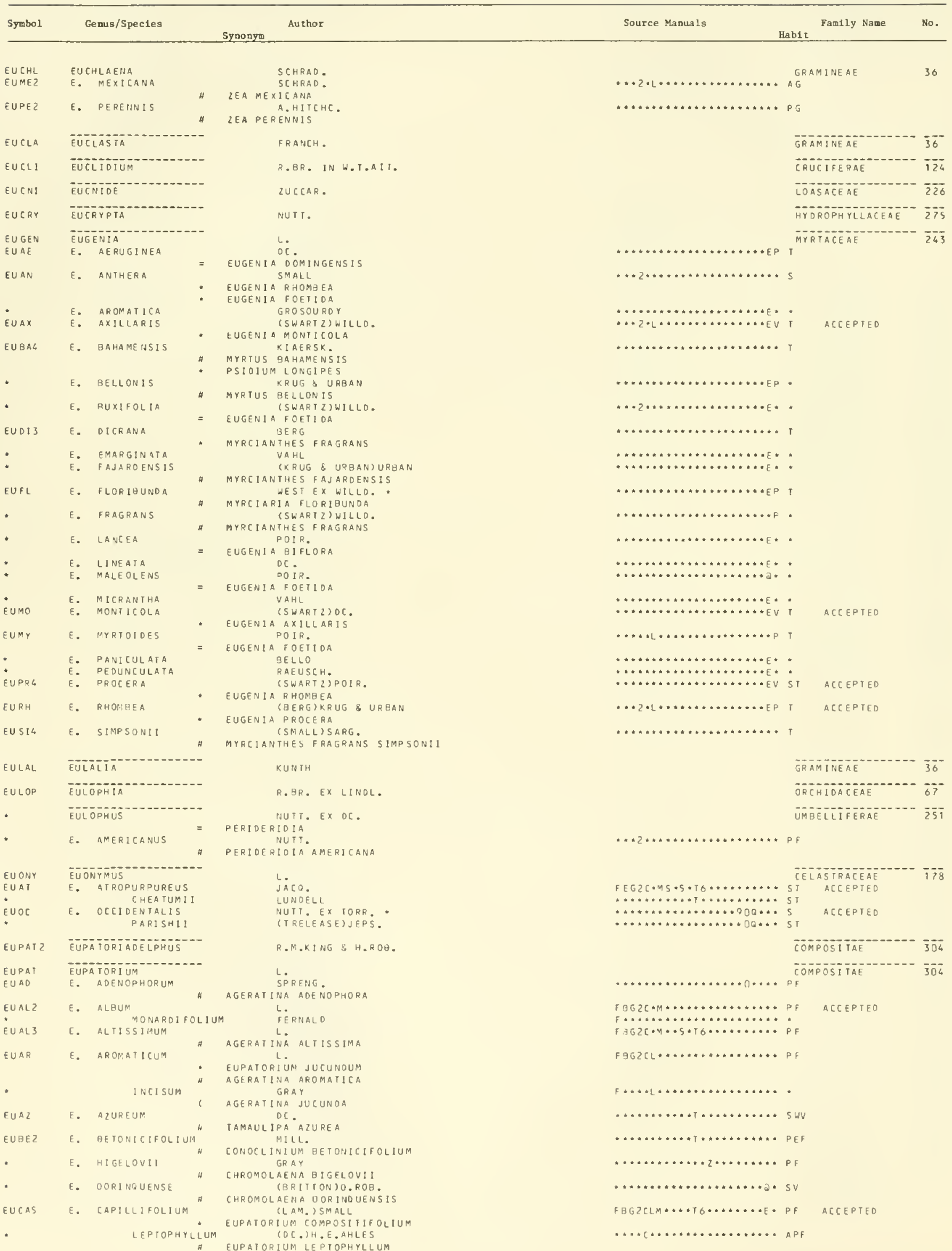




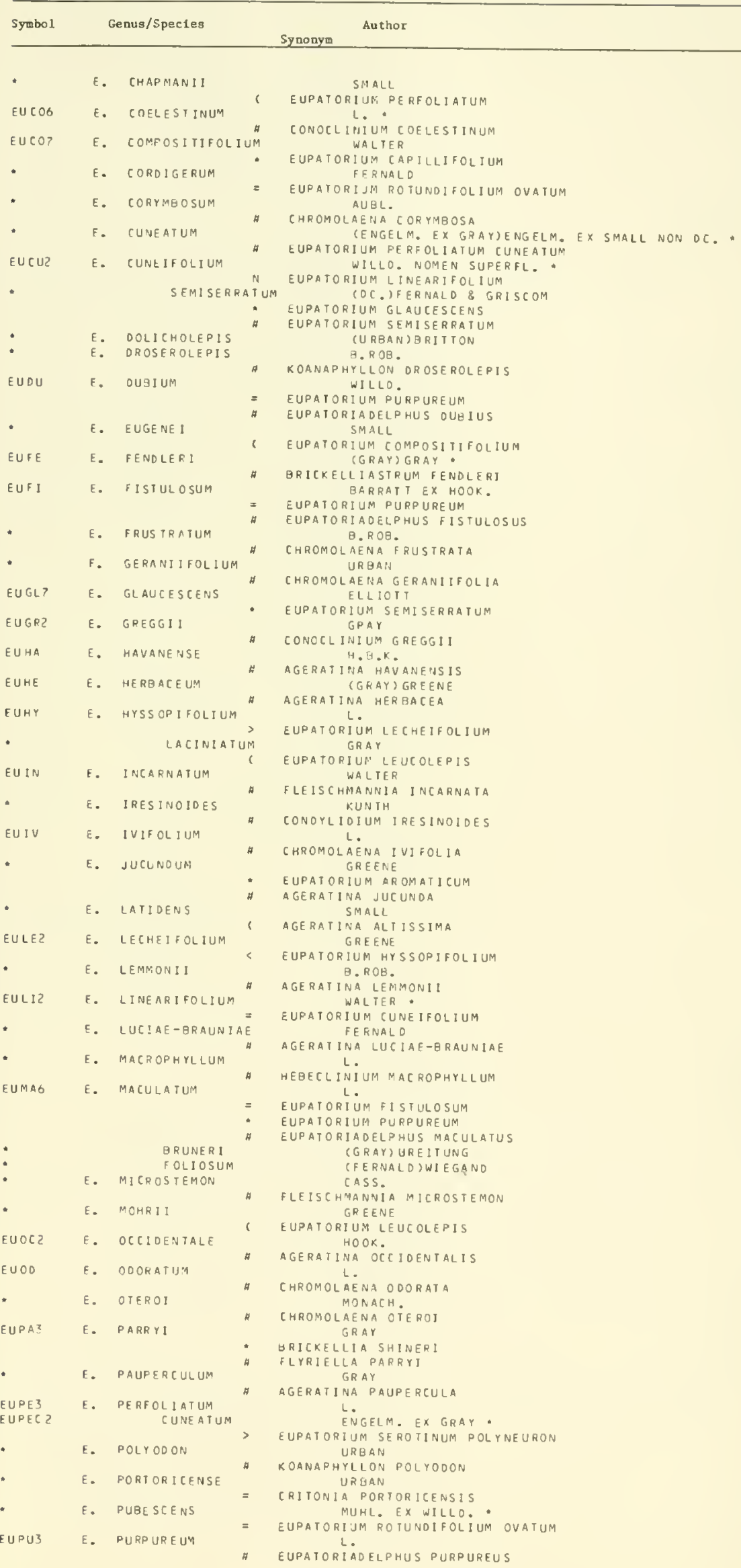

Source Manuals

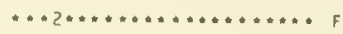

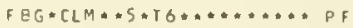

$\cdots 2 C L \cdots \ldots+T 6 * \ldots \cdots * \cdots$ PF

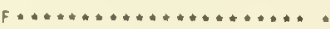

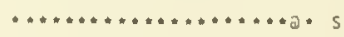

$\ldots . . \ldots \ldots \ldots \ldots \ldots \ldots \ldots \ldots *$

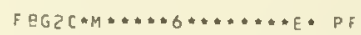

ACCEPTED

F...

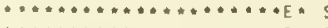

$5 B G+C \cdots \cdots$
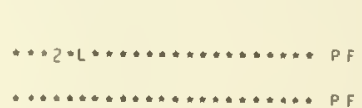

$F B G * C * M * \ldots+T G \cdots \ldots \ldots \ldots \ldots P F$
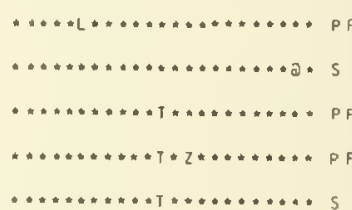

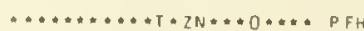

F $B G Z C L M \ldots \ldots T * \ldots \ldots \ldots . . . P F$

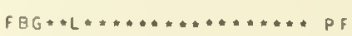

FBGZC.M*.. T6........ PF

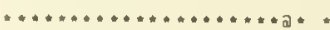

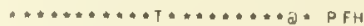

$* * * 2 \ldots \ldots+\ldots+\ldots \ldots \ldots \ldots, F$

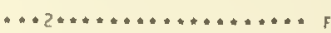

$\ldots \ldots 2 * \ldots \ldots \ldots \ldots \ldots+\ldots \ldots * F$

ACCEPTEO
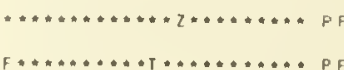

GHG.................

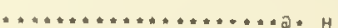

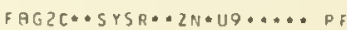

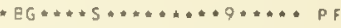

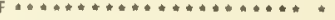

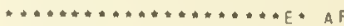

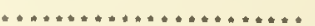

..............90...p p
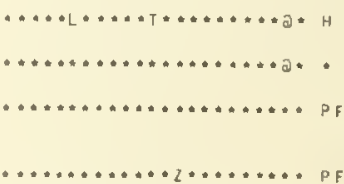

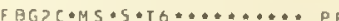
F $B \ldots \ldots M \ldots \ldots \ldots \ldots \ldots \ldots$ $\ldots \ldots \ldots \ldots \ldots+\ldots \ldots \ldots+\ldots * \varepsilon$

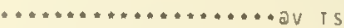

f..2...............

ABG $2 C \bullet M * 5 * T 6 \cdots \cdots \cdots \cdots \cdots B$

\section{ACCEPTEO}

ACCEPTEO

ACCEPTED

ACCEPTEO ACCEPTED
Family N3me

צ्ष०. 


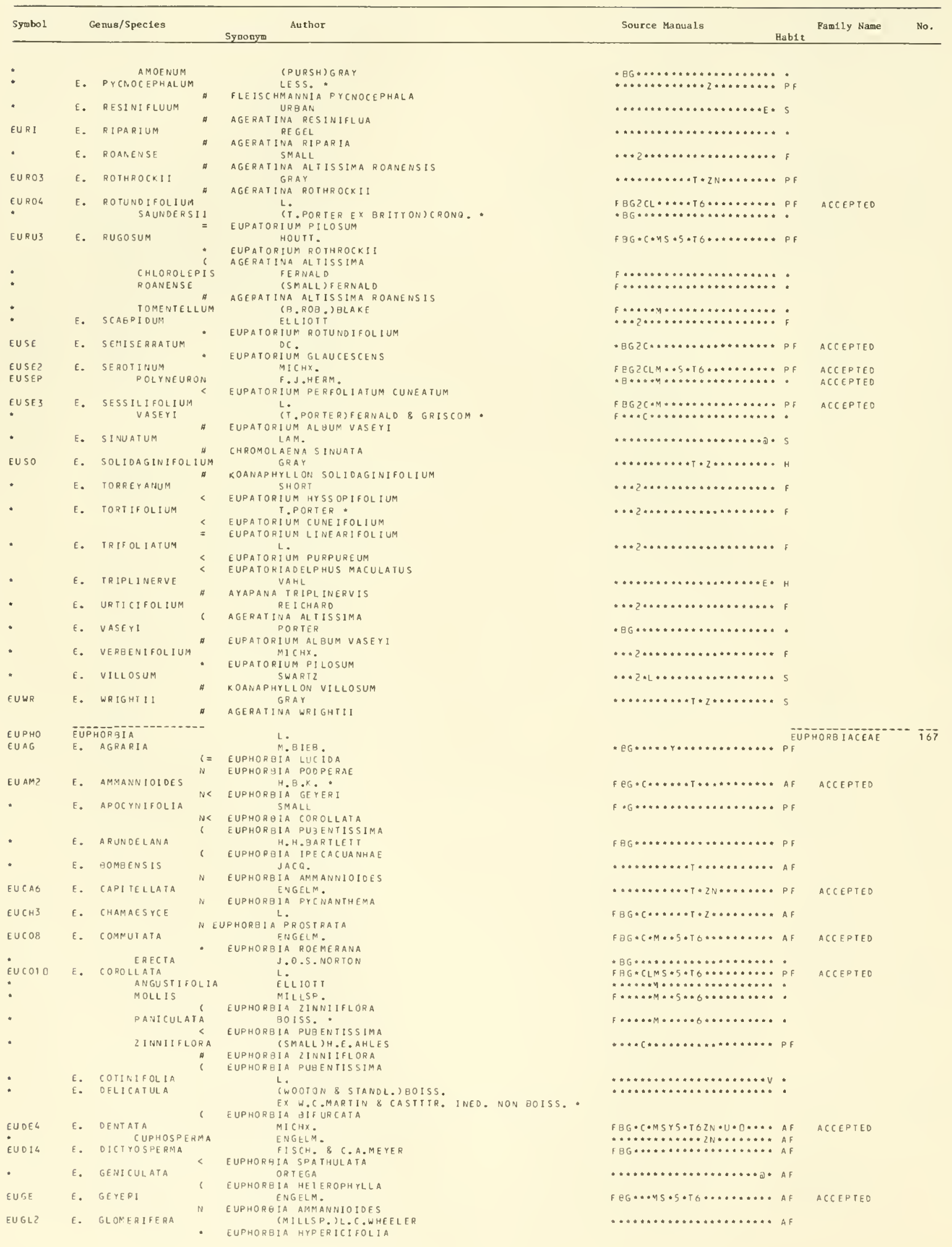




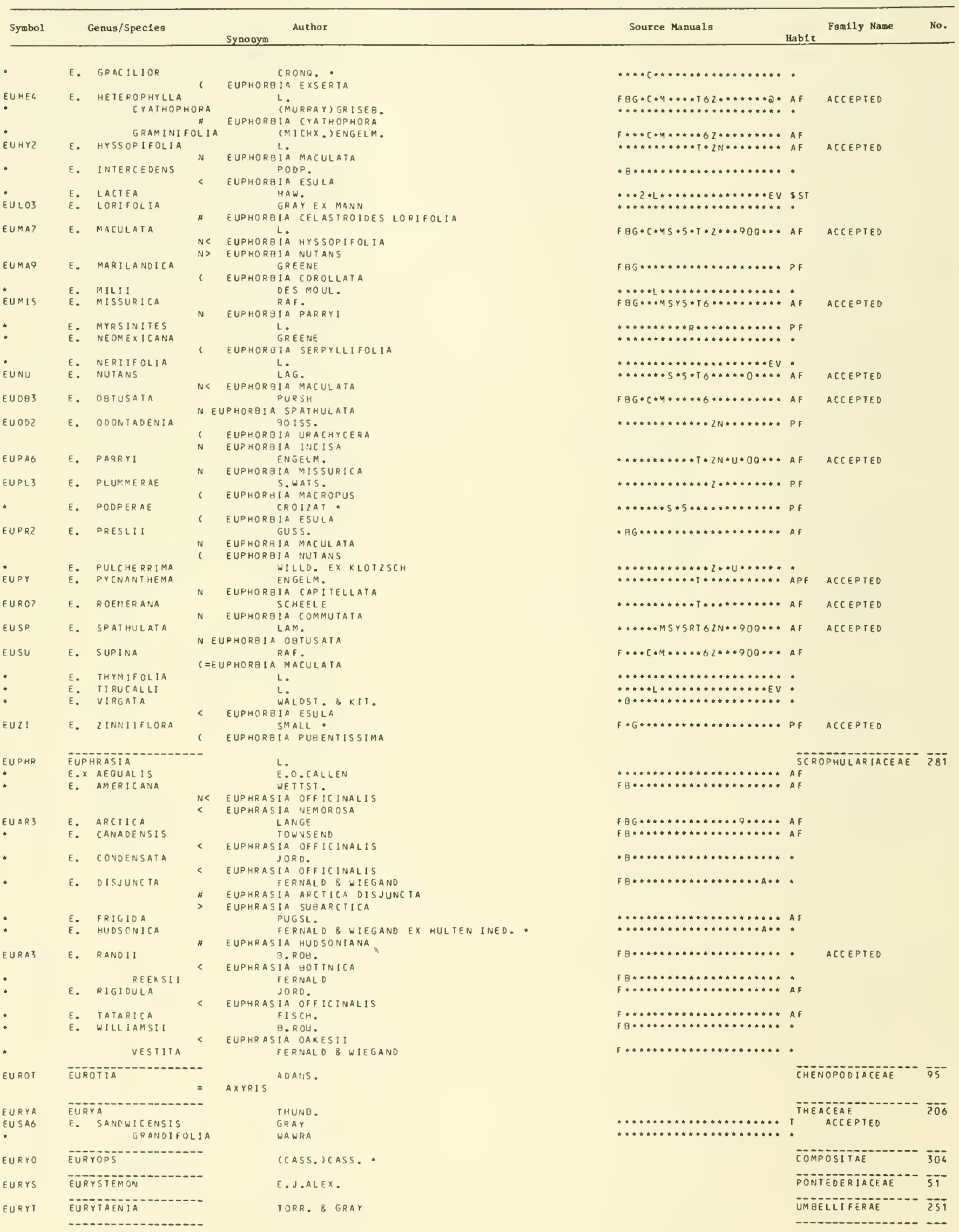




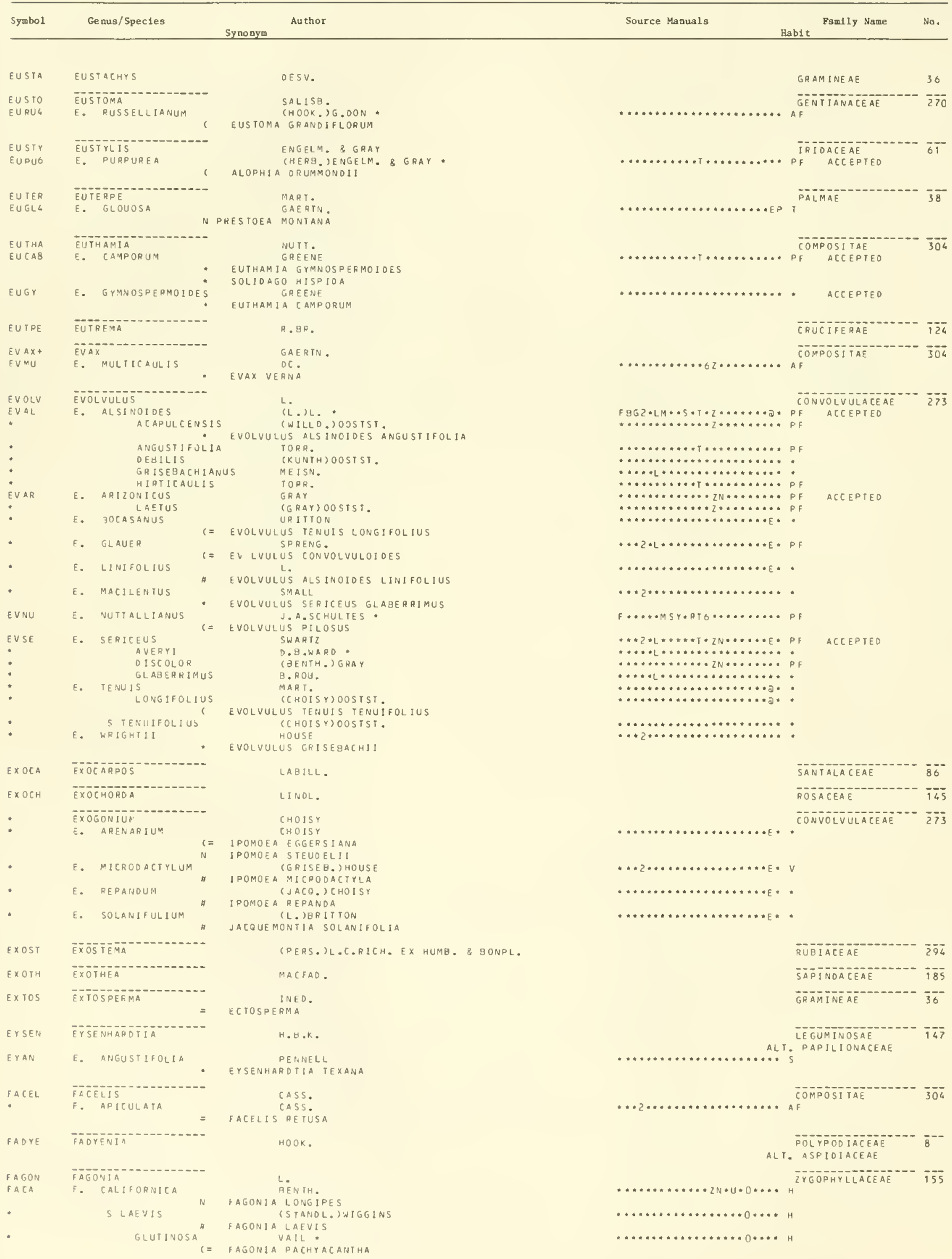




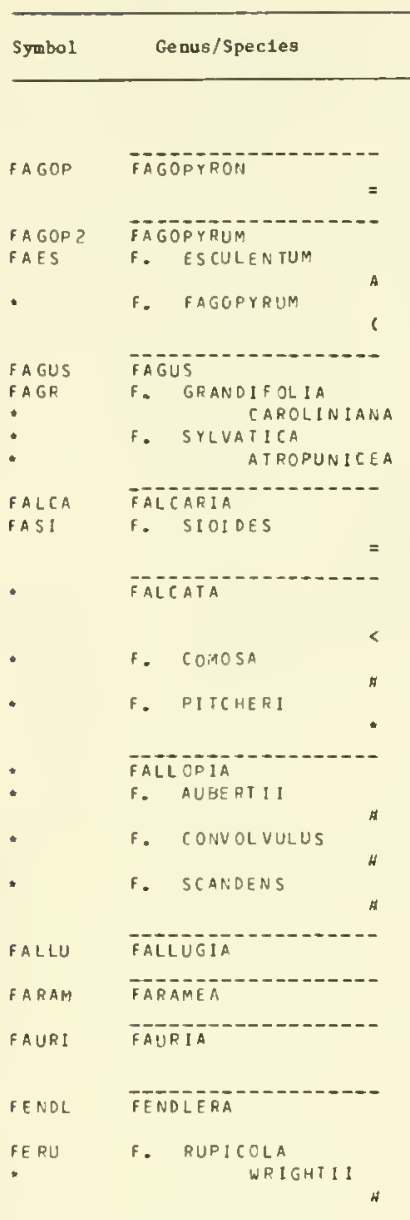

FENOLZ FENDLERELLA

FEROC FEROEATTUS

Eestu bestuca

FEAL FESTUCA ALIAICA

FEBR F. BRACHYPHYLLA =

FECAZ F. CAPILLATA \#

FECO F. CONFUSA

FEDE F. DEPTONENSIS

- f. duriuscula

fEEA F. EASTWOODIAE

FEEL

-

-

$F \in G R$

F. MYUROS

F. OBTUSA
RAF.

MOLLE"

FAGOPYRUM SAGITTATUM

(L.) )KARST.

FAGOPYRUM SAGITTATUM

L.

(LOUDON)FERNALD \& REHD.

LLO

WESTON

$F A B R$.

(WIBEL SASCHERS.

FALCARIA VULGARIS

$$
\text { J.F.GMEL . }
$$

AMPHICARPAEA

AMPHI CARPAEA
CL. IKUNTZE

AMPHICARPAEA BRACTEATA COMOSA (TORR. \& GRAY) KUNTZE.

AMPHICARPAEA BRACTEATA COMOSA

ADANS.

(L. HENRY) J. HOL UB

POLYGONUM AUBERT11!

(L. ) A. LOEVE
POLYGONUM CONVOLVULUS

CL. J. HOLUB

POLYGONUM SCANDENS

ENDL.

AUBL.

FRANCH.

ENGELM. \& GRAY

GRAY

GRAY

FENDLERA WRIGHTII

$$
\text { A. HELLER }
$$

BRITTON \& ROSE

(ENGELM.) ) RRITTON \& ROSE

FEROCACTUS ACANTHODES LECONTEL

TRIN.

FESTUCA SCABRELLA

J.A. \& J.H. SCHULTES

SCMOLAND.

VULPIA BFOMOIDES

FESTUCA LAM.

EESTUCA OVINA CAPILLATA

VULPIA MI GROSTACHYS CONFUSA

(ALL.) IASCHERS. \& GRAEBN.

VULPIA BRCMOIDES

FESTUCA OUINA DURIUSCULA

PIPER
VULPIA MICROSTACHYS CILIATA

$M I$

fESTUCA PPATENSIS

(S C HREB.) WIMM.

FESTUCA RRUNDINACEA

CHUDS.) GRAY

SHUDS.' GRAY

VIPLPIA MIPR.

VULPIA MICROSTACHYS CILIATA

(VASEY)PIPER

FESTUCA SCAERELLA MAJOR

HOLMEN

FESTUCA GCCIDENTALIS

FESTUCA RUBRA

TESTUCA RUT.

VULPIA MYUROS HIRSUTA

NUTT.

FESTUEA ARIDA

VULPIA MI CROSTACHYS

GHOOV. J HOOV.

VULPIA MICROS TACHYS PAUCIFLORA

VULPIA MYUROS

BIEHLER

Source Manuals

Famfly Name

No.

Habit

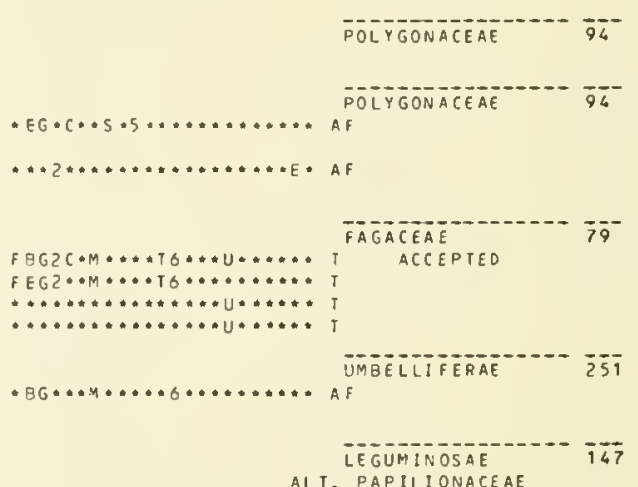

ALT PAPILI ONACEAE

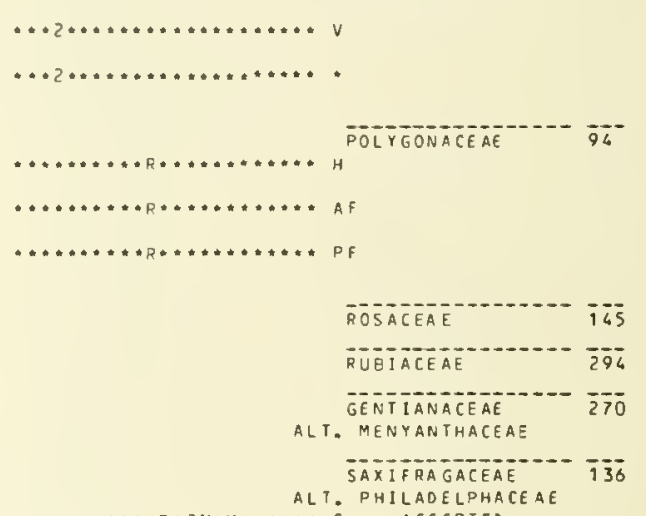

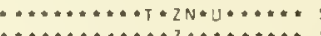

PHILADE LPHACEAE

$\ldots \ldots \ldots \ldots \ldots+\ldots \ldots \ldots \ldots \ldots$

ACCEPTED

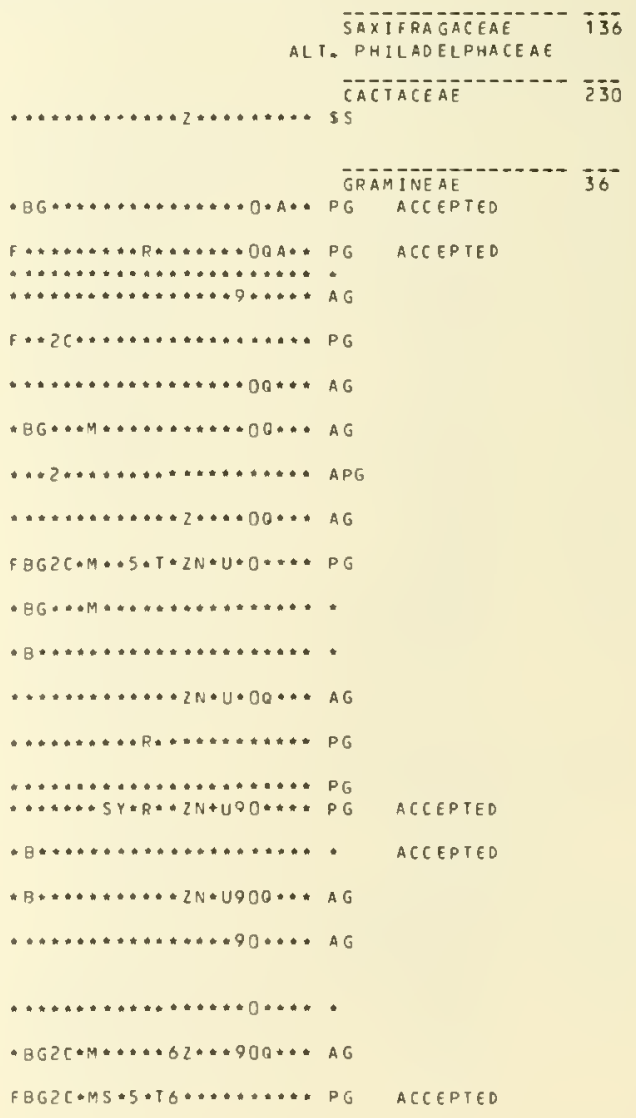




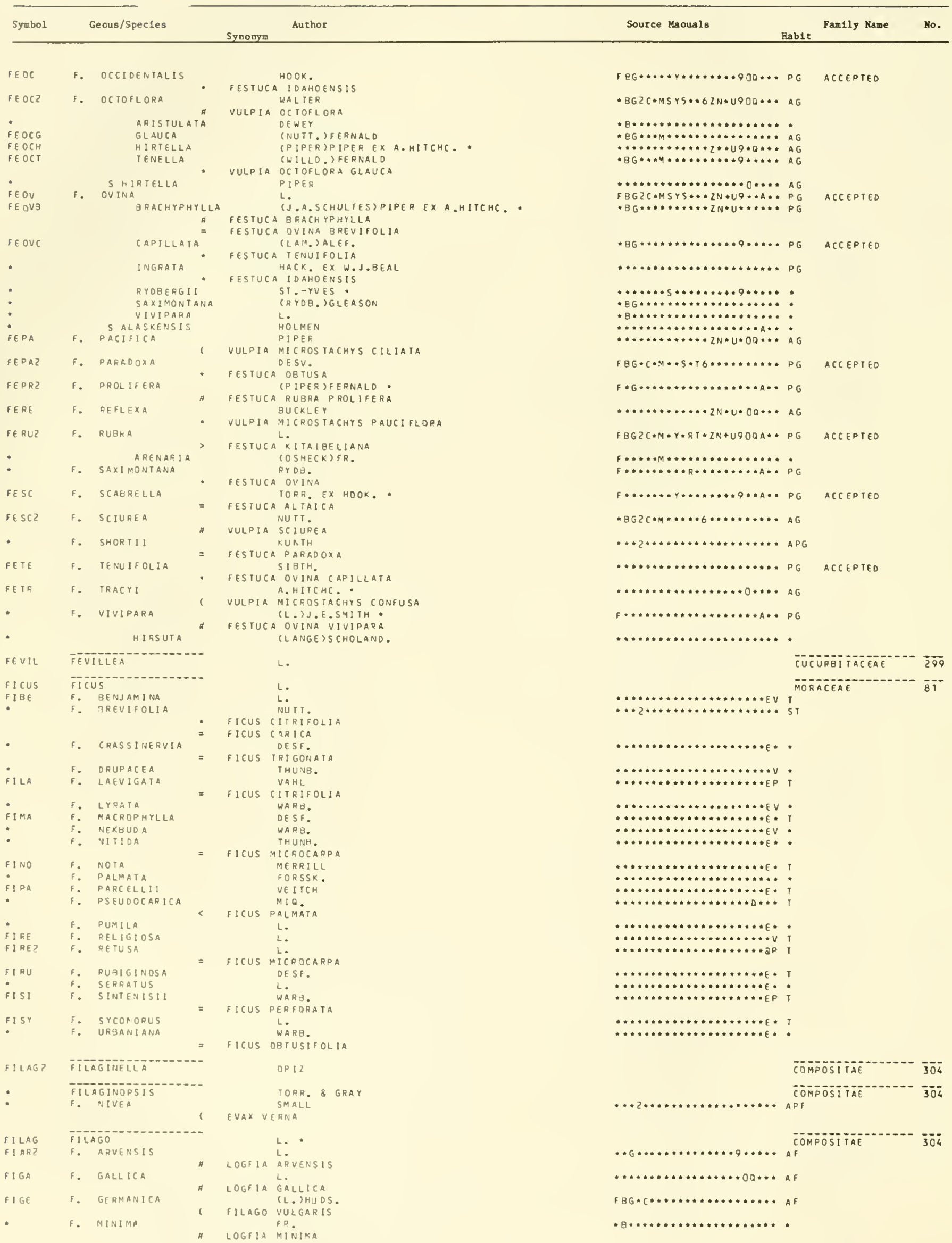


F I LAGO

\begin{tabular}{|c|c|c|c|c|c|}
\hline Symbol & Genus/Species & Synonyw" & Source Manuals & Pam1ly Name & No. \\
\hline - & F. NUTTALLII & $\begin{array}{ll}\text { SHINNERS } \\
\text { - FILAGO PROLIFERA }\end{array}$ & $\ldots \ldots \ldots \ldots \ldots \ldots \ldots \ldots \ldots$ & & \\
\hline - & F. PROLIFERA & * EVAX PROLITERA EX OC.)BRITTON NON POMEL * & $\cdots \ldots \ldots+\ldots \ldots \ldots \ldots \ldots \ldots$ AF & & \\
\hline FILIC & BILICIUM & THWALTES EX J.D.HOOK. & SAPI & INDACEAE & $\overline{185}$ \\
\hline FILIP & BILIPENDULA & M!LL. & ROSA & ACEAE & $\overline{1} \overline{4} \overline{5}$ \\
\hline $\mathrm{FI} \mathrm{HE}$ & F. HEXAPETALA & $\begin{array}{l}\text { G GILIP. EX MAXIM. NOMEN SUPERfl. } \\
\text { Filipendula VULGaris }\end{array}$ & $F B G \ldots+\ldots \ldots \ldots \ldots \ldots \ldots * \ldots \ldots, P F$ & & \\
\hline F $1 M Q R$ & FIMARTSTYLIS & VAHL & $\overline{C Y P E}$ & ERACEAE & $\overline{37}$ \\
\hline- & F. ALAMOSANA & $\begin{array}{l}\text { FERNALD } \\
\text { ( FIMBRISTYLIS ANNUA }\end{array}$ & $* \cdots \cdots \cdots \cdots \cdots z \cdots \cdots \cdots \cdots$ AG & & \\
\hline FI AN & F. ANNUA & $\begin{array}{l}\text { CALL.)ROEM. B J.A. SCHULTES } \\
\text { N FIMERISTYLIS DICHOTOMA }\end{array}$ & 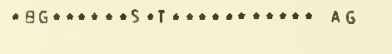 & ACCEPTEO & \\
\hline - & F. ANOMALA & $\begin{array}{l}\text { HOECK. } \\
\leqslant \text { FIMARISTYLIS SPATHACEA } \\
\text { C FIMBRISTYLIS PUBERULA }\end{array}$ & $\cdots 2 \cdots \cdots \cdots \cdots \cdots * \cdots \cdots \cdots P G$ & & \\
\hline - & F. ATOLLENSIS & $\begin{array}{l}\text { H.ST. JOHN } \\
6 \text { FIMERISIYLIS SPATHACEA }\end{array}$ & $\ldots \ldots \ldots \ldots+\ldots \ldots \ldots \ldots \ldots \ldots$ & & \\
\hline I AU2 & $\begin{array}{l}\text { F. AUTUMHALIS } \\
\text { MUCRONULAT }\end{array}$ & $\begin{array}{l}\text { (L.)ROEM. \& J.A.SCHULTES } \\
\text { (MICHX.)FERNALO }\end{array}$ & $F B G 2 C L M+\cdots 5+T 6 \ldots \ldots \ldots \ldots * A G$ & ACCEPTED & \\
\hline$\cdot$ & F. BALOWINIANA & $\begin{array}{l}\text { (J.A.SCHULTES) TORR. } \\
l=\text { EIMBRISTYLIS ANNUA }\end{array}$ & $F \cdots 2 \cdots \cdots \cdots \cdots \cdots \cdots \cdots A G$ & & \\
\hline 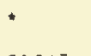 & F. CAPILLARIS & $\begin{array}{l}\text { CL EGRAY } \\
\text { AULEOSTYLIS CAPILLARIS }\end{array}$ & $\cdots \ldots \cdots \cdots \cdots \cdots \cdots, \ldots * A G$ & & \\
\hline FICAB & F. CAROLINIANA & $\begin{array}{l}\text { (LAM.) FERNALD } \\
\text { N FIMERISTYLIS PUEERULA INTERIOR }\end{array}$ & $F B G * A L \cdots S * T \cdots \cdots \cdots \cdots \cdots P G$ & ACCEPTED & \\
\hline FICAG & F. CASTANEA & $\begin{array}{l}\text { (MICHX.) VAHL } \\
\text { FIMERISTYLIS PUBERULA } \\
\text { FIMBRISTYLIS SPATHACEA }\end{array}$ & $F B G 2 \cdot L \cdots \cdot S \cdot T * \ldots \cdots \cdots \cdots P$ PEG & ACCEPTED & \\
\hline FICO & F. COMPLANATA & $\begin{array}{l}\text { (RETZ.)LINK } \\
\text { N FIMERISTYLIS OUINDUANGULARIS }\end{array}$ & $\cdots \ldots \ldots \ldots \ldots \ldots \ldots \cdots, P E G$ & ACCEPTED & \\
\hline 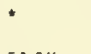 & F. CURASSAVICA & $\begin{array}{l}\text { (BRITTON)ALAIN } \\
\text { BULBOSTYLIS CURASSAVIGA }\end{array}$ & 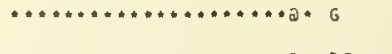 & & \\
\hline FICY & F. CYMOSA & $\begin{array}{l}\text { R.QR. } \\
=\text { FIMBRISTYLIS SPATHACEA }\end{array}$ & $* \ldots \ldots \ldots * \ldots \ldots \cdots * \cdots * a * P G$ & ACCEPTED & \\
\hline f I 01 & F. OICHOTOMA & $\begin{array}{l}\text { (LL SVAHL } \\
\text { FIMBRISTYLIS ANNUA }\end{array}$ & $\cdots \cdots C L M \cdots \cdots{ }^{\prime} \sigma \cdots \cdots \cdots a \cdot$ PAEG & ACCEPTEO & \\
\hline * & F. DIPHYLLA & $\begin{array}{c}\text { (RETZ.) VAHL } \\
l=\text { FIMBRISTYLIS OICHOTOMA }\end{array}$ & $* *+2 \ldots \cdots \cdots \cdots * * \cdots \cdots * \varepsilon \cdot$ & & \\
\hline * & F. DIVARICATA & W. HAM. & $\cdots \ldots \cdots \cdots+\cdots \cdots+\cdots \cdots+\cdots \cdots+\varepsilon$, & & \\
\hline - & F. ORIUMMONOII & $\begin{array}{l}\text { BOECK. } \\
\text { FIMERISTYLIS CAROLINIANA } \\
\text { FIMORISTYLIS SPADICEA }\end{array}$ & $F A n \ldots \ldots+\ldots \ldots \ldots \ldots \ldots \ldots \ldots$ & & \\
\hline * & F. GEMINATA & $\begin{array}{l}\text { (NEES) KUNTH } \\
\text { () FIMERISTYLIS AUTUMNALIS }\end{array}$ & $\cdots \cdots 2 \cdots \cdots \cdots \cdots \cdots \cdots \cdots \cdots+A G$ & & \\
\hline * & F. HARPERI & $\begin{array}{l}\text { ORITTON } \\
\text { FIMBRISTYLIS CAROLINIANA } \\
\text { FIMERISTYLIS SPATHACEA }\end{array}$ & $\cdots \cdots 2 \ldots \ldots \ldots \cdots \cdots \cdots \cdots \cdots * P G$ & & \\
\hline - & F. HIRTELLA & $\begin{array}{l}\text { W. BAM. NON VAHL } \\
\text { BULBOSTYLIS JUNCOIOES }\end{array}$ & 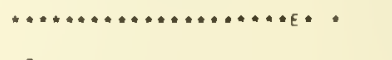 & & \\
\hline - & F. INTERIOR & $\begin{array}{l}\text { 3RITTON } \\
\text { F IMBRISTYLIS PUBERULA INTERIOR }\end{array}$ & $\|, 9 \ldots \ldots \ldots \ldots \ldots \ldots \ldots \ldots$ & & \\
\hline * & F. MONOSTACHYA & $\begin{array}{l}\text { (L.) IHASSK. } \\
\text { ( ABILDGAARDIA OVATA }\end{array}$ & $\cdots \ldots \ldots \ldots \ldots \ldots \ldots \ldots \cdots \cdot P \cdot P G$ & & \\
\hline 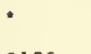 & F. PAPILLOSA & $\begin{array}{l}\text { (XUEKENTH.) ALAIN } \\
\text { (BULBOSTYLIS JUNCIFORMIS }\end{array}$ & 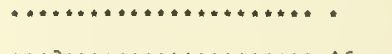 & & \\
\hline FIPE & F. PERPUSILLA & $\begin{array}{l}\text { R.M.HARPER EX SMALL \& BRITTON - } \\
\text { FIMBRISTYLIS VAHLII }\end{array}$ & $\cdots \cdots 2 \cdots \cdots \cdots \cdots \cdots \cdots \cdots \cdots \cdot A G$ & ACCEPTED & \\
\hline - & F. PILOSA N & $\begin{array}{l}\text { VAHL } \\
\text { N FIMBRISTYLIS ANNUA }\end{array}$ & $* *+\cdots+\cdots+\ldots \ldots \ldots+\cdots \cdots+\cdots$ & & \\
\hline - & F. PORTORICENSIS, & $\begin{array}{l}\text { (BEITTON)ALAIN } \\
\text { BULBOSTYLIS PAUCIFLORUS }\end{array}$ & $\ldots \ldots \ldots \ldots+\cdots \cdots \cdots \cdots \cdot P \cdot P G$ & & \\
\hline FIPU & F. PUAE RULA * & $\begin{array}{l}\text { cmichx. Jvahl } \\
\text { * fimeristylis castanea }\end{array}$ & $* *+2 \cdot L \cdots \cdots *+\cdots \cdots \cdots * \cdots *$ PEG & ACCEPTED & \\
\hline FIPUI & INTERIOR & $\begin{array}{l}\text { (ERITTONIKRAL } \\
\text { N FIMERISIYLIS CAROLINIANA } \\
\text { SIMBRISTYLIS SPADICEA }\end{array}$ & $\cdots \cdots \cdots \cdots \cdots \cdot \cdots+\cdots \cdots \cdots *$ PEG & ACCEPTED & \\
\hline fI SP & F. SPADICEA & 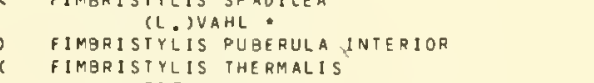 & $\cdots *[\ldots * * * * \ldots+\cdots * \cdots E \cdot P E G$ & ACCEPTED & \\
\hline$F \llbracket S P Z$ & F. SPATHAGEA & $\begin{array}{l}\text { ROIH } \\
\text { FIMERISTYLIS CASTANEA } \\
=\text { FIMBRISTYLIS CYMOSA }\end{array}$ & $\cdots \cdots * L \cdots \cdots \cdots \cdots \cdots \cdots E \cdot P G$ & ACCEPTEO & \\
\hline FITH & F. THERMALIS & $\begin{array}{l}\text { - fimaristilis spadicea } \\
\text { - fistis }\end{array}$ & $\cdots \cdots \cdots \cdots+2 N \cdot U \cdot 00 \cdots P G$ & ACCEPTED & \\
\hline FIVA & F. VAHL 11 & $\begin{array}{l}\text { (LAM. ) LINK } \\
\text { FIMARISTYLIS PERPUSILLA }\end{array}$ & $F B G 2 C \cdot M \cdots+T 62 \cdots \cdots O \cdots$ AEG & ACCEPTED & \\
\hline * & F. VESTITA & $\begin{array}{l}\text { (XUNTH)HEMSL. } \\
\text { a BULBOSTYLIS VESTITA }\end{array}$ & $\ldots \ldots \ldots \ldots \ldots \ldots \ldots \cdots \cdot A G$ & & \\
\hline F I RMI & FI RMIANA & MARSILI & STER & REULIACEAE & $\overline{198}$ \\
\hline$F I P L$ & F. PLATANIFOLIA = & $\begin{array}{l}\text { (L.F.) SCHOTT \& ENDL. } \\
=\text { FIRMIANA SIMPLEX }\end{array}$ & $\cdots 2 c \ldots \ldots \ldots \ldots \ldots \ldots \ldots+\tau^{\top}$ & & \\
\hline$:$ & $\begin{array}{l}\text { FISCHERIA } \\
\text { F. CRISPIFLORA }\end{array}$ & DC. & $\ldots \ldots \ldots \ldots \ldots \ldots \ldots \ldots{ }^{A S C L}$ & LEFIADACEAE & $\overline{2} \overline{72}$ \\
\hline * & FISHLOCKIA & QRITION \& ROSE & ALT. MIM & $\begin{array}{l}\text { UMINOSAE } \\
\text { MOSACEAE }\end{array}$ & $\overline{147}$ \\
\hline * & F. ANEGADENS IS & $\begin{array}{l}\text { a (ORITTON)BRITTON \& ROSE } \\
\text { aCACIA ANEGADENSIS }\end{array}$ & $\ldots \ldots * \ldots \ldots+\cdots+\ldots+\cdots+\cdots+\varepsilon *$ & & \\
\hline
\end{tabular}




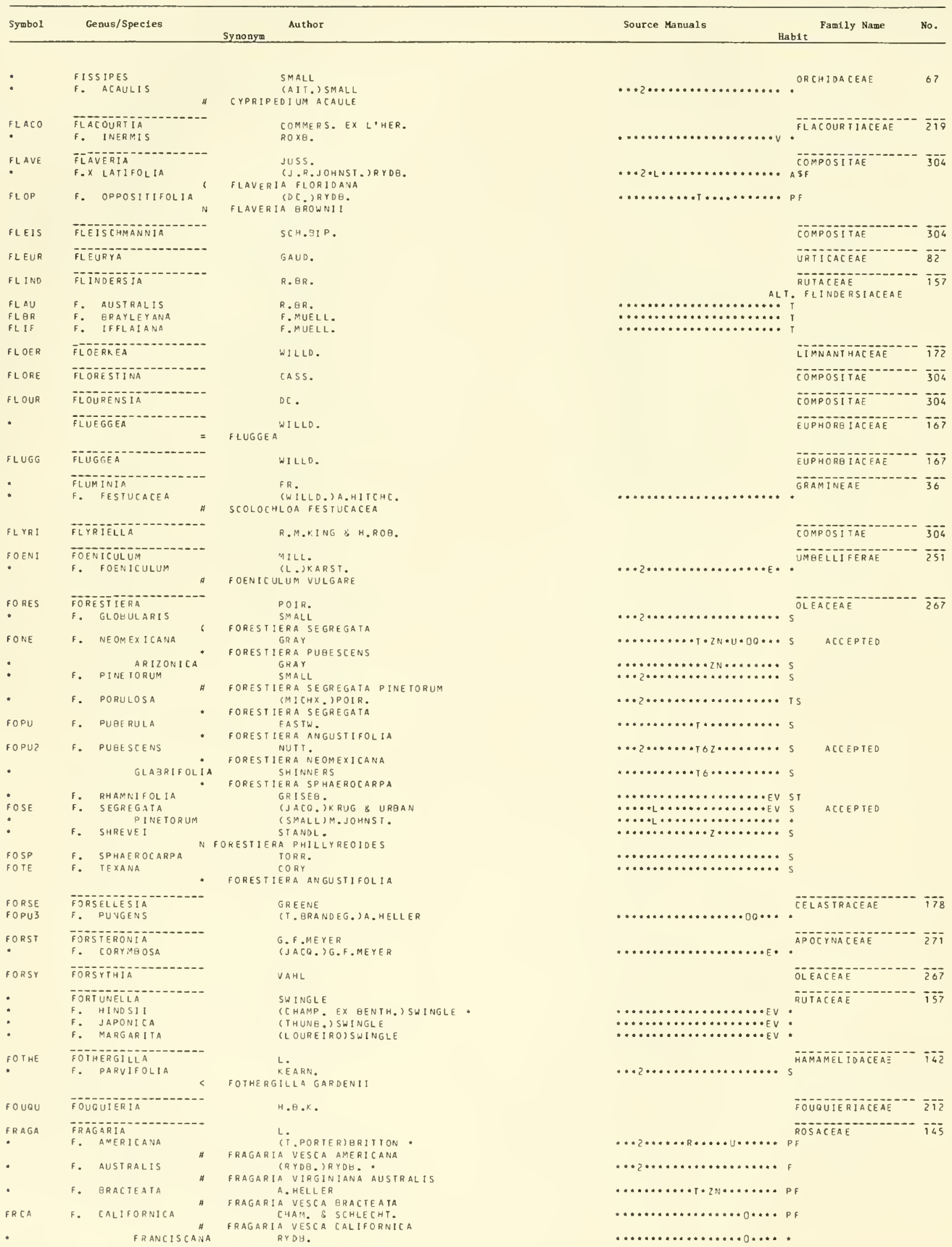


FRAG AR IA

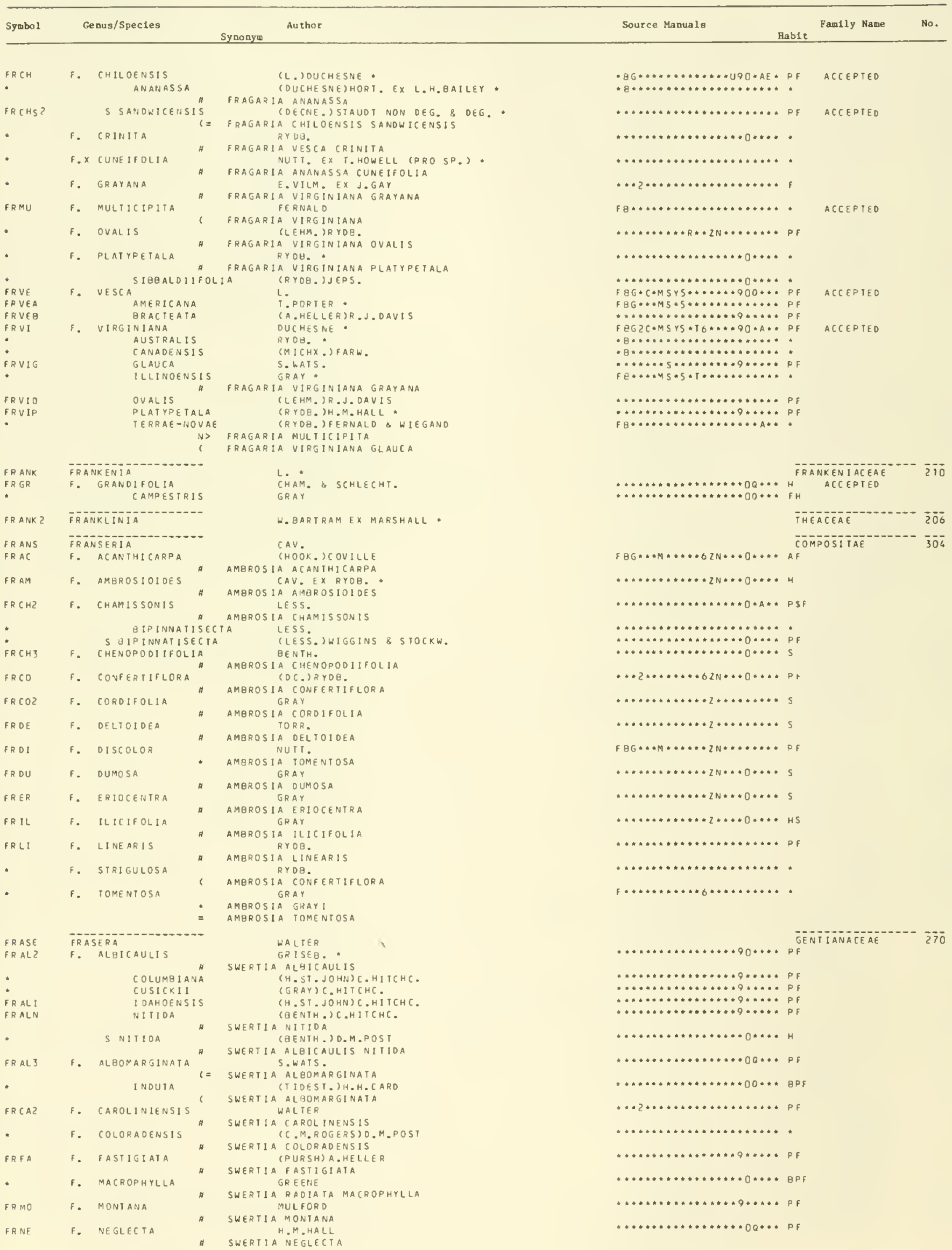




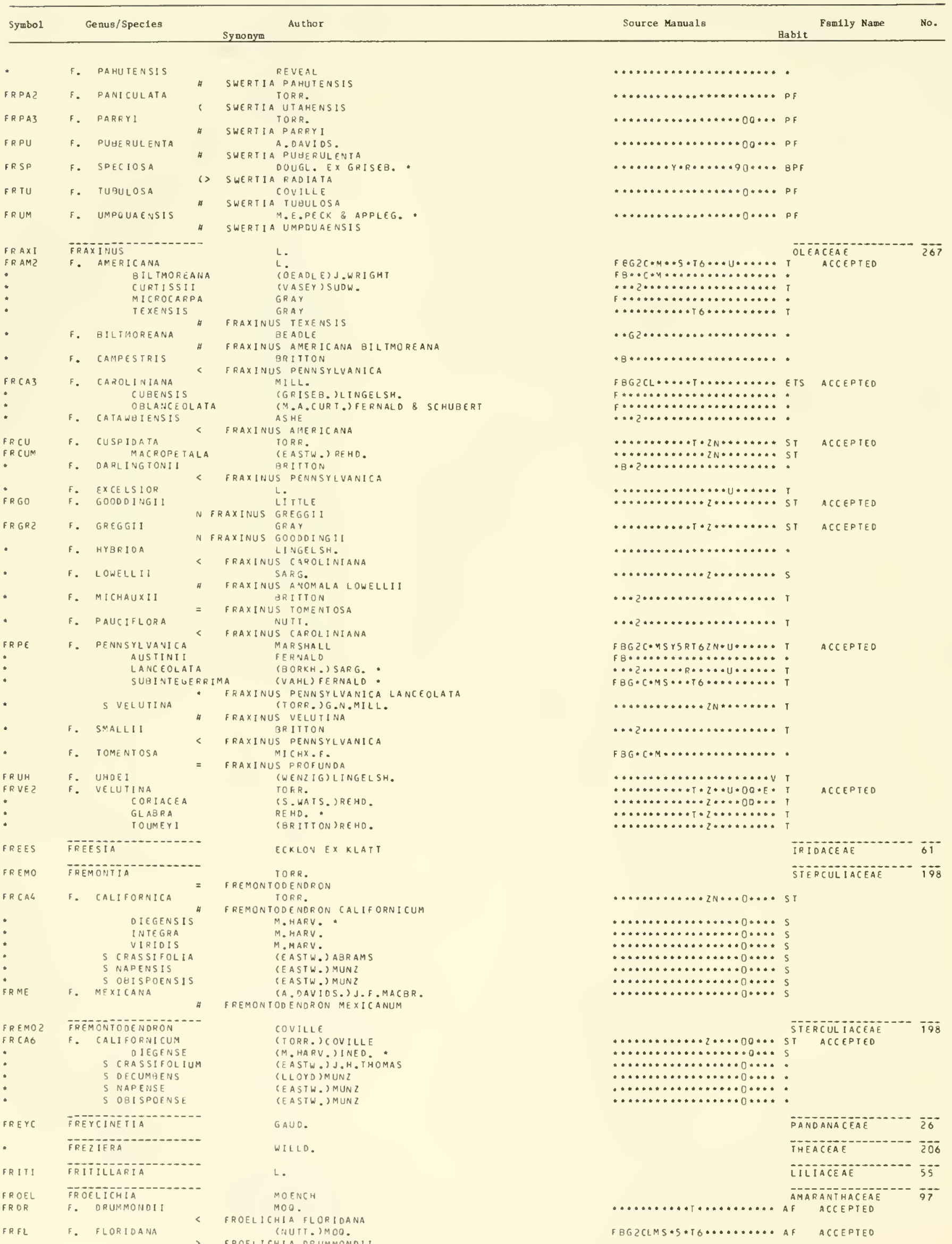




\begin{tabular}{|c|c|c|c|}
\hline Symbol & Genus/Species & & Syno aym \\
\hline FRYXE & FRYXELLIA & & D.N.BATES \\
\hline FUCHS & FUCHSIA & & L. \\
\hline FUIRE & FUIRENA & & ROITB. \\
\hline FUAR & F. GREVISETA & $<$ & $\begin{array}{l}\text { COVILLE } \\
\text { FUIRENA SQUARROSA }\end{array}$ \\
\hline • & F. HISPIDA & $<$ & $\begin{array}{c}\text { ELLIOTT } \\
\text { FUIRENA SQUARROSA }\end{array}$ \\
\hline FULO & F. LONGA & - & $\begin{array}{l}\text { CHAPM. } \\
\text { FUIRENA SCIRPOIDE }\end{array}$ \\
\hline FUPU & F. PUMILA & $=$ & $\begin{array}{l}\text { TORR. } \\
\text { FUIRENA SQUARROSA }\end{array}$ \\
\hline FUSC & F. SCIRPOIDEA & • & $\begin{array}{l}\text { MICHX. } \\
\text { FUIPENA LONGA }\end{array}$ \\
\hline fUSO & F. SOUARROSA & $\begin{array}{l}> \\
=\end{array}$ & $\begin{array}{l}\text { MICHX } \\
\text { FUIRENA BREVISETA } \\
\text { FUIRENA PUMILA }\end{array}$ \\
\hline
\end{tabular}

FUMAR FUMARIA

- f. martilali

F. OCGIOENTALIS

FUNASTRUM

F. CLAUSUM

F. CRISPUM

F. CYNANCHOIDES

SUBTRUNGATUM

F. HETEROPHYLLU,M

F. HIRTELLUM

FUNTU

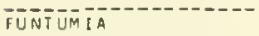

FURCE

FIRCRAEA

F. HEXAPETALA

F. MACROPHYLLA

FURERA

\section{GAHNIA}

\section{GAILLARDIA}

G. AESTIVALIS

FLAVOVIREN

G. CHRYSANTHA

G. DRUMMONDII

G. FASTIGIATA

G. GRACILIS

G. LANCEOLATA

FLAVOVIRENS

G. LUTEA

G. MEARNSI:

G. MULTICEPS

G. PICTA

G. PINNATIFIDA

G. PULCHELLA

G. SEROTI INUM

\section{GALAC GALACTIA}

GAGR G. GRAYI

GAMA G. MACREEI

GA VO

GA WF
CLAVAUD
L. EX W. O.MCDOUGALL INED. WOMEN SUPERFL.

FUMARI OFF ICINALIS

FOURN.

(JACQ.) S CHLECHIER

SARCOSTEMMA CLAUSUM

(OENTH.) SCHLECHTEF

SARCOSTEMMA CRISPUM

(DECNE.) SCHLECHIER

SARCOSTEMNA CYNANCHOIDES

(B.ROB. \& FERNALD) J.F.MACBR.

(ENGELM. ISTANDL.

SARCOSTEMMA CYNANCHOIOES HARTWEGII

(GRAY) SCHLECHTER

SARCOSTEMMA HIRTELLUM

ST APF

VENTENAT

( J ACQ.) SURBAN

C. BAY E R

POCAIS.

$$
\text { J. G.FORST. }
$$

FOUG.

(WALTER) H.ROCK

GAILLARDIA LANGEOLATA

(C. MOHR) CRONO.

GAILLARDIA AFSTIVALIS

CHOOK.)OC.

AILLARDIA PULCHELLA

GREENE

GAILLARUIA LANCEOLATA FASTIGIATA A. NELS.

GAILLARDIA PINNATIFIDA "II I CHX.

GAILLAROI A AESTIVALIS

GATLLAROTA MOHP

GAILLARDIA LUTEA

GREENE

GAILLAPDIA AESTIVALIS

RYD 3.

GAILLARDIA PINNATIFIDA

GREENE

GAILLARDIA PINNATIFIO S'NEET

GAILLARDIA PULCHELLA PICTA

TORR.

GAILLARDIA GRACILIS

GAILLARDIA MEARNSII

GAILLAROIA MULTICEPS

$$
\text { FOUG. }
$$

GAILLARDIA AMBLYODON

GAILLARDIA NEOMEXICANA

(WALTER) H.ROCK

GAILLARDIA LUTEA

$$
\text { P. BR. }
$$

VAIL NOMEN SUPERFL.

GALACTIA HETEROPHYLLA

M. A. CURI.

GALACTIA VOLUBILIS

(L.) BRITTON

GALACTIA WRIGHTI:

GALACTIA MACREEI

GRAY
Source Manuale

Hablt

Pam1ly Name

No.

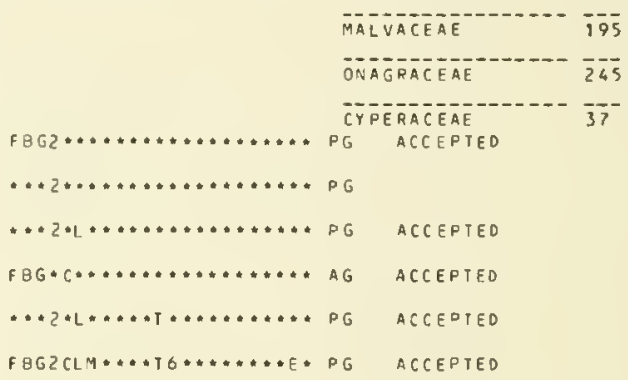
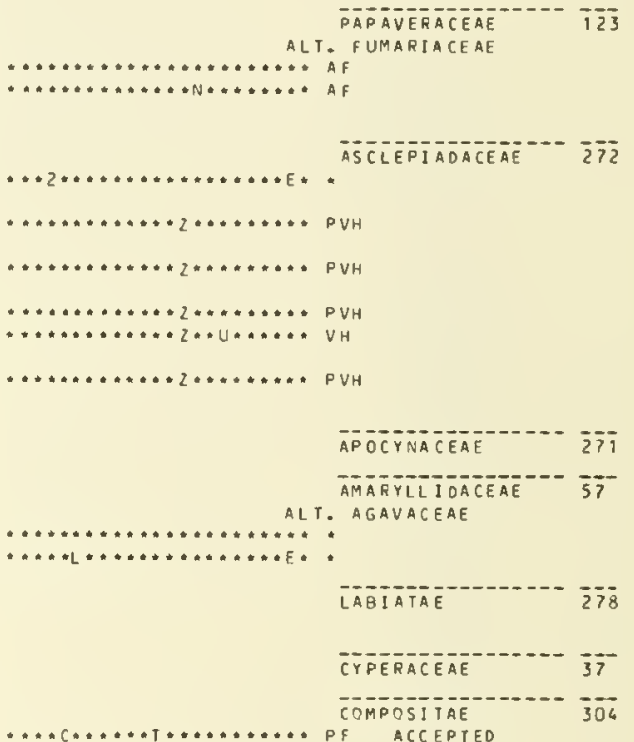

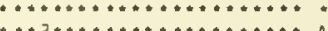

$\cdots+2 \ldots \ldots \ldots \ldots \ldots \ldots \ldots \ldots+\ldots A^{2}$

$\cdots * 2 * \ldots * \ldots * \ldots \ldots \ldots \ldots \ldots$ af

$\ldots \ldots \ldots \ldots \ldots+\ldots \ldots \ldots \ldots, p F$

$\ldots \ldots \ldots \ldots \ldots \ldots \ldots+\ldots \ldots$ PF ACCEPTED

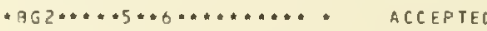

$\bullet 8 G \ldots \ldots \ldots \ldots \ldots+\cdots+\ldots+*$

$F \ldots \ldots \ldots \ldots \ldots+\ldots \ldots \ldots \ldots \ldots *$

................ PF ACCEPTED

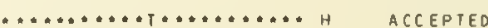

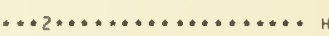

$\cdots \cdots \cdots+\ldots 2 N \cdot U \cdot \cdots$ PFH ACCEPTED

FEG*CLMS.5*T6ZN**00*\#AF ACCEPTED

LEGUMINOSAE

$\overline{147}$ ALT. PAPILIONACEAE

FBG CL * * $+\ldots \ldots \ldots \ldots$ PV ACCEPTED

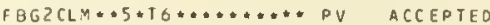

PVFH ACCEPTED 


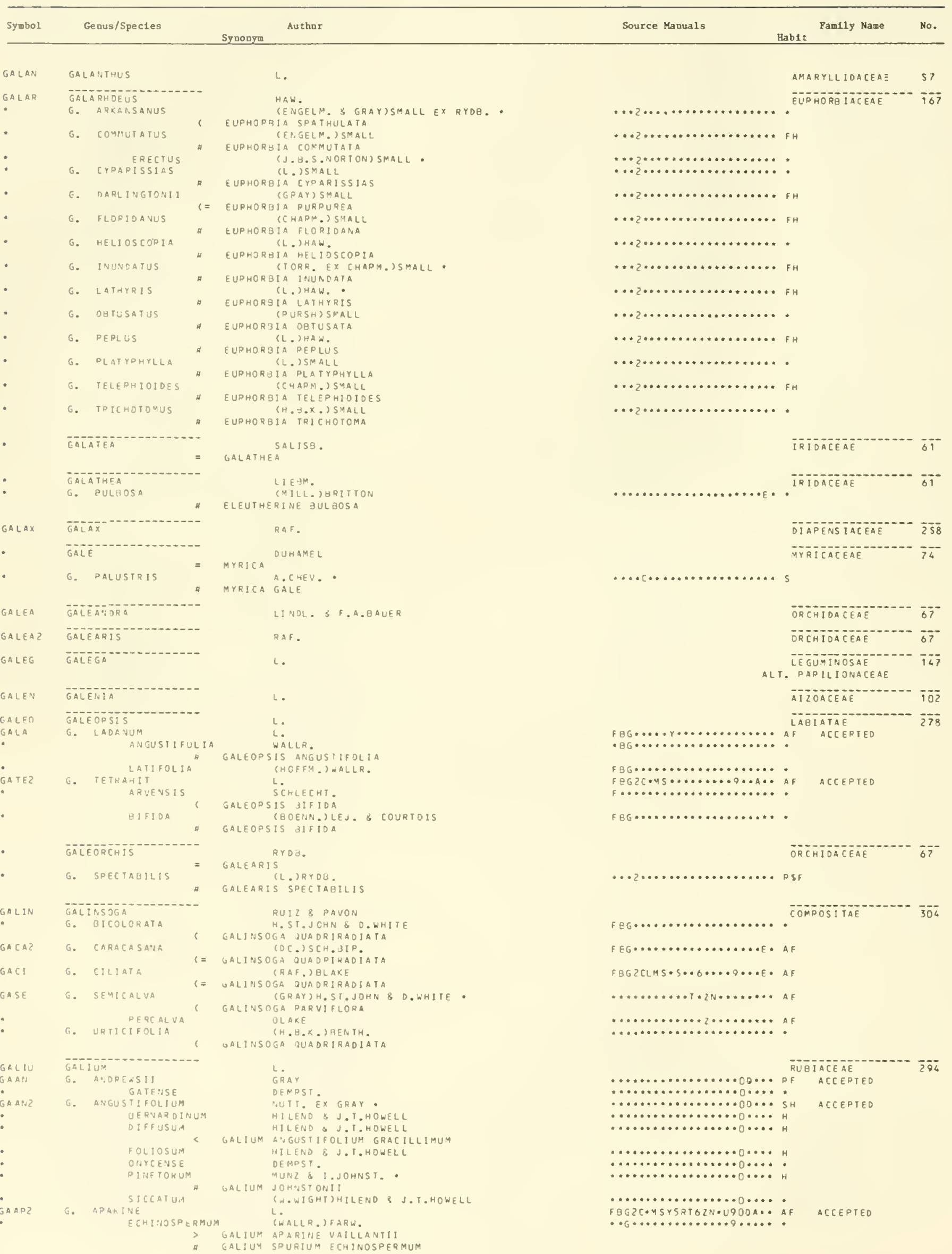




\begin{tabular}{|c|c|c|c|c|c|}
\hline Symbol & Geaus/Species & synonym & Au thor & Sourc & Fam1ly Name \\
\hline
\end{tabular}

VAILLAITIII

G. BERMUDENSE

GAB02 G. BOREALE * HYSSOPIFOLIUM

G. BRANDEGEI

G. BREVIPES

GA BRO

GACA 3

GACAL

*

GA CO2

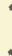

GA G

GAGR2

*

GAKA

GALAL

-

GAMA3

GAMAL

-

GAME3

GAMI

GAMO

Gamo

GAMUZ

GAMUS

-

•

$$
\begin{aligned}
& \text { G. CALIFORNICUM N } \\
& \text { IAIGUELENSE } \\
& \text { G. CATALINENSE } \\
& \text { OUXIFOLIUM }
\end{aligned}
$$$$
\text { G. CLAYTONII }
$$

G. COLORADOEISS

$$
\text { SCAARIUSCULUA }
$$

G. CRUCIATA

G. ERECTUM

G. FILIFOLIUM

G. GRAYANUM

$\#$

- KAMTSCHATICUM
OREGANUM

G. LATIFOLIUM

HISPIDUM

G. MAGNI FOLIUM

G. MATTHEWSI

MAGNIFOLIUM

A

G. MEXICANUM

G ASPERULUM

G. MICROPHYLLUIA

G. MOLLUGO

ERECTUA

G. MULififlorum

G. MUNZII

CARNEUA N

SUBALPINUM

G. NUTTALLII

$$
\text { OVALIFOLIUM }
$$$$
\text { OVALUE }
$$

G. OGTUSUIA

$$
\begin{aligned}
& \text { ND> } \\
& \text { FLORIOANUM } \\
& \text { RAMOSUM }
\end{aligned}
$$

G. PARISIENSE

LEIOCARPUM

G. PUEENS

GRANDE$$
\text { N> }
$$

G. StELLATUM

G. TINCTORIUM$$
\text { SUBGIFLORUM }
$$

G. TRICORNE

NS$$
\text { * Galium SCABRIUSCULUM }
$$

(OC.)W. KOCH

GRAY.

GALIUM MEXICANUM ASPERULUM

GALIUM MEXICANUM ASPERRIMUM

GALIUM HISPIDULUM

(HOFFM, ) DC

$D C$.

( GALIUM TRIFIDUM PUSILLUM

NS GALIUM IINCTORIUM SUBBIFLORUM

TINCTORIUM SUBBIFL

GALIUM IR!FIDUM

HOOK. \& ARN.

(GREENE) JEPS.

GRAY

(GREENE) DEMPST.

GALIUM उUXIFOLIUM

$$
\text { MI CHX. }
$$

GALIUM TINCTORI UM

W. WIGH I :
(EHREND. DEEMPST

GALIUM EMERYENSE

G. DIVARICATUM

(L.)SCOP.

LAM.

HUDS.

ENSE LEIOCARPUM

MOLLUGO EREGTUM

(WIEGANO) SHALL

HISPIDIFOLIUM

G. ROTHROCKII

N) GALIUM OI

GALIUM OIVARI CATUM

ANGUSTIF OLIUM GABRIELENSE

EHRENO.

EHREND.

STELLER EX J.A. \& J.H.SCHULTES *

(BRITTON) PIPER

GALIUM OREGANUM

MI CHX.

SMALL EX BRITTON NOMEN SUPERFL. -

SMALL

COEMPST . IOEMPST.

GALIUM MUNZII

GRAY:

GALIUM MAGNIF OLIUM

GALIUM MUNZI

$$
\text { H. B.K. - }
$$

(GRAY)DEMPST.

URAY

RELEUNIUM MICROPHYLLUM

(HUOS. JOOMIN \& PODP.

KE LLOG G

GALIUM WATSONII

HILENO \& J.T. HOWELL

GALIUM MAGNIFOLIUM

HILEND \& J.T. MOWELL

GALIUM HILENDIAE CARNEUM

OEMPST.

GALIUM HILENDIAE KINGSTONENSE

HILENO \& J.T. HOWELL

GALIUM MYPOTRICHIUM SUBALPINUM

GRAY

DEMPST .

DEMPST:

BI GEL.

GALIUM TINCTORIUM

(WIEGAND)FERNALO

GLEASON

(WIEGANO) PUFF

- I USCH

BOLANDER

(MCCLAT.) IGRAY

GALIUM GRANDE

GRAY

( GALIUM WRIGHTII

(EHREND. JDEMPST. \& EHREND.

GALIUM EMERYENSE

HILEND \& J.T. HOWELL

GALIUM ${ }^{\circ}$

OBTUSUM

$$
\text { OIVERSIFOLIUM GALIUM }
$$

W. WIGHT.

WI EGAND

(WIEGAND)FERNALO

J.STOKES NOMEN SUPERFL.

GALIUM TRICORNUTUM

G. TRIFIOUM N GALIUM GREVIPES

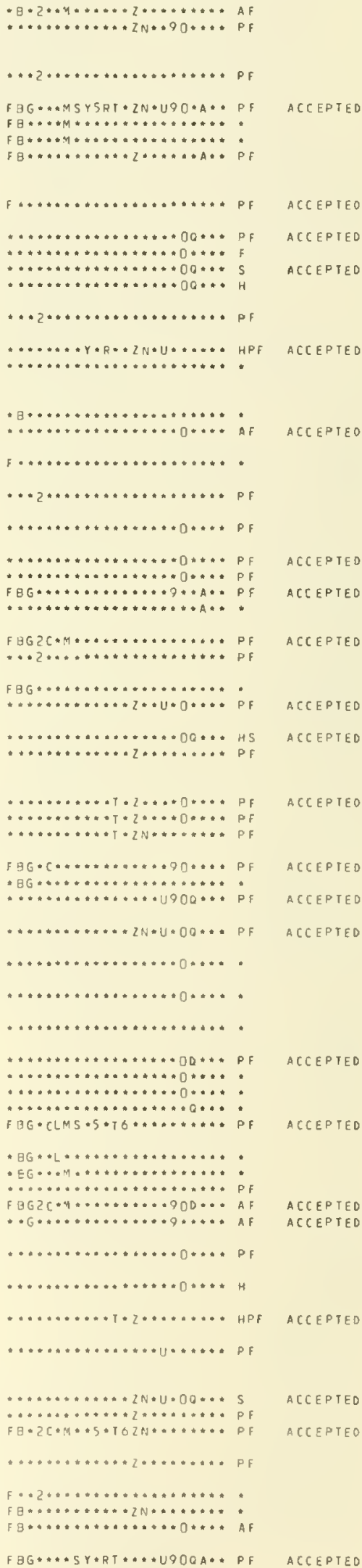




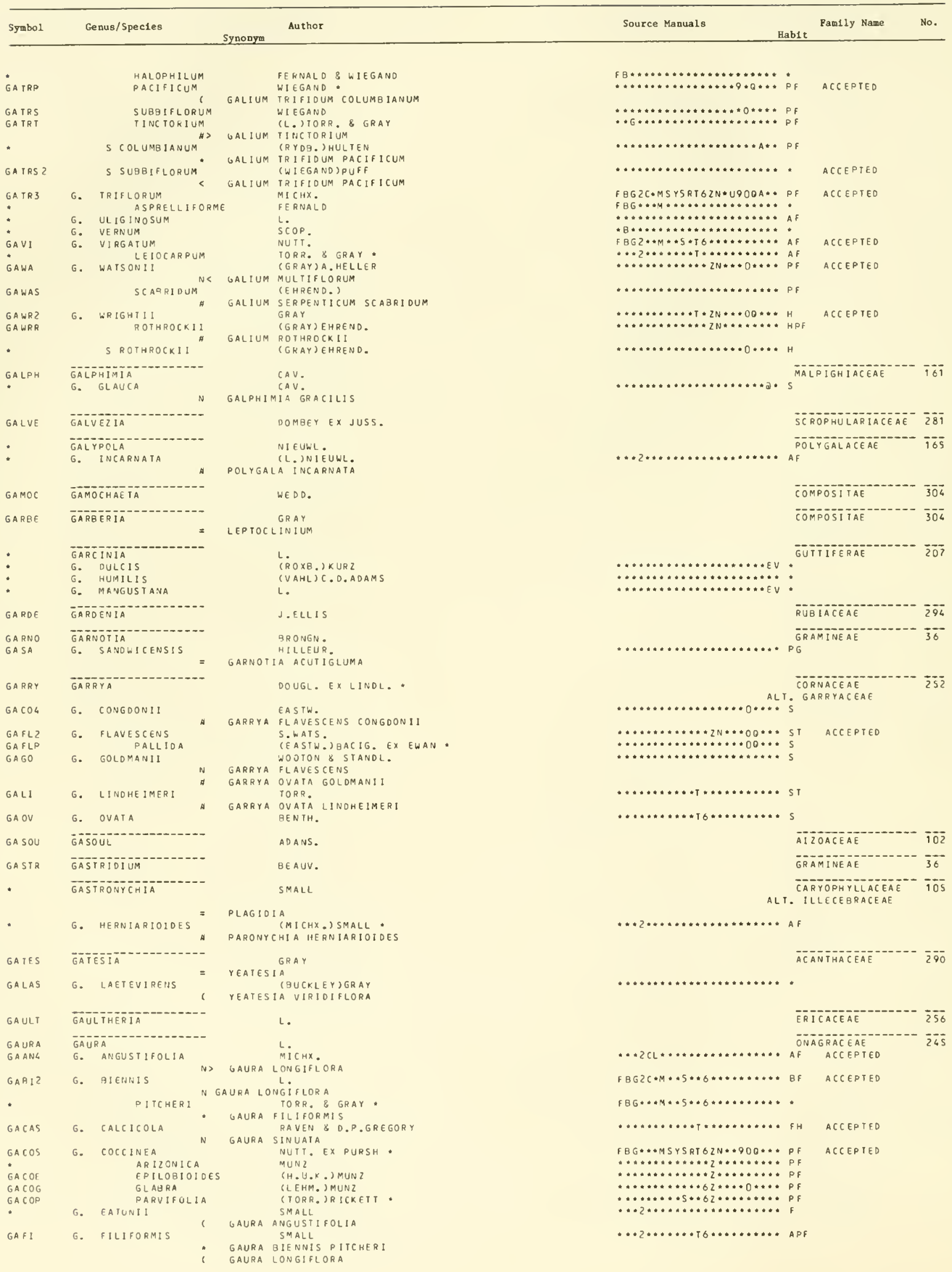




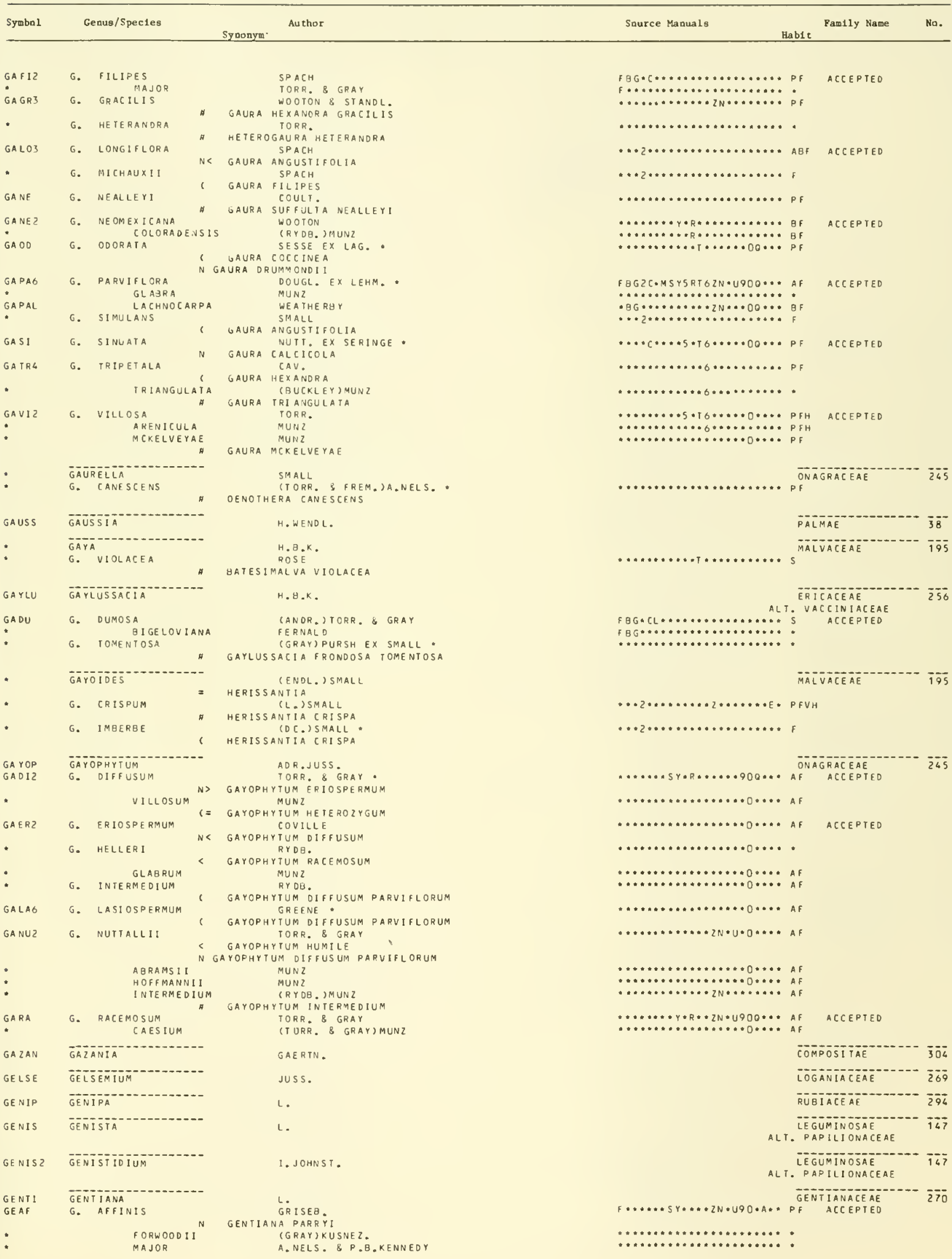




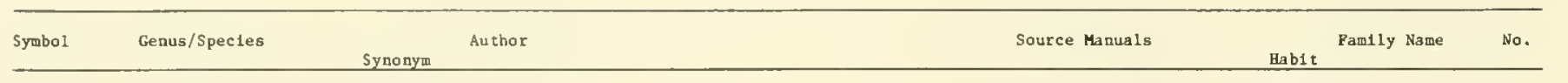

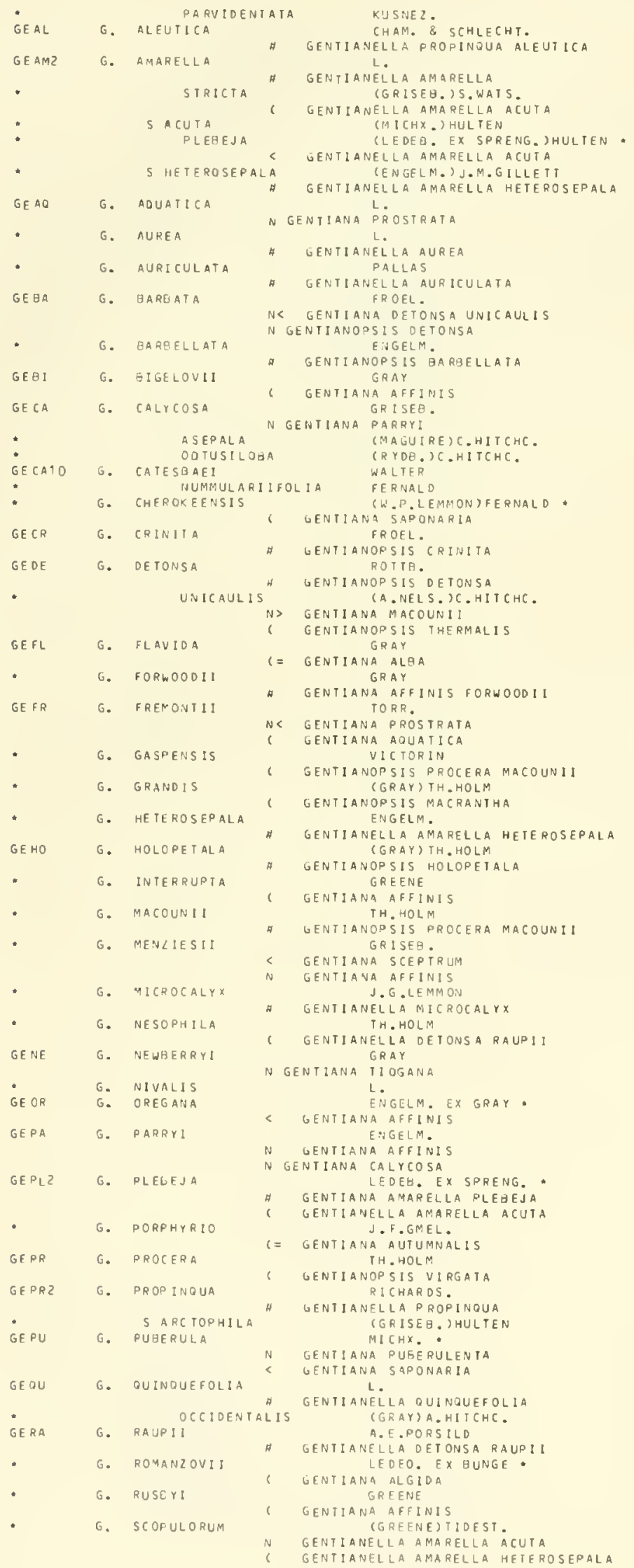

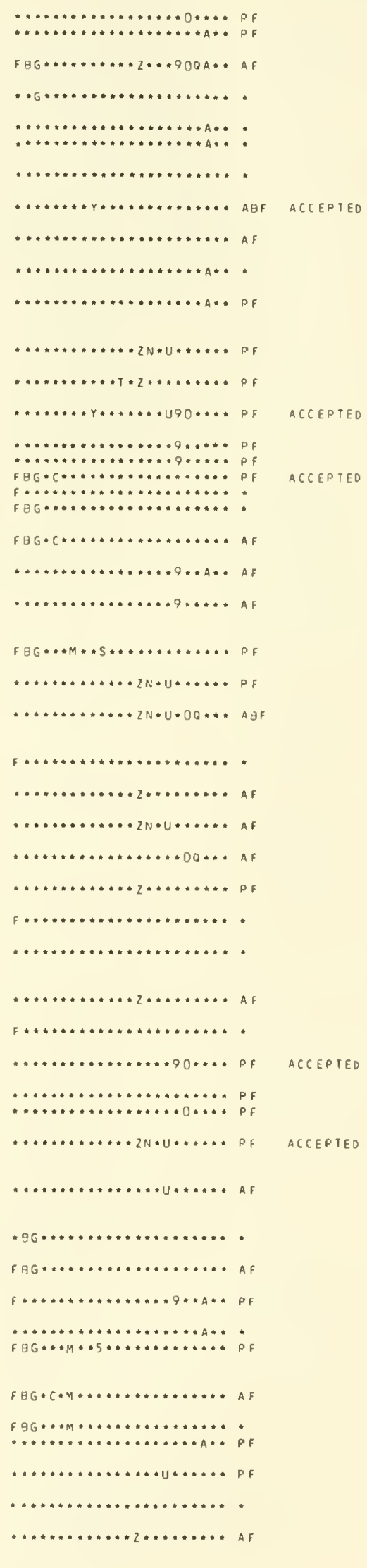


GENT I ANA

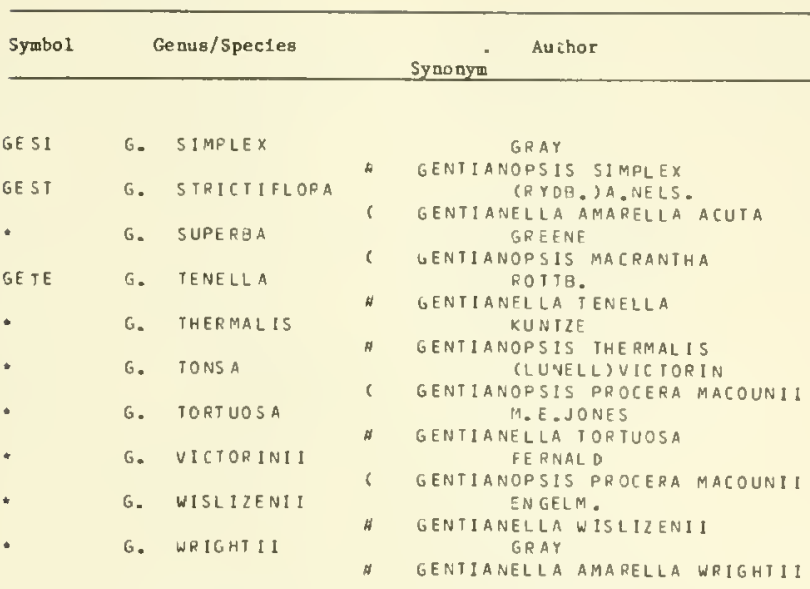

- G. AUREA

g. Sarbellata

G. DETONSA

S ELEGANS

5 RAUP1I

5 SUPERBA

6. OCCIDENTALIS

6. PROPINQUA

$$
\text { ALEUT ICA }
$$

GENTIANOUES

G. ALGIDA

GENITANOPSIS
G. PROLERA

G. PROLERA

GEOBALANUS

G. OBLONGIFOLIUS

G. PALLIDUS

GEOCA

GE OCA

GEOLAULON

GEOFFEA

GEOFFROEA

G. INERMIS

GE OPH

GFOPHILA

G. REPENS

\section{GE OPRUMNON}

G. CRASSICARPUM

G. PLAItense

G. TENNESSEENSE

GERAE

GE $\bar{R} \bar{A} \bar{E} \bar{A}$

GERANIUM

G. ATropurpureum

G. CAESPITOSUM

PARRYI

G. CAROLINIANUM

GE CAS

GE CAC

GEER

GEER2

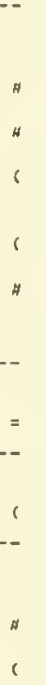

MOENCH

(ENGELM.) J.M, GILLETT

GENTIANOPSIS BARBELLATA

(ROITB.) G.DON

GENI I ANC

(A.NELS.)J.M. GILLET

GENTIANOPSIS THERMAL IS

(A.E.PORSILD) J.M.GILLETT

G RENE) J.M.GILLETT

OPSIS MACRANTH

(GRAY) SMALL
GENIIANELLA OUINQUEFOLIA OCCIDENTALIS

(RICHARDS,) J. M GILLETT

(CHAM, \& SCHLECHT.) WELSH

LOEVE \& LOEVE

(PALLAS)LOEVE \& LOEVE

GENTIANA ALGIDA

$$
M A
$$

(TH.HOLM)MA

GENTIANOPSIS VIRGATA

SMALL

(M! CHX.) SMALL *

LICANIA MI I HAUXII

SMALL.

LICANIA MICHAUXII

MACKENZ.

FERNAL D

SPRENG.

GE OFFROEA

\section{$J A C Q$.}

W. WRIGHT

ANDIRA INERMIS

$0.00 \mathrm{~N}$

(L.) I. JOHNST.

GEOPHILA HERUACEA

\section{RYDB.}

(NUTT.) RYDB.
ASTRAGALUS CRASSIGARPUS

(NUTT, RYDB.

ASTRAGALUS PLATTENSIS

IGRAY EX CHAPM. JRYOB.

ASTRAGALUS TENNESSEENS IS

$$
\begin{aligned}
& \text { TORR. \& GRAY } \\
& \text { L. } \\
& \text { A. HELLER } \\
& \text { EM CAESPITOSUM } \\
& \text { E. JAMES } \\
& \text { UM ATROPURPUREUM } \\
& \text { UM EREMOPHILUM } \\
& \text { CENGELM. IW. WEBER }
\end{aligned}
$$

GERANIUM CAESPITOSUM

GERANIUM ATROPURPUREUM

GERANIUM EREMOPHILUM

GERANIUM FREMONT I :

GERANIUM PARRYI

GERANIUM SPHAEROSPERMUM

$$
\begin{aligned}
& \text { CONFERTIFLORUM TERALI FE } \\
& \text { LONGIPES S. WATS. }
\end{aligned}
$$

G. Geranium BicknelLi l LONGipes

6. ERIAITHUM GERANIUM CAESPITOSUM

GERANIUM PRATENSE

Source Manuals

Fomily Name

No.

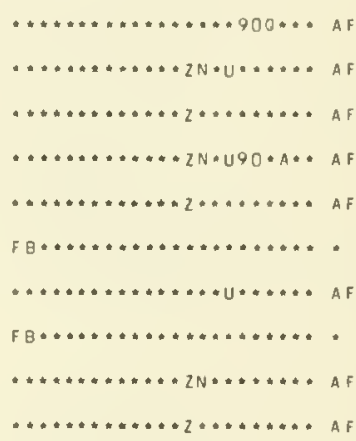

GENTIANACEAE

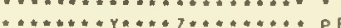

$\ldots \ldots \ldots+\cdots+\cdots+\ldots \ldots$ AF

$\ldots \ldots \ldots+\ldots \ldots+\ldots \ldots \ldots$ AF

$\cdots \ldots \ldots+\ldots \ldots \ldots \ldots \ldots \ldots+\cdots$

$\ldots+2 \ldots * \ldots * \ldots \ldots \ldots \ldots \ldots$ APF

$\ldots \ldots \ldots \ldots \ldots \ldots \ldots \ldots \ldots$ AF ACCEPTED

+....................

GENTIANACEAE

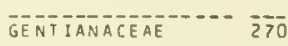

ROSATEAT

ALT. CHRYSOBALANACEAE

$\ldots$ S

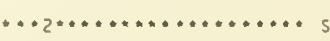

AIZOACEAE

SANTALACEAE

LEGUMINOSAE

ALT. PAPILIONACEAE

ALTEONOSAE

ALT. PAPILIONACEAE

RUBIATEAE

LEGUMINOSAE

ALT. PAPILIONACEAE

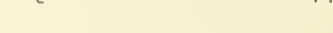

$P F$

$P F$
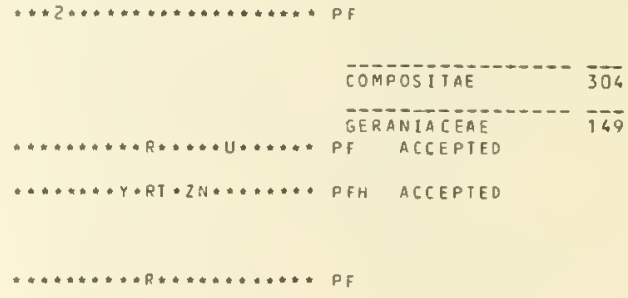

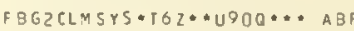

$F B G \ldots+\ldots \ldots \ldots \ldots \ldots \ldots$ AF

$\cdots+\ldots+\ldots \ldots \ldots+\ldots$

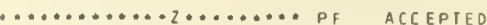

$\ldots \ldots \ldots \ldots \ldots \ldots \ldots \ldots+\ldots \ldots$ 


\begin{tabular}{|c|c|c|c|c|c|c|c|}
\hline Symbol 1 & & Genus/Species & Synonyw & Source Maouals & Bab1t & Famtly Name & No. \\
\hline GEFR? & G. & FREMONTII & $\begin{array}{l}\text { TORR. EX GRAY * } \\
\text { * GERANIUM CAESPITOSUM } \\
\text { GERANIUM PARRYI }\end{array}$ & 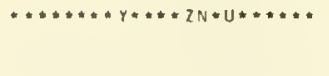 & PF & ACCEPIED & \\
\hline GEFRC & & C OWENI I & (RYDB.) H.D. HARRINGT. * & 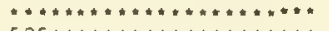 & PF & & \\
\hline : & G. & $\begin{array}{l}\text { NEPA LENSE } \\
\text { THUNBERGII }\end{array}$ & $\begin{array}{l}\text { SWEET } \\
\text { (SIEBOLD \& ZUCCAR.)KUDO }\end{array}$ & 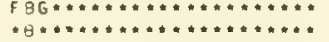 & PF & & \\
\hline - & G. & NERVOS UM & $\begin{array}{l}\text { RYDB. } \\
\text { GERANIUM VISCOSISSIMUM NERVOSUM }\end{array}$ & 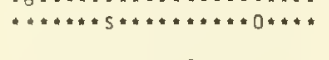 & PF & & \\
\hline GEPAZ & G. & $\begin{array}{l}\text { PARRYI } \\
\text { STRIGOSIUS }\end{array}$ & $\begin{array}{c}\text { (ENGELM.) A. HELLER } \\
\text { " GERANIUM FREMONTII } \\
\text { H. ST. JOHN }\end{array}$ & $\cdots \cdots+\cdots \cdots \cdots+2 N * U * \cdots \cdots * *$ & PF & ACCEPTED & \\
\hline - & G. & STRIGOSIUS & $\begin{array}{l}\text { H.SI.JOHN } \\
\text { L. GERANIUM VISCOSISSIMUM NERVOSUM }\end{array}$ & $\ldots \ldots \ldots \ldots \ldots \ldots \ldots \ldots \ldots \ldots \ldots+$ & A F & & \\
\hline Gerar & $G$. & ACUTA & $\begin{array}{l}\text { BENTH. } \\
\text { (PENNELL) PENNELL }\end{array}$ & 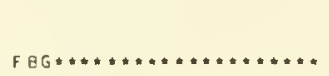 & $S C R O$ & OPHULARIACEAE & 281 \\
\hline GE AP & G. & APHYLLA & $\begin{array}{c}\text { AGALINIS ACUTA } \\
\text { NUIT. }\end{array}$ & $\ldots \ldots \ldots \ldots \ldots \ldots, \ldots, \ldots \ldots \ldots$ & AF & & \\
\hline GEAS & G. & ASPERA & $\begin{array}{l}\text { * AGALINIS APHYLLA } \\
\text { DOUGL. EX BENTH. } \\
\text { AGALINIS ASPERA }\end{array}$ & $F B G * \cdots M * 5 * * 6 * \cdots * \cdots * * *$ & AF & & \\
\hline GE AU & G. & AURICULATA & $\begin{array}{l}\text { MICHX. } \\
\text { TOMANTHERA AURI CULATA }\end{array}$ & $F * * * * 4 * 0, * 6 * * * * \cdots \cdots$ & AF & & \\
\hline * & G. & CADDOENSIS & $\begin{array}{l}\text { (PENNELL)PENNELL. } \\
\text { A AGALINIS (ADDOENSIS }\end{array}$ & $\cdots \cdots+\ldots+\ldots+\cdots+\cdots, \ldots+\cdots$ & $\cdot$ & & \\
\hline * & G. & $C A L Y \operatorname{COS} A$ & $\begin{array}{l}\text { (MACKENZ, \& BUSH)FERNALD } \\
\text { a AUREOLARIA CALYCOSA } \\
\text { K AUREOLARIA FLAVA }\end{array}$ & $F \cdots \cdots \cdots+\cdots \cdots+\cdots+\cdots *$ & $\cdot$ & & \\
\hline * & G. & DECEMLOQA & $\begin{array}{l}\text { GREENE } \\
\text { AGALINIS DECEMLOBA }\end{array}$ & $F B * \ldots \ldots \ldots \ldots \ldots * \ldots \ldots * \cdots$ & • & & \\
\hline GE DEZ & G. & DE NS IFLORA & $\begin{array}{l}\text { BENTH. } \\
\text { TOMANIHERA DENSIFLORA }\end{array}$ & $\cdots \cdots \cdots M \cdots 5 \cdots 6 \cdots \cdots \cdots \cdots \cdots *$ & A F & & \\
\hline GED12 & G. & DISPERSA & $\begin{array}{l}\text { (SMALL)K.SCHUM. } \\
\text { AUREOLARIA FLAVA }\end{array}$ & $\ldots \ldots \ldots \ldots \ldots \ldots+\ldots, \ldots+\ldots+\cdots$ & Af & & \\
\hline GEED & G. & EDWARDSIANA & $\begin{array}{l}\text { (PENNELL) PENNELL } \\
\text { A AGALINIS EDWARDSIANA }\end{array}$ & 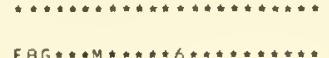 & AF & & \\
\hline GEFA & G. & FASCICULATA & $\begin{array}{c}\text { ELLIOTT } \\
\text { * aGALINIS FASCICULATA }\end{array}$ & 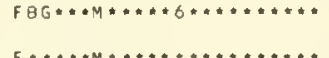 & AF & & \\
\hline • & G. & FLAVA & $\begin{array}{l}\text { LE } \\
\text { We AUREOLARIA FLAVA }\end{array}$ & 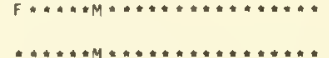 & $*$ & & \\
\hline$\cdot$ & & $C A L Y \operatorname{COS} A$ & $\begin{array}{l}\text { (MACKENZ. \& BUSH)STEYERM. } \\
\text { 4 AUREOLARIA (ALYCOSA }\end{array}$ & 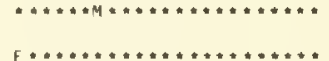 & $*$ & & \\
\hline : & & $\begin{array}{l}\text { MACRANTHA } \\
\text { RETICULATA }\end{array}$ & $\begin{array}{l}\text { (PENNELL)FERNALD } \\
\text { (RAF.) CORY }\end{array}$ & 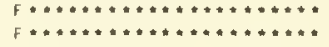 & $\therefore$ & & \\
\hline - & G. & GATESII < & $\begin{array}{l}\text { (BENTH.) PENNELL } \\
\text { AGALINIS SETACEA }\end{array}$ & $\ldots \ldots \ldots+\cdots+\ldots \ldots+\cdots+\cdots * *$ & * & & \\
\hline GEGA & G. & GAITI I GER I & $\begin{array}{l}\text { SMALL } \\
\text { AGALINIS GATTINGER : }\end{array}$ & $F B G \cdots M \cdot \cdots 5 \cdots 6 \cdots \cdots \cdots \cdots$ & AF & & \\
\hline GEGR & G. & $\begin{array}{l}\text { GRANDIFLORA } \\
\text { CI UEREA }\end{array}$ & $\begin{array}{l}\text { BENTH. } \\
\text { AUREOLAR! A GRANDIFLORA } \\
\text { (PENNELL) CORY }\end{array}$ & 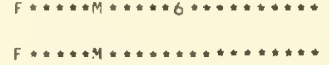 & $P F$ & & \\
\hline$\dot{*}$ & & $\begin{array}{l}\text { CI VEREA } \\
\text { PULCHRA }\end{array}$ & $\begin{array}{l}\text { (PENNELL) CORY } \\
\text { (PENNELL) FERNALO }\end{array}$ & $F \ldots \ldots M \ldots \ldots \ldots \ldots$ & - & & \\
\hline * & G. & HARP ER I & $\begin{array}{l}\text { (PENNELL) PENNELL } \\
\text { AGALINIS HARPERI }\end{array}$ & $\cdots \cdots \cdots \ldots \ldots \ldots \ldots \ldots \ldots \ldots$ & $\cdot$ & & \\
\hline GE HE & G. & HE TE ROPHYLLA & $\begin{array}{l}\text { NUTT } \\
\text { AGALINIS HETEROPHYLLA }\end{array}$ & 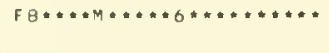 & $A F$ & & \\
\hline GE HOZ & G. & HOMALANTHA & $\begin{array}{l}\text { (PENNELL)PENNELL } \\
\text { AGALINIS TENUIFOLIA }\end{array}$ & $\ldots \ldots \ldots+\ldots+6, \ldots+\ldots+\cdots$ & AF & & \\
\hline - & G. & LAEVIGATA & AUREOLARIA LAEVIGATA & $F \cdots \ldots \ldots \ldots \ldots \ldots \ldots+\cdots \ldots * *$ & • & & \\
\hline GE LI & G. & LINI FOLIA & $\begin{array}{l}\text { NUTT. } \\
\text { AGALINIS LINIFOLIA }\end{array}$ & $F \in G * \ldots \ldots \ldots \ldots \ldots \ldots \ldots * *$ & PF & & \\
\hline - & G. & LOHE IF OLIA & $\begin{array}{l}\text { NUTT. } \\
\text { AGALINIS NUTTALLII }\end{array}$ & $* * * * * * * \cdots * * b * * * * * * * * *$ & $\bullet$ & & \\
\hline GEMA3 & $G$. & MARITIMA & $\begin{array}{l}\text { RAF. } \\
\text { AGALINIS MARITIMA }\end{array}$ & $F B G \ldots \ldots \ldots+\cdots+\ldots \ldots * \cdots$ & AF & & \\
\hline • & & GRANDIFLOR & RA BENTH. & 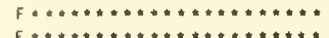 & : & & \\
\hline * & G. & NEOS COTICA & $\begin{array}{l}\text { GREENE } \\
\text { AGALINIS NEOSCOTICA }\end{array}$ & $F * \ldots+\ldots+\ldots+\ldots+\ldots \ldots+*$ & • & & \\
\hline . & G. & OBTUSIFOLIA & $\begin{array}{l}\text { (RAF.) PE NNELL } \\
\text { AGALINIS OBTUSIFOLIA }\end{array}$ & $F B G \ldots \ldots \ldots \ldots \ldots \ldots \ldots \ldots \ldots$ & $\cdot$ & & \\
\hline * & G. & PATULA & $\begin{array}{l}\text { (CHAPM.) GRAY } \\
\text { AUREOLARIA PATULA }\end{array}$ & $\ldots \ldots \ldots \ldots \ldots \ldots \ldots \ldots \ldots \ldots$ & - & & \\
\hline * & G. & PAUPER C ULA & $\begin{array}{l}\text { (GRAY) BRITTON } \\
\text { AGALINIS PAUPERCULA }\end{array}$ & 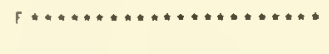 & - & & \\
\hline * & & OOREALIS & (PENNE LL) DEAM & $F \ldots \ldots \ldots \ldots \ldots \ldots \ldots \ldots$ & * & & \\
\hline - & G. & PECTINATA & $\begin{array}{l}\text { (NUTT.) BENTH. } \\
\text { AUREOLARIA PECTINATA }\end{array}$ & 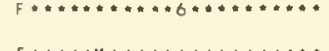 & 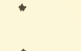 & & \\
\hline • & G. & PEDICULARIA & * AUREOLARIA PEDICULARIA & $F * * * * M * * \cdots * * \ldots \ldots \ldots \ldots$ & $\cdot$ & & \\
\hline - & & AMOIGENS & FERNALD & $f \ldots \ldots \ldots \ldots \ldots \ldots \ldots \ldots$ & $\cdot$ & & \\
\hline - & & AUSTROMONT & ( $P$ ENNELL) FERNALD & $F \cdots * \ldots \ldots * \ldots * \ldots * \ldots \ldots * * *$ & - & & \\
\hline - & & INTERCEDEN & ( $P$ ENNELL) FERNALD & $F \cdots \ldots \ldots \ldots \ldots \ldots \ldots \ldots \ldots \ldots$ & $\cdot$ & & \\
\hline - & & PECTINATA & NUIT & 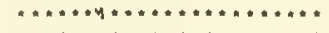 & $\cdot$ & & \\
\hline - & G. & PLURENETII & $\begin{array}{c}\text { ELLIOTT } \\
\text { AGALINIS PLUKENEIII } \\
\text { AGALINIS SEIACEA }\end{array}$ & $\ldots \ldots \ldots \ldots \ldots \ldots \ldots \ldots+\cdots$ & $\cdot$ & & \\
\hline - & G. & PSEUDAPHYLLA & $\begin{array}{c}\text { A AGALINIS SETACEA } \\
\text { (PENNELL)PENNELL } \\
\text { (PGALINIS PSEUDAPHYLLA }\end{array}$ & $\ldots \ldots \ldots \ldots \ldots \ldots \ldots \ldots$ & - & & \\
\hline GEPU3 & G. & PURPUREA & $\begin{array}{l}\text { L. } \\
\text { AGALINIS PURPUREA }\end{array}$ & $F B G \ldots \ldots n * 5 * \ldots * \ldots \ldots \ldots *$ & AF & & \\
\hline - & & NEOSCOTICA & $\begin{array}{l}\text { (GREENE) GLEASON } \\
\text { AGALINIS NEOSCOTICA }\end{array}$ & $* \theta G \ldots \ldots \ldots \ldots \ldots \ldots \ldots \ldots * *$ & * & & \\
\hline GE PUP & & PARVIFLORA & BENTH. & 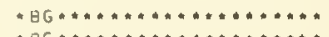 & AF & & \\
\hline$\cdot$ & & $\begin{array}{r}\text { RACEMULOSA } \\
=\end{array}$ & $\begin{array}{l}\text { \{PENNELL\}GLEASON } \\
=\text { AGALINIS VIRGATA } \\
=\text { GERARDIA RACEMULOSA }\end{array}$ & $\theta g_{\ldots} \ldots \ldots \ldots \ldots \ldots \ldots \ldots$ & - & & \\
\hline
\end{tabular}




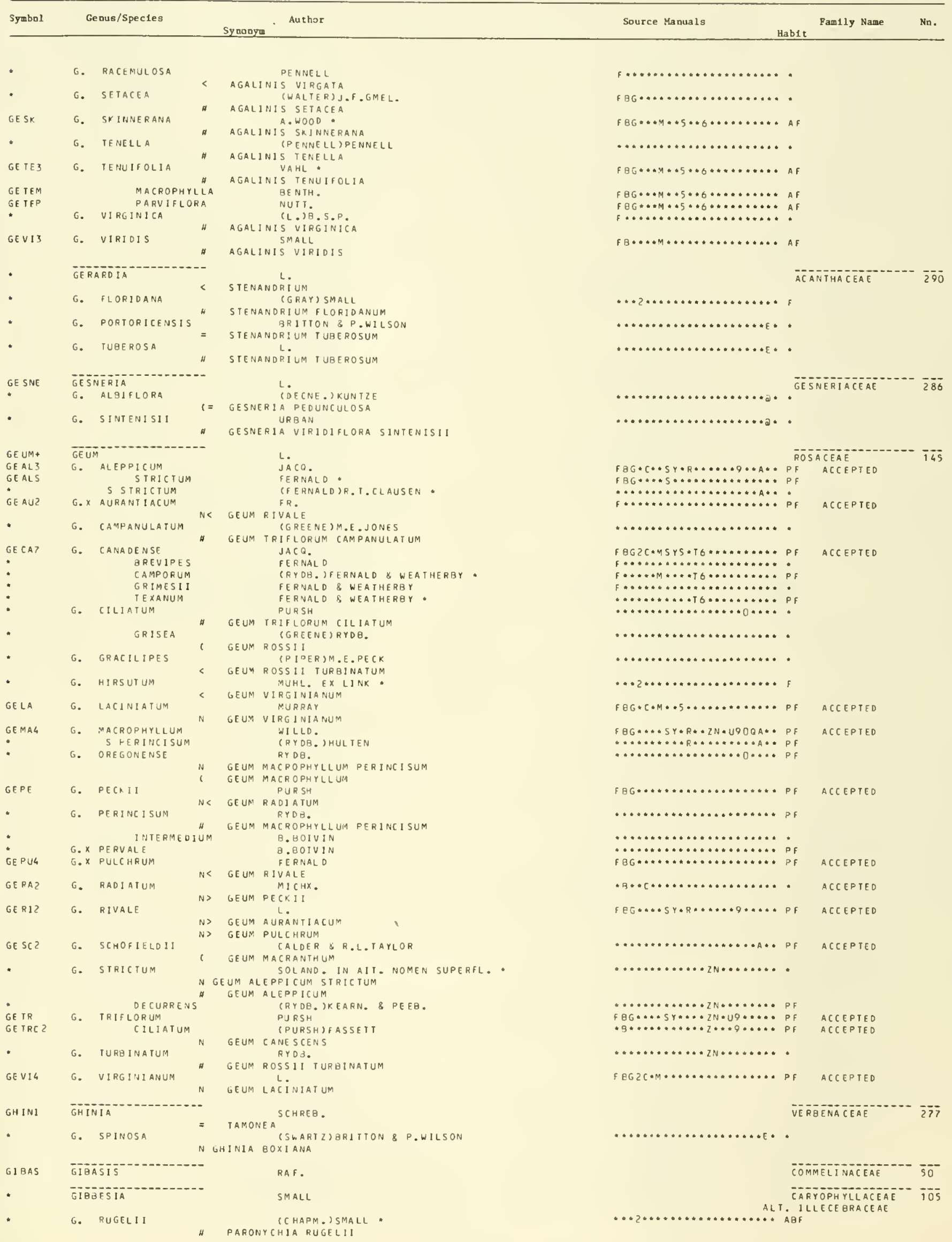




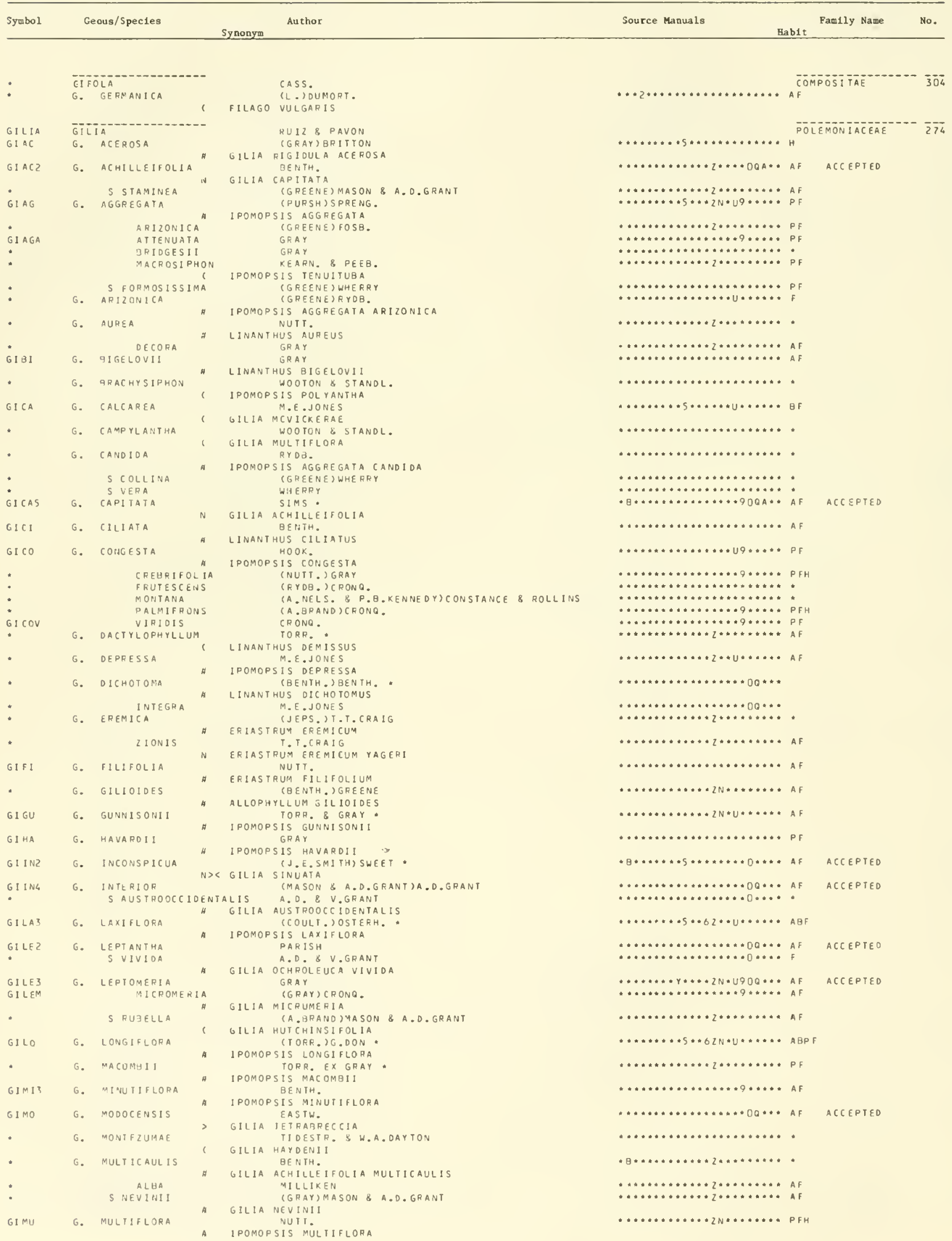


GILIA

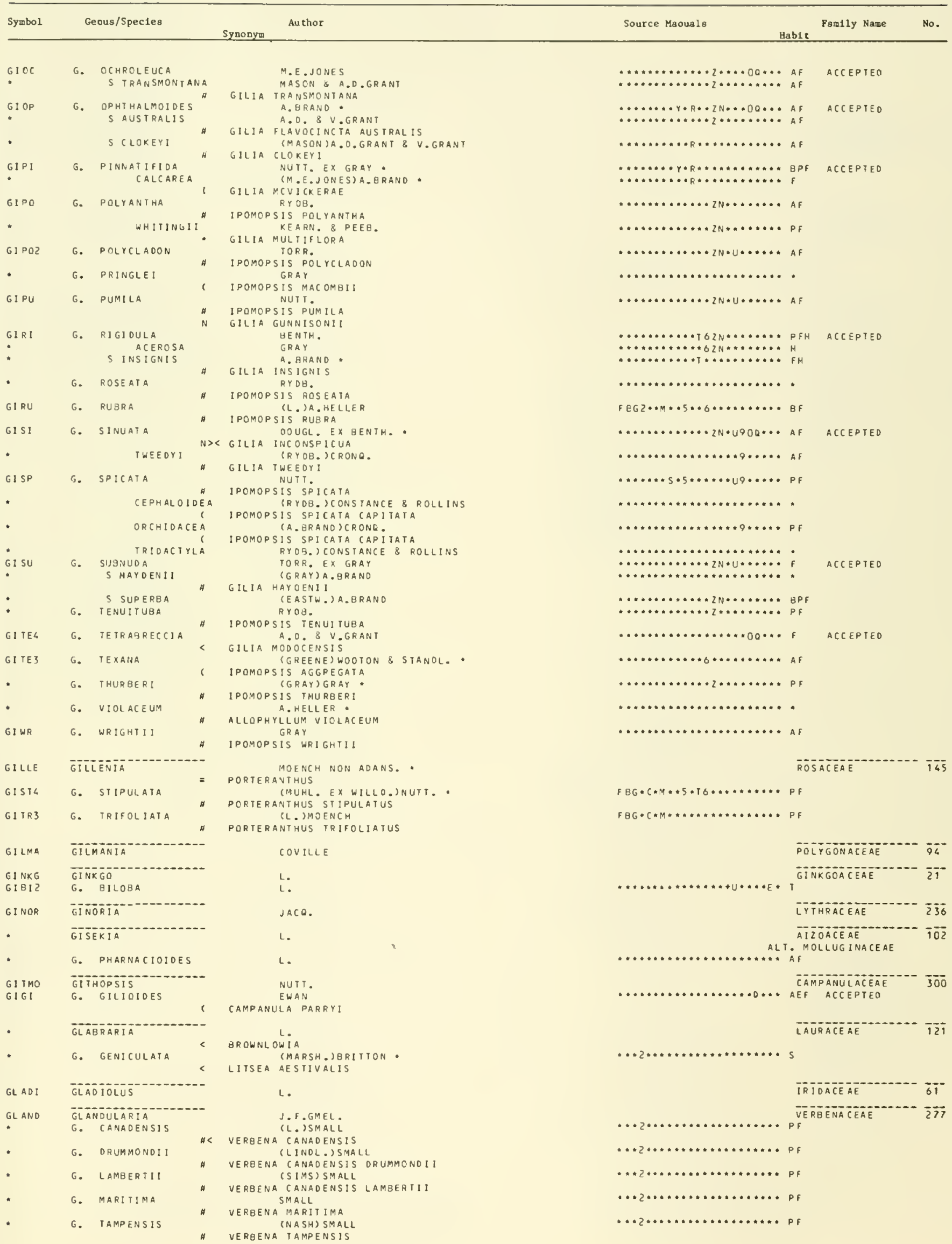




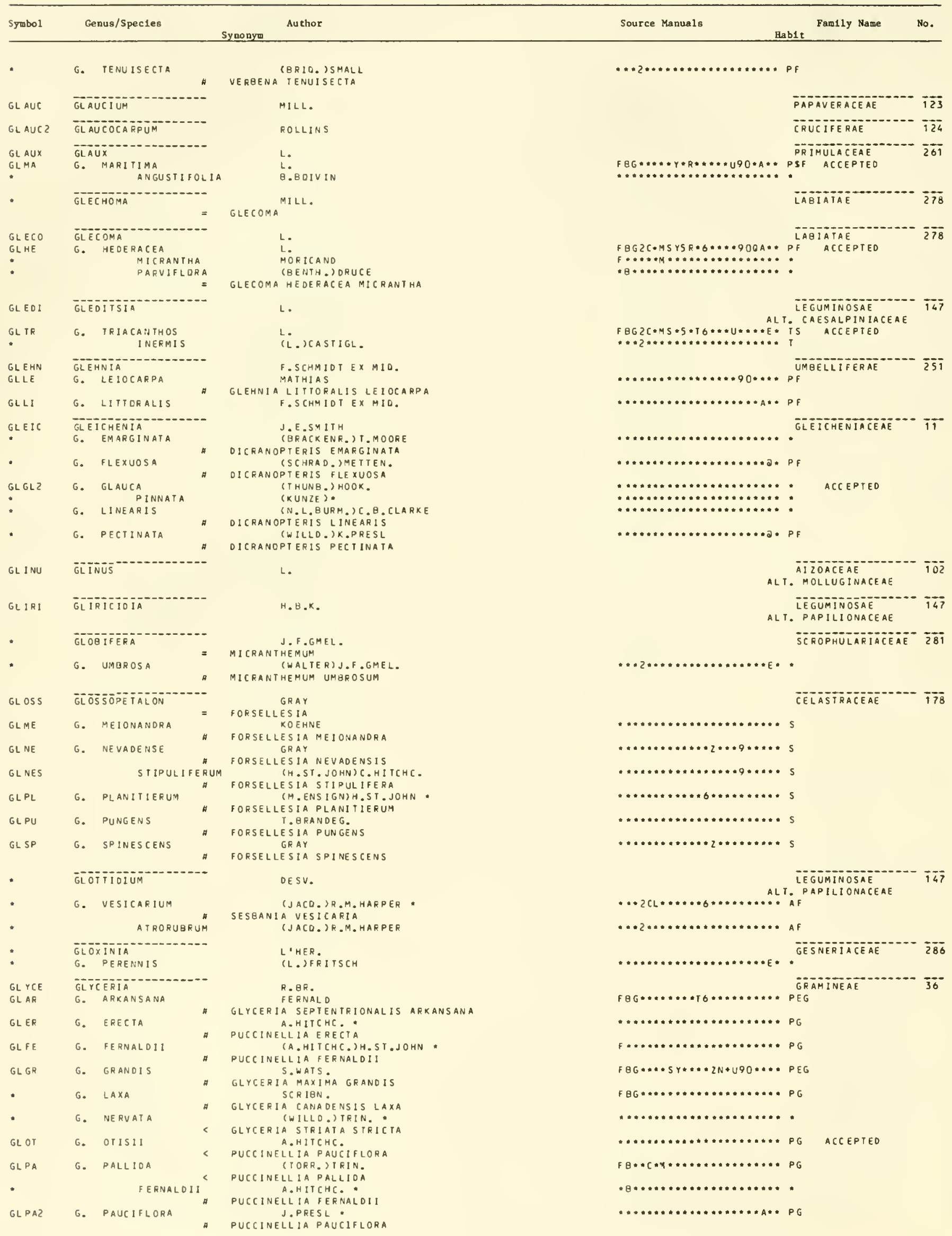


GLYCERIA

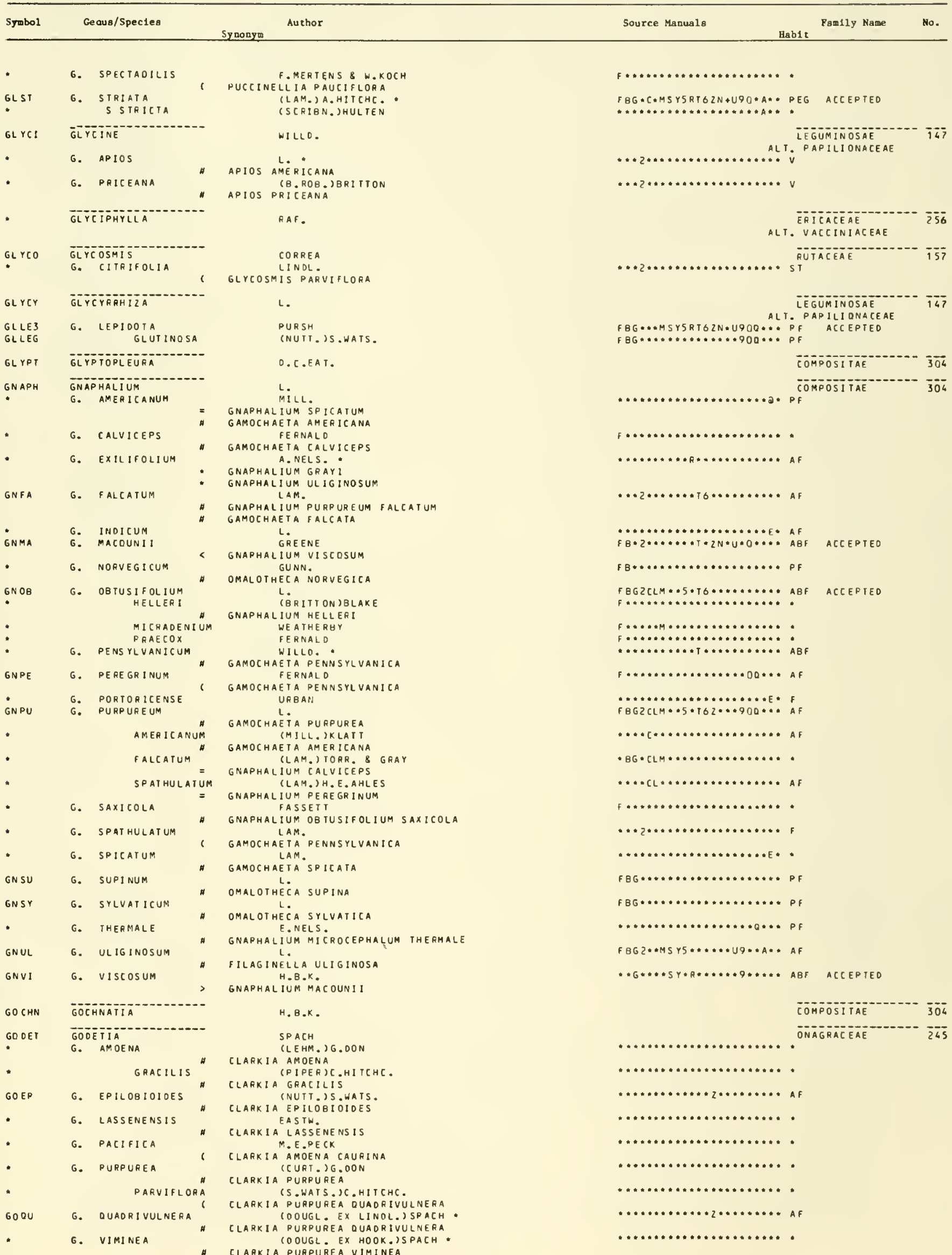




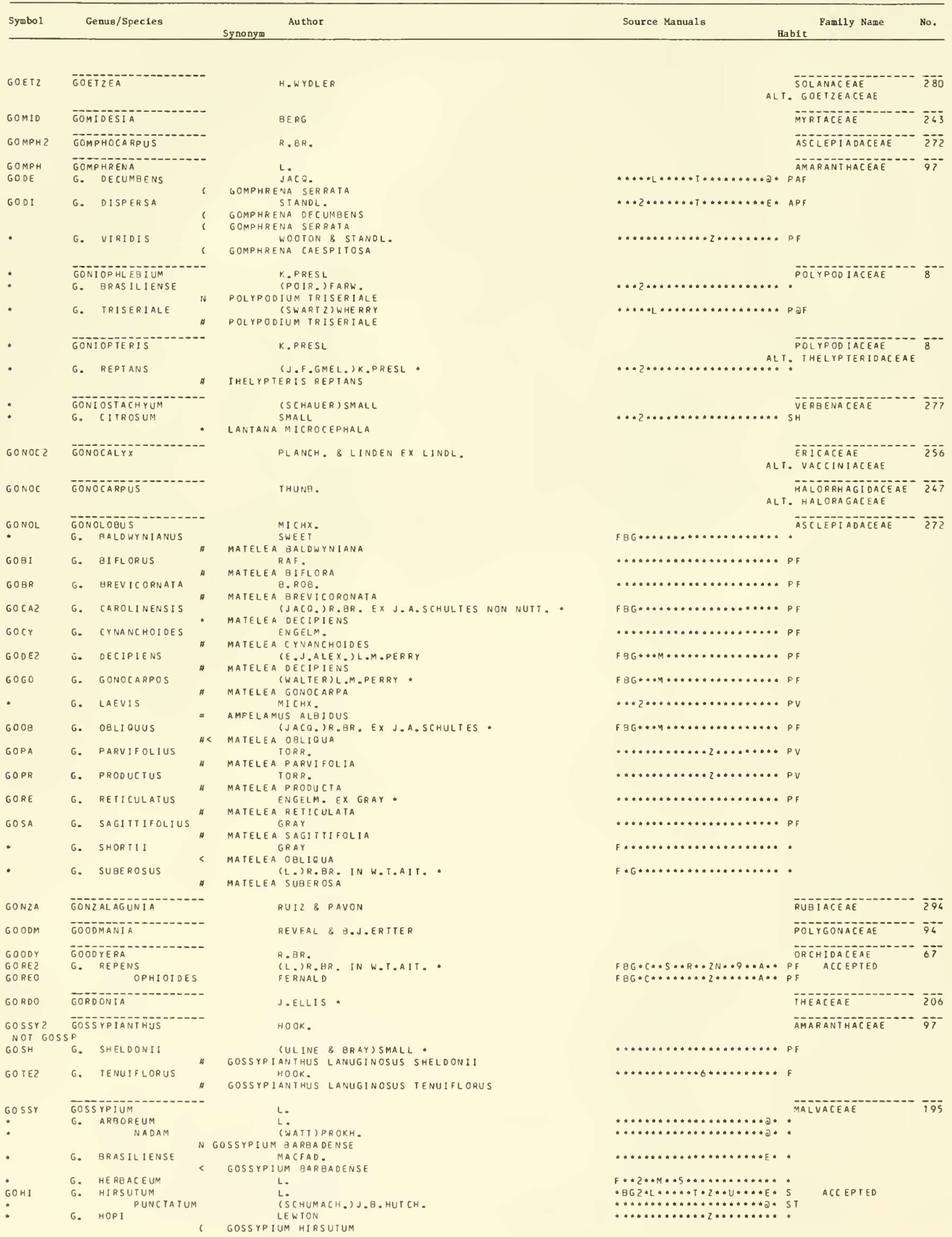




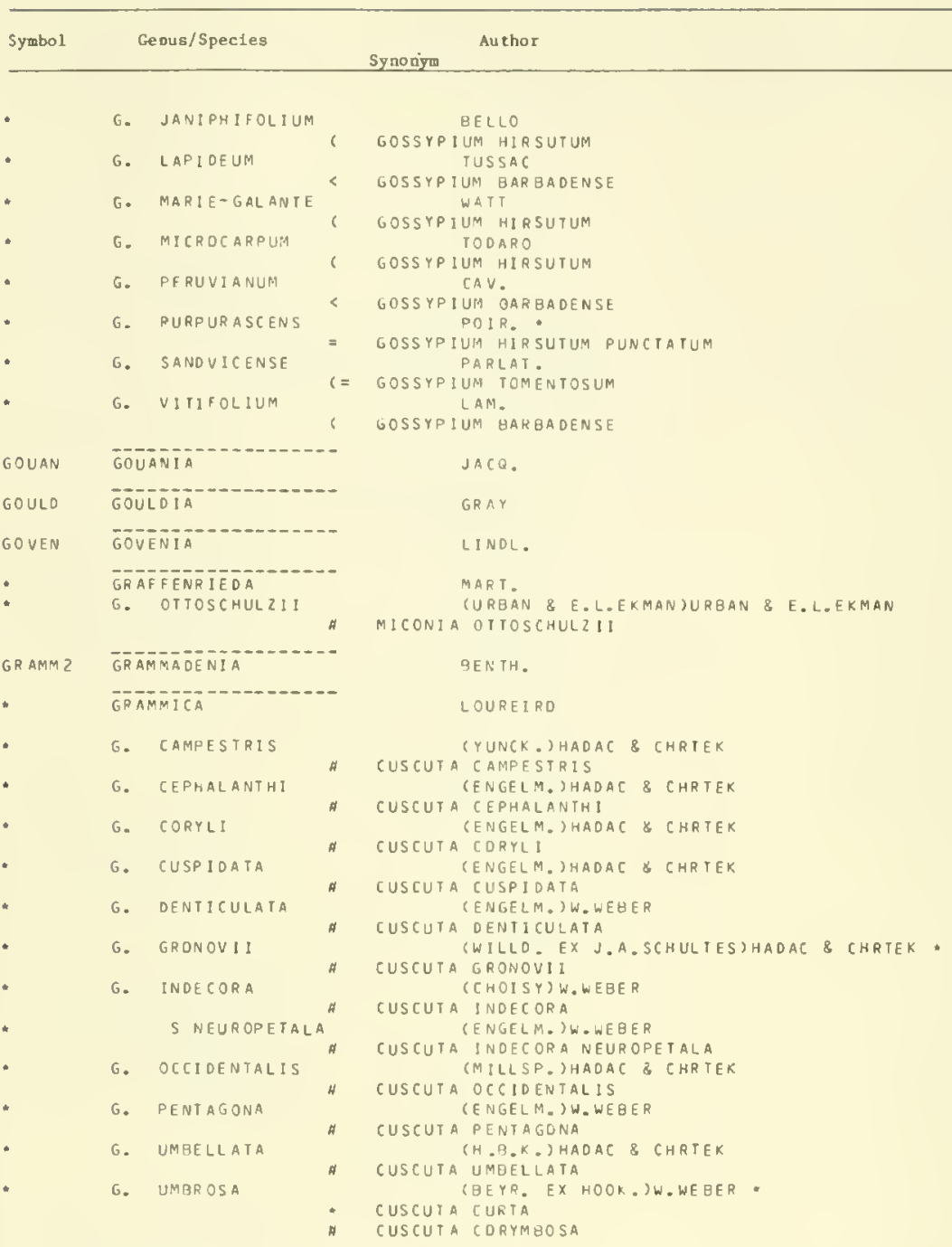

GRAMMT TIS

CUSCUTA CORYMBOSA

$\begin{array}{ll}* & \text { G. HESSII } \\ \text { - } & \text { G. JUBIFORMIS } \\ \text { - } & \text { G. MYOSUROIOES }\end{array}$

$$
\text { AESTUARIORUM GRATI OLA NEGLECTA }
$$

GRAYIA HOOK. \& ARN.

\section{GREENELLA GRAY GR}

G. ARIZONICA GRAY GUTIERREZIA ARIZONICA

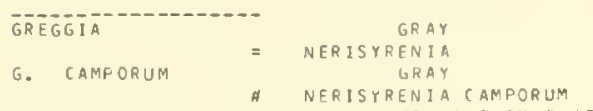

NERISYRENIA CAMPORUM
LINEARI FOLLIA
CS.WATS. JM.E. J ONES A NERISYRENIA LINEARIFOLIA

GREVILLEA
G. BANKSII
GRINDEIIA
G. ARENICOLA

R.BR. EX SALISB.

$R \cdot B R$.

WILLO.

GRINDELIA STRICTA VENULOSA

GRINDELIA INTEGRIF OLIA MACROPHYLLA

Source Mauals Habit
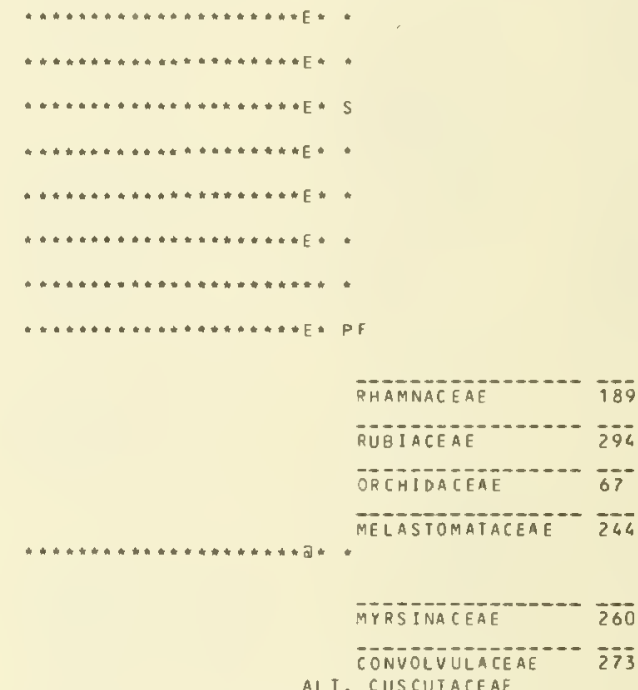
ALT. CUSCUTACEAE

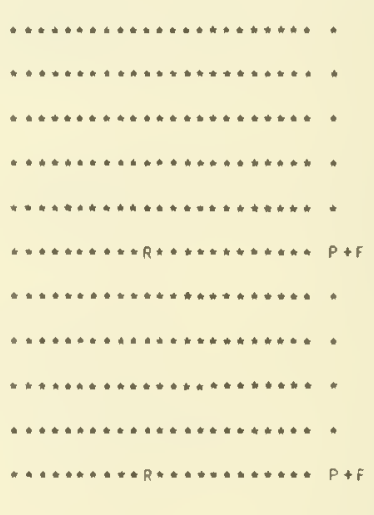

POLYPODTACEAE ALT, GRAMMATIDACEAE

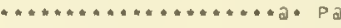

..................a. Pa
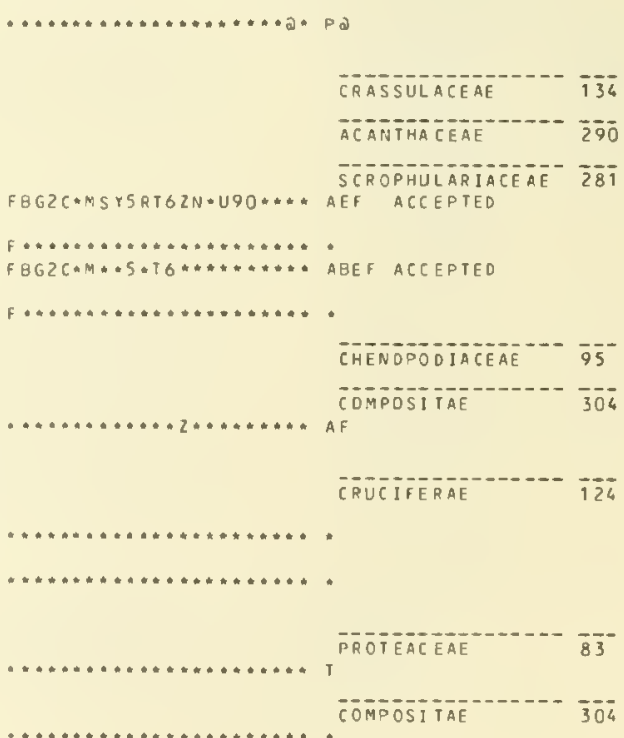


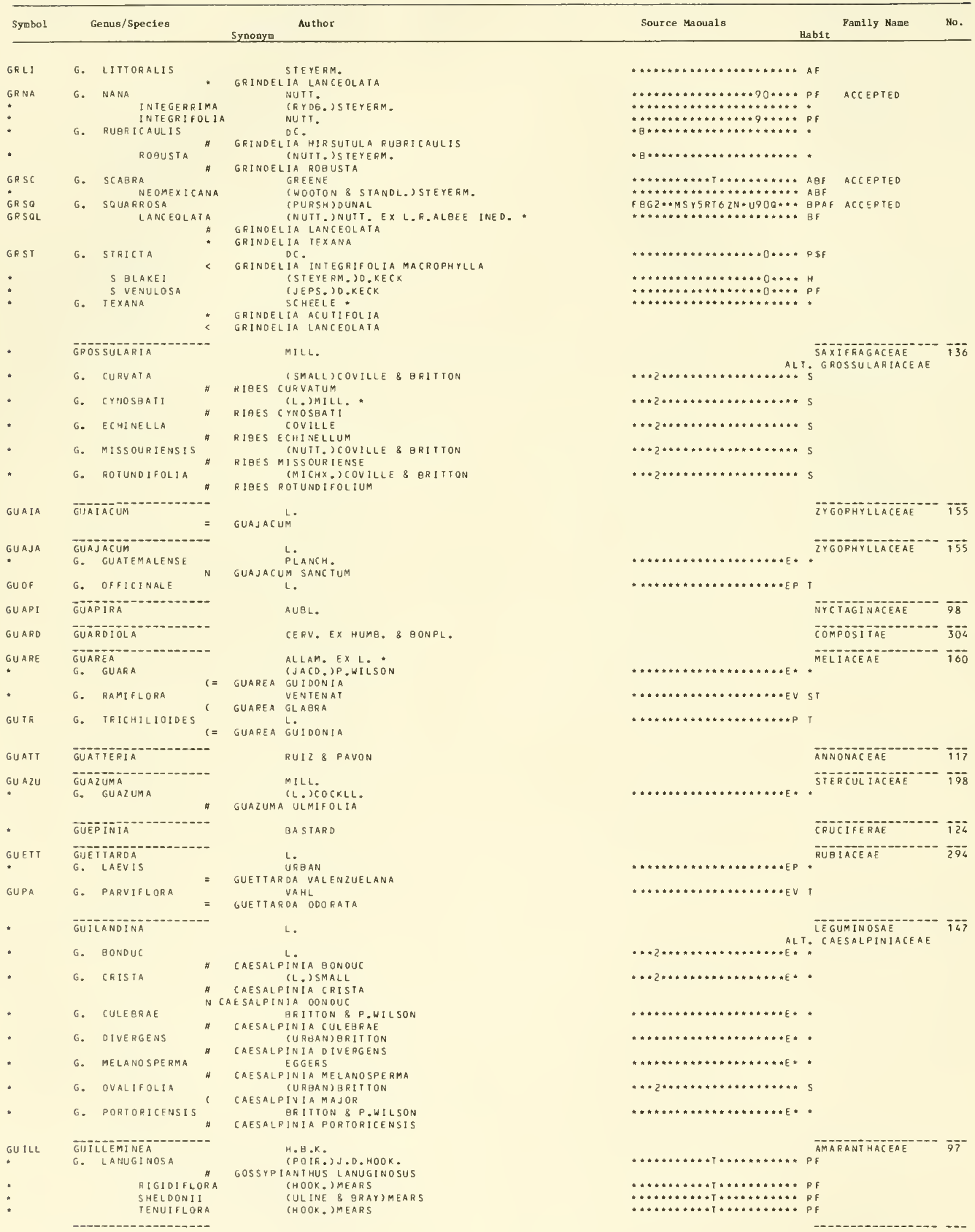




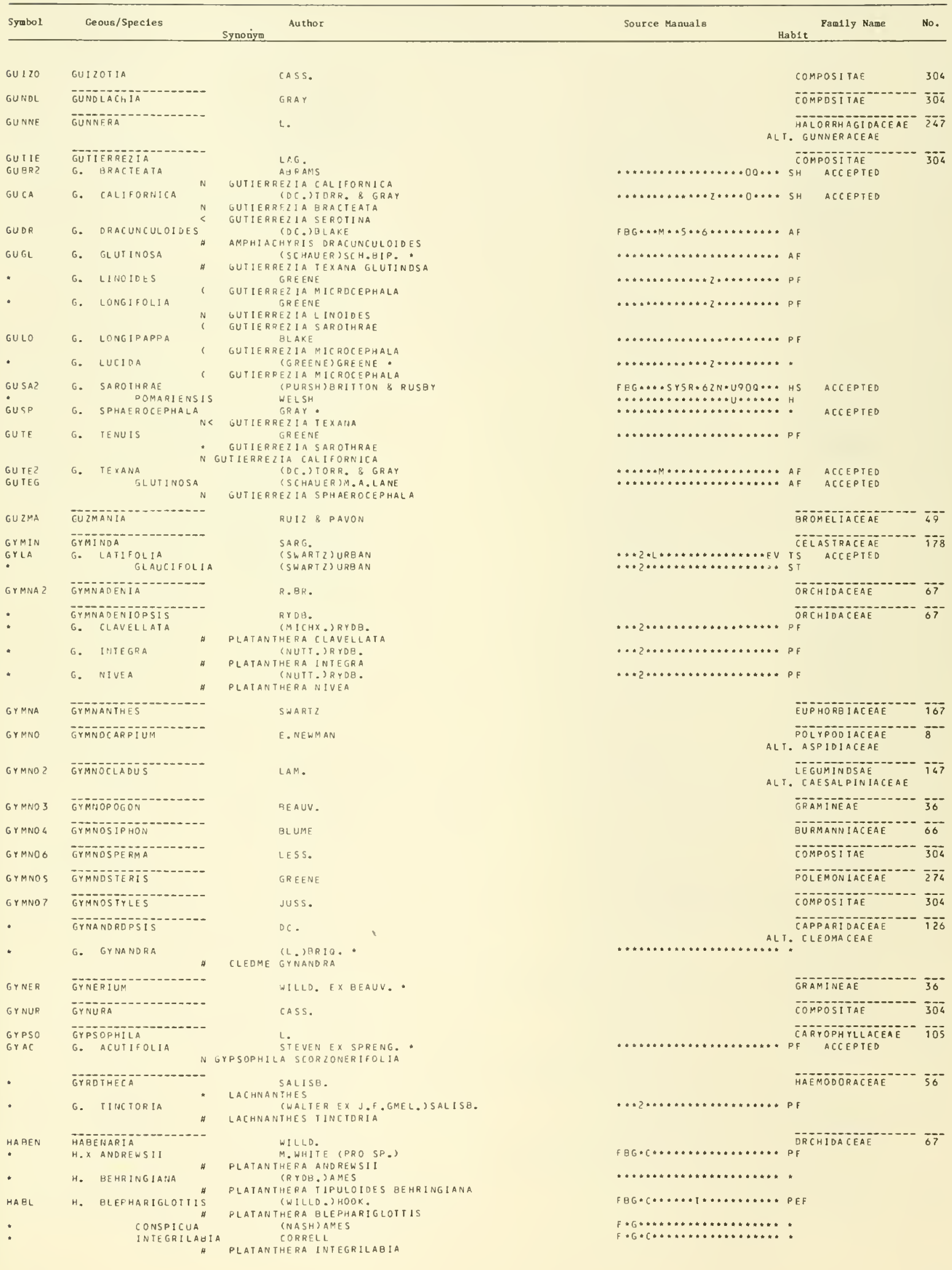




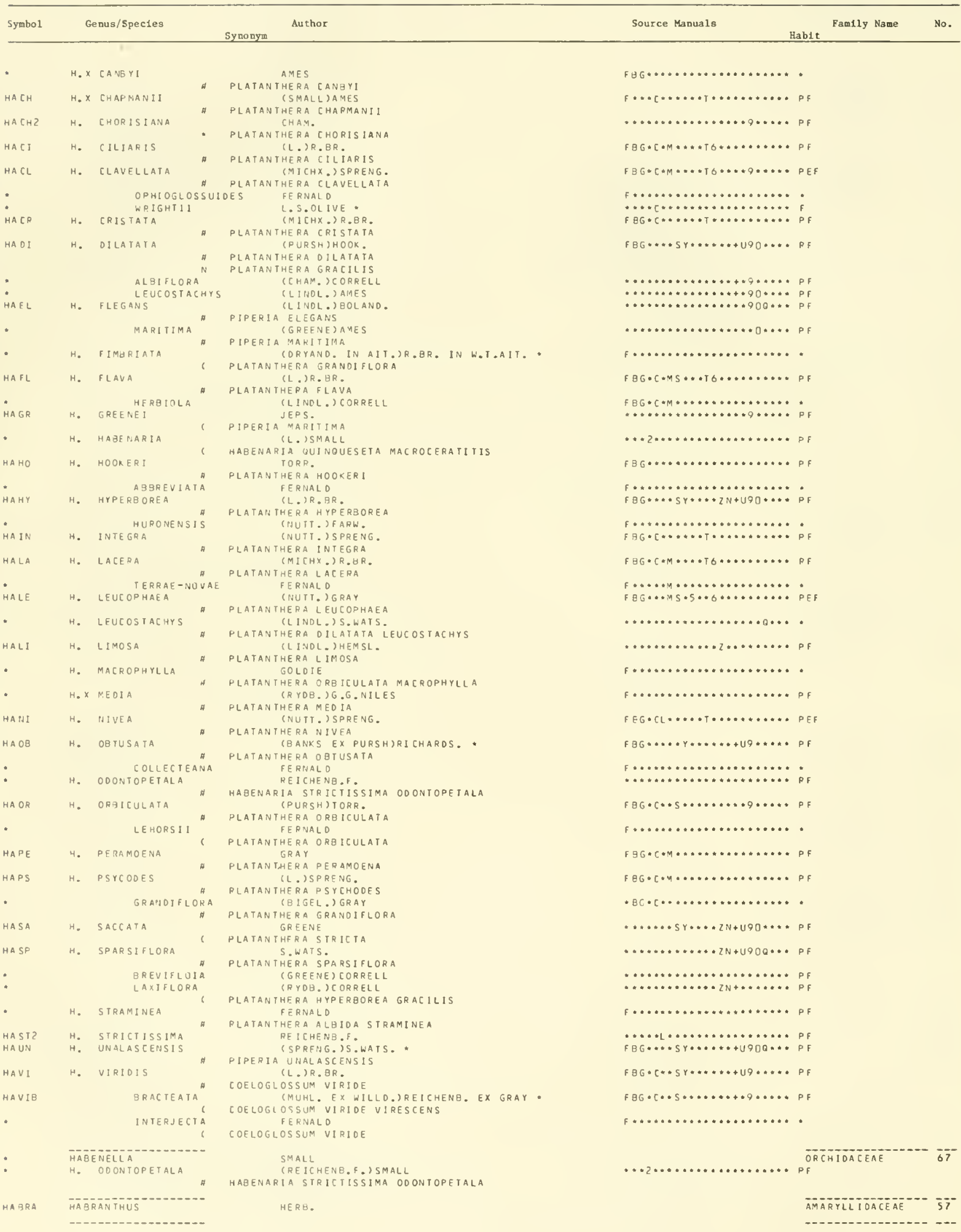




\begin{tabular}{|c|c|c|c|c|c|}
\hline symabol & Genus/Species & Synonym & Source Manuala & Fam1ly Name & No. \\
\hline HACKE & HACKELIA & OPIZ & & AG INACEAE & 276 \\
\hline - & H. AMERICANA & (GPAY) FERNALD & $F B \ldots \ldots \ldots \ldots \ldots \ldots \ldots+\ldots P F$ & & \\
\hline - & H. GRAC ILENTA & $\begin{array}{c}\text { HACKELIA DEFLEXA AMERICANA } \\
\text { (EASTW.) I. JOHNST. }\end{array}$ & $\ldots \ldots \ldots \ldots \ldots \ldots \ldots \ldots \ldots+\ldots$ & & \\
\hline HA JE & H. JESSICAE & $\begin{array}{l}\text { (R.L.MCGREG.)A. BRAND } \\
\text { HACKELIA RIICRANTHA }\end{array}$ & $\cdots \cdots \cdots \cdots \cdots \cdots+U \cdot O \cdot A * P F$ & & \\
\hline $\begin{array}{l}\text { HALEZ } \\
\text { HAPA } \\
\text { - }\end{array}$ & $\begin{array}{l}\text { H. LEPTOPHYLLA } \\
\text { H. PATENS } \\
\quad \text { SEMIGLAURA }\end{array}$ & $\begin{array}{l}\text { (RYDO.) I.JOHNST. } \\
\text { SNUTT.,I. JOHNST. } \\
\text { CRONO. }\end{array}$ & 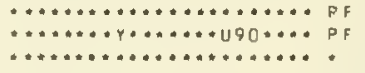 & ACCEPTED & \\
\hline HACKEZ & HACKELOCHLOA & VUNTZE & & MINEAE & 36 \\
\hline HAEMA & HAEMATOXYLON & $\begin{array}{l}\text { L. } \\
\text { HAEMATOXYLUM }\end{array}$ & $A L T, \overline{L E}$ & $\begin{array}{l}\text { UM INOSAE } \\
\text { ESALPINIACE AE }\end{array}$ & $\overline{14} \overline{7}$ \\
\hline HAFMA Z & HAEMAIOXYLUM & L. & $A L T . \overline{L E}$ & IESALPINIACEAE & $\overline{147}$ \\
\hline : & $\begin{array}{l}\text { HAEMOCHARIS } \\
\text { H. PORTORICENSIS }\end{array}$ & $\begin{array}{l}\text { SALISB. EX MART. \& ZUCCAR. } \\
\text { KPUG \& URBAN } \\
\text { LAPLACEA PORTORICENSIS }\end{array}$ & $\ldots * \ldots \cdots \ldots \ldots \ldots \ldots \ldots \ldots \ldots+\varepsilon^{\mathrm{T}_{H}}$ & $\bar{A} \bar{C} \overline{E A} \bar{E}$ & $\overline{2} \overline{0} \overline{6}$ \\
\hline HAENI & $\begin{array}{l}\text { HAENIANTHUS } \\
\text { OBOVATUS }\end{array}$ & $\begin{array}{c}\text { GRISEB. } \\
\text { KR UG \& URBAN } \\
\text { HAENIANTHUS SALICIFOLIUS OBOVATUS }\end{array}$ & $\ldots \ldots \ldots \ldots \ldots \ldots \ldots+{ }^{O L}$ & $A C E A E$ & $\overline{26} \overline{7}$ \\
\hline HASAZ & H. SALICIFOL IUS & GRISE 3 . & $\ldots \ldots \ldots \ldots \ldots \ldots \ldots \ldots \ldots a v$ & & \\
\hline $\begin{array}{l}\text { HALEN } \\
\text { HAOEZ }\end{array}$ & HALENIA & BOKKH. & & IT IANACEAE & $\overline{2} 7 \overline{0}$ \\
\hline HAOEZ & $\begin{array}{l}\text { H. DEFLEXA } \\
\text { GRENTON IANA }\end{array}$ & $\begin{array}{l}\text { (J.E.SMITH)GRISEO. } \\
\text { (GRISEB.)GRAY }\end{array}$ & 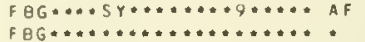 & $A C C E P I E D$ & \\
\hline & 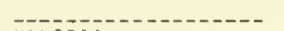 & & & & \\
\hline $\begin{array}{l}\text { HALES } \\
\text { HACA3 }\end{array}$ & HALESIA & J.ELLIS EX L. . & & RACACEAE & 265 \\
\hline $\mathrm{HACA} 3$ & H. CAROLINA, > & HALESIA PARVIFLORA & $F B G 2 C * M \cdots \cdots T_{6} \cdots \cdots \cdots \cdots S T$ & ACCEPTED & \\
\hline HACAM & MONTICOLA & REHD. & $\cdots \ldots \ldots \ldots \ldots \ldots \ldots \ldots \ldots \ldots \ldots, t$ & & \\
\hline - & H. MONTICOLA & $\begin{array}{l}\text { SREHD. ) SARG. } \\
\text { HALESIA (AROL INA MONTI COLA }\end{array}$ & $\cdots * 2 *+M \cdots \cdots \cdots \cdots \ldots \ldots+\cdots \cdots$ & & \\
\hline iApaz & VESTITA & SARG. & $\ldots \ldots \ldots \ldots \ldots \ldots \ldots \ldots \ldots \ldots+\cdots$ & & \\
\hline HAPAZ & H. PARVIFLORA & $\begin{array}{l}\text { MICHX. } \\
\text { HALESIA CAROLINA }\end{array}$ & 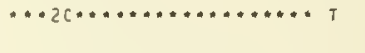 & ACCEP TED & \\
\hline - & H. TETHAPTERA & $\begin{array}{l}\text { J.ELLIS } \\
\text { HALESIA CAKOL INA }\end{array}$ & $* * \cdots+\cdots, \ldots+\cdots+\cdots+\cdots * * * *$ & & \\
\hline - & MONTICULA & SREHD. ) REVEAL \& SELD. & 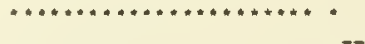 & & \\
\hline : & $\begin{array}{l}\text { HALIMIUM } \\
\text { H. OCCIDENTALE }\end{array}$ & $\begin{array}{l}\text { (DUNAL) SPACH } \\
\text { (GREENE) H.GROSS }\end{array}$ & $\ldots \ldots \ldots \ldots \ldots \ldots \ldots \ldots+\ldots, P^{\bar{C} I}$ & TACEAE & 213 \\
\hline - & HALIMODENDRON & FISCH. EX DC. & $A_{A L T}{ }^{P}$ & $\begin{array}{l}\text { MMINOSAE } \\
\text { PILI ONACEAE }\end{array}$ & $\overline{747}$ \\
\hline * & H. HALODENDRON & (PALLAS)J.VOSS & $\ldots \ldots \ldots \ldots \ldots \ldots \ldots \ldots \ldots$ & & \\
\hline HALIM & HALIMOLOQOS & TAUSCH & & CIFERAE & $12 \overline{4}$ \\
\hline HALOD & HALOOULE & ENDL. & $A L T \cdot \bar{P}$ & $\begin{array}{l}\text { AMOGETONACEAE } \\
\text { MODOCEACEAE }\end{array}$ & 28 \\
\hline HALOG & HALOGETON & C. A. MEYER & & NOPOOIACEAE & $\overline{9} \overline{5}$ \\
\hline HALOP & HALOPHILA & THOUARS & & POCHARITACEAE & 34 \\
\hline : & $\begin{array}{l}\text { H. OVALIS } \\
\text { S HAWA IIANA }\end{array}$ & $\begin{array}{l}\text { (R. BR. I) J.D. HOOK. } \\
\text { (M.S.DOTY \& STONE) DEN HARTOG }\end{array}$ & $\ldots \ldots \ldots \ldots \ldots \ldots \ldots \ldots \ldots \ldots \ldots$ & & \\
\hline & 2 & HALOPHILA HAWAIIANA & & & \\
\hline HALOR & HALORAGIS & J. \& G.FORST. & $A L T \cdot B$ & $\begin{array}{l}\text { ORRAAGIOACEAE } \\
\text { LORAGACEAE }\end{array}$ & $27 \overline{7}$ \\
\hline HAMAM & HAMAMELIS & L. & & A & $\overline{14 \overline{2}}$ \\
\hline HAVEZ & $\begin{array}{l}\text { H. VERNALIS } \\
\text { TOMENTELLA }\end{array}$ & $\begin{array}{l}\text { SARG. } \\
\text { (REHD.)E.J.PALMER }\end{array}$ & $F B * * * 4 * \ldots * 16 \ldots \ldots \ldots * * \cdots s$ & ACCEPTED & \\
\hline HAVIL & $\begin{array}{l}\text { H. VIRGINIANA } \\
\text { PARVIFOLIA }\end{array}$ & $\begin{array}{l}\text { (REHD. )E.J.PALMER } \\
\text { L. } \\
\text { NUTT. }\end{array}$ & FBG & ACCEPTED & \\
\hline & 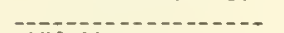 & NUTT. & $F * \ldots \ldots+\cdots+\cdots \cdots+\cdots \cdots+$ & & \\
\hline HAMEL & HA MEL I A & JACO. & & $\triangle A C E A E$ & 294 \\
\hline - & H. ERECTA & $\begin{array}{l}\text { JACQ. } \\
\text { HAMELIA PATENS }\end{array}$ & 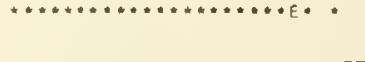 & & \\
\hline - & HAMMARBYA & KUNTZE & & HIDACEAE & 67 \\
\hline - & H. PALUDOSA & $\begin{array}{l}\text { BL. MKUNTZE } \\
\text { MALAXIS PALUDOSA }\end{array}$ & $\cdots \ldots \ldots \ldots+\ldots \ldots \ldots \ldots+A A_{*} \cdots$ & & \\
\hline • & HARALORCHIS & SCMLECHTER & & HIDACEAE & 67 \\
\hline - & H. TENUIS = & $\begin{array}{l}\text { (LINDL.) SCHLECHT. } \\
\text { SPIRANTHES FAWCETIII }\end{array}$ & $* \ldots \ldots \ldots+\ldots+\ldots \ldots \ldots \ldots+\hbar E *$ & & \\
\hline HARLO & HAPLOESTHES & GRAY & & POOSITAE & $\overline{304}$ \\
\hline HA GR 4 & H. GREGGII & GRAY & $\cdots \cdots \cdots \cdots 5 \cdot 16 \cdots \cdots \cdots \cdots+F H$ & & \\
\hline HAPLOZ & $\begin{array}{l}\text { HAPLOPAPPUS } \\
\text { H. ANNUUS }\end{array}$ & $\begin{array}{l}\text { CASS. } \\
\text { (RYDB.) CORY }\end{array}$ & & POSITAE & $\overline{304}$ \\
\hline t & H. ANNUUS & $\begin{array}{l}\text { (RYDQ.) CORY } \\
\text { MACHAERANTHERA ANNUA }\end{array}$ & 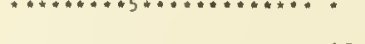 & & \\
\hline HA AU & H. AUREUS & $\begin{array}{l}\text { GRAY } \\
\text { MACHAERANTHERA AUREA }\end{array}$ & $\cdots \ldots \ldots \ldots \ldots \ldots \ldots \ldots \ldots$ AF & & \\
\hline HA AU? & H. AUSTRALIS & $\begin{array}{l}\text { GREENE } \\
\text { MACHAERANTHERA AUSTRALIS }\end{array}$ & $+\ldots \ldots+\ldots \ldots \ldots \ldots \ldots \ldots+\ldots \ldots \ldots \ldots p F$ & & \\
\hline HABLZ & H. BLEPHARIPHYLLUS & $\begin{array}{l}\text { GRAY } \\
\text { MACHAERANTHERA BLEPHARIPHYLLA }\end{array}$ & 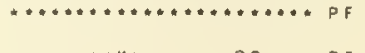 & & \\
\hline HACA 5 & H. CARTHAMOIDES & (HOOK.) GRAY & $\cdots \ldots \ldots \cdots+\cdots \cdots \cdots+q \cdots \cdots P F$ & ACCEPTED & \\
\hline
\end{tabular}




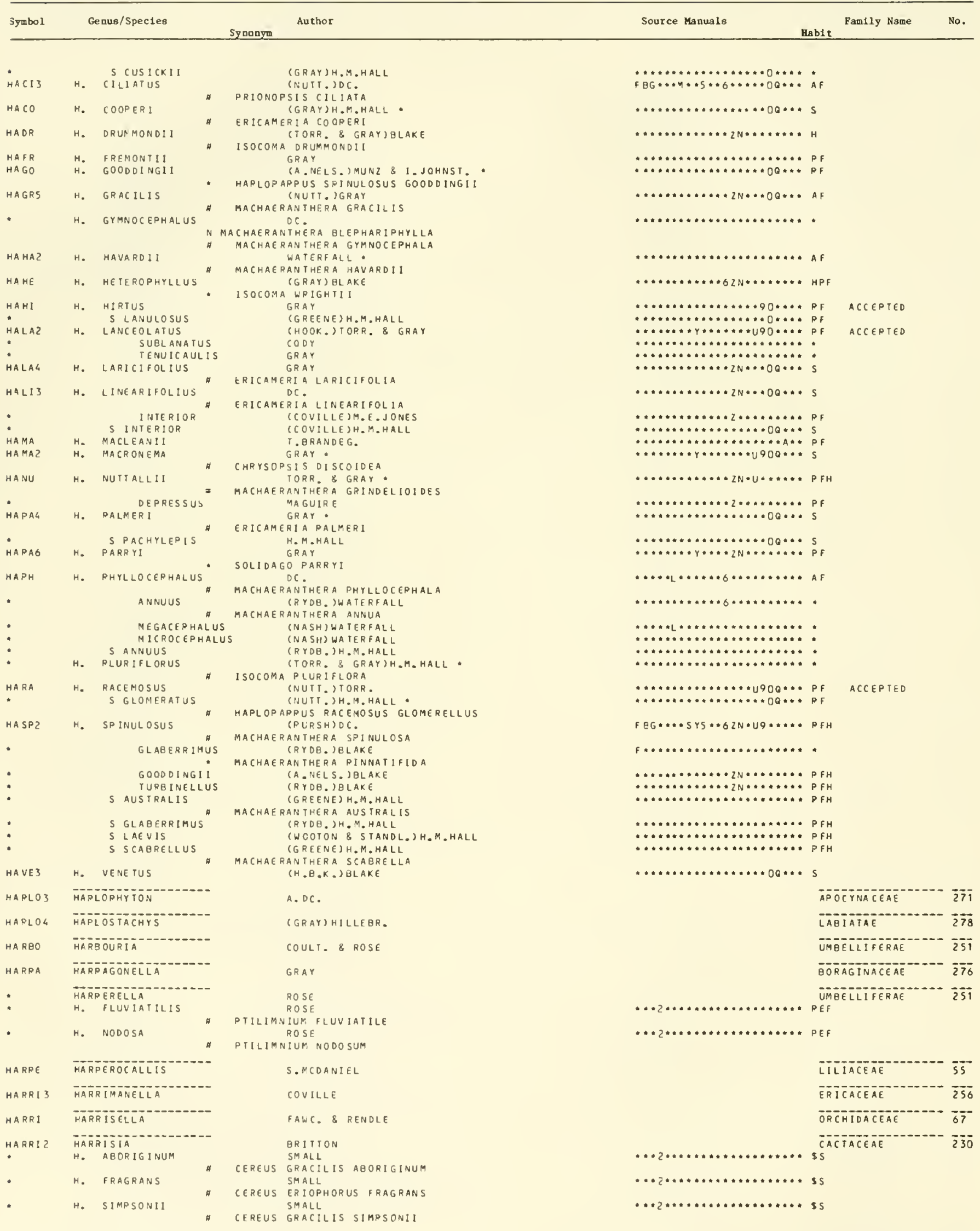




\begin{tabular}{|c|c|c|c|c|c|}
\hline Symbol & Genus/Spec1es & Syonoym & Source Manuals & Hablt Pamily Name & Ro. \\
\hline • & HARRISIELLA & $\begin{array}{l}\text { INED. } \\
=\quad \text { HARRISELLA }\end{array}$ & & ORCHIOACEAE & 67 \\
\hline * & HARTMANIAA & SPACH & & - ONABGRACEAE & $\overline{2} \overline{45}$ \\
\hline • & H. SPECIOSA & $\begin{array}{l}\text { (NUTT. ) SMALL } \\
\text { UENDTHERA SPECIOSA }\end{array}$ & $\cdots \cdot 2 \cdots \ldots \ldots \ldots \ldots \ldots \ldots$ & APF & \\
\hline HARTW & HARTURIGHTIA & GPAY EX S.WATS. & & COMPOSITAE & $30 \overline{4}$ \\
\hline 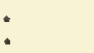 & $\begin{array}{l}\text { HASSEANT HUS } \\
\text { H. BLOCHMANIAE }\end{array}$ & $\begin{array}{l}\text { ROSE } \\
\text { (EASTW.)ROSE }\end{array}$ & & CRASSULACEAE & $\overline{134}$ \\
\hline & 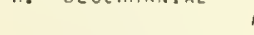 & DUDLEYA GLOCHMANIAE & (n) & & \\
\hline : & $\begin{array}{l}S \text { ORE VIFOLIUS } \\
\text { S INSULAR IS }\end{array}$ & MORAN & ㅅ․ & RSF & \\
\hline : & $\begin{array}{l}\text { S INSULAR:S } \\
\text { H. ELONGATUS }\end{array}$ & $\begin{array}{c}\text { MORAN } \\
\text { ROSE } \\
\text { DUDLEYA MULTICAULIS }\end{array}$ & $\cdots \ldots \ldots \ldots \ldots \ldots \ldots \ldots \ldots \ldots *$ & $\begin{array}{l}\text { R \$F } \\
\text { PSF }\end{array}$ & \\
\hline * & H. NESIOTICUS & a DUDLEYA NESIOTICA & $\ldots \ldots \ldots \ldots \ldots \ldots \ldots \ldots+\ldots$ & PQF & \\
\hline * & H. VARIEGATUS & $\begin{array}{l}\text { (S. WATS. JROSE } \\
\text { DUDLEYA VARIEGATA }\end{array}$ & $\ldots \ldots \ldots \ldots \ldots \ldots \ldots \ldots \ldots$ & RSF & \\
\hline HASTE & HASTEOLA & RAF. & & COMEOSITAE & $\overline{30 \overline{6}}$ \\
\hline HEBE* & HEGE & COMMERS. EX JUSS. & & SCROOHULARIACEAE & $28 \overline{1}$ \\
\hline HE BEC & HEBECLINIUM & $D C$. & & COMPOSITAE & $\overline{306}$ \\
\hline * & HEGESTIGMA & UROAN & $A L I$ & $\begin{array}{l}\text { LEGUMINOSAE } \\
\text { PAPILIONACEAE }\end{array}$ & $\overline{147}$ \\
\hline * & H. CUBENSE & (H.B.X.) UREAN & $\ldots \ldots \ldots \ldots \ldots \ldots \ldots \ldots \ldots \ldots v$ & i & \\
\hline HE CAS & HECASTOCLEIS & GR AY & & COMPOSITAE & $\overline{304}$ \\
\hline HECHI & HECHTIA & KLOTZSCH & & DR OMELIACEAE & $6 \overrightarrow{9}$ \\
\hline HECIS & HECISTOPTERIS & J.SMITH & $A L I$ & $\begin{array}{l}\text { POLYROOIACEAE } \\
\text { T. VITTARIACEAE }\end{array}$ & $8^{--}$ \\
\hline $\begin{array}{l}\text { HE OEO } \\
\text { HE AN }\end{array}$ & $\begin{array}{l}\text { HEDEOMA } \\
\text { H. ANGLLATUM }\end{array}$ & $\begin{array}{l}\text { FERS. } \\
\text { THARP }\end{array}$ & & LABIATAE & $\overline{2} \overline{78}$ \\
\hline HE AN & H. ANGLLATUM & RHODODON CILIATUS & $\ldots \ldots \ldots \ldots \ldots \ldots \ldots \ldots \ldots+\cdots$ & AF & \\
\hline $\begin{array}{l}\text { HECO } \\
\text { HEOR }\end{array}$ & H. COSIATUM & GRAY & $\cdots \cdots \cdots \cdots \cdots+z \cdots \ldots \ldots$ & PF & \\
\hline HE UR & $\begin{array}{l}\text { H. DRUMMONDII } \\
\text { REVERCHONI }\end{array}$ & $\begin{array}{l}\text { GRAY } \\
\text { II HEDEOMA REVERCHONII }\end{array}$ & 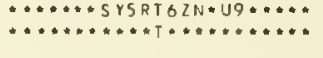 & $\begin{array}{l}\text { PAFH ACCEPTED } \\
\text { H }\end{array}$ & \\
\hline - & SERPYLLIF & OLIUM (SMALL)R.S.IRVING & $\ldots \ldots \ldots \ldots \ldots+\ldots \ldots \ldots \ldots$ & H & \\
\hline - & H. LATUM & $\begin{array}{l}\text { SMALL } \\
\text { ( HEDEOMA REVEQCHONI : }\end{array}$ & $\ldots+\ldots \ldots \ldots \ldots+\ldots+\ldots \ldots+\infty$ & 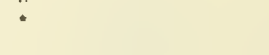 & \\
\hline HE LO & H. LONGIFLORUM & ( HEDEOMA DRUMMONOII & $\ldots \ldots \ldots \ldots \ldots \ldots \ldots \ldots \ldots \ldots$ & PF & \\
\hline * & H. PULCHELLUM & $\begin{array}{l}\text { GREENE } \\
\text { * HEDEOMA COSTATUM PULGHELLUM }\end{array}$ & $\ldots \ldots \ldots \ldots \ldots \ldots \ldots \ldots \ldots$ & $\cdot$ & \\
\hline HETE & H. TEXANUM & $\begin{array}{l}\text { CORY } \\
\text { ( RHODODON CILIATUS }\end{array}$ & $\ldots \ldots \ldots \ldots \ldots \ldots \ldots \ldots \ldots \ldots$ & Af & \\
\hline HE DER & HEDERA & L. & & ARALIACEAE & $25 \overline{0}$ \\
\hline HEOYC & HEDYCHIUM & J. KOENIG & & ZINGIBERACEAE & 63 \\
\hline HE DYO & HE DYOSMUN & SWARTZ & & CHLORANTHACEAE & $\bar{i}$ \\
\hline HE DYOZ & HEDYOTIS & L. & & RUB & $\overline{2} 9 \overline{6}$ \\
\hline$\cdot$ & H. ACEROSA & $\begin{array}{l}\text { GPAY } \\
\text { HOUSIONIA ACEROSA }\end{array}$ & $\ldots \ldots \ldots \ldots+$ เ $\ldots \ldots+\ldots \ldots$ & $H$ & \\
\hline 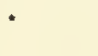 & BIGELOVII & $\begin{array}{l}\text { (GREENM.) W. H. LEWIS } \\
\text { HOUSIONIA ACEROSA POLYPREMOIOES }\end{array}$ & $\cdots \ldots \ldots+\cdots, \ldots \ldots \ldots \ldots$ & H & \\
\hline - & H. AUSTRALIS & $\begin{array}{l}\text { W.H.LEWIS \& D.M.MOORE - } \\
\text { N HOUSTONIA PUSILLA } \\
\text { \& HOUSTONIA MICRANTHA }\end{array}$ & $\cdots \cdots \cdots \cdots \cdots$ * $6 \cdots \cdots \cdots \cdots \cdots$ & Af & \\
\hline • & H. $\operatorname{BOSCII}$ & " OLDENLANDiA BOSCII & 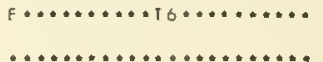 & PF & \\
\hline * & $\begin{array}{l}\text { H. CAERULEA } \\
\text { MINIMA }\end{array}$ & $\begin{array}{l}\text { (L.)HOOK. } \\
\text { * HOUSTONIA CAERULEA } \\
\text { (L.C.EECK)FOSB. }\end{array}$ & $\ldots \ldots \ldots \ldots \ldots \ldots \ldots \ldots \ldots \ldots \ldots$ & $\cdot$ & \\
\hline * & $\begin{array}{l}\text { MINIMA } \\
\text { MINOR }\end{array}$ & $\begin{array}{l}\text { * HOUSTONIA MINIMA } \\
\text { (MICHX.)TORR. \& GRAY }\end{array}$ & $\ldots \ldots \ldots \ldots \ldots \ldots \ldots \ldots \ldots$ & . & \\
\hline & 4. CALUI RISHOIDES & HOUSTONIA PUSILLA & (a) & 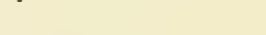 & \\
\hline * & H. CALLIIRICHOIDES & $\begin{array}{l}\text { (GRISEB.)W. H.LEWIS } \\
\text { OLDENLANOIA CALLITRICHOIOES }\end{array}$ & $\cdots \cdots+\llcorner\cdots \ldots \ldots \ldots \ldots \ldots \cdots$ & Af & \\
\hline * & H. CORYMBOSA & $\begin{array}{l}\text { "lli)lam. oldenlandia corymaosa } \\
\text { n }\end{array}$ & $\cdots \cdots\llcorner\cdots \cdots+\cdots \cdots \cdots \cdots \cdots$ & Af & \\
\hline • & H. CRASSIFOLIA & $\begin{array}{l}\text { RAF. } \\
\text { N) HOUSTONIA MINIMA } \\
\text { C HOUSTONIA PUSILLA }\end{array}$ & $\ldots \ldots \ldots \ldots$ ค т $\ldots \ldots \ldots \ldots$ & Af & \\
\hline HECR & H. CROF TIAE & $\begin{array}{l}\text { (ARITTON \& RUSBY)SHINNERS } \\
\text { * HOUSTIDIA CROFTIAE }\end{array}$ & $\ldots \ldots \ldots \ldots \ldots+$ + $\ldots \ldots \ldots \ldots$ & A F & \\
\hline - & H. FASCICULATA & $\begin{array}{l}\text { BERTOL. } \\
\text { OLDENLANDIA FASCICULATA }\end{array}$ & $\ldots \ldots \ldots \ldots \ldots \ldots \ldots \ldots \ldots$ & $\cdot$ & \\
\hline * & H. GREENMANII & $\begin{array}{l}\text { HOSH. } \\
\text { HOUSTONIA PARVIFLORA }\end{array}$ & $\cdots \ldots \ldots \ldots \ldots+1 \cdots \ldots \ldots \ldots$ & $A F$ & \\
\hline * & H. HALE: & $\begin{array}{l}\text { TORR. \& GRAY } \\
\text { ( PENTODON PENTANDRUS }\end{array}$ & $\ldots \ldots \ldots \ldots+\ldots \ldots \ldots \ldots \ldots$ & $\cdot$ & \\
\hline * & H. HUMIFUSA & $\begin{array}{l}\text { GRAY. } \\
\text { HOUSTONIA MUMIFUSA }\end{array}$ & $\ldots \ldots \ldots \ldots+\ldots \ldots \ldots$ & A \& & \\
\hline • & H. INTRICATA & $\begin{array}{l}\text { HOSB. } \\
\text { HOUSTONIA FASCICULATA }\end{array}$ & $\cdots \ldots \ldots \ldots \ldots+\uparrow \ldots \ldots \ldots \ldots$ & s & \\
\hline * & H. LONGIFOLIA & $\begin{array}{l}\text { (GAERTN.) HOOK. } \\
\text { * houstonia longifolia }\end{array}$ & $\ldots \ldots \ldots \ldots \ldots \ldots \ldots \ldots+\ldots \ldots$ & PF & \\
\hline
\end{tabular}




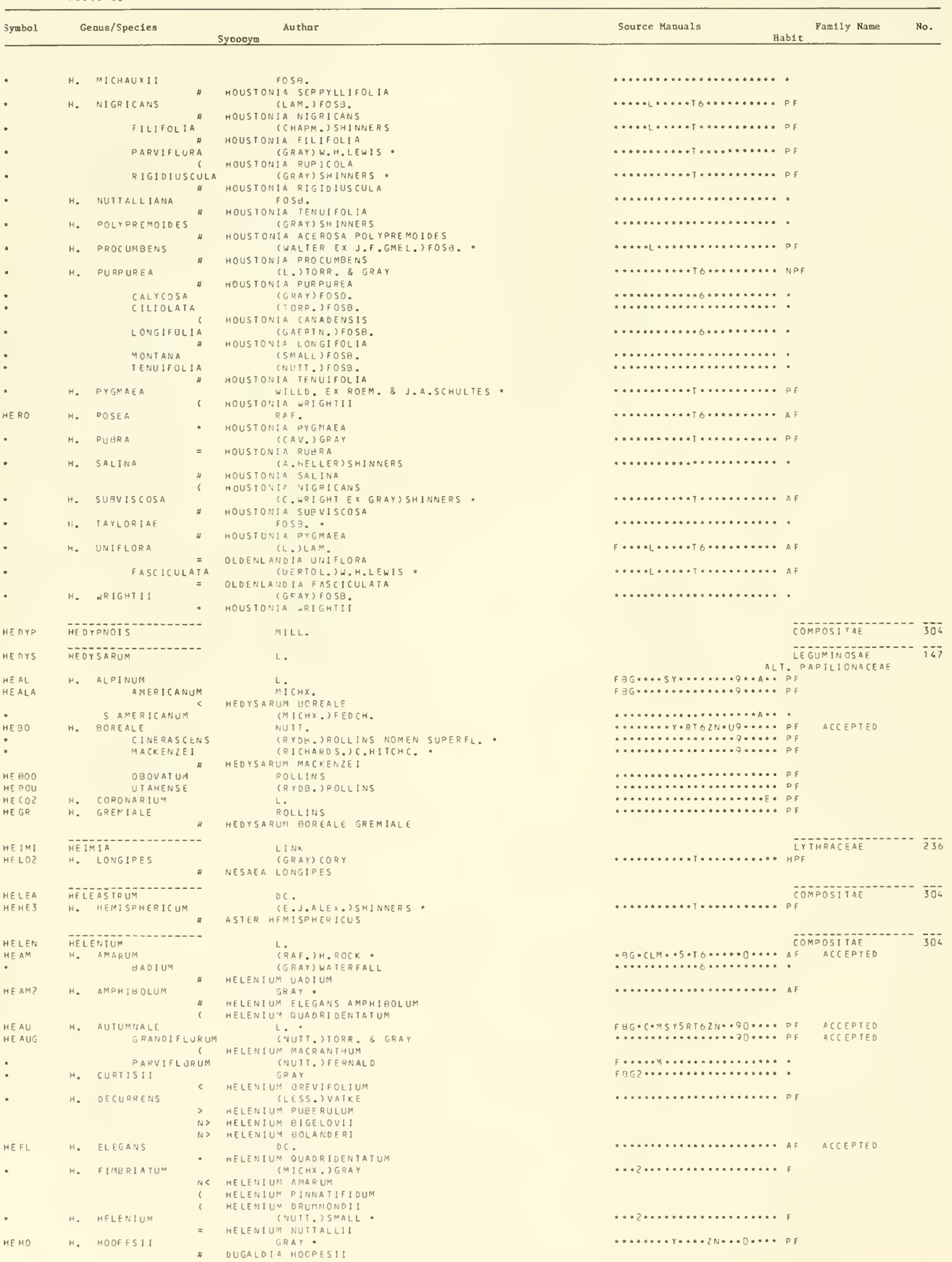




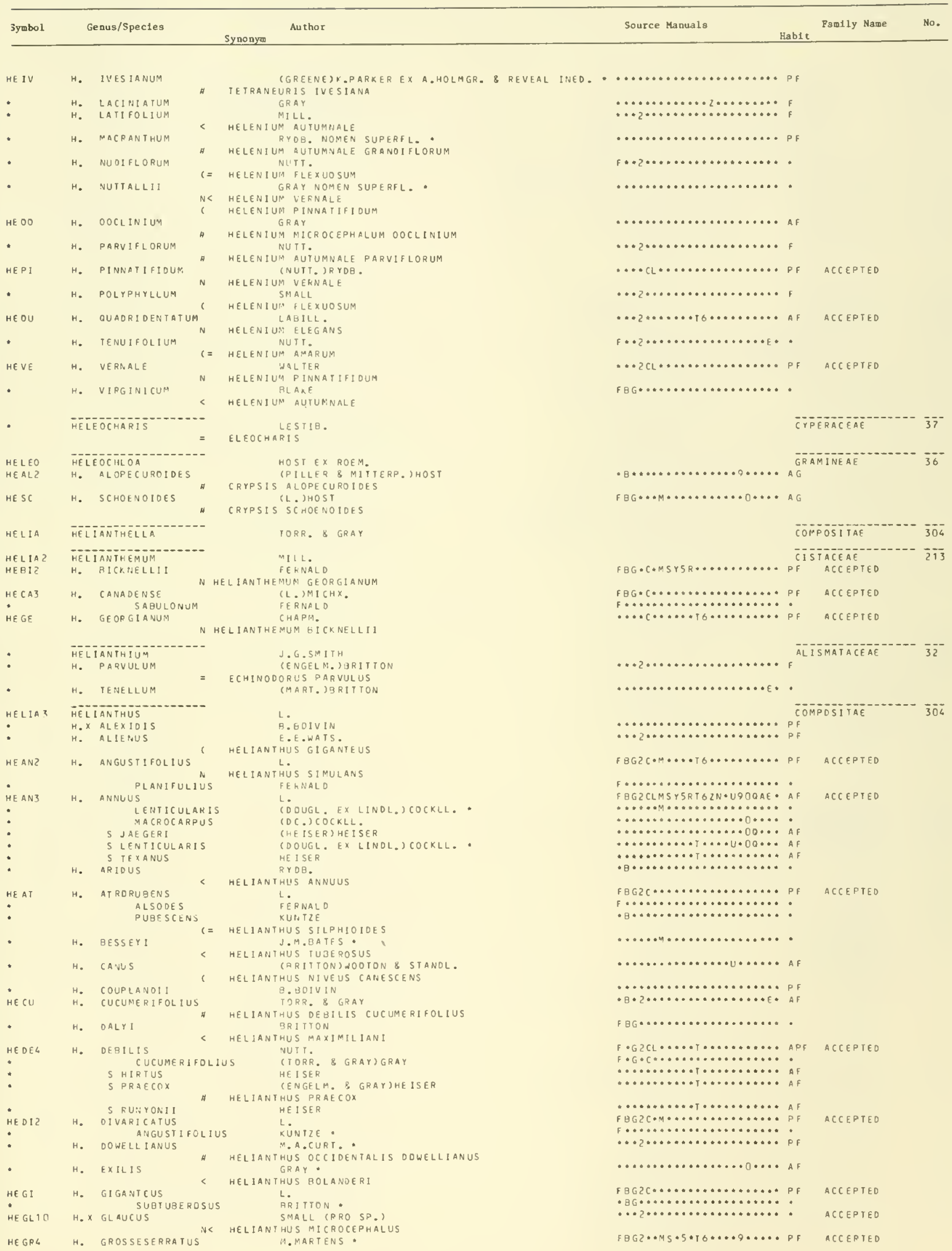




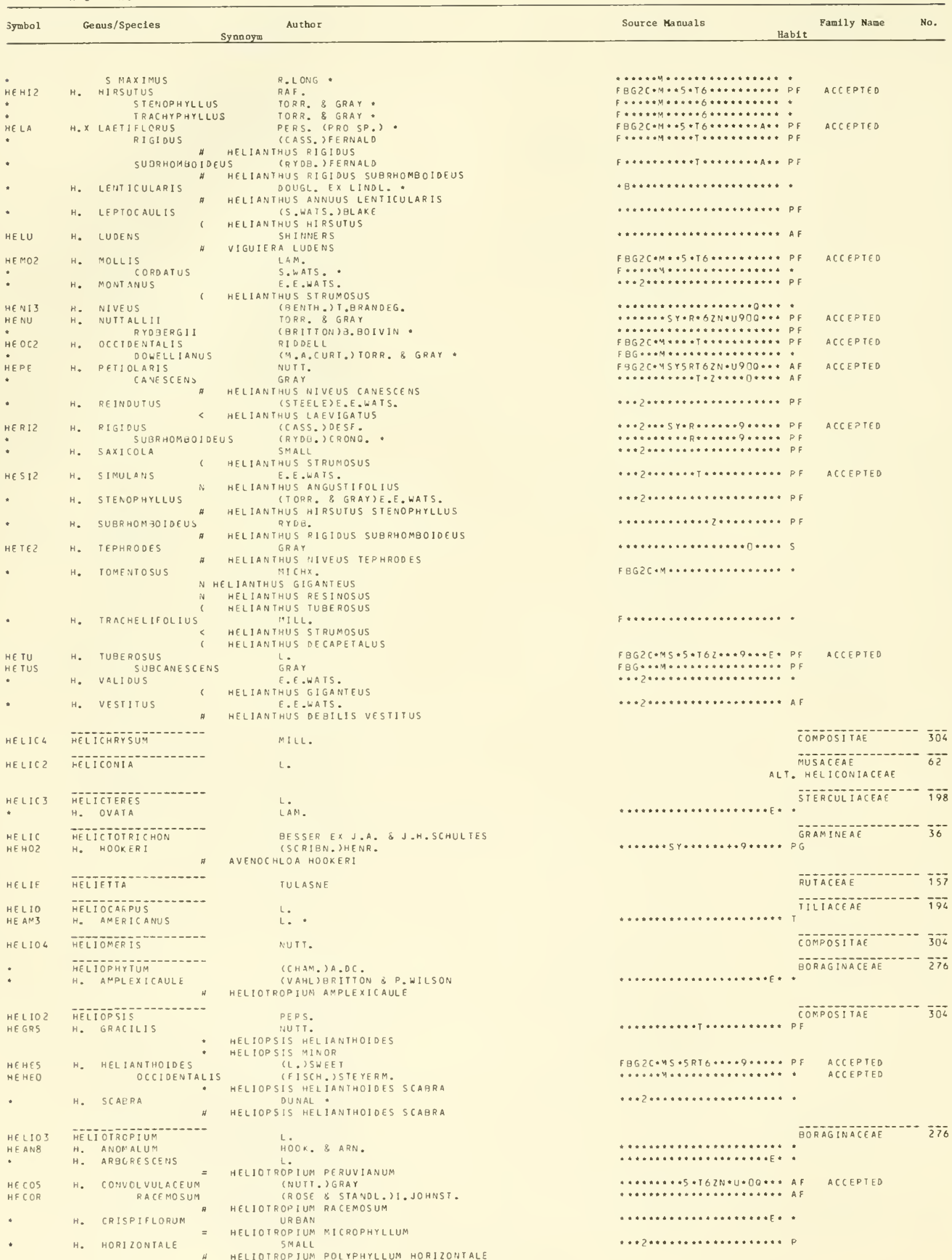


HELI OTROPIUM

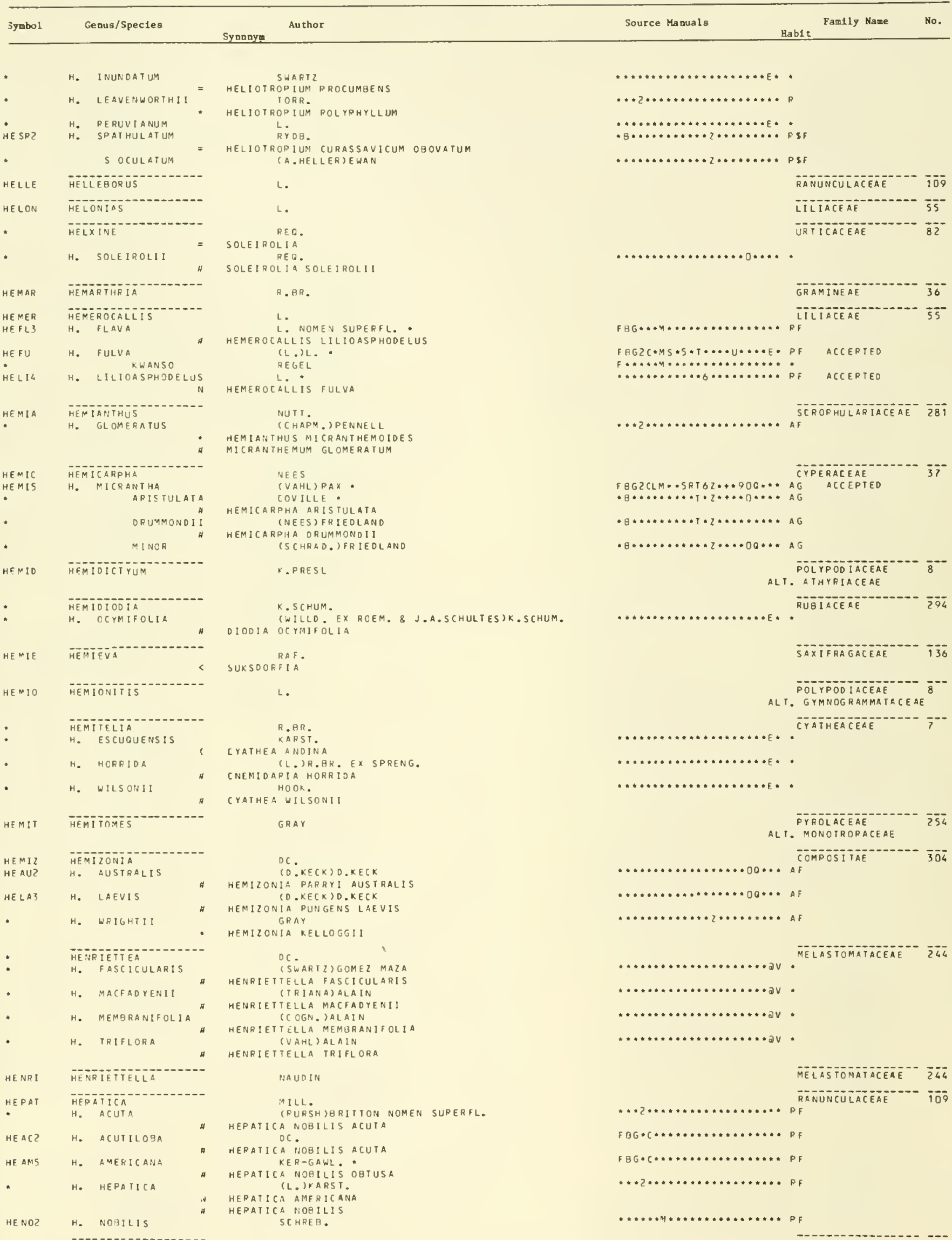




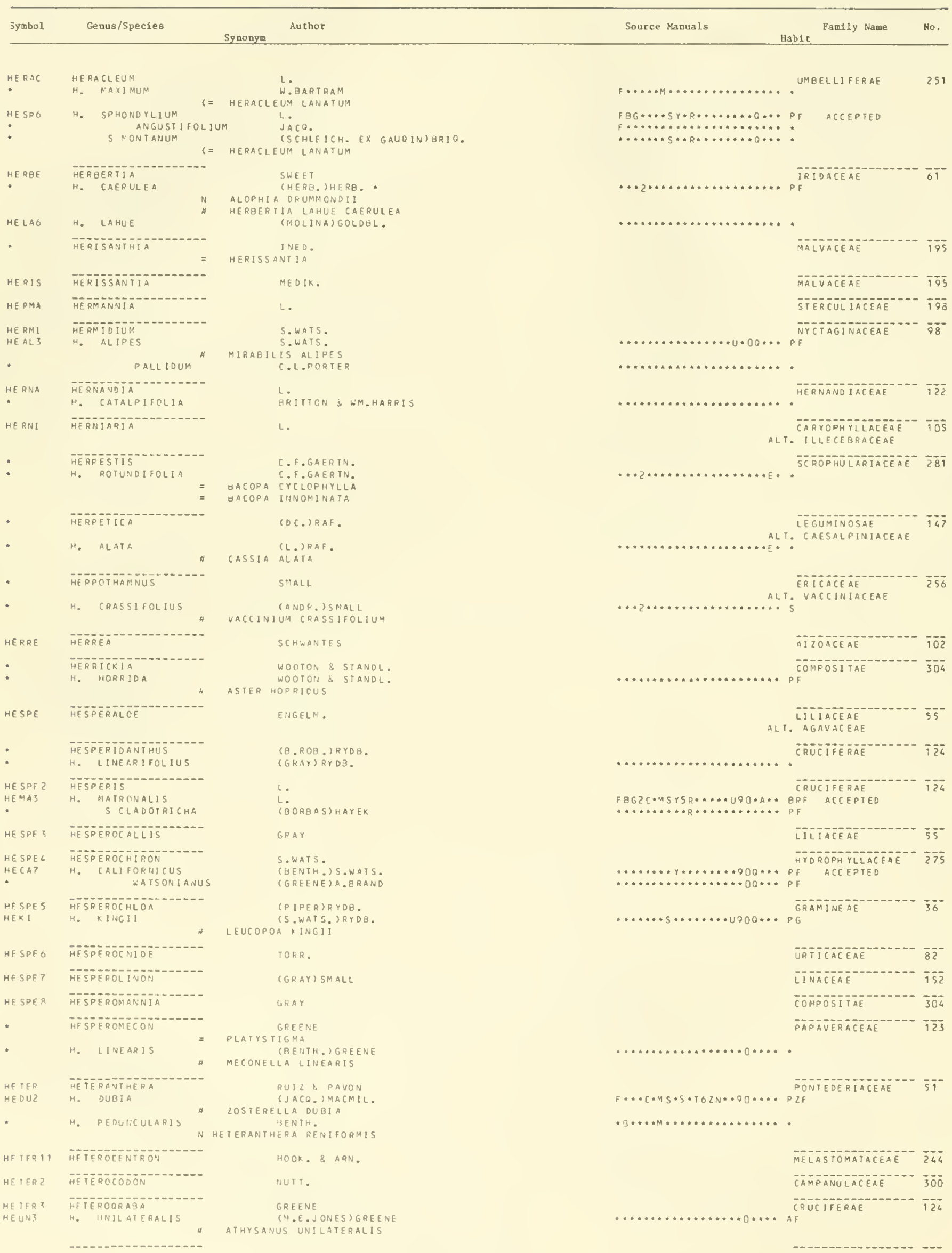


HETEROGAURA

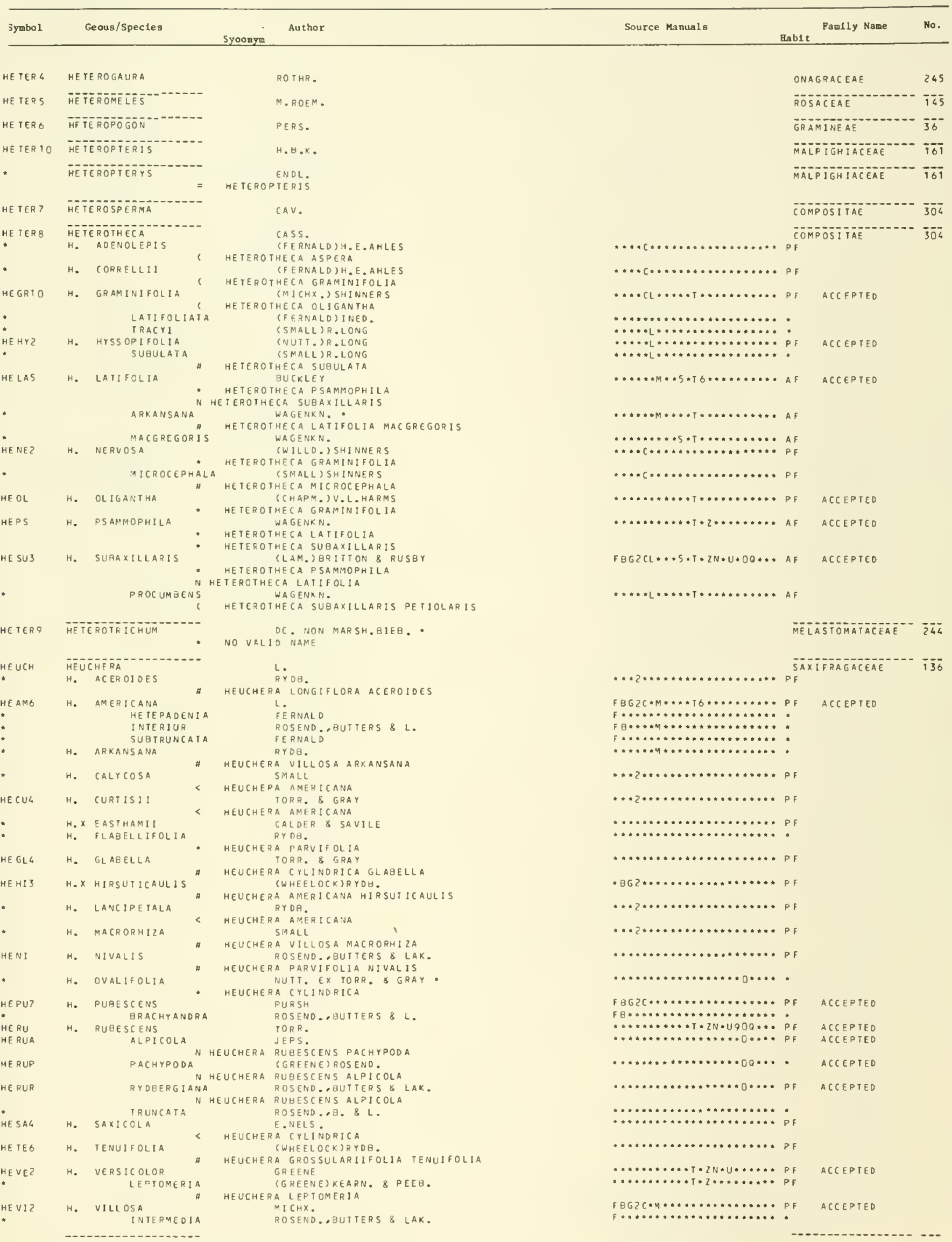




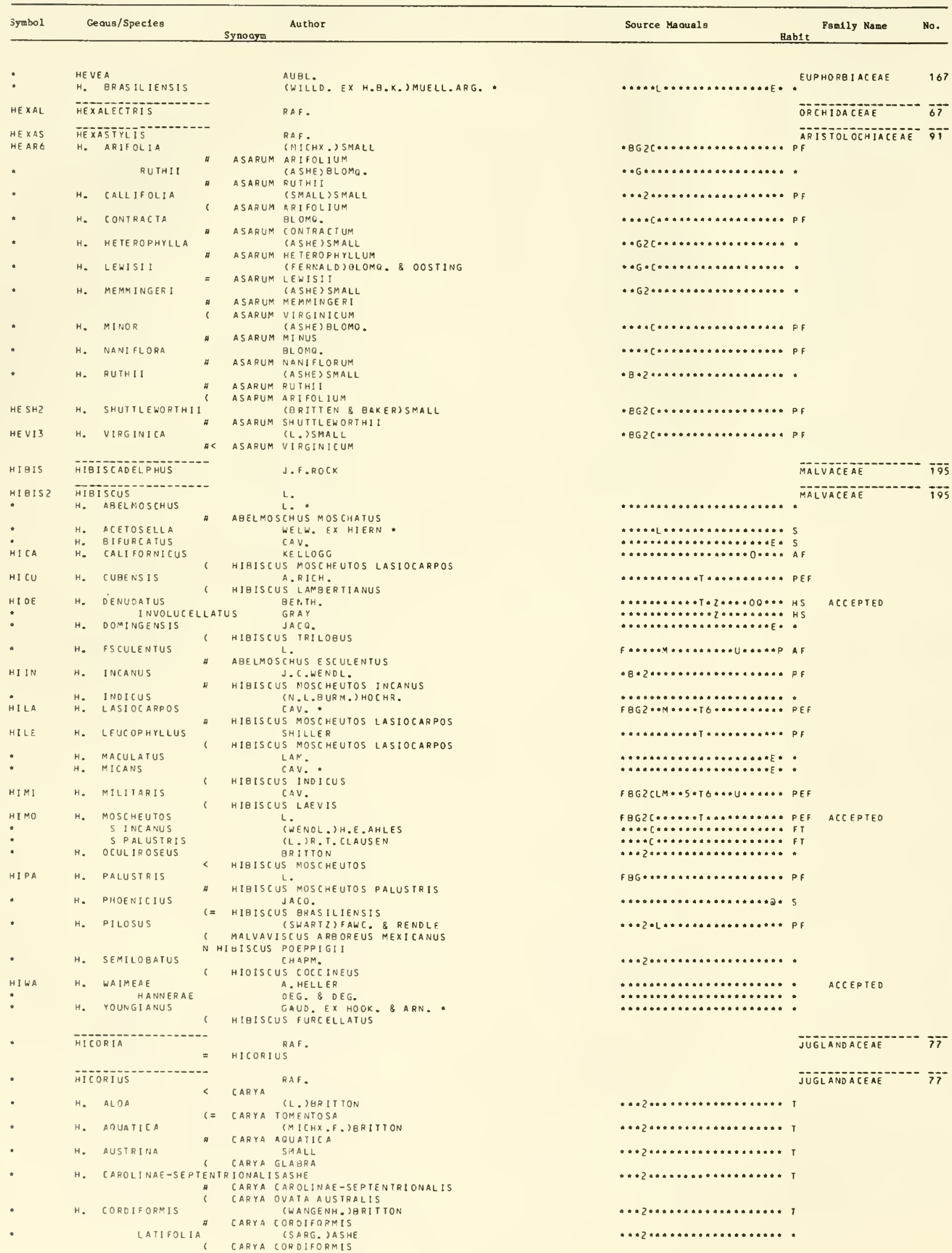




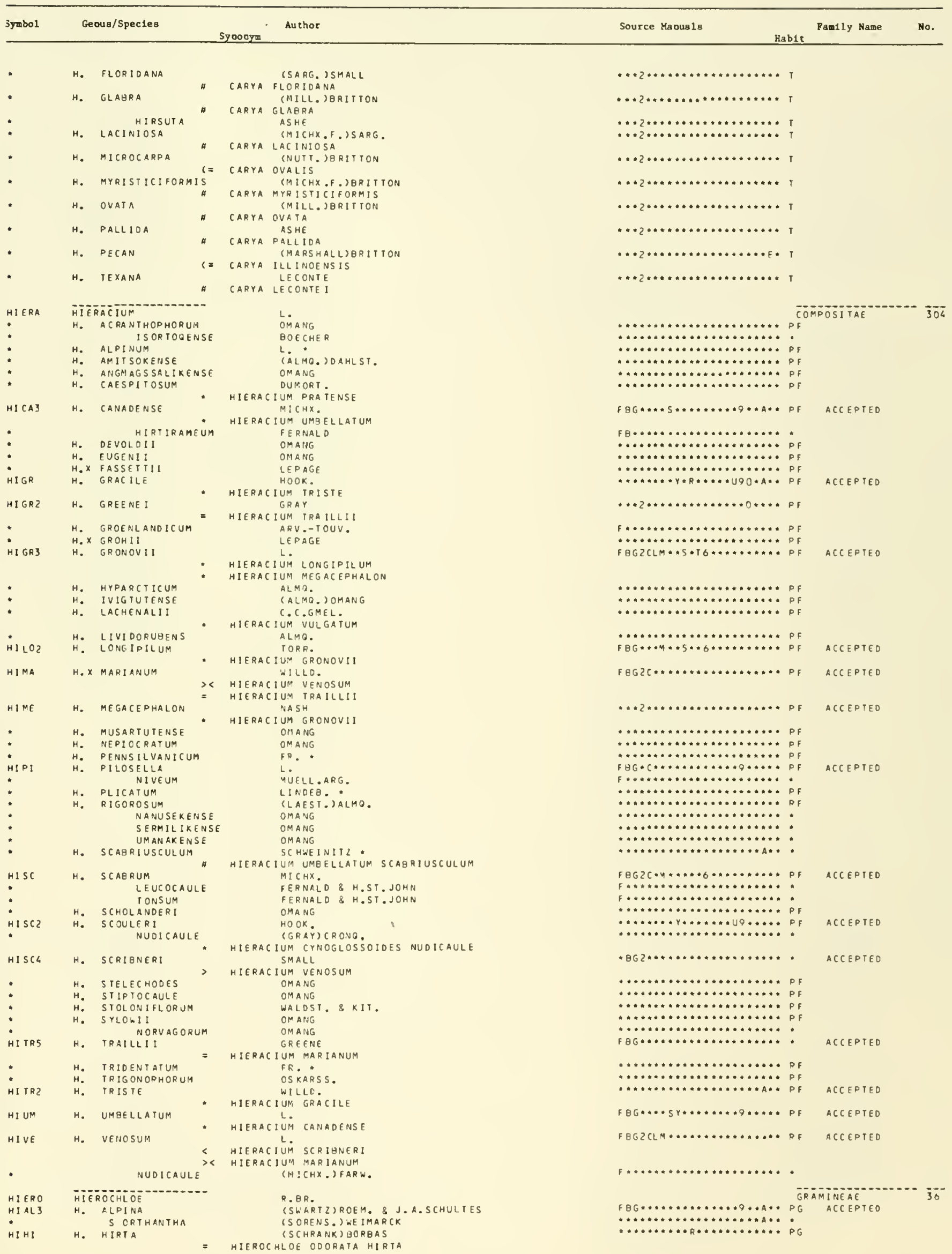




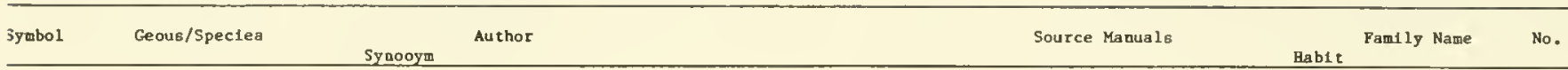

\section{H. NASHII}

HI L A

HILLE

H【LL】

HIPPE

HI PPO 3

- HIPPOCHAETE

H. MYEMAL IS

H. Laevigata

H. VARIEGATA

HIPPOL

HIFPOCRATEA

H. CARI $B A E A$

\section{HIPPOMANE}

\section{HIPPORHAE}

H. RHAMNOIDES

HIPPU

HITE

HI vu2

MIPPURIS

H. TETRAPHYLLA

H. WULGARIS

H. VULGARIS

HI RAE

HI RSH

HIRTE

HIST

HOFFM

HOBR

HOCA

HODE

HOOR2

HOFA

HOJA

HOME

HON!

HOPA

HOFME

HOPL

HOHEN

HOLAC

-

HOLCU

-

HOLL

HOLMS

HOLOC

\section{HIRAEA}

\section{HIRSHFELDIA}

HIRTELLA

HISTIOPTERIS

HOFF MANSEGGIA

H. BRACHYCARRA

H. CAUDATA

H. DENSIFL ORA

H. ORUMMONOII

H. FALCARIA

H. JAMESII

H. MELANOSTICTA

H. MICROPHYLLA

H. PARRY

\section{HOFMEISTERTA}

H. PLURISETA

HOHENBERGIA

HOHENBERGIA

HOLACATITHA

HOLCOPHACOS

H. DISTORIUS

\section{HOLCUS}

H. HALEPEISIS

H. SORGMUM

HOLLISTERI

HOLMSKIOLOIA

HOLOCARPHA

HOLOCARPHA

5 ELONGATA

HOLODISCUS

H. OISCOLOR DUMOSUS
(BICKN.) SKACZM. HIEROCHLOE ODORATA

$$
\begin{aligned}
& \text { H.B.K. } \\
& \text { D. OLIVER } \\
& \text { JACO. } \\
& \text { HERB. } \\
& \text { G.DON }
\end{aligned}
$$

$M I L D E$

CL.) FARW.

EQUISETUM HYEMALE

(A. BRAUN) FARW.

EQUISETUM LAEVIGATUM

(SCHLE ICH. EX F.WEBER \& D.M.H.MOMR) BOERNER EQUISETUM VARIEGAIUM

$$
\text { L. }
$$

URBAN

PRISTIMERA CARIEAEA

L.

L.

L.

HIPPURIS VULGARIS

HIPPURIS TETRAPHYLLA

$$
\begin{aligned}
& \text { JACQ. } \\
& \text { MOENCH } \\
& \text { L. } \\
& \text { (J. AGARDH)J.SMITH }
\end{aligned}
$$

CAV.

GRAY

CAESALPINIA RRACHYCARPA

GRAY

CAESALPINIA CAUDATA

BENTH.

HOFFMANSEGGIA GLAUCA

TORR. \& GRAY

CAESALPIN I A DRUMMONDII

CAY.

HOFEMANSEGGIA GLAUCA

TORR. \& GRAY

CAESALPINIA JAMESII

(SCHAUER)GRAY

CAESALPINIA WOOTONII

TORR.

CAESALPINIA VIRGATA

(E.M. FISHER)B.L.TURNER.

CAESALPINIA PARRYI

$$
\text { WALPERS * }
$$

GRAY

PLEUROCORONIS PLURISETA

J.A. \& J.H.SCHULTES

GRAY

RYD3.

(TORR. \& GRAY)RYDB .

ASTRAGALUS OISTORTUS

SORGHUM HALEPENSE

SORGHUM BICOLOR

$$
\begin{aligned}
& \text { S.WATS. } \\
& \text { REIZ. } \\
& \text { GREENE } \\
& \text { (GRAY) D.KECK } \\
& \text { D.KECK } \\
& \text { (K.KOCH)MAXIM. } \\
& \text { (PUQSH)MAXIM. } \\
& \text { (HUTT. EX HOOK. MAXIM. EX COULT. }
\end{aligned}
$$

A HOLODISCUS DUMOSUS

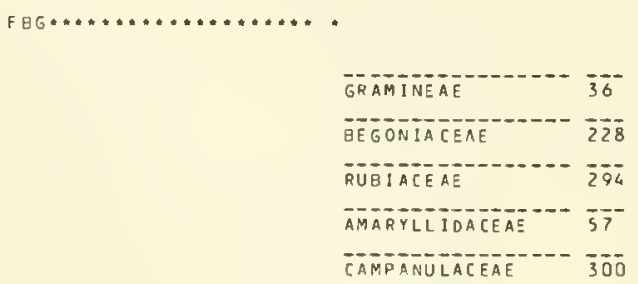

ALT. LOBELIACEAE

EDUISETACEAE

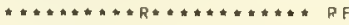

P F

HIPPOCRATEACEAE 179

EUPHORBIACEAE

ELAEAGNACEAE

HIPPURIDACEAE

rzF acCEPTED

$F B G \bullet \cdots S Y \cdot R \backsim Z N \cdot U 8 O Q A \cdots P Z F$ ACLEPTED

MALPIGHIACEAE

CRUCIFERAE

ROSACEAE

POLYPODIACEAE ALT. DENNSTAEDIJACEAE

LEGUMINOSAE

ALT TAESALPINIACEAE

................... pr

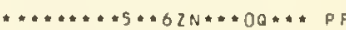

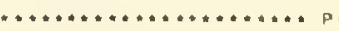

$\ldots \ldots \ldots+\ldots \ldots, 2+\ldots \ldots \ldots+F$

$4 B * \ldots 4 \cdots 5 \cdots 6 Z N \cdots * \ldots * \ldots$ PF

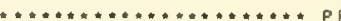

$\ldots \ldots \ldots+\ldots \ldots z * \ldots+00 * \ldots$ s

COMPOSITAE

$\ldots \ldots \ldots \ldots \ldots+2 N * U \cdot 0, \ldots$.

BROMELIACEAE

SIMAROU BACEAE

LEGUMINOSAE ALT. PAPILI ONACEAE

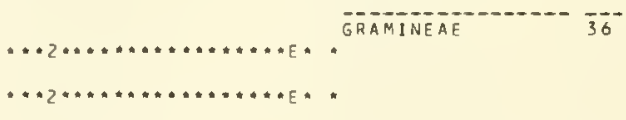

POLYGONACEAE

VERHENACEAE

COMPOSITAE

$\because \ldots \ldots * \ldots \ldots \ldots \cdots 00 \ldots * A$

ROSA $\bar{C} \bar{E} \bar{A} \bar{E}$ $\overline{1} \overline{45}$ 
HOLODISE US

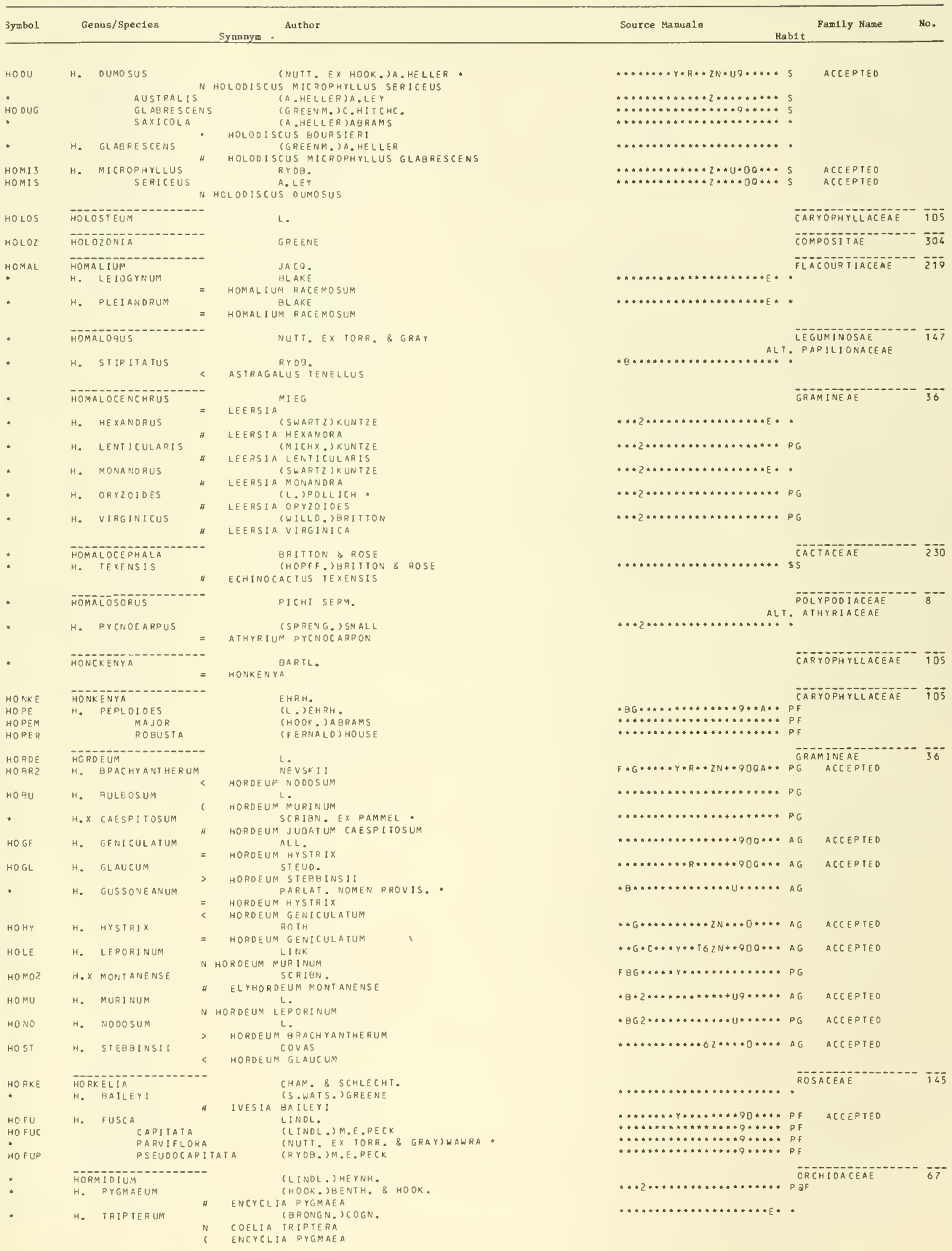




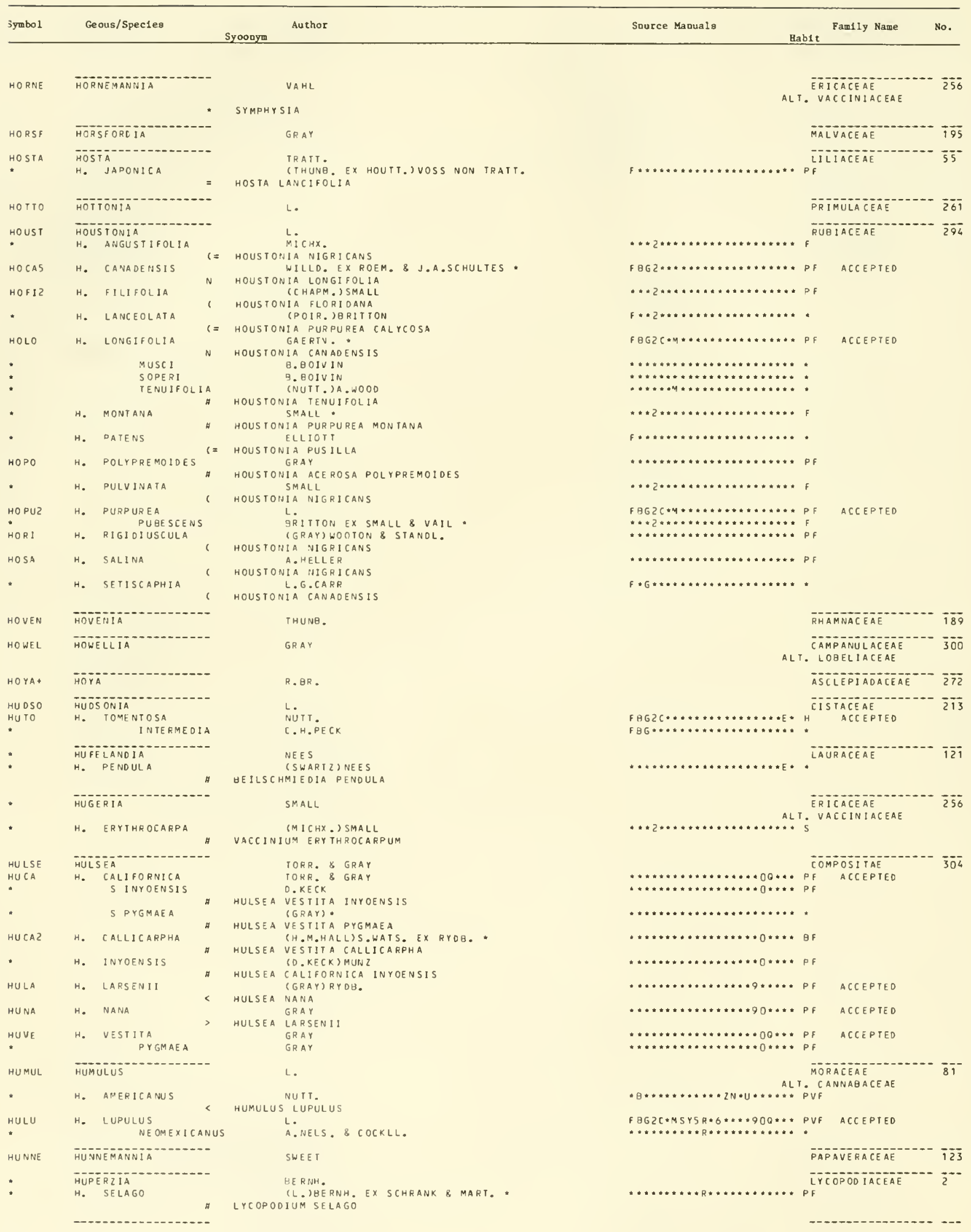




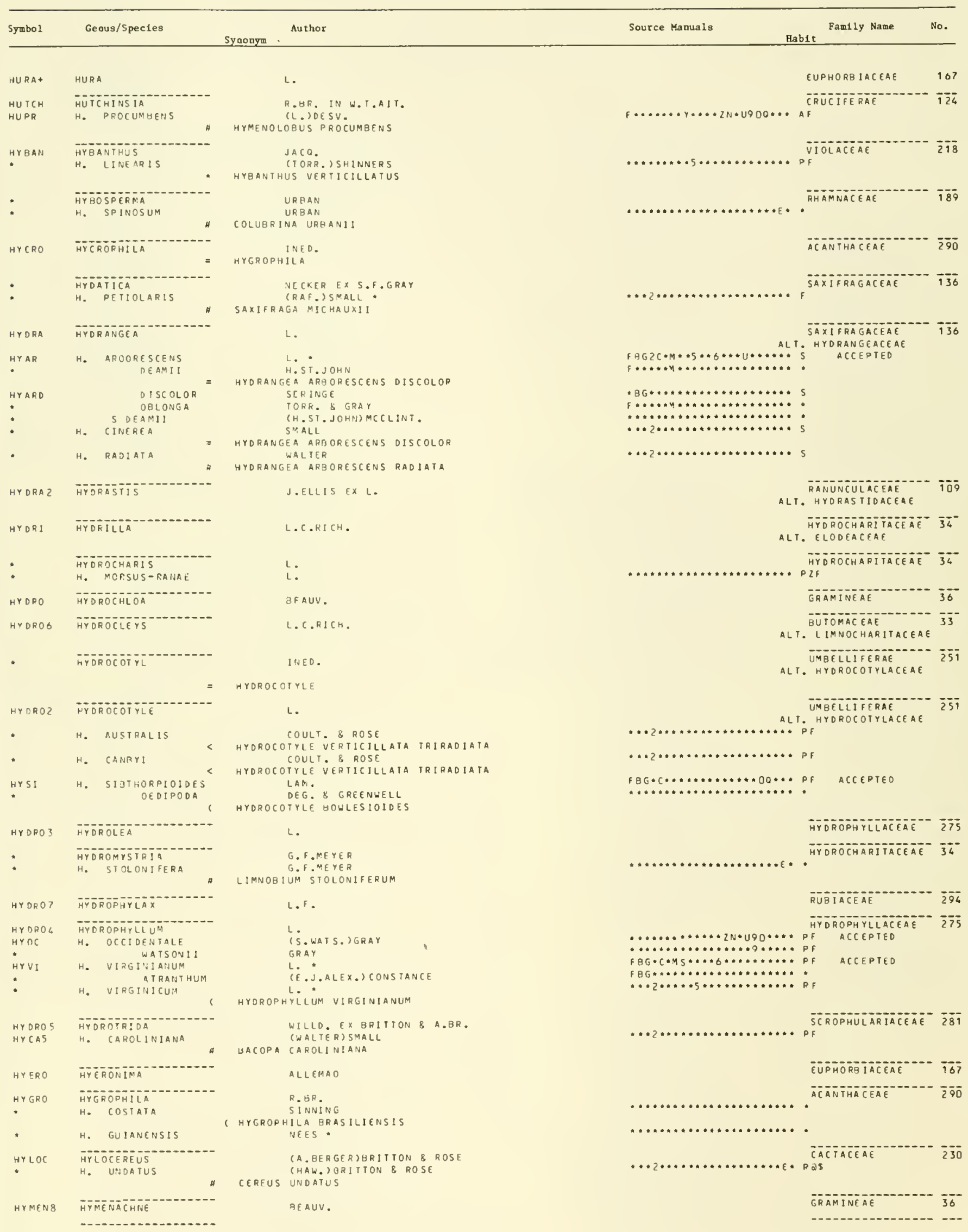




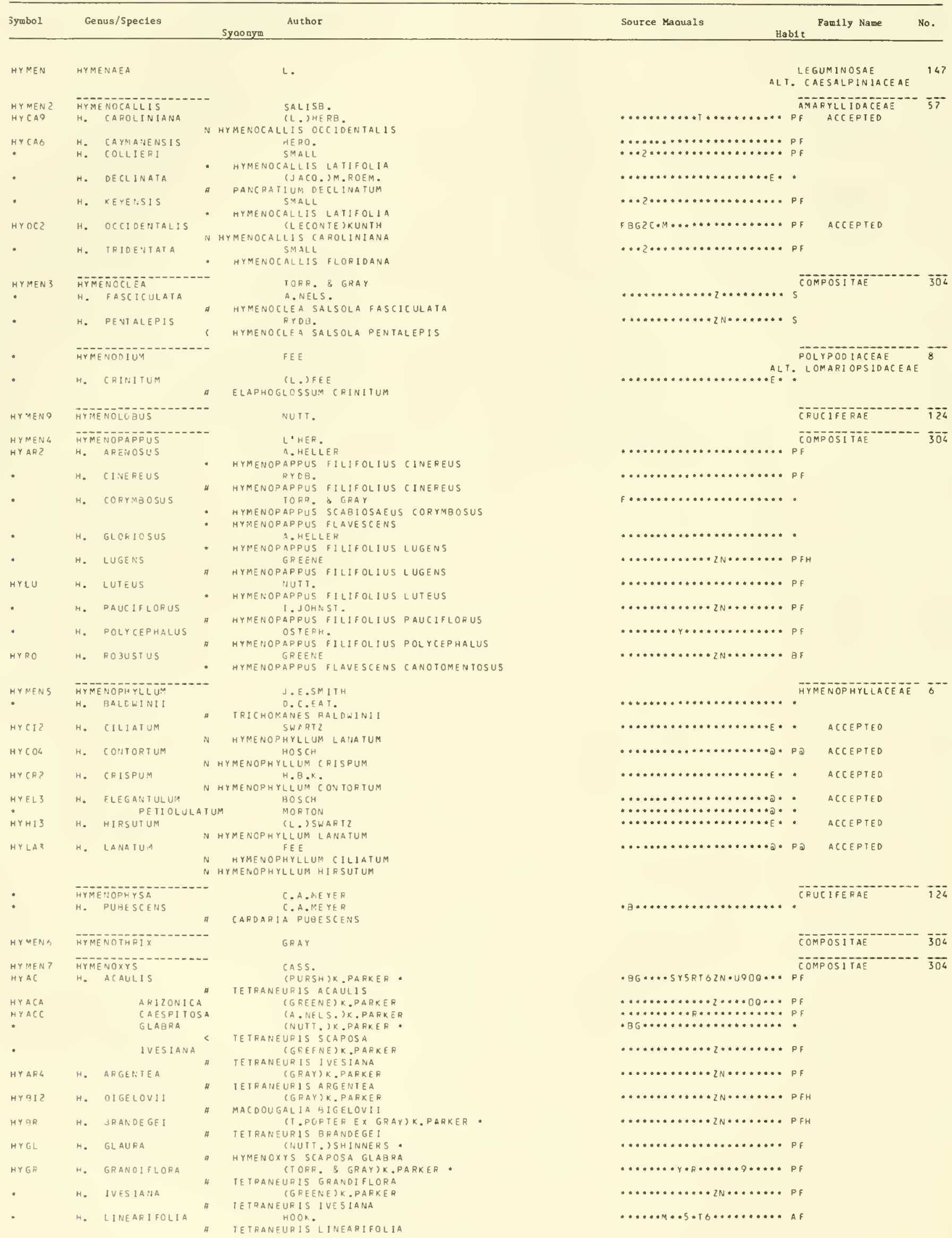




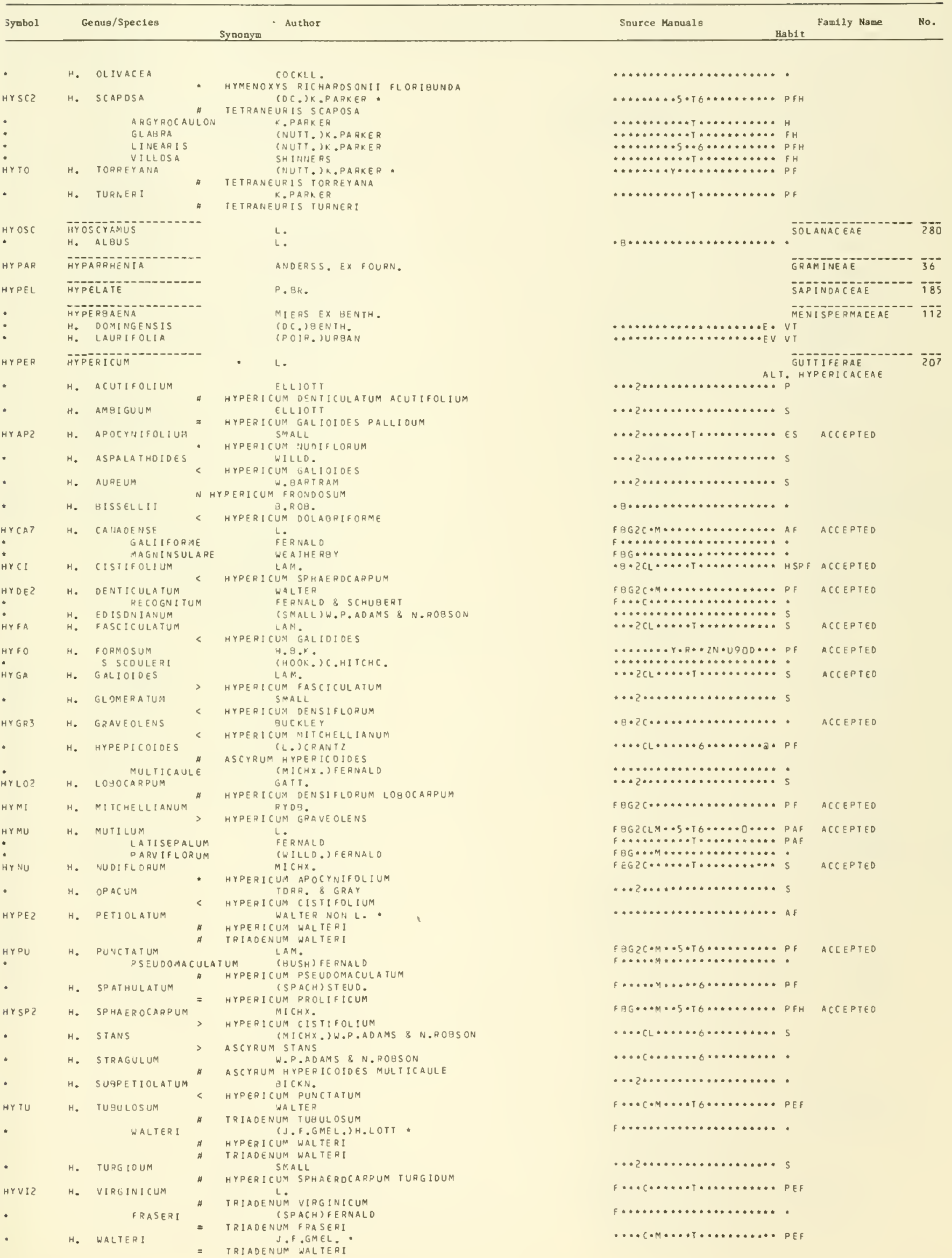




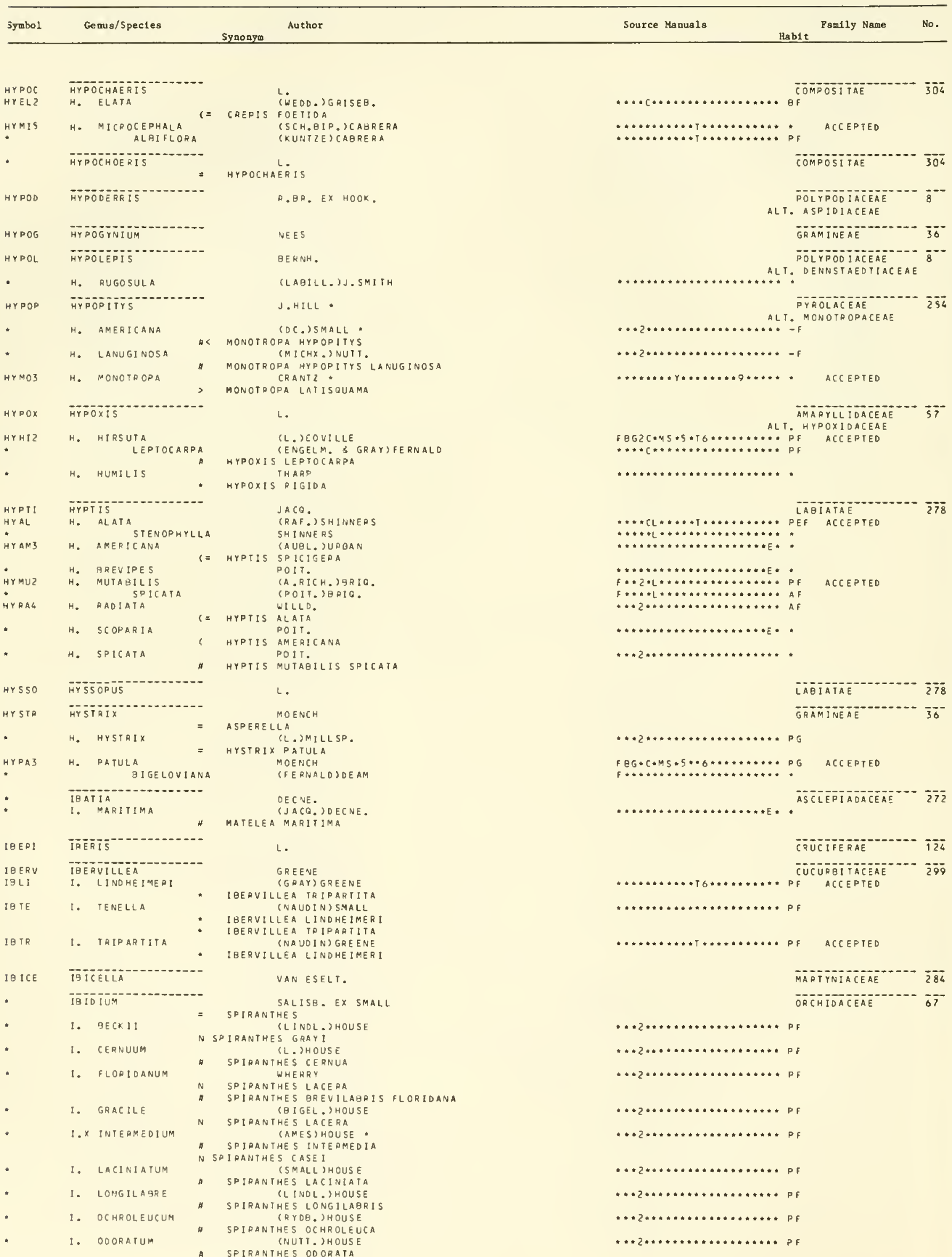




\section{1. ovale \\ 1. Plantagineuin \\ 1. PRAECOX \\ I. TORTILE \\ 1. vernale}

\section{ICACOREA}

1. GLAUCI FLORA

1. GUAOALUPENS IS

1. Luduillensis

I. Paniculata

1. MONTANA

BEAOLE I MOLLIS

1. MONTICOLA

\section{ILIAMNA}

1. COREI

\section{iticicium}

\section{ILYSANTHES}

1. DUB! $A$

1. GRANDIFLORA

1. INEQUAL IS

1. MONTICOLA

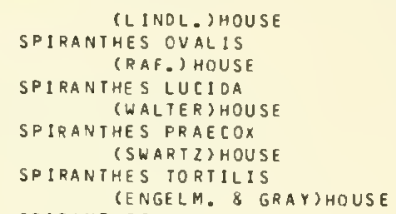

-

I. ALIISSIMA $\quad$ AUEL.

A.NELS. \& J.F.MACBR.

(MICHX.)TORR.

ILEX BEADLEI

(TORR. \& GRAY)H.E.AHLES

* Ilex montana$$
\text { L. }
$$$$
\begin{aligned}
& \text { ASHE } \\
& \text { - ILEX AMBIGUA }
\end{aligned}
$$$$
\text { ILEX DESIDUL }
$$

ILEX DECIDUA

ILEX AMBIGUA

ILEX AMBIGUA MBI GUA N ILEX VOMITORIA

SOLAND. IN AIT.

(WALTER) SARG.

ILEX MYRTIFOL IA
(FERNALD)SMALL

ILEX DECIDUA

ILEX DEEI QUA

SMALL

ILEX CURTISSII

ILEX BUSWELLII

(CHAPM.) H.E.AHLES

ILEX LONGIPES

$$
\text { TORR. \& GRAY . }
$$

(ASHE) FERNALD

(ASHE) FERNALD
EADLE!

ILEX BEADLE !
(GRAY) BR ITION

ILEX BEAOLEI

GRAY NOMEN SUPERFL. .

ILEX MONTANA

(GRAY) BRITTON

ILEX BEADLE!

SOLANO. IN AIT.

(ASHE) ASHE
ARICOLA, ILEX CUMULICOLA

$$
\begin{aligned}
& \text { CILLATA } \\
& \text { CYCLOPHYLLA } \\
& \text { FASTIGIATA } \\
& \text { PAOIFOLIA } \\
& \text { TENUIFOLIA }
\end{aligned}
$$

(L.) GRAY

B.ROB.

( $B I C K N$.) FERNALD

(WILLD.) TORR. \& GRAY

(TORR. ) S.WATS.

\section{GREENE}

IL IAMNA REMOTA

$$
\text { L. }
$$

\section{RAF.}

(L. . BARNHAR T

LINOERNIA DUBIA (NUTT. JEENTH.

LINDERNIA GRANOIFLORA (WALTER) PENNEL

$=$ LINDERNIA ANAGALLIOEA

GRATIOLA BREVIFOLIA

(NUTT.)RAF.

* linoernia monticola

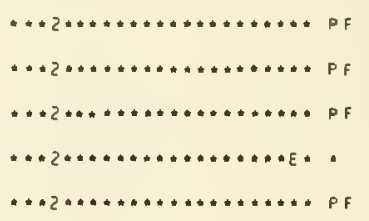

MYRSINACEAE
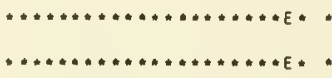

....................
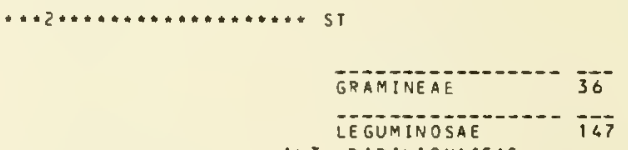

ALT. PAPILIONACEAE
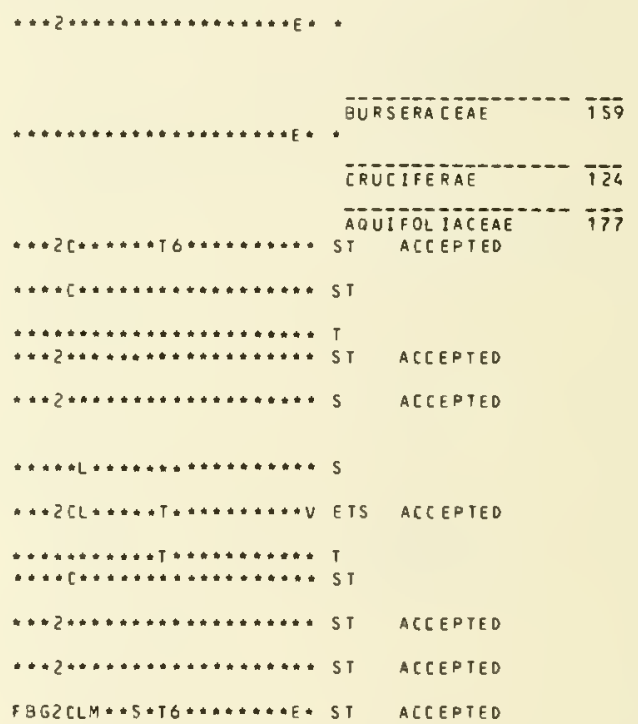

$* * *[* \ldots * \ldots+\ldots * * * \cdots * *$ s

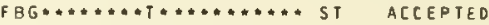

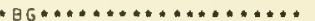

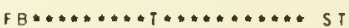

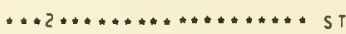

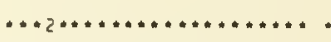

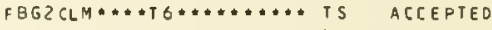

$\cdots+\ldots \ldots+\cdots \cdots+\cdots+\ldots+\cdots$

FBG 2 C $M \# \# * \mathrm{~T} * * * * * * * *$ EST ACLEPTED

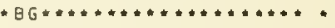

f $Q G \ldots \ldots \ldots \ldots \ldots \ldots \ldots \ldots \ldots \ldots$

F $B G, \ldots M+\ldots \ldots \ldots \ldots \ldots$.

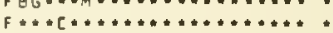
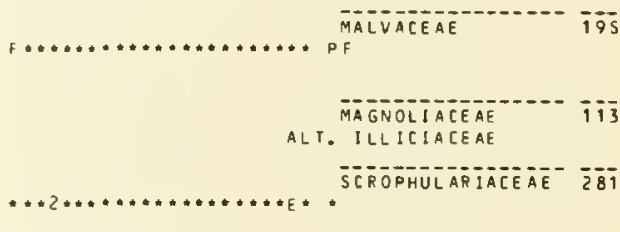

... $2 \ldots \ldots \ldots \ldots \ldots \ldots \ldots+\ldots$

$* *+2 * * \ldots \ldots+\ldots \ldots \ldots \ldots \ldots \ldots F$ 


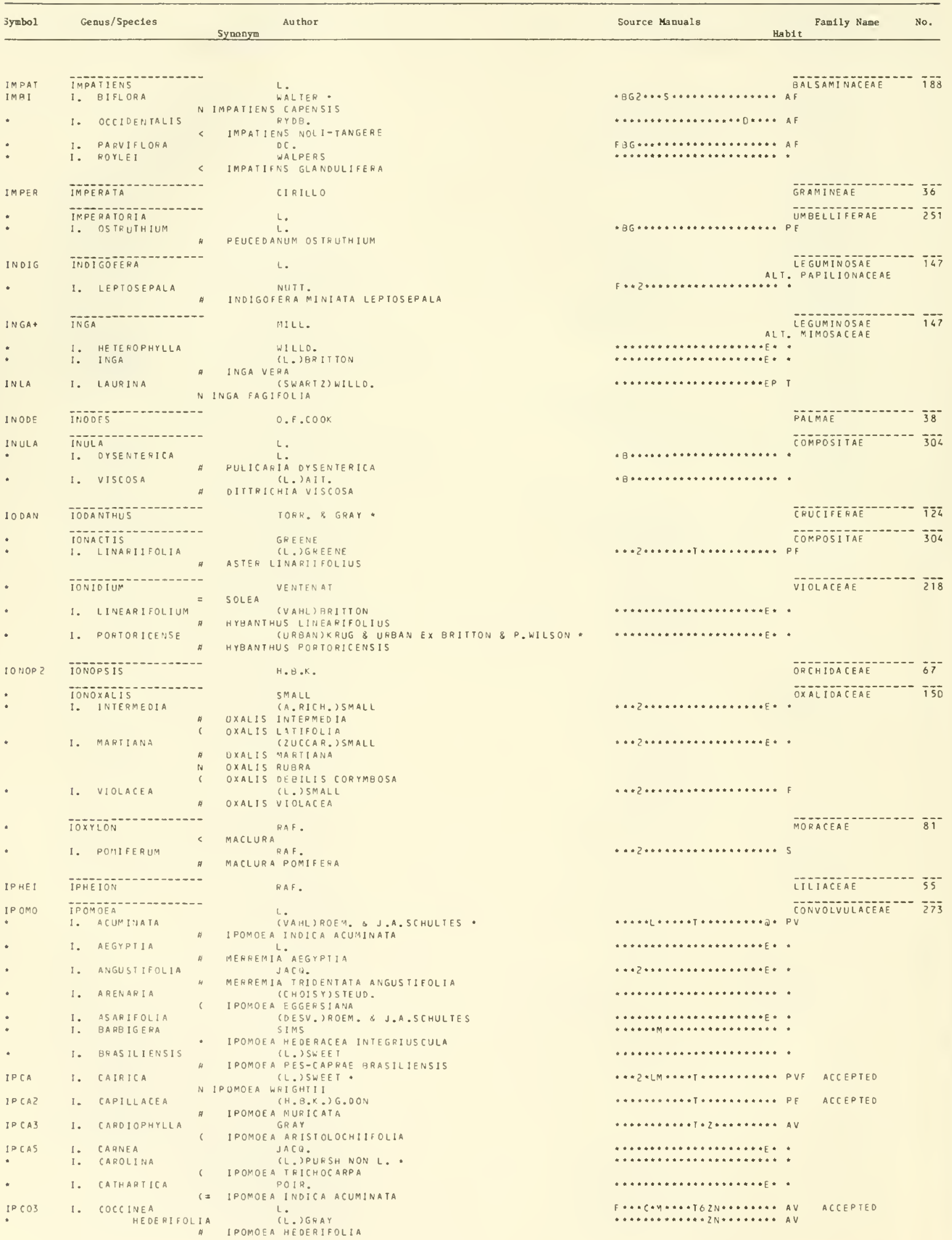




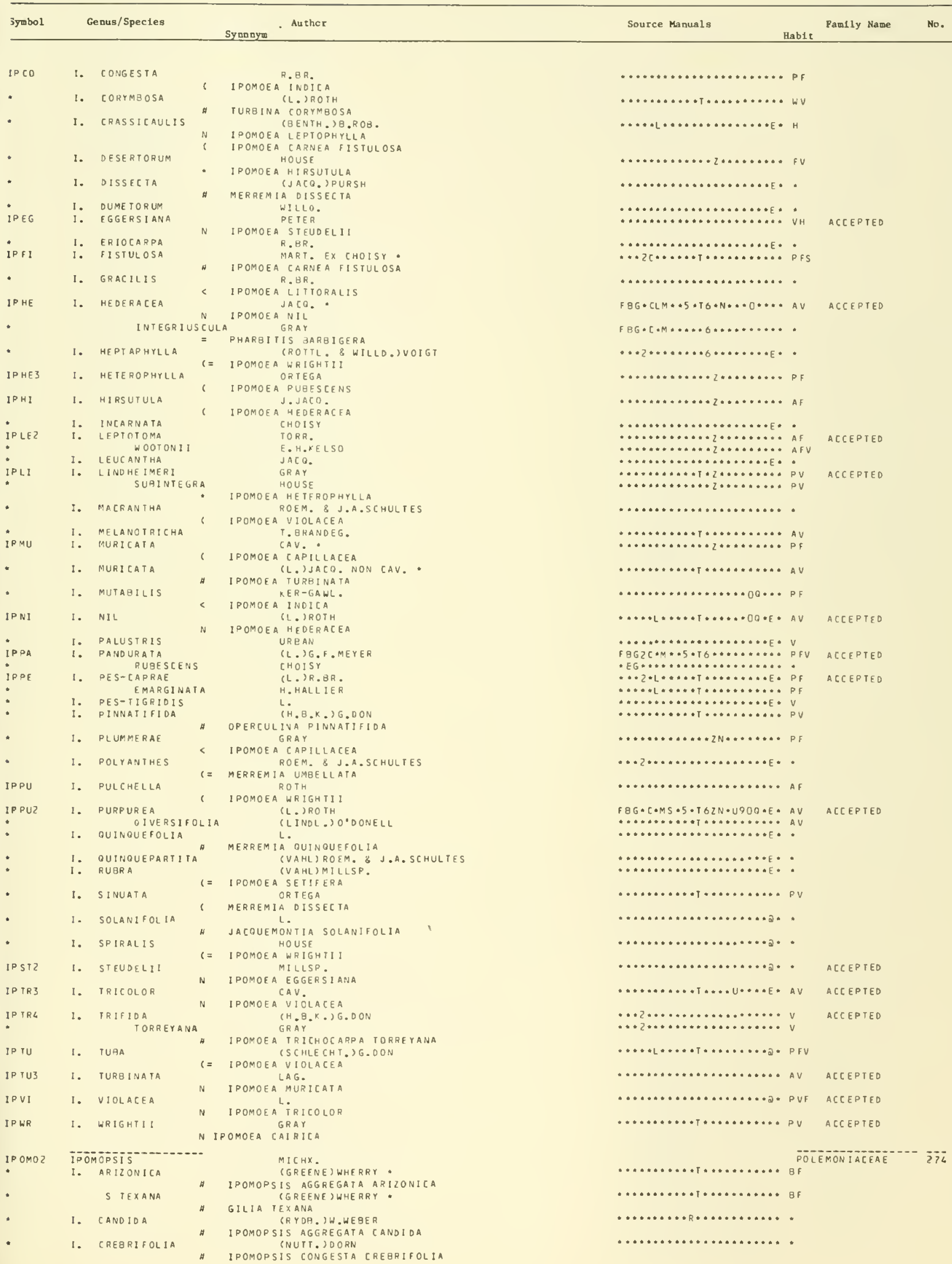




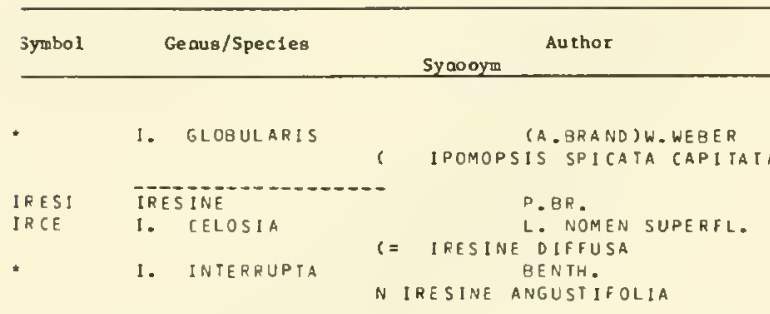

\section{IRIS}

1. ACLEANTHA

1. al abamensis

1. algilinea

l. alsispirtitus

f. altickistata

1. AMNICOLA

I. APHYLLAa

i. ATROCYANEA

J. atroenantha

I. AURALATA

1. aurillinea

I. gifurcata

1. brevipes

1. gucharica

I. callillopha

1. callirhodea

? CAROLINA

1. CARQLINA
i. CERASIOIDES

i. chamaeiris

i. chlorolopha

1. chrYsaeola

1. chrysolopha

1. chrYsophoENicta

1. citricristata

1. citriviola

1. Croc INUBIA

I. cyanantha

1. cyanochrysea

1. DEWINKELERI

i. Dichotoma

i. ecristaia

1. elepHantina

1. fluviatilis

I. FOLIOSA

1. fourchiana

1. fulvaurea

I. fumiflora

t. fumifulva

i. fuscaurea

1. fuscirosea

I. fuscisanguinea

1. fuscivenosa

I. Gatesil

i. gentilliana

1. IANIHINA

1. IOCHROMA

I. Iocranea

1. IODANTHA
N IRESINE ANGUSTIfOLIA

\begin{tabular}{|c|c|c|}
\hline & & SMALL \\
\hline \multirow[t]{2}{*}{$<$} & IRIS & VIRGINICA \\
\hline & & $S M A L L$ \\
\hline$<$ & IFIS & HEXAGONA \\
\hline \multirow[t]{2}{*}{$<$} & IRIS & $\begin{array}{l}\text { E.J.ALEX. } \\
\text { VIRGINICA }\end{array}$ \\
\hline & & SMALL \\
\hline$<$ & IRIS & HEXAGONA SAVANNA RUM \\
\hline \multirow[t]{2}{*}{$<$} & I R IS & $\begin{array}{l}\text { SMALL } \\
\text { GIGANTICAERULEA }\end{array}$ \\
\hline & IRIS & $\begin{array}{l}\text { E.J.ALEX. } \\
\text { VIRGINICA }\end{array}$ \\
\hline$<$ & & SMALL \\
\hline \multirow[t]{2}{*}{$<$} & If IS & VIRGONICA \\
\hline & & SMALL \\
\hline$<$ & IRIS & $\begin{array}{l}\text { VIRGINICA } \\
\text { SMALL }\end{array}$ \\
\hline$<$ & IRIS & $\begin{array}{l}\text { VIRGIIICA } \\
\text { E.J.ALEX. }\end{array}$ \\
\hline$<$ & IRIS & $\begin{array}{l}\text { GIGANTICAERULEA } \\
\text { SMALL }\end{array}$ \\
\hline$<$ & I $R$ IS & $\begin{array}{l}\text { VIRG INICA } \\
\text { SMALL }\end{array}$ \\
\hline$<$ & IRIS & $\begin{array}{l}\text { BREVICAULIS } \\
\text { R. C.FOST. } \\
\text { E.J.ALEX. }\end{array}$ \\
\hline \multirow[t]{2}{*}{$<$} & IRIS & VIRGINICA \\
\hline & IRIS & $\begin{array}{l}\text { E.J.ALEX. } \\
\text { VIRGINICA }\end{array}$ \\
\hline \multirow{2}{*}{$<$} & & RADIUS \\
\hline & IRIS & $\begin{array}{l}\text { E.JALEX. } \\
\text { VIRGINICA }\end{array}$ \\
\hline$<$ & & SMALL \\
\hline$<$ & IRIS & $\begin{array}{l}\text { VIRGINICA } \\
\text { SMALL }\end{array}$ \\
\hline \multirow[t]{2}{*}{$<$} & IRIS & VIRGINICA \\
\hline & & SMALL \\
\hline$<$ & IRIS & VIRGINICA \\
\hline \multirow{2}{*}{ A } & & SMALL \\
\hline & IRIS & VINICOLOR \\
\hline & & SMALL \\
\hline$<$ & IRIS & GIGANTICAERULEA \\
\hline & & SMALL \\
\hline$<$ & IRIS & VIRG INICA \\
\hline$<$ & IRIS & $\begin{array}{l}\text { E.J.ALEX. } \\
\text { VIRGINICA }\end{array}$ \\
\hline$<$ & IRIS & $\begin{array}{l}\text { E.J.ALEX. } \\
\text { VIQGINICA }\end{array}$ \\
\hline & & SMALL \\
\hline$<$ & IRIS & $\begin{array}{l}\text { VIRGINICA } \\
\text { SMALL }\end{array}$ \\
\hline & & $\begin{array}{l}\text { PALLAS } \\
\text { E.J.ALEX. }\end{array}$ \\
\hline$<$ & IRIS & $\begin{array}{l}\text { FULVA } \\
\text { SMALL }\end{array}$ \\
\hline$<$ & IRIS & $\begin{array}{l}\text { GIGANTICAERULEA } \\
\text { SMALL }\end{array}$ \\
\hline$<$ & IRIS & GIGANTICAERULEA \\
\hline * & IRIS & $\begin{array}{l}\text { BREVICAULIS } \\
\text { SMALL }\end{array}$ \\
\hline$<$ & IRIS & $\begin{array}{l}\text { VIRGINICA } \\
\text { SMALL }\end{array}$ \\
\hline$<$ & IRIS & VIRGINICA \\
\hline & & E.J.ALEX. \\
\hline$<$ & $1 \mathrm{R} ! 5$ & $\begin{array}{l}\text { VIRGINICA } \\
\text { SMALL }\end{array}$ \\
\hline$<$ & IRIS & $\begin{array}{l}\text { VIRGINICA } \\
\text { SMALL }\end{array}$ \\
\hline$<$ & IRIS & VIRGINICA \\
\hline & & SMALL \\
\hline & & E. J.ALEX. \\
\hline$<$ & $1 \mathrm{R}$ IS & $\begin{array}{l}\text { VIRGINICA } \\
\text { SMALL }\end{array}$ \\
\hline$<$ & IRIS & $\begin{array}{l}\text { VIRGINICA } \\
\text { R. C.FOST. }\end{array}$ \\
\hline & & E.J.ALEX. \\
\hline$<$ & 1 RIS & VIRGINICA \\
\hline & & E. J.ALEX. \\
\hline$<$ & IRIS & $\begin{array}{l}\text { VIRG INICA } \\
\text { SMALL }\end{array}$ \\
\hline & $1 \% 15$ & VIRGINICA \\
\hline & & SMALL \\
\hline & IRIS & VIRGINICA \\
\hline 8 & IRIS & $\begin{array}{l}\text { E.JALEX. } \\
\text { VIRGINICA }\end{array}$ \\
\hline
\end{tabular}

AMARANTHACEAE

$\# * 2 * L \cdots * * T * \cdots * * \cdots E *$ APFV

Pam11y Name

No.
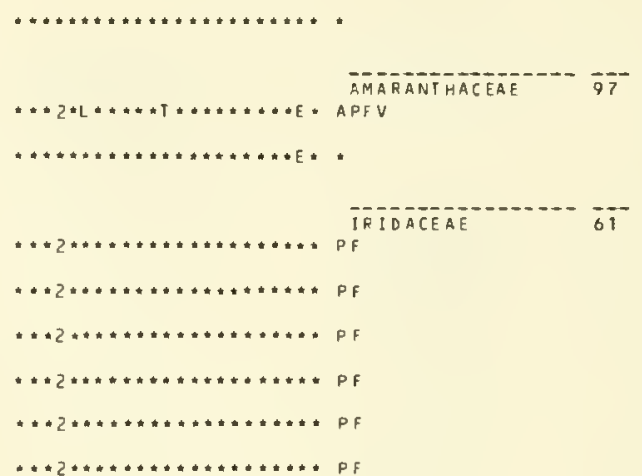

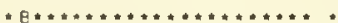

$* * 2+\cdots * * * * * * * * * \cdots *$ PF

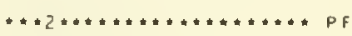

$* *+2 * * * * * * * * * * * * *+* * * P F$

$* * * 2 * * * * * * * \ldots * * * * \ldots, P F$

$* * 2 \cdots * * * * * * * * * \ldots * P F$

$\ldots * 2 * \ldots \ldots \ldots * \ldots \ldots * \ldots * P F$

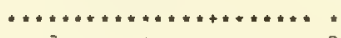

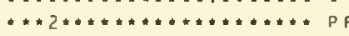

$* * 2 * \ldots * \ldots \ldots * * * * * * * * P F$

$* * * 2 * * * * * * * * * * * * \cdots *$ P

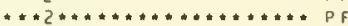

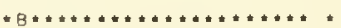

$\ldots+2 \ldots \ldots \ldots \ldots \ldots \ldots \ldots+\ldots$

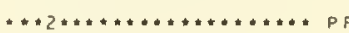

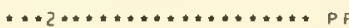

$* * 2 \cdots+\cdots+\cdots+\cdots+\cdots+\cdots P F$

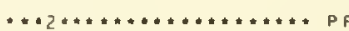

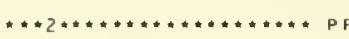

$\ldots+2 \ldots \ldots \ldots+\ldots+\cdots \cdots \ldots+* *$ P

$* * 2 *+\ldots+* * * * * * * * * * * P F$

$* * 2 * * * * * * * * * * * * * * * P F$

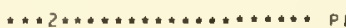

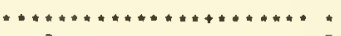

$* * \star 2 * * * * * * * * * * * * * * * * t$ P f

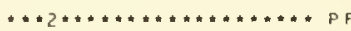

$* * * 2 * * * * * * \cdots * * * * * * \ldots * P F$

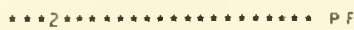

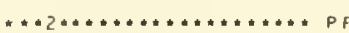

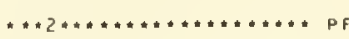

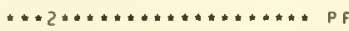

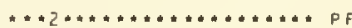

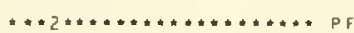

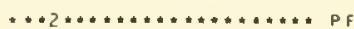

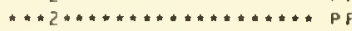

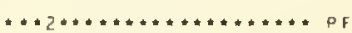

$* * * * \ldots+\ldots+\ldots+\ldots+\ldots \ldots \ldots \ldots$

$* * * 2 * * * * * * * * * * * * * * * * P F$

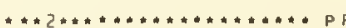

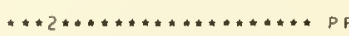

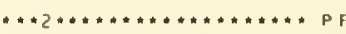




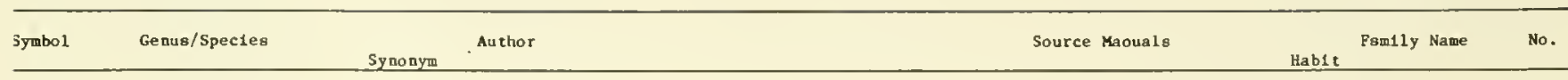

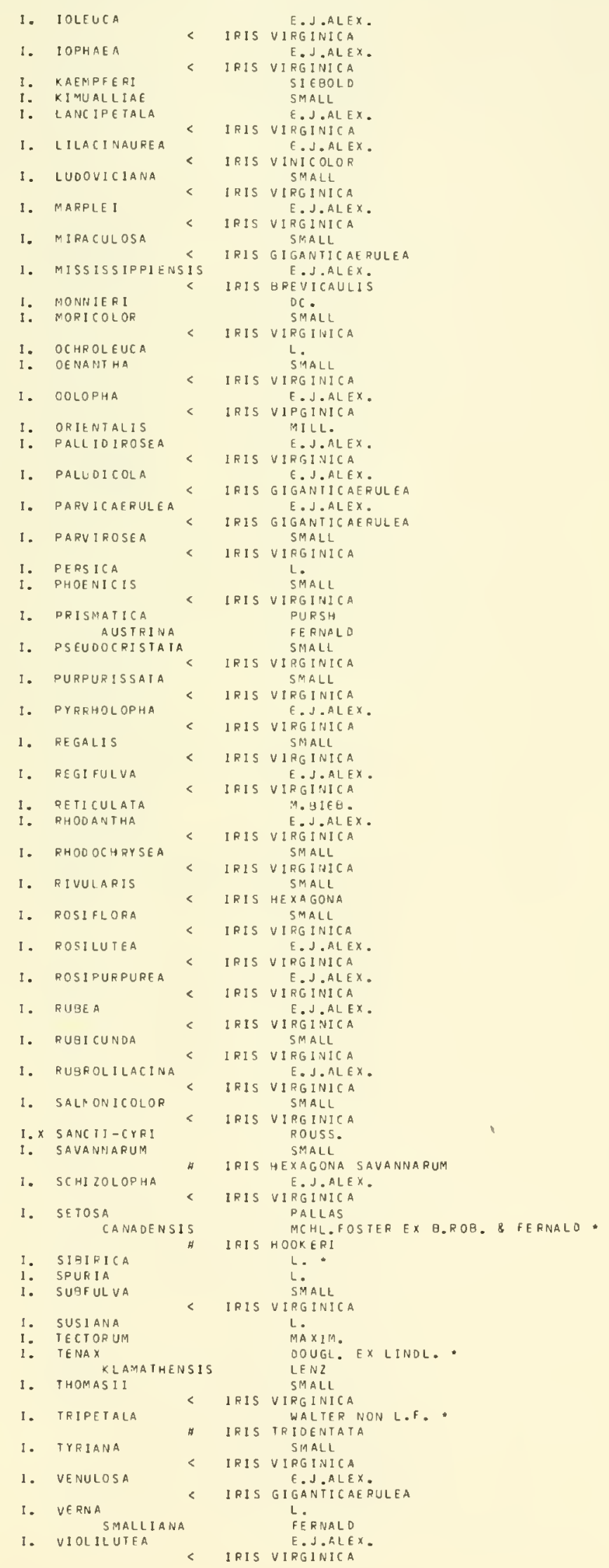

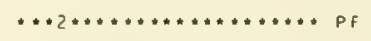

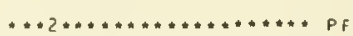

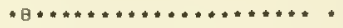
$* * * 2 * * *+* * * * * * * * * * *) P F$

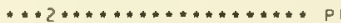

$\ldots \cdot 2 \ldots \ldots \ldots \ldots \ldots \ldots \ldots * P_{F}$
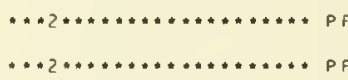

..2............. PF

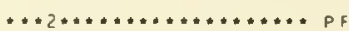

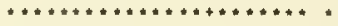
$* * 2 * * * * * * * * * * * * * * * P F$

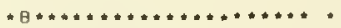

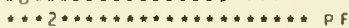

$* * 2 * \cdots * \cdots * * * * * * \cdots \cdots * P F$

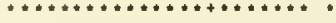
$\ldots, 2+* \ldots \ldots+\ldots+\ldots \ldots \ldots+\ldots$ p

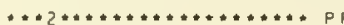

$* *+2 * * *+* * * * * * * * * * P F$

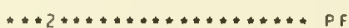

$\because \ldots \ldots \ldots \ldots \ldots \ldots+\ldots \ldots \ldots, \dot{p}$

f $A G 2 C * * * * * * * \cdots+\cdots * * P F$ $f \cdots \ldots+\ldots \ldots \ldots \ldots \ldots \ldots \ldots \ldots$ $* * * 2 * * * * * * * * * * * * * * * * * P F$

$* * 2 * * * * * * * * \ldots \ldots+\ldots * * * P F$

$* * * 2 * * * \ldots \ldots \ldots+\ldots \ldots \ldots \ldots p q$

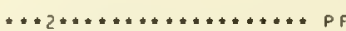

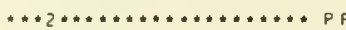

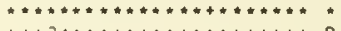
$* \cdots 2 \cdots * \ldots \ldots \ldots \ldots \ldots \ldots \ldots, P F$

$\cdots+2 \ldots * \ldots \ldots \ldots+\cdots \cdots \cdots * P F$

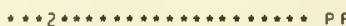

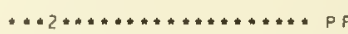

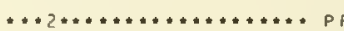

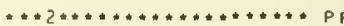

$* * 2 * \ldots * * \cdots \cdots \cdots * * * * * b$

$* * * 2 * * * * * * * * * * * * * * * * * b F$

$\ldots+2 \ldots \ldots \ldots+\cdots * \ldots \ldots \ldots * p$

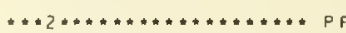

$\ldots+\ldots \ldots+\ldots+\ldots+\ldots+\ldots \ldots+\ldots$ b

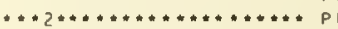

$\ldots * 2 * \ldots * \cdots \cdots * \ldots \ldots * * * * p q$

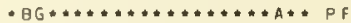
* $B G \ldots \ldots \ldots \ldots \ldots \ldots \ldots \ldots+\ldots * *$

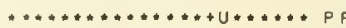
$\ldots \ldots \ldots \ldots \ldots \ldots+\ldots \ldots+\ldots * \ldots$

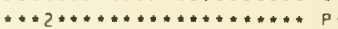
$\cdots \cdots \cdots \cdots+\cdots \cdots+\cdots+\cdots+\infty$

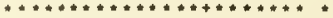
$\cdots \ldots \ldots \ldots \ldots \ldots+90 \cdots \cdots$ PF ACCEPTED

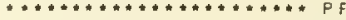

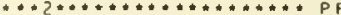

$\ldots \ldots 2 \ldots \ldots \ldots \ldots \ldots * \ldots \ldots \ldots p g$

$* * 2 * * * \ldots * * * * * * * * * * b F$

$* * 2 * * * \ldots+\cdots * \cdots * * * * P F$

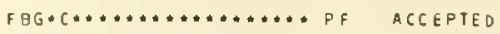

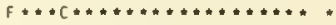

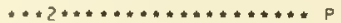

ACCEPTED

ACCEPTEO

ACCEPTED 


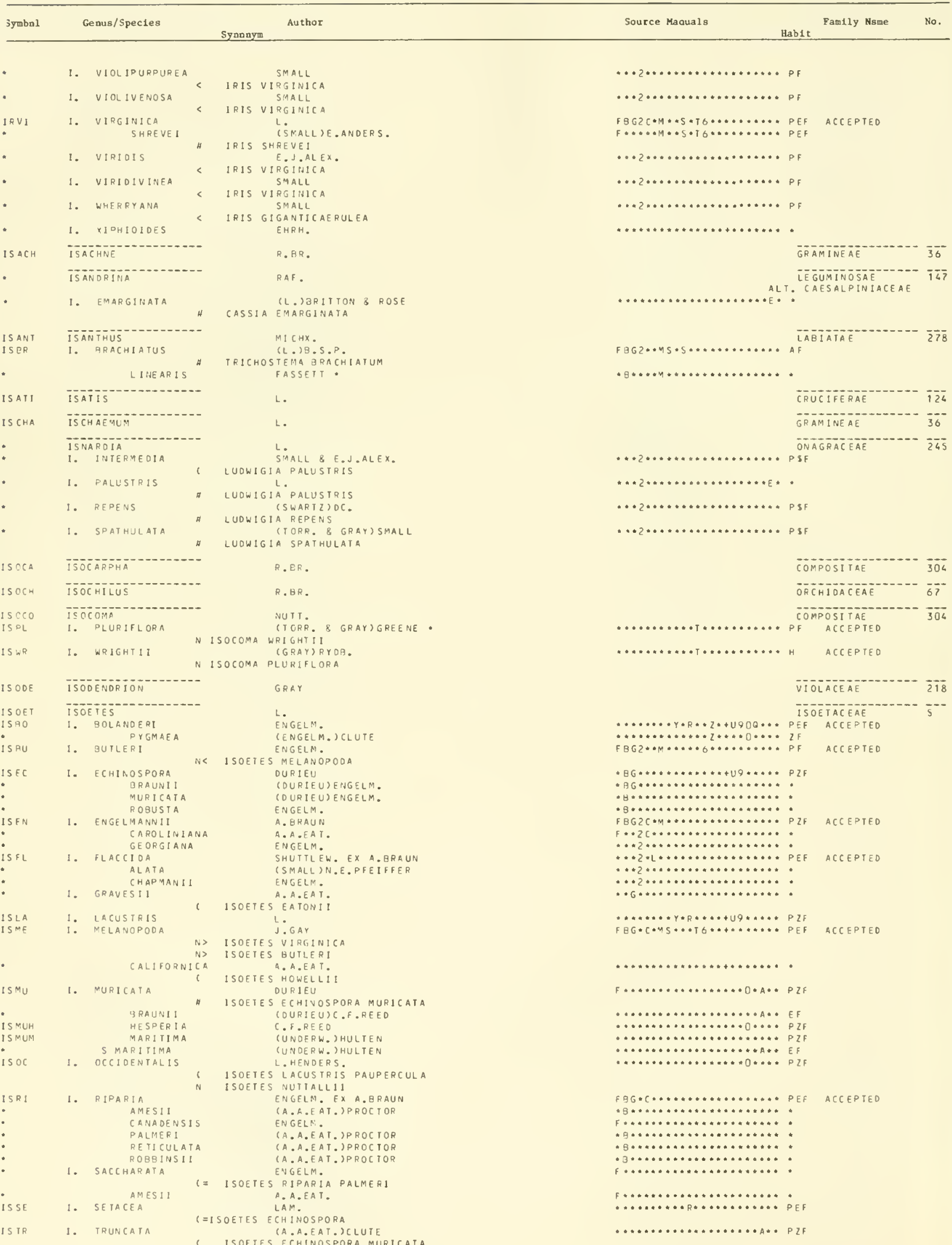




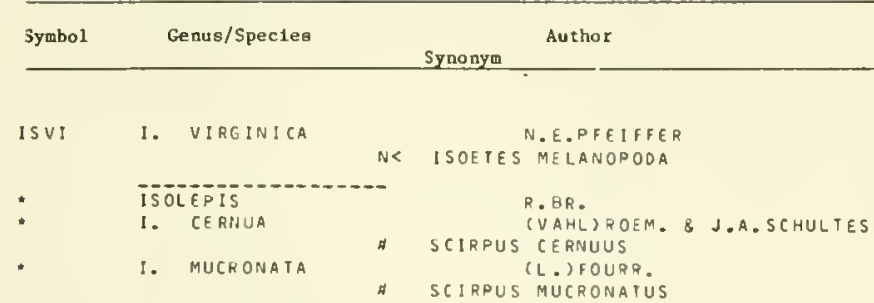

\begin{tabular}{|c|c|}
\hline IS OME & ISOMERIS \\
\hline ISAR & 1. ARBOREA \\
\hline * & ISOPAPPUS \\
\hline * & 1. OIVARICATUS \\
\hline * & 1. VALIDUS \\
\hline IS OPY & ISOPYRUM \\
\hline is a! & 1. BI TERNATUIA \\
\hline ISHA & 1. HALLII \\
\hline IsOC2 & 1. OCCIDENTALE \\
\hline * & 1. SAVILEI \\
\hline ISST & 1. STIPITATUM \\
\hline
\end{tabular}

\section{- TSOTOMa}

* I. LOVGiFlORa

ISOTR

ISOTR

IT EA+

IVAt+

IV AX

*

I ve

-

IVTE

IVES

IVBA

IVBAS
IVGO

IXER

IXST

.

$I \times O P$

I $\times$ ORA

:

:

JABOR

JACAR

-

$J A C O B$

JACA

JACOU

ISOTRIA

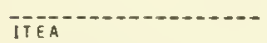

IVล

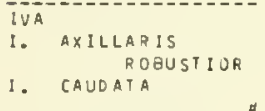

1. CILIATA

1. TEXENSIS

IVESTA

1. BAILEYI

SETOSA

I. GORDONII

IXERIS

1. STOL ONIFERA

\section{ixiotum}

1. WRIGHTII

\section{IXOPHORUS}

IXORA

1. FINLAYSONIANa

1. GRANDIFOLIA

i. THWAITESII

JABOROSA

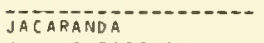

J. ACUTIFOLIA

JACORINIA

J. CANDICANS

JACOUEMONTIA

J. CANESCENS

J. JAHAICENSIS

J. NODIFLORA

J. OBCORDATA
MACROCARPA
NUTr. *

CLEOME NUTT. *

TORR. \& GRAY

CROP I ILON

(NUTT.)TORR. \& GRAY

HAPLOPAPPUS DIVARICATUS

HAPLOPAPPUS VALIDUS

$$
\text { L. (RAF.) TORR. \& GRAY }
$$

ENEMION BITERNATUM

GRAY

ENEMION HALLI!

HOOK. \& ARN.

ENEMION OCCIDENTALE

CALDER \& R.L.TAYLOR

ENEMION SAVILE!

GRAY

ENEMION STIPITATUM

(R. GR. )LINDL.

(L.)K.PRESL
HIPPOOROMA LONGIFLORA

RAF.

(AUST. EX GRAY)RYDB.

HABENARIA SACCATA

L.

L.

PURSH

HOOX.

SMALL

IVA ANNUA CAUDATA

WIILLD.

I VA ANNUA

HLAKE
R.C.JACKS.

IVA ANGUSTIFOLIA LATIOR

TORR. \& GRAY

5. WATS.

S. WATS.

(HOOK, ITORR. \& GRAY

HORKELIA GORDONI!

(CASS.) CASS.

GAAY

LACTUCA STOLONIFERA

EICHLER

(GRISEQ.) EICHLER

EREMOLEPIS WRIGHTII

SCHLECHI .

WALLICH EX G.DON.

ZOLLING. \& MORITZI *

J.D.HOOK.

Juss.

JUSS.

HUMH. \& BONPL.

JACARANDA MIMOS IFOLIA

NEES

(NEES) BENTH. \& HOOK.

CHOISY

(H.B.K.) BENTH.

A CQUEMONTIA PENTANTHA

(JACQ.)H.HALLIER EX SOLERED. *

CONVOLVULUS NOOIFLORUS

- JACOUEMONTIA HAVANENSIS

CONVELVR.) G.DON

CONVOLVULUS NOD IFLORUS

JACOUEMOHIIA OVALIFOLIA OBCORDATA

Source Manuale

Family Name

No.

F $B G \ldots \ldots \ldots \ldots \ldots \ldots \ldots \ldots \ldots \ldots$ ACCEPTED

CYPERACEAE

$P G$

CAPPARI DACEAE

ALT. CLEOMACEAE

$\ldots \ldots \ldots \ldots \ldots 0^{A L}$

COMPOSTTAE

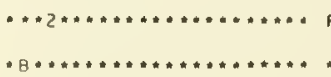

$F B G 2 C+M 5 \cdot 5 \cdot 10 \ldots \ldots \ldots \ldots+B F$
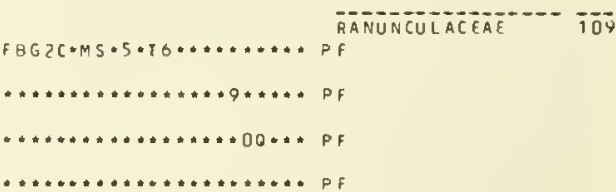

$\ldots \ldots \ldots \ldots * \ldots \ldots * * * 00 * \ldots p F$

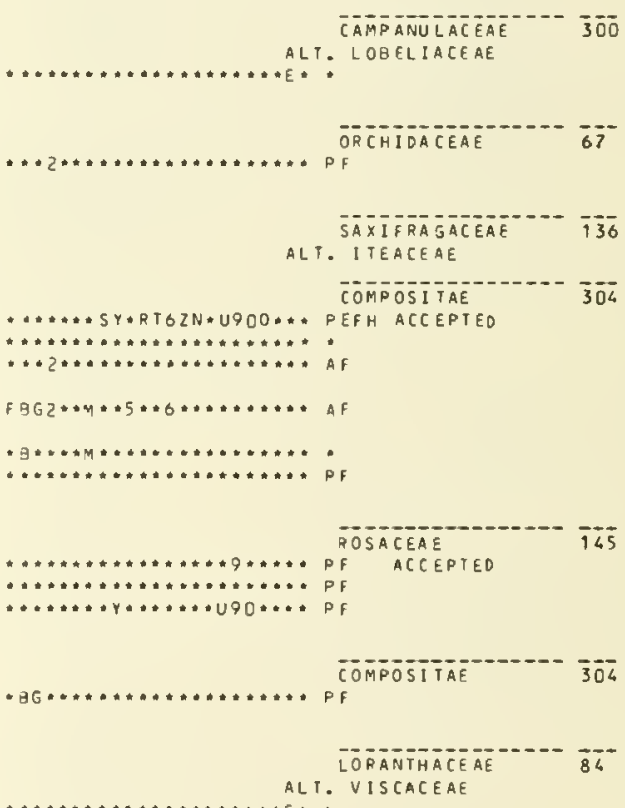
LI. VISCACEAE

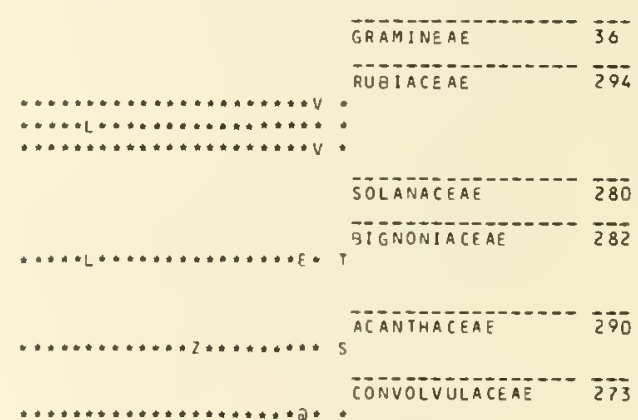

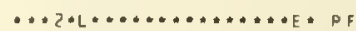




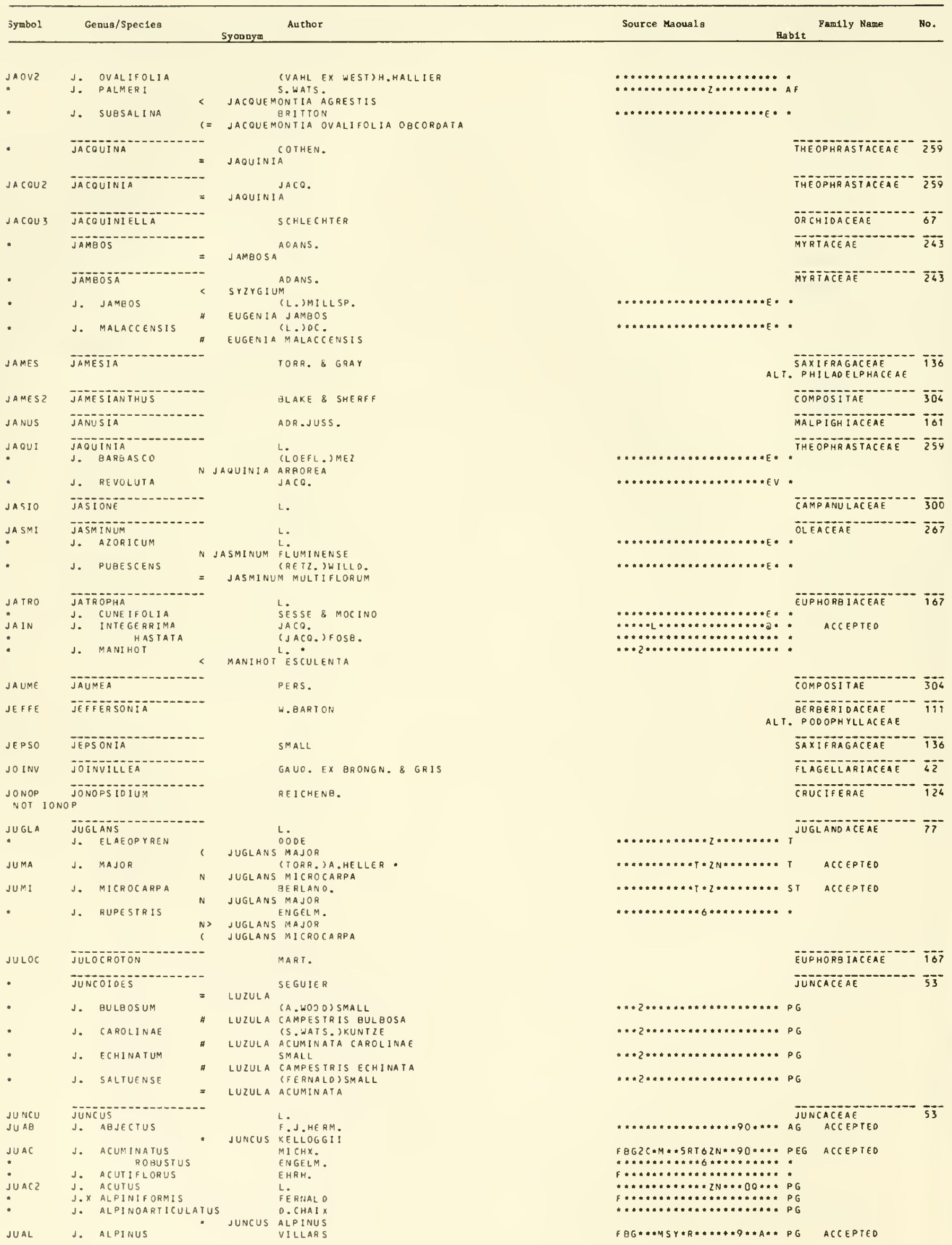




\begin{tabular}{|c|c|c|c|c|}
\hline 3ymbol 1 & \multicolumn{3}{|c|}{ Geaus/Spectes } & \\
\hline - & & & UNICEPS & \\
\hline - & & & NOOULOSUS & \\
\hline JUARZ & J. & $A R C$ & IICUS & \\
\hline - & & $s$ & ALASKANUS & \\
\hline * & & s & A TER & $<$ \\
\hline - & & s & SIT CHENS IS & \\
\hline - & J. & ARI & STULATUS & \\
\hline$\star$ & & & AIFLORUS & \\
\hline JUAR3 & נ. & ARI & ZONICUS & a \\
\hline JUARG & J. & AR T & ICULATUS & - \\
\hline 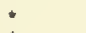 & & & OBTUSATU & \\
\hline * & J. & EAD & IUS & $H$ \\
\hline JU SA & J. & BAL & $\begin{array}{l}\text { TICUS } \\
\text { MEXICANU }\end{array}$ & \\
\hline JUEI & J. & BIf & $\begin{array}{l}\text { SIENOCARD } \\
\text { LDRUS }\end{array}$ & PUS \\
\hline
\end{tabular}

JUER?

JUER 3

JU日R

JUBRS

Ј $\triangle B \cup$

.

JUEUH

JUCA 3

:

JUCA

JU CAG

*

jucos

-

JUD

JUEF

JUEFE

JUEFG

JUEL

JUEA

JUEN

J. BRACHYCEPHALUS

J. erachrphrLLUS

J. brevigaudatus

J. BRYDIDES

J. BufONiUS

CONGDONII

CONGESTUS$$
\begin{aligned}
& \text { ENSI } \\
& \text { FALC } \\
& \text { GERA } \\
& \text { GREEN } \\
& \text { HEMTE } \\
& \text { INTE }
\end{aligned}
$$$$
\text { GRACILIS }
$$

BRUNNESCENS

MONTANUS

$$
\begin{aligned}
& \text { CCATUS } \\
& \text { SITCHENSIS } \\
& \text { ARDII } \\
& \text { PEDICELLATUS } \\
& \text { IENEI } \\
& \text { AENTIUS } \\
& \text { ARIZONICUS } \\
& \text { NEOMEXICANUS }
\end{aligned}
$$$$
\text { Syoonym. }
$$

\section{HAFTM.}

(WAHLENB.) C.LINDMAN EX HULTEN$$
\text { WILLD. }
$$$$
\text { (RYOB.) HULTEN }
$$

JUNCUS BALTICUS MONIANUS

ENGELM.

Juncus MICHX. MaRginatus

(ELLIOTT)SMALL.
JUNCUS BIFLORUS

WIEGAND.

JUNCUS INTERIOR ARIZONICUS

JUNCUS BIFLORUS

ENUELM.
SUKSD.

JUNCUS NEVADENSIS BADIUS

JUNCUS MERTENSIANUS BRDIUS WILLO. Juncus MEXICANUS J.A. \& J.h.sChUltes)KUNtze * BUCHENAU \& FERNALO ELLIOTT.

JUNCUS ARIICULATUS

JUNCUS LONGII

JUNCUS MARGINATUS

IENGELM. JaUCHENAU.
JUNCUS GREVICAUDATUS

JUNCUS INTERIOR

JUNCUS MLNG.

JUNCUS BRACHYCEPHALUS

F.J.HERM.

JUNCUS KELLOGGI $\mathrm{I}$

L.

IS. WATS.) J.T. HOWELL WAHLENB. WUCHENAU \& FERNALD. JUNCUS GUFDNIUS OCCIOENTALIS (SONGEDN \& P. PERRIER) HAYEK J.GAY

juncus subcaudatus

SUBCAUDATUS
FERNALD

FERNALD

F.J.HERM.

JUNCUS KELLOGGII

HOUK.

(ZINZ) HULTEN

COVILLE

JUNCUS NEVAOENSIS COLUMOIANUS

PIPER

C.HITCHC.

gUCHENAU

JUNCUS VALIDUS
ELLIOTT

JUNCUS INTERIOR

JUNCUS TENUIS

WIEGAND
JUNCUS PLATYPHYLLUS

JUNCUS TENUIS OUDLEYI

JUNCUS GR ISCOMII
ENGELM.

JUNCUS EFFUSUS GRACILIS HoOK.

JuNCUS EFUUSUS GRUNNEUS CHAPM.

C.MOHR *

WIKST.

(RYOU.) CRONO.

WIKST.
(RYOLOCRONO.

JUNCUS ENSIFOLIUS MONTANUS
GENGELM.)C.HITCHC.

JUNCUS SAXIMONTANUS

JUNCUS TRACYI

JUNCUS BALTICUS MONTANUS

$$
\begin{aligned}
& \text { E.MEYER } \\
& \text { (BUCHENAUTH }
\end{aligned}
$$

(BUCHENAU) HUL TEN

LOISELEUR

FERNAL D

OAKES \& TUCKERMAN

JUNCUS VASEYI

* Juncus UnCiALIS

JUNCUS KELLDGGI!

WIEGAND
JUNCUS BRACHYPHYLLUS

JUNCUS KANSANUS

JUNCUS DICHOT OMUS

(WI $W$ EAND) F.J.HERM.

RYDB.

JUNCUS REGELII

JUNCUS INTERIOR

INTERIOR
ENGELM.

JUNCUS ABJECTUS

JUNCUS BRYOIDES

JUNCUS CAPILLARIS 


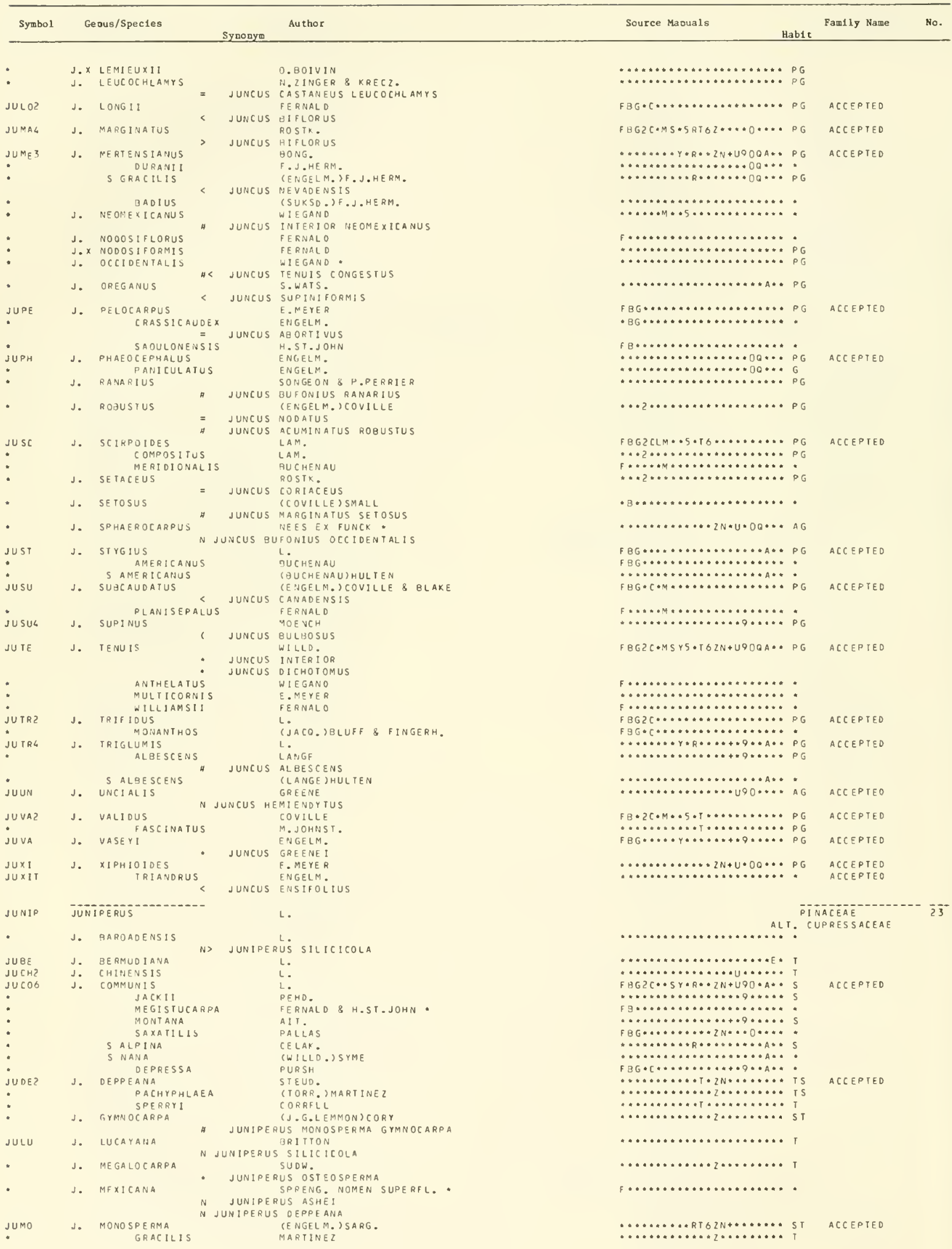




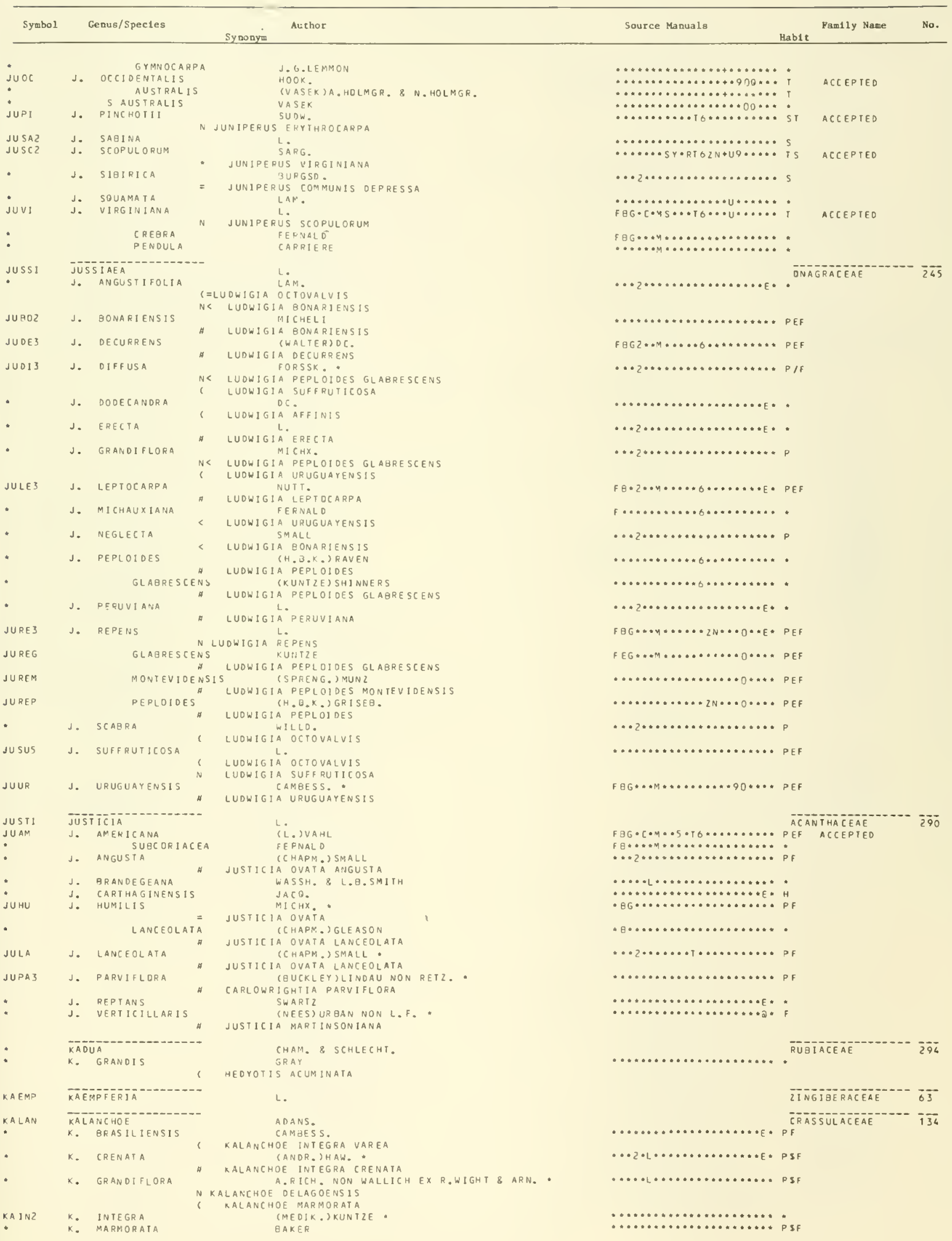


KALANCHOE

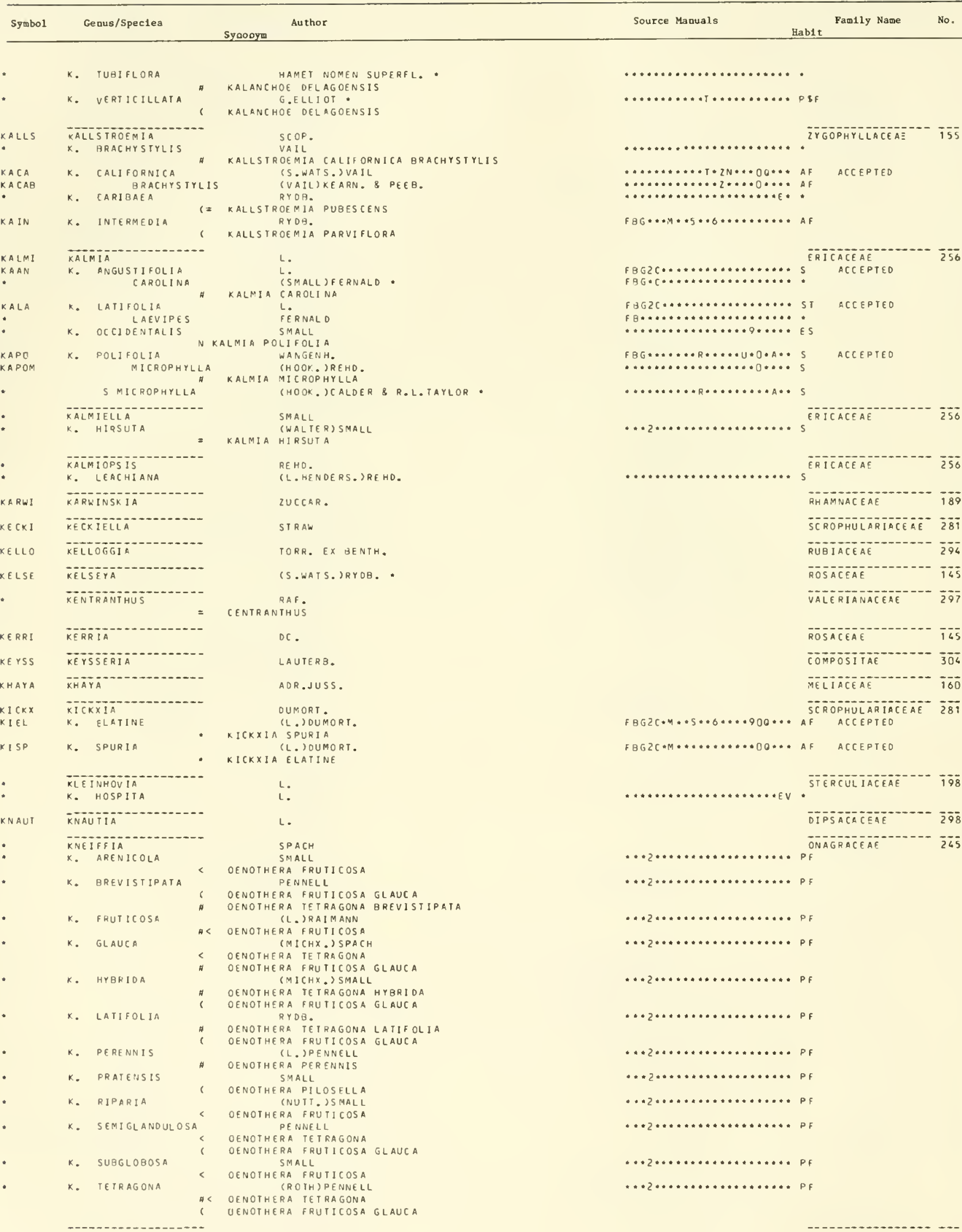




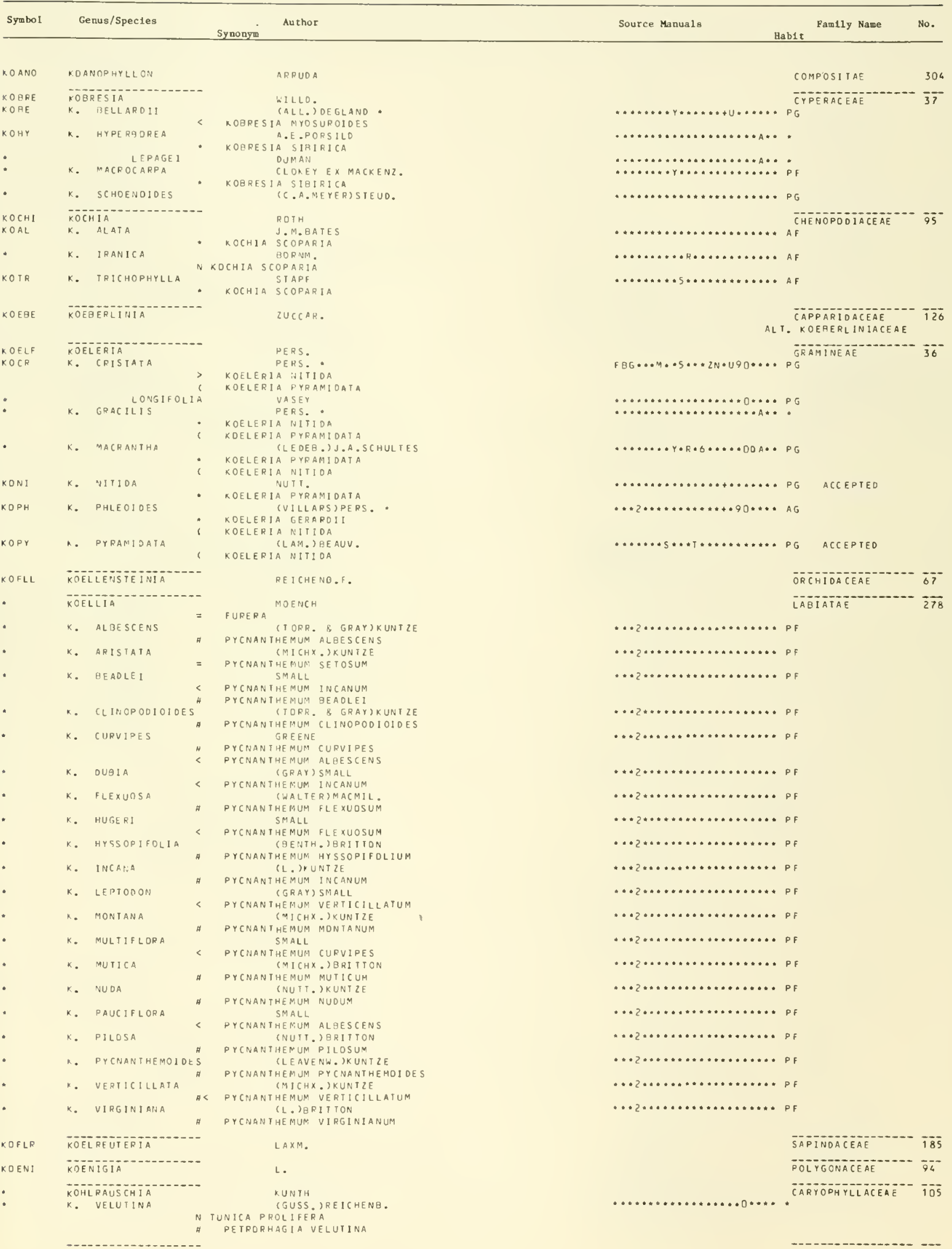




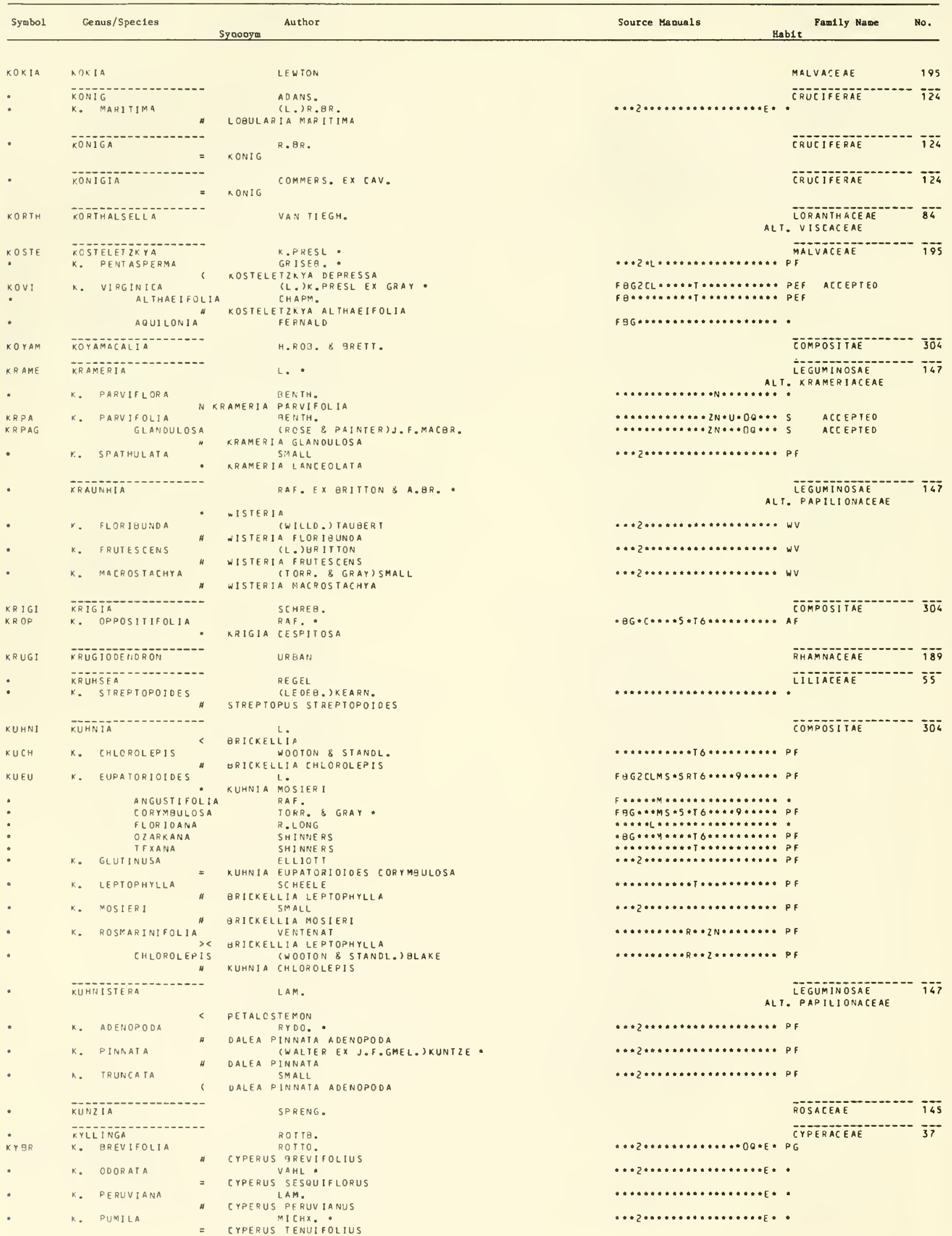




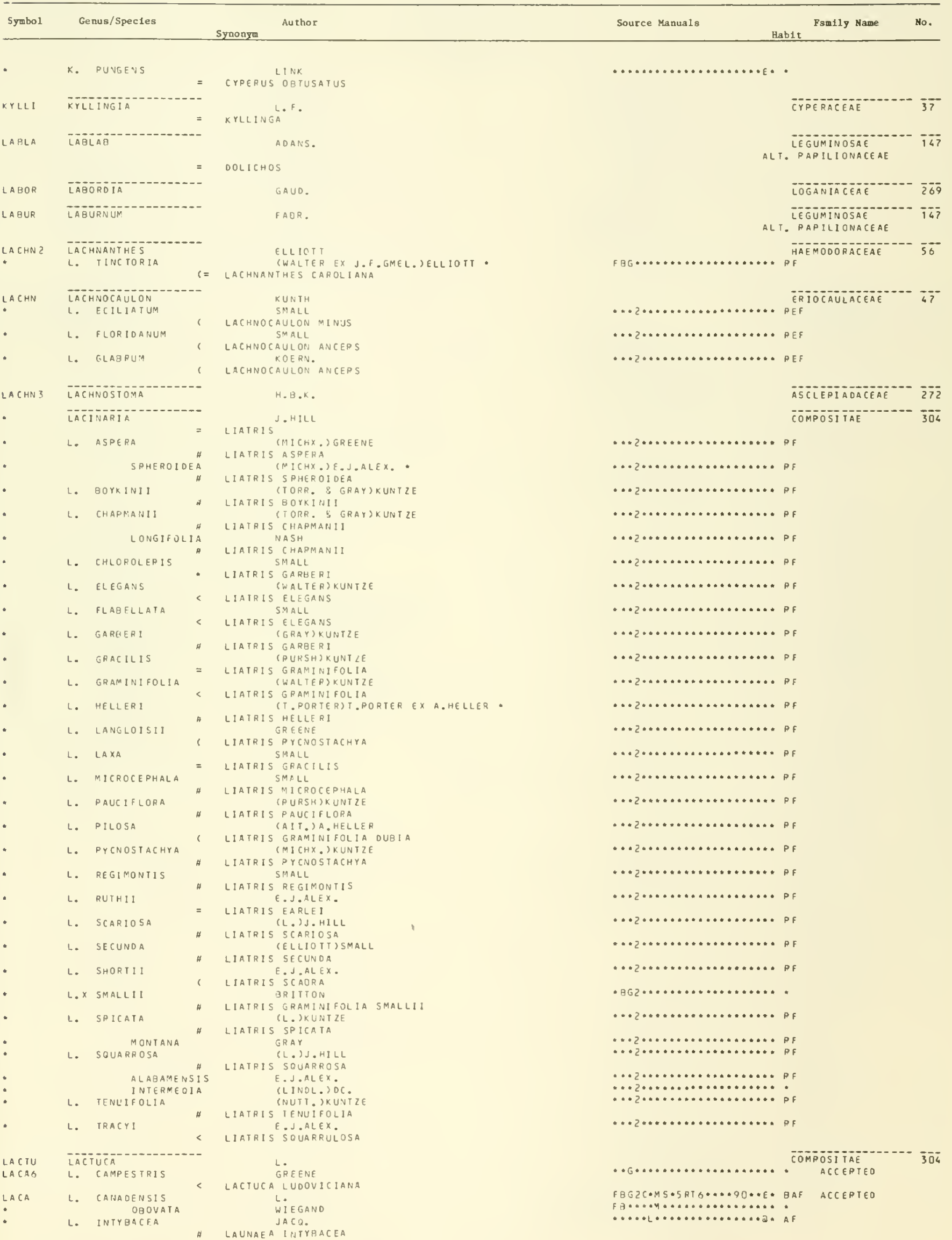




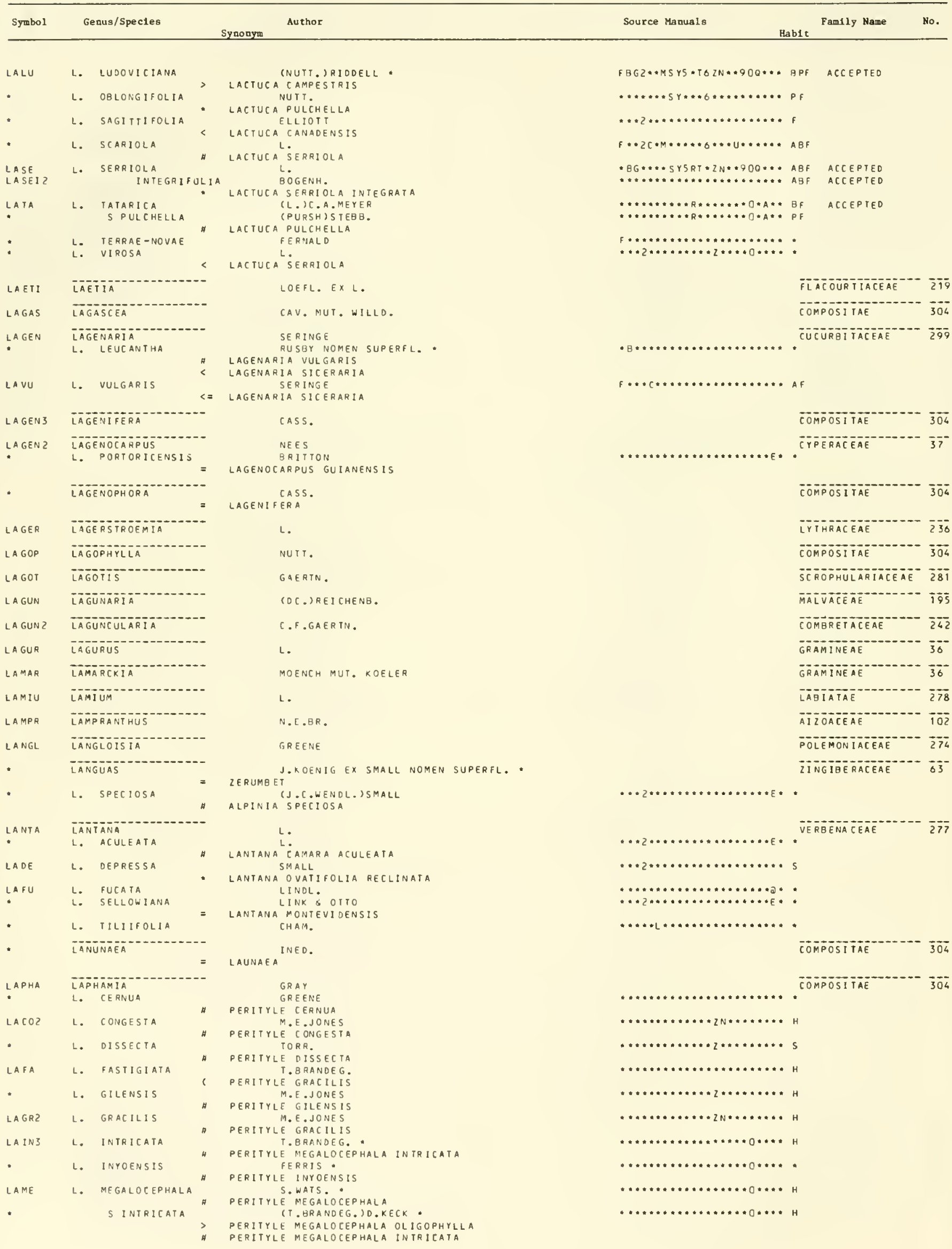




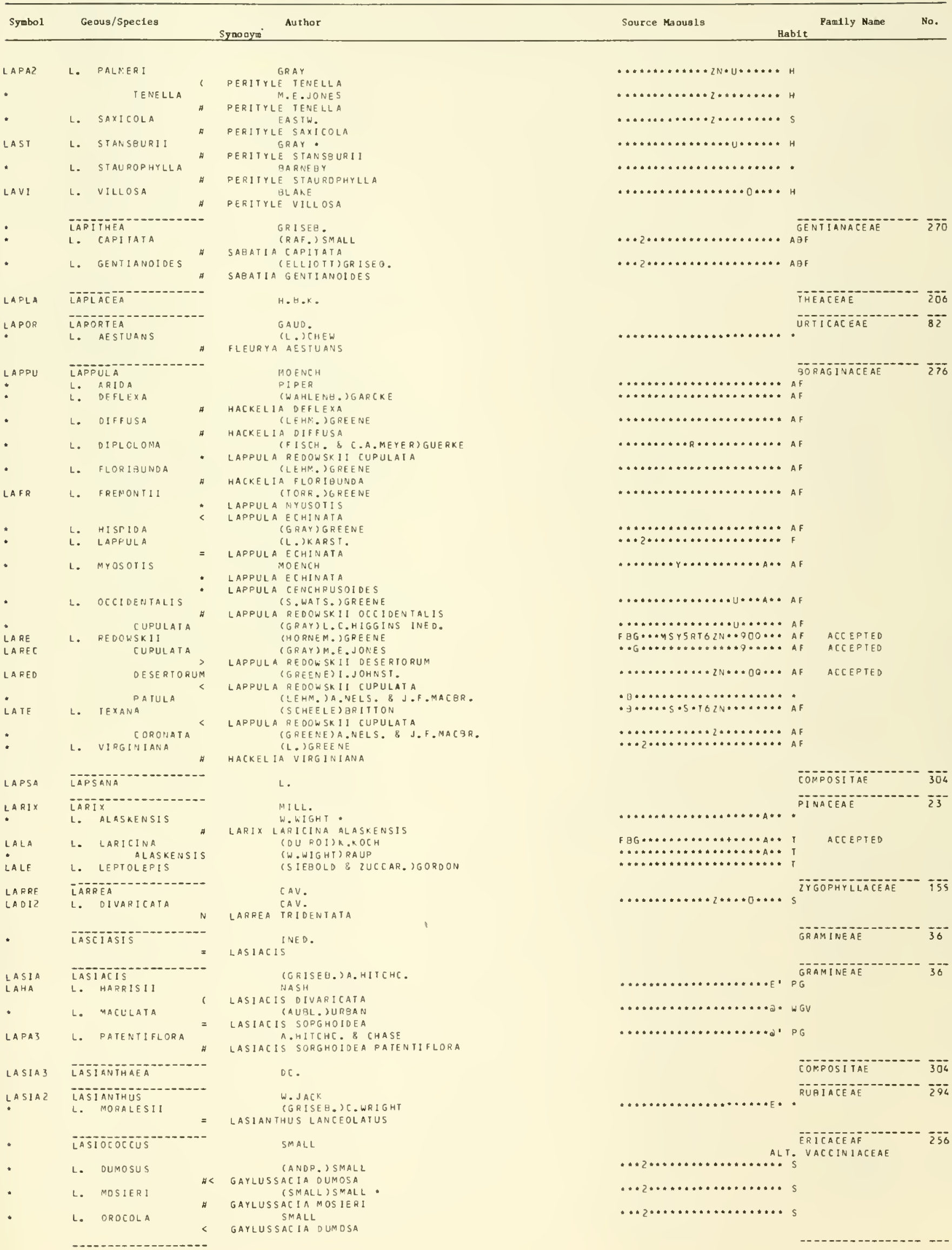




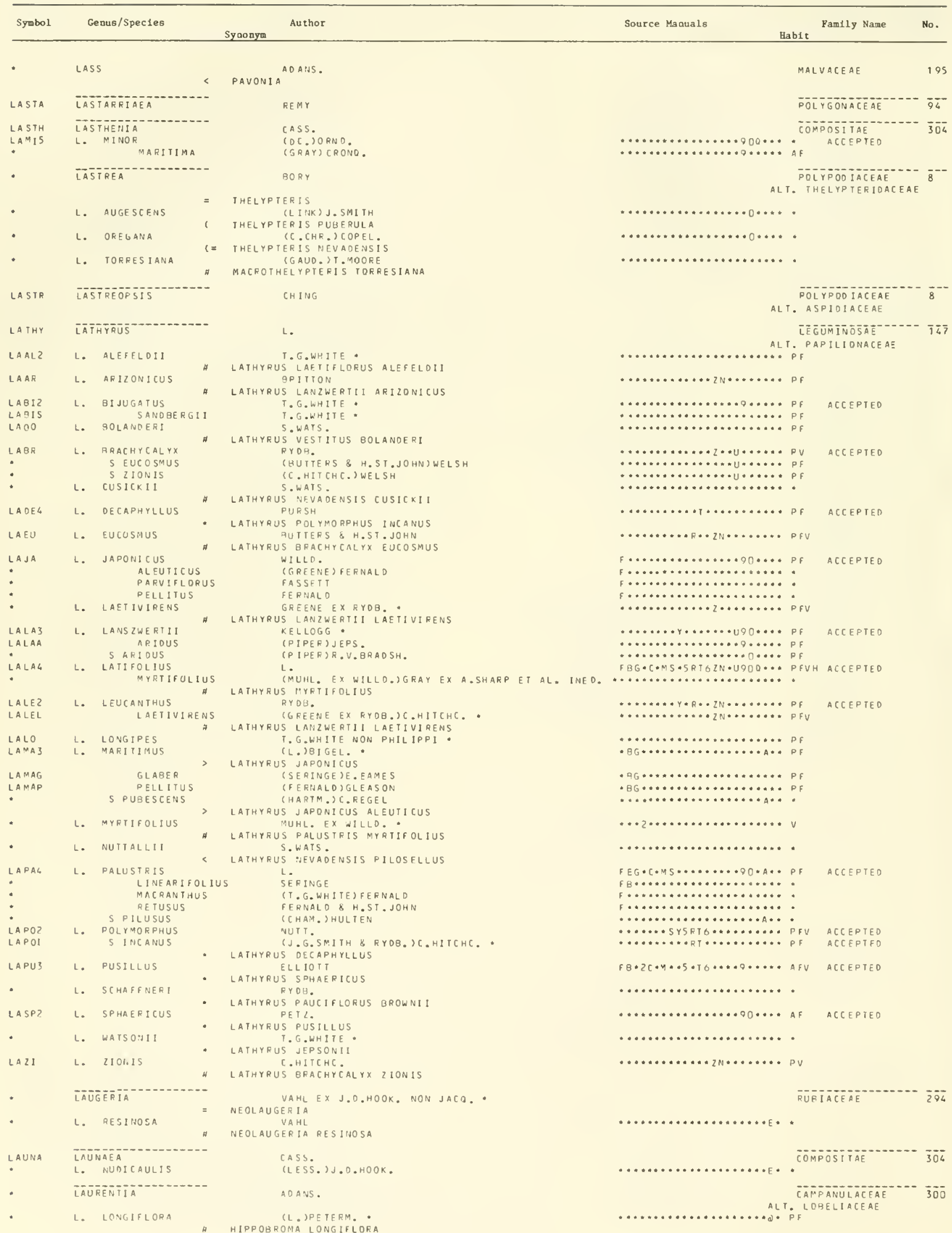




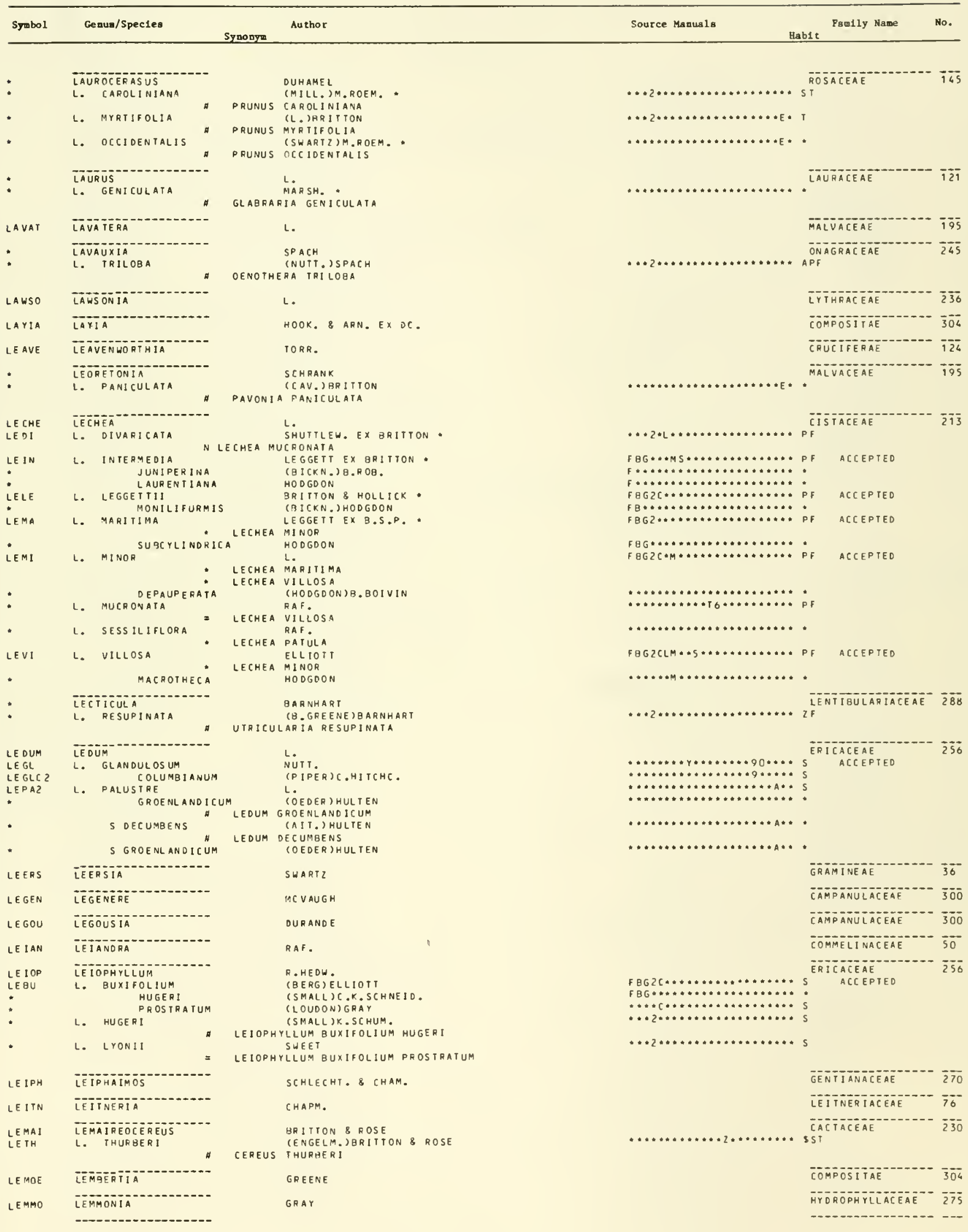




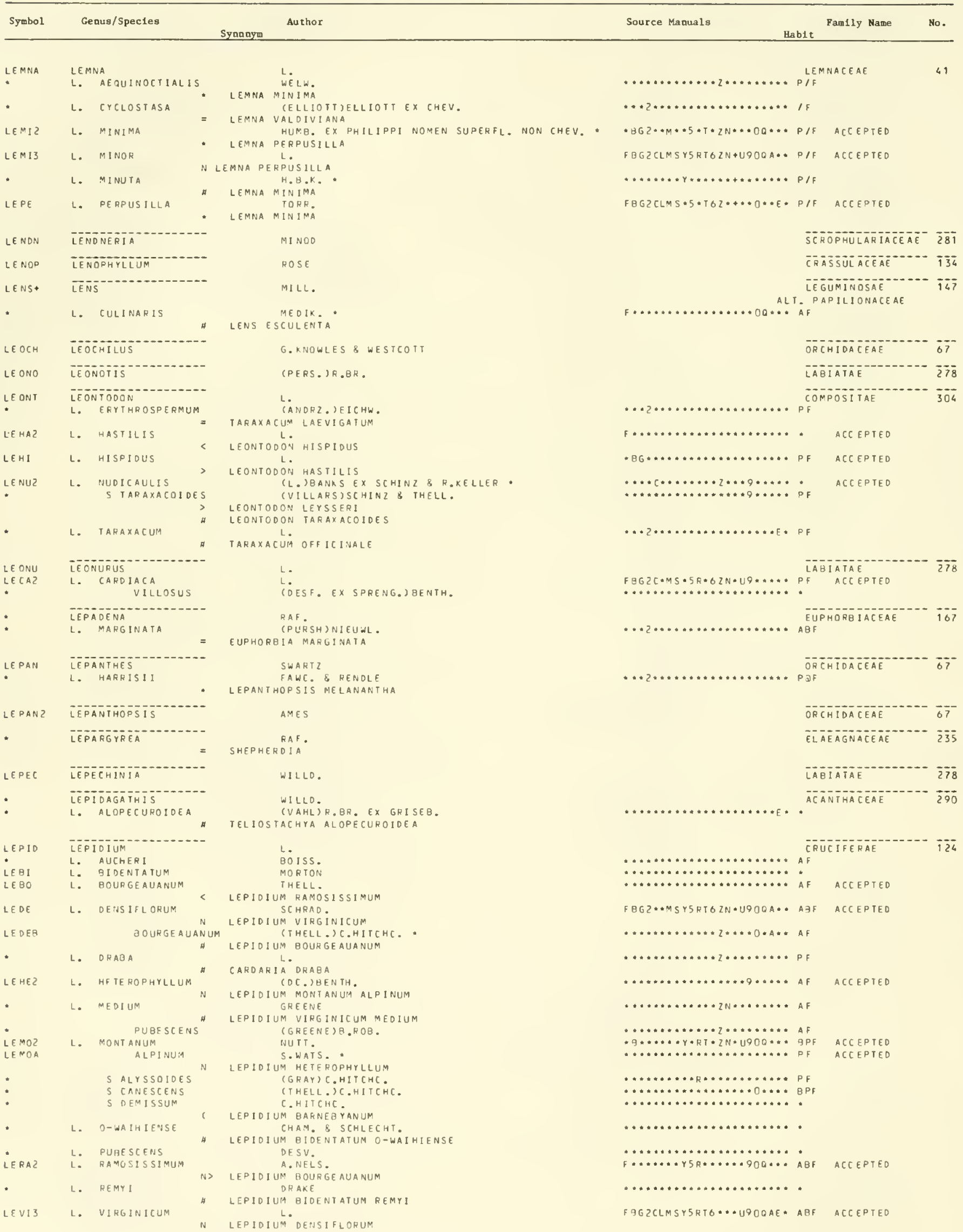




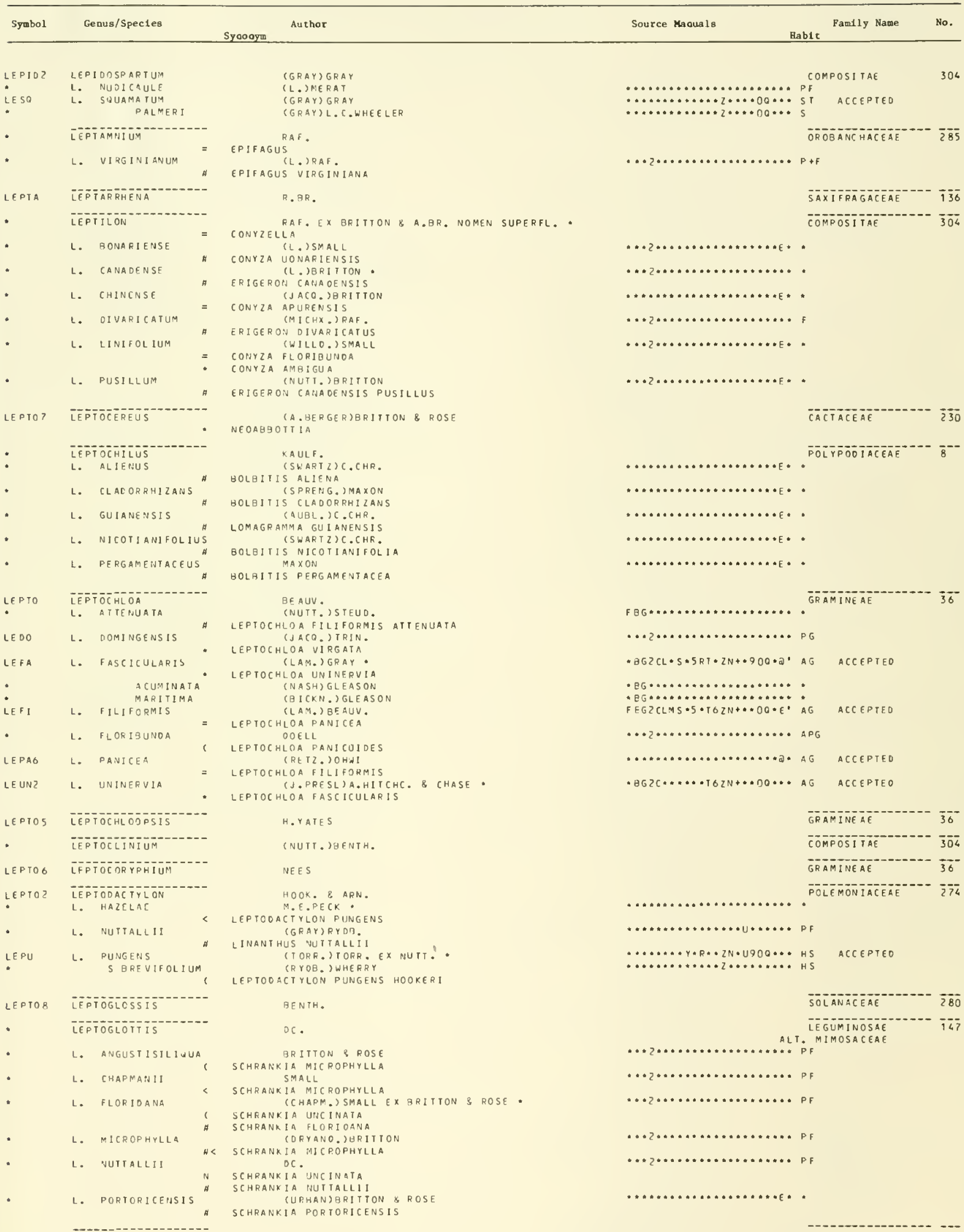




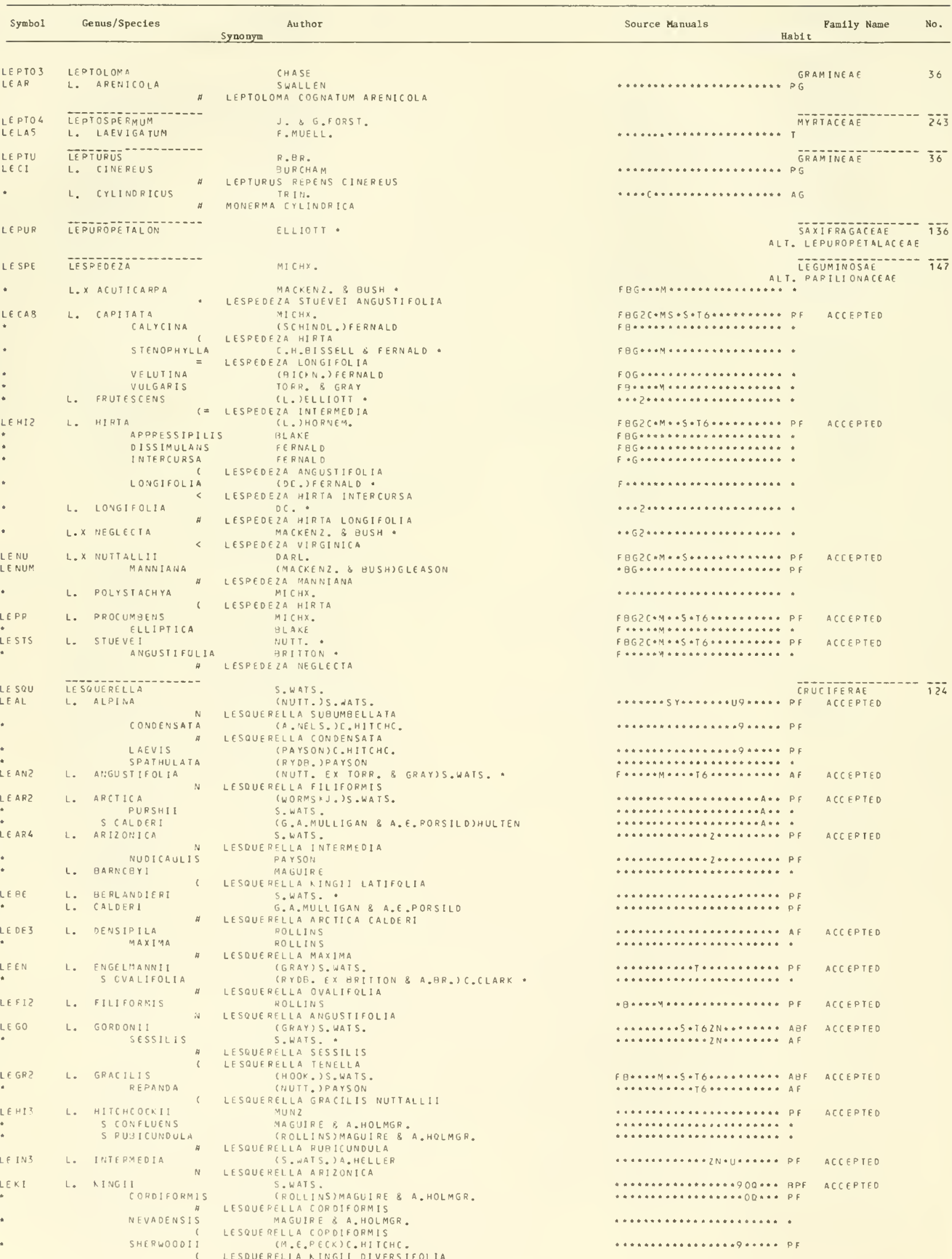




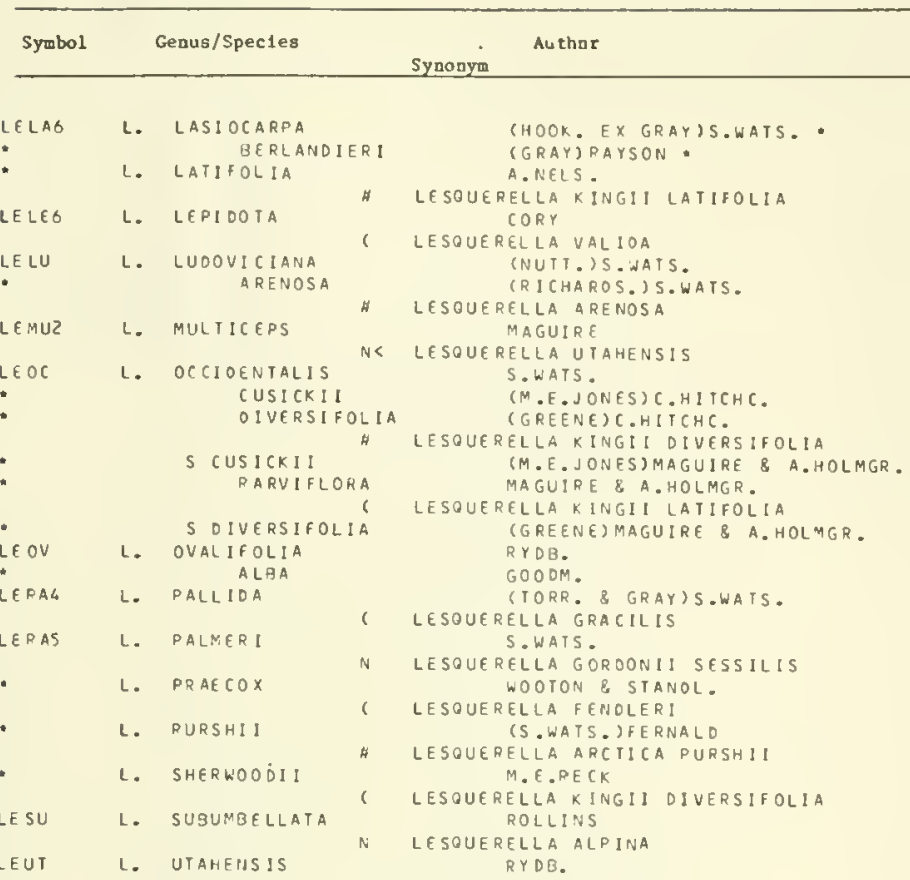

LEUT

LESSI LESSTNGIA

L. GLandulifera

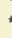

L. TOMENTOSA

L. LEMNOONII

$$
\begin{aligned}
& \text { PECIINATA" } \\
& \text { TOMENTOSA } \\
& \text { ONII }
\end{aligned}
$$$$
\text { PEIRSONII }
$$

L. MICRADENIA

$$
\text { RAMULOSISSIMA }
$$

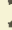

;

L. TENU1S

$$
\text { ARACHNUIOEA }
$$$$
\text { GLABRAI I }
$$

LEUCAE INA

H

LEUCA

LEGL3

- l. latisiligua

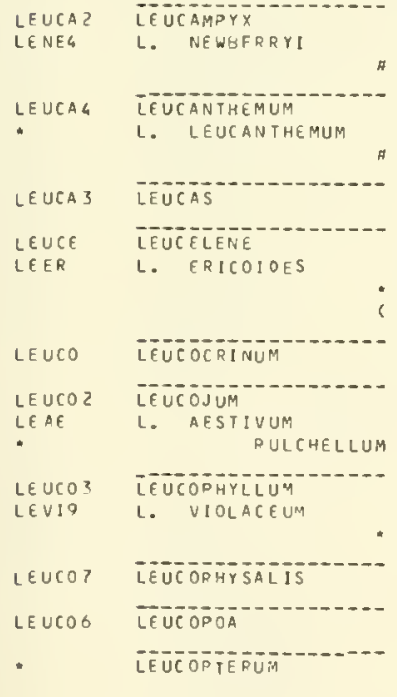

L. PARVIF OLIUM

$$
\text { LESOUERELLA MULTICEPS }
$$

$$
\text { CHAM. }
$$$$
\text { GRAY }
$$

LESSINGIA GERMANORUM GLANOULIFERA (GREENE) JEPS.

(GREENE) FERRIS

GRAY

LESSINGIA GERMANORUM LEMMONII

(J.T. HOWELL) FERR!S

(A. NELS.) FERRIS

LESSINGIA RAMULOSA MICRAOENIA (GREENE) FERR:S

LESSINGIA HOLOLEUCA ARACHNOI DEA

$$
\text { (GRAY) COVILLE }
$$

LESSINGIA GERMANORUM TENUIS

$$
\text { BENTH. }
$$

$$
\text { (L.) GENTH. }
$$

LEUCAEMA LEUCOCEPHALA

(L.) GILLIS.

$$
\begin{aligned}
& \text { (L.) GILLIS } \\
& \text { LEUCAENA LEUCOCEPHALA }
\end{aligned}
$$$$
\text { - LYSILOMA LATLSILIGUA }
$$

GRAY

$$
\text { GRAY }
$$

HYMENOPAPPUS NEWBERRY

$$
\text { MILL. }
$$

CL. RYYOQ. *

LEUCANTHEMUM VULGARE

$R, B R$.

JREENE

(TURP.) GREENE

ASTER BELLUS

ASTER HIRTIFOLIUS

IUTT. EX GRAY.
L.
'SALISO. ISTEYERM. INEO.
HUMB. \& BONPL.
PENIULLL
RYLLUM CANOIOUM
RYOB.
GR ISEB.
SMALL

RHYNCHOSIA

COC.)SMALL

RHYNCHOSIA PARVIFOLIA (O. KECK)FERR!S
Source Manuals

Family Name

No.

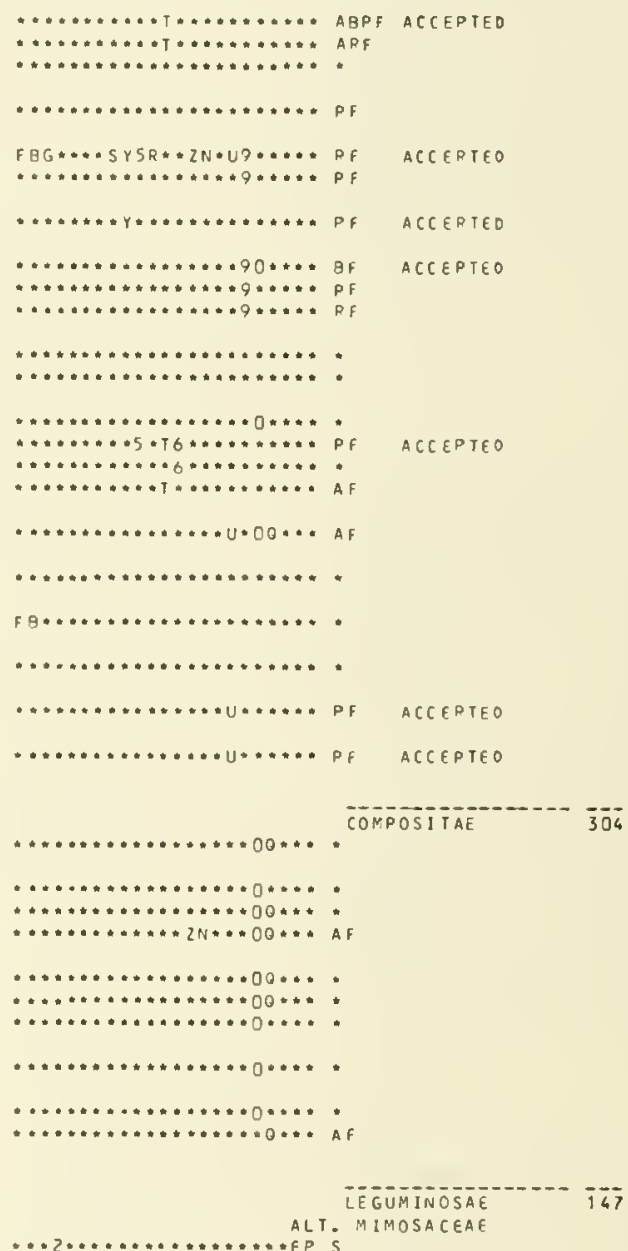
$A L T$

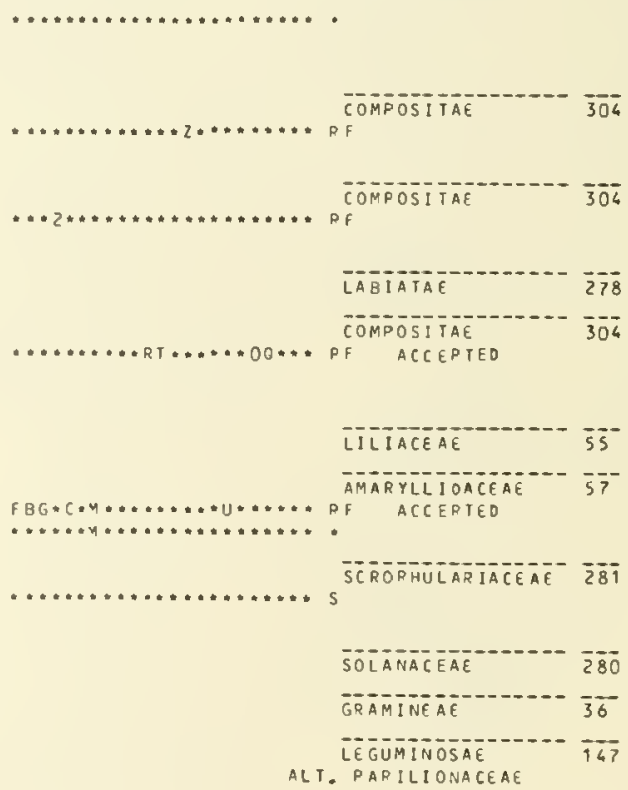

ALT. PARILIONACEAE 


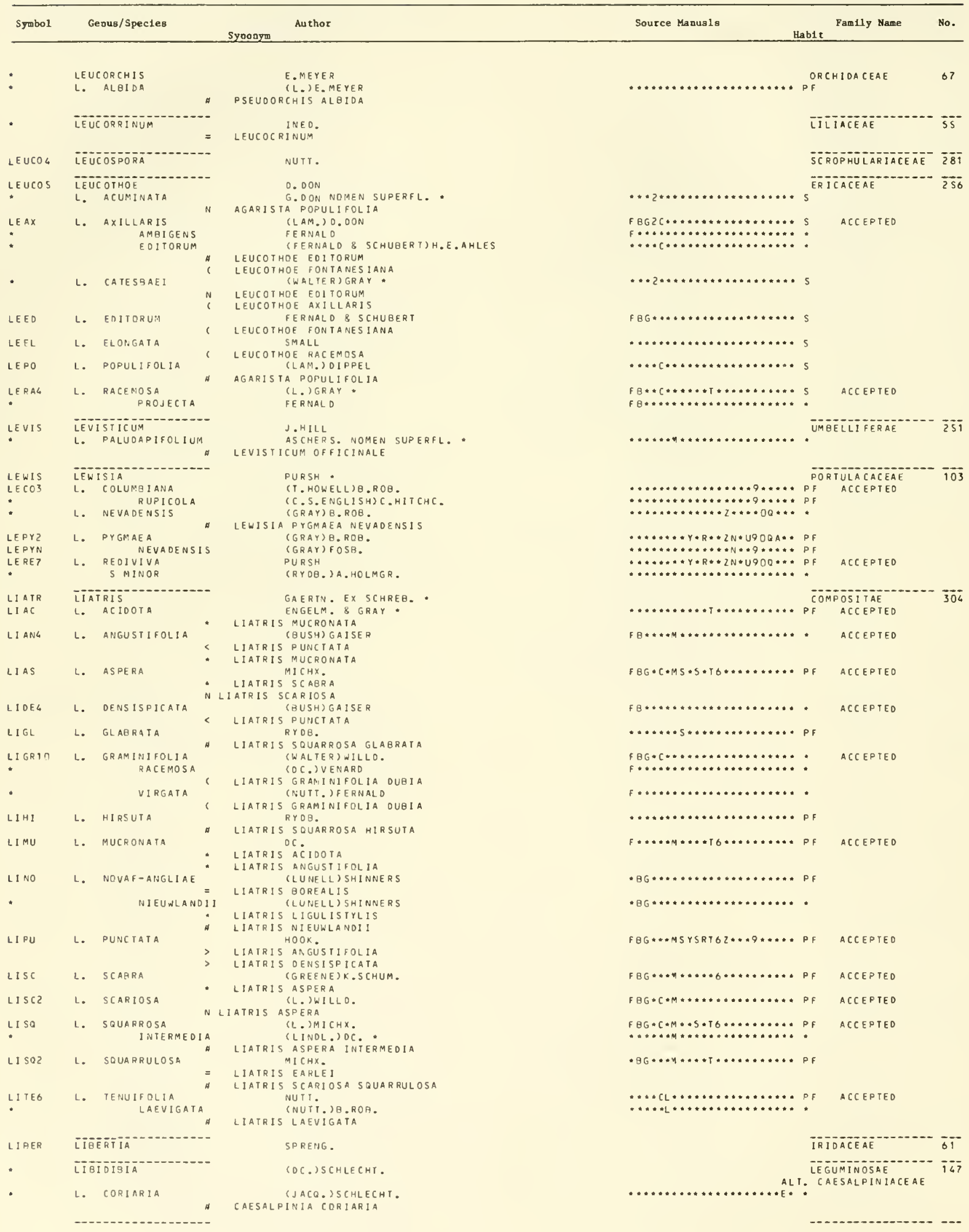



Hab1t

\begin{tabular}{|c|c|c|}
\hline Syabol & Genus/Spectes & Synnnym Author \\
\hline LIBOC & LIBOCEDRUS & ENDL. \\
\hline LICAN & LICANIA & $A \cup B L$. \\
\hline LI CAR & LICARIA & AUBL. \\
\hline * & IIGULARIA & $\begin{array}{l}\text { CASS. } \\
\text { (GRAY) W. WEGER. }\end{array}$ \\
\hline * & L. AMPLECTENS & $\begin{array}{l}\text { (GRAY) W. WEBER. } \\
\text { SENECIO RMPLECTENS }\end{array}$ \\
\hline - & L. BLGELOVII & $\begin{array}{l}\text { (GRAY) W.WEBER } \\
\text { SENECIO BIGELOVII }\end{array}$ \\
\hline - & HALLI! & $\begin{array}{l}\text { (GRAY) W. WEBER INEO. * } \\
\text { SENECIO BIGELOVII HALLII }\end{array}$ \\
\hline * & L. HOLMII & $\begin{array}{l}\text { (GREENE) W.WEBER } \\
\text { SENECIO AMPLECTENS HOLMII }\end{array}$ \\
\hline " & L. PUDICA & $\begin{array}{l}\text { (GREENE) W.WEGER } \\
\text { SENECIO PUDICUS }\end{array}$ \\
\hline * & L. SOLOANELLA & $\begin{array}{l}\text { (GRAY)W.WEBER } \\
\text { SENECIO SOLDANELLA }\end{array}$ \\
\hline * & L. TARAXACOIDES & $\begin{array}{l}\text { (GRAY)W.WEBER * } \\
\text { SENECIO TARAKACOLOES }\end{array}$ \\
\hline Li GUS & LIGUSTICUA & L. \\
\hline LI AP & L. APIIFOLIUM & $\begin{array}{l}\text { (NUTT. EX TORR. \& GRAY)GRAY - } \\
\text { LIGUSTICUM CANGYI }\end{array}$ \\
\hline LICAZ & L. CANBYI & $\begin{array}{l}\text { COULT. \& ROSE } \\
\text { LIGUSTICUM APIIFOLIUM }\end{array}$ \\
\hline LIFI & $\begin{array}{l}\text { L. FILICINUM } \\
\text { TENUIFOLIUM }\end{array}$ & $\begin{array}{l}\text { S.WATS. } \\
\text { (5.WRTS.) MATHIAS \& CONSTANGE }\end{array}$ \\
\hline LI MUZ & $\begin{array}{l}\text { L. MUTELLINOIDES } \\
\text { SALPINUM }\end{array}$ & $\begin{array}{l}\text { LIGUSTICUM TENUIFOLIUM } \\
\text { (CRANIZ) WILLAR } \\
\text { (LEOEB.) THELL. }\end{array}$ \\
\hline
\end{tabular}

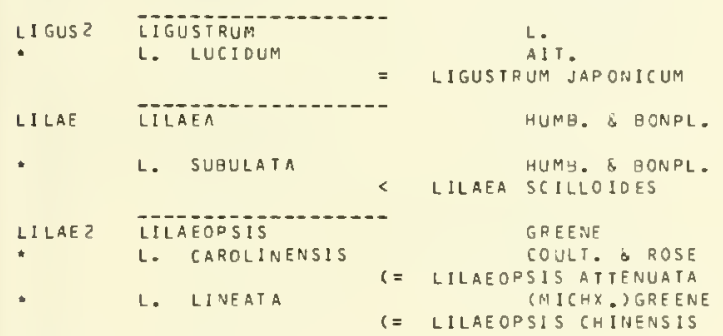

\begin{tabular}{|c|c|c|}
\hline LILIU & Lil & UM \\
\hline$L: C A 3$ & L. & CA A A DE NSE \\
\hline • & & EOLTORUM \\
\hline - & L. & CAROLINIANUM \\
\hline LICO & L. & COLUMB I ANUM \\
\hline - & L. & IRIDOLLAE \\
\hline • & L. & MI CHIGANENSE \\
\hline - & & UNIF L ORUM \\
\hline - & L. & PERAMOENUM \\
\hline LISU & L. & SUPEROUM \\
\hline
\end{tabular}

LILIUM PHILADELPHICUM ANDINUM

$$
\begin{aligned}
& \text { R.HR. } \\
& \text { M.E.PECK }
\end{aligned}
$$

LIMNANTHES FLOCCOSA BELLINGERANA TRELEASE

$$
\text { T. HOWELL }
$$

LIMNANTHES FLOC COSA PUMILA

ITMNIAR HAW.
L. UTAHENSIS RYOB.

\begin{tabular}{|c|c|c|}
\hline \multicolumn{2}{|c|}{ LIMNOBIUM } & L.C.RIGH. * \\
\hline LIMNOOEA & & L. OEWEY \\
\hline LIMNOPHILA & & $R, \forall R$. \\
\hline LIMNORCHIS & & RYDB. \\
\hline L. DILATATA & * & $\begin{array}{l}\text { (PURSH)RYDQ } \\
\text { PLATANTHERA DILATATA }\end{array}$ \\
\hline S ALBIFLORA & & (CHAM.)A.LOEVE \& W.SIMON \\
\hline L. HYPERBOREA & $A$ & $\begin{array}{l}\text { CL. RRYDB. } \\
\text { PLATANTHERA HYPERBOREA }\end{array}$ \\
\hline L. SACCATA & H & $\begin{array}{l}\text { (GREENE)A LOEVE \& W. SIMON } \\
\text { HAQENARIA SACCATA }\end{array}$ \\
\hline SPARSIFLORA & ( & $\begin{array}{r}\text { PLATANTHERA STRICTA } \\
\text { (S. DATS.)RYDB. }\end{array}$ \\
\hline & \# & PLATANTHERA SPARSIFLORA \\
\hline
\end{tabular}

CLAYTONIA PERFOLIATA UTAHENSIS
PINACEAE ALT. CUPRESSACEAE

ROSACEAE

AL T. CHRYSOGALANACEAE

LAURACEAE

COMPOSITAE
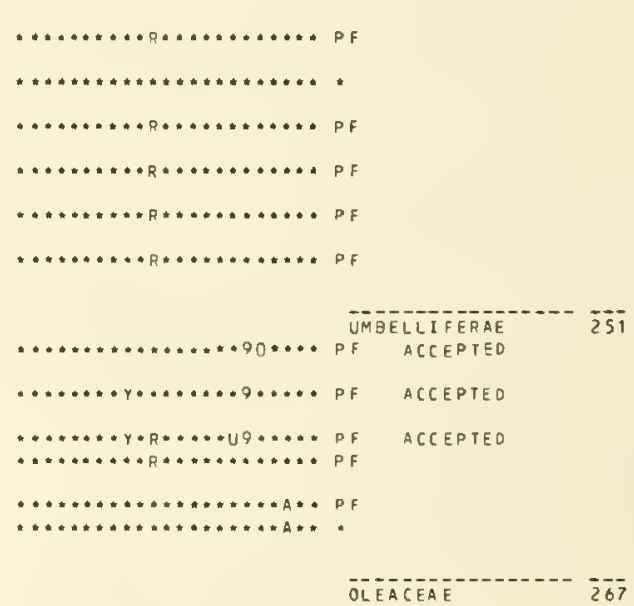

... $2 \ldots \ldots \ldots \ldots \ldots \ldots . . . .4$ JUNCAG INACEAE
ALT. LILAEACEAE

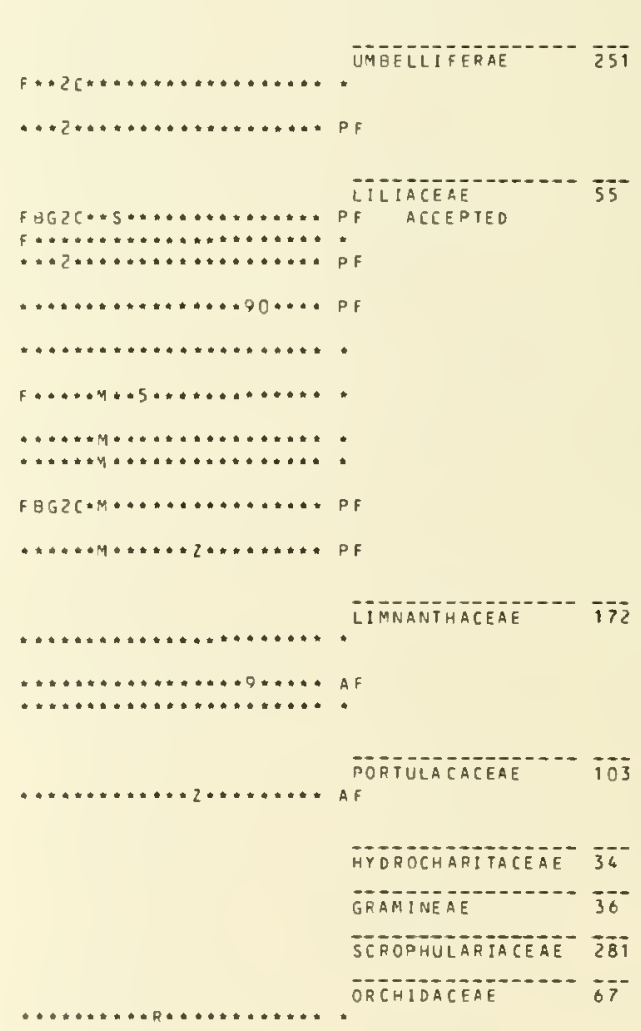

$\ldots \ldots+\ldots \ldots+R * \ldots \ldots \ldots+\ldots \ldots$

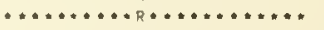




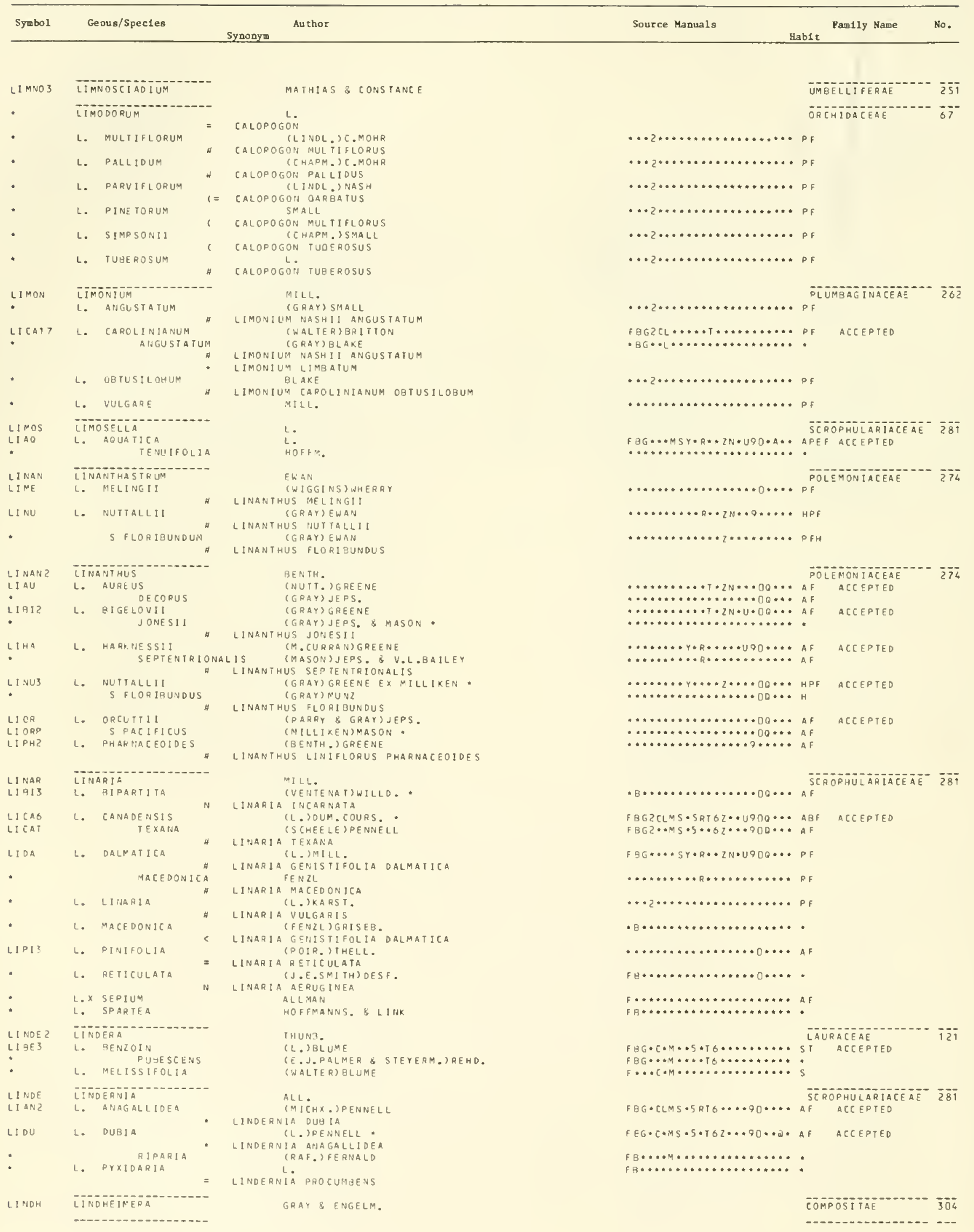




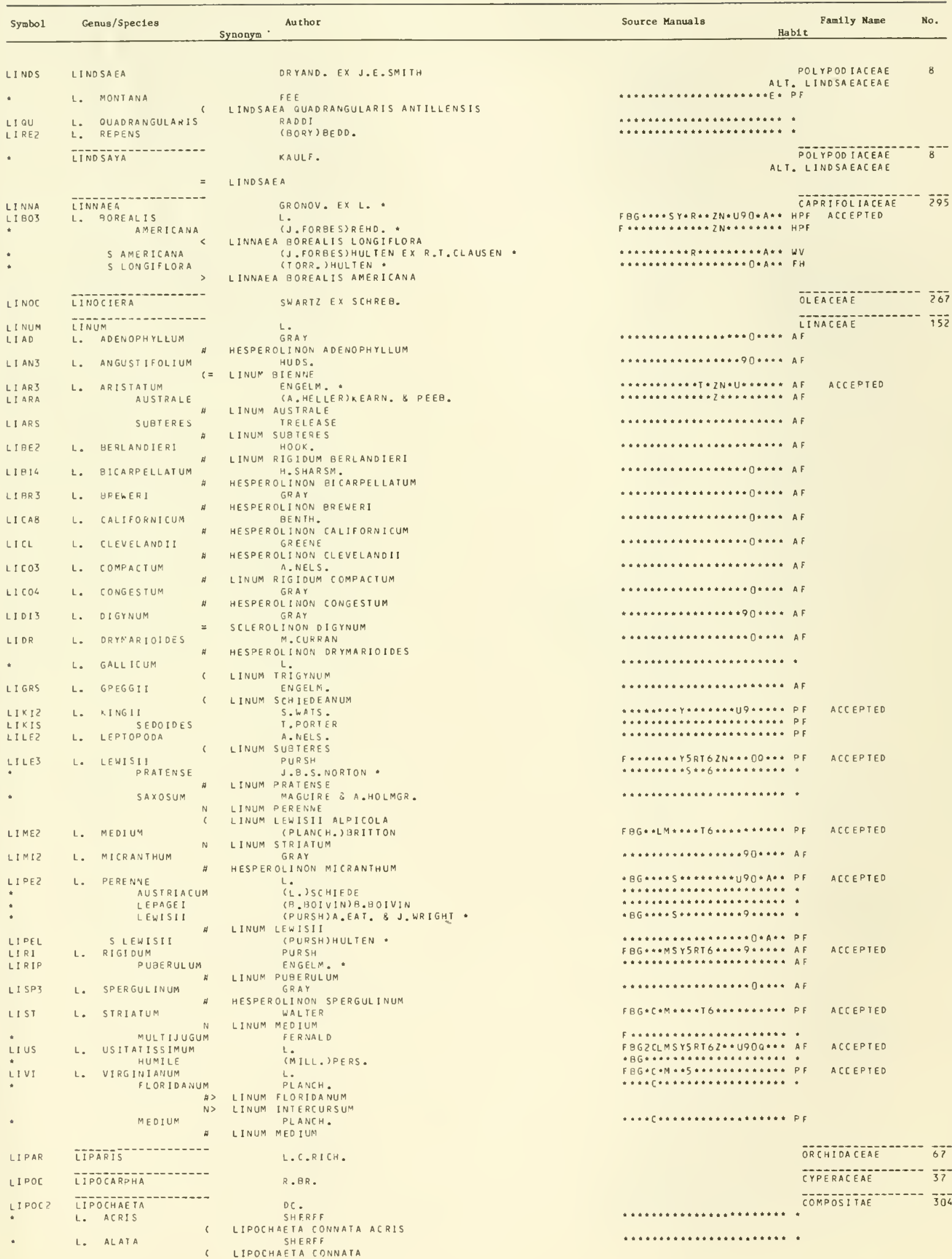




\begin{tabular}{|c|c|c|c|c|c|c|}
\hline Symbol & & nus/Spec1es & Syoonym & Source Manuals & Fanily Name & No. \\
\hline$\cdot$ & & $A C R I O R$ & $\begin{array}{l}\text { SHERFF } \\
\text { LIPOCHAETA CONNATA }\end{array}$ & 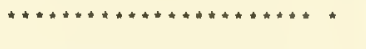 & & \\
\hline * & & PULGHRIOR & $\begin{array}{l}\text { SHERFF } \\
\text { LIPOCHAETA CONNATA }\end{array}$ & $+\ldots+\ldots+\ldots+\ldots+\ldots+\infty \ldots+$ & & \\
\hline LICOCO & L. & CONNATA & $\begin{array}{l}\text { (GAUD.) DC. } \\
\text { HILLEGR. }\end{array}$ & 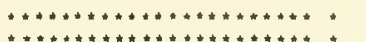 & ACCEPTED & \\
\hline & & LITTORALI & $\begin{array}{l}\text { HILLEQR. } \\
\text { LIPOCHAETA SUCCULENTA }\end{array}$ & & & \\
\hline$*$ & $\mathrm{~L}$. & EXIGUA & $\begin{array}{l}\text { DEG. \& SHERFF } \\
\text { LIPOCHAETA MI CRANTHA EXIGUA } \\
\text { DRARE }\end{array}$ & 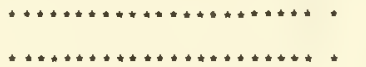 & & \\
\hline • & L. & $\begin{array}{l}\text { FLEXUOSA } \\
\text { FORBES II }\end{array}$ & $\begin{array}{c}6 \text { LIPOCHAETA SUBCORDAIA } \\
\text { SHERFF }\end{array}$ & 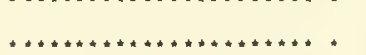 & & \\
\hline & & & LIPOCHAETA ROCKII & & & \\
\hline ¿ & L. & $\begin{array}{l}\text { SHERFF } 11 \\
\text { HASTATA }\end{array}$ & $\begin{array}{r}\text { OEG. B CLAY } \\
\text { HILLEBR. } \\
\text { VERBES INA HASTATA }\end{array}$ & 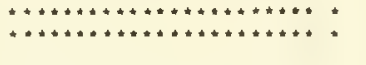 & & \\
\hline L 1 HE 3 & L. & HETE ROPHYLLA & GRAY & 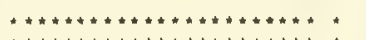 & ACCEPTED & \\
\hline 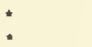 & & $\begin{array}{l}\text { YALVACEA } \\
\text { MOLOKAIEN }\end{array}$ & $\begin{array}{ll} & \text { DEG. \& SHERFF } \\
\text { S IS } & \text { SHERFF }\end{array}$ & 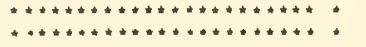 & & \\
\hline L1 ING & L. & INTEGRIFOLIA & (NUTT.) GRAY & 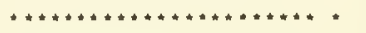 & ACCEPTED & \\
\hline$*$ & & ARGENTEA & SHERFF & 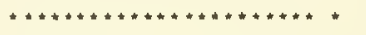 & & \\
\hline * & & $\begin{array}{l}\text { GRACILIS } \\
\text { MAJOR }\end{array}$ & $\begin{array}{l}\text { SHERFF } \\
\text { SHERFF }\end{array}$ & 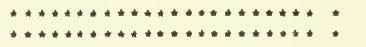 & & \\
\hline • & & MEGACEPHA & DEG. \& SHERFF & 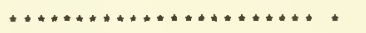 & & \\
\hline • & L. & INTERMEOIA & $\begin{array}{l}\text { DEG B SHERFF } \\
6 \text { LIPOCHAETA SUBCOROATA }\end{array}$ & 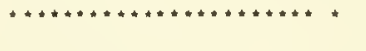 & & \\
\hline • & L. & KAHOOL AWENSIS & $\begin{array}{c}\text { SHERFF } \\
\text { LIPOCHAETA ROCKII }\end{array}$ & 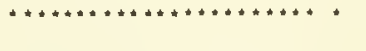 & & \\
\hline • & L. & KAWAIHOAENSIS & $\begin{array}{l}\text { H.ST. JOHN } \\
\text { LIPOCHAETA PERDJIA }\end{array}$ & 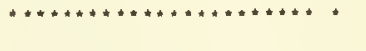 & & \\
\hline LI LAG & L. & LAVARUM & $(G A \cup D) D C$. & 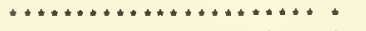 & ACE EPJED & \\
\hline : & & $\begin{array}{l}\text { CONFER IA } \\
\text { HILLEBHAN }\end{array}$ & $\begin{array}{l}\text { SHERFF } \\
\text { SHERFF }\end{array}$ & 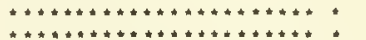 & & \\
\hline * & & $\begin{array}{l}\text { HILLEBHAN } \\
\text { LANAIENSI }\end{array}$ & $\begin{array}{l}\text { SHERFF } \\
\text { SHERFF }\end{array}$ & $\ldots+\cdots++\infty \ldots+\infty+\infty \ldots+\infty+\infty$ & & \\
\hline - & & LONG IF OLI & SHERFF & 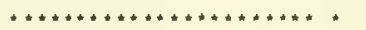 & & \\
\hline - & & MANE LEANA & SHERFF & 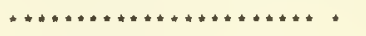 & & \\
\hline * & & OVATA & SHERFF & 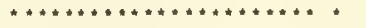 & & \\
\hline • & & SALICIFOL & SHERFF & 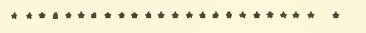 & & \\
\hline+ & & SKOT TSBER & SHERFF & 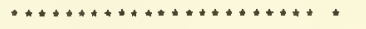 & & \\
\hline - & & STEARNS ! ! & DEG. \& SHERFF & 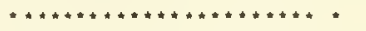 & & \\
\hline LILOZ & L. & LOBATA & CGAUD.) DC. & 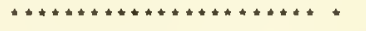 & ACCEPTED & \\
\hline - & & ALBESCENS & SHERFF & 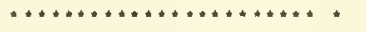 & & \\
\hline : & & AFREVALLI & (DRAKE) SHERFF & 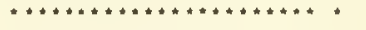 & & \\
\hline * & & DENTICULA & (WAWRA) SHERFF & 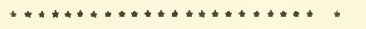 & & \\
\hline - & & GROSSEOEN & $\begin{array}{l}\text { TATA DEG. \& SHERFF } \\
\text { SIPOCHAETA LOBATA LEP TOPHYLLA }\end{array}$ & $+\cdots+\ldots+\ldots+\cdots+\cdots+\cdots+\cdots+*+*$ & & \\
\hline - & & HASTULATA & GHOOK. S ARN.) SHERFF & 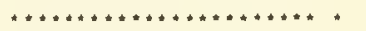 & & \\
\hline * & & INCISIOR & $\begin{array}{l}\text { H. ST J JOHN } \\
\text { LIPOCHAETA CONNATA ACRIS }\end{array}$ & 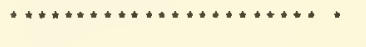 & & \\
\hline * & & MAKENENSI & $\begin{array}{l}\text { S DEG. \& SHERFF } \\
\text { S LIPOCHAETA ROCKII }\end{array}$ & 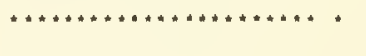 & & \\
\hline * & & MAUNAL OEN & $\begin{array}{l}\text { SHERFF } \\
\text { SIS LIPOCHAETA ROCKII }\end{array}$ & 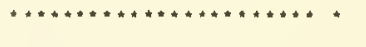 & & \\
\hline - & L. & M I NUSCULA & $\begin{array}{l}\text { DEG \& SHERFF } \\
\text { \& LIPOCHAETA DUBIA }\end{array}$ & $\ldots+\ldots+\ldots+\ldots+\ldots \ldots+\ldots \ldots \ldots$ & & \\
\hline • & L. & NI IHAUENSIS & $\begin{array}{l}\text { H.ST.JOHN } \\
\text { C LIPOCHAETA LOBATA }\end{array}$ & 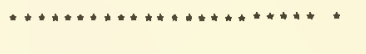 & & \\
\hline • & L. & PROFUSA & $\begin{array}{l}\text { SHERFF } \\
\text { LIPOCHAETA CONNATA }\end{array}$ & $*+*+*+\ldots+\ldots+\ldots \ldots+\ldots \ldots+\cdots$ & & \\
\hline$\star$ & & ROBUSTIOR & $\begin{array}{l}\text { DEG. B SHERFF } \\
\text { D SHE }\end{array}$ & $+*+\infty+++\ldots+*++\ldots+*+* *$ & & \\
\hline LIRO & L. & ROCK 11 & SHERFF & 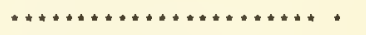 & ACCEPTED & \\
\hline • & & DISSECTA & SHERFF & 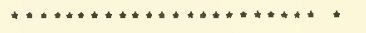 & & \\
\hline - & & SUBOVATA & SHERFF & 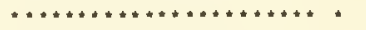 & & \\
\hline${ }_{*}^{*}$ & L. & SCAARA & $\begin{array}{l}\text { H.ST J JOHN } \\
\text { LIPOCHAETA ROCK!I }\end{array}$ & $* \ldots+\ldots+\ldots+\cdots+\infty \ldots+\ldots+\ldots$ & & \\
\hline LISU6 & L. & SUHCORDATA & GRAY & 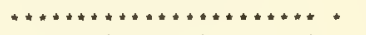 & ACCEPTED & \\
\hline 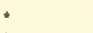 & & ME MERANAC & SHERFF & $\ldots \ldots \ldots \ldots+\ldots \ldots+\cdots+\ldots \ldots \ldots *$ & & \\
\hline * & & POPULIFOL & $\begin{array}{l}\text { SHERFF } \\
\text { IA LIPOCHAETA POPULIFOLIA }\end{array}$ & 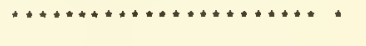 & & \\
\hline LI su? & L. & SUCCULENTA & GHOOK. \& ARN.) DC. & 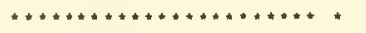 & ACCEPTED & \\
\hline * & & ANGUSTATA & SHERFF & 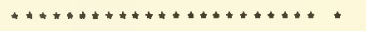 & & \\
\hline • & & SARCLAYI & SHERFF & 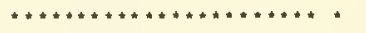 & & \\
\hline • & & DE CURRENS & (GRAY) SHERFF & $\ldots \ldots \ldots \ldots \ldots \ldots \ldots \ldots \ldots$ & & \\
\hline - & & TR If I OA & SHERFF & 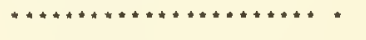 & & \\
\hline LITES & L. & TENUIS & 6 LIPOCHAETA ROCKII & 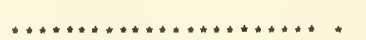 & ACCEPTED & \\
\hline t & L. & SELLINGII & DEG. \& SHERFF & $\ldots \ldots \ldots+\ldots+\ldots+\ldots \ldots \ldots \ldots$ & & \\
\hline - & L. & TRILOBATA & $\begin{array}{l}\text { H.ST.JOHN } \\
\text { LIPOCHAETA LOBATA }\end{array}$ & 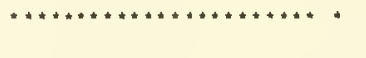 & & \\
\hline LIPPI & 祽 & 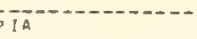 & L. & VE & RBENA TEAE & $2 \overline{7}$ \\
\hline 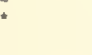 & L. & CUNE I F OLIA & $\begin{array}{l}\text { \# PTORR.)STEUD. } \\
\text { PHYL EUNEIFOLIA }\end{array}$ & 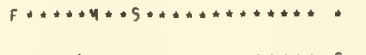 & & \\
\hline - & L. & GFMINA IA & - lippia aliga. & $\ldots \ldots+\ldots \ldots \ldots \ldots \ldots \ldots \ldots, s$ & & \\
\hline * & $\mathrm{L}$. & HELLER I & $\begin{array}{ll}\text { GRITTON } \\
\text { \# LIPPIA MICROMERA HELLER! }\end{array}$ & $\cdots+\cdots+\cdots+\cdots+\cdots+\cdots \cdots+\infty E *$ & & \\
\hline$\bullet$ & L. & INCISA & $\begin{array}{l}\text { SSMALL ITIDEST. } \\
\text { H PHYLA INEISA }\end{array}$ & 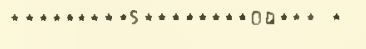 & & \\
\hline * & L. & LANCEOLATA & $\begin{array}{l}\text { H PHYLA INEISA } \\
\text { MICHX. }\end{array}$ & $F \cdots+c * 4 \cdots S \cdots * \cdots * 00 * \cdots$ & & \\
\hline * & & RECOGNIIA & FERNALO \& GRISCOM & $F * \cdots \cdots n+\cdots+* * * * * \cdots \cdots+*$ & & \\
\hline 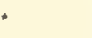 & L. & LIGUSTRINA & CLAG.)BRITTON & 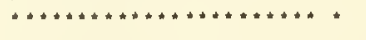 & & \\
\hline - & L. & LYCIOIDES & $\begin{array}{l}\text { N ALUYSIA GRATISSIMA } \\
\text { (CHAM.)STEUD. } \\
\text { - alOYSIA GRATISSIMA }\end{array}$ & 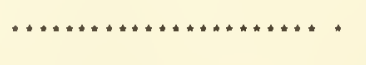 & & \\
\hline+ & L. & MACROSTACHYA & 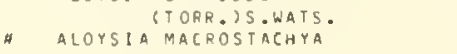 & 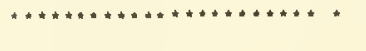 & & \\
\hline
\end{tabular}




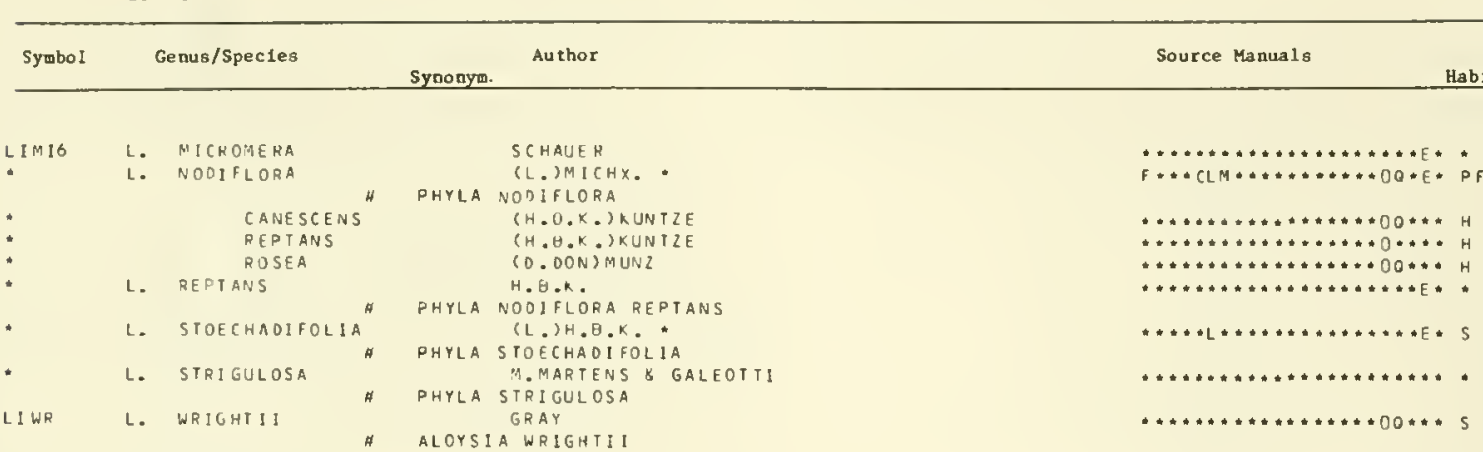

LIOU]

โIAUTOAMAAR

IIRIODENDRON

LISIANTHIUS

LISIANTHUS

\section{LISTERA-}

L. CORDATA

S NEPHROPHYLLA

L. NEPHROPHYLLA

\section{IITHACHNE \\ ITHOCARPUS \\ L. OENSIFLORUS ECHINOIOES}

IITHOCOCTA

L. TENELLUM

UTHOPHIA

LITHOPHRGMA

L. BREVILOTUM

L. BULGIFERUM

L. campanulatum

L. GLAGRUM

L. RUPICOLA

L. SCAORELLUM

L. tripartitum

LITRS

L: THO3

ZITHOSPERMUM

L. CROCEUM

LITOOROCHIA

L. TRIPARTITA

IITTYTSA

IITSEA

UITORELL

L. AMEKICANA

L. UUIFLORA

ИัОYОIA

LOBADIUM

LOBELIA

L. ASSURGENS

L. GRACHYPOOA

L. BRAGTEATA

L. CARDIHALIS GRAMINEA PHYLLOSTACHYA

L. GATIINGERI

1. GEORGIANA

$\angle O C A P$

LOGF
ALOYSIA WRIGHTII

L.

P.BR.

LISIANTHIUS

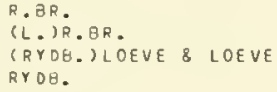

LISTERA CORDATA NEPHROPHYLLA

JEAUV.

SL UME

(HOOK. \& ARN.) REHD.

(R. OR.) ABRAMS

SMALL EX RYOH.

(NUTT.) SMALL

HELIOTROPIUM TENELLUM

SWART 2

CHUTT. ITORR. 8 GRAY *

RYOH.

LITHOPHRAGMA TENELLUM

RYDA.

LITHOPHPAGMA GLAGRUM

T. HOWE LL

LITHOPHRAGMA SCABRELLUM

NUTT.

LITHOPHRAGMA GULGIFERUM

GREENE

LITHOPHRAGMA TENELLUM

GREENE

LITHOPHRAGMA BOLANOERI

GREENE.

LITHOPHRAGMA AFFINE MIXIUM

$\mathrm{L.}$

FE FHALO
LITHOSPERMUM CARULINIENSE

K. PRESL

(SHARTZ)K.PRESL

PTERIS TRIPARTITA

SMALL

LAM.

BERG

FERNAL O

LSTTORELLA UNIFLORA AMERI CANA

(L. ASCHERS.

SALISA. EX REICHENB. *

RAF.

L.

(GRAY)A.DC, EX SMALL.

LOBELIA BERLANDIERI ORACHYPODA

L. Loblit

LLAM.) MCVAUGh.

LOBELIA SPLENDENS

(EHGELM.) MCVAUGH
LOBELIA SPLENDENS

$$
\text { GRAY }
$$

* Lobelia appenoiculata gatitngeri

ME VAUGH

NS LOBELIA ELONGATA
LOBELIA AMOENA
Panily Nome No.

abit

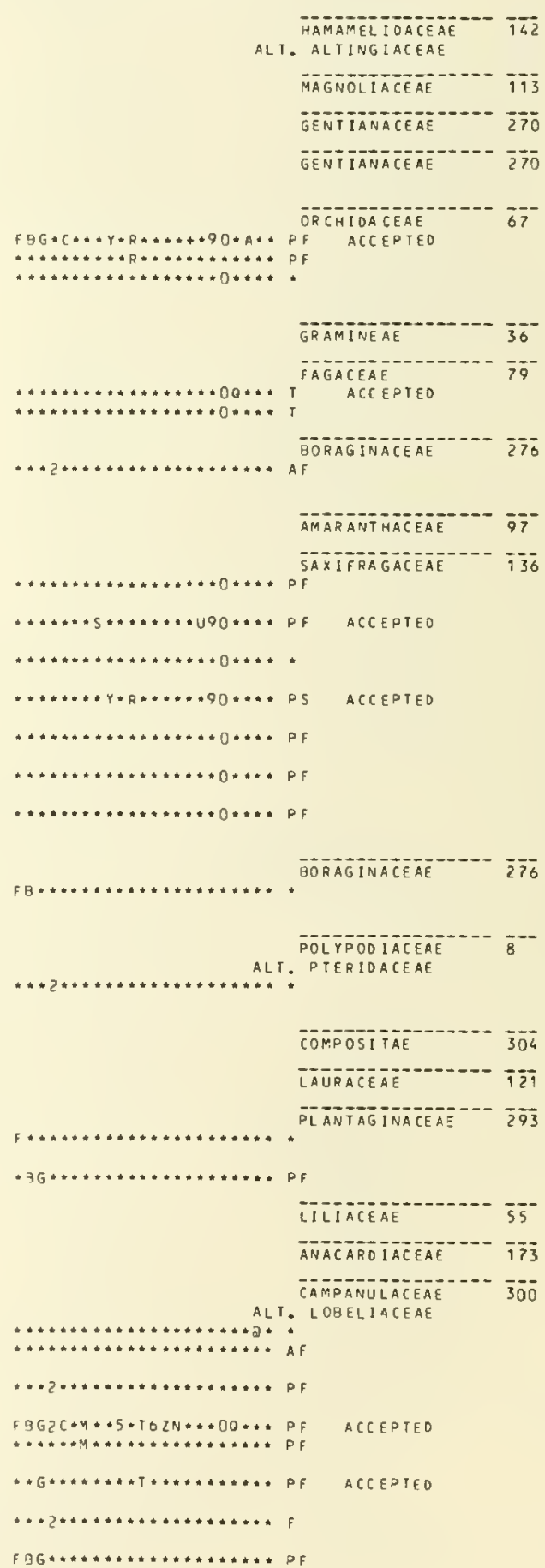




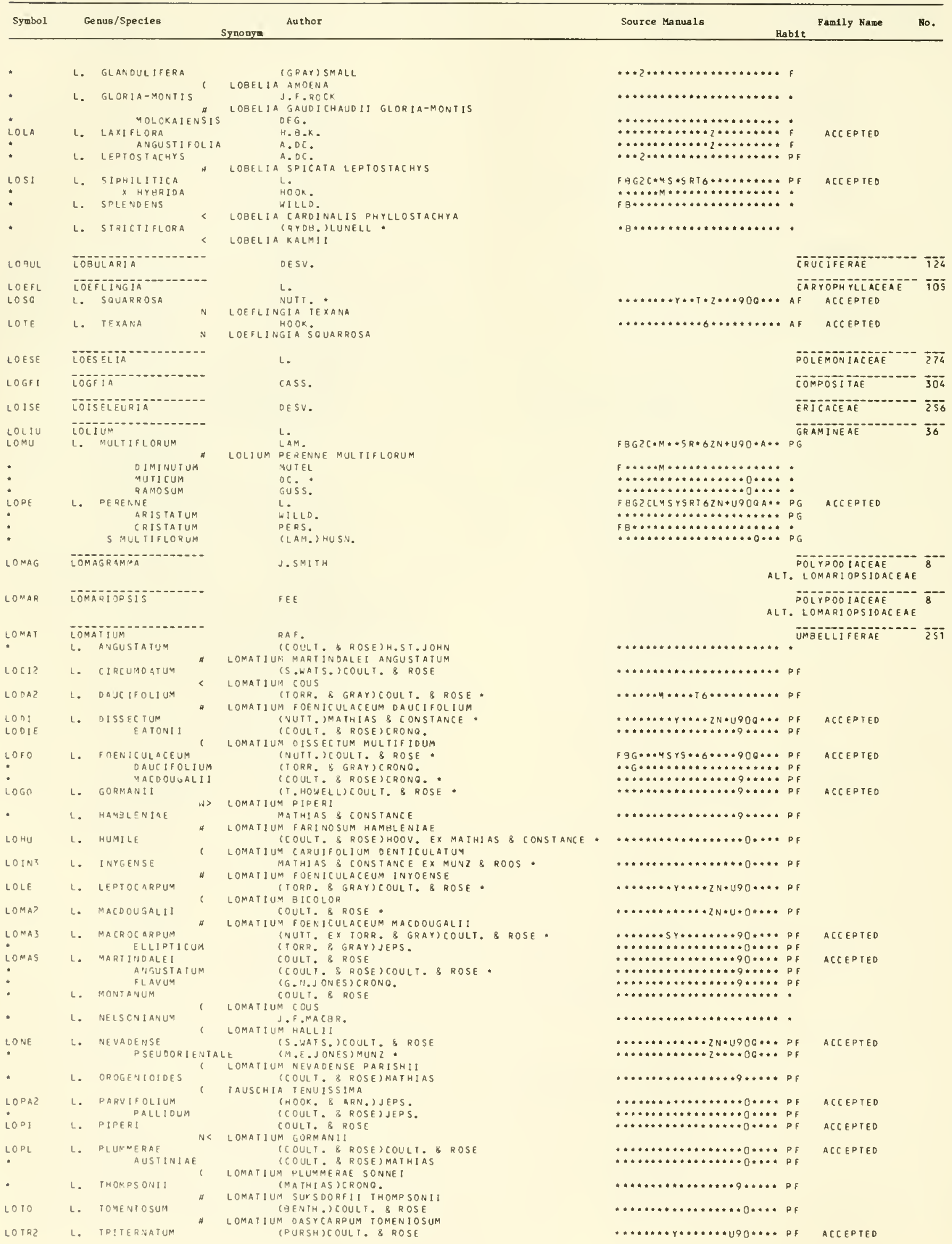


LOMATIUM

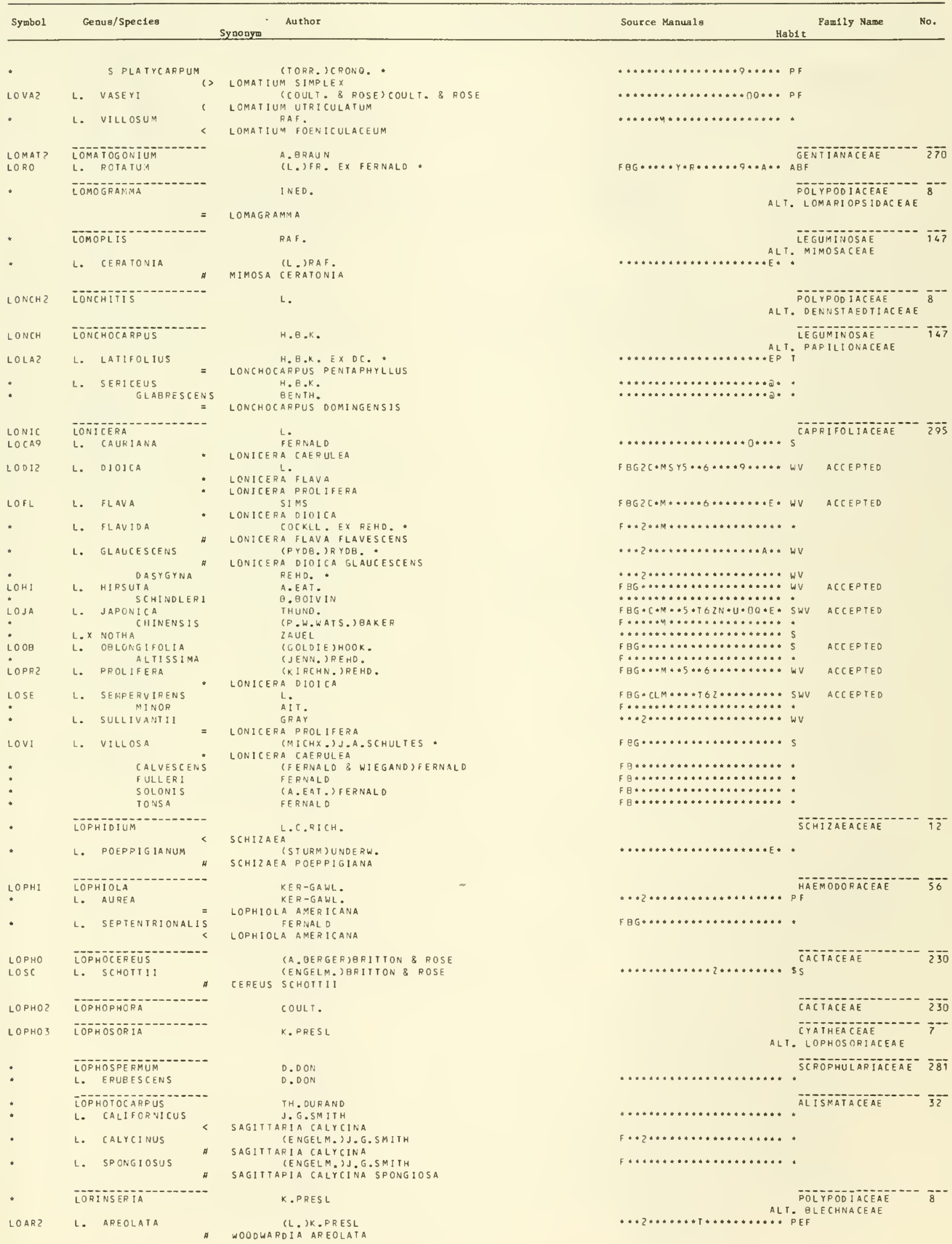




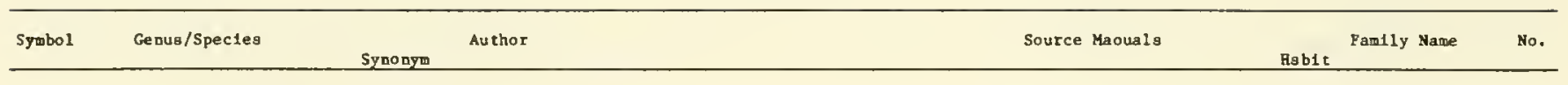

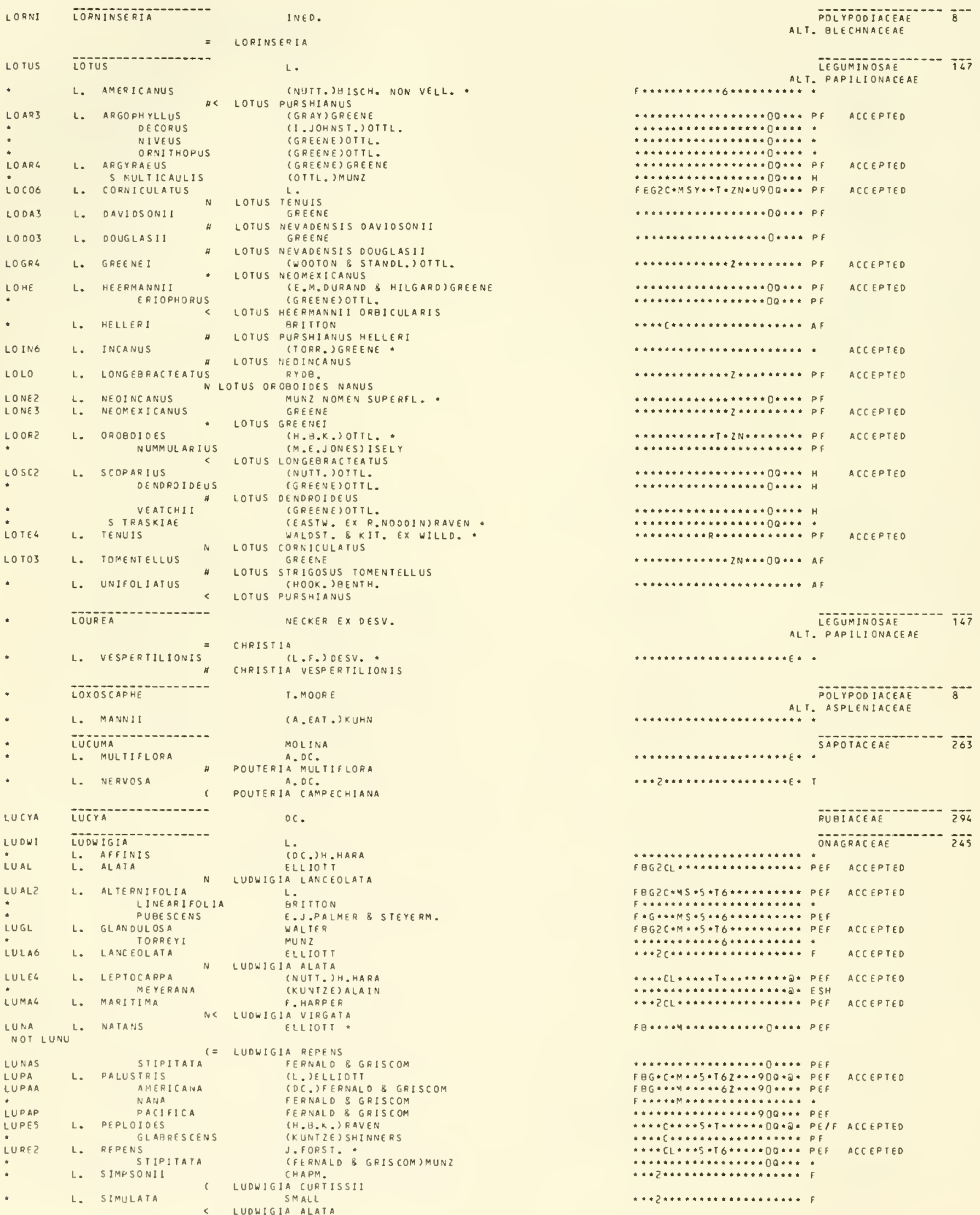




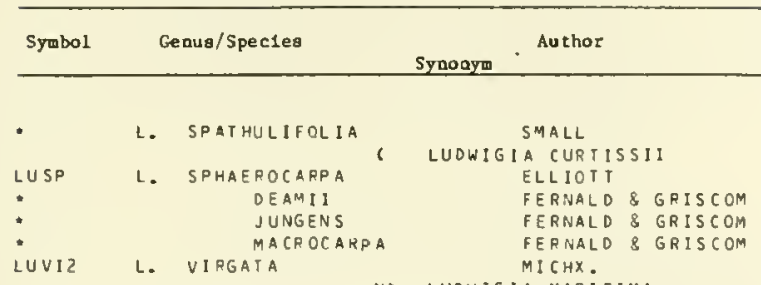

N) LUDWIGIA MARITIMA

LUDWIGIANTHA
L. ARCUATA
L. BREVIPES

LUETK LUETKEA

LUFFA

LU INA

LUNAZ

*

LUST

LUNAN

LUNAR

LUPIN

LUAOZ

LUAL 3

LUAL 5

LUALG

LUAN2

LUARO

LUARZ

-

LUAR3

L. ARGENTEUS

LUETKEA
LUFFA
LUINA

L. NARDOSMIA

GLABRATA

CUNA - -

LUNARIA

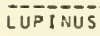

\section{L. aduncus}

L. ALBICAULIS

L. X ALPESTRIS

L. ALPICOLA

L. AMPLUS

$$
\text { APERTUS }
$$

L. ARBUSTUS

CALCARATUS

L. arcticus

TE TONENS IS

.

LUBA

LUBI

?

LUB12

LUBL

LUAU2

LUCA

?

LUC

LU

(WALTER) SMALL

LUDWIGIA ARCUATA

LUOWIGIA GREVIPES

BONG.

MILL.

RENTH.

(GRAY) CRONG.

CACALIOPSIS NARDOSMIA

(PIPEP) CRONQ.

RAINIERA STRICTA

HOOK.

L.

L.

\section{GREENE}

DOUGL. EX HOOK.

LUPINUS ANOERSONII

A. NELS.

LUPINUS ARGENTEUS

GREENE

LUPINUS BAKERI AMPLUS

S. WATS.

LUPINUS ALBICAULIS

LUPINUS APERTUS

(A. HELLER) MUNZ

LUPINUS ANGUSIIFLORUS

DOUGL. EX LINDL. *

(KELLOGG) WELSH.

S. WATS .

LUPINUS BURKE

IE. NELS. C.P.SMITH PURSH

LUPINUS MACULATUS

LUPINUS SITGREAVESII

LUPINUS ALPESIRIS

LUPINUS ROSEOLUS

PARVIFLONUS

RUBRICAULIS
STENOPHYLLUS

LUPINUS PARVIFLORUS

(GREENE) WELSH

L. GAREIGER N LUPINUS SERICEUS

L. BICOLOR

LINOL.

LUPINUS VALLICOLA

(S. WATS.) C.P.SMITH

L. BIDOLEI

MICROPHYLLUS

L. BLUMER I

GREENF

L. BURKEI

L. caudatus

CUTLERI

L. CHIHUAHUENSIS

L. CONCINNUS
L. ANDERSONII

(TORR. \& GRAY) SMALL

B. LONG EX BRIITON,A.BR. \& SMALL.

LUPINUS CAUOATUS ARGOPHYLLUS

L. HENOERS. EX PIPER.

LATIFOLIUS

(A. HELLER) C.P. SMITH

LUPINUS WYETHII TETONENSIS

BOREUS NE LUPINUS ALPESIPIS
(C.P.SMITH)WELSH

DEPRESSUS LUPINUS ARGENTEUS SPATHULATUS
(RYOB.) C.HITGHC.

LUPINUS EVERMANNII

(NUTT. EX HOOK. \& ARN.) C. HITCHC.

(RYDB.)RYOB. EX R.J.DAVIS.

LUPINUS ARGENTEUS TENELLUS

(A. HELLER) C.P. SMITH

L. HENDERS. EX C.P. SMITH.

A RGOPHYLLUS

A GARDHI ANUS

BREVIOR

OPTATUS

ORCUTYII

PALLIDUS

L. CORYMBOSUS

L. CUTLERI 


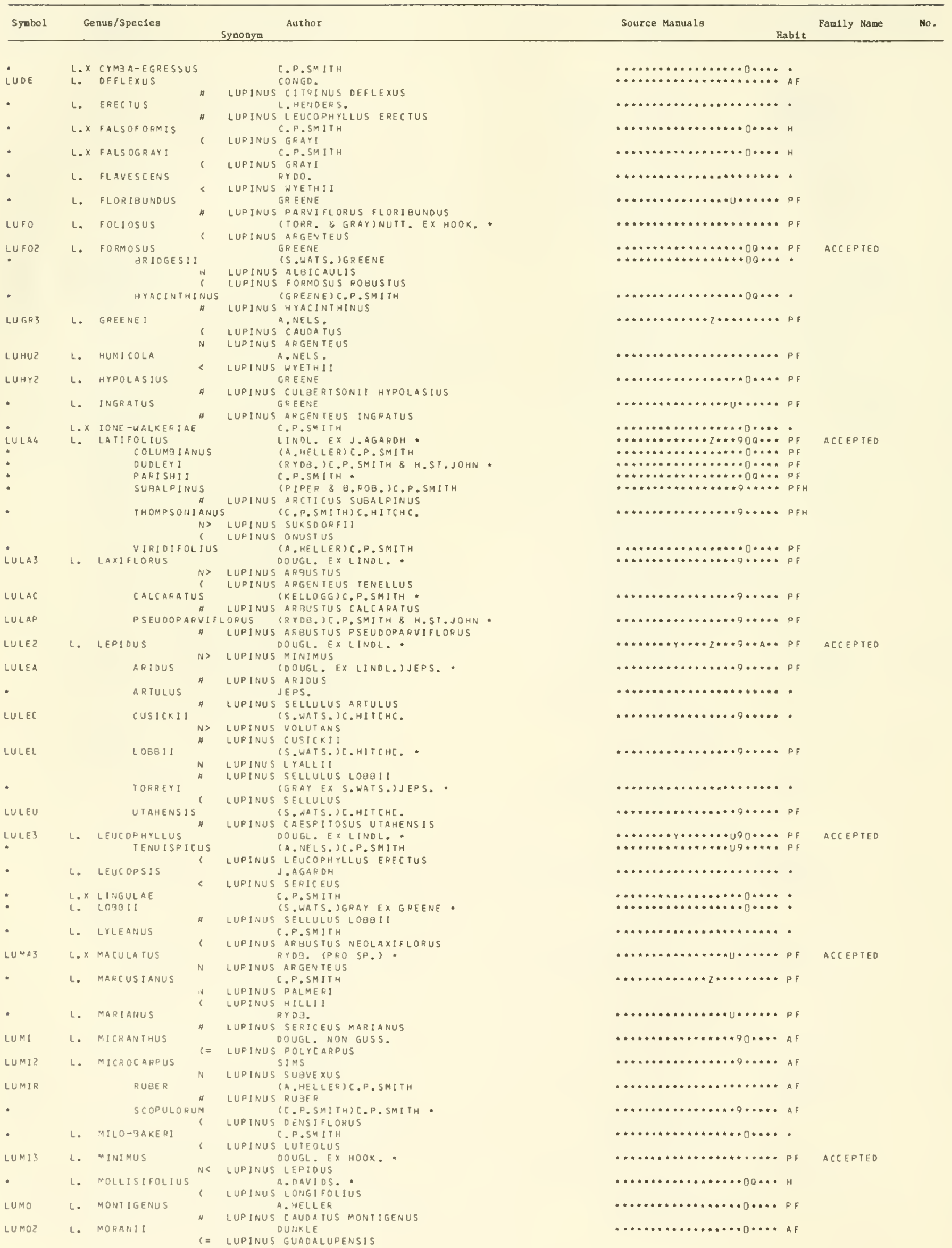




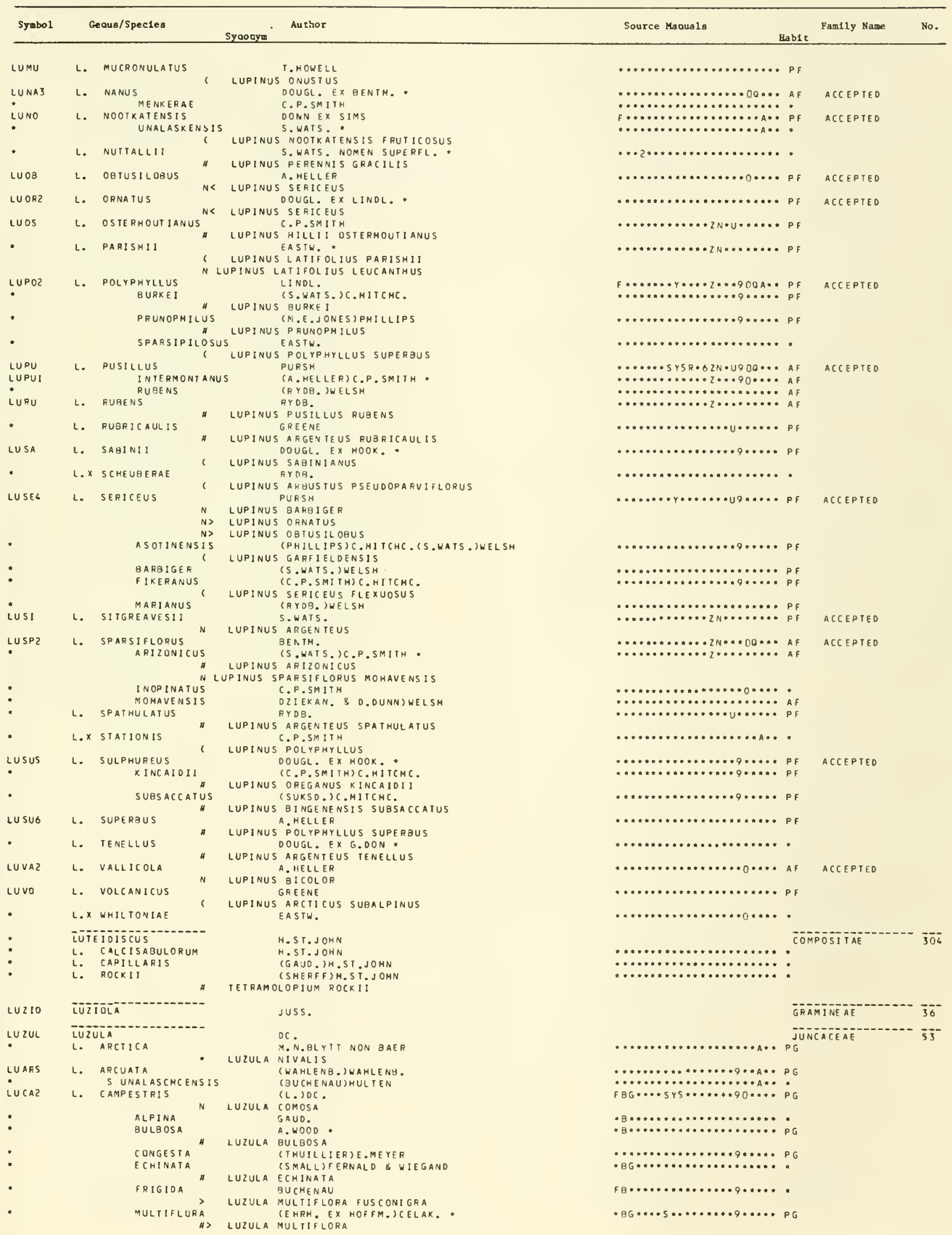




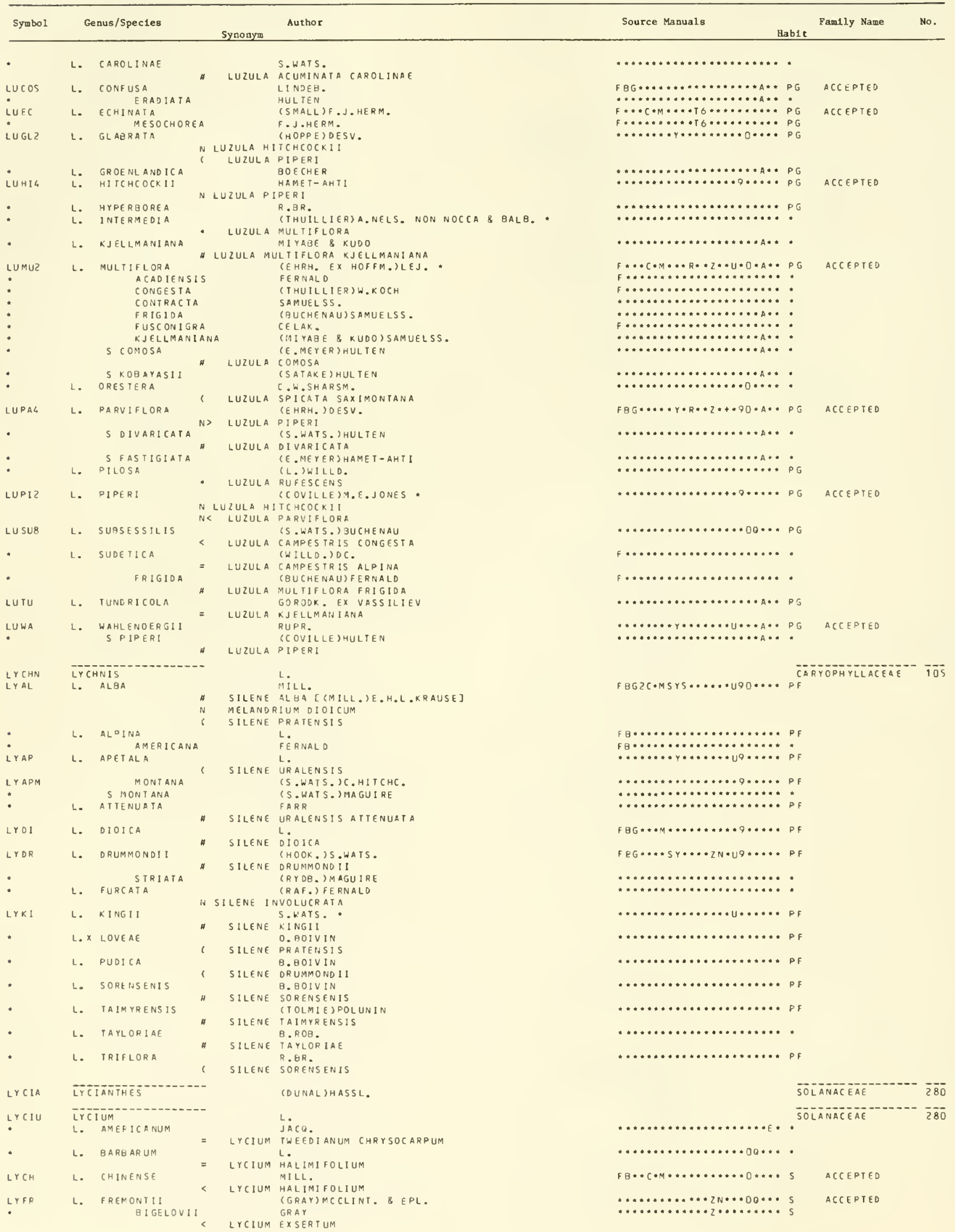




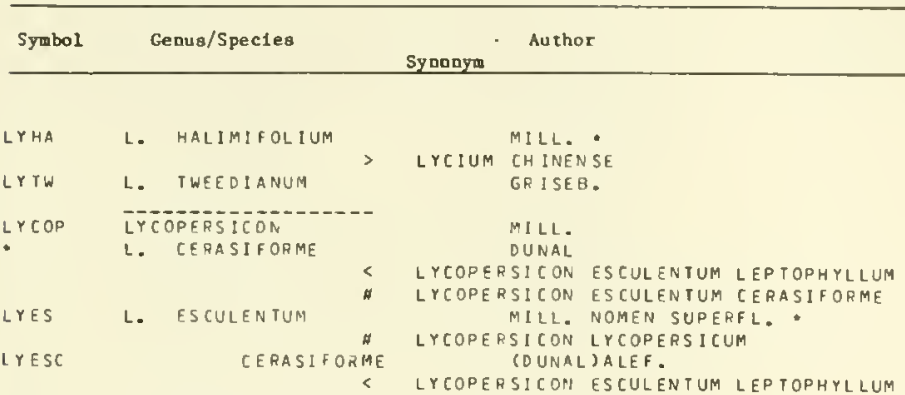

\begin{tabular}{|c|c|c|}
\hline LYCOPZ & \multicolumn{2}{|c|}{ LYCOPODIUM } \\
\hline - & L. & ADPRESSUM \\
\hline LYALZ & L. & ALOPE CUROIDES \\
\hline 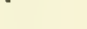 & & PINNA T UM \\
\hline LYAN2 & L. & ANNOT T NUM \\
\hline - & & $A[R] F O L I U$ \\
\hline - & & ALPESTRE \\
\hline
\end{tabular}

*ycl Lo Pungens

\begin{tabular}{|c|c|c|}
\hline & & BREVISPICA TU \\
\hline * & & INTEGEKR IMUM \\
\hline - & & MEGASTACHYON \\
\hline - & & MONOSTACHYON \\
\hline * & & SUAREMUTUM \\
\hline - & & TRISTACHYUM \\
\hline 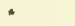 & & 5 YONOSIACHYON \\
\hline $\operatorname{LYCO3}$ & L. & COMPLA IIAIUM \\
\hline - & & $F L A O E L L I F O R M$ \\
\hline - & & HA DERERI \\
\hline$*$ & L. & DU9 I บM \\
\hline$L Y F L$ & L. & FLABELL IFDRME \\
\hline & & ? \\
\hline LYIN & L. & INUN, DAT UM \\
\hline LYINB & & BIGELJVII \\
\hline LYINE & & ELONGATUIM ${ }^{6=}$ \\
\hline & & 6 \\
\hline - & & PI INNAT UM \\
\hline - & & ROBUSTUM \\
\hline
\end{tabular}

LyLu Le LUCIOULUM
OCCIDENTALE

-

LYOB

(Y)

LYSA

LYSE

.

:

;

$\operatorname{Lr} \operatorname{COP} 3$

L. MERIDIONALE

L. OBSEURUM

DENDROI DEUM

L. SABINIFOLIUM
SITCHENSE

L. SELAGO

SITCHEN
PATENS

S APPRESSUIA

L. SETACEUM

\section{LYTOPSIS}

LYCOPG LYCOPUS

LYAM L. AMERICANUS

* LONGII

Lyamz L. amplectens
PUDENS

LYFu L. EUROPAEUS

Mollis
* lo laurentianus

irluz L. lugidus

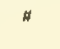

LYCOPODIUM APPRESSUM

L.

LYCOPODIUM PROS TRATUM L.

FERNALD

LYCOPODIUN ANNOTINUM PUNGENS

(PYLAIE) HUL TEN

L.

C.H.PECK

SPRING

FERNALO \& C.H. EISSELL *

HOOK. \& GREV.

VICTOR IN

HOOK.

(SREV. \& HOOK.)SELLING

LERNALO

(HOUSE)B.SOIVIN

ZOEGA

LYCOPODIUM ANNOTINUM PUNGENS

(FERNALO)W. H. BLANCH.

LYCOPODIUM COMPLANATUM FLABELLIFORME

LYCOPODIUM OIGITATUM

L.

LYCOPODIULA APPRESSUM

LYCOPODIUM COPELANDI:

CHAPM.

LYCOPODIUM PROSTRATUM

R. J.EAT.

LYCOPODIUM CDPELANDII

MI CHX.

LYCOPODIUM POROPHILUM

UNDERW. \& LLOYO

LYCOPODIUM CAROLINIANUM MERIDIONALE

(MICHX.) D.C.EAT.

LYCOPODIUM DENDROIDEUM

WILLO.

(RUPR. ) FEANALD

.

*

LYRU

-

$\cdot$

LYUN

LrVI

LYCOR
L. LUGIDUS

SAMERICANUS PUENS
L. PUERAY) HULTEN

L. RUGELLUS 4 LYCOPUS AMPLECTENS PUEENS

MOENCH
LANCEOLATUS BEPNER

L. SESSILIFOLIUS = LYCOPUS RUAELLUS ANGUSTIFOLIUS

L LYCOPUS AMPLEGTENS

L. UMIFLORUS MICHX.

l. virginicus arcepus virginicus

LYCORTS HERB.

TURCZ, EX HENTH.
Source Manuals

Pan1ly Name

No. Habit

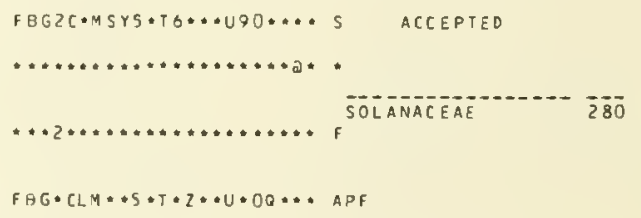

$\ldots \ldots \ldots \ldots \ldots+\ldots \ldots \ldots \ldots . \quad$ ACLEPTED

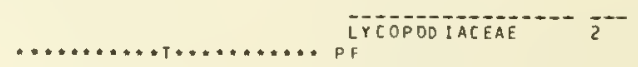

FBG $26 \ldots \ldots \ldots+\ldots \ldots \ldots \ldots$ PF ACEEPTED

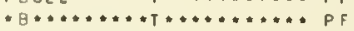

F $A G \cdots \cdots \cdot \gamma * R \cdots \cdots+9 * A * *$ PF ACCEPTED

F $B G \cdots \ldots \ldots \ldots \ldots \ldots \ldots \ldots \ldots$

f...............A*

$\ldots \ldots \ldots \ldots \ldots \ldots \ldots+A * \ldots$

FEG $26 * \ldots \cdots \cdots * * 90 * A *$ PF ACCEPTED

F....................

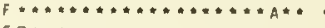

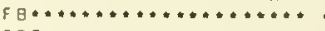

$F B G \ldots \ldots \ldots \ldots \ldots \ldots \ldots+\ldots \ldots+\ldots \ldots$

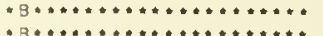

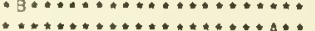

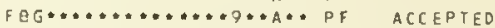

F $B G \ldots \ldots \ldots \ldots \ldots \ldots \ldots \ldots \ldots$

$\ldots \ldots \ldots+\ldots \ldots \ldots \ldots \ldots . . . \ldots$.

$\ldots \ldots \ldots \ldots \ldots \ldots \ldots+\ldots \ldots$.

$\ldots .2[\ldots \ldots \ldots \ldots \ldots \ldots \ldots, p$

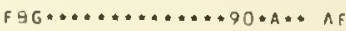

$F B G \cdots \ldots \ldots \ldots \ldots \ldots \ldots \ldots$ AF

ACCEPTED

. $B G \ldots \ldots \ldots \ldots \ldots \ldots \ldots$ A

$\ldots \ldots \ldots \ldots \ldots . . . \ldots . .$.

F...................

$F B G 2 C \cdot M \ldots \ldots \ldots \ldots \ldots \ldots \ldots F F$

f $\mathrm{B} \ldots \ldots \ldots+\ldots+\ldots \ldots \ldots \ldots *$.

ACCEPTED

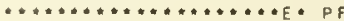

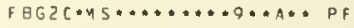

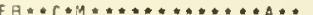

ACCEPTED

E $B G \cdots \ldots \ldots \ldots \ldots \ldots \ldots \ldots+\ldots$

F................

$F B G Z C+M \cdot Y \cdots \ldots \ldots \ldots+Q \cdots A \cdots P F$

FBG.$A M \ldots \ldots \ldots \ldots \ldots \ldots$.

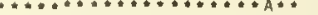

$\ldots \ldots \ldots \ldots \ldots \ldots \ldots \ldots+\ldots, \ldots$

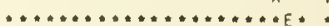

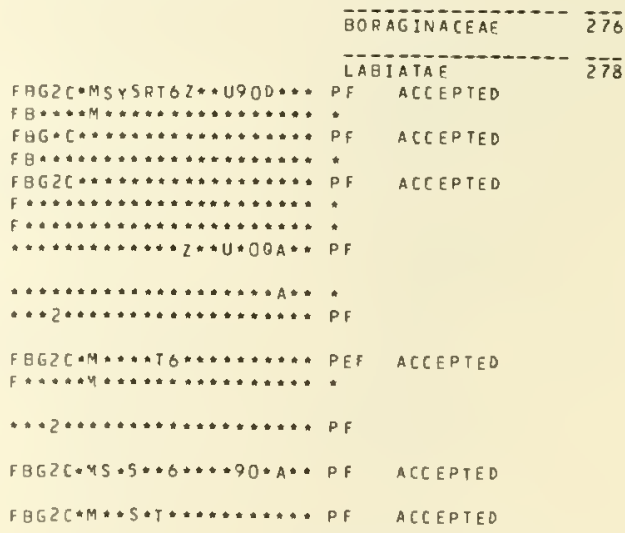

(GRITTON) FERNALD

(A. KERN. ) BRIO

ROLL. - GE RM, 


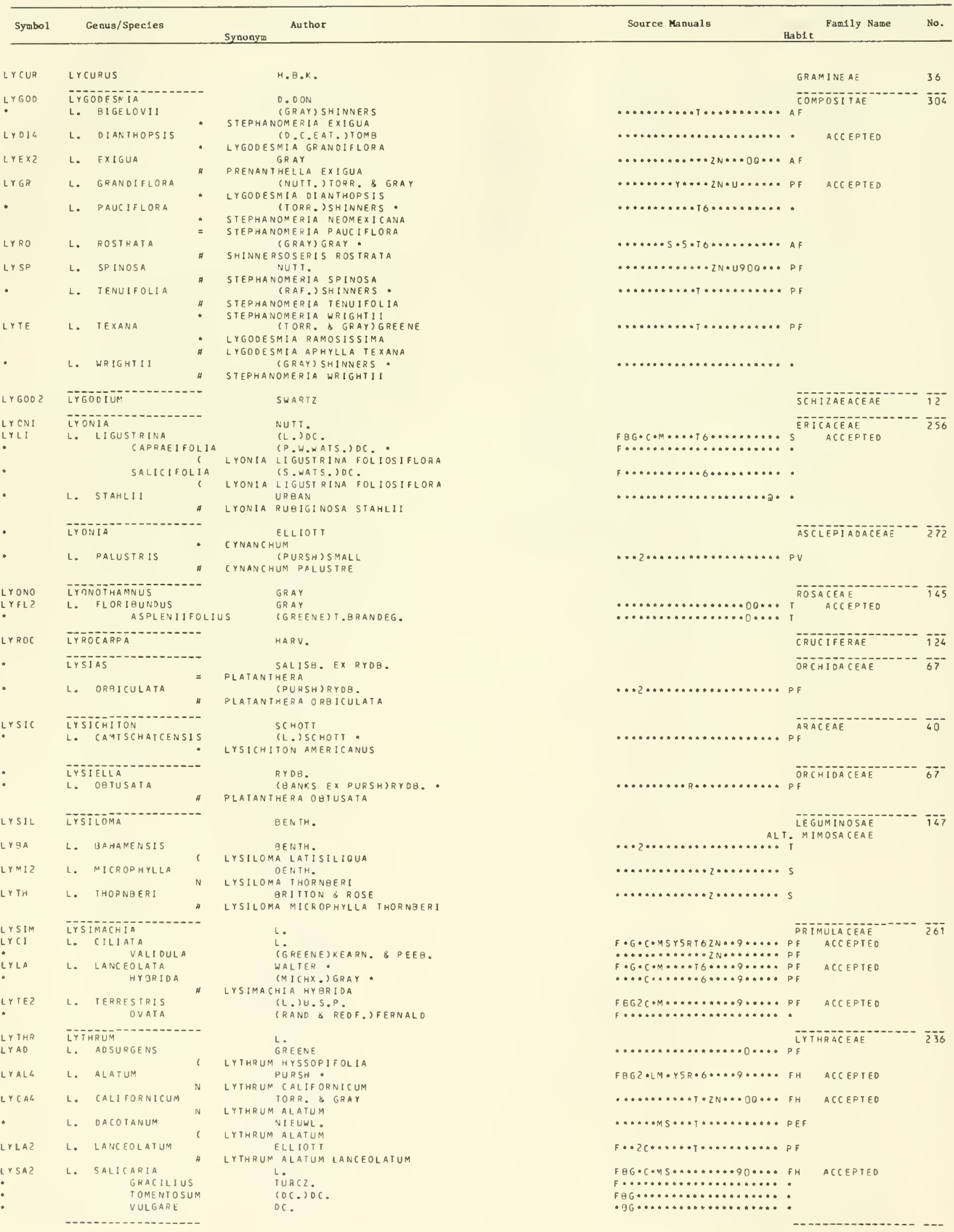




\begin{tabular}{|c|c|c|c|c|c|c|}
\hline Symbol 1 & Genus/Spec1es & Synonym. Author & Source Maouals & Habit & Family Name & No. \\
\hline
\end{tabular}

$\begin{array}{ll}* & \text { MABA } \\ \text { M. CARIBAEA } \\ \text { M. SINTENISII } \\ \text { MACAO MACADAMIA } \\ \text { MACAR MACARANGA } \\ \text { MAGR } \\ \text { MACBR GRANDIFOLIA } \\ \text { MACBRIDEA } \\ \text { M. PULCHRA }\end{array}$

MACDO MACDOUGALIA

MACFA MACFADYENA

\section{MACHA MACHAERANTHERA}

MACHA MACHAERANTHERA

MACAZ M. CANESCENS

MACO M. COGNATA

MACOZ M. COMM!XTA

MAGL M. GLABRIUSCULA

MAGLV

MALE

MAL I

$M A O R$

MAPAIO

MASE 2

MASU 3

MATO

-

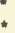

MAWR

MACHA 2

-

MAMA1 2

MAME

MACHA 4

MACHA 3

MACHA 5

MA CLE

MACLU

MACRA

MACRA 2

:

MA CRO

$-$

VILLOSA
M. LEUCANPHEMIFOLIA

M. LINEARIS

M. ORCUTTII

M. PATTERSONII

M. SESSILIFLORA

M. SUGALPINA

M. TORTIFOLIA

M. venusta

HBEREIS

M. WRIGHTII
J. \& G.FORST.

(A. OC.) H IERON.

KRUG \& URBAN

DIOSPYROS SINTENISII

$$
\begin{aligned}
& \text { F. MUELL. } \\
& \text { THOUARS } \\
& \text { (BLANCO) MERRILL } \\
& \text { ELL IOTT EX NUTT. } \\
& \text { ELLIOIT }
\end{aligned}
$$

MACBRIDEA CAROLINIANA

$$
\begin{aligned}
& \text { A. HELLER } \\
& \text { A. DC. }
\end{aligned}
$$

NEES

(GRAY) GREENE
MACHAERANTHERA PATTERSONII

(PURSH) GRAY

MACHAERANTHERA COMMIXTA

MACHAERANTHERA SUBALPINA

(H.M.HALL)CRONO. \& D.KECK

XYLORHIZA COGNATA

GREENE

MACHAERAN THERA SUBALPINA

MACHAERANTHERA CANESCENS

$$
\text { SNUTT.) CRONQ. \& O.KECK }
$$

\# XYLORHIZA GLAERIUSCULA

\# XYLORHIZA GLABRIUSCULA LINEARIFOLIA

(NUTY.) CRONQ. \& D.KECK

(GREENE) GREENE

MACHAERANTHERA CANESCENS

GREENE

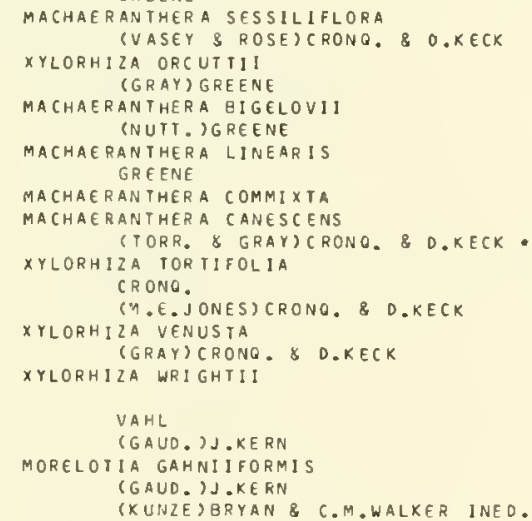

SIS DISCOIDEA

( $B E N T H$. ) URBAN

(L. UURBAN

PHASEOLIJS LATHYROIOES

MUELL. AQG.

(H. ITO) CHING
EBENACEAE
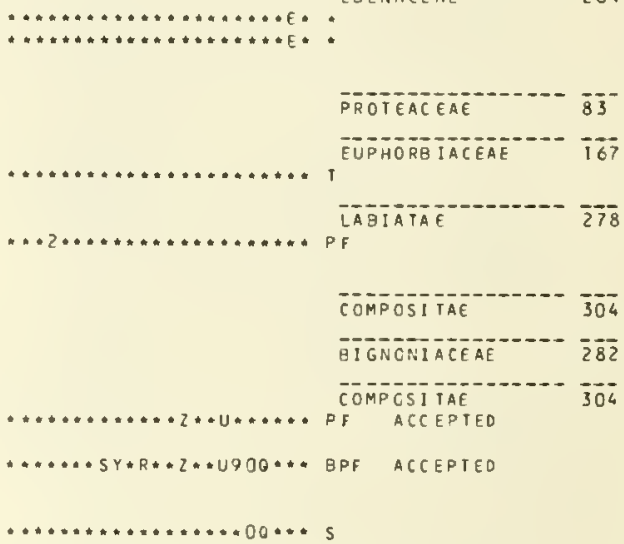

............UQ*... PF ACCEPTED
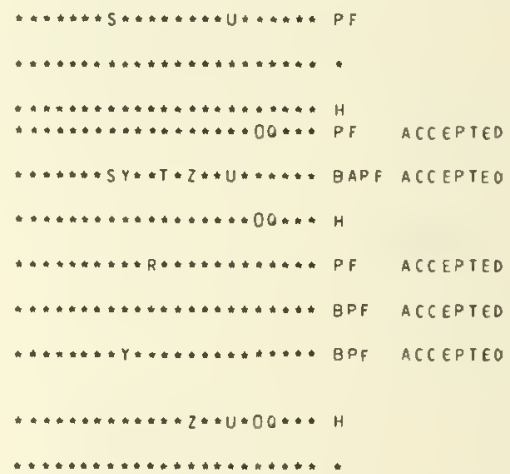

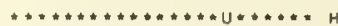
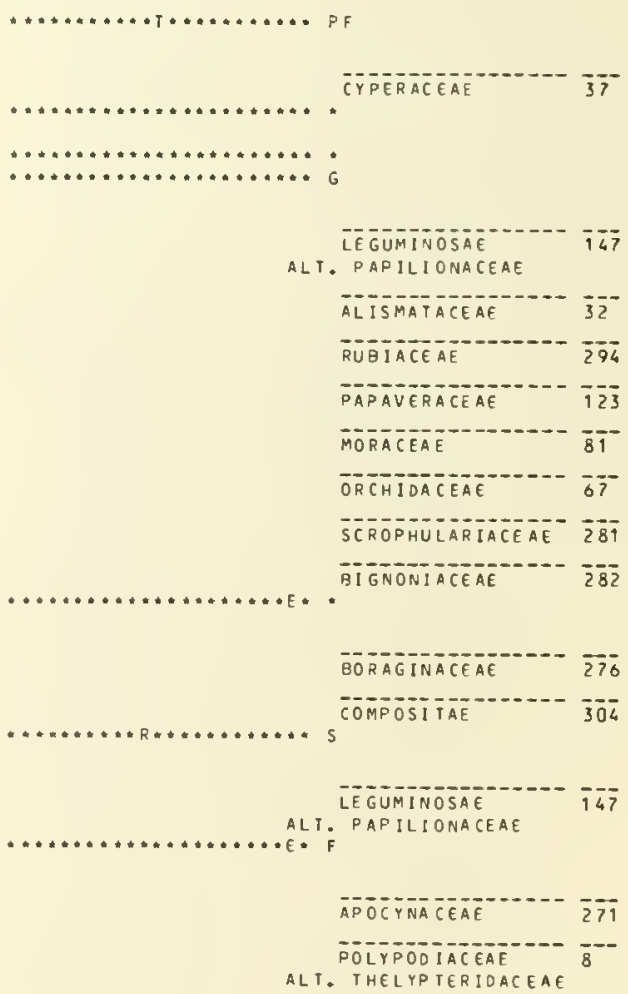

ALT. THELYPTERIDACEAE

264

$\overline{3}^{-}$

78

\section{(1)}

. 


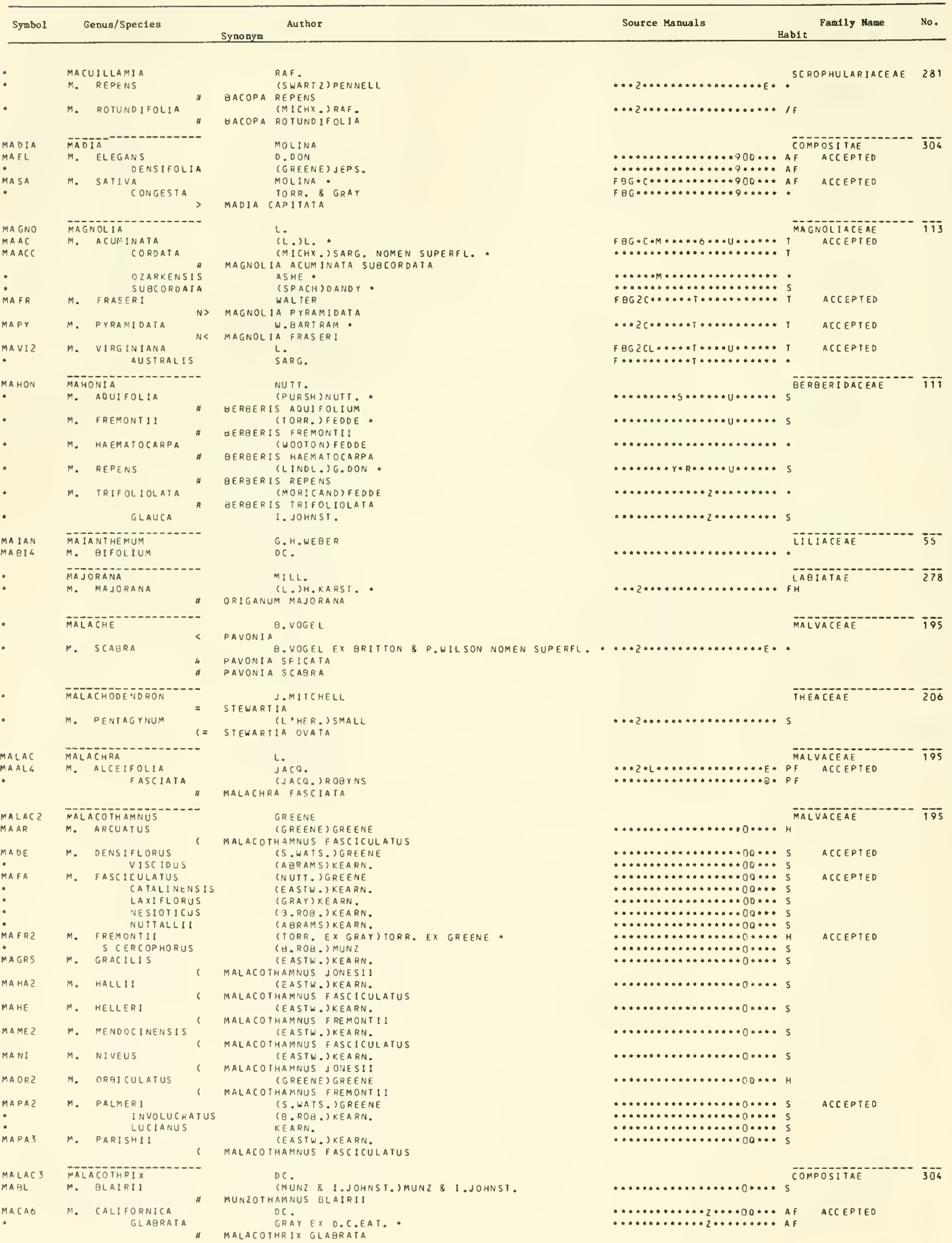




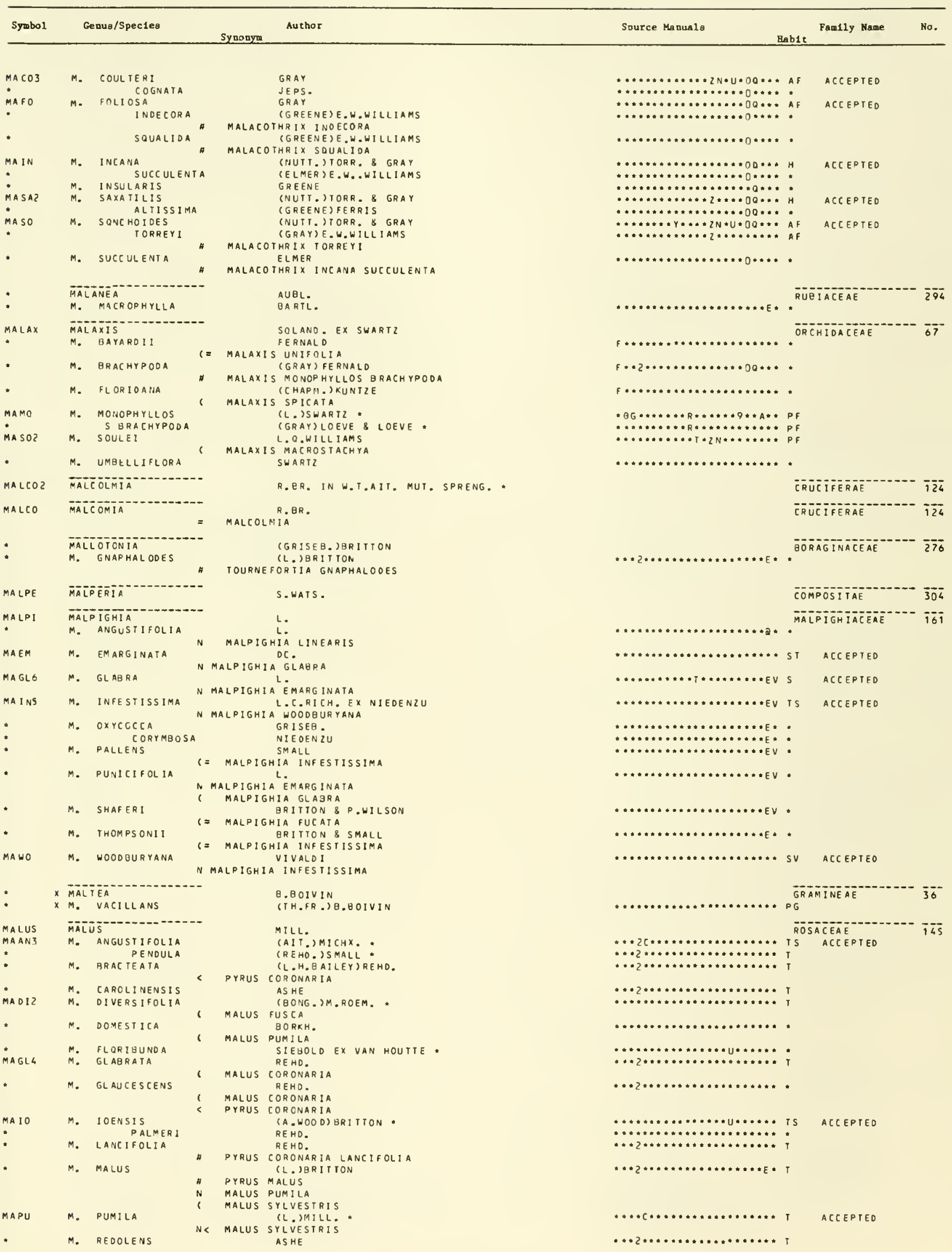




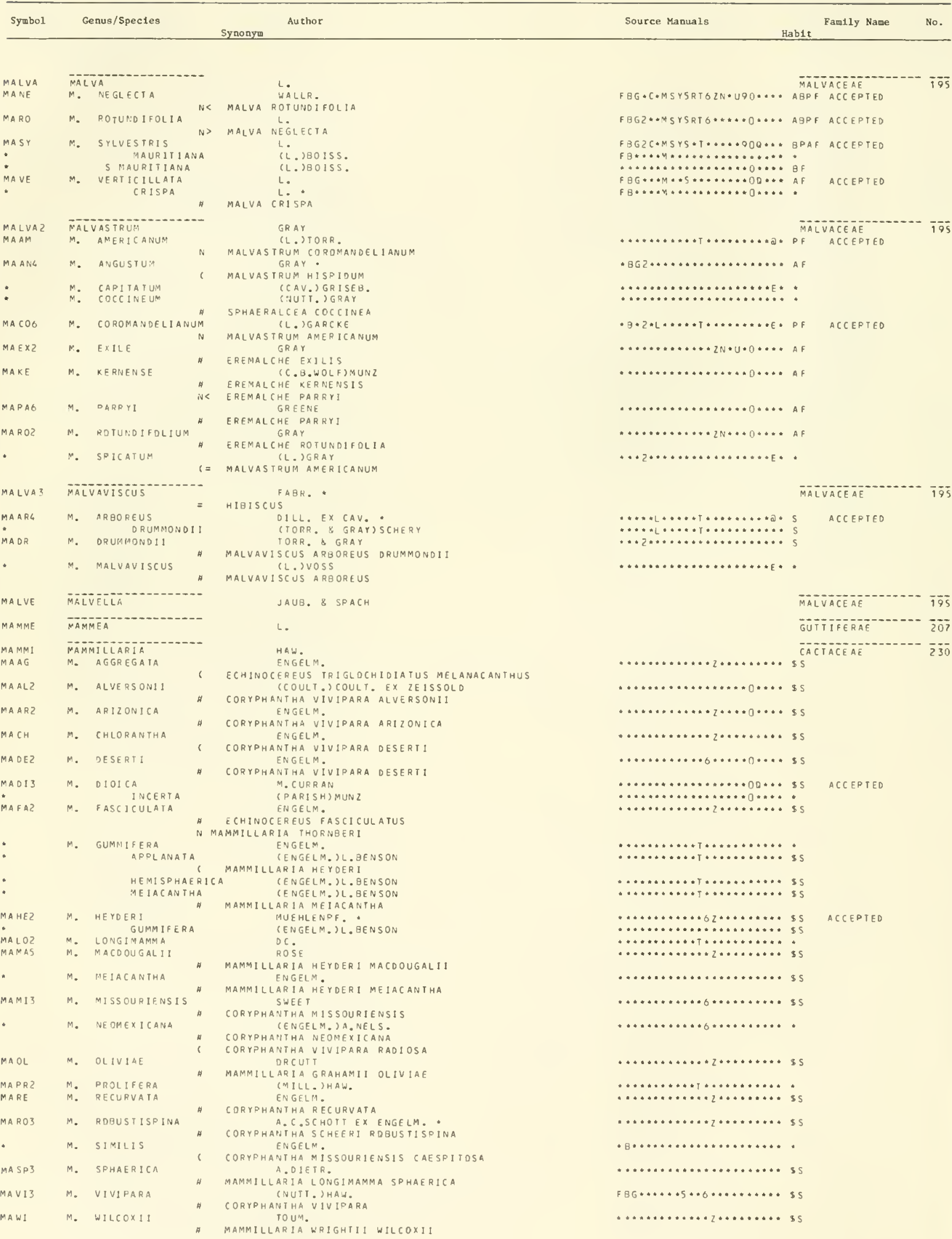


MANCOA

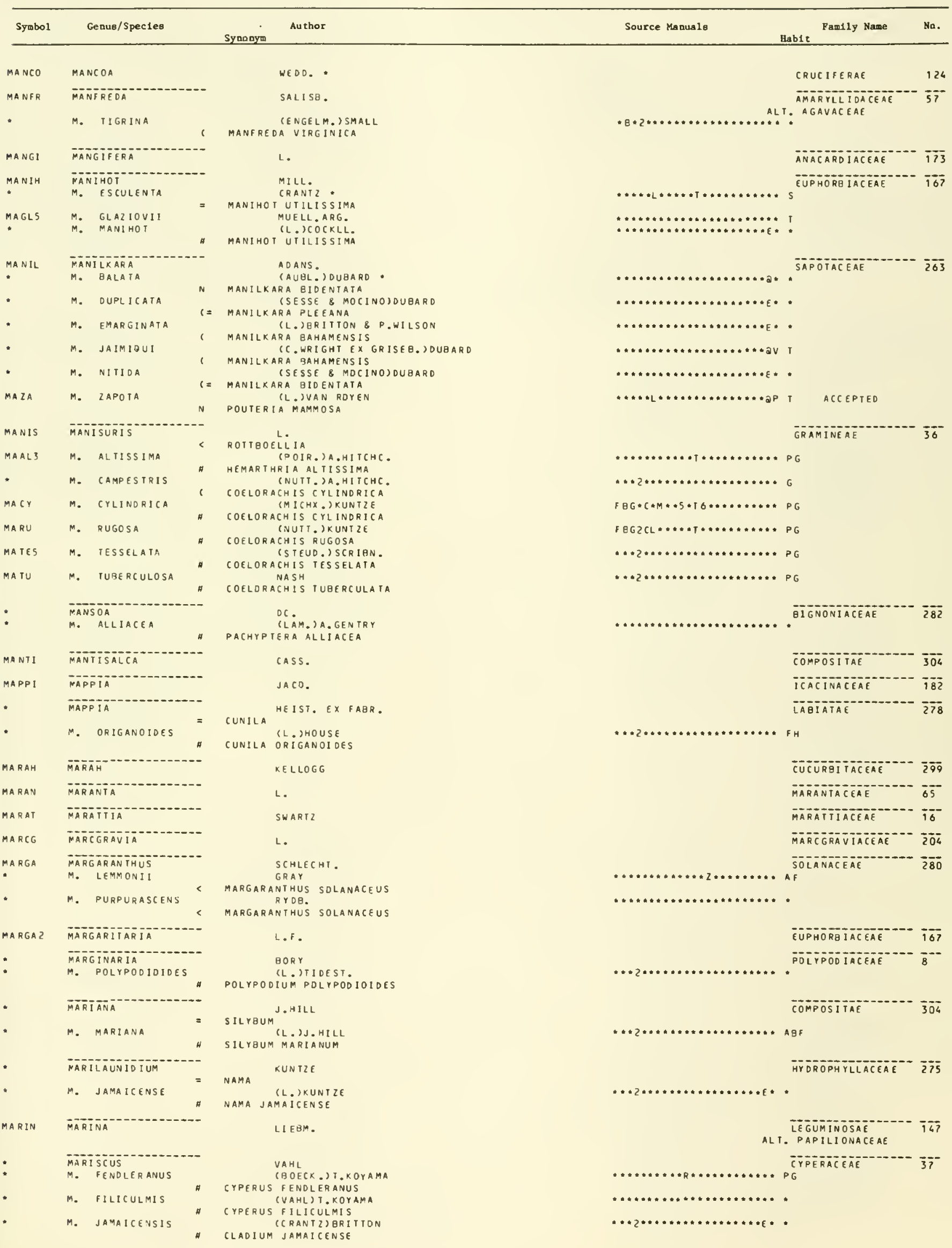




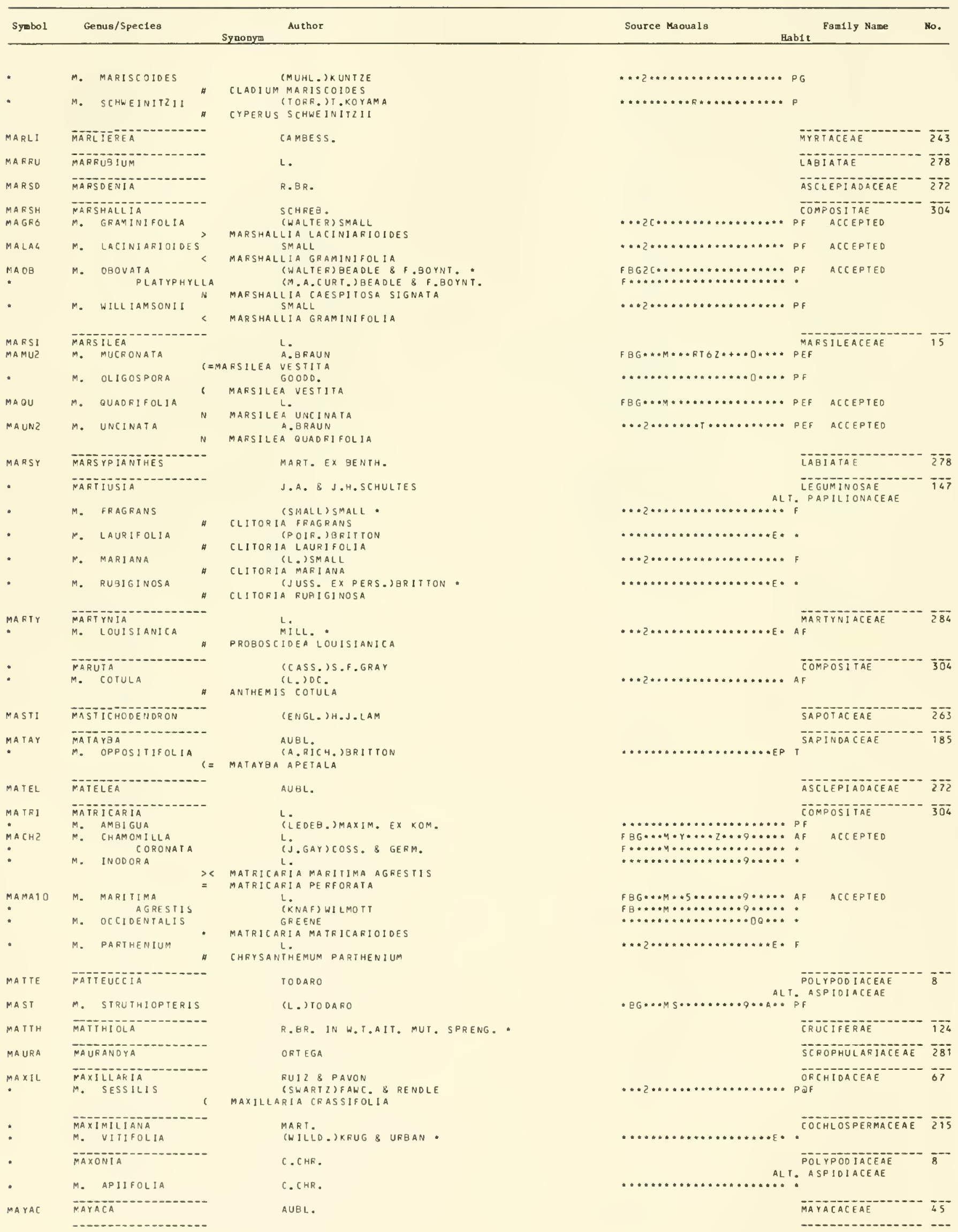




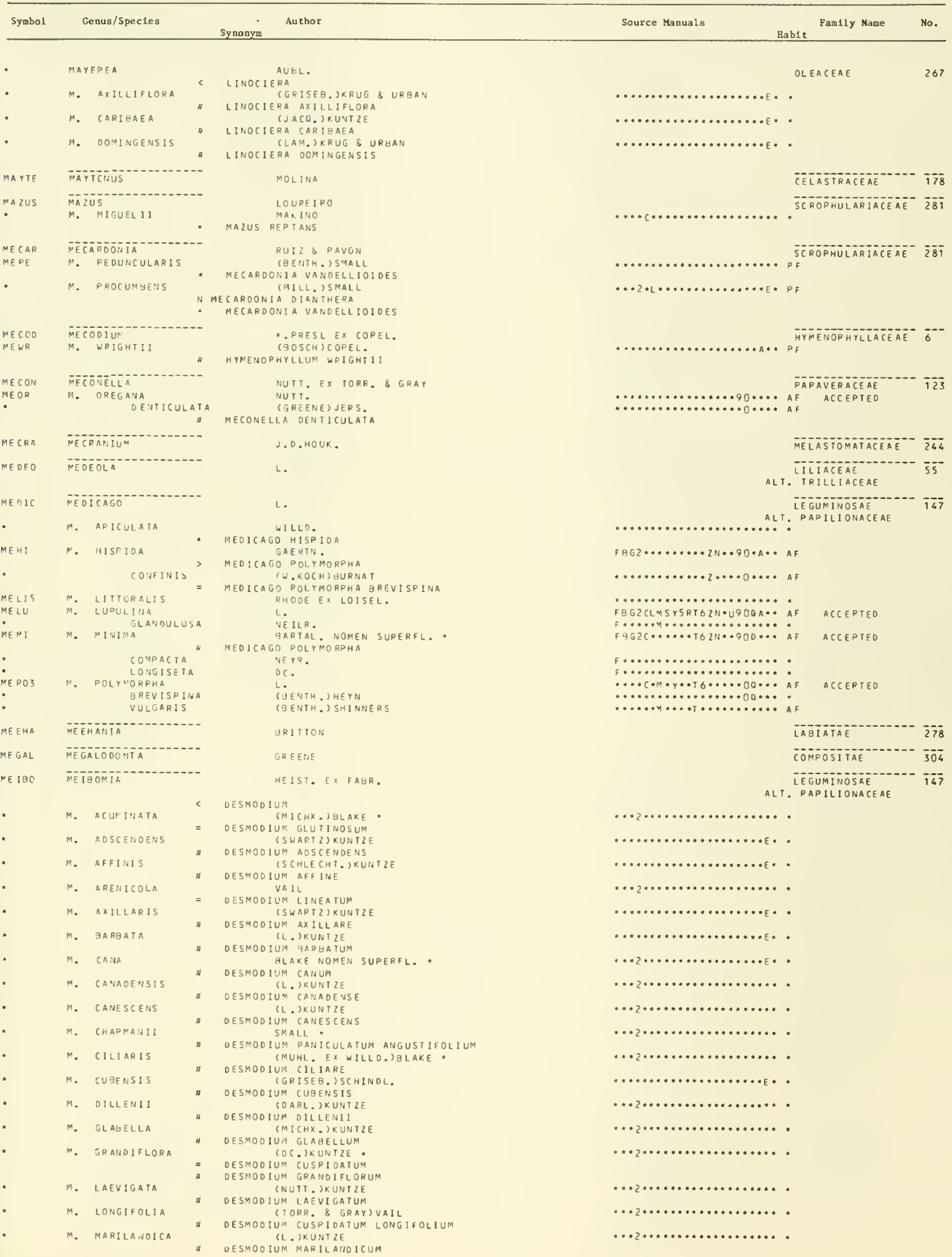




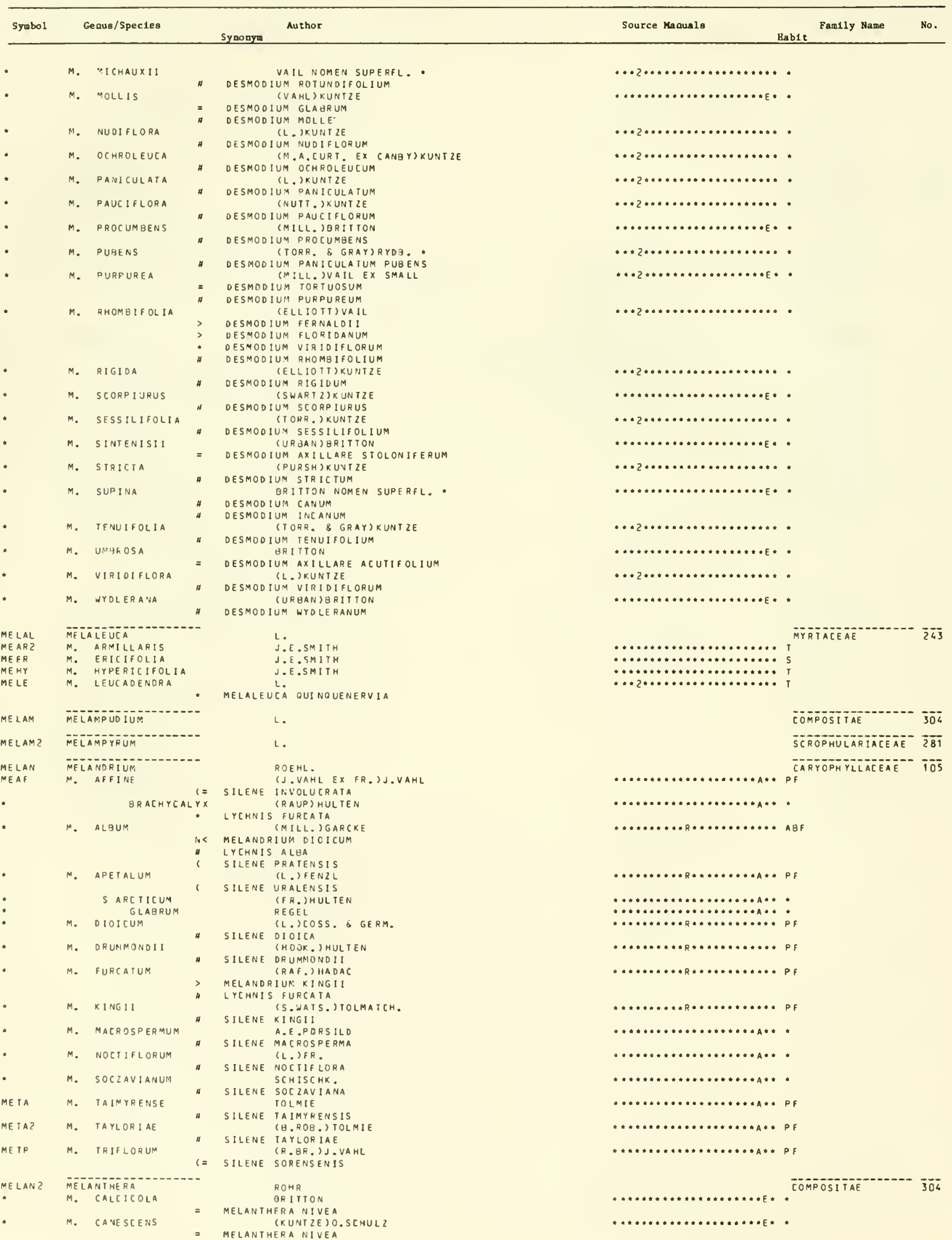




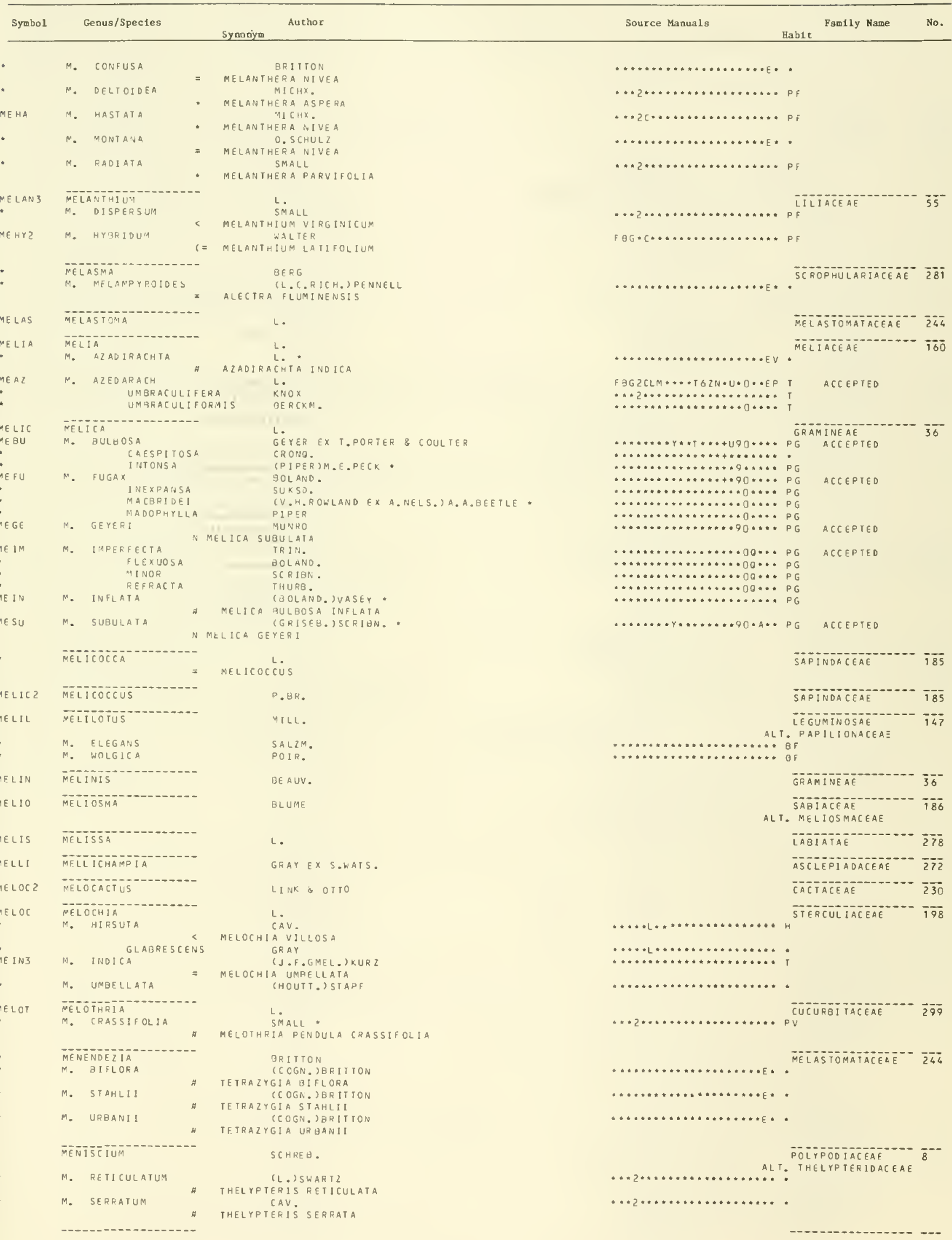




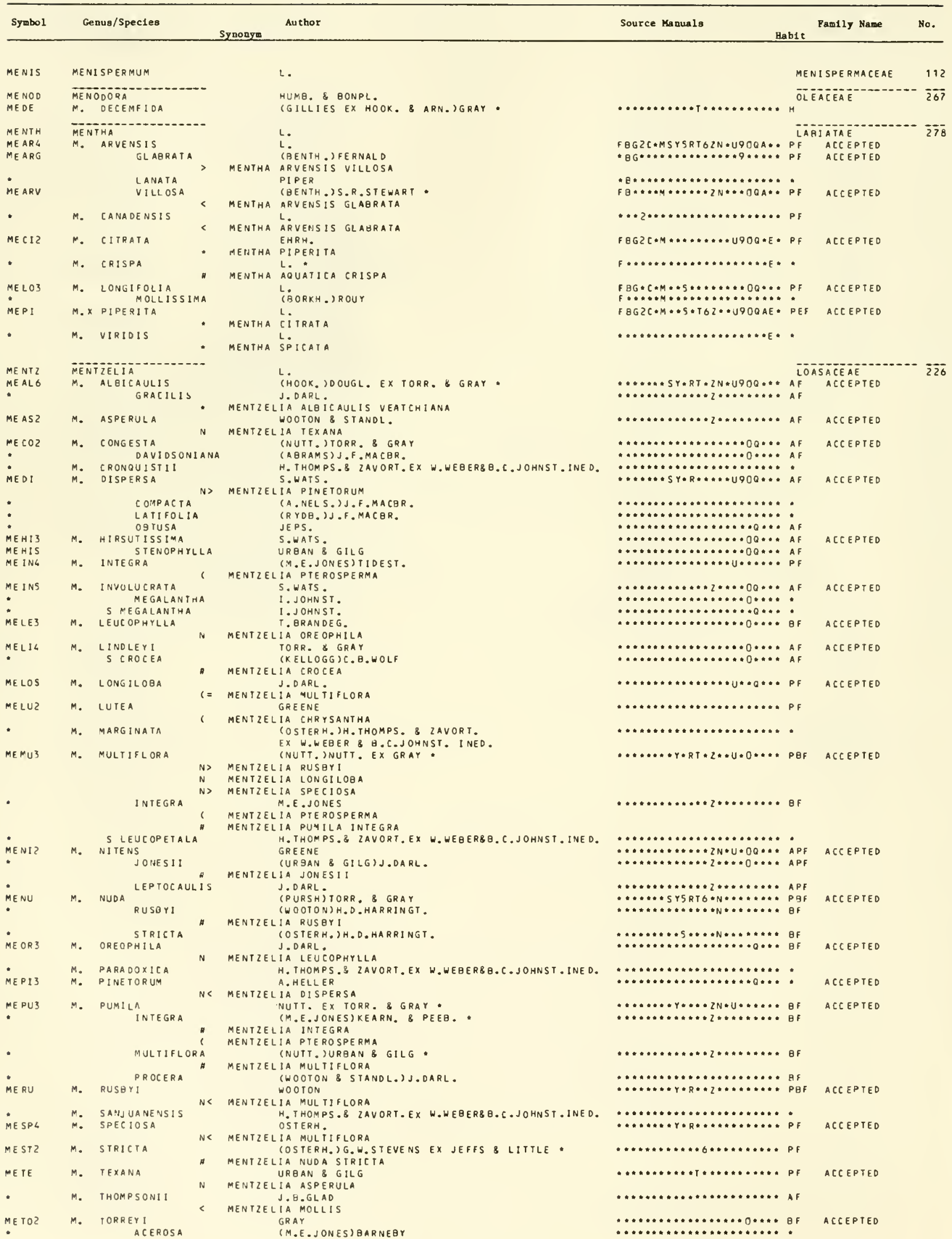


MENT ZELIA

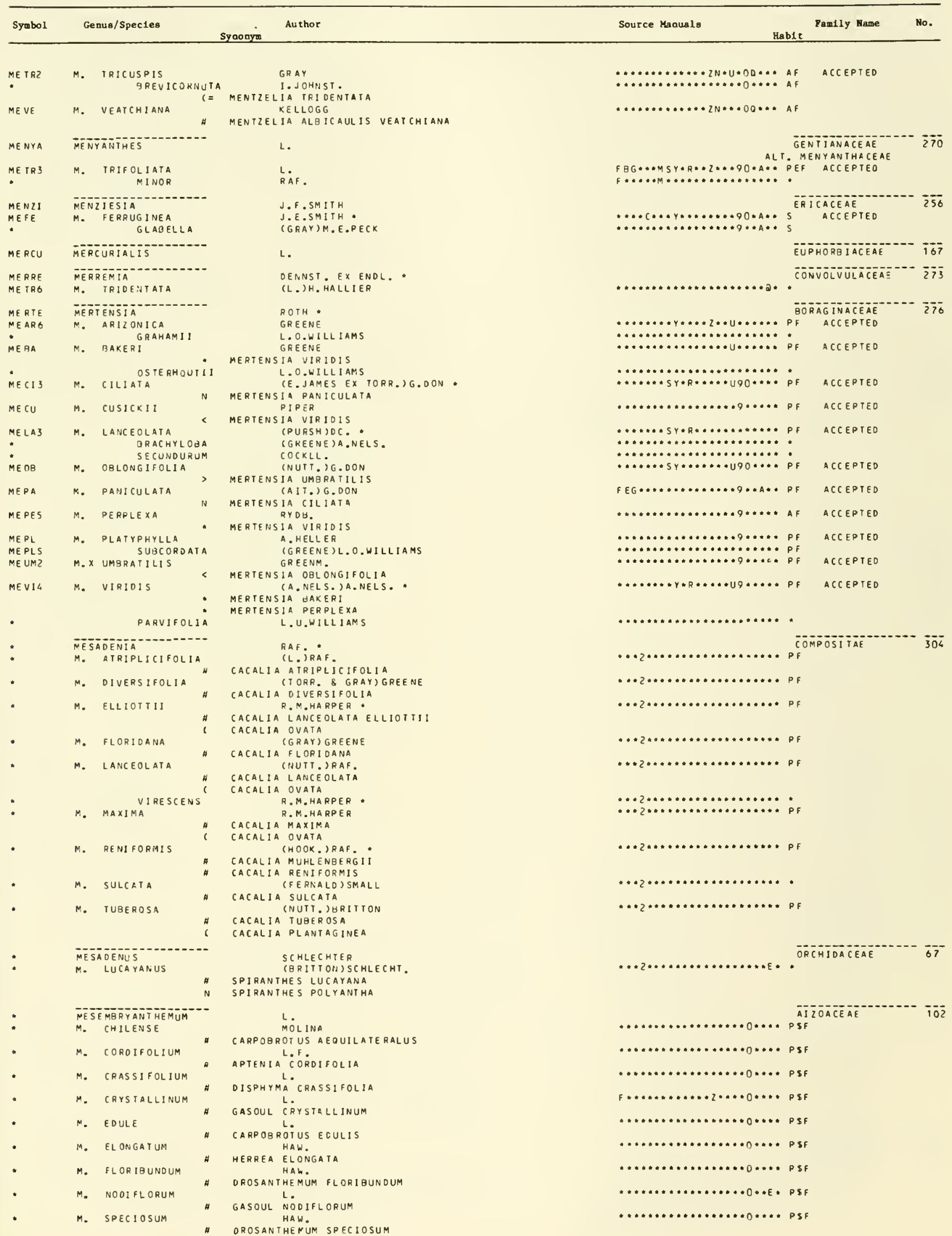




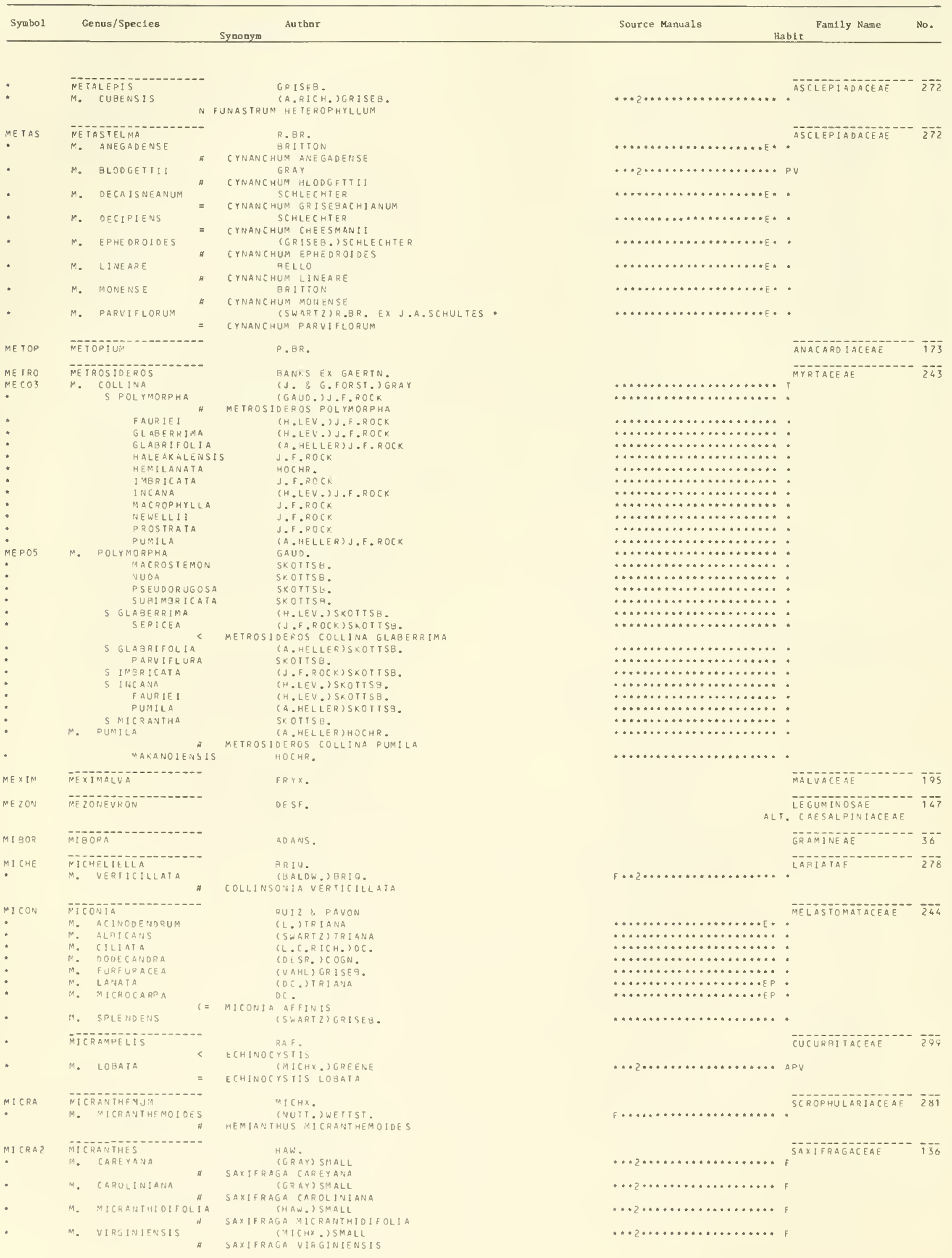




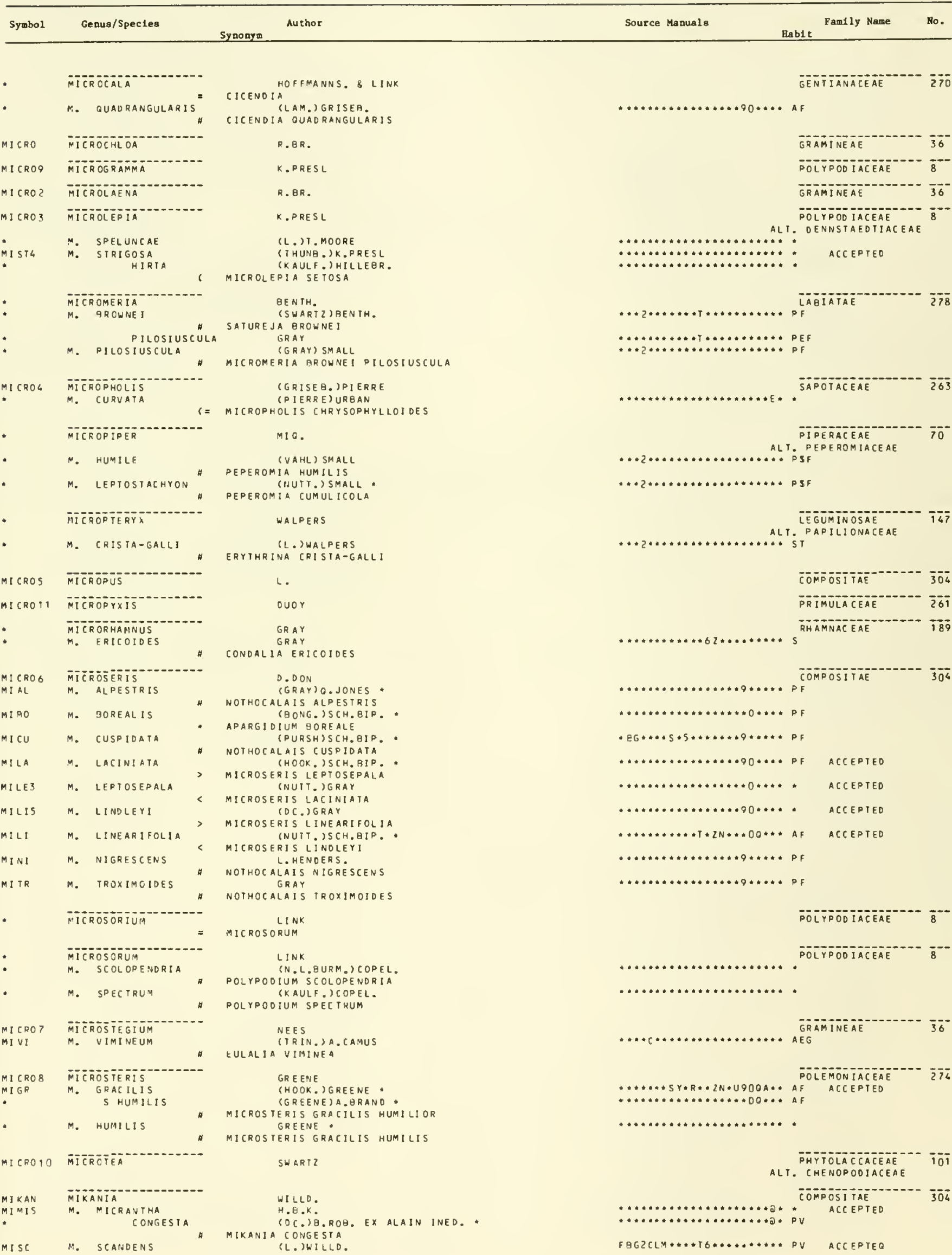




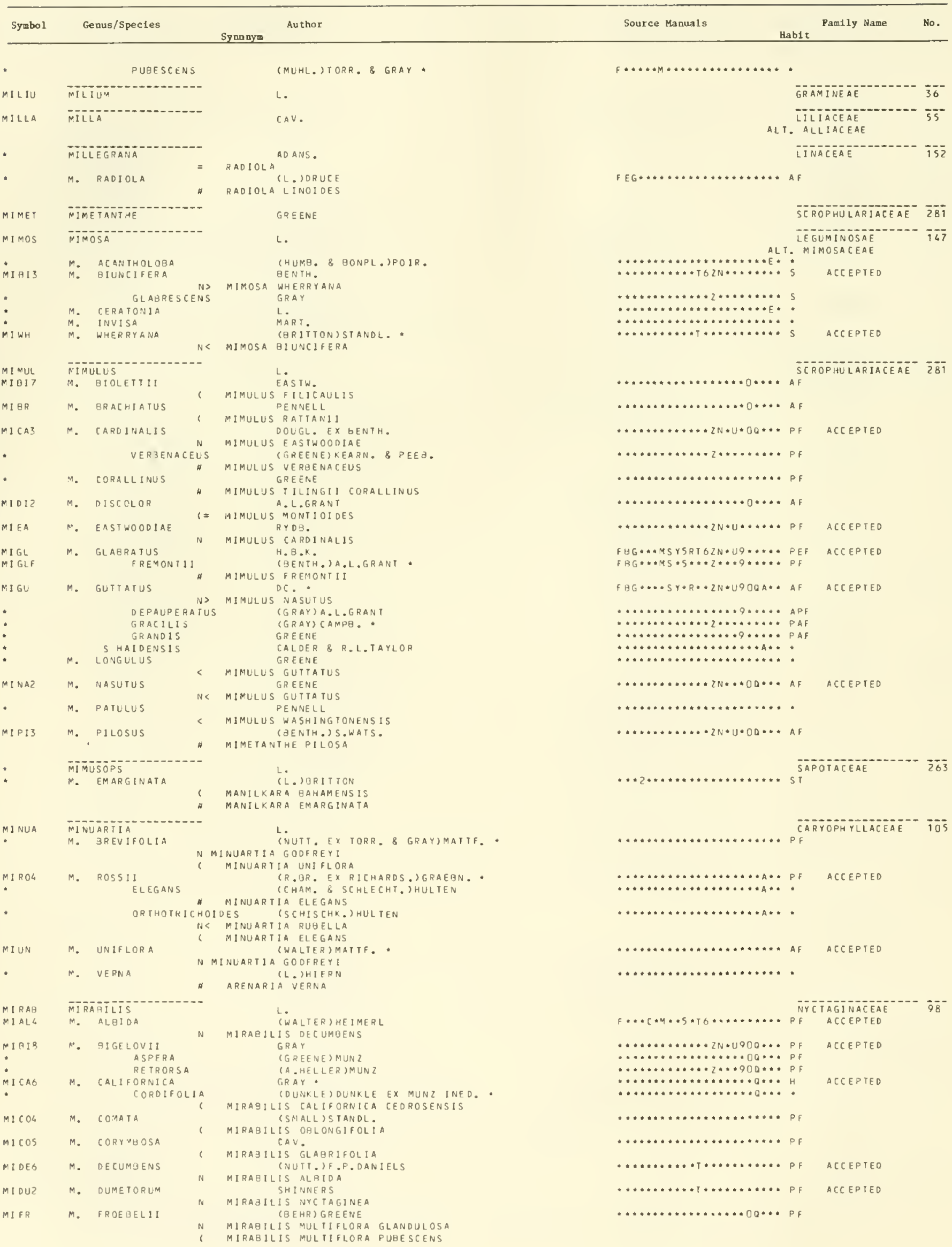




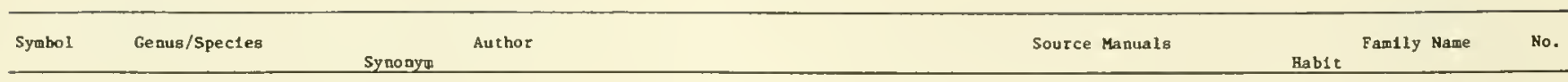

GLAGRATA

\begin{tabular}{|c|c|c|}
\hline HI & M. & HIRSUIA \\
\hline LAG & M. & $\begin{array}{l}\text { LAEVIS } \\
\text { CEOROSENS! } \\
\text { COQDIFOLIA }\end{array}$ \\
\hline 113 & M. & LINEAR IS N \\
\hline 02 & M. & $\begin{array}{l}\text { SUIBHISPIOA } \\
\text { LONG:FLORA } \\
\text { WRIGHTIANA }\end{array}$ \\
\hline & M. & NYCTAGINEA \\
\hline
\end{tabular}

MINY M. NYTAGINEA

MISANTECA

M. TRIANDRA

PISTANTHUS

MITUFLLA

MITELLA

M. GRANDIFLCRA

M. STENOPETALA

MITRACARPUM

MITRACARPUS
M. FRIGIDUS

M. HIRTUS

M. PORTORICENSIS

Mitropsioinm

M. SINTENIS:

MODIOLA

NOEHRINUTA

NOENCHIA

MOHAVEA

MOLDAVICA

". PARVIFLORA

M. THYMIFLORA

MOLINERIA

M. HORTENSIS

MOLINIA

$=$

MOLLUGO

MOLUCCELLA

MOLUCELLA

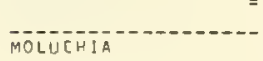

M. PYRAMIDATA

M. TOMENTOSA

MŌMISIA

M. IGUANAEA

M. PALLIDA

MOMORDICA

MONANTHOCHLOE

MONARDA

M. AUSTROMONTANA

*. aradouriana
(STANDL.) JEPS.

MIRAEILIS MULTIFLORA PUBESCENS

MIRAGILIS LINEARIS

(OENTH.) M.CURRAN.

MIRABILIS CALIFORNICA

(SI ANDL.) YUNZ

DUNKLE

(PURSH)HEIMERL *

MIRABILIS HIRSUTA

HE IMEK L

L.

(GRAY EX BRITTON \& KEARN.) KEARN. \& PEEB. (MICHX.) MACMIL.

MIRABILIS DUMETORUM

SCHLECHT. 8 CHAM.

(SWART Z) ME 2

LICARIA IRIANDRA

ANDERSS.

L.

L.

PURSH

TELLIMA GRAND IFLORA

PIPER

MITELLA STAUROPETALA STENOPETALA

ZUCCAR.

MIIRACARPUS

ZUCCAR. EX J.A. \& J.H.SCHULTES

(WILLD. EX ROEM. \& J.A.SCHULTES)K.SCHUM. (L.) OC.

MITRACARPUS ILLOSUS

(URGAN)URAAN

MITRACARPUS FRIGIDUS PORTORICENSIS

ZU RRE T

(KIAERSK.) BURRET

PSIOIUA SINTENISII

MOENCH

L.

EHRH.

GRAY

FABR

PHALU

(NUIT.) HRITTON

ORACOCEPHALUM PARVIFLORUM

(L.) RYDQ.

DRACOCEPHALUM THYMIFLORUM

COLLA

GR ITTON

CURCULIGO CAPITULATA

SCHRANK

L.

L.

MOLUCEELLA

MEDIK.

MELOCHIA

(L. .) ORITTON

MELOCHIA PYRAMIDAT

(L.) IGRITTON

MELOCHIA TOMENTOSA

F. DIETR.

(JACO, ROSE \& STANDL.

CELTIS IGUAHAEA

(TORR.)PLANEH.

CELTIS PALLIOA

L.

E:UGELN.

$\mathrm{L}$.

CI IRI ODORA AUSTROMONTANA

L. C.GECK.

LUSSELIANA [NUTT. EX SIMS]

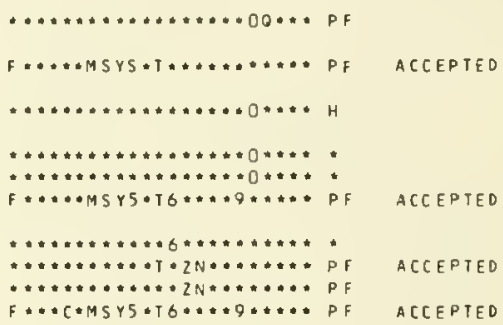

IAURACFAE

$12 \overline{1}$

GRAMINEAE 36

RUBIACEAE

SAXIFRAGACEAE 136

PF

RUBIACEAE

RUBIACEAE

MALVAGEAE

CÄRYOPHYLLACEAE

CARYOPHYLLACEAE

SCROPHULARIACEAE ZBं

LAGIATAE

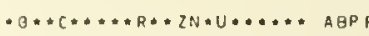

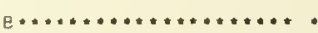

AMARYLLIDACEAE ALT. HYPOXIDACEAE 7 ALT:

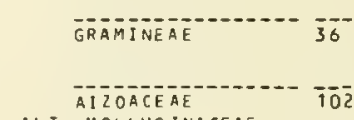

ALT. MOLLUG INACEAE

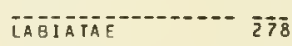

LAGIATAE

STEREUL IACEAEE
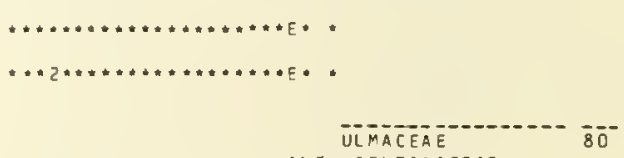

ALT. CELTIDACEAE
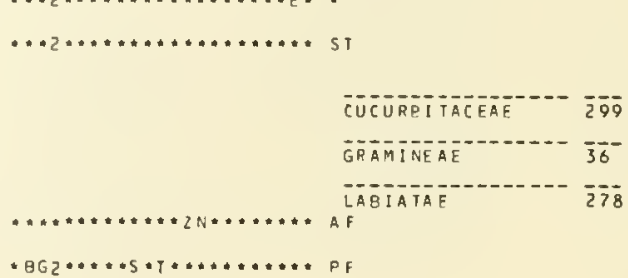


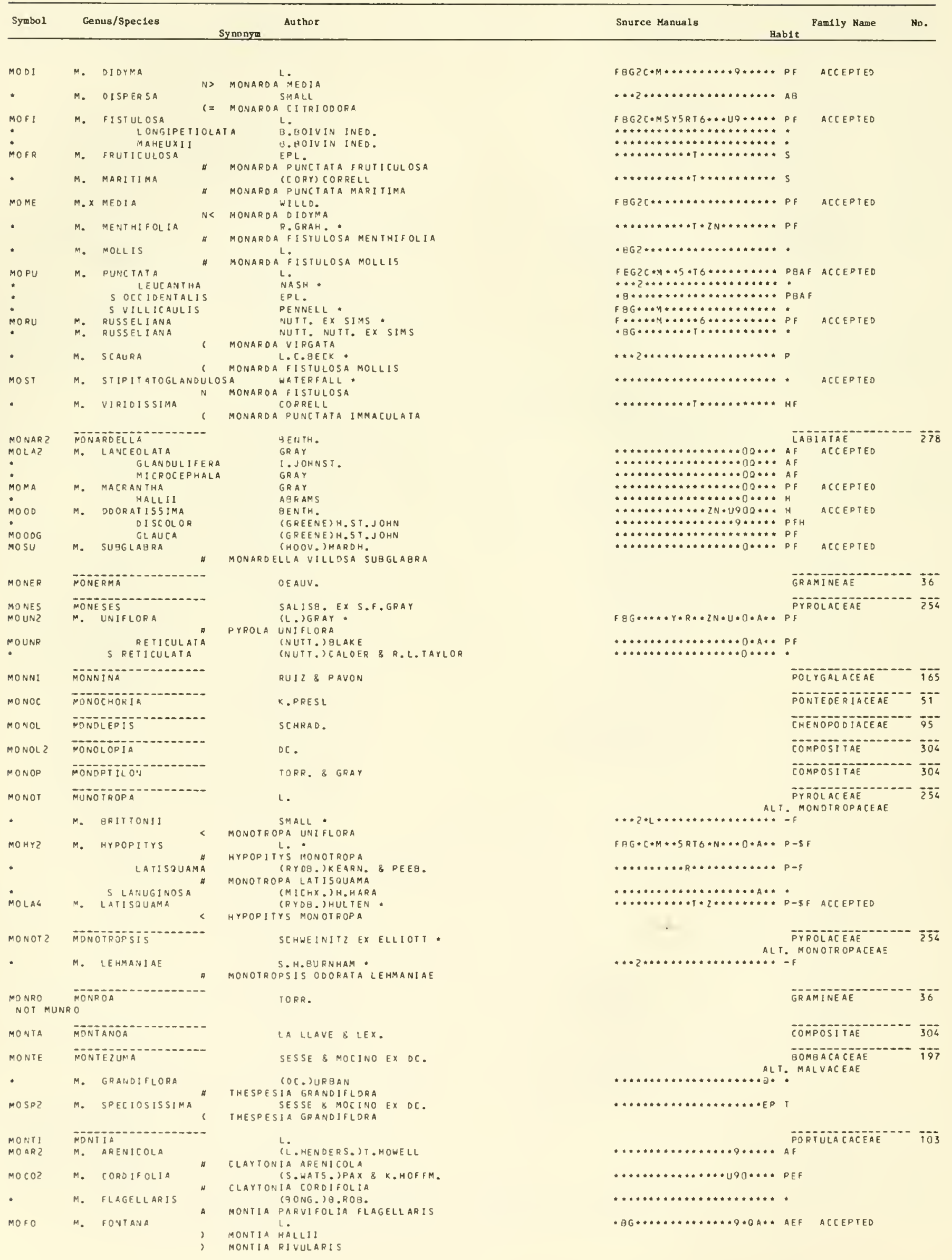




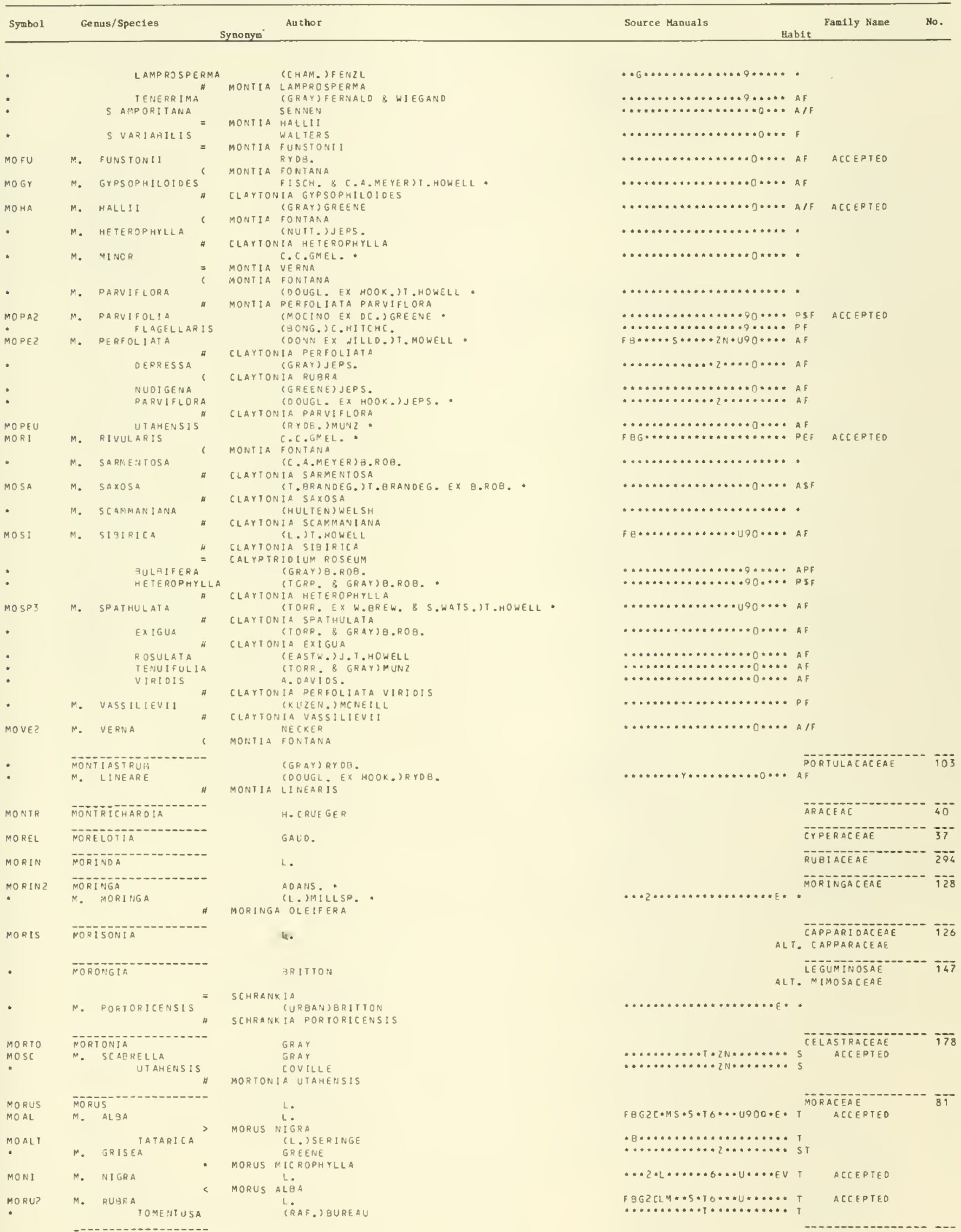




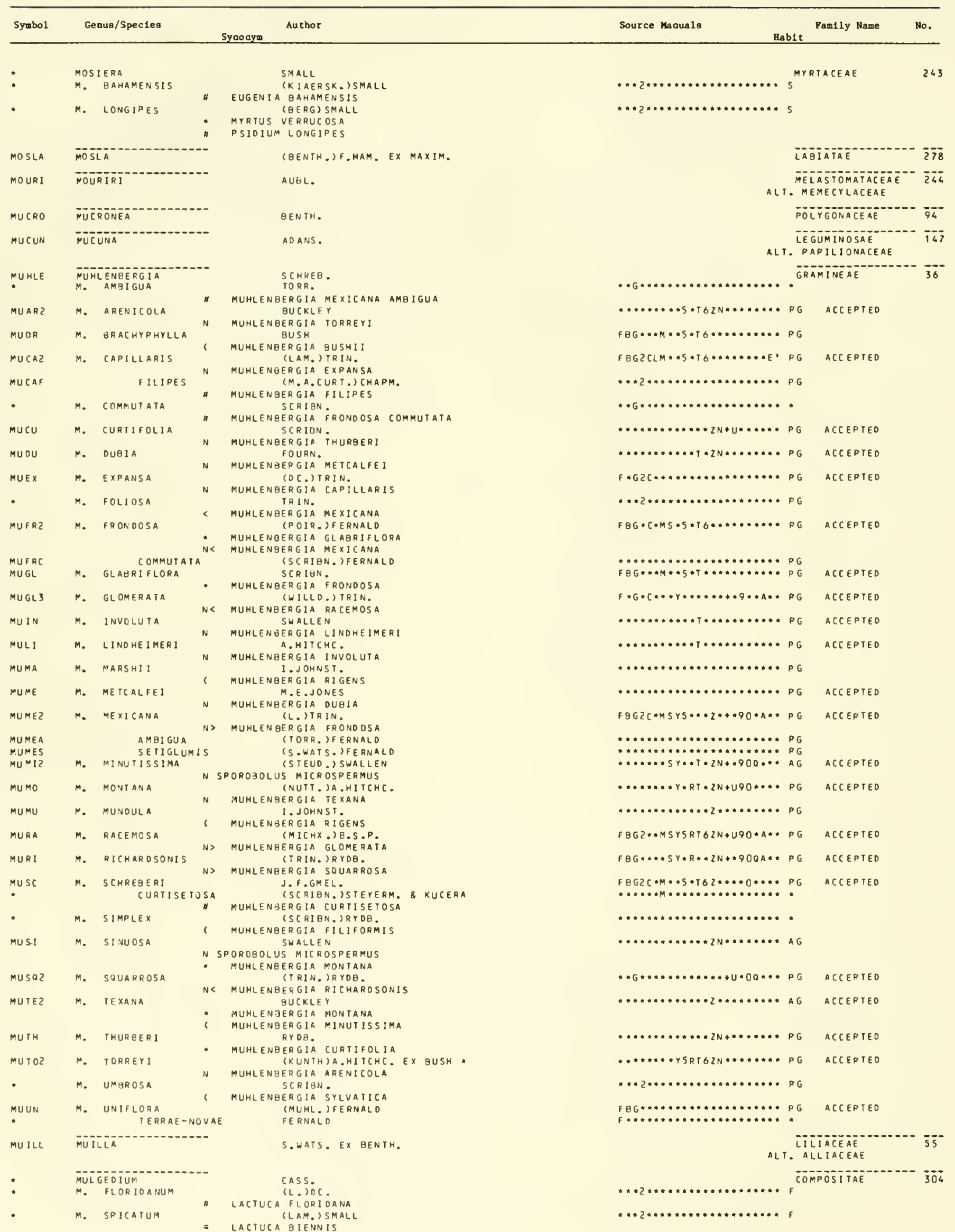




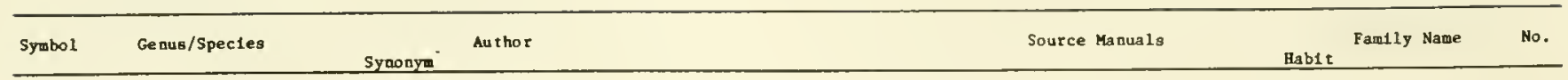

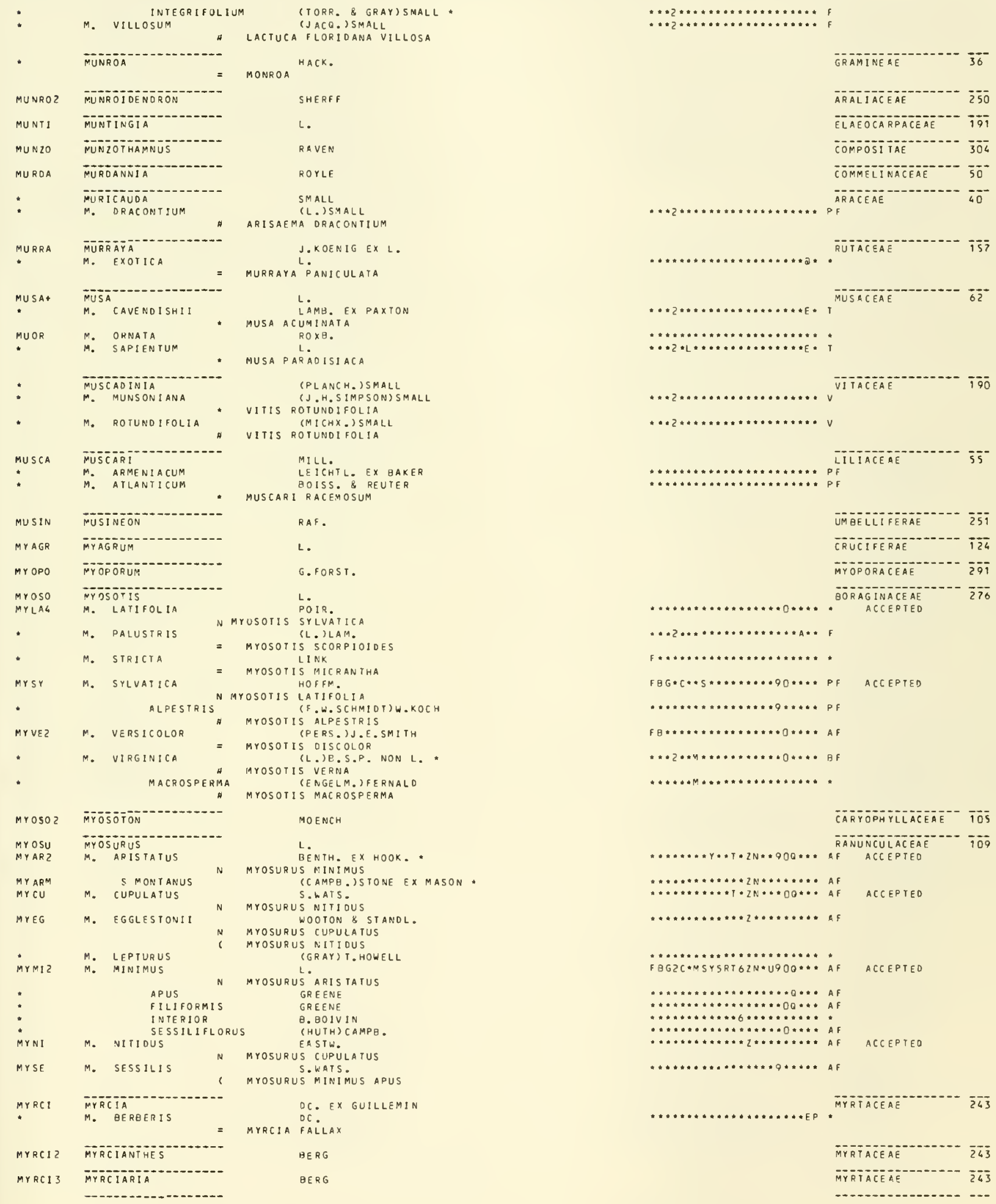




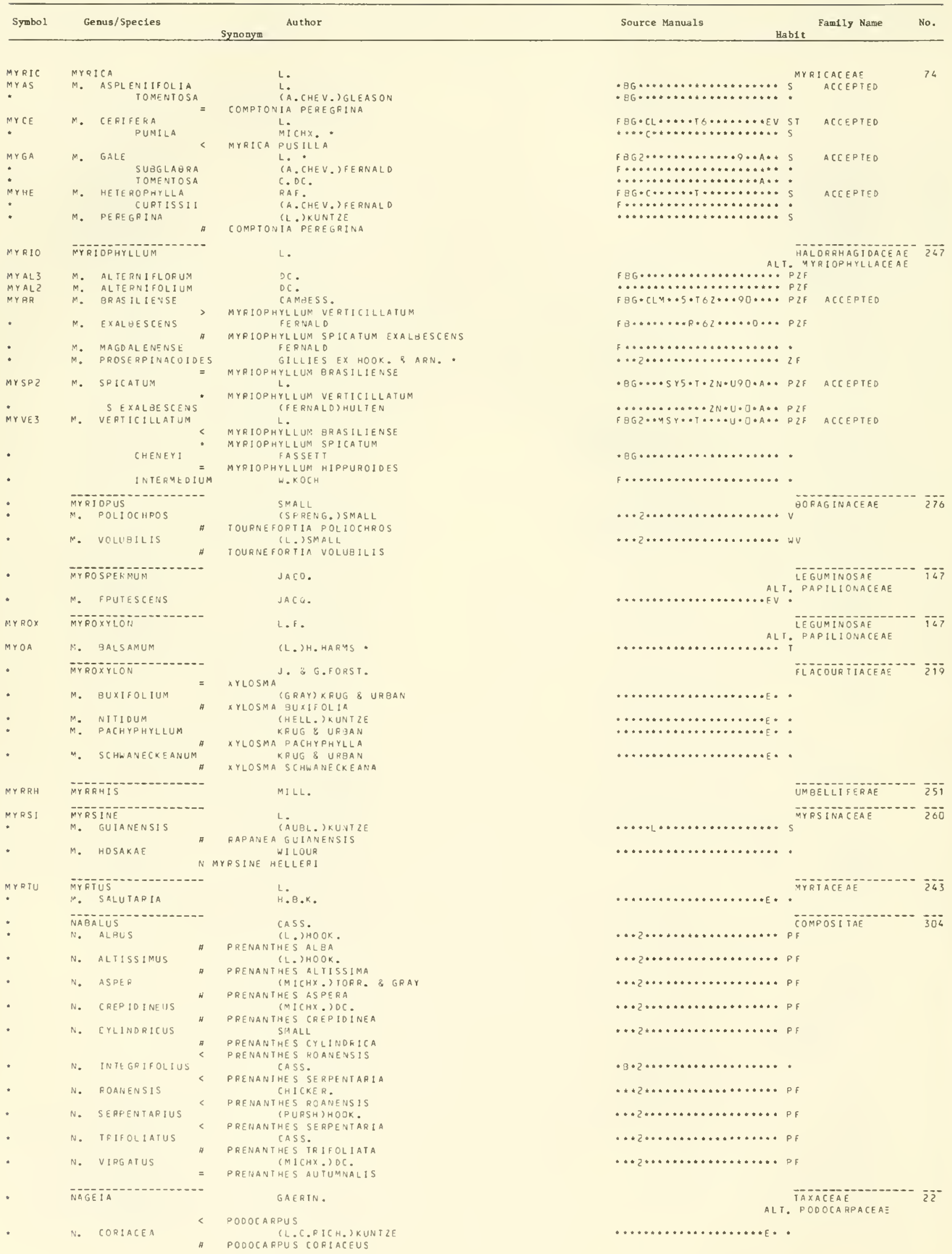




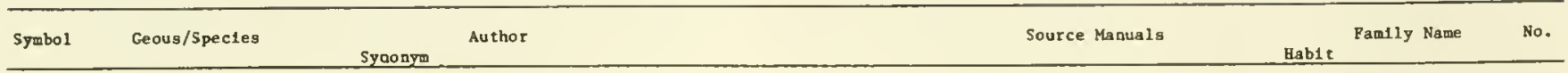

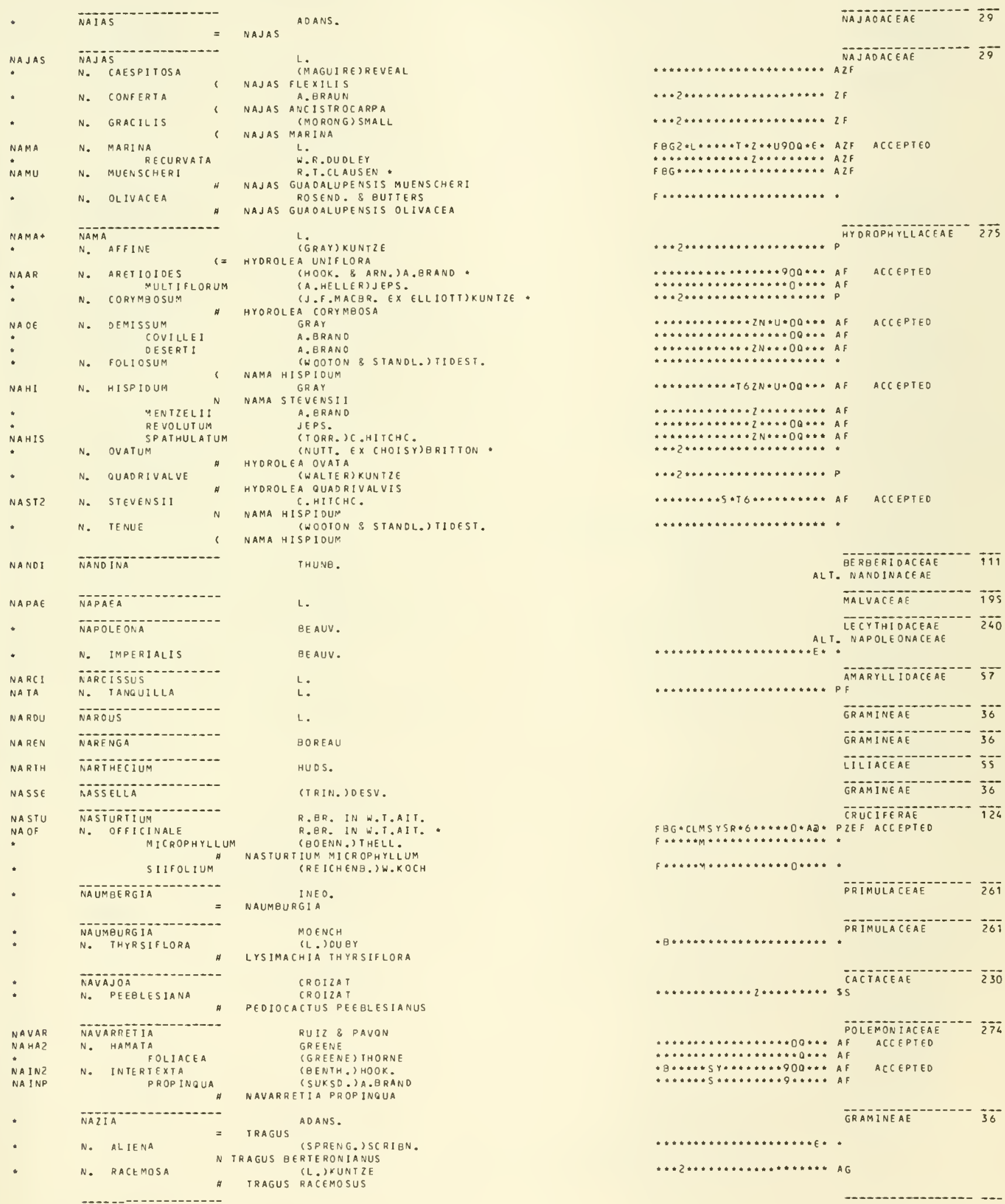




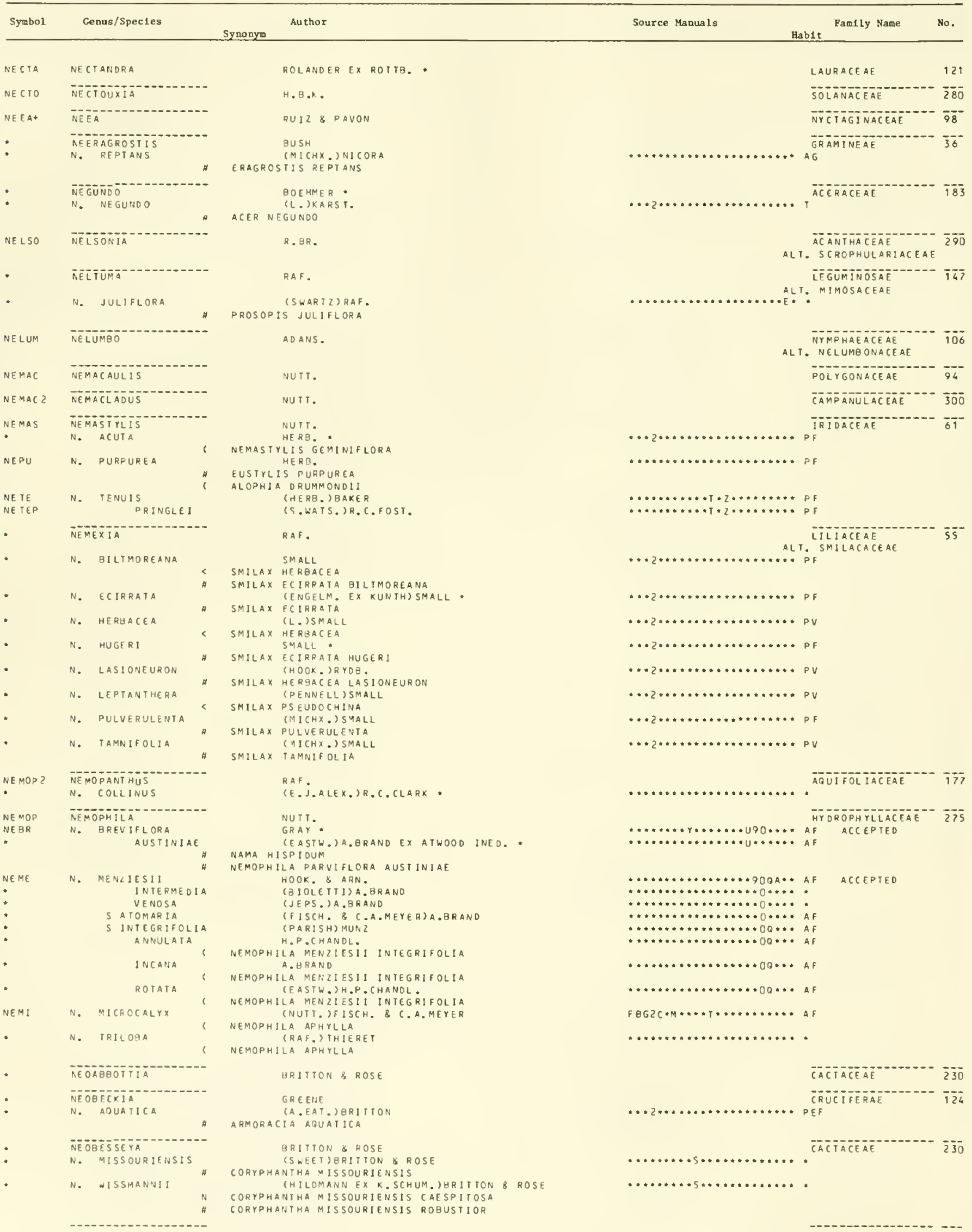




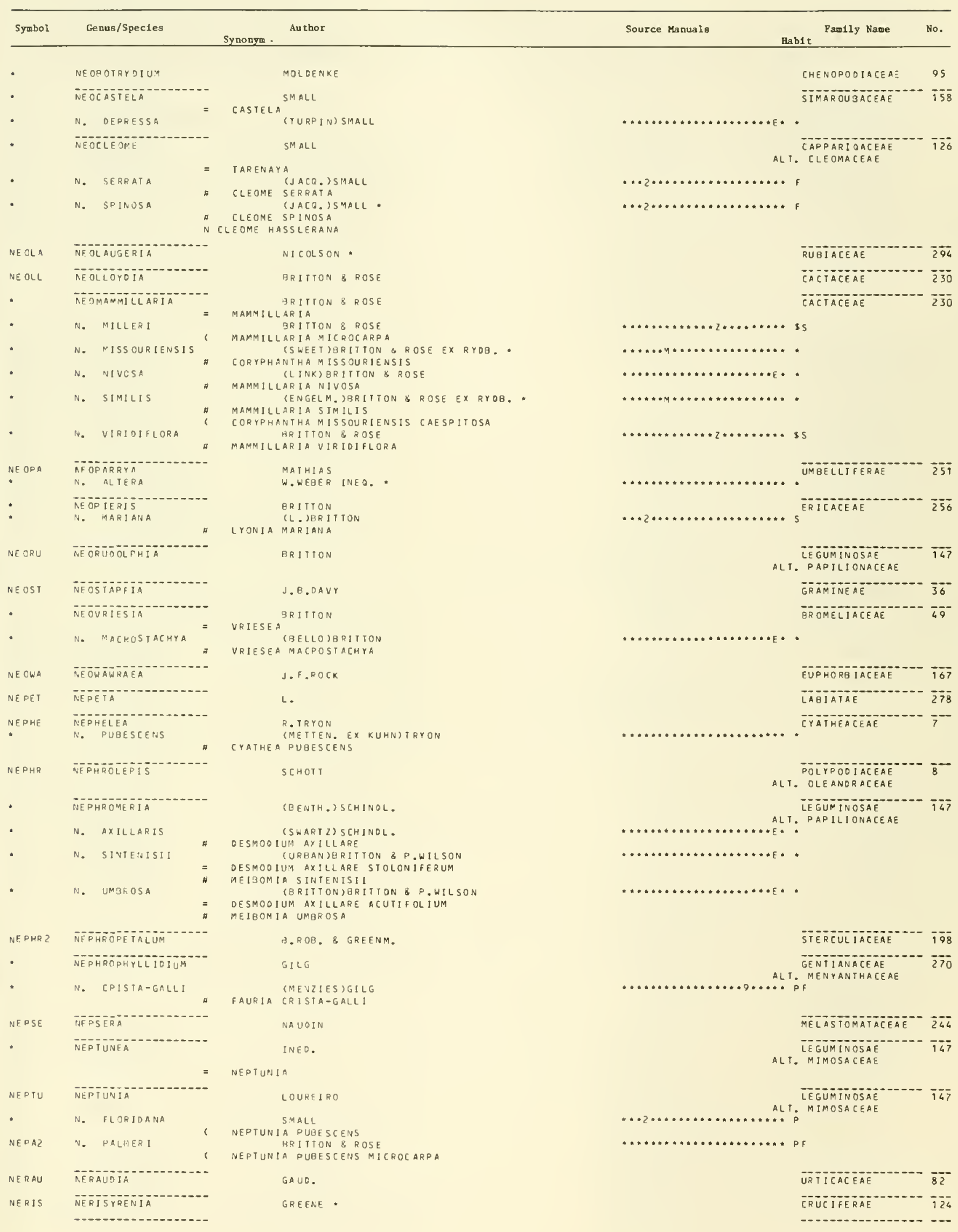




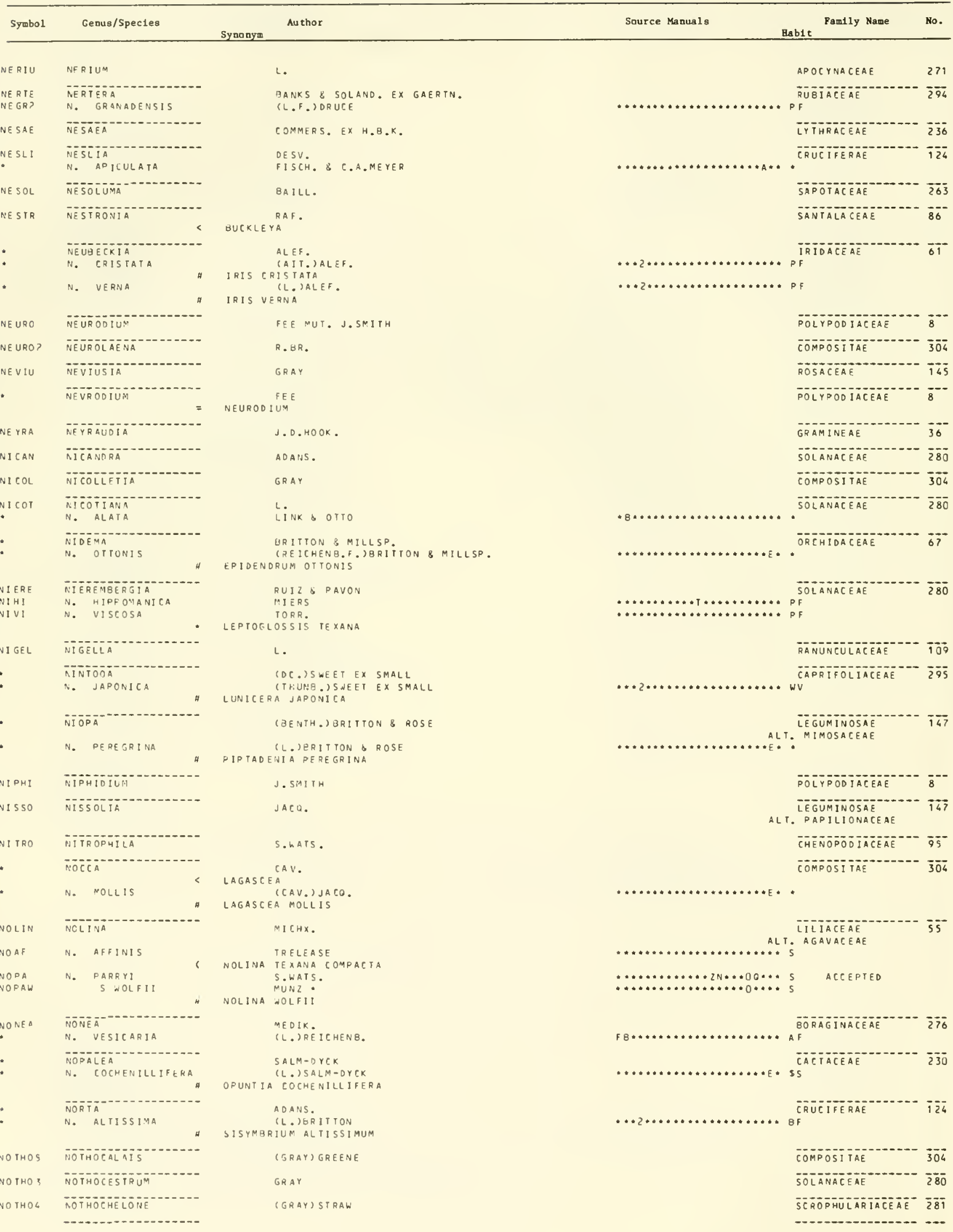




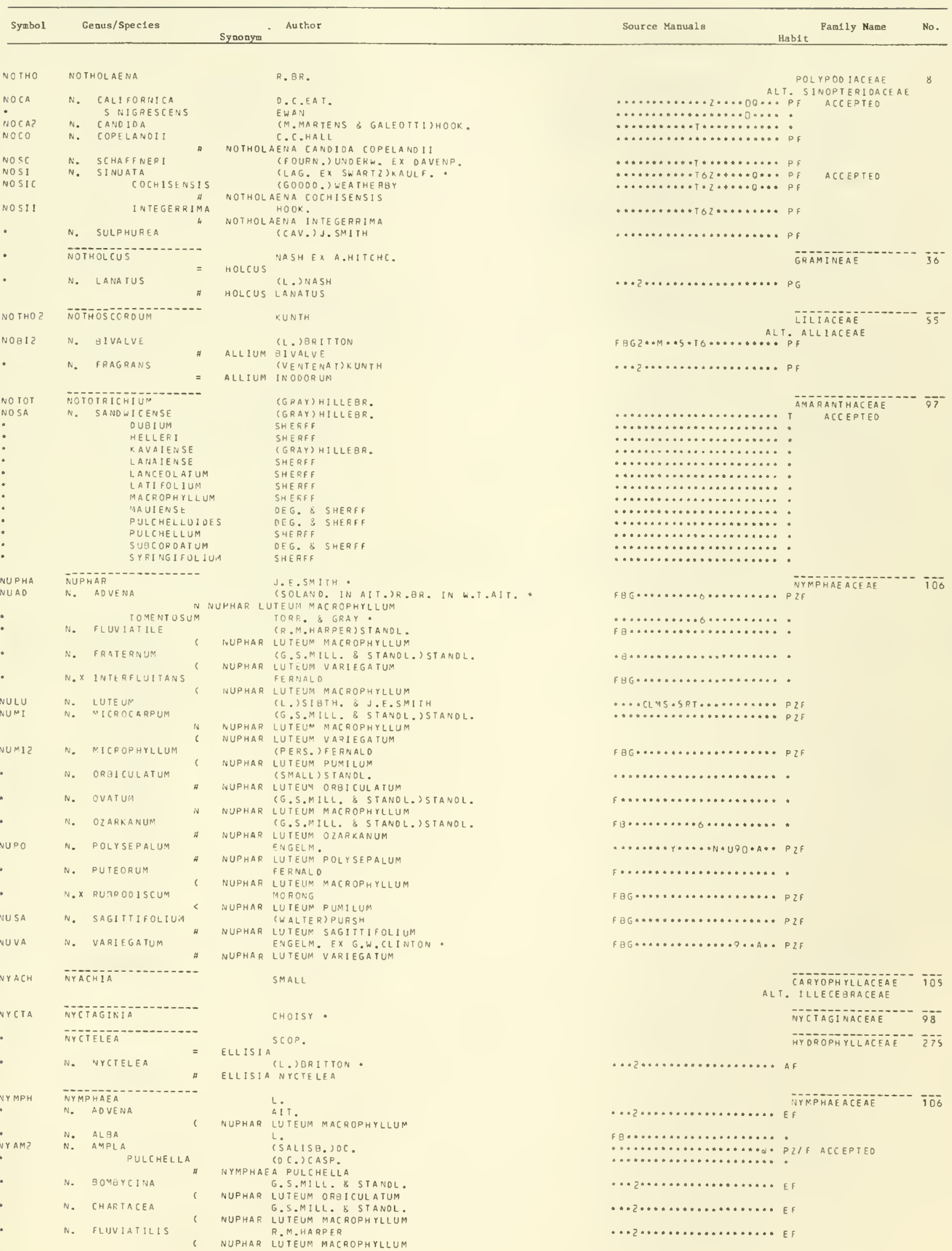




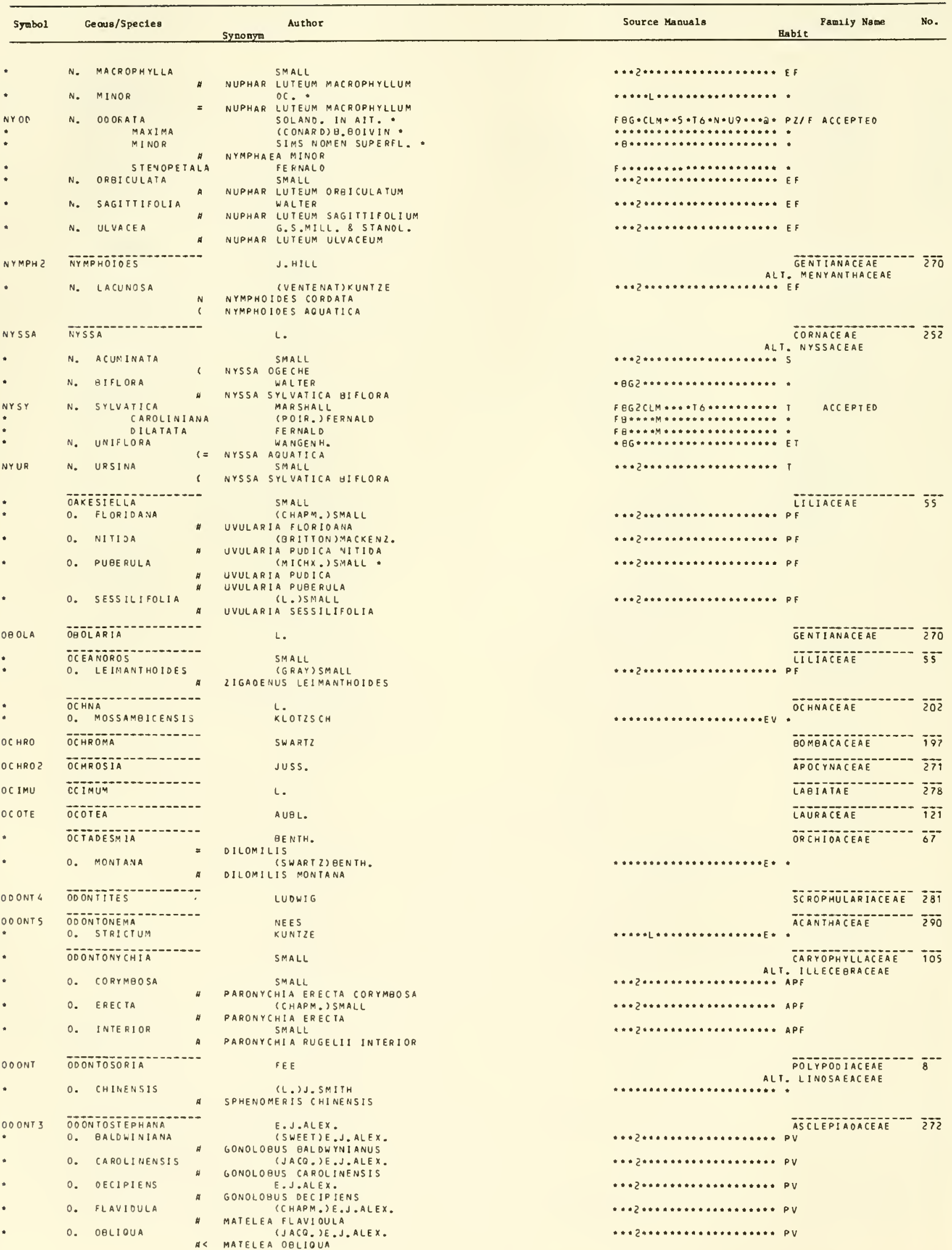


OD ON TOSTEPHANA

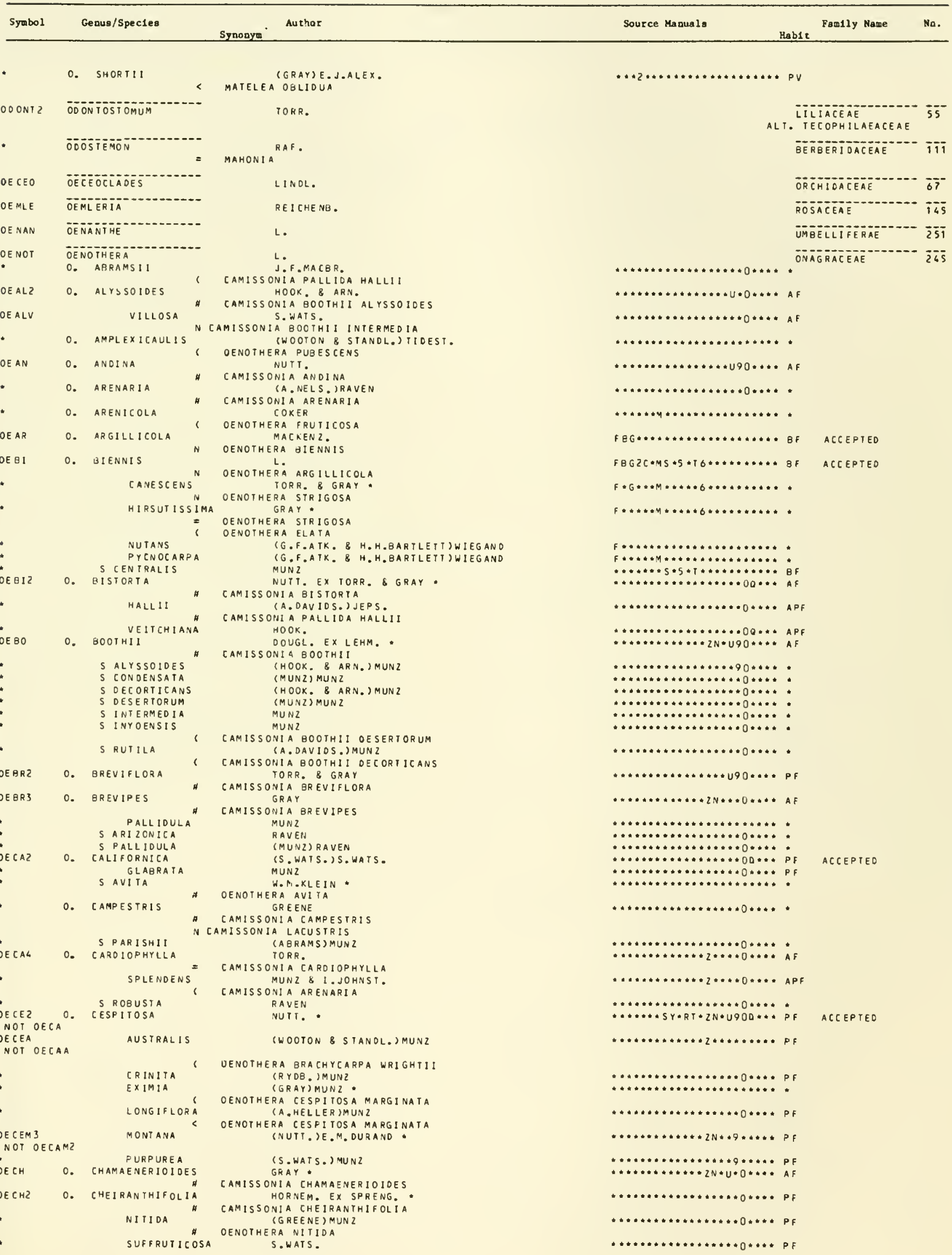




\begin{tabular}{|c|c|c|c|}
\hline Symbol & \multicolumn{3}{|c|}{ Genus/Spectes } \\
\hline - & \multicolumn{3}{|c|}{ S SUFFRUTICODA } \\
\hline OE EL & 0. & & VIFORMIS \\
\hline - & & & $A \cup R A N I I A C A$ \\
\hline - & & & EITRINA \\
\hline • & & & CRUCIFORMI \\
\hline - & & & PEEBLESII \\
\hline - & & & PEIRSON II \\
\hline • & & & RURPURASCE \\
\hline - & & & AURANTIACA \\
\hline - & & & CRUEIFORMIS \\
\hline 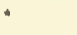 & & $s$ & INTEGRIDR, \\
\hline - & & $\mathrm{s}$ & LANCIFOLIA \\
\hline - & & s & PEIRSONII \\
\hline • & & $s$ & YUMAE \\
\hline OECO & & CON & ITORTA \\
\hline
\end{tabular}

$$
\begin{aligned}
& \text { RAVE: } \\
& \text { DOUGL. EX LEHM. } \\
& \text { UMAETA } \\
& \text { ERILOBIOIDES EAMISSONIA EONTORTA }
\end{aligned}
$$$$
\text { IS. WA IS. IMUNZ }
$$$$
\text { GAMISSONIA GL }
$$$$
\text { HUW }
$$$$
\text { CAMISSONI A CLAVIFORMIS FUNEREA }
$$$$
\text { (RAVEN)RAVEN EX A. HOLMGR. \& REVEAL INEO. }
$$$$
\text { CAMISSONIA CLAVIFORMIS GRUGIFORMIS }
$$$$
\text { SHELLOGG)MUNZ }
$$$$
\text { CAMISSONIA CLAVIFORMIS LANCIFOLIA }
$$$$
\text { MUNZ }
$$$$
\text { SUNZ }
$$$$
\text { CAMISSONIA LLAVIFORMIS INTEGRIOR }
$$$$
\text { EAMISSONIA ELAVIFORMIS CRUELFORMIS }
$$$$
\text { (MUNZ) RAVEN . }
$$$$
\text { (KELLOGG)RAVEN }
$$$$
\text { RAVEN }
$$$$
\text { OENOTHERA CLAVIFORMIS PURPURASCENS }
$$$$
\text { (A. HELLER)RAVEN }
$$$$
\text { (MUNZ) RAVEN }
$$$$
\text { EAMISSONIA STRIGULOSA }
$$$$
\text { (4 CAMISSONIA LACUSTRIS }
$$$$
\text { N CAMISSONIA DEN IATA }
$$

N) CAMISSOHIA PUSILLA

PUBENS CAMISSONIA PARVULA
(S.WATS.]LOVILLE

A CAMISSONIA PUBENS
STRIGULUSA ISCH. 3 E. A. MEYER)MUNZ

C. CRUCIATA CAMISSOHIA STR

I A SIRIGULOSA

NS OENOTHERA PARVIFLORA

SABULONEISIS CAMISSONIA CON

' OENOTHERA PARVIFLORA

STENOPETALA (BICKN.) FERNALD

D. ETENOPHYLLA

DENOTHERA PARVIFLORA

(WOOTON S STANOL.) TIDEST.

OEDF O. DECORTICANS

OENOTHERA ALB ICAULIS

(HOOK. \& ARN.) GREENE

* CONDENSATÁ
DESERTORUM

OE SERTORUM

D. DELESSERTIANA

- DELTOIDES

ARIZONIEA

CI NERACEA

COGNATA

DEEUMBENS

HOWELLIL

HOWELL

S EURFKENSIS

OEDES

D. DFUTATA

CAMISSONIA DOOTHII OECORTILANS

$$
\text { MUNZ }
$$

MUNZ

(A. OAVIOS.) MUNZ

STEUD.

OENOTHERA SPE CIOSA CHILDSII

TORR. S FREM. .

MUNI

OENOTHERA AVI TA ARIZONICA

(JEPS.)MUNZ

(JEPS.)MUVZ .

(S. WATS. IMUNZ

MUNL.

MUNZ" ROOS

OENOTHERA AVI TA EUREKENSIS CAV.

CAMISSONL A DENTATA
CAMISSON:A STRIGULOSA

N CAMISSONIA CONTORTA

OEDEC

CAMPESIRIS

IA CONTORTA

EAMISSONIA LACUSTRIS

CAMISSONIA CAMPESTRIS

GILMANII

JOHNSTONII

PARISHII

O. DEPRESSA

0. EASThOUDIAE

OEFR

0. X ERYTHROSEPALA

Q. FF NOLER

o. fillifolia

OEFR O. FRUTILOSA

* eamesi.

GOOOMAIS II

HUMI FUSA

LINEAR IS

MII CR OCARPA

UNGUICULATA

$O E G R$

OE GR 3

0. GNACILIFLORA

OE GRL

OE GRP

o. GREGGII

[1UNZ

CAMISSONIA KERHENSIS GILMANII $M \cup N 2$

CAMISSONIA KERNENSIS GILMANII

(AORAMS) MUNZ

UENOTHERA SIRIGOSA

OENOTHERA VILLOSA

EAMISSONIA EASTWOOOIAE

$$
\text { HOREAS }
$$

OENOTHERA GLAZI OVIANA

GRAY

CALYLOPHUS HARTWEGII FENDLER I

(EASTW.) TIDEST

CALYLOPHUS HARTWEGII FILIFOLIUS

WLAK

DLLAKE

MU N $A L L E N$

CMICHX.)S.WATS

FER RIALO

FE RNAL D

HOUK. \& ARN

CAMISSONIA GRACILIFLORA GRAY

CALYLOPHUS HARTWEGII PUBESCENS ( $U U$ CKLEY)MUNZ

LAMP ASANA

RRINGLE]

of $\{, U$

O. GUADALUPENSIS

PUU 2

CALYLOPHUS HARTWEGII MACCARTII

$$
\text { S.WATS. }
$$

CAMISSONIA GUADALUPENSIS
Source Manuals

Family Name

No.

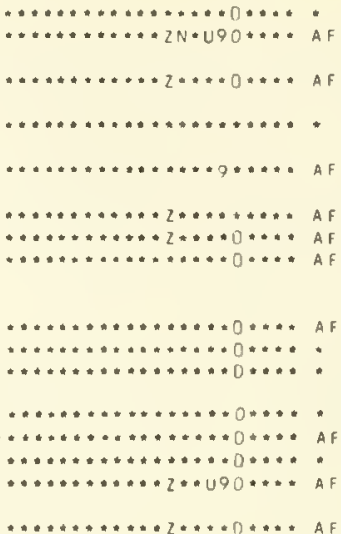

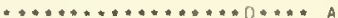

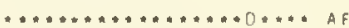

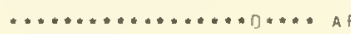

f $B G \cdots \ldots \ldots \ldots \ldots \cdots, \ldots \cdots$

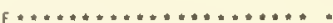

f..................

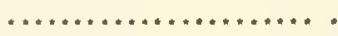

$\cdots \cdots \cdots \cdots \cdots+2 N * U \cdot O+\cdots * A F$

$\cdots \cdots \cdots \cdots+2 N \cdots 0 \cdots \cdots$ AF $\ldots \ldots \ldots \ldots \ldots \ldots \ldots+\ldots \ldots$ AF $\cdots \ldots \ldots \ldots+\ldots \ldots \ldots+0 \cdots \cdots$ AF *...****

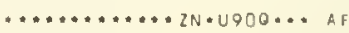
$\ldots \ldots \ldots \ldots \ldots, \ldots \ldots \ldots+\ldots$

AELEPTEO

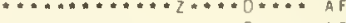

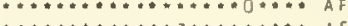

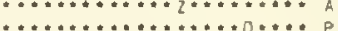

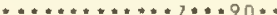
$\cdots \cdots \cdots$

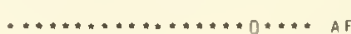

\section{AF}

A F

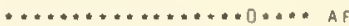

$\ldots \ldots \ldots \ldots \ldots \ldots \ldots+\ldots \ldots$

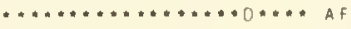
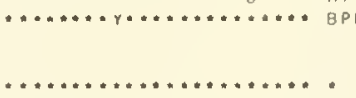

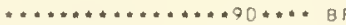

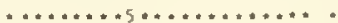

$F B G \cdot[\cdot M \ldots \ldots+\ldots \ldots \ldots \ldots+P F$

$\mathrm{F} E \ldots \ldots \ldots \ldots \ldots \ldots \ldots \ldots \ldots \ldots$

$m, \cdots \cdots \cdots$

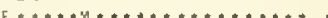

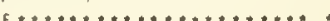

$f \ldots \ldots \ldots \ldots \ldots \ldots \ldots \ldots \ldots \ldots$

$\ldots \ldots \ldots \ldots \ldots \ldots \ldots \ldots A B$

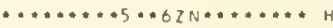

$\ldots \ldots \ldots \ldots \ldots, 2 \mathrm{n} * \ldots \ldots \ldots * \mathrm{PFH}$

$\ldots \ldots \ldots \ldots \ldots \ldots, \ldots \ldots \ldots \ldots$ 


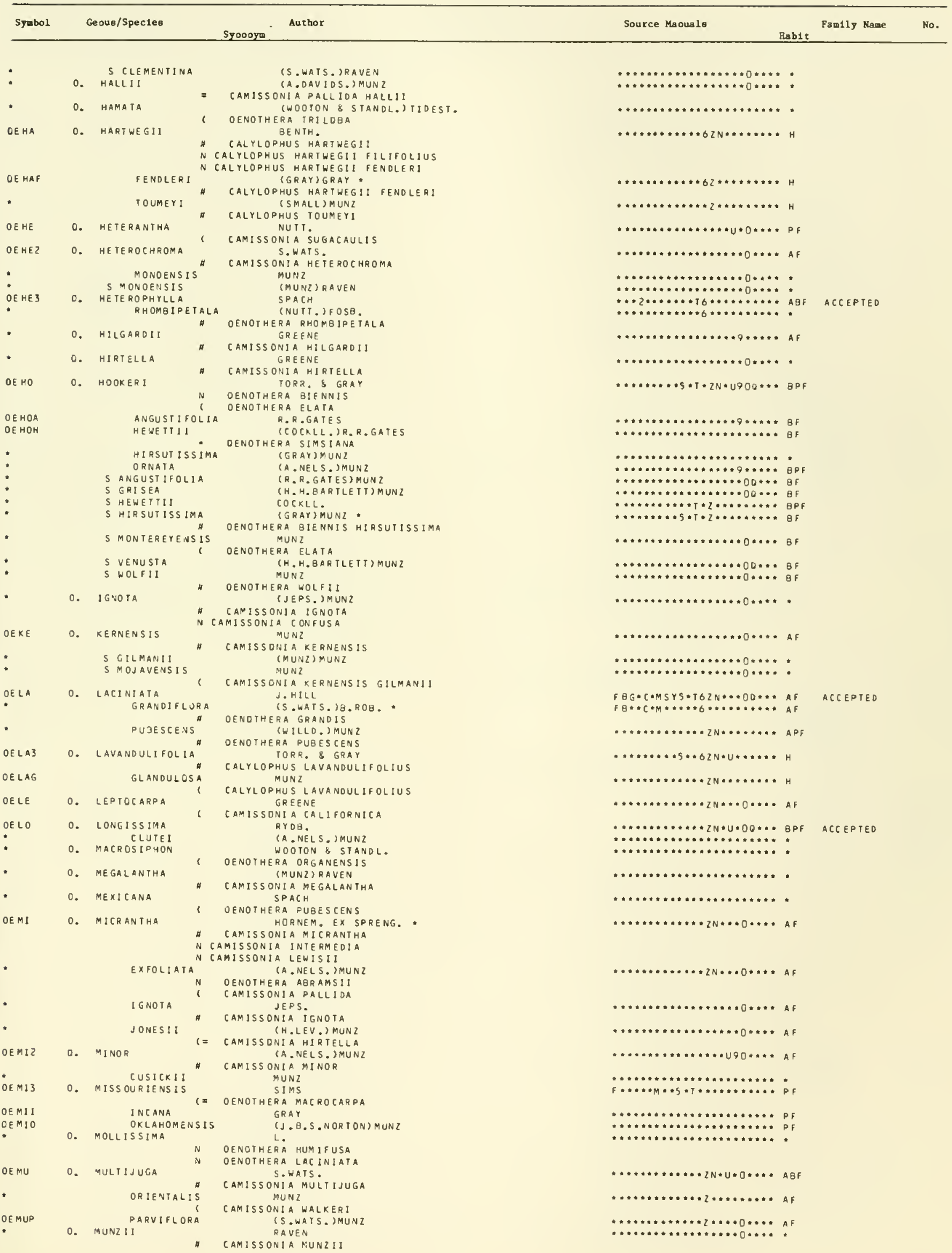




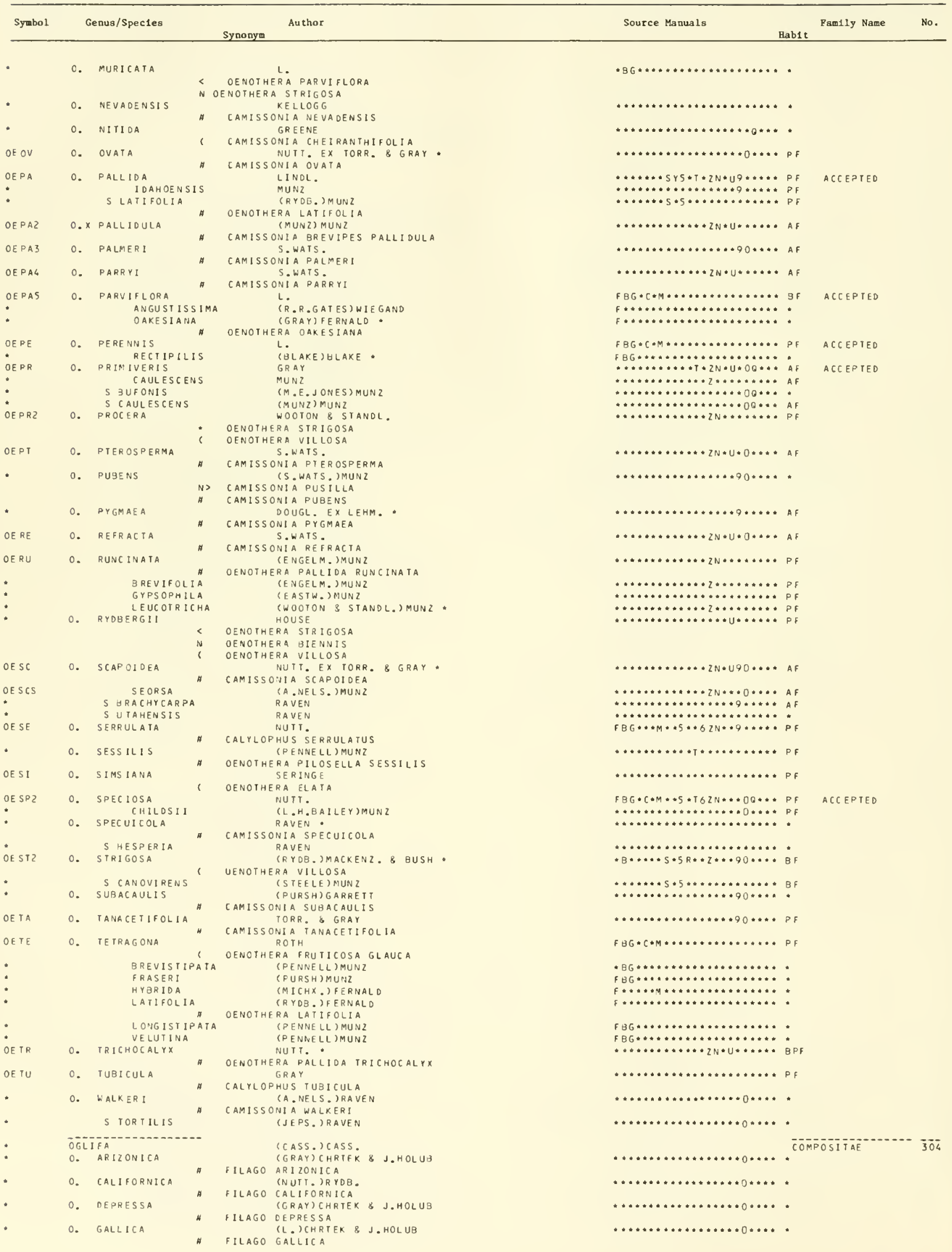




\begin{tabular}{|c|c|c|c|c|c|c|}
\hline Syrabol 1 & Genus/Spectes & $\begin{array}{l}\text { Synonym } \\
\text { Syuthor }\end{array}$ & Source Manuals & Habit & Family Name & No. \\
\hline
\end{tabular}

\begin{tabular}{|c|c|}
\hline OKENI & OKENIA \\
\hline $\begin{array}{l}\text { OLDEN } \\
\text { OL ME }\end{array}$ & $\begin{array}{l}\text { OLOENIANDIA } \\
\text { O. HEREACEA }\end{array}$ \\
\hline OLLAZ & 0. LANCIFOLIA \\
\hline OL SU & $0^{\circ}$. Suaviscosa \\
\hline OLEA+ & $O L E A$ \\
\hline OLEAN & CLEANDARA \\
\hline OL 160 & OLIGOMERIS \\
\hline - & OLIGONEURON \\
\hline - & 0. JACKSONII \\
\hline . & o. rigidum \\
\hline
\end{tabular}

OL NEY

\begin{tabular}{|c|c|}
\hline OLYRA & OLYRA \\
\hline OMALO & OMALOTHECA \\
\hline OMPHA & $\begin{array}{l}\text { OMPHALODES } \\
\text { O. LINIFOLIA } \\
\text { O. VERPVA }\end{array}$ \\
\hline ONCIO & $\begin{array}{l}\text { ONCIOIUN: } \\
\text { O. ENSATUM }\end{array}$ \\
\hline * & O. INTERMEDIUM \\
\hline 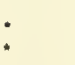 & $\begin{array}{l}\text { b. PRIGNOCHILUIA } \\
\text { O. UNDULATUM }\end{array}$ \\
\hline
\end{tabular}

- bncoja 0. ECHINATA

on OBR

ONOCL

ONSE

-

ON ONI

ONOPO

ONOSM

-

ONMO

ONMOB

-

-

-

:

-

:

.

OPERT

*

OPHIO OPCA

ONOBRYCHIS

\section{CNOCLEA}

o. SENSIBILIS ODTUSILUGATA ONOMIS

UNOPOOPOUM

ONOSMODIUM

o. HISPIOISSIMUM

D. molle macrospermum

D. MOLLE BEJARIENSE SUBSETUSUM

o. OCCIOENTAL SrIVESSaE

Dinterium

o. DENSUIA

o. StrICtuin

OONOOSTIS

o. engel Manni I

o. FOLIOSA

OPERCULINA

o. oISSECTA

o. TUBerosa

DPHTDELOSTUM

o. CALIFORHICUH

O. DE VEROIIEURON
SCHLECHT.\& CHAM.

(i.) D .

OLOENLANOIA LANCIFOLIA

(C. 5 CHUMACH, ) D

OLDENLANDIA HERBACEA
C. WRIGHT EX GRAY

HOUSIONIA SUEVISCOSA

L.

CAV.

CAMAESS.

SMALL

(RAF.) SMALL

SOLIDAGO RIGIDA

(KUNTZE) SMALL

SOLIDAGO PIGIDA GLAGRATA

SOLIDAGO JACKSONII

(L.) SMALL

SOLIDAGO RIGIDA

GRAY

L.

CASS.

MILL.

MOENCH

MOENCH

SWARTZ

LINOL.

ONC1DIUM FLORIDANUM

BERTERO EX SPRENG.

ONC 1 OIUM LEMONIANUM

KRAEN:ZL.

(SWARTZ) SAL ISB.

UNCIDIUM LURI DUM

ONCIOIUM CARTHAGINENSE

FORSSK.

D. OLIVER

MILL.

L.

IS CHKUHR)TORR.

L.

L.

IA C CHX.

ONOSMODIUM MOLLE HISPIDISSIMUM

MACKENZ. 3 GUSH

MICHX.

COC. EX a.oc.) CRONO.

ONOSMODIUM GEJARIENSE

(MACKENZ. B BUSH)CRONO.

ONOSMODIUM SUBSETOSUM

MACKENZ.

ONOSMOOIUM MOLLE OCC IDENTALE

MACKENZ. •

RAULF.

BRACKENR.

ASPIDOTIS OENSA

KUNZE

(NUTT, ) GREENE

(GRAY) GREENE

HAPL OPAPPUS ENGELMANNII

(GRAY) GREENE

MANSO

IA

(JACO.) HOUSE

MERREMIA DISSECTA

"L. . JME ISN.

L. PRANTL

OPHIOGLOSSUM LUSITANICUM CALIFORNICUM

E.ST.JOHN

( uphioglos SUm ivdealcale tenerum

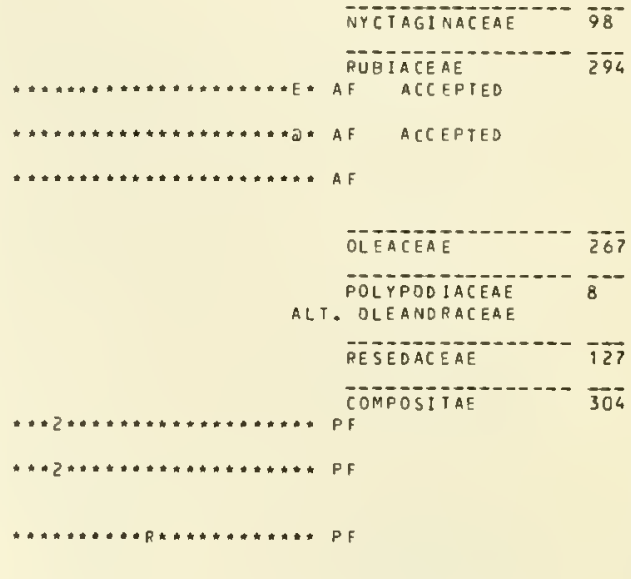

LEGUMINOSAE - T47 ALT. PAPILIONACEAE

GRAMINEAE

COMPOSITAE

BO RAGTINACEAEE

AF

ORTHIOAGEAE

n.t.....................

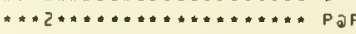

FLACOURTIACEAE

LEGUMINOSAE

ALT. PAPILIONACEAE

POLYPODIACEAE

ALT. ASPIDIACEAE

FGGZC*MS\#.RTO\#......... P. ASPIDIACEAE ACCEPTEO

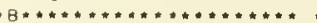

LEGUMINOSAE $\overline{147}$ ALT, PAPILIONACEAE

COMPOSITAE

BORAGINACEAE

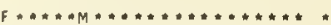

F $3 G 2 C * S Y S R=6 \cdots * * 9 \ldots *$ PF ACCEPTED

*..................... p p

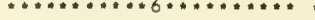

PF

POLYPOOTAGEAE

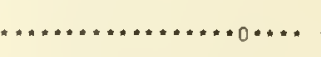

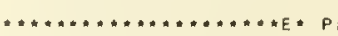

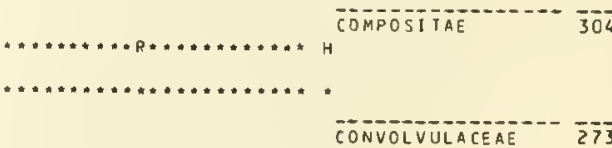

$* \ldots \cdot 2, \ldots \ldots \ldots+\ldots \ldots+\ldots * v$

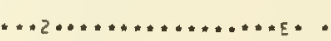

OPHIDGLOSSACEAE 


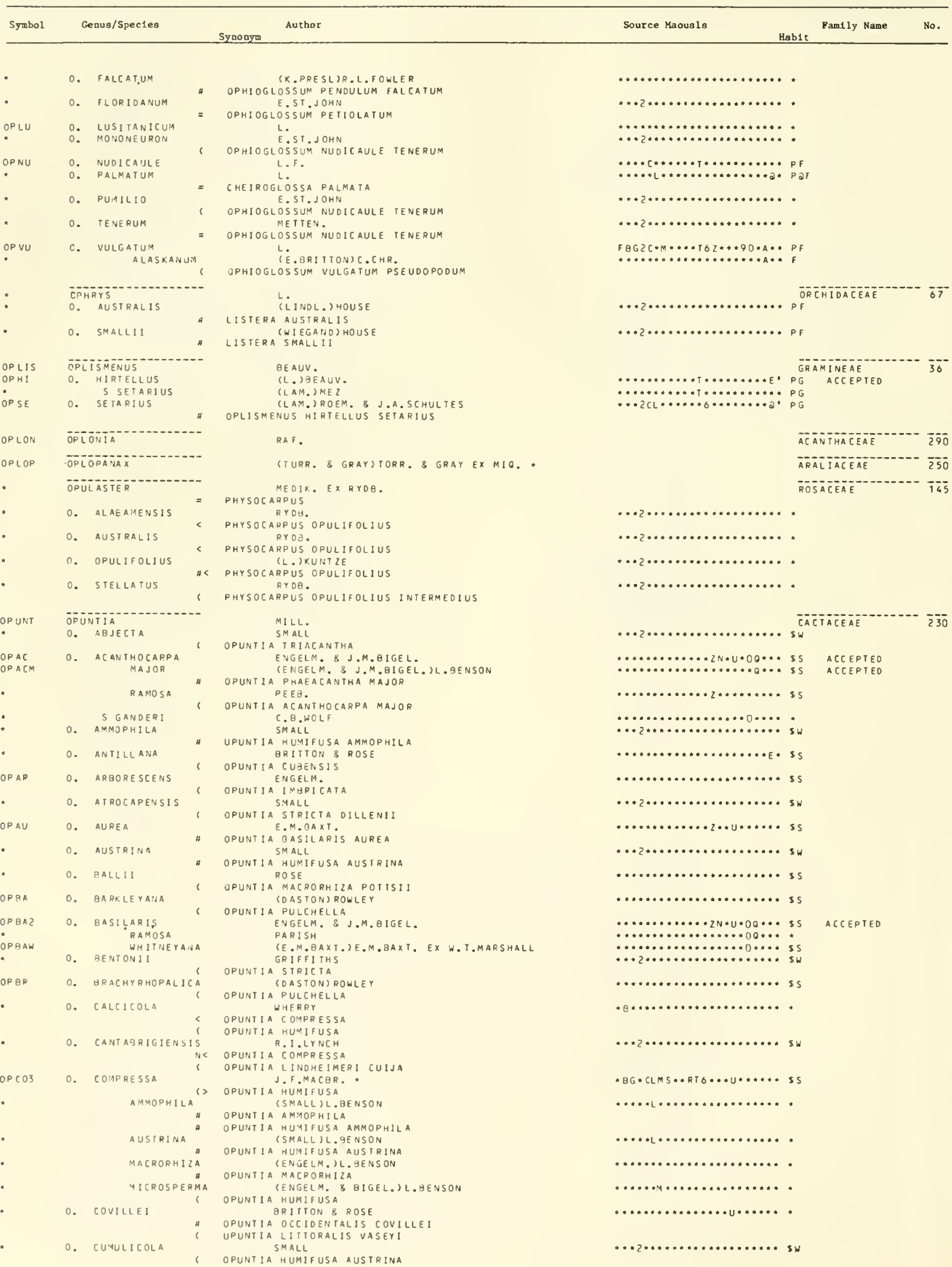




\begin{tabular}{|c|c|c|c|c|c|c|}
\hline Symbol & Geous/Speciea & Syoooym. Author & Source Maouals & $\mathrm{Hab} 1 \mathrm{t}$ & Famlly Name & No. \\
\hline
\end{tabular}

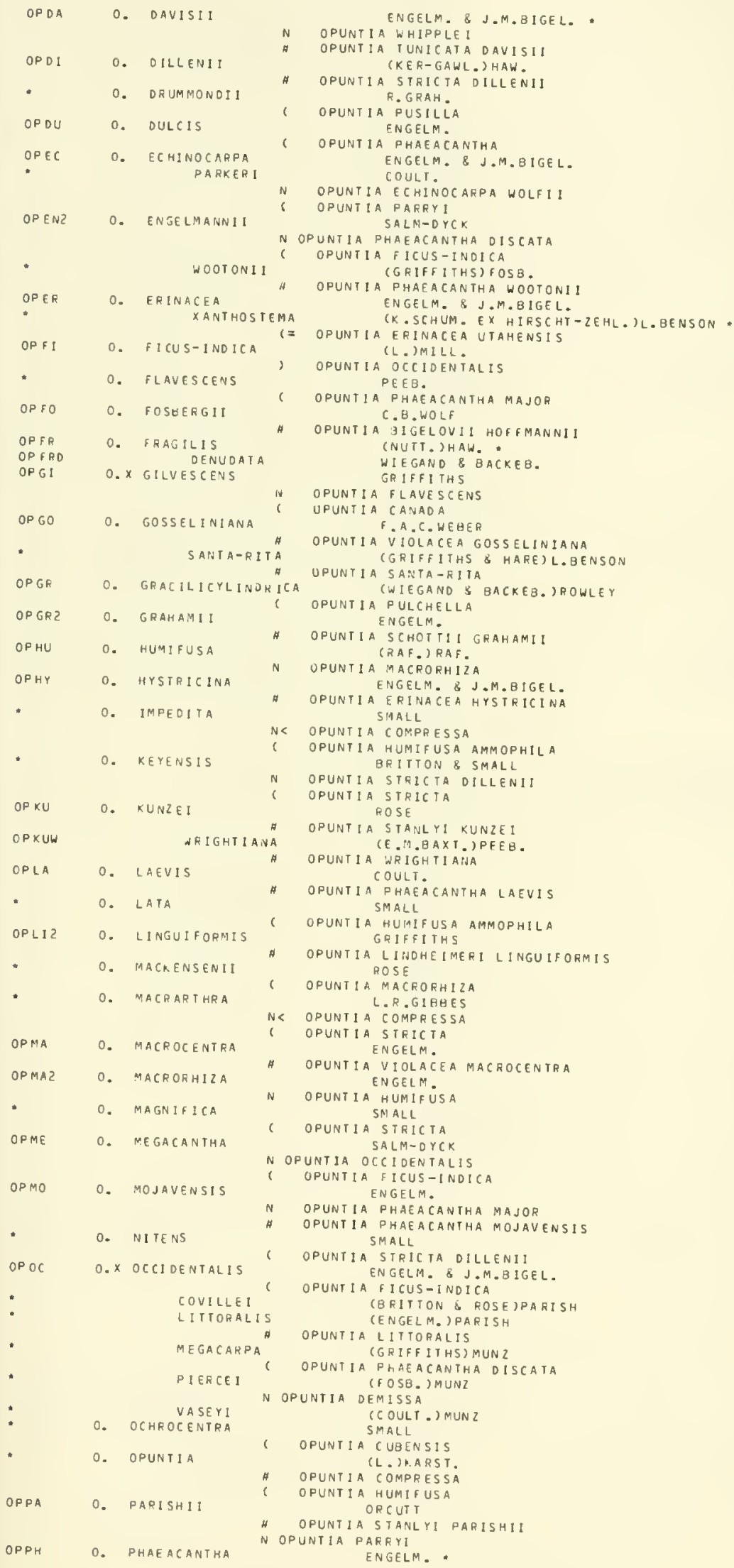

ENGELM. \& J.M.BIGEL.

OPUNTIA WHIPPLE

OPUNTIA TUNICATA DAVISI (KER-GAWL.) HAW.

OPUNTIA STRICTA DILLENII R. GRAH.

OPUNTIA PUSILLA. ENGELM.

OPUNTIA PHAEACANTHA ENGELM. \& J.M.BIGEL. COULT.

OPUNTIA ECHINOCARPA WOLFII

OPUNT IA PARRY I

SALN-DYCK

N OPUNTIA PHAEACANTHA OISCATA

OPUNTIA FICUS-INOICA

(GRIFF ITHS) FOSB.
OPUNTIA PHAEACANTHA WOOTONII

ENGELM. \& 1 M.BIGEL.

(HT - ZEHL.) L. BENSON. ERINACEA UTAHENSIS

$$
\text { (L. .)MLLL. }
$$

OPUNTIA OCCIDENTALIS

PEEB.

OPUNTIA PHAEACANTHA MAJOR

C.B. WOLF

OPUNTIA BIGELOVI 1 HOFFMANNII
(NUTT, ) HAW.

WIEGAND \& BACKEB. GR IFFITHS

OPUNTI I FLAVESCENS

UPUNTI A CANAD F.A.C. WE GER

VIOLACEA GOSSELINIANA

(GRIFFITHS \& HARE)L. BENSON

UPUNTIA SANTA-RIIA

OPUNT IA PEGAND \& BACKEB. )ROWLEY

A PULCHELLA

ENGELM.

OPUNTI A SCHOT TI I GRAHAMI

(RAF.)RAF.

OPUNTIA MACRORHIZA

ENGELM. \& J.M.BIGEL.

OPUNTIA ERINACEA HYSTRICINA$$
\text { SIAALL }
$$

NE OPUNTIA COMPRESSA

OPUNTIA HUMIFUSA AMMOPHILA

BRIITON \& SMALL

OPUNIIA STRICTA DILLENIT

OPUNTIA STRICTA

OPUNT I A STANLYI KUNZEI

OPUNTIA WRIGHTIAINA

$$
\text { COULT. }
$$

* opuntia phaeacantha laevis SMALL

( OPUNTIA HUIAIFUSA AMMOPHILA

A HUIAIFUSA
GRIFFITHS

* opUntia linOHEIMERI linguiformis ROSE$$
\text { OPUNTIA MACRORHIZA }
$$

< OPUNTIA COMPRESSA

6 OPUNTIA STRICTA

- ENGELM.

* opunt la violacea macrocentra

N OPUNT ENGELM.

chall

OPUNTIA STRICTA SALM-DYCK

N OPUNTIA OCCIDENTALIS

OPUNTIA FICUS-INDICA

OPUNTIA PHAEACANTHA MAJOR

OPUNTIA PHAEACANTHA MOJAVENSIS$$
\begin{aligned}
& \text { A PHAEAC } \\
& \text { SMALL }
\end{aligned}
$$

OPUNTIA STRICTA DILLENII ENGELIA. \& J.M.BIGEL.

OPUNTIA FIGUS-INOICA

(BRITTON \& ROSEJPARISH

(ENGELM.) PARISH

OPUNTIA LITTORALIS

(GRIFFITHS) MUNZ

OPUNTIA PHAEACANTHA DISCATA

(F OSB.) MUNZ

(COULT.) MUN 2

( OPUNTIA CUBENSIS

CUBENSIS
CL. IR.ARST

H OPUNTIA COMPRESSA

OPUNTIA HUMIFUSA

ORCUTT

* OPUNTIA STANLYI PARISHII

N OPUNTIA PARRYI ENGELM. .

.

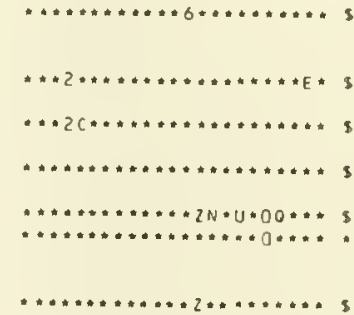

S S

35

is

ACCEPTED

is

$\ldots \ldots \ldots \ldots \ldots \ldots \ldots \ldots \ldots * *$ ss

$\cdots+\cdots \cdots+\cdots \cdots 2 N * U=00 \cdots *$ \&

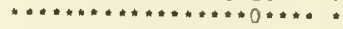

ACCEPTED

ACCEPTED

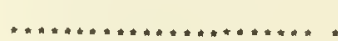

$\ldots \ldots \ldots \ldots \ldots \ldots+\ldots \ldots \ldots$ s

FBG***SYSRTOZN*U90*** IS

H................. is

$\ldots \ldots \ldots \ldots \ldots \ldots \ldots+\ldots \ldots \ldots$ s 5
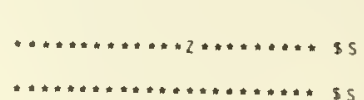

$\ldots \ldots \ldots \ldots+\ldots \ldots \ldots \ldots \ldots \ldots$ s

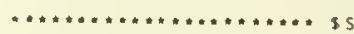

f $B \ldots \ldots \ldots+5 \ldots \ldots \ldots \ldots+$ is
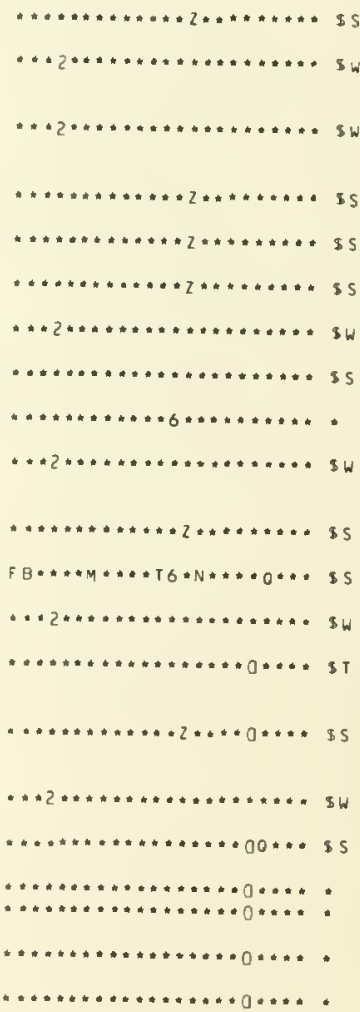

$m * * \ldots+\cdots+\cdots+\cdots+\cdots, 0 \cdots \cdots *$
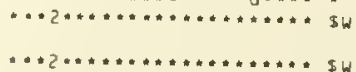

$\cdots \ldots \ldots \ldots+\ldots \ldots+\ldots \ldots$,
ACCEPTED

ACCEPTED

ACCEPTED

ACCEPTED

ACCEPTED 


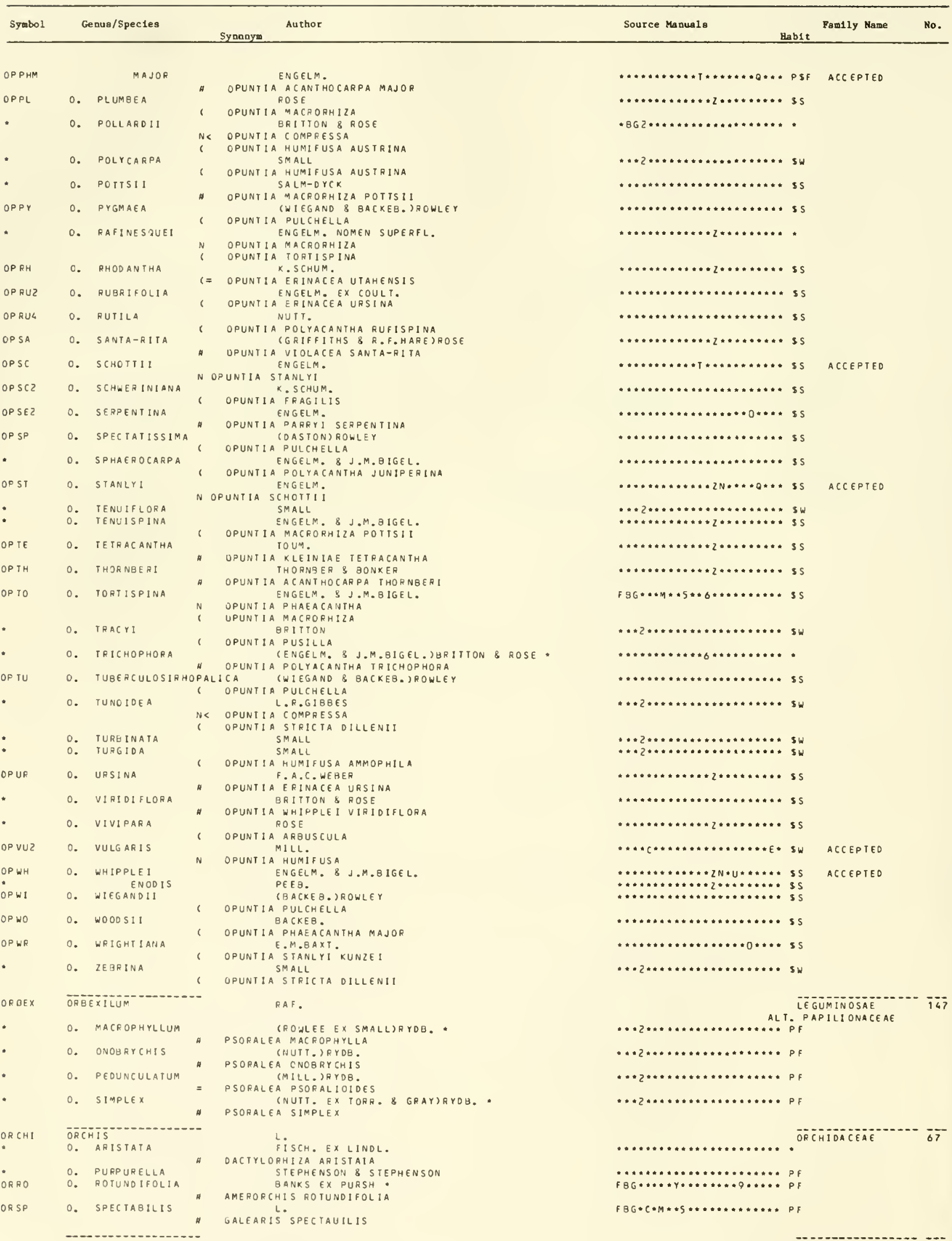




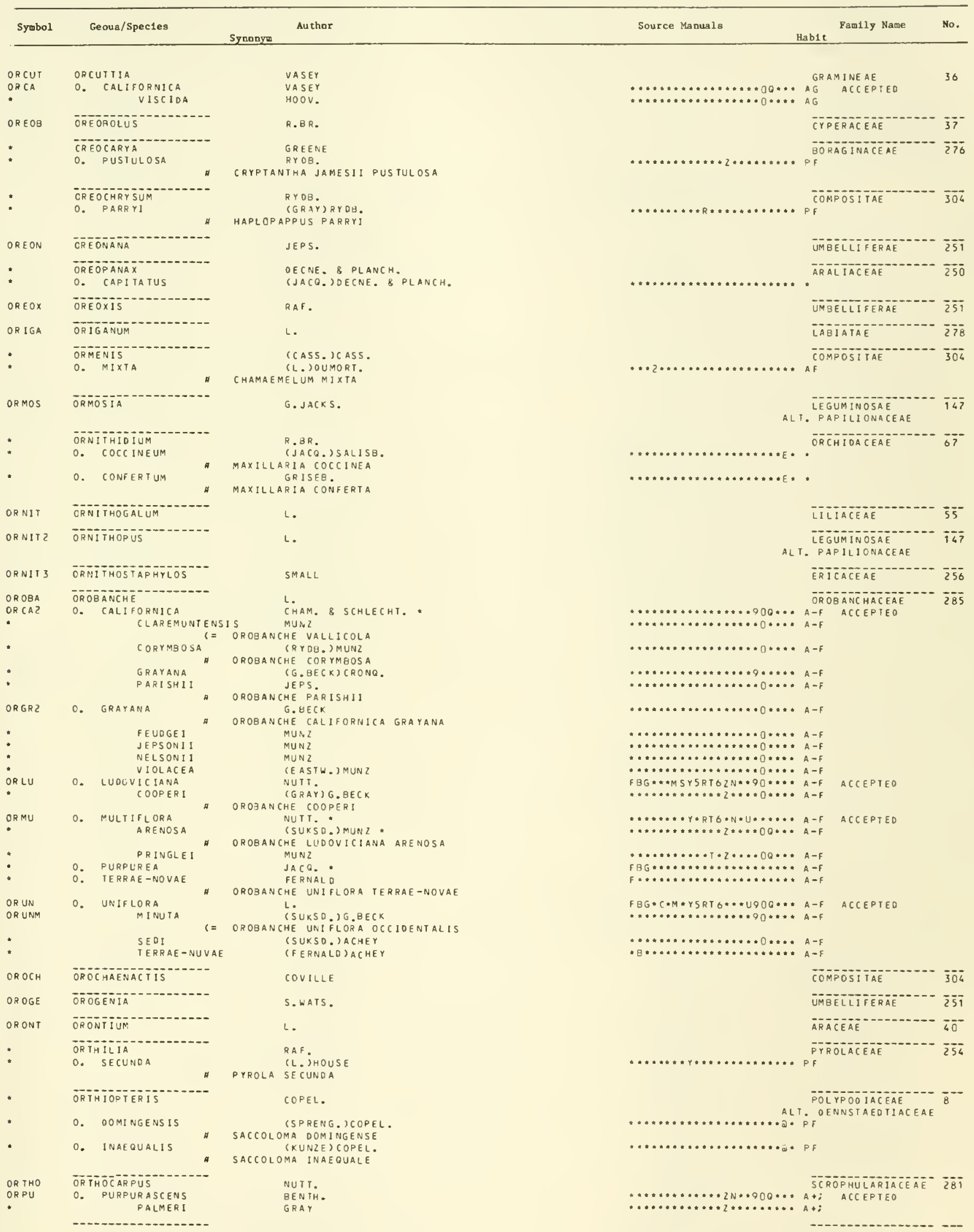




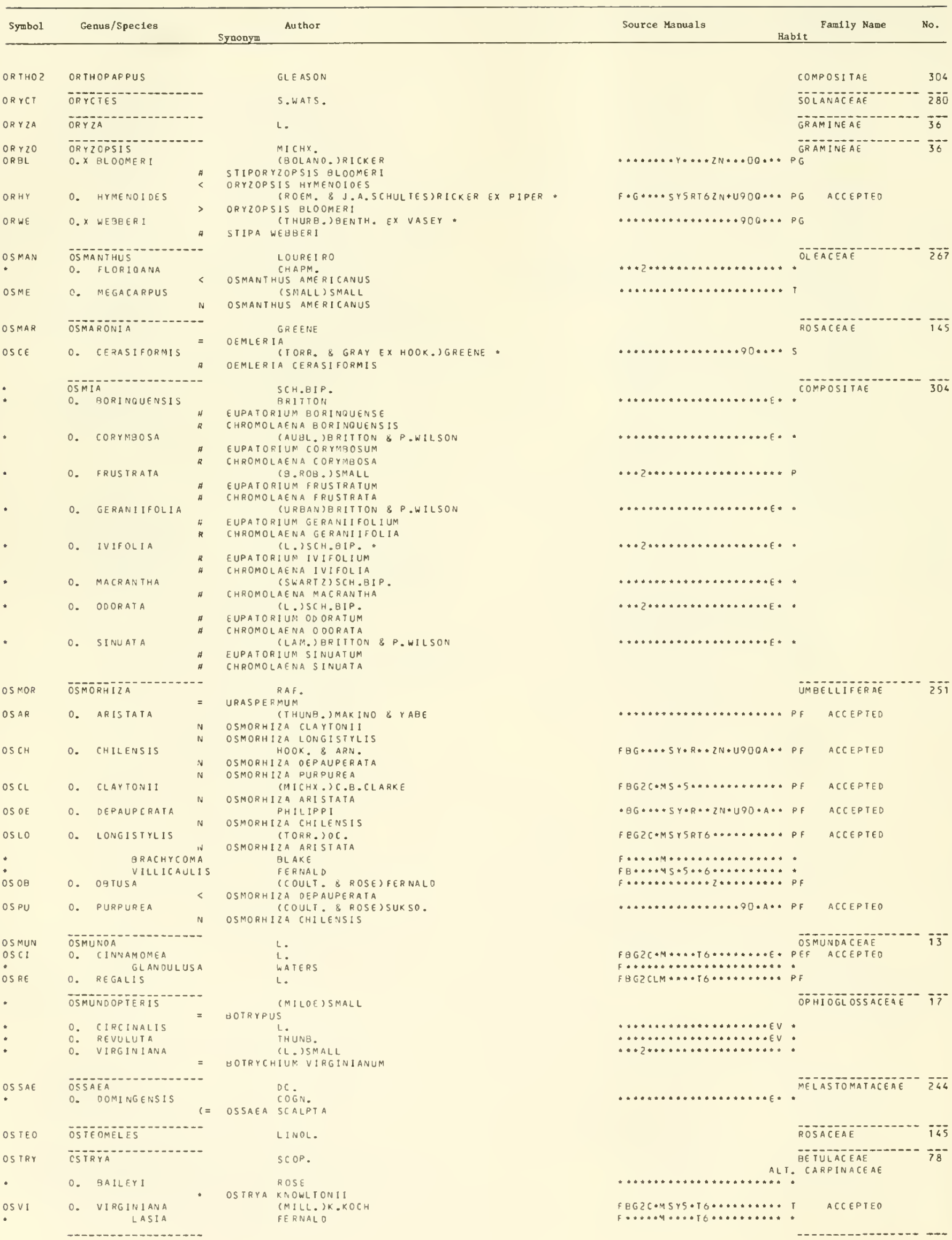




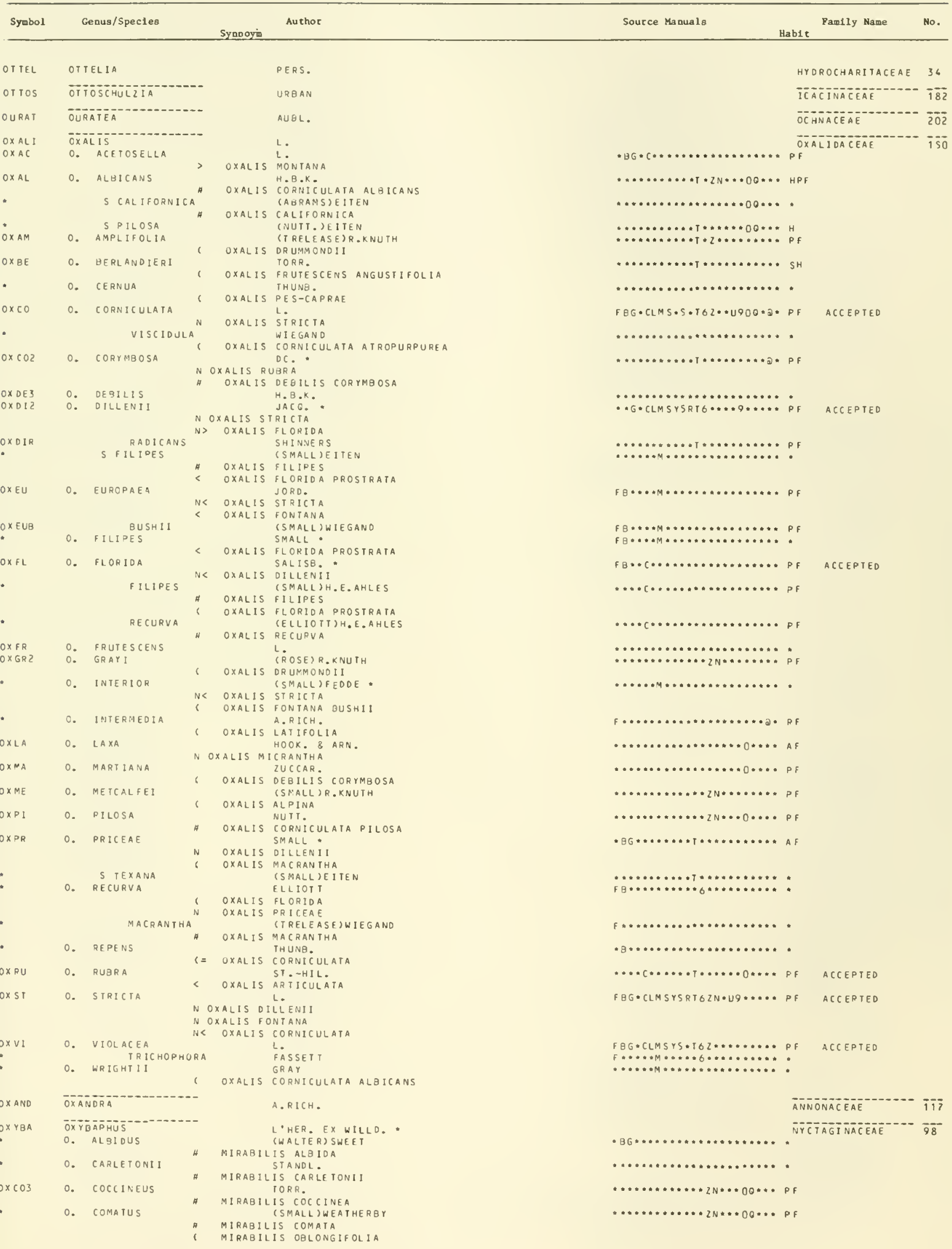




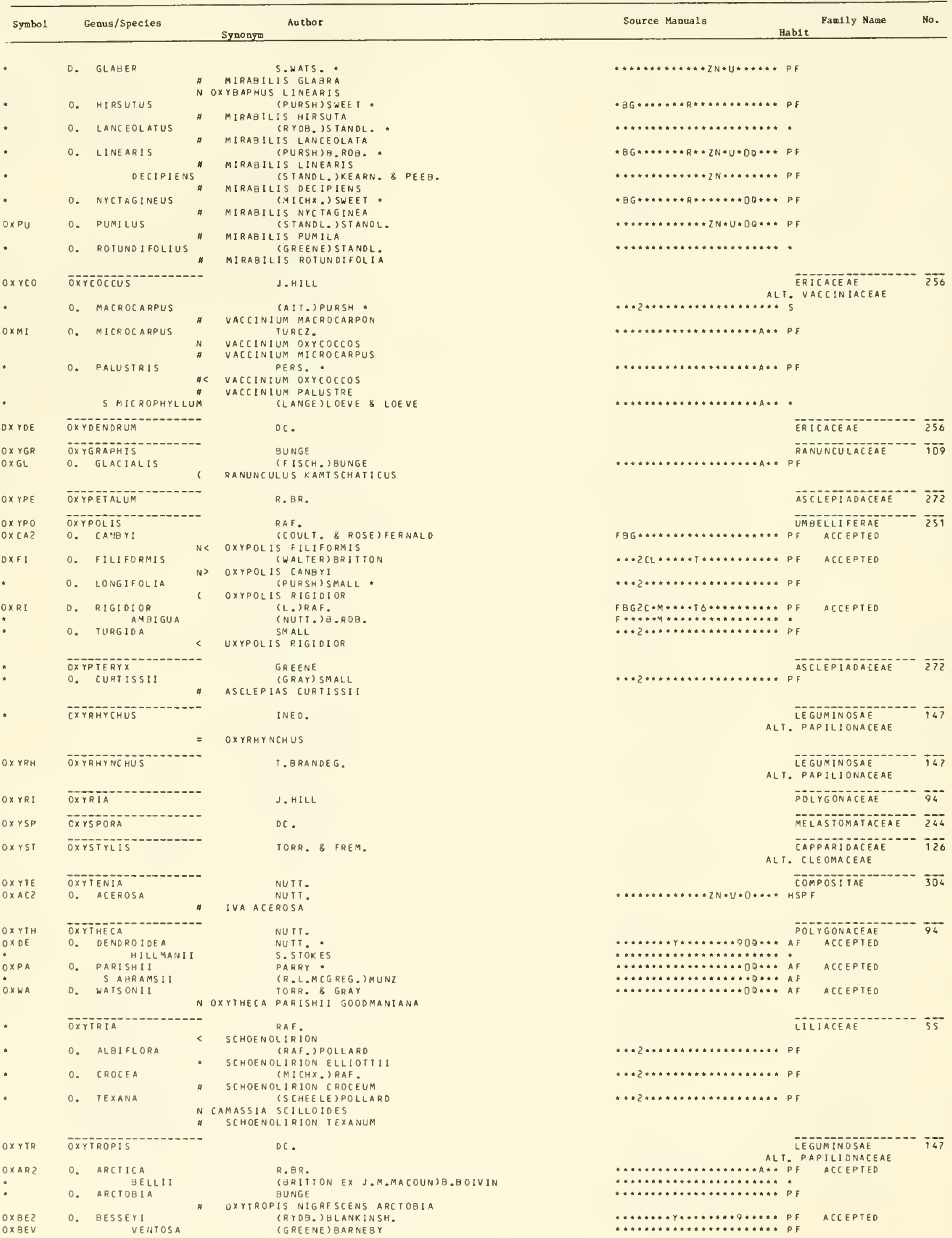




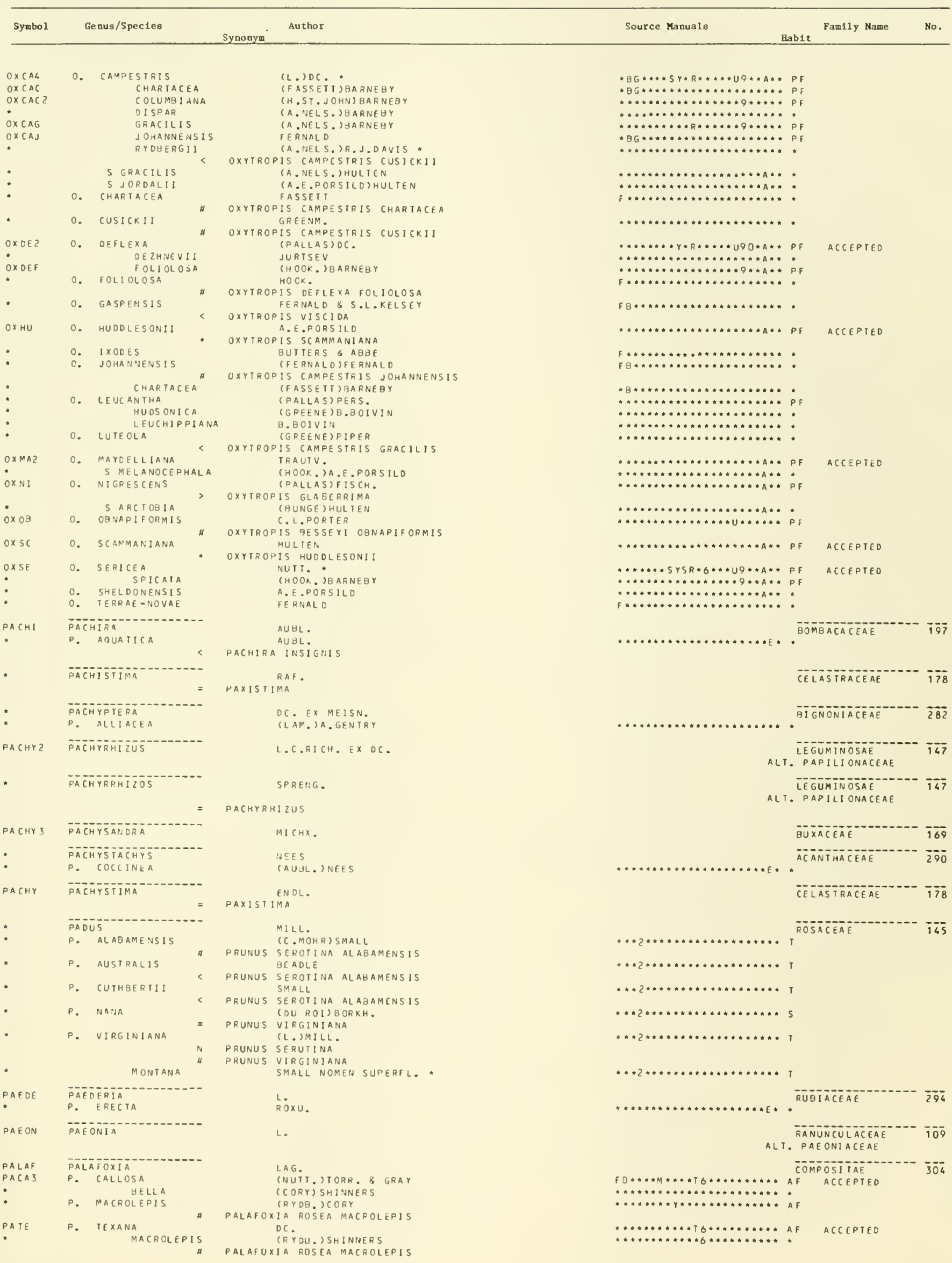


PALAFOXIA

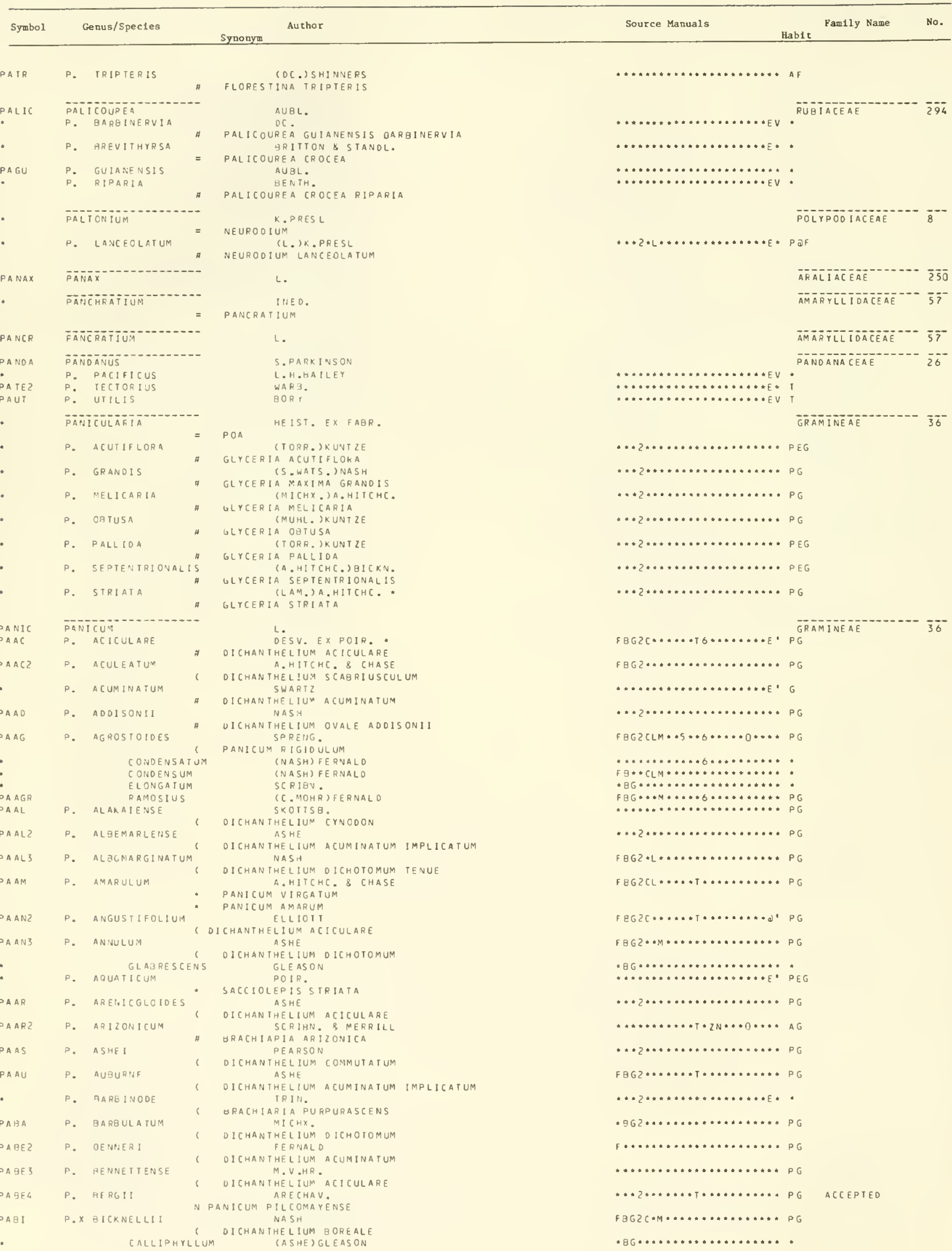




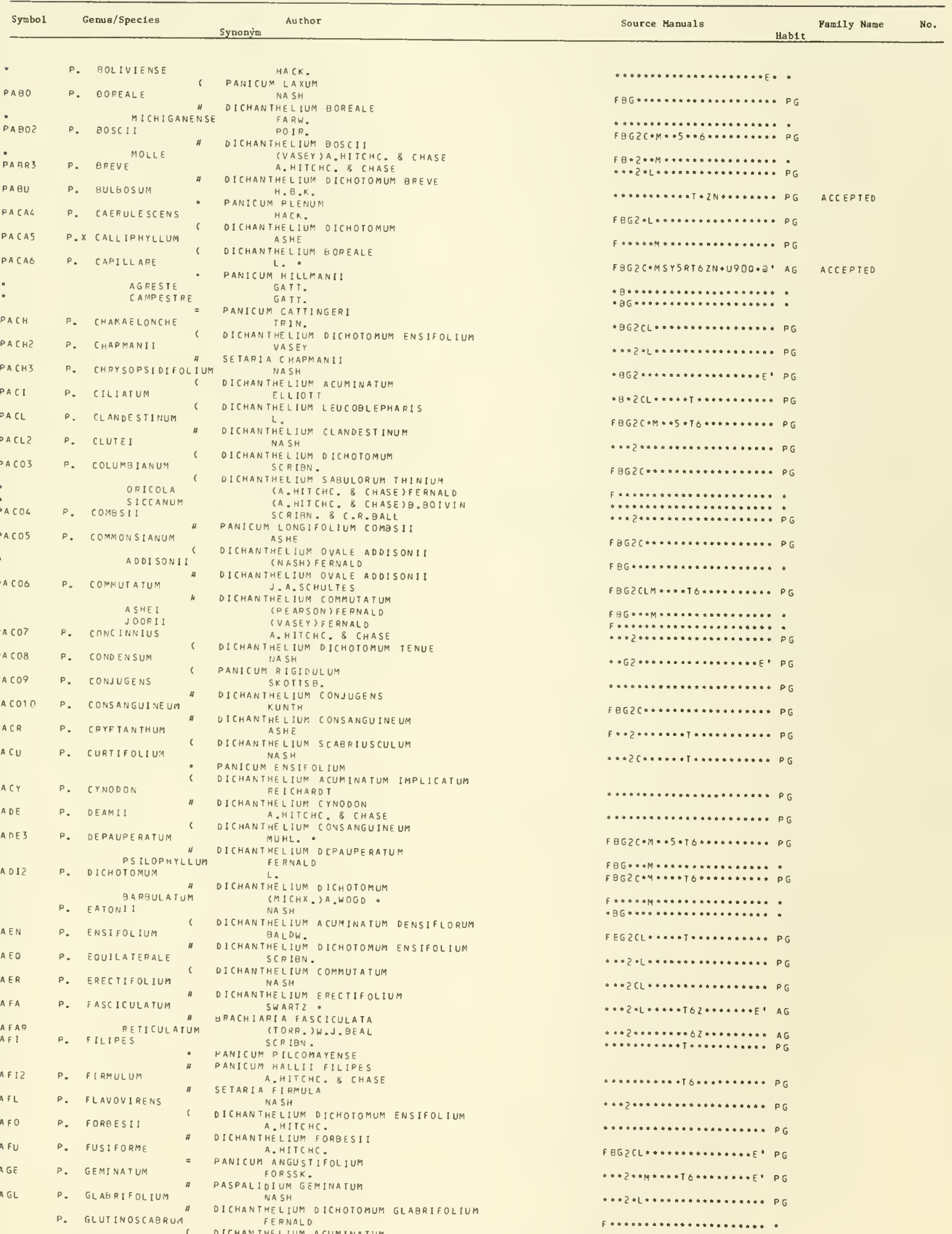




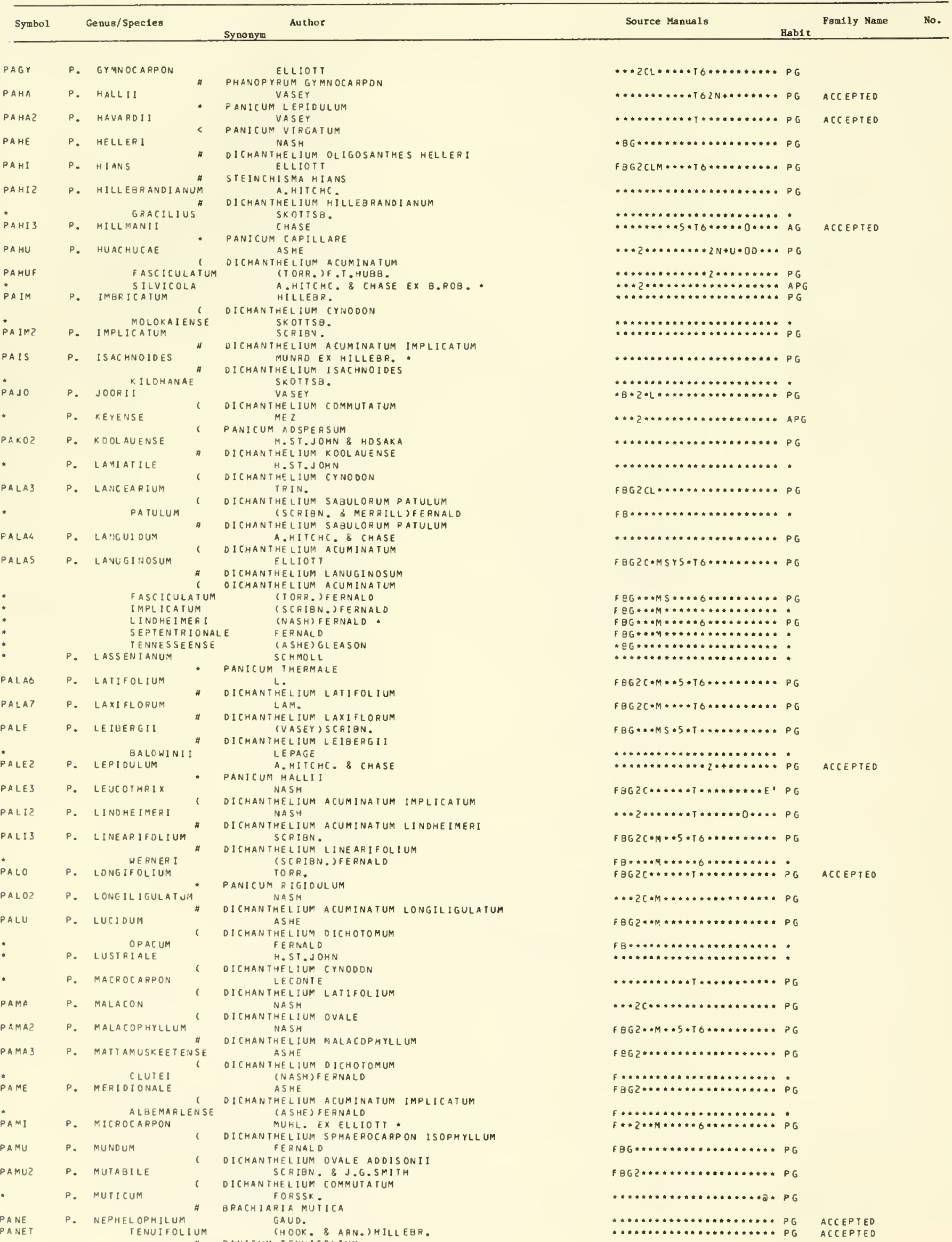




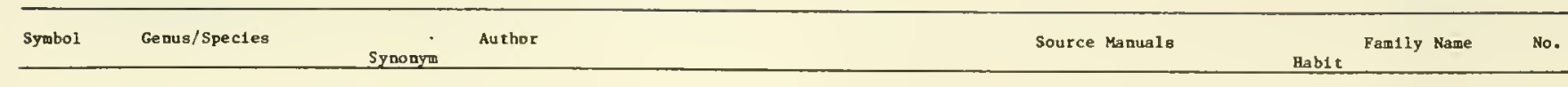

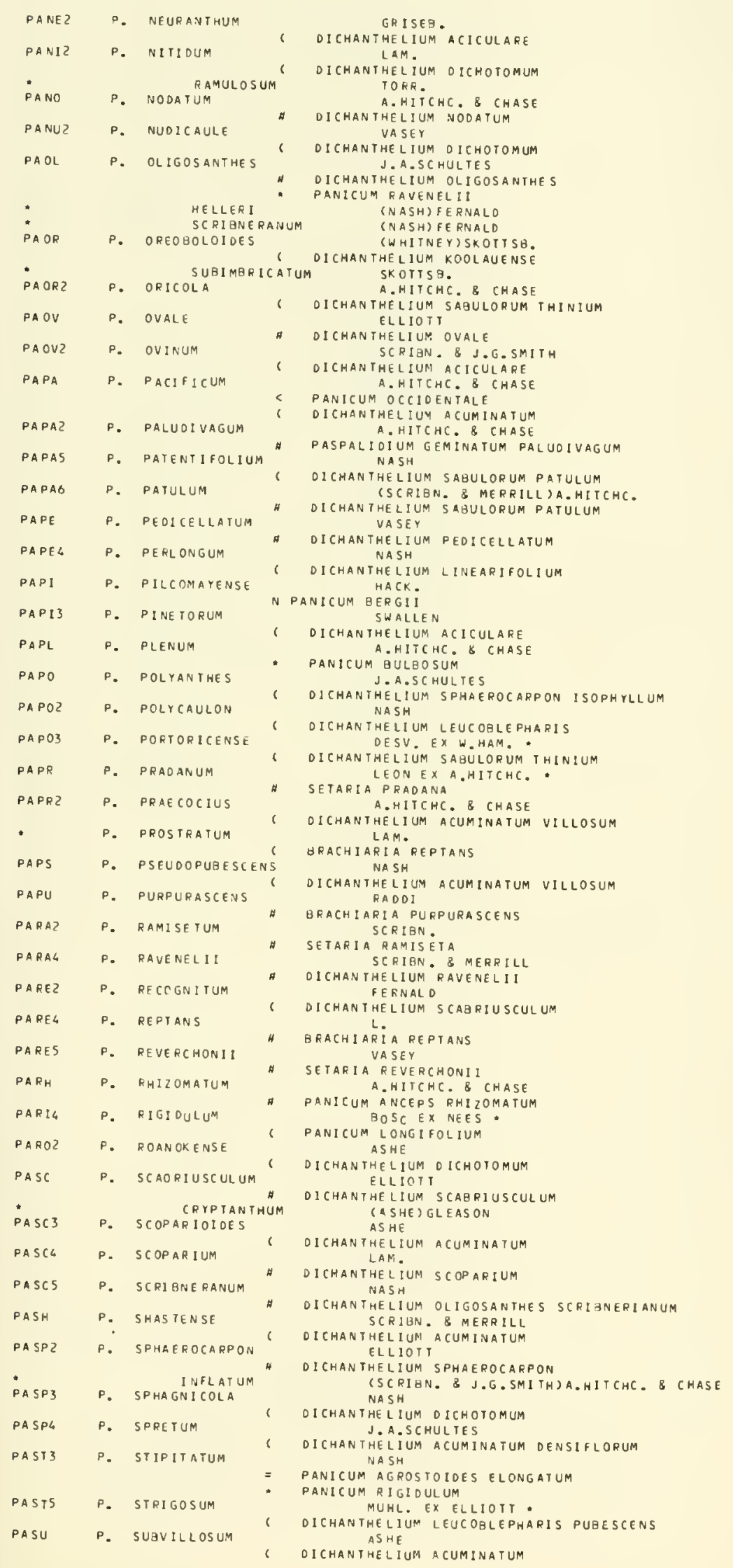
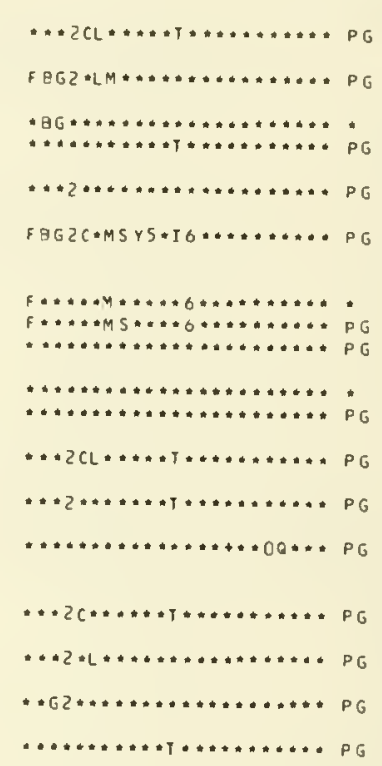

FHG .*4S.5R*6.........PG

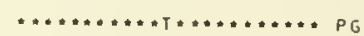
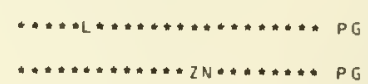

FBG $C+M \ldots+T B * * \ldots \ldots \ldots$ PG

**5*L*\#..........E PG

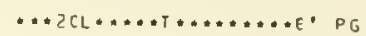

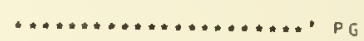

$F B G \ldots$ MS $* 5 \ldots 6 \ldots \ldots \ldots \ldots P G$
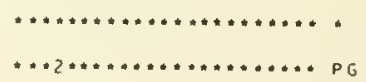

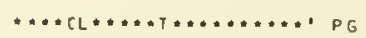

$\ldots \ldots \ldots \ldots+\ldots \ldots \ldots \ldots+P G$

$F B G 2 C * M \ldots \ldots+T O \# \ldots \ldots \ldots P G$

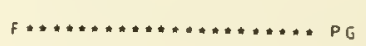

$\cdots \cdot * \ldots \ldots *+\ldots \ldots \ldots \ldots E$. $A G$

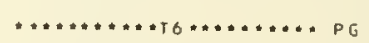

$* * 2 * 1 * \ldots \ldots \ldots \ldots \ldots \ldots \ldots$ PG

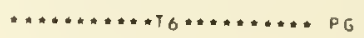

f $B G z \cdot \ldots \ldots \ldots \ldots \ldots \ldots \ldots P_{G}$

FEg $2 C \ldots \ldots \ldots \ldots \ldots \ldots \ldots$ PG

. $\theta G \ldots \ldots \ldots \ldots \ldots \ldots \ldots \ldots \ldots \ldots$

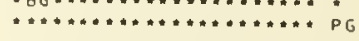

FBG $2 C+M \cdots 5 \cdot T 6 \cdots \ldots \cdots E \cdot P G$

- $B G 2 \ldots \ldots+\ldots+2 N \cdot 490 \cdots . . . P G$

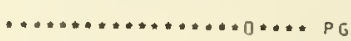

F $E G Z C * M \cdots 5 * T 6 \ldots * * \ldots * P G$

\& $B G_{5} \cdots M \cdots \cdots+\ldots \ldots \ldots \ldots *$ *

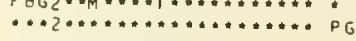

FBG $2 \ldots \ldots \ldots+\ldots \ldots \ldots \ldots+P G$

$F * 2 C \cdot M+\ldots \ldots \ldots \ldots \ldots \ldots \ldots P G$

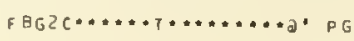

\& $9 G \ldots * 4 \ldots \ldots \ldots \ldots \ldots \ldots * P G$
ACCEPTEO

ACCEPTEO

ACCEPTED 


\begin{tabular}{|c|c|c|c|c|c|}
\hline Symbol & Genus/Spectes & Synonym & Source Manuals & Family Name & No. \\
\hline PATEG & P. TENHESSEENSE & $\begin{array}{l}\text { ASHE } \\
\text { DICHANIHELIUM LANUGINOSUM FASCICULATUM } \\
\text { DICHANTHELIUM ACUMINATUM }\end{array}$ & $\cdots \cdot 2 \cdots \cdots \cdots \cdots \cdot 2 \cdot+\cdots \cdots \cdots p G$ & & \\
\hline PATES & P. TENLE & 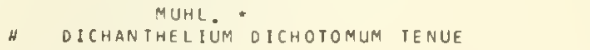 & 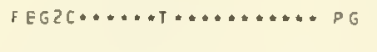 & & \\
\hline PATE? & P. TEXANUM & $\begin{array}{l}\text { BUCKLEY } \\
\text { * BRACHIARIA TEXANA }\end{array}$ & $* B * 2 C * M * 5 \cdot T 62 \cdots \cdots * * * A G$ & & \\
\hline $\begin{array}{l}\text { PATH } \\
\text { PATHZ }\end{array}$ & $\begin{array}{l}\text { P. THERMALE } \\
\text { P. THUROWII }\end{array}$ & $\begin{array}{l}\text { BOLAND. } \\
\text { DIGHANTHELIUIF ACUMINATUM } \\
\text { SC?IEN. \& J.G.SMIJH }\end{array}$ & $\cdots \cdots \cdots \cdots \cdots \cdots+0 \cdots \cdots P G$ & & \\
\hline PATR5 & P. TRIFOL IUM & $\begin{array}{l}\text { * DICHANTHELIUM ACUMINATUM THUROWI: } \\
\text { NASH }\end{array}$ & $F B G Z \cdot L \ldots \ldots \ldots \ldots \ldots \ldots \ldots F G$ & & \\
\hline PATS & P. TSUGETORUM & $\begin{array}{l}6 \text { DIGHANTHELIUM DICHOTOMUM TENUE } \\
\text { NASH } \\
\text { DICHANTHELIUM SABULORUM PATULUM }\end{array}$ & $\ldots * 2 * \ldots \ldots \ldots \ldots \ldots \ldots \ldots * P G$ & & \\
\hline PATU & P. TUCKERMANII & $\begin{array}{l}\text { FERMAL D } \\
\text { * PANICUM PHILADELPHICUM TUCKERMANII }\end{array}$ & $F * G * * \ldots \cdots \cdots \cdots \cdots \cdots \cdots \cdots * A G$ & ACCEPTED & \\
\hline PAUTZ & $\begin{array}{l}\text { P. UTOWANAEUM } \\
\text { P. VERNALE }\end{array}$ & $\begin{array}{l}\text { SCRIBV. } \\
\text { a SETARIA UTOWANAEA } \\
\text { A.HITCHC. \& CHASE }\end{array}$ & 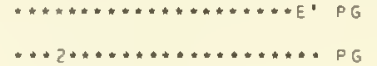 & & \\
\hline PAVE & P. VERNALE & $\begin{array}{l}\text { A.HITCHC. \& CHASE } \\
\text { C DICHANTHELIUM DICHOTOMUM ENSIFOLIUM } \\
\text { NASH }\end{array}$ & 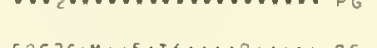 & & \\
\hline PAVI & $\begin{array}{l}\text { P. VILLOSISSIMUA } \\
\text { OSEUDOPUE }\end{array}$ & $\begin{array}{l}\text { NASH } \\
\text { CEICHANTHEL IUM ACUMINATUM VILLOSUM } \\
\text { BESCENS }\end{array}$ & 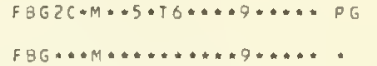 & & \\
\hline * & SCOOAR 101 & $\begin{array}{l}\text { (ASHE) FERNALD } \\
\text { ( DICHANTHELIUM ACUMINATUM }\end{array}$ & $F \forall G \ldots \ldots \ldots \ldots \ldots \ldots \ldots \ldots \ldots$ & & \\
\hline PAVIZ & P. VIRGATUM & > PANICUM HAVARDII & FEGZCLMSYSF* $2 Z N+U * \ldots \ldots P G$ & ACCEPIED & \\
\hline PAWE & D. LEE33ERANUM & $\begin{array}{l}\text { NASH } \\
\text { DICHANTHELIUM SASULORUM PATULUM }\end{array}$ & $* * 2 C * M \cdots \cdots * \cdots * * * * * P G$ & & \\
\hline PAWE 2 & P. WERNER I & $\begin{array}{l}\text { SCFIBN - } \\
\text { OICHANTHELIUM LINEARIFOLIUM }\end{array}$ & 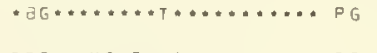 & & \\
\hline PAWI & P. WILCOXIANUM & $\begin{array}{l}\text { VASEY } \\
\text { DICHANTHELIUM OLIGOSANTHES WILCOXIANUM }\end{array}$ & $F B G * \cdots M S * 5 * 6 * \cdots \cdots \cdots \cdots P G$ & & \\
\hline PAWIZ & $\begin{array}{r}\text { OREITUNGI } \\
\text { P. WILMINGTONENSE }\end{array}$ & $\begin{array}{l}\text { B. BOIVIN } \\
\text { ASHE } \\
\text { DICHANTHELIUM OVALE ADOLSONII }\end{array}$ & $\cdots \ldots \ldots \ldots \ldots \ldots \ldots \ldots \ldots \ldots+\dot{p}_{G}$ & & \\
\hline PAWR & P. WRIGHTIANUM & $\begin{array}{l}\text { " SCRIBN. } \\
\text { " DICHANTHELIUM A CUMINATUM WRIGHTIANUM }\end{array}$ & $F E G 2 C \ldots \ldots \ldots \ldots \ldots \ldots \cdots+P G$ & & \\
\hline$P A \times A$ & P. XALAPENSE & $\begin{array}{l}\text { H.E.K. } \\
\text { ( DICHANTHELIUM LAKIFLORUM }\end{array}$ & $\cdots * 2 \cdot L \cdots \cdots * * \cdots+\cdots * * * P G$ & & \\
\hline PAXAZ & $\begin{array}{l}\text { STRICTIRAR } \\
\text { P. XANTHOPHY SUM }\end{array}$ & $\begin{array}{l}\text { A.HEUT ITCHC. \& CHASE } \\
\text { GRAY }\end{array}$ & 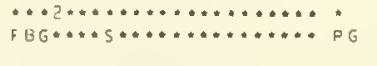 & & \\
\hline$P A Y A$ & P. YADKINENSE & $\begin{array}{l}\text { H DICHANTHELIUP XANTHOPHYSUM } \\
\text { ASHE } \\
\text { \& DICHANTHELIUM OICHOTOMUM }\end{array}$ & $F B G 2 \cdots \eta \cdots \cdots \cdots \cdots \cdots \cdot P G$ & & \\
\hline * & D. ZIZANIOIDES & $\begin{array}{l}\text { KUNTH } \\
\text { 6 ACROCERAS ORYZOIDES }\end{array}$ & $\ldots \ldots \ldots \ldots \ldots \ldots \ldots \ldots \ldots$ & & \\
\hline $\begin{array}{l}\text { PAPAV } \\
\text { PAAL }\end{array}$ & $\begin{array}{l}\text { PAPAVER } \\
\text { P. ALASKANUM }\end{array}$ & LULTEN & $\ldots$ & AVERACEAE & $i 2 \overline{3}$ \\
\hline PAAL4 & P. ALASKANUM & 6 PAPAVER LAPPONICU/A OCCIDENTALE & $\cdots \cdots \cdots \cdots \cdots * \cdots \cdots \cdots \cdot \cdots * P F$ & & \\
\hline $\begin{array}{l}\text { PAAP } \\
\text { PAAPM }\end{array}$ & P. APULUIA MICRANTHUI & $\begin{array}{l}\text { TENORE } \\
\text { IM (BOREAU)FEDOE }\end{array}$ & $\because \ldots \ldots \ldots \ldots \ldots \ldots \ldots \cdots \cdots \cdots$ AF & & \\
\hline PAAF 3 & P. ARGEMONE & $\begin{array}{c}\text { N PAPAVER HYBRIOUM } \\
\text { L. }\end{array}$ & $* B_{G} * \ldots \ldots \ldots \ldots+\ldots 0 * \ldots * A F$ & & \\
\hline * & P. CORRWALLISENSI & $\begin{array}{l}\text { D. LOEVE } \\
\text { PAPAVER LAPPOHICUM OCCIOENTALE }\end{array}$ & $\ldots \ldots \ldots \ldots \ldots \ldots \ldots \ldots+p_{f}$ & & \\
\hline * & $\begin{array}{l}\text { P. CROCEUM } \\
\text { P. FREEOMANIANUIA }\end{array}$ & $\begin{array}{l}\text { LEOEB. } \\
\text { O. LOEVE }\end{array}$ & 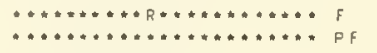 & & \\
\hline PAHUZ & P. HULTENII & $\begin{array}{l}\text { 6APAVER LAPPONICUM OCCIOENTALE } \\
\text { G.KNABEN } \\
\text { PAPAVER MACOUNII }\end{array}$ & $\ldots \ldots \ldots \ldots \ldots \ldots \ldots \ldots+\ldots \ldots$ & & \\
\hline * & SALMONI 60 & LOR HULTEN & $\because \ldots \ldots \ldots \ldots \ldots \ldots \ldots$ A $\cdots$ & & \\
\hline - & P. KEELEI & $\begin{array}{l}\text { A.E.PORS ILO } \\
\text { PAPAVER MACOUNII }\end{array}$ & $\ldots \ldots \ldots \ldots \ldots \ldots \ldots k+\ldots \ldots+p F$ & & \\
\hline 0 & P. KLUANENSE & $\begin{array}{l}\text { D. LOEVE } \\
\text { (PAPAVER LAPPONICUM OCCIOENTALE }\end{array}$ & 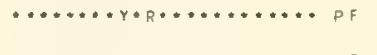 & & \\
\hline PALAQ & $\begin{array}{l}\text { P. LAPPON ICUM } \\
\text { SPORSILDII }\end{array}$ & $\begin{array}{c}\text { (TOLMJE)NORDH. } \\
\text { G.KJABEN } \\
\text { ( PAPAVER LAPPONICUM OCCIDENTALE }\end{array}$ & $\because \ldots \ldots * \ldots \ldots \ldots \ldots \ldots \ldots \ldots A+P F$ & & \\
\hline * & P. MCCCINNELLII & HULIEN & $\ldots \ldots \ldots \ldots \ldots \ldots \ldots A, P F$ & & \\
\hline 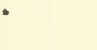 & P. NIGRCFLAVUM & $\begin{array}{l}\text { D. LOEVE } \\
\text { ( PAPAVER LAPPONICUM occidentale }\end{array}$ & $\cdots \ldots \ldots \ldots \ldots \ldots \ldots \ldots, p F$ & & \\
\hline PANU3 & $\begin{array}{l}\text { P. NUDICAULE } \\
\text { RADICATUM }\end{array}$ & 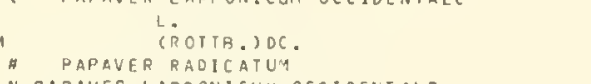 & 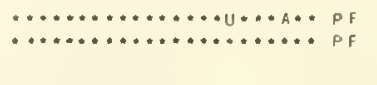 & $A C C E O T E D$ & \\
\hline PAPY & P. PYGMAEUM & $\begin{array}{l}\text { N PAPAVER LAPPONICUM OCCIOENTALE } \\
\text { BYDO. } \\
\text { PAPAVER RLINUM }\end{array}$ & $\cdots \cdots \cdots \cdots \cdots \cdots+\cdots, \ldots p q$ & & \\
\hline PARA5 & P. RADICAIUM & $\begin{array}{l}\text { ROTPB. } \\
\text { N PAPAVER LAPPONICUM OCCIDENTALE }\end{array}$ & $\ldots \ldots \ldots \ldots \ldots \ldots \ldots \ldots \ldots p h$ & & \\
\hline PA WA & $\begin{array}{l}\text { P. WALPOLEI } \\
\text { SULPHUREO }\end{array}$ & $\begin{array}{l}\text { OME.PORSILO } \\
\text { OMACULATA HULTEN }\end{array}$ & $\because \ldots \ldots \ldots \ldots \ldots \ldots \ldots \ldots+\cdots, P E$ & ACCEPTEO & \\
\hline PAPPO & $\begin{array}{l}\text { PAPPOPHORUM } \\
\text { P. ALORECUROIOEUM }\end{array}$ & $\begin{array}{l}\text { SCHREB. } \\
=\quad \text { PAPL } \\
=\quad \text { PAPPOPHDRUIM PAPPIFERUM }\end{array}$ & $\ldots \ldots \ldots \ldots \ldots \ldots \ldots \cdot e^{\overline{G R}}$ & MINEAE & $\overline{36}$ \\
\hline PAMU 3 & P. MUCRONULATUM & $\begin{array}{l}\text { MEFS } \\
\text { N PAPPOPHORUM VAGINATUM }\end{array}$ & $F \cdot \cdots \cdots+5 \cdot T \cdot 2 \cdots \cdots \cdots+P G$ & & \\
\hline - & PAPYRIUS & L LAMOUSSOME IIA & & YACEAE & $\overline{8} \overline{9}$ \\
\hline - & P. PAPYRIFERA & $\begin{array}{l}\text { CL. iKuntze } \\
\text { * brousSONETIA PAPYRIFERa }\end{array}$ & $* * 2+\cdots * * \cdots * * * * \cdots * * \quad 1$ & & \\
\hline
\end{tabular}


PARALABAIIA

P. PORTORICENSIS

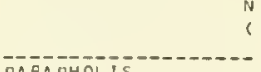

PARATHES IS

P. SERFULATA

\section{PARE PITUCELLIA}

PARIETARIA

P. FLORIDANA

PARI

PAFL 3

PAHES

P. HESPERA

PANU4

PAPES

-

PARIS

-

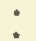

PAR

\section{PAT}

PARME PARMENTIERA

PARME PARMENTIERA
P. ACULEAIA

PACE P. ACULEATA

- P. EDULIS

PARNA PARNASSIA

- p. califorinica

PACA13 P. CAROLINIANA

PAGL3 P. GLAUCA

- P. grandiflora

- P. intermedia

PAPAB

-

PAPAO

PARON

PAAR4

$\because$

PAFA3

PAFAB

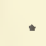

PAPAI 0

PARI2

PAV 16

-

.

\section{P. PALUSIRIS}

S NEOGAEA

P. PARUIFLORA

\section{PARONYCHIA}

P. ARGYROCOMA

S ALBimoniana

P. DIEHOTOMA

P. FASTIGIAIA

P. NUdATA

P. PARKSII

P. RIPARIA

P. VIRGINIEA

PARDSELA SCDPARIA

P. ALOPECUROIDES

P. DOMINGENSIS

P. ENNEANDRA

P. FLORIDANA
PIERRE

BRITTDN \& P.WILSON

POUIERIA DICTYONEURA FUERTESI!

POUTERIA DICTYDHEURA

$$
\text { HUв日. }
$$

(A.DC.) J.D.HOOK

(A.DC. J.D.HOOK
(SWARTZ)MEZ

PARAIHESIS GRENULATA

VIVIANI

NUंT.

PARIETARIA DBTUSA

PARIETARIA PRAETERMISSA

PARIEIARIA HESPERA

B.D.HINTON.

PARIETARIA PENSYLVAN!CA

SMALL

PARIETARIA FLDRIDANA

RYDA. EX SMALL.

PARIETARIA PENSYLVANICA OBIUSA

MUHL. EX WILLD.

(RYDE. EX SMALL ISHINNERS.

GRAY

ADANS.

BFITION EX SMALL .

(L.)ST.-HIL.

HIBISCUS TILIAGEUS

$$
\text { R.BR. }
$$

MEFRILL

L.

$D C$.

(H. H. K.) SEEM

SEEM.

PARMENTIERA ACULEATA

\section{L.}

\section{(GRAY) GREENE}

PARNASSIA PALUSTRIS CALIFORNICA

$M I C H X$.

PARNASSIA GLAUCA

RAF.

PARNASSIA CAROLINIANA

DC. EX A.J.SHARP ET AL. INED. NON RAF.

PAFNASSIA GRANDIFOLIA RYDB.

PARNASSIA FIMBRIATA INIERMEDIA

PARNASSIA PARVIFLORA

(FERNALD) HULTEN

$$
\begin{aligned}
& \text { CFERNA } \\
& D C \text {. }
\end{aligned}
$$

PARNASSIA PALUSIRIS

MILL. .

(MICHX.) NUTT .

(FERNALD)MAGUIRE

(L. )NUTT.

PARONYCHIA VIRGINICA SCOPARIA

(FAF.) FERNALD

(A.WOOD) FERNALD

PARONYCHIA MONIANA

CORRELL

PARONYCHIA MONIICOLA

CORY

PARONYCHIA VIRGINICA PARKS!

CHAPM.

PARONYCHIA BALDWINII RIPAEIA

SPRENG.

(SMALL) CORY

CAV .

(WILLD, RYDB.

DALEA ALOPECURDIDES

DALEA LEPDFINA

DDC.)MILLSP.

DALEA CARTHAGINENSIS DDMINGENSIS

(NUTT.) BRITTON

DALEA ENVEANDFA

DALEA CARTHAGINENS IS FLDRIDANA
SAPOTACEAE

263

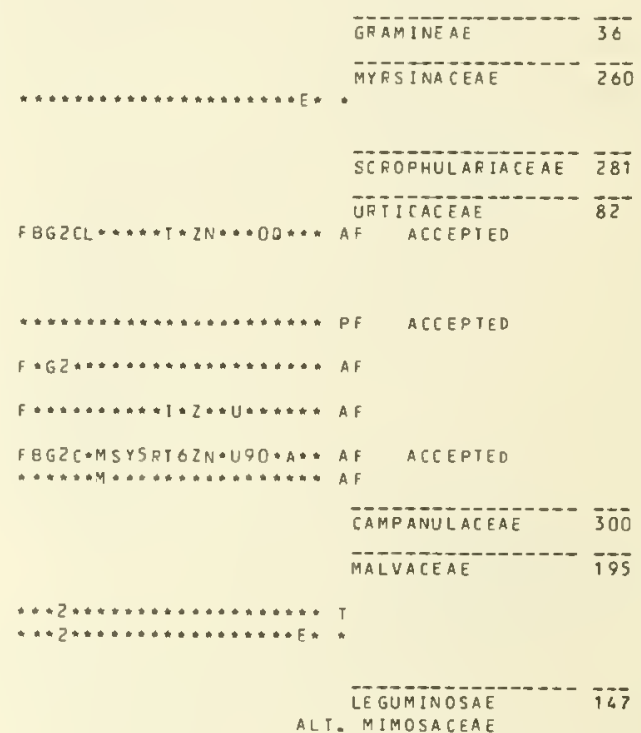
ALT.

LEGUMINOSAE

ALT. CAESALPINIACEAE

BIGNONIACEAE

$\ldots \ldots \ldots+\ldots \ldots \ldots \ldots \ldots$ EV $s$

$\ldots \ldots \ldots \ldots \ldots \ldots+\ldots \ldots+\cdots, \ldots$

SAXIFRAGACEAE

ALT. PARNASS IACEAE

$\bullet \bullet \cdot 2[\ldots \ldots \ldots \ldots \ldots \ldots \ldots *$ ACCEPTED

FEG.............. PF ACCEPIEO

$\ldots \ldots \ldots \ldots+\ldots \ldots \ldots+\ldots \ldots *$.

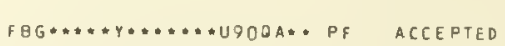

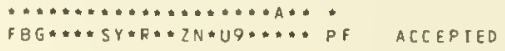

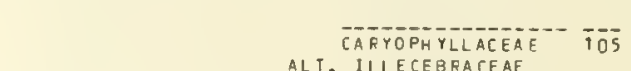
ALT. ILLECEBRACEAE

F $B G 2 C \ldots \ldots \ldots \ldots \ldots \ldots \ldots \ldots$ PF ACCEPTED

$\mathrm{F} B G \ldots \ldots \ldots \ldots \ldots \ldots \ldots \ldots \ldots$

$* * 2+\cdots * \cdots \cdots+* * * \cdots+*_{A P F}$

$F B G * C \cdot M \cdots 5 * T 6 \cdots \ldots \ldots \ldots A F$ ACEEPTED

$\mathrm{f} B \mathrm{G} * \ldots \ldots \ldots+\ldots \ldots \ldots \ldots \ldots$

$\ldots \ldots \ldots \ldots+\ldots \ldots \ldots \ldots$

$\ldots \ldots \ldots \ldots+\ldots \ldots \ldots \ldots+\ldots F$

$\mathrm{f} B G \cdot \mathrm{C} \cdots \ldots \ldots \ldots \ldots \ldots \ldots+\mathrm{P} F$

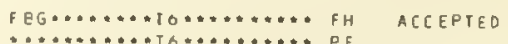

LEGUMINOSAE ALT. PAPILIONACEAE

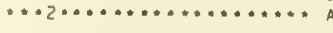

Af

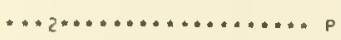

$\ldots * 2 \cdots * \ldots \ldots+\ldots \ldots+\ldots \ldots p$ 


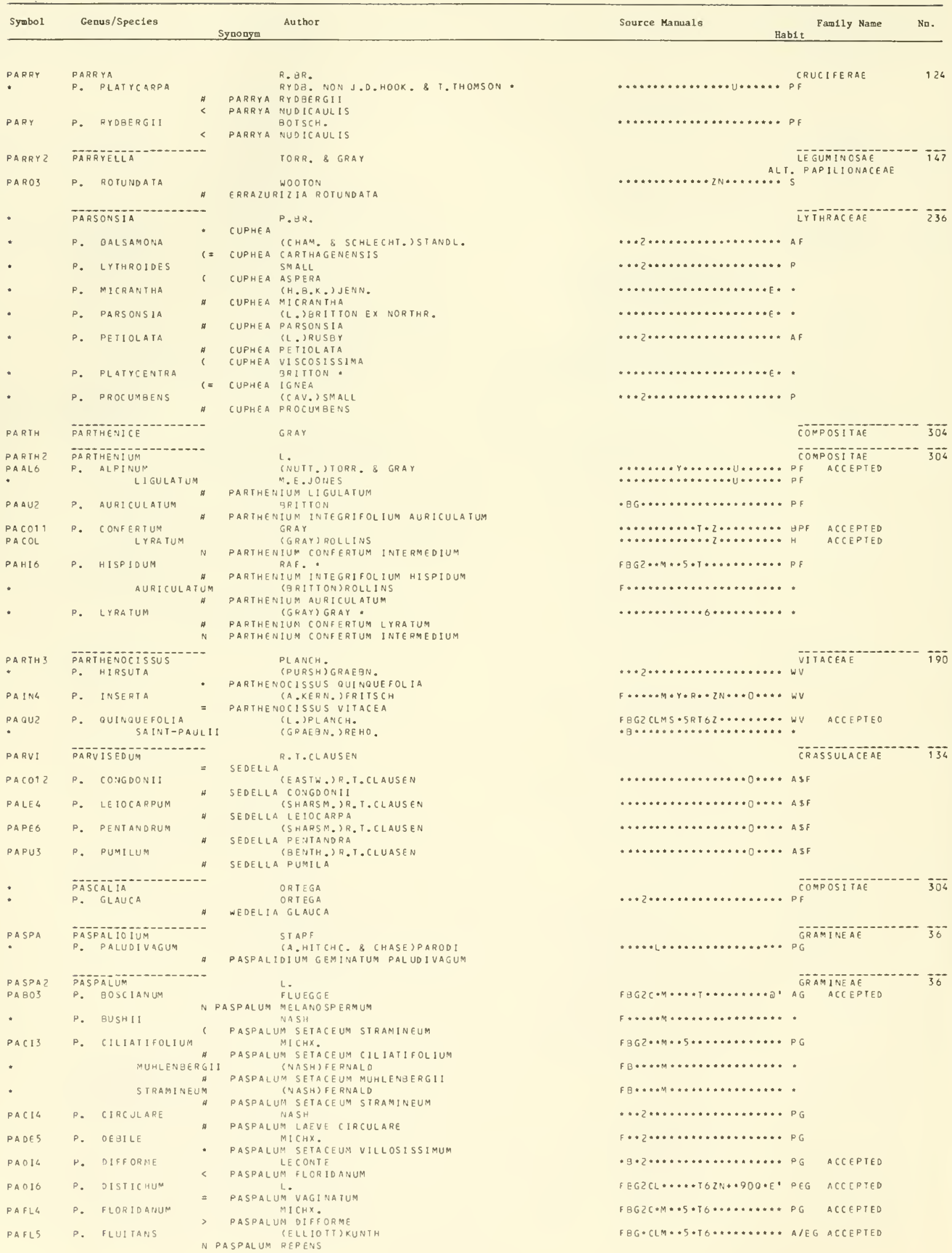




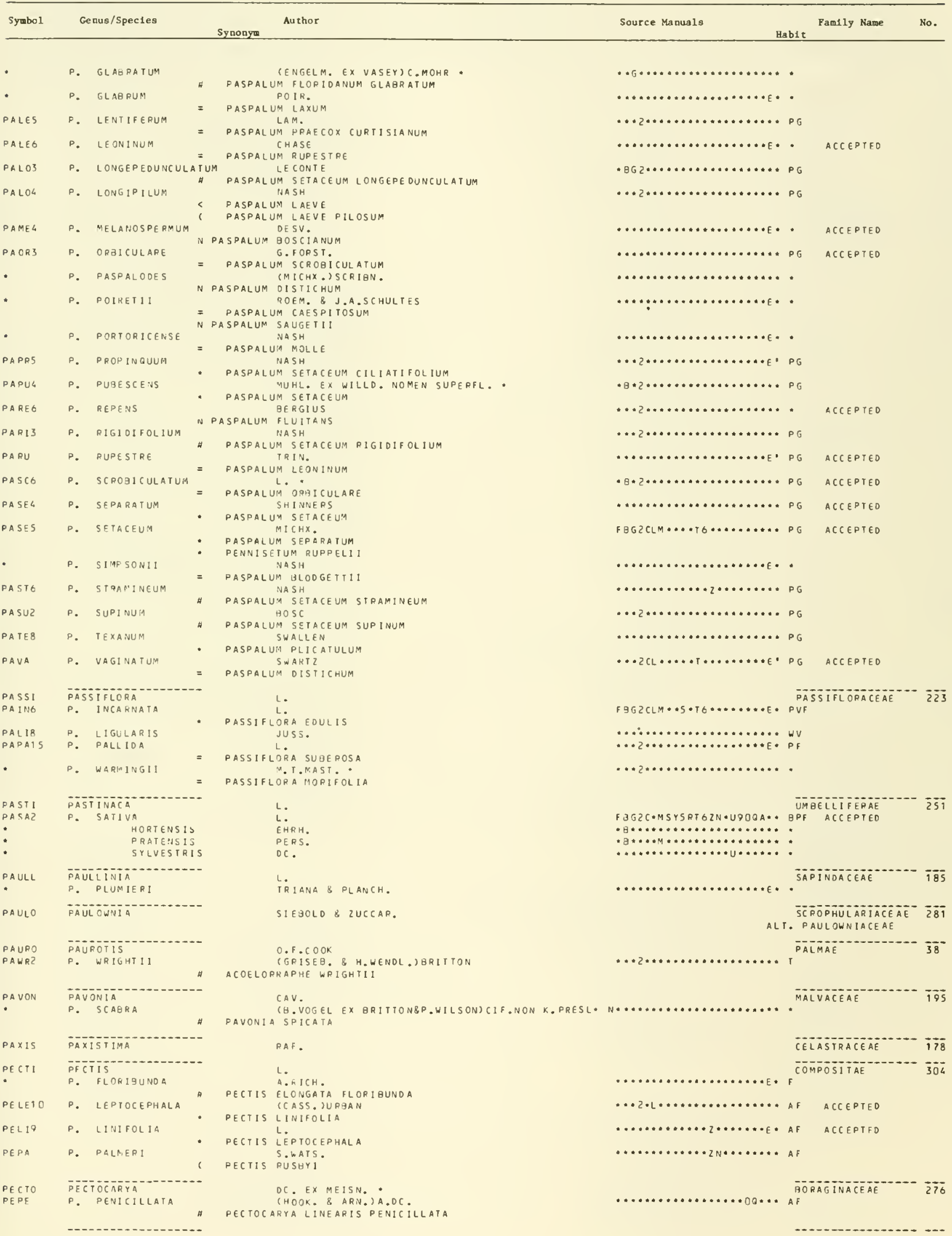




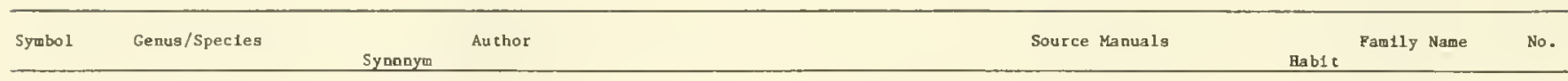

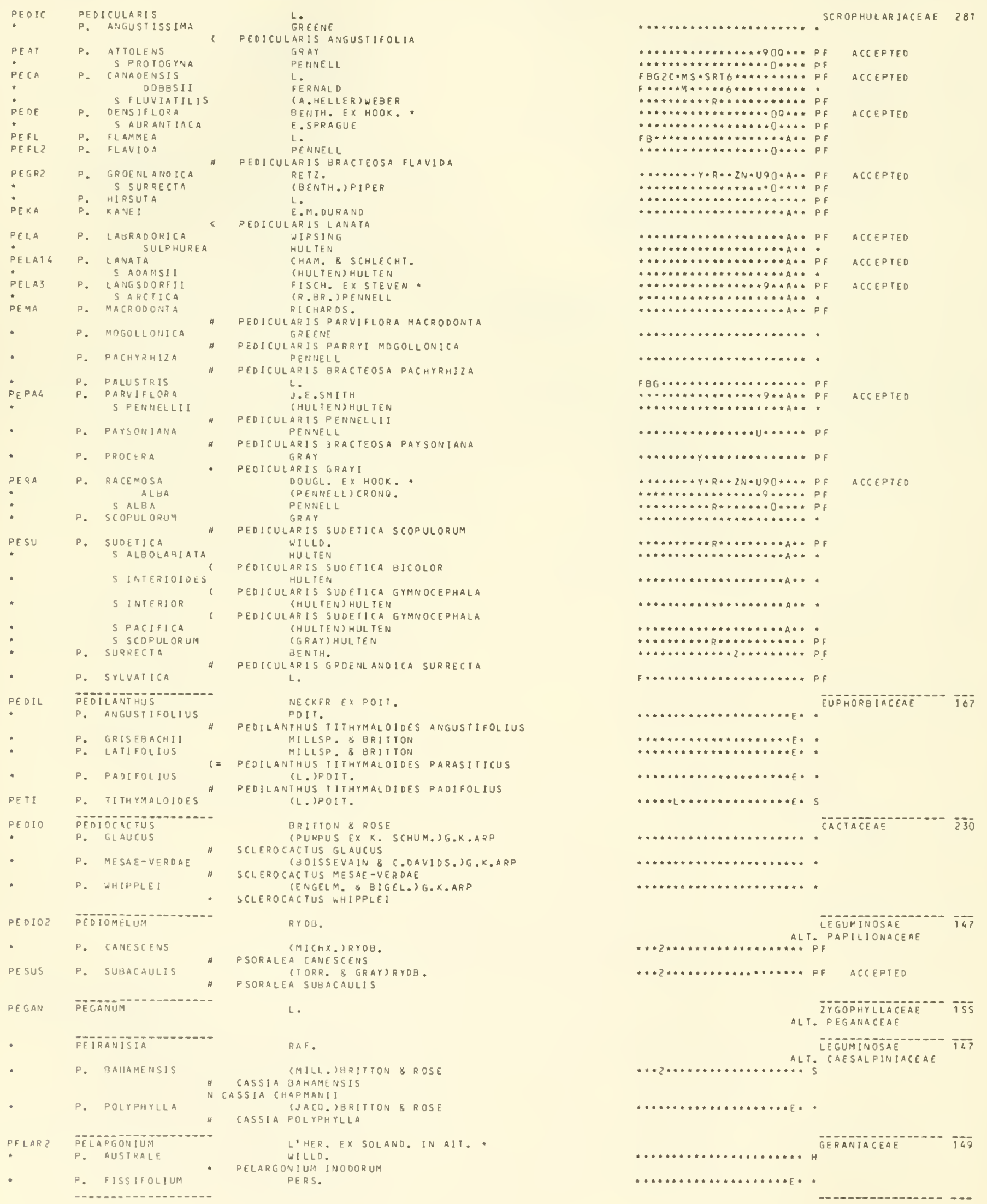


PELEA

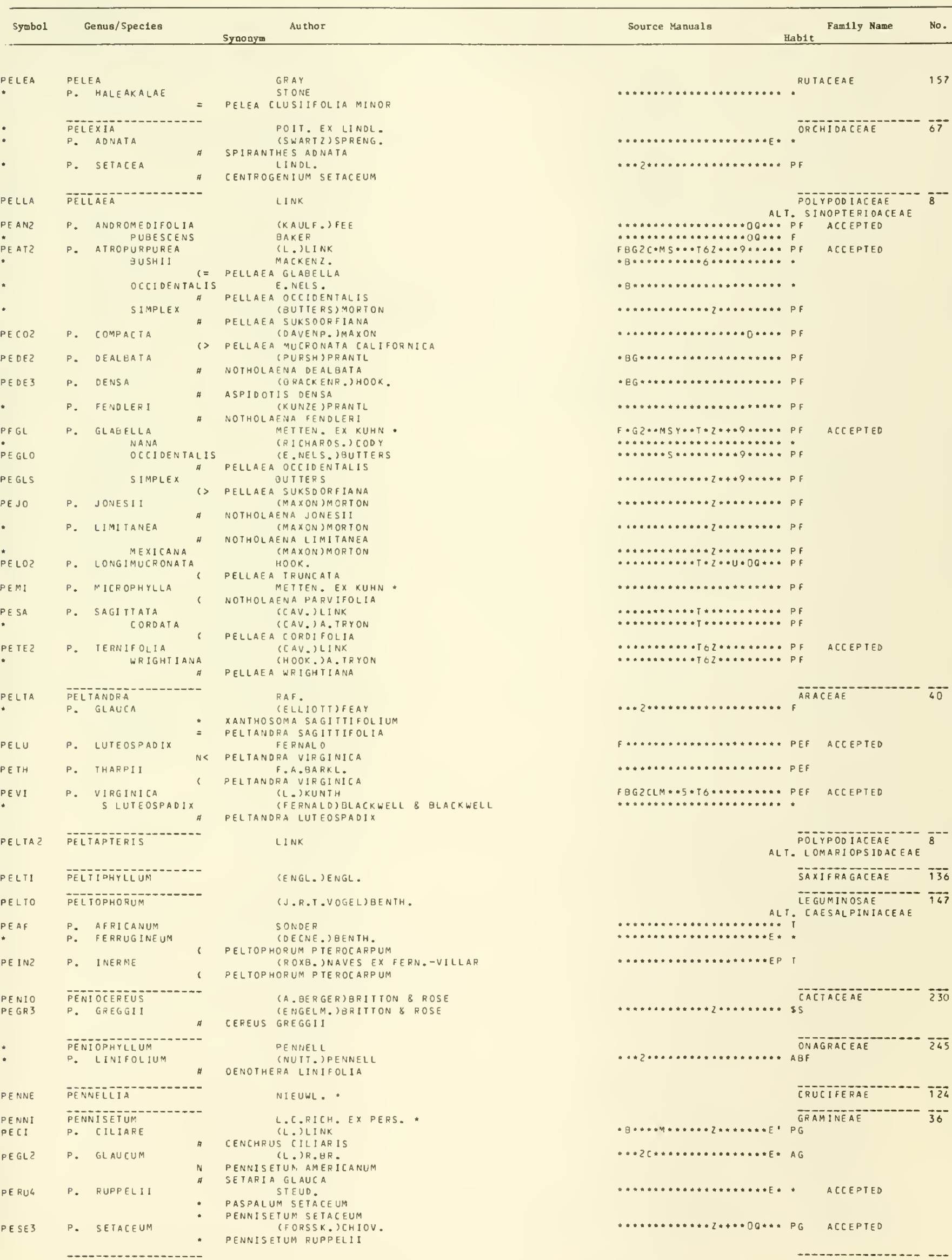




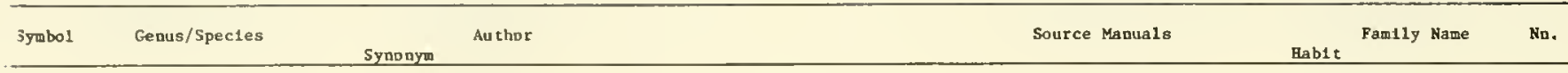

\begin{tabular}{|c|c|c|c|c|}
\hline \multirow{3}{*}{$\begin{array}{l}\text { PENST } \\
\text { PE AL4 }\end{array}$} & \multicolumn{3}{|c|}{ PENSTEMON } & \\
\hline & P. & ALLUVIORUM & & PENNELL \\
\hline & & & $>$ & PENSTEMON DEAMII \\
\hline PEANG & P. & $\begin{array}{l}\text { ANGUSTIFOLIUS } \\
\text { S CAUDATUS }\end{array}$ & & NUTT. EX PURSH : \\
\hline - & & S VENOSUS & & $\begin{array}{l}\text { (A. BELLER) D. KECK } \\
\text { D. KECK }\end{array}$ \\
\hline & & & \# & PENSTEMON VENOSUS \\
\hline PE ANS & P. & ANTIRRHINOIOES & & AENTH. \\
\hline - & & & H & KECKIELLA ANTIRRHINOIDES \\
\hline PEAR & P. & $\begin{array}{l}\text { S MICROPHYLLL } \\
\text { ARKANSANUS }\end{array}$ & & $\begin{array}{l}\text { (GRAY) D.KECK } \\
\text { PENNELL }\end{array}$ \\
\hline & & & $N<$ & PENS TEMON PALLIOUS \\
\hline & & & $>$ & PENSTEMON WHERRYI \\
\hline PEAT3 & P. & ATTENUATUS & & DOUGL. EX LINDL. * \\
\hline * & & MILITARIS & & (GREENE) CRONO. \\
\hline * & & PALUSTRIS & & (DEHNELL) CRONG. \\
\hline PEBAZ & & PSEUDOPRO & OCERL & (RYOB.) CROND. \\
\hline PE BAZ & P. & BARBATUS & & (CAV.)ROTH \\
\hline * & & PUBERULUS & & GRAY \\
\hline * & & TORREYI & & (BENTH.) GRAY \\
\hline 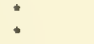 & & TRICHANDE & & GRAY \\
\hline - & P. & BRADEURII & & PURSH \\
\hline & & & r & PENSTEMON GRANDI FLORUS \\
\hline * & P. & BF AIN DE GE I & A & $\begin{array}{l}\text { (T.PORTER)T.PORTER EX RYOB. } \\
\text { PENSTEMON ALPINUS BRANOEGEI }\end{array}$ \\
\hline * & P. & BREVIBARBAIUS & & $\begin{array}{l}\text { PENSTEMON ALPINUS BRANOEGEI } \\
\text { CROSSWH. INEO. }\end{array}$ \\
\hline & & & r & PENSTEMON TRI FLORUS INIEGRIFOLIUS \\
\hline PE BRO & P. & BREVIFLORUS & & LINDL. \\
\hline * & & $S G L A B R I S E P A L$ & " & KECKIELLA BREVIFLORA \\
\hline PE BR1O & P. & $\begin{array}{l}\text { S GLABRISEPAL } \\
\text { BREVISEPALUS }\end{array}$ & LUS & $\begin{array}{l}\text { D. KECK } \\
\text { PENNELL }\end{array}$ \\
\hline & & & N & PENSTEMON PALLIDUS \\
\hline PE BR7 & P. & BR IDGESII & & GRAY \\
\hline * & & AMPLEXICA & AULIS & MONNET \\
\hline - & P. & BRITTONIORLM & & PENNEL L \\
\hline & & & $<$ & PENSTEMON CANESCENS \\
\hline PECAL & P. & $\begin{array}{l}\text { CAESPII OSUS } \\
\text { SUFFRUTIC }\end{array}$ & cosus & $\begin{array}{l}\text { NUTT, EX GRAY * } \\
\text { GRAY }\end{array}$ \\
\hline & & & H & PENSTEMON TUSHARENSIS \\
\hline PECAS & P. & CALCAREUS & & T. BRANDEG. \\
\hline & & & N & PENSTEMON PETIOLATUS \\
\hline$P E\{A\}$ & P. & CALYCOSUS & & SMALL \\
\hline & & & $\mathrm{N}$ & PENSTEMON DIGITALIS \\
\hline : & P. & CAMPANULATUS & & $\left(C A V_{.}\right) W I L L Q$. \\
\hline - & P. & CAUDATUS & & A. HELLER \\
\hline - & & & \# & PENSTEMON ANGUSTIFOLIUS CAUDATUS \\
\hline PECi3 & P. & $\begin{array}{l}\text { CINERASCENS } \\
\text { CINEREUS }\end{array}$ & & $\begin{array}{l}\text { GREENE } \\
\text { PIPER }\end{array}$ \\
\hline & & & 6 & PENSTEMON HUMILIS \\
\hline PECO4 & P. & COBAEA & & NUT T. \\
\hline 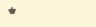 & & PURPUREUS & & PENNELL * \\
\hline PECO6 & P. & CONFERTUS & & DOUGL - \\
\hline - & & PROCERUS & & SOOUGL. EX R.GRAH.) COVILLE \\
\hline PECO 7 & P. & confusus & 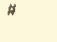 & $\begin{array}{c}\text { PENSTEMON PROCERUS } \\
\text { M.E.JONES }\end{array}$ \\
\hline - & & $S$ PATENS & & (M.E.JONES)D.KECK. \\
\hline & & & * & PENSTEMON PATENS \\
\hline - & P. & CONGESTUS & & (M.E. JONES) PENNELL \\
\hline PECO8 & & & * & PENSTEMON PACHYPHYLLUS CONGESTUS \\
\hline PECO8 & P. & CORDIFOLIUS & & BENTH. \\
\hline$P E C O Q$ & P. & CORYMBOSUS & 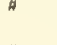 & $\begin{array}{l}\text { KECKIELLA CORDIFOLIA } \\
\text { BENTH. EXA.DC. }\end{array}$ \\
\hline & & & * & KEEKIELLA CORYMBOSA \\
\hline PECRS & P. & CRAN OALLII & & A. NELS. \\
\hline - & & $S \times Y L U S$ & & A. NELS. \\
\hline & & & 6 & PENSTEMON CRANDALL $I$ PROCUMBENS \\
\hline PECYZ & $P \cdot$ & CYANAN THUS & & HOOK. \\
\hline PEOAZ & P. & $\begin{array}{l}\text { S LONGIFLORUS } \\
\text { OAVIOSONII }\end{array}$ & & $\begin{array}{l}\text { PENNELL } \\
\text { GREENE }\end{array}$ \\
\hline$\cdot$ & & MENZIESII & & (D.KECK) CRONG. \\
\hline * & & PRAETERIT & & CRUND. \\
\hline PEDE 10 & P. & DEAMII & & PENNELL \\
\hline & & & $<$ & PENSTEMON ALLUVIORUM \\
\hline PEDE 6 & P. & OEUSTUS & & DOUGL. EX LINOL.. \\
\hline & & HE TERANDE & & (TORR. \& GRAY) [RONG. \\
\hline - & & VARIAB ILI & & (SUKSD.) CRONG. \\
\hline - & P. & OIFFUSUS & & DOUGL. EX LINDL. • \\
\hline PED! & P. & & 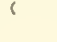 & $\begin{array}{c}\text { PENSTEMON SERRULATUS } \\
\text { NUTT. }\end{array}$ \\
\hline & & D.GITALIS & N & PENSTEMOA CAL YCOSUS \\
\hline$P \subseteq E A$ & P. & EATONII & & GRAY \\
\hline * & & UNDOSUS & & M.E.JONES \\
\hline PFFR 3 & P. & FRUTICOSUS & & (PURSH) GREENE \\
\hline PEFRS & & SCOULEKI & & (LINOL.) (RONG. * \\
\hline PEFRS 2 & & SERRATUS & & (D. KECK) CRONO. \\
\hline PEGA & P. & GAIKONER: & & HOOK. \\
\hline - & & OREGANUS & & GRAY \\
\hline PE GL4 & P. & GLANDULOS US & & DOUGL.: \\
\hline * & & CHELANEISS & is & (D. KECK) CRONO. * \\
\hline PE GRS & P. & GRACILIS & & NUTT. \\
\hline - & & WI SCONENS & is & (PENNELL) FASSETT \\
\hline - & P. & GRIFFIIJII & & A. NELS. \\
\hline & & & $\zeta=P E$ & ENSTEMON OLIGANTHUS \\
\hline * & P. & HELLER I & & SMALL \\
\hline & $P$ & & 6 & PENSTEMON TRIFLORUS INTEGRIFOLIUS \\
\hline 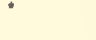 & P. & HESPERIUS & 6 & $\begin{array}{r}\text { M. E.PECK } \\
\text { PENSTEMON RYDBE }\end{array}$ \\
\hline PE HE 3 & P. & HE TEROPHYLLUS & & $\begin{array}{l}\text { PENSTEMON RYDBERGII } \\
\text { LINDL. }\end{array}$ \\
\hline
\end{tabular}

CROPHULARIACEAE 281

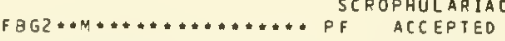

$\# * \# * * S Y S R * * 2 N * U * * * * * * P F$

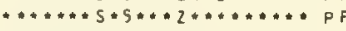

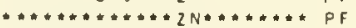

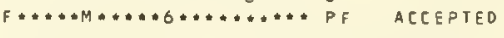
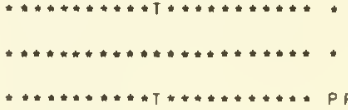

$\cdots \cdots \cdots \cdots \cdots \cdots \cdots \cdots+\cdots \cdots$

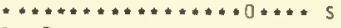

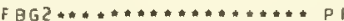

$\cdots * \cdots \cdots * * * 2 \mathrm{~N} * \mathrm{U} * \mathrm{OQ} \cdots *$ HPF

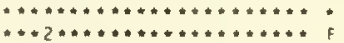

$\# * * * *+Y * *+2 N * U * * \cdots * P F$

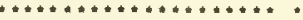
ACCEPTED

$* * * * * * \cdots * * * * * 00 * * * b=$

$F B G 2 * * M * \ldots \ldots \ldots+\cdots \cdots \cdots * P F$

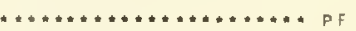

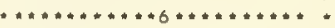

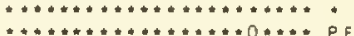

$F B G * * M * * S * T 62 N * U * * * * * P F$

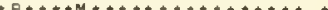

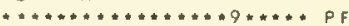

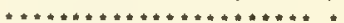

$\cdots \cdots+\ldots+\cdots+\ldots+\cdots U * O Q \cdots+P F$

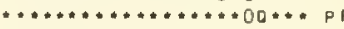

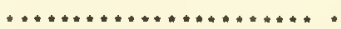

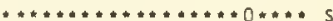

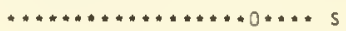

$\ldots \ldots \ldots \ldots \ldots \ldots \ldots+U_{* \ldots \ldots} \ldots p F$

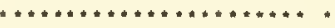

$\omega * * * * * * Y * \ldots \ldots * * U * * * * * * P F$ $\ldots+\ldots \ldots+\ldots$ $\cdots \ldots \ldots \ldots \ldots \ldots+\cdots \cdot 9+\ldots, P F H$

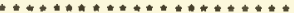

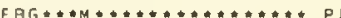

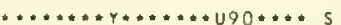
$* * * * * * * * * * * * * * 9 * * * *$ HPF

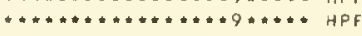

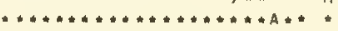

$F B G 2 C * M * * 5 * 16 * \cdots \cdots+\cdots * * P F$

$* * * * * * * * \cdots Z N \cdot U * 00 * * P P F$

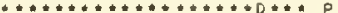

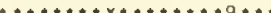

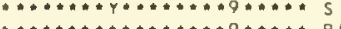
0.00a

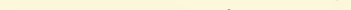

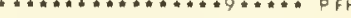

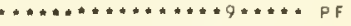

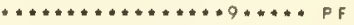
$F B G * \cdots+5 Y+R \cdots \cdots+9 \cdots \cdots * P F$ $F B G+\ldots \ldots+\ldots \ldots \ldots+\ldots \ldots+\ldots ;$ ACCEPTEO ACCEPTED

ACCEPTED

ACEEPTED

ACCEPTED

ACCEPTED ACCEPTED

ACCEPTED ACCEPTED ACCEPTED ACCEPTED ACGEPTED ACCEPTED ACLEPIED ACCEPTED

ACEEPTEO ACCEPTED ACCEPTED

ACEEPTED ACCEPTED ACCEPTED 


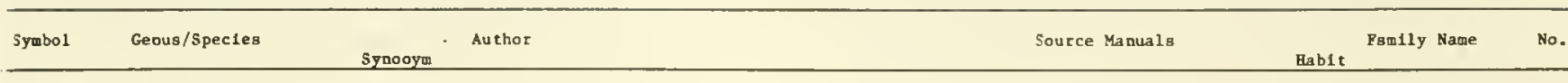

- austral is

PEHI P. HIRSUTUS

- p. holmgreni

PEJA P. JAMESII

5 BREVICULUS

PELAT

PELAR P. LAETUS ROEZLII

PELAB

PELE

PELI2 P. LINARIOIOES

- 5 TAOSENSIS

* 5 VIRIDIS

P. MENZIESII

\section{- SDAVIOSONII}

PEMI

PEMI 3

PEMO3

PEMOS

PEMOL

PE MU 8

PENE

PENI3

PEOR3

PEOR

P

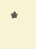

-

PEPE 13

PE PRZ

:

$:$

:

PE PU1 3

PER I

-

PERO

*

PERY

Pe suz

PETES

- 5 SEPTENTrionalis

P. TOLMIEI 5 BRACHYANTHUS
5 FORMOSUS

S FORMOSUS
P. TOREEYI

P. UTAHEHSIS

P. VARIABILIS

P. VENOSUS

P. VIRGATUS

S ARIZONICUS

PEWH 3 P. WHERKYI

PENTAZ
MUNZ \& I.JOHNST.

(L. IWILLD.

PENSTEMON LAEVIGATUS

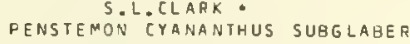

$$
\text { BENTH. }
$$

PENSTEMON BREVICULUS

GRAY
(REGEL) JEPS.

SOLAND

PENSTEMON HIRSUTUS

GRAY

KECKIELLA LEMMONI: GRAY

D. KECK

PENSTEMON CRANDALLII TAOSENSIS (D. KECK) O.KECK EX A.HOLMGR. \& REVEAL INED. "

PENSTEMON DAVIDSONII MENZIESII

PENSTEMON FRUTI COSUS
(GREENE) PIPER

PENSTEMON DAVIOSONI GRAY KECKIELLA ANTIRRHINOIDES MICROPHYLLA

N PENSTEMON BARNEGYI

N PENSTEMON JANISHIAE

$$
\begin{aligned}
& \text { EASTW. } \\
& \text { D. KECK }
\end{aligned}
$$

PENSTEMON MAR[USI]

GREENE

(D.KECK) CRONO. PENNELL

PENSTEMON ARKANSANUS

(DOUGL. EX LINOL.) TRAUTV.

NOTHOCHELONE NEMOROSA

DOUGL. EX BENTH.

(PENNELL) CRONO.

GREENE

PENSTEMON RYDBERGI

SMALL

PENSTEMON BREVISEPALUS

PENSTEMON ARKANSANUS

BUCKLEY
PENSTEMON PALLIDUS

MLN.)MACIMIL.

PENSTEMON LAEVIGATUS

T. BRANDEG.

PENSTEMON CALCAREUS

DOUGL. EX R.GRAH.

(M. E. JONES)A.NELS.

(PENNELL )CRONO.

(PENNELL) CRONO.

(A. NELS.) CRONO
(HOOK. I CRONO.

(HOOK.) D.KECK

LINDL.

PENSTEMON CAMPANULATUS

A. NELS.

PENSTEMON VIRGATUS

DOUGL. EX LINDL.

(D. RECK) CRONO.

(D.KECK) CRONO.

GRAY

KECKIELLA ROTHROCKII

(ABRAMS) D.KECK

A. NELS.

(PENMELL)D.KECK

PYO3.

PENSTEMON UTAHENSIS

PENSTEMON [RANDALLI]

TORR. EX GRAY *

KECKIELLA TERNATA

(MUNZ \& 1.JOHNST.) D.KECK

HOOK.

MON PROEERUS TOLMIE

(PENNE LL) D.KECK

(A. NELS.) D.KEC

BENTH.

PENSTEMON BARBATUS TORREY I

EAST'S.

PENSTEMON SUBGLABER

SUKSD.

PENSTEMON DEUSTUS VARIABILIS

(D. KECK) REVEAL .

PENSTEMON ANGUSTIFOLIUS VENOSUS GRAY

PENSTEMON PUTUS

(GRAY) D.KECK

PENSTEMON DEAVER I

PENNELL.

PENSTEMON ARKANSANUS

TAUSCH

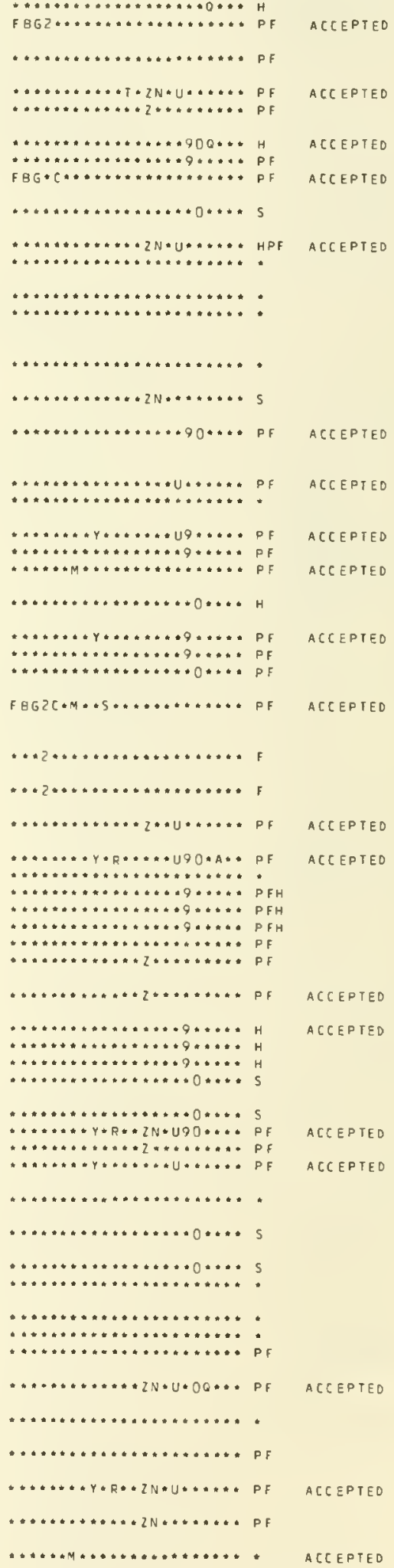

ACLEPTED 


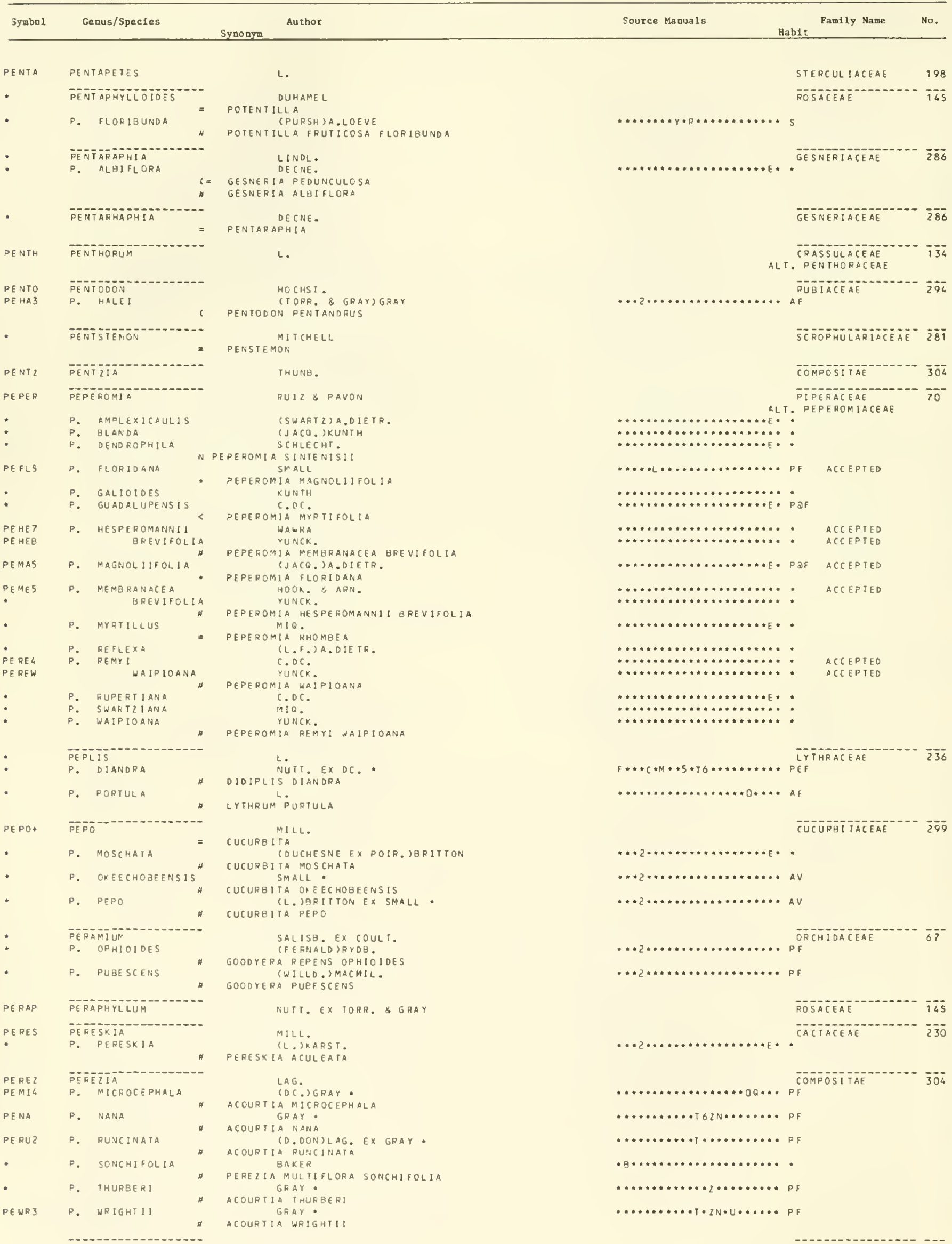




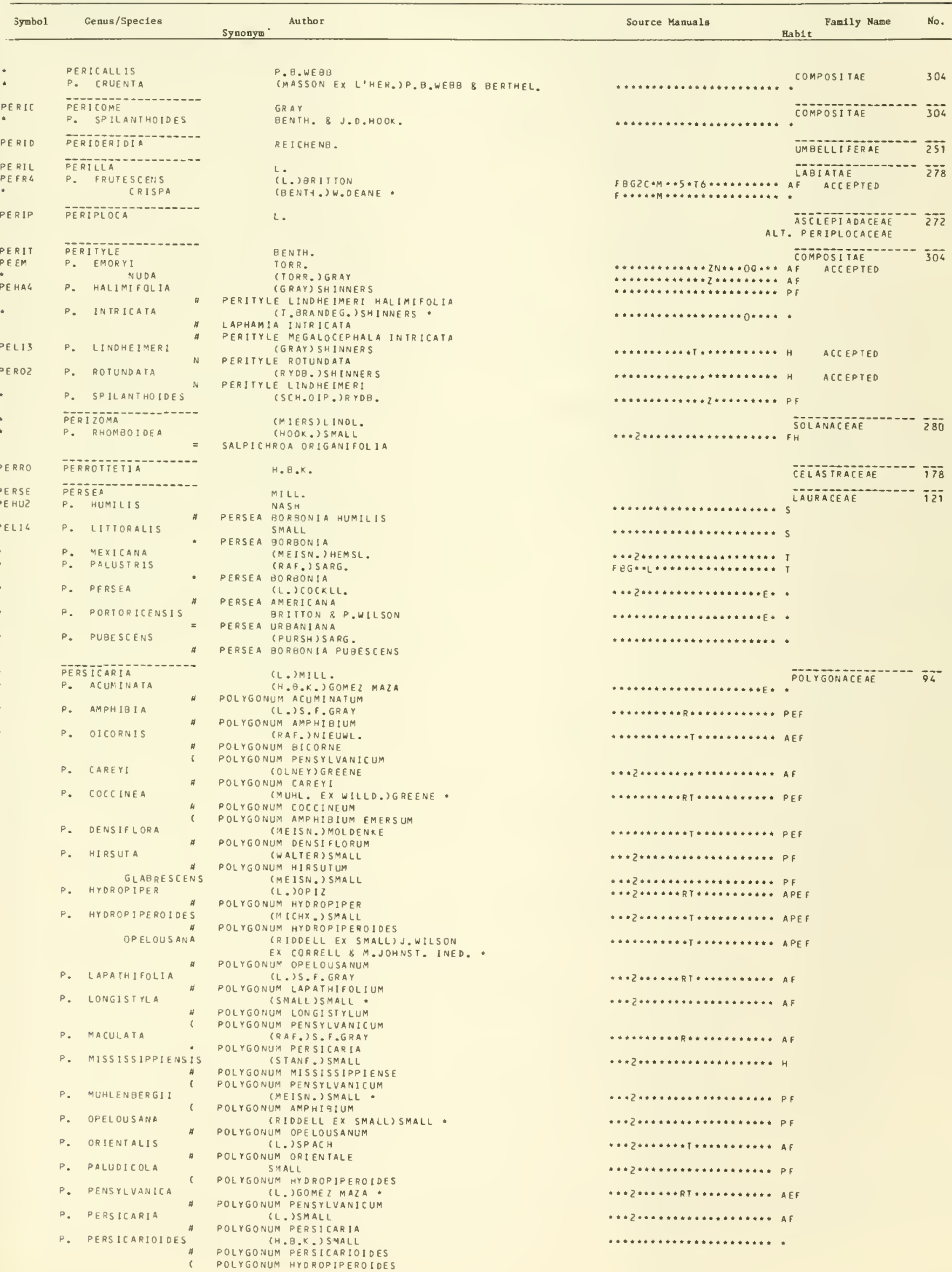




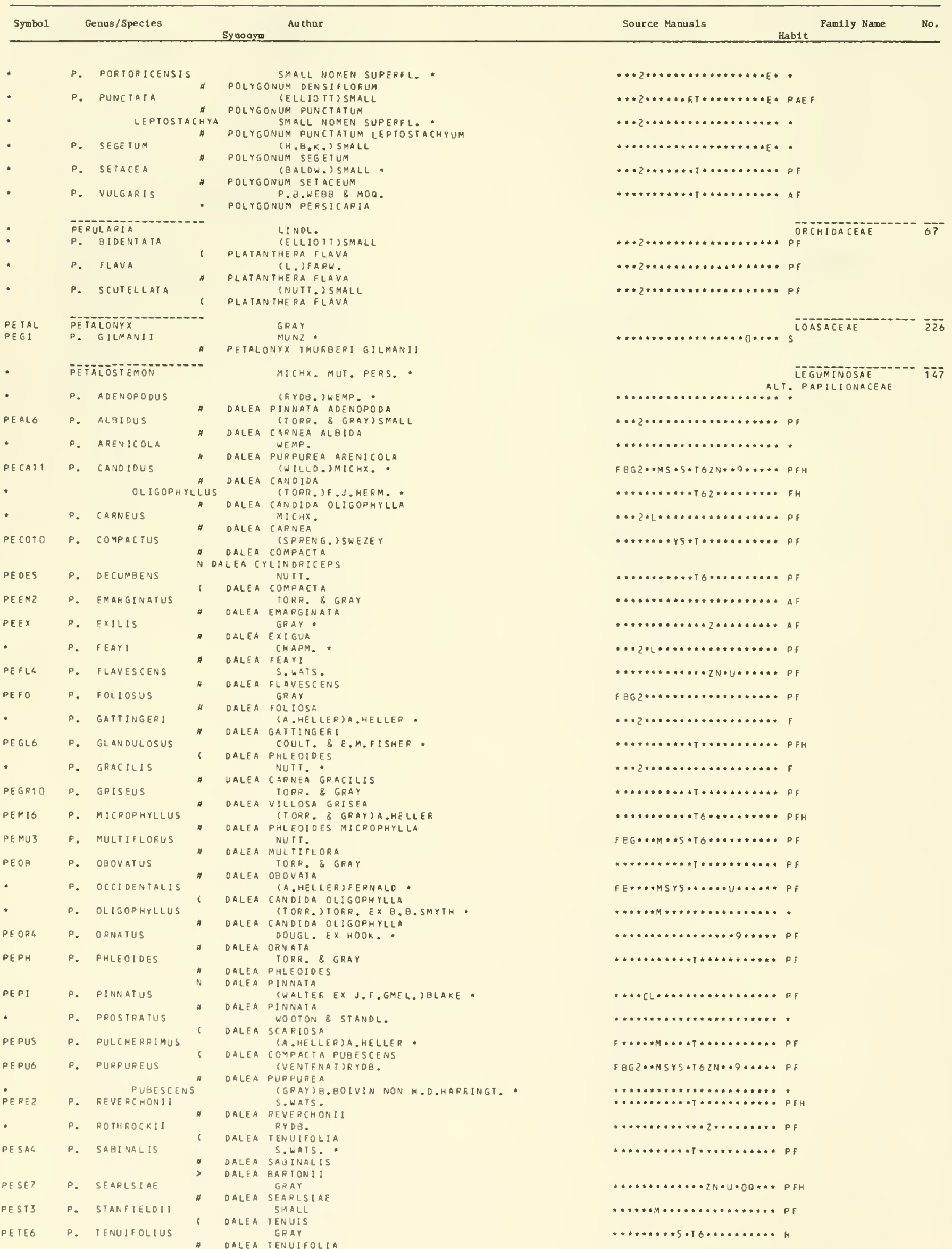




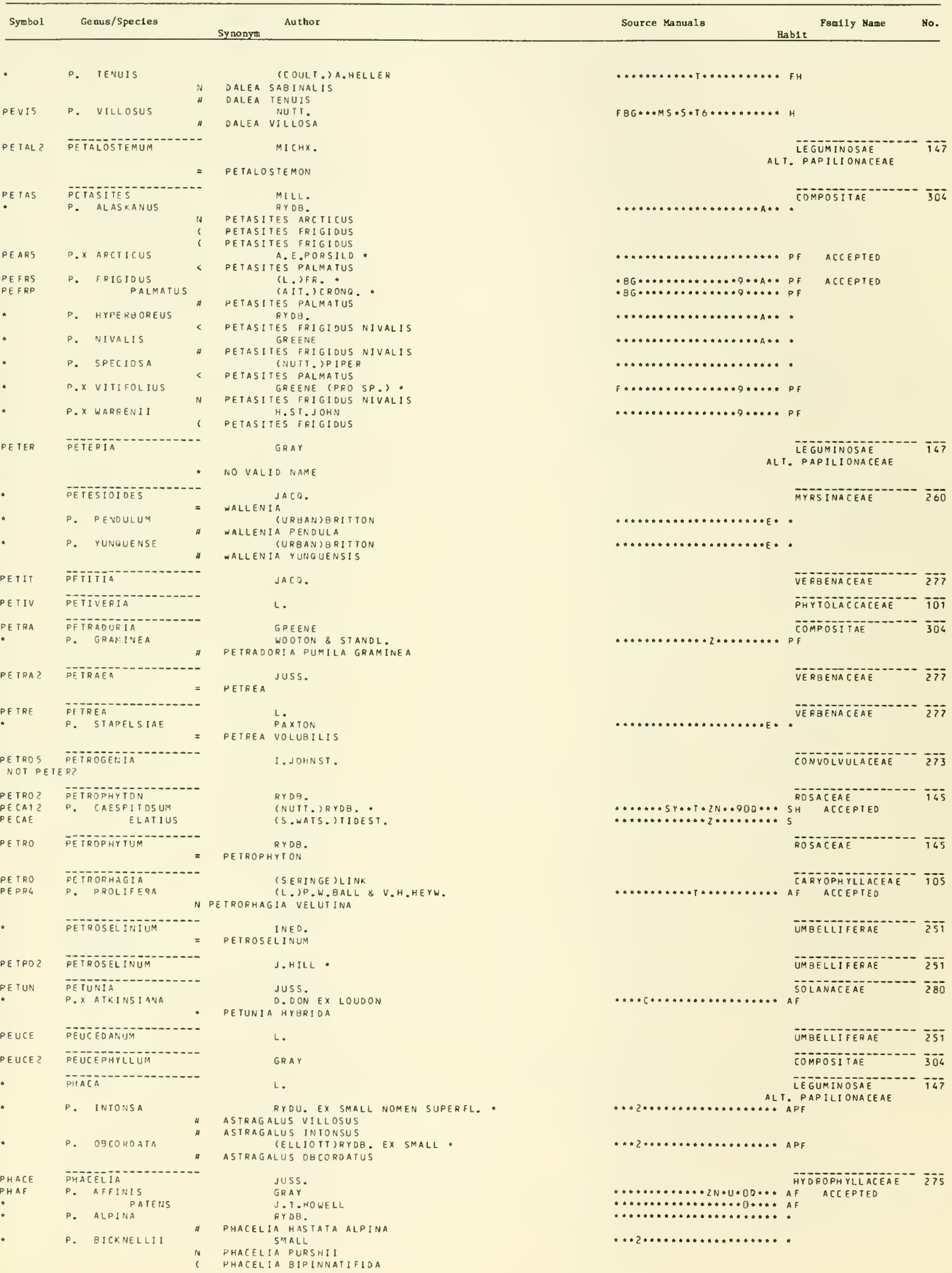




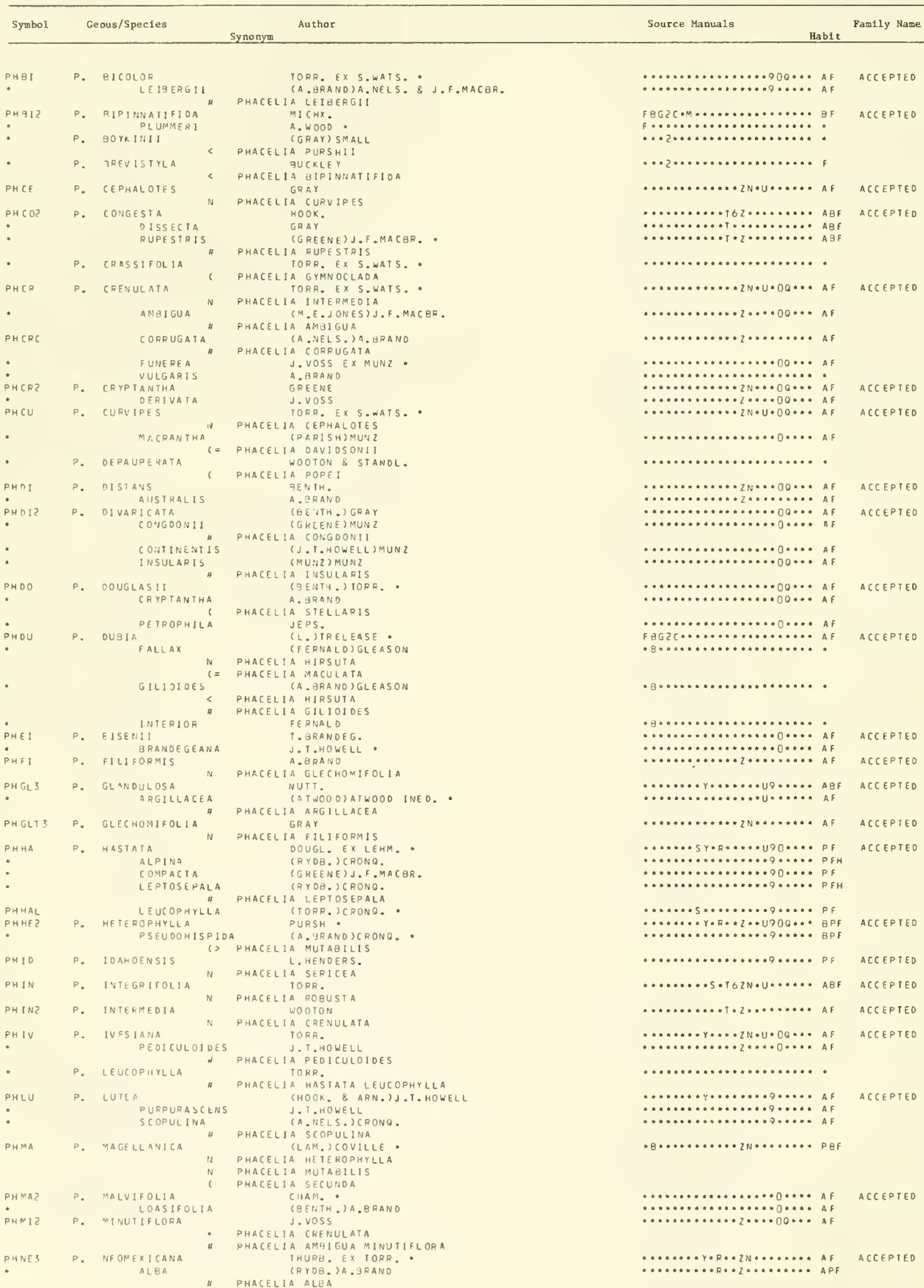


PHACELIA

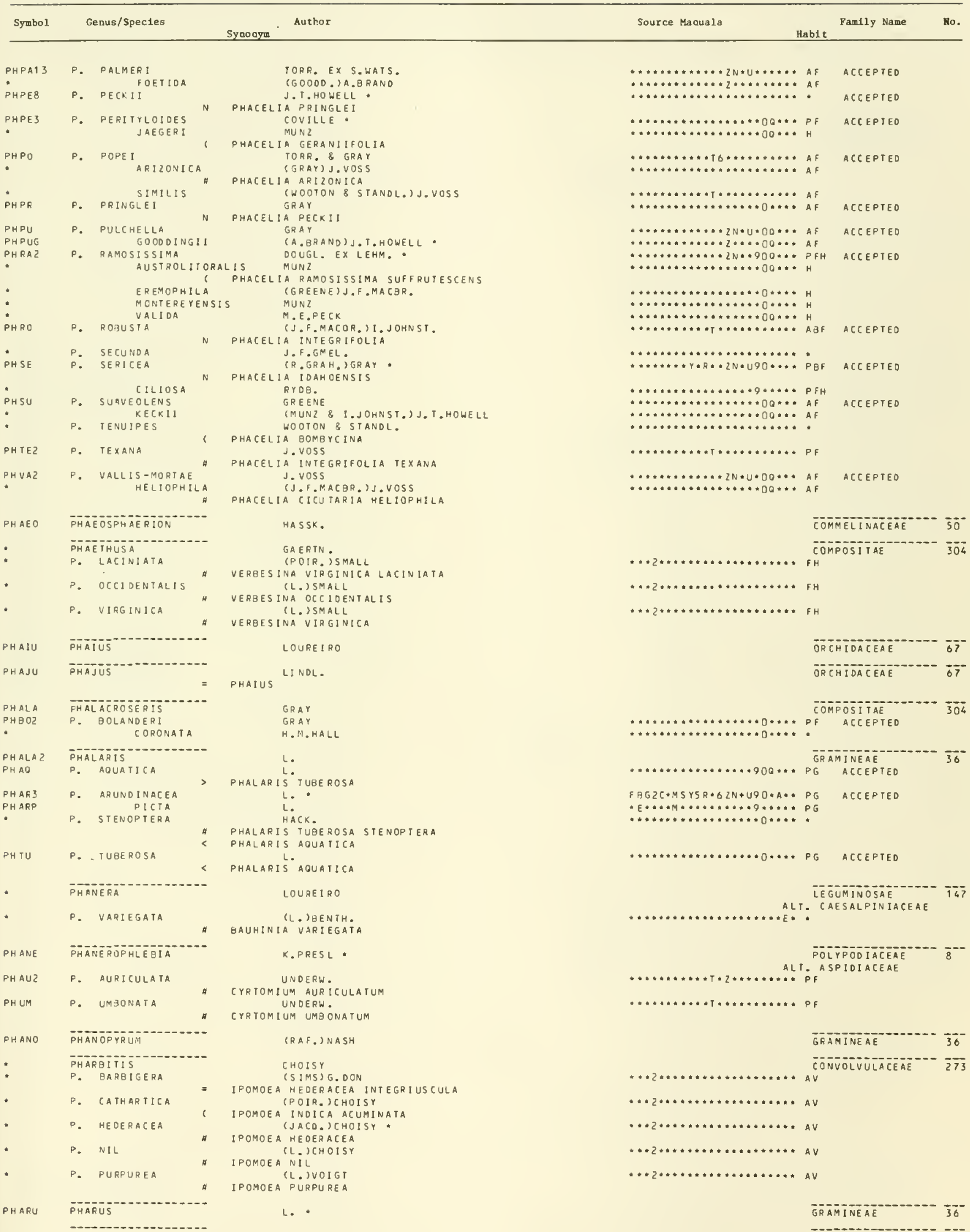




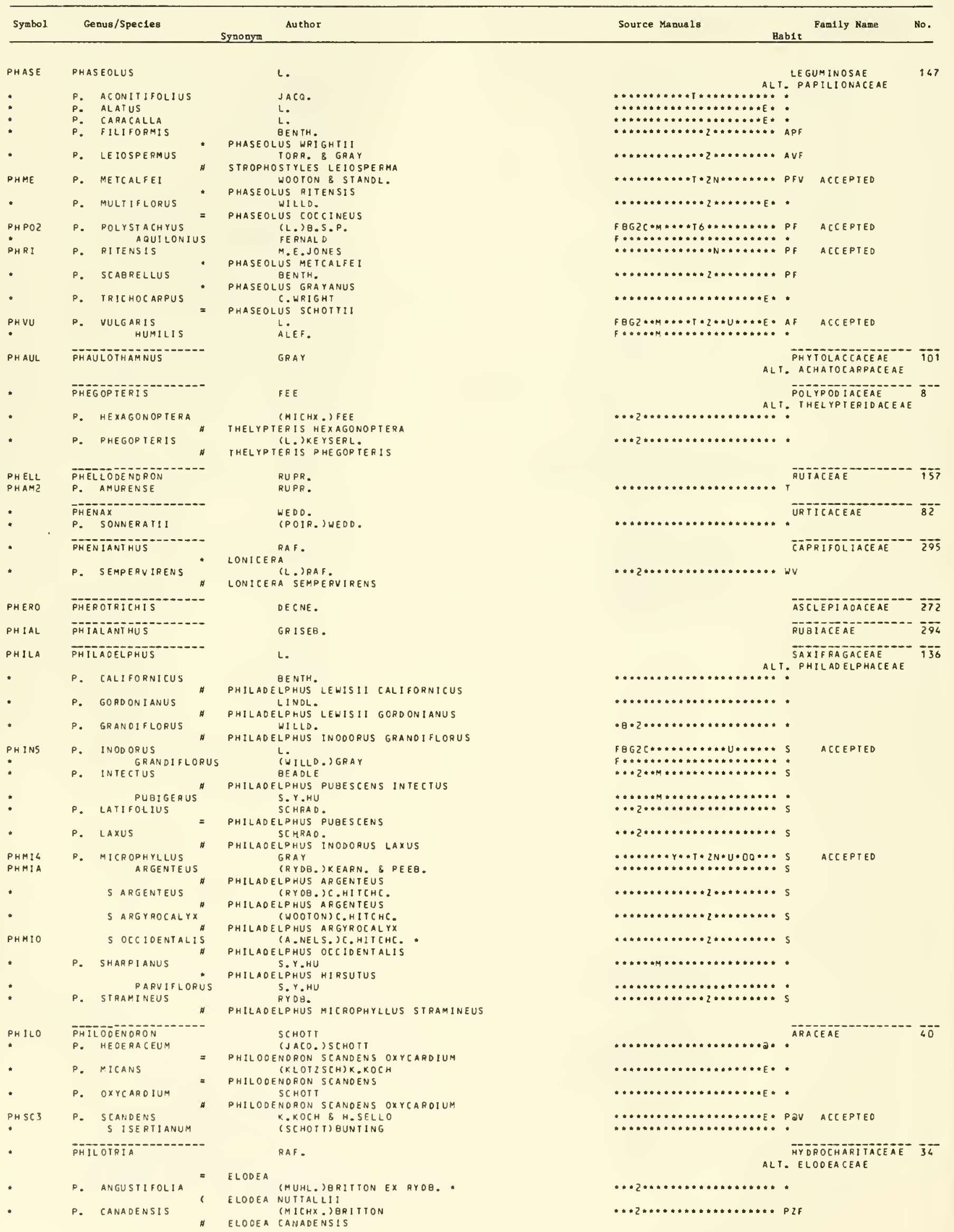




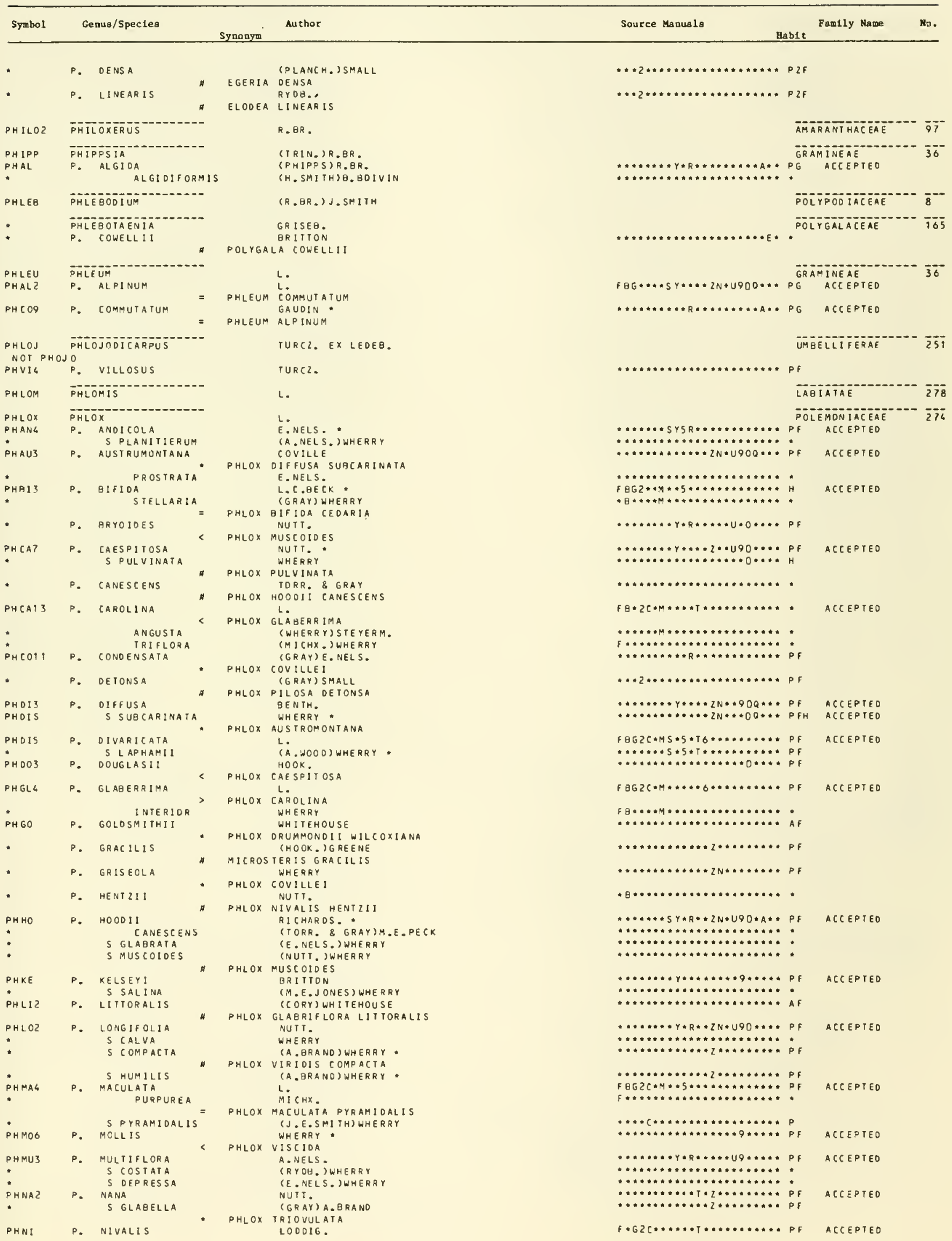




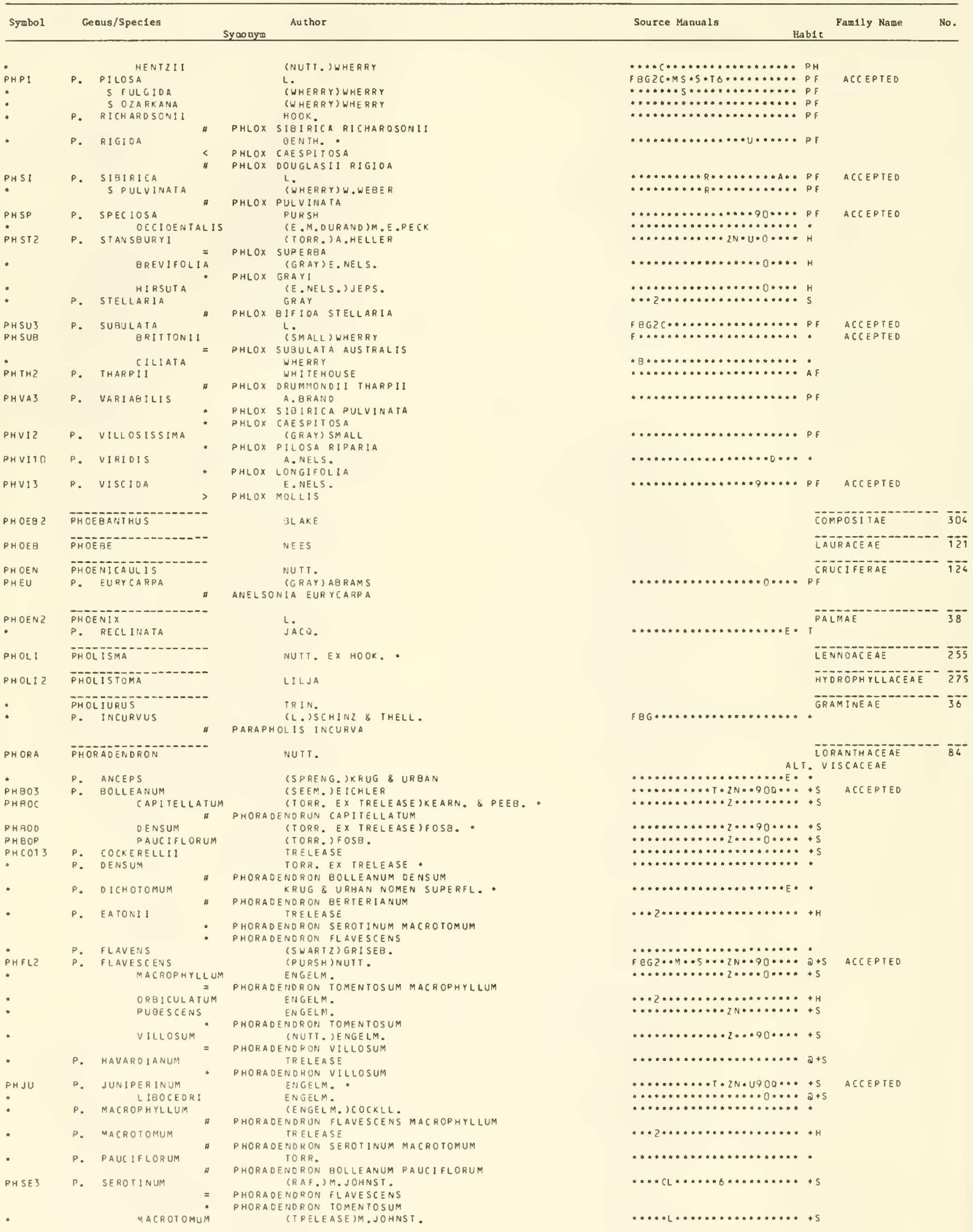




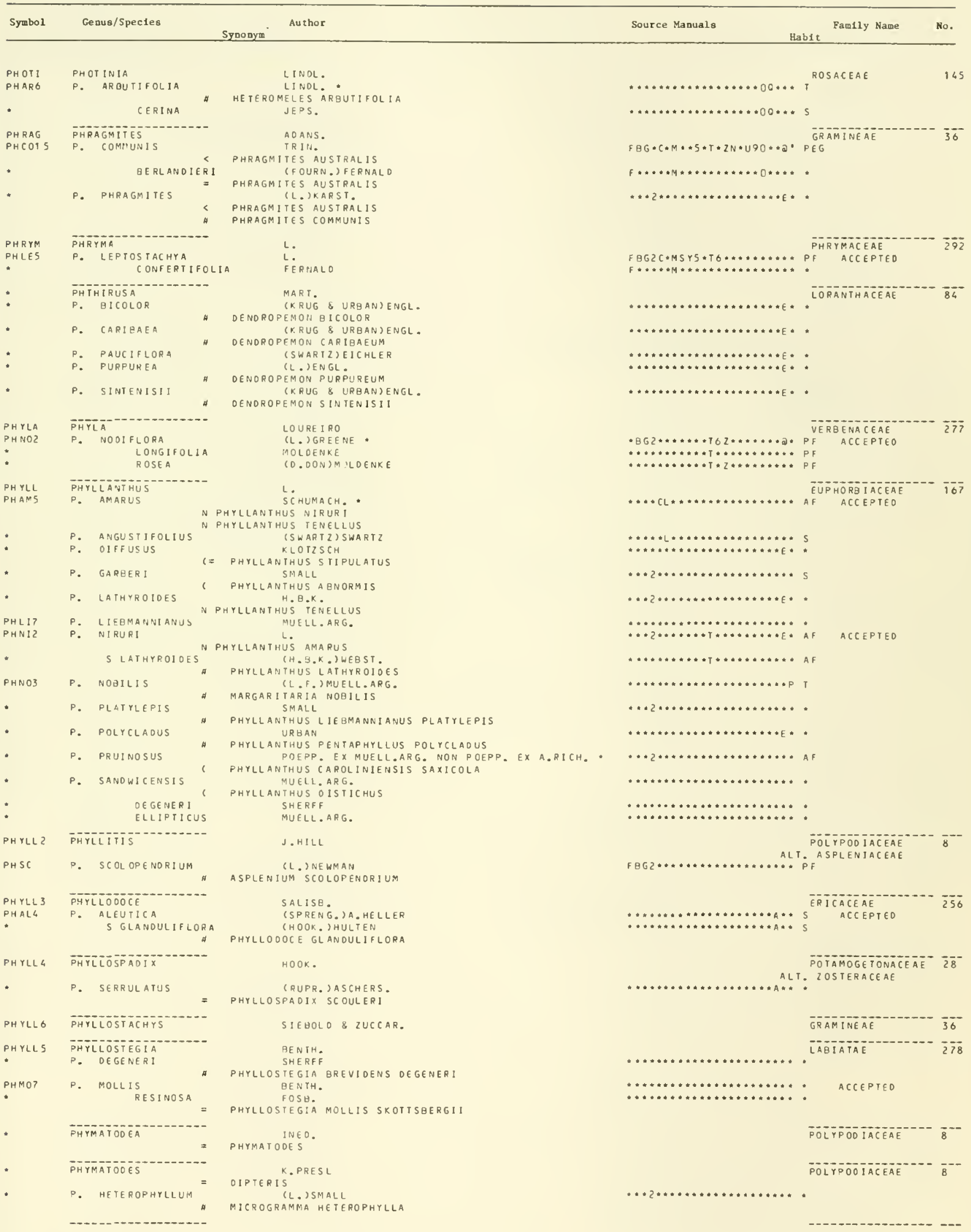




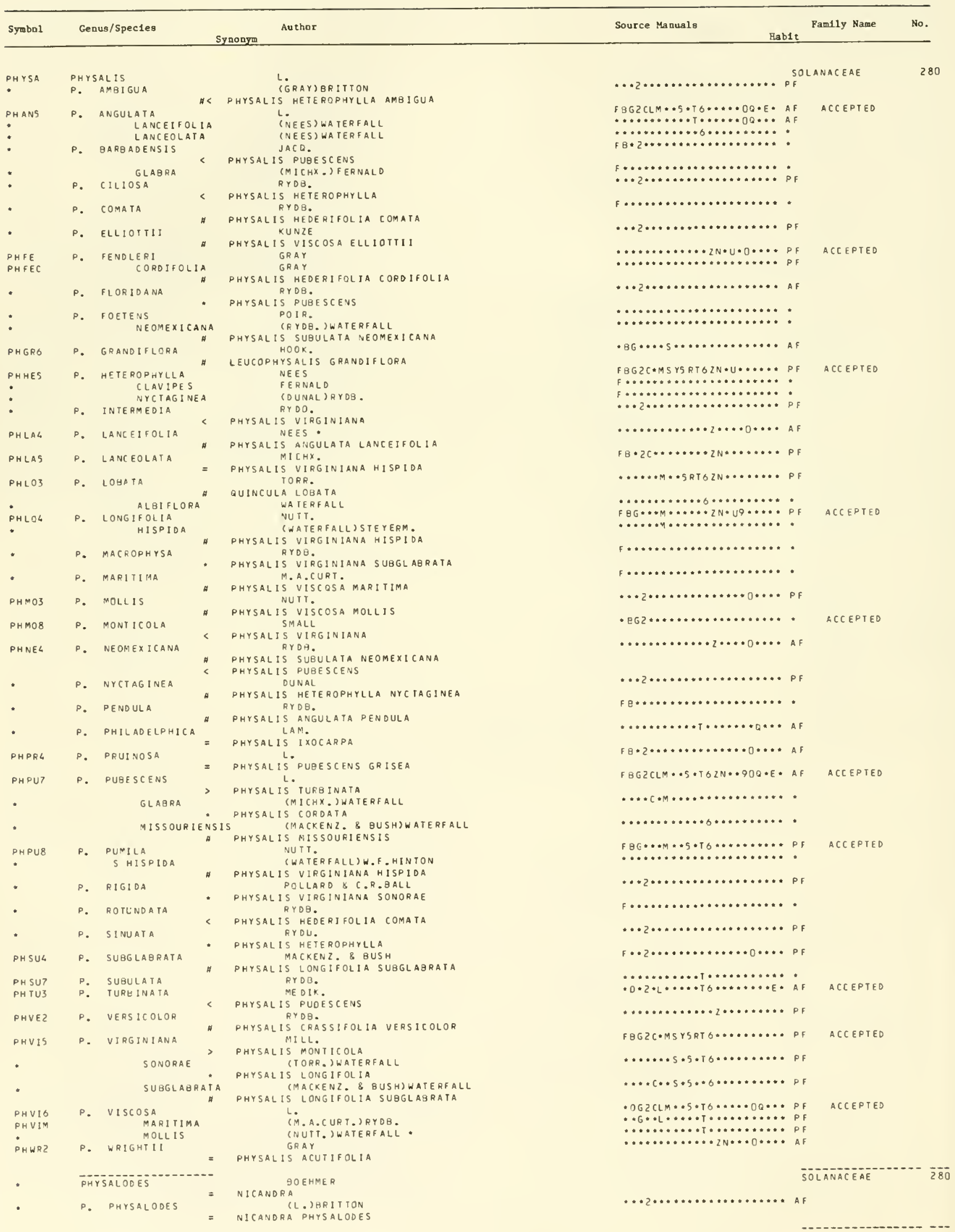




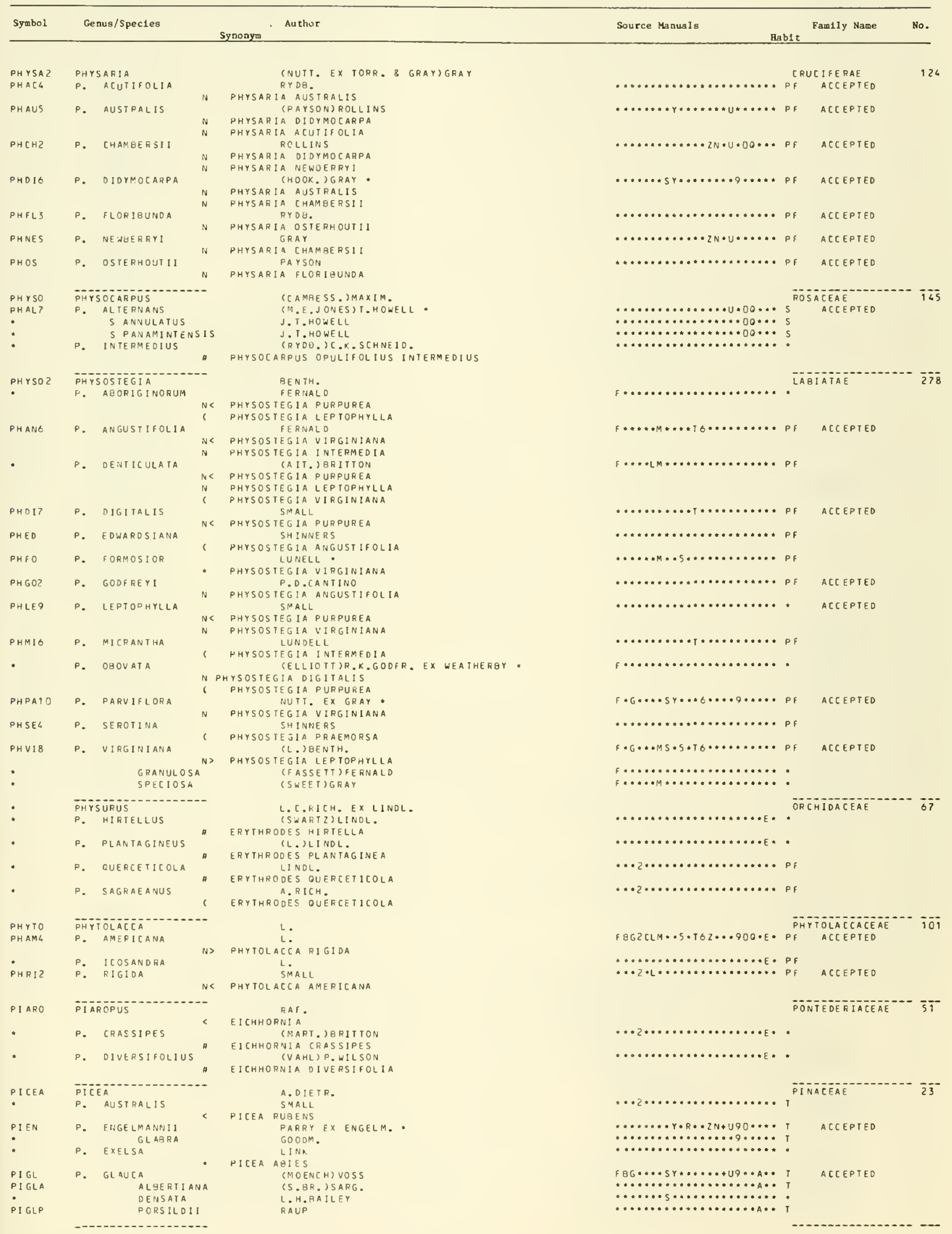




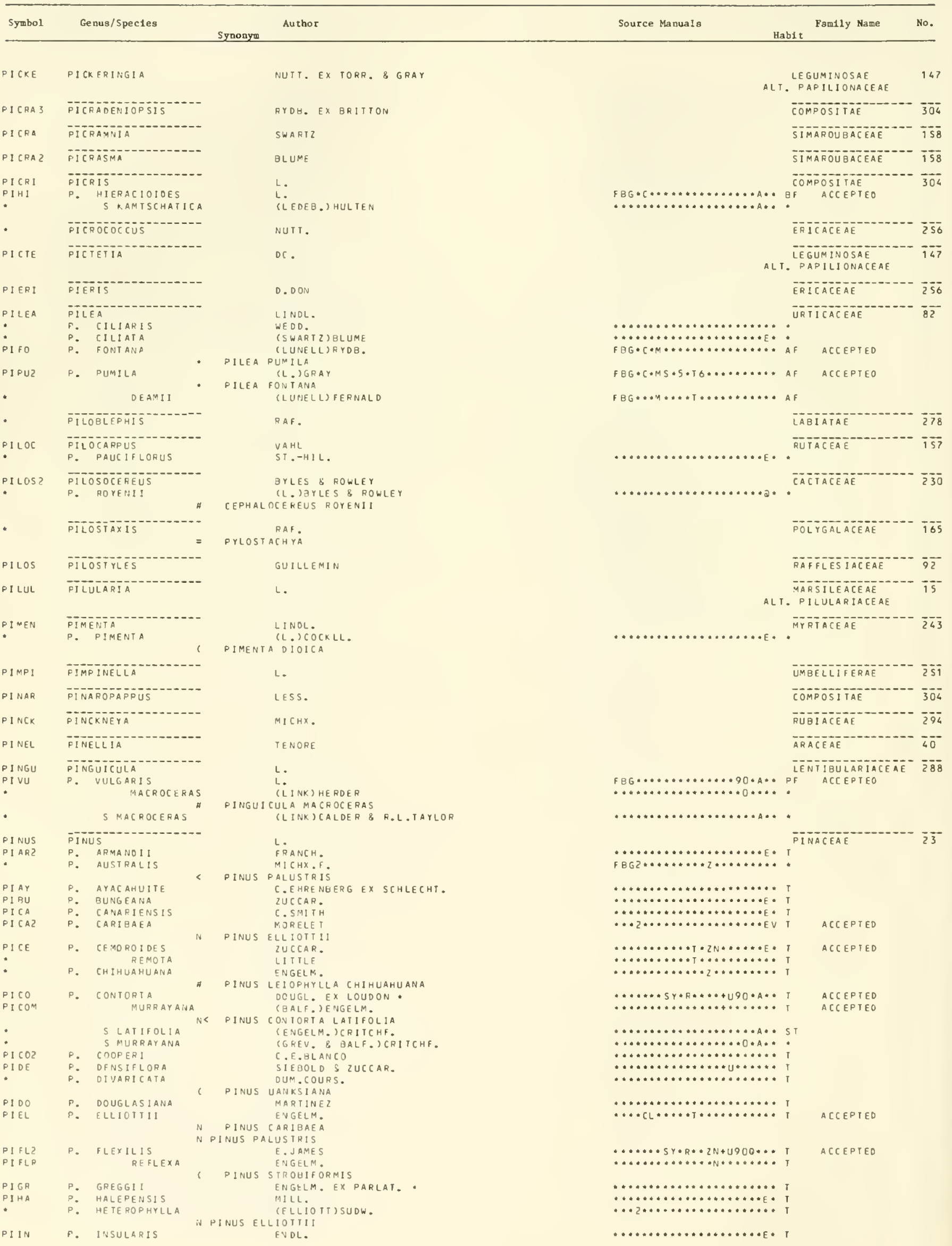




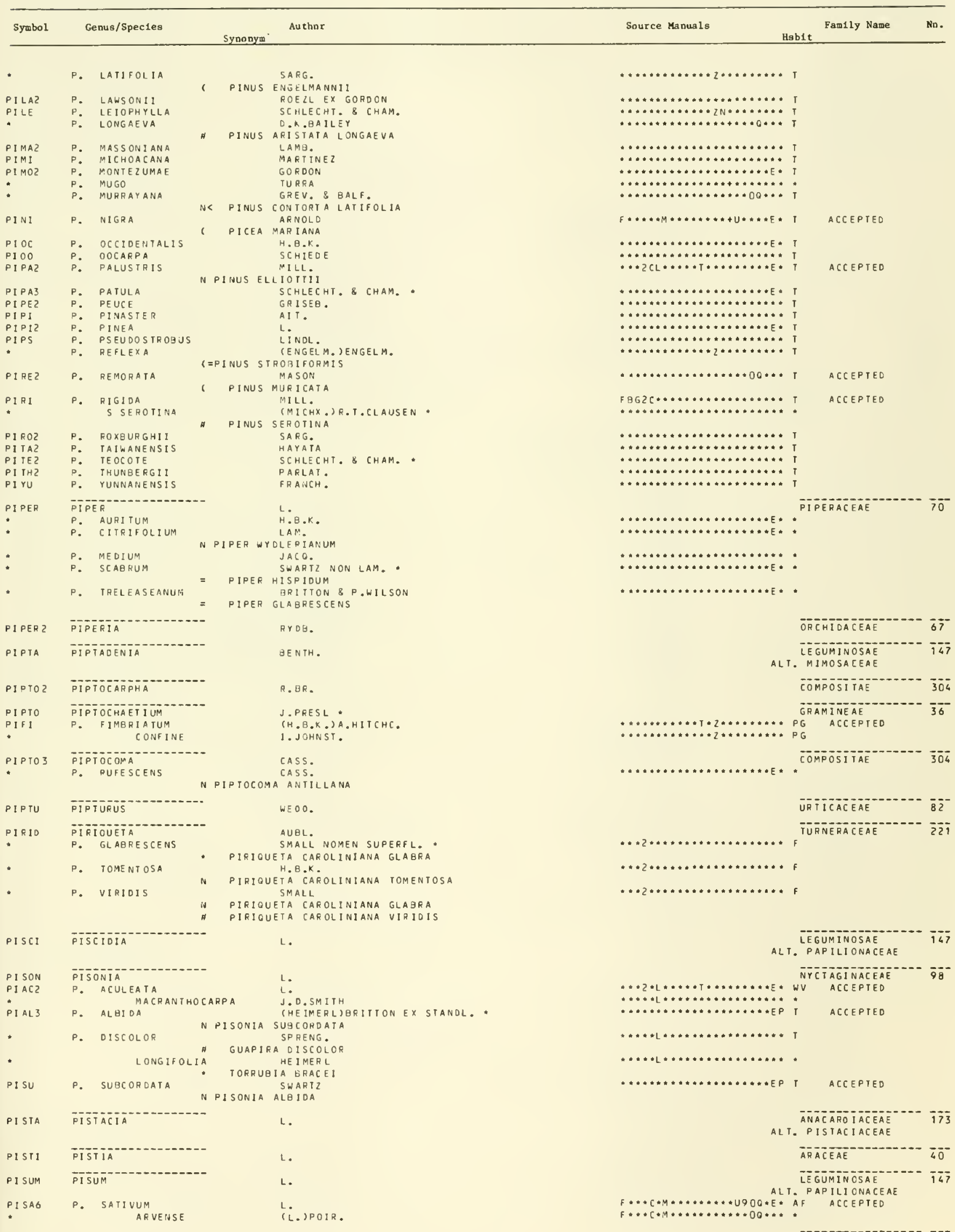




\begin{tabular}{|c|c|c|c|c|c|}
\hline Symbol & Genus/Spectes & Synonym & Source Manuals & Fantly Name & No. \\
\hline - & PITAVIA & MOLINA & \multicolumn{2}{|c|}{ RUTACEAE } & 157 \\
\hline PITCA & PITCAIRNIA & L'HER - & \multicolumn{2}{|c|}{ B̈ROMELIACEAE } & $\bar{q}$ \\
\hline * & P. LATIFOLIA & $\begin{array}{l}\text { SOLANO. IN AIT. } \\
\text { PITCAIRNIA ANGUSTIFOLIA }\end{array}$ & \multicolumn{2}{|l|}{ 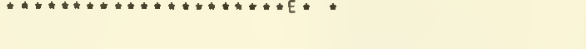 } & \\
\hline PITCH & PITCHERIA & nUTT. & \multicolumn{2}{|c|}{ ALT. PAPILIONACEAE } & $\overline{14} \overline{7}$ \\
\hline PITHE & PITHECELLOBIUM & MART. & \multicolumn{2}{|c|}{$\begin{array}{l}\text { LEGUMINOSAE } \\
\text { ALT. MIMOSACEAE }\end{array}$} & $\overline{147}$ \\
\hline 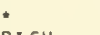 & P. GRACILIFLORUM & BLAKE & $* * * * L \ldots \ldots \ldots \ldots \ldots \ldots+\ldots, s$ & (a) & \\
\hline PI GU & $\begin{array}{l}\text { P. GUADALUPENSE } \\
\text { P. PARVIFOLIUM N }\end{array}$ & $\begin{array}{l}\text { (PERS.) CHAPM. } \\
\text { PITHECELLOHIUM KEYENSE } \\
\text { BENTH. NOMEN SUPERFL. }\end{array}$ & 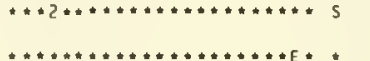 & & \\
\hline PITto & $\begin{array}{l}\text { P. PARVIFOLIUM } \\
\text { PITTOSPORUM }\end{array}$ & $\begin{array}{l}\text { BENTH. NOMEN SUPERFL." } \\
\text { BANKS EX SOLANO. }\end{array}$ & 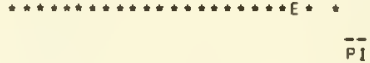 & TOSPORALEAE & $\overline{13} \overline{7}$ \\
\hline 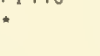 & P. CAULIFLORUM < & $\begin{array}{l}\text { MANN } \\
\text { PITTOSPORUA CONFERTIFLORUM }\end{array}$ & $\ldots+\ldots+\cdots+\ldots+\ldots+\ldots+\cdots+\cdots *+$ & & \\
\hline - & CLAOANTHOIOES & S SHERFF & 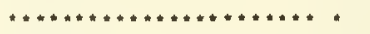 & & \\
\hline$*$ & FULVUM $=$ & $\begin{array}{l}\text { HILLEGR. } \\
\text { PITTOSPORUM CLAOANTHUM }\end{array}$ & 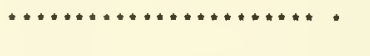 & & \\
\hline PIft? & PEOICELLATUM & SHERFF & 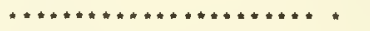 & & \\
\hline PI $[$ L & P. CLAOANTHUM & $\begin{array}{l}\text { SHERFF } \\
\text { (SKOTTSH.) SHERFF }\end{array}$ & 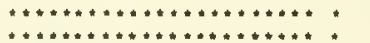 & ACCEPTEO & \\
\hline & RETICULATJM & $\begin{array}{l}\text { 'SKOTTSH. S SHERFF } \\
\text { PITTOSPCRUM CLADANTHUM SKOTTSBERGII }\end{array}$ & 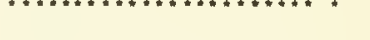 & & \\
\hline$P I[04$ & $\begin{array}{l}\text { P. CONFERTIFLORUM } \\
\text { LONGIPES }\end{array}$ & $\begin{array}{l}\text { GRAY } \\
\text { SHERFF }\end{array}$ & 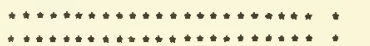 & ACCEPTEO & \\
\hline$*$ & MICROPHYLLUM & SHERFF & 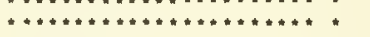 & & \\
\hline PIGL4 & P. GLAGRUM GLOMERATUM & HOOK. $\triangle$ ARN. & 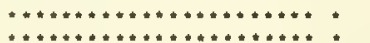 & ACCEPTEO & \\
\hline & $\begin{array}{r}\text { GLOMERATUM } \\
=\end{array}$ & $\begin{array}{l}\text { (HILLEBR. ISHERFF } \\
\text { PITTOSPORUM GLABRUM SPATHULATUM }\end{array}$ & 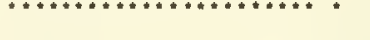 & & \\
\hline * & $\begin{array}{r}\text { TINIFOLIUM } \\
=\end{array}$ & $\begin{array}{l}\text { SHERF } \\
\text { PITIOSPORUM INS IGNE HILLEBRANOI I }\end{array}$ & $* * \ldots \ldots \ldots+\ldots \ldots \ldots \ldots \ldots \ldots \ldots$ & & \\
\hline * & P. HALOPHILOIOES = & $\begin{array}{l}\text { SHERFF } \\
\text { PITTOSPORUM CONFERTIF LORUM }\end{array}$ & $* \ldots+\ldots+\ldots \ldots \ldots+\ldots \ldots \ldots$ & & \\
\hline$\cdot$ & P. HELLER1 = & $\begin{array}{l}\text { SHERF } \\
\text { PITTOSPORUM ACUMINATUM }\end{array}$ & $* \ldots \ldots \ldots \ldots \ldots \ldots \ldots \ldots \ldots$ & & \\
\hline$P ! I N 3$ & P. INSIGNE & HILLEBR. & 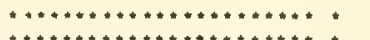 & ACCEPTEO & \\
\hline 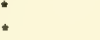 & $\begin{array}{l}\text { LYDGATEI } \\
\text { MICRANTHUM }\end{array}$ & $\begin{array}{l}\text { SHERFF } \\
\text { SHERFF }\end{array}$ & 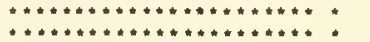 & & \\
\hline & $=$ & PITTOSPCRUM GLABRUM SPATHULATUM & & & \\
\hline P IKA] & $\begin{array}{l}\text { P. KAUAIENSE } \\
\text { PHAE OLARPUM }\end{array}$ & $\begin{array}{l}\text { H:LLEBR. } \\
\text { SHERFF }\end{array}$ & 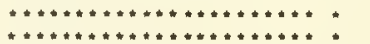 & ACCEPTEO & \\
\hline * & REPENS & SHERFF & $+\ldots+\ldots+\ldots$ & & \\
\hline PI SU3 & P. SULCATUN & SHERFF & 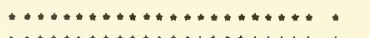 & ACCEPTEO & \\
\hline PITES & $\begin{array}{l}\text { RUMICIFOLIUM } \\
\text { P. IERPINALIOIOES }\end{array}$ & $\begin{array}{l}\text { SHERFF } \\
\text { PLANCH EX GRAY }\end{array}$ & 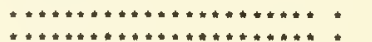 & & \\
\hline 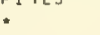 & $\begin{array}{l}\text { LANAIENSE } \\
\text { LANST }\end{array}$ & SHERFF & 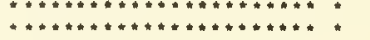 & ACCEPTEO & \\
\hline • & MACROCARPUM & SHERFF & 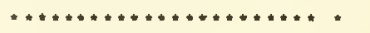 & & \\
\hline • & MACROPUS & SKOTTSB. & 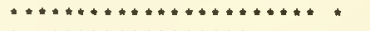 & & \\
\hline • & MAUIENSE & SHERFF & 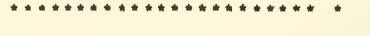 & & \\
\hline • & PITropsis & NUTT. & & POSIIAE & 304 \\
\hline . & P. ASPERA & $\begin{array}{l}\text { CSHUTTLEW.2SMALL } \\
\text { HETEROTHECA ASPERA }\end{array}$ & $\cdots+2 \cdots+*+\infty+\cdots+\cdots+\cdots+* * b F$ & & \\
\hline - & P. FLEXUOSA & $\begin{array}{l}\text { (NASH) SMALL } \\
\text { HETEROTHECA FLEXUOSA }\end{array}$ & 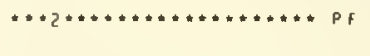 & & \\
\hline$\cdot$ & P. GRAMINIFOLIA & $\begin{array}{l}\text { (MICHX.) NUTI. } \\
\text { HETEROTHECA GRAMINIFOLIA }\end{array}$ & $* *+2 * * *+* \cdots+\cdots+\cdots+\ldots+* P F$ & & \\
\hline * & LAIIFOLIA & FERNALO & 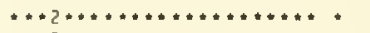 & & \\
\hline * & P. MICROCEPHALA & $\begin{array}{l}\text { SMALL } \\
\text { HETEROTHECA MII ROCEPHALA }\end{array}$ & 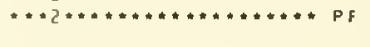 & & \\
\hline * & P. OLIGANTHA & $\begin{array}{c}\text { CCHAPM. I SMALL } \\
\text { HETEROTHECA OLI GANTHA }\end{array}$ & $\cdots \cdots 2 \cdots \cdots \cdots \cdots \cdots \cdots \cdots \cdots *$ bq & & \\
\hline * & P. PINIFOLIA & $\begin{array}{l}\text { (ELLIOTT ) NUTT. } \\
\text { HETEROTHECA PINIFOLIA }\end{array}$ & 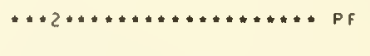 & & \\
\hline * & P. RUTHII & $\begin{array}{l}\text { (SMALL) SMALL } \\
\text { HETEROTHECA RUIHII }\end{array}$ & 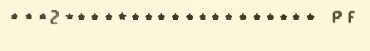 & & \\
\hline - & P. TRACYI & $\begin{array}{l}\text { SMALL } \\
\text { HETEROTHECA GRAMINIFOLIA TRACYI }\end{array}$ & $* n+2 * \ldots+\ldots+\cdots \cdots+\ldots \ldots \ldots+p F$ & & \\
\hline PITYO & PITYOPUS & SMALL & ALI. $\bar{P} \bar{Y}$ & $\begin{array}{l}\text { OLACEAE } \\
\text { NOTROPACEAE }\end{array}$ & $\overrightarrow{2} \bar{s}$ \\
\hline * & PITYOTHAMNUS & SMALL & $\overline{A N}$ & ONACEAE & $\overline{17}$ \\
\hline - & P. ANGUSI IFOLIUS & $\begin{array}{l}\text { SMALL } \\
\text { ASIMINA LONGIFOLIA }\end{array}$ & $* * 2 * * * * * * * \cdots * \cdots * \cdots * \omega s$ & & \\
\hline - & P. INCARNUS & $\begin{array}{l}\text { (W. BARTRAM) SMALL * } \\
\text { ASIMINA INCARNA }\end{array}$ & $* * * 2 \cdots * * * * * \cdots * * * * * * s$ & & \\
\hline - & P. OBDVATUS & $\begin{array}{l}\text { CWILLO..2SMALL } \\
\text { ASIMINA OUOVATA }\end{array}$ & $* *+2 * \cdots * \cdots * \cdots * * * * * * * * s$ & & \\
\hline * & P. PYGMAEUS & $\begin{array}{l}\text { (W. BARTRAM) SMALL } \\
\text { ASIMINA PYGMAEA }\end{array}$ & $* \cdots 2 \cdots \cdots * \cdots * * * \cdots * * * * s$ & & \\
\hline - & P. RETICULATUS & $\begin{array}{l}\text { (SHUTTLEW. EX CHAPM.) SMALL * } \\
\text { ASIMINA RETICULATA }\end{array}$ & $* \cdots+2 * \cdots * \cdots * \cdots \cdots \cdots * \cdots \cdots * s$ & & \\
\hline • & P. TETRAMERUS & $\begin{array}{l}\text { CSMALL ISMALL } \\
\text { ASIMINA TETRAMERA }\end{array}$ & $\cdots * 2 \cdots * \cdots \cdots+\cdots \cdots \cdots * * s$ & & \\
\hline PIIYR & PITYROGAAMMA & LINK & ALT. $\bar{P}$ & MNOOIACEAE & $\overline{8}$ \\
\hline - & P. TARTAREA & $\begin{array}{l}\text { (CAV.) MAXON } \\
\text { PI TYROGRAMMA EUENEA }\end{array}$ & 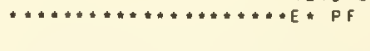 & & \\
\hline PIT I P & $\begin{array}{l}\text { P. TRIAIVGULARIS } \\
\text { PALLIOA }\end{array}$ & $\begin{array}{l}\text { (KAULF.) MAXON } \\
\text { WEATHERBY }\end{array}$ & $* * * * * * * * * 2 *+U 9 O Q+* * P F$ & ACEEPTEO & \\
\hline & $\cot$ & PITYROGRAMMA PALLIOA & (⿻) & & \\
\hline 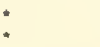 & $\begin{array}{l}\text { SEMIPALLIOA } \\
\text { VIRIOIS }\end{array}$ & $\begin{array}{l}\text { J. T.HOWELL } \\
\text { HOOV. }\end{array}$ & 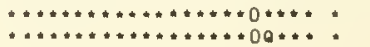 & & \\
\hline
\end{tabular}




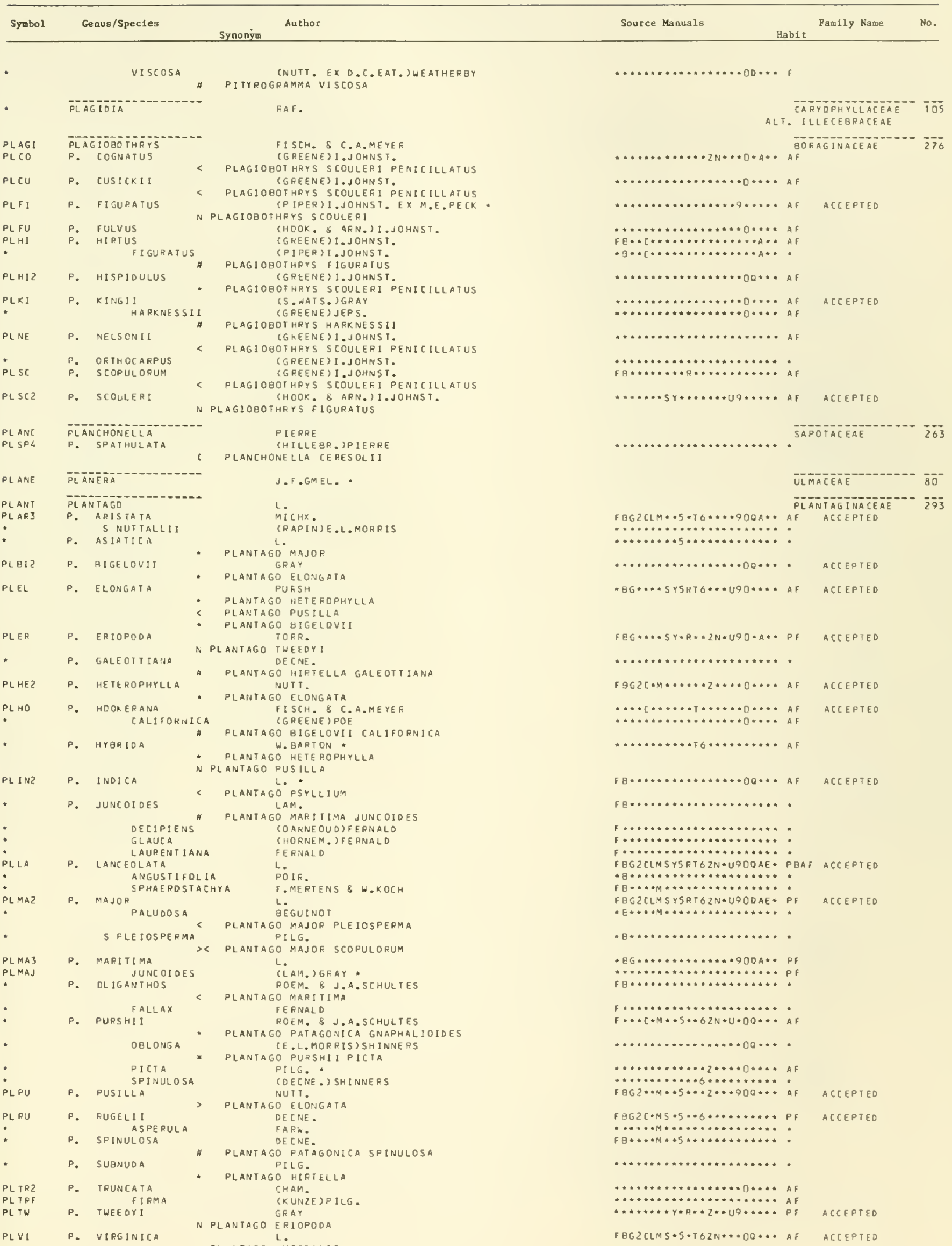




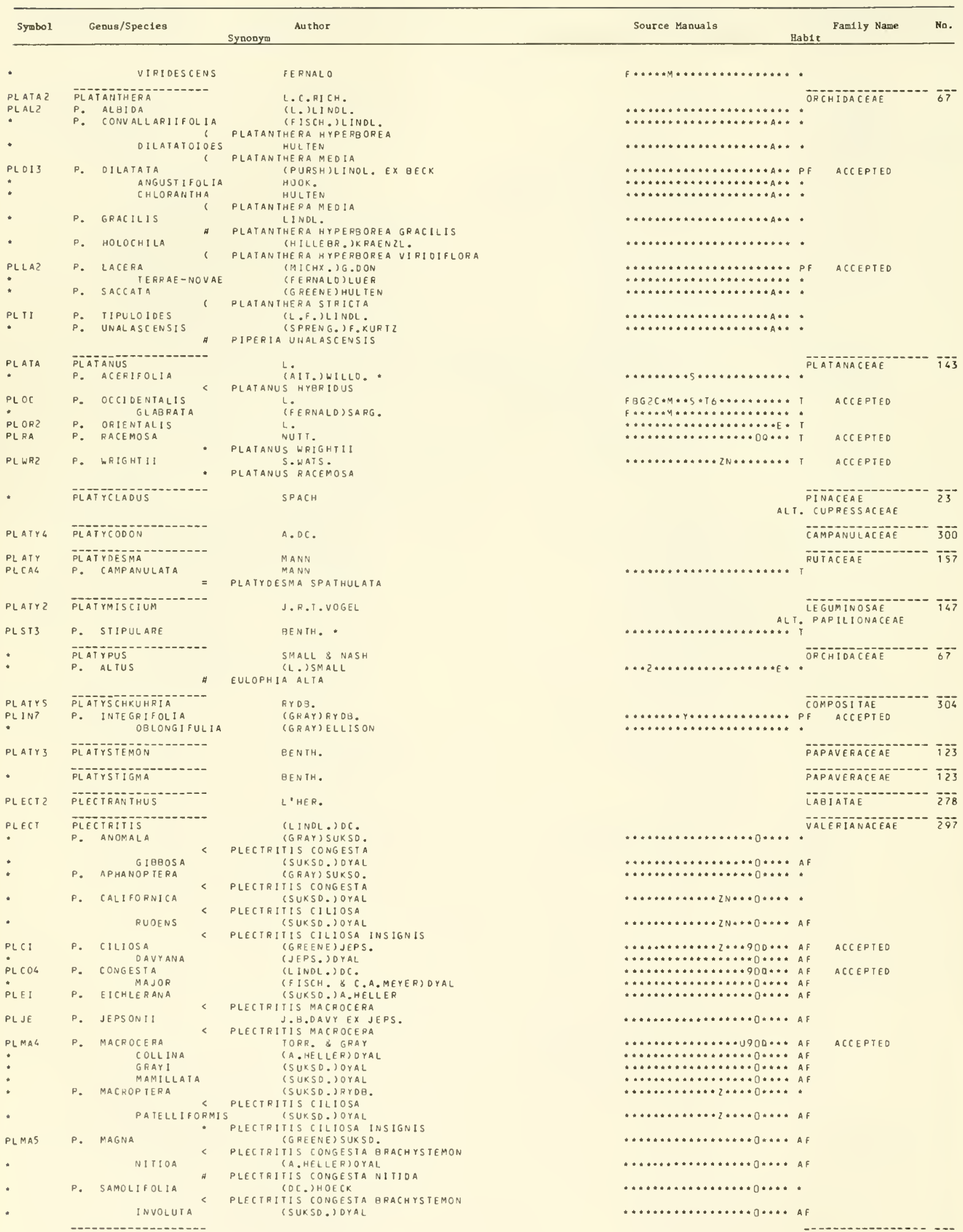




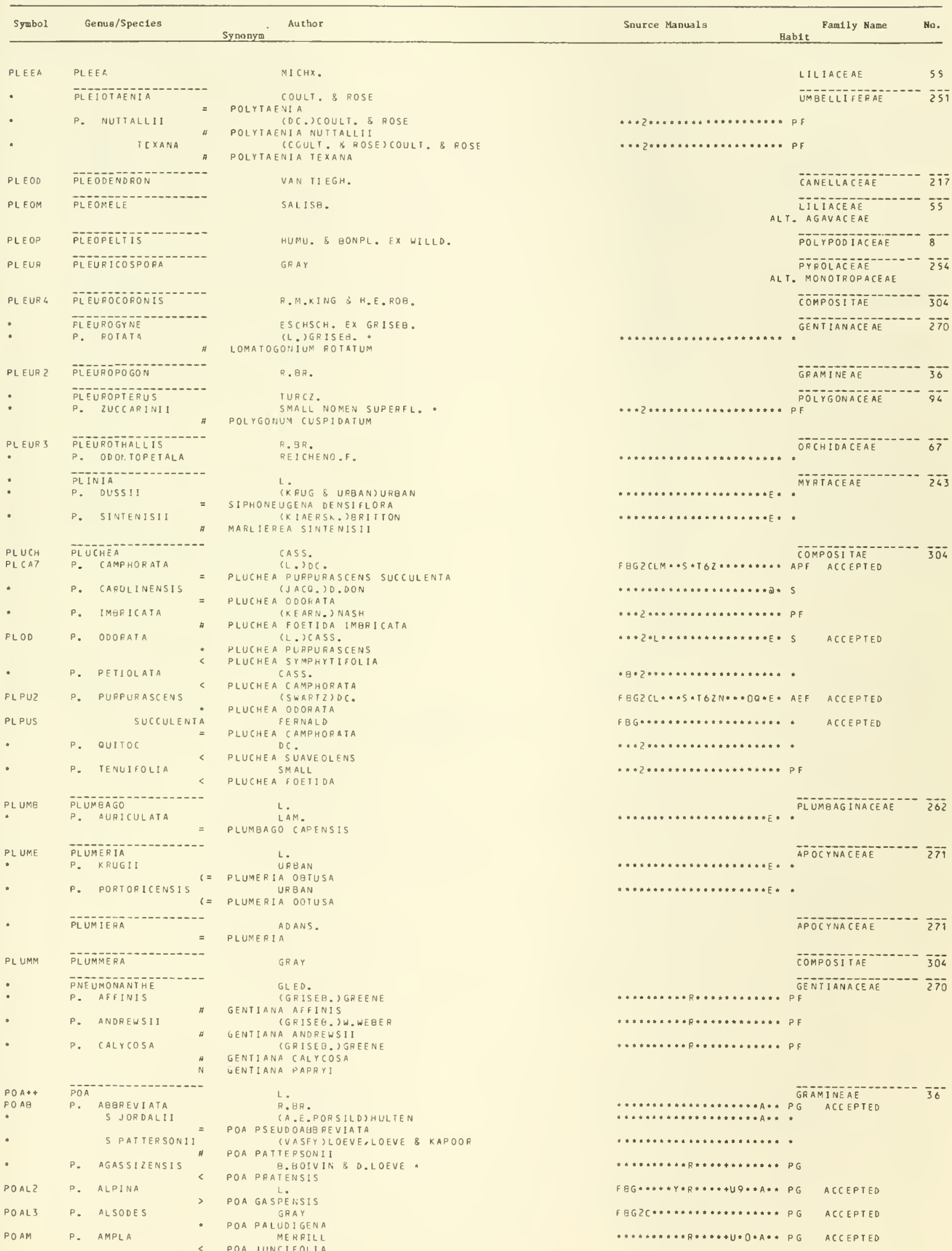




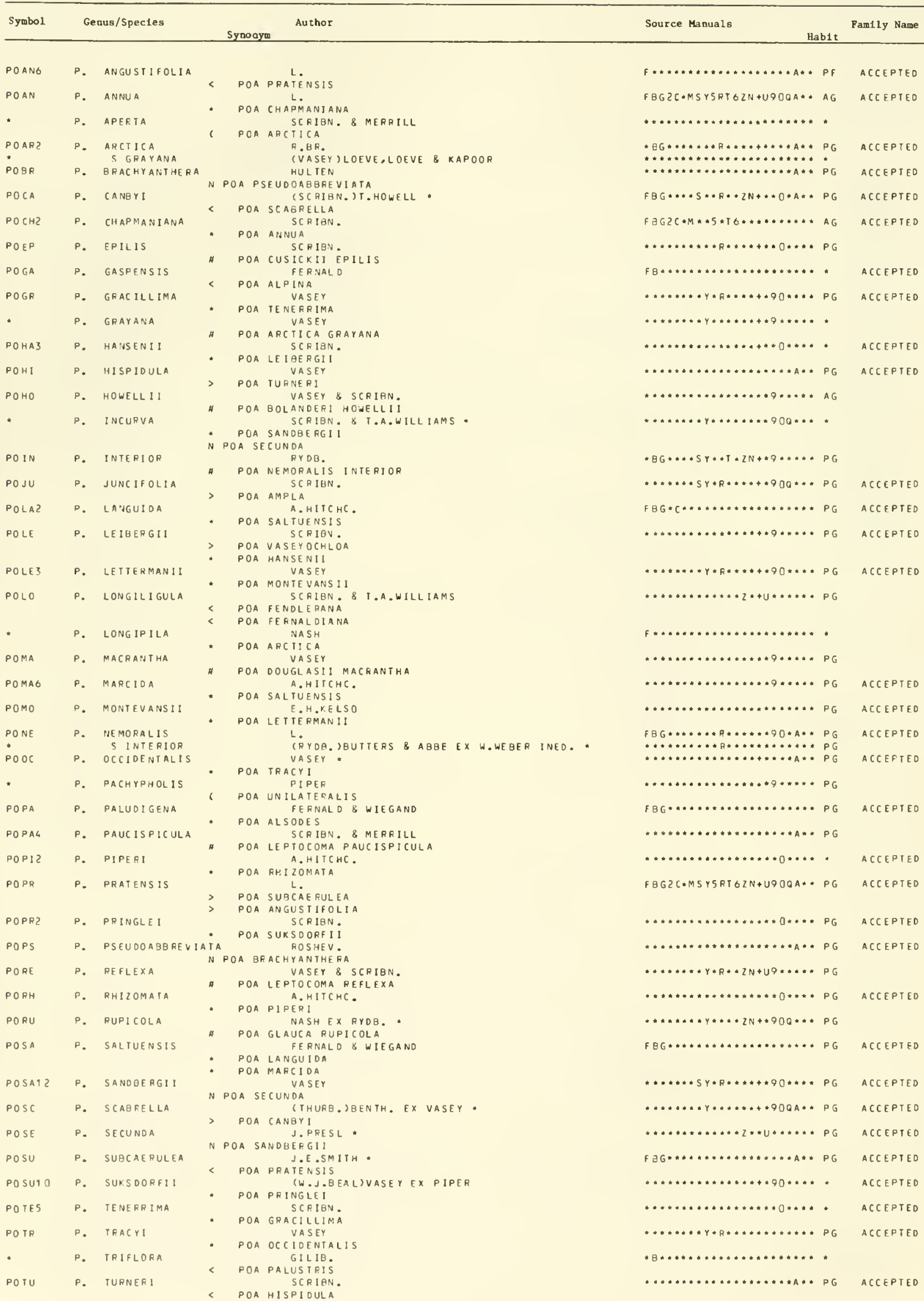




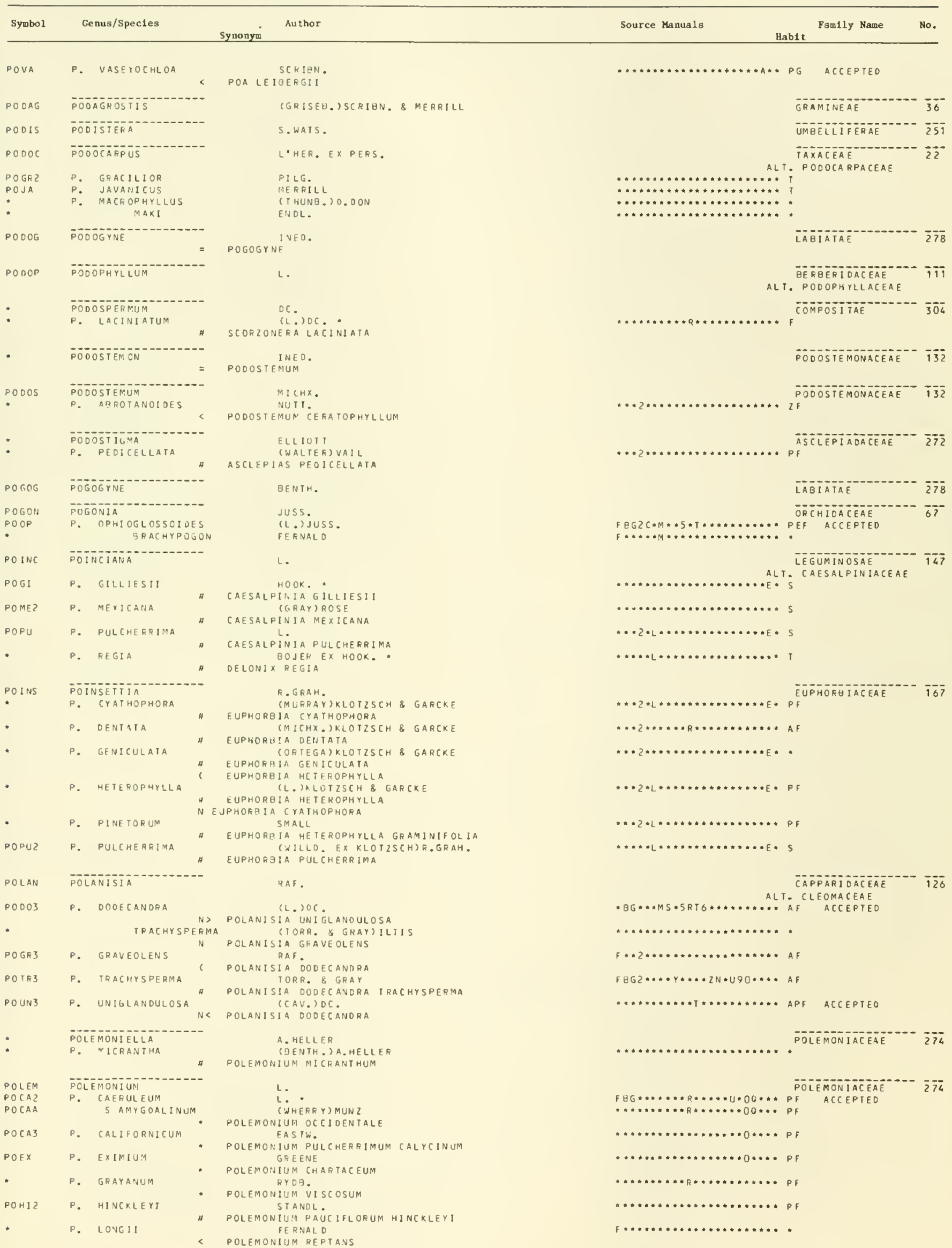




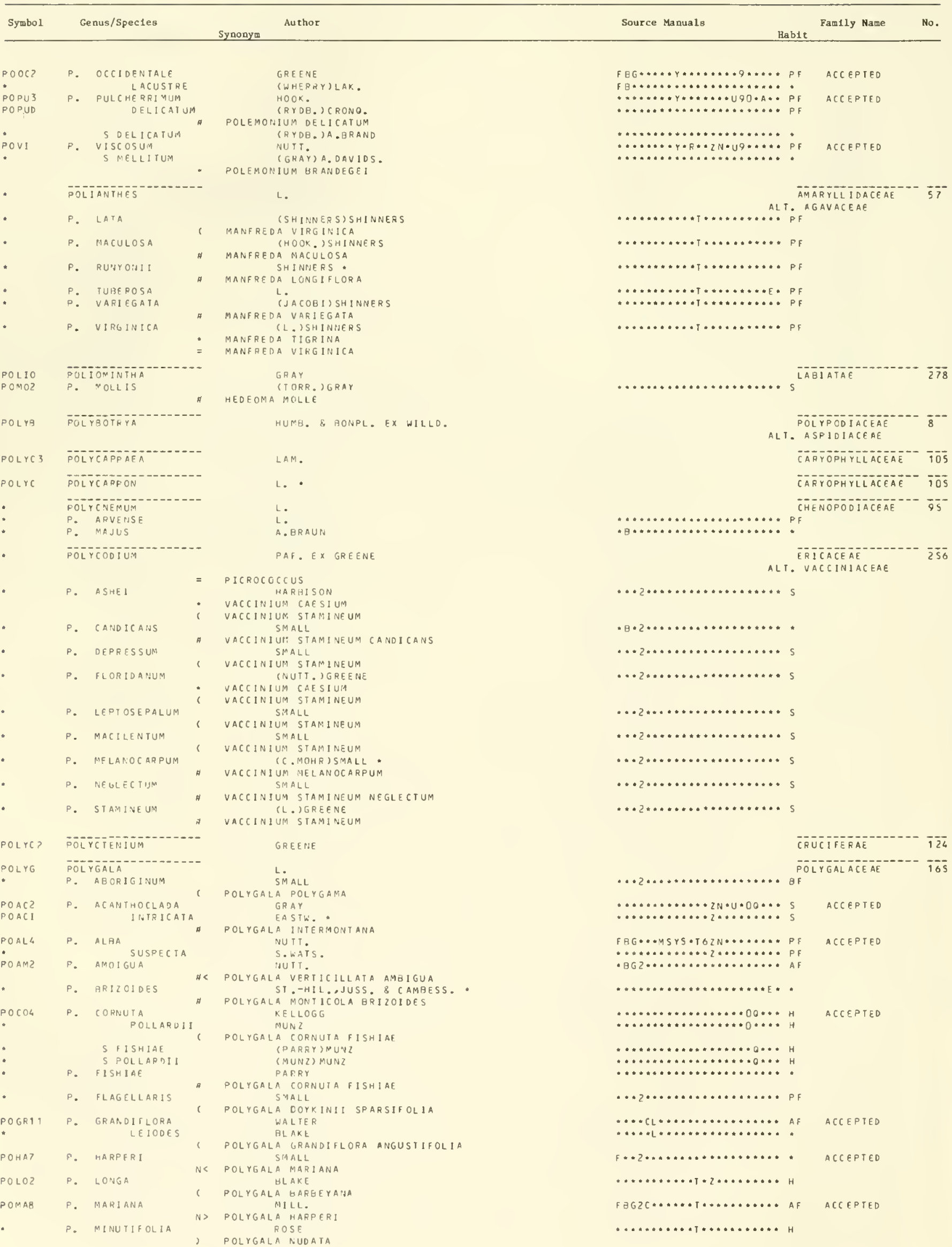




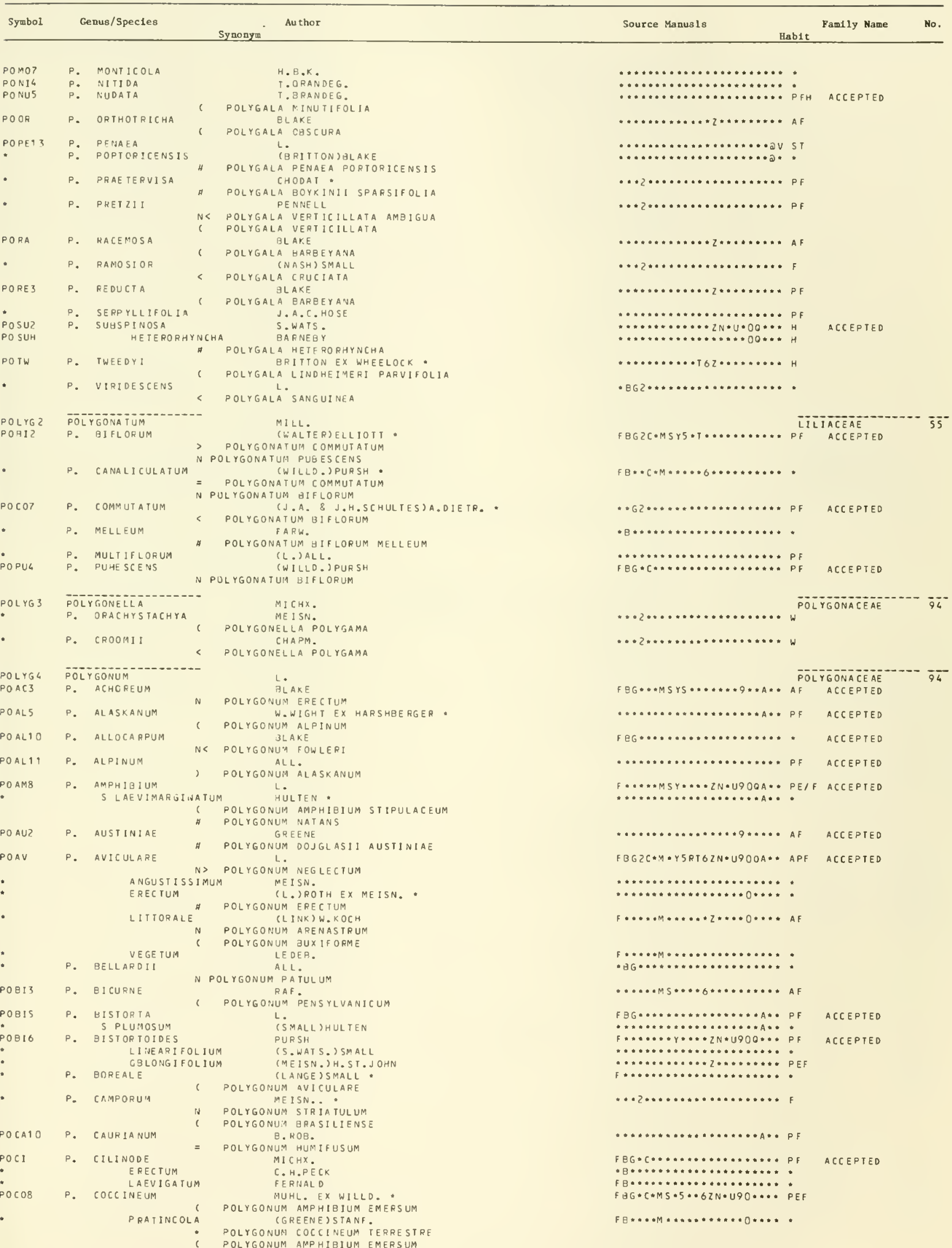




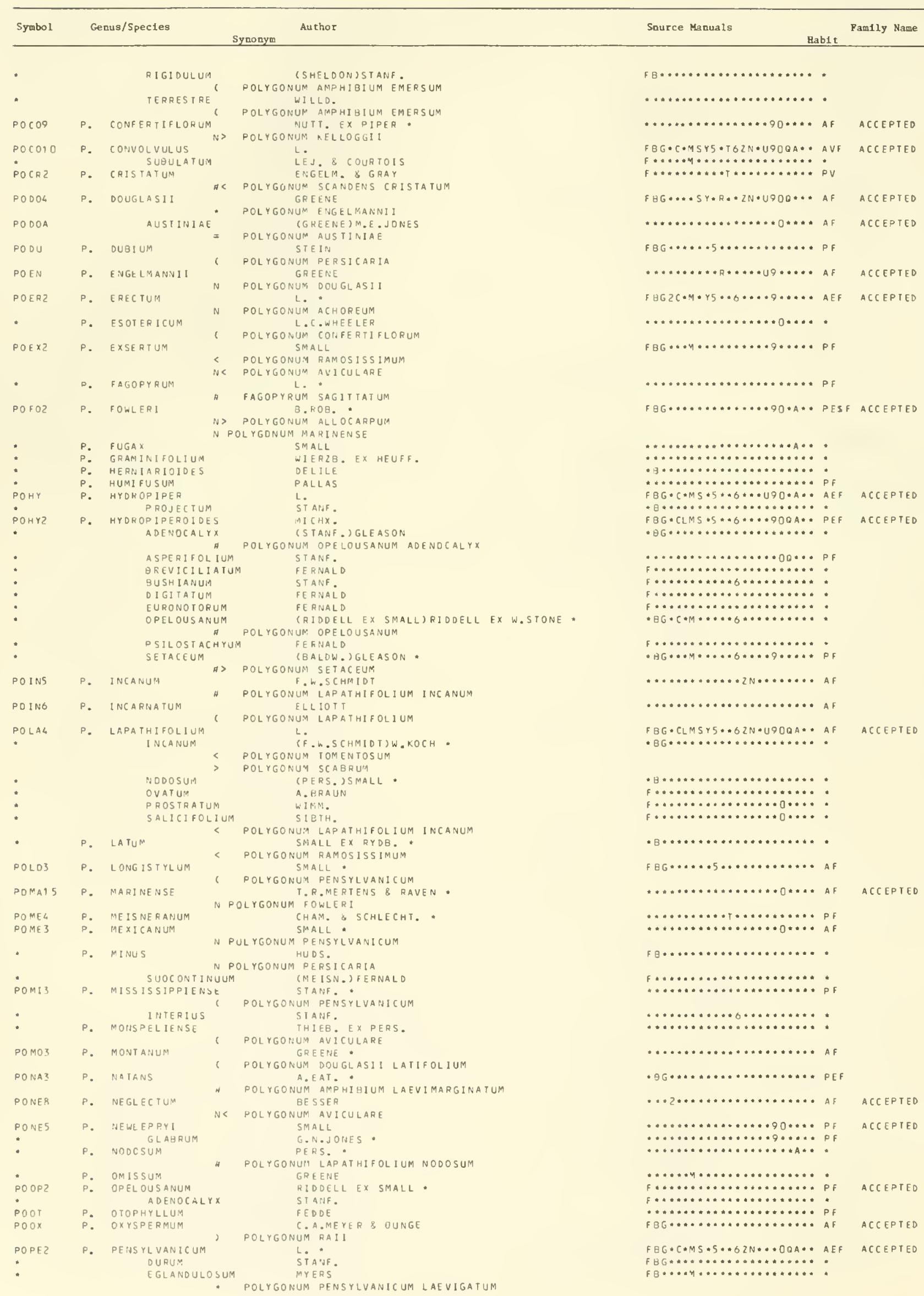




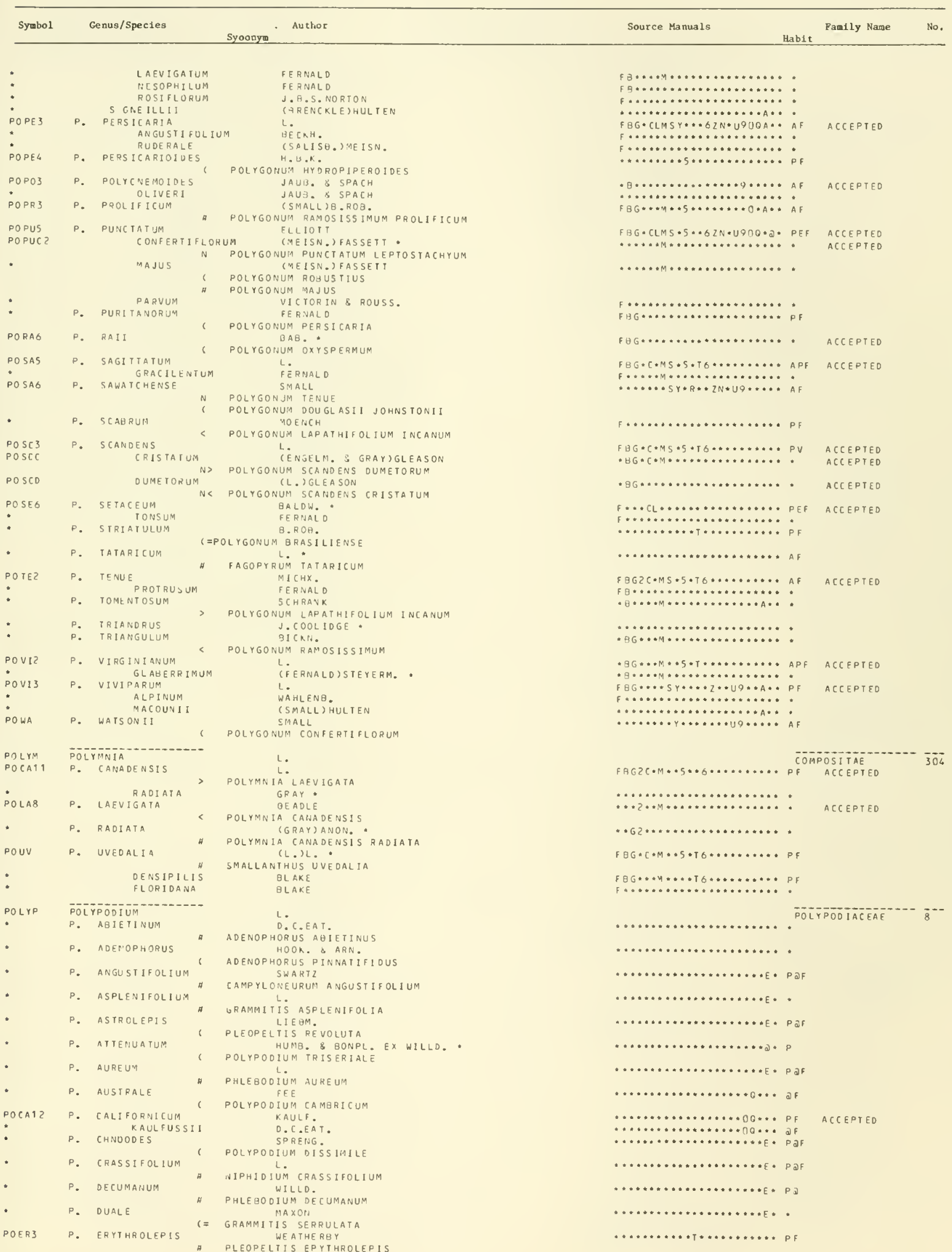




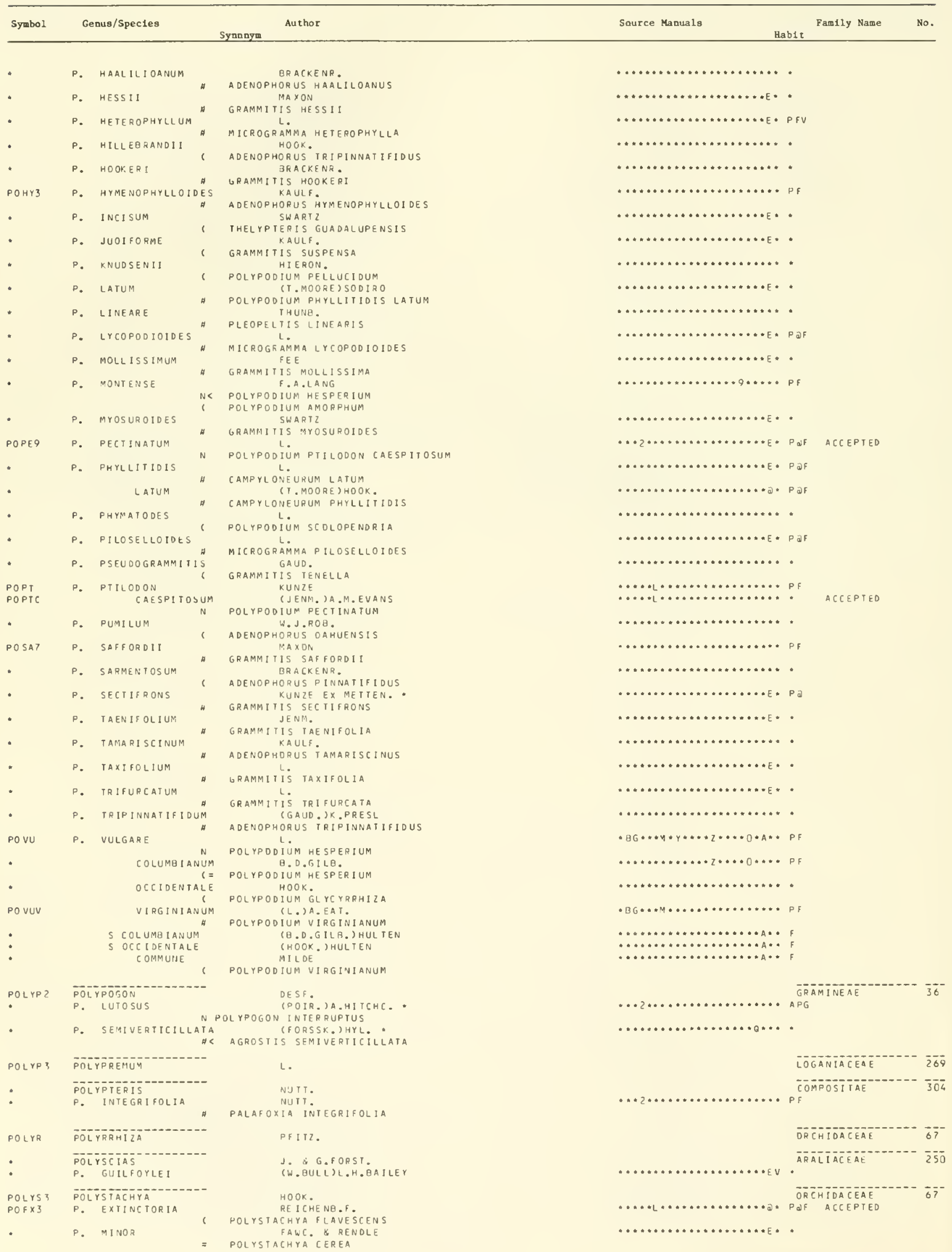


POLYSTACHYA

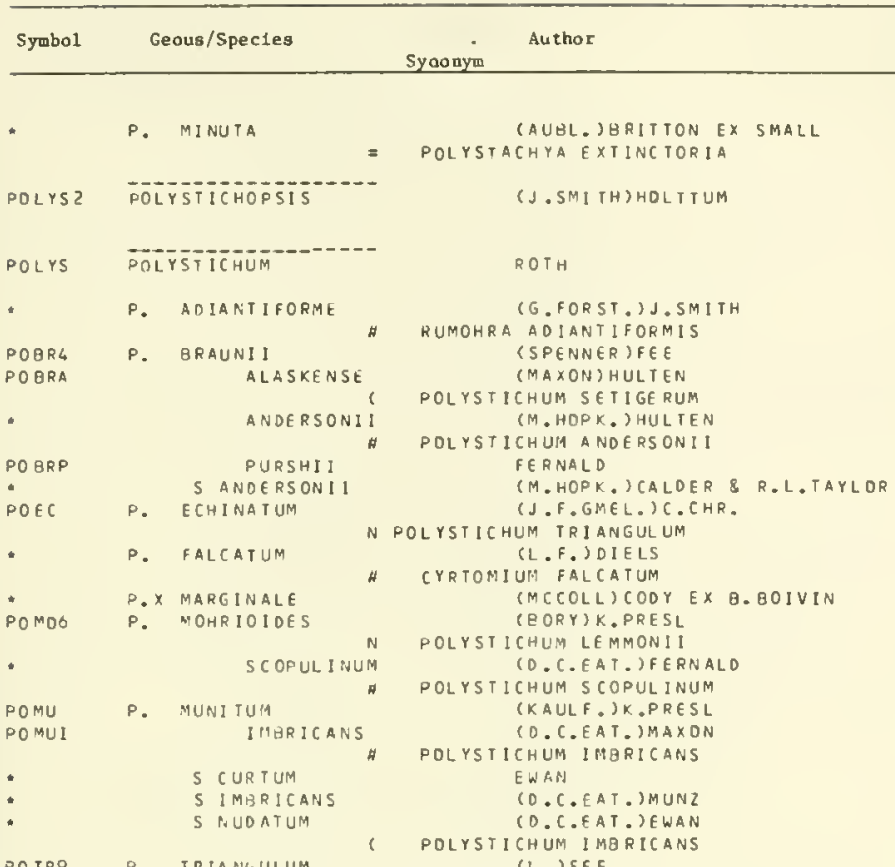

POTRQ P. TRIANGULUM POLYSTICHUM IMERICANS

POLYT POLYTAENIA
PONUL P. NUTIALLII
TEXANA

POLYTL

POLYTL POLYTOCA

POLYT3 POLYTRIAS

$\begin{array}{ll}\text { POLYTS } & \text { POLYTRIAS } \\ \text { POAML } & \text { P. AMAURA }\end{array}$

PONCI PONCIRUS

PONGA

$$
\text { RAF. }
$$

(BERTERO EX OC.)BRITTON \& RDSE *

P. MACRACANTHOIDES

P. TORTUOSA

$$
\text { i }
$$$$
\text { ACACIA IL.)RAF." }
$$
SAFG.

POPULUS HINCKLEYANA<smiles>[CH]1C=CC1</smiles>

(LAUCHE) OTTO A IT.

POPULUS CANESCENS BUNGE

POPULUS FREMONTII ARIZONI CA

N PDPULUS FREMONT II MESETAE

P. balsamifera n populus di DitoIdoes

MICHAUXII

= POPULUS BALSAMIFERA SUBCOROATA

SUGCOROATA

P. $X$ RERNAROII

P.X BESSEYANA

P. BOLLEANA
HYLL.
HYLSA

3. BOIVIN

POPULUS JACKI

POPULUS DELTOIOES MONILIFERA

LAUCHE

populus alga bolleana
Source Mauals

Family Name

No.

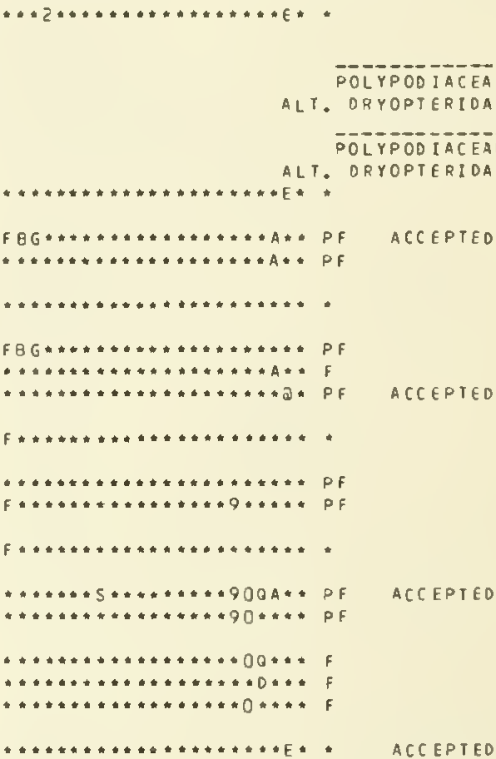

FBG UMBELLIFERAE UMBELLIFERAE

$\ldots * \ldots * \ldots * * * 0 * \ldots \ldots * * \ldots *$.

POLYPODIACEAE
ALT. VITTARIACEAE

GRAMINEAE

$* * * * * * * * * * \cdots * * \cdots * * E *$ b

\section{RUTACEAE}

LEGUMINOSAE-D-D-77

ALT. CAESALPINIACEAE

PONTEOERIACEAE

F $B G 2 C * M * * S * T 6 \cdots \cdots * \cdots *$ PEF ACCEPTEO

PEF

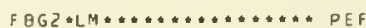

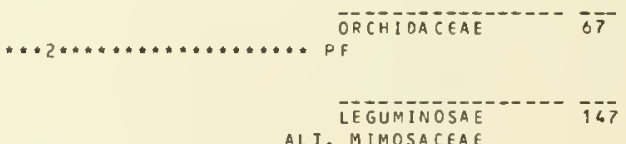
$A L T$

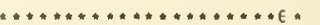

SALICACFAE

ALT. POPULACEAE

\#.....SY*RT*ZN*U90*\#* T ACCEPTED

F BG 2 C*MS $* 5 * T 6 * \cdots$ U9OD* T

$\ldots \ldots \ldots+\ldots \ldots \ldots$........

ACCEPTEO

F $\mathrm{gG} \ldots \ldots \ldots \ldots+\ldots \ldots \ldots \ldots \ldots$

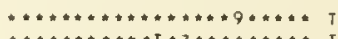

$n * \ldots * \ldots \ldots+t \cdot 2 \ldots \ldots \ldots \ldots+1$

$F B G 2 * * * 5 Y * R * \ldots * 90 * A * T$

ACC EPIEO 


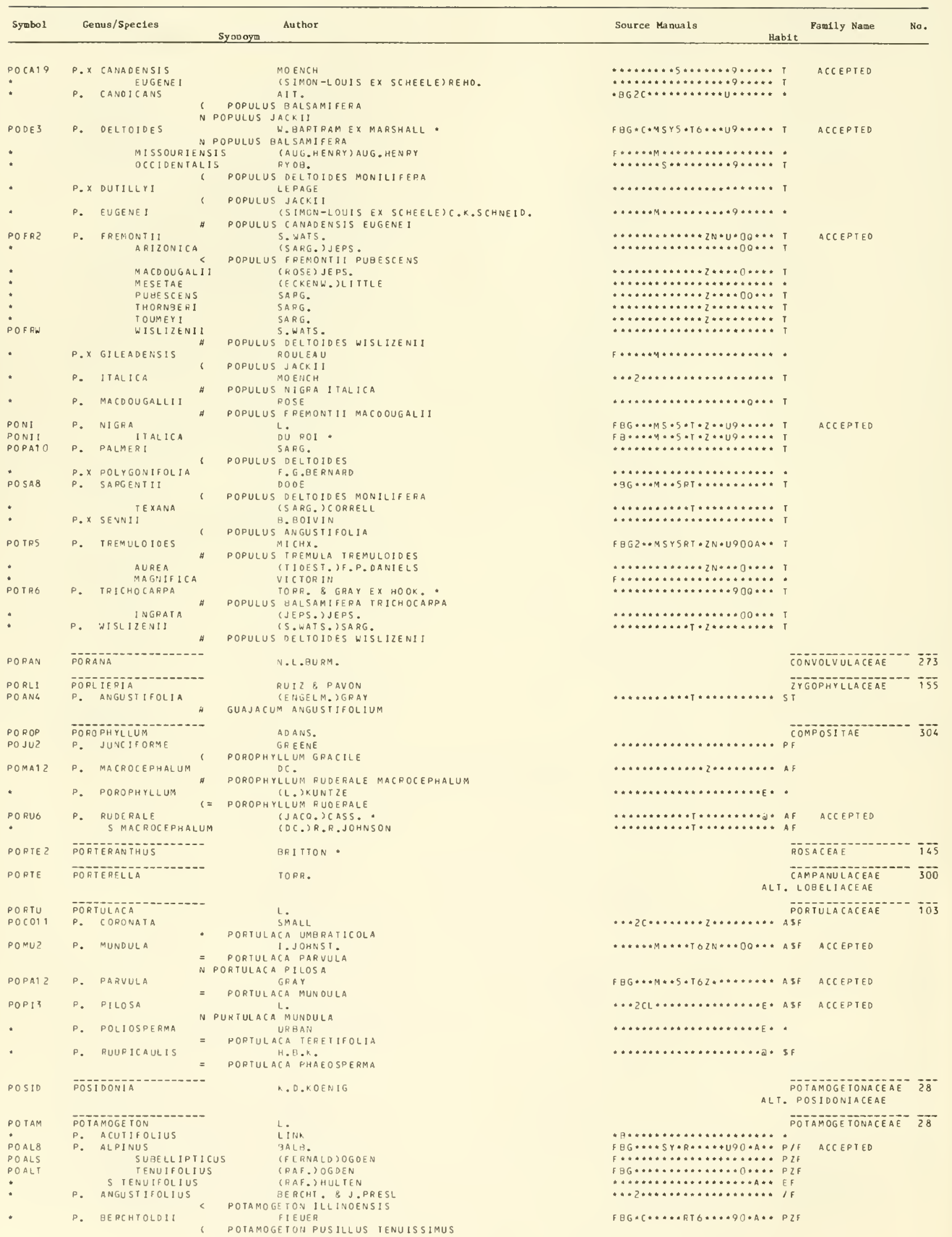




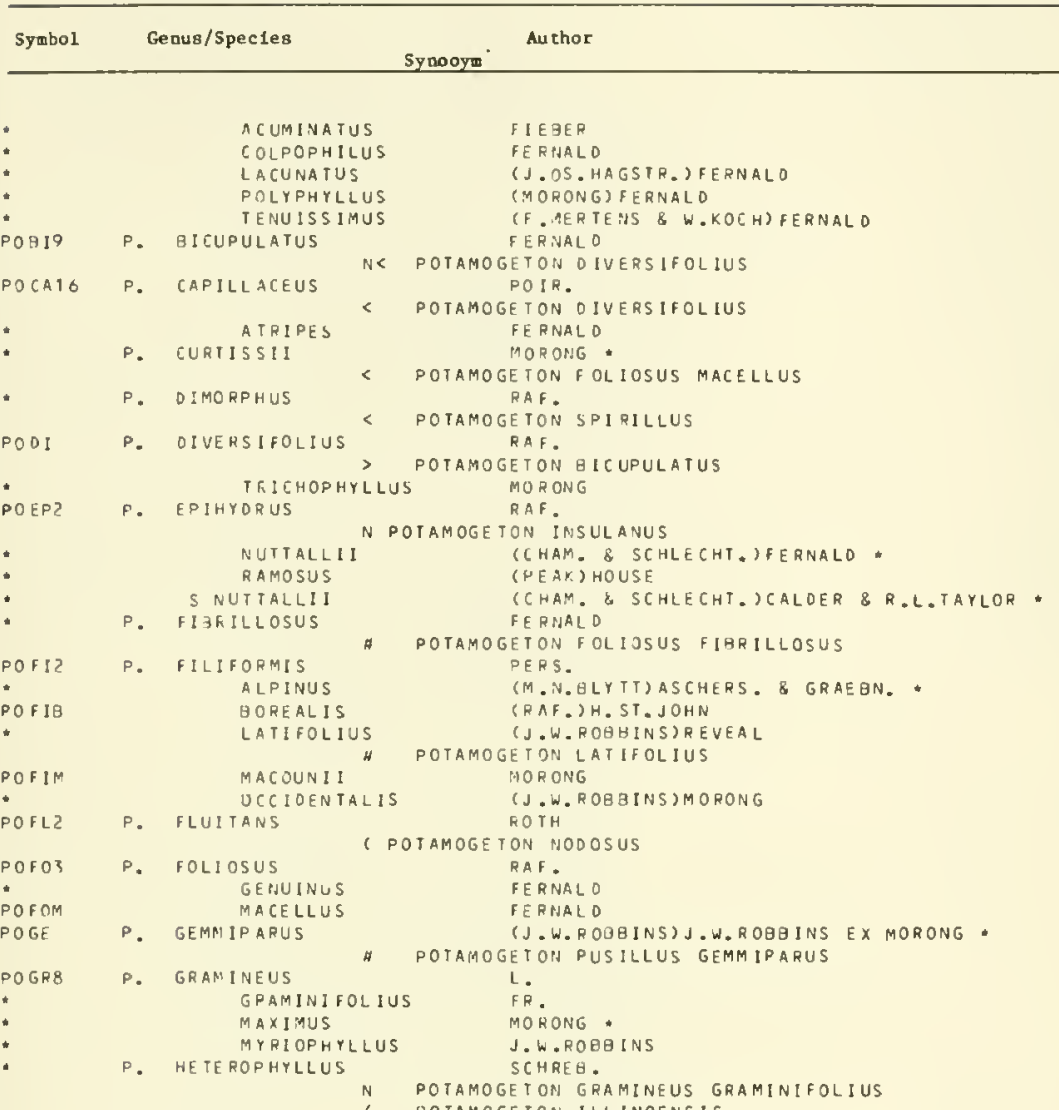

P. INSULANUS

P. INTERR UPTUS

P. LUCERS

P. X METHYENSIS

P. OQLONGUS

P. PANORMITANUS

POPEG P. PECTINATUS

LAIIFOLIUS

POPE?

POPEB

POPU?

"

POTEN

POANB

POANS

-

PO ANS

POA

.

Source Maquals

Hab1t

Family Name No.

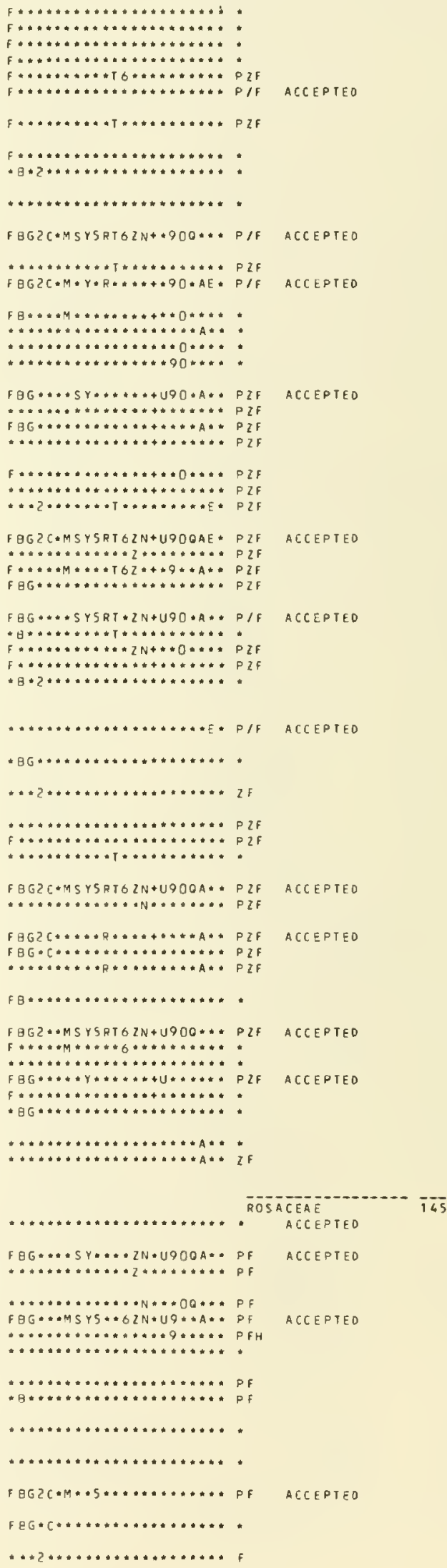




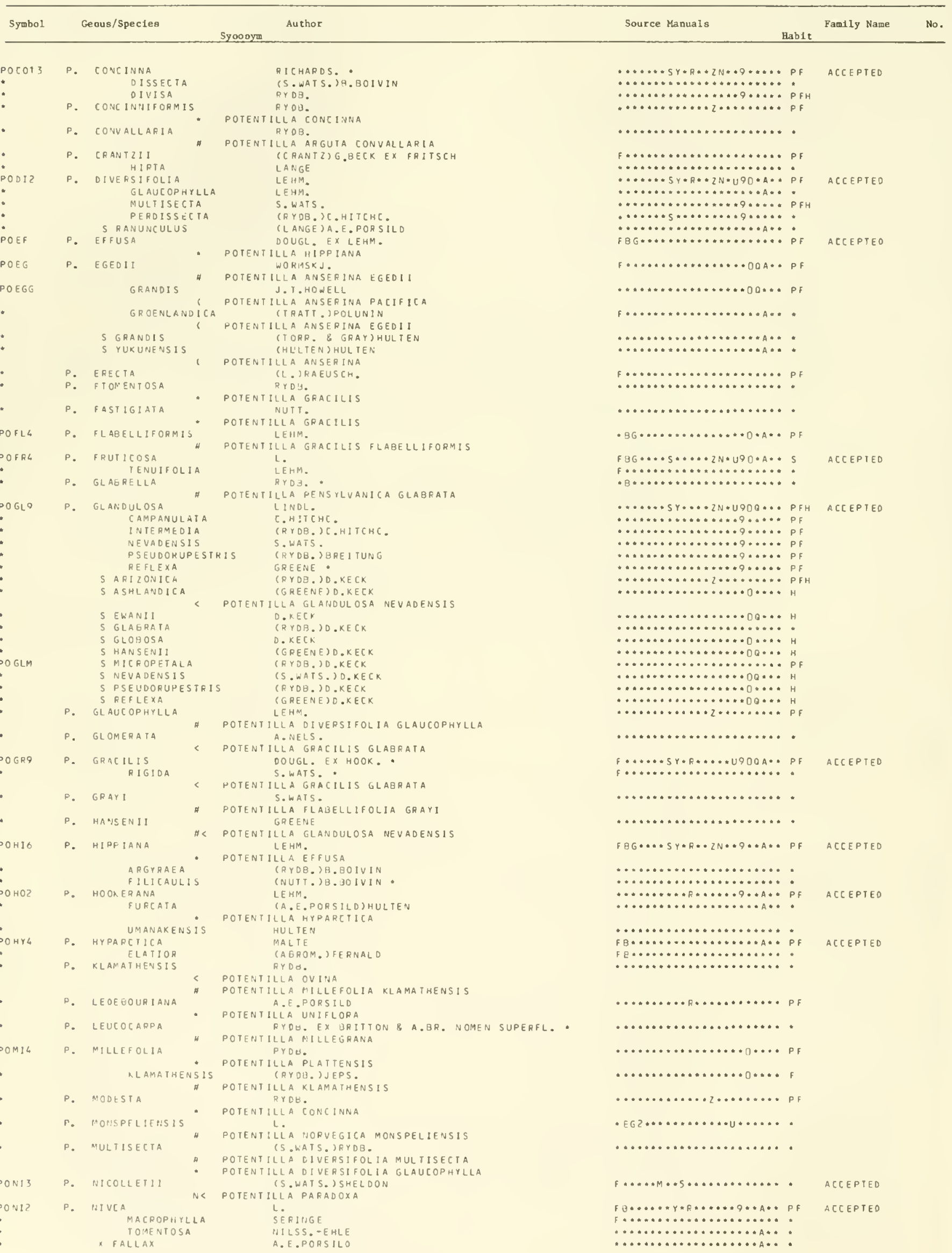




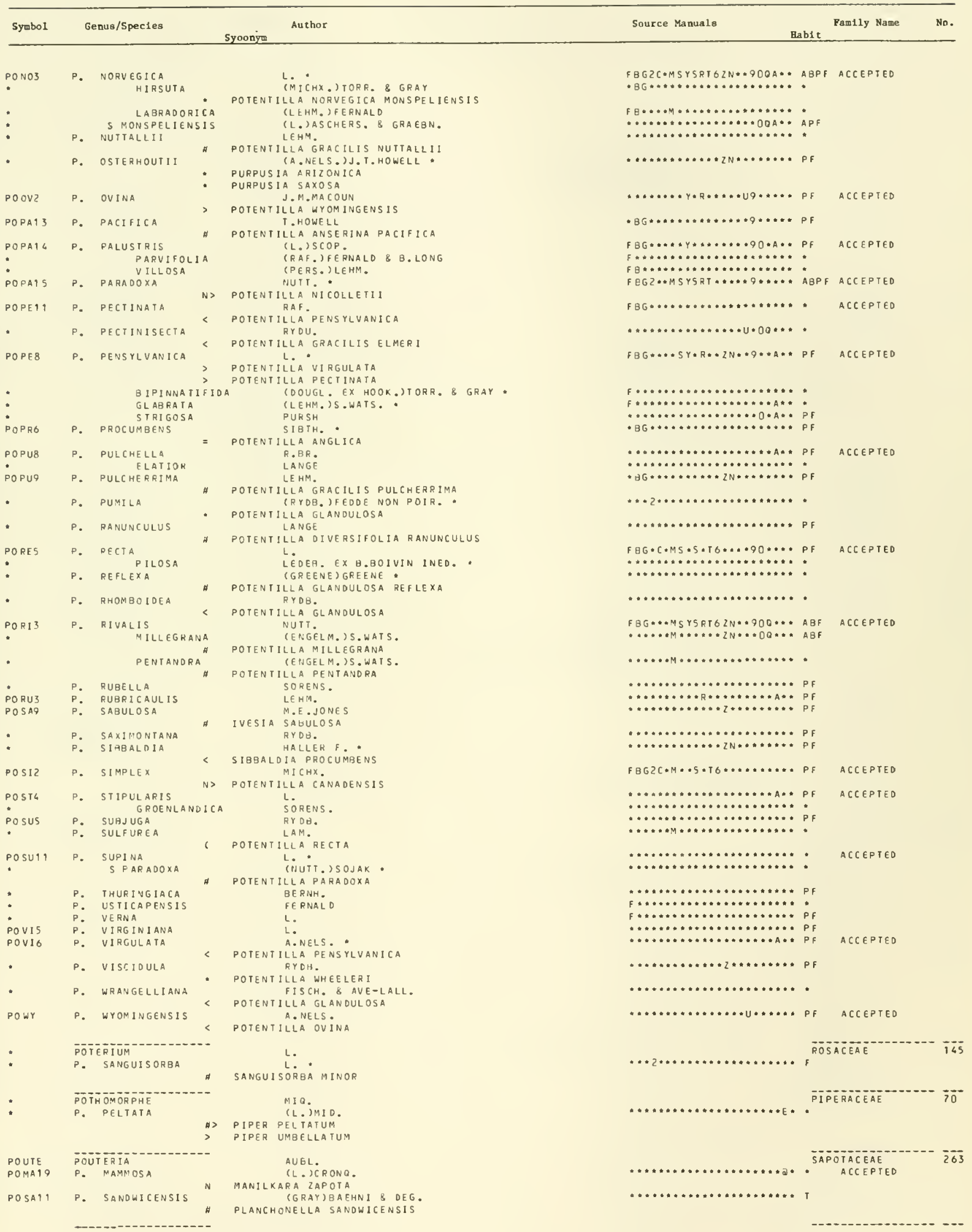




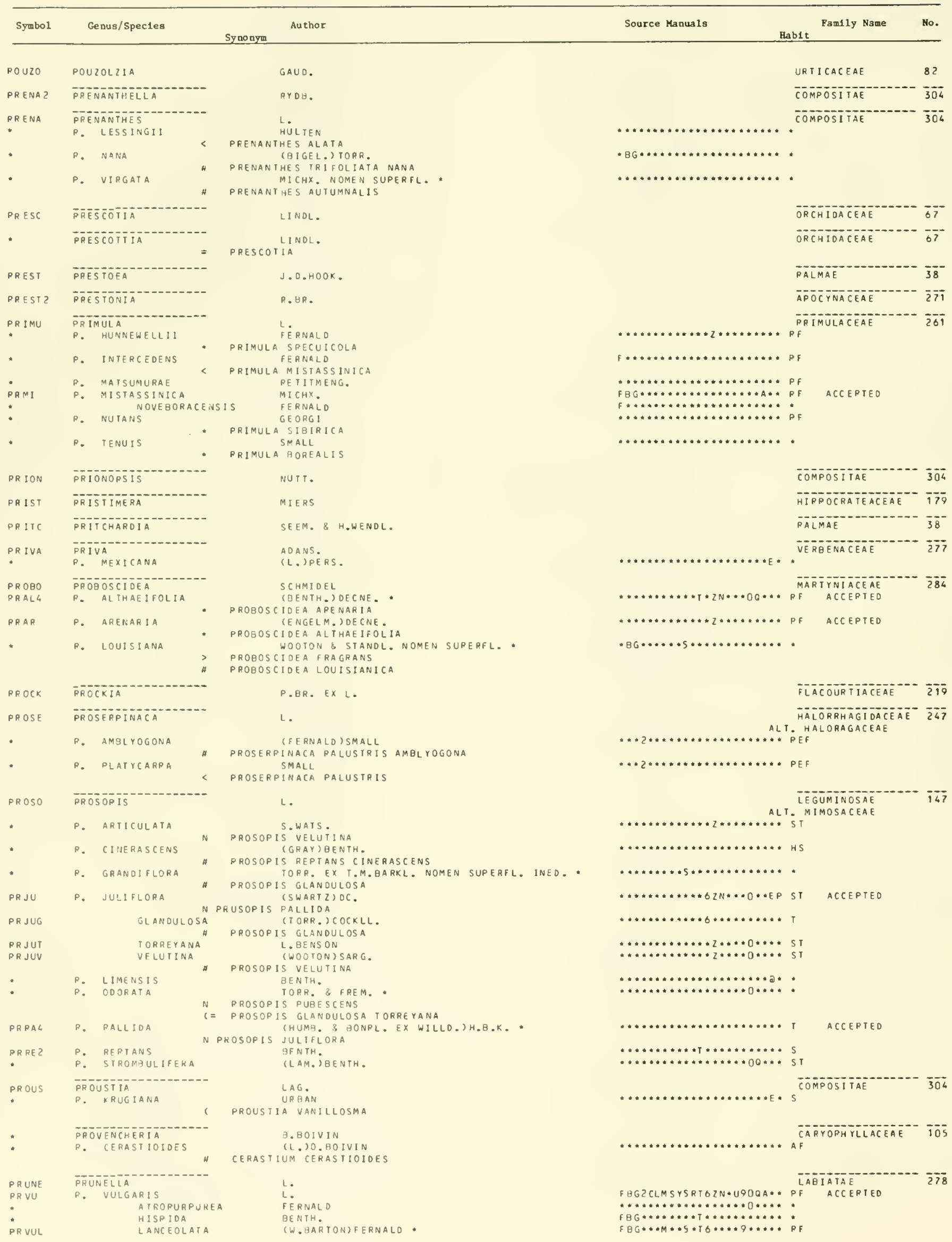




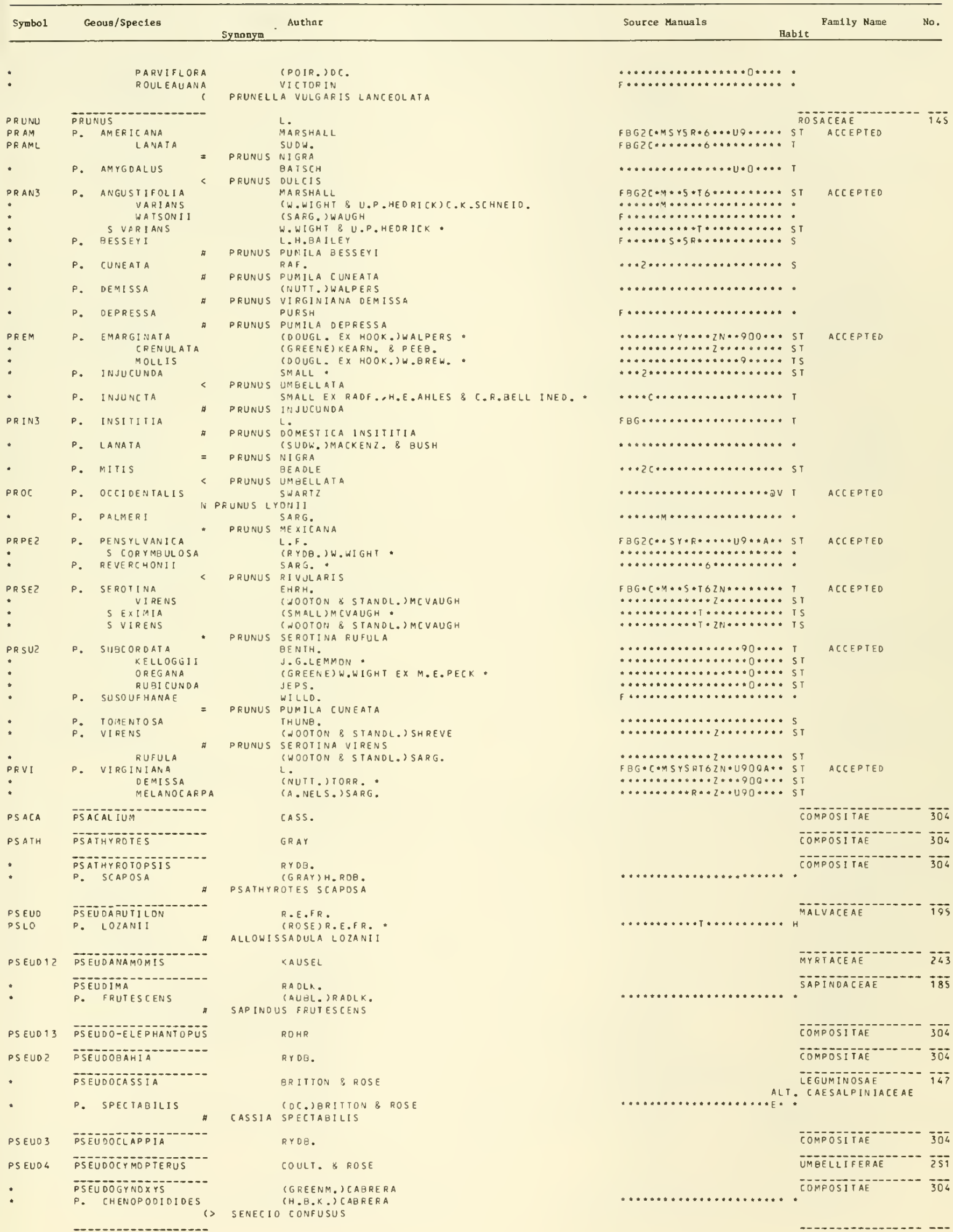




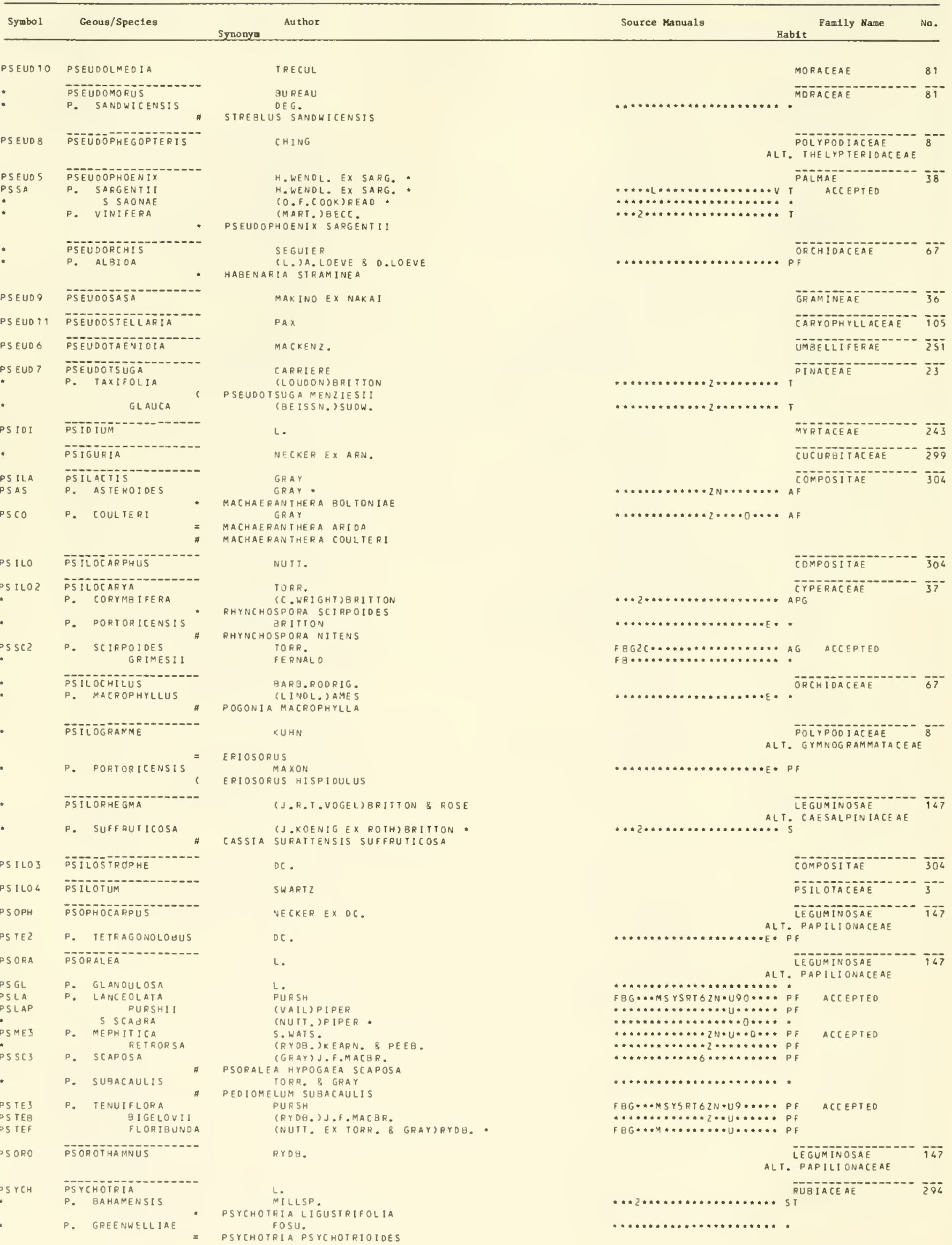


Source Manuals

Synonym

CBAILL.) URBAN

PSYCHOTRIA GUADALUPENSIS GROSOUROYANA COC. SHOWARO GRAY

(CHAM. \& SCHLECHY.)FOSB.

$\begin{array}{lll}\text { PS GU2 } & \text { P. GUADALUPENS } \\ \text { PSIN } & \text { P. INSULARUM }\end{array}$

PSKA P. KADUANA

- LONGISSIMA

P. LAXA

P. LEPI OCARPA

P. MYRTIPHYLLUM

P. PATENS

P. PINULARIS

P. PORTORICENSIS

P. UNOATA

PTELE PTELEA
PTAN P. ANGUSTJFOLIA

$\begin{array}{ll}\text { PTELE } & \text { PTELEA } \\ \text { PTAN } & \text { P. ANGUSTJFOLIA }\end{array}$

COGNATA
P. BALDWINII

MICROSARPA

P. MONTICOLA

P. PALLIDA

P. SEKKATA

P. STRAMINEA

P. TRIFOLIATA ANGUSTIFOLIA DEAM!

MOLLIS TOMENIUSA

5 ANGUSTIFOLIA PERSICIFOLIA

5 PALLIDA

5 POL YAOENIA

\section{PTERALYXIA}

\section{PTERETIS}

P. PENSYLVANICA

H

MATTEUCCIA

PTERIOIUM

H

PSYCHOT

(J.F. ROCK)FOSB

$$
\text { SWART } 2
$$

SHILLEQR.JFOSE.

FIA KADUANA

SWART
SWARTZ

PSYCHOTRIA DEFLEXA

SESSE \& MOCINO

PSYCHOTRIA MICRODON

TR 1 A M
DC.
JACU.

PSYCHOTRIA NERVOSA

BENTH.

PIELEA TRIFOLIATA ANGUSTIFOLIA

PTELEA CRENULATA (GREENE) KEARN. \& PEEB. TORR.

PTELEA TRIFOL IATA SMALL

PIELEA TRIFOLIATA

GREENE

PTELEA TQIFOLIATA

GREENE

trifol Iata pallida

SMALL

PIELEA TRIFOLIATA

GREENE

PIELEA PALL

L.

L.BA JLE

NIEUWL.

TRIFOLIATA MOLLIS

TORR. \& GRA

L.

(BENTH.) V.L. BAILEY

(GREENE) V.L.OAILEY

(GREENE) V.L. BA ILEY

(UREENE) V.L.BAJLEV

K. SCHUM.

RAF.

PIERI

PTAD

*

P. AQUILINUM
CHAMPLAINENSE
LAINUGINOSUM

- S lanuelnosum

P. ARAC HHOJOEUM

- P. CAUDATUM a PTERIDIUM AQUILINUM arachNoIdoEum

H

(BONG. SHULTEN

(WILLD.) FERNALO

MATTEUCCIA STRUTHIOPTERIS PENSYLVANICA

GLEO. EX SCOP.

¿L. , KUHN

B. BOIVIP

H PTERIDIUM aQUILINUI

PTERIS CAUDAT A

PTERIZ PTERTS L.

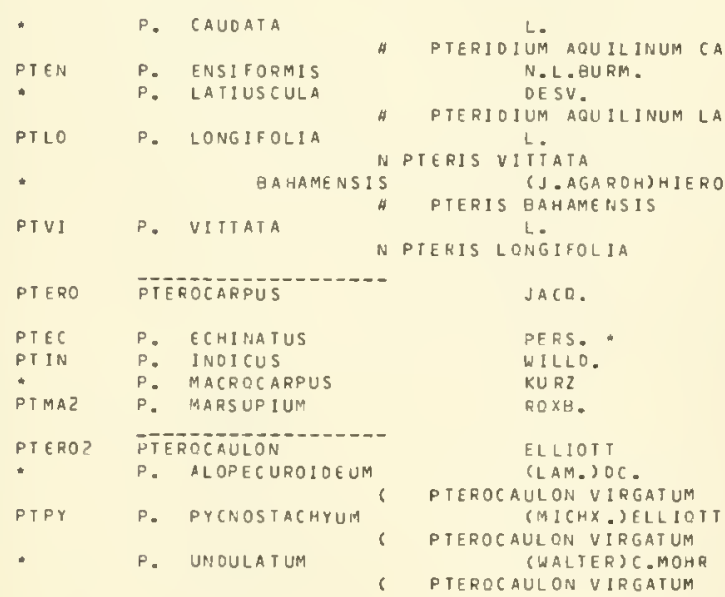
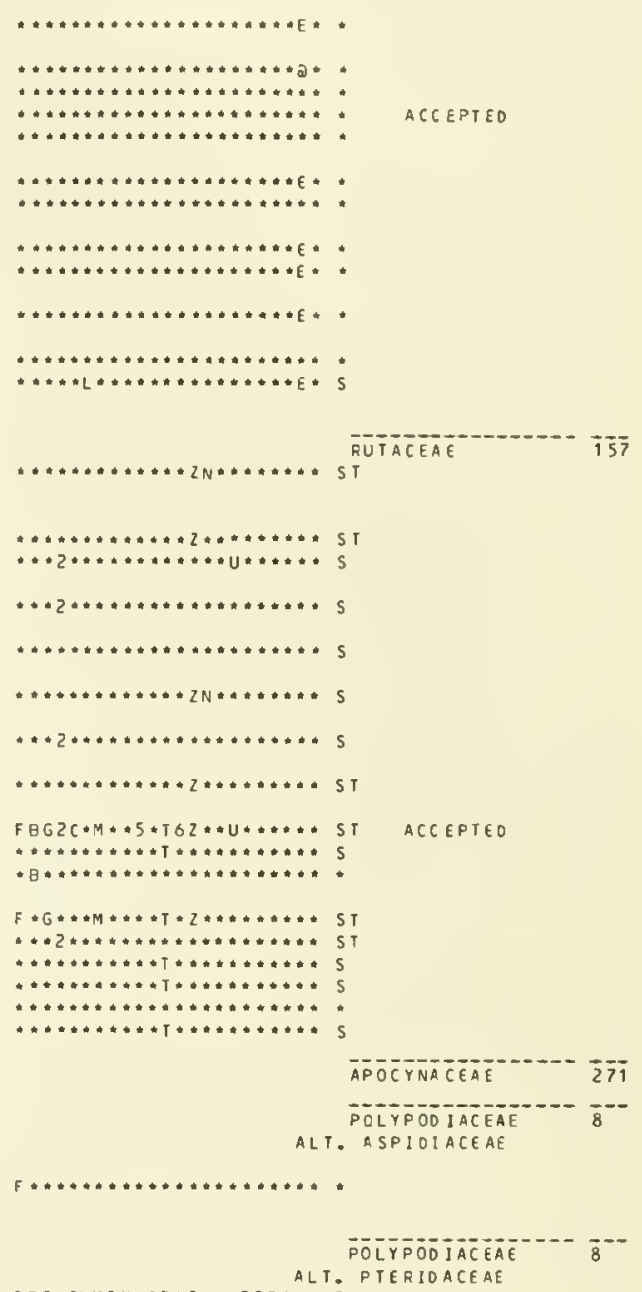
FBG CLMSY*RTGZ*+U9ORALE PF

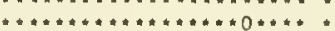

$\ldots \ldots \ldots \ldots \ldots+\cdots+\ldots+\cdots+A+*$

$*+*+*+\cdots+* *+\cdots * * * * E *$

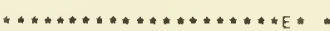

POLYPOOTACEAE ALT . PTERIDACEAE

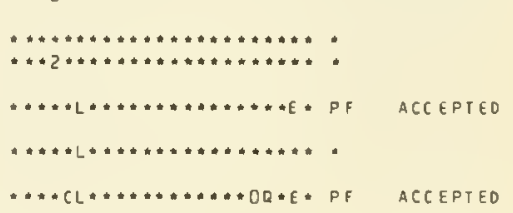

LEGUMINOSAE

ALT. PAPILIONACEAE

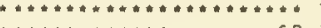

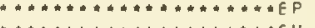

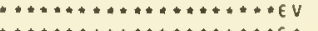

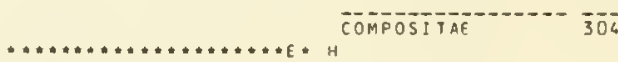

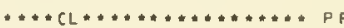

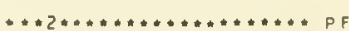




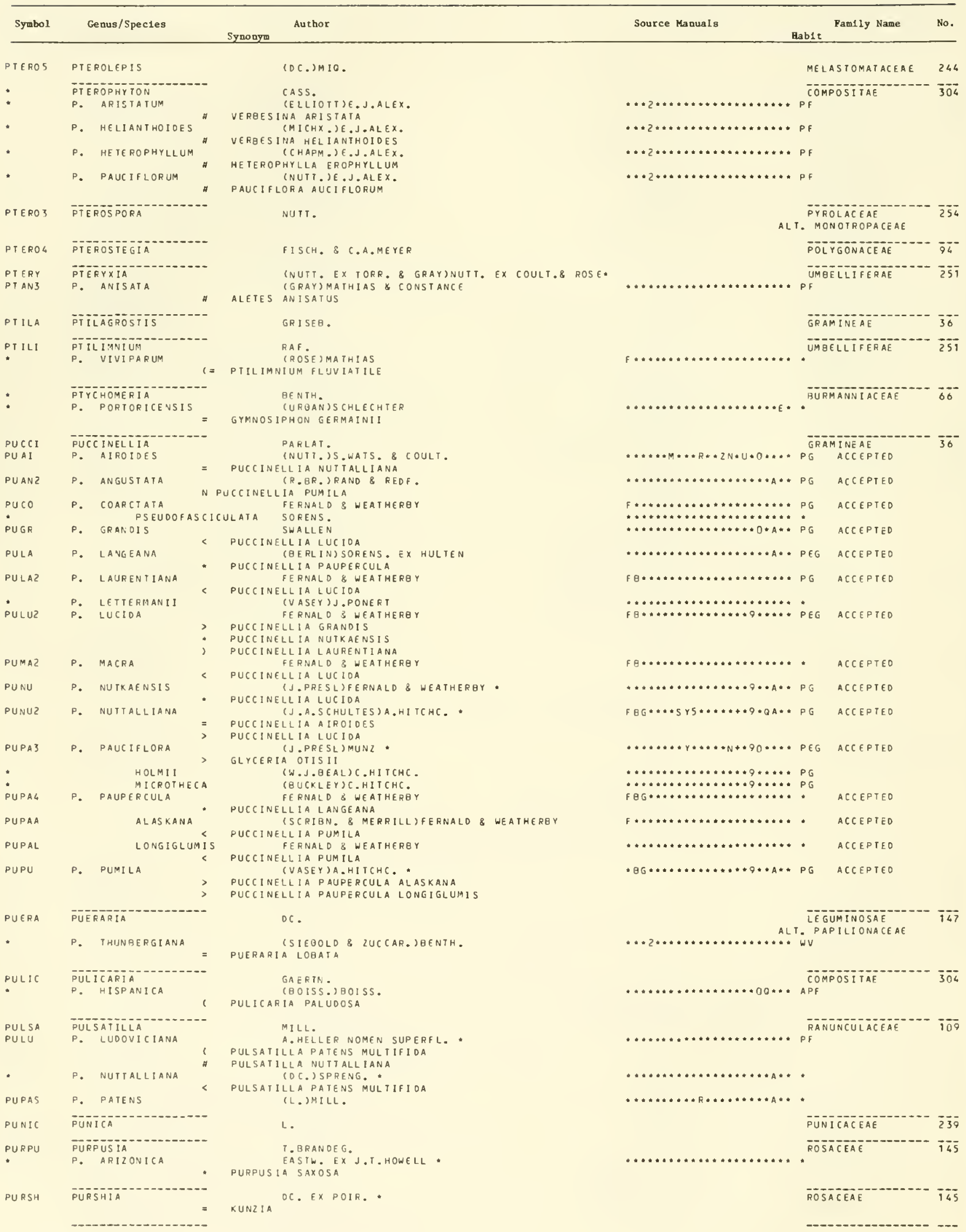


PYCNANTHEMUM

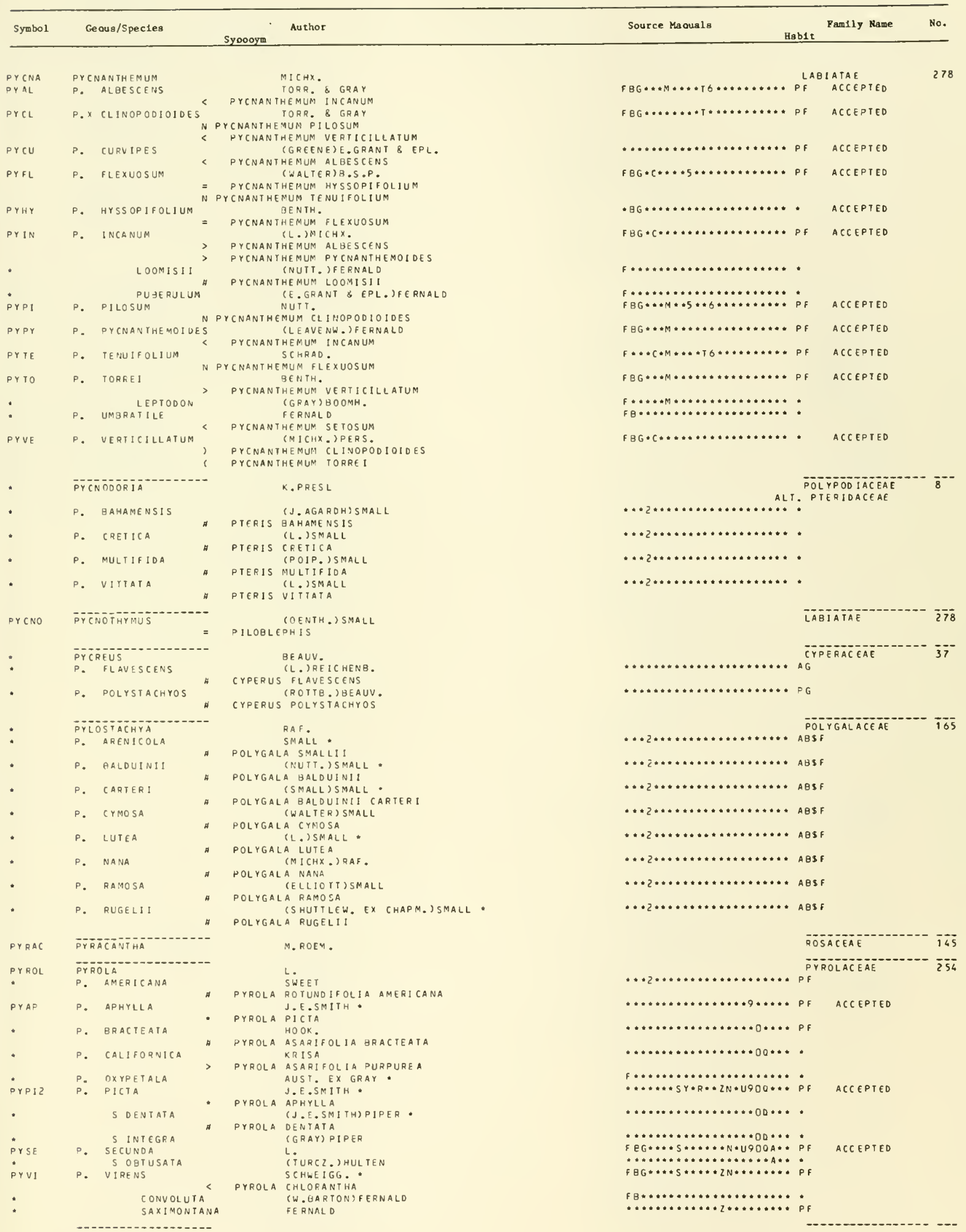




\begin{tabular}{|c|c|}
\hline Symbol & Genus/Species \\
\hline ROS & $\begin{array}{l}\text { PYROSTEGIA } \\
\text { P. IGNEA }\end{array}$ \\
\hline $\begin{array}{l}R P H \\
\text { Y CAZ }\end{array}$ & $\begin{array}{l}\text { PYRRHOPAPPUS } \\
\text { P. CAROLINIANUS } \\
\text { GEORGIANU }\end{array}$ \\
\hline GE & P. GE ISERI \\
\hline Muz & P. MULTICAULIS \\
\hline RO2 & P. ROIHROCKII \\
\hline & P. SCAPOSUS \\
\hline & P. SESSAEANUS \\
\hline & $\begin{array}{l}\text { PYRROCOPA } \\
\text { P. CLEMENT IS }\end{array}$ \\
\hline & P. CROCEA \\
\hline & P. INTEGPJFOLIA \\
\hline & P. LAINCEOLLATA \\
\hline & P. UNIFLORA \\
\hline
\end{tabular}

PYRUL

PYPUS

कर RULAR!A

\section{PYRUंड}

P. X ALPINA

P. AMERICANA

F. AMGLLSTIFOLIA

P. ARBUIIFOLIA

P. X APSENII

P. AUCUPARIA

P. BACCATA

P. GRACTEATA

P. CORONARIA

\section{DASYCALYX} LANCIFULIA

P. OECCRA GROENLANOICA

P. $X$ FALLAX

P. FLOPIJUNOA

P. FUSCA

P.. glaucescens

P. X HYGFIOA

P. TOENSIS

$$
\begin{aligned}
& \text { PUSHII } \\
& \text { PALMERI }
\end{aligned}
$$$$
\text { P.X JACVI }
$$

P. LanCifolia

P. MALL'S

P. MELANOCARPA

P. $\times$ MIXTA

P. P PLATYCARPA

P. PPUI:IFOLIA

P. RIVLLAPIS

P. X SOULARDII
K. PRESL

(VAHL)ORESL

PYROSTEGIA VENUSTA

$D C$.

('NALTER) DC.

( $S H I N N E R S$ ) H. E. AHLES

PYRRHOPAPPUS GEORGIANU

SHINNERS

PYRRHOPAPPUS MULTICAULIS GEISERI

$D C$.

PYRRHOPAPPUS ROTHROCKII

GRAY

PYPRHOPAPPUS MULTICAULIS

OC.

PYARHOPAPPUS GRANOIFLORUS

$(O . D O N) D C$.

HOOK.

rY $P$ O

HAPLOPAPPUS CLEMENTIS

(GRAY) GREEPE.

HAPLOPAPPUS CROCEUS

(I PORTER EX GRAY) GREENE *

HAPLOPAPPUS INTEGRIFOLIUS

(HOOK.) GREENE

HAPLOPAPPUS LANCEOLATUS

(HUOR.) GREENE

HAPLOPAPOUS UNI FLORUS

$$
\text { MICHX. }
$$

$L$.

SOroAR OUILLO.

WILLO.

(MARSHALL) DC.

AMEKICAN

$A ! T$.

MALUS ANGUSTIFOLIA

REHO.) L.H.BAILEY

ARONIA ARBUTIFOL

(GRTTTON) ARSENE

SOREARONIA ARSENII

L.) GGAERIN.

SORBUS AUCUPARIA

L.

MALUS BACLATA

PYRUS COROHAPIA

MALUS BRACIEATA

MALUS PLAIYCARPA

MALUS CORONARIA

$$
\text { (REHO.) FERNALD }
$$

(REHO.)FERNALD

PYRUS LANCIFOLIA

(SARG.)HYL.

SORBUS DECORA

(C. . . SC MNE 1D. F FERNALD

SORBUS GROENLANDICA

(C.R.SCHNE ID.) FERNALD

SORBARONIA FALLAX

ARONIA PRUNIFOLIA

ARONIA PREF

MALUS FUSCA

PYRUS CREHO.) L.H.BAILEY

MYRUS CORONARIA

MALUS GLAUCESCENS

MOENCH

(A. WOOO) L.H.BAILEY

WALUS IOENSIS

MALUS SOULARDI!

CREHO.) L.H. BAILEY

(REHD.) L.H.BAILEY

(REHD.) FERNALD

SORBARONIA JACKI

(REHO. L.H.BAILEY

PYRUS CORONARIA LANCIFOLIA

L. "

MALUS PUMILA

MALUS SYLVESTRIS

( IAICHX.)WILLO.

ARONIA PRUNIFOLIA

FERNALD

SORGARONIA SORHIFOLIA

L. H.AAILEY

MALUS PLATYCARPA

WILLO.

MALUS PRUNIFOLIA

OOUGL. EX HOOK.

MALUS FUSCA

$$
\text { L.H.BAILEY }
$$

MALUS SOULAPDI!
Source Manuals Habit

Family Name

No.

BIGNONIACEAE 282

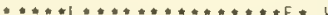

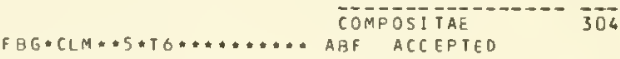

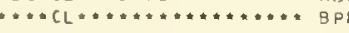

$\cdots * \cdots * * \cdots * * * 6 * \cdots \cdots * * \cdots * A$

$\cdots \cdots \cdots \cdots+T * 2 N \cdots \cdots \cdots$ PAF ACCEPTED

$\ldots \ldots \ldots \ldots+\ldots \ldots \ldots$. $\ldots \ldots$ accepteo

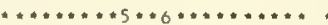

$\ldots \ldots \ldots \ldots+\ldots \ldots \ldots \ldots$ A $\ldots \ldots$

COMPOSITAE

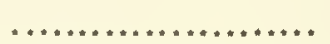

$\ldots \ldots \ldots \ldots \ldots \ldots \ldots+\ldots$

$* \ldots \ldots+\ldots \ldots \ldots \ldots \ldots \ldots$
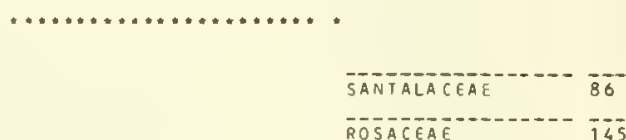

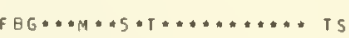

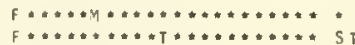

$f \ldots \ldots \ldots+\ldots \ldots \ldots+\ldots \ldots+$ t

$F \ldots \ldots \ldots \ldots \ldots \ldots \ldots \ldots \ldots$

f $3 G \ldots \ldots \ldots \ldots \ldots \ldots \ldots$

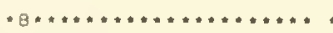

$F B G \cdots \cdots \cdots+\ldots \ldots+\cdots \cdots * * *$

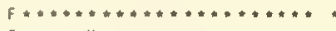

$F \ldots \cdots+4 \ldots \ldots \ldots \ldots \ldots \ldots * *$

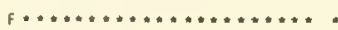

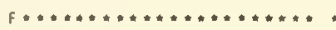

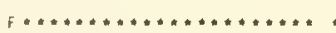

$F \cdots \cdots+\ldots+\ldots \ldots+\ldots \ldots \ldots+$

$\ldots \ldots \ldots \ldots+\ldots \ldots \ldots+\ldots, \ldots, s t$
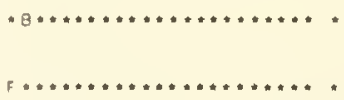

$F B G \cdots M S \cdot 5 \cdot T 6 \ldots \ldots \ldots \ldots \ldots$ IS

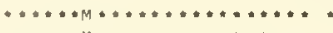

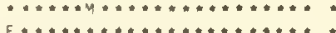
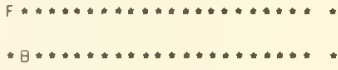

$F B G \cdots \cdots M * 45 \cdots 6 \cdots \cdots 9 \cdots \cdots+1$

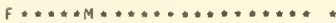

$f, \ldots \ldots \ldots \ldots \ldots \ldots \ldots \ldots$.
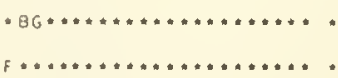

$\mathrm{F} B \mathrm{G} \cdots * \mathrm{M} \ldots \ldots \ldots \ldots \ldots \ldots \ldots$ 


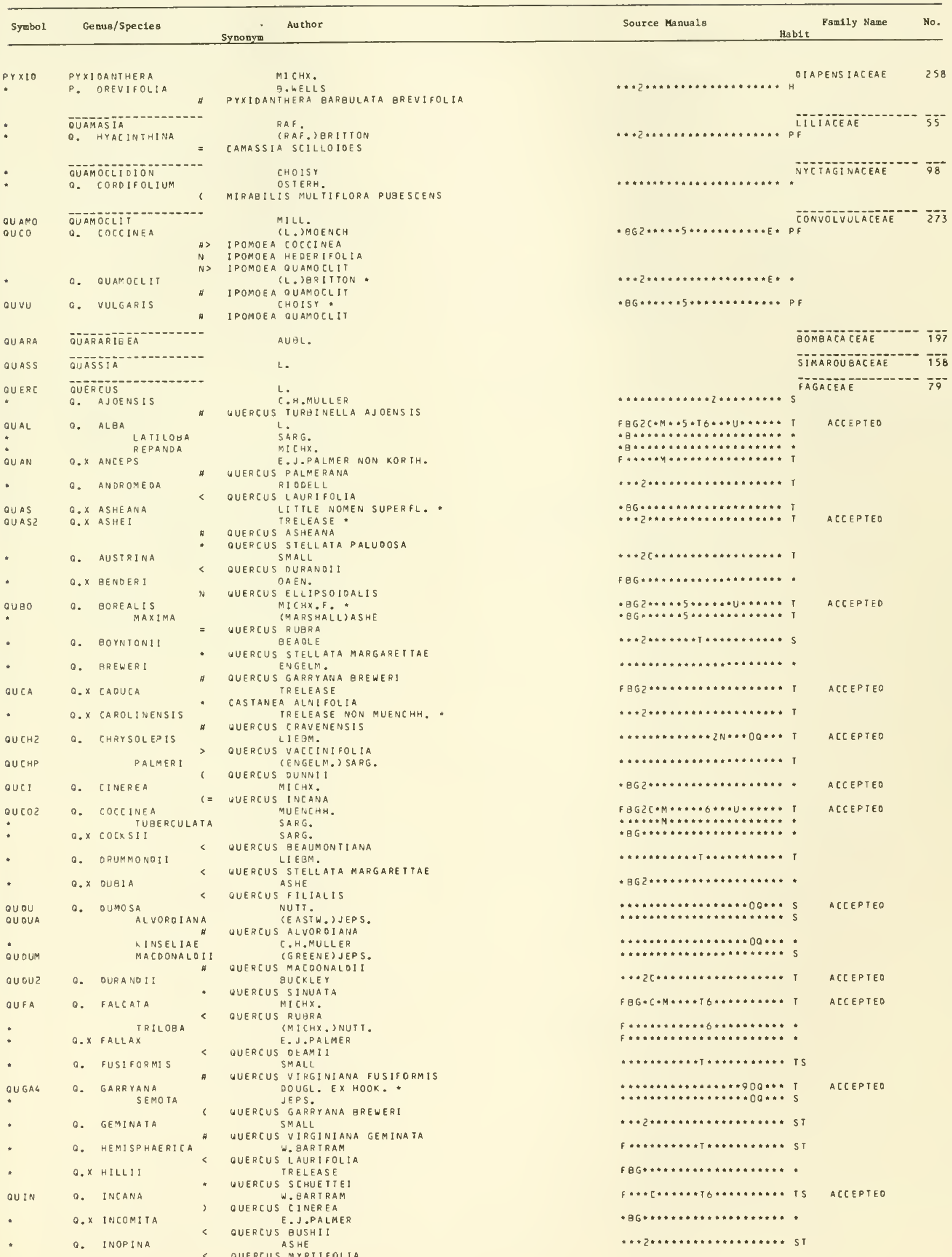




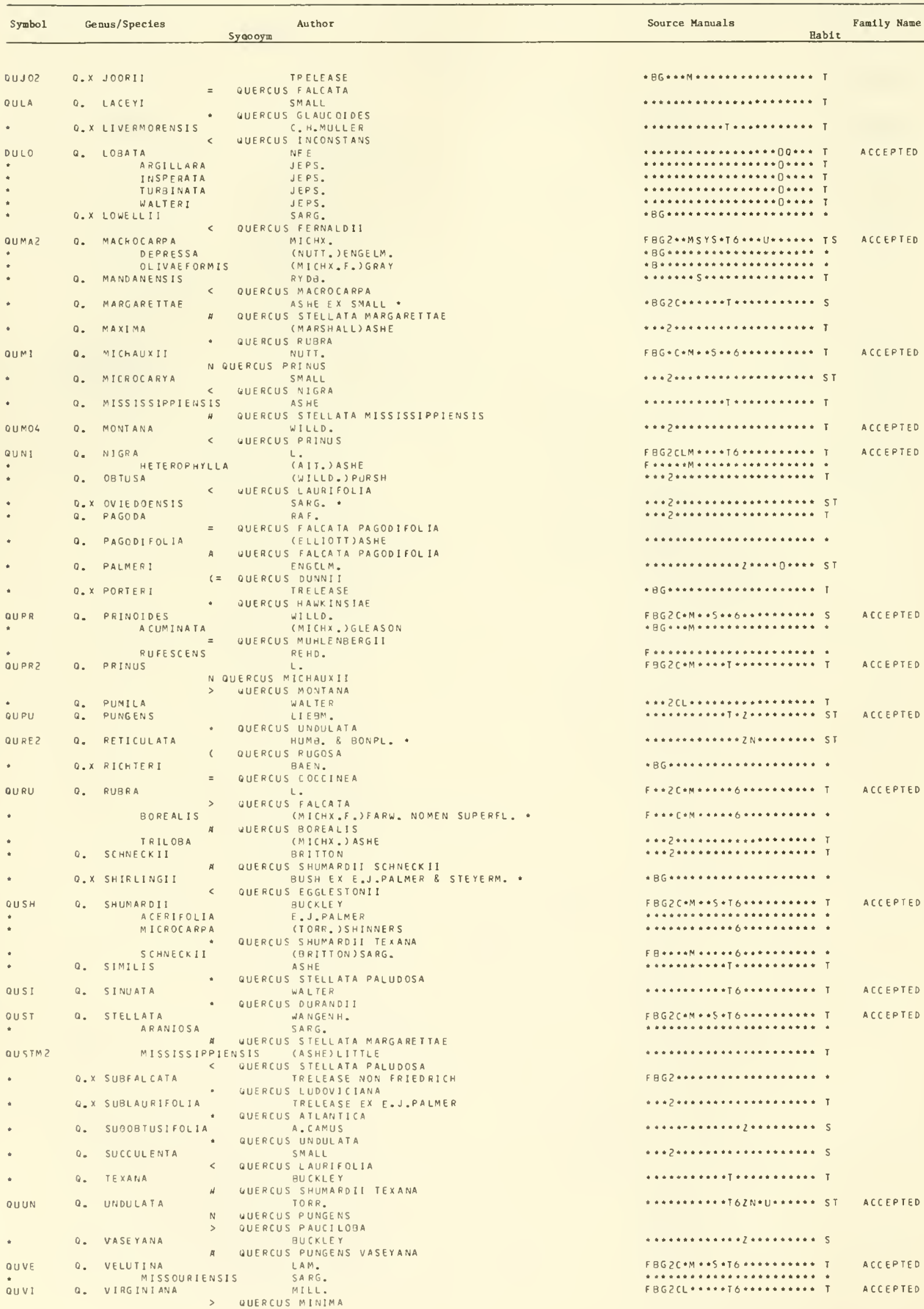




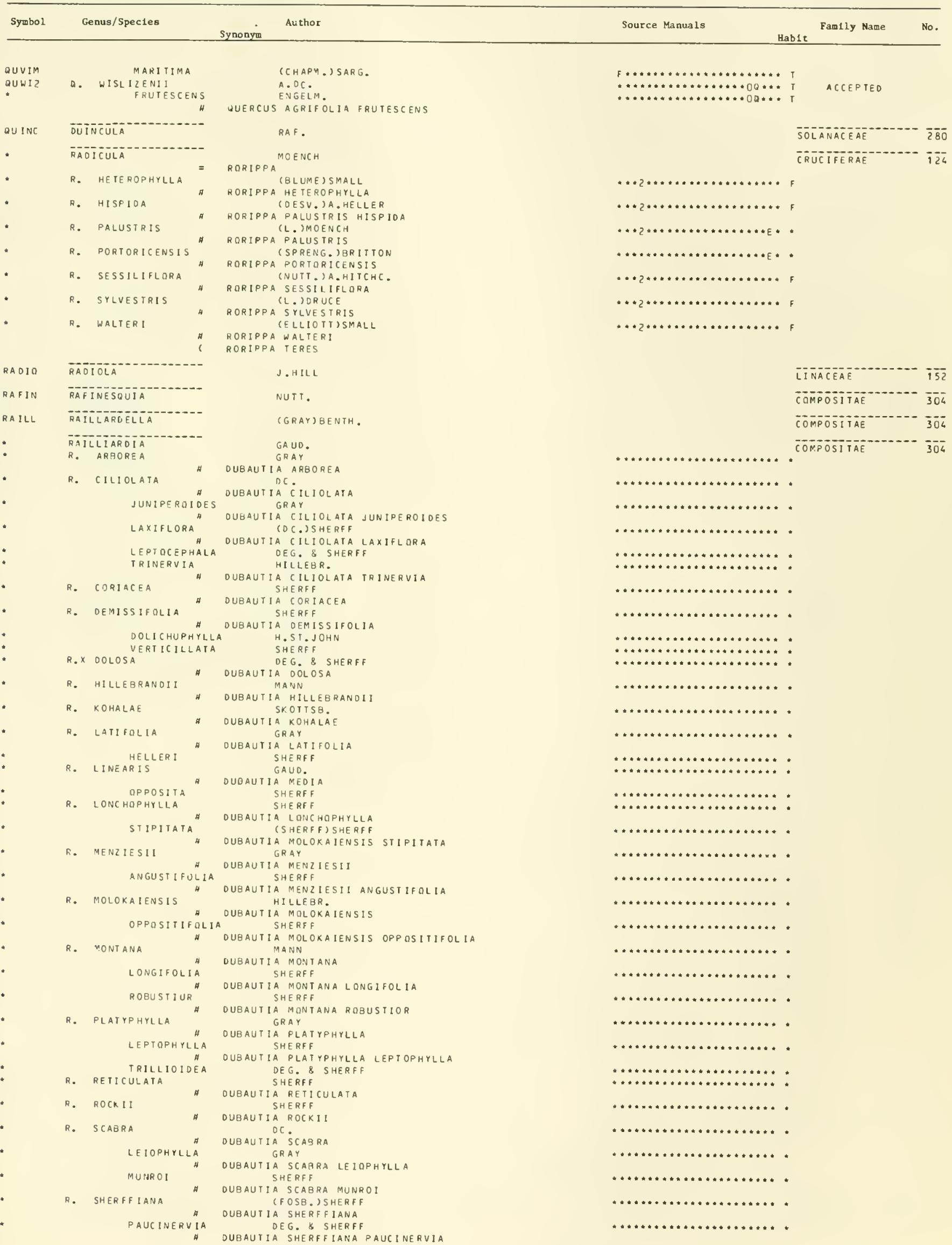




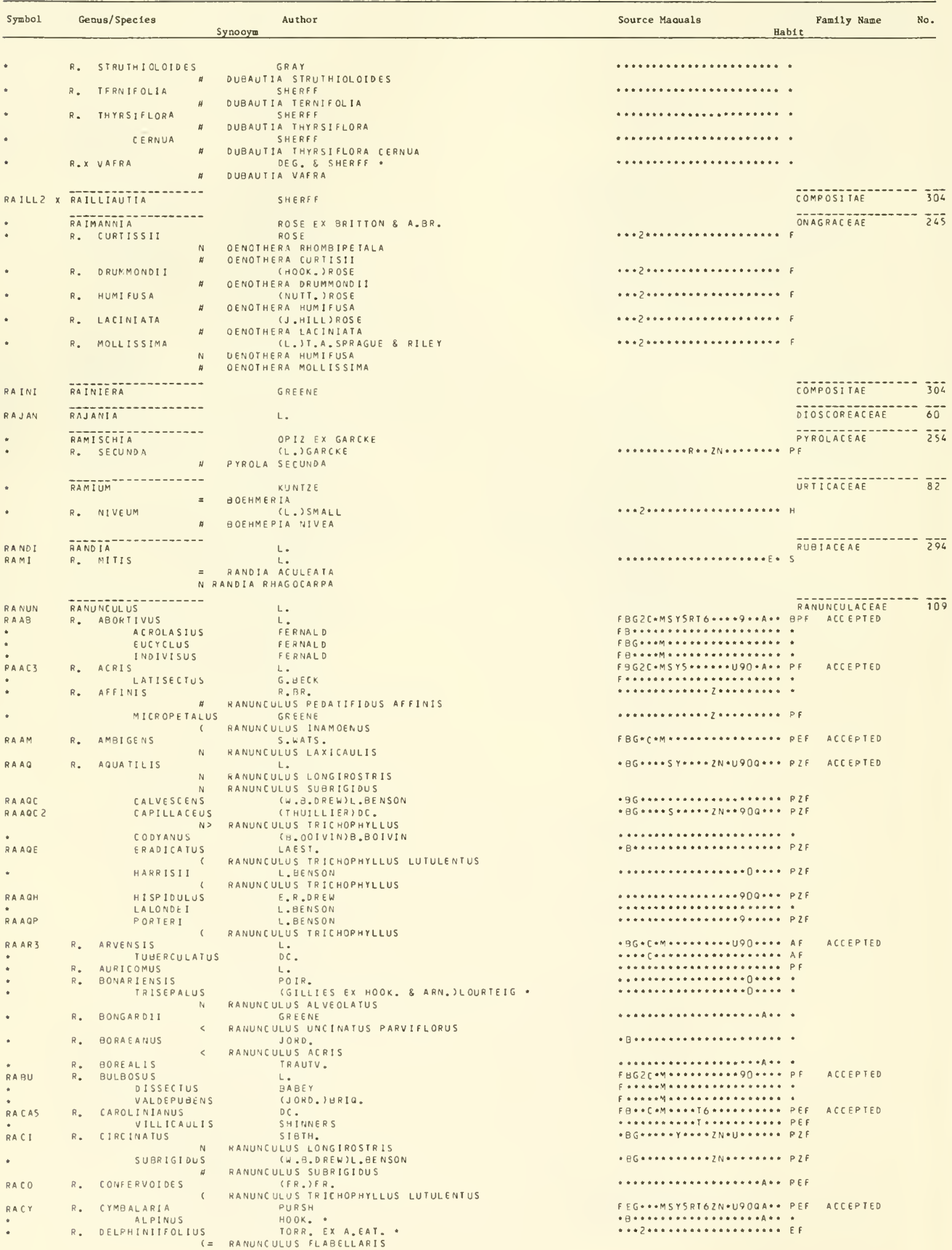


RANUNCULUS

\begin{tabular}{|c|c|c|c|}
\hline Syubol & & Senua/Speciea & Sraonye \\
\hline RAES & R. & ESCHSCHOL TZII & SCHLECHT. \\
\hline & & $\mathrm{N}$ & RANUNCULUS NIVALIS \\
\hline * & & ALPINUS & IS.WATS.)C. HITCHC. \\
\hline RAESE & & EXIMIUS & $\begin{array}{l}\text { HANUNCULUS AOONEUS ALPINUS } \\
\text { (GREENE) } L \text {. BENSON }\end{array}$ \\
\hline & & N< & RANUNCULUS ESCHSCHOLIZII SUKSDORFII \\
\hline RAESS & & SUKSDORFII & (GRAY) L. OENSON \\
\hline & & $N>$ & RANUNCULUS ESCHSCHOLIZII EXIMIUS \\
\hline RAFA & $\mathrm{R}$. & FASCICULARIS & MUHL. EX BIGEL. • \\
\hline$:$ & & APRICUS & (GREENE) FERNAL D \\
\hline RAFL? & R. & $\begin{array}{l}\text { CULE IFORMIS } \\
\text { FLAMMULA }\end{array}$ & $\begin{array}{l}\text { ( SMALL)L. BENSON } \\
\text { L. }\end{array}$ \\
\hline • & & ANGUSTIFOLIU & WALLR. \\
\hline - & & SAMOLIF OLIUS & (GREENE) L. BENSON \\
\hline & & 1 & RANUNCULUS FLAMMULA OVALIS \\
\hline RAGE & R. & GELIOUS & KAAEL. \& KIR. \\
\hline - & & S GRAYI & ( GRITTON) HULTEN \\
\hline - & & SHUMAGINENSI & HULTEN \\
\hline RA GL? & R. & GLACIALIS & L. \\
\hline RAGM & R. & GMELINII & $O C$. \\
\hline - & & PROLIFICUS & $\begin{array}{l}\text { (FERNALO)H. HARA } \\
\text { (FER }\end{array}$ \\
\hline - & & S PURSHII & $\begin{array}{l}\text { RANUNCULUS GMELINI I HOOKERI } \\
\text { (RI I CHAROS.) HULTEN }\end{array}$ \\
\hline & & 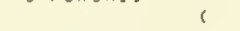 & RANUNCULUS GMELINII HOOKERI \\
\hline$R \wedge G R$ & R. & GRANDIS & HONDA \\
\hline * & R. & GRAY 1 & BRITTON \\
\hline & & n & RANUNCULUS GELIDUS GRAYI \\
\hline RAHI & R. & HISPIDUS & MI CHX. \\
\hline * & & FALSUS & FERNALD \\
\hline & & $\mathrm{c}=$ & RANUNCULUS HISPIDUS MARILANDICUS \\
\hline RAHYZ & R. & HYPERGOREUS & ROTTE. - \\
\hline - & & S ARNELL $! 1$ & SC HEUT 2 \\
\hline - & & S INTERTEXIUS & (GREENE) KAPOOR \& L OEVE \\
\hline & & \# & RANUNCULUS NATANS INTERTEXTUS \\
\hline RAIN & R. & INAMOENUS & GREENE \\
\hline . & & ALPEOPHILUS & (A. NEL S.) L. BENSON \\
\hline * & & SUAAFFINIS & (GRAY)L.BENSON \\
\hline RALAZ & R. & LAXIGAULIS N N & $\begin{array}{l}\text { (TORR. इ GRAY) DARBY } \\
\text { RANUNCULUS AMBIGENS }\end{array}$ \\
\hline * & R. & Limosus & $\begin{array}{l}\text { NUTT. } \\
\text { RANUNCULUS GMELIHI LIMOSUS }\end{array}$ \\
\hline * & R. & LINOHE IMERI & ENGELM. \\
\hline RALO2 & $R$. & LONGIROSTRIS & $\begin{array}{l}\text { RANUNCULUS PUSILLUS } \\
\text { GOOR. } \\
\text { RANUNCULUS AQUAIILIS }\end{array}$ \\
\hline RAMAZ & R. & MACOUN I I & BRITTON \\
\hline - & & OREGANUS & GRAY \\
\hline RAMA3 & $R_{\text {. }}$ & MACRANTHUS & SCHEELE \\
\hline & & N< & RANUNCULUS ORTHORHYNCHUS PLATYPHYLLUS \\
\hline RAMIZ & $R_{0}$ & MICRANTHUS & NUTT. \\
\hline - & & CYMBALISTES & (GREENE) FERNALD \\
\hline - & & DELITESCENS & (GREENE) FERNAL D \\
\hline - & R. & MISSISSIPPIENSIS & SMALL \\
\hline & & N< & $\begin{array}{l}\text { RANUNCULUS AMBIGENS } \\
\text { RANUNCULUS LAXICAULIS }\end{array}$ \\
\hline
\end{tabular}

Source Manuals

Fanlly Nam

No.

RANA R. NATAMS

R. NIVALLS COS LAXICAULIS

$$
\text { C.A.MEYER }
$$

RANUNCULUS ESCHSCHOLIZII

RANUNCULUS SULPHUREUS

$$
\text { ELL IOTT }
$$

RANUNCULUS LAXICAULIS

RANUNCULUS PUSILLUS

RAF.

R. OATUSIUSCULUS

RANUNCULUS AMBIGENS NU TT. HULTEN

RAOC R. OCCIOENTALIS

S HELSONII

RANUNCULUS OCCIOENTALIS NELSONI

(OC.) HULTEN

S ALASCHENSIS

R. PALMATUS GRAY

RANUNCULUS MACRANTHUS (L. BENSON) HULTEN (LL BENSON)
ELLIOTT

RANUNCULUS CAROLINIANUS

RANUNCULUS HISPIDUS MARILANDICUS

R. PARVULUS

R. PEOATIFIDUS

$S$ AFFINIS

R. PYGMAEUS

PETIOLULATUS
S SABINEI

$(=$ RANUNCULUS SARDOUS

J. SMI H H

( R. BR.) HULTEN

WAHLENB.

WA

(R. BR.) HULTEN

R. RECONDITUS

\#

RANUNCULUS SABINEI

A.NELS. \& J.F.MACBR.

r. recurvatus POIR.

R. REPENS A DPRESSIPILI ERECTUS GLABRATUS LINEAR I LOBUS PLENIFLORUS

R. REPTALS
R. ROSUS

$$
\text { POIR. }
$$

WEATHERGY

D.

$O C$.

$O C$.

FERNALD

LAMOITE

RANUNCULUS FLAMMULA FLLIFORMIS

(HOOK.) TORR. \& GRAY

INTERMEOIUS OVAL IS

ACCEPTED

ACCEPTED

ACCEPTED

ACCEPTED F $6 G * \cdots * Y * * 2 N * 900 \cdots *$ PEF ACCEPTED

$F \ldots \ldots \ldots \ldots \ldots \ldots \ldots \ldots \ldots * \ldots * \ldots$

$\ldots \ldots \ldots \ldots * R * \ldots \ldots ., \ldots A \ldots P F$

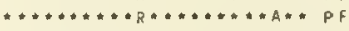

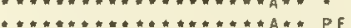

FBG …YPR \#\#UQ\#A* PEF ACCEPTEO $\mathrm{R} B \cdots \ldots \ldots+\ldots \ldots \ldots \ldots \ldots+\ldots$

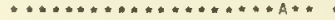

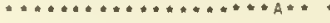

F BGZC*MS*5*6*...... PF ACCEPTED

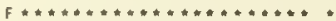

F....... R.... $\cdots \cdots \cdots \cdots \cdots \cdots \cdots \cdots \cdots \cdots \cdots \cdots$ $\ldots \ldots \ldots+R+\ldots \ldots \ldots \ldots+P E$

$\ldots \ldots+S Y \cdot R * Z N \cdot U 9 \ldots \ldots P F$

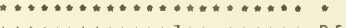

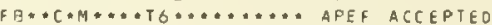

$\bullet \theta * \cdots * \cdots * \ldots * \ldots \ldots \ldots * \ldots$.

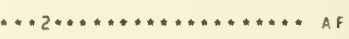

FBG...MS.5.T6\#. U9.... PZIF ACCEPTED

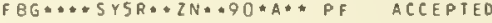
$* \ldots *+* *+\ldots \ldots+9 * \ldots *$ PAF ........ I. ZN....... PEF ACCEPTED

F $8 G 2 C \cdot M S * 5 * 6 * \cdots * * * *$ PF ACCEPTED

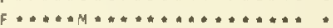
$\because * 2 * \ldots \ldots \ldots \ldots \ldots \ldots \ldots \ldots$ Af

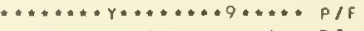

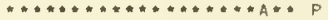

ACCEPTED
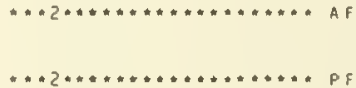

$\cdots * * * * * 4 * * * \cdots * 900 A * * P F$

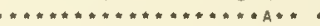

ACCEPTEO $* * * * Y * \cdots * * \cdots 90 * A * * P F$ $\cdots \ldots+\ldots+\ldots \cdots+90 \cdots \cdots P F$

ACCEPTED ACCEPIED

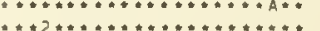

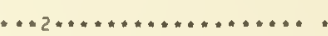

$F B \cdot \cdots \cdots Y+R \cdots Z N \cdot U, \cdots A \cdots P F$

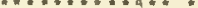
$E B G * * Y * R \cdots \cdots+Q * A * P F$

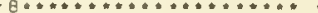

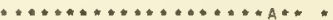

$\ldots \ldots \ldots+\ldots \ldots \ldots \ldots \ldots 9 \ldots \ldots . .+P F$

$F 8 G 5 C \cdot M * * 2 * 16 \cdots \cdots Q * \ldots * P F$ F, acCEPTED

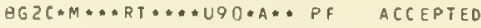

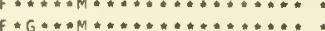

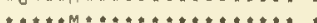
$F \cdot G \cdots \cdots \cdots * T \cdots \cdots 90 \cdots \cdots$ PF

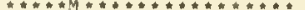
$F \cdots \ldots \ldots \ldots R * \ldots \ldots \ldots A * * P E Z F$

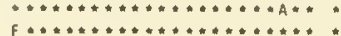




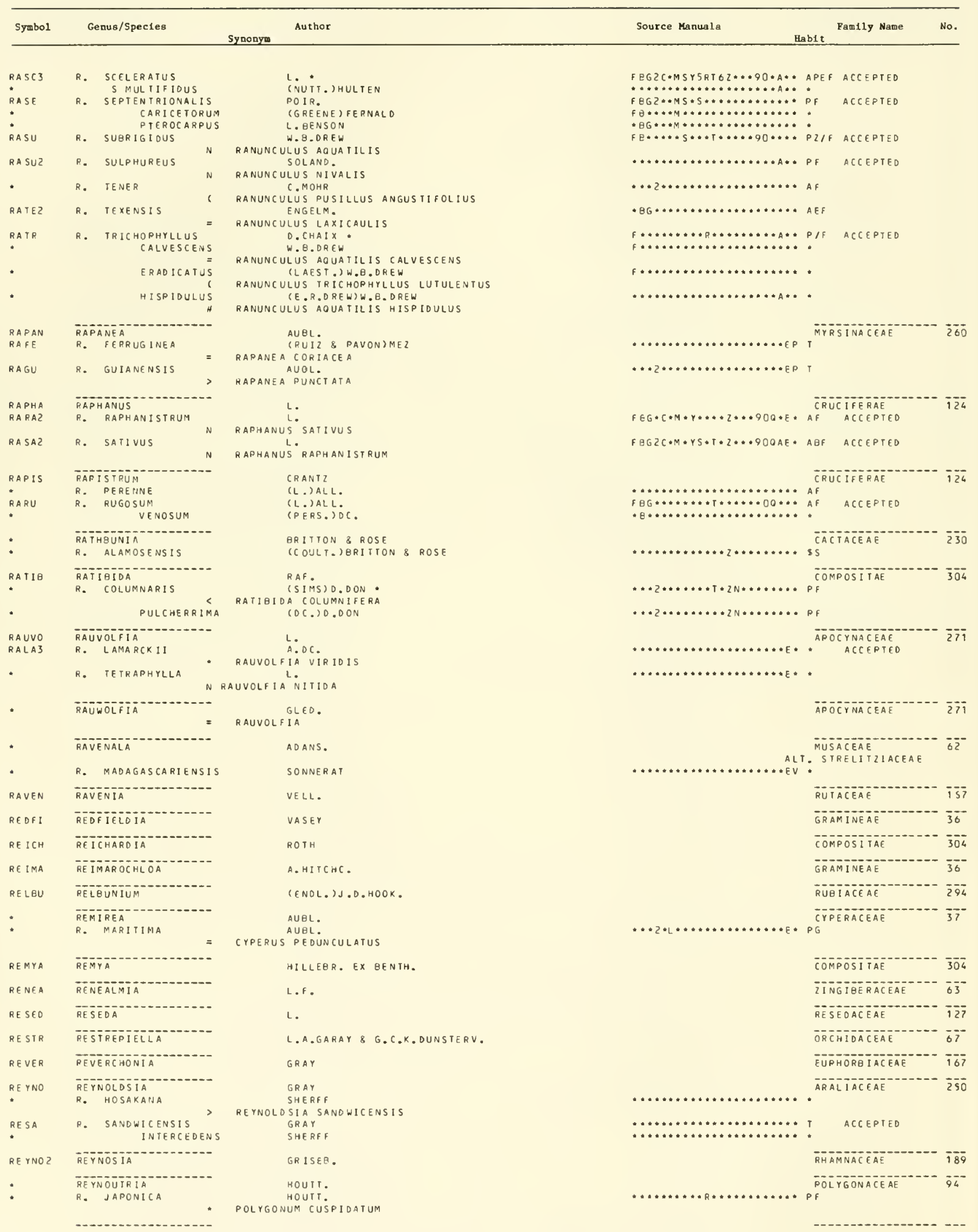




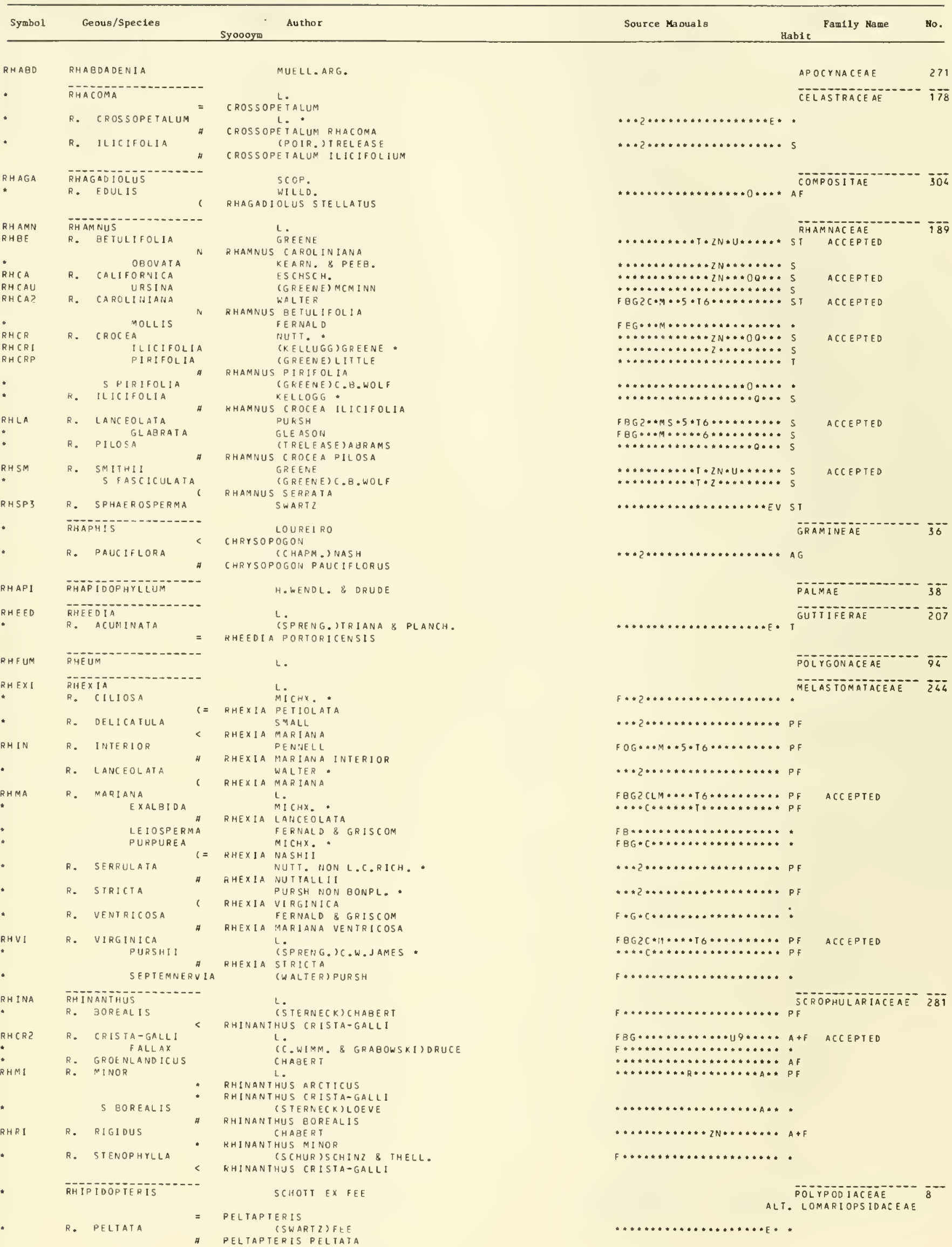




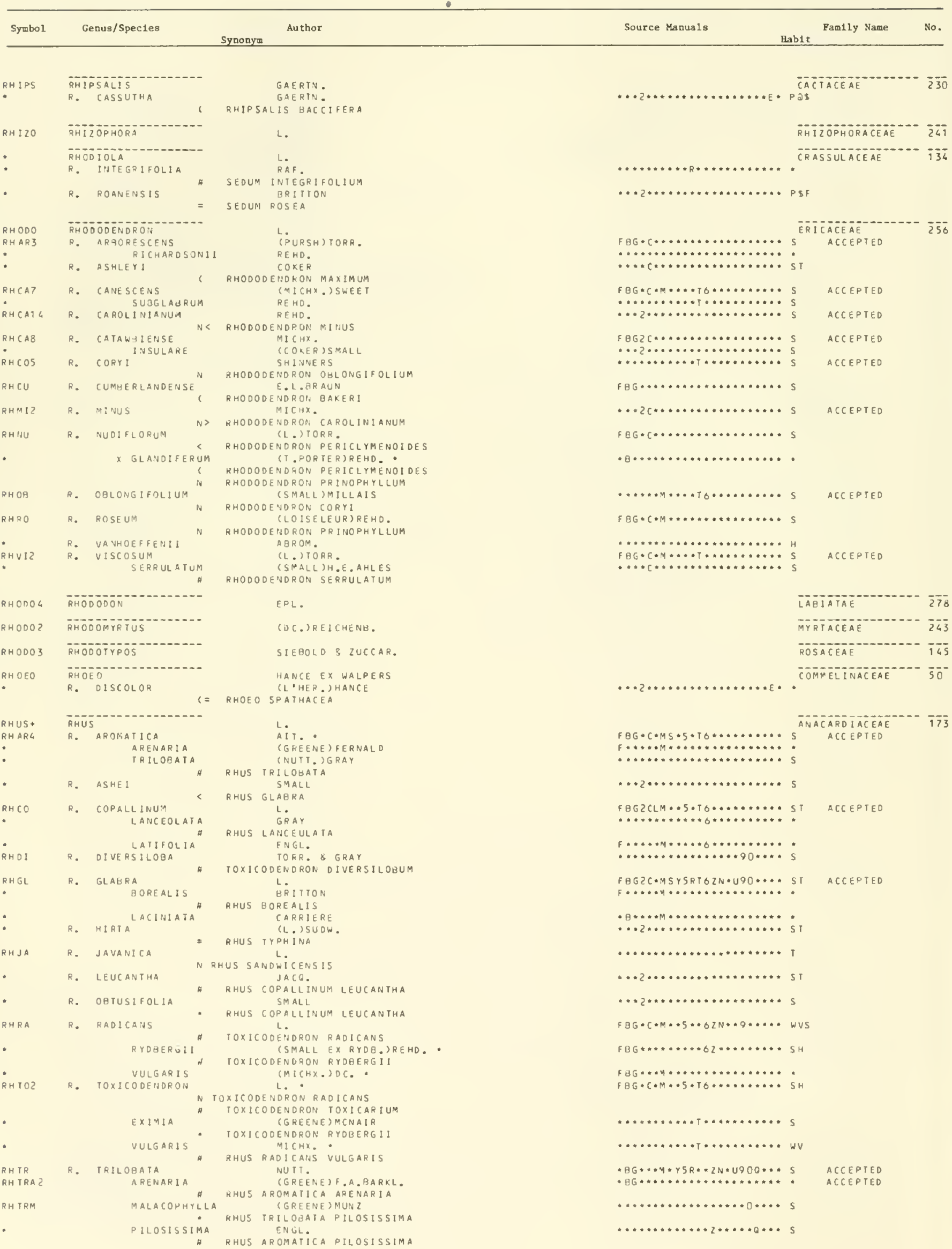




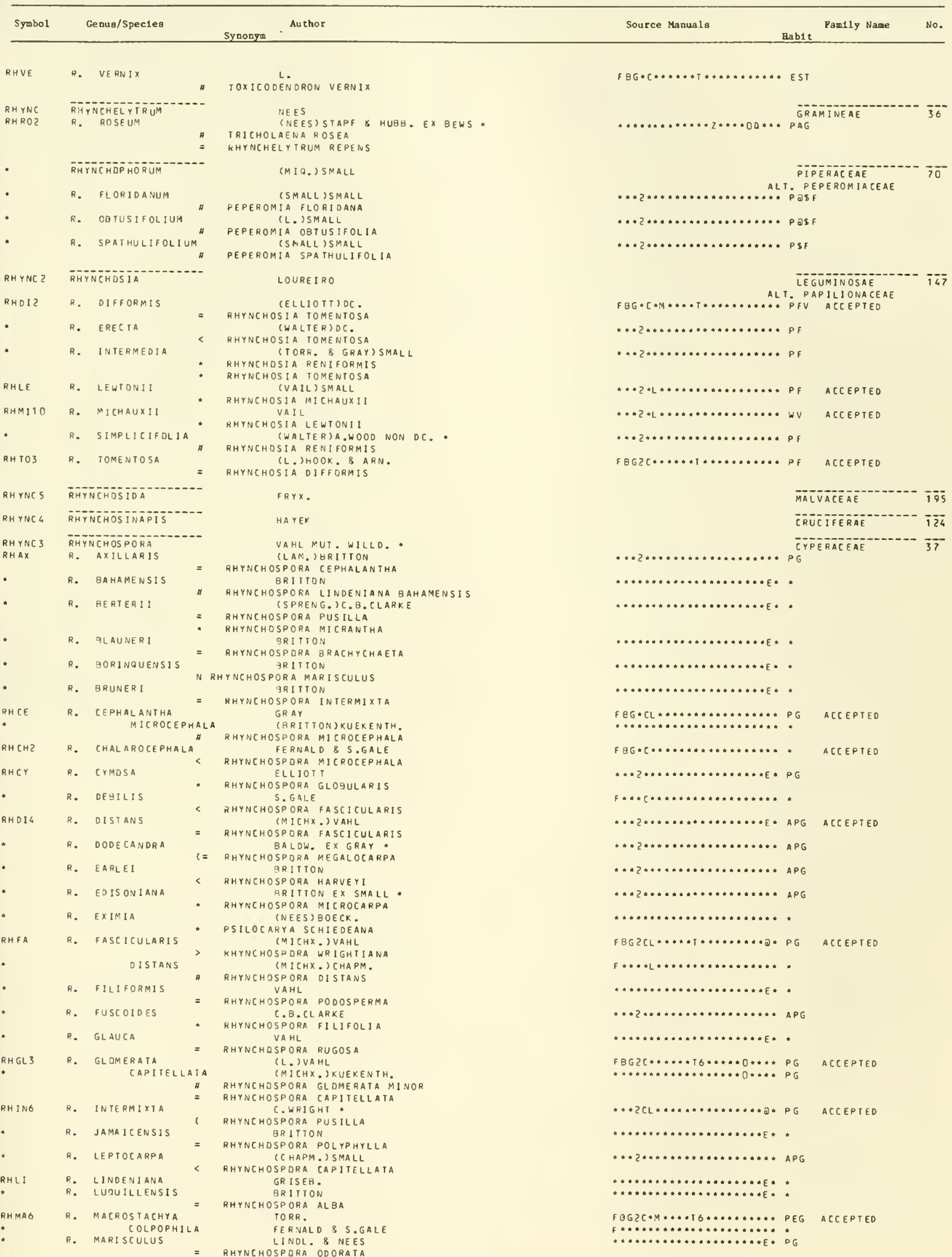




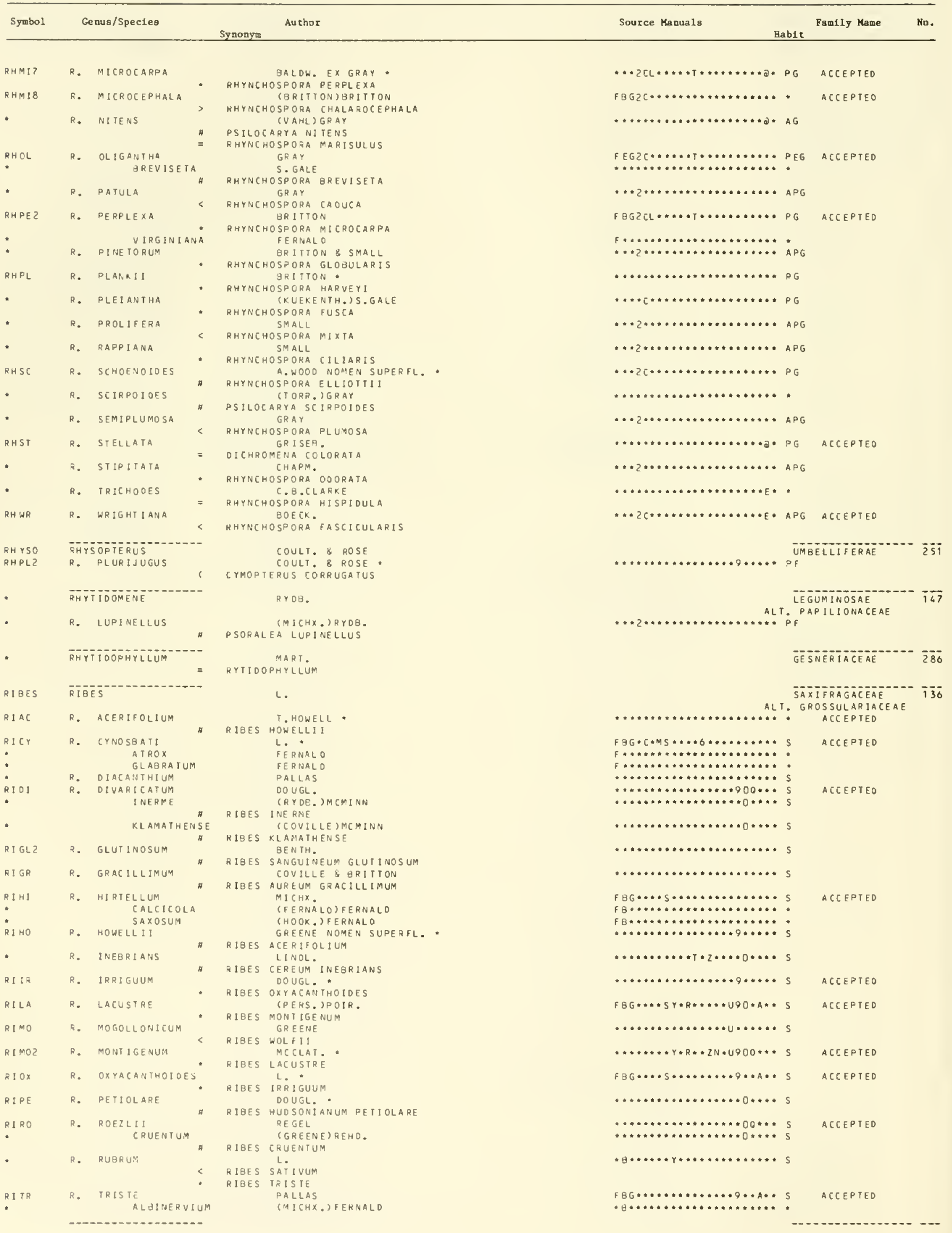


RICHARDIA

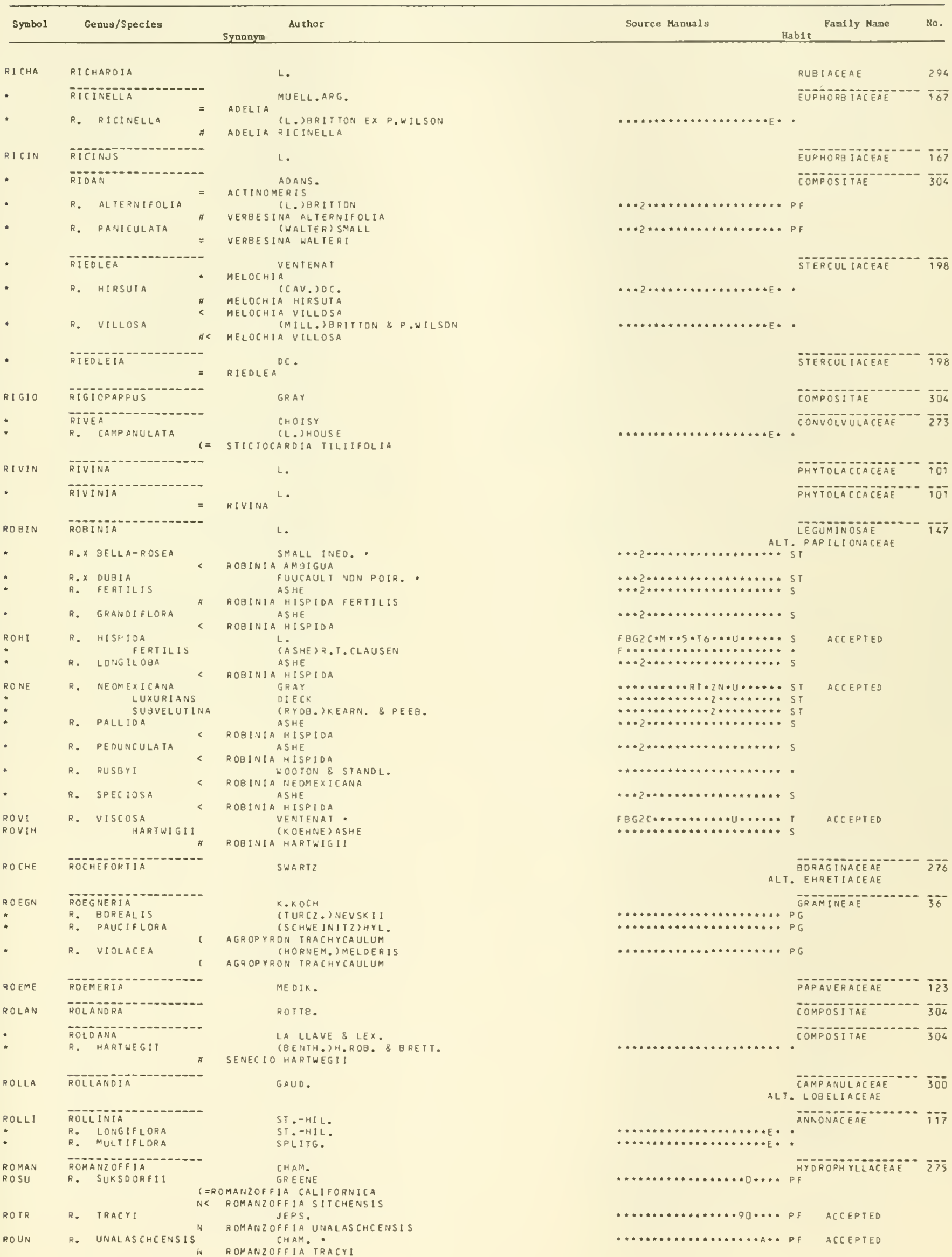




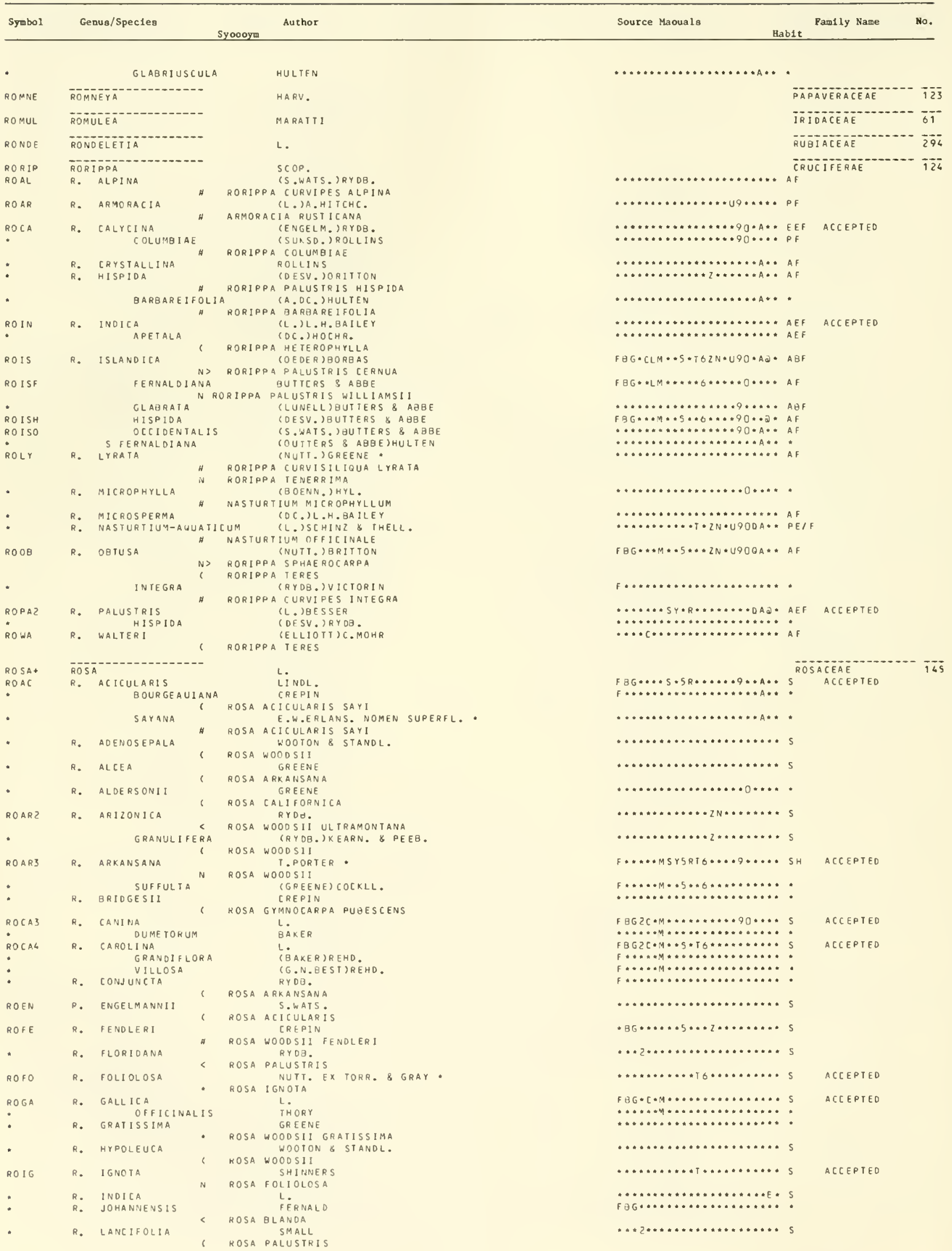




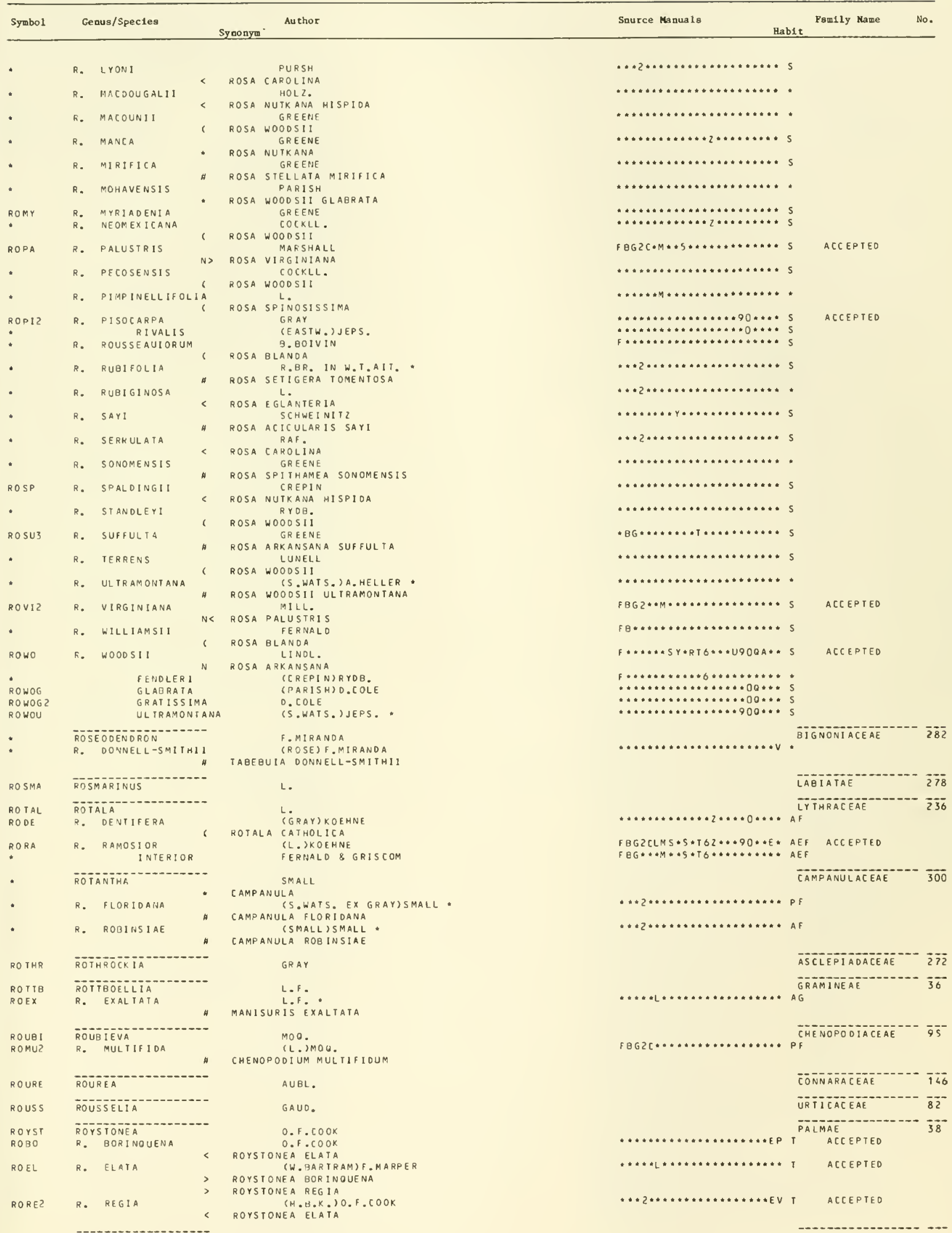




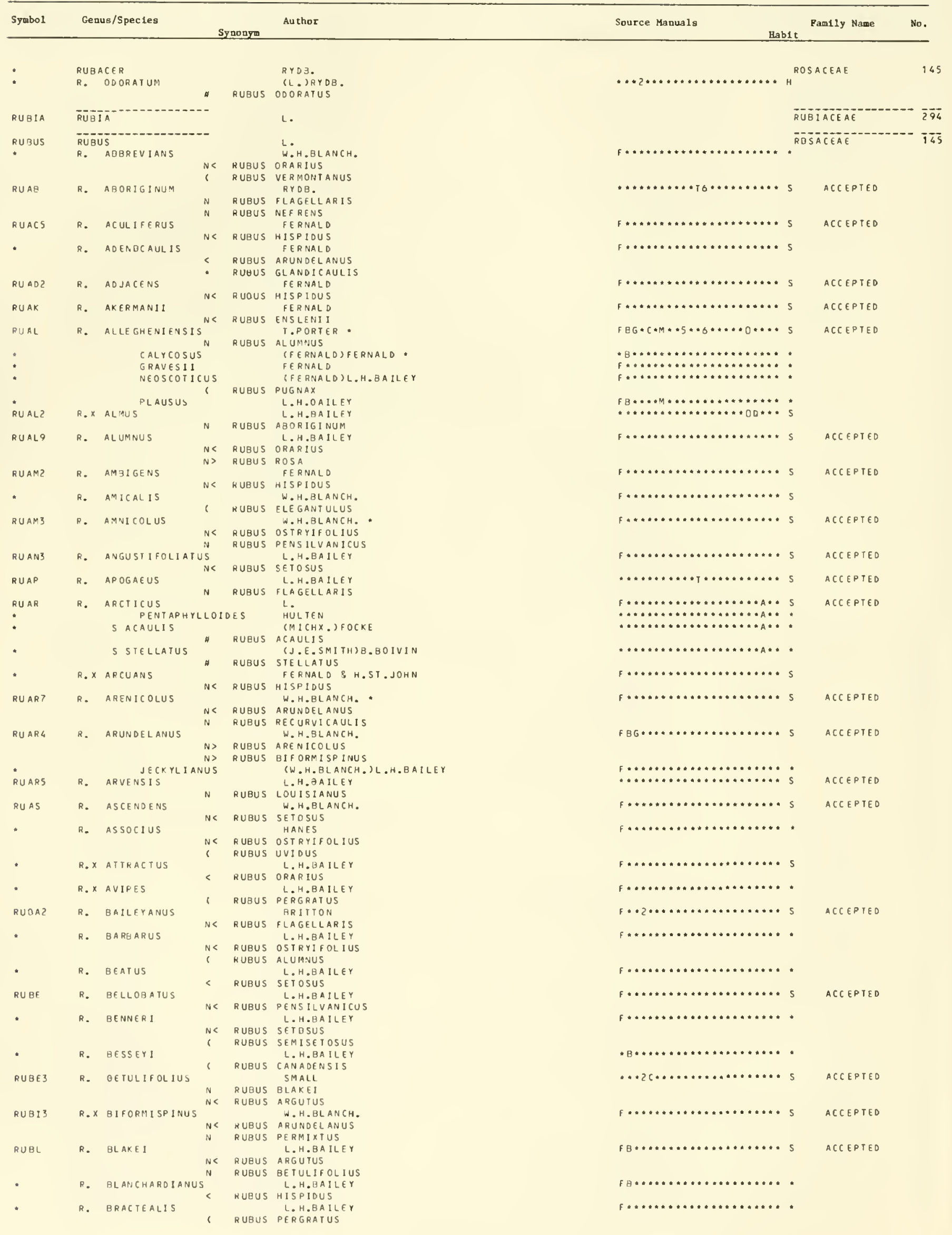




\begin{tabular}{|c|c|c|c|c|}
\hline Symbol & Genus/Species & Synonyw" & Source Manuals & Pamlly Name \\
\hline
\end{tabular}
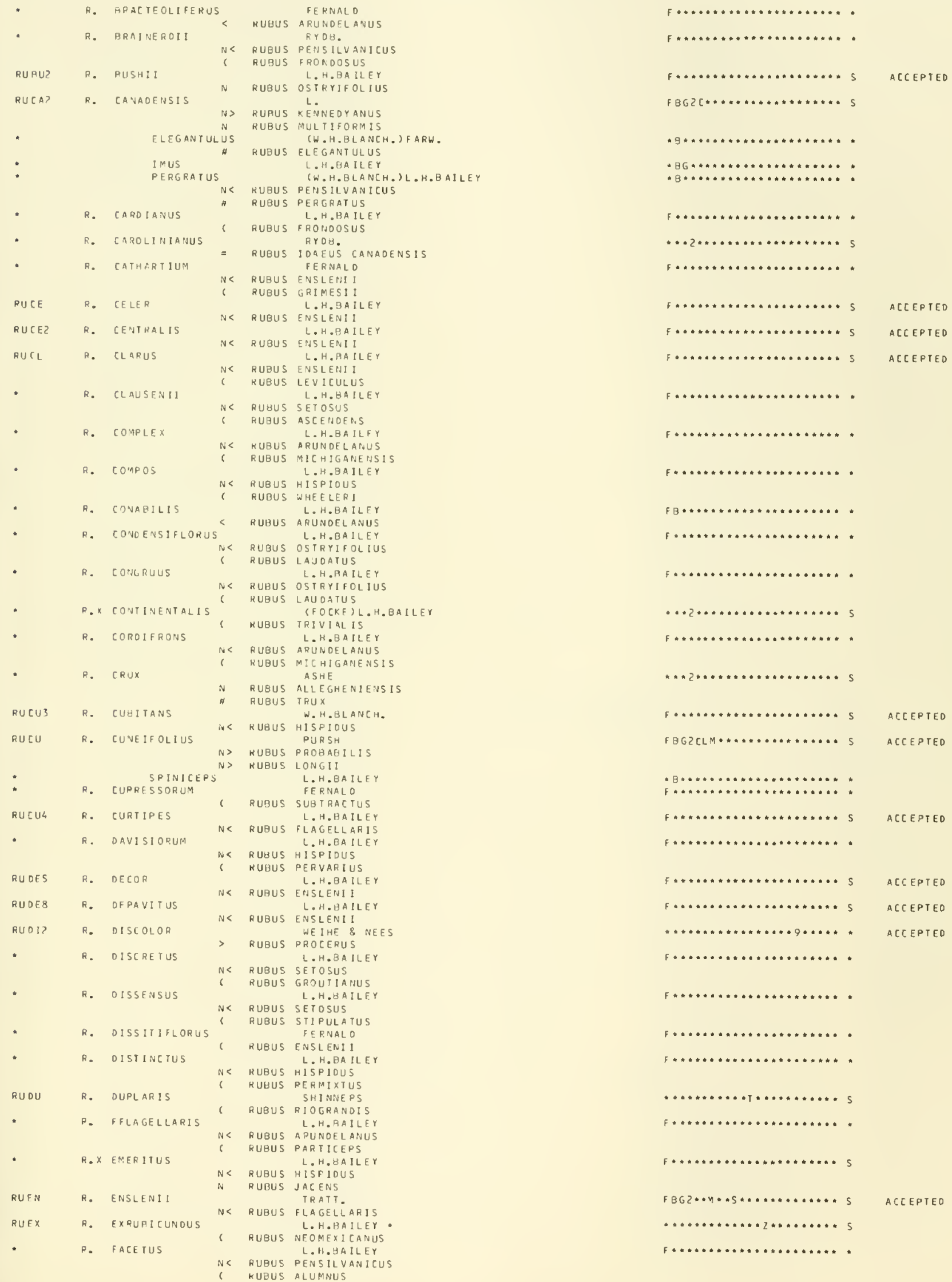


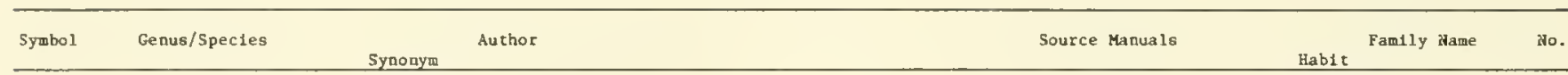

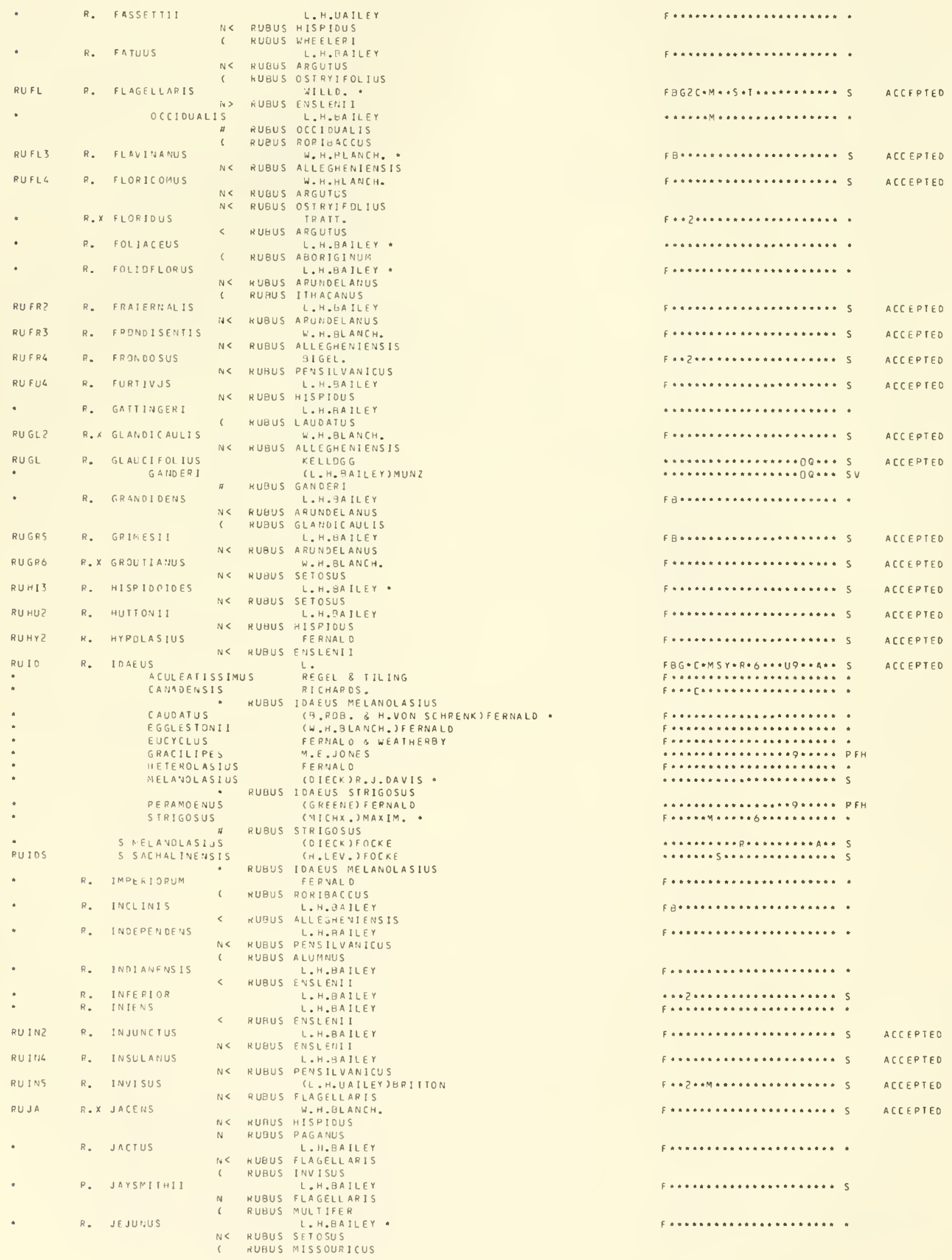




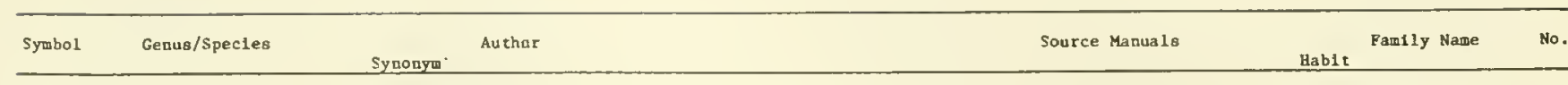
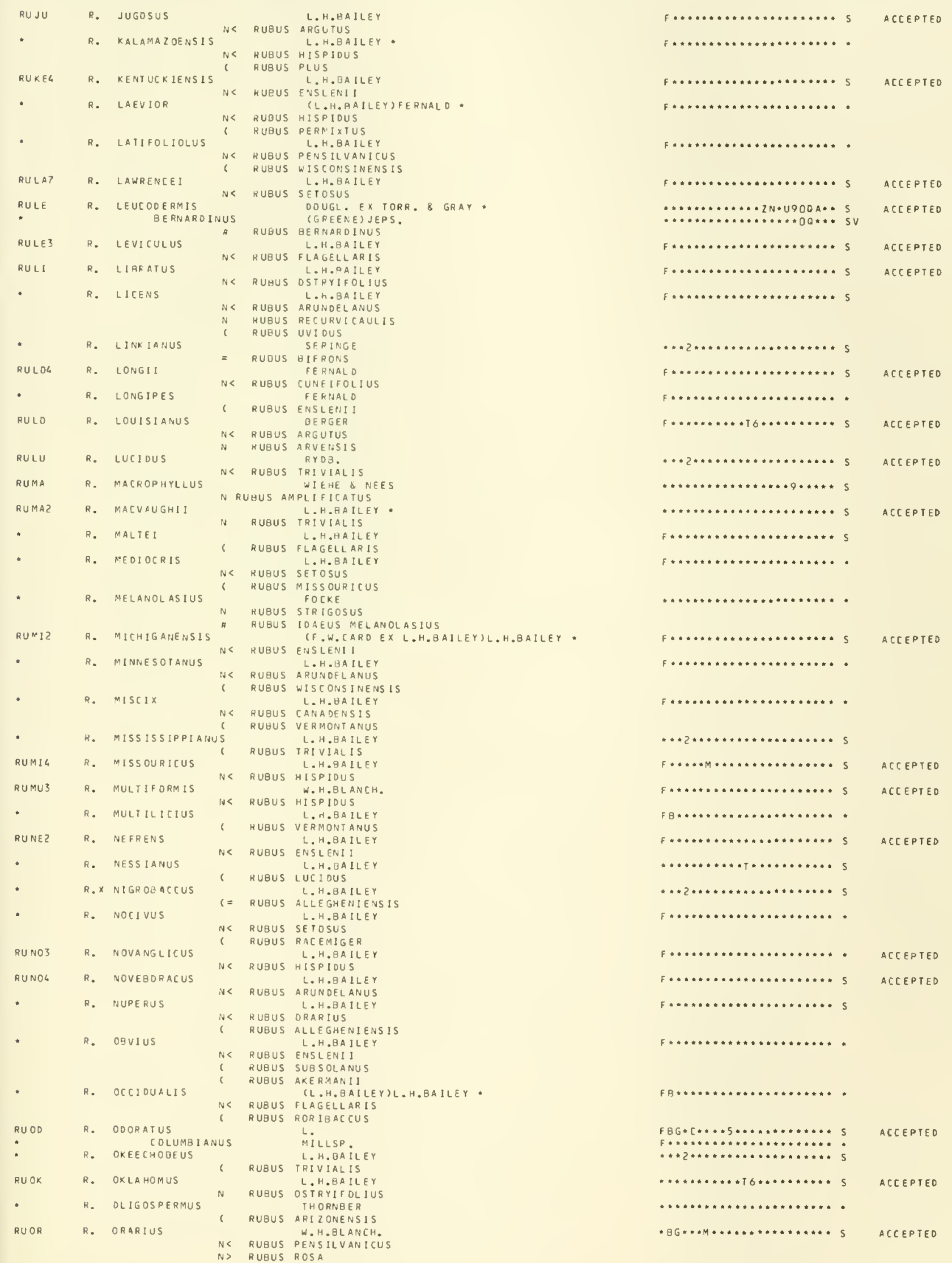


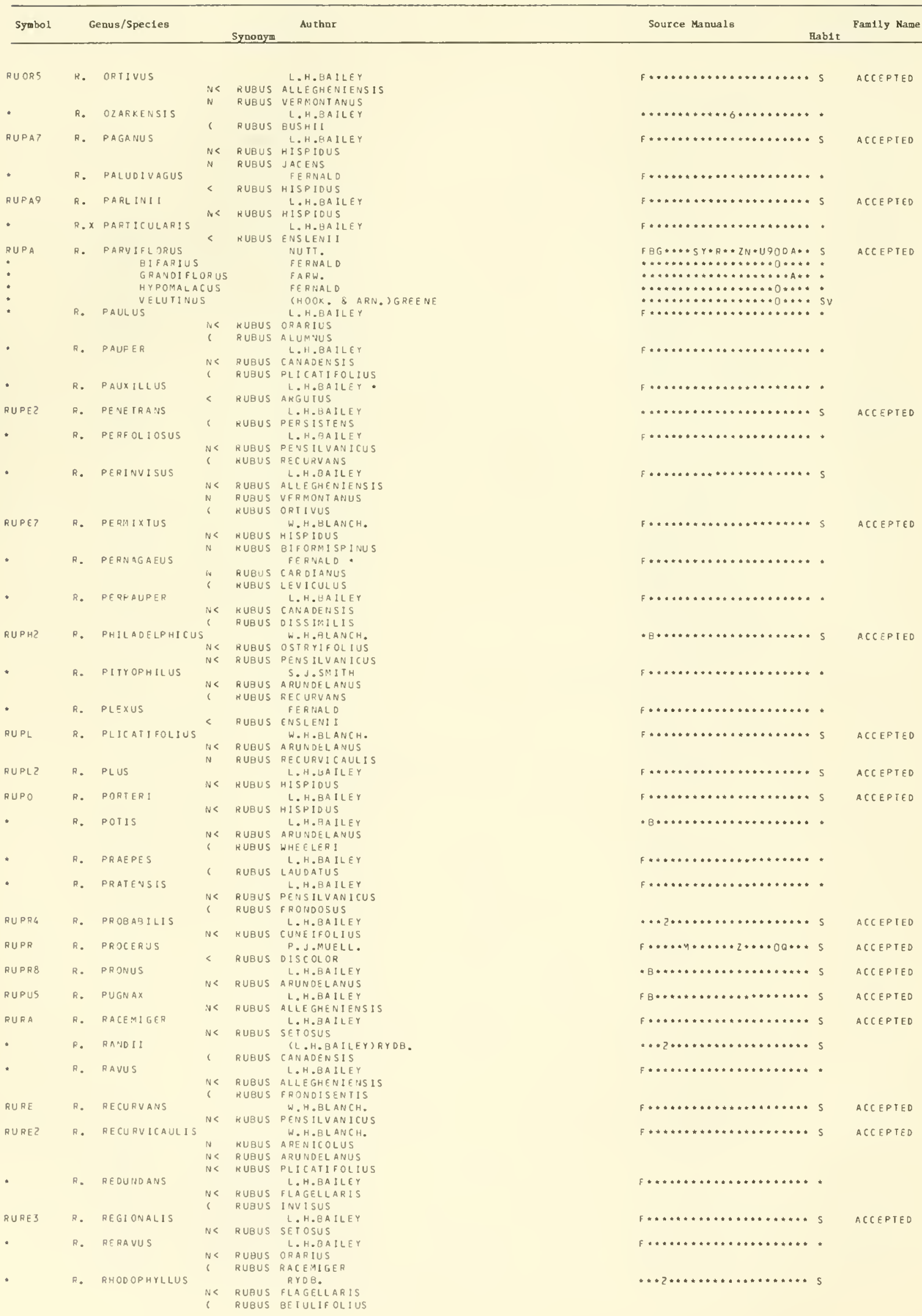




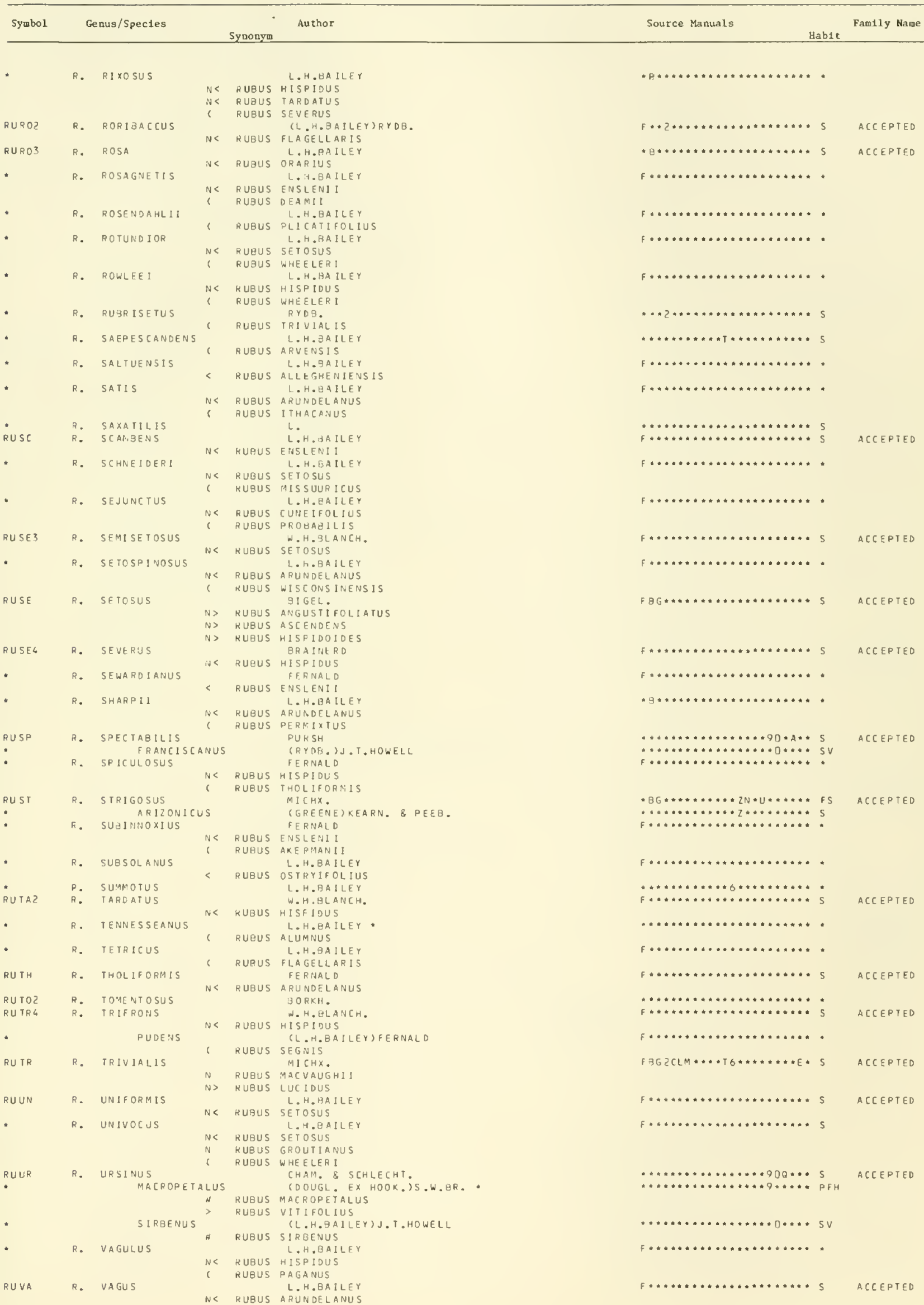


RUgus

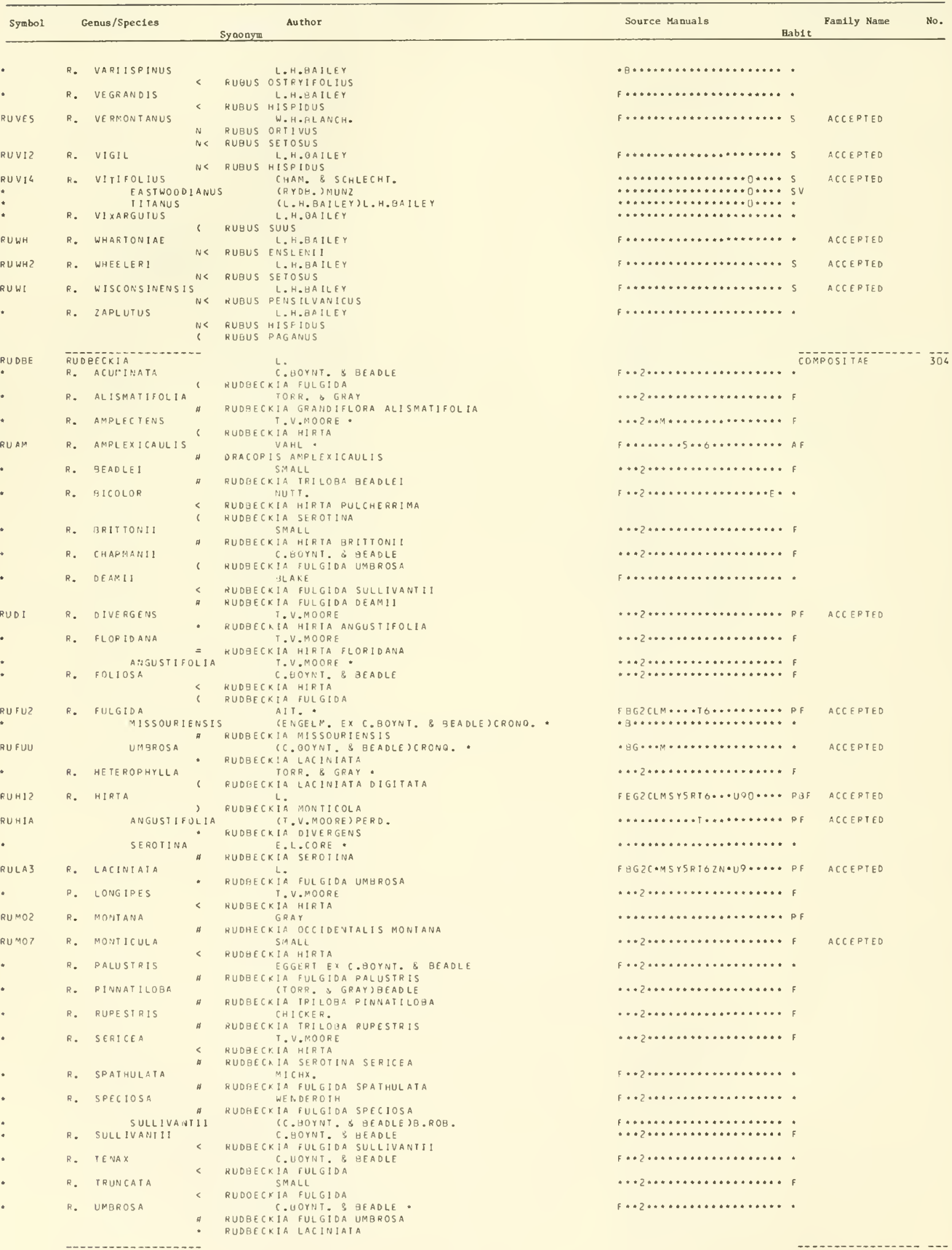


RUELL】A

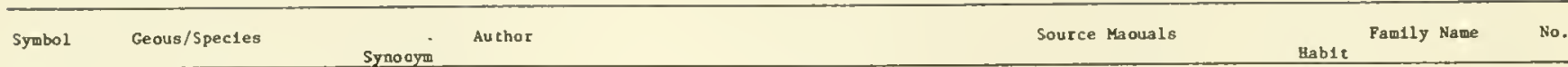

\begin{tabular}{|c|c|c|c|c|c|c|c|}
\hline \multirow{3}{*}{$\begin{array}{l}\text { RUFLL } \\
R \cup[A G\end{array}$} & RUEL & LL:A & L. & & & NTHACEAE & 290 \\
\hline & R. & CAROLINIENSIS & (WALTER EX J.F.GMEL.) STEUO. * & $F B G * C L \cdots \cdots * T \ldots \ldots \ldots \ldots \ldots *$ & $\mathrm{PF}$ & ACCEPTED & \\
\hline & & $\mathrm{N}$ & RUELL:A HUMILIS & & & & \\
\hline$*$ & & CHELONI FURMIS & FERMALD & 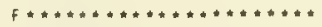 & - & & \\
\hline - & & DENTATA & (NEES) FERNALD & $F \cdots \cdots * * * \ldots \ldots \ldots \ldots \ldots \ldots$ & - & & \\
\hline$*$ & & MEMBRANACEA & FER RAL D & 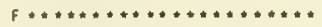 & - & & \\
\hline - & & NANELLA & FERNAL D & $f \cdots \ldots \ldots \ldots \ldots \ldots \ldots \ldots+\infty$ & - & & \\
\hline RUCAS & & SALICINA & FERNAL O & 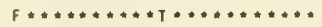 & PF & & \\
\hline RUCAS 2 & & SEMICAL VA & FERNAL D & 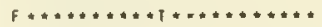 & PF & & \\
\hline - & & SERRULATA & THARP \& F.A. . BARKL. & 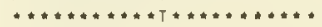 & PF & & \\
\hline RUC 1 & R. & CILIOSA & PURSH & $\cdots+2 c \ldots \ldots+\cdots+\cdots+\cdots+\cdots$ & P F & & \\
\hline & & $N \gg<$ & RUELLIA HUMILIS & & & & \\
\hline & & \# & RUELLIA CAROLINIENSIS CILIOSA & & & & \\
\hline$R \cup O A$ & R. & OAVISIORUM & THARP \& F.A.BARKL. & 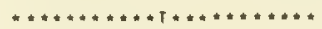 & PFH & & \\
\hline & & encos & RUELLIA NUOIFLORA OCCIDENIALIS & & & & \\
\hline RU OR2 & R. & ORUSHELII & $\begin{array}{l}\text { FHARP \& F.A.BARKL. } \\
\text { RUELLIA NUDIFLORA }\end{array}$ & 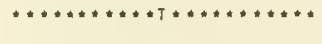 & PF & & \\
\hline . & & MACROCARPA & THARP \& F.A.BARKL. & $\# \ldots+\cdots+\cdots+\cdots$ & PF & & \\
\hline RU HU & R. & HUMILIS N & $\begin{array}{l}\text { NUTT. } \\
\text { RUELLIA CAROL INIENSIS }\end{array}$ & 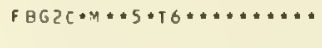 & PF & ACCEPTED & \\
\hline - & & CALVESCENS & FERNAL O & 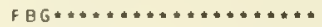 & * & & \\
\hline RUHUD & & OEPAUPERAIA & THARP \& F.A.BARKL. & 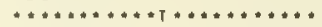 & $\mathrm{PF}$ & & \\
\hline RU HUE & & EXPANSA & FERNAL D & 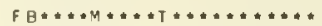 & PF & & \\
\hline RUHUF & & FRONDOSA & FERNALD & 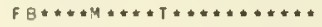 & PF & & \\
\hline RUHUL & & LONGIFLORA & (GRAY) FERNALD & $f B * \cdots+M \cdots * T * \cdots \cdots \cdots * *$ & PF & & \\
\hline 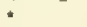 & R. & HYSRIOA & PURSH & 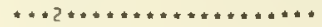 & f & & \\
\hline & & 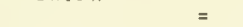 & RUELLIA CAROLINIENSIS HETEROMORPHA & & & & \\
\hline RUIN & R. & INTERMEOIA & E.LEONARO & 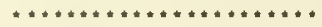 & PF & & \\
\hline * & R. & LORENTZIANA & GPISEA. & 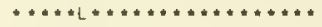 & PF & & \\
\hline & & ( & RUELLIA CILIATIFLORA & & & & \\
\hline RUME & R. & METZIAE & THARF . & 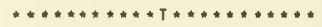 & PFH & ACEEPTED & \\
\hline RUMEM & & MARSHII & THARP \& F.A.BARKL. & $\ldots \ldots+\cdots+\ldots \ldots+\cdots+\cdots * \ldots+\cdots$ & $\mathrm{PF}$ & & \\
\hline RUMU & R. & MUELLER! & PHARP \& F.A.BARKL. & $\ldots \ldots \ldots \ldots \ldots \ldots \ldots \ldots \ldots \ldots \ldots$ & PF & & \\
\hline & & (2) & RUELLIA METZIAE & & & & \\
\hline RUNU & R. & NUOI FLORA & (GRAY)URBAN * & 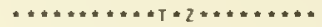 & HPF & ACCEPTED & \\
\hline RUNUH & & HISPIDULA & SHINNERS & $\ldots \ldots \ldots \ldots \ldots+1, \ldots \ldots \ldots \ldots$ & PF & & \\
\hline * & R. & OCCI DENTALIS & (GRAY) THARP \& F.A.BARKL. & 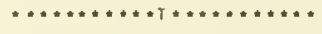 & $P F$ & & \\
\hline RUPAZ & R. & PANICULATA & RUELLIA NUDIFLORA OCLIOENTALIS & 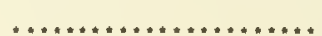 & $\mathrm{PF}$ & & \\
\hline 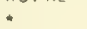 & R. & PARU IFL ORA & (NEES) BRITTUN & 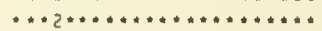 & f & & \\
\hline & & (2) & RUELLIA CAROLINIENSIS & & & & \\
\hline & & . & RUELLIA HUPILIS & & & & \\
\hline RUPEL & R. & PEOUNCULATA & TORR E EX GRAY . & 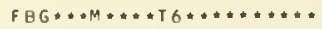 & PF & ACCEPTEO & \\
\hline & & N) & RUELLIA PURSH IANA & & & & \\
\hline - & R. & PINE TORUM & FEKNAL D . & 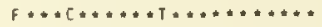 & PF & & \\
\hline & & enc & RUELLIA PEDUNCULATA PINETORUM & & & & \\
\hline RUPUZ & R. & PURSHIANA & FERNALD & 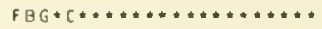 & PF & ACLEPTEO & \\
\hline & & N $<$ & RUELLIA PEDUNCULATA & & & & \\
\hline RURU & R. & RUNYON I: & THARP \& F.A.BARKL. & 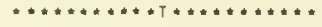 & PFH & ACCEPTED & \\
\hline - & & aERLANOIERI & THARP 3 F.A.GARKL. & $\ldots \ldots+\cdots \cdots \cdots \uparrow+\ldots \ldots \ldots \ldots$ & GH & & \\
\hline * & R. & SERPENS & NEES & 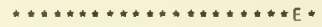 & - & & \\
\hline RUST3 & R. & STRICTOPANICULATA & TYARP \& F.A.GARKL. & 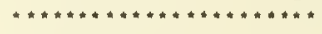 & PF & & \\
\hline * & & SUCEULENTA & RUELLIA NUDIFLORA OECIOENTALIS & & & & \\
\hline • & R. & SUCCULENTA & SMALL & $* * 2 * \cdots \cdots \cdots \cdots \cdots * \cdots \cdots \cdots$ & PF & & \\
\hline RU YU & R. & YUCATANA & $\begin{array}{r}\text { RUELLIA CAROLINIENSIS SUCCULENTA } \\
\text { (E.LEONARD)THARP B F.A.BARKL. }\end{array}$ & & D. 5 & & \\
\hline & ". & YULATANA & $\begin{array}{l}\text { (E.LEONARD)THARP \& F.A.BARKL. } \\
\text { RUELLIA NUDIFLORA YUCATANA }\end{array}$ & 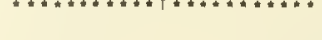 & P & & \\
\hline - & RUFA & ACER & SMALL & & $\overline{A C}$ & RACEAE & $\overline{8} \overline{3}$ \\
\hline - & R. & CAROLINIANUM & (W L LER ) SMALL & 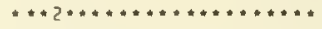 & $\boldsymbol{I}$ & & \\
\hline & & enter & ACER RUERUM TRILOBUM & & & & \\
\hline * & R. & ORUMMONDII & (HOOK. \& ARN. EX NUTT.) SMALL * & 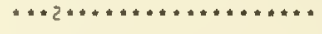 & r & & \\
\hline - & R. & RUDRUM & $\begin{array}{c}\text { ACER RUBRUM DRUMMONDII } \\
\text { (L.) SMALL }\end{array}$ & 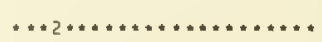 & $\mathbf{T}$ & & \\
\hline & & * & ACER RUBRUM & & & & \\
\hline RUGEL & RUGE & ELIA & SHUTTLEW. EX CHAPM." & & $\overline{c o}$ & OOSITAE & 304 \\
\hline RUMEX & $\overrightarrow{R U M E}$ & Ex - - - - - - - - n & L. & & PO & YGONACEAE & $9 \overline{4}$ \\
\hline RUACZ & R. & ACETCSA & L. & $F B G * * * * 5 * \ldots \ldots * 9 * \star A * *$ & PF & ACCEPTED & \\
\hline$*$ & & S ALPESTRIS & (SCOP.) LOEVE & $\cdots \ldots+\ldots \ldots \ldots \ldots \ldots+\cdots$ & 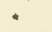 & & \\
\hline & & $=$ & RUMEX ACETOSA LAPPONICUS & & & & \\
\hline$R U A C 3$ & R. & ACETOSELLA & L. & $F B G \cdot[+45 Y 5+T 6 Z N \cdot U 900 A \cdots$ & PF & ACLEPTED & \\
\hline - & & PYRENAEUS & (POURRET) TIMBAL & 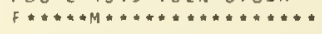 & 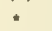 & meetrito & \\
\hline * & & S ANGIUCARPUS & ( MUKBECK) MURBECK & $\ldots \ldots \ldots \ldots \ldots \ldots \ldots \ldots, \ldots$ & - & & \\
\hline & & * & RUMEX ANGIDCARPUS & & & & \\
\hline * & ค. $x$ & ALEXIOIS & D. BOIVIN & $\ldots \ldots \ldots \ldots \ldots \ldots \ldots+\cdots \ldots$ & PF & & \\
\hline * & R. & AQUATICUS & L. & 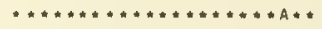 & • & & \\
\hline * & R. & BERLANOIERI & $\begin{array}{l}\text { MEISH. } \\
\text { RUMEX CHRYSOCARPUS }\end{array}$ & $\ldots+2 * \ldots \ldots \ldots \ldots \ldots \ldots \ldots$ & $\mathbf{f}$ & & \\
\hline - & $R$. & CALIFORNICUS & $\begin{array}{l}\text { RECHEX } \\
\text { RECH. }\end{array}$ & $\ldots \ldots \ldots+\cdots+2 N \cdots 00 * \cdots$ & $P F$ & & \\
\hline & & - & RUMEX SALICIF OLIUS & & & & \\
\hline$\bullet$ & R. & CONFERTUS & AI LLO. & $\ldots \ldots \ldots \ldots \ldots \ldots \ldots \ldots \ldots \ldots$ & PF & & \\
\hline * & R. & CRASSUS & RECH.F. & $\# \cdots+\ldots+*+*+\cdots+* * 00 * \ldots$ & - & & \\
\hline & & 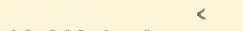 & RUMEX SALICIFDLIUS & & & & \\
\hline RU OEZ & R. & DENS IFLORUS & OSTERH . & 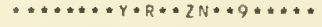 & PF & ACEEPTED & \\
\hline & & $<$ & RUMEX DCCIOENTALIS & & & & \\
\hline RUEL & $\mathrm{R}$. & ELLIPTICUS & GRCENE & $\cdots \ldots+\ldots+\cdots \cdots+z \cdots+\cdots++*$ & PF & & \\
\hline & & - & RUMEX ALTISSIMUS & & & & \\
\hline$\star$ & R. & FENESTRATUS & GREENE & $F \cdots \cdots+\cdots \cdots \cdots+\cdots \cdots+\cdots \cdots$ & - & & \\
\hline & & $50 \mathrm{sec}$ & RUMEX OCCIOENTALIS & & & & \\
\hline RU FL? & R. & FENNICUS & MURBECK & 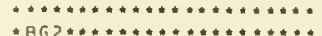 & $P F$ & & \\
\hline RU FL? & R. & FLORIOANUS & $\begin{array}{l}\text { MEISN. } \\
\text { RUMEX VERTICILLATUS }\end{array}$ & 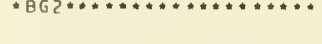 & PF & ACEEPTED & \\
\hline * & R. $x$ & FRAIN TONIS & 8. $301 \mathrm{~V}: \mathrm{N}$ & 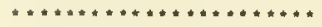 & PF & & \\
\hline RUHY & R. & HYME NOSEPALUS & TORR. & $\cdots \cdots \cdots \cdots * 4 \cdots T 6 Z N=U * 00 * \cdots$ & PF & ACCEPTED & \\
\hline . & & SALINUS & (A. DELL.) RECH.F. & 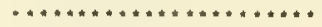 & - & & \\
\hline$R \cup L 02$ & R. & L.DNG IFOLIUS & oC. & 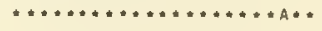 & PF & & \\
\hline
\end{tabular}

RUMEX OOMESTICUS 
RUMEX

\begin{tabular}{|c|c|c|c|c|c|c|c|}
\hline Syrebol & & nus/Specles & Synonym & Au thor & Source Manuals & Fan1ly Name & No. \\
\hline RUMAL & R. & $\begin{array}{l}\text { MARIIIMUS } \\
\text { FUEGINUS }\end{array}$ & & L. & 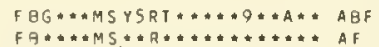 & ACCEPIEO & \\
\hline & & a & RUMEX F & FUE G INUS & & & \\
\hline - & & S FUEGINUS & & (PHILIPPI) HUL TEN & $* \ldots \ldots \ldots \ldots \ldots+\cdots \cdots * A * *$ & & \\
\hline RUMEZ & $\mathrm{R}$. & MEXICANUS & RUMEX & $\begin{array}{l}\text { ME ISN. } \\
\text { TRIANGULIVALVIS }\end{array}$ & $F B G * * M S * S * T \cdot Z N * * * * A * * P F$ & ACCEPTED & \\
\hline * & & SUBARCIICU & & (LEPAGE)B.BOIVIN & 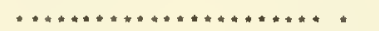 & & \\
\hline - & R. & NEMATOPOOUS & * RUMEX & $\begin{array}{l}\text { RECH.F. } \\
\text { OCCIOENTALIS }\end{array}$ & $\cdots \ldots \ldots \ldots \ldots \cdots z \cdots \cdots \cdots, p F$ & & \\
\hline RUOC 3 & R. & OCCIDENTALIS, & > RUMEX & $\begin{array}{l}\text { S. WATS } \\
\text { OENSIFLORUS }\end{array}$ & $* B G \cdots \cdots$ SY5R*2**U90***PF & $A C C E P T E D$ & \\
\hline $\begin{array}{l}\text { RUPAG } \\
\text { RUPAG }\end{array}$ & R. & $\begin{array}{l}\text { PAUCIFOLIUS } \\
\text { GRACILESCE }\end{array}$ & ENS & $\begin{array}{l}\text { NUIT. EX S. WATS. } \\
\text { (RECH.F.) RECH.F. }\end{array}$ & 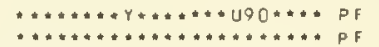 & ACCEPTED & \\
\hline RUPES & R. & PERSICARIOIOES & & L. & $F B G 2 \ldots \ldots \cdots \cdots+\cdots, \ldots 00 \cdots A F$ & & \\
\hline$R \cup P R 2$ & R. & PRACCOX & $\begin{array}{l}\text { a } \\
\text { RUMEX } \\
\text { RUMEX }\end{array}$ & $\begin{array}{l}\text { MARITIMUS PERSICARIOIDES } \\
\text { RYOE. } \\
\text { OENSIFLORUS }\end{array}$ & 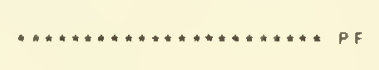 & & \\
\hline RUPY & $R$. & PYCNANTHUS & - Rumex & $\begin{array}{l}\text { RECH.F. } \\
\text { OENSIFLORUS }\end{array}$ & $\ldots \ldots \ldots \ldots \ldots \ldots+* \ldots \ldots * * P F$ & & \\
\hline RUSA & R. & $\begin{array}{l}\text { SALICIFOLIUS } \\
\text { TRIANGULIV }\end{array}$ & VALVIS & $\begin{array}{l}\text { WE INM. } \\
\text { (DANSER)C.HITCHC. } \\
\text { TRIANGULIVALVIS }\end{array}$ & 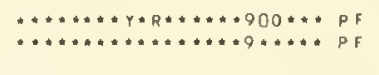 & ACCEPTEO & \\
\hline RUSAT & & S TRIANGULIVAL & LuIS & $\begin{array}{l}\text { DANSER } \\
\text { DANSER }\end{array}$ & 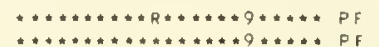 & & \\
\hline$\cdot$ & & MEXICANUS & RUMEX & $\begin{array}{l}\text { (MEISN.) C.HITCHC. } \\
\text { MEXICANUS }\end{array}$ & $\ldots \ldots \ldots \ldots \ldots \ldots q \ldots \ldots p$ & & \\
\hline * & & MONT [GENIT & TUS RUMEX L & $\begin{array}{l}\text { JEPS. } \\
\text { UTAHENS IS }\end{array}$ & $* * \ldots * * * * * * * * 9 * * * * P F$ & & \\
\hline RUS1 & R. & SIBIRICUS & * RUMEX & $\begin{array}{l}\text { HULTEN } \\
\text { MEXICANUS }\end{array}$ & $\cdots *+\cdots * * * * * * * * A * * P F$ & & \\
\hline RuSU? & R. & SUBALPINUS & * Rumex r & $\begin{array}{l}\text { M. E. JCNES } \\
\text { PYCNANTHUS }\end{array}$ & $\ldots \ldots \ldots \ldots \ldots \ldots \ldots \ldots$ & & \\
\hline * & R. & TENUI F OLIUS & * RUMEX & $\begin{array}{l}\text { (WALLR.) A. LOEVE } \\
\text { ACE IOSELLA }\end{array}$ & $\ldots \ldots \ldots \ldots \ldots \ldots \ldots \ldots q \ldots$ & & \\
\hline - & R. & TRAIS ITORIUS & $<\quad$ RUMEX & $\begin{array}{l}\text { RECH:F. } \\
\text { SALIGIFOLIUS }\end{array}$ & $\ldots \ldots \ldots \ldots \ldots \ldots \ldots+0_{*} \ldots$ & & \\
\hline RUTR3 & R. & TRIANGULIVALVIS & RUMEX A & $\begin{array}{l}\text { (DANSER) RECH.F. } \\
\text { MEXICANUS }\end{array}$ & $\cdot B G \cdots \cdots \cdots \cdots+\cdots \cdot U * 00 * *$ PF & ACCEPTEO & \\
\hline * & & OREOPATHUM & & RECH.F. & $\ldots \ldots \ldots \ldots \ldots \ldots \ldots+\cdots, 0 \cdots \ldots$ & & \\
\hline - & R. & UTAHE IS IS & * RUMEX : & $\begin{array}{l}\text { RECH.F : } \\
\text { MEXICANUS }\end{array}$ & $\cdots \cdots \cdots+\cdots \cdots+\cdots \cdots, A \cdots P F$ & & \\
\hline RUVES & R. & VERTIC!LLATUS, & RUMEX & FLORIOANUS & FBGZCLM **5*T6*********PEF & $A C C E P I \in O$ & \\
\hline RUMOH & & OHRA & & RADOI & $A L T . \overline{P O}$ & $\begin{array}{l}\text { AVALLIACEAE } \\
\text { AYODIACEAE }\end{array}$ & 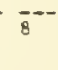 \\
\hline RUNYO & Ru & YONIA & & ROSE & ALT. A A $\bar{A}$ & $\begin{array}{l}\text { ARYLLIOACEAE } \\
\text { GAVACEAE }\end{array}$ & $5 \overline{7}$ \\
\hline RULO3 & R. & LONGIFL ORA & MANFRE & $\begin{array}{l}\text { RUSE. } \\
\text { EA LONGIFLORA }\end{array}$ & 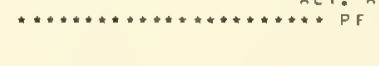 & & \\
\hline RUPPI & RUT & 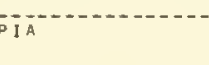 & & L. & ALT. R & $\begin{array}{l}\text { TAMOGETONACEAE } \\
\text { UPPIACEAE }\end{array}$ & $\overline{2} 8$ \\
\hline 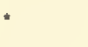 & R. & ANOMALA & $=$ RUPOIA & $\begin{array}{l}\text { OSTENF. } \\
\text { MARITIMA }\end{array}$ & 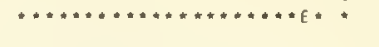 & & \\
\hline RUMAS & R. & MARITIMA & & L. & FBGZCLMSYSRT6**+U9OOAE* PZF & ACIEPTEO & \\
\hline * & & BREVIROSTR & & J. AGAROH & $F \ldots \ldots \ldots \ldots \ldots \ldots \ldots \ldots$ & & \\
\hline * & & EXIGUA & & FERNALO \& WIEGAND & 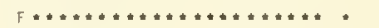 & & \\
\hline$*$ & & OBL IOUA & & (SCHUR) S SCHERS. \& GRAEBN. & $F_{\theta} \cdots \ldots \ldots \ldots \ldots \ldots+\ldots \ldots \ldots$ & & \\
\hline * & & SPIRALIS & & MORIS . & $\| B G * * * \ldots \ldots \ldots \ldots++\ldots+\ldots * *$ & & \\
\hline * & & SUACAPITAT & & FERNALO \& WIEGAND & $F_{\ldots} \ldots \ldots \ldots \ldots \ldots \ldots \ldots \ldots$ & & \\
\hline RUSP3 & R. & SPIRAL IS & $\begin{array}{ll}= & R U P P I A \\
= & R U P P I A\end{array}$ & $\begin{array}{l}\text { L. EX DUMORT. NOMEN SUPERFL. } \\
\text { MAEITIMA SPIRALIS } \\
\text { CIRRHOSA }\end{array}$ & $* * * * * * * * * \cdots * * * * \pi * 0 * A * * P Z F$ & & \\
\hline RUSSE & & $S E L I A$ & & JACO. & & ROPHULARIACEAE & 281 \\
\hline$\cdot$ & R. & JUNCEA & - RUSSEL & $\begin{array}{l}\text { ZUCCAR. } \\
\text { IA EQUISETIFORMIS }\end{array}$ & $* \cdots 2+* \ldots \ldots \ldots+\ldots+\cdots+\cdots+p p$ & & \\
\hline . & & SELLIIA & $=\quad$ RUSSEL & INED. & & ROPHULAR IACEAE & $2 \overline{8}$ \\
\hline RUTA+ & $\overline{R U}$ & 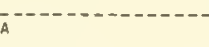 & & L. & & TACEAE & $\overline{15} \overline{7}$ \\
\hline • & & CHOSPORA & = & $\begin{array}{l}\text { VAHL } \\
\text { HOSPORA }\end{array}$ & & PERACEAE & $\overline{3} \overline{7}$ \\
\hline RYTIO & & IOOPHYLLUM & & MART. & & SNERIACEAE & $\overline{2} \overline{86}$ \\
\hline - & $\overrightarrow{R Y}$ & ILIx & HACKELC & $\begin{array}{l}\text { RAF. EX A.HITCHC. } \\
\text { OCHLOA }\end{array}$ & & AMINEAE & $\overline{36}$ \\
\hline - & R. & GRANULARIS & HACKELC & $\begin{array}{l}\text { CL. 2SKEELS } \\
\text { LOCHLOA GRANULAR IS }\end{array}$ & $\cdots+\cdots \cdots \cdots \cdots \cdots \cdots \cdots+A G$ & & \\
\hline SABAL & $\overrightarrow{S A}$ & $\bar{A} \bar{L}^{-\infty-m-\infty-\infty+\infty-\infty}$ & & ADANS. & & LMAE & $\overline{38}$ \\
\hline ? & s. & GERR UDANA & N SAUAL OO & $\begin{array}{l}\text { L.H.BAILEY } \\
\text { OMLNGENSIS }\end{array}$ & $\ldots \ldots \ldots \ldots \ldots \ldots \ldots \ldots \ldots+p$ & & \\
\hline • & & BLACKBURNIANUM & N SAUAL DON & $\begin{array}{l}\text { GLAZEBR. } \\
\text { OMINGENSIS }\end{array}$ & $\ldots \ldots \ldots \ldots \ldots \ldots \ldots \ldots \ldots$ & & \\
\hline * & 5. & OEERINGIANA & - sabal & $\begin{array}{l}\text { SMALL } \\
\text { MINOR }\end{array}$ & $\cdots * 2 * * \cdots * * * * * * * * * * * T$ & & \\
\hline - & 5 . & DO:11 NGENS IS & & BECC. & $\ldots \ldots \ldots \ldots \ldots+\ldots \ldots \ldots \ldots v$ & & \\
\hline - & s. & JAMESIANA & - sabal f & $\begin{array}{l}\text { SMALL } \\
\text { PALMETTO }\end{array}$ & 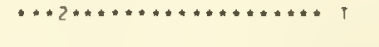 & & \\
\hline$S A L O$ & & LOUISIANA & $<$ SABAL, & $\begin{array}{l}\text { (OAREY)M.L. BOMHARO } \\
\text { MINOR }\end{array}$ & 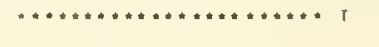 & & \\
\hline SATE & s. & TEXANA & SABAL & $\begin{array}{l}\text { CO.F.COOK.) BECC. } \\
\text { MEXICANA }\end{array}$ & $\ldots \ldots \ldots \ldots+\ldots \ldots \ldots \in E \cdot T$ & & \\
\hline
\end{tabular}




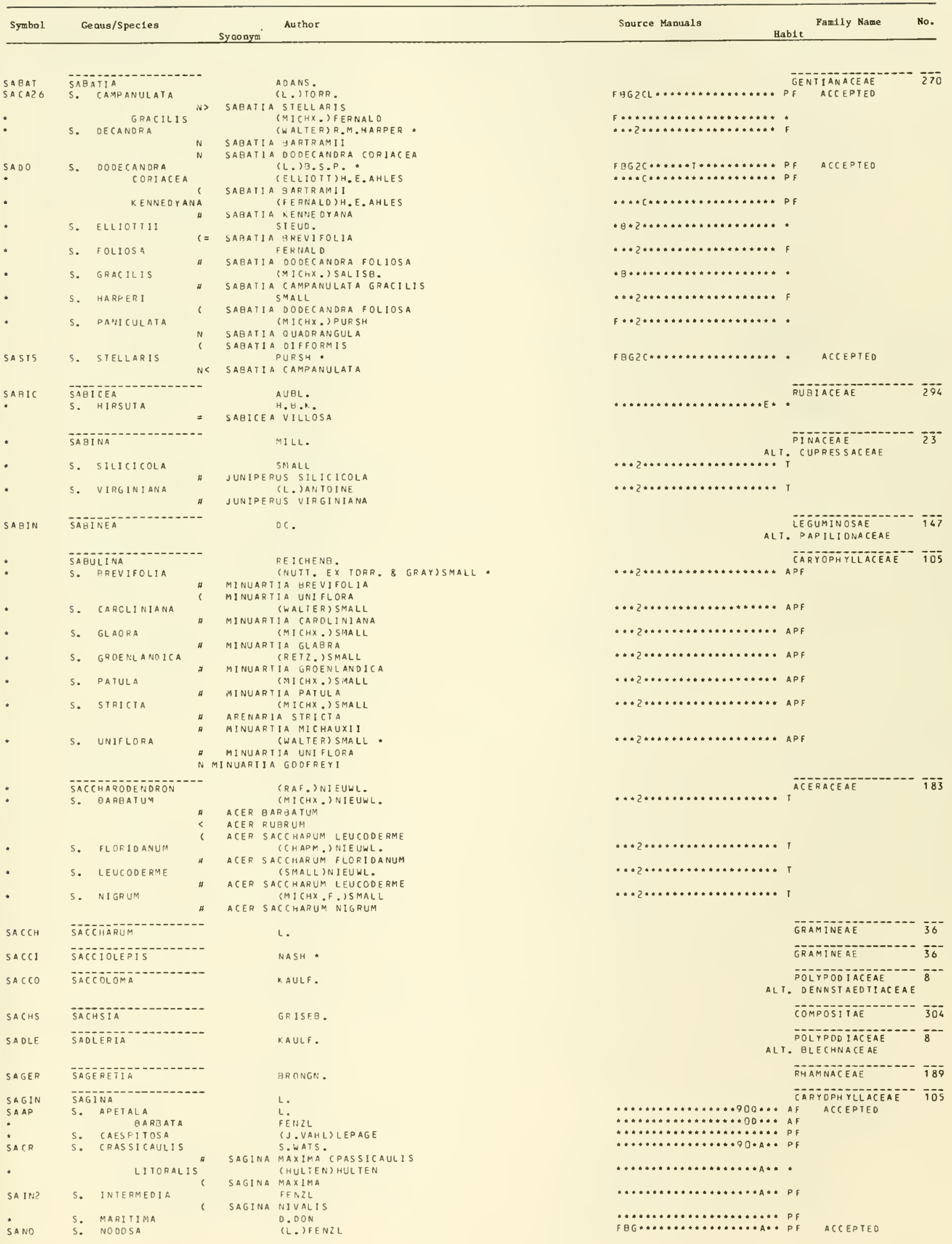




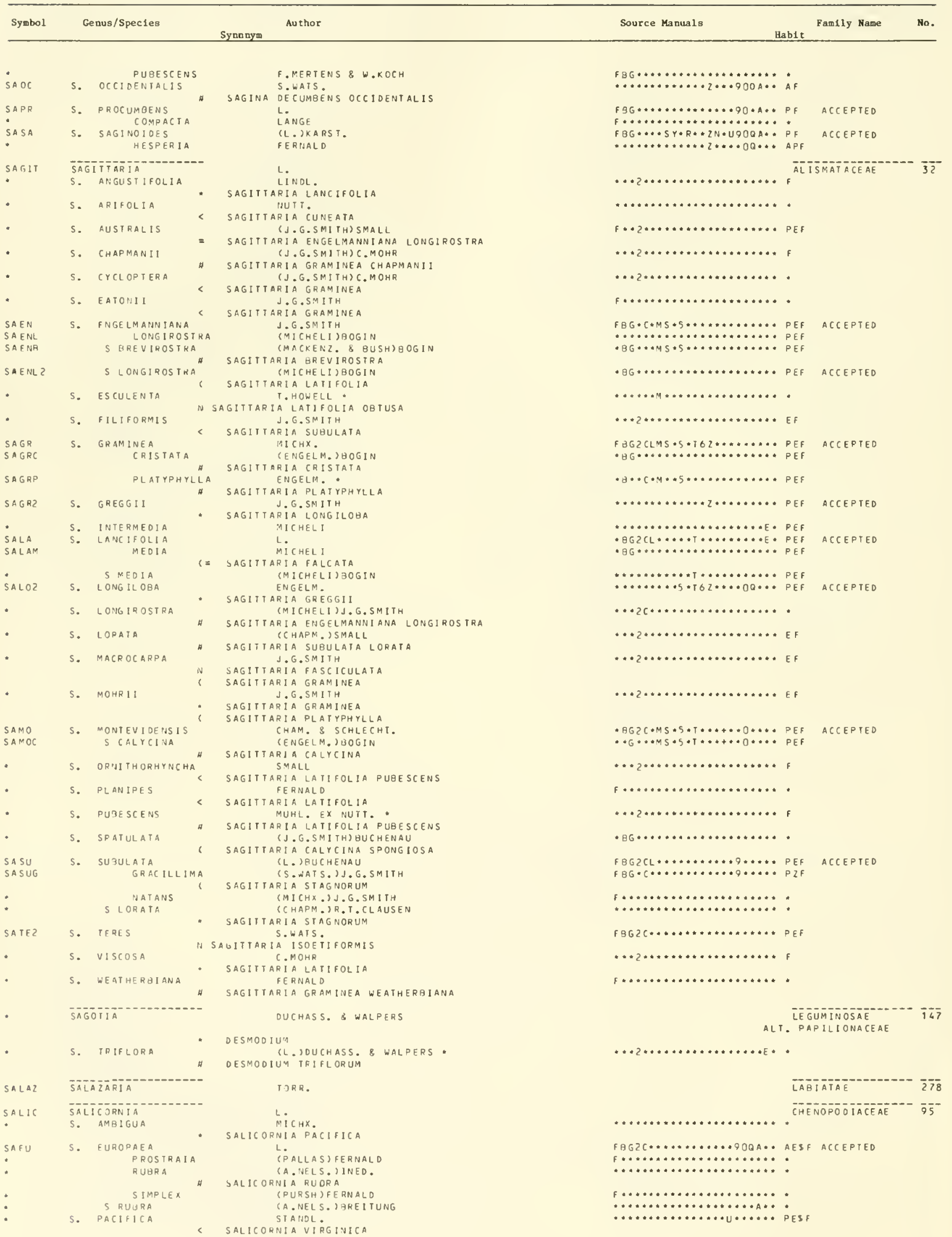




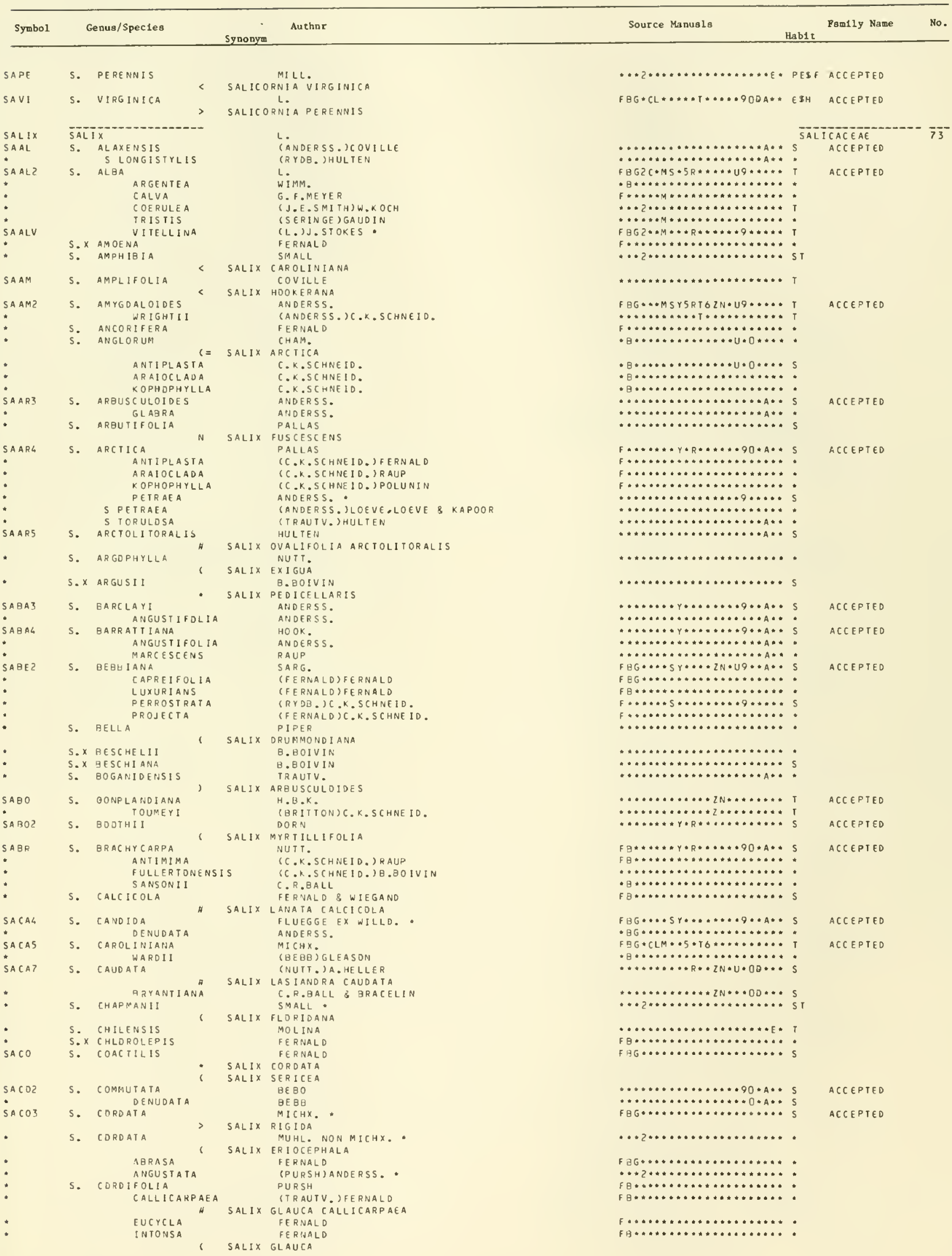




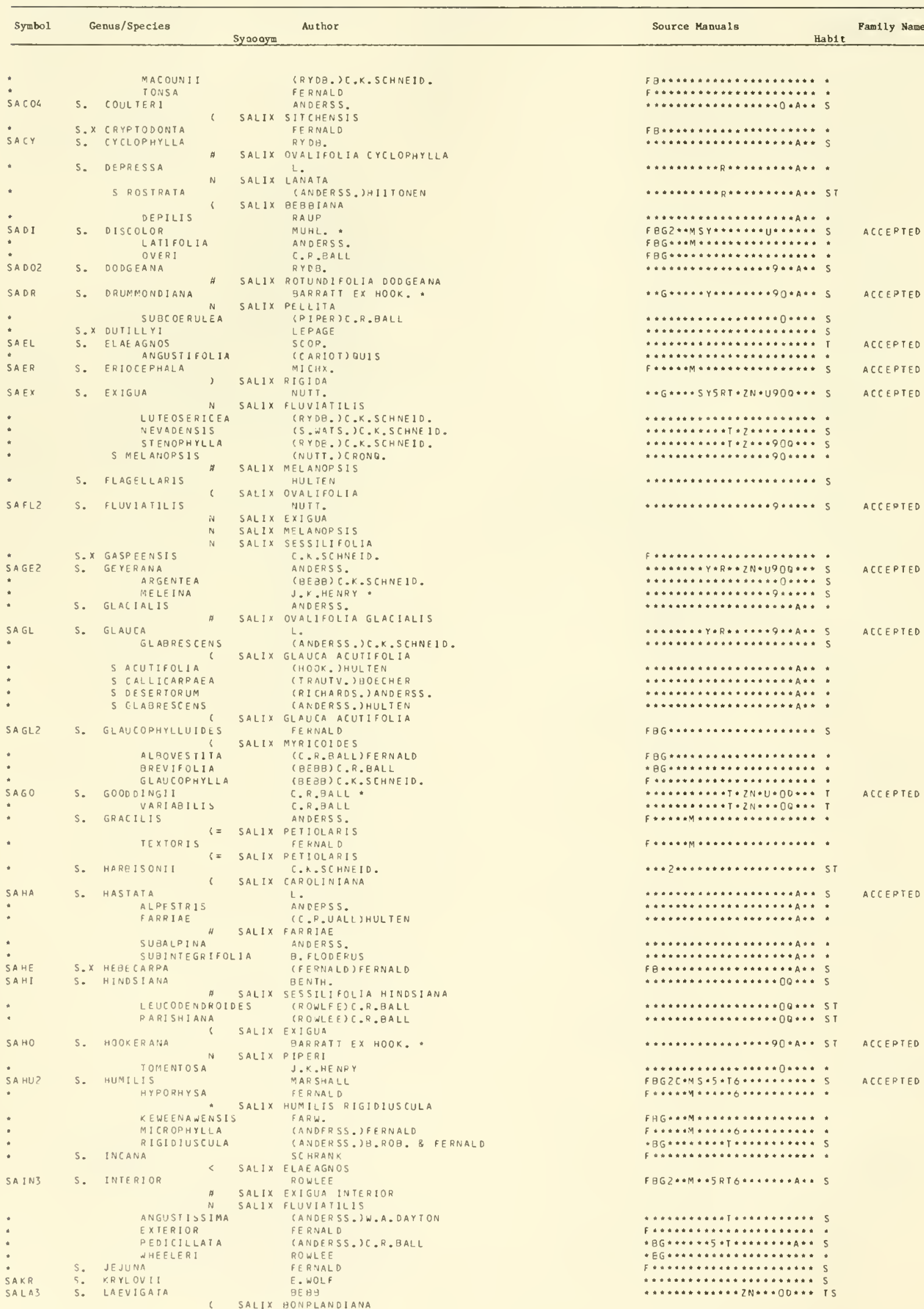


SALIX

\begin{tabular}{|c|c|c|c|c|c|c|c|c|}
\hline Symbol & & eaus/Species & Synonym & Au thor & Source Manuals & Habit & Fandly Name & No. \\
\hline & \multicolumn{2}{|r|}{ ARAQUIPA } & & $\begin{array}{l}\text { (JEPS.)C.R.BALL } \\
\text { BONPLANDIANA }\end{array}$ & $\cdots \cdots \cdots \cdots \cdots+\cdots \cdot 00 * \cdots$ & - TS & & \\
\hline \multirow[t]{3}{*}{ SALAG } & \multirow[t]{3}{*}{ s. } & LANATA & SALIX & $\begin{array}{l}\text { BONPLANDIANA } \\
\text { L. }\end{array}$ & & & & \\
\hline & & & SALIX & RICHARDSONII & $\ldots \ldots \ldots \ldots \ldots \ldots * \cdots+\cdots * A_{* *}$ & $s$ & & \\
\hline & & & SALIX $X$ & & $\ldots \ldots \ldots \ldots \ldots \ldots+\ldots \ldots \ldots A+$ & - $s$ & & \\
\hline \multirow[t]{3}{*}{ SALAS } & \multirow[t]{3}{*}{ s. } & LASI ANDRA & SALIX & $\begin{array}{l}\text { SENTH } \\
\text { SER ISSIMA }\end{array}$ & $\cdots \cdots \cdots+Y \cdots+2 N \cdots 900 A \cdots$ & - ST & ACCEPTEO & \\
\hline & & ABRAMSII & & C.R.BALL & 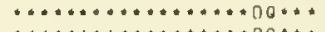 & - $T$ & & \\
\hline & & LANCIFOLIA & & (ANOERSS.) BEBB & 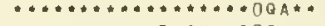 & - $T$ & & \\
\hline$A L A G$ & \multirow[t]{3}{*}{ s. } & $\begin{array}{l}\text { LASIOLEPIS } \\
\text { BLGELOVII }\end{array}$ & & $\begin{array}{l}\text { BENTH. } \\
\text { (TORR.) BEBB }\end{array}$ & $\ldots \ldots \ldots \ldots+2 N \cdots 900 \cdots$ & $\begin{array}{l}S \\
S T\end{array}$ & ACCEPIEO & \\
\hline & & BRACELINIA & & C.R.BALL : & $\ldots \ldots \ldots \ldots+T+2 \ldots \ldots 00 \ldots$ & ST & & \\
\hline & & SANOBERGII & & (RYDA.)C.R.BALL & $\cdots \cdots * \cdots * * * * * * * * 0 * * * *$ & ST & & \\
\hline & s. $x$ & LAURENTIANA & SALIX & $\begin{array}{l}\text { FERNALD } \\
\text { GLAUCOPHYLLOIDES }\end{array}$ & $F \theta \cdots \ldots \ldots \ldots \ldots \ldots \ldots \cdots \cdots$ & s & & \\
\hline & s. & LE IOLEPIS & SALIX & & $F \ldots \ldots \ldots \ldots \ldots \ldots \ldots \ldots+\ldots$ & - & & \\
\hline & s. & LONGIPES & $\begin{array}{l}\text { SALIX } \\
\text { SALIX }\end{array}$ & $\begin{array}{l}\text { VESTITA } \\
\text { SHUTILEW. } \\
\text { CAROLINIANA }\end{array}$ & $\ldots * 2+\cdots+\ldots \ldots \ldots \ldots \ldots \ldots \ldots \ldots$ & $\cdot$ & & \\
\hline SALU & s. & LUCIOA & LIA & $\begin{array}{l}\text { MUHL. } \\
\text { ANOERSS. }\end{array}$ & 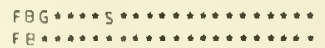 & $\mathrm{r}$ & ACCEPTED & \\
\hline SALU2 & s. & LUTEA & N) SALIX & $\begin{array}{l}\text { NUTT: } \\
\text { RIGIOA }\end{array}$ & $F * \cdots \cdots * Y=R * Z N * U=O O A * *$ & $s$ & ACCEPTEO & \\
\hline & & FAMELICA & & C. K. BALL & $\ldots \ldots \ldots \ldots \ldots \ldots \ldots \ldots$ & - & & \\
\hline & & PLATYPHYLL & H SA SALIX & $\begin{array}{l}\text { LIGULIFOLIA } \\
\text { C.R. GALL }\end{array}$ & $\ldots \ldots \ldots \ldots \ldots \ldots$ & . & & \\
\hline & & WATSONII & & $\begin{array}{l}\text { C.R. } . \text { PALL } \\
\text { (BEQR) JEPS. }\end{array}$ & $\ldots+* \ldots \ldots \ldots \ldots+000 *$ & $\dot{s}$ & & \\
\hline & s. & MACC ALLIANA & & ROWLEE & $\ldots \ldots \ldots \ldots \ldots \ldots \ldots \ldots \ldots A \cdots$ & s & & \\
\hline SAMA & s. & MACHENZIEANA & & (HOOK.) BARRATT EX ANDERSS. * & $\cdots \cdots \cdots \cdots \cdots \cdots+\cdots \cdot A \cdot \cdots$ & - $\mathrm{T}$ & & \\
\hline & & & $\begin{array}{l}\text { SALIX } \\
\text { SALIX }\end{array}$ & $\begin{array}{l}\text { RIG I DA MACKENZIEANA } \\
\text { LUTEA }\end{array}$ & & & & \\
\hline & & & N SALIX PR & OLIXA & & & & \\
\hline & S. & MARGINATA & $<\quad S A L I X$ & $\begin{array}{l}\text { C. WIMM. EX SMALL NON C.WIMM. EX ANDERSS. } \\
\text { NIGRA }\end{array}$ & $* \cdots+2 \ldots+\cdots \cdots \cdots \cdots \cdots \cdots$ & S $\mathbf{T}$ & & \\
\hline SAME? & s. & MELANOPSIS N & N $\quad$ SALIX & $\begin{array}{l}\text { CAROLINIANA } \\
\text { NLTT. }\end{array}$ & $\ldots \ldots \ldots, y \cdots \cdots+\cdots+0, \ldots$ & & & \\
\hline the & & GOLANDERAN & & (ROWLEE) C.K.SCHNEID. & $\ldots \ldots \ldots \ldots \ldots+\ldots+00 \ldots$ & ST & ACCEPTEO & \\
\hline & & GRACILIPES & & $C . R \cdot B A L L$ & $\ldots \ldots \ldots \ldots \ldots+\cdots+\cdots, \ldots$ & ST & & \\
\hline • & & TENERR I NA & & (L. . HENDERS.) C.R.BALL & $\ldots \ldots \ldots \ldots \ldots \ldots \ldots+\cdots+0 \cdots \cdots$ & : & & \\
\hline & & & SALIX & $E \times I G U A$ & & & & \\
\hline - & s. & MISSOURIENSIS & & BEEB & $\ldots \ldots \ldots \ldots \ldots \ldots \ldots \ldots$ & - $\cdot$ & & \\
\hline & & & SALIX & ERI OCEPHALA & & & & \\
\hline & & & SALIX & $\begin{array}{l}\text { CORDATA } \\
\text { RIGIDA }\end{array}$ & & & & \\
\hline SAMOZ & s. & MONTICOLA & SAL I X & $\begin{array}{l}\text { RIGIDA } \\
\text { REB3 }\end{array}$ & $\ldots \ldots \ldots+S Y+R+\ldots \cdots, \ldots \ldots$ & $s$ & ACCEPTED & \\
\hline SAMY & s. & $\begin{array}{l}\text { MYRS INIFOLIA } \\
\text { MYRIILLIFOLIA }\end{array}$ & & $\begin{array}{l}\text { SALISB. } \\
\text { AN DERS\$. }\end{array}$ & $\ldots \ldots \ldots \ldots \ldots \ldots \ldots \ldots$ & s $s$ & & \\
\hline & & MYRTILLIFOLIA & SALIX & $\begin{array}{l}\text { ANDERS\$. } \\
\text { BOOTHII }\end{array}$ & 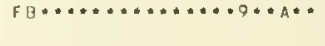 & $s$ & ACCEPTED & \\
\hline - & & BRACHYPODA & & FERNAL D & $F \varepsilon+\ldots \ldots \ldots \ldots \ldots \ldots \ldots$ & . & & \\
\hline & & & SALIX & BALLII & & & & \\
\hline : & & PSEUDOMYRS & SINI I IS & (ANDERSS.) C.R.BALL & $* \ldots \ldots \ldots \ldots \ldots \ldots \ldots \ldots \ldots * *$ & * & & \\
\hline SANI & s. $x$ & NE OFOR 3ES 11 & & TOEPFF. & $\ldots \ldots \ldots \ldots \ldots \ldots \ldots \ldots$ & - $\cdot$ & & \\
\hline SANI & s. & NI GRA & & MARSHALL * & $F B G 2 C * M \cdots+5 * T 6 \cdots \cdots U * \cdots * *$ & $\mathrm{~T}$ & ACCEPTEO & \\
\hline : & & LINDHE I MER & & C.K.SCHNEIO. & $\cdots \cdots \cdots+\cdots+\top * \cdots \cdots+\cdots$ & T $T$ & & \\
\hline - & & VENULOSA & & (ANDERSS. JBEBB & $\ldots \ldots \ldots \ldots \ldots+\ldots \ldots \ldots+\ldots+$ & $\cdot$ & & \\
\hline SAN] 2 & s. & NIPHOCLADA & SALIX & $\begin{array}{l}\text { GOODDINGII } \\
\text { RYDB. }\end{array}$ & $\ldots \ldots \ldots+\ldots \ldots \ldots \ldots+\cdots * A * *$ & s & & \\
\hline & & & SALIX & BRACHYCARPA NIPHOCLADA & & & & \\
\hline : & & $M E X[A E$ & & (C.R.BALL)HULTEN & $\cdots \ldots \ldots \ldots \ldots \ldots \ldots \ldots \ldots \ldots+A \cdots$ & - & & \\
\hline SANI3 & & MUR IE I & & (HULTEN) HUL TEN & $\because * \cdots \cdots+\cdots \cdots \cdots+\cdots$ & * * & & \\
\hline SANI 3 & s. & NIVALIS & & HOOK. & 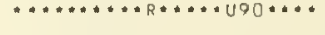 & s $s$ & & \\
\hline - & & SAXIMONTAR & NA SALIX & $\begin{array}{l}\text { RETICULAYA NIVALIS } \\
\text { (RYDB.)C.K.SCHNEIO. }\end{array}$ & & - 5 & & \\
\hline * & s. & NOVAE-ANGLJAE & & ANOERSS. & $\ldots \ldots \ldots \ldots \ldots \ldots \ldots \ldots$ & is & & \\
\hline & & & SALIX & MYRTILLIFOLIA PSEUDOMYRSINITIS & & & & \\
\hline - & s. & OBIUSATA & & FERNAL D & $F \cdots \ldots \ldots \ldots \cdots \cdots \cdots+\cdots * *$ & * & & \\
\hline SAPAB & 5. & PADOPHYLLA & SALIX & $\begin{array}{l}\text { MYRICOIDES } \\
\text { RYOB. }\end{array}$ & $\ldots \ldots \ldots \ldots \ldots Z_{N} \cdots \cdots A \cdots$ & - $s$ & & \\
\hline & & & SALIX & MON T I COL A & & & & \\
\hline * & s.x & PARALEUCA & SALIX & $\begin{array}{l}\text { PSEUDOMONTISOLA PADOPHYLLA } \\
\text { FERINLLD }\end{array}$ & & & & \\
\hline SAPAL & s. & PARKSI ANA & & C.R.BALL & $\ldots \ldots \ldots \ldots+\ldots$ & $\dot{s}$ & & \\
\hline SAPE? & & PEOICELLARIS & SAL IX & $E \times I G \cup A$ & 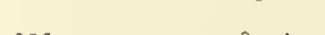 & & & \\
\hline SAPEZ & S. & & & PURSH & $F B G \cdots \cdots \cdots \cdots \cdots \cdot \cdot \cdots \cdot A \cdots$ & * $s$ & ACCEPTEO & \\
\hline - & & $\begin{array}{l}\text { HYPOGLAUCA } \\
\text { HYPOLEUCA }\end{array}$ & & FERNALD & 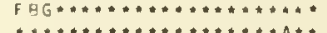 & * & & \\
\hline - & & $\begin{array}{l}\text { HYPOLEUCA } \\
\text { TENUESCENS }\end{array}$ & & $\begin{array}{l}\text { FERNALD } \\
\text { FERNALD }\end{array}$ & $F$ & : & & \\
\hline - & s. & PEDUNCULATA & & FERNALD & $f B \ldots \ldots \ldots \ldots+\ldots \ldots \ldots \ldots$ & : & & \\
\hline & & & SALIX & DISCOLOR & & & & \\
\hline * & s. & PENNATA & $S A L J X$ & $\begin{array}{l}\text { ORUMMONDIANA } \\
\text { C.R.BALL }\end{array}$ & & . & & \\
\hline & & & SALJX & PHYLICIFOLIA PENNATA & ( & 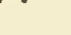 & & \\
\hline & & & SAL IX & PLAN!FOLIA & & & & \\
\hline SAPES & s. & PETIOLARIS & & J.E.SMITH & 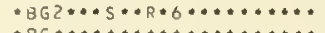 & $s$ & ACCEPIEO & \\
\hline 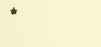 & & ANGUST I FOL & LIA & ANDERSS. & $* 9 G \ldots \ldots+\ldots+\ldots \ldots \ldots \ldots$ & - & & \\
\hline - & s. & PETFOPHILA & SAL IX & $\begin{array}{l}\text { LUC IDA ANGUSTIFOLIA } \\
\text { RYDU. }\end{array}$ & $\ldots \ldots \ldots \ldots \ldots \ldots \ldots \ldots \ldots$ & * & & \\
\hline & & & $A<\quad S A L I X$ & ARCTICA PETRAEA & (a) & 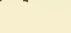 & & \\
\hline SAPHZ & s. & PHYLICIFOLIA & & LIDNIFOLIA & $\cdots G \cdots \cdots S \cdots R \cdots \cdots \cdots Q \cdots A *$ & - $s$ & & \\
\hline & & ANIFUL & SALIX & $\begin{array}{l}\text { PLANIFOLIA } \\
\text { (PURSH) C. HITCHC. }\end{array}$ & $\ldots \ldots \ldots s+\ldots \ldots+\cdots, a \ldots$ & - $s$ & & \\
\hline
\end{tabular}


SALIX

\begin{tabular}{|c|c|c|c|c|c|c|c|c|}
\hline Sywbol & Geot & ous/Species & Synonye & Authar & Source Manuals & labit & Fanily Name & No. \\
\hline SAPHP & & $S P L A N I F O L I A$ & & (PURSH) IITONEN * & $* \ldots \ldots \cdots \cdots R \bullet \ldots \ldots q \cdots A \cdots$ & $s$ & & \\
\hline : & & MONICA & & (ВEOB) JEPS. & $* * *+* * * * * * * * *+90 * * * *$ & $*$ & & \\
\hline ¿API & & PENNATA & & (C.R.BALL) CRONG. & $\ldots \ldots \ldots \ldots \ldots \ldots \ldots * \theta_{*+* * *}$ & s & & \\
\hline SAPI & s. & PIPER I & SALIX & $\begin{array}{l}\text { OEBB } \\
\text { HOOKERANA }\end{array}$ & $* * \ldots+* \ldots * * * * * 90 * * *$ & $s$ & ACCEPIEO & \\
\hline SAPLZ & s. & PLANIFOLIA & & PURSH & FBG $\cdots \cdots * Y \cdots * \cdots+* * 0 * * \star *$ & $s$ & ACCEPTEO & \\
\hline SAPO & s. & $\begin{array}{l}\text { YUKONENSIS } \\
\text { POLARIS }\end{array}$ & & (C.K. SCHNEID.) ARGUS & 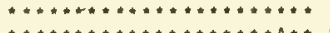 & $\mathrm{s}$ & & \\
\hline 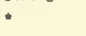 & & S PSEUDOPOLARIS & & $\begin{array}{l}\text { WAHLENB. } \\
\text { (B. FLOOERUS) HULTEN }\end{array}$ & 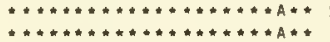 & $\begin{array}{l}s \\
s\end{array}$ & ACCEPTEO & \\
\hline - & & GLABRATA & & HULIEN & $\ldots \ldots \ldots \ldots \ldots \ldots \ldots \ldots+\cdots A_{*}$ & s & & \\
\hline$\cdot$ & s. & PSEUDOCORDATA & & (ANDERSS.) RYOB. - & $\cdots+* * * \cdots \cdots+2 N * U * 0 * \cdots *$ & 5 & & \\
\hline & & $\stackrel{N}{l=}$ & $\begin{array}{l}\text { SALIX } \\
\text { SALIX }\end{array}$ & $\begin{array}{l}\text { BOOTHII } \\
\text { MYRTILLIFOLIA }\end{array}$ & & & & \\
\hline - & s. & PSEUDOLAPPONUM, & SALIX & $\begin{array}{l}\text { VON SEEMEN } \\
\text { GLAUCA VILLOSA }\end{array}$ & $\ldots+\ldots+\ldots+\ldots+\cdots+u * \ldots+\cdots$ & $s$ & & \\
\hline SAPS & s. & PSEUDOMONIICOLA & SALIX & $\begin{array}{l}\text { C.R.BALL } \\
\text { MOVTICOLA }\end{array}$ & 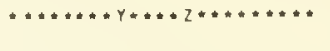 & $\mathrm{s}$ & ACCEPTEO & \\
\hline • & & PADOPHYLLA & SALIX & $\begin{array}{l}\text { CRYOB. C.R.OALL } \\
\text { MONTICOLA }\end{array}$ & 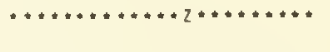 & $s$ & & \\
\hline$*$ & S. & PSEUDDMYRSINITES & SALIX & $\begin{array}{l}\text { ANOERSS. } \\
\text { MYRTILLIFOLIA PSEUOOMYRSINITIS }\end{array}$ & $\ldots \ldots+\ldots+\ldots+\ldots+\ldots+\ldots+\ldots$ & $\cdot$ & & \\
\hline SAPU & s. & PULCHRA & SALIX & $\begin{array}{l}\text { CHAM. } \\
\text { PLANIFOLIA PULCHRA }\end{array}$ & 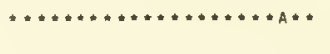 & $s$ & & \\
\hline 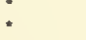 & & $\begin{array}{l}\text { PALMERI } \\
\text { YUKONENSIS }\end{array}$ & & $\begin{array}{l}\text { C.R.BALL } \\
\text { C.K.SC HNE IO. }\end{array}$ & $\cdots \cdots \cdots \cdots \cdots \cdots \cdots$ & $\cdot$ & & \\
\hline & & 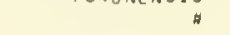 & SALLX & $\begin{array}{l}\text { C.K.SCHNE IO. } \\
\text { PLANIFOLIA YUKONENS IS }\end{array}$ & 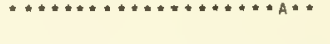 & • & & \\
\hline SARE? & s. & & & L. & $F \cdots \cdots \cdots \cdot Y \cdot R * \cdots \cdots \cdots * A * *$ & s & ACCEPTEO & \\
\hline : & & $\begin{array}{l}\text { GI GANI I E OLIA } \\
\text { SEMICALVA }\end{array}$ & & $\begin{array}{l}\text { C.R.GALL } \\
\text { EERNALO }\end{array}$ & 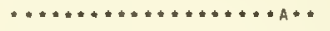 & $\star$ & & \\
\hline SA REO & & $\$$ OROICULARIS & & $\begin{array}{l}\text { FERNALQ } \\
\text { (ANDERSS.) B. FL ODERUS. }\end{array}$ & 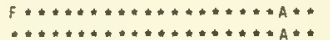 & $\dot{s}$ & & \\
\hline SARIL & s. & RIGIOA & & MUHL. & $F B G \cdots+M S \cdot 5 \cdots \cdots \cdots 90 \cdots *$ & s & ACCEPTEO & \\
\hline - & & ANGUSTATA & SALIX & $\begin{array}{l}\text { LUTEA } \\
\text { ANDERSS. }\end{array}$ & & & & \\
\hline & & A & SALIX & $\begin{array}{l}\text { ANDERSS. } \\
\text { CORDATA ANGUSTATA }\end{array}$ & 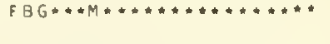 & • & & \\
\hline - & & $\begin{array}{r}\text { MACKENZIEANA } \\
\text { N) }\end{array}$ & A SALIX & $\begin{array}{l}\text { (KOOR.) CRONO. } \\
\text { MONOCHROMA }\end{array}$ & $\ldots \ldots \ldots+\ldots+\cdots+\cdots * 9+\cdots \cdots$ & $s$ & & \\
\hline * & & $\begin{array}{l}\text { NS } S \\
\text { MACROGEMMA }\end{array}$ & SALIX $P R$ & $\begin{array}{l}\text { OLI } \times A \\
\text { (C.R.BALL) } C R O N G \text {. }\end{array}$ & & & & \\
\hline & & MAL KODE & SALIX & $\begin{array}{l}\text { (C.R.BALL)CRONG. } \\
\text { LUTEA }\end{array}$ & 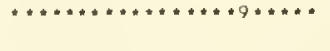 & & & \\
\hline . & & VESTITA = & SALIX & $\begin{array}{l}\text { ANOERSS. } \\
\text { ERI OCEPHALA }\end{array}$ & $\beta^{\beta} G_{*} \ldots \ldots \ldots \ldots \ldots \ldots \ldots \ldots$ & $\cdot$ & & \\
\hline * & & WATSONII & & ( GEEB) CRONG. & $\ldots \ldots \ldots s+\ldots \ldots \ldots q, \ldots$ & $\cdot$ & & \\
\hline * & s. s. & SAXIMDNTANA & SALIX & $\begin{array}{l}\text { RYOB. } \\
\text { RET!CULATA NIVALIS }\end{array}$ & $\ldots \ldots \ldots+\ldots+\ldots+\ldots+\ldots+$ & • & & \\
\hline - & $5 . x 5$ & SCHNE I DER I & & B. BOIVIN & 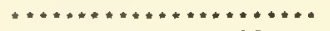 & s & & \\
\hline SASE & s. s & SCOULERANA & & BARRATT EX HOOK. & $\# \ldots \cdots S Y * R * Z N * U 9 O O A *$ & ST & ACCEPTED & \\
\hline * & & COETANA & & $C \cdot R \cdot B A L L$ & $* \ldots \ldots+\ldots \ldots+\ldots+\cdots * 0 * A * *$ & ST & & \\
\hline SASEZ & s. & SERISSIMA N N & SALIX & $\begin{array}{l}\text { (L.H.BAILEY) FERNALO } \\
\text { LASIANDRA }\end{array}$ & $F B G \cdots \cdots S \cdots R * \cdots \cdots * * A \cdots$ & s & ACCEPIED & \\
\hline SASE3 & s. & SESSILIFOLIA N & SALIX & $\begin{array}{l}\text { NUTTE } \\
\text { FLUVIATILIS }\end{array}$ & $*+\ldots+\cdots+\ldots+\ldots+4, \ldots+$ & $\mathrm{s}$ & ACCEPTEO & \\
\hline - & s. $x$ s $-10-10$ & SIMULANS & & FERNALO & $* \beta * * * * * * * * *+\cdots+\cdots+\cdots *$ & $\bullet$ & & \\
\hline SASI? & 5. & SITCHENSIS & & SANSON EX BONG. * & $* \ldots \ldots * \cdots * * * * * 90 * A * *$ & $s$ & ACCEPTEO & \\
\hline SA SPZ & s. & $\begin{array}{l}\text { A J ANENSIS } \\
\text { SPUENOPHYLLA }\end{array}$ & & $\begin{array}{l}\text { ANDERSS. } \\
\text { A. SKVORT2 }\end{array}$ & 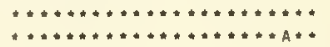 & $\dot{s}$ & & \\
\hline & & S & SALIX & EXIGUA & $* \cdots \cdots+\cdots+\cdots+\cdots * * * * A * *$ & 5 & ACCEPTEO & \\
\hline 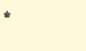 & & S PSEUDOTORULOSA & SALIX & $\begin{array}{l}\text { A. SKVORTZ } \\
\text { ARCIICA TORULOSA }\end{array}$ & $\ldots \ldots \ldots \ldots+\cdots \cdots \cdots+\cdots+A *$ & * & & \\
\hline * & s.x & STENOC ARPA & & FERNALD & $* B_{*} \ldots \ldots \ldots \ldots \ldots * \cdots \cdots *$ & $\bullet$ & & \\
\hline SASTL & s. s. & STOL ONIFERA & SALIX & $\begin{array}{l}\text { COVILLE } \\
\text { PARALEUCA }\end{array}$ & $* * * * * * * *+* * * * * * A * *$ & $s$ & ACCEPTED & \\
\hline * & s. 5 & SUACOERULEA & & PIPER & 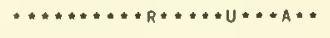 & $s$ & & \\
\hline . & s. & SYRTICOLA & SALIX & $\begin{array}{l}\text { DRUMMONO IANA SUOCOERULEA } \\
\text { FEQNALD }\end{array}$ & 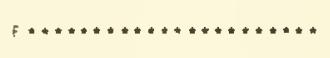 & $\bullet$ & & \\
\hline SATA & s. 1 & IAXIFOLIA & SALIX & $\begin{array}{l}\text { COROATA } \\
\text { H.B.K. }\end{array}$ & 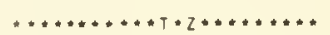 & T & ACCEPIED & \\
\hline$\cdot$ & & MICPOPHYLLA & & (SCHLECHT. \& CHAM.) C.K.SCHNELO. & 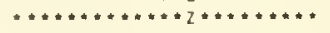 & st & & \\
\hline SATRZ & s. & TRISTIS = & SALIX & $\begin{array}{l}\text { AITIIS MICROPHYLLA } \\
\text { HUMILIS }\end{array}$ & 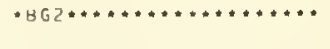 & $s$ & & \\
\hline SAVE & s. 1 & VESTITA & & PURSH & $F_{\theta} \ldots \ldots \ldots \ldots \ldots \ldots+q_{* \cdots *}$ & $s$ & ACCEPTEO & \\
\hline * & & ERECTA & & ANDERSS. & 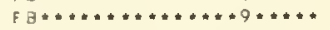 & 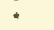 & & \\
\hline - & & PSILOPHYLLA & & FERNALO \& H.ST.JOHN & $f \cdots \ldots \ldots \ldots \ldots \ldots \ldots \ldots \ldots$ & - & & \\
\hline - & S. $x$ & WAGHORNEI & & RYDB. & $F \cdots+\ldots \ldots \ldots+\ldots \ldots \ldots+\ldots+$ & 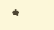 & & \\
\hline * & s. & WAROIL & SALIX & $\begin{array}{l}\text { BEBII } \\
\text { CAROLINIANA WAROII }\end{array}$ & $\cdots G \ldots \ldots \ldots \ldots \ldots \ldots * \ldots * *$ & • & & \\
\hline$\cdot$ & S. $x$ & WIEGANOII & & FERNAL D & $\xi \ldots \ldots \ldots \ldots \ldots \ldots+\ldots+* * *$ & $s$ & & \\
\hline SAWO & s. & WOLF II & & BE 90 & $\cdots * \cdots \cdots+r \cdot R * \cdots \cdots \cup \vee q \cdots \cdots$ & $\mathrm{s}$ & ACCEPTEO & \\
\hline * & & I DAHOENSIS & & $C . R \cdot B A L L$ & $\ldots \ldots \ldots \ldots \ldots \ldots \ldots+\cdots+\cdots * *$ & $s$ & & \\
\hline - & s. & WRIGMTII & SALIX & $\begin{array}{l}\text { ANDERSS. } \\
\text { AMYGDALOIDES WRIGHIII }\end{array}$ & 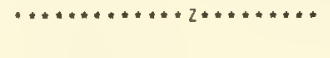 & I & & \\
\hline SALME & $\overline{S A L M}$ & $M E A$ & & $D C$. & & & MPOSITAE & $\overline{304}$ \\
\hline SALPI & $\overline{S A L P}$ & PICHROA & & MIERS & & & LANACEAE & $\overline{2} 8 \overline{0}$ \\
\hline SARH & s. & RHOM SOIDEA & SALPIC & $\begin{array}{l}\text { (GILLIES \& HOOK.)MIERS * } \\
\text { CHROA ORIGANIFOLIA }\end{array}$ & $\cdots+\cdots+\cdots+\cdots+\infty *+0 \cdots+$ & PFV & & \\
\hline - & $\overline{S A L} \bar{P}$ & INGOSTYLIS & & $\operatorname{SMALL}$ & & & IDACEAE & $\overrightarrow{6} \dot{1}$ \\
\hline * & s. & COELESTINA & SPHENO & $\begin{array}{l}\text { (W. BARTRAM) SMALL } \\
\text { OSTIGMA COELESTINUM }\end{array}$ & $* *+2 * * * * *+* * * * * \cdots+* *$ & $P F$ & & \\
\hline SALSO & SALS & $S O L A$ & & L. & & & ËNOPODIATEEAE & $-7 \overline{9}$ \\
\hline$\star$ & s. & IBERICA & SALSOLA & $\begin{array}{l}\text { SENNEN \& PAU } \\
\text { KALI }\end{array}$ & $\cdots * \cdots * S * R * \ldots \cdots *+Q \cdots$ & Af & & \\
\hline SAKA & s. & KALI & & L. & FEG $2 C L M * Y S * T 62 N * 490 * * * *$ & AF & ACCEPTED & \\
\hline
\end{tabular}




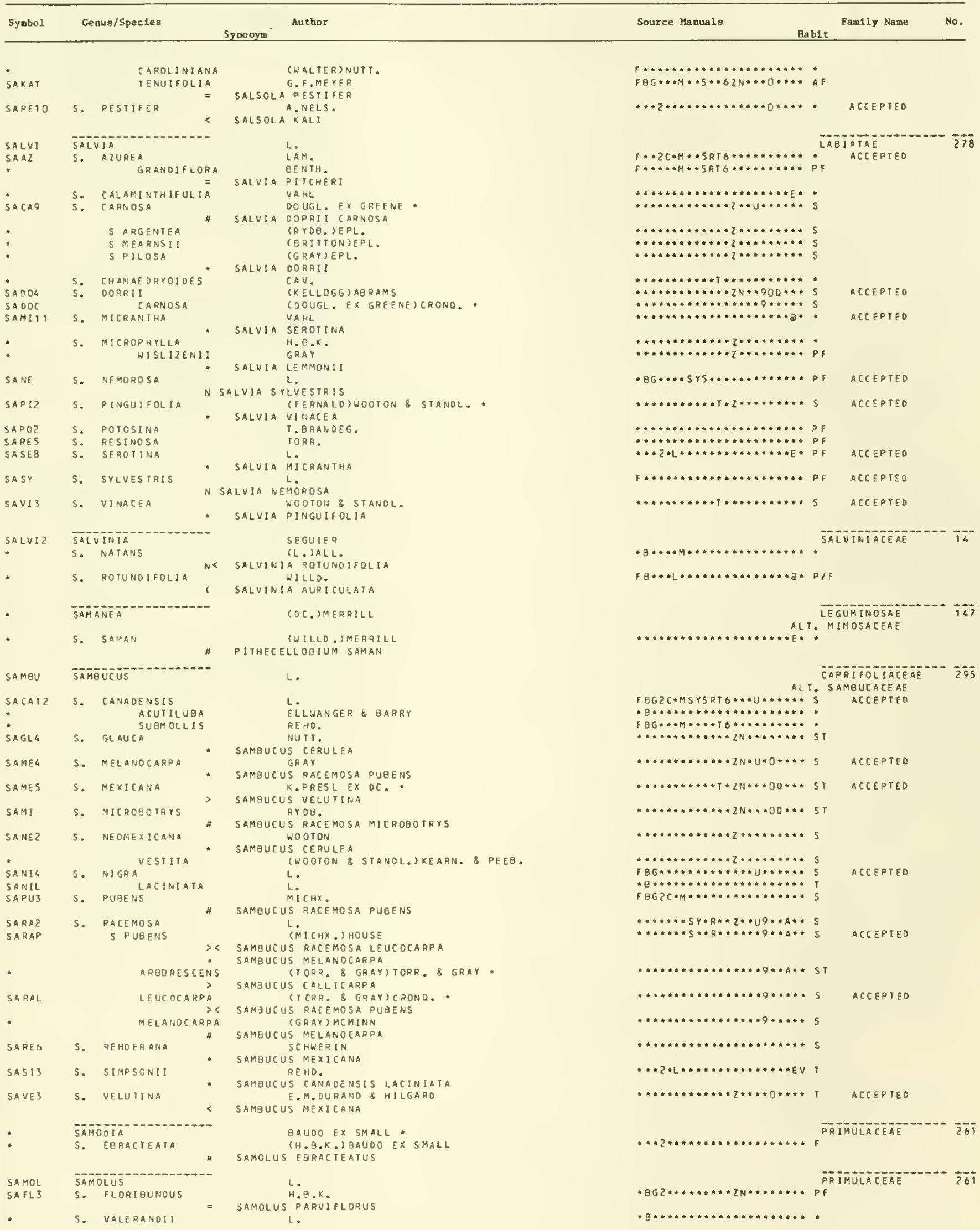




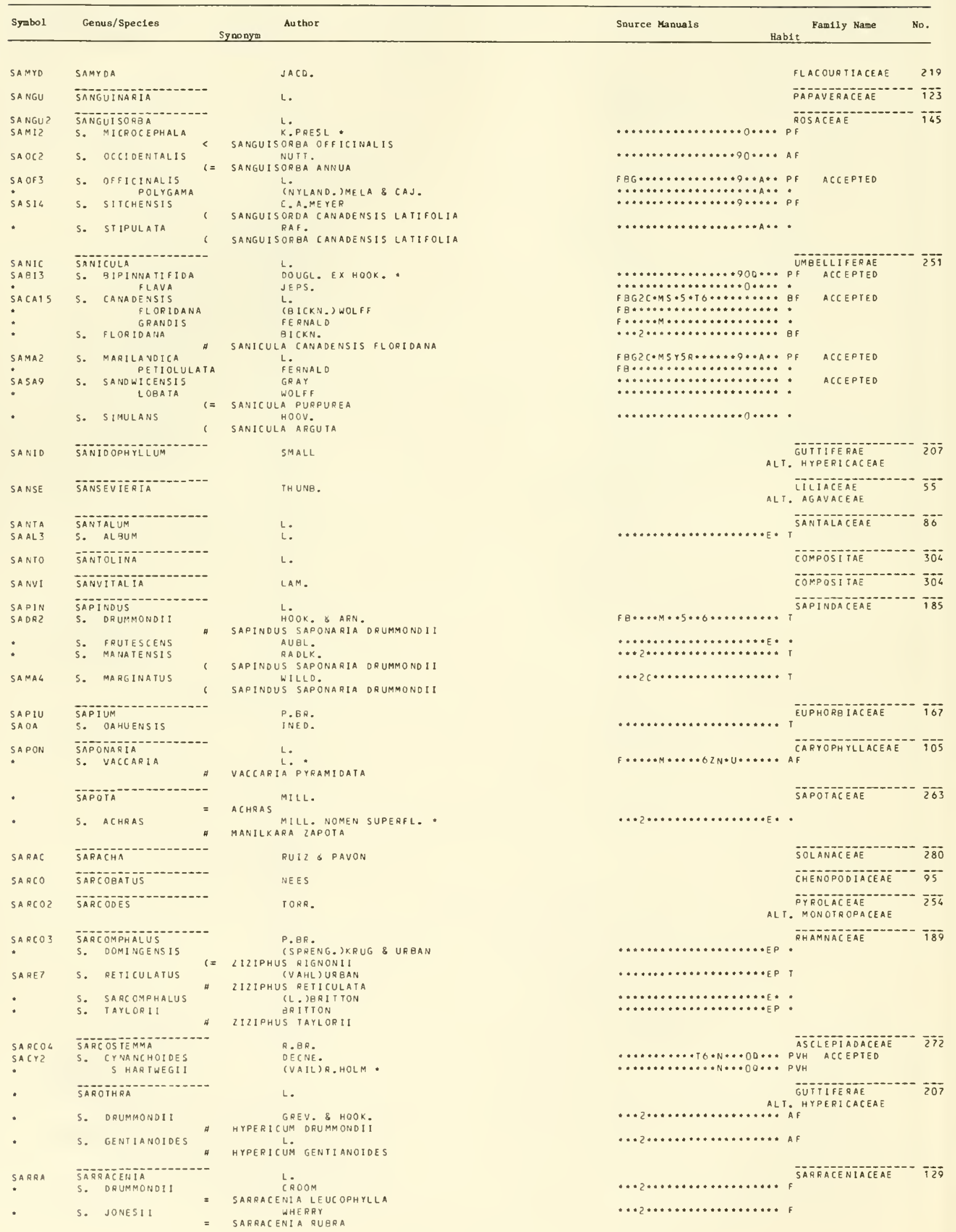




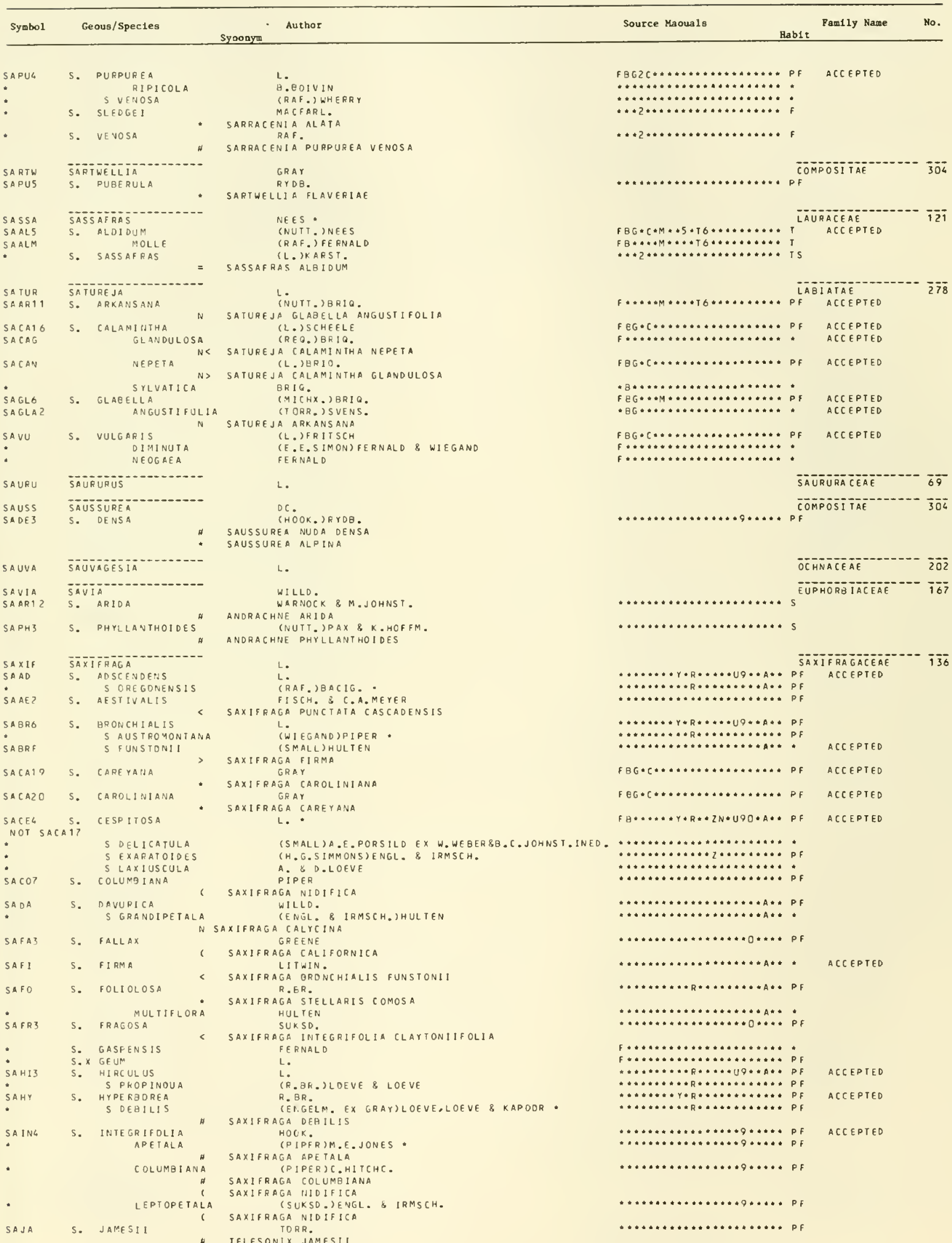




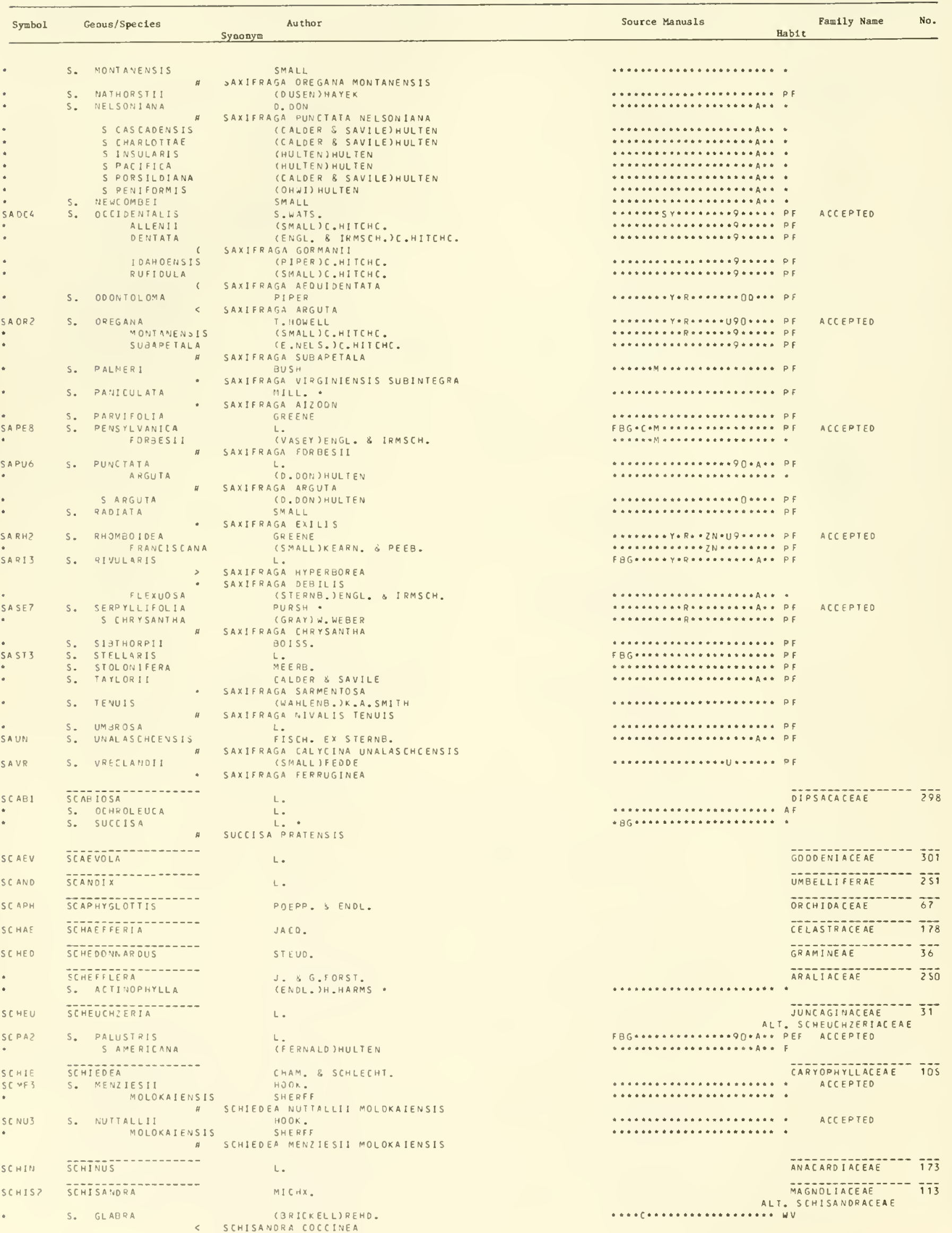




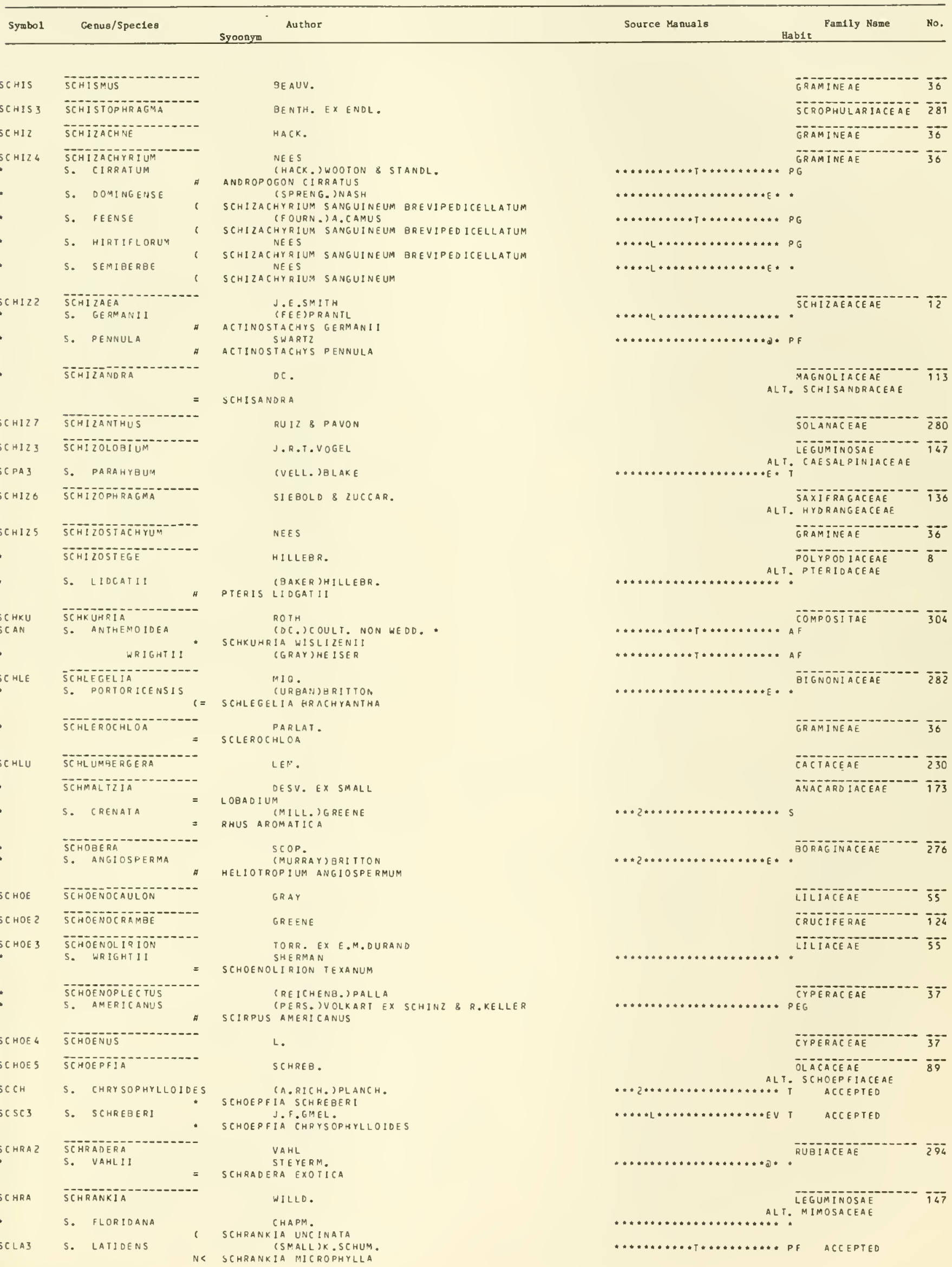




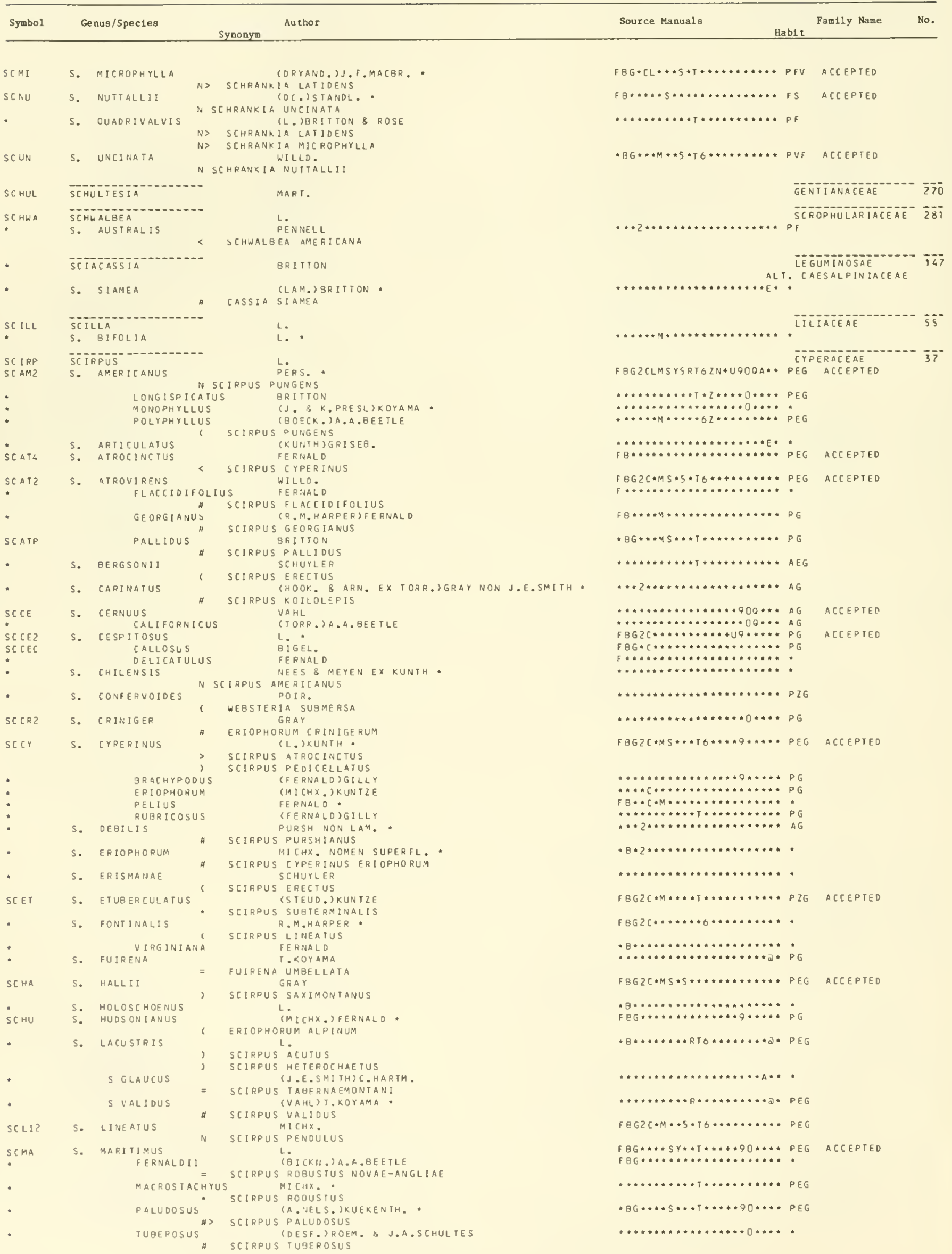




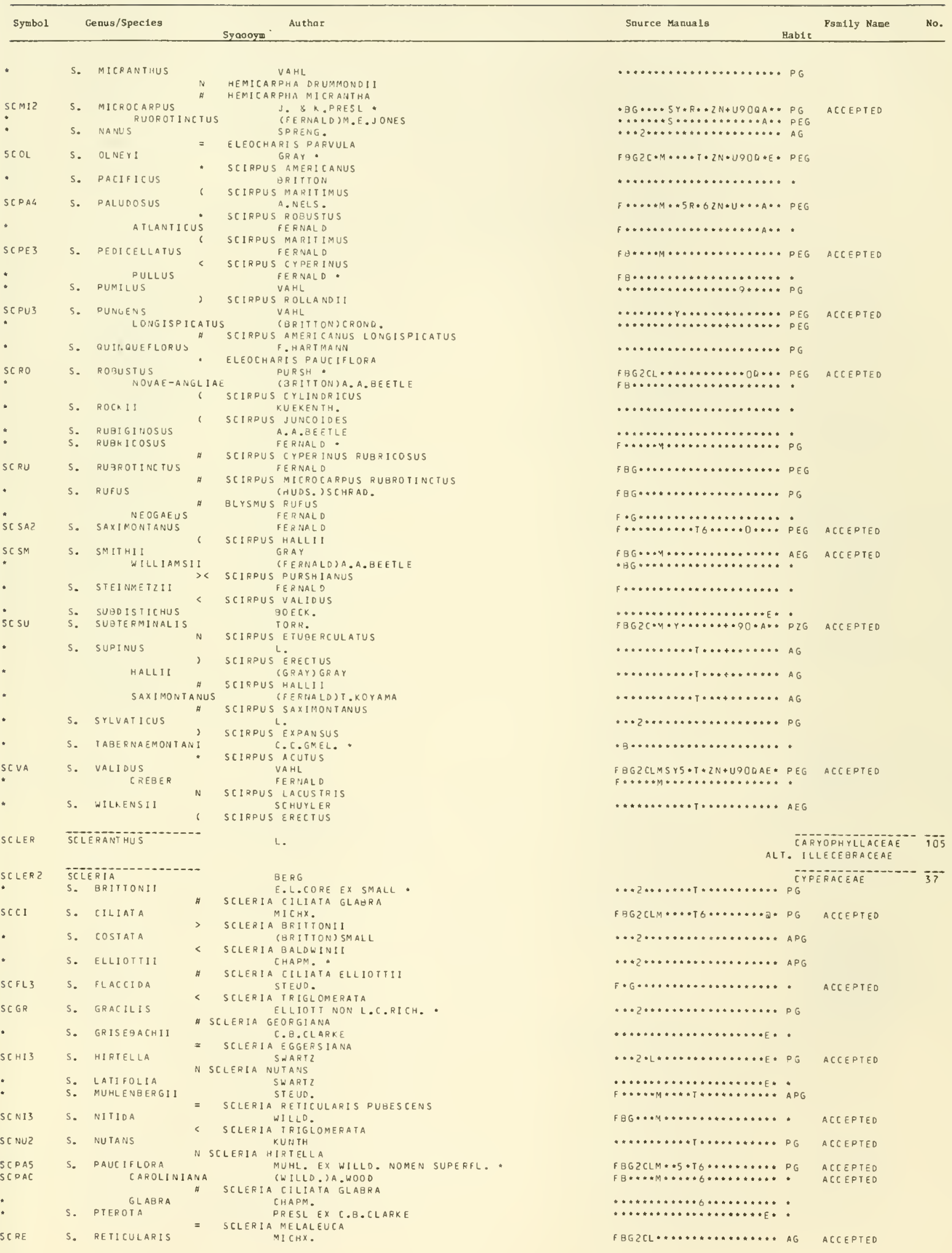




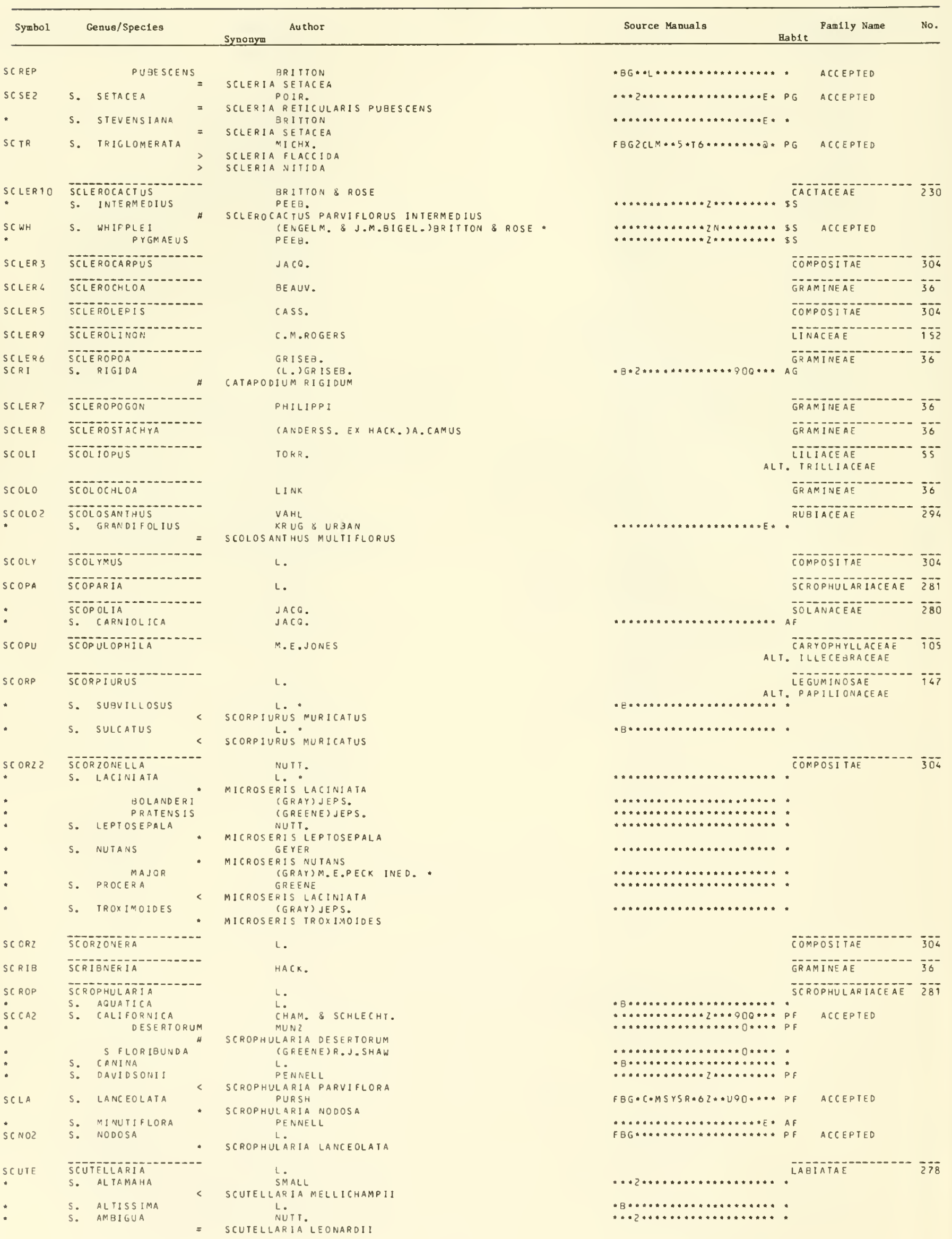




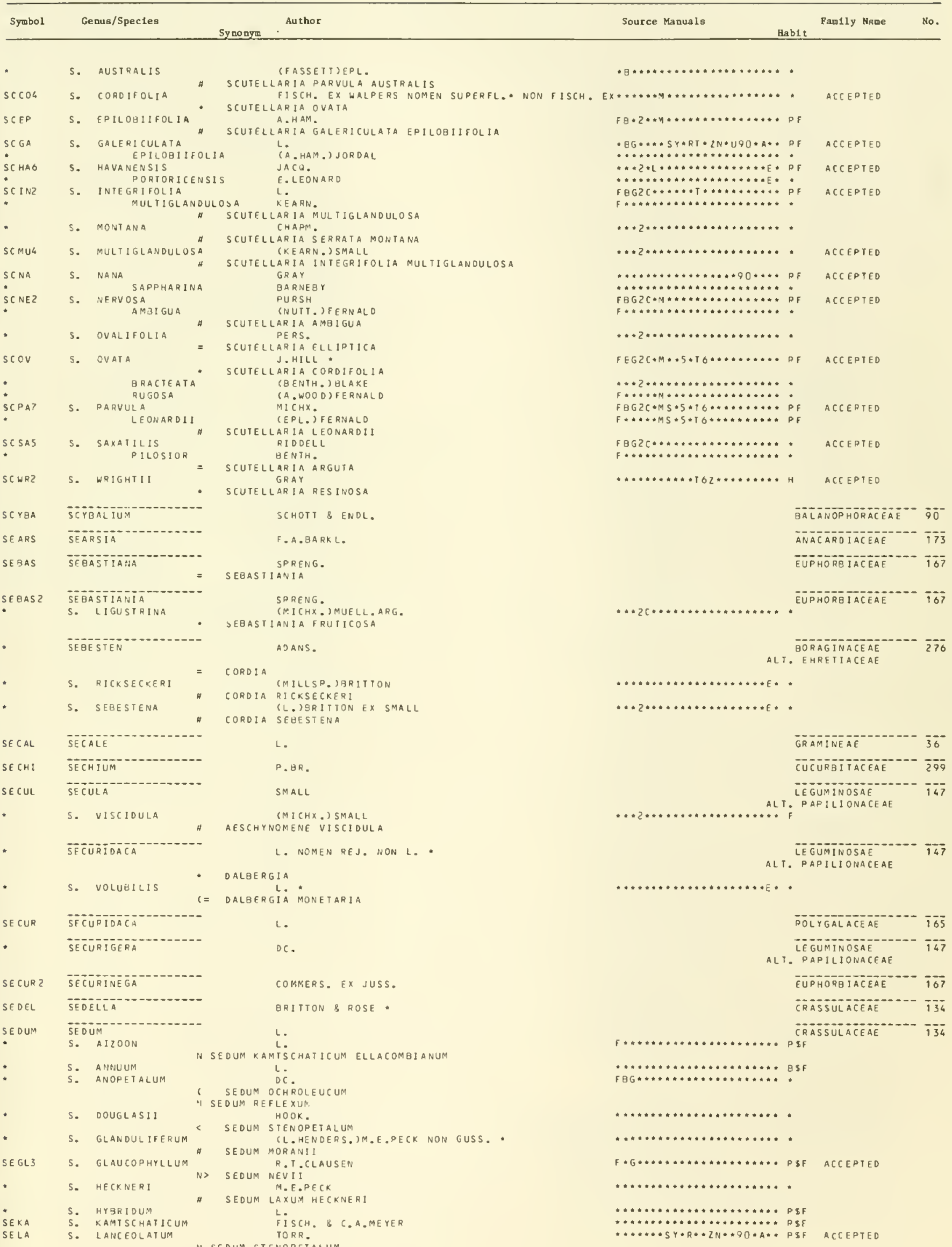




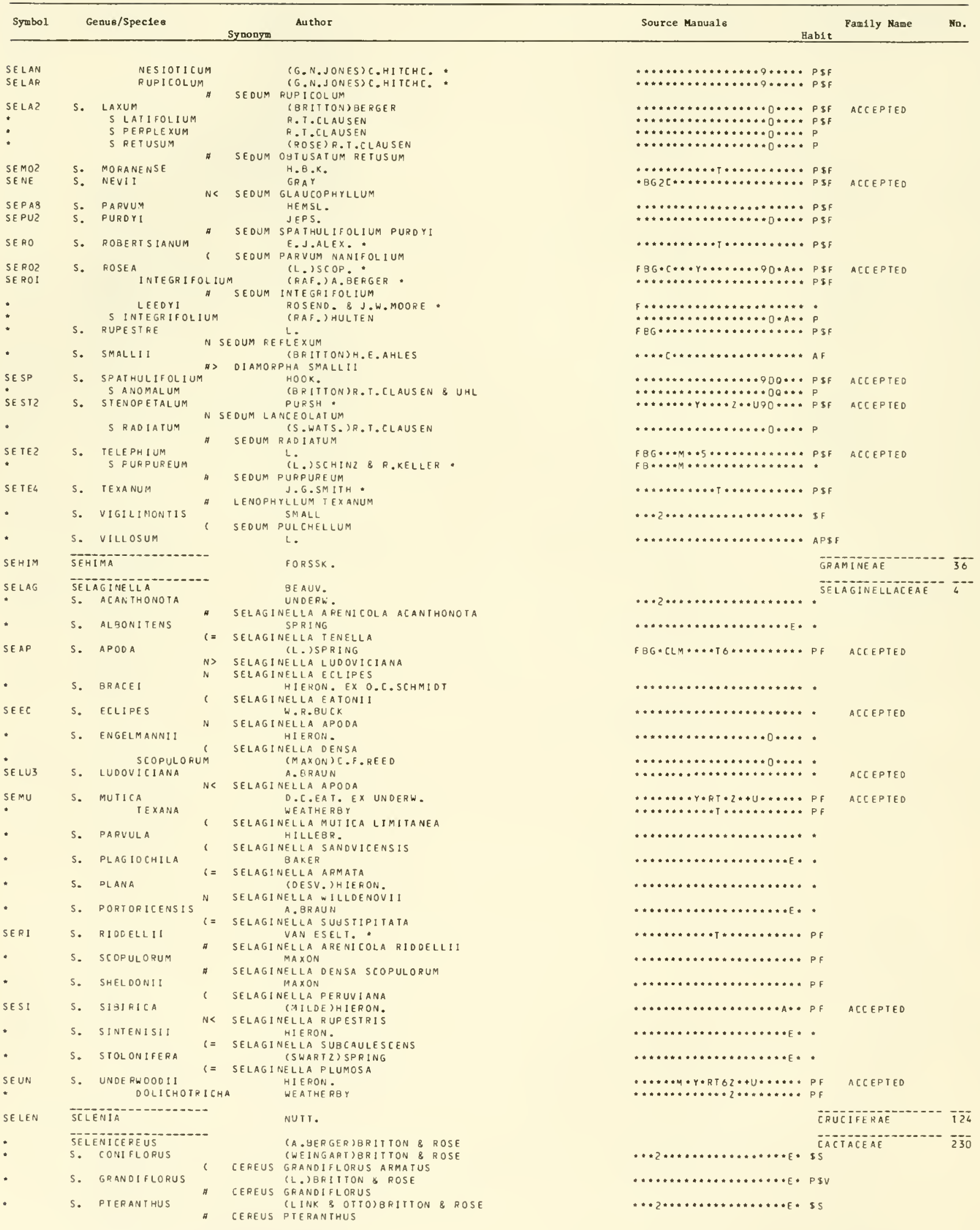




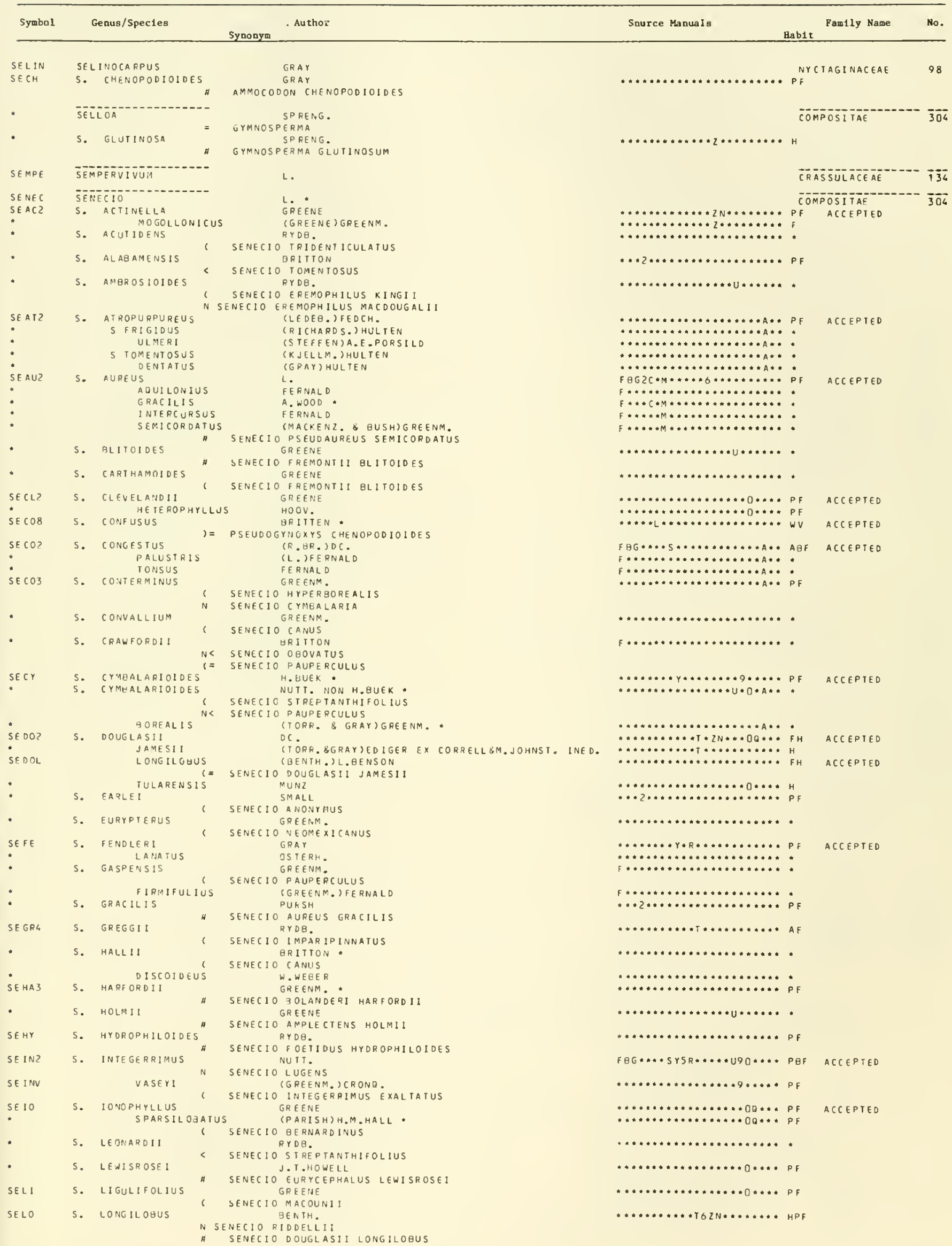




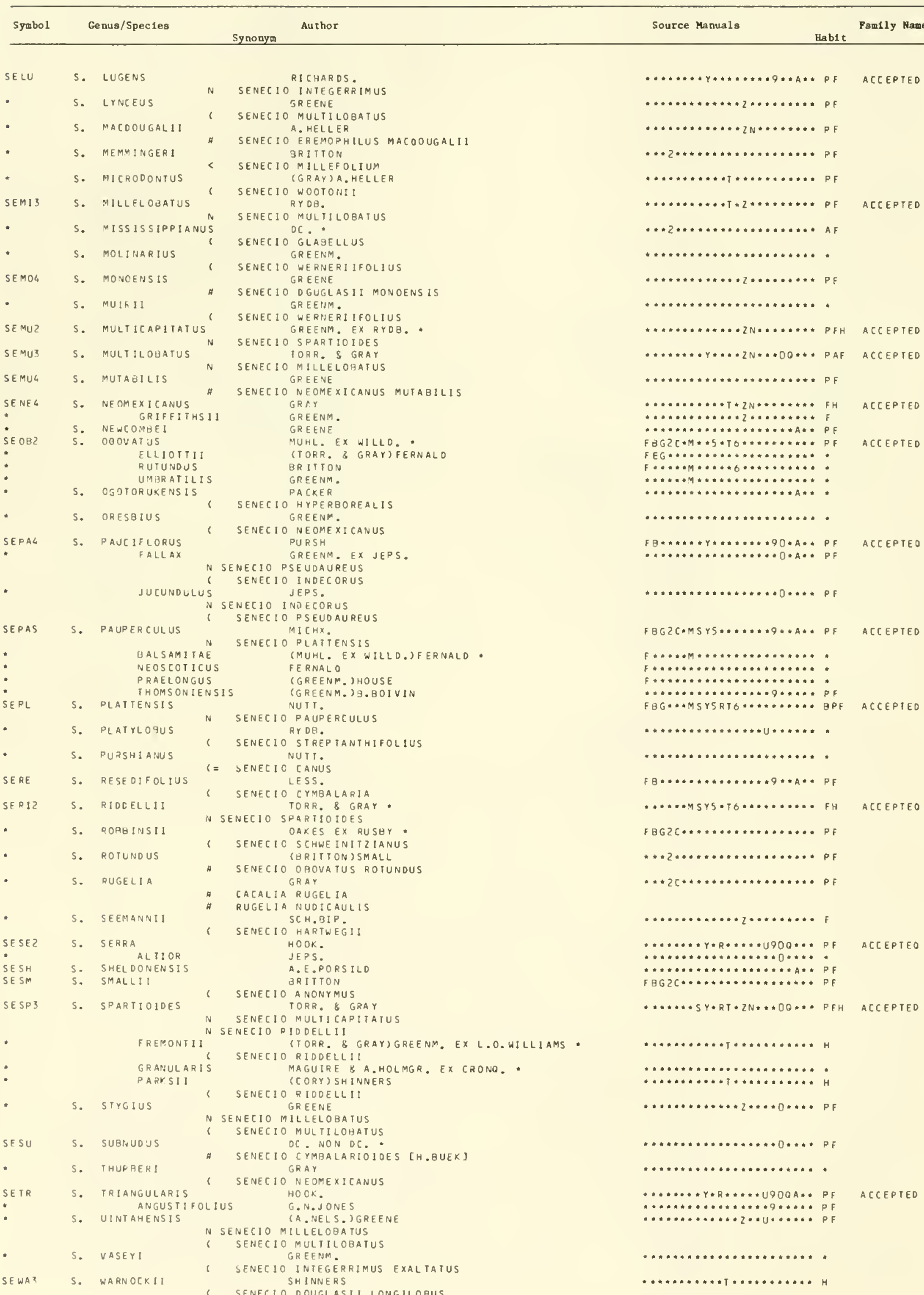




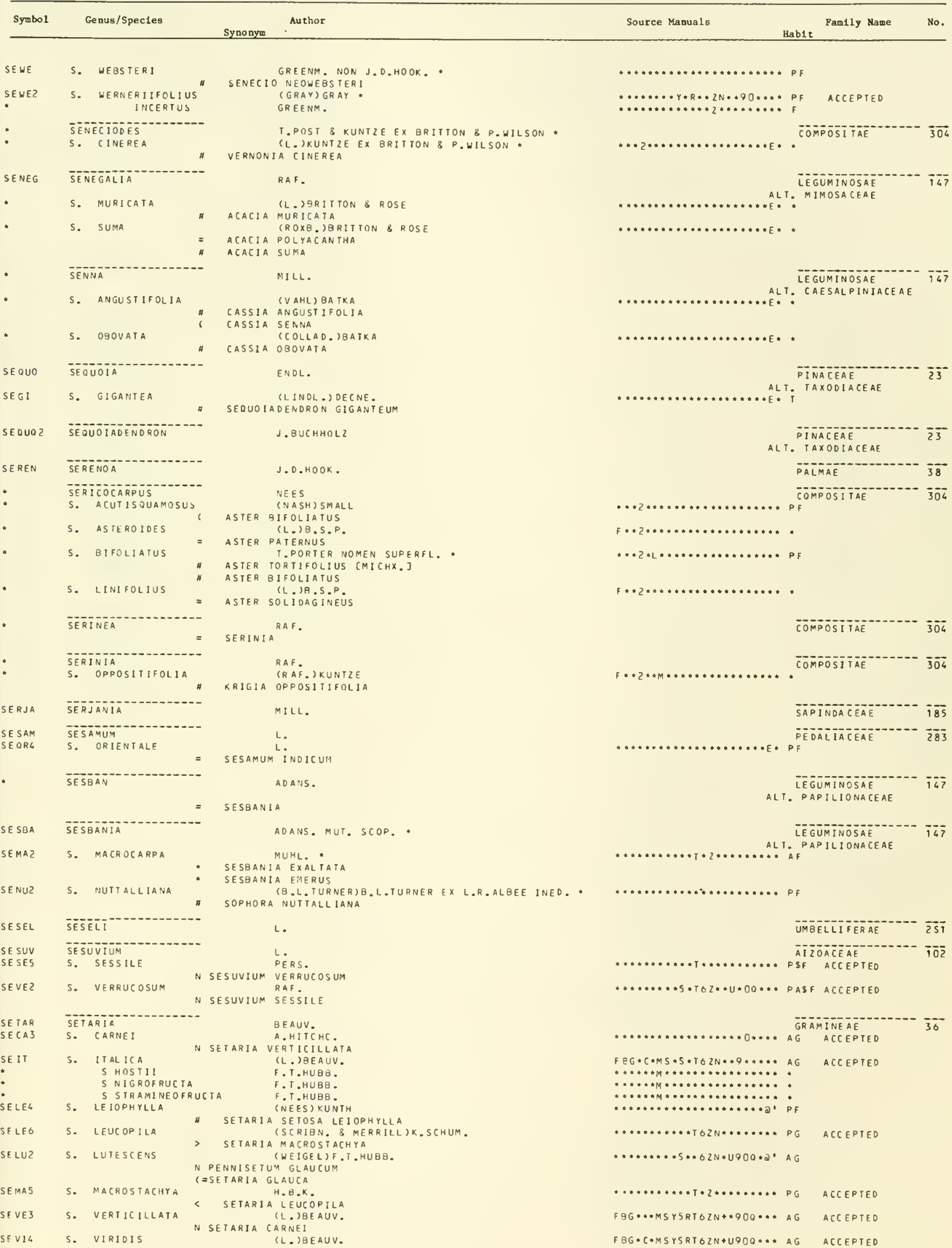




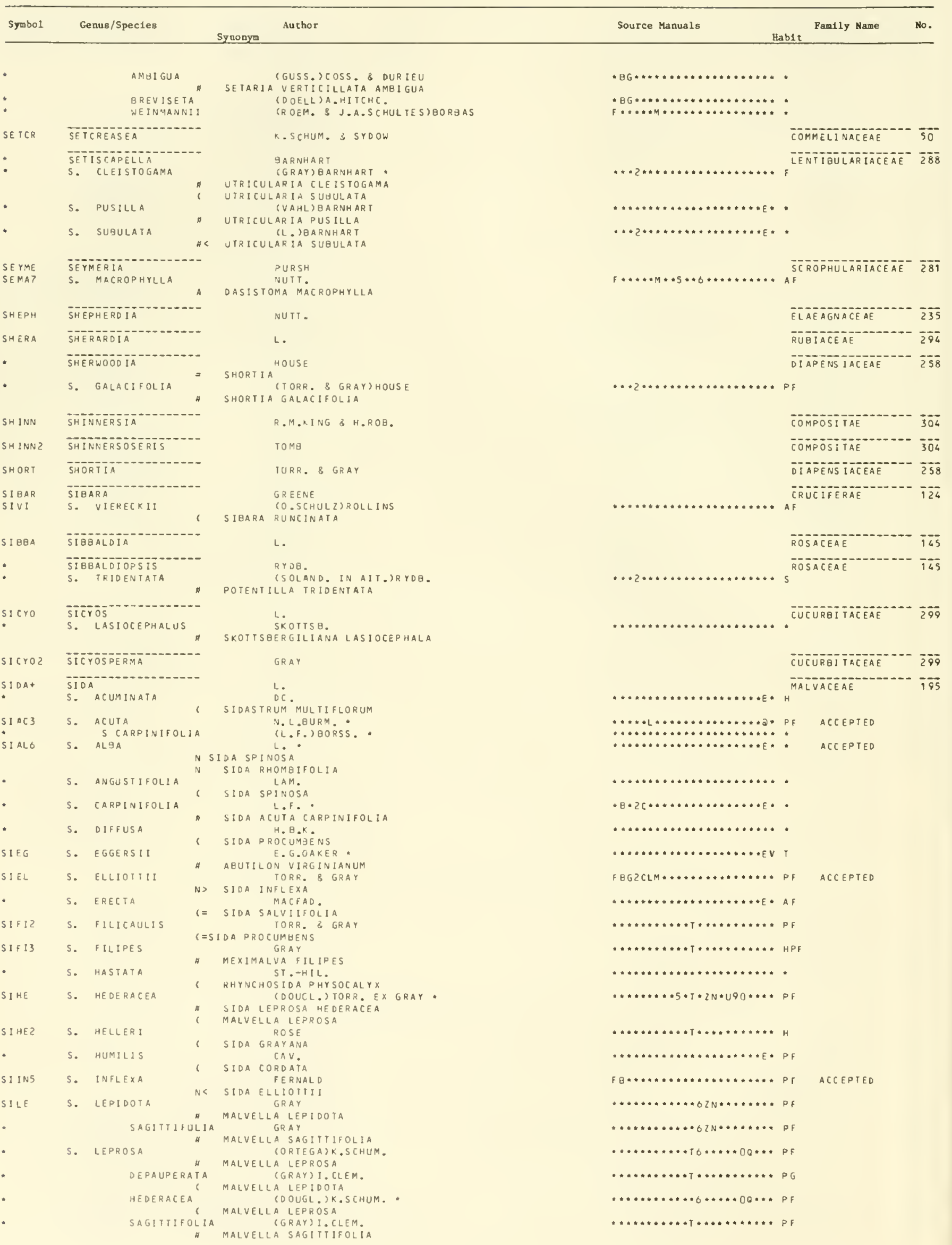




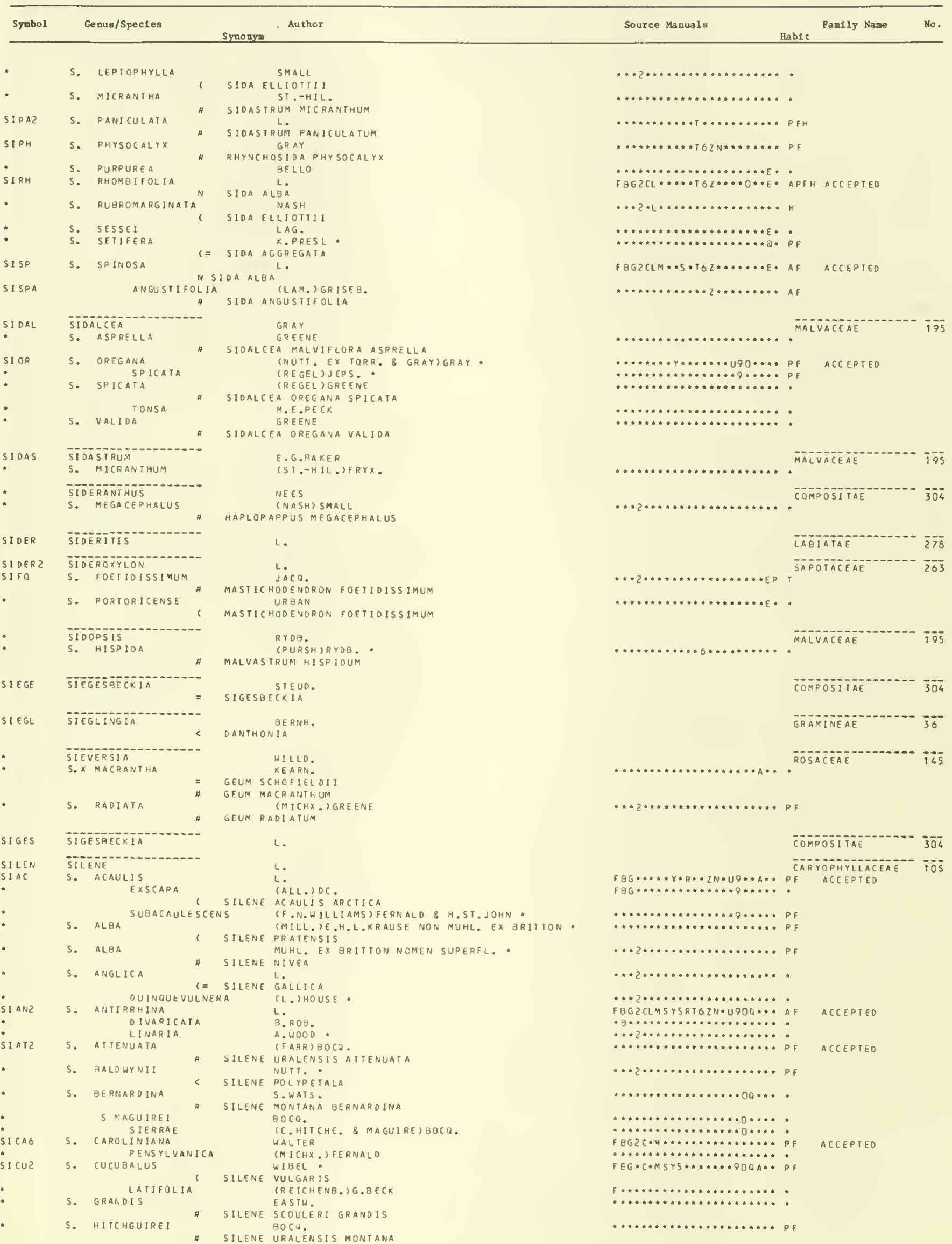




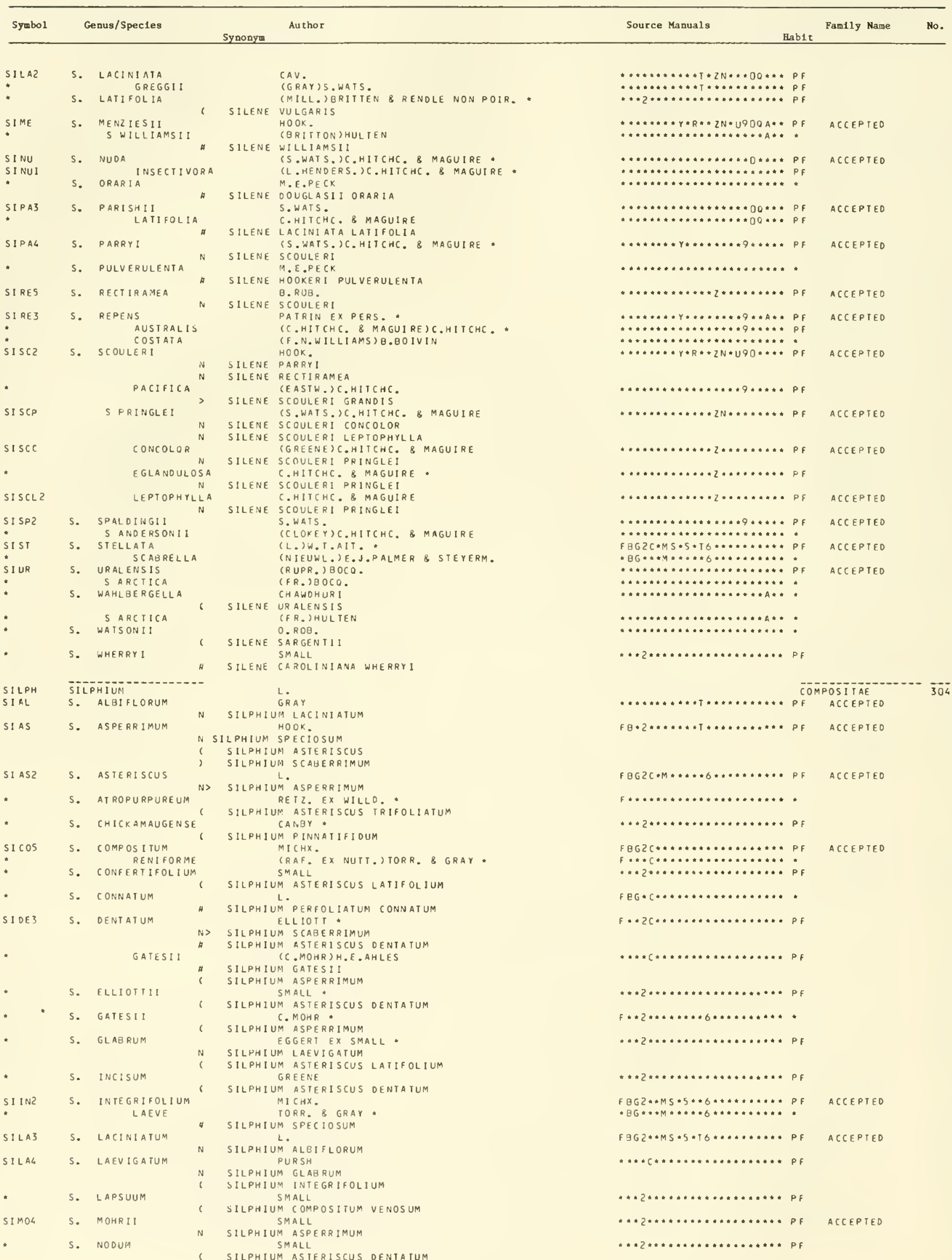




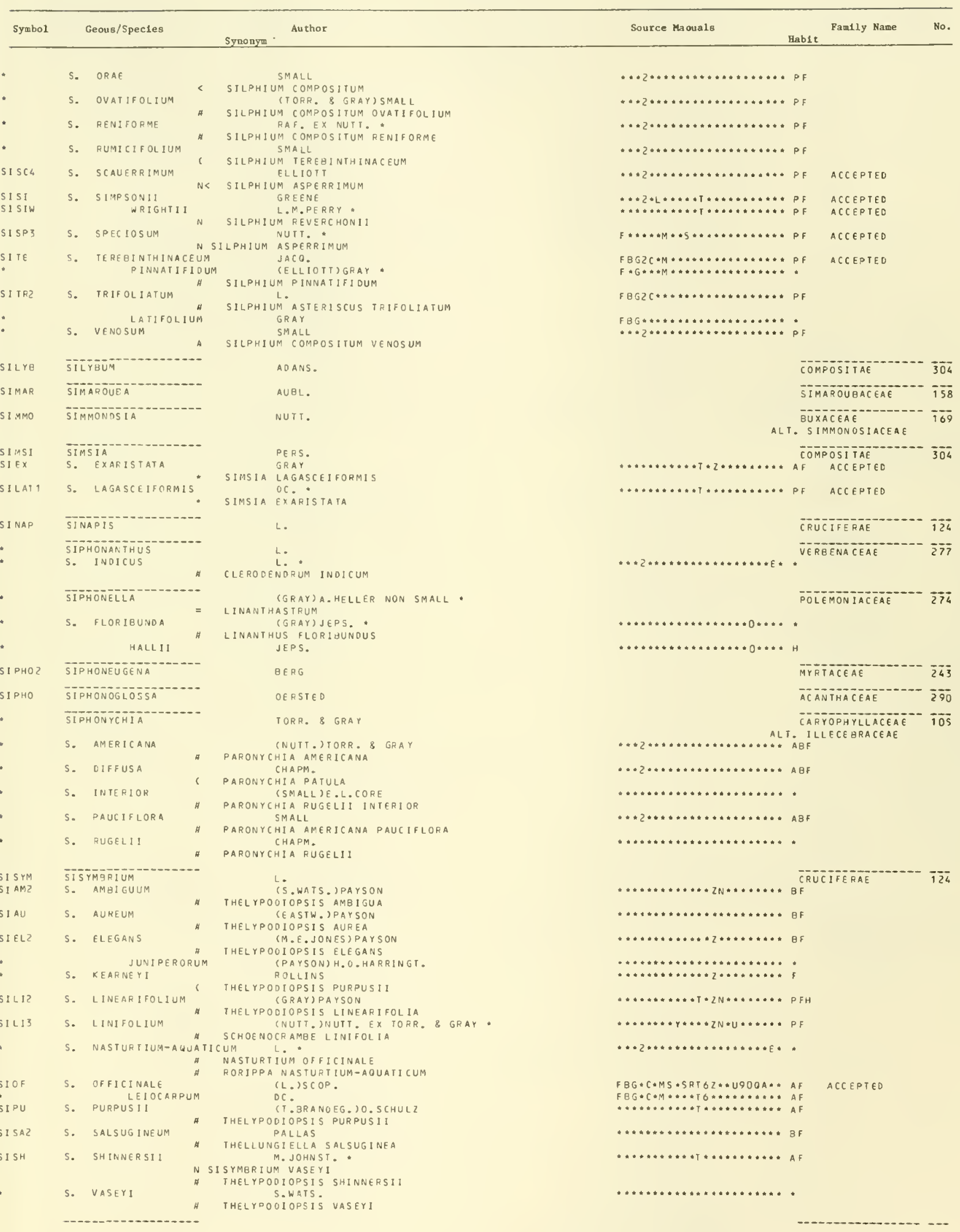




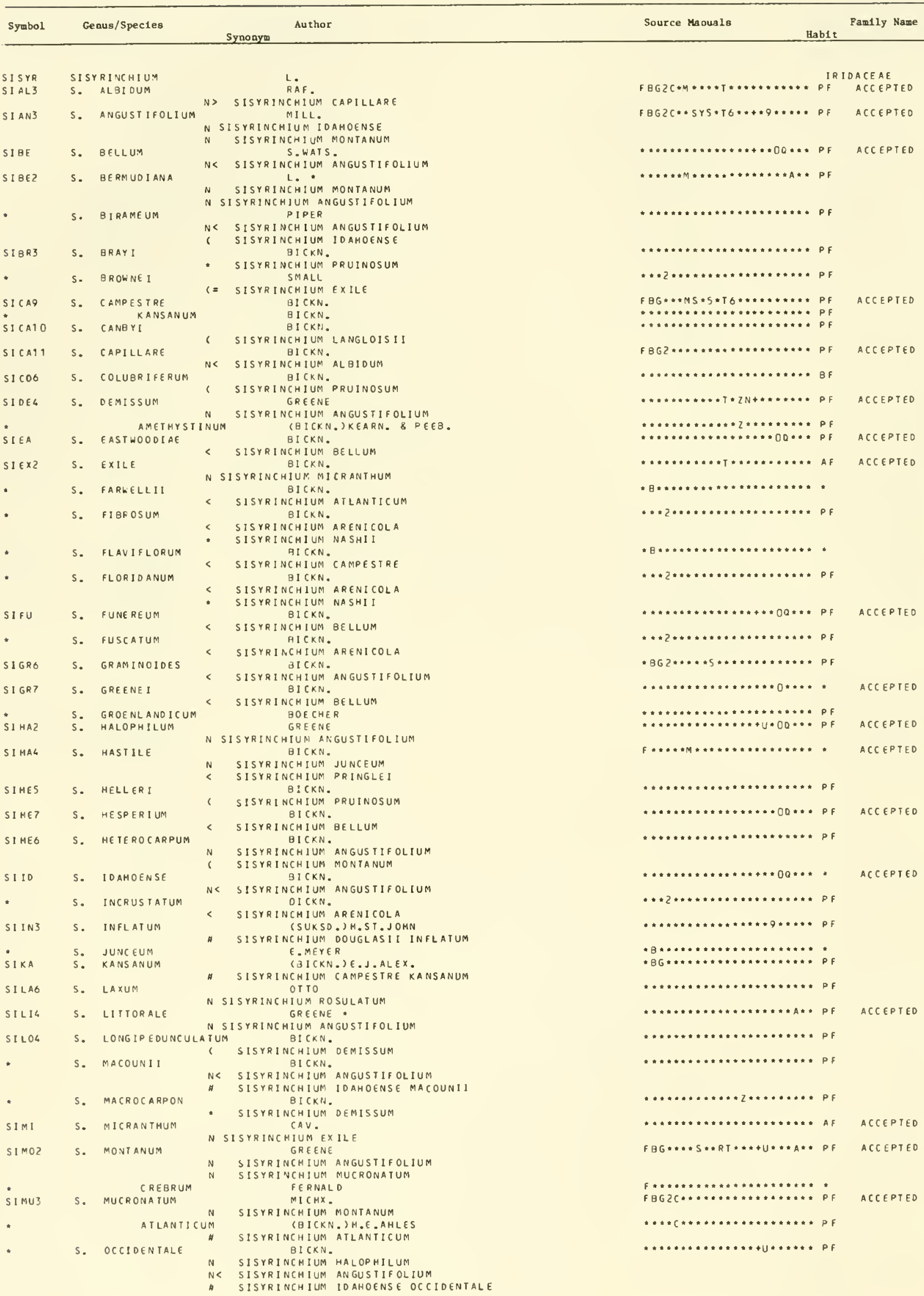




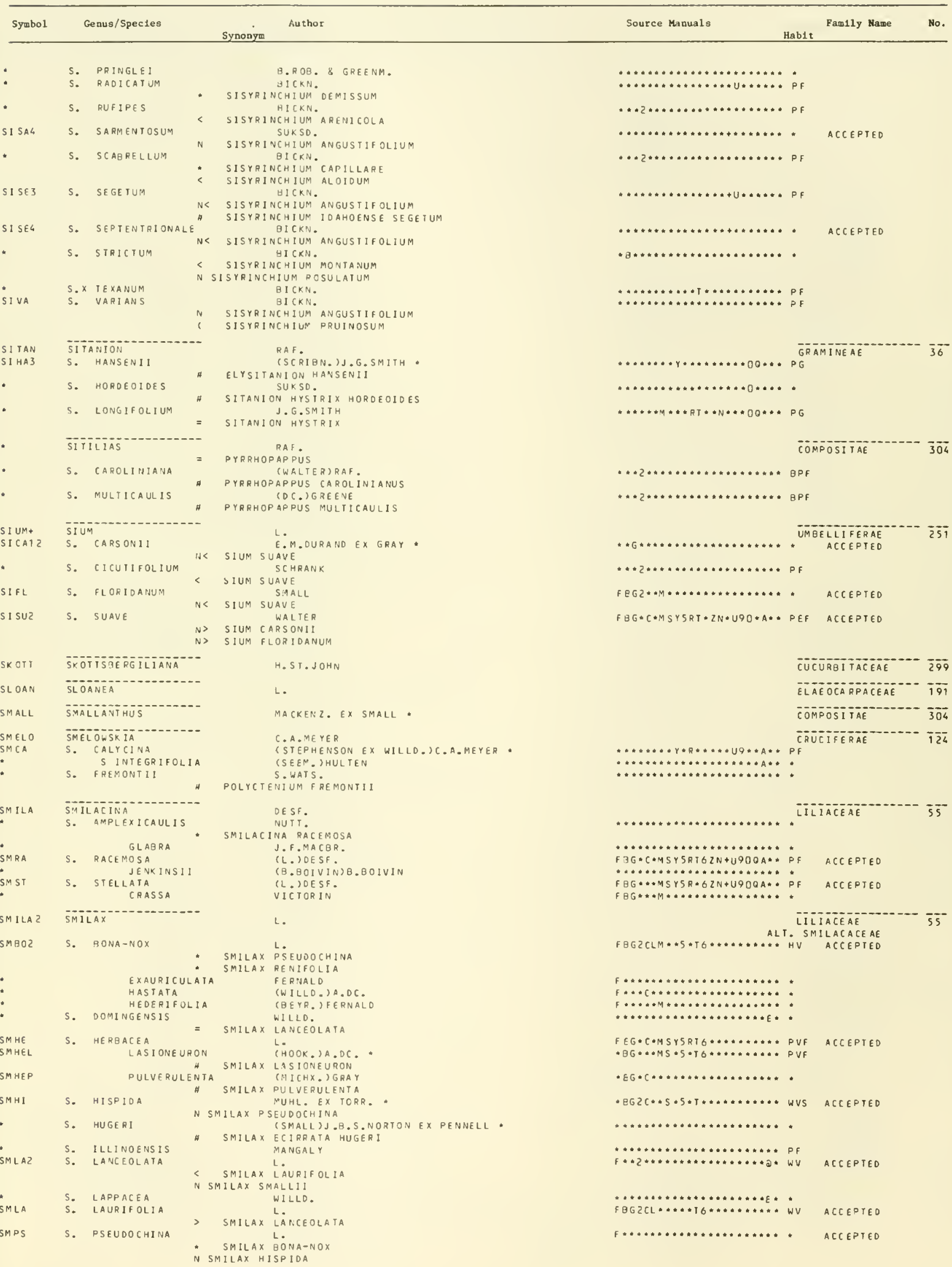




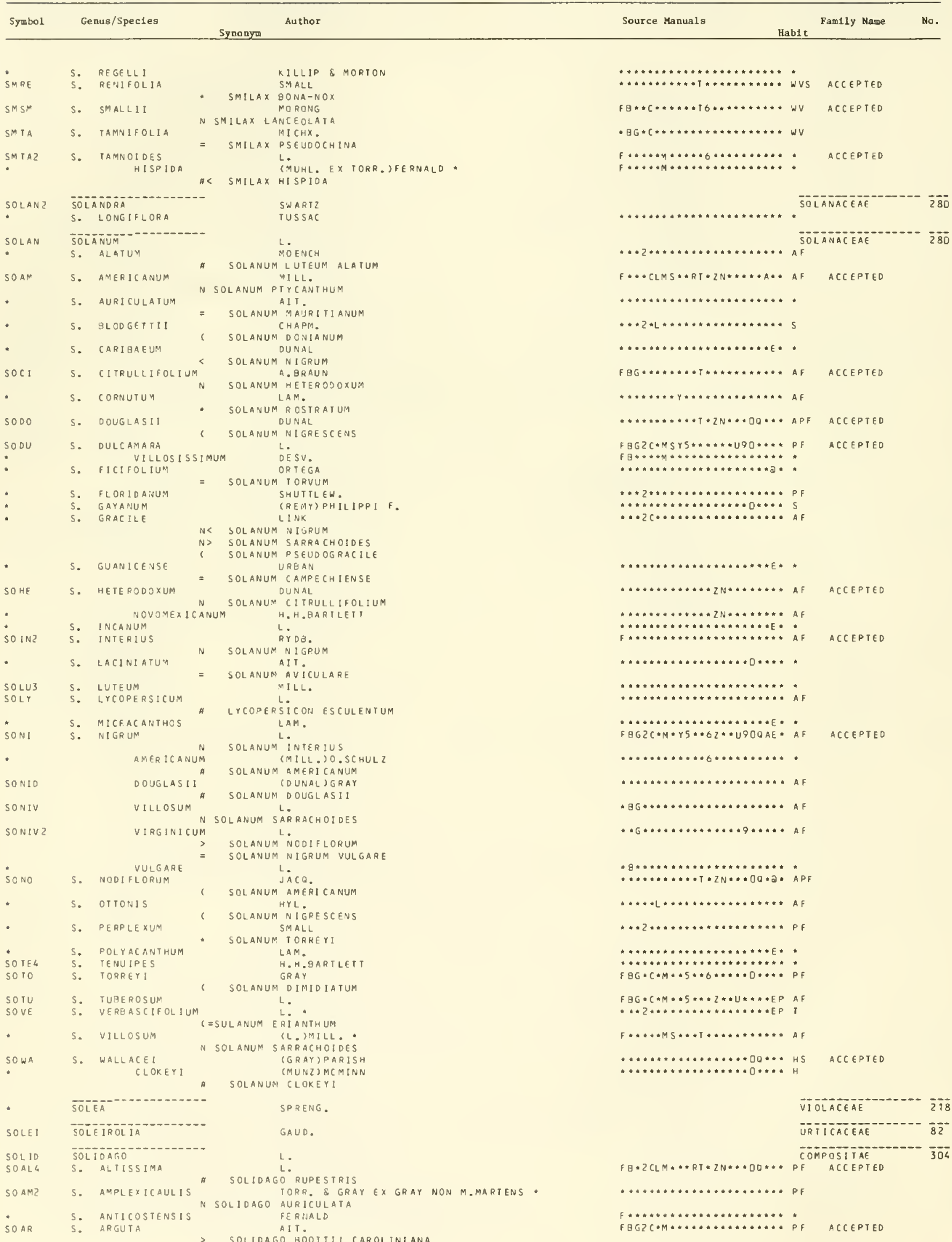




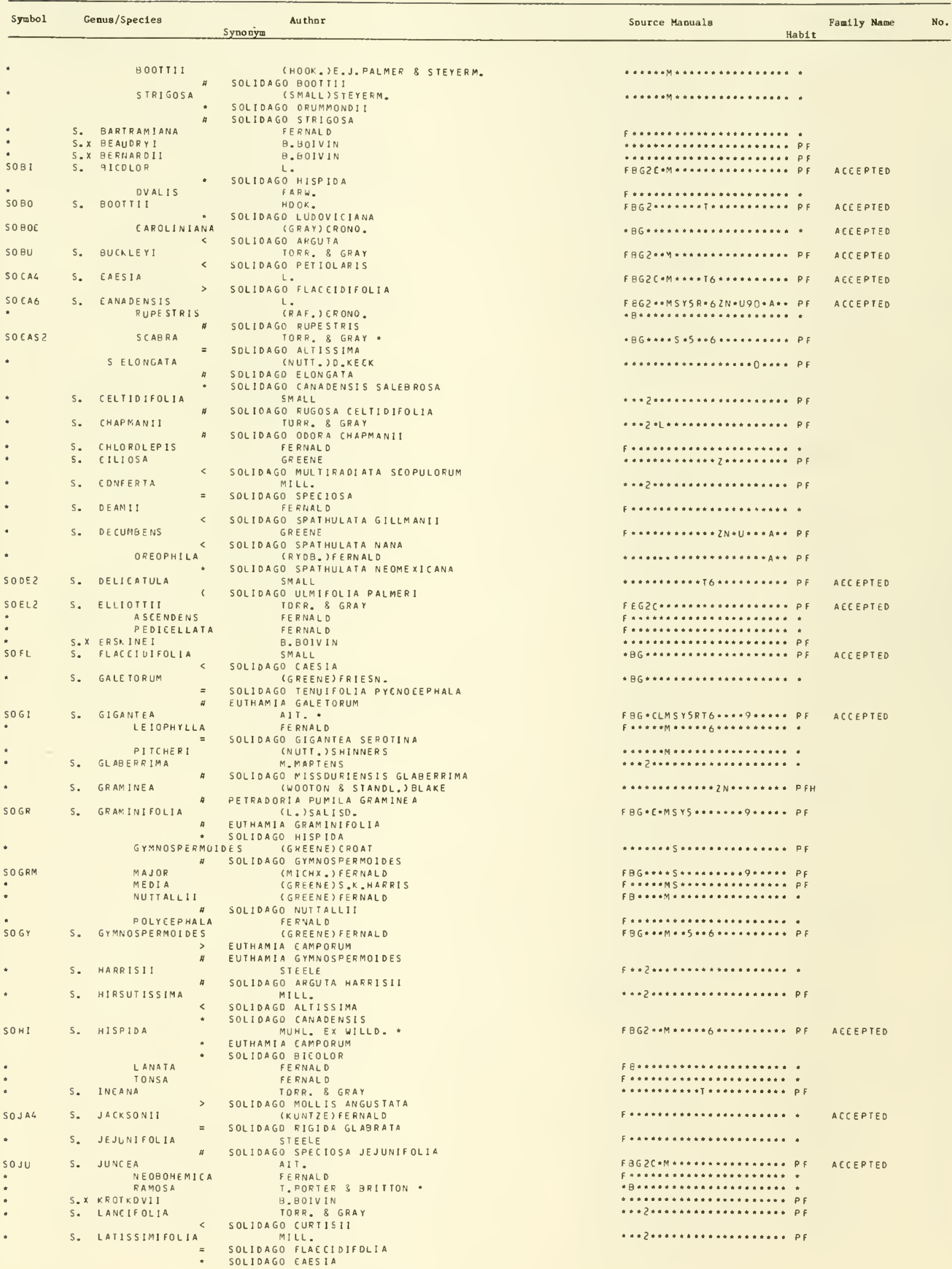




\begin{tabular}{|c|c|c|c|c|c|}
\hline Symbol & & nus/Spectes & Synonym & Source Manuals & Pam1ly Name \\
\hline SOLE & s. & LEPIOA $<$ & $\begin{array}{l}\text { DC. } \\
\text { SOLIOAGO CANADENSIS SUBSERRATA }\end{array}$ & $F \cdots \cdots \cdots \cdots \cdots \cdots \cdots \cdots \cdots * * A * * P F$ & \\
\hline - & & ELONGATA & $\begin{array}{l}\text { (NUTT.) FERNALD } \\
\text { * SOLIDAGO ELGNGATA }\end{array}$ & $F \ldots \ldots \ldots \ldots \ldots \ldots \ldots \ldots+n n_{*}$ & \\
\hline - & & FALLAX & $\begin{array}{l}\text { - SERNAL } \\
\text { - SOLIDAGO CANADENSIS SALEBROSa }\end{array}$ & $F \cdots \cdots \cdots \cdots \cdots \cdots \cdots+\cdots+\cdots * *$ & \\
\hline SOLEZ & S. & $\begin{array}{l}\text { MOLINA } \\
\text { LEPTOCEPHALA }\end{array}$ & $\begin{array}{l}\text { FERNALD } \\
\text { TORR. \& GRAY } \\
\text { EUTHAMIA LEPTOCEPHALA }\end{array}$ & 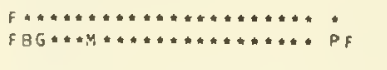 & \\
\hline SOLU & s. & LUDOVICIANA & $\begin{array}{l}\text { (GRAY)SMALL } \\
\text { - SOLJDAgo BOOTIII }\end{array}$ & 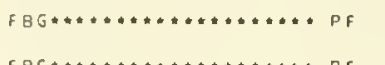 & ACCEPTED \\
\hline SOMAC & s. & $\begin{array}{l}\text { MACROPHYLLA } \\
\text { THYRSOIDEA }\end{array}$ & $\begin{array}{l}\text { PURSH } \\
\text { (E.MEYER) FERNALD }\end{array}$ & 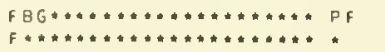 & ACCEPTED \\
\hline * & s. $x$ & MAHEUXII & B. BOIVIN & $* \ldots+\ldots+\ldots+\ldots \ldots \ldots \ldots+\ldots$ pq & \\
\hline * & S. & MAXONII & $\begin{array}{l}\text { POLLARO } \\
<\quad \text { SOLIOAGO SPATHULATA RANDII } \\
\text { - SOLIOAGO ROANENSIS }\end{array}$ & 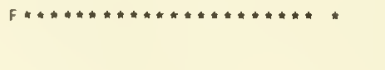 & \\
\hline - & s. & MENS L IS & FERNALO & $f \ldots \ldots \ldots+\ldots+\ldots \ldots \ldots \ldots \ldots$ & \\
\hline$\cdot$ & s. & $M E X I C A N A$ & SOLIOAGO SEMPERVIRENS MEXICANA & $\cdots * 2 \cdots * n+* * * * \cdots * \cdots * \cdot E * P F$ & \\
\hline SOMI & s. & MICROCEPHALA & $\begin{array}{l}\text { (GREENE) QUSH } \\
\text { > EUTHAMIA MINOR }\end{array}$ & $F B G * C L * \ldots * \hbar \cdots \ldots \ldots \ldots * P F$ & \\
\hline - & s. & MICROPHYLLA & $\begin{array}{l}\text { SMaLl } \\
\text { - Soljoago oelicatula }\end{array}$ & 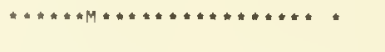 & \\
\hline * & S. & MILLERANA & $\begin{array}{l}\text { MACKENZ. } \\
\text { SOLIDAGO PET]OLARIS }\end{array}$ & 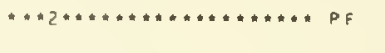 & \\
\hline SOM12 & s. & $\begin{array}{l}\text { MISS OURIENSIS } \\
\text { GLABERRIMA }\end{array}$ & $\begin{array}{l}\text { NUTT. } \\
\text { (M.MARTENS)ROSEND. \& CRONQ. }\end{array}$ & FBG**MSYSRTGZN* $90 * * * P F$ & ACCEPTEO \\
\hline SOMU & s. & $\begin{array}{l}\text { MULTIRADIATA } \\
\text { PARVICEPS }\end{array}$ & AIT ERALO & 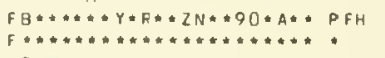 & ACCEPTED \\
\hline - & s. & NEGLECTA & $\begin{array}{l}\text { TORR. \& GRAY } \\
\text { SOLIOAGO ULIGINOSA }\end{array}$ & 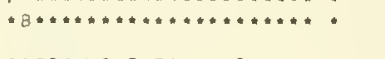 & \\
\hline SONE & S. & $\begin{array}{l}\text { NEMORALIS } \\
\text { OE CEMFLORA }\end{array}$ & $\begin{array}{l}\text { AIT. } \\
\text { COC.)FERNALO }\end{array}$ & $F B G Z C * M S Y 5+16 \cdots * 9 * \cdots * P F$ & ACCEPTEO \\
\hline & & & $\begin{array}{l}\text { SOLIDAGO NEMORALIS LONGIPETIOLATA } \\
\text { SOLIDAGO DECEMFLORA }\end{array}$ & & \\
\hline * & s. & NE OMEXI C ANA & $\begin{array}{l}\text { (GRAY) WOOTON STANOL. } \\
\text { SOLIOAGO SPATHULATA NEOMEXICANA }\end{array}$ & 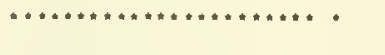 & \\
\hline * & s. & NEUFOLEPIS & $\begin{array}{l}\text { FERNALO } \\
\text { * SOLIOAGO ARGUTA NEUROLEPIS }\end{array}$ & $F \cdots \ldots \ldots+\cdots+\ldots+\ldots \ldots \ldots \ldots \ldots *$ & \\
\hline 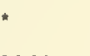 & s. & NOTABILIS & $\begin{array}{l}\text { mackenz. } \\
\text { - solidago auriculata }\end{array}$ & $\cdots \cdot 2 C * * \cdots \cdots * \cdots * \cdots \cdots \cdot P F$ & \\
\hline SoOC & s. & OCGIDENTALIS & $\begin{array}{l}\text { CNUTT. ITORR. } 8 \text { GRAY } \\
\text { " EUTHAMIA OCCIDENTALIS }\end{array}$ & $\cdots \cdots \cdots Y * \cdots Z N * 900 \cdots P F$ & \\
\hline SOPAZ & s. & PATULA & - SOlidago RIGIDA WILLD. & $F B G \cdot C+M \cdots \cdots \cdots \cdots \cdots \cdots+P F$ & ACCEPTEO \\
\hline SOPAS & & STRICTULA, & $\begin{array}{l}\text { TORR. \& GRAY } \\
\text { SOLIOAGO SALICINA } \\
\text { MIGHX. }\end{array}$ & $F Q G * C * M \cdots \cdots * \cdots \cdots+\cdots \cdots \cdots *$ & ACCEPTEO \\
\hline SOPA3 & S. & PAUC IFLOSCULOSA & $\begin{array}{l}\text { MICHX. } \\
\text { CHRYSOMA PAUCI FLOSCULOSA } \\
\text { FERNALO }\end{array}$ & $\cdots \cdots, \cdots \cdots \cdots \cdots \cdots * \ldots * \cdots+s$ & \\
\hline • & s. & PERL ONGA & $\begin{array}{l}\text { FERNALO } \\
\text { SOLIDAGO BOOTTII }\end{array}$ & $F \cdots \cdots+\cdots \cdots \cdots \cdots \cdots \cdots \cdots \cdots \cdots \cdots *$ & \\
\hline SOPE & s. & $\begin{array}{l}\text { PETIOLARIS } \\
\text { WAROII }\end{array}$ & $\begin{array}{l}\text { AIT. } \\
\text { SOLIDAGO BUCKLEYI } \\
\text { CORITTON)FERNALD }\end{array}$ & $F B G * C * M * * 5 * T G * \cdots \cdots \cdots * P F$ & ACCEPTEO \\
\hline * & & $\begin{array}{l}\text { WAROII } \\
\text { PETI OLAIA }\end{array}$ & $\begin{array}{l}\text { (ORITTON) FERNALD } \\
=\text { SOLIOAGO ANGUSTA } \\
\text { MILL. }\end{array}$ & 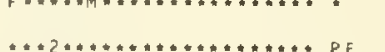 & \\
\hline * & s. & $\begin{array}{l}\text { PETIOLATA } \\
\text { PETRADORIA }\end{array}$ & $\begin{array}{c}\text { MILLE } \\
<\text { SOLIDAGO SIRICIA } \\
\text { OLAKE }\end{array}$ & $\ldots \ldots \ldots \ldots$ & \\
\hline * & s. & $\begin{array}{l}\text { PETRADORIA } \\
\text { PRUINOSA }\end{array}$ & $\begin{array}{l}\text { - PETRAOORIA PLiMILA } \\
\text { GREENE }\end{array}$ & 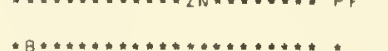 & \\
\hline * & s. & PRUINOSA & SOLIDAGO CANADENSIS GILVOCANESCENS & (6) & \\
\hline • & s. & PUBENS & $\begin{array}{l}\text { M.A.CURI : } \\
\text { a SOLJDAGO CURTISII PUBENS }\end{array}$ & 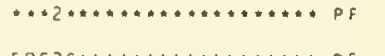 & \\
\hline SOPU & s. & $\begin{array}{l}\text { PUBERULA } \\
\text { PULVERULEN }\end{array}$ & NTA NUTT. SCHAPM. & 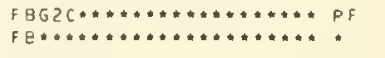 & A CCEPIEO \\
\hline • & s. & PULCHRA & $\begin{array}{l}\text { SOLIOAGO PUBERULA PUBERULENTA } \\
\text { SMALL } \\
\text { SOLIOAGO STRIETA }\end{array}$ & 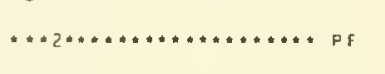 & \\
\hline - & s. & PULVERULENTA & $\begin{array}{l}\text { NUTT. } \\
\text { " SOLIOAGO PUBERULA PULVERULENTA } \\
\text { " EUTHAMIA PULVERULENTA }\end{array}$ & $\cdots * 2 * \ldots \ldots n+\ldots \ldots \ldots * \cdots * P F$ & \\
\hline * & s. & PURSHII & $\begin{array}{l}\text { T.PORTER. } \\
=\text { SOLIDAGO ULIGINOSA PERACUTA }\end{array}$ & 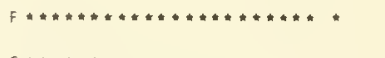 & \\
\hline - & s. & RACEMDSA & $\begin{array}{l}\text { GREENE } \\
\text { SOLIDAGO SPATHULATA RACEMOSA }\end{array}$ & 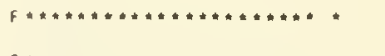 & \\
\hline - & & GILLMANII & (GRAY) FERNALO & 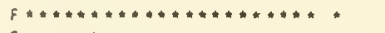 & \\
\hline ॥ & s. & RANOII & $\begin{array}{l}\text { (T.PORTER)BRITTON: } \\
\text { SOLIDAGO SPATHULATA RANDI: }\end{array}$ & $F \ldots \ldots \ldots \ldots \ldots \ldots+\hbar * \hbar \hbar \hbar \hbar \hbar \hbar *$ & \\
\hline SORE & s. & REMOTA & $\begin{array}{l}\text { (GREENE)FRIESN. } \\
\text { * euthamia REMOIA }\end{array}$ & $F B G \ldots \ldots * \ldots \ldots \ldots \ldots+\ldots+\cdots * P F$ & \\
\hline SOR12 & s. & RIGIDA & - solidago patula & 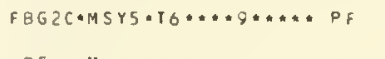 & ACCEPTED \\
\hline SORIG & & GLABRATA $=$ & $\begin{aligned} \text { E. L.BRAUN: } \\
\text { SOLIOAGO JACK SONI: }\end{aligned}$ & 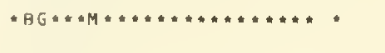 & ACCEPTED \\
\hline - & s. & FIGIDIUSCULA & $\begin{array}{l}\text { (TORR. \& GRAY)T.PORTER * } \\
\text { a SOLIDAgO SPECIOSA RIGIDIUSCULA }\end{array}$ & $\cdots * 2 * \ldots+\ldots+\ldots \ldots \ldots \ldots \ldots$ PF & \\
\hline $50 R 02$ & s. & $\begin{array}{l}\text { ROANENSIS } \\
\text { MONTICOLA }\end{array}$ & $\begin{array}{l}\text { TPORTER. } \\
\text { (TORR. \& GRAY) FEFNALO }\end{array}$ & $f B G 2 c * \ldots \ldots \ldots \ldots \ldots \ldots * * * * f f$ & ACCEPTEO \\
\hline SORUZ & s. & $\begin{array}{l}\text { RUGOSA } \\
\text { ASPERA }\end{array}$ & $\begin{array}{l}\text { MILL. * } \\
\text { (AIT.) FERNALD }\end{array}$ & $F \in G * C * M * \cdots * T 6 \cdots \ldots \ldots \ldots * P F$ & ACCEPTEO \\
\hline SORUC & & CELTIOIFOL & $\begin{array}{l}\text { LIA (SMALL) FERNALD } \\
\text { S SOLIDAGO ULM!FOLIA }\end{array}$ & 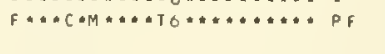 & ACCEPTED \\
\hline SORUA & & $S$ ASPERA & $\begin{array}{l}\text { (AIT.) CRONG. } \\
=\text { SOLIOAGO ALTISSIIAA }\end{array}$ & $F B G \ldots \cdots M+\ldots \ldots+\ldots+\ldots \ldots \ldots$ & ACCEPTEO \\
\hline SORU3 & s. & RUPESTRIS & $=$ SOLIOAGO ALIISSIMA & 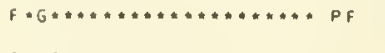 & ACCEPTED \\
\hline SOSA2 & s. & SALICINA & $\begin{array}{l}\text { ELLIOTT } \\
<\text { SOLIOAGO PATULA STRICTULA }\end{array}$ & $F * 2 * * \cdots \cdots * T \cdots * * \cdots * * * P F$ & ACCEPTED \\
\hline
\end{tabular}




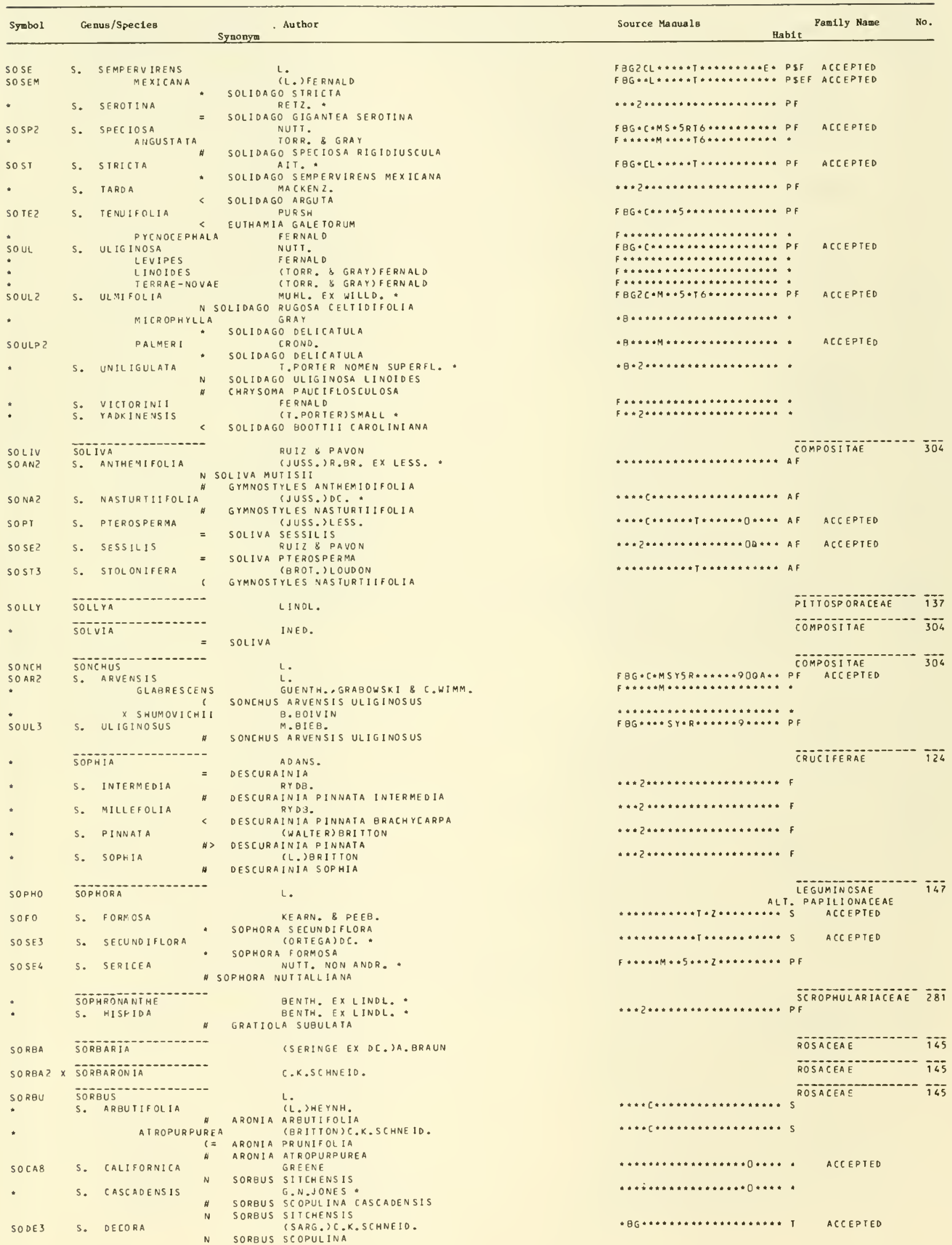




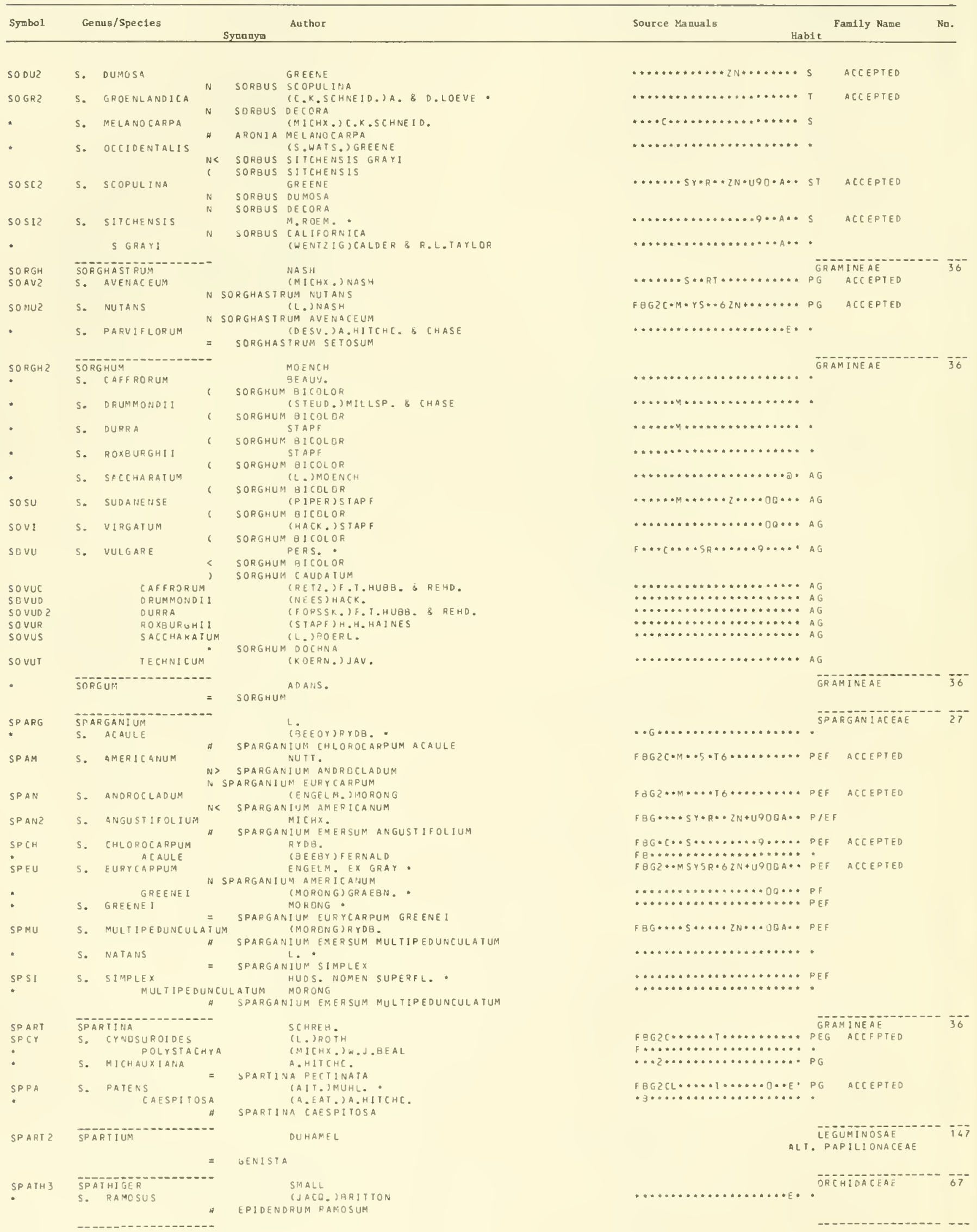




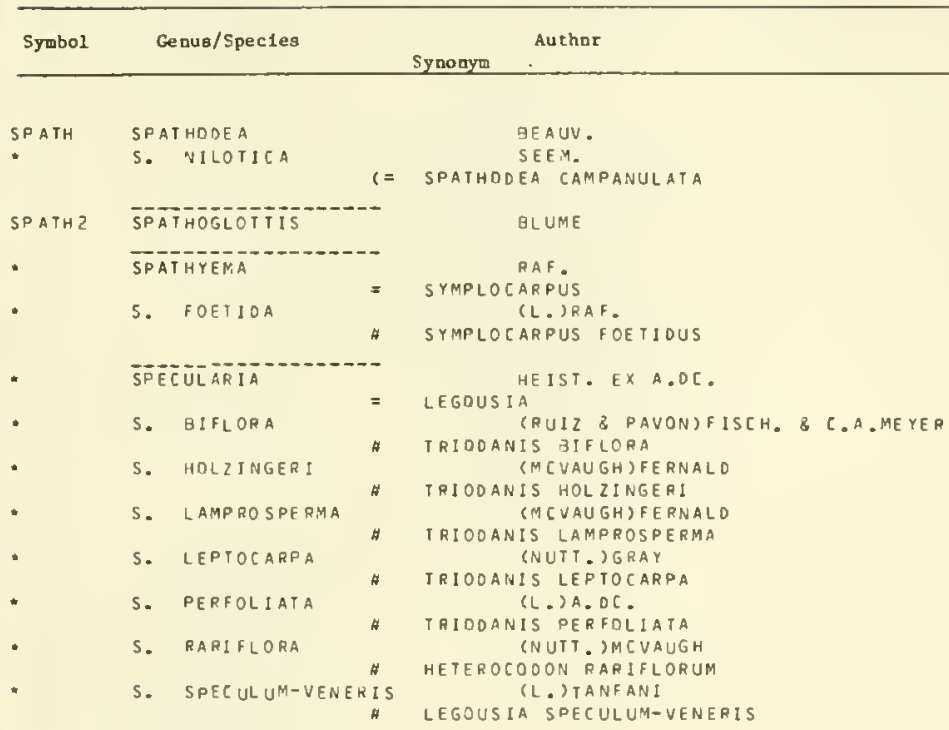

L.

(PERS.) J. 8 K. PRESL *

(PERS,)D.DON

S. CANADE:NSIS

N SPERGULARIA MARIVA

OCCIDENTALIS DIANDRA R.ROSSB.
S. CGUSS.)BOISS.

S. DIANDAA ND SPERGULARIA SALSUGINEA

S. LEIOSPERMA ND SPERGULARIA SALSUGINEA
(KIVDO.)F.SCHMID:

5. MAGROTHECA SPERGULARIA MARINA
(HORNEM.) HEYNH.

LEUCANTHA (GREENE)B.RDB.

LONGISTYLA R.ROSSB.

SPMAZ S. MARINA N SPERGULARIA IANADENSIS

(L.) GRISEB.

- TENUIS (GREENE)R.ROSSB.

5. SALSUGIHEA SOUNGE)FENZL

SPERM SPCOT

SPERMACOCE

S. CONFUSA

S. HISPIDA

S. KEYENSIS

5. DC YMOIOES

5. RADICANS

S. RIPARIA

S. SUFFRUTESCENS

S. TENUIOR

S SPERGULARIA DIANORA

SPTE

-

SPERM 2

* ERM

SP HAE

SPAMC

:

SPANB to

S. ANGUSTIFDLIA CUSPIOATA

LOBATA

S. COCEINEA

DISSECTA

ELATA

S. DIGITATA

S. EMORYI

TENUIPES

SPEM

SPFE

*

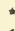

RENDLE
N SPERMACOCE TENUIOR L.

SMALL

Spermacoce tenuior floridoana

II. L BURM.

- borrerla dCYMDioes

AUBL.

CHAM, \& S[HLECHT

SPERMacole tenuloo

S BUKRERIA LAEVI

N SPERMACOCE CONFUSA

(URBAN)R.LONG

- spermacore florioana

* borreria verticillata

PAF. RUTT, SB. ROA.

$=$ SPERMOLEPIS INERMIS

ST. -HIL.

GRAY

(MUINZ \& I. JOHNST.) KEARN.

(GRAY) FERNALO *

MALVASTRUP ANGUSTUM

MALVASTRUP: HISPIDUM

(CAV.) G. ODN

GRAY

(WOOTON) KEARN

(NUTT.) RYDB.

(NUTT.) GARREIT

(E.G. OAKER) KEARN.

(GREENE) RYOB.

(WOOTON \& STANDL.) KEARN.

TORR.

SPHAERALCEA AMBIGUA

(ROSE) KEARN.

(KEARN.) KEARN.

KCOCKLL.) KEARN.

GRAY

(KEARN, ) KEARN.

(KEARN, ) KEARN.

(WOOTON STANOL.) KEARN.

(KEARN.) KEARN.

(HOOK. \& ARN.)RYOB.

(TORR. EX GRAY) KEARN.
JACQ.

SPHAER ALCEA EMORYI
Source Manuals Hs bi

Fam1ly Name

No.

BI GNONI A CEAE

282

ORCHIOACEAE

ARACEAE

CAMPANULACEAE

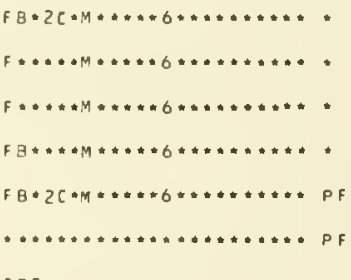

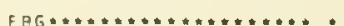

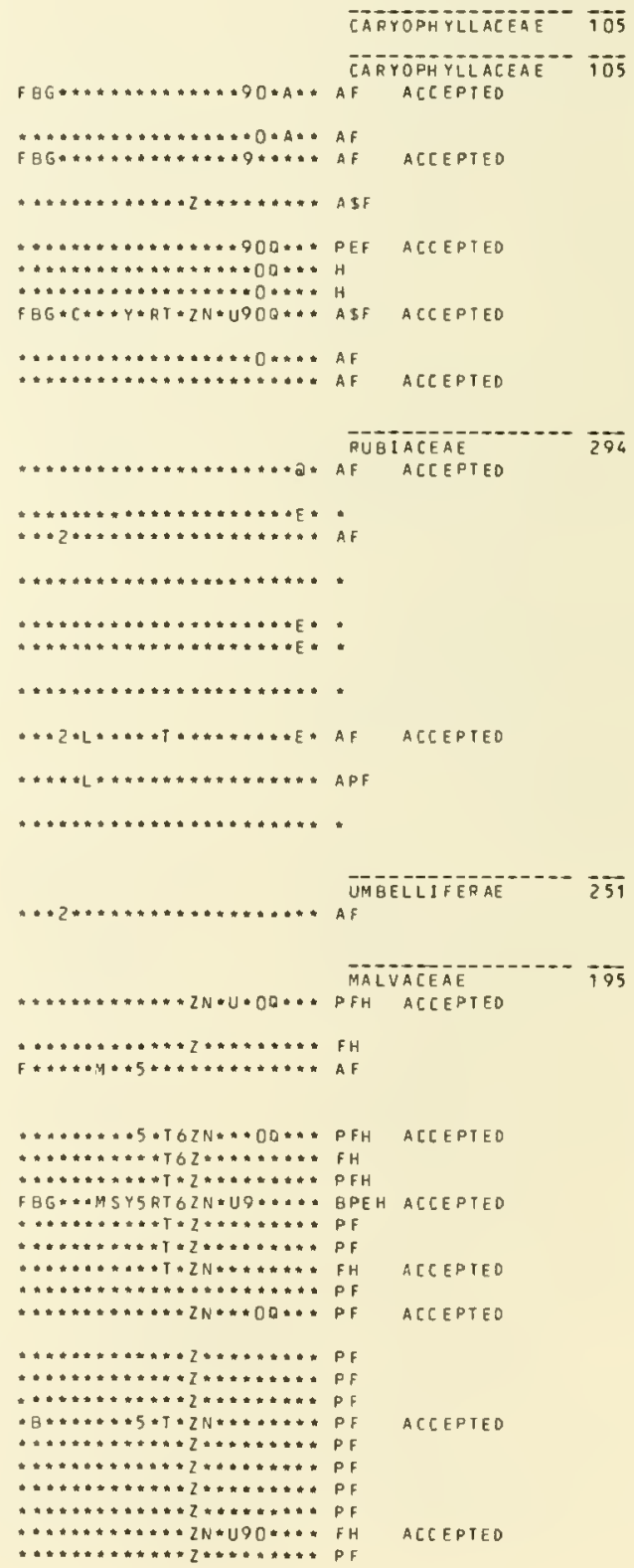




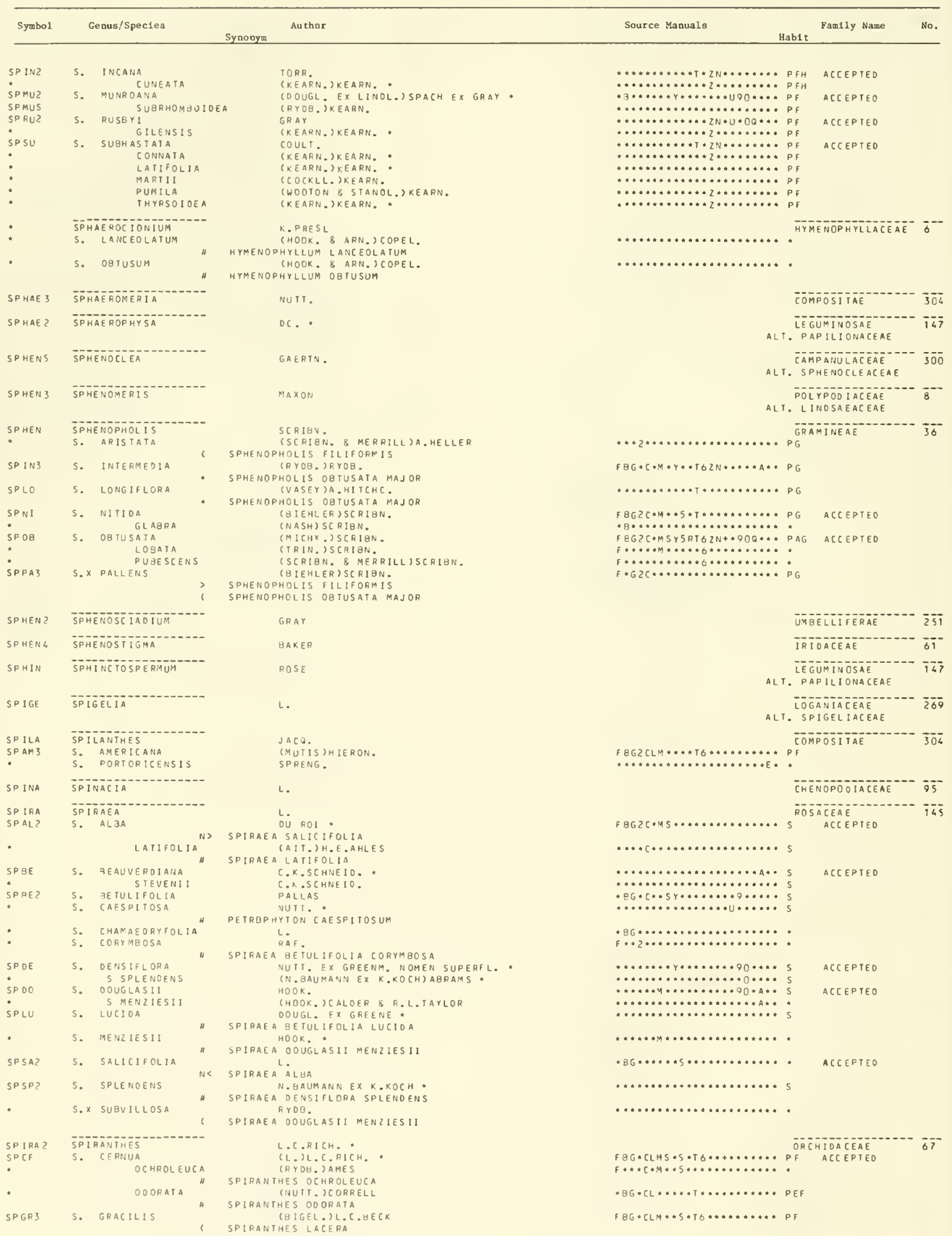




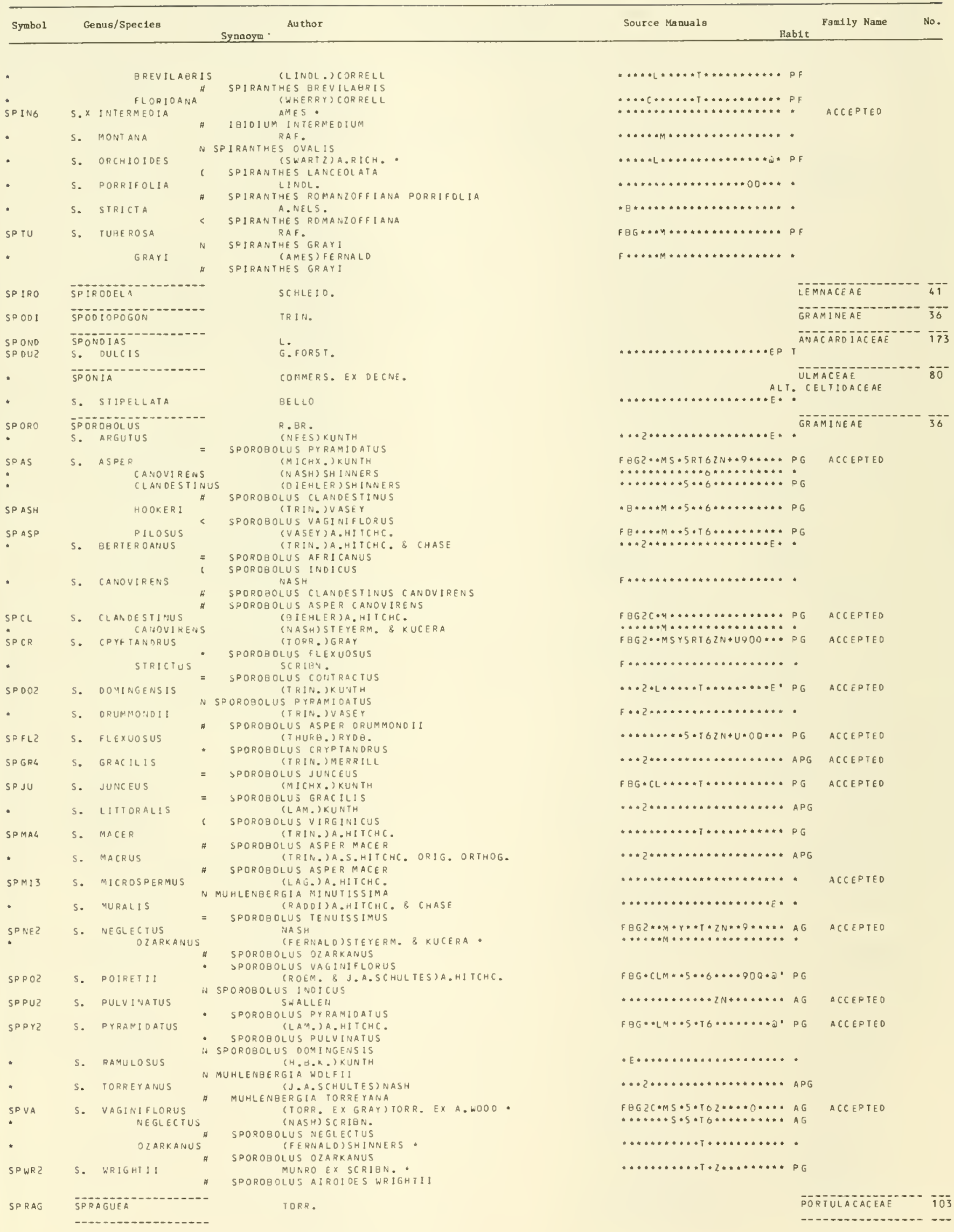




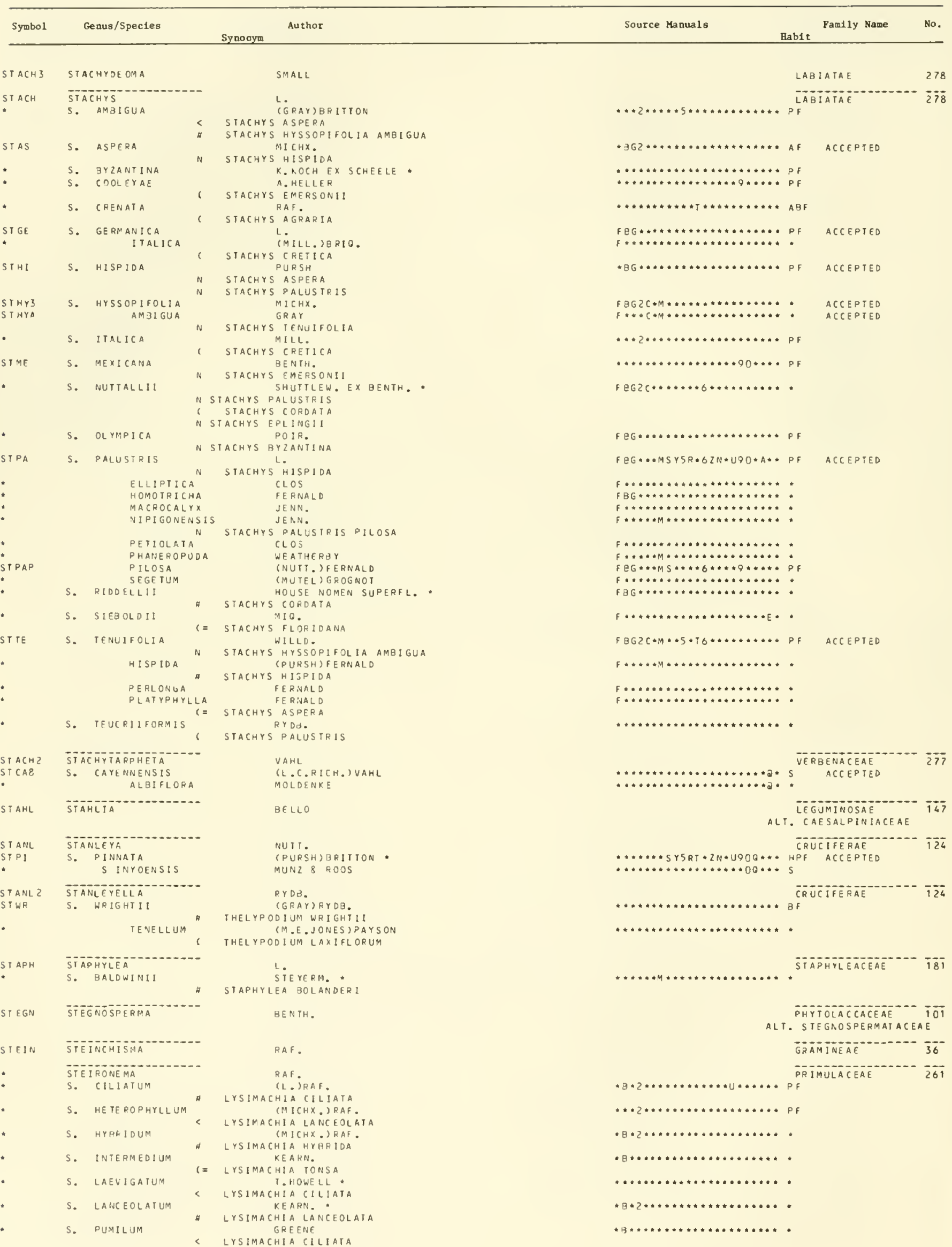




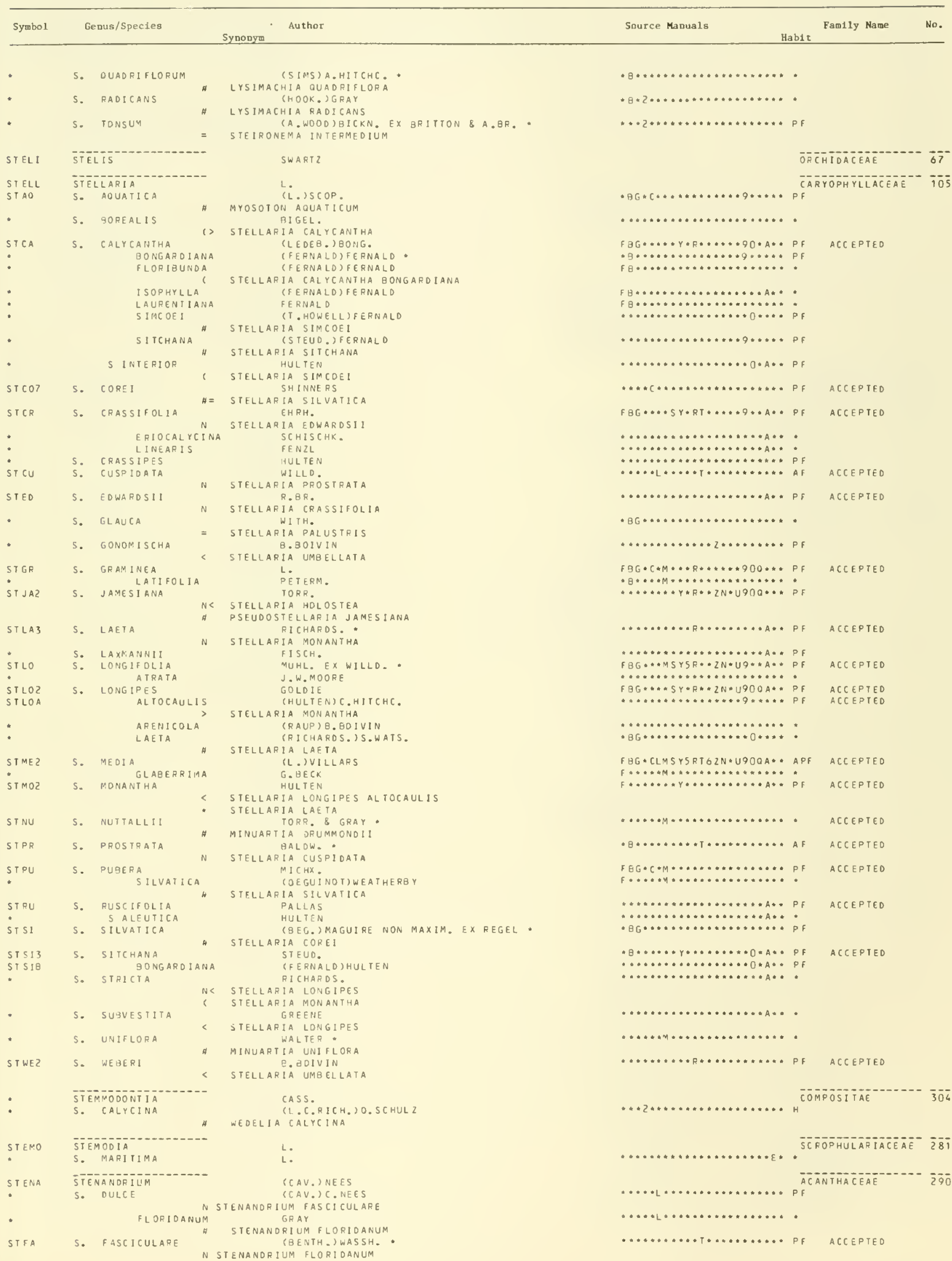




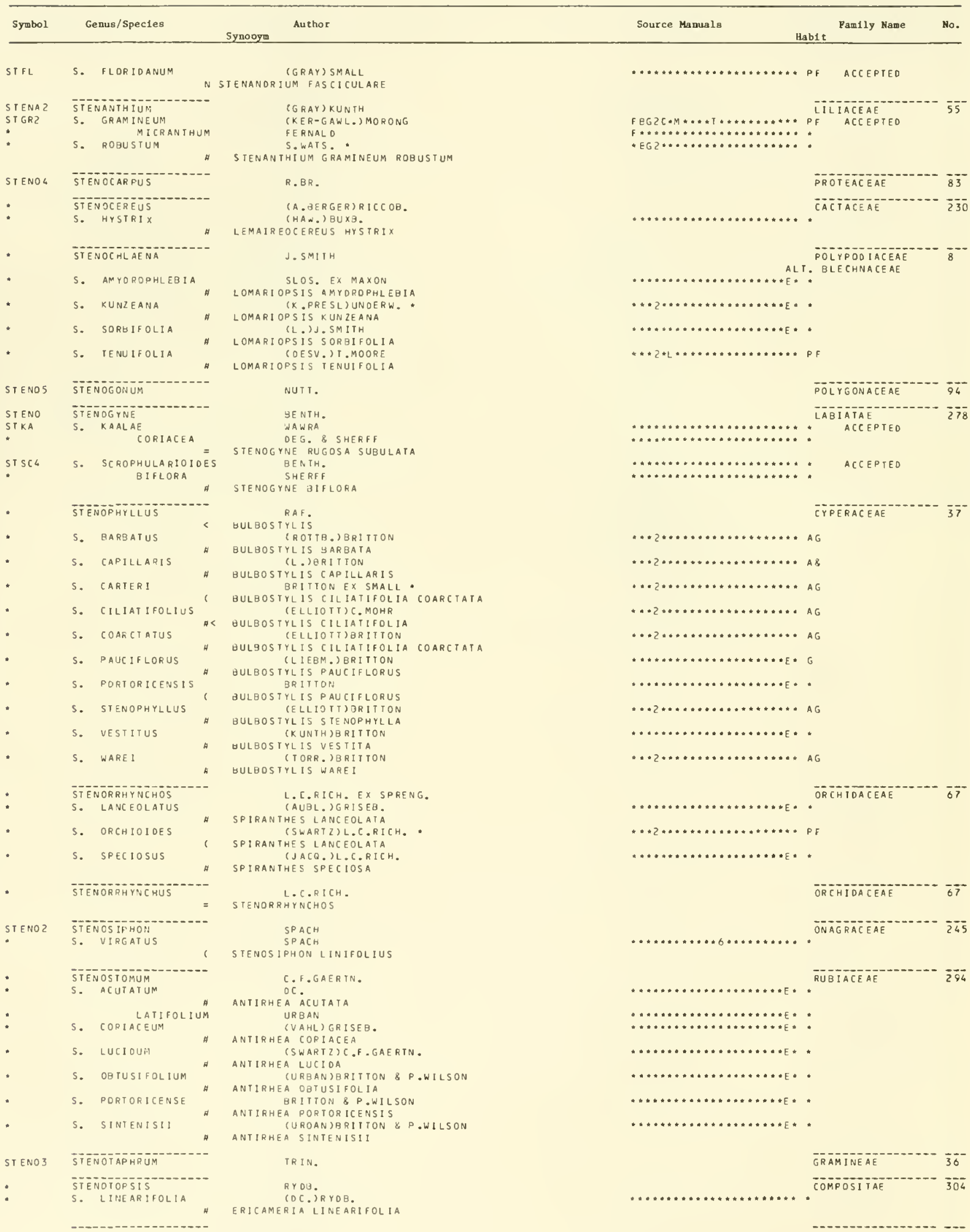




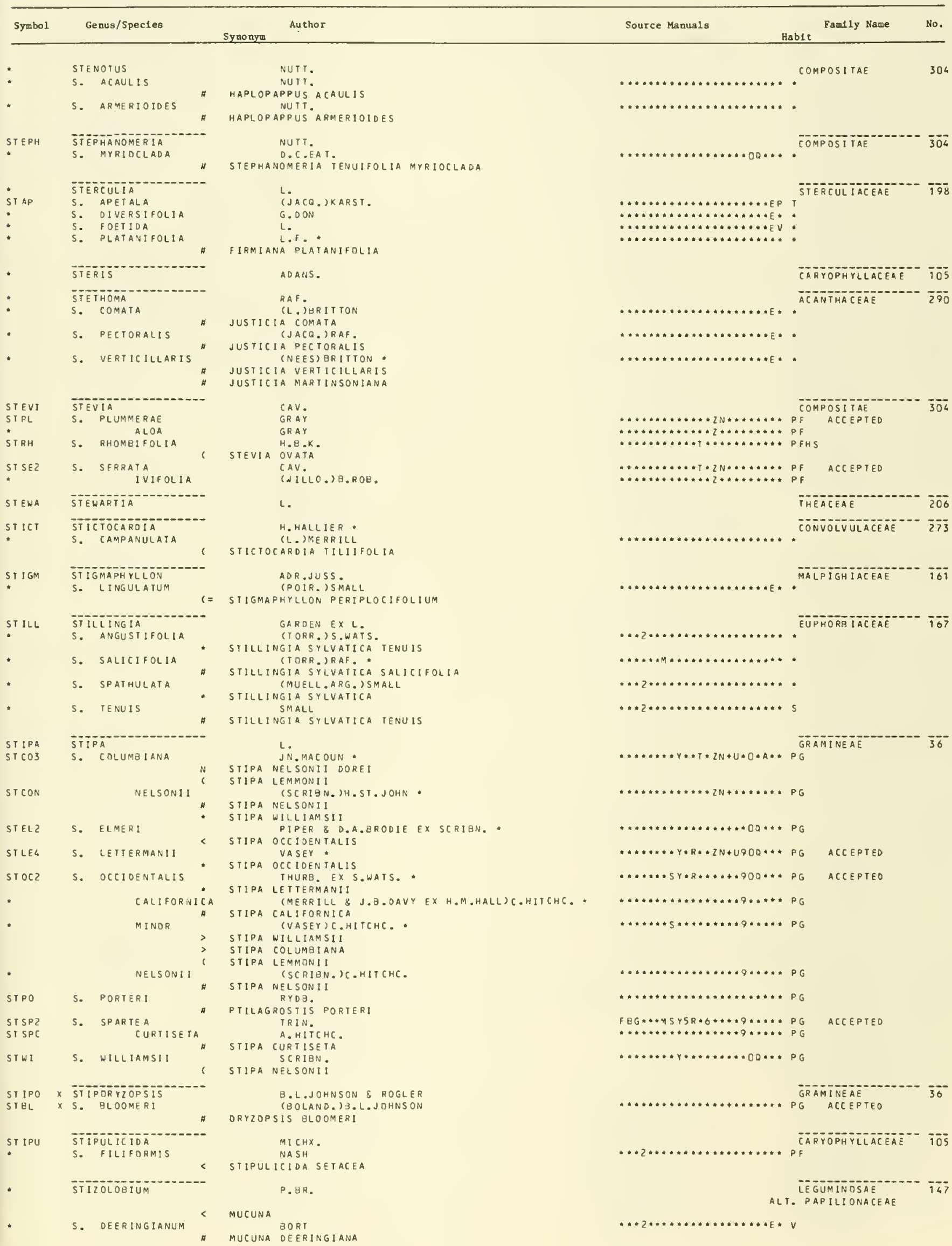


ST IZOLOBIUM

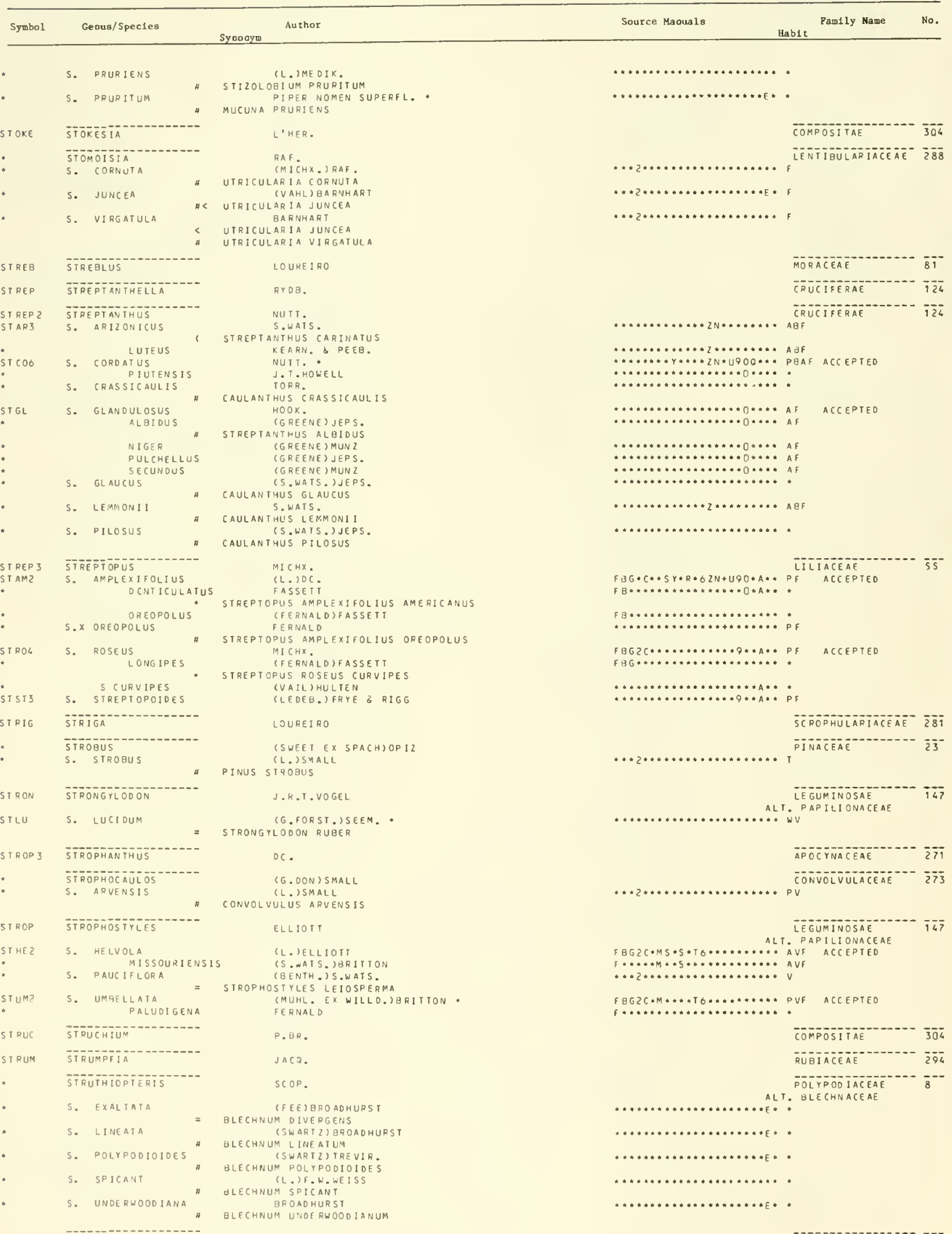




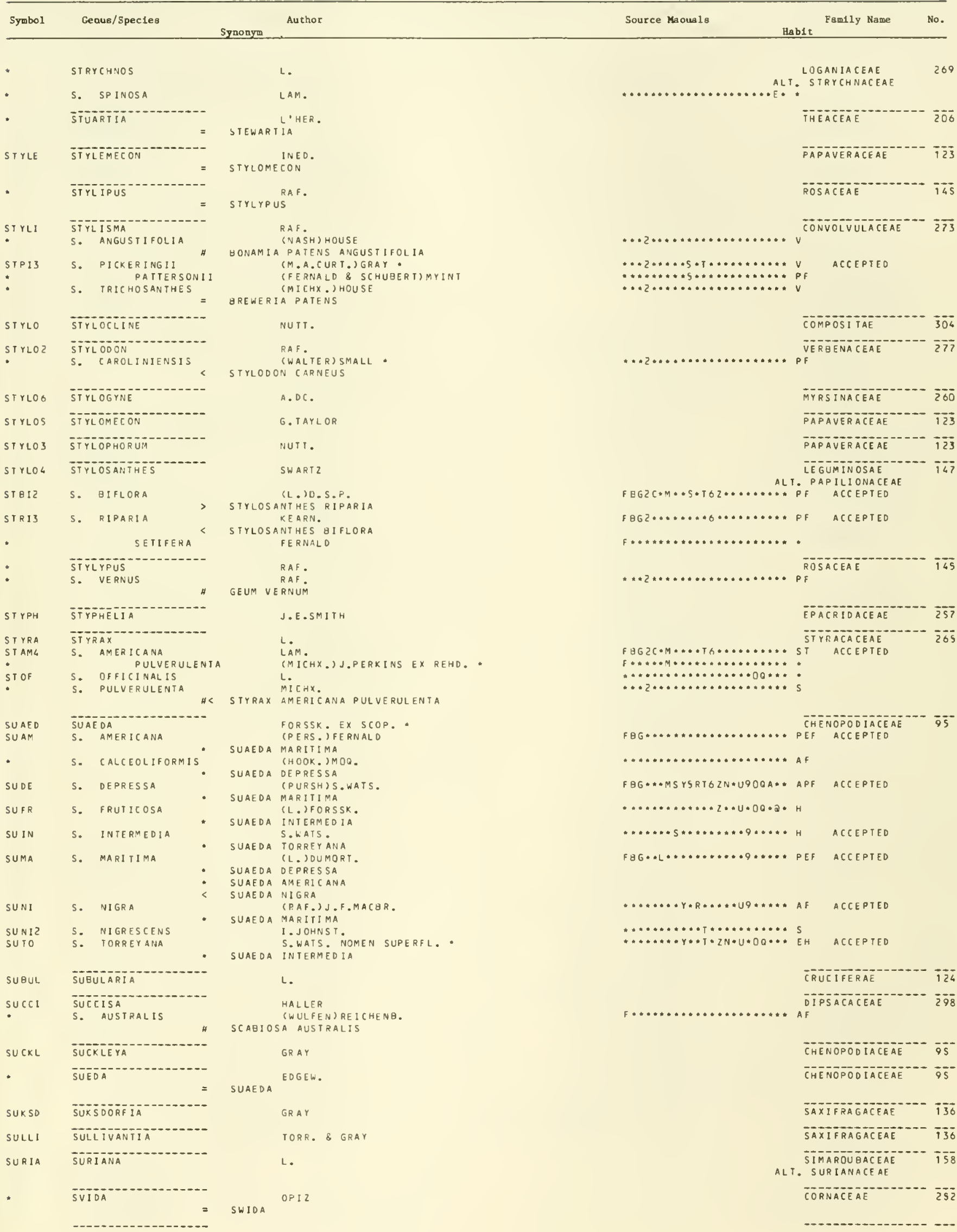




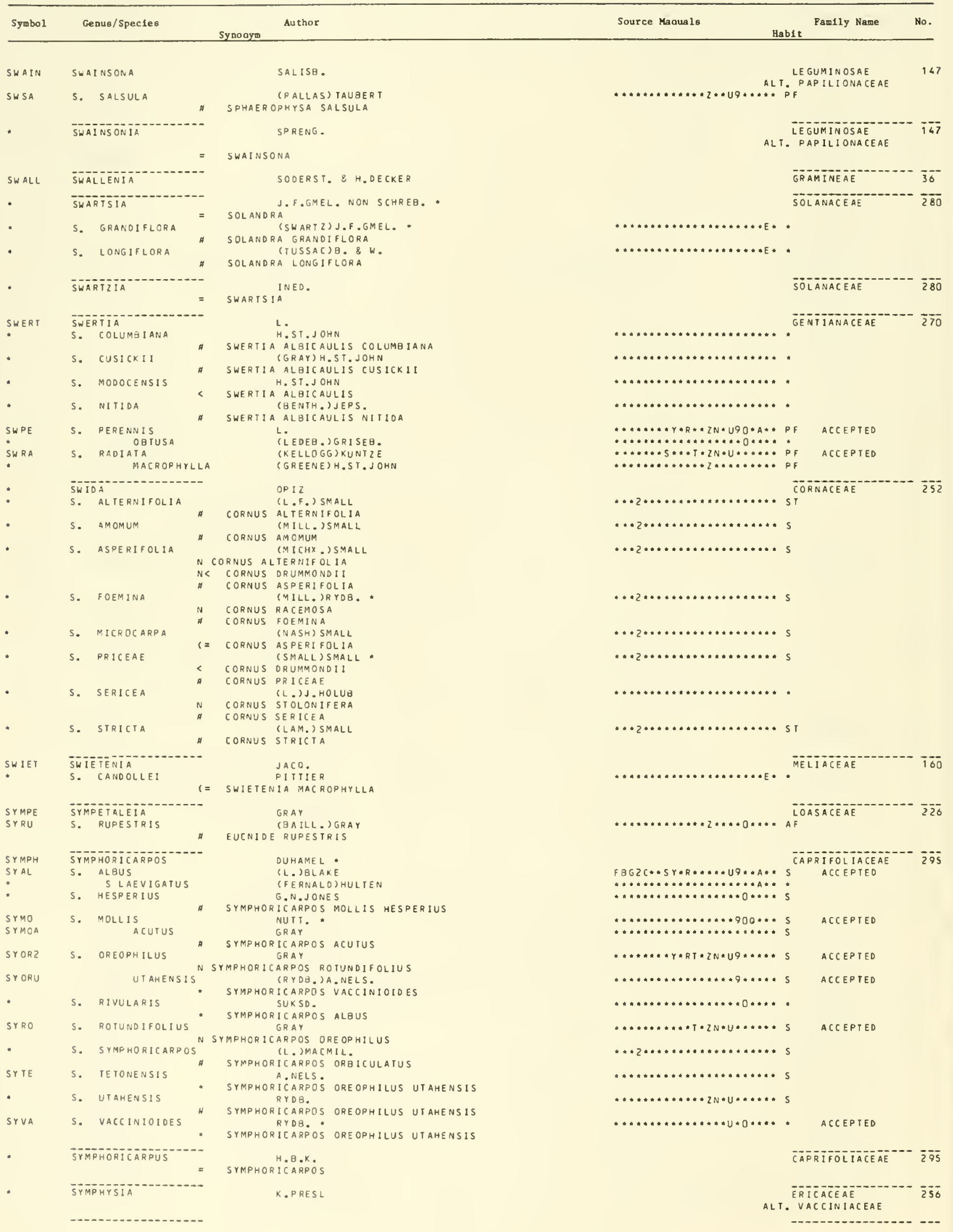




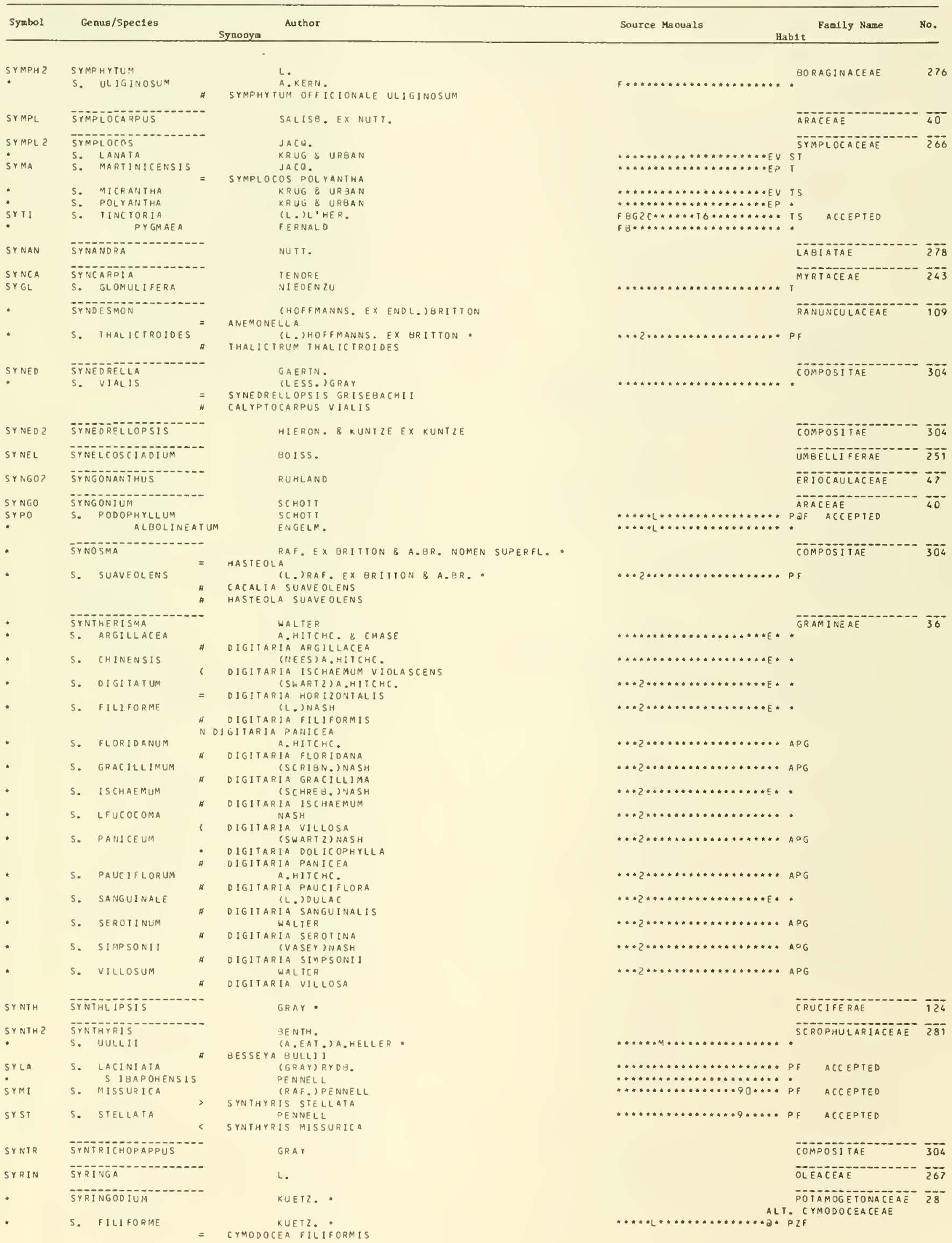




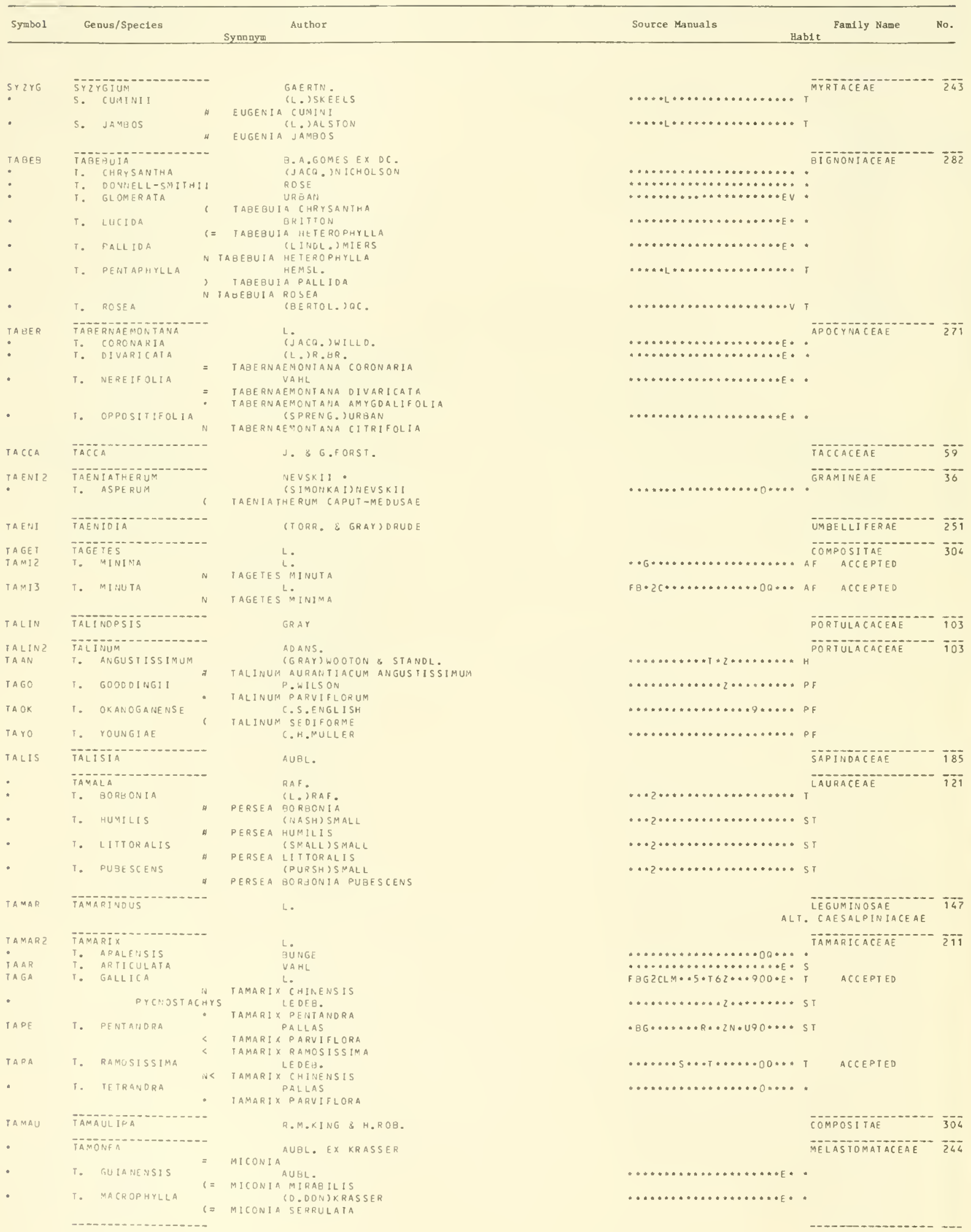




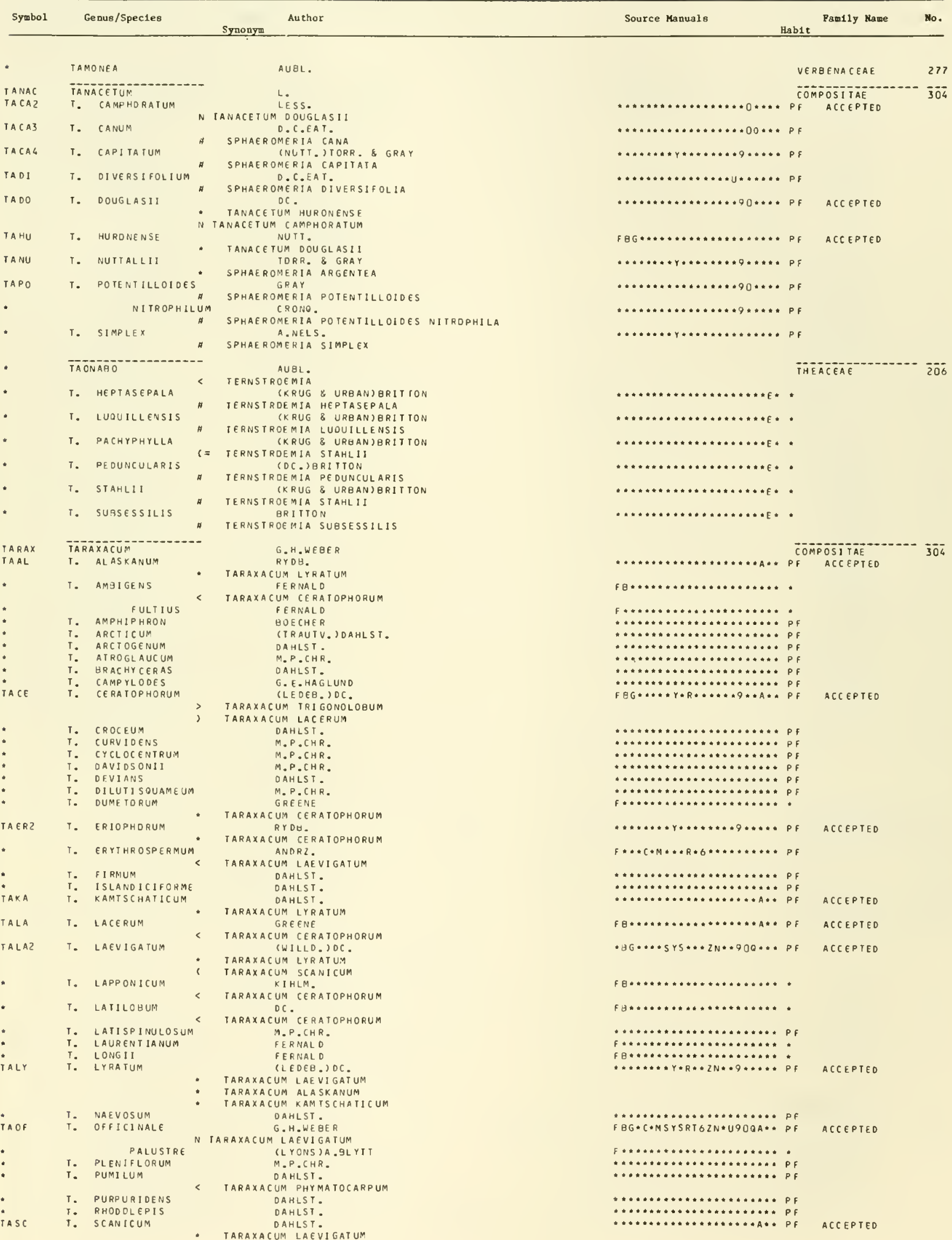




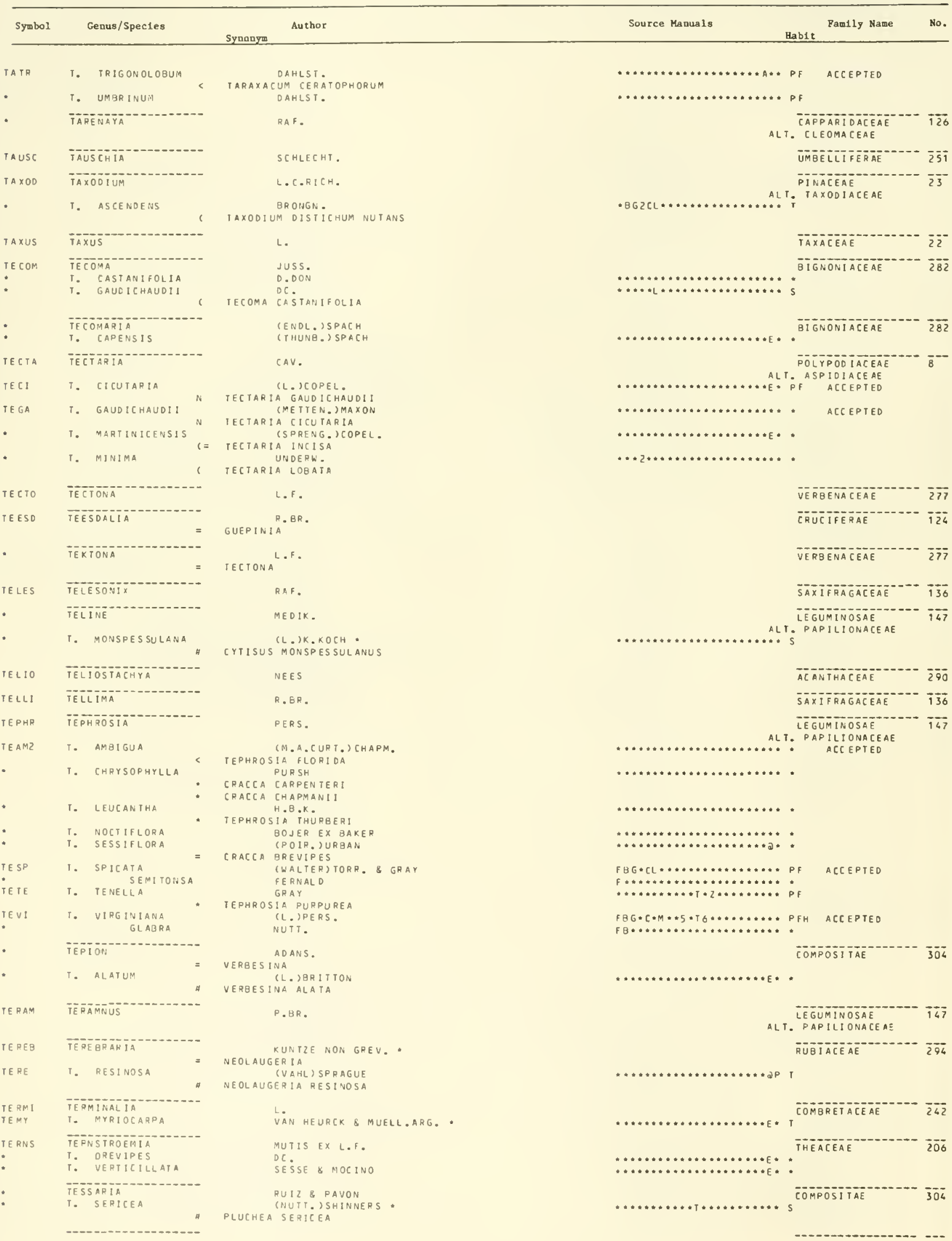




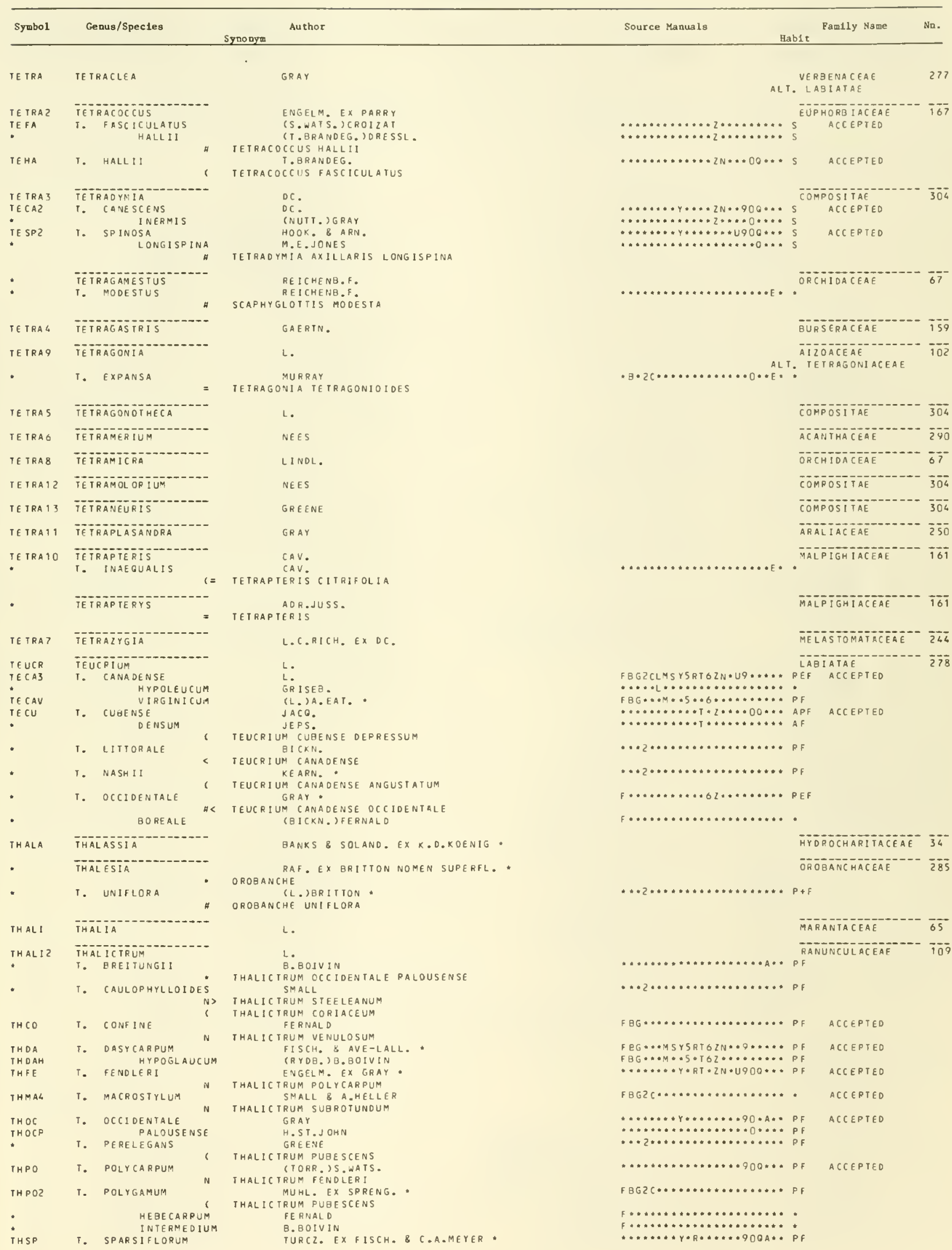




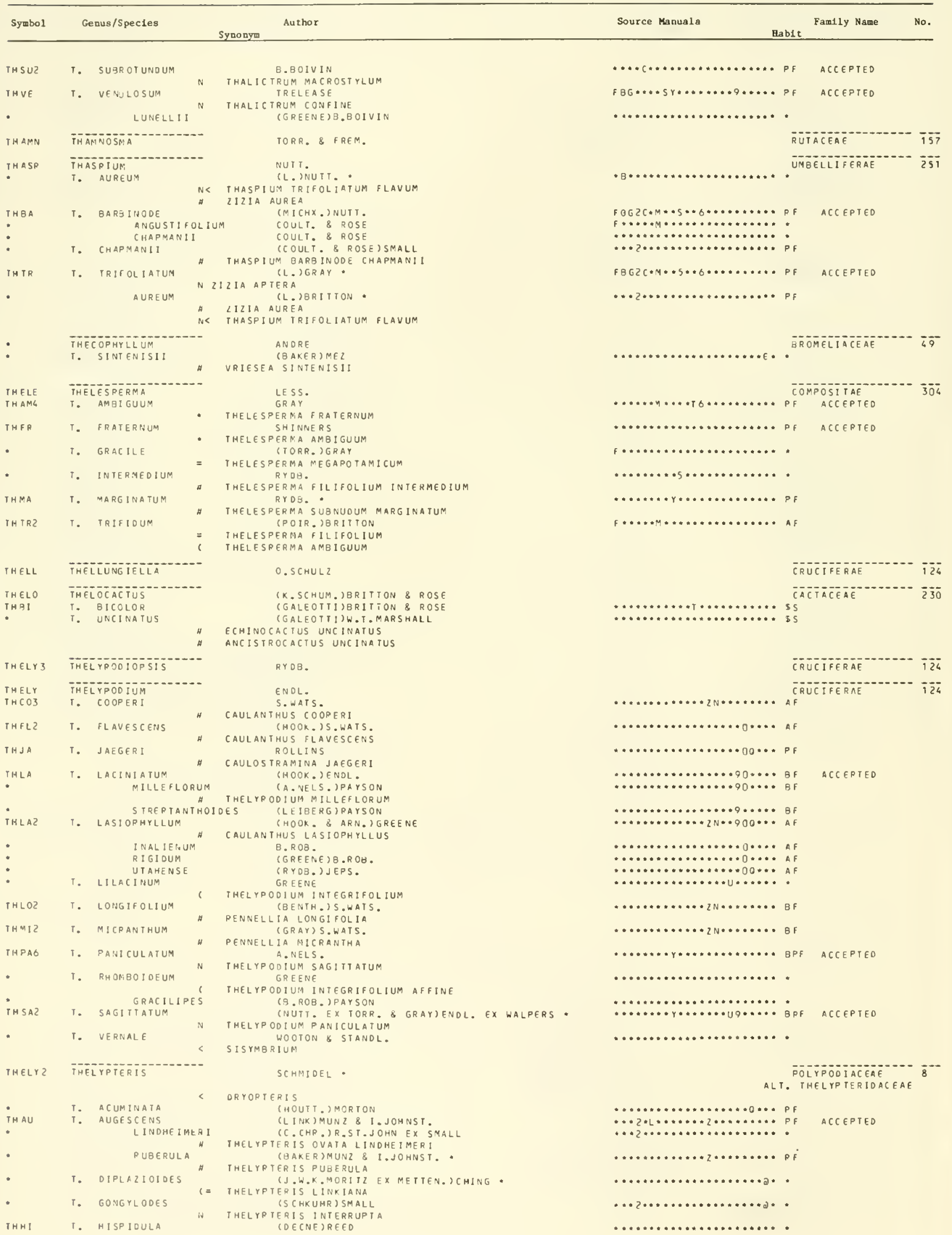




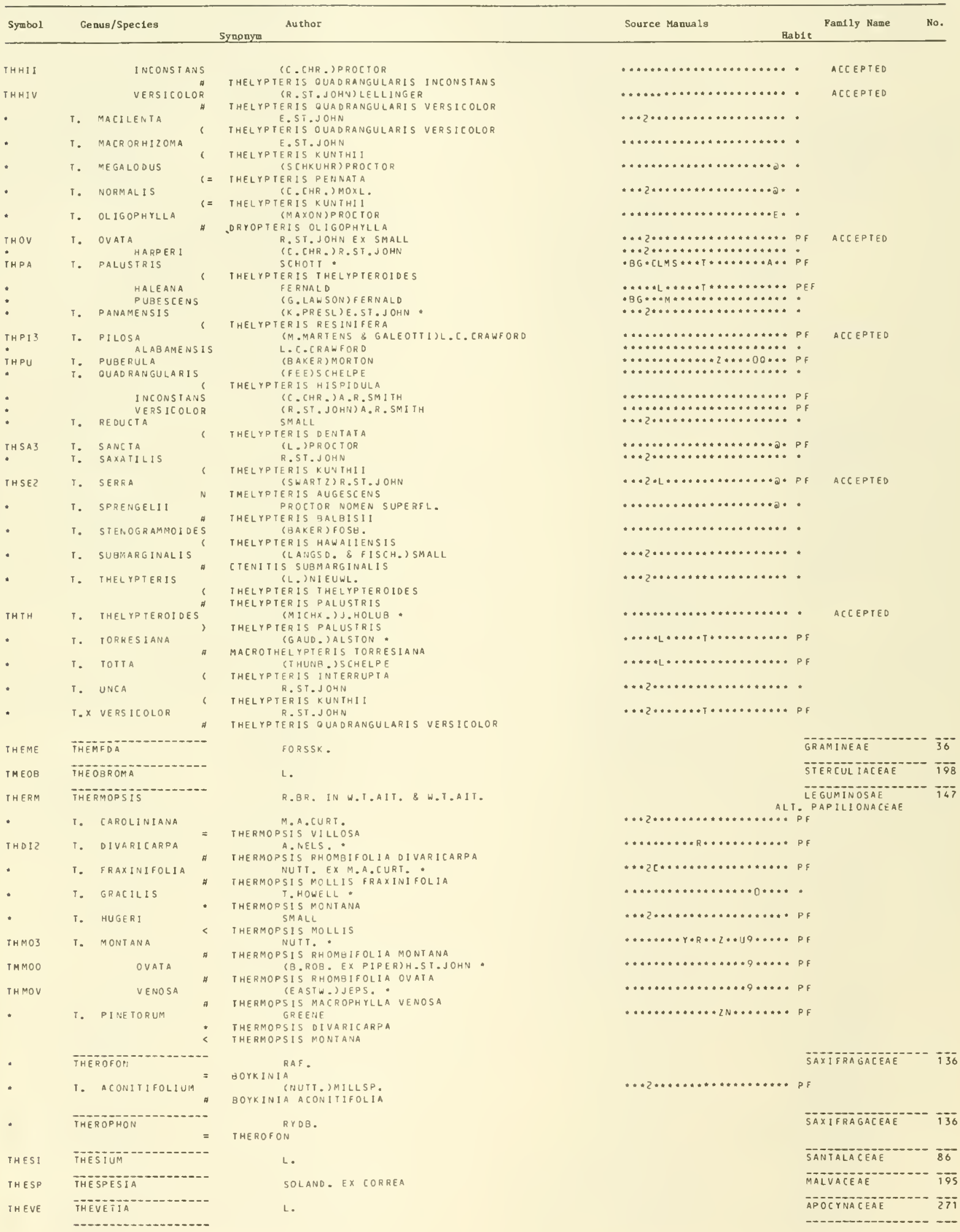


THIBAUDIA

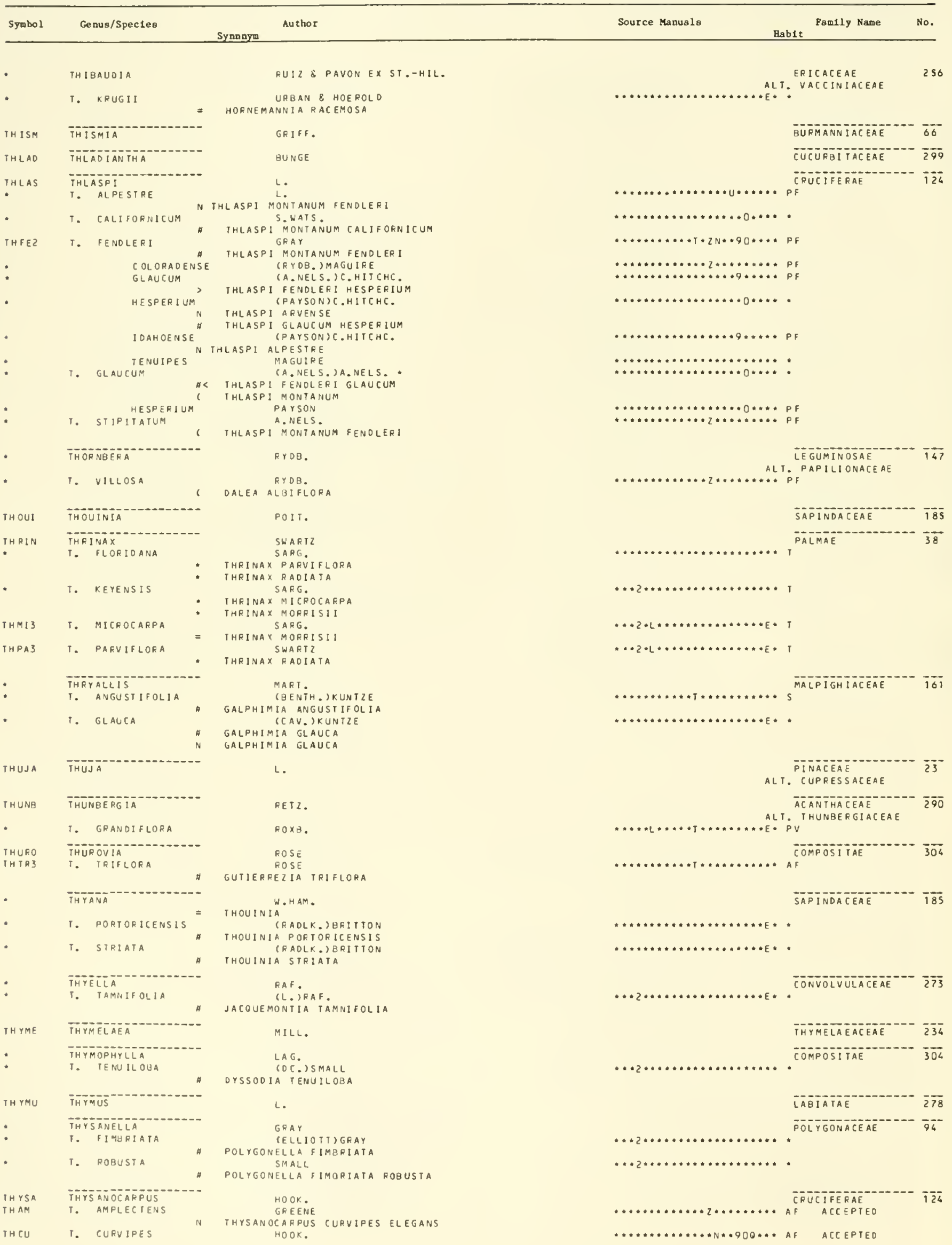




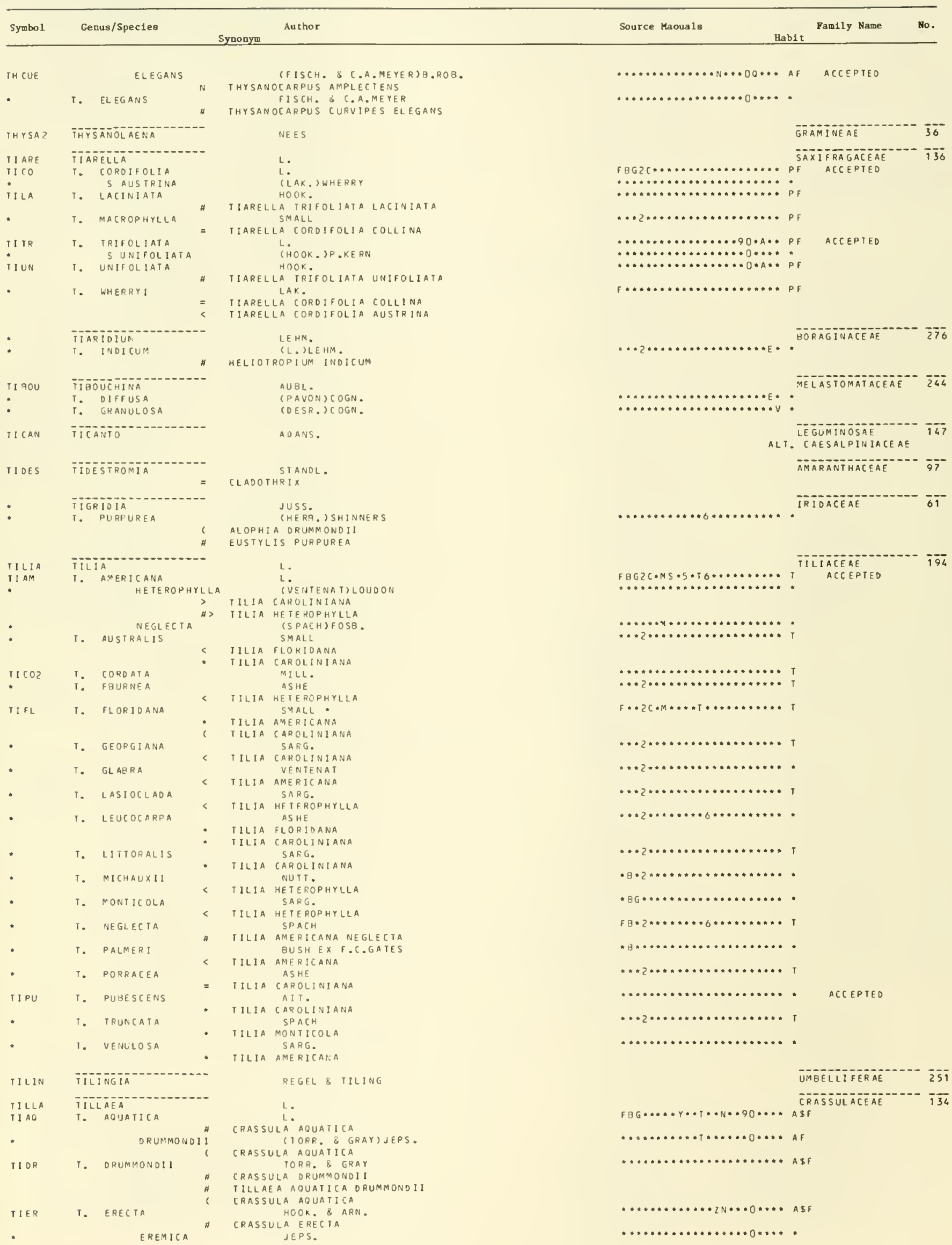




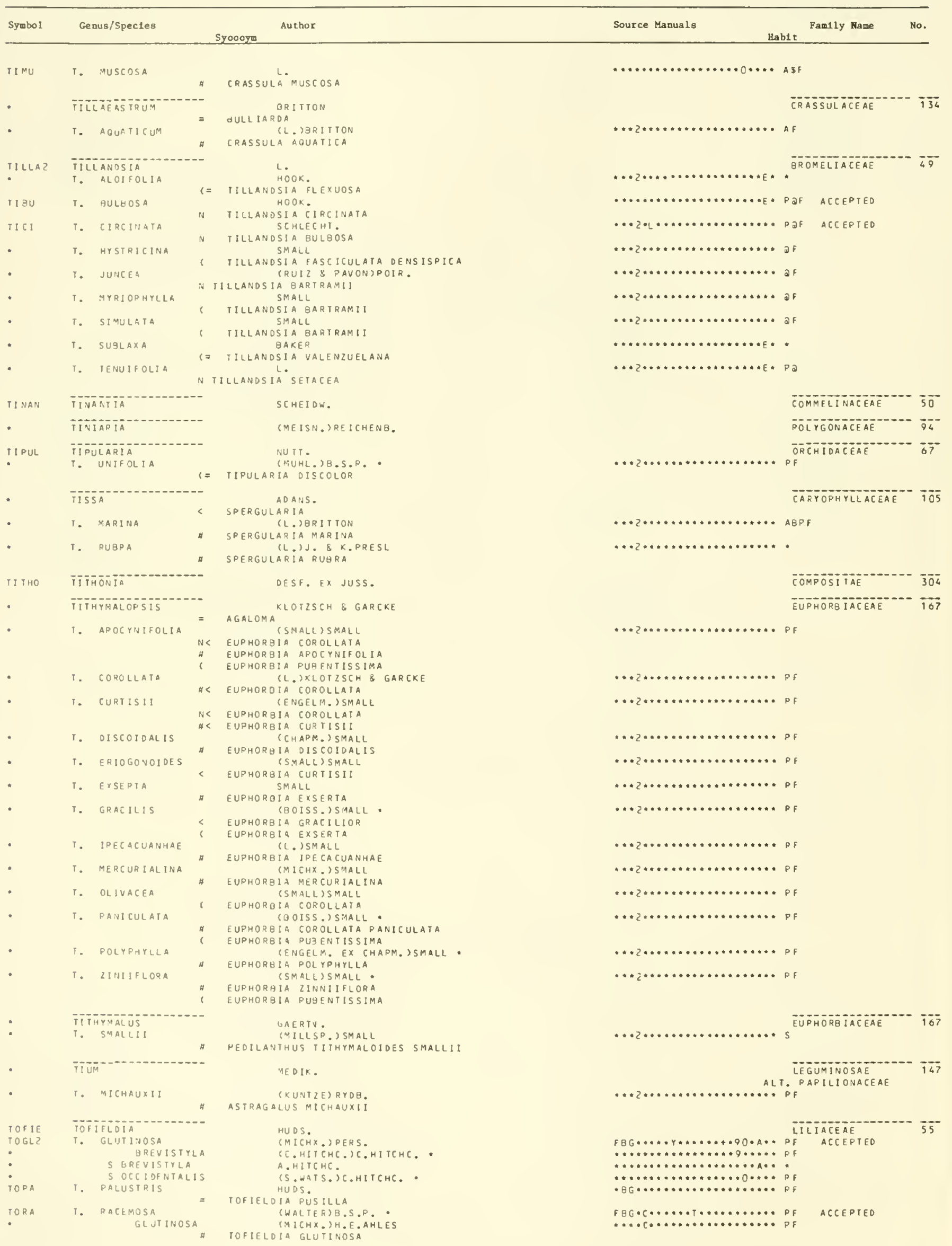




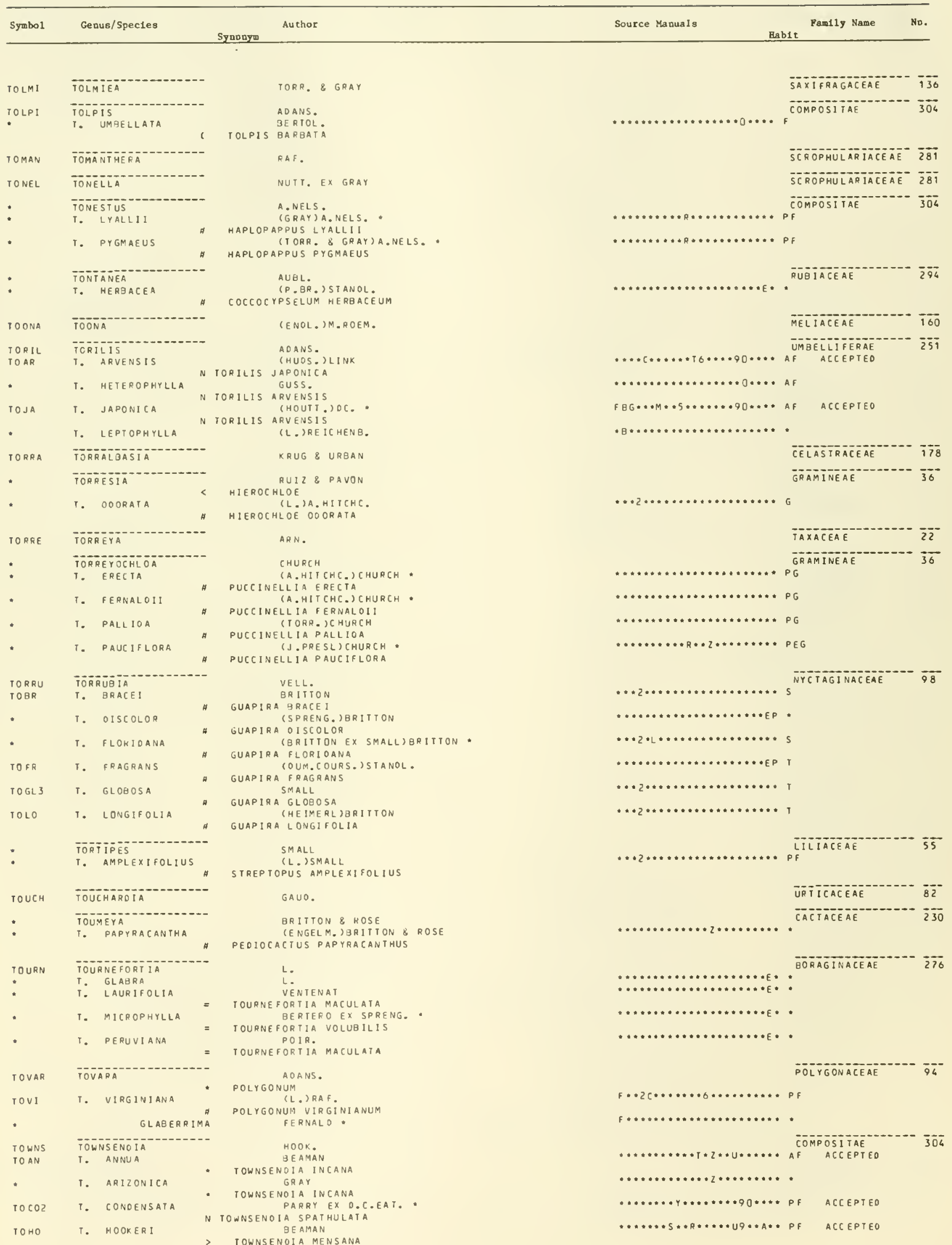


TOWNSEND:A

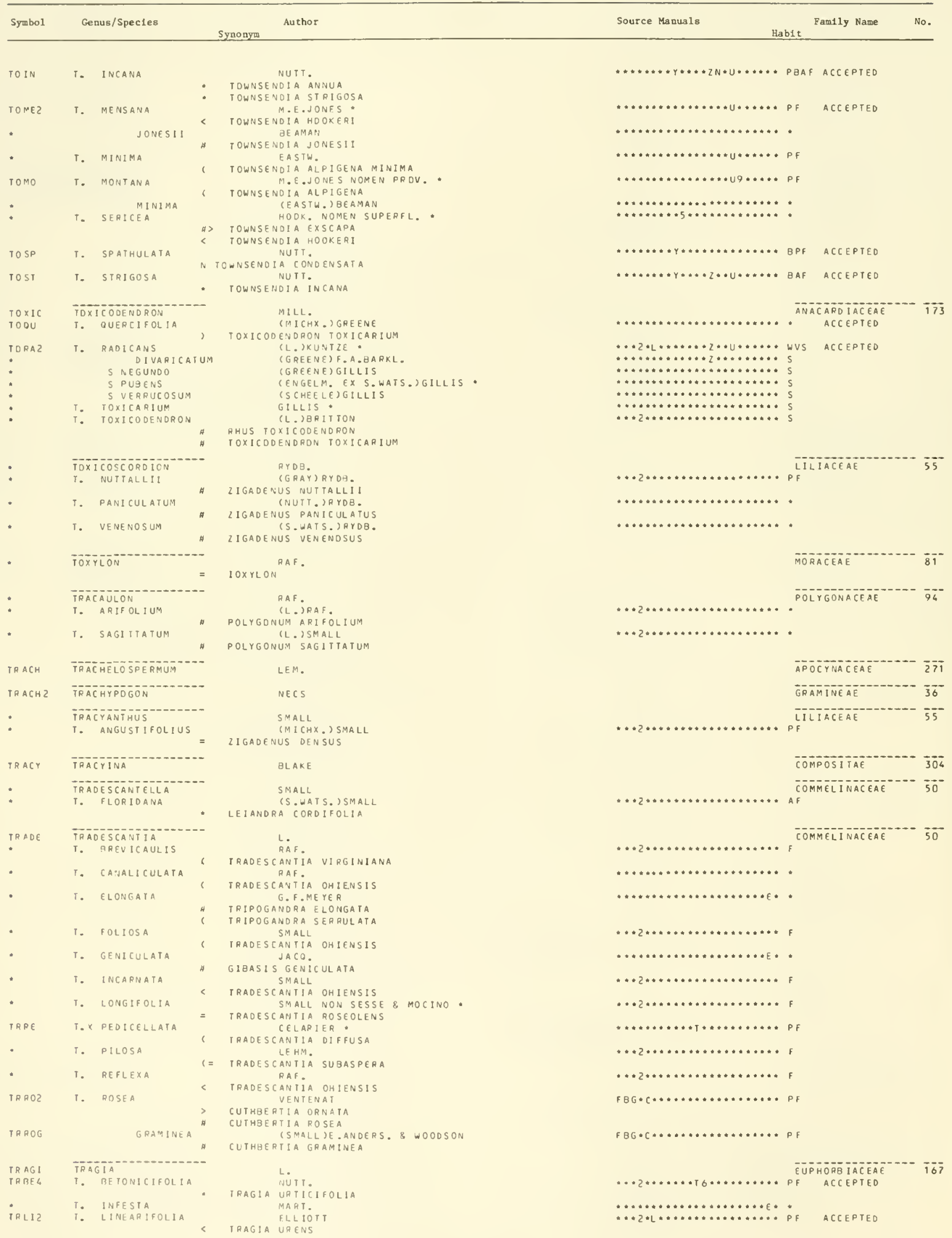




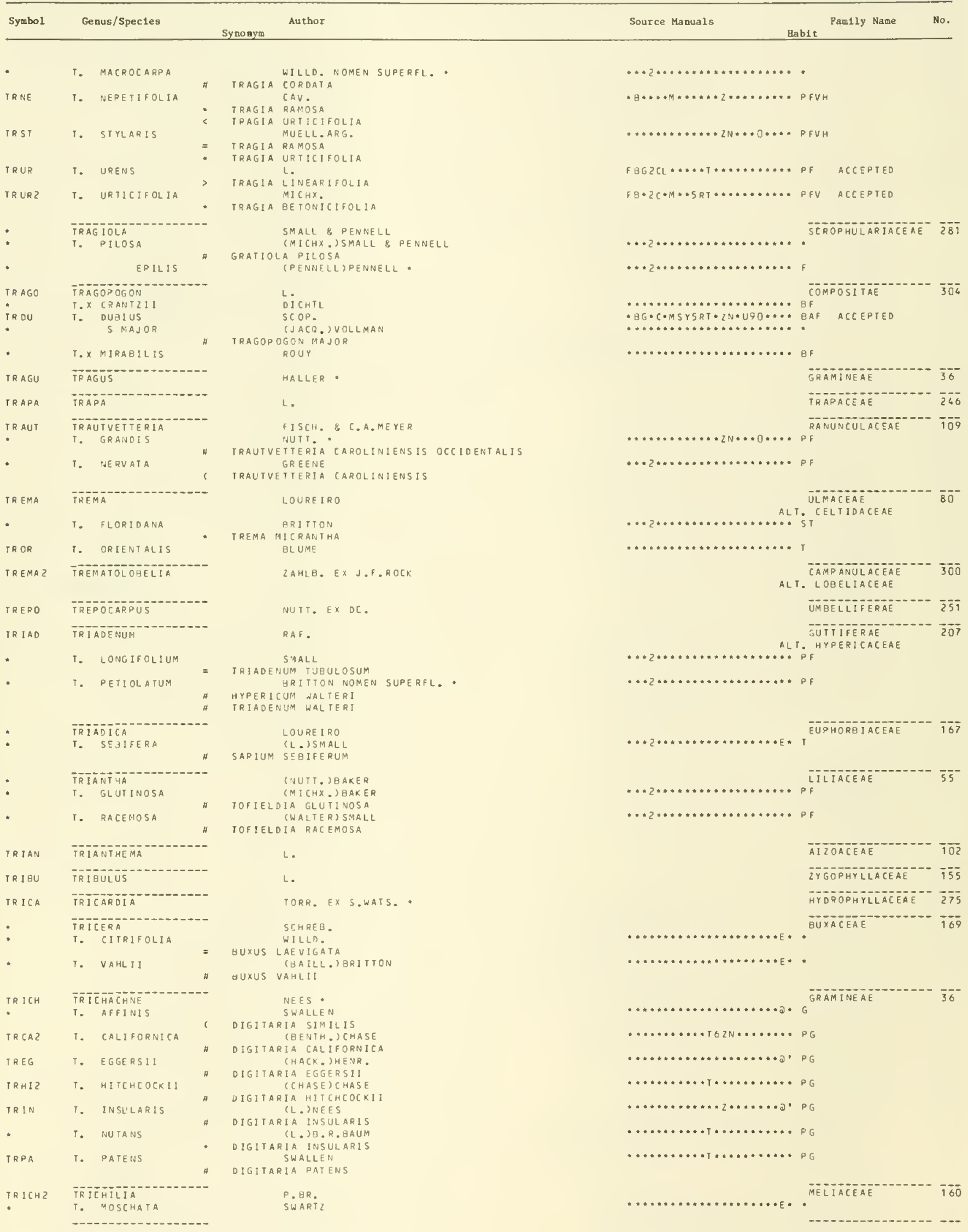




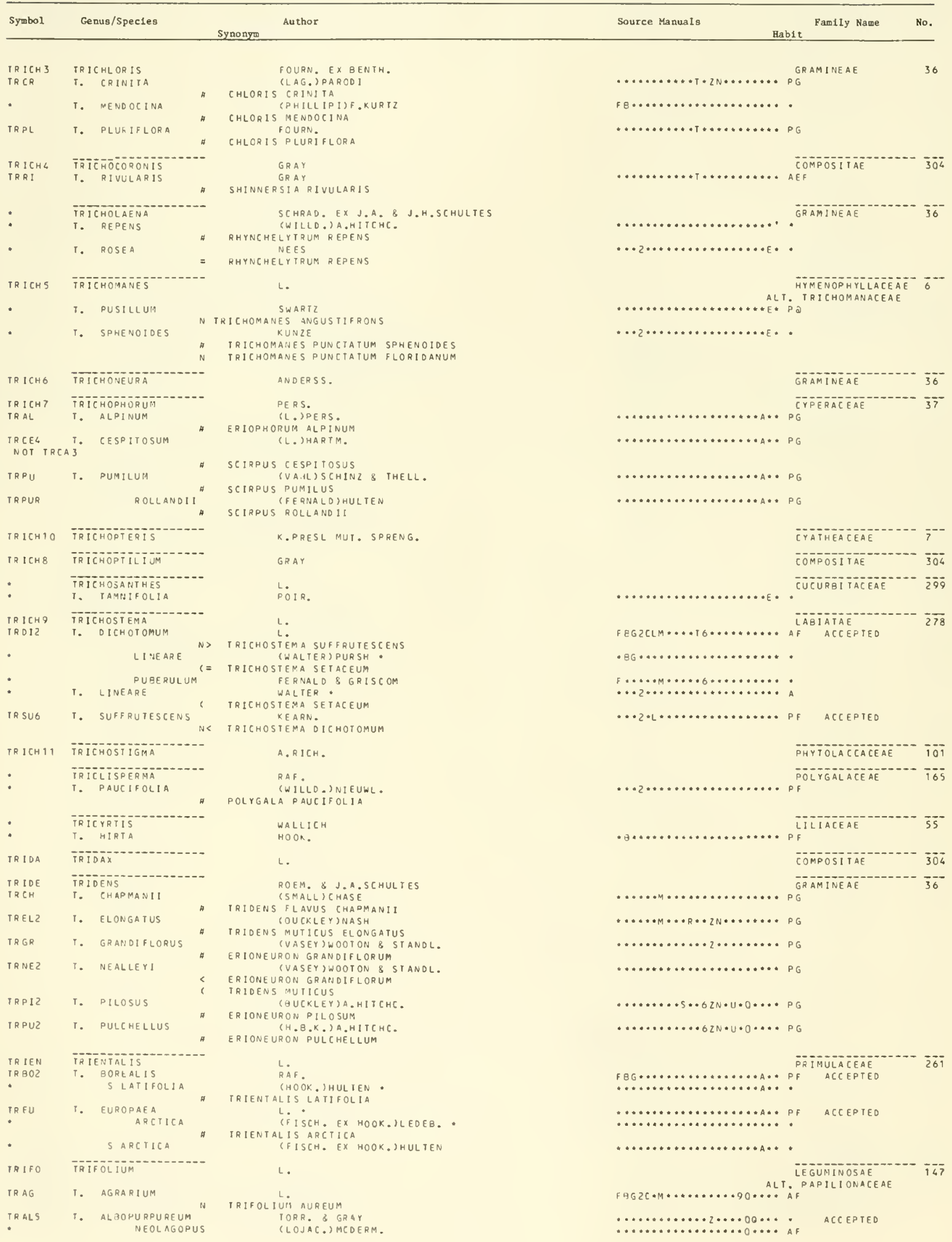




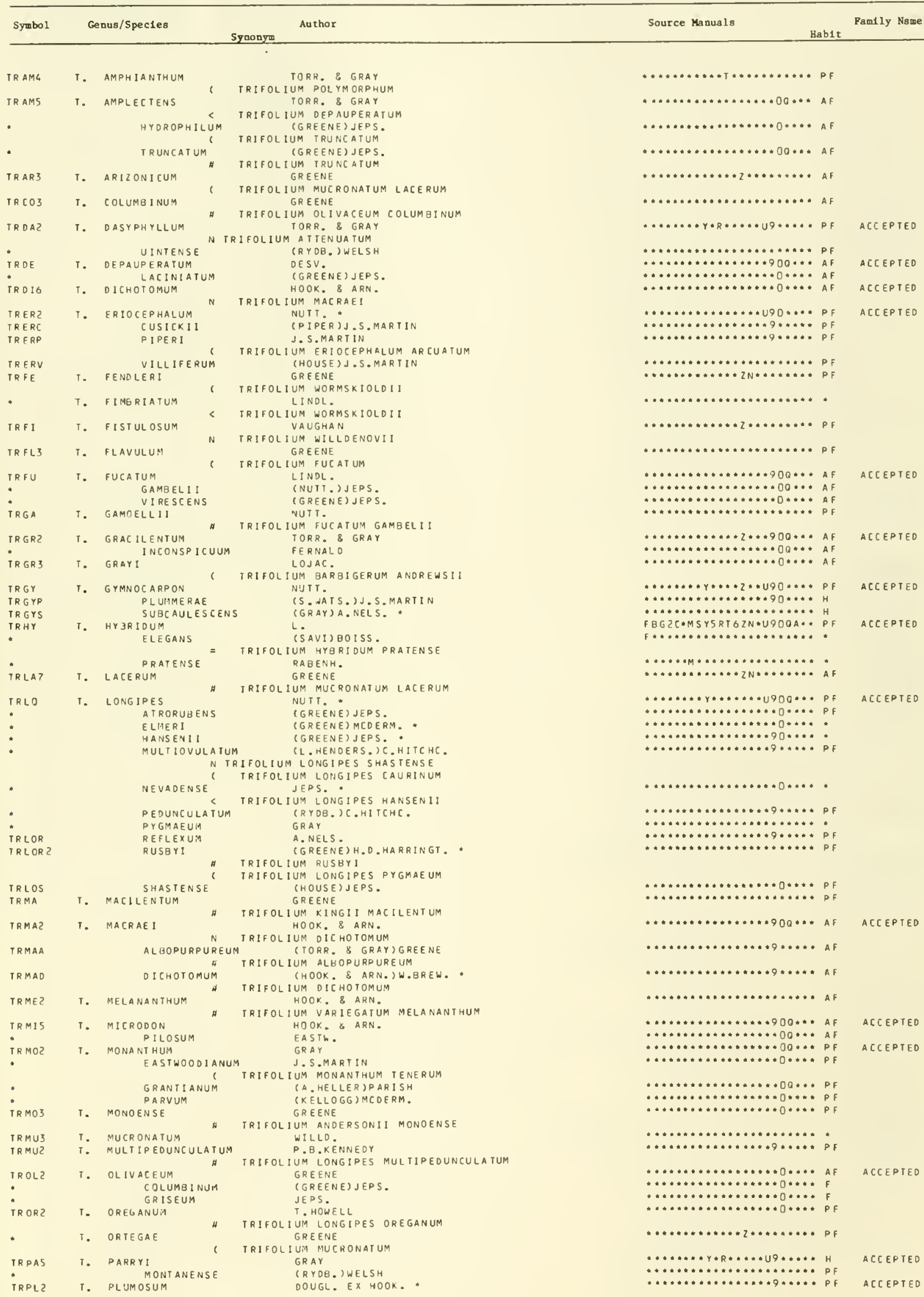




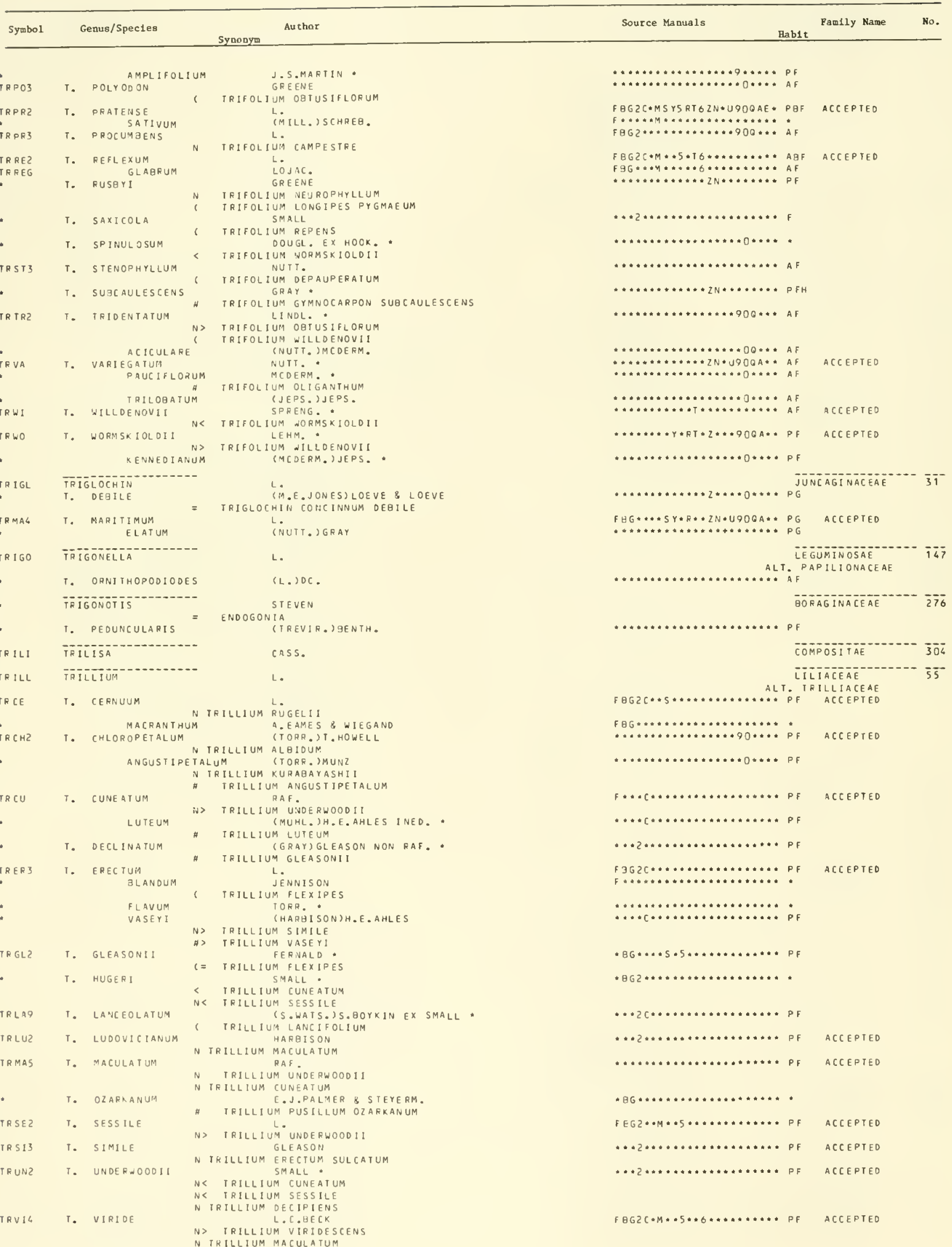


TR ILL I UM

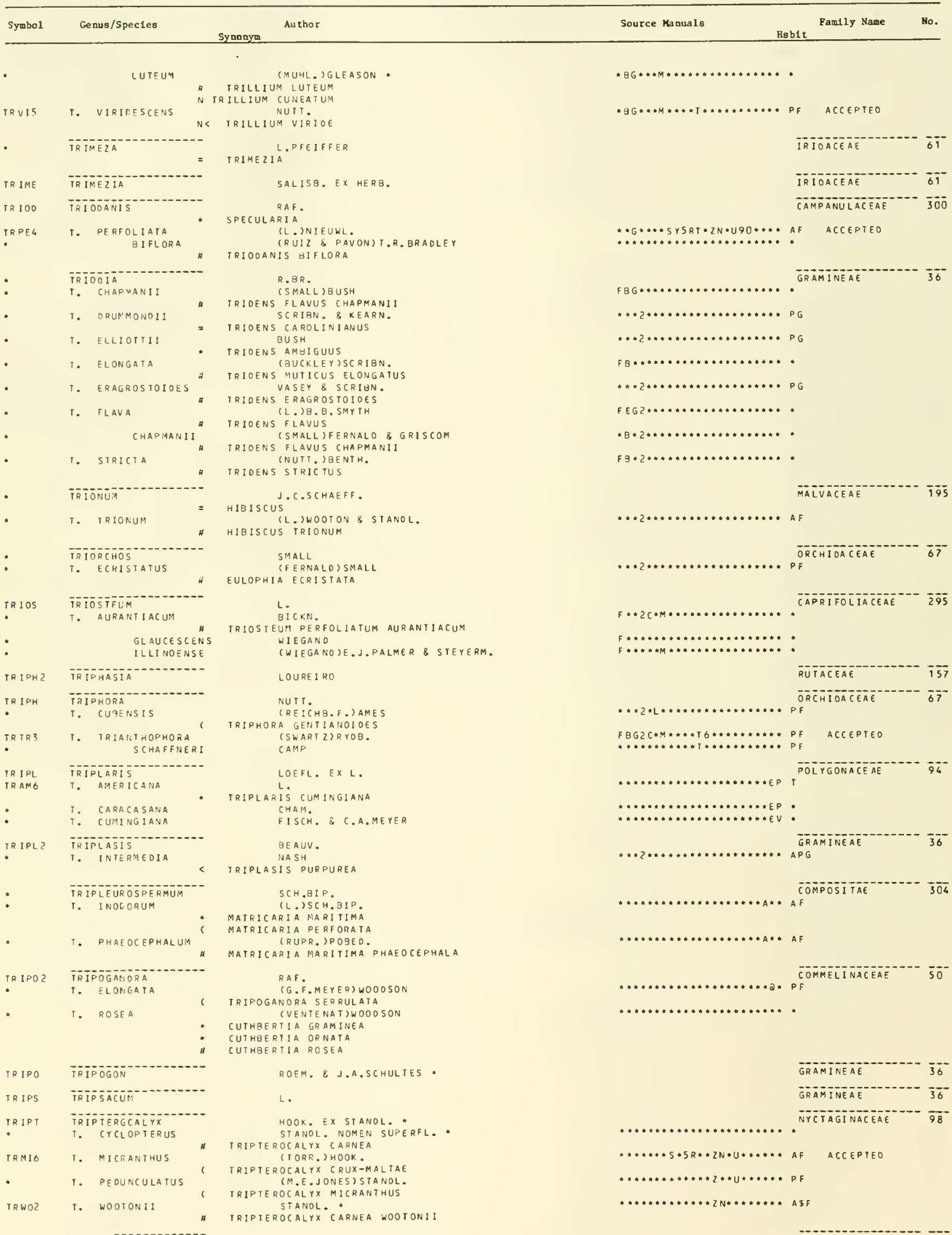




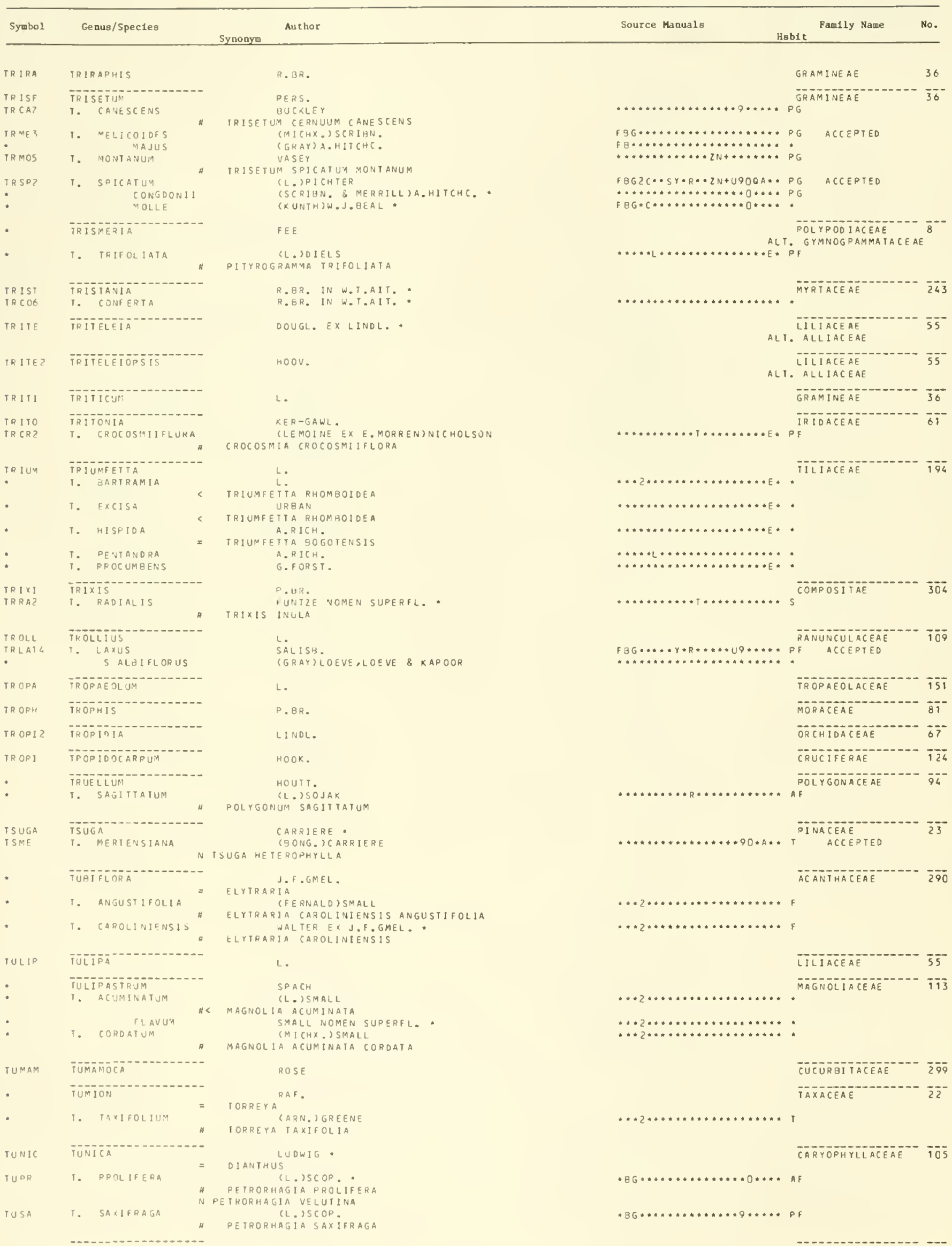




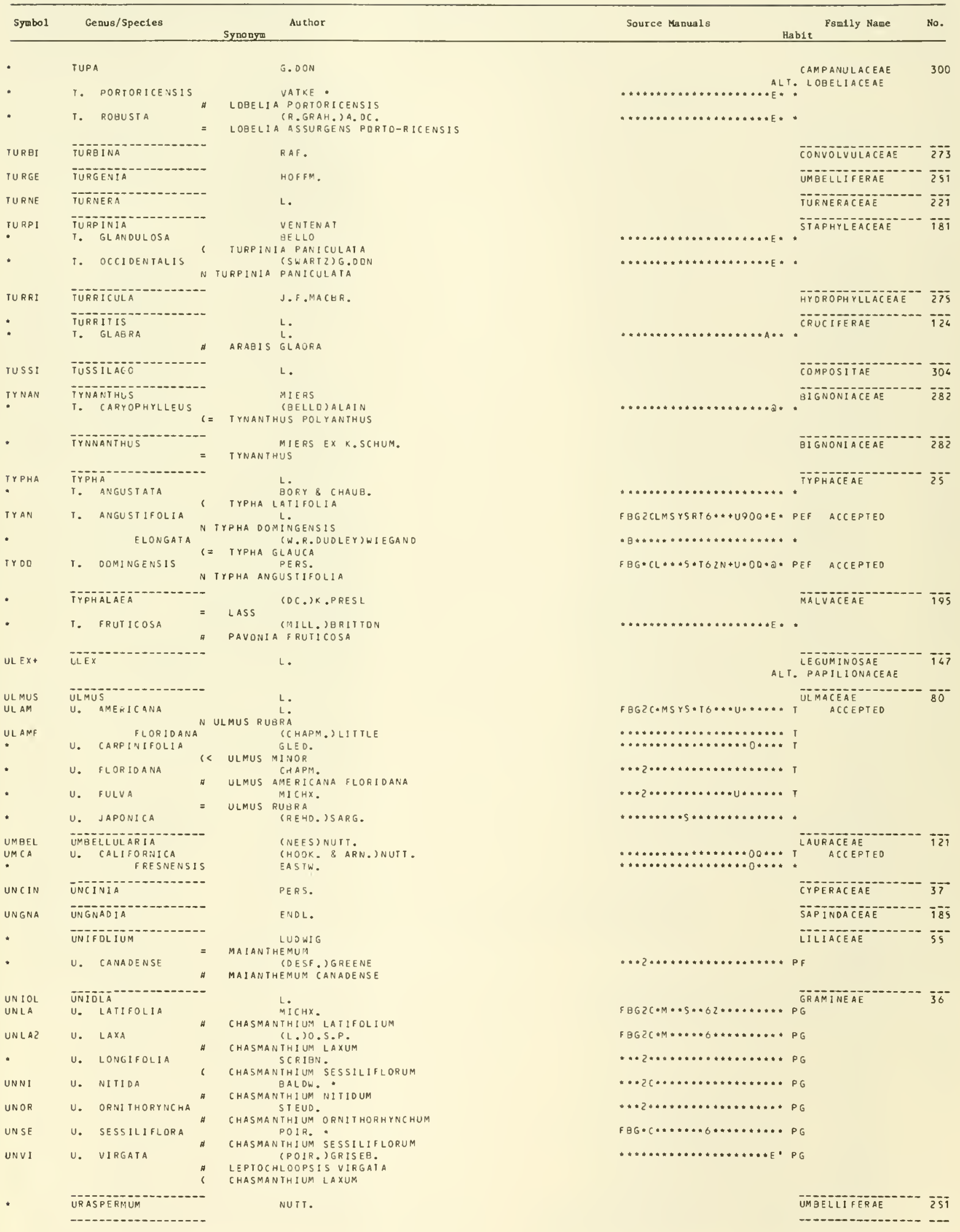




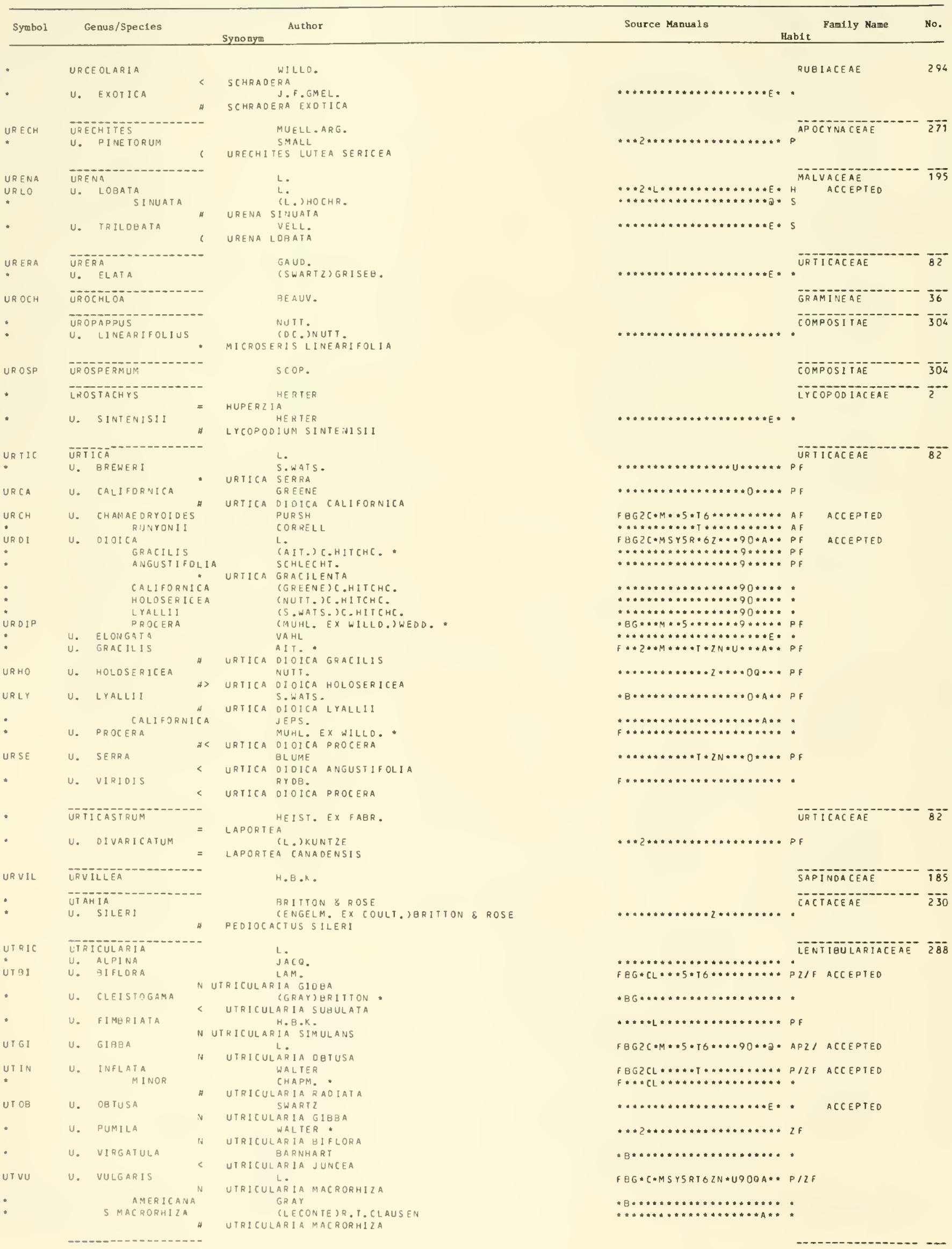




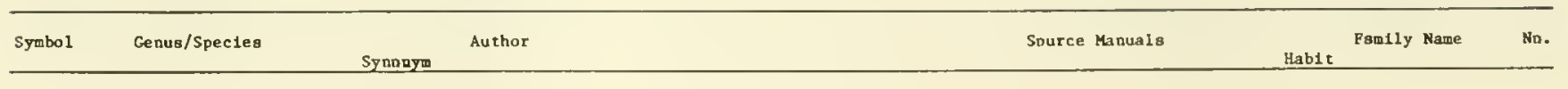

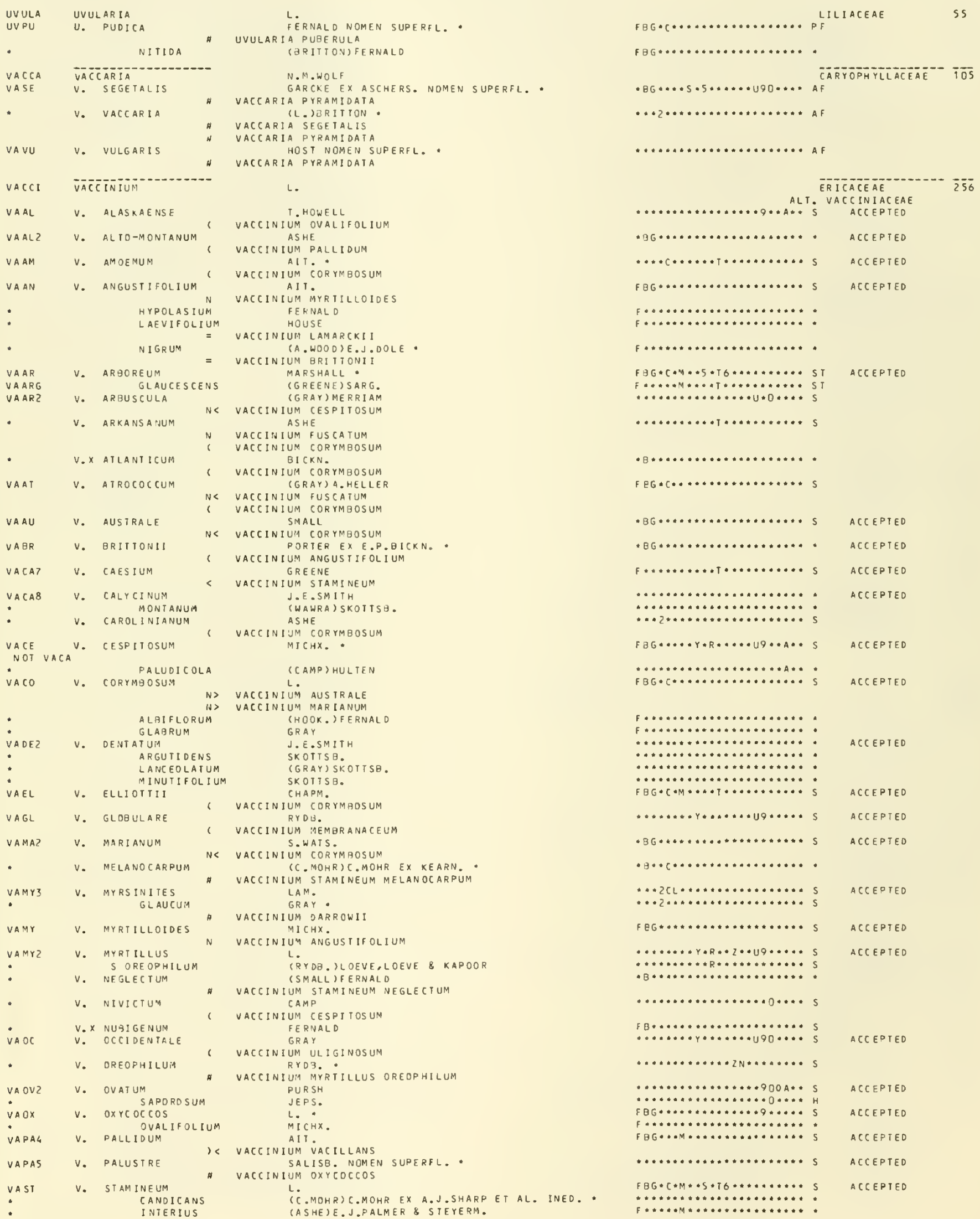




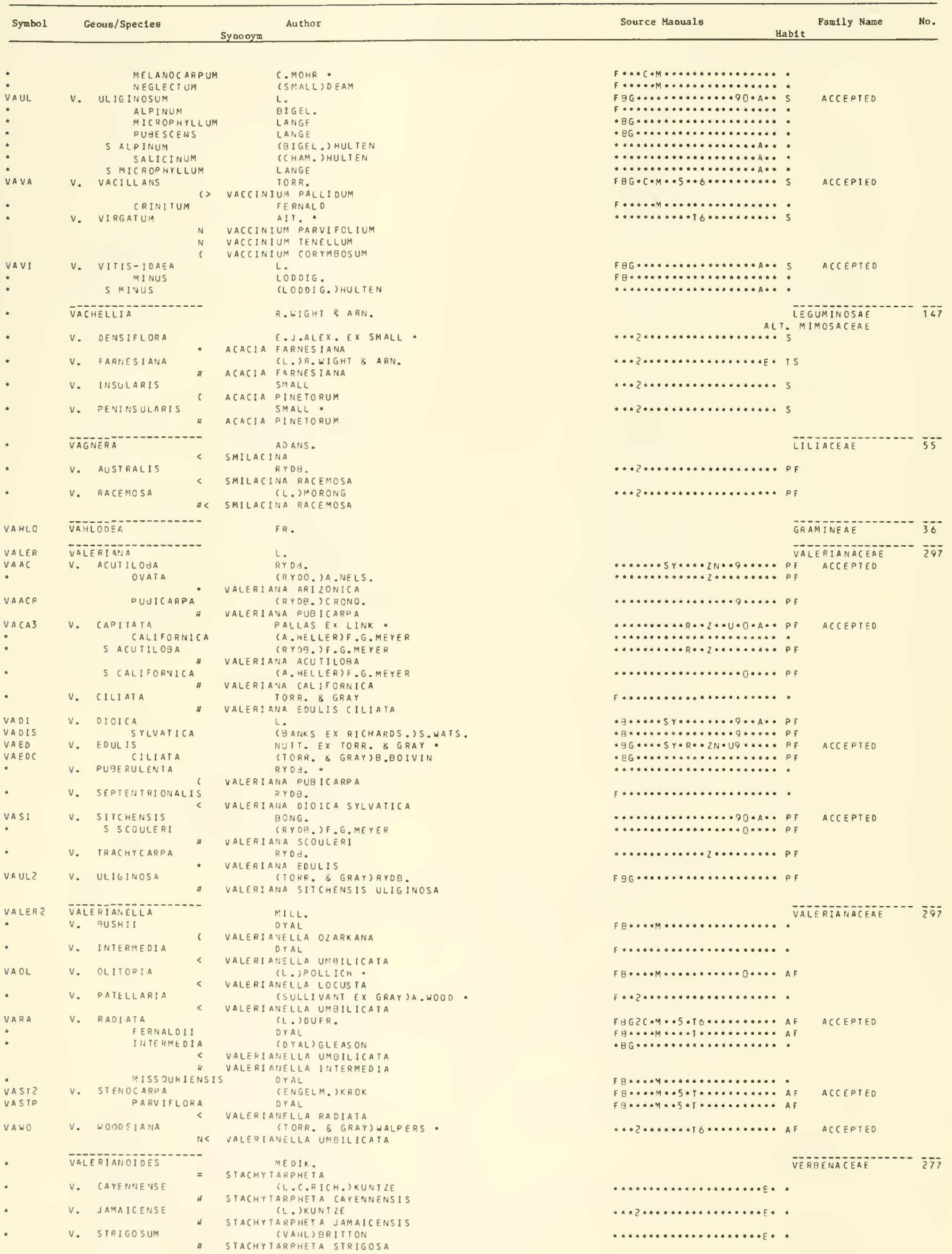




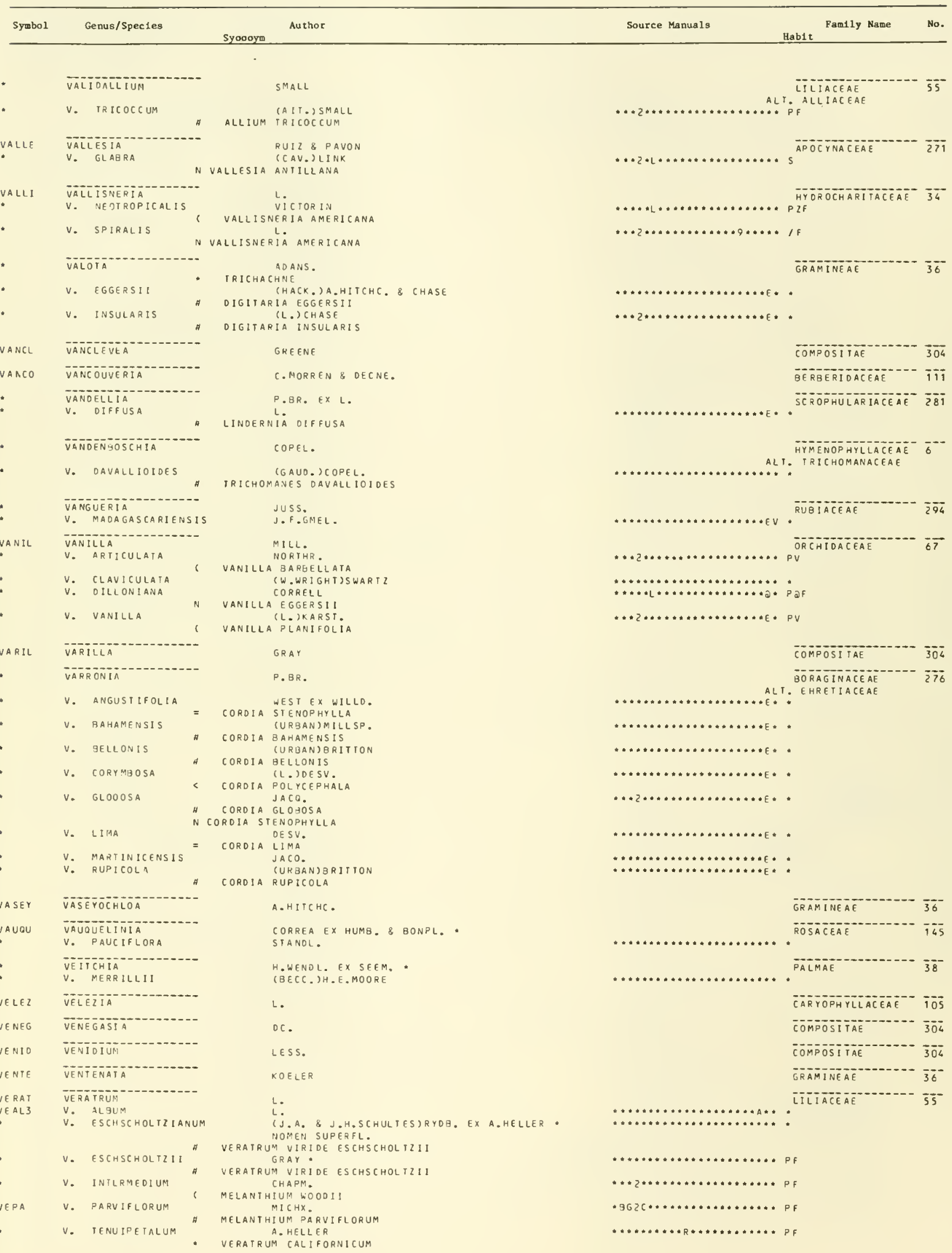




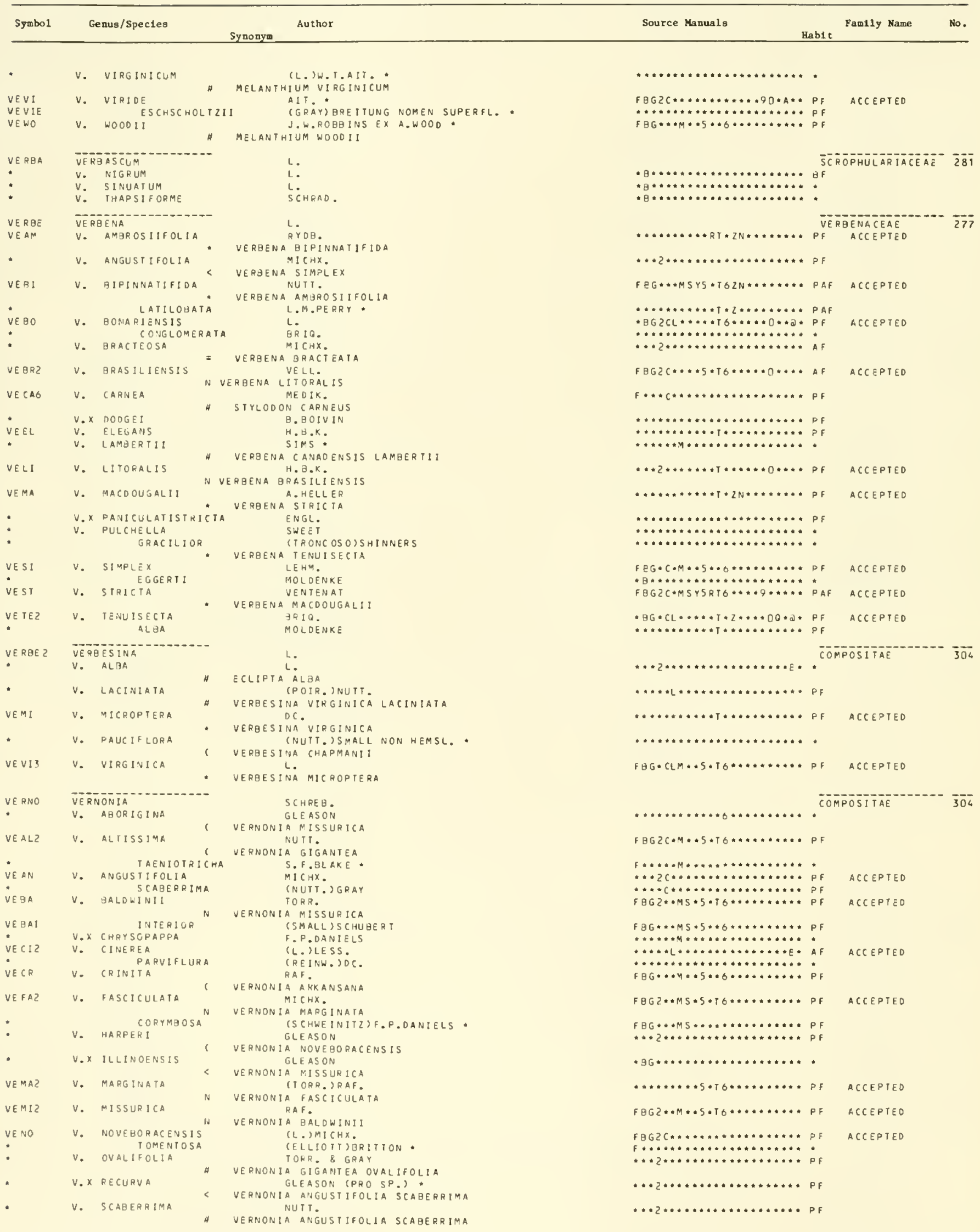




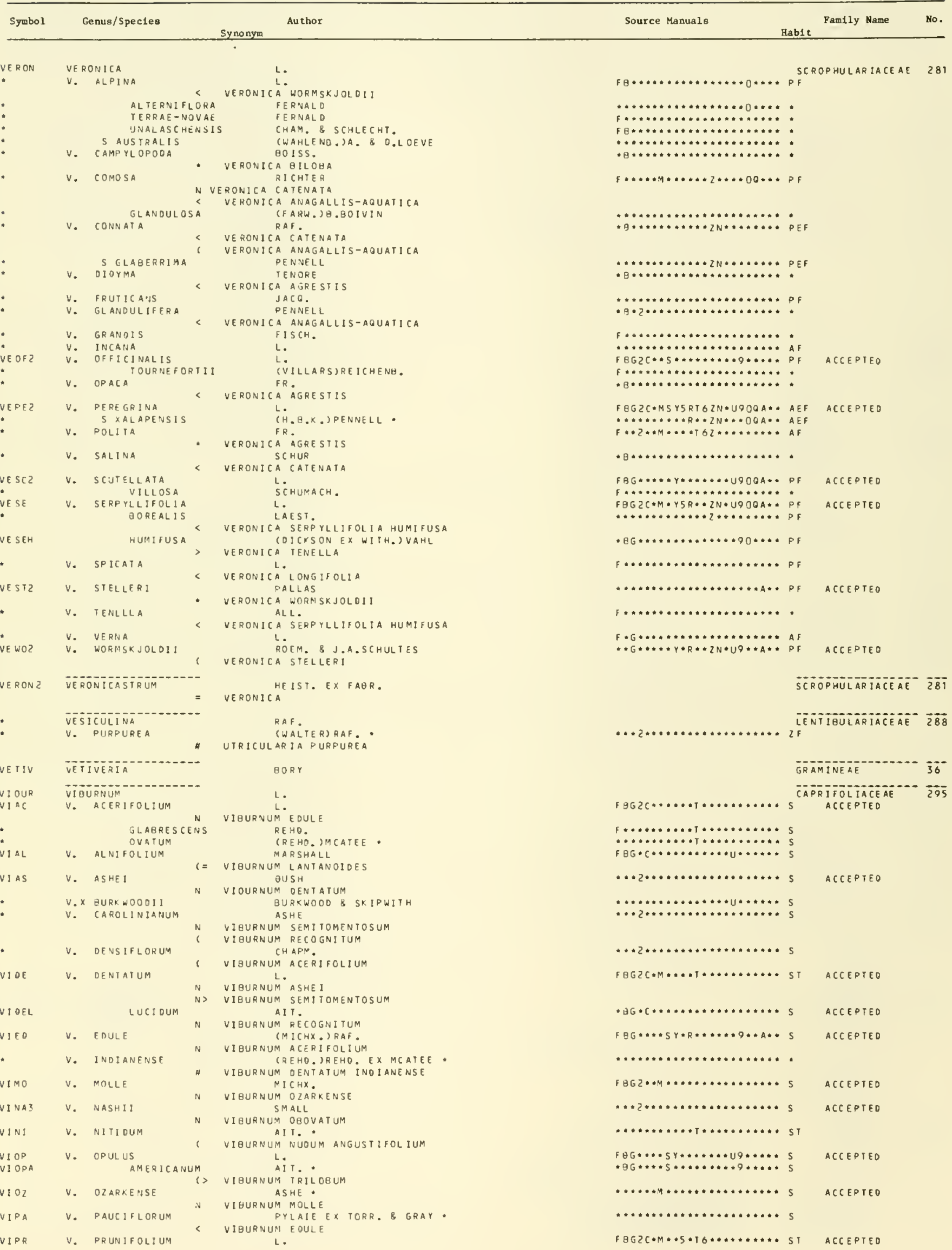




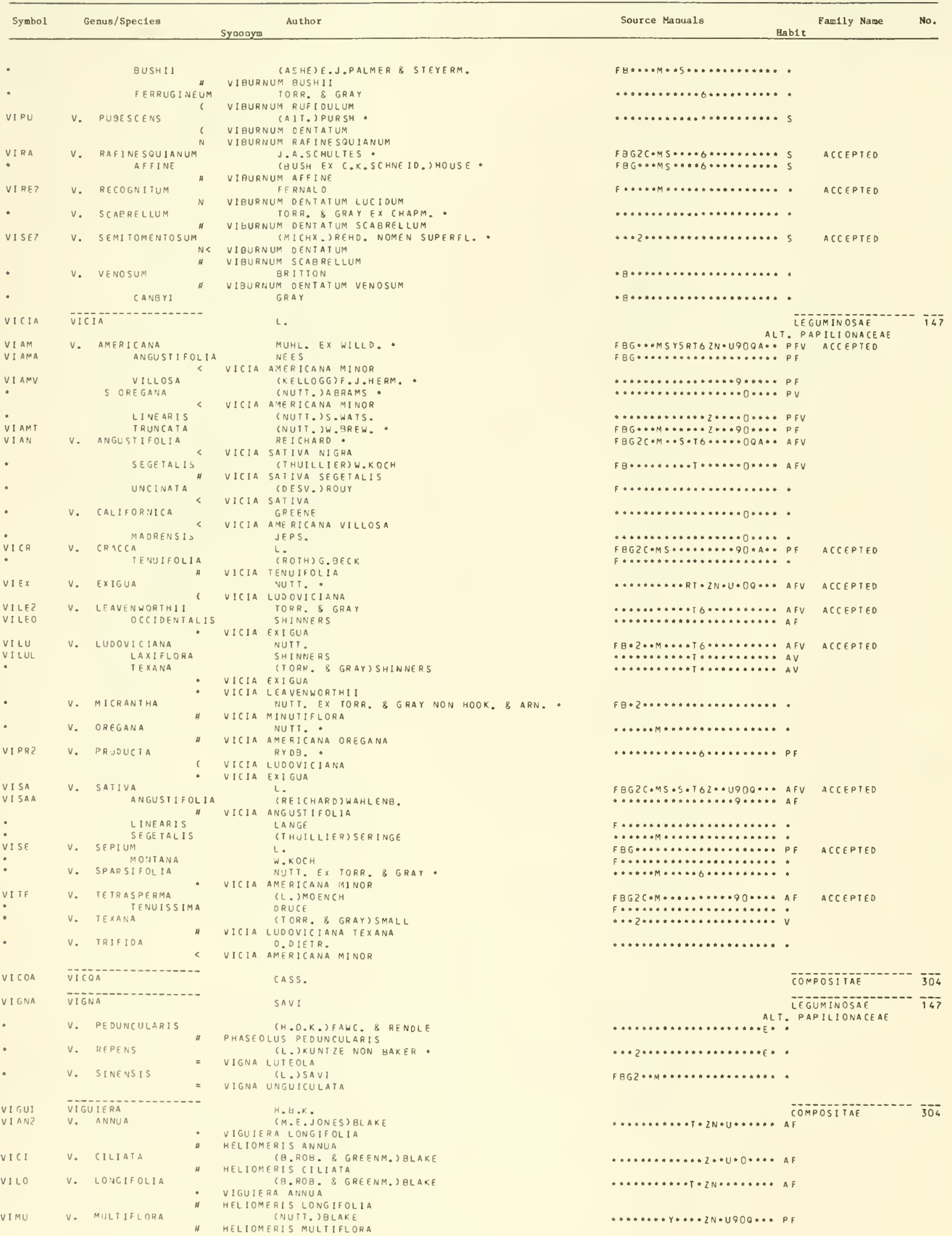




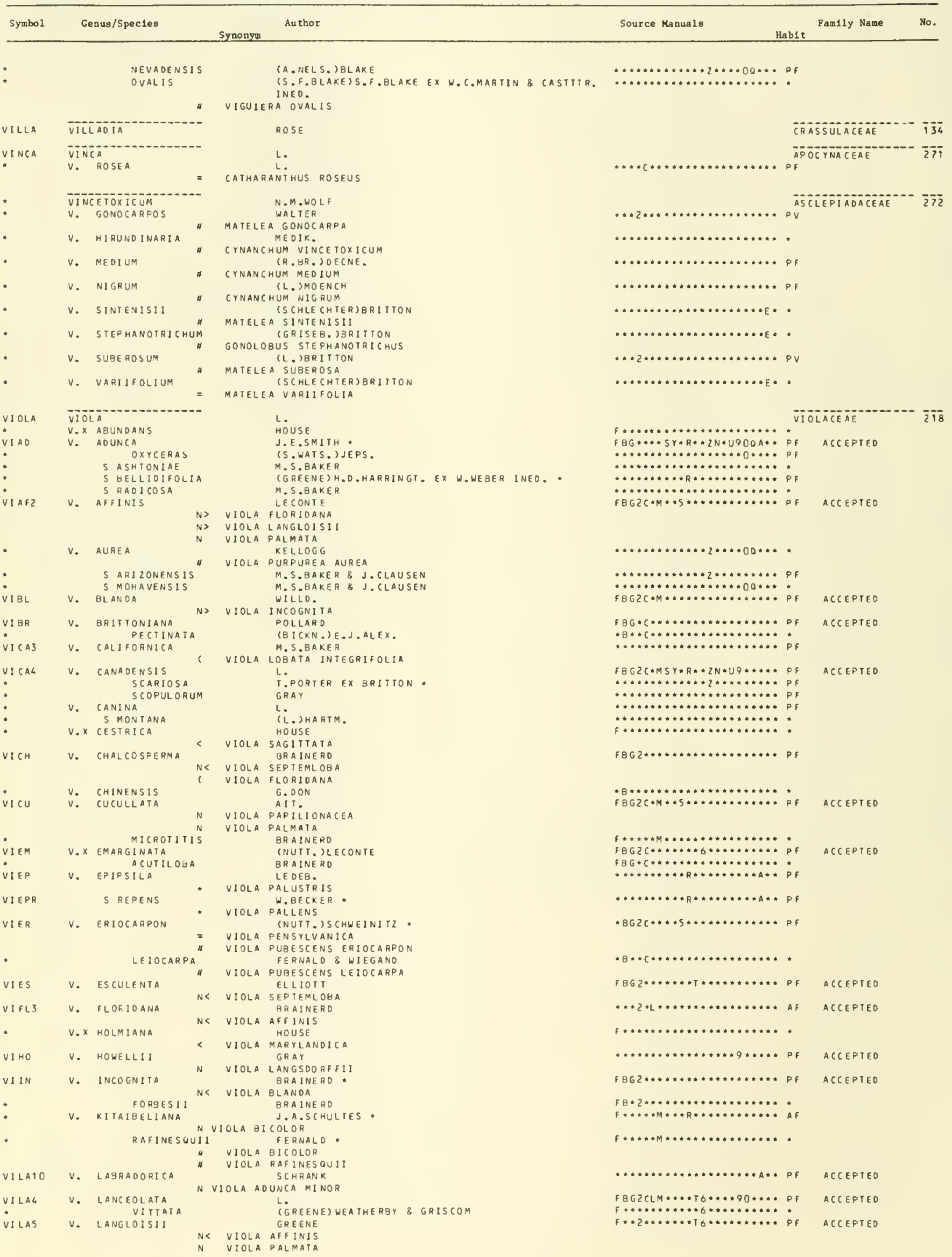




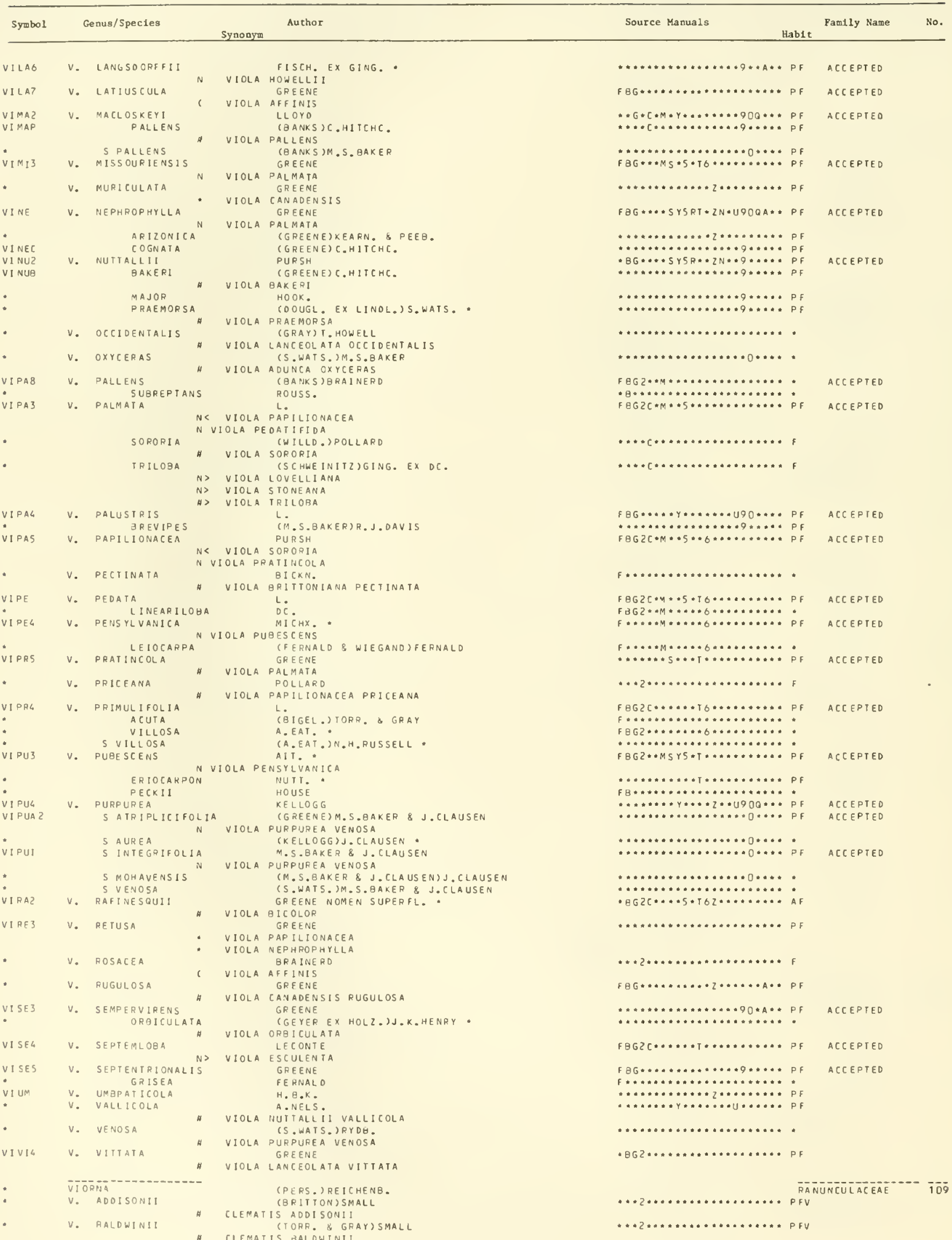




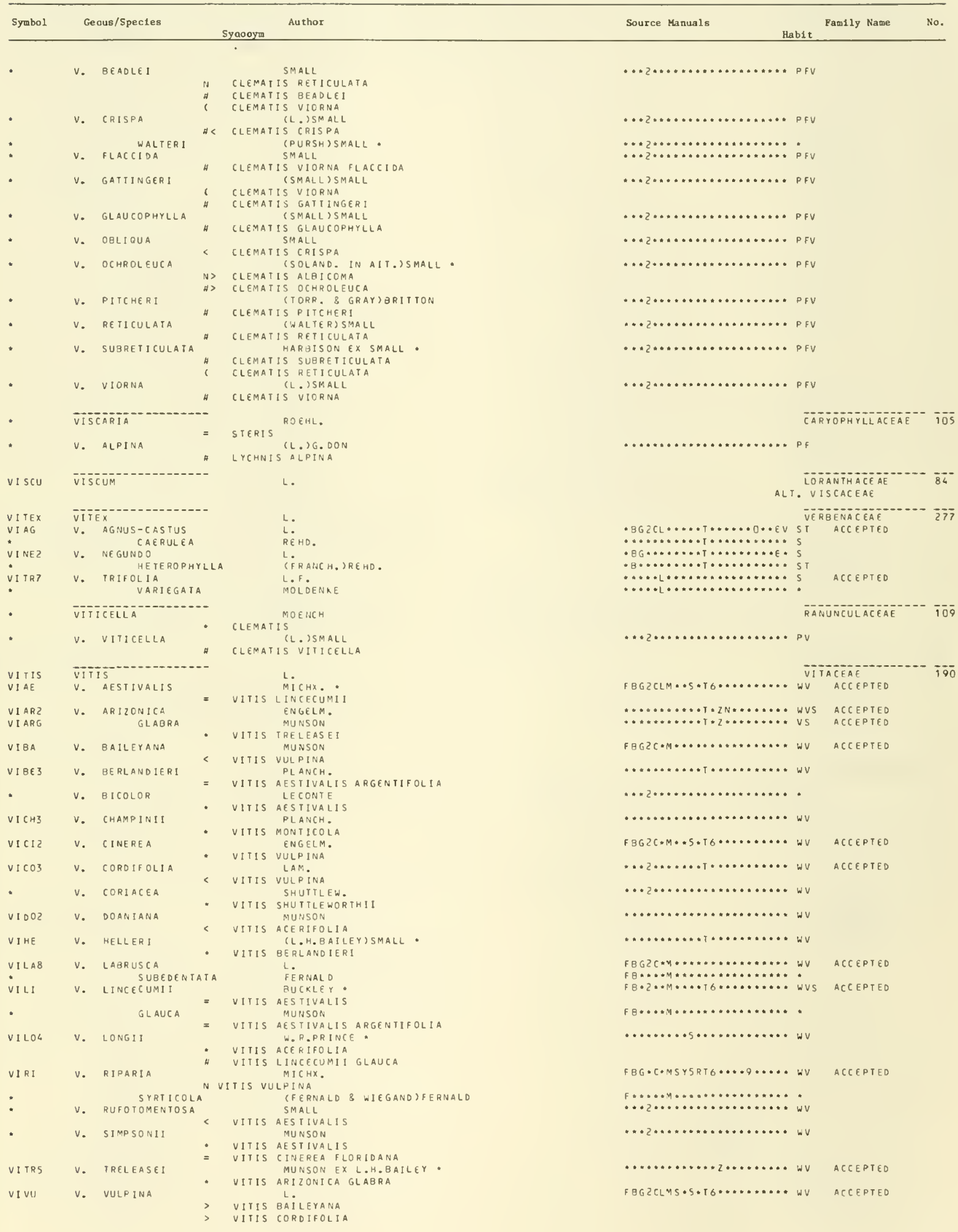


VITTARIA

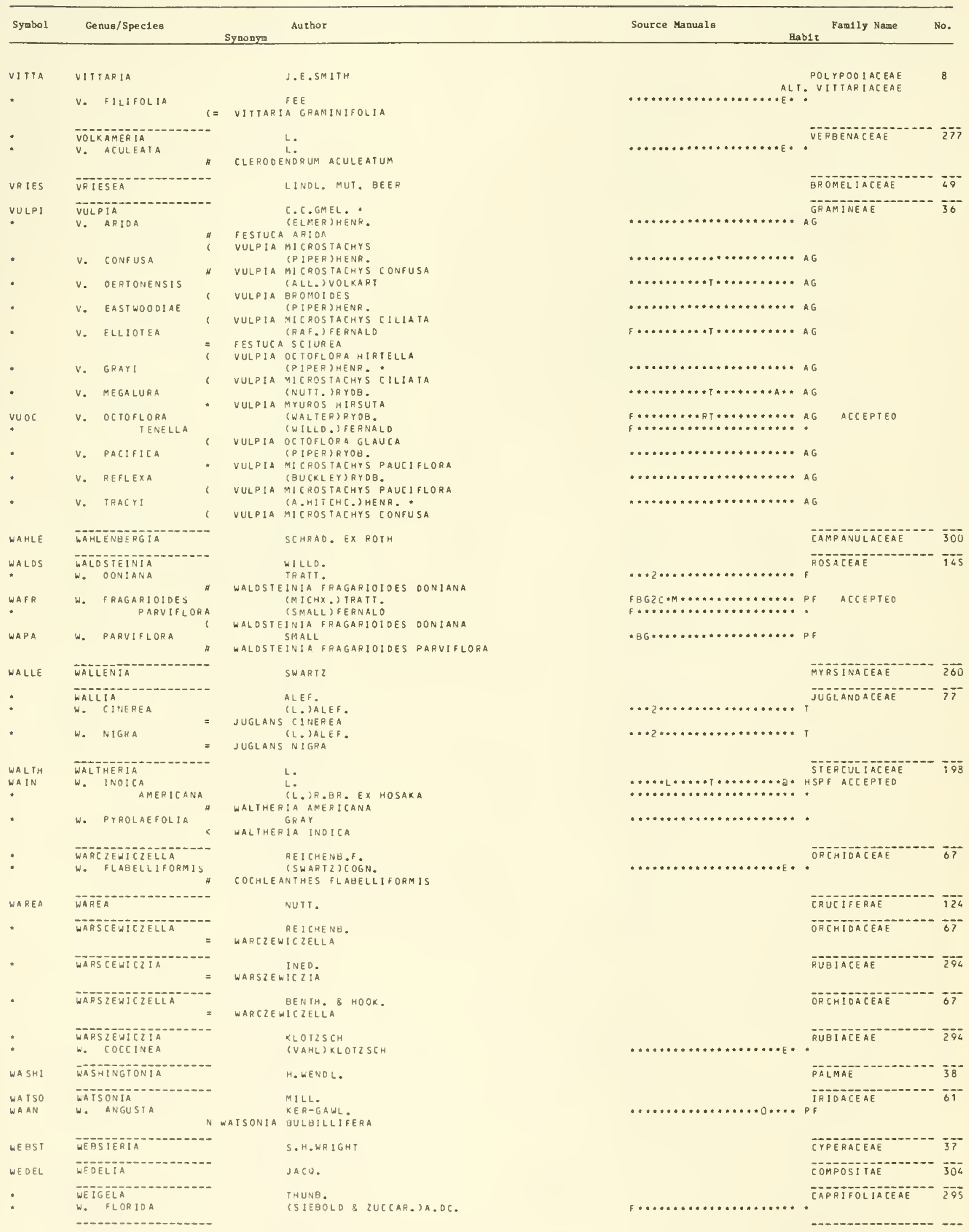


WE IGEL I A

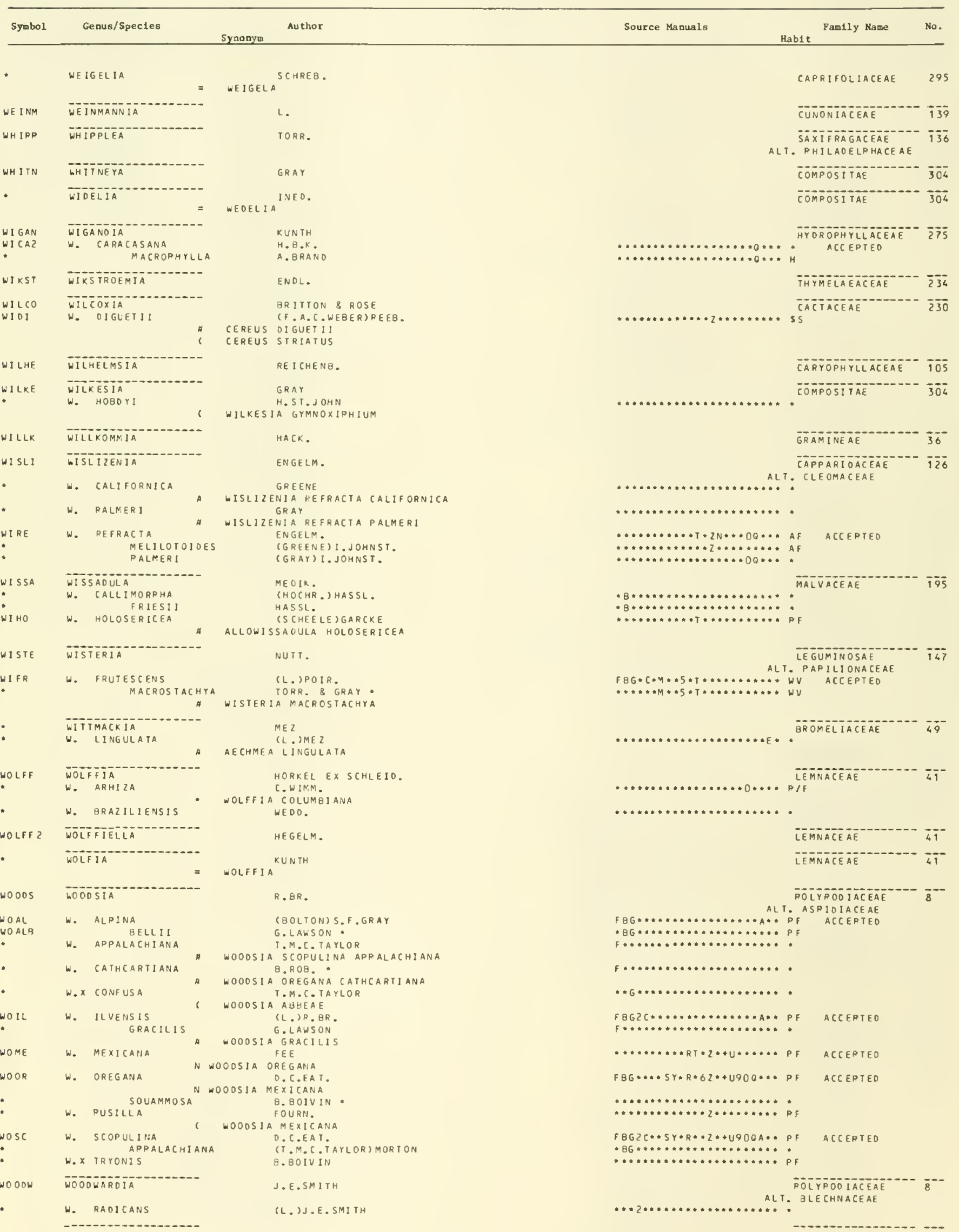




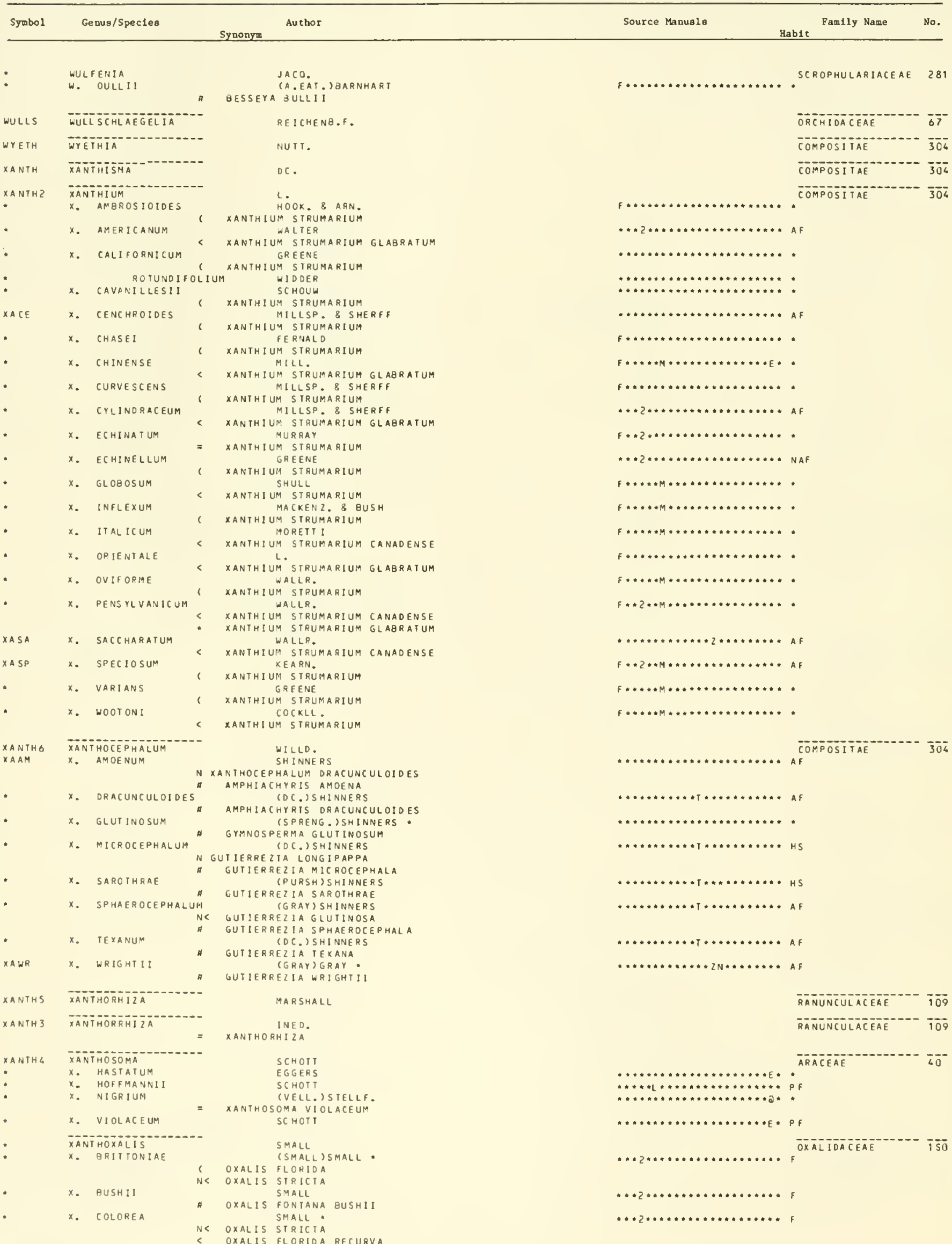




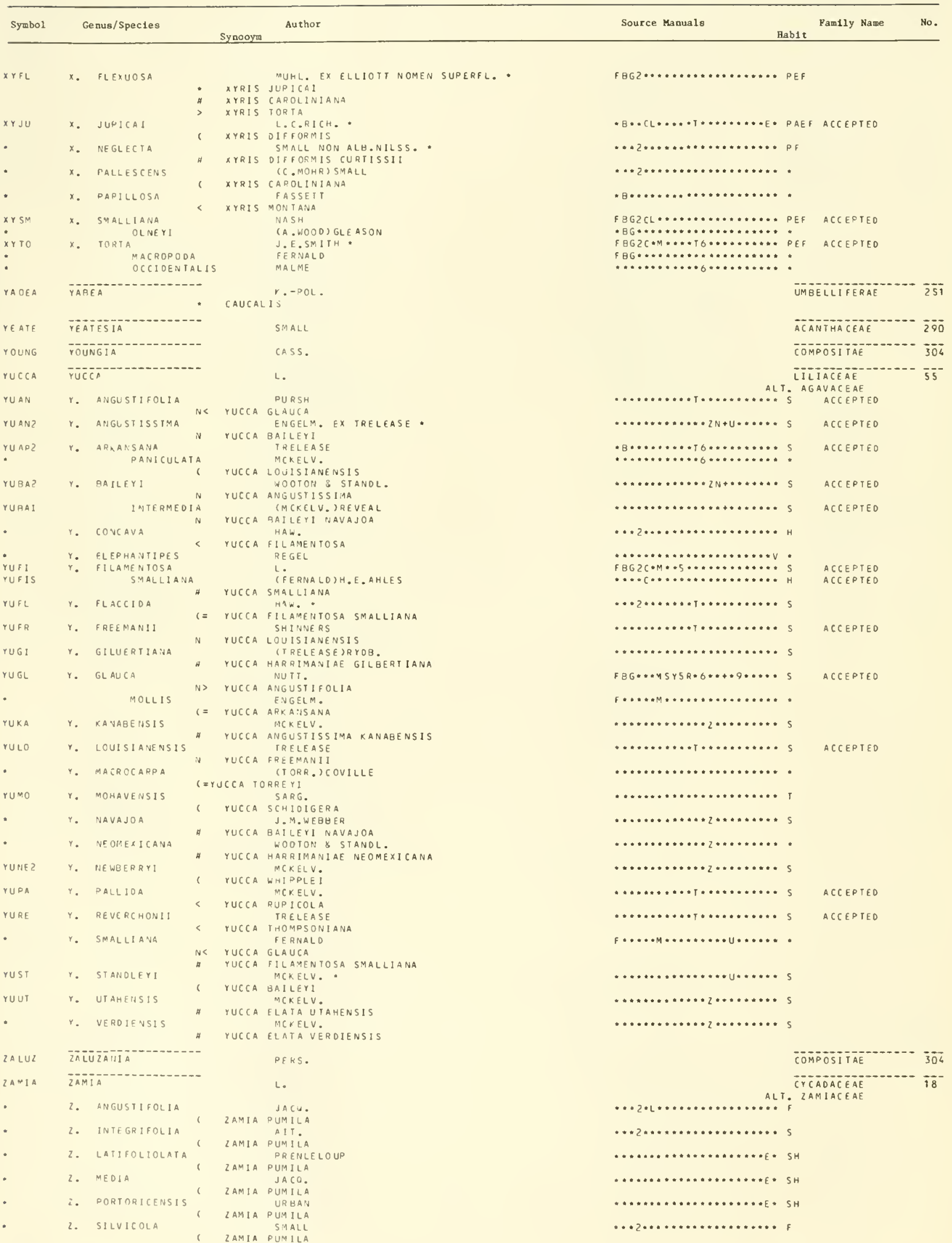




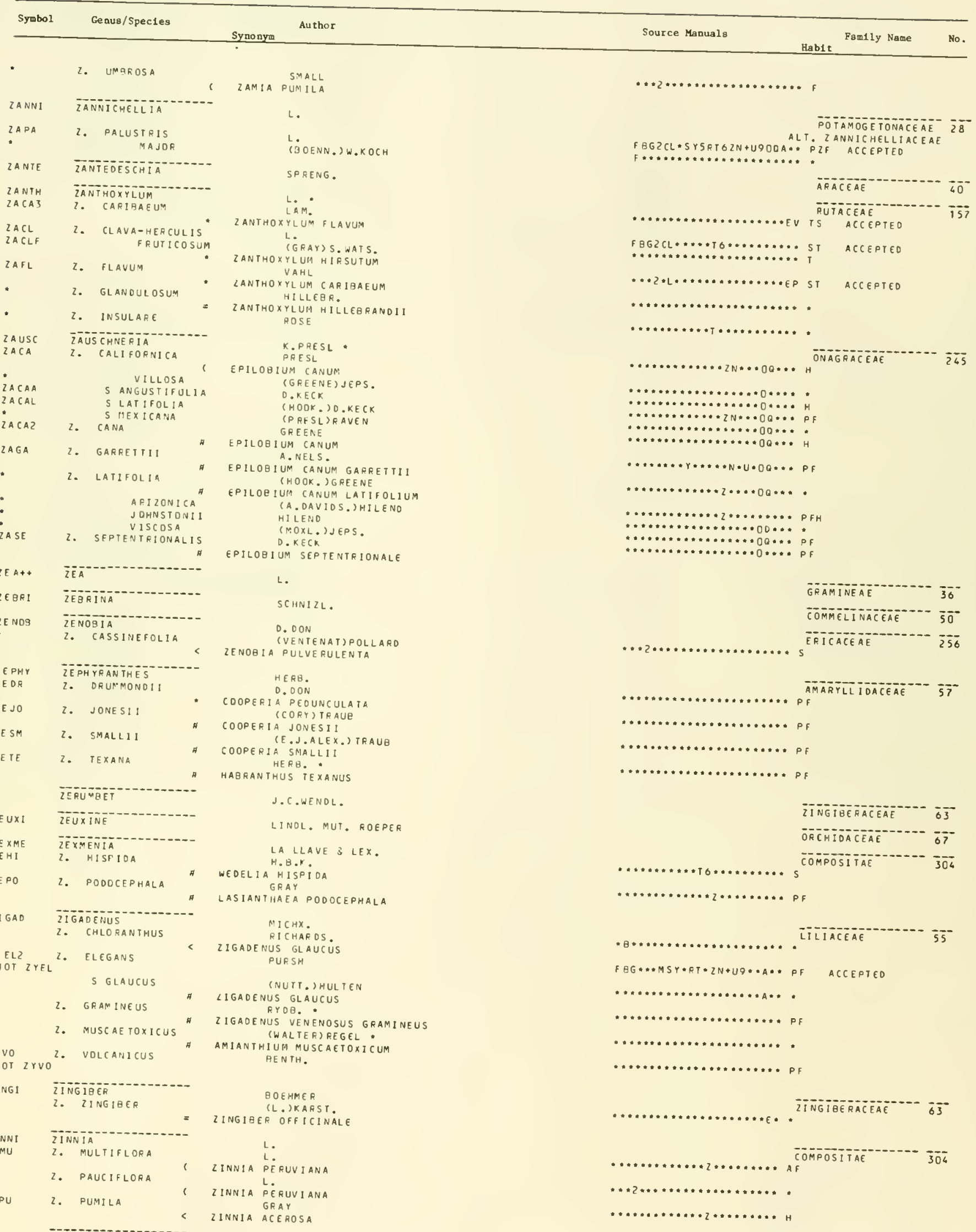




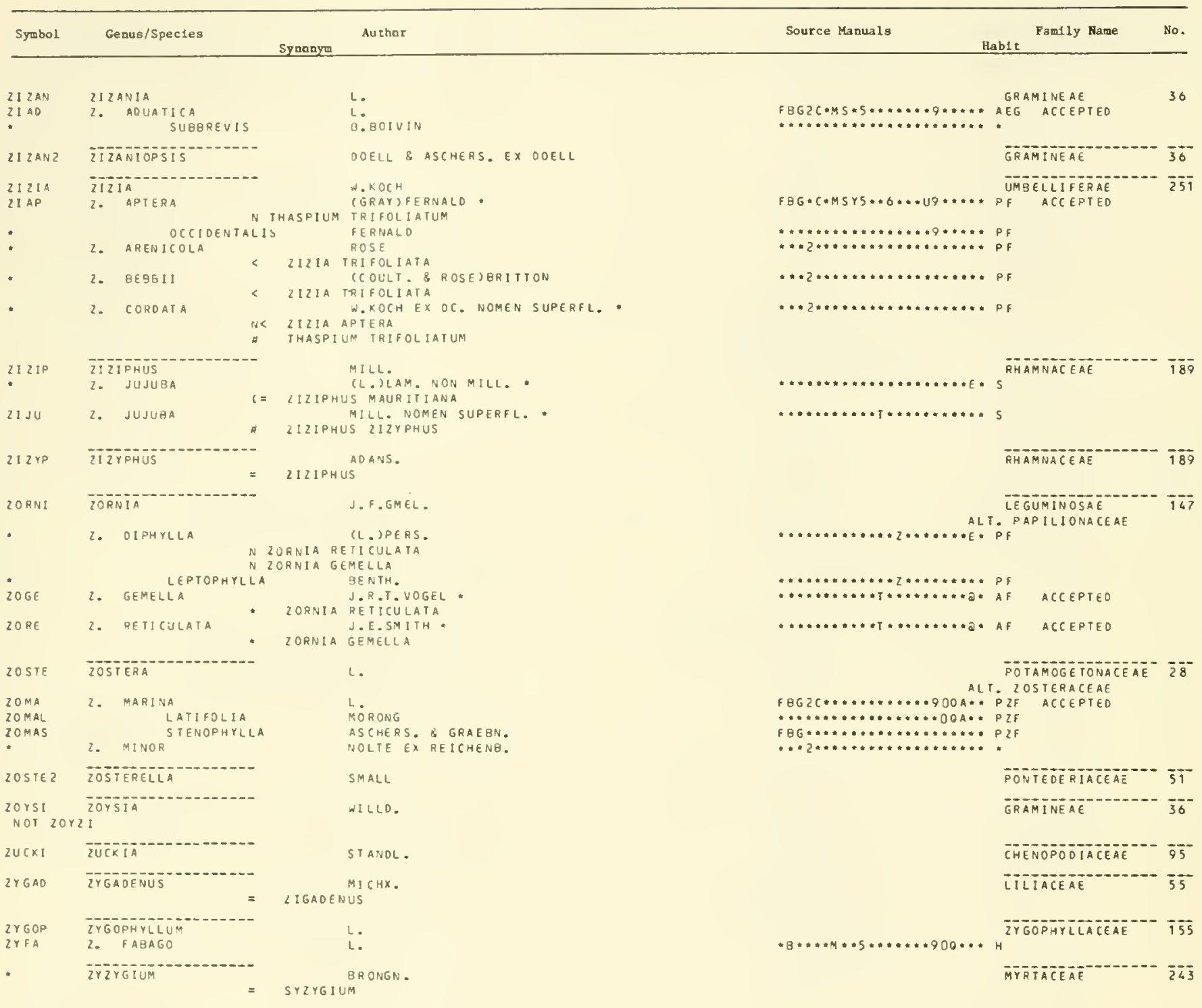




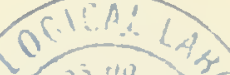

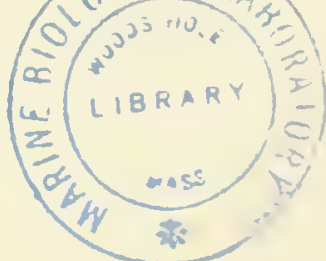


